\title{
Engineering Conference
}

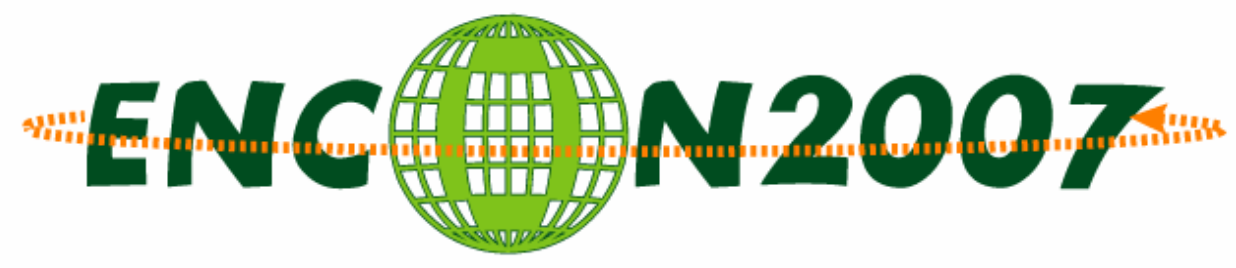

Proceedings of the $1^{\text {st }}$ Engineering Conference: Energy and Environment (EnCon 2007)

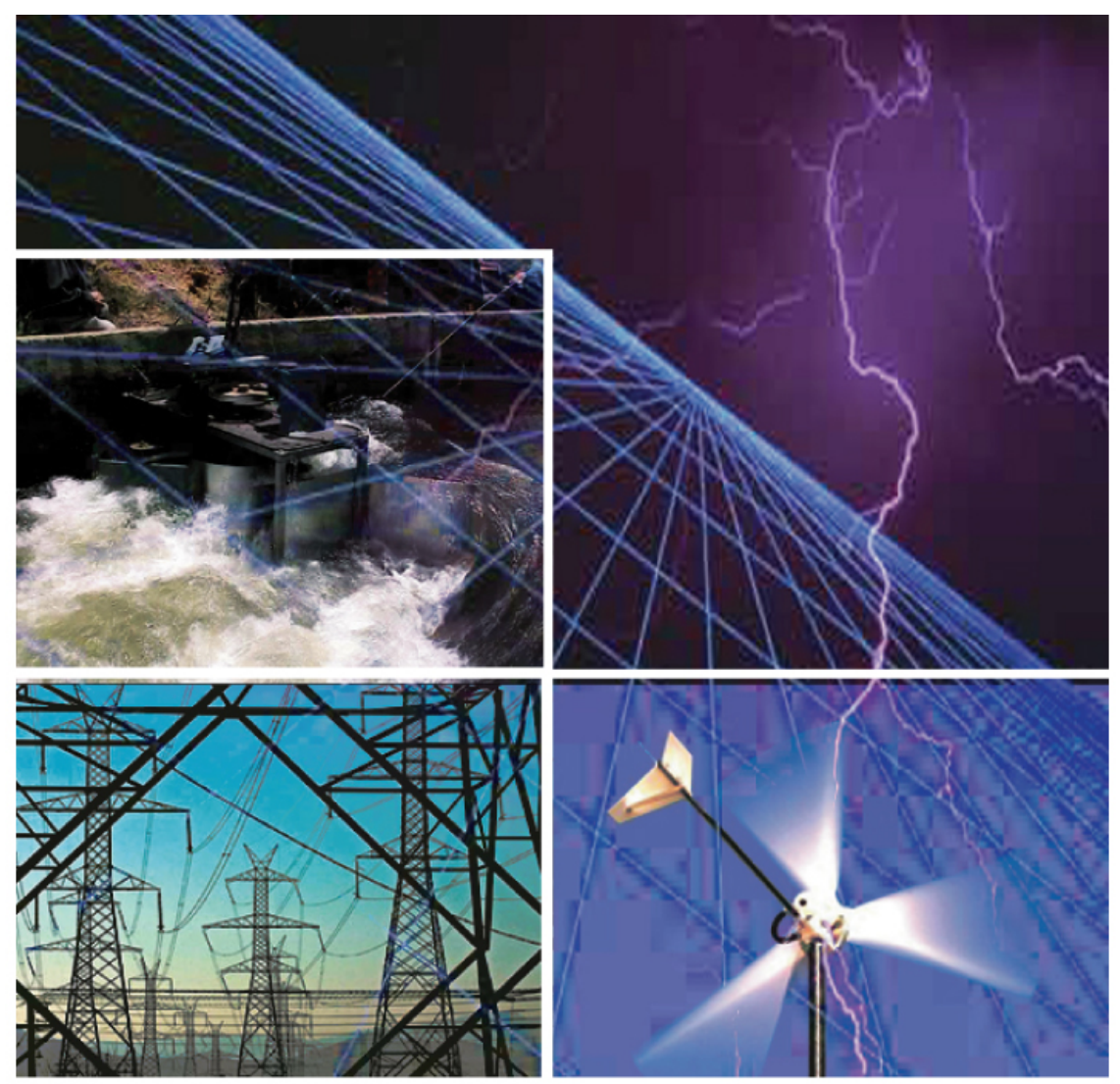

$27^{\text {th }}-28^{\text {th }}$ December 2007

Kuching, Sarawak, Malaysia

Editors:

Chee Khoon Ng

Mohammad Omar Abdullah

Andrew Ragai Henry Rigit

Mohammad Shahril Osman

Abu Saleh Ahmed

Mahbub Hasan

Puong Ling Law 


\title{
Organising Committee
}

\author{
Advisor \\ Prof. Khairuddin Ab Hamid \\ Co-Advisor \\ Dr Azhaili Baharun \\ Chairperson \\ Assoc. Prof. Dr Ng Chee Khoon \\ Co-Chairpersons \\ Assoc. Prof. Ir. Dr Law Puong Ling \\ Ir. Dr Andrew Ragai Henry Rigit \\ Secretary \\ Dr Siti Noor Linda Taib \\ Assistant Secretary \\ Mdm. Rosmina Ahmad Bustami \\ Treasurer \\ Mr. Norhuzaimin Julai \\ Assistant Treasurer \\ Mdm. Dyg Azra Awg Mat \\ Chairperson of Technical Committee \\ Dr Mohammad Omar Abdullah
}

Chairperson of Venue and Publicity Committee

Assoc. Prof. Dr Sinin Hamdan

\section{Chairperson of Programme and Protocol Committee}

Dr Mohammad Shahril Osman

\section{Chairperson of Logistics Committee}

Mr. Abu Bakar Ibrahim

Committee Members

Dr Abu Saleh Ahmed

Dr Mahbub Hasan

Ron Aldrino Chan Buking

Supporting and Technical Staff of the Faculty of Engineering 


\title{
Proceedings
}

\section{$1^{\text {st }}$ Engineering Conference: Energy and Environment (EnCon 2007)}

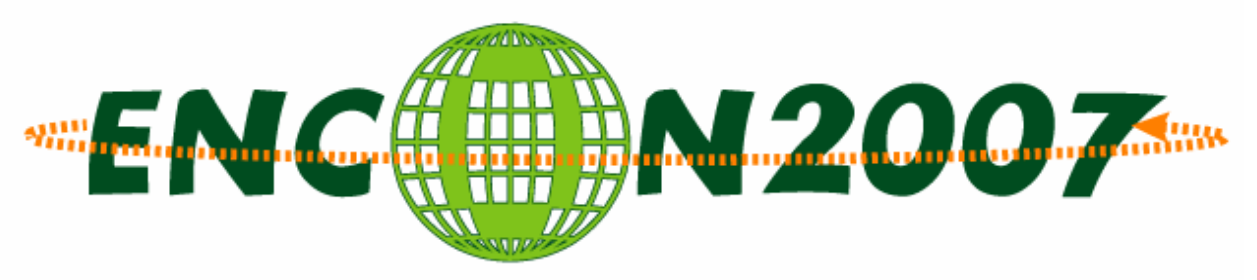

\author{
$27^{\text {th }}-28^{\text {th }}$ December 2007 \\ Kuching, Sarawak, Malaysia
}

Edited by:

Chee Khoon Ng

Mohammad Omar Abdullah

Andrew Ragai Henry Rigit

Mohammad Shahril Osman

Abu Saleh Ahmed

Mahbub Hasan

Puong Ling Law 
Orders:

Faculty of Engineering

Universiti Malaysia Sarawak

94300 Kota Samarahan

Sarawak

Malaysia

Email: ckng@feng.unimas.my

Proceedings: Engineering Conference

Edited by: Chee Khoon Ng, Mohammad Omar Abdullah, Andrew Ragai Henry Rigit, Mohammad Shahril Osman, Abu Saleh Ahmed, Mahbub Hasan, Puong Ling Law

ISBN: 983-9257-34-XXXXX

All rights reserved (C) 2007. No part of these proceedings shall be reproduced, stored in a retrieval system, or transmitted in any form or by any means, electronic, mechanical, photocopying, recording or otherwise, without prior permission of the copyright holder.

No responsibility is assumed by the publisher for any injury and/or damage to persons or property as a matter of products liability, negligence or otherwise, or from any use or operation of any methods, product, instruction or ideas contained in the material herein.

Cover design and printing: DIMENSION ADVERTISING AND DESIGN HOUSE

PRINTED IN KUCHING, SARAWAK, MALAYSIA 


\title{
Powering Sarawak into the Future - The Way Forward
}

\author{
Tan Sri Datuk Amar Abdul Aziz bin Dato Hj. Husain
}

\section{THE FUTURE - AN OUTLOOK OF THE ELECTRICITY DEMAND AND SUPPLY SCENARIO IN SARAWAK AND MALAYSIA}

According to a recent report of the Jawatan Perancangan Perlaksanaan Pembekalan Elektrik dan Tarif (JPPPET), a steering committee on energy planning in Malaysia coordinated by the Kementerian Tenaga, Air, dan Telekomunikasi (KTAK), the power and energy demand in Malaysia will grow at a rapid rate for the next fifteen years or so. In Peninsular Malaysia alone, the peak power and energy demand growth is expected to be between 3.5-5\% per annum, from the present 13.6 GW and 79.4 TWh to about 23GW and 135 TWh in year 2020. In Sarawak, the forecasted demand growth rate is even more impressive where the peak power and energy demand for the same period is $5-6 \%$ per annum, from $800 \mathrm{MW}$ and 5.1 TWh to 1.4GW and 9.1TWh. Yet these forecasts are conservative considering only the organic growth aspect of the electricity demand in Malaysia and excludes the large industries expected to become key consumers in the energy-sector here. As you are aware, the Federal government had recently identified several regions in Peninsular Malaysia, for example the Iskandar Development Region, and targeted them as strategic development areas to gain competitive edge over our neighbors. The full development of these regions will inevitably require large quantum of power to fuel the economic activities within them. Similarly, the Sarawak government had also recently enunciated a plan to develop the central region of the state stretching between Similajau near Bintulu and Tg.Manis near Sarikei, into an energy-intensive industrial corridor. It is envisaged that within five years after the industrial corridor is officially launched, expected to be sometime very soon, the power requirement within the corridor will be at least 1500MW.

While the aggressive pace of industrial development has exacerbated the increase in electricity demand in Malaysia, on the other hand the supply side is struggling to catch up with the demand. At just the organic growth rate mentioned earlier, there is a need to plant up 700MW and 50MW of new generation capacity year on year in Peninsular Malaysia and Sarawak, respectively. The new generation capacity requirements are even more when the bulk

Tan Sri Datuk Amar Abdul Aziz bin Dato Hj. Husain is the Group Managing Director of Sarawak Energy Berhad, Wisma SESCO, Petra Jaya, 93673 Kuching, Sarawak. electricity demand is considered. For example, in Sarawak the demand is expected to reach $7000 \mathrm{MW}$ by 2020 , taking into consideration the requirements of the energy-intensive industries, and export to Peninsular Malaysia and neighboring countries. To make matters worse, the existing indigenous fuel resources used for power generation in the country are rapidly dwindling. This adverse situation is particularly true for natural gas, which according to some optimistic estimates will only last for a maximum of thirty years at the current production rate and proven reserves. Yet, the current power generation in Malaysia is heavily dependent on natural gas - about $67 \%$ and $60 \%$ of the generation capacity in Peninsular Malaysia and Sarawak, respectively, are based on open-cycle gas turbine or combined-cycle plants, which consumes nearly $61 \%$ or 1333 mmscfd of the domestic gas production. Further, the escalating price of crude oil to a current USD93/barrel from a mere USD23/barrel in 2003, and possibly edging towards a US100/barrel in the near term, will intensify the shift to natural gas usage and the increased domestic and external demand will inevitably make it very competitive to secure supply for power generation. This has also strengthened the entrepreneurial lobby to conserve the gas reserves for international export markets where it derives a higher economic value than that from domestic consumption, thereby making its availability for domestic power generation even less. Coal is also a major fuel for power generation in the country, second only to natural gas. In Peninsula Malaysia and Sarawak, coal-fired generation constitutes about $22 \%$ of the generation mix. With the depleting gas supply in Peninsula Malaysia, coal based power generation will likely increase its stake in the generation mix unless other energy resources such as the hydropower in Sarawak or nuclear energy are extensively exploited. In Sarawak, the coal resources are indigenous but much of the coal supply in Peninsula Malaysia is imported from external sources. This dependence of foreign sources has raised national concern about the long-term impact of escalating or volatile international market coal price on electricity costs in the country and more importantly, concern about the fuel supply security vis a vis national security.

Even while the future outlook of the supply and demand situation in Malaysia doesn't appear to be optimistic, this is not all the challenges that the power industry is expected to encounter. The international community is now very worried about global warming due to the greenhouse effect. 
This grave concern will consequently lead to increasing legislation and enforcement of more stringent environmental protection standards. In the context of power generation, sophisticated gaseous emission control and clean fuel technologies will have to be used in power plants to comply with the requirements, which will inevitably result in higher energy production costs due to additional capital investment on environmental protection equipment. With rising energy production costs due to escalating fuel and higher capital investment costs while electricity tariffs are capped by regulatory control, independent generators and power utility companies will be increasingly challenged to increase organizational efficiency and productivity to maintain profitability and stay competitive. There is also an increasing entrepreneurial lobby and political will to unbundle the local electricity supply industry in the belief that consumers will have more choices of electricity suppliers which will consequently introduce a freely competitive electricity market that ultimately dictates efficient electricity pricing. While such objective has been achieved by certain electricity market models in developed countries, deregulation has to be introduced with caution in developing regions such as Sarawak, since its power infrastructure is still rudimentary and there is still limited access to electricity supply in rural areas which will likely be perpetuated by a competitive electricity market model that rules out the unfavorable economics of electricity supply to geographically remote areas. Yet every corporate citizen in Sarawak whose economic activity has an impact on the socio-economic development of the rural areas must accept its measure of social responsibility to contribute. Thus balancing between this responsibility and business interests will be a challenge in a competitive electricity business environment. Meanwhile, electricity consumers are also clamoring for higher reliability and quality in the electricity supply. For example, in the Samajaya free-trade industrial zone in Kuching there is high expectation and requirement by the electronics and semi-conductor industries within for such power standards. In the near future when even more investors that are currently being aggressively wooed by the State administration set up hightech manufacturing facilities and factories in the State, the requirement for highly reliable and quality electricity supply will be even greater. In a utility power supply environment where the typical industry standards on power reliability and quality are met for the majority of consumers, there will be a significant cost impact to provide a custom-grade electricity supply for such specialized consumers. Thus, maintaining costs while tightening on the technical performance standards will be another major challenge that the local power industry has to contend with.

These aforementioned challenges may seem daunting but how the local power industry fares in the future will depend on how successful it can tackle them. Our vision of the future must then be focused on embracing these challenges and what needs to be done to surmount them should shape our developmental objectives and strategic plans for the long term. I believe Sarawak possesses vast indigenous energy resources that can be tapped to meet the electricity supply and demand challenges, the human resources with the potential and capacity to be highly skilled, to learn, innovate, and develop to meet the technical challenges. Above all, in the face of challenges, our people and nation have always exhibited our finest innate human quality - the resolve, determination, and resilience to overcome and succeed.

In the remaining of this address, we will take stock of the various indigenous energy resources that Sarawak possesses and how we can consolidate and utilize these to meet the supply and demand challenges. We will also outline the key developmental policies and strategic plans that are needed to power and propel us forward to tackle these challenges. We will then conclude by discussing briefly the tremendous benefits that our people and country can gain from these activities.

\section{TAKING STOCK AND CONSOLIDATION - INDIGENOUS RESOURCES AND POWER INFRASTRUCTURE NEEDS TO MEET THE POWER DEMAND \& SUPPLY}

Sarawak is blessed with abundant natural resources such as oil, gas, coal, hydro, and renewable sources. Apart from gas, which has been developed in Sarawak since 1992 as a major fuel for power generation due to its low economic cost, the other resources have largely been unexploited for power generation. However, with the role of gas for power generation expected to diminish in years to come due to increasing competition for secure supply of the depleting resource, coal and hydro resources in Sarawak will feature prominently in the power generation development in Sarawak and Malaysia as a whole. In fact, the hydroelectric potential in Sarawak may be the only feasible, economic, and sustainable fuel option left to harness for power generation to sustain the rapidly developing energyintensive industries in the long term.

Hydroelectric potential is the largest indigenous renewable energy resource in Malaysia. The total amount of technically exploitable potential in Malaysia is 123 TWh per year. About $70 \%$ of this lies in Sarawak, attributed to its abundant rainfall and topography characterized by numerous rivers flowing between steep, narrow, interconnected ridges of up to 1,200 metres high. In a detailed survey on hydropower potential carried out in the early 1980's under a technical assistance program offered by the German government, a total of 51 hydro projects with an exploitable potential of $20 \mathrm{GW}$ and annual energy output of $87 \mathrm{TWh}$ were identified. The power from these projects, which are concentrated in the interior of Sarawak, constitutes the major portion of the unexploited hydropower resources in Malaysia.

Among the 51 sites, the Bakun and Murum hydroelectric projects with respective generating capacity of $2400 \mathrm{MW}$ and 1000MW are two of the large projects for which feasibility studies have confirmed their technical and economic viabilities. As you may be aware, the Bakun hydroelectric project is currently being constructed and is expected to be completed by early 2010. About 2000MW of the power generated from the Bakun hydro plant will be exported to Peninsular Malaysia by High Voltage Direct Current transmission by 2015. In Sarawak, an aluminium smelter located in Balingian near to Mukah will begin production in 2009 with a modest initial power consumption of $90 \mathrm{MW}$ but the demand is expected to increase to 630MW by 2011 and ultimately $1100 \mathrm{MW}$ by 2020 . Another large aluminium smelter located in Similajau near to Bintulu is also expected to begin production by late 2010, requiring 
about 900MW by 2011. With this anticipated increase in the bulk power demand, the supply from the Bakun hydro plant alone will be inadequate and the Murum hydroelectric project needs to be developed. Plans are actively underway to develop and implement the project in order to complete it by late 2013. In fact, the combined firm generation capacity from the Bakun and Murum hydro plants is insufficient to meet the total demand by 2015 and thus the Baram hydroelectric project with a 1000MW generation capacity will also have to be developed concurrently to complement them by then. Plans are also currently underway to carry out the necessary feasibility investigation to confirm the technical and economic viability of the Baram hydroelectric project. The State administration is also currently developing an energy-intensive industrial corridor along the coastal belt stretching between Tg. Manis and Similajau. Energy-intensive industries such as paper and pulp production, solar panel manufacturing, and mineral processing such as iron, zinc, nickel, and silicon smelting are expected to be set up within this region. Beyond 2015, there are also further prospects to export additional 10002000MW to Peninsular Malaysia in view of the expected crunch on gas supply and uncertainty of coal price by then. There is also a lucrative market to export power to neighboring regions such as $100 \mathrm{MW}$ to Sabah, 150MW to West Kalimantan, and 100MW to Brunei by 2013. With such massive growth in the bulk power demand, it will be necessary to develop further hydroelectric projects, notably those in the Upper and Lower Rejang basin such as Baleh, Pelagus, Metjawa, Linau, Belepeh, Balui, just to name a few with a total combined generation capacity of about 3000MW so that by 2020 the total installed generation capacity in Sarawak is expected to exceed 10,000MW.

Coal is also abundantly found in Sarawak with an estimated 500 millions tones of inferred reserve. The three major coalfields in Sarawak are the Silantek, Merit-Pila, and the Mukah-Balingian formation. Presently only about 0.9 million tones of coal are consumed annually for power generation by the existing 200MW Sejingkat coal-fired power plant in Kuching. With the 270MW Matadeng coalfired power plant in Mukah expected to be in operation by 2009, the total coal consumption in the State for power generation will then increase to about 2.1 million tones annually. This is just a small fraction of the available coal reserve in Sarawak and thus there is still a lot of potential to harness our coal resources for power generation. Between 1984-1987, the Geological Survey Malaysia carried out an exploration and evaluation exercise in the Tebulan and Merit block in the Merit Pila coalfield near to Nanga Merit by the Upper Rejang river, as part of an effort to map and develop an inventory of the coal reserves in Sarawak. The investigation established a total reserve of about 150 million tones of which about 130 million tones are measured or indicated reserve. Contingent on detailed technical and economic feasibility studies, this amount of reserve will potentially be able to sustain a mine-mouth coal-fired power plant of 1200MW generating capacity for an economic lifespan of 25 years. Thus there is also a plan to construct a 4x300MW coal-fired power plant in the Nanga Merit area to be part of the overall generation development strategy to meet the anticipated bulk demand growth by 2015 and which will also balance the then predominantly hydro generation mix which is inherently susceptible to unexpected power output shortfall due to the vagaries of rainfall and hydrology.

Biomass and solar energy in particular are also emerging as renewal fuel resources for our generation development in line with the Federal Government's fuel diversification policy formulated in the $8^{\text {th }}$ Malaysia Plan to utilize all types of renewable resources including biomass, biogas, municipal waste, solar, mini-hydro and wind as the fifth fuel resource for small renewable power generation. For Sarawak, a target of $4 \%$ of the total installed generation or about 100MW is planned to be achieved by 2015. The use of biomass as a renewal fuel resource in Sarawak is a logical choice since about $25 \%$ of the 328 palm oil mills in Malaysia are located in Sabah and Sarawak. It is envisaged that the future growth of the palm oil plantations and mills will be largely concentrated in Sarawak and Sabah since large areas of forested land that can be converted to oil palm plantations are still available as compared to the already saturated land usage in Peninsular Malaysia. On the other hand, the utilization of solar energy is deemed a good choice of renewal fuel resource since there is almost constant sunlight the whole year in Sarawak, and the increased usage and demand for solar energy equipment will also spur the development of a local photovoltaic industry such as solar panel manufacturing, polysilicon processing, etc. The photovoltaic business opportunity is tremendous since the world market business potential in grid-connected photovoltaic is forecasted to be RM500 billion by 2015. The current world production capacity is about $5 \mathrm{GW}$ while a 810GW demand is expected by 2010.

In parallel with the generation development strategy that takes account of the fuel resources availability in Sarawak, there is also a need to identify the concomitant transmission infrastructure so as to evacuate and reticulate the generated power efficiently to their off-take locations. For this purpose a main $500 \mathrm{kV}$ transmission system is envisaged to be required in the long term since most of the hydro potential sites are in the far reaches of their river basins and transmission of the bulk power from these sites involve long distances. The transmission infrastructure is expected to be constructed in stages in coordination with the hydropower projects development, so that a $500 \mathrm{kV}$ transmission backbone spanning about $600 \mathrm{~km}$ between Bunut and Kuching will be fully developed by 2020 . The $500 \mathrm{kV}$ backbone will also be located within designated land corridors that are strategically routed to maintain orderly future development of transmission systems in Sarawak as well as achieving maximum transmission efficiency of bulk generation to the load centers. Within the major river basins where all the potential hydro sites have a large quantum of aggregate power output, the $500 \mathrm{kV}$ backbone will branch off to the upper reaches to evacuate the hydropower tapped and marshaled by $275 \mathrm{kV}$ sub-branches. Within other minor river basins where all the potential hydro sites have lesser quantum of aggregate power output, $275 \mathrm{kV}$ sub-branches directly tap and marshal the hydropower and feed it directly by a $275 \mathrm{kV}$ main branch into the main $500 \mathrm{kV}$ backbone.

\section{THE WAy ForWARD - Key STRATEgIES TO MEET THE CHALLENGES}

With an understanding of the power supply and demand challenges, and identifying our available energy resources 
and power infrastructure options to provide the solutions, we must then follow on to define our key developmental objectives and also develop the strategic implementation plans to achieve them.

In a general context, one of our key developmental objectives is to meet the long term power demand growth in Malaysia and the neighboring region, driven by the traditional organic and emerging energy-intensive loads within Sarawak, the export demand to Peninsular Malaysia and neighboring countries. Our strategic implementation plan is to develop the vast hydroelectric potential as the primary and coal as the secondary fuel resource for power generation to derive a reliable, efficient, sustainable, and cost-competitive energy source that will position Sarawak as a premier energy supplier in the region. A second key developmental objective is to comply with the government's policy of utilizing renewal resources in the country for power generation to contribute to environmental preservation. For this our strategic implementation plan is to develop the biomass from oil palm plantations and solar energy for power generation, this being motivated by the rapidly emerging oil palm industry in Sarawak and the potential spin off business opportunity in the photovoltaic industry. A third key developmental objective is to ensure a highly adequate, secure, and quality power supply which are increasingly becoming requisites of potential investment, and which are also crucial to the economy and manufacturing sector as they become more ICT-driven and geared toward higher technology. Our strategic implementation plan to achieve this is to develop in the long term a highly reliable and efficient transmission network that has adequate capacity and security to transport the hydro and coal generation to the load centers. For this purpose, the development of a second $275 \mathrm{kV}$ and a $500 \mathrm{kV}$ transmission grid in the intermediate and long term respectively, is part of this plan.

In a specific context, these key developmental objectives and strategic implementation plans can be quantified by the following:

- To meet the power demand growth of the four major load drivers through a 30-year generation development strategy to install 10,000MW of hydro and coal generation by 2020 and 20,000MW by 2037 .

- To fulfill the government policy on utilization of renewal resources for power generation to contribute to environmental preservation, by targeting to install at least $4 \%$ of the total generation capacity in Sarawak or $100 \mathrm{MW}$ to be based on renewal fuel by 2015 .

- $\quad$ To ensure a highly reliable, quality, and efficient power supply through a 30-year transmission development strategy to construct a second $275 \mathrm{kV}$ transmission grid by 2012 and a $500 \mathrm{kV}$ transmission backbone by 2020 . The use of power-electronics based FACTS equipment at the transmission and distribution level will also be included to achieve the power quality that is required by the specialized consumers.

- $\quad$ To recruit the human resources, train and develop them to acquire the knowledge-base, expertise, skills, and competency that are necessary to take on leading roles in the implementation of the strategic plans.

New institutional changes and structures are also required to ensure efficient implementation of the strategic plans to quickly attain the key developmental objectives. In this respect, the recent corporate restructuring of Sarawak Enterprise Corporation Berhad and the Sarawak Electricity Supply Corporation to become a new single entity Sarawak Energy Berhad - will blend the commercially and technically oriented psyche of the former and latter respectively, into a new organisational culture that is needed to succeed in a competitive, technically demanding, and business-driven environment which is rapidly emerging. Also under the restructuring exercise, the setting up of the engineering services and new business development departments which house the hydropower, renewal energy, and transmission projects divisions, creates the organizational structures and mechanisms to achieve the key developmental objectives.

Human resource training and development in the areas of power engineering, corporate and large projects financing, are also essential to equip ourselves with the prerequisites for the appropriate technology application and the new roles we are expected to play. No longer must we be satisfied to act as apprentice or counterpart to expatriate personnel in our work environment, instead we must quickly learn, develop the capabilities, and become masters in power system planning, engineering design and construction, operations and maintenance, and project management and implementation to take control of and assume key roles in the large-scale power infrastructure development work that we will undertake. Ultimately, we must be able to develop the knowledge-base, expertise, skills, and competency that are recognized and marketable as consultancy and technical services to others beyond our corporate boundary. Along with the development of these capabilities, we must then also evolve the employee career development and upgrading path, and the rewards and incentives system, to be able to attract and more importantly to retain the skills and expertise in order to compete and take on the leading roles in the activities associated with achieving the key objectives.

\section{WHAT'S FOR SARAWAK - THE OPPORTUNITIES AND POTENTIAL BENEFITS}

Ultimately, one question lingers in our minds - what's in it for the ordinary citizen and the country when we have attained all that we have set out to achieve. Indeed the opportunities and benefits will be many.

Firstly, the sustainable revenues to the country from the royalty payments, the direct and indirect taxation on the energy sales, and the return on investment to the shareholders of the corporate entities involved in the development, will be tremendous. Imagine that if just 1 sen per $\mathrm{kWh}$ in revenue is made from the 20,000MW of generation capacity developed, this would mean a staggering RM 1.75 billion in revenue from the total energy sales per year.

Employment opportunities created by the power infrastructure development work will also be significant. It is envisaged that many professionals and skilled labor in engineering, management, finance and administration are required in the various stages of the power infrastructure development, like project development, design and engineering, construction, operation and maintenance.

With the implementation of large-scale hydropower and transmission projects many types of material resources and 
professional services, viz. food supply, housing, construction material, transportation, engineering consultancy services, financial and legal services, civil infrastructure construction, and a host of other supporting services will be required. Thus there will be plenty of opportunities for the local business community, particularly with the concurrent implementation of a number of those large-scale projects.

The power infrastructure development is also expected to stimulate the socio-economic development in the State, particularly in the interiors. Access and transportation of heavy equipment need major roads to be constructed to the hydropower sites, which will also make the interior areas of Sarawak more accessible and spur economic activities and development of electricity and water supply, and communications infrastructure more viable.

From the institutional development and training provided, the exposure to and direct involvement in the initial power infrastructure development projects, our local engineering professionals will develop the confidence, experience, knowledge, and skills in managing latter projects by themselves. In general, the competency level of the power engineering profession in Sarawak will be raised to a higher level, and in time to come we will be able to market our expertise in engineering planning, design, and construction, project management, and operations and maintenance to others beyond Sarawak.

In conclusion, the future power industry will pose many challenges but learning from and finding the solutions to overcome them will bring about many social, economic, and technical benefits and opportunities for our people and country. The exposure to these challenges will empower us to face the future confidently and facilitate us to equip ourselves with the expertise and knowledge to succeed in a highly competitive and technically demanding environment. We will prosper from these challenges through the economic and technical benefits gained and indeed they will provide us the impetus to power our way forward into the future. 


\title{
Environment and Energy: Our Legacy and Our Future
}

\author{
Mohammad Siphon
}

\section{INTRODUCTION}

Thomas Malthus, in his most famous theory on population growth in 1798 predicted then that population increase would overrun food supply due to fixed supply of land as human population increases in exponential manner compared to arithmetic increase of food due to land availability. His theory was however greatly shadowed by the technological advancement in agriculture that kept population increase continually checked with sufficient food supply. Despite being refuted by many, the theory actually never nullified, it remained, and in this modern day, we are arguably facing the possibility of this to happen, albeit in many other forms.

\section{THE PARADIGM SHIFT}

The general environment as have been naturally perceived, was regarded as abundant, self repairing, and little attention was put into using and managing them wisely, let alone sustainably. Over the last 5 decades, population boom, industrial revolution and modernization have led to unprecedented use and depletion of natural resources, as well as equally, the unparalleled pollution and contamination of the general environment. Climate change, which was unheard of before, become a threat so imminent! that, civilizations as we know it today, is potentially facing its own antithesis. We are paying the high price now when we all have to deal with lots of these once abundant environmental assets, from becoming continually polluted if not depleted, in terms of economic impacts. The sustainable use of our natural resources, are the key, cardinal fundamental management strategies in mitigating and possibly halt, the adverse proliferations of environmental impacts that our forefathers might have had an oversight.

\section{ENERGY AND ENVIRONMENTAL STRESSES}

Befitting the theme of the conference, energy has been the lifeline of human evolution, from the discovery of flame by ancient man, energy is now in its all forms usable are the very reason of our existence. We all know too well that fossil fuel, from where we derive most of our energy

Mohammad Siphon is the Operations Manager of Trienekens (Sarawak) Sdn. Bhd., 216 Jalan Sungai Tapang, Lot 2040 Block 15 MTLD, 93250 Kuching, Sarawak. P.O. Box No. 3105, 93760 Kuching, Sarawak, Malaysia. resources will become scarce in the next thirty to fifty years. Unfortunately, there is not yet a definite answer to this dilemma. Search of alternative energy resources continue. Unfortunately, as civilizations develop, so does the amount of energy needed and the amount of wastes generated consequently.

\section{ENERGY DEMAND - MALAYSIA}

Sarawak maximum demands of electricity has been steadily rising from $554 \mathrm{MW}$ in year 2000 to $741 \mathrm{MW}$ in 2005 and is projected to continue rising (Energy Commission, 2006). The same upward trend could also be observed too, both in the Peninsula Malaysia (9712 MW to 12,493 MW) and the state of Sabah (373 MW to $533 \mathrm{MW}$ ). In the same period, sales of energy for domestic consumption rises from 669 GWh to 992 GWh as the increase in the domestic users jumped from 270,299 consumers to 348,377 consumers. The increasing trend of power demand and usage reflected above could only mean that the burden of power generation will not potentially reduce, but will remain progressively increasing, so does the demand for resources needed to generate these power needs, either through renewable means or through continued reliance on fossil fuels. These resources are however nonrenewable and within the next 30 to 50 years, the reality of running out of these precious commodities, is beyond comprehension.

Interestingly, at the national level, the power-generating plant mix in Malaysia comprises of gas (64.7\%), coal (25.6\%), Hydro (6.1\%), Diesel (2.6\%) and the rest are biomass $(0.6 \%)$ and others, giving a high $92.9 \%$ reliance on fossil fuel (Energy Commission, 2006). At this seemingly high dependence on fossil fuel, we definitely need to initiate strategies to diversify our energy resources.

\section{WASTES GENERATION}

During the same period (2000 - 2005), Kuching city alone generated approximately 900,000 MT of solid wastes (Trienekens, 2006) with annual average of about 130,000 MT. It has been projected that the Kuching city will be producing solid wastes at a staggering rate of between 155,000 MT to a high of 290,000 MT in the year 2020 (NREB/DANIDA, 2001). At the national level, Malaysia generated a whopping 7.34 million tons of solid wastes in 2005 while recycling rates hovered at only a mere 5\% (WMAM, 2006). Given these statistics, the burden of ensuring that the wastes are and will be properly managed 
and treated, recycled or disposed will be very much shouldered by the authorities, the waste managers, and ultimately the public as additional stresses to the public funds and resources, while at the same time deplete the current capacity to manage them longer. It is worth noting too that, industries in Malaysia as a whole generate about 549,000 MT of scheduled wastes in 2005 with $1.5 \%$ of the wastes originated and disposed in Sarawak (EQR DOE, 2006). These added, warrant additional stresses to the environment soon as they are treated and disposed.

\section{SOLID WASTE DisPOSAL}

The majority of the wastes disposal facilities in Malaysia as well as Sarawak, are still the open dumpsites that lack the much needed environmental protection systems - barriers, liners - as well as the proper management and operations protocol. Due to the high capital and operating costs in the acquisition of other appropriate alternative disposal techniques like incineration plant, land-based wastes disposal in Malaysia and Sarawak will remain a necessity for some foreseeable years to come, from short to medium term planning and implementation period, despite issues on sustainability. In this perspective, it is indeed critical that we build our capacity of being able to put in place and implement a sustainable system of land-based disposal facilities now in order to ensure that we do not repeat the failures of our past, of delineating the same to mere chores. Dumpsites are no longer an acceptable form of disposal means for solid wastes, they are by far some of the most persistent sources of contamination if not dealt with in appropriate manner. Newly designed sanitary landfill will need to be engineered together with the base-sealing liner of appropriate specifications and design, able to withstand the wastes loadings on which it will be subjected to.

\section{RIVER/WATER QUALITY}

Rivers are indeed our lifeline, not only in river transport but for hosts of other functions, from subsistence to irrigation. Lost of functionalities in their services, could bring our daily livelihoods to a standstill. Our rich ecosystems could be adversely affected, potentially disrupting the vital food chains, and economic importance. Rivers in Sarawak were undeniably progressively polluted by the various sources of pollution (NREB, 2004), from untreated sewage discharge to siltation due to soil erosion as a result of land clearing and land developments. It was reported too that Sarawak rivers are primarily contaminated with faecal bacteria due to the severe pollution by black water (toilet waste), overflowing septic tanks as well as hanging toilets. In Malaysia, 146 river basins monitored by the Department of Environment via 1085 of their monitoring stations revealed that about half of the river basins were polluted or slightly polluted (EQR DOE, 2006). Pollution sources were largely attributed to mainly sewage treatment plants (46.9\%), manufacturing industries (45.7\%), animal farms (4.8\%) and agrochemicals (2.6\%). Left unchecked, these sources could eventually further degenerate our river waters beyond our practical usage, necessitating higher treatment costs. Environmental awareness must be geared higher so as to ensure that these needs of managing our sources of pollution transcends generations and remained in perpetual consciousness.

\section{ENVIRONMENT AND ENERGY}

Environment is without doubt where we all have coexisted with our other natural resources since time immemorial, from which we have exploited and derived our energy needs to date. Terminal depletion in any one of them, could be a catastrophe to us all, if not a disaster. In essence, the truth is, if we do not take actions on our accord now, we probably run out of that place where we could grow our food to continue our sustenance and use the energy resources to aid our lives for the same sustenance. There is no other replacement for what we have had now and then, it is the only one. While all is not lost now, or yet, we could not be or becoming complacent either.

\section{WAY FORWARD}

Let us all look at our opportunities to put our environmental challenges now into perspective, walk into our actions and agenda for which we all know is very important for continued sustainability of what have been equally achieved in this country - our economic prowess, our societies' structural integrity and our diverse unified cultures. We could then say that Thomas Malthus have indeed erred in his prepositions on population and food productions. 


\section{Contents}

\section{Keynote Papers}

Powering Sarawak into the Future - The Way Forward

Tan Sri Datuk Amar Abdul Aziz bin Dato Hj. Husain

Environment and Energy: Our Legacy and Our Future

Mohammad Siphon

\section{Technical Papers}

Highly Efficient Bioenergy Production from Woody Biomass

Abu Saleh Ahmed, Keisuke Makino, Sinin Hamdan, Khairuddin Ab Hamid, Ayub Md Som and Masjuki Hj Hassan

Fuel Penalty from Periodic Rich Combustion of a Diesel Fuelled Engine during Lean $\mathrm{NO}_{X}$ Trap Purging

Ahmad J. Alimin, Stephen F. Benjamin and Carol A. Roberts

Pyrolysis Characteristics and Kinetics of Coconut Coir and Shell Mixtures with Sub-Bituminous Coal using

Thermogravimetric Analysis

Azil Bahari Alias, Zulkifli Abdul Rashid, Norazah Abd Rahman, Siti Shawalliah Idris, Khudzir Ismail, Mohd Azlan Mohd Ishak, and Wan Azlina W.Ab. Karim Ghani

Construction and Laboratory Testing of Parabolic Types Solar Cooker

Erwan Sulaiman, Kok Boon Ching and Chessda Uttraphan

Electric Energy Saving for Residential Sector

K. Ramar, Gobbi R., Normiza Nor, A. Arunagiri, E.E. Ngu, C.M. Kok, H.C. Voon and Lawrence W.

Performance Analysis of Savonius
H. H. Al-Kayiem, A. E. Sa'ad Al-deen

Production of Biodiesel from Palm Oil Using Montmorillonite KSF as a Heterogeneous Catalyst

Thermal Characteristics of Eutectic Mixture of Capric-Lauric Acids as Phase Change Material (PCM) in

Gypsum Board

Rozanna Dewi, Luqman Chuah A., Salmiah A., Thomas Choong S.Y. and Sa’ari Musthapa

ASEAN'S Biomass Fired Grid-Connected Cogeneration Plants: The Way Forward

Mohamad Fadzil Adnan @ Nan, Rusmi Alias and Nasrin Abu Bakar

Design and Development of Liquid Spray Gas Scrubber for Odour Control

Mohd Faizal Mohideen Batcha, Lai Dyi Yih and Vijay R. Raghavan

M. Z. Zainon and A. Serizawa

Performance Evaluation of Constant Frequency Torque Controller-Based and SVM-Based of Direct Torque 
Method

I. Daut, R. Chan Bahaudin, C.M. Hadzer, S.F. Siraj, S. Hardi and N. Hashim

Simulation of Diesel Engine Fueled by Compressed Natural Gas Performance Using GT-Power

Rosli A. Bakar, Semin and Abdul R. Ismail

Voltage Sag and the Sensitive Load

I. Daut, C.M. Hadzer, S. Hardi, R. Chan Bahaudin, S.F. Siraj and N. Hashim

The Performances of RBD Palm Stearin as Cold Metal Working Lubricant by Plane Strain Extrusion Test S. Syahrullail, S. Kamitani and K. Nakanishi

Refrigeration Efficiency Improvement via Heat Transfer Enhancement

R. Thiruselvam, Mohammad Zainal Yusof, Vijay R. Raghavan

Exergy Analysis of a Cogeneration and Cooling Plant

C. Rangkuti, A. L.Tamiru, S. I. Gilani, and M. Amin

Identification of Microorganisms in an Anaerobic Baffled Reactor (ABR) for Methane Production

A. Malakahmad, S.M. Zain, N.E. Basri \& F. Suja’

Power Consumption Pattern of Consumer in Kuching Sarawak

S.S.Ngu, L.C.Kho, J. Annie, C.J. Chai and Leslie K.P. Chai

Water Impact Analysis over Distribution Pipelines Network

Abdurazzag Ali Aburas, Manal I Alfellah and Salem Ali Reheil

A Study on Electric Energy Usage at the Residential Area

Asmarashid Ponniran, Erwan Sulaiman, Siti Amely Jumaat, Maizul Ishak, Megat Azahari Chulan and Suhaimi Saiman

Measurement of Insulation Resistance of Line Insulator under Adverse Conditions

M. A. Salam, Nurul Fadzlinah, Shaera and Zuharni

Evaluation of UTHM Administrative Office Block Cooling Load through Dynamic Simulation

Ismail Abdul Rahman, Kamarul Aini Mohd Sari, and Nur Hanisah Mat Tasir

Gasification Behaviour of Malaysian Biomass: Sugarcane Waste

Barranco, R., Cooper, M., Hanson, S., Lee, K. U., Low, K. S. and Teo, C. H.

Estimation of Solar Energy Potential in East Malaysia by Using Satellite Images

Ayu Wazira Azhari, Kamaruzzaman Sopian, Abdul Haqi Ibrahim

Mesoporous Solid Base Catalyst for the Heterogeneous Transesterification of Palm Oil to Biodiesel

A. Z. Abdullah, N. Razali, K. T. Lee, B. Salamatinia and H. Mootabadi

The Rheology Properties of Sabah Bentonite at High Temperature Environment

Sonny Irawan, Ismail B Mohd Saaid, Norrulhuda Bt Hj Mohd Taib and Sultan Mohammed Al-Rawhani

Analysis of Field Distribution of a Cylindrical Permanent Magnet Linear Generator

Wijono and Hamzah Arof

Study of Roof Evaporative Cooling by Flooding, Misting and Sprinkler Spraying Systems

Chu Chi Ming, Ong Kein Kok and Tan Liang Harn

Burner Flame Observation by Using High Speed Video Image

A.K. Amirruddin, M.M. Rashidi and S.M. Sapuan

Physical Properties of Local Palm Oil Clinker and Fly Ash

Mohd Hilton Ahmad and Nurazuwa Mohd Noor

Design, Development and Performance Evaluation of Parabolic Trough Solar Collector in Tropical Regions

Syed Ihtsham-ul-Haq Gilani, Chalilullah Rangkuti, Aklilu Tesfamichael, and Tsen Wee Yew 
as a Biofuel

Abdullah, N.A., Ramli, S., Ayub, M.A., Abdul-Talib, S., Mohd Tajuddin, R., Atan, I., Jaafar, J. and Baki, A.

Development of a Cost-Effective Op-Amp Based Digital Power Factor Meter

B.C. Kok, C.S. Tan, and E. Sulaiman

Development of a Microcontroller-Based Power Factor Corrector

C. Uttraphan, B.C. Kok, K.H. Lai, and S.A. Zulkifli

The Effects of Linear and Non Linear Loads to the Three Phase Four Wire Distribution Systems

Mohd Izhar A.Bakar

Dynamic Performance Analysis of a Practical PI Regulated AC Variable Speed Drive

Rajendran.S , M.K.A. Ahmed Khan, Gobbi.R, K.Y. Loh and A. Kareem

Modeling of a Three Phase Linear Generator by Using Finite Element Magnetics Method Rajendran S., H. Arof and M.K.A. Ahmed Khan

Extraction Methodology and Biogas Quality From Palm Oil Mill Effluent

F. Awang, J. Ahmad, A. Saifizul and M. Z. Zainon

Solar Energy Technologies

Andhe Satyanarayana Murthy

Overview on the Infrastructure Planning of Hydrogen Energy in Malaysia

Profit Optimization of a Refrigerated Gas Plant

Nooryusmiza Yusoff, M. Ramasamy, Suzana Yusup

EnergyPlus ${ }^{\circledR}$ Simulation Program to Evaluate the Performance of Sustainable Buildings? Ooi Koon Beng and Azhaili Baharun

Biodiesel Production from Different Used Vegetable Cooking Oil as Environmental Recycling Process and

Economic Source-A Review

A.B.M. Sharif Hossain, Amru Nasrulhaq Boyce, Abu Saleh Ahmed, Haji Mohamed A. Majid, S. Chandran, and R. Zuliana

Development DC Converter for Low Voltage High Current Applications Using CUK DC-DC Converter

W. Salah, and S. Taib and A. Al-Mofleh

Computational Fluid Dynamics (CFD) Simulation of a Downdraft Gasifier Using Wood Residue as Biomass

Fuel

Mahbub Hasan, Shanti Faridah Salleh and Raymond John

Prospects of Biogas Production Utilizing the Sewage Waste Water

Finite Element Analysis of Thermal Gradient of Transformer Windings 
Heat Transfer Study of a Progressive Freeze Concentration System for the Treatment of a Simulated

Wastewater with Various Type of Insulator

M. Jusoh, R. Mohd. Yunus, M.A. Abu Hassan, and M.I. Abdul Razif

Hydrodynamics Studies Of Sand/Palm Shells Binary Mixtures In Compartmented Fluidized Bed Gasifier

V.S. Chok, L.F.B. Chin, S.K. Wee, W.W. Tang, A. Gorin, H.B. Chua, H.M. Yan

$\mathrm{CO}_{2}$ Emissions: Impacts and Forecasting in Malaysia

Z.Z. Noor, N.S. Mohd Safaai, H. Hashim and Z. Ujang

cylinder Engine

M.K. Hassan, I. Aris, S.Mahmod, R. Sidek, K. N. Zainuddin, N.A. Alina, H.H. Masjuki, M. Redzuan and M.A. Kalam

Experimental Tests and Simulations Of Compressed Natural Gas Direct Injection (CNGDI) Single Cylinder

Engine

I. Aris, M.K. Hassan, S. Mahmod, R. Sidek, K.N. Zainuddin, N.A. Alina, H.H. Masjuki, M. Redzuan and M.A. Kalam

Electricity Planning Under $\mathrm{CO}_{2}$ Mitigation: A Canadian Case Study

H. Hashim, Peter Douglas, Ali Elkamel and Eric Croiset

A Surge Suppressor Model for Increasing the Energy-Handling Capability in High Voltage Protection

Muhammad Saufi Kamarudin and Erwan Sulaiman

Initial Study on the Operation, Energy Usage and Improvement Opportunities of an Ice-Thermal District

Cooling System

Mohammad Omar Abdullah, Samsudin Bin Sulaiman, Mohamad Odil Sabri

The Study of Vortex Formation after a Bluff Body Due To Wind Velocities with the Effect of underneath Wavy

Surface

Jamaluddin Md Sheriff, Kahar Osman and Asral

The Use of Fly Ash to Reduce the Environmental Impact of Concrete

D. Hardjito

Environmental Effect Due to the Energy Consumption of Refrigerator-freezer

M. Hasanuzzaman, R. Saidur, H.H. Masjuki and M.M. Hasan

Flow Characterization of Gas-Solid Mixture in Fluidized Bed Combustion

Rosyida Permatasari, Mohammad Nazri bin Mohd Jaafar, Tholudin bin Mat Lazim. Mohammad Sayuthy bin Samsudin

Marwan Affandi and Mat Nawi Wan Hassan 
J. Labadin and A.R.H. Rigit

Single Phase Multilevel Power Inverter 
Azizul Mohamad, Abdul Sattar Megat Husin and Daniel William

Solutions of Water and Tetrahydrofuran

Khalik M. Sabil, Nadia Oujamaa, Johannes M. Bruining, Geert-Jan Witkamp, and Cor J. Peters

Thermodynamic Analysis of a Closed Rankine Cycle Solar Power Generator

A 3-stage Wastewater Treatment System: Part II. Performance Evaluation

Yin Wee Oon, Puong Ling Law, Chee Khoon Ng, Lock Hei Ngu and Kien Kuok Wong 


\title{
Highly Efficient Bioenergy Production from Woody Biomass
}

\author{
Abu Saleh Ahmed, Keisuke Makino, Sinin Hamdan, Khairuddin Ab Hamid, Ayub Md Som and \\ Masjuki Hj Hassan
}

\begin{abstract}
The more efficient use of biomass is expected to solve the global crisis such as depletion of fossil fuel and global warming. Woody biomass, including agriculture residues, wood chips, municipal solid wastes, paper wastes, etc., has already been transferred to bio-ethanol and bio-diesel in some cases and used as energy related products, although many issues such as efficiency and productivity still exist to be overcome. Xylose is one of the major fermentable sugars present in woody biomass, the second most abundant carbohydrate polymer in nature to glucose. The efficient fermentation of xylose is required to develop economically viable processes for producing biofuels such as ethanol from woody biomass. In this study, the bioengineering was applied to generate enzymes with completely reversed coenzyme specificity and recombinant yeasts containing those engineered enzymes were developed for construction of an efficient biomass-ethanol conversion system. Saccharomyces cerevisiae transformed with the native genes encoding xylose reductase (XR), xylitol dehydrogenase (XDH) from Pichia stipitis and the endogenous gene xylulokinage (XK) ferments xylose to ethanol but has not yet been applied to the industrial bio-process due to the unfavorable excretion of xylitol. Intercellular redox imbalance caused by the different coenzyme specificity of XR (using NADPH/NADH with preference for NADPH) and XDH (exclusively using $\mathrm{NAD}^{+}$) has been thought to be one of the main factors of xylitol excretion. Introduction of $\mathrm{NADP}^{+}$dependent XDH generated in this study prevented the xylitol excretion probably because of maintaining the intercellular redox balance. Ethanol fermentation was measured in batch culture under anaerobic conditions. The significant enhancement was found in Y-ARSdR strain, in which NADP ${ }^{+}$dependent XDH was expressed; $86 \%$ decrease of unfavorable xylitol excretion with $41 \%$ increased ethanol production, when
\end{abstract}

Abu Saleh Ahmed is with the Department of Mechanical and Manufacturing Engineering, Faculty of Engineering, Universiti Malaysia Sarawak, 94300 Kota Samarahan, Sarawak, Malaysia (phone: +6082583299; fax: +6082583410; e-mail: aasaleh@ feng.unimas.my).

Keisuke Makino is with the Institute of Advanced Energy, Kyoto University, Gokasho, Uji, Kyoto 611-0011, Japan (kmak@iae.kyotou.ac.jp).

Sinin Hamdan is with the Department of Mechanical and Manufacturing Engineering, Faculty of Engineering, Universiti Malaysia Sarawak, 94300 Kota Samarahan, Sarawak, Malaysia (e-mail: hsinin@feng.unimas.my).

Khairuddin $\mathrm{Ab}$ Hamid is with the Faculty of Engineering, Universiti Malaysia Sarawak, 94300 Kota Samarahan, Sarawak, Malaysia (e-mail: khair@cans.unimas.my).

Ayub Md Som is with the Faculty of Chemical Engineering, Universti Technologi Mara, 40450 Shah Alam, Malaysia (ayu522@salam.uitm.edu.my).

Masjuki Hj Hassan is with the Faculty of Engineering, University of Malaya, 50603 Kuala Lumpur, Malaysia (e-mail: masjuki@um.edu.my). compared with the references strain expressing the wild-type XDH. The Y-ARSdR strain produced a maximum of $7.02 \mathrm{~g} / \mathbf{l}$ ethanol with yield of $0.46 \mathrm{~g} / \mathrm{g}$ from $5 \mathrm{~g}$ glucose/liter plus $15 \mathrm{~g}$ xylose/liter. The yield of ethanol from consumed sugars reached $90 \%$ of the theoretical yield. Measurement of intracellular coenzyme concentrations suggested that maintenance of the NADPH/NADP ${ }^{+}$and $\mathrm{NADH} / \mathrm{NAD}^{+}$ratios are important for efficient ethanol fermentation from biomass sugars by recombinant $S$. cerevisiae.

Keywords: biomass, bioethanol, recombinant yeast, woody biomass, biomass-ethanol conversion

\section{INTRODUCTION}

Xylose is one of the major fermentable sugars present in lignocellulosic biomass, the second most abundant carbohydrate polymer in nature to glucose. The efficient fermentation of xylose is required to develop economically viable processes for producing biofuels such as ethanol from biomass [1]. Although a few xylose fermenting yeasts are found in nature [2], [3], Saccharomyces cerevisiae is used universally for industrial ethanol production because of the ability to produce high concentrations of ethanol and high inherent ethanol tolerance. However, the native $S$. cerevisiae can not ferment xylose, so engineering $S$. cerevisiae for xylose utilization has focused on adapting the xylose metabolic pathway from the xylose-utilizing yeast Pichia stipitis [4], [5]. In this organism, xylose is converted into xylulose by two oxidoreductases. First, xylose is reduced to xylitol by an NADPH/NADH-linked xylose reducatase (XR) [6], and then xylitol is oxidized to xylulose by an NAD-linked xylitol dehydrogenase (XDH) [7]. Finally, xylulokinase (XK) phosphorylates xylulose into xylulose-5-phosphate, which is metabolized further via the pentose phosphate pathway (PPP) ('figure 1'). Many researchers attempt at engineering xylose metabolism expressed only native XR and XDH from $P$. stipitis in $S$. cerevisiae because $S$. cerevisiae can ferment xylulose [8]. Recombinant $S$. cerevisiae expressing native XR and XDH could grow on xylose, but ethanol production from xylose was not significant because a substantial portion of the consumed xylose was converted into xylitol [9].

The most straightforward metabolic engineering strategy is the expression of bacterial xylose isomerase (XI) gene, so that xylose can directly be converted to xylulose with no coenzyme. There have been numerous unsuccessful attemts to express XI genes from various bacteria in S. cerevisiae 
except a thermophilic enzyme [10], [11], we focused on the fungal xylose metabolic pathway in this study. XR and $\mathrm{XDH}$ in this fungal pathway are necessary for $S$. cerevisiae to ferment xylose to ethanol because of a lack of genes encoding these enzymes in $S$. cerevisiae. Several studies reported that $S$. cerevisiae transforming the genes encoding $\mathrm{XR}, \mathrm{XDH}$ from $\mathrm{P}$. stipitis and the endogenous gene $\mathrm{XK}$, a most potent recombinant strain, has not yet been applied to the industrial bio-process due to the unfavorable excretion of xylitol [12], [13]. Because intercellular redox imbalance caused by the different coenzyme specificity of XR (with $\mathrm{NADPH}$ ) and XDH (with NAD) has been thought to be one of the main factors of xylitol excretion. The introduction of the protein engineered $\mathrm{XDH}$ (with NADP) of $P$. stipitis generated in this study prevented this xylitol excretion by maintaining the intercellular redox balance.

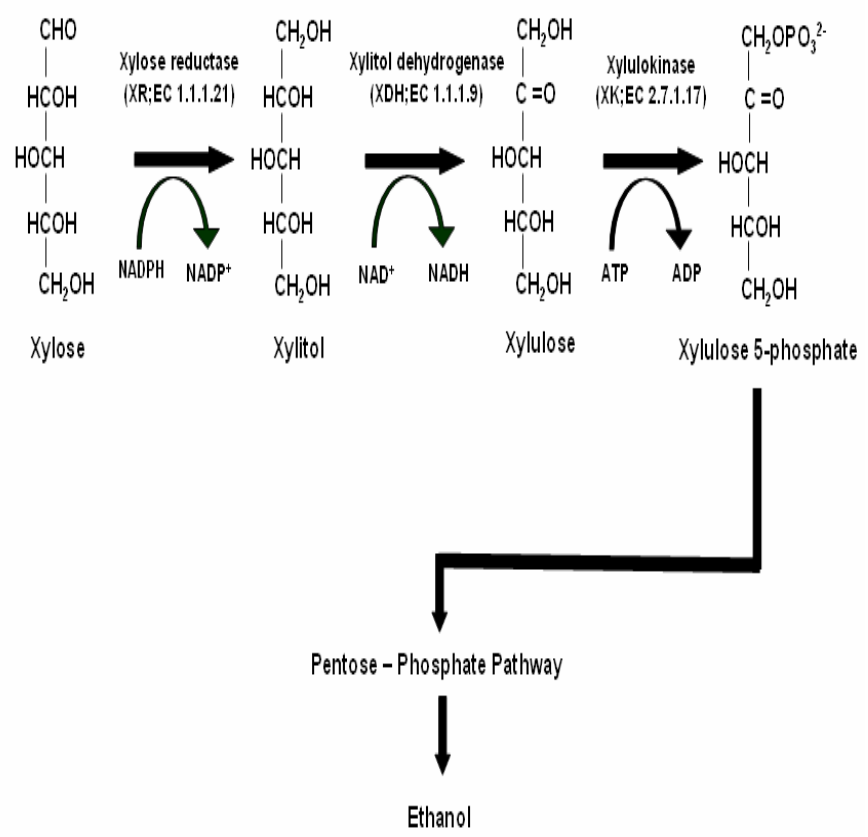

Figure 1 Metabolic pathway from xylose to ethanol.

In spite of many attempts for modification of coenzyme specificity, only a few of them can be judged to be fully reversal, in terms of obtaining a mutant enzyme as catalytically efficient as the wild type [14], [15]. In our previous study [16], site-directed mutagenesis for complete reversal of coenzyme specificity was performed on $\mathrm{NAD}^{+}$dependent $\mathrm{XDH}$ from $P$. stipitis to generate $\mathrm{NADP}^{+}$ dependent $\mathrm{XDH}$ and these mutated XDHs were characterized in vitro. In the present study, we constructed recombinant yeasts that were transformed with the genes encoding the protein engineered XDH (with completely NADP-dependent) and a wild type XR of $P$. stipitis and characterized those recombinant yeasts based on the enzyme activity and fermentation ability of xylose to ethanol. The protein engineered enzymes were expressed significantly in $S$. cerevisiae as judged by the enzyme activity in vitro. Ethanol fermentation was measured in batch culture under anaerobic conditions. Introduction of NADP-dependent $\mathrm{XDH}$ was shown to prevent the xylitol excretion probably because of maintaining the intercellular redox balance.

\section{MAterials AND METHODS}

\section{Materials and media}

S. cerevisiae D452-2 (MATa leu2 his3ura3can1) [17] was used as the recipient yeast strain for the expression of Pichia stipitis XDH and XR. S. cerevisiae was grown in YPD medium (10 g/l yeast extract, $20 \mathrm{~g} / 1$ peptone, and $20 \mathrm{~g} / \mathrm{l}$ glucose) for transformation. In all other experiments, a defined minimal medium (6.7 $\mathrm{g}$ of yeast nitrogen base without amino acid with 5 g glucose per liter) was used. Leucine, Histidine, and Uracil were added at $20 \mathrm{mg} / \mathrm{l}, 20$ $\mathrm{mg} / \mathrm{l}$, and $5 \mathrm{mg} / \mathrm{l}$ respectively for strain D452-2. Bacterial strains were grown in Luria-Bertani medium (yeast extract 5 $\mathrm{g} / \mathrm{l}$, peptone $10 \mathrm{~g} / \mathrm{l}$, and sodium chloride $10 \mathrm{~g} / \mathrm{l}$ ). Transformants were selected by adding ampicillin (50 $\mathrm{mg} /$ liter). For growth on solid media $20 \mathrm{~g}$ of agar per liter was added.

\section{Yeast strain transformation}

Yeast transformation was performed using the lithium acetate method [18]. Yeast cells were incubated overnight in YPD medium before being streaked on selective medium. S. cerevisiae transformants were selected on agar plates with minimal medium containing $20 \mathrm{mg} / \mathrm{l}$ histidine, $20 \mathrm{mg} / \mathrm{l}$ leucine and $5 \mathrm{mg} / \mathrm{l}$ uracil.

\section{Preparation of crude cell extract}

The recombinant yeast strains were grown in minimal medium at $30^{\circ} \mathrm{C}$. The cells were harvested by centrifugation at $5000 \mathrm{rpm}$ for 10 minutes. The cells were resuspended in disintegration buffer $100 \mathrm{mM}$ sodium phosphate ( $\mathrm{pH} 7.0$ ) containing $1 \mathrm{mM} \mathrm{MgCl}_{2}, 0.5 \mathrm{mM}$ EDTA and $0.5 \mathrm{mM}$ dithiothreitol (DTT) and vortexed together with an equal volume of glass beads ( $0.5 \mathrm{~mm}$ diameter) twice for $5 \mathrm{~min}$ at $4^{\circ} \mathrm{C}$. Cell debris and glass beads from the cell extract were separated by centrifugation at $15000 \mathrm{rpm}, 10 \mathrm{~min}, 4^{\circ} \mathrm{C}$ and the remaining supernatant was used for enzyme determinations.

\section{Enzyme assays}

Enzyme activities were measured by using a V-550 model spectrophotometer (JASCO Corporation, Japan) operating at $340 \mathrm{~nm}$ and $35^{\circ} \mathrm{C}$. The standard assay mixture for $\mathrm{XDH}$ contained $50 \mathrm{mM} \mathrm{MgCl}$ and $300 \mathrm{mM}$ xylitol in $50 \mathrm{mM}$ Tris- $\mathrm{HCl}$ ( $\mathrm{pH}$ 9.0); for XR contained $60 \mathrm{mM}$ sodium phosphate and $133 \mathrm{mM}$ xylose [13]. All reactions were started by addition of $0.10 \mathrm{ml}$ of a $20 \mathrm{mM} \mathrm{NAD/NADP}$ solution for XDH and $0.10 \mathrm{ml}$ of a $1.5 \mathrm{mM}$ NADPH/NADH solution for XR to a final volume of $1.0 \mathrm{ml}$ (standard assay condition). One unit of enzyme activity refers to $1 \mu \mathrm{mol}$ of $\mathrm{NAD}(\mathrm{P})^{+} \mathrm{H}$ produced $/ \mathrm{min}$. Protein concentrations were determined by the method of Lowry et al. [19] with bovine serum albumin as a standard.

\section{Preliminary batch fermentation in shake flask}

After precultivation of recombinant yeast strains in $3 \mathrm{ml}$ minimal medium in $15 \mathrm{ml}$ falcon tube for 3 days, yeast cells were aerobically cultivated for 3 days at $30^{\circ} \mathrm{C}$ in $100 \mathrm{ml}$ minimal medium in $300 \mathrm{ml}$ flask in incubator at $200 \mathrm{rpm}$. The cell pellets were collected by centrifugation for $10 \mathrm{~min}$ at $5000 \mathrm{rpm}$ and $4^{\circ} \mathrm{C}$, and were washed once with a solution containing $9 \mathrm{~g}$ of $\mathrm{NaCl} /$ liter. The cell pellets were then inoculated into $200 \mathrm{ml}$ fermented medium $(6.7 \mathrm{~g}$ of yeast 
nitrogen base without amino acid per liter with $5 \mathrm{~g}$ of glucose/liter plus $15 \mathrm{~g}$ of xylose/liter and appropriate amino acid). Ethanol fermentation was carried out as batch cultures in $200 \mathrm{ml}$ flask with anaerobic condition in incubator at 150 rpm. The flasks were sealed with two layers of Saran wrapping paper to allow fermentation to occur anaerobically. Samples $(1 \mathrm{ml})$ of the fermentation broth were removed at intervals and stocked at $-35^{\circ} \mathrm{C}$ for analyses of substrates and fermentation products.

\section{Batch fermentation in bioreactor}

A high performance bioreactor (BioFlo 110, New Brunswick Scientific Co.) was used for anaerobic batch fermentation. After precultivation of recombinant yeast strains in $5 \mathrm{ml}$ minimal medium in $15 \mathrm{ml}$ falcon tube for 3 days, yeast cells were aerobically cultivated for 3 days at $30^{\circ} \mathrm{C}$ in $300 \mathrm{ml}$ minimal medium in 1 liter flask in incubator at $200 \mathrm{rpm}$. The cell pellets were collected by centrifugation for $10 \mathrm{~min}$ at $5000 \mathrm{rpm}$ and $4^{\circ} \mathrm{C}$, and were washed once with a solution containing $9 \mathrm{~g}$ of $\mathrm{NaCl} /$ liter. The cell pellets were then inoculated into fermented medium $(6.7 \mathrm{~g}$ of yeast nitrogen base without amino acid per liter with $5 \mathrm{~g}$ of glucose/liter plus $15 \mathrm{~g}$ of xylose/liter and appropriate amino acid). Silicone antifoam $0.5 \mathrm{ml} /$ liter was added to the media before autoclaving. Anaerobic cultivation took place in a 1.3 liter bioreactor with an initial working volume 1 liter. The temperature was maintained at $30^{\circ} \mathrm{C}$. The $\mathrm{pH}$ was controlled at 5.5 by addition of $2 \mathrm{M} \mathrm{NaOH}$ and $1 \mathrm{M} \mathrm{H}_{2} \mathrm{SO}_{4}$. The agitation speed was constant at $500 \mathrm{rpm}$.

\section{Measurement of intracellular coenzyme contents}

Intracellular coenzymes were extracted from cells growing under oxygen-limiting conditions in minimal medium with $10 \mathrm{~g}$ of xylose/liter plus $10 \mathrm{~g}$ of glucose/liter. A $20 \mathrm{ml}$ sample of yeast culture was withdrawn and sprayed into $80 \mathrm{ml} 60 \%(\mathrm{v} / \mathrm{v})$ methanol at $-40^{\circ} \mathrm{C}$. After this quenching step, coenzymes were extracted in $50 \mathrm{mM}$ potassium phosphate ( $\mathrm{pH} 5.0$ ) (for $\mathrm{NAD}^{+}$and $\mathrm{NADP}^{+}$) or 50 $\mathrm{mM}$ Tris/ $\mathrm{HCl}(\mathrm{pH} 9.0)$ (for $\mathrm{NADH}$ and NADPH). The contents of $\mathrm{NAD}^{+}, \mathrm{NADH}, \mathrm{NADP}^{+}$and NADPH in the samples were determined by monitoring the absorbance difference at $339 \mathrm{~nm}$ using the followed coupling enzymes at room temperature [20]: for $\mathrm{NAD}^{+}$, alcohol dehydrogenase (from baker's yeast, Sigma); for NADP ${ }^{+}$, glucose-6phosphate dehydrogenase (type XV from baker's yeast, Sigma); for NADH, glycerol-3-phosphate dehydrogenase (from rabbit muscle, Wako); for NADPH, glutamate dehydrogenase (from beef liver, Oriental Yeast).

\section{Analysis of fermentation products}

Ethanol concentration was measured using gas chromatography. The gas chromatograph (model GC -14B, Shimadzu Corporation, Kyoto, Japan, fitted with a flame ionization detector) was operated under the following conditions: glass column $(2.0 \mathrm{~m}$ by $3.2 \mathrm{~mm})$ packed with Thermon-3000 (Shimadzu); temperature of column $70^{\circ} \mathrm{C}$, injector $200^{\circ} \mathrm{C}$, and detector $250^{\circ} \mathrm{C}$; and nitrogen carrier gas flow rate $25 \mathrm{ml} / \mathrm{min}$. A high-performance liquid chromatograph (Tosoh Corporation Ltd., Japan) equipped with an RI detector was used to analyze the concentrations of glucose, xylose, xylitol, glycerol, and acetic acid. A BioRad type HPX-87H ion-exclusion column was used. The mobile phase was $5 \mathrm{mM} \mathrm{H}_{2} \mathrm{SO}_{4}$ at a flow rate of $0.4 \mathrm{ml} / \mathrm{min}$.
Cell growth was monitored by measuring the absorbance at $600 \mathrm{~nm}$ using a spectrophotometer (model U-2001, Hitachi, Japan).

\section{RESULTS}

\section{Expression of $P$. stipitis XR and XDH mutants in $S$. cerevisiae}

Plasmids pPGK-WT, pPGK-ARS, pPGK-ARSdR, pPGKC4, pPGK-C4/ARS, and pPGK-C4/ARSdR for XDH expression in yeast were constructed by inserting the wildtype (WT) or corresponding mutated XDH expression region [16] into a yeast expression vector, pPGK [21], which is a $2 \mu \mathrm{m}$ DNA-based shuttle vector containing a phosphoglycerate kinase (PGK) promoter and terminator for expression, a multicloning site, and the URA3 gene as a yeast selectable marker. To construct a plasmid for XR expression in yeast, the XR coding region [22] was first inserted into $\mathrm{pPGK}$ vector. Then the XR coding region with PGK promoter and terminator was excised from the plasmid and reinserted into YEpM4 [23] vector, which is a $2 \mu \mathrm{m}$ DNA-based shuttle vector containing a multicloning site LEU2 gene as a yeast selectable marker to yield pPGK-XR. S. cerevisiae D452-2 (MATa leu2 his3ura3canl) [17] was used as the recipient yeast strain for the expression of XDH and XR. D452-2 was transformed with pPGK-XR and then D452-2 containing $\mathrm{pPGK}-\mathrm{XR}$ was further transformed with pPGK-WT, pPGK-ARS, pPGK-ARSdR, pPGK-C4, pPGK$\mathrm{C} 4 / \mathrm{ARS}$, and $\mathrm{pPGK}-\mathrm{C} 4 / \mathrm{ARSdR}$ to construct recombinant yeast strains Y-WT, Y-ARS, Y-ARSdR, Y-C4, Y-C4/ARS, and Y-C4/ARSdR respectively. pPGK and YEpM4 were transformed into D452-2 to construct $\mathrm{Y}-\mathrm{Vector}$ as a control strain.

\section{Effect of PsXDH mutants in S. cerevisiae}

All the recombinant $S$. cerevisiae strains constructed in this study grew normally in the minimal medium (data not shown). The recombinant strains were cultured in the minimal medium, harvested and then the crude extracts were prepared using glass beads as described in materials and methods. XDH and XR activities in the crude extracts were measured spectrophotometrically. As shown in 'table 1 ', Y-ARS showed higher enzyme activity with $\mathrm{NADP}^{+}$ compared with Y-WT (more than 8-fold higher values of YWT). Y-ARSdR that contains quadruple mutant XDH showed 13-fold enzyme activity with $\mathrm{NADP}^{+}$compared with Y-WT.

Table 1. Activities of XR and XDH in recombinant yeast strains

\begin{tabular}{llll}
\hline Strain & XR $\left(\mathrm{Umg}^{-1}\right)$ & $\mathrm{XDH}\left(\mathrm{U} \mathrm{mg}^{-1}\right)$ & \\
\cline { 3 - 4 } & & $\mathrm{NAD}^{+}$ & NADP $^{+}$ \\
\hline Y-Vector & $0.010 \pm 0.001$ & $0.024 \pm 0.006$ & $0.022 \pm 0.005$ \\
Y-WT & $0.041 \pm 0.002$ & $0.074 \pm 0.006$ & $0.032 \pm 0.006$ \\
Y-ARS & $0.051 \pm 0.005$ & $0.046 \pm 0.002$ & $0.256 \pm 0.022$ \\
Y-ARSdR & $0.049 \pm 0.009$ & $0.048 \pm 0.004$ & $0.426 \pm 0.050$ \\
Y-C4 & $0.050 \pm 0.003$ & $0.381 \pm 0.029$ & $0.045 \pm 0.002$ \\
Y-C4/ARS & $0.045 \pm 0.008$ & $0.046 \pm 0.032$ & $0.709 \pm 0.054$ \\
Y-C4/ARSdR & $0.037 \pm 0.003$ & $0.048 \pm 0.004$ & $0.350 \pm 0.020$ \\
\hline
\end{tabular}

$\mathrm{Y}-\mathrm{C} 4$ that contained the additional zinc introduced $\mathrm{XDH}$ showed 5-fold enzyme activity with $\mathrm{NAD}^{+}$compared with Y-WT. Y-C4/ARS and Y-C4/ARSdR showed, respectively, 
22-fold and 11-fold XDH enzyme activity with $\mathrm{NADP}^{+}$. The $\mathrm{XR}$ activities in these recombinant yeast were observed at al most the same levels ('table 1'). The XDH and XR activities observed in control strain were probably due to endogenous $\mathrm{XDH}$ and XR activities.

\section{Effect of NADP-Preferring XDH mutant in Ethanol Fermentation}

Ethanol production and xylitol excretion of the recombinant yeasts grown in an incubator with shaker at $30^{\circ} \mathrm{C}$ were measured. One set of fermentation $(100 \mathrm{ml})$ was started with $5 \mathrm{~g} / 1$ glucose and $15 \mathrm{~g} / 1$ xylose ('figure 2'). In case of $\mathrm{Y}$-Vector, a increase of ethanol concentration was found in only initial phase (3-25 h), which may be due to glucose consumption. Y-WT produced ethanol at $2.22 \mathrm{~g} / 1$ and excluded xylitol at $1.42 \mathrm{~g} / 1$. Y-C4 produced ethanol at $2.58 \mathrm{~g} / \mathrm{l}$ (higher than Y-WT), whereas the strain still excluded xylitol at a relatively high amount $(1.24 \mathrm{~g} / \mathrm{l})$. YARSdR produced the highest amount of ethanol at $2.97 \mathrm{~g} / \mathrm{l}$ and excreted the lowest amount of xylitol at $0.16 \mathrm{~g} / \mathrm{l}$. YARS, Y-C4/ARS, and Y-C4/ARSdR produced ethanol at $2.79,2.82$, and $2.91 \mathrm{~g} / 1$, respectively. In all these recombinant yeast strains, glucose was completely fermented in 25 to $40 \mathrm{~h}$. Of the xylose, $25-58 \%$ was fermented in $68 \mathrm{~h}$.

Since Y-ARSdR gave the highest ethanol production and the lowest xylitol excretion, it was further investigated in a high performance bioreactor under anaerobic conditions and compared with Y-WT. Y-ARSdR produced ethanol at 7.02 $\mathrm{g} / 1$ with a yield of $0.46 \mathrm{~g}$ of ethanol/g of total consumed sugars ('figure 3'). The yield of ethanol from consumed sugars reached $90 \%$ of the theoretical yield and xylitol excretion was below $0.35 \mathrm{~g} / \mathrm{l}$. Xylitol yield was calculated at $0.2 \mathrm{~g} / \mathrm{g}$ of consumed sugars. Glucose was completely fermented in $15 \mathrm{~h}$. Of the xylose, $68 \%$ was consumed in 72 h. A small amount of glycerol $(0.28 \mathrm{~g} / \mathrm{l})$ and acetic acid $(0.27 \mathrm{~g} / \mathrm{l})$ was produced mainly during the glucose consumption phase. When compared with Y-WT, YARSdR showed $86 \%$ decrease of unfavorable xylitol excretion with $41 \%$ increased ethanol production.

\section{Intracellular coenzyme levels}

We measured the intracellular concentrations of $\mathrm{NADH}$, $\mathrm{NAD}^{+}, \mathrm{NADPH}$, and NADP ${ }^{+}$in cells of recombinant strains in the late exponential growth phase ('table 2'). No significant differences in the concentrations of the four coenzymes were observed between samples in early, middle and late exponential growth phases (data not shown). Intracellular redox states were estimated by the ratios of $\mathrm{NADH} / \mathrm{NAD}^{+}$and NADPH/NADP ${ }^{+}$(referred to as $\mathrm{R}_{\mathrm{NAD}(\mathrm{H})}$

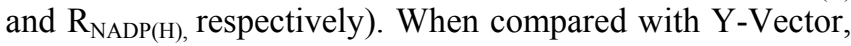
the $\mathrm{R}_{\mathrm{NADP}(\mathrm{H})}$ of $\mathrm{Y}-\mathrm{WT}$ was threefold higher, while $\mathrm{R}_{\mathrm{NAD}(\mathrm{H})}$ was twofold less. It is noteworthy that Y-ARSdR has $\mathrm{R}_{\mathrm{NAD}(\mathrm{H})}$ and $\mathrm{R}_{\mathrm{NADP}(\mathrm{H})}$ values more similar to $\mathrm{Y}$-Vector than does $\mathrm{Y}-\mathrm{WT}$, indicating that the NADP preferring mutation in PsXDH led to maintenance of redox balance in yeast cells.
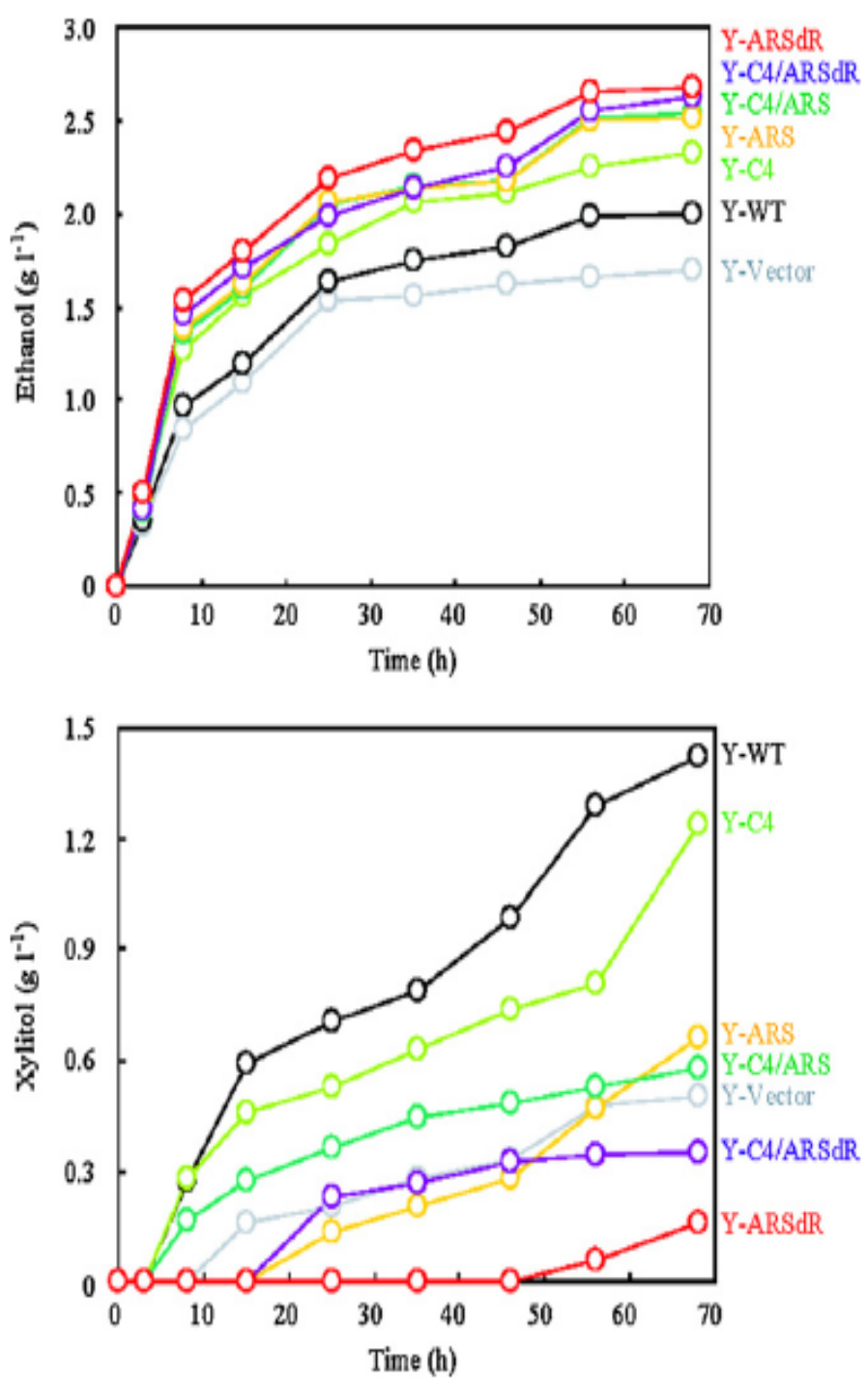

Figure 2. Time dependent ethanol production (upper panel) and xylitol excretion profiles (bottom panel) of recombinant yeasts grown in shaker flasks

\section{DISCUSSION}

We applied the protein engineering to generate enzymes with completely reversed coenzyme specificity and developed recombinant yeasts containing those engineered enzymes for construction of an efficient biomass-ethanol conversion system. Most interesting insight in this study is to estimate the effect modifications of PsXDH (in vitro) [16] on fermentation of xylose to ethanol (in vivo). One of functional modifications in $\mathrm{PsXDH}$ is thermostabilization by introduction of an structural zinc ion. Three thermostable mutants containing this $\mathrm{C} 4$ mutation (C4, C4/ARS and $\mathrm{C} 4$ /ARSdR) were expressed much more highly than each parent enzyme in $S$. cerevisiae cells, similar to in E. coli cells. Walfridsson et al. [5] reported that the overexpression of XDH in S.cerevisiae by a "promoter-modification" could prevent xylitol formation, although only a slight enhancement of ethanol yield was achieved. 


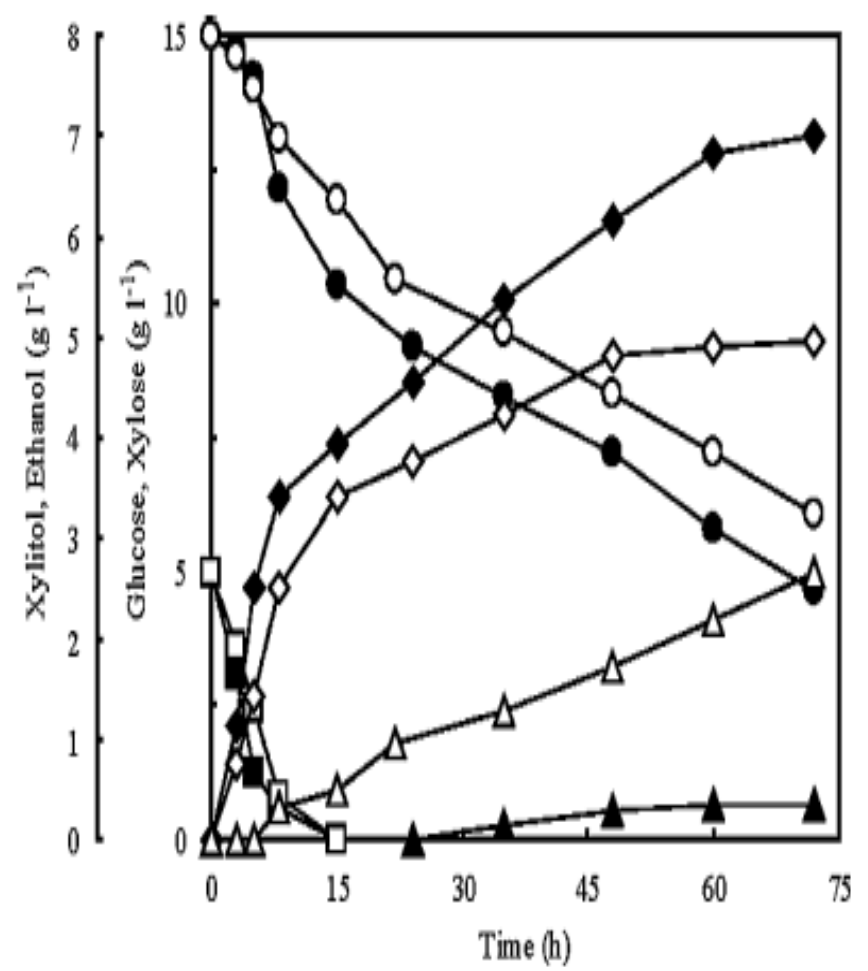

Figure 3. Time dependent xylose (circle) and glucose (square) utilization and ethanol (rhomboid), and xylitol (triangle) concentration profiles of Y-WT (open symbol) and Y-ARSdR (closed symbol) grown in a bioreactor. Small amount of glycerol and acetic acid were not shown (see in text)

Table 2. Intracellular concentration of coenzymes in recombinant yeast cells

\begin{tabular}{|l|l|l|l|l|l|l|}
\hline \multirow{2}{*}{ Strain } & \multicolumn{3}{|l|}{ Intracellular concn ( $\mu$ mol/g dry weight of biomass) } & \multirow{2}{*}{$\mathrm{R}_{\text {NADH }}{ }^{*}$} & \multirow{2}{*}{$\mathrm{R}_{\text {NADPHI }}$} \\
\cline { 2 - 5 } & NAD $^{+}$ & NADH & NADP $^{+}$ & NADPH & & \\
\hline Y-Vector & $6.58 \pm 0.11$ & $3.19 \pm 0.29$ & $3.41 \pm 0.18$ & $5.24 \pm 0.01$ & $0.49 \pm 0.01$ & $1.54 \pm 0.08$ \\
\hline Y-WT & $8.26 \pm 0.16$ & $2.69 \pm 0.08$ & $1.68 \pm 0.04$ & $8.24 \pm 0.14$ & $0.33 \pm 0.01$ & $4.92 \pm 0.18$ \\
\hline Y-ARSdR & $6.45 \pm 0.13$ & $3.02 \pm 0.01$ & $5.55 \pm 0.04$ & $7.53 \pm 0.19$ & $0.47 \pm 0.01$ & $1.36 \pm 0.04$ \\
\hline
\end{tabular}

*Ratio of $\mathrm{NADH} / \mathrm{NAD}^{+}$

qRatio of NADPH/NADP ${ }^{+}$

The similar tendency is observed between Y-WT and Y$\mathrm{C} 4$, while such as positive effect is not found in Y-C4/ARS and Y-C4/ARSdR, compared with Y-ARS and Y-ARSdR. Another functional modification of $\mathrm{PSXDH}$ in this study is to shift coenzyme specificity toward $\mathrm{NADP}^{+}$. Although both Y-ARS and Y-ARSdR mutants show similar reversal coenzyme specificity toward $\mathrm{NADP}^{+}$(under coenzymesaturated condition in vitro), $\mathrm{K}_{\mathrm{m}}$ value for $\mathrm{NAD}^{+}$of $\mathrm{ARSdR}$ mutant is 13-fold higher than that of ARS mutant [16], which seems to be due to more efficient fermentation ability in Y-ARSdR (in particular, decrease of xylitol excretion), compared with Y-ARS. Intracellular concentration of coenzyme may optimize for $\mathrm{NADP}^{+}$by $\mathrm{XDH}$ in ARSdR: $22 \%$ decrease of $\mathrm{NAD}^{+}$and $230 \%$ increase of $\mathrm{NADP}^{+}$ compared those of WT. In summary, that the change of coenzyme dependency is more effective than the increase in enzyme activity by stabilization.
As described in the Introduction, an alternative strategy for maintaining intracellular redox balance is to utilize the xylose-fermenting pathway via XI. Recently, it was first reported that XI from the fungus Piromyces sp. strain E3 resulted in significant activity in $S$. cerevisiae cells [11]. However, comparison of xylose-fermenting ability by XR$\mathrm{XDH}$ - and XI-carrying recombinant $S$. cerevisiae strains revealed that the $\mathrm{XR}-\mathrm{XDH}$ xylose utilization pathway is much better than the XI pathway [24]. In this study, we introduced only exotic XYL1 and XYL2 genes into $S$. cerevisiae. On the other hand, in addition to the metabolic engineering described above, the introduction and overexpression of other endogenous genes, including xylulokinase $(X K)$, transketolase $(T K L 1)$, transaldolase (TAL1) and several hexose-transporter (HXT1-7) genes, have been attempted to enhance the pentosephosphate pathway and/or xylose uptake [1]. Combined approaches of these strategies and our strategy using the proteinengineered enzyme should be considered to achieve more effective ethanol production from xylose by recombinant $S$. cerevisiae.

\section{ACKNOWLEDGMENT}

This work was supported by the the Ministry of Education, Science, Sports and Culture, Japan.

\section{REFERENCES}

[1] Jeffries, T.W., and Jin, Y.S. 2004. Metabolic engineering for improved fermentation of pentoses by yeasts. Appl Microbiol Biotechnol. 63, 495-509.

[2] Jeffries, T. W. 1983. Utilization of xylose by bacteria, yeasts, and fungi. Adv. Biochem. Eng. Biotechnol. 27, 1-32.

[3] Kurtzman, C. P. 1994. Molecular taxonomy of the yeasts. Yeast. 10, $1727-1740$

[4] Jin, Y. S., Lee, T. H., Choi, Y. D., Ryu, Y. W., and Seo, J. H. 2000. Conversion of xylose to ethanol by recombinant Saccharomyces cerevisiae containing genes for xylose reductase and xylitol dehydrogenase from Pichia stipitis. J. Microbiol. Biotechnol. 10, 564-567.

[5] Walfridsson, M., Anderlund, M., Bao, X., and Hahn-Hägerdal, B. 1997. Expression of different levels of enzymes from the Pichia stipitis XYL1 and XYL2 genes in Saccharomyces cerevisiae and its effects on product formation during xylose utilization. Appl Microbiol Biotechnol. 48, 218-224.

[6] Verduyn, C., Van Kleef, R., Frank, J., Schreuder, H., Van Dijken, J. P., and Scheffers, W. A. 1985. Properties of the NAD(P)H-dependent xylose reductase from the xylose-fermenting yeast Pichia stipitis. Biochem. J. 226, 669-677.

[7] Rizzi, M., Harwart, K., Erlemann, P., Buithanh, N. A., and Dellweg, H. 1989. Purification and properties of the NAD -xylitoldehydrogenase from the yeast Pichia stipitis .5. J. Ferment. Bioeng. 67, 20-24.

[8] Chiang, L.-C., Gong, C.-S., Chem, L.-F., and Tsao, G. T. 1981. DXylulose fermentation to ethanol by Saccharomyces cerevisiae. Appl. Environ. Microbiol. 42, 284-289.

[9] Tantirungkij, M., Izuishi, T., Seki, T., and Yoshida, T. 1994. Fedbatch fermentation of xylose by a fast-growing mutant of xyloseassimilating recombinant Saccharomyces cerevisiae. Appl. Microbiol. Biotechnol. 41, 8-12.

[10] Walfridsson, M., Bao, X., Anderlund, M., Lilius, G., Bulow, L., and Hahn-Hägerdal, B. 1996. Ethanolic fermentation of xylose with Saccharomyces cerevisiae harboring the Thermus thermophilus xylA gene, which expresses an active xylose (glucose) isomerase. Appl. Envir. Microbiol. 62, 4648-4651.

[11] Kuyper, M., Harhangi, H.R., Stave, A.K., Winkler, A.A., Jetten, M.S., de Laat, WT., den Ridder, J.J., Op den Camp, H.J., van Dijken, J.P., and Pronk, J.T. 2003. High-level functional expression of a fungal xylose isomerase: The key to efficient ethanolic fermentation of xylose by Saccharomyces cerevisiae? FEMS Yeast Res. 4.1, 69-78.

[12] Ho, N.W.Y., Chen Z., and Brainard A.P. 1998. Genetically Engineered Saccharomyces Yeast Capable of Effective 
Cofermentation of Glucose and Xylose. App Environ Microbiol. 64,1852-1859.

[13] Eliasson, A., Christensson, C., Wahlbom, F.C., and Hahn-hägendal, B. 2000. Anaerobic Xylose Fermentation by Recombinant Saccharomyces cerevisia Carrying XYL1, XYL2, and XKS1 in Mineral Medium Chemostat Cultures. App Environ Microbiol. 66.8, 33813386.

[14] Jeppsson, M., Bengtsson, O., Franke, Katja., Lee, H., Hahn-Hägerdal, B., and Gorwa-Grauslund, M.F. 2005. The expression of a Pichid stipitis xylose reductase mutant with higher $\mathrm{K}_{\mathrm{M}}$ for NADPH increases ethanol production from xylose recombinant Saccharomyces cerevisiae. Biotechnol Bioeng.

[15] Metzger, M.H., and Hollenberg, C.P. 1995. Amino acid substitutions in the yeast Pichia stipitis xylitol dehydrogenase coenzyme-binding domain affect the coenzyme specificity. Eur J Biochem. 228.1, 50-54.

[16] Watanabe, S., Kodaki, T., and Makino, K. 2005. Complete Reversal of Coenzyme Specificity of Xylitol Dehydrogenase and Increase of Thermostability by the Introduction of Structural Zinc. J. Biol. Chem. 280, $10340-10349$

[17] Kurztman, C.P., 1994. Molecular taxonomy of the yeasts. Yeast. 10, $1727-1740$.

[18] Gietz, D., Jean, A.S., Woods, R.A., and Schiestl, R.H. 1992 Improved method for high efficiency transformation of intact yeast cells. Nucleic Acids Research. 20.6, 1425.

[19] Lowry, O.H., Rosebrough, N.J., Farr, A.L., and Randall, R.J. 1951 Protein measurement with the folin phenol reagent. J. Biol. Chem. 193, $265-275$.

[20] Bergmeyer, H. U. 1985. Nicotinamide-adenine dinucleotides and dinucleotide phosphates (NAD, NADP, NADH, NADPH). In Methods of Enzymatic Analysis, $3^{\text {rd }}$ edn, vol. VII. Weinheim: VCH.

[21] Kang, Y. S., Kane, J., Kurjan, K., Stadel, J. M., and Tipper, D. J. 1990. Effects of expression of mammalian $\mathrm{G} \alpha$ and hybrid mammalian-yeast $\mathrm{G} \alpha$ proteins on the yeast pheromone response signal transduction pathway. Mol. Cell. Biol. 10, 2582-2590.

[22] Amore, R., Kötter, P., Kuster, C., Ciriacy, M., and Hollenberg, C. P. 1991. Cloning and expression in Saccharomyces cerevisiae of the $\mathrm{NAD}(\mathrm{P}) \mathrm{H}-$ dependent xylose reductase-encoding gene $x y 11$ from the xylose-assimilating yeast Pichia stipitis. Gene, 109, 89-97.

[23] Nikawa, J., sass, P., and Wigler, M. 1987. Cloning and characterization of the low-affinity cyclic AMP phosphodiesterase gene of Saccharomyces cerevisiae. Mol. Cell. Biol. 7, 3629-3636.

[24] Karhumaa, K., Sanchez, R. G., Hahn-Hägerdal, B., and GorwaGrauslund, M. F. 2007. Comparison of the xylose reductase-xylitol dehydrogenase and the xylose isomerase pathways for xylose fermentation by recombinant Saccharomyces cerevisiae. Microb Cell Fact, 6, 5 . 


\title{
Fuel Penalty from Periodic Rich Combustion of a Diesel Fuelled Engine during Lean $\mathrm{NO}_{\mathrm{X}}$ Trap Purging
}

\author{
Ahmad J. Alimin, Stephen F. Benjamin and Carol A. Roberts
}

\begin{abstract}
Compression ignition engines produce excessive $\mathrm{NO}_{\mathrm{X}}$ emissions compare to spark ignition engines. Lean $\mathrm{NO}_{\mathrm{X}}$ trap system is currently being investigated as an aftertreatment device to suppress the $\mathrm{NO}_{\mathrm{X}}$ emissions from compression ignition engines. The application of this aftertreatment device requires alternating lean and rich exhaust gas mixture, in order to produce the necessary reducing agents necessary for purging the LNT system. In this study an engine testbed was set-up which consisted of a 4-cylinder light-duty diesel engine, a diesel oxidation catalyst (DOC) and an LNT system. The LNT system purging utilised in-cylinder enrichment method based on DSPACE system, which controlled the engine management system (EMS) and the main engine operating parameters. The enrichment method used open-loop control system, to provide different storage/purge cycles for the LNT system. During enrichment, extra fuelling was needed to produce the required rich exhaust mixture. Emissions test at low operating temperature using this method had shown the capability of this in-cylinder enrichment method to produce the required periodic rich exhaust mixture for the LNT system. Nevertheless, the occurrences of periodic rich combustion also led to the increase in fuel penalty. Therefore, an acceptable trade-off between emissions reduction and fuel penalty is necessary.
\end{abstract}

Keywords: diesel engine, $\mathrm{NO}_{\mathrm{X}}$ emissions, lean $\mathrm{NO}_{\mathrm{X}}$ trap, incylinder enrichment method, fuel penalty

\section{INTRODUCTION}

Diesel engines operate under lean conditions and reduction of $\mathrm{NO}_{\mathrm{X}}$ to $\mathrm{N}_{2}$ is difficult due to the presence of excess $\mathrm{O} 2$ in the exhaust stream [1]. Various methods of reducing $\mathrm{NO}_{\mathrm{X}}$ emissions have been attempted and among them are development of systems to improve fuel mixture and combustion, and development of new post-combustion treatment devices [2]:

Dr. A. J. Alimin is with the Department of Automotive and Plant Engineering, Faculty of Mechanical and Manufacturing Engineering, Universiti Tun Hussein Onn Malaysia, 86400 Parit Raja, Batu Pahat, Johor (Phone: +6074537868; fax: +6074536080; e-mail: ajais@uthm.edu.my).

Prof. S. F. Benjamin is the Director of Automotive Engineering Applied Research Group, Faculty of Engineering and Computing, Coventry University, Priory Street, Coventry CV1 5FB, UK (e-mail: s.benjamin@coventry.ac.uk).

Dr. C. A. Roberts is a Research Fellow with the Automotive Engineering Applied Research Group, Faculty of Engineering and Computing, Coventry University, Priory Street, Coventry CV1 5FB, UK (e-mail: carol.roberts@coventry.ac.uk).
Lean $\mathrm{NO}_{\mathrm{X}}$ trap (LNT) is one of the post-combustion treatment devices that can treat $\mathrm{NO}_{\mathrm{X}}$ emissions, as it has certain advantages over other diesel after-treatment devices such as selective catalytic reduction (SCR) and catalysed diesel particulate filter [3], [4]. However, the LNT system requires periodic regeneration under all driving condition to prolong the performance and durability of the trap, as it has a finite trapping capability [5]. Nevertheless, LNT system is sulphur sensitive and it is difficult to achieve rich exhaust mixture that is necessary for regenerating the trap. Previous study has shown that the cost of LNT for diesel fuelled vehicles is much higher than the SCR technology and therefore, optimum system design is critical [6].

LNT catalysts are typically composed of Pt-group metal, which plays an important role in the reduction-oxidation process and a basic adsorbent or base-metal-oxide (BMO) that is responsible for providing the storage capacity. The chemical reactions that occur on the LNT catalyst are very complex and involve the reaction of acidic gas (nitrogen dioxide- $\mathrm{NO}_{2}$ ) with the BMO to form nitrate or nitrosospecies on the surface of the catalyst, desorption of $\mathrm{NO}_{\mathrm{X}}$ during regeneration and reduction with $\mathrm{CO}$ or $\mathrm{H} 2$ [1], [4], [7].

The operating factors that can influence the LNT performance, apart from the combinations of the Pt-group metals, are: the composition of the exhaust gas during lean and rich conditions; corresponding air-fuel ratios; exhaust gas temperatures; and also the duration of the lean and rich cycles [7]-[12].

For the LNT system to operate effectively, it requires optimisation of key engine operating parameters as part of the reduction process, since there are insufficient reducing agents in the rich pulse that are able to completely reduce the $\mathrm{NO}_{\mathrm{X}}$ levels. Integrated control of exhaust gas recirculation (EGR) and turbocharging has been shown to augment the reduction of $\mathrm{NO}_{\mathrm{X}}$ emissions [13]. It has been reported that the recommended desorption and reduction of $\mathrm{NO}_{\mathrm{X}}$ is when the exhaust lambda $(\lambda)$ value is around $0.87-$ 0.90 , where higher $\mathrm{CO}$ levels give shorter desorption times [14]. Previous study, on the expected emission control technologies that will be implemented in the coming years, has indicated that LNT will play an important role in curbing the $\mathrm{NO}_{\mathrm{X}}$ emissions, especially for the application in light-duty vehicles [17].

In this paper, the results of exhaust emissions and fuel 
penalty from periodic rich combustion experiments of an LNT system, using in-cylinder enrichment method are presented.

\section{EXPERIMENTAL SET-UP}

In the experimental works, a 4-cylinder diesel engine was used, equipped with a common rail injection system, an EGR system, and an intake throttle body. The EMS, which was comprised an engine control unit (ECU) and an injection control unit (ICU), and the throttle body were connected to the DSPACE control tool to enable the generation of the periodic rich combustion. The EMS was also connected to a GREDI system that served as the calibration tool. Throughout the tests, the engine used a very low sulphur diesel fuel. The engine specification is given in 'table 1 '.

Table 1. Specifications of the test engine

\begin{tabular}{|l|l|}
\hline \multicolumn{1}{|c|}{ Items } & \multicolumn{1}{c|}{ Description } \\
\hline Engine capacity & $1998 \mathrm{cc}$ \\
\hline Rated power output & $96 \mathrm{~kW}$ at $3800 \mathrm{rpm}$ \\
\hline Rated torque & $330 \mathrm{Nm}$ at $1800 \mathrm{rpm}$ \\
\hline
\end{tabular}

The turbo outlet of the engine was linked to an exhaust aftertreatment test rig that consisted of a long diffuser followed by a flow straightener upstream of a diesel oxidation catalyst (DOC) and an LNT system. Both the DOC and LNT used Pt-group metal as the main catalytic compounds. Figure 1 shows the layout for the experimental set-up and the details of the sampling points on the exhaust aftertreatment test rig are illustrated in 'figure 2'.

The control algorithm set-up within the DSPACE system allowed the control on the intake throttle body, EGR, fuel injection quantities and timings for each of the Pilot, Main and Post injections. In addition to that, it can also produce different cyclic regeneration sets (different durations of alternating lean and rich operations), even though only in open-loop condition. For characterising the exhaust emissions, CAMBUSTION fast response analysers were used during each storage and regeneration phases, with response time of less than 10 milliseconds. Data logging for all the measurements from the engine and the emissions analysers was performed concurrently at frequency of 50 $\mathrm{Hz}$, using Froude-Consine TEXCEL data logger.

In this research work, the experiment was conducted under steady-state condition, at an engine speed of 1500 $\mathrm{rpm}$ and a torque setting of $48 \mathrm{Nm}$. Emissions were sampled after the engine temperature and the catalyst beds temperatures had approximately stabilised. The exhaust gas temperature was around $250-280{ }^{\circ} \mathrm{C}$. The lean and rich durations, for trap storage and regeneration, were set at 60 seconds and 6 seconds respectively. The results from this test are then compared with the findings from previous work.

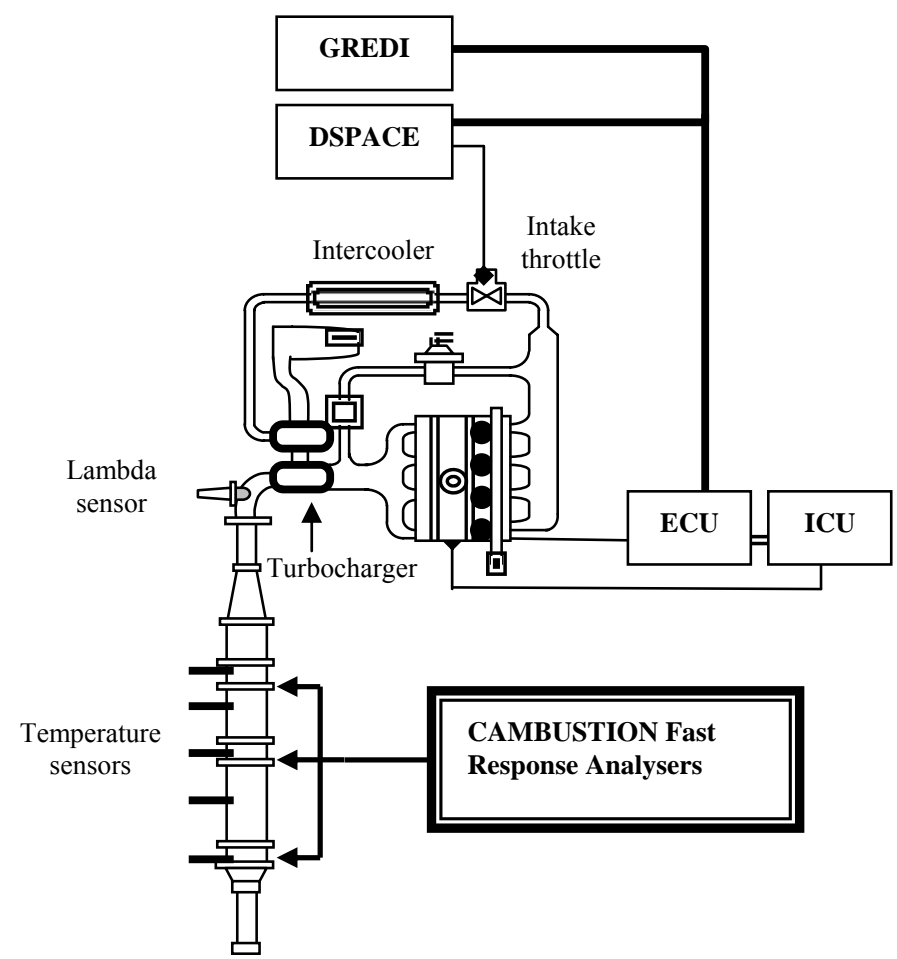

Figure 1. Schematic of the system set-up.

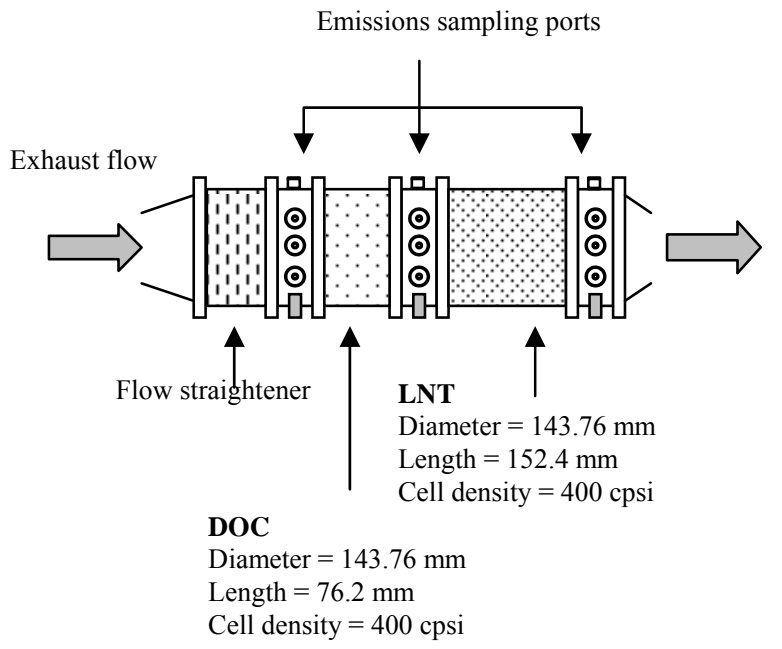

Figure 2. Details of the LNT test rig

\section{RESUlTS AND DisCUSSION}

'Figure 3' shows a series of $\mathrm{NO}_{\mathrm{X}}$ storage and purging cycles (or lean and rich cycles), which are repetitive and indicate the capability of the in-cylinder enrichment system to periodically purge the LNT system. The LNT purging events are indicated by the cyclic changes of the exhaust lambda values. 


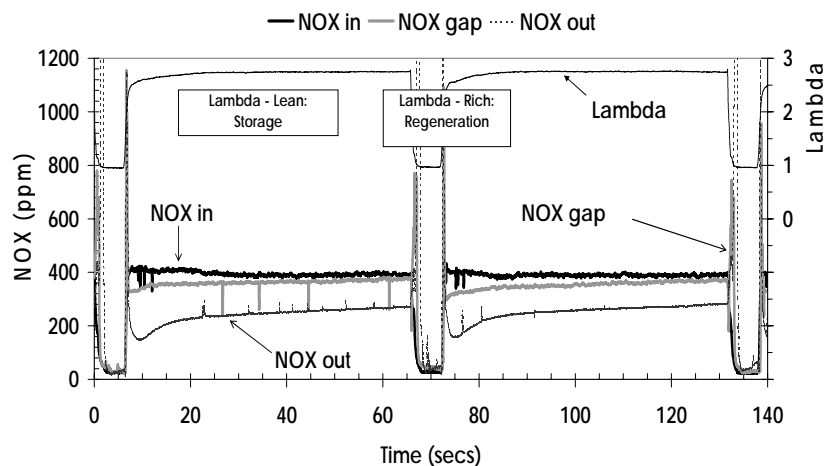

Figure 3. LNT storage and regeneration cycles (peaks $\mathrm{NO}_{\mathrm{X}}$ at out are truncated).

The individual plots for $\mathrm{NO}, \mathrm{NO}_{2} \mathrm{CO}$ and $\mathrm{CO}_{2}$ emissions, during storage and regeneration, are shown respectively in 'figures 4, 5, 6 and 7'.

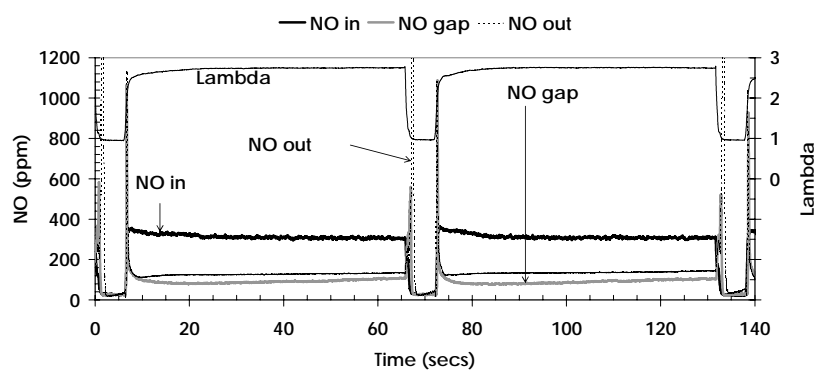

Figure 1. NO emissions during storage and regeneration (peaks NO at out are truncated)

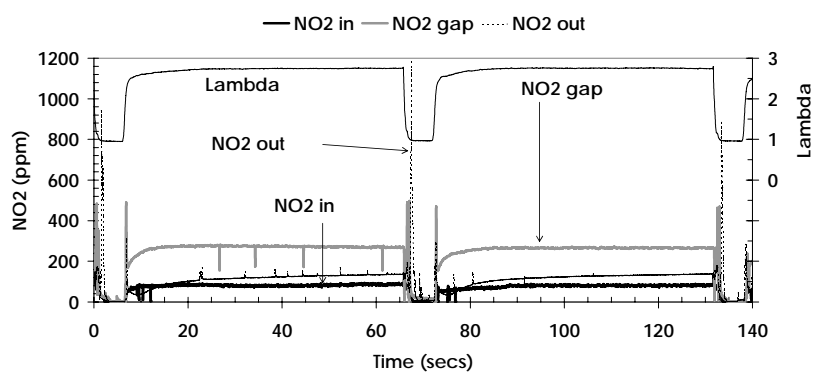

Figure 2. $\mathrm{NO}_{2}$ emissions during storage and regeneration (peaks $\mathrm{NO}_{2}$ at out are truncated)

Immediately after purging, $\mathrm{NO}_{\mathrm{X}}$ emissions after the LNT increased steadily before starting to stabilise after around 15 seconds, as the trap was started to fill. On average the amount of $\mathrm{NO}_{\mathrm{X}}$ emitted by the engine during lean operation was around 430-450 ppm and consisted mostly of NO (see figure 4). During the storage period, almost half of the total $\mathrm{NO}_{\mathrm{X}}$ that went into the LNT system, around 200-250 ppm, was successfully stored.

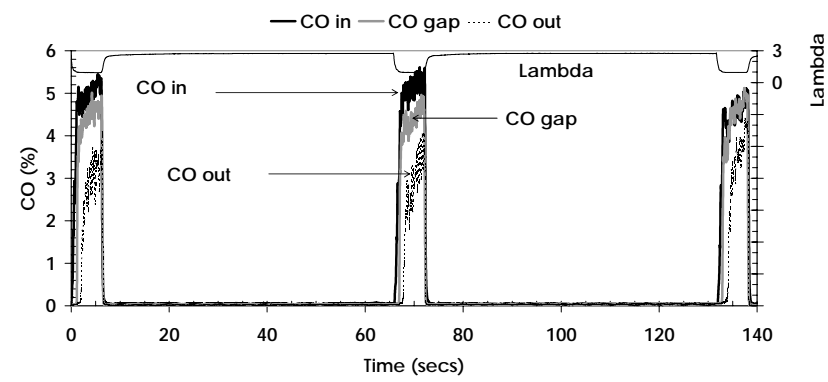

Figure 3. CO emissions during storage and regeneration

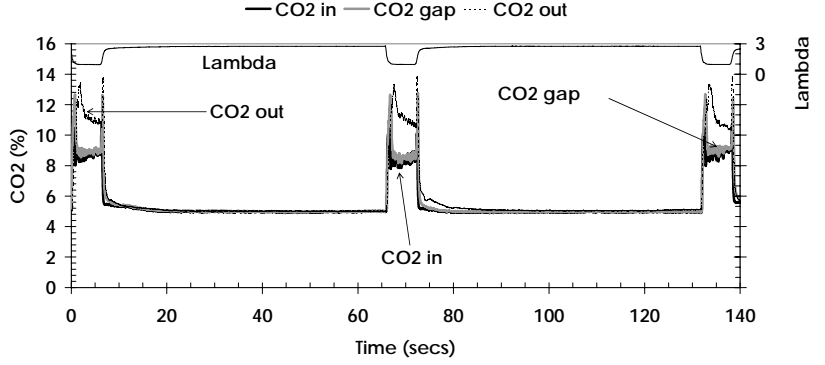

Figure 4. $\mathrm{CO}_{2}$ emissions during storage and regeneration

Referring to the $\mathrm{NO}$ and $\mathrm{NO}_{2}$ plots, ('figures 5 and 6') all the individual plots shown are repetitive, which indicate the capability of the LNT system to store and reduce the incoming $\mathrm{NO}_{\mathrm{X}}$ emissions. Although there are some cycle to cycle variations, similar features are repeatable and identifiable during the storage and purging events. The large amount of $\mathrm{NO}$ from the engine was oxidised into $\mathrm{NO}_{2}$ by the DOC as shown by the increase of $\mathrm{NO}_{2}$ to around 280-300 ppm and reduction of NO to around 100-120 ppm, measured at the gap (post DOC before LNT). The lower $\mathrm{NO}_{2}$ trace observed post LNT suggests that almost all the incoming $\mathrm{NO}_{2}$ from the DOC has been stored by the LNT during the lean period, whereas, the LNT was not storing the NO emission from the DOC in both cases, as the levels for both the NO inside the gap and at out (post LNT) are almost similar. Hence, the DOC proved its capability to oxidise the NO from the engine and the LNT functioned by storing mainly the $\mathrm{NO}_{2}$ emission during the lean period.

During the regeneration events, two significant $\mathrm{NO}_{\mathrm{X}}$ breakthroughs or spikes were observed after the LNT (refers to figure 3 ). These were observed at the start and the end of every regeneration period, during the changeover from rich to lean and vice versa, and were present in every cycle. The $\mathrm{NO}_{\mathrm{X}}$ breakthroughs consisted mainly of $\mathrm{NO}$, rather than the $\mathrm{NO}_{2}$. Theis et al. [12] stated that at operating temperatures around $250{ }^{\circ} \mathrm{C}$, the $\mathrm{NO}_{\mathrm{X}}$ release can be ascribed to low $\mathrm{NO}_{\mathrm{X}}$ reduction activity. The existences of these $\mathrm{NO}_{\mathrm{X}}$ spikes during regeneration were not detected in previous studies on LNT, for example in the studies from Theis et al. [12], Bögner et al. [15] and Li et al. [16].

Throughout the lean period, the CO level was very low and only increased drastically during the regeneration period (refers to figure 6). $\mathrm{CO}$ emissions were much lower after the LNT than after the DOC during regeneration, and the $\mathrm{CO}$ was partially consumed at the beginning of the regeneration period before starting to increase again, although not reaching the same level as the incoming $\mathrm{CO}$ from the DOC. The significant consumption of $\mathrm{CO}$ emissions during the regeneration indicates that the $\mathrm{CO}$ acts as a primary reductant for the purged $\mathrm{NO}_{\mathrm{X}}$. This is comparable to the findings from the study by West et al. [17], as well as previous lab-scaled studies on the roles of $\mathrm{CO}$ as the reducing agent by Abdulhamid et al. [18].

$\mathrm{CO}_{2}$ emissions were almost equal during the lean period at each sampling point, before they started to rise only during the rich period; see 'figure 7'. The increase of $\mathrm{CO}_{2}$ emissions at the beginning of the regeneration period could be associated to the partial consumption of $\mathrm{CO}$ and at that particular time. $\mathrm{CO}_{2}$ emissions began to drop after that time and started to increase again as the combustion mixture 
started to switch from rich to lean.

Fuel usage analysis during lean, rich and in a complete lean/rich cycle for the test condition described above (60L6R) and test at 60 seconds lean and 3 seconds (60L3R), which has been described previously [19], are depicted in 'figure 8'. In this figure, the term 'Fuel consumed_1 cycle' refers to the combined fuel consumption during lean and rich periods for a particular lean/rich cycle. As can be seen, the total amount of fuel being consumed was also increased as the regeneration period became longer.

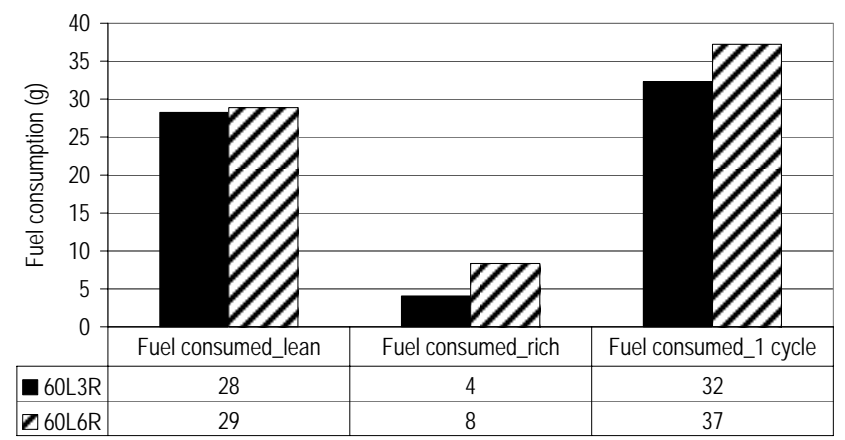

Figure 5. Fuel consumption during lean, rich and a complete lean/rich cycle under different test conditions

Using the information on 'figure 8', an analysis on the fuel penalty for each test condition was performed and the results are as shown in 'figure 9'. The fuel penalty is defined as the ratio between the additional fuel consumption due to regeneration during a complete lean/rich cycle duration to the total fuel consumption during the same duration but with the engine running in lean condition only. The effect of periodic lean/rich cycle on the overall engine fuel conversion efficiency (or thermal efficiency) is also illustrated in 'figure 9'. In this analysis, the term 'Fuel conv eff-lean' refers to the fuel conversion efficiency of the engine during the storage period and was calculated based on the fuel specific consumption when the engine was running lean only. Similarly, the term 'Fuel conv eff-rich' indicates the fuel conversion efficiency during regeneration only and was calculated using the fuel specific consumption of the engine when it was running rich.

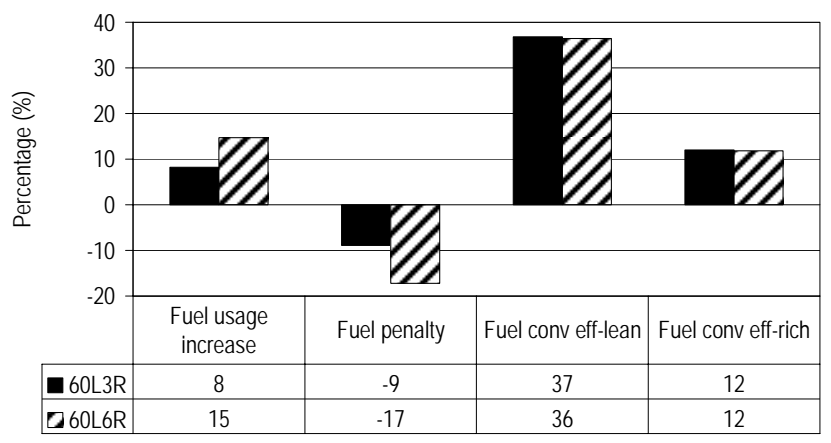

Figure 6 . The percentage fuel usage increase, fuel penalty and engine fuel conversion efficiency due to regeneration for a complete storage/regeneration cycle under different test conditions

From the figure above, significant increase in fuel usage was observed for both tests, 60L3R and 60L6R, which caused fuel penalty of $9 \%$ and $17 \%$ respectively. During lean the engine produced comparable fuel conversion efficiency for all test conditions, between $34-38 \%$ during lean and around $11-12 \%$ during rich. The addition of a regeneration stage caused the engine fuel conversion efficiency to drop. This was due to the fact that the periodic in-cylinder enrichment system for the emission tests used high amount of fuel to purge the LNT.

The results from the tabulated data can be interpreted together with their LNT system instantaneous trapping efficiencies, shown in 'figure 10'.

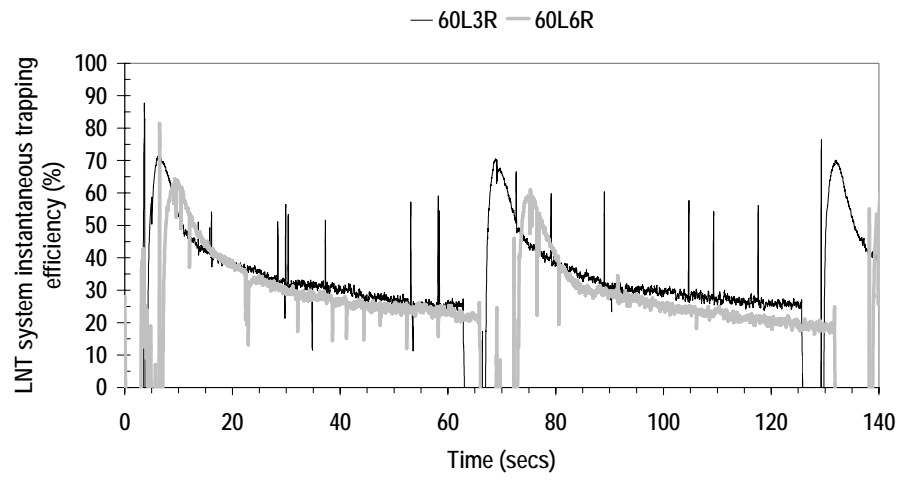

Figure 7. LNT system instantaneous trapping efficiency for 60L3R and 60L6R tests

From 'figure 10', both emission tests were operating at system trapping efficiency of within $20-70 \%$. The trapping efficiency was the highest immediately after regeneration before it began to drop gradually with time, during the storage period. Although large amount of fuel was added during the regeneration period in test $60 \mathrm{~L} 6 \mathrm{R}$, the system trapping efficiency was still at a comparable level with the system trapping efficiency of test 60L3R. This would indicate that adding extra fuel or increasing the fuel injection duration would not necessarily improve the LNT system trapping performance.

\section{CONCLUSION}

The experimental results have shown the ability of the incylinder enrichment method to provide periodic lean and rich combustion, required for the operation of the LNT system. The approach used in developing the in-cylinder enrichment technique for this study, which was based on the fuel injections properties and intake air throttling, was only one of many possible options for generating rich combustion but was used because it was the simplest.

The fast response emissions analysers had also effectively displayed the detail of the events that occurred during the storage and regeneration periods, particularly during the lambda changeover, either from lean to rich or from rich to lean.

From those emissions results, it can be confirmed that the $\mathrm{NO}_{\mathrm{X}}$ storage and reduction process had been successfully carried out using the developed in-cylinder enrichment method. Nevertheless, fuel penalty analysis has implied that if the in-cylinder enrichment technique was chosen to purge the LNT system, appropriate fuel injection properties must be used. This is important to avoid excessive loss in the fuel conversion efficiency, as well as to achieve the acceptable trade-off between LNT system performance and fuel penalty. 
It is recommended that future work in this area, should involve further experiments under different engine operating conditions.

\section{ACKNOWLEDGMENT}

The authors would like to thank ArvinMeritor, Ford, Jaguar Land Rover and Johnson Matthey, for their technical and financial support.

\section{REFERENCES}

[1] Epling, William S., Parks, James E., Campbell, Greg C., Yezerets, Aleksey, Currier, Neal W. and Campbell, Larry E. (2004) - Further evidence of multiple $N O_{X}$ sorption sites on $N O_{X}$ storage/reduction catalysts, Catalysis Today, 96, 21-30, available online www.elsevier.com/locate/cattod.

[2] Armas, O; Baltesteros, R; Martos, F.S. and Agudelo, J.R. (2004) Characterization of light duty diesel engine pollutant emissions using water-emulsified fuel, Fuel, Vol. 84, Number 7-8, available online www.sciencedirect.com.

[3] West, Brian H. and Sluder, C. Scott (2000) - NO $O_{X}$ adsorber performance in a light-duty diesel vehicle, SAE2000-01-2912, International Fall Fuels and Lubricants, Meeting and Exposition, Baltimore, Maryland, 16-19 ${ }^{\text {th }}$ October 2000.

[4] Fang, Howard L.; Huang, Shyan C.; Yu, Robert C., Wan, C.Z. and Howden, Ken (2002) - A fundamental consideration on $\mathrm{NO}_{X}$ adsorber technology for diesel application, SAE2002-01-2889, Powertrain and Fluid Systems, Conference and Exhibition, San Diego, California, USA, 21-24 ${ }^{\text {th }}$ October 2002.

[5] Guyon, M.; Blanche P.; Bert, C.; Phillippe, L. and Messaoudi, I. (2000) - $\mathrm{NO}_{X}$-Trap system development and characterization for diesel engines emission control, SAE 2000-01-2910, SAE International Fall Fuels and Lubricants, Meeting and Exhibition, Baltimore, USA, 16-19 ${ }^{\text {th }}$ October 2000.

[6] Warren, James P. (2001) - Diesel aftertreatment systems: 2004-2007, A Cost Assessment, SAE2001-01-1948, International Spring Fuels and Lubricants, Meeting and Exhibition, Orlando, Florida, 7-9 2001.

[7] Olsson, Louise; Fridell, Erik; Skoglundh, Magnus and Andersson, Bengt (2002) - Mean field modelling of $\mathrm{NO}_{X}$ storage on $\mathrm{Pt} / \mathrm{BaO} / \mathrm{Al}_{2} \mathrm{O}_{3}$, Catalysis Today, Vol. 73, pp263-270.

[8] Takahashi, Naoki; Shinjoh, Hirofumi; Ijima, Tomoko; Suzuki, Tadashi; Yamazaki, Kiyoshi; Yokota, Koji; Suzuki, Hiromasa; Miyoshi, Naoto; Matsumoto, Shin-ichi; Tanizawa, Tsuneyuki; Tanak, Toshiaki; Tateishi, Syu-shi and Kasahara, Kouichi (1996) - The new concept 3-way catalyst for automotive lean-burn engine: $N O_{X}$ storage and reduction catalyst, Catalysis Today, Vol. 27, pp 63-69.

[9] Kobayashi, Takeshi; Yamada, Teiji and Kayano, Kunihide (1997) Study of $N O_{X}$ trap reaction by thermodynamic calculation, SAE970745, SAE International Congress and Exposition, Detroit, Michigan, USA, 24-2 $7^{\text {th }}$ February 1997.

[10] Fridell, Erik; Skoglundh, Magnus; Westerberg, Björn; Johansson and Smedler, Gudmund (1999) - NOX storage in barium-containing catalysts, Journal of Catalysis, Vol. 183, pp196-209.

[11] Mahzoul, H.; Brilhac; J.F. and Gilot, P. (1999) - Experimental and mechanistic study on $\mathrm{NO}_{X}$ adsorption over $N \mathrm{O}_{X}$ trap catalysts, Applied Catalysis B: Environmental, Vol. 20, pp47-55.

[12] Theis, Joseph R.; Ura, Justin A.; Li, John J.; Surnilla, Gopichandra G.; Roth, John M. and Goralski Jr., Christian T. (2003) - NOX release characteristics of lean no $o_{x}$ traps during rich purges, SAE2003-011159, SAE World Congress, Detroit, Michigan, USA, 3-6 $6^{\text {th }}$ March 2003.

[13] Capobianco, M. (2001) - Optimum control of an automotive direct injection diesel engine for low exhaust emissions, Proc. Inst. Mech. Engineers-Part D, Journal of Automobile Engineering, Vol. 215, 2001

[14] Johnson, Timothy V. (2002) - Diesel emission control: 2001 in Review, SAE2002-01-0285, SAE 2002 World Congress, Detroit, Michigan, 4-7 ${ }^{\text {th }}$ March, 2002.

[15] Bögner, W.; Krämer, M.; Krutzsch, B.; Pischinger, S.; Voigtländer, D.; Wenninger, G.; Wirbeleit, F.; Brogan, M.S.; Brisley, R.J. and Webster, D.E. (1995) - Removal of nitrogen oxides from the exhaust of a lean-tune gasoline engine, Applied Catalysis B: Environmental, Vol. 7, pp153-171.

[16] Li, Yuejin; Roth, Stan, Yassine, Mahmoud, Beutel, Tilman, Dettling, Joe and Sammer, Chris (2000) - Study of factors influencing the performance of a $\mathrm{NO}_{X}$ trap in a light-duty diesel vehicle, SAE2000-
01-2911, SAE International Fall Fuels and Lubricants, Meeting and Exhibition, Baltimore, USA, 16-19 $9^{\text {th }}$ October 2000.

[17] West, Brian; Huff, Shean; Parks, James; Lewis, Sam; Choi, Jae-Soon; Partridge, William and Storey, John (2004) - Assessing reductant chemistry during in-cylinder regeneration of diesel lean $\mathrm{NO}_{X}$ traps, SAE2004-01-3023, Powertrain and Fluid Systems Conference and Exhibition, Tampa, Florida USA, 25-28 $8^{\text {th }}$ October 2004.

[18] Abdulhamid, Hussam; Fridell, Erik and Skoglundh, Magnus (2005) The reduction phase in $\mathrm{NO}_{X}$ storage catalysis: Effect of type of precious metal and reducing agent, Applied Catalysis B: Environmental, Vol. 62, pp319-328.

[19] A. J. Alimin, C.A. Roberts and S. F. Benjamin (2006) - A NOX trap study using fast response emission analysers for model validation, SAE2006-01-0685, Special Publication, SP2023, Society of Automotive Engineers, April 2006. 


\title{
Pyrolysis Characteristics and Kinetics of Coconut Coir and Shell Mixtures with Sub- Bituminous Coal using Thermogravimetric Analysis
}

\author{
Azil Bahari Alias, Zulkifli Abdul Rashid, Norazah Abd Rahman, Siti Shawalliah Idris, Khudzir Ismail, \\ Mohd Azlan Mohd Ishak, and Wan Azlina W.Ab. Karim Ghani
}

\begin{abstract}
Combustion of coal contributes to $\mathrm{CO}_{2}$ emissions, a major cause of global warming. A means of reducing the $\mathrm{CO}_{2}$ emissions is by minimizing coal combustion by implementing co-firing of coal and biomass. Combustion of biomass alone emits less $\mathrm{CO}_{2}$, but the energy produced is less than that generated by coal alone. Hence, co-firing of coal with biomass is an excellent compromise between $\mathrm{CO}_{2}$ emissions and energy production. Thermal behaviour during pyrolysis of coal, biomass materials (coconut coir and shell) and coal/biomass blends prepared at different weight ratios $(80: 20,50: 50$ and $20: 80$ ) were studied by thermogravimetry (TG) at different heating rates $\left(20,40\right.$ and $\left.60{ }^{\circ} \mathrm{C} / \mathrm{min}\right)$. The thermal events of coconut shell and coconut coir during pyrolysis were mainly contributed by hemicellulose and cellulose. The thermal events of coal/coconut shell and coal/coconut coir blend during pyrolysis, however, showed the domination of coconut shell and coir pyrolysis at lower temperature, with the coal pyrolysis (i.e. volatile matter released) occurred at much higher temperature. Apparently, no interactions were seen between the coal, coconut shell and coconut coir during pyrolysis, indicating a general lack of synergistic effects. As such, the yield of the major pyrolysis products is linearly proportional to the weight ratio of biomass and coal in the blend mixture in an additive manner at a constant heating rate. Likewise, an increased in the coal reactivity was observed with increasing heating rates as the coal ratio increases in the blends. First order equations were used to determine the biomass and coal component thermal decomposition kinetics. The results, thus far, showed that the 50:50 wt\% blends, with respect to the coal volatile matter released, revealed the lowest activation energies of 83.7 and $85.0 \mathrm{~kJ} / \mathrm{mol}$ for coal/coconut shell and coal/coconut coir blend,
\end{abstract}

Azil Bahari Alias, Zulkifli Abdul Rashid, Norazah Abd Rahman and Siti Shawalliah Idris are with the University Technology MARA, Faculty of Chemical Engineering, 40450 Shah Alam, Selangor, Malaysia (phone: +603-55436342; fax: $\quad+603-55436300$; email: azilbahari@salam.uitm.edu.my).

Khudzir Ismail is with the University Technology MARA, Faculty of Applied Sciences, 02600 Arau, Perlis, Malaysia.

Mohd Azlan Mohd Ishak is with the University Technology MARA, Faculty of Applied Sciences, 02600 Arau, Perlis, Malaysia.

Wan Azlina W.Ab. Karim Ghani is with the University Putra Malaysia, Department of Chemical and Environmental Engineering, 43400 UPM, Serdang, Malaysia. respectively. These findings will provide useful data for power generation industries for the development of cofiring options using coal/biomass blends.

Keywords: pyrolysis, thermogravimetry, coconut coir and shell, coal

\section{INTRODUCTION}

Biomass energy (bioenergy) is derived from organic matter. Coconut coir and shell is an example of a source of biomass energy. Biomass resources such as agricultural residues (i.e. coconut coir and shell) abundant in most countries especially developing countries, and have strong potential as fuels for green power generation. In practice, about half of the agricultural residues are utilized for energy generation which contributes $20 \%$ of the primary energy demand industries. The role of biomass is presently limited in power development, but opportunities exist for increasing its share. It is estimated that by 2050 biomass could provide nearly $38 \%$ of the world's direct fuel use and $17 \%$ of the world's electricity [1].

Combustion of fossil fuels such as coal contributes to $\mathrm{CO}_{2}$ emission that can cause global warming effect to the environment. An alternative means of reducing the $\mathrm{CO}_{2}$ emission is by minimizing the combustion of coals by utilizing the co-firing of coal and biomass fuels which is wood waste. The rationale is that the thermal utilisation of biomass can contribute to the reduction of $\mathrm{CO}_{2}$ emissions as the same amount of $\mathrm{CO}_{2}$ is extracted from the atmosphere during the growth period of the plants as it is released by combustion $\left(\mathrm{CO}_{2}\right.$ balance). Hence, in order to achieve a significant $\mathrm{CO}_{2}$ reduction by substituting the fossil fuels, a large quantity of biomass is needed for power generation [2].

Pyrolysis of coal, biomass and coal/biomass blends is a relatively new area of research. Several researchers have investigated the pyrolysis and combustion behaviour of biomass, coal and coal/biomass blends [3,4,5]. Recently, Vamvuka, et. al., [6] developed a kinetic modeling for the volatile matter released during the pyrolysis of several biomass (i.e. olive kernel, forest and cotton residues) blends 
with lignite using thermogravimetry (TG). Their findings revealed that the biomass possess higher thermochemical reactivity with shorter devolatilisation times in comparison to the lignite. In another work, Moghtaderi, et.al. investigated the pyrolytic behaviour of coal/woody biomass (i.e. pine dust) blends over a wide range of heating rates and temperatures using tubular reactors in an attempt to simulate the pulverized fuel boilers [7]. Importantly, they found that the total yield of the major pyrolysis products were linearly proportional to the blending ratio, indicating no synergistic effect between coal and biomass. Likewise, Vuthaluru [8] also reported the same observation during the investigation on the thermal behaviour during co-pyrolysis of coal/biomass (i.e. wood waste and wheat straw) using thermogravimetry.

The aims of this work were to investigate the thermal behaviour of coal, coconut coir, coconut shell and coal/coconut coir and coal/coconut shell blends during pyrolysis process using thermogravimetry. The thermogravimetric technique was chosen to identify the thermal events occurred during pyrolysis and to obtain the kinetic data during the process. The effect of varying heating rates on the reactivity of coal and coconut coir and shell will be reported.

\section{MATERIALS AND METHODS}

\section{A. Coal, Coconut Coir and Coconut Shell Preparation}

Senakin sub-bituminous coal and biomass (coconut shell and coir) originated from Kalimantan, Indonesia and Selangor, Malaysia, respectively, were selected in this study. The samples were open air dried for 2 to 3 days to remove moisture and to ease crushing. These samples were pulverized into powder and were sieved into specific particle size of less than $212 \mu \mathrm{m}$. Sieving was accomplished by shaking the ground coal and biomass in a Endecotts Shaker Model EFL2 MK3 for 30 minutes and dried in a vacuum oven set at $80^{\circ} \mathrm{C}$ overnight and were kept in a tightly screw cap bottle.

\section{B. Instrumental Analysis}

The elemental analyses and calorific values of the coal, coconut coir and shell were performed using a Thermo Finnigan Flashed 1112 analyzer and Ika-works C5000 calorimeter, respectively. Blends of coal to coconut coir and shell were prepared in three different weight percents (i.e. 20:80, 50:50 and 80:20). The pyrolysis on the coal, coconut coir, coconut shell and their blends were performed using thermogravimetric analyser Metler Toledo/TGA/ SDRA51e under inert nitrogen gas at ambient pressure with constant flow-rate of $50 \mathrm{ml} / \mathrm{min}$, and at different heating rates of 20 , 40 , and $60^{\circ} \mathrm{C} / \mathrm{min}$ in the temperature range of $25-900^{\circ} \mathrm{C}$. The sample $(20 \mathrm{mg})$ was weighed directly into ceramic crucible and the temperature was kept isothermal for one minute until steady condition was obtained before ramping to the desired temperature. The experiments were replicated at least twice to obtain reproducibility. The ultimate and proximate analyses of the coal, coconut coir and coconut shell were presented in Table 1.

\section{RESULTS AND DISCUSSION}

\section{A. Thermal Decomposition of Pure Samples}

The TG and DTG curves for the thermal decomposition of coconut coir, shell and coal during pyrolysis at heating rate of $20^{\circ} \mathrm{C} / \mathrm{min}$ are shown in Figures 1 and 2, respectively. Apparently, the TG curves for coconut coir, shell and coal showed an initial decrease in the weight at temperature below $200^{\circ} \mathrm{C}$ that were due to the release of moisture remaining in the samples. This initial decrease in weight loss corresponds to the first curve in the differential weight loss (DTG) profiles for coconut coir, shell and coal as shown in Figures 1 and 2, respectively. Hence, this curve was not taken into consideration for the determination of thermal events in both wood waste and coal. The thermal events for the decomposition of coconut coir, shell and coal were defined as the region where the slope of TG curve is constant [2]. DTG profiles for the coconut shell decomposition revealed two major thermal events at temperature ranging from $275-320^{\circ} \mathrm{C}$ and $345-385^{\circ} \mathrm{C}$ that were due to volatile matter released from the degradation of hemicellulose and cellulose, respectively [9]. Similarly, DTG profiles for coconut coir also revealed two major thermal events at temperature ranging from $205-230^{\circ} \mathrm{C}$ and $295-395^{\circ} \mathrm{C}$ that were due also to volatile matter released from the degradation of hemicellulose and cellulose, respectively. On contrary, the DTG profiles for the coal decomposition showed only one major thermal event at temperature range of $380-580^{\circ} \mathrm{C}$ and was attributed to the released of coal volatile matter [2].

Table 1. Ultimate and proximate analyses of Senakin coal, coconut coir and coconut shell

\begin{tabular}{|c|c|c|c|}
\hline Fuel & $\begin{array}{c}\text { Senakin } \\
\text { coal }\end{array}$ & $\begin{array}{l}\text { Coconut } \\
\text { Coir }\end{array}$ & $\begin{array}{l}\text { Cocon } \\
\text { ut } \\
\text { shell }\end{array}$ \\
\hline $\begin{array}{l}\text { Proximate } \\
\text { analysis } \\
\text { (wt \% db) }\end{array}$ & & & \\
\hline Volatile matter & 29.2 & 68.4 & 66.6 \\
\hline Fixed carbon & 54.02 & 18.9 & 21.8 \\
\hline Ash & 14.78 & 2.2 & 1.6 \\
\hline Moisture & 2 & 10.5 & 10 \\
\hline $\begin{array}{l}\text { Ultimate analysis } \\
\text { (wt\% db) }\end{array}$ & & & \\
\hline Carbon & 70.02 & 30.3 & 42.3 \\
\hline Hydrogen & 8.5 & 5.3 & 5.4 \\
\hline Nitrogen & 2.21 & 0.2 & 0.01 \\
\hline Sulfur & 0.8 & 0.01 & 0.01 \\
\hline $\begin{array}{l}\text { Oxygen } \\
\text { (by different) }\end{array}$ & 18.47 & 64.2 & 52.3 \\
\hline $\begin{array}{l}\text { Calorific values } \\
\text { (MJ/kg) }\end{array}$ & 29.4 & 16.5 & 18 \\
\hline
\end{tabular}

Based on the method described previously [10], it was deduced that the DTG peak height, $\mathrm{R}$ and maximum peak temperature, Tmax is directly and inversely proportional to the reactivity, respectively. Hence, from the DTG profiles of coconut coir, shell and coal decompositions, it appears that 
the volatile matter released of the former occurred at lower peak temperature with higher peak height indicating the high reactivity of the biomass materials in comparison to coal during pyrolysis condition.
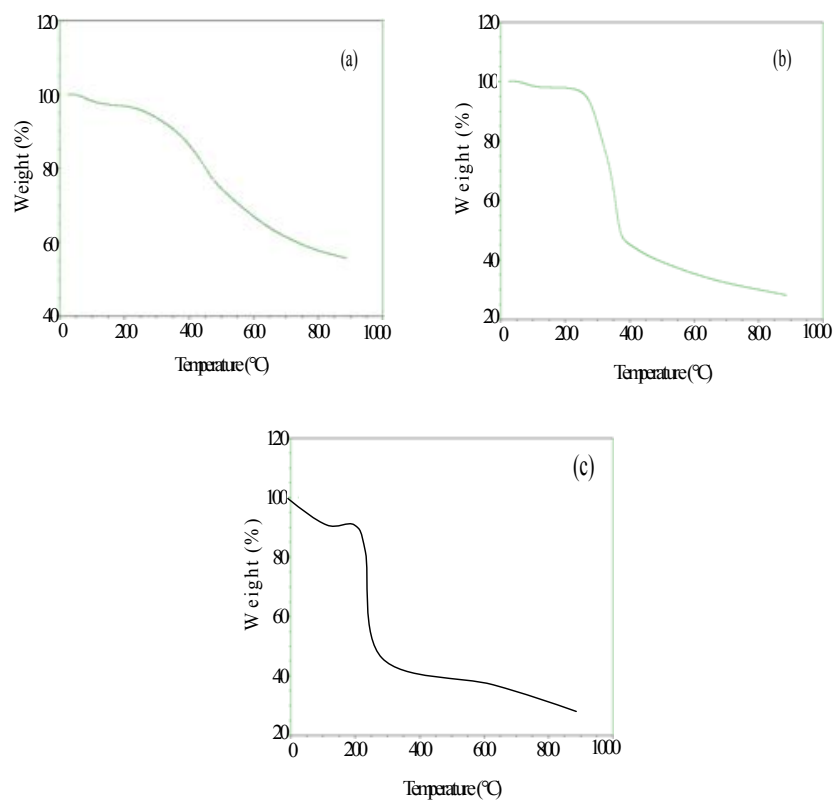

Figure 1. TG curves of (a) coal; (b) coconut shell; and (c) coconut coir
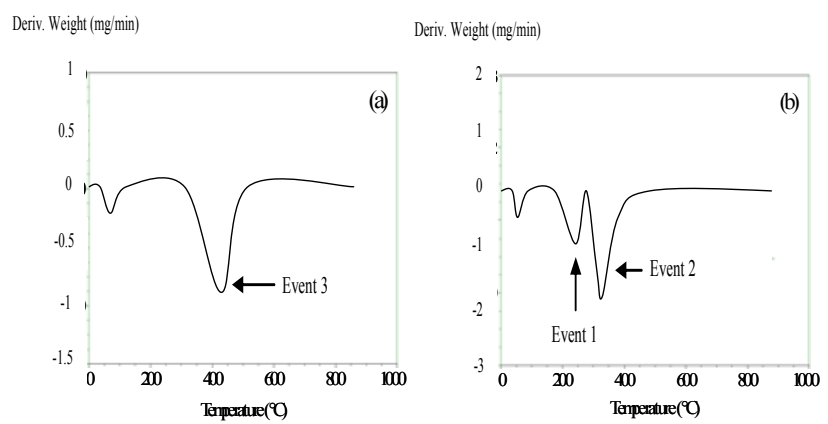

Deriv. Weight (mg/min)

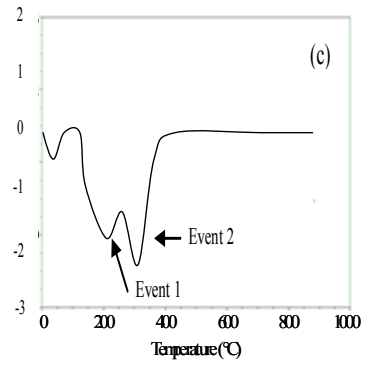

Figure 2. DTG curves of (a) coal; (b) coconut shell; and (c) coconut coir

\section{B.Thermal Decomposition of Coal/Coconut Shell and Coal/Coconut Coir Blends}

Figure 3 shows the typical DTG profiles for the thermal decomposition of 50:50 coal/coconut shell and coal/coconut coir blends during co-pyrolysis condition at $20^{\circ} \mathrm{C} / \mathrm{min}$ heating rate. The DTG profiles revealed three thermal events. The first and second thermal events correspond to the volatile matter released from degradation of hemicellulose and cellulose in the coconut shell and coir respectively, with the second event being assigned to the volatile matter released from decomposition of coal that occurred at higher temperature regime. Table 2 shows the reactivity of coal, coconut shell, coconut coir and their blends. From Table 2, it was observed that the DTG peak height $(\mathrm{R})$ for the first and second thermal events decreased with increasing in the percentage of the coal in the blends. These observations indicated that the reactivity for the volatile matter released from degradation of cellulosic materials of the blends was slightly reduced by the presence of coal. Similarly, the third thermal event for the volatile matter released from coal decomposition in the blends showed a slight decreased in peak height with increasing in the percent of coal in the blends. Thus, it seems that the amount of volatile matter released from the coconut shell, coir and coal is directly proportional to the percent of respective fuels that presence in the blends, indicating lack of interaction between the three fuels during pyrolysis. The results agree reasonably well with those of slightly similar studies [2].
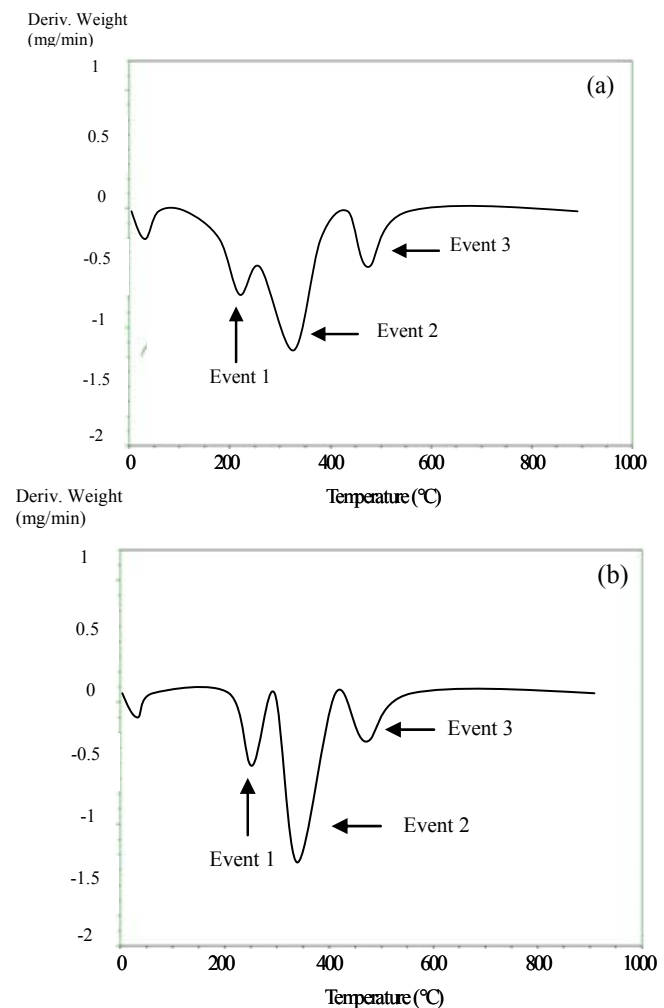

Figure 3. Thermal events for 50:50 wt $\%$ of (a) coal and coconut shell; and (b) coal and coconut coir blends

\section{Heating Rate Effects}

The overall reactivity of coal, coconut shell, coconut coir and their blends during pyrolysis were calculated based on the mean reactivity, $\mathrm{Rm}$ by using a parameter $(\mathrm{R} / \mathrm{Tmax}) 100$ to calculate for each peak, adding the share of any secondary peaks or shoulders present in the thermal profile [11]. In this calculation the rate of weight loss $\left(\mathrm{mg} \cdot \mathrm{min}^{-1}\right)$ was used, giving the dimensions for the mean reactivity as mg. $\min ^{-1}{ }^{\circ} C^{1}$. The mean reactivity $(\mathrm{Rm})$ values for all the pure and blend samples are previously displayed in Table 2 .

Evidently, Tmax, R and Rm for the first, second and third thermal events increased with increasing heating rates (i.e. 
20, 40 and $60^{\circ} \mathrm{C} / \mathrm{min}$ ) during pyrolysis of coal, coconut shell, coconut coir and its blend, indicating an increased in reactivity with increasing heating rates. However, the amount of percent volatile matter released (VM) differs between the two fuels. Coconut coir and coal blends generate the highest volatile yields in comparison to that of coconut shell and coal blends regardless of heating rates and followed those of the parent fuels in an additive behaviour. Further, as the heating rate increased from 20 to $60^{\circ} \mathrm{C} / \mathrm{min}$, Tmax and $\mathrm{R}$ of the thermal events gradually increased and approached to a similar value to that of pure samples. Again, this indicates that the pyrolytic behaviour of the blends followed those of the parent fuels in an additive behaviour. On the other hand, the total volatile matter released presents a decreasing trend as the heating rate increases and Tmax shifts toward higher temperature at a higher heating rate. This behaviour can be explained on the basis of heat transfer and medium diffusion. Since the pyrolysis experiment at higher heating rate has a shorter reaction time (in the same temperature region), the evolution is shifted toward higher temperature. The same observation was also been reported by Zhang et. al. on pyrolysis of pulverized China coal [12]. Thus, under pyrolysis condition, the three fuels seem to undergo independent thermal degradation process without any chemical interaction in the solid phase.

Table 2 . The reactivity of coal, coconut shell, coconut coir and their blends

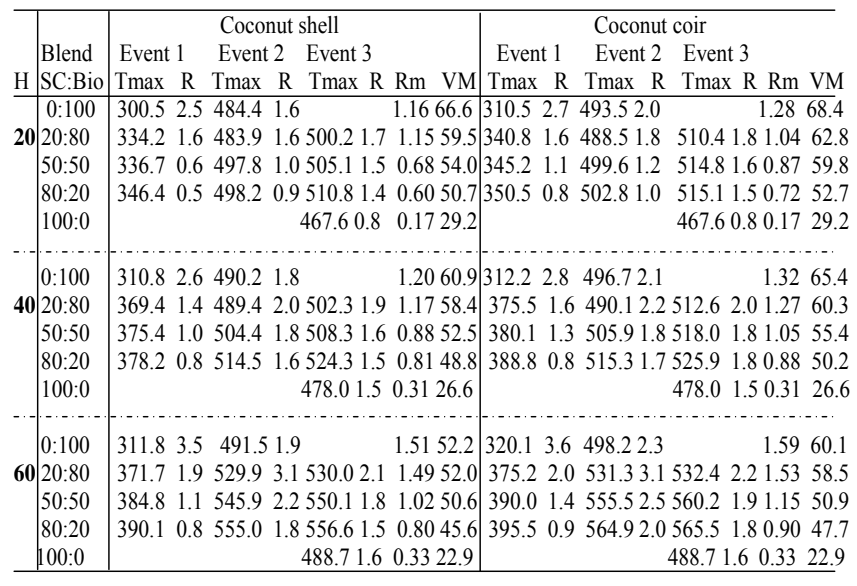

$\mathrm{H}=$ Heating rate $\left({ }^{\circ} \mathrm{C} / \mathrm{min}\right) ; \mathrm{SC}=$ Senakin coal; Bio $=$ Biomass materials; $\mathrm{Tmax}=$ Maximum peak temperature $\left({ }^{\circ} \mathrm{C}\right) ; \mathrm{R}=$ Peak height $(\mathrm{mg} / \mathrm{min}) ; \mathrm{Rm}=$ Mean reactivity $\left(\mathrm{mg} / \mathrm{min}^{\circ} \mathrm{C}\right) ; \mathrm{VM}=\%$ Volatile matter released.

\section{D.Effect of Blending on Char Yields}

Figure 4 represents the percent of char yield with increasing weight percent of coal in the blend during copyrolysis. Obviously, it can be seen that the percent of char yield increased with increasing in percent of coal in the blend. Likewise, as the percent of coconut shell and coir increased in the blend, the percent of char yield decreased accordingly. This trend is due to the high volatile content and low fixed carbon content in the both coconut shell and coir with comparison to coal. Moreover, the strength of the molecular structure of the fuels differs from one another. The polymeric materials in the coconut shell and coir constitute of mainly hemicellulose and cellulose that are linked together by relatively weak ether bonds that are less resistant to the heat at low temperatures [13]. On contrary, the coal macromolecular structure is mostly comprises of dense polycyclic aromatic hydrocarbons with strong aromatic bondings that are more resistant to the heat [14]. Consequently, a small amount of fragmented polycyclic aromatic compounds are expected to result from the immobile phase of coal. Thus, the mass loss is lower during the pyrolysis of coal alone, in comparison to co-pyrolysis of blends involving the coconut shell and coir. Hence, by increasing the percent of coal in the blend, the volatile content decreases and the fixed carbon increase. These result to an increased in the char yield with blends containing higher proportion of coal during co-pyrolysis. These observations clearly indicate the lack of chemical interactions and no synergistic effects between the fuels in the solid phase. These findings are consistent with other researchers reported in the literatures $[5,6,7]$.

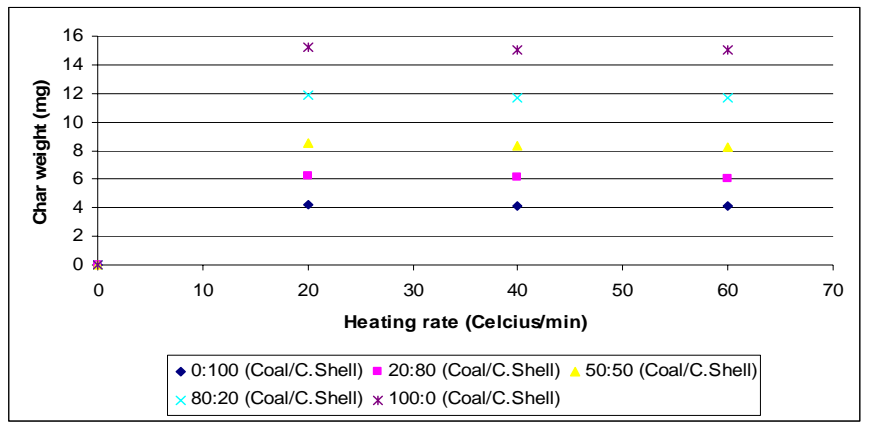

(a)

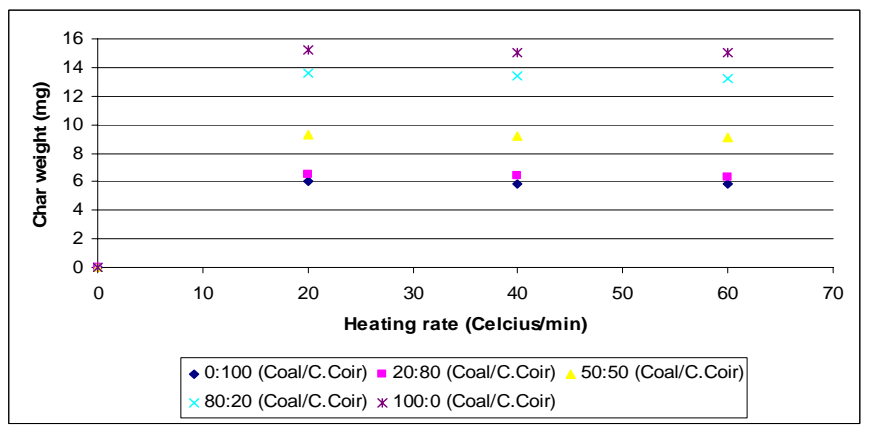

(b)

Figure 4. Percent of char yield of (a) coal and coconut shell (C.Shell); and (b) coal and coconut coir (C.Coir) and their blends

\section{E. Blending Effects on Activation Energy}

The kinetics of thermal decomposition reactions of carbonaceous materials is complex involving a large number of reactions in parallel and in series. The data obtained from TGA besides providing general information on the overall reaction kinetics, could also be used as a tool for providing comparison of kinetic data of various reaction parameters such as temperature and heating rate. Moreover, only a single sample and considerably fewer data are needed to obtain kinetics over entire temperature range in a continuous manner. In this work, temperatures at different heating rates were used to obtain the kinetic parameters of coal, coconut shell, coconut coir and their blends during pyrolysis and co-pyrolysis conditions. The dynamic heating rate from TGA provides one of the high- 
resolution approach by which both the heating rate and rate of weight loss continuously vary during decomposition. The decomposition kinetics obtained from dynamic heating rate TGA experiments using a derivation of the Arrhenius equation first published by Seferis and Salin [15] and the equation is as follows.

$$
\left(\frac{\mathrm{d} \alpha}{\mathrm{dt}}\right)=\mathrm{Z} \exp \left(-\mathrm{E}_{\mathrm{a}} / \mathrm{RT}\right)(1-\alpha)^{\mathrm{n}}
$$

By applying a second derivative and natural log to the Arrhenius equation against temperature produced equation (2):

$\ln \left(\mathrm{H}_{\mathrm{r}} / \mathrm{T}^{2}\right)=\left(-\mathrm{E}_{\mathrm{a}} / \mathrm{R}\right)(1 / \mathrm{T})+\ln \left(\frac{\mathrm{ZR}}{\mathrm{E}_{\mathrm{a}}} \mathrm{n}(1-\alpha)^{\mathrm{n}-1}\right)$

Where: $\quad \mathrm{H}_{\mathrm{r}}=$ Heating rate at the peak $\left({ }^{\circ} \mathrm{C} / \mathrm{min}\right)$

$\mathrm{T}=$ Temperature at the peak $(\mathrm{K})$

$\mathrm{Z}=$ Pre-exponential factor $(1 / \mathrm{seconds})$

$\mathrm{E}_{\mathrm{a}}=$ Activation energy $(\mathrm{J} / \mathrm{mole})$

$\mathrm{R}=$ Gas constant $(8.314 \mathrm{~J} /$ mole $\mathrm{K})$

$\mathrm{n}=$ Reaction order (dimensionless)

$\alpha=$ Fraction of decomposition

Assuming: $\alpha$ is constant

$\mathrm{dH}_{\mathrm{r}} / \mathrm{dT}$ is zero at the peak maximum $\mathrm{d}(\mathrm{d} \alpha / \mathrm{dT}) / \mathrm{dT}$ is zero.

Hence, the activation energy can be determined directly by plotting in $\ln \left(\mathrm{H}_{\mathrm{r}} / \mathrm{T}^{2}\right)$ versus $1 / \mathrm{T}$ provides a straight line with an intercept of $\ln \left(\mathrm{ZR} / \mathrm{E}_{\mathrm{a}}\right)$ and slope of $\left(\mathrm{E}_{\mathrm{a}} / \mathrm{R}\right)$, thus enabling determination of activation energy $\left(E_{a}\right)$.

The kinetic parameters obtained from the pyrolysis and co-pyrolysis of coconut shell, coconut coir, coal and its blend are summarized in Table 3. Obviously, the activation energies decreased with increasing percentage of coal in the blends for the first thermal event (i.e. degradation of hemicellulose) with minimum values of 58.4 and 66.0 $\mathrm{kJ} / \mathrm{mol}$ of coal/coconut shell and coal/coconut coir, respectively, being achieved with $80 \mathrm{wt} \%$ of coconut shell and coir. Similarly, the activation energy of the reaction for the second thermal event (i.e. decomposition of cellulose) in the blend is at minimum with $80 \mathrm{wt} \%$ coal with 80.9 and $68.5 \mathrm{~kJ} / \mathrm{mol}$, respectively, being achieved with $80 \mathrm{wt} \%$ of coconut shell and coir. As for the third thermal event (i.e. decomposition of coal), the minimum values of 83.7 and $85.0 \mathrm{~kJ} / \mathrm{mol}$ of coal/coconut shell and coal/coconut coir, respectively, were observed with blending ratio of $50 \mathrm{wt} \%$ of coal/coconut shell and coal/coconut coir. The activation energy for the pyrolysis of coal alone was higher by $150 \mathrm{~kJ} / \mathrm{mol}$ of energy with comparison to the $50 \mathrm{wt} \%$ coal/coconut shell and coal/coconut coir blend. This implies that to ensure less energy required for decomposition the coal during co-pyrolysis condition, a $50 \mathrm{wt} \%$ of coal to coconut shell and coal/coconut coir content are recommended in the blend.
Table 3: The activation energy, $\mathrm{E}_{\mathrm{a}}$ of coal, coconut shell, coconut coir and their blends

\begin{tabular}{lcccc}
\hline Feeds & Blend ratios & \multicolumn{3}{c}{$\mathrm{E}_{\mathrm{a}}(\mathrm{kJ} / \mathrm{mol})$} \\
& & Event 1 & Event 2 & Event 3 \\
\hline Coal & 100 & - & - & 231.8 \\
Coconut shell & 100 & 227.3 & 280.4 & - \\
Coconut coir & 100 & 263.8 & 302.2 & - \\
Coal/coconut shell & $20: 80$ & 71.1 & 82.1 & 128.6 \\
& $50: 50$ & 60.4 & 83.0 & 83.7 \\
& $80: 20$ & 58.4 & 80.9 & 106.0 \\
Coal/coconut coir & $20: 80$ & 75.1 & 80.7 & 188.2 \\
& $50: 50$ & 68.3 & 68.6 & 85.0 \\
& $80: 20$ & 66.0 & 68.5 & 90.1 \\
\hline
\end{tabular}

\section{CONCLUSION}

Thermogravimetric studies showed that the thermal degradation of the coconut shell and coconut coir occurred at lower temperature as comparison to the coal sample. Shorter devolatilization time is needed for the coconut shell and coir to decompose during pyrolysis. Obviously, lacks of interaction were seen between coal, coconut shell and coconut coir in solid phase by looking at the profiles of char remains after pyrolysis. The coconut shell and coir are more reactive than coal in most cases. The presence of the coconut shell and coir in the blends enhanced the reactivity of the coal. Three thermal events were observed in the blended fuels, whereby first thermal event was dominated by the decomposition of hemicellulose materials and second thermal event was contributed by degradation of cellulose materials and third thermal event was contributed by decomposition of coal. The 50:50 blend, with respect to the coal volatile matter released showed the lowest activation energy for both coal/coconut shell and coal/coconut coir blend and again revealed that the presence of the coconut shell and coir conducive to the reduction of activation energy of coal.

\section{ACKNOWLEDGMENT}

The authors would like to thank the Institute of Research, Development \& Commercialization (IRDC), University Technology MARA and Faculty of Chemical Engineering for their support.

\section{REFERENCES}

[1] http://www.nrdc.org/air/energy/renewable/biomass.asp, 7 March 2007.

[2] Ismail, K., Zakaria, Z. and Ishak, M.A.M., Proceedings of Brunei International Conference on Engineering and Technology 2005 (BICET 2005), 2005, 229.

[3] Kastanaki, E., Vamvuka, D., Grammelis, P. and Kakaras, E., Fuel Processing Technology, 2002, 159.

[4] Pan, Y.G., Velo, E., and Puigjaner, L., Fuel, 1996, 412.

[5] Caballero, J.A., Marcilla, A., and Conesa, J.A., Journal of Anal. Appl. Pyrolysis, 1997, 75.

[6] Vamvuka, D., Kakaras, E., Kastanaki, E., and Grammelis, P., Fuel, 2003, 1949.

[7] Moghtaderi, B., Meesri, C., and Wall, T.F., Fuel, 2004, 745.

[8] Vuthaluru, H.B., Bioresource Technology, 2004, 187.

[9] L. Helsen and E. Van den Bulck, J. Anal. Appl. Pyrolysis 44 (1997) 75-88.

[10] Kastanaki, E. and Vamvuka, D., Fuel, 2006, 1186.

[11] Ghetti, P., et al. Thermal analysis of biomass, Vol. 75, 1996, 5.

[12] Zhang, C., Jiang, X., Wei, L. And Wang, H., Energy Conversion and Management, 2007, 797.

[13] Blazej, A.,Kosik, M., Phytomass, Ellis Horwood, England, 1993.

[14] Smith, L.H., Smoot, L.D., Fletcher, T.H., The structure and reaction process of coal. Plenum Press, New York, 1994.

[15] J. Seferis and Salin, J. Appl. Poly. Sci., 1992. 


\title{
Construction and Laboratory Testing of Parabolic Types Solar Cooker
}

\author{
Erwan Sulaiman, Kok Boon Ching and Chessda Uttraphan
}

\begin{abstract}
Solar cooker is a delightful alternative to conventional cooking methods. As the rates of LPG Cylinders hit the roof and due to the fact that the conventional cooking fuels become scarce and cause considerable damage to the environment, there has been an increase in the demand for an alternative, environmental friendly cooking fuel. Meeting this demand is the unique solar cooker that utilizes solar power to cook up a meal. A research to develop, test and evaluate various types of solar cooker was carried out with the major objective to look into its suitability as an alternative means of cooking. There are ten models of parabolic solar cooker that have been built and tested to boil some amount of water in Peninsular Malaysia environment and the results shown that the fastest time in boiling the water is by using twelve angle sided cooker. In addition to the evaluation of the solar cooker based on the experimental results, the feasibility and possible modification in the design to improve the efficiency are also discussed.
\end{abstract}

Keywords: parabolic, solar cooker, twelve angle sided cooker

\section{INTRODUCTION}

Cooking can be considered as part and parcel of important domestic activity thus making significant demands on energy supplies. Any successful attempt to substitute exhaustible fuels currently in use for domestic cooking with renewable energy sources is therefore welcomed because of its value in finding a permanent answer to domestic energy demand [1]. Applications that harness solar energy is practicable include the direct use or conversion of solar thermal energy.

A conventional design for a solar cooker basically consist of an insulated black box with a glass cover for letting in solar radiation and trapping it through the greenhouse effect, has long been known and its use is widespread. In order to get the high temperature to heat water or cook food, it is necessary to concentrate large amount of sunlight on a small light-absorbing area [2].

The basic idea is to focus the sun's rays using reflected area inside the cooker. Figure 1 (a) shows the concept used to build a solar cooker with a black pot located at the base of the cooker. Meanwhile, figure 1 (b) shows the sun's light ray reflects to a focus point hanging from the base of the

Erwan Sulaiman, Kok Boon Ching and Chessda Uttraphan are currently with Department of Electrical Power Engineering, Faculty of Electrical and Electronic Engineering, University of Tun Hussein Onn Malaysia, 86400, Batu Pahat, Johor. (Phone: 07-4537608; Fax: 07-4536060; email: erwan@uthm.edu.my, bckok@uthm.edu.my, chessda@uthm.edu.my) cooker. When the heat from the sun hit the solar cooker inner surface constructed with aluminum foil, the heat will reflect to the black pot and heat the pot constantly.

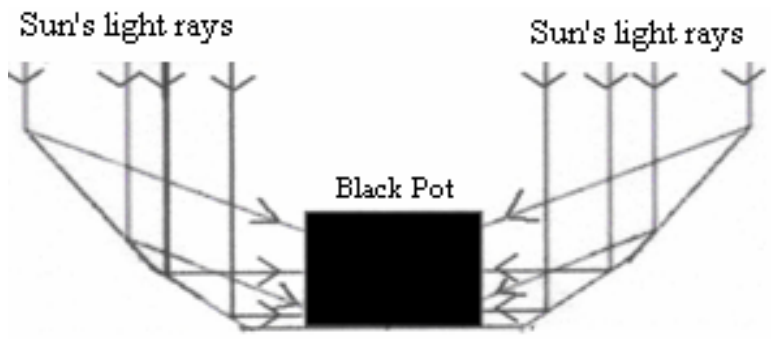

(a)

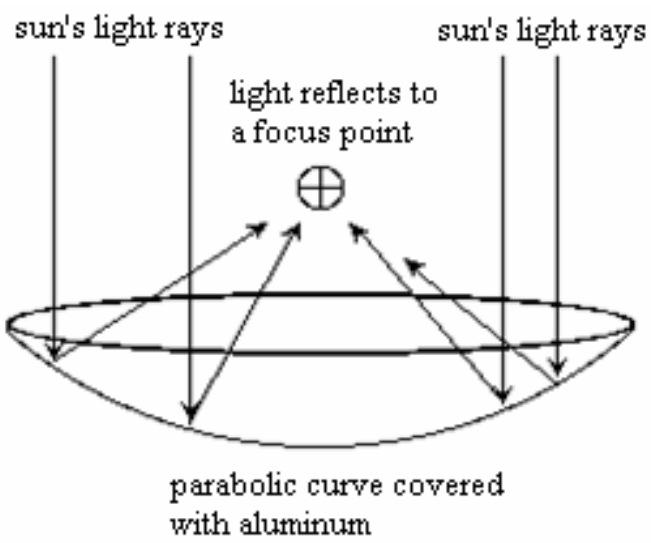

(b)

Figure 1. The concept of reflected heat for solar cooker

The most common approach is to use curved reflective panel. A reflector with a parabolic curve will concentrate sunlight on a very small spot to start a fire. Simple solar oven and cooker are used around the world in both commercial kitchens and people's home. Solar cooker can be easily made with everyday materials such as cardboard and tinfoil.

A solar cooker also is a good example of the "greenhouse effect" at work. Short wave radiation from the sun passes easily the glazing and absorbed by the dark surfaces inside the cooker and may become a delightful alternative to conventional cooking method. The solar cookers available today really functional and deserve serious evaluation by a much larger audience especially when the sunshine period is appreciably long and easily obtained.

Furthermore, cooking with solar cooker is smoke free and therefore increases life expectancy. Housewife's responds that 
they have more time for other works as by using solar cooker they has no worry on fire and also less dangerous to children. Nevertheless there are also some disadvantages of using solar cooker since it cannot be used to cook breakfast and meals in the evening when there is no sunlight.

\section{CONVERSION OF ENERGY}

The energy available for cooking is the balance remaining after energy storage and heat losses are subtracted from energy entering the solar cooker. Application of the first law of thermodynamics (conservation of energy) and dimensional analysis provides the following equation:

$$
\begin{aligned}
& \mathrm{P}_{\mathrm{u}}=\mathrm{X}_{\mathrm{o}}+\mathrm{X}_{1} \mathrm{~A}_{\mathrm{i}} \mathrm{I}_{\mathrm{T}}-\mathrm{X}_{2} \mathrm{U}_{\mathrm{L}} \mathrm{A}_{\mathrm{u}} \Delta \mathrm{T}_{\mathrm{u}}+\mathrm{X}_{3} \mathrm{KtdT}_{\mathrm{a}}-\mathrm{X}_{4} \mathrm{~L}^{3} \mathrm{C}_{\mathrm{v}} \Delta \mathrm{T}_{\mathrm{t}} \\
& \pm \mathrm{X}_{5} \mathrm{KA}_{\mathrm{i}} \mathrm{I}_{\mathrm{T}}
\end{aligned}
$$

Only the constant, $X_{t}$, was assigned an expected value. This value, 0.15 , is the efficiency of a solar cooker determined from earlier experiments (Funk, 1992).

The constant $\mathrm{X}_{2}$ reflects the dependence of heat loss coefficient on temperature differences. The heat loss coefficient can usually be approximated with a linear relationship when the temperature difference is less than $100 \mathrm{C}$ because irradiative heat transfer is small relative to forced convection. If $\mathrm{X}_{2}$ is other unity, it indicates a discrepancy between heat loss measured in the wind tunnel and experienced in the field.

The constant $X_{3}$ attempts to quantify the effect of internal heat transfer. It requires knowledge of thermal gradients within the absorber plate, information which is not available. This approach is also limited because it does not account for contact resistance. The largest problem is the interdependence of the internal heat transfer (absorber plate conductivity) and oven loading (pot quantity and mass of contents). Thermal conductivity has less influence on performance when the load is distributed (Funk and Larson, 1994).

Losses arising from internal thermal inertia are accounted for by the equation where the constant $\mathrm{X}_{4}$ adjusts for variation in mass and heat capacity of construction materials. The time rate of change of absorber temperature may not be known, but is probably similar to that of the fluid in the pots if there is no warm-up period. Judging thermal inertia to be small for the cookers tested, this parameter was expected to be small. It was dropped from the equation as a means of simplification, to reduce the number of parameters to the most significant ones and to contain the present experimentation to a feasible scope. Yet, thermal inertia is important in predicting performance for solar cookers incorporating thermal storage, and may be worth examining future experimentation.

Finally, the relationship between clearness index (ratio of extraterrestrial to observed beam radiation) and concentration (ratio of intercept to aperture area) may be significant because reflectors primarily make use of beam radiation Diffuse radiation is a greater proportion of total radiation when the clearness index is low. Diffuse radiation is not effectively utilized by reflectors of imaging concentrators since it is impossible to focus a non-point source. The final constant, $\mathrm{X}_{5}$ attempts to quantify clearness index effects.

\section{SOlAR COOKER DESIGN}

There are three main principles incorporated into solar cooking which are fundamental to any cooker. These principles are reflection of the greatest possible amount of sunlight to the food, converting these light waves to heat and effectively retaining the heat by insulation.

For the reflection, the aim is to make the cooker that can be adjusted to the sun's varying positions to capture enough light to cook using the reflector that will direct the sun's rays onto the pot. The shape of the parabolic model is made such that it reflects only on a single point where the food is to be placed.

For the conversion, the aim is to make the cooker that can effectively absorb and convert the sun's rays into heat by using pots made of dark material. Dark-colored materials absorb more heat while light-colored reflect the sun's rays. If a shiny pot is used, the rays are reflected out of the cooker allowing no light energy to be converted to heat. On the contrary, the black pot absorbs the rays and converts them to heat.

In this experiment, ten different designs of solar cookers have been constructed using several tools and basic materials such as an umbrella, a cardboard, an aluminum foil and a black pot as shown in Figure 2. The focal length of each solar cooker has been calculated using parabolic equation to get the best location for collection of directed and reflected sun's light rays i.e.,

$y=a x^{2}+b x+c$

Each prototype of the solar cooker has been tested to boil some amount of water ranging from $200 \mathrm{ml}$ to $650 \mathrm{ml}$ in Peninsular Malaysia environment with a minimum temperature of $24^{\circ} \mathrm{C}$ to maximum of $33{ }^{\circ} \mathrm{C}$.

The testing of each solar cooker has been made on the same day at about 11.00 am to $3.00 \mathrm{pm}$ local time continuously from $12^{\text {th }}$ to $16^{\text {th }}$ April 2007. When the water start to boil i.e., the temperature of the water reach $100{ }^{\circ} \mathrm{C}$, the boiling time is noted.

\section{RESULTS}

Table 1 and table 2 show the average results for all solar cookers that have been tested for five days. It shows that double angle twelve sided (DATS) solar cooker give the fastest time to boil i.e., 8 to 20 minutes for amount of water ranging from $200 \mathrm{ml}$ to $650 \mathrm{ml}$. Meanwhile, figure 3 shows the plot of boiling time versus amount of water.

The results derived from Figure 3 shows that the fastest time to boil water is by using double angle twelve sided cooker with equivalent to $\mathrm{Y}=1.25 \mathrm{X}+6.73$ where $\mathrm{Y}$ is the boiling time, and $\mathrm{X}$ is the amount of water. Meanwhile, the longest time to boil water is by using trapezium box cooker with equivalent to $\mathrm{Y}=14.93 \mathrm{X}+41.2$ respectively. 


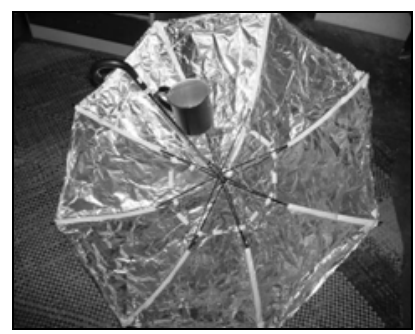

8-Sided umbrella

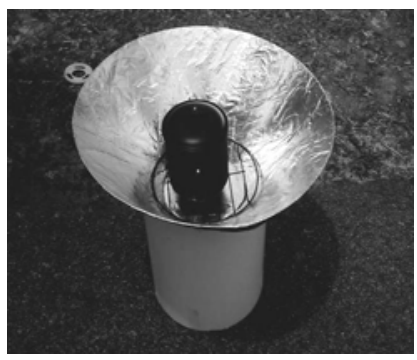

Parabolic I

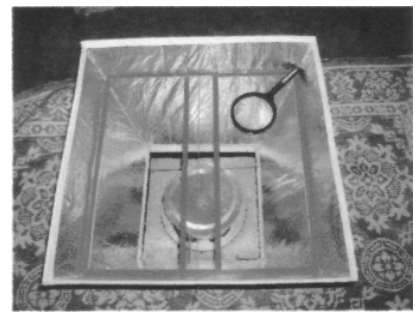

Trapezium box

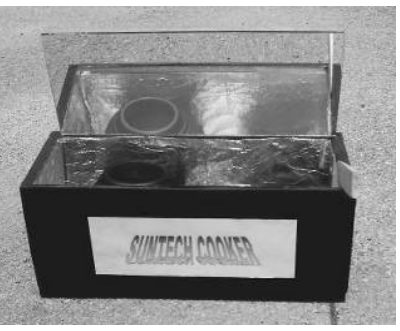

Box with glass

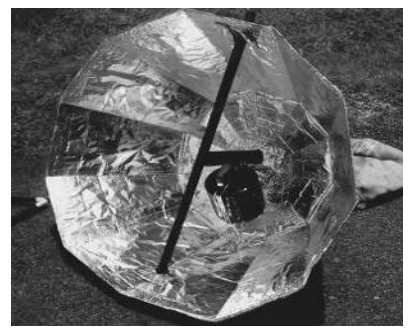

Parabolic II

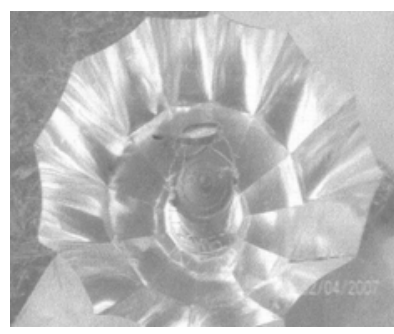

Double angle 12-sided

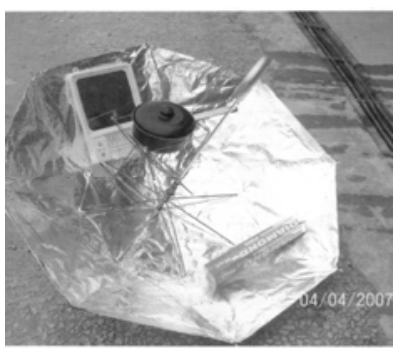

10-Sided umbrella

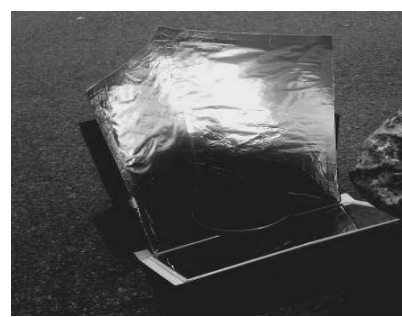

Reflective Box

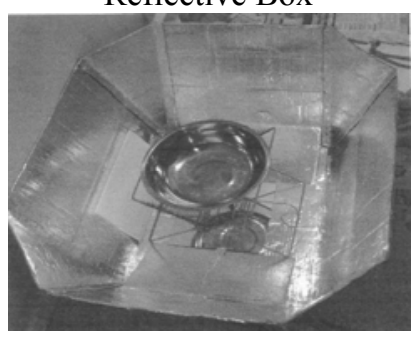

8-Sided box

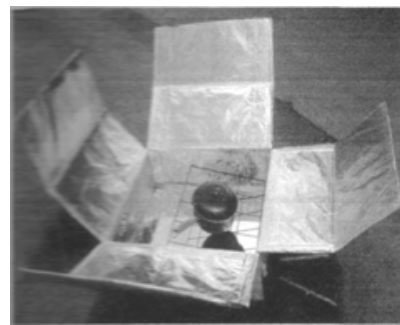

Box with 4-sided wing
Figure 2. Ten different construction of solar cooker
Table 1. Data I

\begin{tabular}{|c|c|c|c|c|c|}
\hline \hline \multirow{2}{*}{$\begin{array}{c}\text { Water } \\
(\mathrm{ml})\end{array}$} & \multicolumn{5}{|c|}{ Time (minute) } \\
\cline { 2 - 6 } & DATS & $\begin{array}{c}\text { Parabolic } \\
1\end{array}$ & $\begin{array}{c}\text { 10 Sided } \\
\text { Umbrella }\end{array}$ & $\begin{array}{c}\text { Parabolic } \\
2\end{array}$ & $\begin{array}{c}\text { 8 Sided } \\
\text { Umbrella }\end{array}$ \\
\hline \hline 200 & 8 & 13 & 25 & 33 & 43 \\
\hline 250 & 9 & 15 & 29 & 39 & 57 \\
\hline 300 & 11 & 16 & 34 & 42 & 73 \\
\hline 350 & 12 & 18 & 38 & 47 & 92 \\
\hline 400 & 13 & 19 & 43 & 55 & 113 \\
\hline 450 & 14 & 21 & 47 & 61 & 127 \\
\hline 500 & 15 & 22 & 52 & 73 & 140 \\
\hline 550 & 16 & 24 & 56 & 83 & 153 \\
\hline 600 & 18 & 25 & 60 & 90 & 161 \\
\hline 650 & 20 & 28 & 64 & 98 & 169 \\
\hline \hline
\end{tabular}

Table 2. Data II

\begin{tabular}{|c|c|c|c|c|c|}
\hline \hline \multirow{2}{*}{$\begin{array}{c}\text { Water } \\
(\mathrm{ml})\end{array}$} & \multicolumn{5}{|c|}{ Time (minute) } \\
\cline { 2 - 6 } & $\begin{array}{c}\text { Box 4 } \\
\text { Wing }\end{array}$ & $\begin{array}{c}\text { Box with } \\
\text { glass }\end{array}$ & $\begin{array}{c}8 \text { Sided } \\
\text { Box }\end{array}$ & $\begin{array}{c}\text { Cone } \\
\text { Shape }\end{array}$ & $\begin{array}{c}\text { Trapezium } \\
\text { box }\end{array}$ \\
\hline \hline 200 & 22 & 42 & 49 & 51 & 53 \\
\hline 250 & 28 & 43 & 51 & 58 & 67 \\
\hline 300 & 43 & 45 & 67 & 69 & 83 \\
\hline 350 & 49 & 46 & 70 & 74 & 102 \\
\hline 400 & 54 & 47 & 84 & 88 & 123 \\
\hline 450 & 61 & 49 & 92 & 95 & 137 \\
\hline 500 & 69 & 51 & 106 & 110 & 150 \\
\hline 550 & 76 & 53 & 111 & 117 & 163 \\
\hline 600 & 84 & 55 & 114 & 124 & 172 \\
\hline 650 & 92 & 58 & 124 & 131 & 183 \\
\hline \hline
\end{tabular}

Boiling Time (min) vs Amount of Water (ml) (min)

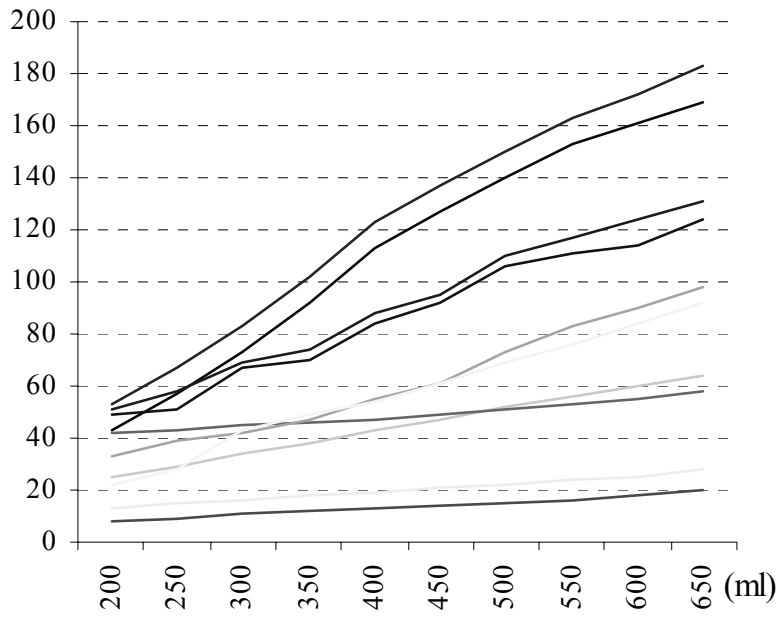

Figure 3. Boiling time (min) vs. amount of water (ml)

\section{CONCLUSION}

Solar cooker are the most common way that can offer a practical solution for solar energy. It must place out in the open area in order to get the maximum sunlight that depends on the equipment used to build it. In addition, it must be made to be efficient enough, robust, convenient to use and sufficiently cheap for rural people in developing countries. 
The best concept of solar cooker is parabolic type base on the basic principle of reflection. The Doubled-AngleTwelve-Sided solar cooker obtains the fastest time to boil water which is ranging from 8 minutes for $200 \mathrm{ml}$ to 20 minutes at $650 \mathrm{ml}$. It is a renewable source that has never ending and environmentally friendly.

\section{ACKNOWLEDGEMENT}

The authors wish to express their gratitude to the staff of Power System Laboratory, Universiti Tun Hussein Onn Malaysia and all supporting staff for their encouragement and assistance in the laboratory works.

\section{REFERENCES}

[1] Tamara Dwyer, 1999. 'Types of Solar Oven' Website: www.exoticblades.com

[2] H. P. Garg, J. Prakash. Solar Energy: Fundamental and Applications, Mc Graw Publishing, 2000.

[3] John Schaeffer, Doug Pratt. Solar Living Source Book: The Complete Guide to Renewable Energy Technologies and Sustainable Living, Gaiam Real Goods; 11th edition. August 2001.

[4] Kevin R. Porter, Solar Cooking Archive: The Next Generation. Solar Cookers and Food Processing International Conference proceedings, 2006.

[5] A Parabolic Solar Cooker for Unattended Cooking, Li-Yan Zhu., Yim K.Kim. http://solarcooking.org/umattendedparabolic.htm

[6] Innovative 'Balcony Model of Concentrating Solar Cooker', Ajay Chandak., Deepak Dubey., Rahul Kulkarni.

[7] Chances and Limitations of Solar Cookers, Agnes Klingshirn

[8] Varieties of Solar Cooker Devices and Uses, Shyam S. Nandwani

[9] Parabolic Solar Concentrators for Cooking, Food Processing and Other Applications, Deepak Gadhia., Shirin Gadhia.

[10] The Solar Cooker Development and Applications in China. Chen Xiofu, Han Tingcun.

[11] Shyam S. Nandwani, Book- La Cocina/Horno Solar. Hagala Usted Mismo, FUNDACION, Universidad Nacional, Costa Rica. 2004 (Revised Edition). 120 pages.

[12] SunSmile. Proposal for Development of a hybrid solar oven for rural Morocco, December 2004 


\title{
Electric Energy Saving for Residential Sector
}

\author{
K. Ramar, Gobbi R., Normiza Nor, A. Arunagiri, E.E. Ngu, C.M. Kok, H.C. Voon and Lawrence W.
}

\begin{abstract}
As electrical energy is mostly produced by burning fossil fuels, this emits carbon-dioxide into the atmosphere, trapping heat and increasing global temperature. So there is a crying need to cut down the energy consumption in various sectors to reduce this greenhouse gas (carbondioxide) emission which causes environmental degradation and also to save fuel for future generation. In this paper we concentrate on the energy saving in residential sector as this sector consumes a bulk of electrical energy (34\% in Malaysia) produced. The objective of this paper is to present information about the energy consumed by some of the important household appliances in normal operating mode and standby mode, and the cost of energy bill for a period of one month.
\end{abstract}

Keywords: energy, energy bill, electric appliances and residential

\section{INTRODUCTION}

The standard of living of people is directly related to the electric energy consumption. Increasing use of electric energy causes a drain on our ever depleting natural resources. According to Asia Pacific Energy Research Center (APERC) the energy consumption in this region is expected to grow at the rate of $4.7 \%$ per year and at this rate the indigenous oil will last only for another 12 years or so. Also, as electrical energy is mostly produced by burning fossil fuels, this emits carbon-dioxide into the atmosphere, trapping heat and increasing global temperature. So there is a crying need to cut down the energy consumption in various sectors to reduce this greenhouse gas (carbondioxide) emission which causes environmental degradation and also to save fuel for future generation. In this paper we concentrate on the energy saving in residential sector as this sector consumes a bulk of electrical energy $(34 \%$ in Malaysia) produced.

The number of electrical devices/appliances we use in our houses increases day by day. Even though most of us know how to use the electrical appliances many do not have knowledge about their energy consumption pattern resulting in either unnecessarily using appliances which consume lot of energy or unknowingly waste energy while using essential devices. In order to save energy consumption without sacrificing comfort people must know some facts about the energy consumption details of household appliances. By monitoring and controlling the operation of the appliances the energy consumption can be reduced. The

This work was supported in part by The Associated Chinese Chambers of Commerce and Industry of Malaysia (ACCCIM)

All authors are with the Multimedia University, Jalan Multimedia, 63100, Cyberjaya, Malaysia (corresponding author, K. Ramar: 60383125473; fax: 603-83183029; e-mail: ramar@mmu.edu.my). objective of this paper is to present some facts on the energy consumption pattern of some important household appliances and give suggestions to save energy. Saving wasteful energy not only reduces the monthly energy cost of our houses but also contributes to the reduction of greenhouse gas emission and environmental degradation. According to Malaysia Energy Commission (Suruhanjaya Tenaga) 5\% saving in energy eliminates 3.4 million tons of carbon- dioxide emission per year.

This paper gives information about the energy consumed by some of the important household appliances in normal operating mode and standby mode, and the cost of energy bill for a period of one month. The cost is worked out based on the rate prevailing in Malaysia for residential sector. Standby electricity is the energy consumed by appliances when they are not performing their main functions. Many people are not aware of the fact that many modern electrical appliances such as Television, Personal Computers, etc. consume energy when they are in standby mode. Based on the study, recommendations are given to reduce energy consumption without sacrificing the standard of comfort. One will be amazed to know how easy it is to save energy around his home often without spending a cent by following the simple suggestions presented.

The energy consumption details of the various appliances have been obtained by performing tests using wattmeter to measure the power and computing energy by multiplying power and the estimated hours of usage. In the case of appliances which absorb intermittently varying power such as Refrigerators, Air conditioners, etc. energy consumption is calculated based on the load curves. Besides using the test results we have also collected a few details from the internet.

The appliances considered for the study are grouped into four categories as follows: Group I: Living Room Appliances, Group II: Kitchen Appliances, Group III: Bedroom \& Bathroom Appliances, Group IV: Other Common Appliances.

For each item the name of the appliance, its ratings, measured power consumption and the total energy consumption for normal and other modes of operations based on the assumed usage are presented. Also, the total energy cost is computed based on the electricity consumption charges for home users in Malaysia. As the total energy consumption may exceed the first slab limit of 200 units, the average of the first two slab rates (25.35 sen) is used in the calculation.

A list of suggestion to reduce the energy consumption is given for each appliance. In the concluding section, a table is presented. The table gives the energy consumption 
details, the energy cost for all the important appliances, the percentage consumption of each group and the total monthly energy cost for a standard house with 4 persons. The table also presents the same if some of the energy saving suggestions are carried out.

\section{LIVING ROOM APPLIANCES}

\section{A. Personal Computer (Desktop)}

The desktop type personal computer normally consists of, at least, a central procession unit (CPU), a monitor, a keyboard and a mouse. In this study, two Pentium-4 (one branded and one cloned), one Pentium-3 (branded) and one Pentium-2 (branded) computers have been used for energy measurement.

It is found that the branded Pentium- 4 computer when it is in normal operating mode consumes approximately $80 \mathrm{~W}$ for $0 \%$ CPU usage and $150 \mathrm{~W}$ for $100 \%$ CPU usage. Its average consumption is calculated to be $115 \mathrm{~W}$. Similarly Pentium-3 and -2 computers consume an average power of about $30 \mathrm{~W}$.

In standby mode, Pentium-4 computer consumes negligible power compared to Pentium-2 and Pentium-3 computers, which consume $17 \mathrm{~W}$ and $20 \mathrm{~W}$ respectively. In addition, we have found that the cloned Pentium- 4 computer consumes a staggering $53 \mathrm{~W}$ in standby mode. Keeping the computers in standby mode for a long time when they are not in active use adds to electricity cost.

Monitor: It is found that the Cathode Ray Tube (CRT) 14" monitor consumes about $35 \mathrm{~W}$ compared to Liquid Crystal Display (LCD) 14" monitor, which consumes only 4.5 W. Besides, the larger is the size of the monitor, higher is the power consumption. For example, a 17" CRT monitor consumes $22 \%$ higher power compared to a 14 " CRT monitor in normal operating condition.

Recommendations

a) Most of the household work may be done using Pentium-3 personal computer.

b) The computers (Pentium-2 and -3 models) shall be switched off when they are not in use.

c) The standby mode power consumption shall be checked when purchasing a computer. Even a small amount of standby mode power may cost a lot when it is left switched on.

d) When an application program is running other applications windows shall be closed to minimize the CPU usage. Less $C P U$ usage results in less power consumption

e) Computer accessories use electricity even when they are not in use. Each computer component shall be plugged into a power socket that can be shut off, to avoid wasting electricity in 'standby' mode.

\section{B. Printers}

Three commonly used printers namely Laser Jet Printer, Dot Matrix Printer and Bubble Jet Printer (called inkjet printer) have been studied. The selection of a printer mainly depends on the quality, volume, and speed of printing required. The Laser jet printer is expensive but it gives the best printing quality and second in the list is Bubble jet printer. Dot matrix printer is usually used for printing where the volume of printing is more important than quality and therefore it gives the lowest printing cost per page.

Laser jet printer consumes more power compared to other printers. It requires about $440 \mathrm{~W}$ to initialize when it is first switched on. The printer consumes about $200 \mathrm{~W}$ during printing regardless of the number of pages. Monthly energy consumption for daily printing usage of 1 hour will be about $5.6 \mathrm{kWh}$.

Dot matrix printers consume $5.8 \mathrm{~W}$ during normal operating mode, and require no extra power for initialization. Therefore, printing for one hour daily using this printer saves energy up to $97 \%$ compared to the Laser Jet printer.

Bubble jet printers consume the least energy compared to the other two. It consumes $2.5 \mathrm{~W}$ during normal operating mode.

All printers consume power during standby mode.

Recommendations

a) The printers shall be switched off when they are not in use to avoid standby energy loss.

b) If printing volume is low, consider using bubble jet printer.

c) The laser jet printer shall not be switched off and start again if it is to be used within a short period of time as for every time it is switch on, a lot of power is wasted for initialization.

\section{Laptop}

The laptop computer is portable compare to desktop computer. In this study, Pentium-4 and Pentium-3 Laptops have been used for measurement and analysis.

A laptop computer is a good choice compared to a desktop computer as the laptop consumes far less energy. The Pentium-4 laptop tested in the laboratory consumes 13 $\mathrm{W}$ for $0 \% \mathrm{CPU}$ usage and $30 \mathrm{~W}$ for $50 \% \mathrm{CPU}$ usage (normal operating mode). In other words, a laptop computer at the normal operating mode consumes only $26 \%$ of power consumed by the desktop computer (Pentium-4). The power has been measured by connecting the Laptop with the fully charged battery to the mains.

Recommendations

a) Use Laptop (if affordable) as it is portable and it consumes significantly lower energy compared to a desktop computer

b) When the laptop is not in use, the power supply shall be switched off.

\section{Television}

In this study, Flat screen CRT 21" and 29" TVs have been used for measurement. 29" CRT TV consumes $130 \mathrm{~W}$ during normal operation. It is found that increasing contrast, color, brightness, sharpness, and volume increases power consumption. For example, setting them towards $100 \%$ than usual $50 \%$ setting, it consumes additional $15 \mathrm{~W}$. Increasing the volume from zero to full the TV consumes an additional power of $10 \mathrm{~W}$. In standby mode, 29" CRT TV consumes 5 W.

A 21" CRT TV consumes $45 \mathrm{~W}$ during normal operation. In addition, it draws different power for different settings. Higher volume setting causes higher power consumption. In standby mode, it consumes $1.2 \mathrm{~W}$.

\section{Recommendations}

a) TV contrast, picture, brightness, sharpness, volume etc. shall be set to as minimum for comfortable viewing as possible.

b) The TV shall be switched off at the power socket to reduce energy wasted during standby mode.

\section{E. Astro Decoder}

Astro is the region's leading cross-media operator with direct-to-home satellite television services in Malaysia. The 
decoder consumes about $10 \mathrm{~W}$ both in normal operating and standby modes. It is recommended by the service provider that the decoder is left in standby mode when not in use. Therefore, the decoder consumes power for all 24 hours per day. The total energy consumption per month will be 6.72 units.

\section{F. HOME Audio system}

A 55-W audio system has been tested to understand the power consumption at various operating modes.

The system can be set to three different functions during normal operation, namely "CD", "Tape" or "Radio". In "CD" function mode, it consumes $15 \mathrm{~W}$ when no CD is playing and the power increases by $33 \%$ when a $\mathrm{CD}$ is playing. In "Tape" function it consumes $8 \mathrm{~W}$ when no cassette is playing and it draws an additional $2 \mathrm{~W}$ when a cassette is playing. Finally, when the system is tuned to "Radio" mode, it consumes $9 \mathrm{~W}$. When the micro system is in standby mode, it consumes $3 \mathrm{~W}$.

It is found that the system consumes higher power when volume is increased. Beside, ejecting and inserting CD consume additional power.

Recommendations

a) The volume shall be set to a minimum comfortable audible level. High volume setting unnecessarily increases energy consumption.

b) When not in use the power supply shall be switched off to eliminate standby mode power consumption

\section{G.DVD Player}

Digital Video Disk (DVD) player is very similar to a Compact Disk (CD) player, but DVD has much larger data capacity. A standard DVD holds about seven times more data than a CD does. This huge capacity means that a DVD has enough room to store a full-length, MPEG-2-encoded movie, as well as a lot of other information. Hence, in this study, a standard DVD player has been tested tested.

DVD player consumes $4.85 \mathrm{~W}$ during normal operating mode and $2.3 \mathrm{~W}$ during standby mode. The player consumes additional power to perform additional task such as forward/reverse, next/previous etc.

Recommendations

a) The player shall be switched off when it is not in use.

\section{H.Vacuum Cleaner}

A standard domestic vacuum cleaner with different settings has been used for measurement. Vacuum cleaner consumes additional power of $20 \mathrm{~W}$ when the dust compartment is not empty.

Recommendations

a) Clean the dust compartment before using the vacuum cleaner.

b) Use appropriate setting to save energy.

\section{KITCHEN APPLIANCES}

\section{A. Automatic Rice Cooker}

An automatic rice cooker has been tested to find out the power consumption during cooking mode and warming mode.

The readings are obtained to cook 2 cups of rice with 3 cups of water. "Keep-warm" mode consumes about $45 \mathrm{~W}$ of power and consumes about 50 per cent of energy per month if the food is kept in warm mode for 2 hours per day. Recommendations

a) Avoid using "Keep warm" mode to save energy. Food can be re-heated if required.

\section{B. Electric Oven}

An electric radiant type oven is normally used for backing food items only. The energy consumption depends on the hours of usage.

For the data in the energy consumption has been calculated assuming that the oven is used for one hour per day and 15 days per month.

\section{Recommendations}

a) The oven shall be turned off 10 minutes before finishing - the oven will remain hot, long enough to complete the job.

b) The seal on the oven door shall be checked. A good seal will hold a piece of paper in place when the oven door is closed.

c) The oven shall be fully utilized where possible and plan on cooking several dishes simultaneously.

\section{Electrical Air Pot and Kettle}

People use boiled water for making coffee/tea and for drinking. Here Electric air pot and electric kettle are tested. 3.6-liters of water is boiled using the above appliances.

\section{Recommendations}

a) Electric kettle consumes less energy compared to electric air pot to boil the same quantity of water and hence kettle shall be used for boiling water.

\section{Cooking Appliances (Stoves)}

An electric stove, a gas stove with natural gas connection and a gas stove with LPG connection are compared here.

The same quantity of rice (two cups of rice with 3 cups of water) has been cooked with the above three stoves for comparison. It was observed that an electric stove with $720-\mathrm{W}$ heater element took approximately 15 minutes to cook, on the other hand, the gas stove using natural gas and LPG gas took approximately 20 minutes to do the same job. The total calorie of heat supplied by LPG and the natural gas to do the same job was the same. Based on this study, it is estimated that if a gas stove takes about 3 hours per day to do all the cooking jobs for a house with 4 members then the electric stove will take about 2 hours and 15 minutes. Also, it is observed that the efficiency of gas stove is only $45 \%$ if we assume that the efficiency of electric stoves is $90 \%$. The test details are given in the table below.

The cost is worked out based on the following rates prevailing in Malaysia.

Cost of Electrical Energy: RM 0.2535 per kWhr (Average)

Cost of Natural Gas : $\quad$ RM $0.75 / \mathrm{m}^{3}$

Cost of LPG: $\quad$ RM $1.45 / \mathrm{kg}$

Recommendations

a) Based on the cost of gas and electricity prevailing in Malaysia the gas stove with natural gas is more economical to use.

b) Only marginal difference in cost between gas stove with $L P G$ and electric stove is noticed. So, the choice of appliance usually depends on a number of factors including price, appearance, kitchen layout and personal preference.

c) Smaller electric appliances (such as frying pans and deep fryers) can be cheaper to run than both electric and gas stoves when shorter cooking periods are required.

d) Microwave ovens use less energy, so a microwave oven can be used instead of an electric stove or oven for reheating. 
e) In general, the larger the cooking appliance and the longer the cooking time, the more expensive cooking costs will be.

f) Small appliances tend to be more efficient and should be used wherever possible for cooking or heating smaller amounts of food.

g) A pressure cooker saves up to 2/3 cooking time, saves energy and preserves the goodness in your food.

h) A slow-cook pot also uses a great deal less energy.

\section{E. Refrigerator}

A three-door 66-kg refrigerator has been tested. Recommendations

a) The temperature shall be set as cold as you need; check manufacturer's recommendations.

b) Freezers shall not be let to build up more than $6 \mathrm{~mm}$ of frost. Defrost regularly to keep freezers working their best.

c) The old inefficient refrigerator running additionally for occasional use shall be avoided. It will increase your electricity bill.

d) The refrigerator shall not be overfilled, as this blocks air circulation. Conversely, a full freezer will perform better than an empty one.

e) The refrigerator's door seal shall be checked by closing the door over a piece of paper which is half in and half out of the refrigerator. If the paper can be pulled out easily, the hinge may need adjustment or the seal may need replacing.

f) Refrigerator shall be kept out of direct sunlight, and allow at least 5 centimeters all around (or as recommended by the manufacturer) to allow heat to escape from the compressor and condensing coil. If possible position your fridge away from cooker or any other source of heat, or at least leave a good gap between them.

g) Allow hot food to cool before putting them in the refrigerator.

h) Loading or unloading a fridge or freezer shall be done quickly as the cold air escapes and it costs more to get it back to a low temperature. For the same reason, the door shall not be opened unnecessarily and never leave it ajar.

i) Using a fridge or freezer that is too large also wastes energy.

j) If fridge or freezer is more than ten years old very significant savings can be made by replacing it with a modern efficient model. Apart from the technical improvements that have taken place during that period, there is the fact that the cabinet insulation tends to deteriorate with time and the compressor then has to work harder to maintain a low temperature.

\section{BEDROOM \& BATHROOM APPLIANCES}

\section{A. Air Conditioner (Split type)}

One split type air conditioner has been studied. This unit was fitted in a room of $3.86 \mathrm{~m}$ length, $3.12 \mathrm{~m}$ width and $2.80 \mathrm{~m}$ height. This room contains one window and one door. Using the remote control the temperature of the room may be adjusted from $20^{\circ} \mathrm{C}$ to $29^{\circ} \mathrm{C}$.

Mainly two experiments were conducted one with the temperature control setting at $25^{\circ} \mathrm{C}$ and the other at $20^{\circ} \mathrm{C}$. The unit was on for 10 hours with one person inside the room. It was noted that the power consumption at standby mode is almost zero. It is assumed that all the three bedrooms in the house (one in each room) are fitted with air conditioners for calculating energy consumption reported in the table.

\section{Recommendations}

a) During nights the thermostat shall be set at $25^{\circ} \mathrm{C}$ and light blanket shall be used to cover the body rather than setting the thermostat at $20^{\circ} \mathrm{C}$ and using thick blanket. This will reduce the energy cost by approximately $29 \%$.

b) The insulation of the air conditioned rooms shall be kept effective by sealing the door frames and window frames. Put false ceiling if the height of the room is more than 8 feet $(2.4$ $m$ ). This reduces the volume of the air to be cooled and hence reduces the energy cost.

c) The air conditioner shall be switched off when you are not in the room.

d) Ceiling fans shall be installed and use them to supplement or even as an alternative to air conditioner as ceiling fans generally use very little electricity.

e) Carpets can be used on floors to provide floor insulation.

f) Blinds, shades and window curtains shall be kept closed when the air conditioner is on. Thick window curtains shall be used if possible which will reduce the heat radiation into the room.

g) Unnecessary lights in the room where air conditioner is on shall be turned off. (They produce heat which works against the air conditioner.)

h) Leafy trees shall be planted on the sunny side of your house. This will cool the rooms and reduce the energy cost of air conditioners.

i) Air-conditioner unit shall not be installed in direct sunlight.

j) Filters shall be maintained bi-weekly or follow the manufacturer's instruction to reduce energy consumption.

\section{B. Hair Dryer}

One hair dryer with two power levels has been studied. For the same time duration, the power level 2 consumes $50 \%$ more energy than that of power level 1 . For an average usage of 30 minutes per day at power level 1 the energy consumption is $0.284 \mathrm{kWh}$, and it is $0.565 \mathrm{kWh}$ at power level 2. However, to produce the same dryness, the usage time with power level 2 will be shorter than with power level 1. It has been found out that the energy consumed to produce the same dryness with power level 1 is $20 \%$ lower than with power level 2 .

\section{Recommendations}

a) Towel your hair as dry as possible before using hair dryer.

b) Use power level 1, since it can reduce energy consumption by $20 \%$ than the power level 2 .

\section{C.Instant Water Heater (with or without pump)}

Two types of instant water heaters, one with pump and the other without pump have been studied. The heaters have two settings for heating power and two settings for the speed of water. The low water speed is $0.048 \mathrm{litre} / \mathrm{s}$ and high water velocity is 0.064 litre/s. These instant water heaters are mainly used for taking shower bath. As both water heaters consume almost the same amount of energy for the same settings, the results of one type alone is given here.

It is assumed that 50 liters of water is required per day to take a shower bath twice a day for one person. The power consumption will be the same for a particular power setting whatever may be the water speed setting. Of course, the higher the speed setting, the lower will be the temperature of the water. For comfortable shower, higher speed setting is preferable. For this speed setting it has been found that the water temperature is $39^{\circ} \mathrm{C}$ for medium power setting and $42^{\circ} \mathrm{C}$ for high power setting. The medium power setting has been found to give comfortable water temperature for taking shower bath. 


\section{Recommendations}

a) Instant water heater is more energy efficient compared to storage type water heater.

b) High water velocity with medium power setting shall be used which finishes the shower quickly and saves around $40 \%$ of energy.

c) Taking a shower instead of a tub bath, will save around $60 \%$ in water heating cost. This is because, it requires more water (around 75-125 liters) to fill up the tub every time a person is having a bath, as compared to the average water usage of 25 liters for a shower.

\section{Steam iron and Dry iron}

Two types of irons (Dry iron and steam iron) have studied. Both types have many power level settings marked as nylon, silk, cotton and wool etc. For this study, the power setting suitable for 'cotton' was used.

The readings obtained are for two different types of power rating. It is found that the percentage ON period of the dry iron is $33 \%$ and for the steam iron it is $40 \%$. Therefore, for irons with same power rating, the dry iron will consume less energy.

Recommendations

a) The dry iron shall be used as it consumes less energy.

b) The iron shall be switched off when not in use.

\section{E. Washing Machines}

One top loading washing machine and one front loading machine have been tested. We assume 12 wash per month for a normal house in preparing the table.

The top loading machine takes one hour per wash and the front loading machine takes 2 hours per wash.

\section{Recommendations}

a) Always use cold water for the RINSE cycle. Using warm or hot water for the RINSE cycle does not get your clothes any cleaner.

b) Front-loading washers consumes more energy than top loading washing machines, but they use roughly half the water per load, and are more effective at squeezing the water out of the clothes - which lowers the electricity costs for drying them.

c) The machine shall be run with full loads whenever possible but not to overload the machine. For small loads use half load button or quarter load button, if the machine has these features, to reduce water consumption.

d) Because we are in a hot country we can use "cold" water for normal washing or warm water for washing badly soiled clothes. A whopping $85-90 \%$ of the energy used by washing machines is for heating the water. A lot of energy can be saved by lowering the water temperature. Also hot water shrinks and fades your clothes, and wears them out more quickly.

e) As we are in a hot country avoid using dryer for the washed clothes. Clothes shall be dried outside the house.

\section{OTHER COMMON APPLIANCES}

\section{A. Ceiling and Table Fans}

One ceiling fan ( 3 blades, blade length, $62.5 \mathrm{~cm}$ and blade width, $13 \mathrm{~cm}$ ) in a room was studied. The speed regulators of the fan contained 5 settings.

As expected, the higher the speed setting of the fan, the higher is the energy consumption. The energy consumption at the speed setting of 5 (full speed) is around $70 \%$ higher than that at the speed setting 1 . For an average usage of 15 hours per day the energy consumption is $0.57 \mathrm{kWh}$ at the speed setting 3 and $0.848 \mathrm{kWh}$ at the full speed setting of 5 . It is assumed that there are 5 fans in a house, two in the hall and one in each bedroom for the energy calculation for the table.

One table fan with three speed setting is studied. The energy consumption at the speed setting of 3 (full speed) is $12 \%$ higher than that at the speed setting 1 . For an average usage of 20 hours per day the energy consumption is 0.64 $\mathrm{kWh}$ at the speed setting 2 (the average speed setting) and $0.68 \mathrm{kWh}$ at the full speed setting 3 .

Recommendations

a) The fan shall be cleaned regularly to reduce energy consumption.

b) Medium speed setting (Setting 3 for ceiling fan) which gives comfortable air circulation with lower energy consumption shall be considered compared to full speed setting.

c) Switch off the fan when you are not in the room.

\section{B. Lamps}

Some commonly used lamps have been studied to know their energy consumption pattern and illumination level. In order to produce same illumination different lamps will consume different power. To do the comparative study we have tested the following five different types of lamps. They are: Incandescent Lamps (Filament Lamp), Compact Florescent Integrated Lamps, CFIL (Energy saving lamps), Compact Florescent Non Integrated Lamps (Energy saving lamps), Florescent Lamps and Halogen Lamps

The light illumination of a lamp has been measured using a lux meter at a point which is at a distance of one meter directly below the lamp. The power consumption has been measured using the wattmeter. Based on the readings the following recommendation are made.

Recommendations

a) Use compact florescent integrated lamps compared to compact florescent non-integrated lamps.

b) Replace traditional incandescent bulbs with compact fluorescent (CFIL) lamps. They cost a bit more, but they use two-thirds less energy and can last up to ten times longer than incandescent lamps.

c) Conventional florescent lamps of $36 \mathrm{~W}$ will be preferable if we want bright illumination in a room as we require two CFIL to get the same illumination and there is no saving in energy.

d) If illumination by one compact florescent lamp (CFIL) is enough in a room then CFIL is preferred.

e) Wherever bright illumination is required halogen lamp may be used. As the light from the halogen lamp is yellow in colour people do not prefer in living or reading rooms. In such places halogen lamps may be installed to provide additional lighting along with other lamps.

f) Use electronic ballast in florescent lamps as it saves about $50 \%$ energy compared to normal ballast.

\section{CONCLUSION}

Table 1 presents the energy consumption details of various appliances in a typical house with a family of four. Second and third columns gives the energy consumption details without taking into effect the energy saving options. The total monthly energy cost is worked out based on the rates prevailing in Malaysia. Last two columns gives the energy consumption details taking into consideration some of the energy saving tips presented in the earlier sections 
(All the appliances are in Normal operating mode only).

Table 1. Energy consumption for a typical house with energy saving suggestion

\begin{tabular}{|l|c|c|c|c|}
\hline \multicolumn{1}{|c|}{ Appliance } & $\begin{array}{c}\text { Energy } \\
\text { per month } \\
(\mathrm{kWh})\end{array}$ & $\begin{array}{c}\text { Cost } \\
(\mathrm{RM}) \\
\text { Without } \\
\text { Saving }\end{array}$ & $\begin{array}{c}\text { Energy } \\
\text { per month } \\
(\mathrm{kWh})\end{array}$ & $\begin{array}{c}\text { Cost } \\
(\mathrm{RM}) \\
\text { With Saving } \\
\text { Options }\end{array}$ \\
\hline $\begin{array}{l}\text { Personal Computer and } \\
\text { Accessories }\end{array}$ & 36.53 & 10.26 & 9.76 & 2.65 \\
\hline Entertainment Appliances & 60.59 & 17.01 & 50.26 & 13.64 \\
\hline Vacuum Cleaner & 62.4 & 17.52 & 48.00 & 13.03 \\
\hline Automatic Rice Cooker & 8.1 & 2.27 & 5.40 & 1.47 \\
\hline Electric Oven & 36 & 10.11 & 36.00 & 9.77 \\
\hline Stoves and Kettle & 59.73 & 16.77 & 32.64 & 8.86 \\
\hline Refrigerator & 52.13 & 14.64 & 52.13 & 14.15 \\
\hline Air-Conditioner & 471.6 & 132.40 & 336.60 & 91.38 \\
\hline Instant water heater & 71.79 & 20.16 & 47.08 & 12.78 \\
\hline Hair Dryer & 11.29 & 3.17 & 9.07 & 2.46 \\
\hline Iron & 16.8 & 4.72 & 10.29 & 2.79 \\
\hline Washing Machine & 3.17 & 0.89 & 1.23 & 0.33 \\
\hline Fans and Lamps & 300.53 & 84.38 & 194.76 & 52.87 \\
\hline & $\mathbf{1 1 9 0 . 6 6}$ & $\mathbf{3 3 4 . 2 8}$ & $\mathbf{8 3 3 . 2 2}$ & $\mathbf{2 2 6 . 2 0}$ \\
\hline
\end{tabular}

Please note that the saving in cost with energy saving option is $32.33 \%$. It will be more if some of the non quantifiable energy saving suggestions are also taken into consideration.

\section{REFERENCES}

[1] Ar Chan Seong Aun. "Energy Efficiency - Designing Low Energy Buildings Using Energy 10." Pertubuhan Arkitek Malaysia, CPD Seminar,7 August 2004.

[2] Brahmanand Mohanty. "Standby power losses in household electrical appliances and office equipment." Regional Symposium on Energy Efficient Standards and Labeling. 29-31 May 2001 Bangkok, Thailand.

[3] "Energy Efficiency Fact Sheet 6". sunderland.gov.uk . 22 Feb. 2006. Sunderland City Council. 15 July 2006 $<$ http://www.sunderland.gov.uk/Public/Editable/Themes/Housing/Ho using-in-Sunderland/energy-efficiency.asp>

[4] "Energy Efficient In the Home." theiet.org. The Institution of Engineering and Technology. 10 June 2006 $<$ www.theiet.org/publicaffairs/energy/saveitnew.pdf $>$

[5] "How to Be Energy Efficient". eagagroup.com EAGA Partnership. 6 June $2006<$ www.eagagroup.com>

[6] "How to Save Money on your Energy Bills." energysmart.com. Energy Smart Information Centre. 15 June 2006 $<$ www.energysmart.com.au>

[7] Leona K. Hawks, "Kitchen Appliances." Online Posting. Utah State University: Extension Publications \& Knowledge Base . 5 June 2006 $<$ http://extension.usu.edu/files/publications/publication/HI 18.pdf $>$

[8] McMaster Institute for Energy Studies, Canada "Conserve Energy and Save Money." 2 June 2006. <www.energy.gov.on.ca>

k) "Saving Energy At Home." edf-energy.com. 5 June 2006. Energy Co. Ltd. 20 June $2006 . \quad \mathrm{http}: / /$ www.edf-energy.com/edfenergy/showPage.do?name=homeenergy.energyefficiency.savingstips .til

[9] Sustainable Energy Development Office's Energy Smart Line, Australia " Live Energy Smart - Cooking." 5 June 2006. $<$ www.sedo.energy.wa.gov.au>

[10] The Hong Kong \& China Gas Co. Ltd., "Save Energy In Your Home." 5 June 2006. 17 July 2006

[11] http://www.epd.gov.hk/epd/eindex.html 


\title{
Performance Analysis of Savonius Wind Rotor for Directed Flow Operation
}

\author{
H. H. Al-Kayiem, A. E. Sa'ad Al-deen
}

\begin{abstract}
The Savonius wind rotor is commonly used in a free air stream environment and is installed vertically. In the present work, the rotor is subjected to mathematical modeling and numerical analyses to study it's suitability for energy conversion in artificial wind generator. In this application, the rotor is installed horizontally. Three installation configurations are proposed, modeled mathematically and analyzed numerically. In the first proposed configuration, the entire rotor is subjected to the wind stream. In the second configuration, the flow is diverted to be captured by the down concave vane, only. In the third configuration, a guide plate is inserted to apply as a casing for the rotor. Some of the obtained results have been compared with previous experimental results to verify the adopted numerical analysis. The third configuration has produced considerable enhancement in the turbine performance. The power coefficient is increased by $34 \%$ and the torque coefficient is increased by $70 \%$ compared with the traditional configuration. The analyses confirm the possibility of using the modified wind rotor for the artificial wind generator, as well as further applications requiring ducted installation of the rotor.
\end{abstract}

Keywords: wind energy, wind rotor, Savonius, numerical modeling

\section{INTRODUCTION}

Solar energy is one of the array of alternative energy options that is being vigorously pursued by a number of countries in an effort to reduce their dependence on fossil based-fuel. Also, it is to be used as energy source in remote areas where other energy sources are not available, as well as it is environmentally friendly. There is no involvement of fossil fuels in the energy gathering from wind or solar. The wind has been used as an energy source for thousands of years all across the world. Very early wind machines are vertical axis structure and they are used for pumping water in irrigation projects. Horizontal axis wind machines were developed by the Arab nation and their use became widespread throughout the Islamic world [1].

In addition to its use in vertical installation in open atmosphere, the vertical axis turbines are used in many

This work was supported in part by Universiti Teknologi PETRONAS.

Hussain H. Al-Kayiem is Associate professor in Mechanical Engineering with the Universiti Teknologi PETRONAS, 31750 Tronoh, Perak, Malaysia (phone: 605368 7008; fax: 605 3656461; e-mail: hussain_kayiem@ petronas.com.my).

Afreen E. Sa'ad Aldeen was with Al-Mustansirya University, Baghdad, Iraq (phone: +964 4158351, e-mail::musteng1985@yahoo.com). further applications. Both drag and lift turbines have been investigated, mainly for use in Oscillating Water Column (OWC). An example is the Darrieus turbine with three or for thin blades airfoil section in an OWC plant. Some stand-alone prototypes have been tested including $5 \mathrm{~kW}$ Darrieus turbine in the KurushimaStrait, Japan. The concept of installing a number of vertical axis turbines in a tidal fence is being pursued in Canada, with plans to install a 30 MW demonstration system in the Philippines. [2]

Ref. [3] used the Savonius turbine in horizontal installation as a bi-directional turbine in an OWC system to convert the wave energy shown in figure 1.

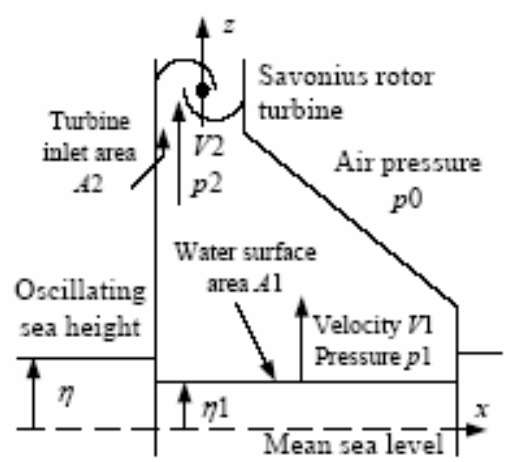

Figure 1. Oscillating Water Column (OWC) plant diagram using Savonius rotor [3]

He carried out mathematical analysis of the system including the ducted Savonius rotor.

This paper reports numerical analysis of Savonius wind rotor proposed to be used as energy converter in a rectangular shaped thermal solar chimney.

\section{The Proposed SOlar CHIMnEY}

A thermal solar chimney with rectangular cross section can be implemented using external wall facing the sun as a collector. The wall is to be covered by good solar absorbing material. Cement wall, for example, painted by black color paint could be used as the collector. Canopy cover, made of glass, then, to be installed to provide transparency media for the solar radiation. The wall and the canopy are spaced by reasonable distance to create rectangular flow passage for the warmed air. The structure represents thermal solar chimney. By installing Savonius rotor at the top of the chimney, power can be extracted from the air stream created inside the chimney. Analysis of the rotor performance for such application is necessary since many blade geometries 
can be used to design the Savonius rotor, and, many installation options may improve the rotor performance [4]. Schematic of the proposed thermal solar chimney is shown in figure 2.

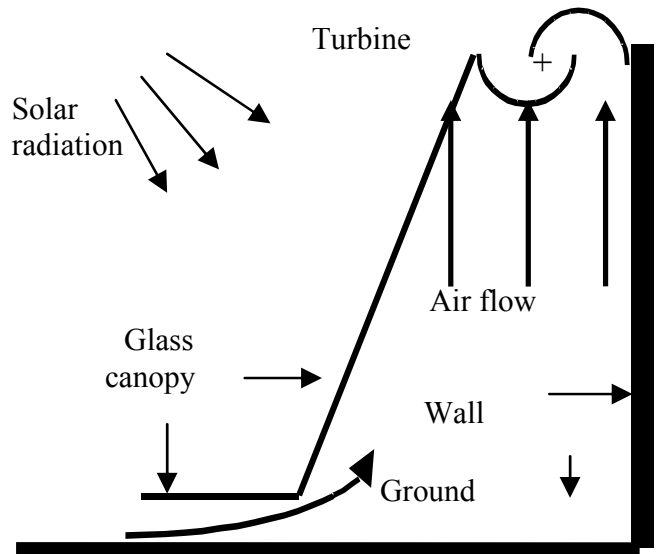

Figure 2. Thermal solar chimney diagram using Savonius rotor

The input solar energy to the system is the total amount of heat absorbed by the system, $q_{\text {total }}$. It can be calculated as the sum of radiation heat transfer to the wall, $q_{\text {wall }}$ and to the ground, $q_{\text {ground }}$, as

$$
q_{\text {total }}=q_{\text {ground }}+q_{\text {wall }}
$$

where

$$
q_{\text {ground }}=I \cdot \tau_{\text {glass }} \cdot \alpha_{\text {ground }} \cdot A_{\text {ground }}
$$

and

$$
q_{\text {wall }}=I \cdot \tau_{\text {glass }} . \alpha_{\text {wall }} \cdot A_{\text {wall }}
$$

$I$, is the solar intensity in $\mathrm{W} / \mathrm{m}^{2}, \tau_{\text {glass }}$ is transmissivity of the glass, $\alpha_{\text {wall }}$ and $\alpha_{\text {ground }}$ are the absorbtivity of the wall and the ground respectively, and $A_{\text {wall }}$ and $A_{\text {ground }}$ in $\mathrm{m}^{2}$ are the wall and ground areas respectively. The power extracted by the turbine, $P_{t}$ is governed by the fundamental wind turbines equation:

$$
P_{t}=C_{p} P_{w}=C_{p} \cdot \frac{1}{2} \rho \cdot V^{3} A_{\text {rotor }}
$$

Where $C_{p}$ is the power coefficient represent the ability of the rotor for energy conversion.

All the available data of the Savonius turbine is related to open atmosphere operation. The objective of the present work is to provide data for ducted Savonius turbines in horizontal installation as in the tidal power stations, the OWC power stations and in the proposed solar chimney pilot. The analyses are carried out numerically on three different configurations of installations.

\section{SAVONIUS WIND TURBINE}

A Savonius rotor is a drag-type of vertical axis wind turbine (VAWT). The power conversion is governed by the traditional wind power conversion. Savonius wind turbines typically only have an efficiency of around 15\% i.e. just $15 \%$ of the wind energy hitting the rotor is turned into rotational mechanical energy when operate in open atmosphere[5]. This wind turbine turns slowly but generates a high Torque. It is a low cost structure although the conversion is also low.

The traditional Savonius rotor is shown with the corresponding dimensions in figure 3. The dimension $S$ is called the offset and $R$ is the vane radius of rotation, and $d$ is the geometrical diameter of the vane. With $h$ is the turbine axial length, and $D$ is the rotor diameter, the wind capturing area becomes:

$$
A_{\text {rotor }}=h(2 d-S)=h D
$$

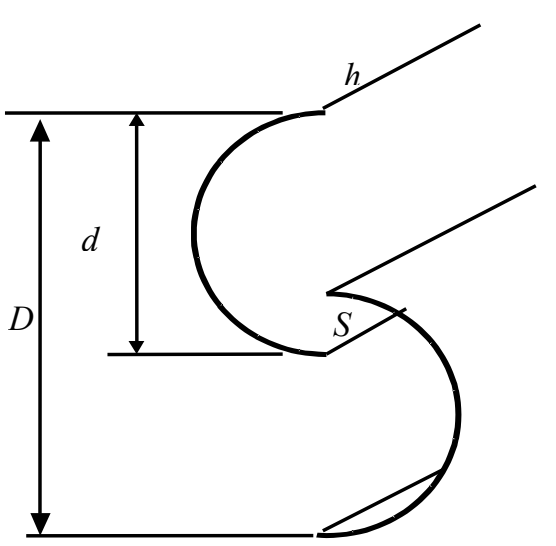

Figure 3. Geometries of traditional Savonius rotor

The conversion performance of the rotor is highly dependent of the offset value, $S$. Experimental analysis by [6] are reported for $-0.3<s / d<0.6$ in steps of 0.1 . Among the 10 tested offsets, they have claimed highest conversion at $s / d$ equal to 0.2 . An optimum offset is found to be within 0.15 to $1 / 6$. The latest value has been reported by many other investigators, e.g.; [6] and [7], and was adopted in the present analysis.

\section{NUMERICAL MODELING}

Three different installation configurations of the rotor are considered in the present analysis as will be discussed later. The analysis is carried out numerically. Each vane is subdivided into $\mathrm{N}$ subdivision, segment. Each segment is representing partial area of the vane subjected to air flow. The equations governing the flow are the continuity and the momentum as:

The momentum

$$
\sum F=\frac{\partial}{\partial t} \int_{c v} \rho \cdot V \cdot d v+\int_{c s} \rho \cdot V \cdot \vec{V} \cdot d A
$$

The continuity

$\frac{\partial}{\partial t} \int_{c v} \rho d v+\int_{c s} \rho V . d A=0$

The flow is assumed steady, $\partial / \partial \mathrm{t}=0$, and 
incompressible, $\rho=$ constant. The wind velocity vector on the segment of area is analyzed to normal and tangential components as in figure 4. The normal component is the drag producer on the element after subtraction of the vane velocity component in the same direction caused by the rotational speed. Summation of the drag forces on the $\mathrm{N}$ segments represents the total drag on the vane. The performance characterization of the rotor requires the torque rather than the force as will discussed later.

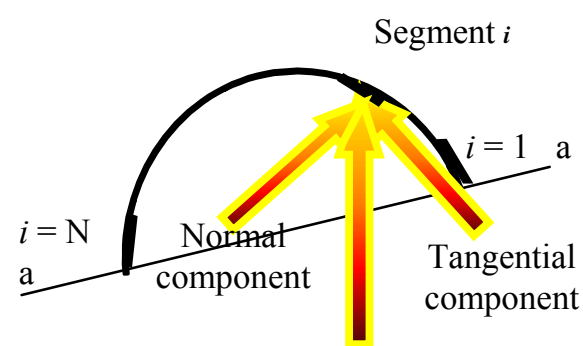

Wind velocity vector

Figure 4 . The wind velocity vectors on the segment.

\section{A. Segmentation Criteria}

The position of segment $i$ is vary from $i=1$ at the outer radius up to $i=\mathrm{N}$ at the inner radius along the half circle of the vane surface. The vane location itself is variable since it rotates. Modeling of the forces acting on the segments requires detailed velocity vectors analysis and detailed angles definition. Three angles, $\beta, \psi$, and $\alpha$ shown in Figure 5, and are identified as:

$\beta$, is the rotation position angle of the axis line aa with respect to the $\mathrm{x}$-axis.

$\psi$, is the segment $i$ position angle with respect to the axis of rotation (AoR).

$\alpha$, is the segment $i$ position angle with respect to the geometrical centre of the vane.

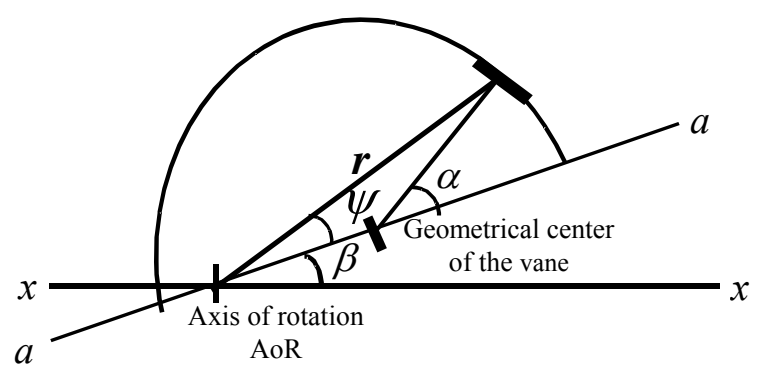

Figure 5. Angles defining the segment position w.r.t. the axis of rotation and geometrical centre of the vane

The values of the angle $\alpha_{\mathrm{i}}$ varies from $(180 / 2 \mathrm{~N})^{\circ}$ at $i=1$ up to $(180-(180 / 2 \mathrm{~N}))^{\circ}$ at $i=\mathrm{N}$. The angle $\psi_{\mathrm{i}}$ can be obtained from the triangles geometries as:

$$
\psi_{i}=\sin ^{-1}\left[\left(0.5 d \times \sin \alpha_{i}\right) / r_{i}\right]
$$

The radius of rotation, $r_{i}$, of segment $i$ is evaluated as:

$$
r_{i}=\left[\begin{array}{l}
\left(0.5 d \times \sin \alpha_{i}\right)^{2} \\
+\left(0.5\left(d-S+d \times \sin \alpha_{i}\right)\right)^{2}
\end{array}\right]^{0.5}
$$

\section{B. Modeling of the Forces}

The wind velocity is analyzed as shown in figure 5 . The created Drag force on the segment, $i$ is obtained by applying the momentum principle. The segment $i$ rotate as part of the rotor with peripheral velocity, $V_{r}$. Accordingly, the momentum equation on the segment becomes:

$$
\begin{aligned}
F_{i} & =\dot{m}\left(V_{w}-V_{r, i}\right)_{n} \\
& =\rho A_{s}\left(V_{w . n, i}-V_{r . n, i}\right)^{2}
\end{aligned}
$$

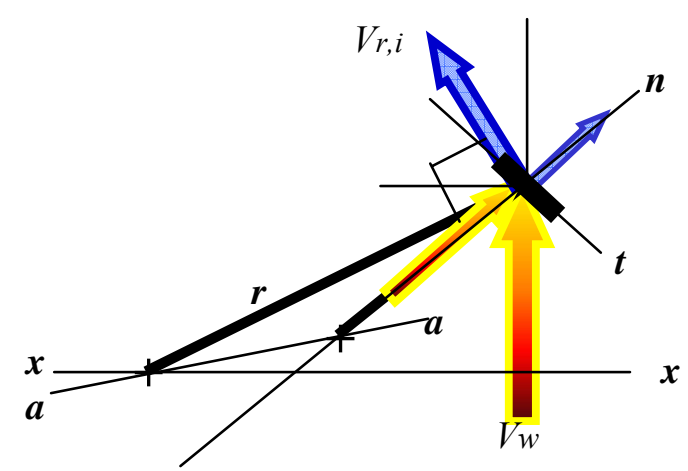

Figure 6. Velocity analysis to obtain the drag force on the segment

The normal velocities components of the wind, $V_{w . n, i}$, on the segment area are obtained with the aid of figure 6 , as:

$$
V_{w . n, i}=V_{w} \sin \left(\beta_{j}+\alpha_{i}\right)
$$

and, the normal velocity component, $V_{r . n, i}$, resulted from the rotation, in the normal direction to the segment, is derived as:

$$
V_{r . n, i}=V_{r}\left[\begin{array}{l}
\cos \left(\beta_{j}+\psi_{i}\right) \sin \left(\alpha_{i}+\beta_{j}\right) \\
-\sin \left(\beta_{j}+\psi_{i}\right) \cos \left(\alpha_{i}+\beta_{j}\right)
\end{array}\right]
$$

where, $V_{r}=\omega r_{i}$. The offset area $(S \times h)$ contributes as an obstacle to the wind flow to hit the internal surface of concave down vane, 1 . In the same time, it reflects the flow from the internal surface of vane 1 to the internal surface of concave up vane, 2 . The flow streaming is shown in figure 7. 

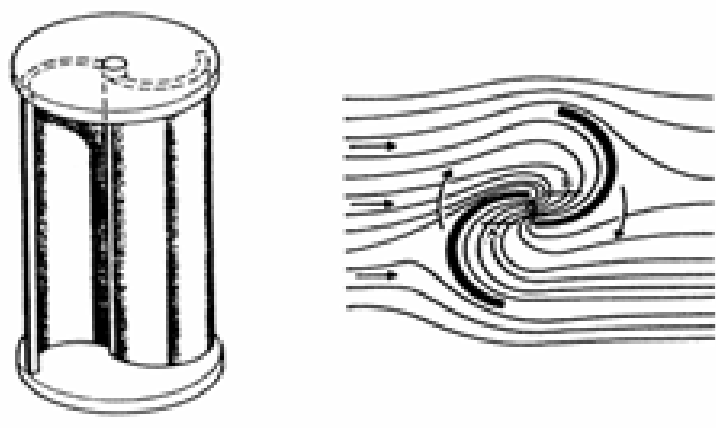

Figure 7. The Flow orientation due to the offset, $S$ [4]

The analysis includes mathematical modeling of the flow deflection from the concave down vane to concave up cup in the space denoted by "the offset, $S$ ", as shown in figure 8 .

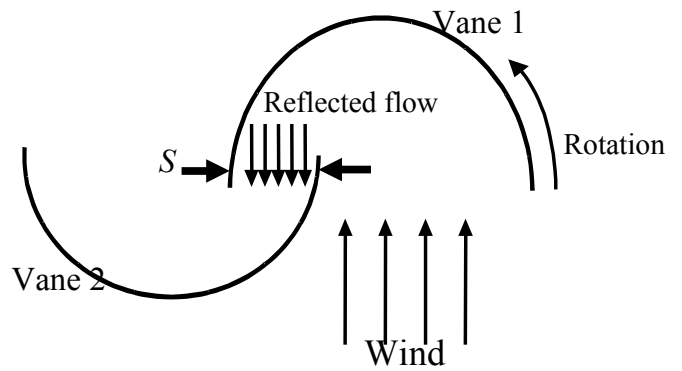

Figure 8. Modeling of the reflected flow in the offset portion

\section{Computational Procedure}

The modeling of the force on the entire segments of the two vanes has been converted into a computer program. The set of equations have been solved simultaneously to characterize the rotor at different tip speed ratio, $\lambda$. To select the optimum number of segmentation, preliminary tests has been carried out by selecting $\mathrm{N}=5,10 \ldots$ up to 100 in steps of 5. The results are found to be stable and accurate at $\mathrm{N}=$ 40 with certainty of $95 \%$ based on the deviation from the mean of $\mathrm{C}_{\text {Tmax. }}$ at $\mathrm{N}=100$. The total drag force applied on the rotor is the summation of the segments drags of the two vanes.

The Torque, $T_{j}$, applied on the rotor at rotational position angle, $\beta_{j}$, is evaluated as:

$$
T_{j}=\sum_{N=1}^{N=40} T_{i, \text { vane } 1}+\sum_{N=1}^{N=40} T_{i, \text { vane } 2}
$$

Where

$$
T_{i}=F_{i} \times L_{i}
$$

And $L_{i}$ is the perpendicular distance from the AoR to the normal vector on the segment $i$ evaluated as

$$
L_{i}=r_{i} \times \sin \left(\alpha_{i}-\psi_{i}\right)
$$

The position variation of the segment $i$ due to the rotation is identified by the angle $\beta$ made by the axis a-a and the axis $\mathrm{x}-\mathrm{x}$. For one revolution of the rotor, the angle $\beta$ is varied from $0^{\circ}$ up to $360^{\circ}$. The index $j$ is the sub divider of $\beta$ and is selected to subdivide the complete revolution into 36 positions. Accordingly, $\beta=0$ at $j=1$ and increased in steps of $10^{\circ}$ to close complete revolution with $\beta=360$ at $j=36$.

A computer program was written using Quick Basic programming language to solve the set of equations which allow the evaluation of the parameters of each segment. Two Sets of $\mathrm{N}$ equations are arranged in matrix format to evaluate the Torque on vane 1 and vane 2 . The procedure starts with $\beta=0$ at $j=1$. The two sets are solved by matrix inversion resulting in 40 values of Torque for each vane. The some according to equation 12 represents the resulted Torque of the rotor at $\beta=0$. The procedure is repeated by setting $\beta=10$, i.e.; $j=2$. Another vale of the rotor Torque is obtained. The Torque evaluation is repeated for a completed rotation from $j=1$ to $j=36$ and, then the average is considered as:

$$
T=\frac{1}{36} \sum_{1}^{36} T_{j}
$$

The Torque coefficient is evaluated as

$$
C_{T}=\frac{T}{1 / 2 \rho v^{2} A_{\text {rotor }}(D / 2)}
$$

And the power coefficient is evaluated from

$$
C_{p}=\lambda C_{T}
$$

\section{Discussion OF Results}

The aim of the present analysis is to probe the highest performance can be obtained from ducted Savonius wind rotor in a solar chimney. Three configurations, that the rotor can be installed as in figure 9, are subjected to the present numerical analysis.

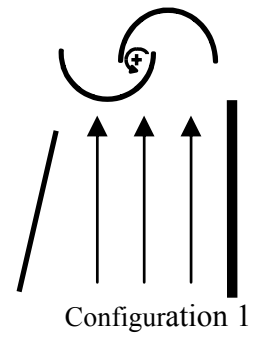

Configuration 1

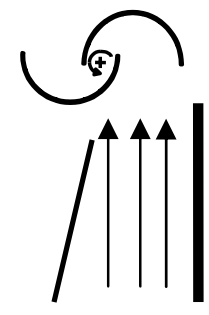

Configuration 2

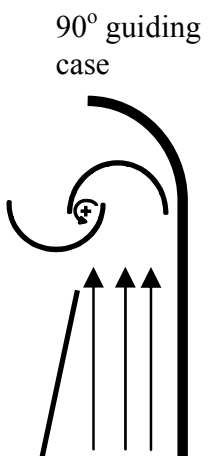

Configuration 3
Figure 9. The three configurations subjected to analysis

The results have been verified by comparison with experimental investigations reported by previous researchers. Different experimental values of the maximum power coefficient have been reported for the Savonius rotor. They are ranging from 0.14 to 0.25 and the maximum power coefficient occurred within TSR ranging from 0.9 to 1.1. The difference is caused mainly by the fabrication procedures and the value of the offset $S$. Table 1 shows a 
comparison between the present numerical analysis and previous experimental results obtained by further works.

Table1. Comparison of calculated $\mathrm{C}_{\mathrm{p} \text {,max }}$ with experimental results

\begin{tabular}{|l|c|c|}
\hline Reference & $\mathrm{C}_{\mathrm{p} \text { max. }}$ & $\mathrm{TSR}$, \\
\hline Present Numerical Analysis & 0.21 & 0.9 \\
Ref. 5, Experimental & 0.17 & 0.93 \\
Percival and Datta, 2004, Experimental & 0.22 & 1.1 \\
Blackwell et al., 1978, Experimental & 0.24 & 0.92 \\
Gourieres, 1982, Experimental & 0.15 & n. a. \\
\hline
\end{tabular}

The trend of the power coefficient, $\mathrm{C}_{\mathrm{p}}$, evaluated from the present work is similar to the trend of the experimental results gained by other works (e.g., Ref. 6 and Ref. 7) as shown in figure 10. This demonstrates the validity of the numerical procedure adopted in the present analysis.

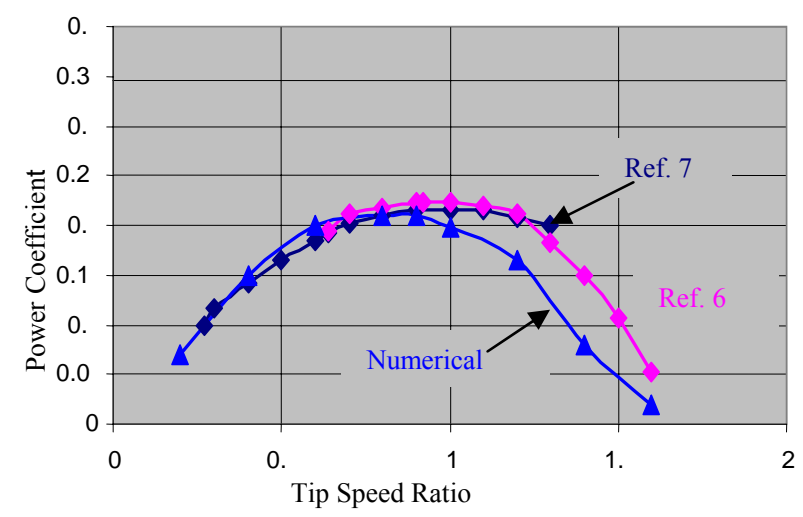

Figure10. Comparison between the predicted and the experimental power coefficient data verses the TSR

The results obtained numerically for the ducted installation are demonstrating higher values of power coefficient than the case of open air installation. The value the maximum power coefficient as 0.12 , in fact, is considered high when compared with the common experienced data from experimentation. This is due to the assumption that the entire flow rate is captured by the turbine area. In open atmosphere operation, part of the flow diverted to the sides of the turbine as shown schematically in figure 7.

The two assumed modifications of the installation (configurations 2 and 3 ) demonstrate appreciable contribution on the performance of the rotor. The maximum power coefficient is increased by $12 \%$ when the down concave vane is isolated from the air stream approaching the rotor. The drag force exerted on the down concave is eliminated and only the upward concave is contribute in the final drag force evaluation.

The idea of guiding the flow up to $90^{\circ}$ along the rotation path, as shown in figure $9-$ configuration 3 , has enhancement the rotor performance considerably. The maximum power coefficient is increased by $34 \%$ compared with the maximum power coefficient of configuration 1 . The enhancement of the maximum power coefficient is presented in Table 2.

For a TSR, $\lambda$, ranging from 0.2 up to 1 , the predicted value according to configuration 3 results in higher performance compared with the other configurations. While, for TSR higher than 1, the power coefficient obtained from configuration 2 demonstrates higher values of power coefficient. The results of the power coefficient for the three analyzed configurations are shown in figure 11.

Table2. The enhancement in the power coefficient at different configurations compared with configuration 1

\begin{tabular}{|c|c|c|}
\hline Configuration & $\mathrm{C}_{\text {p.max. value }}$ & $\begin{array}{c}\text { \% of increase } \\
\text { in } \mathrm{C}_{\text {p.max. }}\end{array}$ \\
\hline 2 & 0.24 & 12 \\
\hline 3 & 0.32 & 34 \\
\hline
\end{tabular}

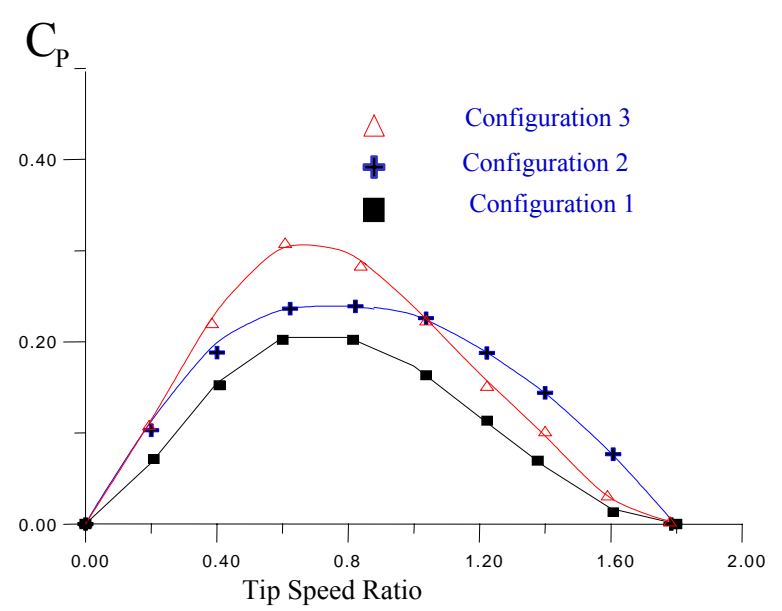

Figure 11. The power coefficient verses the TSR for the three configurations

One of the advantages of the Savonius rotor is the capability to produce high Torque which can be exploited to produce higher rotational speed by gear system. The analysis of the present work shows high enhancement in the Torque coefficient value by using the proposed mounting configurations. The results of the Torque coefficient of the three configurations are shown in figure 12. The results show that the maximum Torque coefficient is not affected highly when operating under the conditions of configuration 2. Large increase in the Torque coefficient value is obtained when operating the turbine as proposed in configuration 3 . The percents of enhancements in the Torque coefficient are shown in table 3 .

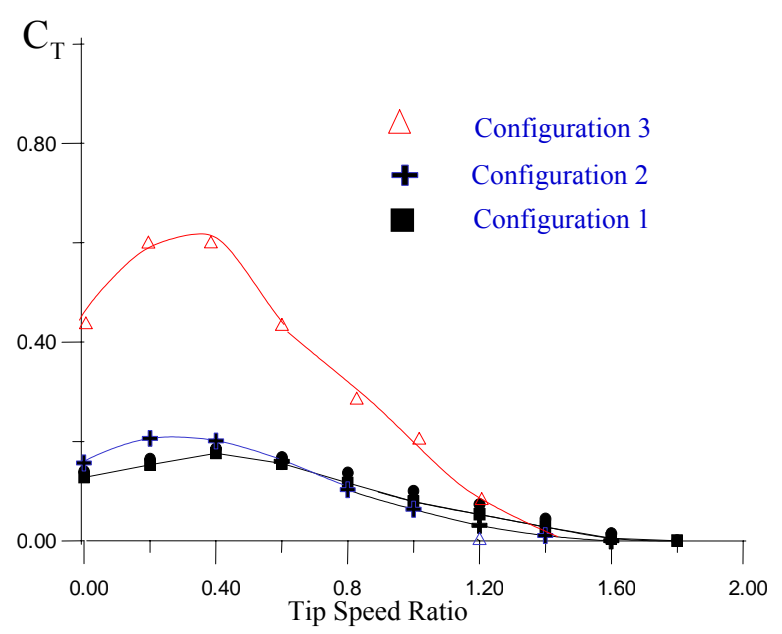

Figure 11. The Torque coefficient verses the TSR for the three configurations 
Table 3. The enhancement in Torque coefficient at different configurations compared with configuration 1

\begin{tabular}{|c|c|c|}
\hline Configuration & $\mathrm{C}_{\text {T.max. value }}$ & $\begin{array}{c}\text { \% of increase in } \\
\mathrm{C}_{\text {T.max }}\end{array}$ \\
\hline 2 & 0.2 & 10 \\
\hline 3 & 0.59 & 70 \\
\hline
\end{tabular}

\section{CONCLUSION}

Savonius wind rotor is modeled and analyzed by Numerical technique. The rotor is proposed to be installed horizontally as part of a solar chimney pilot. Three configurations of installation are included in the analysis. The numerical results are verified by comparing with previous experimental data. Good agreement is obtained which confirm the validity of the numerical modeling. The proposed modifications have shown considerable enhancement in the rotor performance mainly the one with flow guiding plate. The rotor can be used in connection with gear system to operate electrical motor for power generation since it produces relatively high Torque.

\section{REFERENCES}

[1] W. Shephered, and D. W. Shehpered, Energy Studies, Imperial College Press. 2003.

[2] O. Yaakob, T. M. Ariff and M. A. Abdul Mukti, "Prospects for ocean energy in Malaysia", CIEE2006 conference, UNITEN, Bangi, Malaysia, 28-30 Aug. 2006. PP62-68.

[3] D. G. Dorrell and W. Fillet, "Investigation of a small-scale segment oscillating water column utilizing a Savonius rotor turbine", CIEE2006 conference, UNITEN, Bangi, Malaysia, 28-30 Aug. 2006. PP23-29.

[4] A. E. Sa'addeen, Enhancement of Savonious Wind Rotor, MSc Dissertation, College of Engineering, Al-Mustansirya University, Baghdad, Iraq,2005.

[5] Savonius Wind Turbines, Wind, http://www.reuk.co.uk/wind.htm

[6] M. C. Percival, P. K. Datta, "The development of a vertical turbine for domestic electricity generation “, European Wind Energy Conference,22-25 Nov., London, 2004(available on line).

[7] B. B. Blackwell, R. Sheldahl, and L V. Felts, "Wind tunnel performance data for two and three bucket Savonius rotor", Journal of Energy, 1978; 2:160-164.

[8] D. Le Gourieres, Wind Power Plant Theory and Design; Pergamon Press Ltd, 1982. 


\title{
Production of Biodiesel from Palm Oil Using Montmorillonite KSF as a Heterogeneous Catalyst
}

\author{
J. Kansedo, K. T. Lee, S. Bhatia
}

\begin{abstract}
The production of biodiesel has become popular nowadays as a result of the increasing demand for a clean, safe and renewable energy. Biodiesel is made from natural sources such as vegetable oils and animal fats. The conventional method of producing biodiesel is by reacting vegetable oil with alcohol in the presence of homogenous catalyst $(\mathrm{NaOH})$. However, this conventional method has some limitations such as the formation of soap, usage of lot wash water and complicated separation processes. On the other hand, heterogeneous process using solid catalysts has a lot of advantages over the homogenous methods. This study focused on the production of biodiesel from palm oil using montmorillonite KSF as the heterogeneous catalyst. Cooking oil and methanol were used as the raw materials. The heterogeneous transesterification process was studied using design of experiment (DOE), specifically response surface methodology (RSM) based on four-variable central composite design (CCD) with $\alpha$ $=2$. The transesterification process variables were reaction temperature, $x_{1}\left(50-190{ }^{\circ} \mathrm{C}\right)$, reaction period, $x_{2}(60-300 \mathrm{~min})$, methanol/oil ratio, $x_{3}(4-12 \mathrm{~mol} / \mathrm{mol})$ and amount of catalyst, $x_{4}(1-5 \%$ wt $)$. It was found that the conversion of palm oil to biodiesel can reach up to $78.69 \%$ using the following reaction conditions; reaction temperature of $155{ }^{\circ} \mathrm{C}$, reaction period at 120 min, ratio of methanol $/$ oil at $10: 1 \mathrm{~mol} / \mathrm{mol}$ and percentage of catalyst at $4 \%$. From this study, it was shown that montmorillonite KSF catalyst can be used as a solid catalyst for biodiesel production replacing the conventional homogenous process.
\end{abstract}

Keywords: biodiesel, transesterification process, heterogeneous catalysts, montmorillonite KSF

\section{INTRODUCTION}

Due to increasing crude petroleum oil prices and the effect of global warming, a search for alternative fuels has

All authors are with the School of Chemical Engineering, Universiti Sains Malaysia Engineering Campus, 14300 Nibong Tebal, Seberang Perai Selatan, Pulau Pinang, Malaysia.

K. T. Lee is the corresponding author. (chktlee@eng.usm.my), Tel: 045996467. Fax: 04-5941013. J. Kansedo.(jib_zbz@yahoo.com).

S. Bhatia. (chbhatia@eng.usm.my). gained significant attention over the years. Among the various sources of alternative energy, renewable energy such as solar, hydro and biomass present a promising alternative to replace the conventional fossil fuels. Since Malaysia is the largest producer of palm oil in the world, this commodity presents a promising alternative to be converted to bio-diesel as a source of renewable energy, replacing the petroleum-based diesel oil.

In the recent years, a number of studies have shown that vegetable oil such as palm oil can be used as a source of bio-diesel fuels [1]-[4]. However, the direct utilization of vegetable oil in diesel engine can lead to a number of problems such as poor fuel atomization, poor cold engine start-up, gum and other deposit formation. Consequently, considerable efforts have been made to develop alternative bio-diesel fuels that have the properties and performance similar to the petroleum-based diesel fuels. The most promising way to overcome these problems is the transesterification of triglycerides present in vegetable oil such as palm oil to fatty acid alkyl esters.

Transesterification, also called alcoholysis, is the reaction of an oil with an alcohol to form esters and glycerol [2]-[4]. This process has been widely used to reduce the viscosity of vegetable oil. In the transesterification process, the triglycerides in the vegetable oil will react with alcohol to form mixture of fatty acid alkyl esters and glycerol [2-4]. The fatty acid alkyl esters produced from this process is known as bio-diesel which has become more attractive recently because it has almost the same properties and characteristic of petroleum-based diesel. Several studies have shown that bio-diesel produced from vegetable oil have viscosity close to petroleum-based diesel fuel [1], [5], [6]. Their volumetric heating values are a little lower compared to the petroleum-based diesel fuel, but they have high cetane and flash point values.

The transesterification process includes the reaction between oil and alcohol, with the presence of catalyst as shown in the equation (1) below.

$\mathrm{TG}+3 \mathrm{R}$ 'OH $\leftrightarrow 3$ Alkyl esters (biodiesel) + Glycerol

where TG is triglycerides and $\mathrm{R}^{\prime} \mathrm{OH}$ is alcohol.

Transesterification process is catalyzed by catalysts with both, acidic and basic sites [2]-[5]. Currently, all the 
transesterification process goes through the homogenous transesterification process by mainly using potassium hydroxide as the catalyst [2]. The Malaysian Palm Oil Board (MPOB) research centre has also successfully developed this technology [9]. In the homogeneous transesterification process, the catalysts used are in the same phase as the reactants/ products. However, homogeneous transesterification process using potassium hydroxide presents certain disadvantages. Among some of the disadvantages are [6], [7]:

a. The formation of soap in the product mixture. This has led to additional cost required for the separation of soap from the bio-diesel.

b. The separation of soap requires several steps that makes the overall process complex.

c. Since the catalyst and the reactants/products are in similar phase, the separation of products (bio-diesel) from the catalyst becomes complex.

d. The formation of soap also led to the loss of triglycerides molecules that can be used to form biodiesel.

These disadvantages make the homogeneous transesterification process complex and uneconomical. Therefore, there is a need to develop a novel technology for the conversion of palm oil to bio-diesel using alternative route that can overcome the limitations of the homogenous transesterification process. One of the possible routes is by using heterogeneous transesterification process. In heterogeneous transesterification process, the catalysts are in different phase from the reactants/products. The advantages of heterogeneous transesterification process are as follows [5]:

a. It is a simple process whereby the products of the reaction only consist of glycerol and bio-diesel. Since glycerol and bio-diesel are essentially immiscible in each other, the separation processes are very simple. The glycerol produced has a high demand in the pharmaceutical industries.

b. Since the catalyst and the products/reactants are in different phases, separation process is simpler.

c. There is no formation of soap, and therefore there is no additional cost required for its separation.

Based on this information, it is obvious that the heterogeneous transesterification process overcomes all the limitations of the homogenous transesterification process. Recent researches have shown that there are various types of catalyst that can be used in the heterogeneous transesterification process. Jitputti et al. [6] studied the transesterification of coconut oil and palm kernel oil using zirconium oxide-based and zinc oxide-based catalyst, while Suppes et al. [10] used zeolite and metal catalyst for the conversion of soybean oil to bio-diesel. In other research, $\mathrm{Na} / \mathrm{NaOH} / \mathrm{Al}_{2} \mathrm{O}_{3}$ [8], [15] and metal complexes [12] are used as the catalyst.

Other researches have investigated various heterogeneous catalysts such as zeolites [10], [28], metal oxides [11], [19], [21], [22], [25], [29]-[32], metal complexes [12], tungstated zirconia [13], resins (acid and basic) [14, 16], $\mathrm{NaOH}-$ alumina catalyst [15], sulfonic-acid functionalized [17], activated calcium oxide [19], anionic ion-exchange resin [20], solid super acid [21], amorphous zirconia [20,22], calcium based compounds [23,31,32], alumina-supported potassium iodide [24], $\mathrm{KF}-\mathrm{ZnO}$ [25], Mg-Al hydroalcites
[26], potassium loaded on alumina [27], zinc modified with alkali earth metals [31], vanadyl phosphate [18], enzymes and etc. These researches have used various natural oils such as soybean oil, rapeseed oil, sunflower oil and etc.

However only limited that use palm oil as the feed stock. Since palm oil is the cheapest vegetable oil in the world, therefore the aim of this study is to convert palm oil to biodiesel using montmorillonite KSF as the heterogeneous catalyst.

\section{METHODS}

\section{a. Materials}

Purified palm oil was purchased from Yee Lee Edible Oils Sdn Bhd, Malaysia and methanol from R \& M Chemicals, UK. Montmorillonite KSF, methyl myristate, methyl palmitate, methyl stearate, methyl oleate, methyl linoleate (as standard references) and methyl heptadecanoate (internal standard) was obtained from Fluka Chemie, GmbH, Riedstr Germany. These methyl esters were used for the analysis of the product.

\section{b. Experimental Design}

The design of experiment (DOE) for the transesterification processes was developed using DesignExpert software, in which response surface methodology (RSM) was applied. The RSM was based on four-variable central composite design (CCD) with $\alpha=2$. The variables were reaction temperature $\mathrm{x}_{1}\left(50-190{ }^{\circ} \mathrm{C}\right)$, reaction period $\mathrm{x}_{2}$ (60-300 $\mathrm{min})$, ratio of oil $/ \mathrm{methanol} \mathrm{x}_{3}(4-12 \mathrm{~mol} / \mathrm{mol})$ and amount of catalyst $\mathrm{x}_{4}(1-5 \% \mathrm{wt})$. Table 1 shows the complete DOE matrix with experimental results for the transesterification processes. Table 2 shows the actual values of coded experimental variables used in Table 1 .

\section{c. Experimental Procedure}

The transesterification processes were carried out in a low pressure batch reactor with a magnetic stirrer, as shown in the Figure 1. Mixture of palm oil, catalyst and methanol was charged into the reactor. The mixing intensity of the magnetic stirrer was set at 190-200 rpm. The reaction temperature, duration, ratio of oil/methanol and amount of catalyst (montmorillonite KSF) were set according to the values as proposed in the DOE.

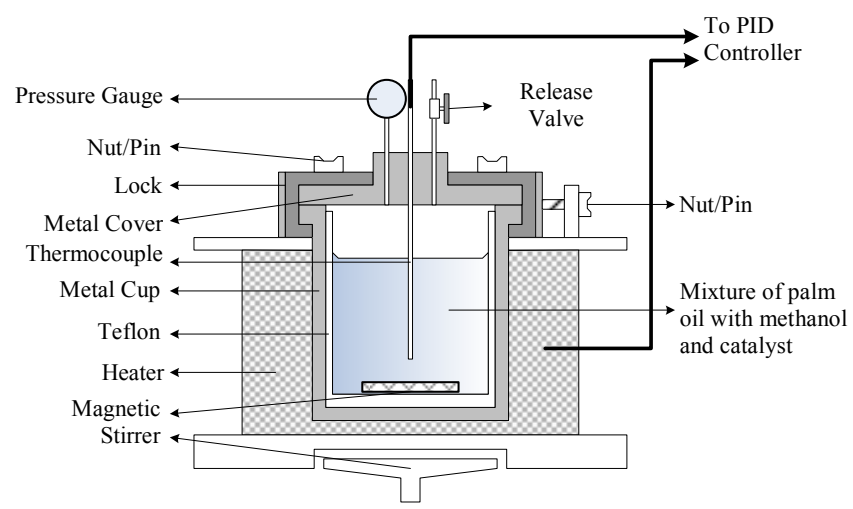

Figure 1. Schematic diagram of the low pressure batch reactor for the transesterification process

\section{d. Analytical Method}

The resulting products from the transesterification processes were analyzed using Gas Chromatography (GC) 
to identify the presence of methyl esters (biodiesel) in the sample. Methyl heptadecanoate was used as internal standard for the calculation of the conversion. The conversion of the transesterification processes were calculated as mol ratio of methyl esters produced to palm oil used.

\section{RESUlTS AND DISCUSSION}

The experimental result (conversion) of the transesterification processes is shown in Table 1. Apart from that, the conversion values predicted using the model developed by the DOE software is also shown in Table 1.

Table 1: The DOE experimental matrix and results for the transesterification processes

\begin{tabular}{|c|c|c|c|c|c|c|}
\hline \multirow[b]{2}{*}{ Run } & \multicolumn{4}{|c|}{ Level } & \multirow{2}{*}{$\begin{array}{c}\text { \#Exp. } \\
\text { Conv. } \\
(\%)\end{array}$} & \multirow{2}{*}{$\begin{array}{c}\text { *Pre. } \\
\text { Conv } \\
(\%)\end{array}$} \\
\hline & $\mathrm{x}_{1}$ & $\mathrm{x}_{2}$ & $\mathrm{x}_{3}$ & $\mathrm{x}_{4}$ & & \\
\hline KSF 01 & +1 & +1 & +1 & -1 & 71.63 & 74.29 \\
\hline KSF 02 & +1 & +1 & -1 & -1 & 59.1 & 62.73 \\
\hline KSF 03 & +1 & -1 & +1 & +1 & 78.69 & 81.35 \\
\hline KSF 04 & -1 & +1 & -1 & +1 & 5.07 & 8.70 \\
\hline KSF 05 & +1 & -1 & -1 & +1 & 55.1 & 58.73 \\
\hline KSF 06 & -1 & -1 & +1 & -1 & 3.92 & 6.58 \\
\hline KSF 07 & -1 & +1 & +1 & +1 & 5.43 & 8.09 \\
\hline KSF 08 & -1 & -1 & -1 & -1 & 2.9 & 6.53 \\
\hline KSF 09 & -2 & 0 & 0 & 0 & 0.79 & 0.00 \\
\hline KSF 10 & +2 & 0 & 0 & 0 & 74.34 & 71.20 \\
\hline KSF 11 & 0 & -2 & 0 & 0 & 14.49 & 11.35 \\
\hline KSF 12 & 0 & +2 & 0 & 0 & 60.05 & 56.91 \\
\hline KSF 13 & 0 & 0 & -2 & 0 & 36.52 & 32.41 \\
\hline KSF 14 & 0 & 0 & +2 & 0 & 51.39 & 49.22 \\
\hline KSF 15 & 0 & 0 & 0 & -2 & 32.54 & 29.40 \\
\hline KSF 16 & 0 & 0 & 0 & +2 & 61.98 & 58.84 \\
\hline KSF 17 & 0 & 0 & 0 & 0 & 55.07 & 54.50 \\
\hline KSF 18 & 0 & 0 & 0 & 0 & 50.88 & 54.50 \\
\hline KSF 19 & 0 & 0 & 0 & 0 & 55.75 & 54.50 \\
\hline KSF 20 & 0 & 0 & 0 & 0 & 55.07 & 54.50 \\
\hline KSF 21 & 0 & 0 & 0 & 0 & 55.75 & 54.50 \\
\hline
\end{tabular}

\# Experimental conversion in percentage

* Predicted conversion in percentage

Table 2. The actual values of the parameters for each level

\begin{tabular}{|l|c|c|c|c|c|}
\hline Variables & -2 & -1 & 0 & +1 & +2 \\
\hline Reaction temperature, $\mathrm{x}_{1}$ & 50 & 80 & 120 & 155 & 190 \\
\hline Reaction period, $\mathrm{x}_{2}$ & 60 & 120 & 180 & 240 & 300 \\
\hline Ratio oil/methanol, $\mathrm{x}_{3}$ & $1:$ & $1: 6$ & $1: 8$ & $\begin{array}{c}1: 1 \\
0\end{array}$ & $1: 12$ \\
& 4 & & & 0 & \\
\hline Amount of catalyst, $\mathrm{x}_{4}$ & 1 & 2 & 3 & 4 & 5 \\
\hline
\end{tabular}

Units $-\mathrm{x}_{1}\left({ }^{\circ} \mathrm{C}\right), \mathrm{x}_{2}(\mathrm{~min}), \mathrm{x}_{3}(\mathrm{~mol} / \mathrm{mol}), \mathrm{x}_{4}(\% \mathrm{~g})$

Based on Table 1 above, the highest conversion was achieved at a reaction temperature of $155^{\circ} \mathrm{C}$, reaction period of $240 \mathrm{~min}$ or $4 \mathrm{hr}$, oil/methanol ratio of $1: 10$ and $\%$ catalyst of $4 \% \mathrm{wt}$ of oil. Whereas the lowest conversion was obtained at a reaction temperature of $50{ }^{\circ} \mathrm{C}$, reaction period of $3 \mathrm{hr}$, oil $/$ methanol ratio of $1: 8$ and $\%$ catalyst of 3 $\%$ wt of oil.
Visual inspection of the data trend shown in Table 1 indicates that the maximum conversion occurs at the border of the experimental variables selected for this study. This finding was also supported by the model developed using the DOE software whereby no maximum conversion was located within the experimental domain examined. Therefore, optimum experimental conditions to obtain the highest conversion cannot be derived (except for the experimental conditions at the border of the design variables). Thus, the model developed by the DOE software was used to facilitate a straight forward examination of the effects of the variables and their interaction towards the conversion of the transesterification process. Figure 2, 3 and 4 show the interactions between the parameters/variables used in the transesterification processes.

Referring to figure 2, both oil/methanol ratios showed increasing conversion of palm oil to biodiesel with increasing reaction temperature. Apart from that, higher ratio of oil/methanol, 1:10, gives higher conversion whereas lower ratio of oil/methanol, 1:6, gives lower conversion of palm oil to biodiesel. This behavior is in agreement with those reported in the literature Technically, the equilibrium of the reaction will shift to the right (equation 1), with the additions of more heat or when more methanol is used in the reaction.

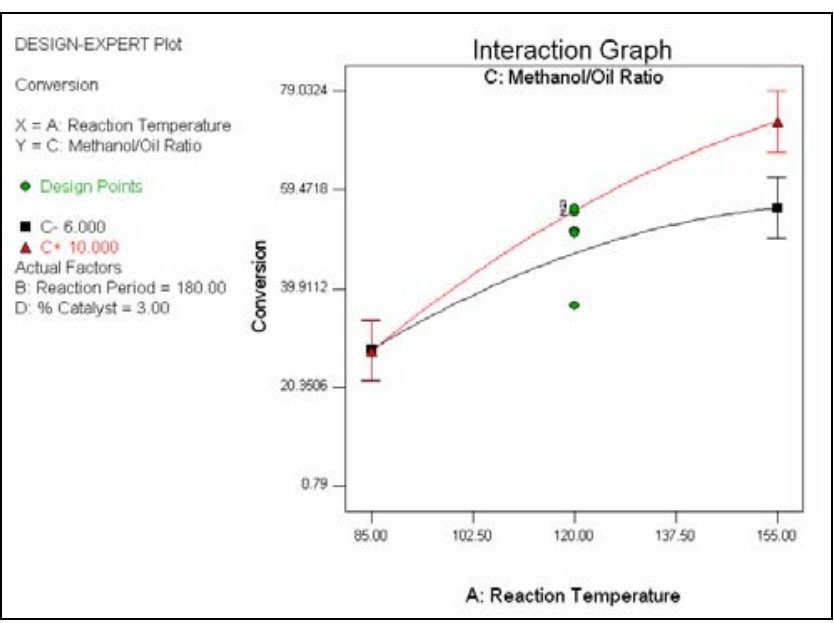

Figure 2. Interaction between the reaction temperature and methanol/oil ratio towards conversion

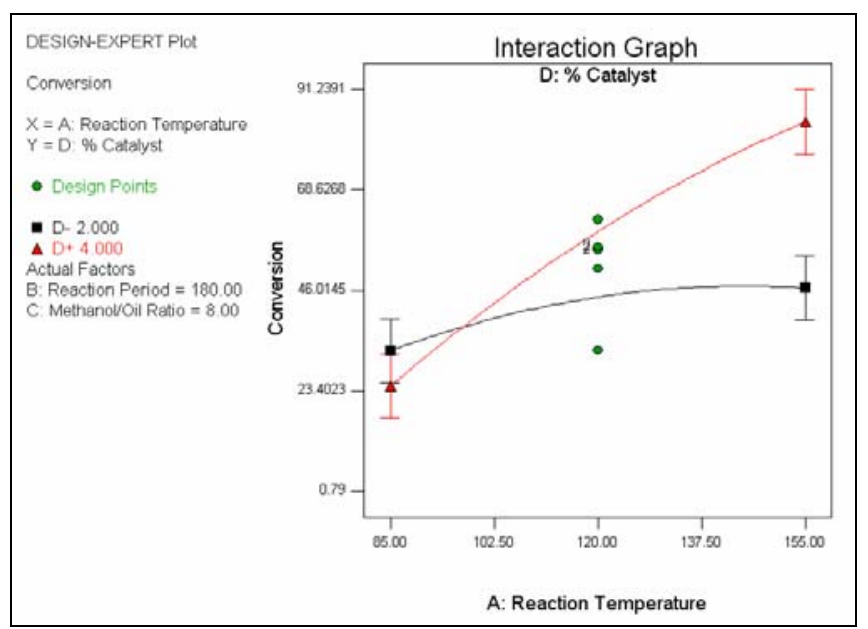

Figure 3. Interaction between reaction temperature and $\%$ of catalyst towards conversion 
It is generally perceived that higher percentage of catalyst used will results in higher conversion of triglycerides to biodiesel because of higher availability of active sites. However, this perception may not always be true. Referring to figure 3, the conversion of oil to biodiesel was found to be lower at $4 \%$ catalyst as compared to $2 \%$ catalyst when the reaction temperature was lower than $95^{\circ} \mathrm{C}$. This may suggest the real interactions between the supplied energy (heat) and the active site on the catalyst itself. The active sites on the catalysts may require a certain amount of energy to be activated. At lower temperature, smaller percentage of catalyst, $2 \%$, will reach the activation energy more quickly than the $4 \%$ catalyst because the ratio of active site to energy (heat) is smaller. In other words, the total energy required to activate the whole active sites on the $2 \%$ catalyst is much smaller than those for $4 \%$ catalyst. Thus, lower temperature (small energy) may just be enough to activate the whole active site on the $2 \%$ catalyst but not for the $4 \%$ catalyst because of the assumption of fair distribution of energy to all active sites. Thus, the transesterification reactions by $2 \%$ catalysts will commence sooner, while the $4 \%$ catalyst will show delay in transesterification reactions. However, as activation energy for all catalyst in the $4 \%$ is fully achieved at higher temperature, the reactions will proceed rapidly and subsequently exceeds to that of $2 \%$ catalyst. Thus, only when the reaction temperature is sufficient to activate all the active sites of the catalyst, then only the perception that high amount of catalyst will results in higher conversion applies.

As in figure 4 , at shorter reaction period, $2 \%$ catalyst showed a significant lower conversion of palm oil to biodiesel compared to that of $4 \%$ catalyst. However, the conversion by $2 \%$ catalyst continues to steadily increase until it subsequently exceeds to that of $4 \%$ catalyst after 210 minutes of reaction. The $4 \%$ catalyst showed a fairly constant conversion for most of the time and slightly dropped when the reaction period is beyond 210 minutes. From this behavior, it can be said that the equilibrium of the reactions is reached rapidly with $4 \%$ catalyst, and after reaching the equilibrium, the transesterification reactions may become stagnant or start to reverse. The equilibrium of transesterification reactions by $2 \%$ catalyst may only reached after longer reaction time because of smaller active site availability, thus defines the staging increment of conversion by $2 \%$ catalyst from $120 \mathrm{~min}$ to $240 \mathrm{~min}$. After all, the total rate of reactions, be it transesterification or reversed reaction, may directly be influenced by the total active sites of the catalyst, whereby higher active sites will accelerate both reactions.

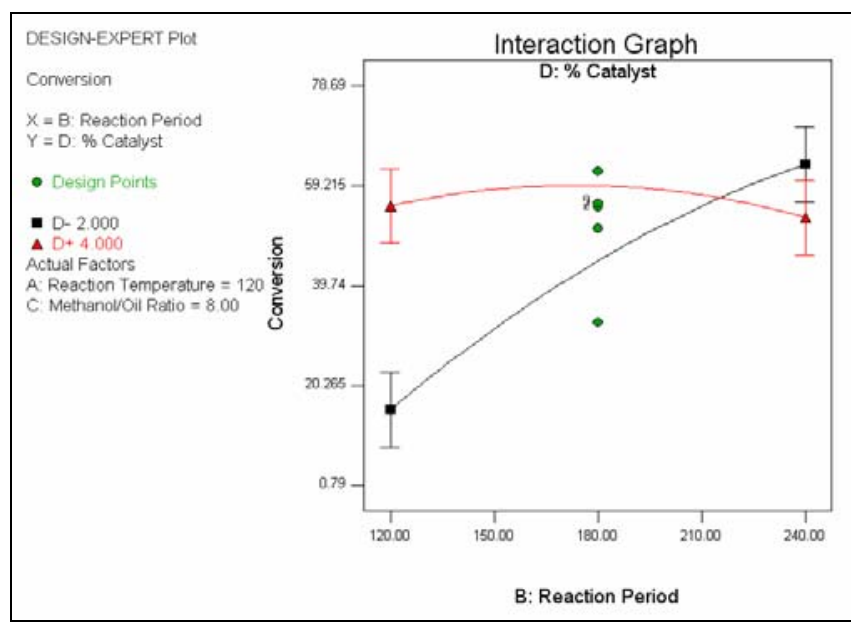

Figure 4. Interaction between reaction period and $\%$ of catalyst towards conversion

The ability of montmorillonite KSF to catalyze the transesterification reactions, at any rate, is due to the acidic properties of the catalyst itself. Montmorillonite KSF has free acid of 8-12\%, and its surface partially laid with $\mathrm{H}_{2} \mathrm{SO}_{4} \cdot \mathrm{H}_{2} \mathrm{SO}_{4}$ is known as a typical strong acid, and since the transesterification reactions can be catalyzed with acid or base, therefore the strong acidity of this montmorillonite KSF will definitely contribute to the high conversion of palm oil to biodiesel.

\section{CONCluSions}

Based on the experimental result obtained, it can be concluded that montmorillonite KSF could be used as an effective catalyst for the conversion of palm oil to biodiesel. However, future research on this catalyst is still needed to investigate wider range of the parameters, and also new possible parameters which can be included in the reaction process such as the mixing intensity, reaction pressure and so forth.

\section{ACKNOWLEDGMENT}

The authors would like to thank Universiti Sains Malaysia for providing fund via Short Term Grant A/C No: 6035157 for this project.

\section{REFERENCES}

[1] Abreu, F.R., Lima, D.G., Hamu, E.H., Wolf, C. and Suarez, P.A.Z. 2004. Utilization of metal complexes as catalysts in the transesterification of Brazilian vegetable oils with different alcohols. Journal of Molecular Catalysis A: Chemical, Vol 209 (1-2), 29-33.

[2] Ma, F., and Hanna, M.A. 1999. Biodiesel production: a review. Bioresource Technology (70) 1-15.

[3] Fukuda, H., Kondo, A., and Noda, H. 2001. Biodiesel Fuel Production by Transesterification of Oils. Journal of Bioscience and Bioengineering. Vol. 92, No. 5, 405-416

[4] Van Gerpen, J. 2005. Biodiesel processing and production. Fuel Processing Technology (86) 1097- 1107.

[5] Barnwal, B.K. and Sharma, M.P. 2005. Prospect of biodiesel production from vegetable oils in India. Renewable and Sustainable Energy Reviews, Vol 9 (4), 363-378.

[6] Jitputti, J., Kitiyanan, B., Bunyakiat, K., Rangsunvigit, P. and Jenvanitpanjakul, P. 2004. Proceedings of the Joint International Conference on Sustainable Energy and Environment, Hua Hin, Thailand, 1-3 December, 267-271.

[7] Karmee, S.K. and Chadha, A. 2005. Preparation of biodiesel from crude oil of Pongamia pinnata. Bioresource Technology, Vol 96 (13), 1425-1429. 
[8] Kim, H.J., Kang, B.S., Park, Y.M., Kim, D.K., Lee, J.S. and Lee, K.Y. 2004. Transesterification of vegetable oil to biodiesel using heterogeneous base catalyst. Catalysis Today, Vol 93-95, 315-320.

[9] MPOB (Malaysian Palm Oil Board) webpage. www.mpob.gov.my. (date excess: $8^{\text {th }}$ April 2005)

[10] Suppes, G.J., Dasari, M.A., Doskocil, E.J., Mankidy, P.J. and Goff, M.J. 2004. Transesterification of soybean oil with zeolite and metal catalysts. Applied Catalysis A: General, Vol 257 (2), 213-223.

[11] Mazzochia, C., Modica, G., Kaddouri, A., and Nannicini, R., 2004. Fatty acid methyl esters synthesis from triglycerides over heterogeneous catalysts in the presence of microwaves. C. R. Chimie (7) $601-605$.

[12] Ferreira, D.A.C., Meneghetti, M.R., Meneghetti, S.M.P. and Wolf, S.R. 2007. Methanolysis of soybean oil in the presence of tin (IV) complexes. Applied Catalysis A: General (317) 58-61.

[13] López, D.E., Suwannakarn, K., Bruce, D.A. and Goodwin Jr, J. G. 2007. Esterification and transesterification on tungstated zirconia: Effect of calcination temperature. Journal of Catalysis (247) 43-50.

[14] López, D.E., Goodwin Jr, J. G., and Bruce, D.A. 2007. Transesterification of triacetin with methanol on Nafion ${ }^{\circledR}$ acid resins. Journal of Catalysis (245) 381-391.

[15] Arzamendi, G., Campoa, I., Arguinarena, E., M. Sanchez, Montes, M. and Gandia, L.M. 2007. Synthesis of biodiesel with heterogeneous $\mathrm{NaOH} /$ alumina catalysts: Comparison with homogeneous $\mathrm{NaOH}$ Article in press.

[16] Marchetti, J.M., Miguel, V.U. and Errazu, A.F. 2007. Short communication. Heterogeneous esterification of oil with high amount of free fatty acids. Fuel (86) 906-910.

[17] Guerreiro, L., Castanheiro, J.E., Fonseca, I.M., Martin-Aranda, R.M, Ramos, A.M., and Vital, J. 2006. Transesterification of soybean oil over sulfonic acid functionalised polymeric membranes. Catalysis Today (118) 166-171.

[18] Di Serio, M., Cozzolino, M., Tesser, R., Patrono, P., Pinzari, F., Bonelli, B., and Santacesaria, E. 2007. Vanadyl phosphate catalysts in biodiesel production. Applied Catalysis A: General (320) 1-7.

[19] M. Lopez Granados, M.D. Zafra Poves, D. Martın Alonso, R. Mariscal, F. Cabello Galisteo, R. Moreno-Tost, J. Santamaria, and J.L.G. Fierro. 2007. Biodiesel from sunflower oil by using activated calcium oxide. Applied Catalysis B: Environmental (73) 317-326.

[20] Shibasaki-Kitakawa, N., Honda, H., Kuribayashi, H., Toda, T., Fukumura, T., and Yonemoto, T. 2007. Biodiesel production using anionic ion-exchange resin as heterogeneous catalyst. Bioresource Technology (98) 416-421.

[21] Furuta, S., Matsuhashi, H., and Arata, K. 2004. Biodiesel fuel production with solid superacid catalysis in fixed bed reactor under atmospheric pressure. Catalysis Communications (5) 721-723.

[22] Furuta, S., Matsuhashi, H., and Arata, K. 2006. Biodiesel fuel production with solid amorphous-zirconia catalysis in fixed bed reactor. Biomass and Bioenergy (30) 870-873.

[23] Gryglewicz, S. 1999. Rapeseed oil methyl esters preparation using heterogeneous catalysts. Bioresource Technology (70) 249-253.

[24] Xie, W., and Li, H. 2006. Alumina-supported potassium iodide as a heterogeneous catalyst for biodiesel production from soybean oil Journal of Molecular Catalysis A: Chemical (255) 1-9.

[25] Xie, W., and Huang, X. Synthesis of biodiesel from soybean oil using heterogeneous KF/ZnO catalyst. Catalysis Letters Vol. 107, Nos. 1-2, February 2006.

[26] Xie, W., Peng, H., and Chen, L. 2006. Calcined Mg-Al hydrotalcites as solid base catalysts for methanolysis of soybean oil. Journal of Molecular Catalysis A: Chemical (246) 24-32.

[27] Xie, W., Peng, H., and Chen, L. 2006. Transesterification of soybean oil catalyzed by potassium loaded on alumina as a solid-base catalyst. Applied Catalysis A: General (300) 67-74.

[28] Xie, W., Huang, X., and Li, H. 2006. Short communication. Soybean oil methyl esters preparation using $\mathrm{NaX}$ zeolites loaded with $\mathrm{KOH}$ as a heterogeneous catalyst. Article in press.

[29] Liu, X., He, H., Wang, Y., and Zhu, S. 2007. Transesterification of soybean oil to biodiesel using $\mathrm{SrO}$ as a solid base catalyst. Catalysis Communications (8) 1107-1111.

[30] Liu, X., He, H., Wang, Y., Zhu, S., and Piao, X. 2007. Transesterification of soybean oil to biodiesel using $\mathrm{CaO}$ as a solid base catalyst. Article in press.

[31] Yang, Z., and Xie, W. 2007. Soybean oil transesterification over zinc oxide modified with alkali earth metals. Fuel Processing Technology (88) 631-638.

[32] Huaping, Z., Zongbin, W.U., Yuanxiong, C., Ping, Z, Shijie, D., Xiaohua, L., and Zongqiang, M. 2006. Preparation of Biodiesel
Catalyzed by Solid Super Base of Calcium Oxide and Its Refining Process. Chin J Catal, 27(5): 391-396. 


\title{
Thermal Characteristics of Eutectic Mixture of Capric-Lauric Acids as Phase Change Material (PCM) in Gypsum Board
}

\author{
Rozanna Dewi, Luqman Chuah A., Salmiah A., Thomas Choong S.Y. and Sa'ari Musthapa
}

\begin{abstract}
Thermal characteristics of some eutectic mixtures of fatty acids as phase change materials (PCM) for passive solar building heating and cooling application have been studied previously. This study looked at the effect of using capric - lauric acids eutectic mixture with a composition of 65 : $35 \mathrm{w} / \mathrm{w} \%$ as PCM in gypsum board. Capric - lauric acids eutectic mixture has melting point of $17.480 \mathrm{C}$ and latent heat of $133.08 \mathrm{~kJ} \mathrm{kg-1}$. The melting point is considered suitable to maintain a comfortable temperature in a space having a moderate ambient temperature. Gypsum board samples were immersed for 1 hour in the PCM and the thermal characteristics before and after immersion were analysed. The $12.5 \mathrm{~mm}$ thick gypsum board was found to have PCM content of $25.6 \%$ of the PCM and the Differential Scanning Calorimetric (DSC) showed that the melting point and latent heat were $17.90 \mathrm{C}$ and $33.1 \mathrm{~kJ} \mathrm{~kg}-1$, respectively. Meanwhile for $6 \mathrm{~mm}$ thickness the PCM content was $29.2 \%$, the melting point was $18.10 \mathrm{C}$ and the latent heat was $36.8 \mathrm{~kJ} \mathrm{~kg}-1$. From the result it can be seen that the thermal characteristic of PCM gypsum board has the same properties as the PCM. Indeed, the immersion process also did not affect the physical properties of gypsum.
\end{abstract}

Keywords: phase change materials (PCMs), thermal characteristic, oleochemicals, fatty acids, eutectic mixtures

\section{INTRODUCTION}

Rapid development has lead to huge demand on energy. In an attempt to conserve energy and reduce dependency on fossil fuels and also to reduce the greenhouse gas emission, it is essential to seek effective means of reducing peaks in power consumption and to shift portions of the load from

Luqman Chuah A. is with Department of Chemical \& Environmental Engineering, Faculty of Engineering, Institute of Tropical Forestry and Forest Products, University Putra Malaysia 43400 UPM Serdang, Selangor D.E, Malaysia (e-mail: chuah@eng.upm.edu.my; phone: +60389466288; fax: +60386567120 )

Rozanna Dewi is with Department of Chemical \& Environmental Engineering, Faculty of Engineering, University Putra Malaysia 43400 UPM Serdang, Selangor D.E, Malaysia

Salmiah A. is with Advanced Oleochemical Technology Division (AOTD), Malaysian Palm Oil Board (MPOB),Lot 9 and 11, Jalan P/14 Seksyen 10, 43650 Bandar Baru Bangi, Selangor D.E Malaysia.

Thomas Choong S. Y. is with Department of Chemical \& Environmental Engineering, Faculty of Engineering, University Putra Malaysia 43400 UPM Serdang, Selangor D.E, Malaysia

Sa'ari Musthapa is with Department of Chemical \& Environmental Engineering, Faculty of Engineering, University Putra Malaysia 43400 UPM Serdang, Selangor D.E, Malaysia periods of maximum demand. Storage of thermal energy, hence, becomes an important aspect in engineering application, especially in energy conservation in buildings.

Thermal energy storage in domestic solar space heating and cooling application has been given attention since it can utilise this renewable energy to reduce the greenhouse gas emissions. It also provides a reservoir of energy to adjust the mismatch between peak and off peak time and meet the energy demand at all times. Thermal energy storage is basically classified as latent, sensible and chemical energy storage. The concept of using latent heat storage as energy saving vehicle provides the advantages of storing a large amount of energy in a small mass/volume and the phase transition occurring at nearly constant temperature.

In Phase Change Materials (PCMs), latent heat storage stores heat as latent heat of fusion during phase changes that undergo melting and solidification process. To gain a comfortable temperature in a space, the PCM can be employed to absorb heat when melting and release it when solidify. For a material to be used as PCM, some of the following criteria need to be fulfilled: high heat of fusion, high thermal conductivity, high density, high specific heat, congruent melting, small volume changes during phase changes, chemical stability, non corrosive, non poisonous, non flammable and non explosive. It is also noteworthy to take into consideration the availability as well as cost [1].

The selection of PCM has recently been directed towards the use of low melting organic materials in an effort to avoid some of the problems inherent in inorganic phase change materials, such as supercooling and segregation. Special attention has been given to fatty acids since they can easily be obtained from renewable sources such as oils/fats [2].

Fatty acids show solid-liquid transitions within narrow temperature ranges. They possess some superior properties over other PCMs such as melting congruency, good chemical stability, non-toxicity and suitable melting temperature range for solar passive heating and cooling applications. In the liquid phases, these materials have surface tensions in the order of 20-30 dyne $\mathrm{cm}^{-1}$ and are therefore high enough to be retained in the structure of the host material. These materials possess elevated latent heat of transition and high specific heat (in the range 1.9-2.1 $\mathrm{J} \mathrm{g}^{-1}$ $\left.{ }^{\circ} \mathrm{C}\right)$. They also exhibit only small volume changes during melting or solidification (example: melting dilatation is around $0.1-0.2 \mathrm{ml} \mathrm{g}^{-1}$ ). Because of the protected carboxyl group, fatty acids based PCMs are chemically, heat and 
colour stable, low corrosion activity and non-toxic [3]. Table 1 shows that the potential fatty acids that can be used as PCMs.

PCM can be utilised as a single component or eutectic mixtures (binary mixtures that exhibit fixed melting/solidification points at a certain composition between two single components and act as single component). In passive solar application, three methods are proposed for the incorporation of a thermal storage material within the construction element: encapsulation of the storage material in high-density polyethylene pellets mixed with a gypsum board material, direct incorporation and simple immersion of conventional board in molten PCM. The latter method contributes to the economic impact of the passive solar wall [4].

Several works have been carried out in order to investigate the thermal properties of the binary mixtures of fatty acid and its compatibility with the building materials. Study that carried out by Feldman and Banu [2], investigated thermal characteristics of some PCM and found eutectic mixture of butyl palmitate and butyl stearate (49 : $41 \%$ weight) with melting point of $17^{\circ} \mathrm{C}$ and latent heat of fusion of $140 \mathrm{~kJ} / \mathrm{kg}$, to be compatible with gypsum wallboard. Nikolić et al [5] analysed thermal properties of methyl stearate, methyl palmitate and their binary mixtures use for solar storage in gypsum wallboard. No obvious changes in the thermal performance of materials were recorded after 50 thermal cycles. Their findings indicate that the latent heat of fusion has contributed to the overall heat storage capacity in a wallboard impregnated with the esters and their mixtures. Besides that, Shapiro et al [6] has shown several PCMs to be suitable for introduction into gypsum wallboard with possible thermal storage applications for the Florida climate. These materials were mixtures of methylesters, methyl palmitate, and methyl stearate and mixtures of short chain acids and capric and lauric acid. Although these materials had relatively high latent heat capacity, the temperature ranges required in achieving the thermal storage did not fall sufficiently within the range of comfort for buildings in hot climates. Rudd [7] analysed coconut fatty acids as PCM in gypsum and found that for absorption of 25 $\%$ weight of acids, the melting point is $24.9^{\circ} \mathrm{C}$ and latent heat is $22.26 \mathrm{~kJ} / \mathrm{kg}$. Room scale test showed that the PCM wallboard has an average thermal storage capacity of 24.2 $\mathrm{kJ} / \mathrm{kg}$. This varies by only $8.7 \%$ compared to the latent heat recorded by DSC. An comprehensive review on fatty acids being used as PCMs are reported by Rozana et al [8].

In this work, a simple immersion of gypsum board in eutectic mixture of capric-lauric acids (65: $35 \%$ weight) as phase change material will be studied. Gypsum is a nonmetallic mineral, found as a rock composed of $70.1 \%$ calcium sulphate and $20.9 \%$ water by weight. In construction industry, it is widely used as ceiling, roofing and wall. It is considered as a good supporting medium for phase change material since approximately $41 \%$ of the wallboard volume is air voids.

Thermal energy storage in domestic solar space heating and cooling application has been given attention since it can utilise this renewable energy to reduce the greenhouse gas emissions. It also provides a reservoir of energy to adjust the mismatch between peak and off peak time and meet the energy demand at all times. Thermal energy storage is basically classified as latent, sensible and chemical energy storage. The concept of using latent heat storage as energy saving vehicle provides the advantages of storing a large amount of energy in a small mass/volume and the phase transition occurring at nearly constant temperature.

In Phase Change Materials (PCMs), latent heat storage stores heat as latent heat of fusion during phase changes that undergo melting and solidification process. To gain a comfortable temperature in a space, the PCM can be employed to absorb heat when melting and release it when solidify. For a material to be used as PCM, some of the following criteria need to be fulfilled: high heat of fusion, high thermal conductivity, high density, high specific heat, congruent melting, small volume changes during phase changes, chemical stability, non corrosive, non poisonous, non flammable and non explosive. It is also noteworthy to take into consideration the availability as well as cost [1].

The selection of PCM has recently been directed towards the use of low melting organic materials in an effort to avoid some of the problems inherent in inorganic phase change materials, such as supercooling and segregation. Special attention has been given to fatty acids since they can easily be obtained from renewable sources such as oils/fats [2].

Fatty acids show solid-liquid transitions within narrow temperature ranges. They possess some superior properties over other PCMs such as melting congruency, good chemical stability, non-toxicity and suitable melting temperature range for solar passive heating and cooling applications. In the liquid phases, these materials have surface tensions in the order of 20-30 dyne $\mathrm{cm}^{-1}$ and are therefore high enough to be retained in the structure of the host material. These materials possess elevated latent heat of transition and high specific heat (in the range 1.9-2.1 J g ${ }^{\circ} \mathrm{C}$ ). They also exhibit only small volume changes during melting or solidification (example: melting dilatation is around $0.1-0.2 \mathrm{ml} \mathrm{g}^{-1}$ ). Because of the protected carboxyl group, fatty acids based PCMs are chemically, heat and colour stable, low corrosion activity and non-toxic [3]. Table 1 shows that the potential fatty acids that can be used as PCMs.

PCM can be utilised as a single component or eutectic mixtures (binary mixtures that exhibit fixed melting/solidification points at a certain composition between two single components and act as single component). In passive solar application, three methods are proposed for the incorporation of a thermal storage material within the construction element: encapsulation of the storage material in high-density polyethylene pellets mixed with a gypsum board material, direct incorporation and simple immersion of conventional board in molten PCM. The latter method contributes to the economic impact of the passive solar wall [4].

Several works have been carried out in order to investigate the thermal properties of the binary mixtures of fatty acid and its compatibility with the building materials. Study that carried out by Feldman and Banu [2], investigated thermal characteristics of some PCM and found eutectic mixture of butyl palmitate and butyl stearate (49 : $41 \%$ weight) with melting point of $17^{\circ} \mathrm{C}$ and latent heat of fusion of $140 \mathrm{~kJ} / \mathrm{kg}$, to be compatible with gypsum wallboard. Nikolić et al [5] analysed thermal properties of methyl stearate, methyl palmitate and their binary mixtures use for solar storage in gypsum wallboard. No obvious changes in the thermal performance of materials were 
recorded after 50 thermal cycles. Their findings indicate that the latent heat of fusion has contributed to the overall heat storage capacity in a wallboard impregnated with the esters and their mixtures. Besides that, Shapiro et al [6] has shown several PCMs to be suitable for introduction into gypsum wallboard with possible thermal storage applications for the Florida climate. These materials were mixtures of methylesters, methyl palmitate, and methyl stearate and mixtures of short chain acids and capric and lauric acid. Although these materials had relatively high latent heat capacity, the temperature ranges required in achieving the thermal storage did not fall sufficiently within the range of comfort for buildings in hot climates. Rudd [7] analysed coconut fatty acids as PCM in gypsum and found that for absorption of 25 $\%$ weight of acids, the melting point is $24.9^{\circ} \mathrm{C}$ and latent heat is $22.26 \mathrm{~kJ} / \mathrm{kg}$. Room scale test showed that the PCM wallboard has an average thermal storage capacity of 24.2 $\mathrm{kJ} / \mathrm{kg}$. This varies by only $8.7 \%$ compared to the latent heat recorded by DSC. An comprehensive review on fatty acids being used as PCMs are reported by Rozana et al [8].

In this work, a simple immersion of gypsum board in eutectic mixture of capric-lauric acids (65: $35 \%$ weight) as phase change material will be studied. Gypsum is a nonmetallic mineral, found as a rock composed of $70.1 \%$ calcium sulphate and $20.9 \%$ water by weight. In construction industry, it is widely used as ceiling, roofing and wall. It is considered as a good supporting medium for phase change material since approximately $41 \%$ of the wallboard volume is air voids.

\section{EXPERIMENTAL METHOD}

\section{Materials}

Fatty acids used in this work are capric and lauric acids with the purity of 0.99 , supplied by Palm-Oleo Sdn.Bhd, Malaysia. The chemicals were used without any further purification .Capric acid has melting point of $31^{\circ} \mathrm{C}$, whereas for lauric acid is $44^{\circ} \mathrm{C}$. The eutectic mixture of capric-lauric acids in the weight ratio of 65.5:35 was prepared by melting the acids together at temperature $80^{\circ} \mathrm{C}$ [9].

Gypsum boards with thickness of $12.5 \mathrm{~mm}$ are used in this study. Gypsum board was prepared by mixing gypsum with water in the ratio of $1.25: 1$ and poured into $20 \mathrm{x} 20 \mathrm{x}$ $1.25 \mathrm{~cm}$ size mould. After stiffening, the gypsum samples were cut into $6 \times 15 \times 1.25 \mathrm{~cm}$ in size. Physical properties of gypsum samples before and after immersion in PCM were tested.

\section{Experimental Method}

The thermal properties of eutectic mixture of lauricstearic acids such as melting point, solidification point and latent heat were first characterised. Gypsum board samples ( $6 \times 15 \times 1.25 \mathrm{~cm}$ size) were immersed for 1 hour in eutectic mixture of acids at $60^{\circ} \mathrm{C}$. After immersion, the samples were taken out, dried and the thermal characteristics of PCMgypsum board were analysed.

\section{Thermal Analysis Using DSC}

Differential scanning calorimetric (DSC) Perkin-Elmer Thermal Analysis Seri 7 was used to measure the thermal characteristics of eutectic mixture and PCM-gypsum board. Samples were weighted in a sealed aluminium pan with a mass of $2.5-10 \mathrm{mg}$. The DSC thermal analysis was performed in the temperature range of $-10-+80^{\circ} \mathrm{C}$ with a heating rate of $5^{\circ} \mathrm{C} \mathrm{min}^{-1}$ and under constant stream of nitrogen at atmospheric pressure. The melting temperature of the PCM, $\mathrm{T}_{\mathrm{m}}$, corresponds to the onset temperature obtained by drawing a line at the point of maximum slope of the leading edge of the peak. The latent heat, $\Delta \mathrm{H}_{\text {fus, }}$, was calculated as the area under the peak by numerical integration. In addition, melting or solidification peak temperatures are defined as the temperature of the points, which are located furthest from the base line.

\section{RESUlTS AND DISCUSSION}

The DSC analysis for eutectic mixtures of capric-lauric acids $(65: 35 \mathrm{w} / \mathrm{w} \%)$ shows a sharp peak with no secondary peak or hump in the temperature range of $-10-+80^{\circ} \mathrm{C}$. It can be seen from Figure 1 that melting temperature, latent heat and melting temperatures range of the eutectic mixture are $17.482^{\circ} \mathrm{C}, 133.081 \mathrm{~J} \mathrm{~g}^{-1}$ and $14.863-22.714^{\circ} \mathrm{C}$, respectively. The melting point of eutectic mixtures is lower than those of the single acids. However, the latent heat is high enough that they can be comparable to other PCMs, such as salt hydrates and polyalcohols, which are between $100-250 \mathrm{~J} \mathrm{~g}^{-1}$. Thermal characteristics of solidification process as determined by DSC are shown in Figure 2, which show that the solidification point of $15.364^{\circ} \mathrm{C}$, latent heat of $131.951 \mathrm{~J} \mathrm{~g}^{-1}$ and solidification temperatures range $16.171-$ $9.192^{\circ} \mathrm{C}$, respectively. The temperature transitions between melting and solidification processes are 17.482 and $15.364^{\circ} \mathrm{C}$ and these are considered close. The solid-liquid phase transition was reversible, as it can be seen that heat absorbed as latent heat of fusion was released as heat of solidification (with a small heat loss). Figures 3 and 4 illustrate the DSC thermogram for $12.5 \mathrm{~mm}$ gypsum board loaded with $25.6 \%$ of acid for heating and cooling, respectively.

\section{CONCLUSION}

Eutectic mixture of capric-lauric acids $(65: 35 \mathrm{w} / \mathrm{w} \%)$ has melting point of $15.364^{\circ} \mathrm{C}$, latent heat of $131.951 \mathrm{~J} \mathrm{~g}^{-1}$ and close temperature transition. When impregnated in gypsum board, the thermal characteristics of the mixtures are practically unchanged, with sharp peak and no additional peak or hump occurs. Indeed, the immersion process does not affect the physical characteristics of gypsum board. It is found that eutectic mixture of capric-lauric acids possess a great potential as a phase chase material for thermal energy storage applications.

\section{ACKNOWLEDGEMENT}

The authors would like to thank Advanced Oleochemical Technology Division (AOTD), Malaysian Palm Oil Board (MPOB) and Palm-Oleo Sdn.Bhd, Malaysia, for their supports of this work. 


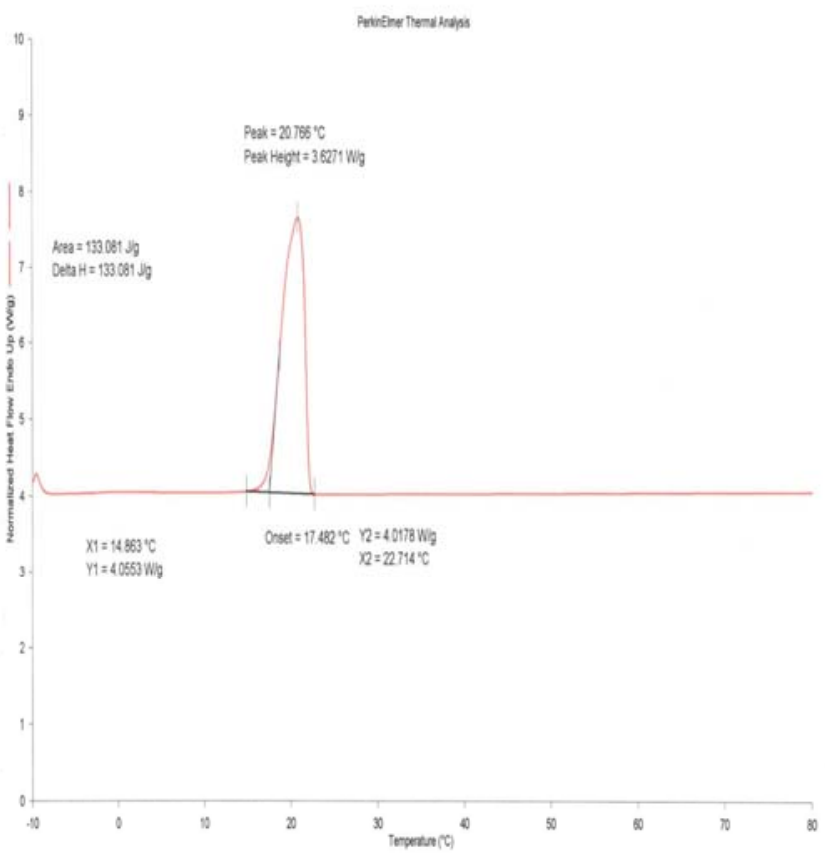

Figure 1. DSC thermogram of eutectic mixture of capriclauric acids $(65: 35 \mathrm{w} / \mathrm{w} \%)$ scanned at $5^{\circ} \mathrm{C} \mathrm{min}^{-1}$ (Heating)

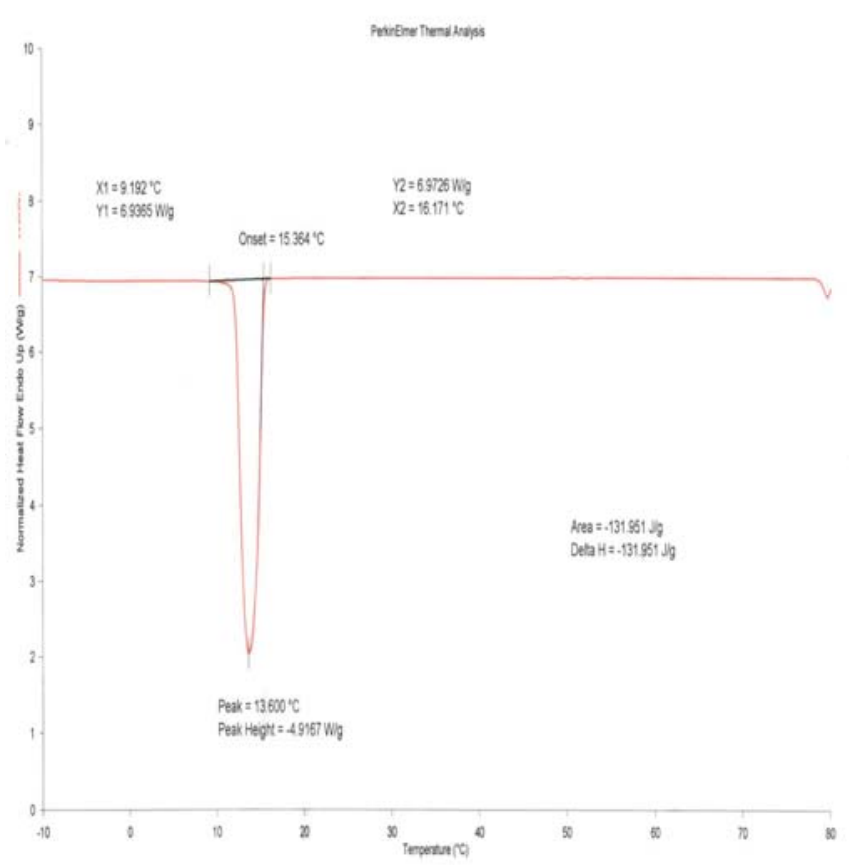

Figure 2. DSC thermogram of eutectic mixture of capriclauric acids $(65: 35 \mathrm{w} / \mathrm{w} \%)$ scanned at $5^{\circ} \mathrm{C} \mathrm{min}^{-1}$ (Cooling)

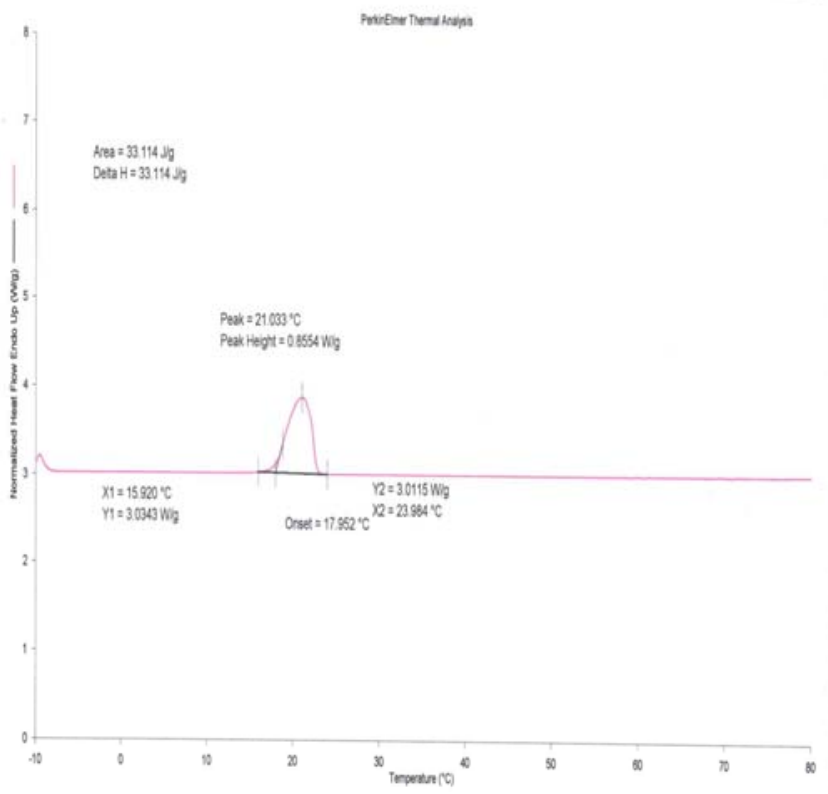

Figure 3. DSC thermogram of PCM-gypsum board 12.5 $\mathrm{mm}$ thickness scanned at $5^{\circ} \mathrm{C} \mathrm{min}^{-1}$, loading $25.6 \%$ weight (Heating)

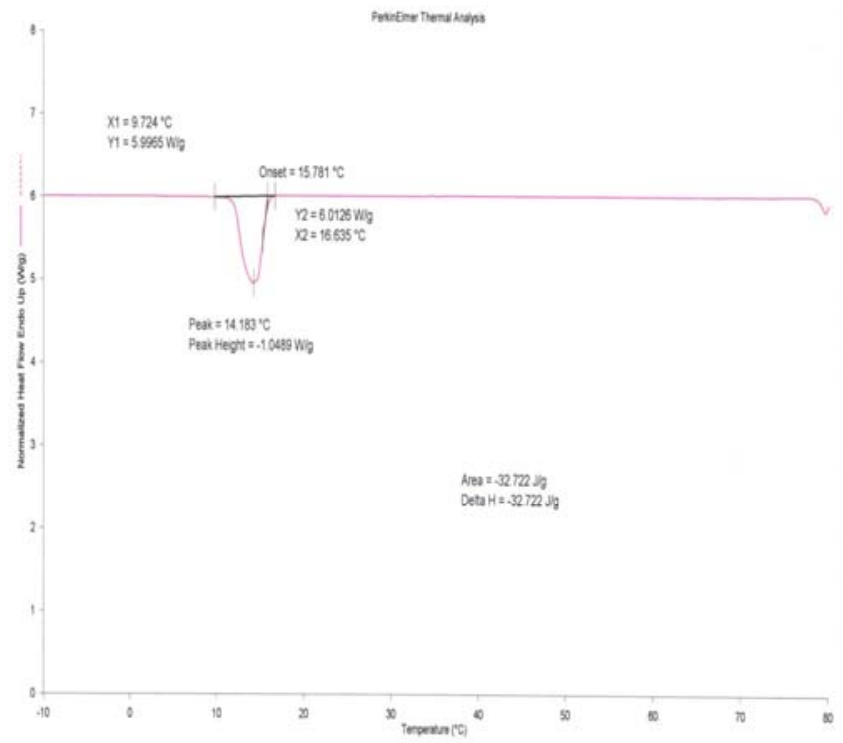

Figure 4. DSC thermogram of PCM gypsum board $12.5 \mathrm{~mm}$ thickness scanned at $5^{\circ} \mathrm{C} \mathrm{min}^{-1}$, loading $25.6 \%$ weight

(Cooling)

\section{REFERENCES}

[1] G. Beghi , Thermal Energy Storage, D. Reidel Publising Company, Dordrecht, Holland,1982.

[2] D. Feldman and D. Banu, DSC analysis for the evaluation of an energy storing wallboard, Thermochimica Acta, 272 (1996) 243- 251.

[3] D. Feldman, D. Banu and D. Hawes, Low chain esters of stearic acids as phase change materials for thermal energy storage in building, Solar Energy Materials and Solar Cells, 36 (1995) 311-322.

[4] B. Zelba, J. Marin, L. F. Cabeza, H. Mehling, Review on thermal energy storage with phase change; materials, heat transfer analysis and application, Applied Thermal Engineering, 23 (2003) 251-183.

[5] R. Nikolić, M. Marinović-Cincović, S. Gadzurić and I. J. Zsigrai, New materials for solar thermal storage-solid/liquid transitions in fatty acid ester, Solar Energy Materials and Solar Cells, 79(2003) 285-292.

[6] M. M. Shapiro, D. Feldman, D. Hawes and D. Banu, Thermal storage in drywall using organic phase-change material, Passive Solar Journal, 4(4) (1987) 419-438. 
[7] A. F. Rudd, Phase change material wallboard for distributed thermal storage in buildings, ASHRAE Transaction, Vol. 99, Part 2 (1993) 3724.

[8] D. Rozanna, T. G. Chuah, A. Salmiah, M. Sa'ari , Thomas S. Y. Choong and R. Medyan. Fatty acids as phase change materials
(PCMs) for thermal energy storage application. Int. J. of Green Energy, 1(4) (2004) 1-19.

[9] A. Sari, H. Sari, A. Onal, Thermal properties and thermal reliability of eutectic mixtures of some fatty acids as latent heat storage materials, Energy Conversion and Management, 45 (2004) 365-376.

Table 1. Oleochemical with potential use as PCM [4-9]

\begin{tabular}{|c|c|c|c|c|}
\hline Compound & $\begin{array}{l}\text { Melting } \\
\text { temperature } \\
\left({ }^{\circ} \mathrm{C}\right)\end{array}$ & $\begin{array}{l}\text { Heat of } \\
\text { fusion } \\
(\mathrm{kJ} / \mathrm{kg})\end{array}$ & $\begin{array}{l}\text { Thermal conductivity } \\
(\mathrm{W} / \mathrm{m} \mathrm{K})\end{array}$ & Density $\left(\mathrm{kg} / \mathrm{m}^{3}\right)$ \\
\hline Propyl palmiate & $10^{\mathrm{a}}$ & $186^{\mathrm{a}}$ & n.a & n.a \\
\hline Isopropyl palmiate & $11^{\mathrm{a}}$ & $95-100^{\mathrm{a}}$ & n.a & n.a \\
\hline $\begin{array}{l}\text { Capric-lauric acids } \\
+ \text { pentadecane } \\
(90: 10)\end{array}$ & $13.3^{\mathrm{a}}$ & $142.2^{\mathrm{a}}$ & n.a & n.a \\
\hline Isopropyl stearate & $14-18^{\mathrm{a}}$ & $140-142^{\mathrm{a}}$ & n.a & n.a \\
\hline Caprylic acid & $\begin{array}{l}16^{\mathrm{a}} \\
16.3^{\mathrm{a}} \\
16.7^{\mathrm{c}}\end{array}$ & $\begin{array}{l}148.5^{\mathrm{a}} \\
149^{\mathrm{a}}\end{array}$ & $\begin{array}{l}0.149{\left.\text { (liquid, } 38.6^{\circ} \mathrm{C}\right)^{\mathrm{a}}}^{\mathrm{a}} 0.145{\left.\text { (liquid, } 67.7^{\circ} \mathrm{C}\right)^{\mathrm{a}}}^{\mathrm{a}} \\
0.148\left(\text { liquid, } 20^{\circ} \mathrm{C}\right)^{\mathrm{a}}\end{array}$ & 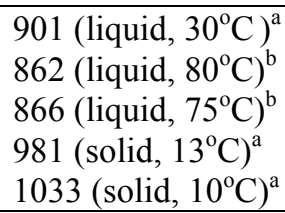 \\
\hline $\begin{array}{l}\text { Capric-lauric acids } \\
(65 \mathrm{~mol} \%-35 \mathrm{~mol} \%)\end{array}$ & $18.0^{\mathrm{a}}$ & $148^{\mathrm{a}}$ & n.a & n.a \\
\hline Butyl stearate & $19^{\mathrm{a}}$ & $\begin{array}{l}140^{\mathrm{a}} \\
123-200^{\mathrm{a}}\end{array}$ & n.a & n.a \\
\hline $\begin{array}{l}\text { Capric-lauric acids } \\
(45-55 \%)\end{array}$ & $21^{\mathrm{a}}$ & $143^{\mathrm{a}}$ & n.a & n.a \\
\hline Dimethyl sabacate & $21^{\mathrm{a}}$ & $120-135^{\mathrm{a}}$ & n.a & n.a \\
\hline $\begin{array}{l}34 \% \text { Miristic acid }+ \\
66 \% \text { Capric acid }\end{array}$ & $24^{\mathrm{a}}$ & $147.7^{\mathrm{a}}$ & $\begin{array}{l}0.164\left(\text { liquid, } 39.1^{\circ} \mathrm{C}\right)^{\mathrm{a}} \\
0.154\left(\text { liquid, } 61.2^{\circ} \mathrm{C}\right)^{\mathrm{a}}\end{array}$ & $\begin{array}{l}888\left(\text { liquid, } 25^{\circ} \mathrm{C}\right)^{\mathrm{a}} \\
1018\left(\text { solid, } 1^{\circ} \mathrm{C}\right)^{\mathrm{a}}\end{array}$ \\
\hline Vinyl stearate & $27-29^{\mathrm{a}}$ & $122^{\mathrm{a}}$ & n.a & n.a \\
\hline Capric acid & $\begin{array}{l}32^{\mathrm{a}} \\
31.5^{\mathrm{a}} \\
31.6^{\mathrm{c}}\end{array}$ & $\begin{array}{l}152.7^{\mathrm{a}} \\
153^{\mathrm{a}}\end{array}$ & 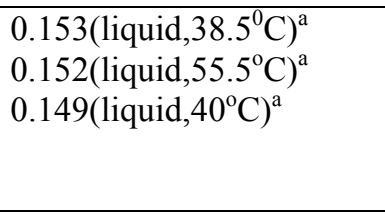 & $\begin{array}{l}878\left(\text { liquid, } 45^{\circ} \mathrm{C}\right)^{\mathrm{a}} \\
886\left(\text { liquid, } 40^{\circ} \mathrm{C}\right)^{\mathrm{a}} \\
858\left(\text { liquid, } 75^{\circ} \mathrm{C}\right)^{\mathrm{b}} \\
853\left(\text { liquid, } 80^{\circ} \mathrm{C}\right)^{\mathrm{b}} \\
1004\left(\text { solid, } 24^{\circ} \mathrm{C}\right)^{\mathrm{a}}\end{array}$ \\
\hline $\begin{array}{l}\text { Methyl-12 hydroxy- } \\
\text { stearate }\end{array}$ & $42-43^{\mathrm{a}}$ & $120-126$ & n.a & n.a \\
\hline Lauric acid & $\begin{array}{l}42-44^{\mathrm{a}} \\
44^{\mathrm{a}} \\
44.2^{\mathrm{c}}\end{array}$ & $\begin{array}{l}178^{\mathrm{a}} \\
177.4^{\mathrm{a}}\end{array}$ & 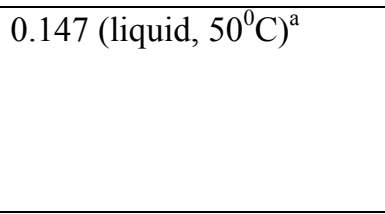 & $\begin{array}{l}862{\left.\text { (liquid, } 60^{\circ} \mathrm{C}\right)^{\mathrm{a}}} \\
870{\left.\text { (liquid, } 50^{\circ} \mathrm{C}\right)^{\mathrm{a}}} \\
852{\text { (liquid, } 75^{\circ} \mathrm{C} \text { ) }}^{\mathrm{b}} \\
848{\text { (liquid, } 80^{\circ} \mathrm{C} \text { ) }}^{\mathrm{b}} \\
1007{\text { (solid, } 24^{\circ} \mathrm{C} \text { ) }}^{\mathrm{a}}\end{array}$ \\
\hline Myristic acid & $\begin{array}{l}49-51^{\mathrm{a}} \\
54^{\mathrm{a}} \\
58^{\mathrm{a}} \\
54.4^{\mathrm{c}}\end{array}$ & $\begin{array}{l}204.5^{\mathrm{a}} \\
187^{\mathrm{a}} \\
186.6^{\mathrm{a}}\end{array}$ & n.a & $\begin{array}{l}861{\left.\text { (liquid, } 55^{0} \mathrm{C}\right)^{\mathrm{a}}} \\
849{\left.\text { (liquid, } 75^{\circ} \mathrm{C}\right)^{\mathrm{b}}} \\
844{\left.\text { (liquid, } 80^{\circ} \mathrm{C}\right)^{\mathrm{b}}} \\
990{\left.\text { (solid, } 24^{0} \mathrm{C}\right)^{\mathrm{a}}}\end{array}$ \\
\hline Palmitic acid & $\begin{array}{l}64^{\mathrm{a}} \\
61^{\mathrm{a}} \\
63^{\mathrm{a}} \\
62.9^{\mathrm{c}}\end{array}$ & $\begin{array}{l}185.4^{\mathrm{a}} \\
203.4^{\mathrm{a}} \\
187^{\mathrm{a}}\end{array}$ & 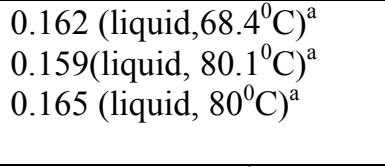 & 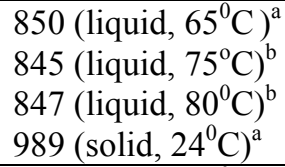 \\
\hline Stearic acid & $\begin{array}{l}69^{\mathrm{a}} \\
60-61^{\mathrm{a}} \\
70^{\mathrm{a}} \\
69.6^{\mathrm{c}}\end{array}$ & $\begin{array}{l}202.5^{\mathrm{a}} \\
186.5^{\mathrm{a}} \\
203^{\mathrm{a}}\end{array}$ & 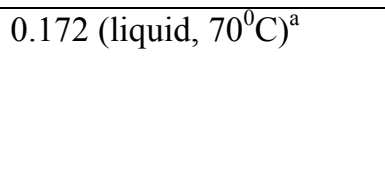 & $\begin{array}{l}848{\left.\text { (liquid, } 70^{\circ} \mathrm{C}\right)^{\mathrm{a}}} \\
843{\left.\text { (liquid, } 75^{\circ} \mathrm{C}\right)^{\mathrm{b}}} \\
839{\left.\text { (liquid, } 80^{\circ} \mathrm{C}\right)^{\mathrm{b}}}^{\mathrm{b}}{\left.\text { (solid, } 24^{\circ} \mathrm{C}\right)^{\mathrm{a}}}^{2}\end{array}$ \\
\hline
\end{tabular}




\title{
ASEAN'S Biomass Fired Grid-Connected Cogeneration Plants: The Way Forward
}

\author{
Mohamad Fadzil Adnan@ Nan, Rusmi Alias and Nasrin Abu Bakar
}

\begin{abstract}
ASEAN with its ten member countries has a total population of more than 567 million. With same weather condition, similar agricultural products are produced namely from the palm oil industry, rice industry, and sugarcane industry. Through ASEAN cooperation, there have been significant changes and improvement particularly in the field of renewable energy especially from biomass residues utilization through cogeneration process. Although biomass cogeneration projects are nothing new but currently these projects are connected to the grid. These biomass grid connected cogeneration plants use bagasse and palm oil residues as fuel with capacity up to $63 \mathrm{MW}$. These environmentally friendly projects have been implemented within the region with the objectives to develop, increase awareness and improve the socio-economic status of ASEAN. There are several advantages in optimizing biomass residues as an energy source due to the high oil prices such as reducing fossil fuel usage and green house gases emission and eligibility for Clean Development Mechanism (CDM) Funding. Thus, this paper will highlight how such projects can be easily duplicated throughout the region and it should be the way forward by optimizing biomass residues utilisation.
\end{abstract}

Keywords: ASEAN, biomass, grid connected and cogeneration

\section{INTRODUCTION}

The Association of Southeast Asian Nations, commonly known as ASEAN is a geo-political and economic organization countries located in Southeast Asia. Since its formation in 1967, ASEAN has grown to ten member countries and comprises of Brunei, Cambodia, Indonesia, Laos, Malaysia, Myanmar, the Philippines, Singapore, Thailand and Viet Nam. ASEAN was formed with the objective to accelerate the economic growth, social progress, cultural development among its member countries and promote regional peace.

As shown in 'table 1', ASEAN is currently one of the most active regional associations in the world with a population of about 567 million people and Gross Domestic product (GDP) per capita of US\$1,890. As of Sept. 2007, ASEAN had a combined GDP of about US\$ 1,072 billion growing at an average rate of around $6.0 \%$ per annum. With continued economic and population growth (average annual population growth is $1.5 \%$ ), optimisation of local resources is crucial to reduce the strain to natural resources available.

\section{AsEan Biomass Resources Availability}

ASEAN region is endowed with huge biomass resources as it is home to the world's largest producers of palm oil, sugar, rice, and timber products. These activities give rise to large quantities of by-products that become a nuisance as the needs to dispose them properly. The biomass residues are generated from the four agricultural sectors mentioned earlier are namely bagasse from the sugar industry, empty fruit bunches, palm fibre and shell from the palm oil industry, rice husk from the rice industry and offcuts and saw dust from the wood industry. The effective use of these waste by-products alone is thought to hold much potential for the regions' future electricity production.

Based on a study conducted in ASEAN, it was found that there are 100 millions tones of wood and agro industrial wastes are produced annually. These residues alone constitute a primary energy equivalent of 25 million tones of oil equivalent (MTOE). However, only up to half of these biomass residues are used for heat and power generation, normally using outdated and inefficient technology due to limited demand and financial constraint. Most of these factories will incinerate these excess biomass residues or use them for landfill, causing further environmental problems.

The potential for power generation from biomass in the ASEAN region amounts to about $5.8 \mathrm{GW}$ installed capacity as shown in 'table 2'. It should be noted that this figure does not take into account the potential power generation from one other major ASEAN country which is Thailand and related activities such as biogas produced from anaerobic digestion from palm oil effluent (POME) of palm oil industries which have a huge potential.

'Figure 1' shows the types of biomass residues available in some ASEAN countries from various agricultural activities mention earlier. It can clearly be seen that there are great similarities in the type of biomass residues generated such as rice husk, wood residues and palm oil. As rice is the staple food in ASEAN, rice husk would be available in all ASEAN countries but the biggest potential would be in Indonesia, the Philippines and Viet Nam. Whereas bagasse is predominantly available in Thailand, the Philippines, Indonesia and Vietnam.
Ir. Mohamad Fadzil Adnan @ Nan, Rusmi Alias and Nasrin Abu Bakar are with Universiti Teknologi MARA, Shah Alam, Selangor, Malaysia (Tel: 03-55436311, fax: 03-55436300, e-mail: mfadzil740@salam.uitm.edu.my). 
Table 1. Total land area, Population, annual population growth and GDP growth rate for ASEAN countries

\begin{tabular}{|c|c|c|c|c|c|}
\hline Country & $\begin{array}{c}\text { Total land area } \\
(, 000 \mathrm{~km} 2)\end{array}$ & $\begin{array}{c}\text { Population } \\
(, 000)\end{array}$ & $\begin{array}{c}\text { Annual population } \\
\text { Growth }(\%)\end{array}$ & $\begin{array}{c}\text { GDP } \\
\text { US \$ mill. }\end{array}$ & $\begin{array}{c}\text { GDP growth } \\
\text { rate }(\%)\end{array}$ \\
\hline $\begin{array}{c}\text { Brunei } \\
\text { Darussalam }\end{array}$ & 5.8 & 383 & 3.49 & $11,571.8$ & 5.1 \\
\hline Cambodia & 181 & 13996 & 2.45 & $7,256.3$ & 10.8 \\
\hline Indonesia & 1891 & 222051 & 1.30 & $364,258.8$ & 5.6 \\
\hline Lao PDR & 237 & 6135 & 2.53 & $3,521.8$ & 8.3 \\
\hline Malaysia & 330 & 26686 & 2.14 & $156,924.2$ & 5.9 \\
\hline Myanmar & 677 & 57289 & 2.30 & $11,951.0$ & 7.0 \\
\hline Philippines & 300 & 86910 & 1.96 & $117,457.1$ & 5.3 \\
\hline Singapore & 0.697 & 4484 & 3.27 & $132,273.4$ & 7.9 \\
\hline Thailand & 513 & 65233 & 0.73 & $206,645.1$ & 5.0 \\
\hline Viet Nam & 330 & 84222 & 1.33 & $60,965.2$ & 8.2 \\
\hline ASEAN & $4,464,456$ & 567,557 & 1.5 & $1,072,824.7$ & 6.0 \\
\hline
\end{tabular}

Table 2. Potential for biomass power production (in MW) for some ASEAN countries

\begin{tabular}{|c|c|c|c|c|c|c|}
\hline Countries & Paddy & $\begin{array}{c}\text { Palm } \\
\text { Oil }\end{array}$ & Wood & $\begin{array}{c}\text { Sugar } \\
\text { Cane }\end{array}$ & Coconut & Total \\
\hline Indonesia & 450 & 260 & 275 & 360 & 250 & 1800 \\
\hline Lao PDR & 60 & & & 8 & & 68 \\
\hline Malaysia & 156 & 2400 & 55 & & & 2600 \\
\hline Philippines & 360 & & & 540 & 20 & 920 \\
\hline Viet Nam & 150 & & 5 & 200 & & 400 \\
\hline
\end{tabular}

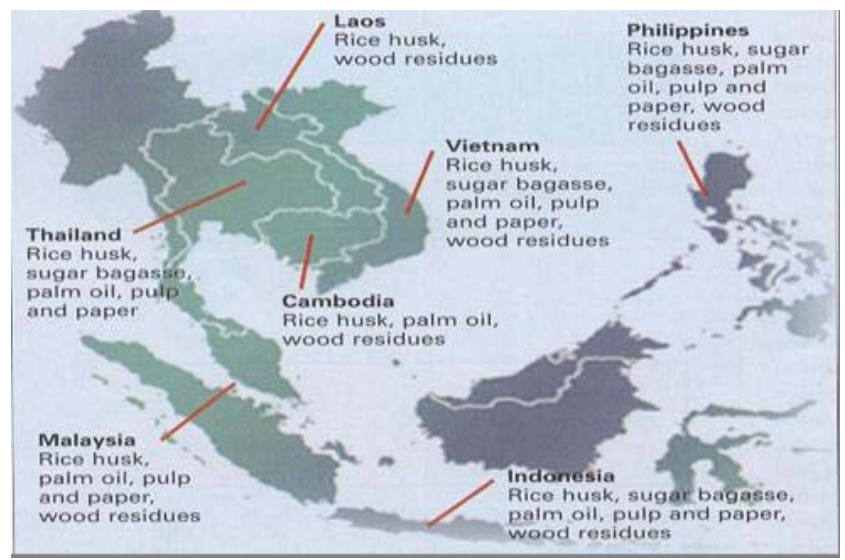

Figure 1. Types of biomass residues in ASEAN region

\section{A. Sugar Mills}

There are over than 150 sugar mills in ASEAN producing over 26 millions tons of bagasse annually, representing an energy equivalent of about 4.6 MTOE. However, most existing technologies are very old and inefficient, often generating low pressure steam. There is a need to install boiler system which bigger capacity and pressures greater than 40 bar for better energy recovery.

A little less than one-third of raw sugarcane is turned into bagasse. By processing one tonne of sugarcane, an average of $290 \mathrm{~kg}$ of bagasse can be produced, which is capable of producing about $100 \mathrm{kWh}$ of energy. On the other hand, 0.4 tonne of steam and 25 to $30 \mathrm{kWh}$ of electricity are required to process a tonne of sugarcane (see 'figure 2').

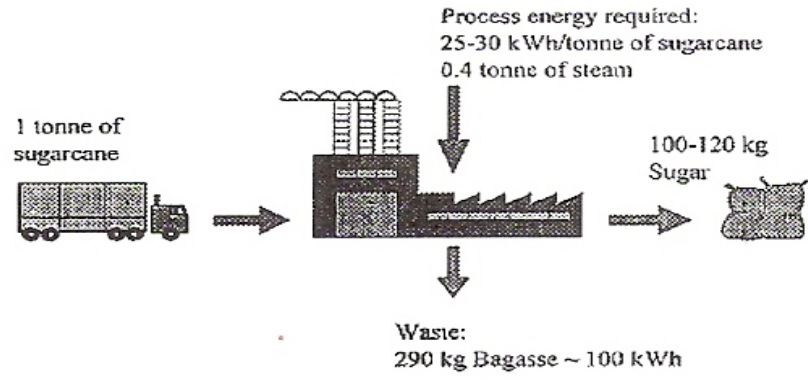

Figure 2. Cogeneration potential in sugar industry

\section{B. Palm Oil Mills}

The palm oil industry is one of the agro-industrial sectors which produce quite substantial of biomass residues. As much as $44 \%$ of the fresh fruit bunches (FFB) are turned into biomass residues such as empty fruit bunches (23\%), and fibre and shell (21\%). (see 'figure 3'). Over 40 million tonnes of FFB are processed annually and more than $75 \%$ of this is processed in Malaysia and Indonesia.

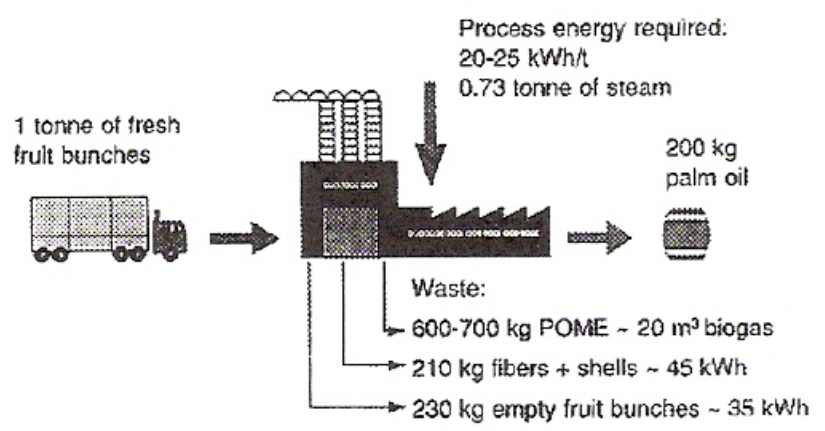

Figure 3. Cogeneration potential in palm oil industry

To process one tonne of FFB requires on average 20 to 25 $\mathrm{kWh}$ of energy and 0.73 tonne of steam, a palm oil mill could export two to three times as much as its self consumption. However, since most of these mills are isolated and not connected to grid, the mill is design to be energy self sufficient.

\section{ASEAN BIOMASS FIRED GRID CONNECTED PlantS}

Over the years there are a number of biomass fired cogeneration projects that have been developed in ASEAN. However there are a number of projects that are unique as they are grid connected as implemented by the EC-ASEAN 
COGEN Programme Phase III or simply COGEN3. COGEN is one of the longest collaboration programme between ASEAN and European Countries (EC) as it started in 1991 when COGEN Phase I was launched. COGEN3 objective was to promote efficient and proven biomass, gas and coal cogeneration, using European cogeneration equipment.

Throughout ASEAN, COGEN3 has eight Full Scale Demonstration Projects (FSDPs) using various types of biomass residues as fuel such as bagasse, palm oil shell, fibre and empty fruit bunches, woodwastes from tree trimming activities and rice husk. These demonstration projects were each given a maximum grant of $€ 400,000$ or $15 \%$ of the equipment costs whichever is lower.

Three of those projects are located in both Thailand and Malaysia, and two of them are in Singapore. The total installed capacity of these plants is more than $100 \mathrm{MW}$ with $68 \mathrm{MW}$ of them connected to the grid. The location of these FSDPs is shown in 'figure 4'.

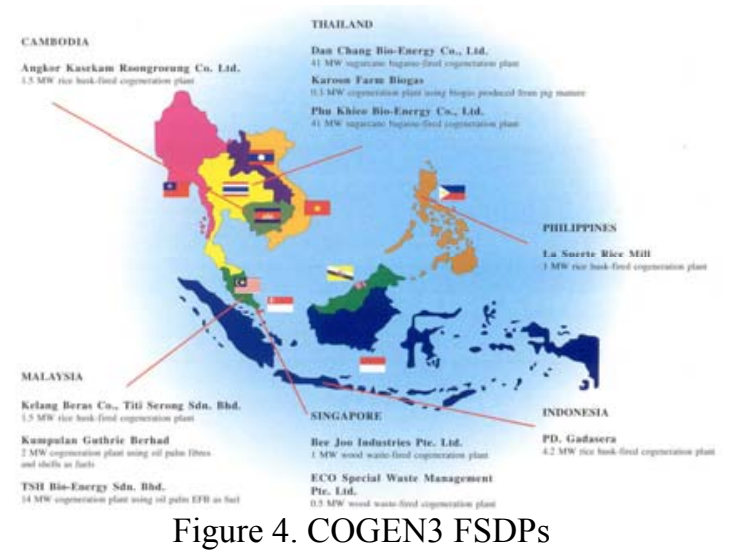

\section{A. Dan Chang Bio-Energy in Thailand}

Dan Chang Bio-Energy Co. Ltd. (DC) is one of subsidiaries of the Mitr Phol Sugar Group (MPSG) in Thailand. For many years MPSG has been the leader in the sugar industry, not only in Thailand but in the whole ASEAN region. The group is continuously improving its sugar process to enhance production capacity and increase in productivity. Over the years, it has gained a lot of experience in the sugar business that is not only competent in sugar production but also in product development. The plant is located in Dan Chang, Suphanburi province which is in central Thailand. Design, construction and operation of the waste to energy plant are taken care of Alstom Power (Thailand) Ltd.

The idea to utilize the bagasse came as in 2004 when the total daily crushing capacity of the group stands at just over 100,000 tonnes. This results in 12,000 tonnes of sugar and 32,000 tonnes of bagasse is generated, which is a major biproduct of the sugar cane crushing activity. Moreover, the group plans to increase its crushing capacity to 120,000 tonnes of cane per day in the near future.

Since one of the group "value driven excellence" goals is to take care of the environment, they chose to invest in high quality and pollution free systems to safeguard the environment. This has inspired them to develop a "clean and efficient" biomass power business in the sugar industry. Moreover, bagasse is a natural and renewable energy source which is clean and also helps to improve the quality of life of people within the vicinity.

The 41 MWe cogeneration plant is designed to cover all the steam and electricity requirements of the whole sugar complex. Process steam will be extracted for the sugar milling and refining process in the plant. Excess electricity of $25 \mathrm{MWe}$ will be exported to the national grid through a firm contract for 21 years for further distribution to the local community. During its schedule maintenance, electricity will be bought from the grid for basic office facilities and general requirements.

The power plant consists of a 41 MWe extraction condensing steam turbo-generator with two vibrating grate boilers with an hourly capacity of 120 tonnes of steam at 68 bar and $510^{\circ} \mathrm{C}$ each. The total investment cost is $€ 35.5$ million excluding civil works, building foundations and financing costs. The expected pay back period of the plant is about 5 years after being commissioned.

In addition to bagasse, the plant also uses other types of biomass residues such as sugarcane leaves and rice husks as energy which they bought from the nearby farmers. This not only helps the farmers to increase their income but also prevent further environmental damage caused by open burning once harvesting is completed.

\section{B. TSH's Palm Oil Residues Grid Connected Cogeneration Plant in Malaysia}

TSH Bio-Energy (TSH) is one of the subsidiaries of TSH resources Bhd. (TSHRB). THSRB is involved in various agricultural activities such as oil palm plantation, palm oil milling, timber products, cocoa processing, forest management and power generation. The plant is located in Kunak, Sabah which is in the Borneo island.

The biomass fired cogeneration plant capacity is $14 \mathrm{MWe}$ with a steam flowrate of 80 tonnes per hour at 66.5 barg and $402{ }^{\circ} \mathrm{C}$. The plant has the status of Small Renewable Energy Programme (SREP) project, where it will gain the SREP benefits such as tax exemptions. SREP is one of the Malaysian government initiatives in promoting the Fifth Fuel Policy. Fifth Fuel Policy was introduced during the Eighth Malaysian Plan (2000 to 2005) where renewable energy is added as the main grid connected energy source in addition to natural gas, coal, oil and hydro. Under the SREP project, the plant can export up to $10 \mathrm{MWe}$ of electricity to the grid without the needs to have Environmental Impact Assessment (EIA).

TSH sells its excess power at a selling rate of $21 \mathrm{sen} / \mathrm{kWh}$ through a 21 years renewable energy purchase agreement (REPA) for further distribution to the local community. During its schedule maintenance, electricity will be bought from the grid for basic office facilities and general requirements.

The plant consists of various components such as fuel conveying system, water-cooled inclined vibrating membrane grate, water tube steam boiler, an automatic deashing system, and a multi-valve steam turbine. For the steam, 25 tonnes of steam will be used for palm oil processing which is adjacent to it. TSH intends to utilize this excess capacity for further downstream processing such as pulp and paper process.

The total investment cost is about $€ 9$ million excluding civil works, building foundations and financing costs. The 
expected pay back period of the plant is about 4 years after being commissioned.

For fuel resources, TSH will use empty fruit bunches (EFB) as the main fuel besides fibre and shell for the cogeneration plants. This scenario is totally different from other palm oil mills which use only fibre and shell as fuel for its cogeneration plant and they are not connected to the grid. Currently, most EFB is used for mulching.

This project will also provide socio-economic benefits to the surrounding area where up to 40 locals will be employed for the plant operation. It is estimated that by embarking on this technology, 40,000 to 50,000 tonnes of carbon dioxide $\left(\mathrm{CO}_{2}\right)$ will be mitigated annually. $\mathrm{CO}_{2}$ reduction is the result of the biomass energy plant replacing the electricity supplied by the local power producers which relies mainly on diesel as fuel.

\section{The Needs to Optimise Biomass Residues UTILISATION}

There are several advantages in optimizing biomass residues as an energy as source. They are:

i) Savings in fuel and electricity costs,

ii) Optimise biomass residues availability,

iii) Remove uncertainty of increasing oil prices, and

iv) Eligibility for Clean Development Mechanism (CDM) Funding.

\section{A. Savings in Fuel and Electricity Costs}

By utilising biomass residues as fuel for the cogeneration plant, the project developer would save expenditure in fuel and electricity costs as through cogeneration process; both products are achieved with just one source of fuel. This is even more for plants which have to pay to dispose them as biomass residues which are previously considered as a waste has been turned into a resourceful energy source. Being grid connected, the additional revenue could be valuable if excess power is available. This would also enable the plants to insulate themselves from any fluctuations in electricity and fuel costs.

\section{B. Optimise Biomass Residues Availability}

As demonstrated by COGEN3 FSDPs biomass residues such as palm oil wastes and bagasse have shown that they can be used as fuel for plant usage and connected to the grid. Biomass residues waste such as palm's shell and fibre and bagasse have good calorific value of about 15 to 20 $\mathrm{MJ} / \mathrm{kg}$. which is relatively high. Most ASEAN countries have these biomass residues readily available and in abundant quantities, thus it is wise to optimise their resources through a more efficient process such as cogeneration. However, most of the times they are used inefficiently and extra costs do incur to pay contractors for disposal. The usage of there biomass residues for power generation would enable to reduce dependencies on other type of fuel such as diesel or natural gas.

\section{Remove Uncertainty of Increasing Oil Prices}

As mention earlier, oil prices have breached the US\$80 per barrel limit on $20^{\text {th }}$ September 2007 and increases more than $44 \%$ since $20^{\text {th }}$ March 2007. Economists have forecasted that the price would remain high and could even reaches US\$100 per barrel due to various reasons such as instability in Iraq and Iran economic sanctions. With such high oil prices, most ASEAN will certainly felt this impact on their gross domestic product (GDP) and economic growth as they are net oil importer. Thus, it is timely to make full use of the biomass resources that are readily available to reduce the oil price hike impact to ensure the current economic growth is maintain.

\section{D.Eligibility for Clean Development Mechanism (CDM) Funding}

The Clean Development Mechanism (CDM) is one of the mechanisms resulted from the Kyoto Protocol which allows developing countries to obtain financial resources and stateof the art technology from industrialised country in order to mitigate their green house gases emission. Starting from 1997 in Kyoto, Japan where the Protocol was adopted, calling for stronger action in reducing green house gases (GHG) emission in the post year 2000 period. Under this protocol, developed countries such as those from Europe, have a legally binding commitment to reduce their collective emissions of the GHG by at least 5\% compared to 1990 levels by the period of 2008 to 2012 . The protocol has established an emission trading regime called CDM to facilitate countries to fulfill their commitments.

$\mathrm{CDM}$ is a flexible mechanism that involves both developed and developing countries. It allows developed nations to achieve part of their reduction obligations by developing projects that reduce GHG emissions in developing countries such as those in ASEAN. This mechanism also assists the developing countries in achieving sustainable development projects which are beneficial to them. The GHG benefits of each CDM projects will be quantified in standard units, to be known as "Certified Emission Reductions" (CER).

As biomass residues are considered to be "zero" carbon dioxide generators, it is eligible for such funding. Thus, the agricultural sector is expected to reap more benefits, as demand for the biomass residues would increase. This CDM would encourage them to operate efficiently.

\section{CONCLUSION}

ASEAN has continued for further demand for electricity due to its strong economic growth. Thus, the need to develop new power plants to meet the growing demand is the driving force behind many regulatory and institutional changes that transform the electricity sector in most ASEAN countries. With uncertainty and high oil prices, it is wise that ASEAN country to focus its attention to their nonconventional energy resources that are readily available namely biomass resources.

The concept of cogeneration using biomass residues is nothing new in ASEAN. However, expertise in cogeneration technology to ensure optimisation of biomass residues such as rice husk and palm fibre and shell is indeed rather low.

With the assistance from ASEAN partners such as European countries (EC), there are already a number of biomass fired FSDPs that are connected to the grid. These plants have been operating well and could act as a showcase in ASEAN. For instance, Thailand has two $41 \mathrm{MW}$ projects based on bagasse and Malaysia has one $14 \mathrm{MW}$ which uses 
palm oil residues. These plants have been a major economic contributor to the country by reducing electricity demand and self-sustaining.

These FSDPs could be a showcase of such technology, which can be duplicated easily throughout the region, as the nature of biomass residues is almost the same. Assistance from EU and other collaborating partners are very much welcome to ensure continues information dissemination and market awareness of the latest technology. It is hope that with such cooperation and natural resources availability, the ASEAN region will optimize these resources utilization to grow and move forward.

\section{ACKNOWLEDGEMENT}

The author would like to thank all parties involved in making this paper a success namely COGEN3, SIRIM Berhad, MPOB and colleagues in University Technology MARA (UiTM).

\section{REFERENCES}

[1] Co-generation \& On-Site Power production, Nov-Dec. 2003 and JulyAug. 2004.

[2] Ecobusiness Opportunities in Asia and How Government, Nongovernmental Organisations, and Industry Can Participate, Edwin Khew, Green Productivity, Asian Productivity Organisation, Japan 1997. p. 483-490.

[3] Cogeneration-Cleaner Technology for Simultaneous Production of Heat and Power, M F Adnan, $5^{\text {th }}$ ASEAN S\&T Week, Hanoi, Viet Nam, 12-14 Oct. 1998.

[4] Assessment of Energy Utilisation, Dr H Bathish, Renewable Energy Roadshow, $7^{\text {th }}$ Aug. 2007, Kuala Lumpur.

[5] Key ASEAN Macroeconomics indicator, ASEAN Trade Database as of 18 July 2007.

[6] Biomass fired grid-connected cogeneration plants in ASEAN, Asia Pacific Tech Monitor, MF Adnan, Jul-Aug. 2007, pp. 29-34

[7] Kyoto Protocol and Clean development mechanism, Mr M Kishioka, Effective Energy Systems for Sustainable Development, $29^{\text {th }}$ Aug. 2006, Bangkok, Thailand. 


\title{
Design and Development of Liquid Spray Gas Scrubber for Odour Control
}

\author{
Mohd Faizal Mohideen Batcha, Lai Dyi Yih and Vijay R. Raghavan
}

\begin{abstract}
One of the major factors affecting the productivity of workers in industry is quality of air in the working ambience. The quality of air can be characterized by means of microbial contaminants, chemicals, gases, allergen, odour or any mass or energy stressor that can induce health issues. This study is concerned with the presence of excessive detergent odour in industrial laundries. Odours are basically chemicals dissolved in air which we may be perceived by the sense of olfaction and always referred to as unpleasant smell. As for the case of industrial laundries, the effect of detergent odour becomes significant as cleaning processes simultaneously discharge high detergent concentration to the surrounding air which may lead to serious health issues apart from reducing workers' performance. Therefore, a design of liquid spray gas scrubber had been proposed in this work as a means to overcome the problem. A prototype has been designed and developed to prove the working principle of the scrubber. By taking that the scrubbing efficiency is independent of the chemical specie, a solution of ammonium hydroxide $\left(\mathrm{NH}_{4} \mathrm{OH}\right)$ was used as a source of odour as alternative to detergent. This is due to the unavailibity of tools to measure detergent odour while concentration of $\mathrm{NH}_{4} \mathrm{OH}$ in the scrubbing liquid, i.e. water can be easily determined. From the tests conducted, scrubbing efficiency up to $36 \%$ is obtained in a single stage, which is encouraging considering that the scrubber head was a simple shower nozzle. With improved designs of the spray, the scrubber unit may be used to improve indoor working environments.
\end{abstract}

Keywords: air quality, gas scrubber, odour, ammonium hydroxide

\section{INTRODUCTION}

One of the major factors affecting the productivity of workers in industry is the quality of air in the working ambience. The quality of air (or usually termed Indoor Air Quality) can be characterized by means of measuring the microbial contaminants (mould, bacteria, etc.), chemicals (toxicants, radon, etc.), gaseous (carbon monoxide, sulphur dioxide, etc.), allergen, odour or any mass or energy stressor that can introduce health concerns. This study is related to the presence of excess detergent odour in industrial laundries as reported by [1] and [2] in their respective case studies conducted on several "Dr Clean" laundry outlets around Johore. Their findings conclude two significant factors affecting workers productivity, namely heat stress

Mohd Faizal Mohideen Batcha, Lai Dyi Yih and Vijay R. Raghavan are from Faculty of Mechanical Engineering \& Manufacturing, University Tun Hussei\n Onn Malaysia, 86400 Parit Raja, Batu Pahat, Johor (e-mail: mfaizal@uthm.edu.my). and excessive detergent odour. Therefore, the present work proposes a liquid spray gas scrubber design to overcome the excessive detergent odour inside the laundry stores.

Odours are chemicals dissolved in air which we may perceive by the sense of olfaction. Odours always refer to unpleasant smell, and generally regarded as a nuisance. In the case of industrial laundries, the effect of odour becomes even more significant. The cleaning processes which release a significant amount of detergent concentration to the surroundings may lead to serious health issues apart from reducing workers performance, human discomfortand furthermore, may harm workers' health.

By treating the above statements as problems requiring solution, an odour control mechanism is necessary in such environments. The existing odour control technology actually works on the principle of removing odours-causingchemical compounds from the foul air stream by physical or chemical means. Wet water scrubber is one of the methods of odour control. Wet water scrubber is a system with high reliability and high efficiency that are relatively low in cost.

A design of a liquid spray gas scrubber had been proposed in this work. To prove the working principle and concept of the designed scrubber, a prototype has been developed. A few tests conducted had shown good scrubbing efficiency apart from the potential to be commercialized in working ambience which needs control.

\section{LITERATURE REVIEW}

Detergents belong to the group of consumer products, which are necessary for cleanliness, health and hygiene. Detergents are mixtures of several ingredients such as surfactants, builder, filler, anti-redeposition agent, perfume, dye and fluorescent - whitening agent. Increase of detergent use for both industrial and domestic purposes has resulted in rapid deterioration in air quality and water quality. The environmental risk of detergent effluents associated with manufacture, use and disposal of chemicals had always been one of the biggest environment issues around the globe.

In industry, the pollution of these effluents is mainly due to the residual products in the reactor, which have to be washed away in order to use the same facility for the manufacture of other products. As for domestic use, the detergent is commonly called as laundry detergent which is used for cleaning of clothes and fabrics. Detergents commercialized for hand use are normally neutral or slightly acid in order to protect human skin. However, a lot of household and industrial cleaning formulations have a high 
$\mathrm{pH}$, being highly corrosive for skin and for laboratory instrumental [3].

An odour is a chemical dissolved in air, generally at a very low concentration, which we perceive by the sense of olfaction. Odours also called smells, which can refer to both pleasant and unpleasant. Stench is specifically used to describe an unpleasant odour. On the other hand, the terms fragrance, scent, or aroma are used primarily by the food and cosmetic industry to describe a pleasant odour, and are sometimes used to refer to perfumes [4]. Odours always refer to unpleasant smell, and generally regarded as a nuisance and commonly perceived to be those 'foul smelling substances' found as fugitive emissions from a variety food, chemical or wastewater treatment operations. But, when a fragrance is synthetic, such as perfume, scented shampoo, scented deodorant, or other common products, a person can actually be allergic to the oil derivatives. The reaction can be anywhere from a slight headache to anaphylactic shock, which can result in death. When allergy occurs, the person may have a slight intolerance, environmental illness or some other similar negative effect.

Odour intensity is the strength of the perceived odour sensation and is related to the odorant concentration, which is an entirely different category of measurement [5]. The intensity of an odour is perceived directly without any knowledge of the odorant concentration or of the degree of air dilution of the odorous sample needed to eliminate the odour. The following equation defines the relationship between the odour intensity (I) and concentration (C) where $\mathrm{k}$ is a constant and $n$ is the exponent.

$$
\begin{aligned}
\mathrm{I}(\text { perceived }) & =\mathrm{k}(\mathrm{C})^{n} \\
\text { or } \quad \log \mathrm{I} & =\log \mathrm{K}+n \log (\mathrm{C})
\end{aligned}
$$

This is known as Stevens' Law or the power law [5]. As for odours, $n$ ranges from about 0.2 to 0.8 , depending on the odorant. This is an important concept that is related to the basic problem of reducing the odour intensity of a substance by air dilution or other means. Figure 1 shows the data [6] for different chemicals as a $\log$ to $\log$ plot, where the concentration, in parts per billion, as the abscissa varies with the right-side ordinate show as relative odour intensity. The spacing of the numbers on the relative-odour-intensity scale is based on data available for methyl sulphide and the four italicized odorants (IIT Research Institute data). The slope of the straight line is equal to $n$, which is the evident that the effect of dilution on the odour intensity of methyl sulphide is much lesser than that for hydrogen sulphide.

Odour control is part of air pollution control, which can be generally described as a "separation" technology [7]. The pollutants, whether gaseous, aerosol, or solid particulate, are separated from a carrier gas, which is usually referred to as air [8]. Usually the term "gas scrubber" is used to describe the technology. To overcome air-pollutant based problems, odour control methods should be applied, and by separating these pollutant substances from air, human health issues will be overcome. There are several available methods to control odours, with a variety of advantages, disadvantages and degree of cost-effectiveness. Some of these methods are thermal oxidizers, biological systems, wet scrubbing systems and activated carbon.

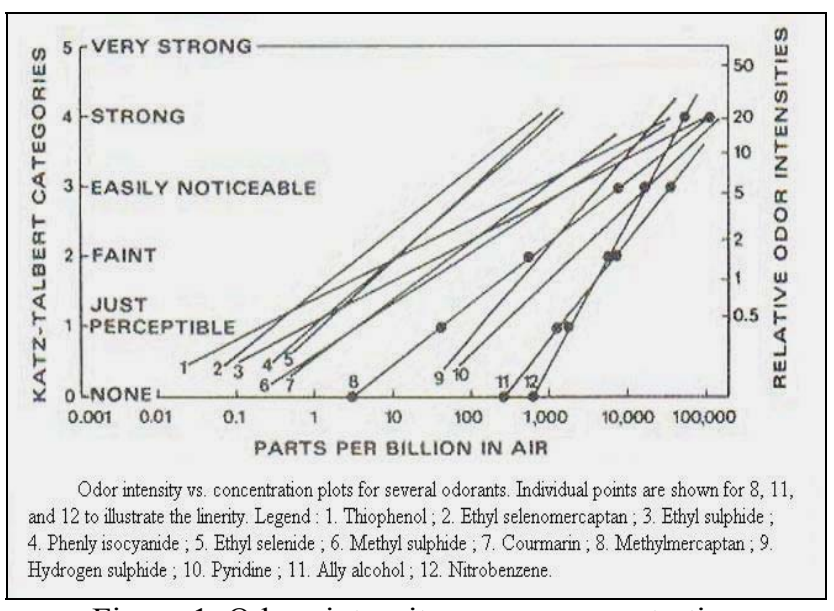

Figure 1. Odour intensity versus concentration

The present work focuses on wet scrubbing systems. Usually a wet scrubbing system is used under situations where the contaminant cannot be removed easily in dry form, soluble gases are present or soluble wet particulates are present. The contaminant might undergo some subsequent wet process such as recovery, wet separation or settling or neutralization. The pollution control system must be compact, and the contaminants are mostly safely handled wet rather than dry [9]. There are several types of available wet scrubbing system, and can be roughly be grouped into seven major categories: venturi scrubbers, mechanicallyaided scrubbers, pump-aided scrubbers, wetted-filter-type scrubbers, tray-type or sieve type scrubbers, spray towers, and hybrid scrubbers.

A wet scrubber uses capture techniques. It removes the particles from the gas by capturing the particles in liquid (usually water) droplets and separating the droplets from the gas stream. The droplets act as conveyors of the particulates out of the gas stream. There are three mechanisms that wet scrubbers use to capture the particulates [7,9]: "impaction" of the particles directly into a target droplet, "interception" of the particles by a target droplet as the particle comes near the droplet and "diffusion" of the particle through the gas surrounding to the "target droplet" until the particle is close enough to be captured. The three mechanisms are shown in Figures 2, 3 and 4 respectively.

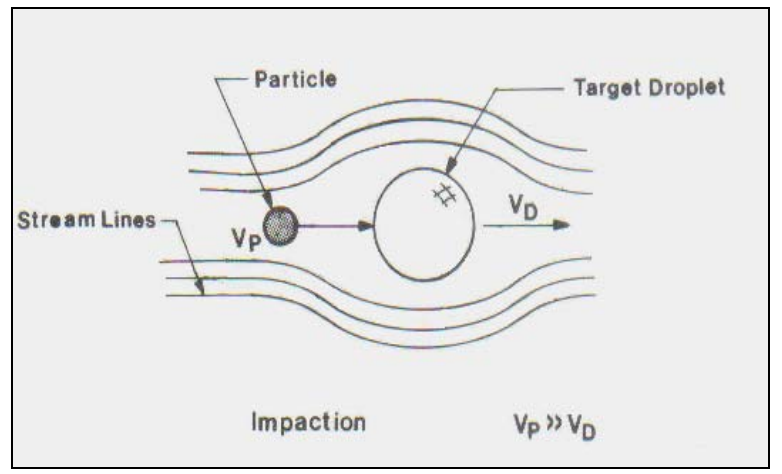

Figure 2. Direct impaction into a droplet [9] 


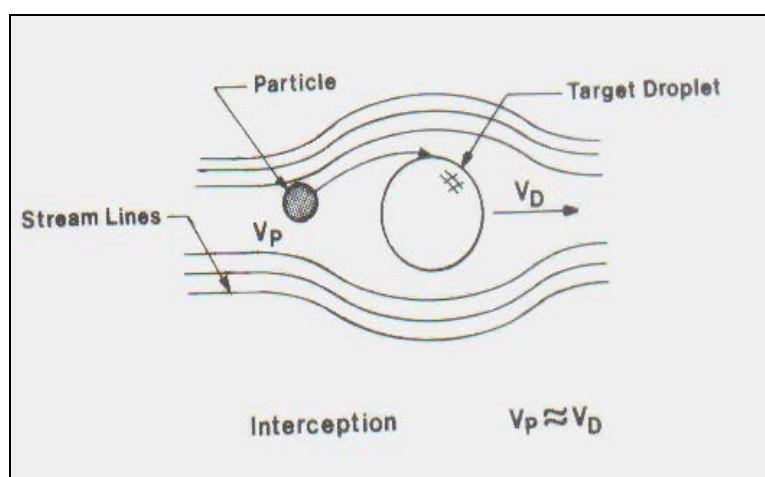

Figure 3. Interception [9]

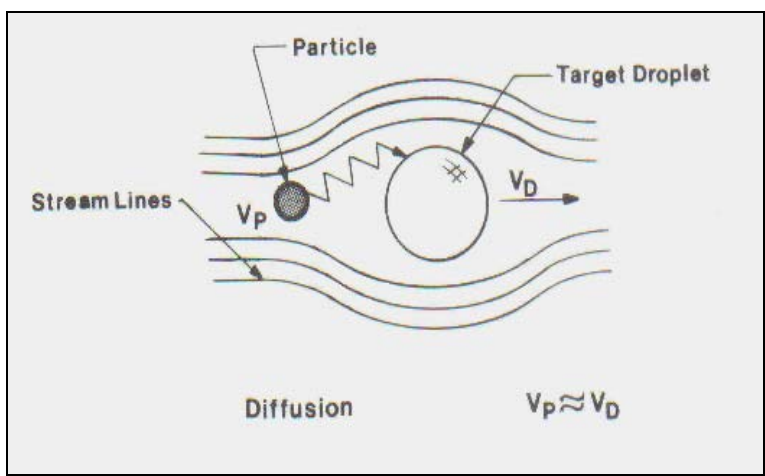

Figure 4. Diffusion [9]

"Target droplet" is the key word in wet scrubbing systems. A successful scrubber creates and controls the droplet dispersion effectively. Almost all high-efficiency wet scrubbers are proficient at creating target droplets, by accelerating gases to high velocity, injecting liquid, then pneumatically shearing the liquid into a fine spray, or use mechanical energy such as spinning disk or high-pressure sprays to create the target droplet diffusion. The goal is to cause the tiny pollutant particle to be lodged inside the collecting droplet and then to remove the larger droplet from the gas stream. There are two general ideas in creating a wet scrubbing system:

i. The smaller the target droplet, the smaller the size of particulate that can be captured.

ii. The more densely the droplets are packed, the greater is the probability of capture.

\section{Methodology}

A theoretical framework for the idea of developing a wet water scrubber system had been undertaken based on literature review of the current wet water scrubber system. The idea in developing this system is to spray scrubbing liquid (e.g. water) onto the surface which has large area per unit volume (such as fill material in cooling towers) whereby the odorous gas is being forced to flow over the surface as well. The simultaneous contact between the odorous gas and the scrubbing liquid will result in capturing of odorous substance at certain concentration. These process will reduce the amount of odour concentration in the gas, thus enable us to determine the scrubbing efficiency of the whole system.

The proposed system consists of 3 main sub-assemblies, namely the odour generator, scrubbing unit and scrubbing liquid collector. The odour generator operates atmospheric air is blown through sparger, in which air is bubbled through detergent solution with certain concentration thus creating detergent odour gas. This odourous gas then flows directly into the scrubbing unit where gas and scrubbing liquid which is sprayed by an array of nozzles comes into contact simultaneously. Contact between these two fluids creates scrubbing action in which the droplets of scrubbing liquid capture the odour generating particles from the gas. Augmentation of scrubbing action is achieved by enlarging the total surface area of contact between the odourous gas and scrubbing liquid, by means of using a large surface area to volume material, similar to fill materials used in cooling towers. Finally, the scrubbing liquid is collected and filtered before the same liquid is pumped inside the scrubbing unit [10]. The process is illustrated in Figure 5.

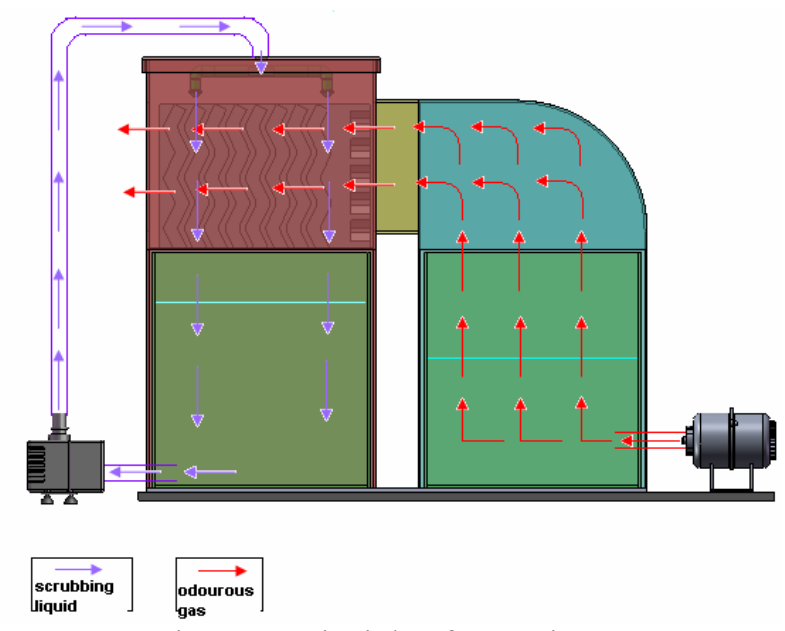

Figure 5. Principle of operation [10]

However, in this work, the composition of chemicals which creates odour in commercial detergents could not be identified. This is due to the inability to extract information from the manufacturer as they are kept as industrial secrets. As a result, the corresponding target droplet inside the scrubbing liquid could not be established. However, a criterion to determine the scrubbing efficiency is required to evaluate the performance of the proposed liquid scrubber. Therefore, the principle of dependence of scrubbing efficiency to chemical specie is applied [10]. By this principle, the scrubbing efficiency is taken to be independent of any particles or contaminants. As such, ammonium solution in water or ammonium hydroxide $\left(\mathrm{NH}_{4} \mathrm{OH}\right)$ which features pungent odour is used to generate odour in this work. $\mathrm{NH}_{4} \mathrm{OH}$ is diluted in distilled water with a concentration of 1:50, which is then bubbled with air to generate vapour and forced to flow inside the scrubber. The efficiency of the scrubber is basically a ratio of $\mathrm{pH}$ of $\mathrm{NH}_{4} \mathrm{OH}$ captured to $\mathrm{pH}$ of $\mathrm{NH}_{4} \mathrm{OH}$ injected;

$\eta_{\text {scrubber }}=\frac{p H_{\text {captured }}}{p H_{\text {injected }}}$

$p H_{\text {injected }(t=i)}=p H_{\text {solution }(t=i-1)}-p H_{\text {solution }(t=i)} i=1,2, \ldots, 15$

$p H_{\text {captured }(t=i)}=p H_{\text {water }(t=i)}-p H_{\text {water }(t=i-1)} i=1,2, \ldots, 15$

where $t$ are in minutes.

\section{RESUltS AND DISCUSSION}

The wet water scrubber system consists of two main chambers with other parts such as nozzles, fill material, 
pump and blower. It can be divided into three subassemblies; Sub-assembly A is where the scrubbing process takes part, Sub-assembly B is where the detergent odour being carries out from the detergent contaminated water, and Sub-assembly $\mathrm{C}$ works as a connector path, allowing detergent odour to flow from Sub-assembly B to Subassembly A. These sub assemblies and the scrubber's individual parts are shown in Figure 6.

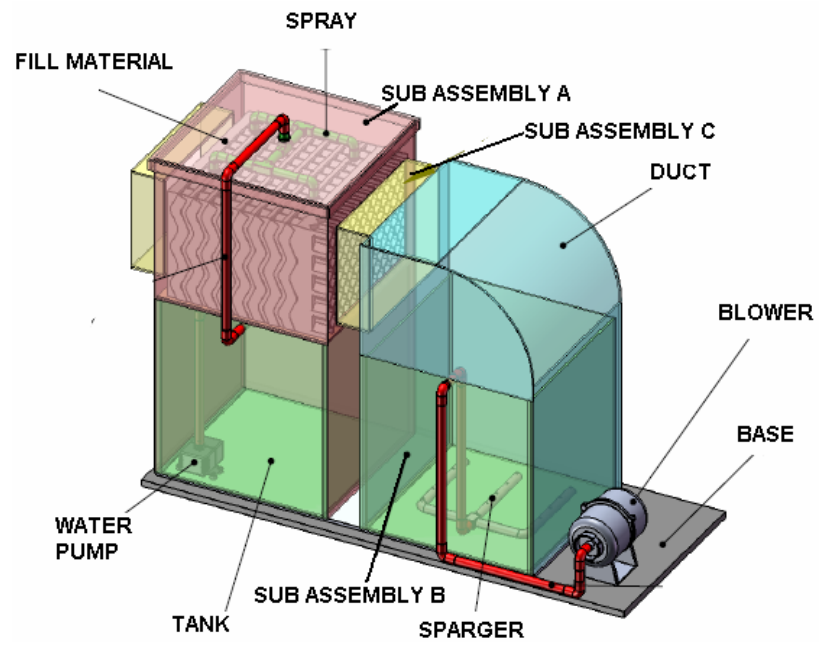

Figure 6. Wet water scrubber system [10]

Ammonium solution $\left(\mathrm{NH}_{4} \mathrm{OH}\right)$ which is used in this work is a corrosive liquid and upon vaporization, it becomes toxic by inhalation and is hazardous. Therefore it is important to determine a safe operating condition during experiments. This is achieved by diluting the ammonium hydroxide with distilled water in a ratio of 1:50. This enables a minimum volume of ammonium hydroxide used but sufficient to provide necessary odour. From this ratio, 10 litres of water is used with 0.2 litres of $\mathrm{NH}_{4} \mathrm{OH}$. The chamber and connections are perfectly sealed to avoid any gas leakage. The experiment is run for 15 minutes where both initial values of $\mathrm{pH}$ injected and captured are recorded simultaneously. The results are shown in Table 1.

Table 1. Results

\begin{tabular}{|c|c|c|c|c|}
\hline $\begin{array}{c}\text { Time } \\
\text { (minute } \\
\text { s) }\end{array}$ & $\begin{array}{c}\text { pH in } \\
\text { Chamber } \\
\text { B }\end{array}$ & $\begin{array}{c}\text { pH in } \\
\text { Chamber } \\
\text { A }\end{array}$ & $\begin{array}{c}\text { pH of } \\
\text { Scrubbing } \\
\text { Liquid }\end{array}$ & $\begin{array}{c}\text { Efficiency } \\
(\%)\end{array}$ \\
\hline $\mathrm{t}=0$ & 9.24 & 8.82 & 6.43 & 14.95 \\
\hline $\mathrm{t}=5$ & 9.18 & 8.73 & 6.82 & 19.07 \\
\hline $\mathrm{t}=10$ & 9.11 & 8.68 & 7.17 & 22.16 \\
\hline $\mathrm{t}=15$ & 9.07 & 8.89 & 7.68 & 12.95 \\
\hline $\mathrm{t}=20$ & 8.99 & 8.64 & 7.79 & 29.17 \\
\hline $\mathrm{t}=25$ & 9.04 & 8.67 & 8.01 & 35.92 \\
\hline $\mathrm{t}=30$ & 8.92 & 8.81 & 8.11 & 13.58 \\
\hline
\end{tabular}

During experiments, observations shown that the flowrate of $\mathrm{NH}_{4} \mathrm{OH}$ vapor are smaller than expected. The main reason is due to the small amount of air supplied by the blower. The large cross sectional area of ducts reduces the velocity, and the level of water combined with fill material imposes large pressure drop. Therefore, amount of $\mathrm{NH}_{4} \mathrm{OH}$ vapor flowing through the scrubbing unit becomes low. As a result, amount of $\mathrm{NH}_{4} \mathrm{OH}$ removed from the vapor also becomes relatively small affecting the total scrubbing efficiency. However, the pressure drop by water is unrealistic for a real application of scrubber as this is only necessary for lab experiments.

\section{CONCLUSION}

From the results, a maximum scrubbing efficiency of $35.92 \%$ was obtained. Although this efficiency is actually far from typical liquid scrubber's efficiency which ranges more than $90 \%$, several conclusions can be drawn. First, determination of a scrubber's efficiency can be generalized by taking the efficiency to be independent of chemical specie. Second, the fill material may increase the total surface of scrubbing area but costs pressure drop significantly. Therefore, appropriate type of custom made fill material can be considered in the scrubbing unit if the blower size is a constraint. Third, the array and height of nozzle which sprays the scrubbing liquid also plays important role to ensure equal distribution of scrubbing liquid with maximum wetted area. Hollow-cone type of spray must be avoided.

Several improvements can also be considered in the current design of scrubber. Swirling flow of gas entering the scrubber unit can improve scrubbing efficiency significantly as gas and scrubbing liquid became better in contact. The swirling motion can be obtained through devices such as custom made gas swirler; similarly as being used in internal combustion engines for fuel saving and creating homogenous air-fuel mixture for better combustion. One can also consider counter-flow type of scrubbing unit to increase the reaction time and area of contact between gas and scrubbing liquid as both fluid now flows in opposing directions.

\section{ACKNOWLEDGMENT}

The author wish to acknowledge the Ministry of Science, Technology and Innovation, Malaysia for funding this research under Fundamental Research Grant, vote 0260 and University Tun Hussein Onn Malaysia (UTHM).

\section{REFERENCES}

[1] Nur Azhani Ishak, "Effects of Heat Stress on Laundry Workers: A Case Study in Ergonomics View", Bachelor Engineering Thesis, Kolej Universiti Teknologi Tun Hussein Onn, 2006.

[2] Zulhilman Dor, "Further Investigation of Heat Stress on Laundry Workers: An Ergonomics Viewpoint", Bachelor Engineering Thesis, Universiti Tun Hussein Onn Malaysia, 2006.

[3] Josep F. Ventura-Gayete, Miguel de la Guardia, Salvador Garrigues, "Attenuated Total Reflectance Infrared Determination of Sodium Nitrilotriacetate in Alkaline Liquid Detergents." Department of Analytical Chemistry, University de València, Spain

[4] American Society of Heating, Refrigerating and Air Conditioning Engineers (ASHRAE), “ASHRAE Handbook 2005, Fundamentals Chapters, F13: Odours.” 2005.

[5] Anthony J. Buonicore, Wayne T. Davis, "Air Pollution Engineering Manual" Air \& Waste Management Association, Van Nostrand Reinhold, New York, 1992: 147-154.

[6] A. Dravnieks, "Odor Perception and Odorous Air Pollution." J. TAPPI, 55:737-742, 1972.

[7] Kenneth C. Schifftner, "Air Pollution Control Equipment Selection Guide.” Lewis Publisher, A CRC Press Company, 2002.

[8] H. Saito, "Assessment of Industrial VOC Gas-Scrubber Performance." Lawrence Livermore National Laboratory, 2004.

[9] Anthony J. Buonicore, Wayne T. Davis, "Air Pollution Engineering Manual" Air \& Waste Management Association, Van Nostrand Reinhold, New York, 78-88, 1992.

[10] Lai Dyi Yih, "Study on a Liquid Spray Gas Scrubber", Bachelor Engineering Thesis, Univeriti Tun Hussein Onn Malaysia, 2007. 


\title{
Analytical Prediction of Critical Heat Fluxes in Forced Convective Boiling for Power Plant Applications
}

\author{
M. Z. Zainon and A. Serizawa
}

\begin{abstract}
In relation to the design and safety analysis of the various industrial equipment involving the boiling heat transfer and two-phase flow, particularly in the power plants, an analytical study was carried out to predict the $\underline{\text { Critical }} \underline{\text { Heat }}$ Fluxes (CHF) in forced convective boiling in a straight pipe. The three-fluid model and appropriate CHF models were used to study the flow characteristics and calculate the $\mathrm{CHF}$ in wide range of flow condition which was set close to the condition of typical steam plant operation. Comparisons of predicted CHF were carried out with the experimental data from Risø database. In this work, it was found that the constitutive equation of droplet entrainment and deposition play very important roles in the prediction for annular dispersed flow regime where the CHF occurred from the film dryout. Since these correlations were developed based on different flow condition and flow model, there are considerable discrepancies among these correlations. Therefore, the sensitivity of these correlations to the CHF prediction was evaluated and some recommendations for appropriate constitutive equation were made.
\end{abstract}

Keywords: flow boiling, heat transfer, power plant, safety design, analytical prediction

\section{INTRODUCTION}

Gas-liquid two-phase flow and heat transfer with a change in phase is of considerable importance in a whole range of applications. Many industrial processes rely on these phenomena for materials processing or for energy transfer; e.g., petroleum processing, paper pulping, power plant, and chemical industries, in refrigeration, air-conditioning and gas liquefaction. Starting in the 1950 s, intensive studies of this area were made in the context of the nuclear industry. Although the nuclear industry work has declined there is still a strong driving force with respect to the other steam plant applications. A generalized solution methodology for two-phase flow especially in force convection boiling problem has remained as an elusive goal, mainly because gas-liquid flow systems combine the complexities of turbulence with those of deformable interfaces.

Flow boiling occurs when all the phases are in bulk flow together in a channel; e.g., vapor and liquid flow in a pipe. The multiphase flow may be classified as adiabatic, i.e., without or with heat addition at the channel wall. In this work, analyses were

M. Z. Zainon is a Lecturer at the Department of Mechanical Engineering, University of Malaya, 50603 Kuala Lumpur, Malaysia, (phone: 03-7967 6876; Fax: 03-79675317; email: zzainon@yahoo.com).

A. Serizawa is a Professor Emeritus of Kyoto University. He is also an alumnus of Department of Nuclear Engineering, Kyoto University. focused on the adiabatic flow of which some examples are such as flow in the riser tubes of steam generators and boiler tubes in power plants or in the coolant channels between nuclear fuel elements in boiling water reactor. During the boiling, flow patterns change due to vapor production on the heating wall and flow together in the mainstream of liquid.

Hence the force convective boiling is an important means of heat transfer in the practical engineering owing to its ability to transport thermal energy with high efficiency. However, a loss of this ability might lead to a serious damage of heat transfer systems, especially in the steam power plant. The optimization of operating condition and the ability to obviate potentially dangerous conditions requires the ability to predict accurately its behavior in such process. A lot of forced convection boiling studies have been performed so far by many researchers. Based on those experimental results, important parameters in boiling heat transfer such as void fraction [1,2], critical heat flux [3 7] etc, various flow patterns and behavior of two-phase flow have been carried out. Among this, critical heat flux (CHF) is one of the most important parameter of the heat transfer equipment. This parameter determines the limitation of heat removal from heating wall, which is a controlling parameter of the heat transfer equipment. Based on those previous researches, considerable knowledge has been accumulated and a number of correlations have been proposed and a used in designing of heat transfer equipment. However, many of them have been made to correlate the burnout of CHF for flows inside of round tubes, annuli, outside of the rod bundles, etc. It is therefore, probably close to the truth to say that due to the complexity of included phenomena, no correlation for burnout heat flux has yet been evolved that is applied universally to the data for various kinds of fluids and conditions and there are no assurances of the validity of extrapolation of these correlations beyond the experimental conditions on which are based on.

From a practical point of view, prediction of CHF in annular flow is one of the most important mechanisms, for channels of reasonable length, the first occurrence of CHF may likely occur in this region. There is also a general agreement that the the mechanism leading to CHF depends on the flow quality. At high qualities, the flow is annular and CHF is caused by dryout of the liquid film on the heated surface. For this case, the predictive method based on physical model of film dryout with use of threefluid model (vapor, droplet and liquid film) has been developed with favorable agreement of experimental data $[8,9]$ where the droplet deposition and entrainment play very important roles. With respect to this reason, detailed evaluations were specifically carried out for the effects of droplet deposition and entrainment rate correlations to well predict the dryout-type CHF under various flow conditions. Using developed models and correlations, CHF in annular dispersed flow for wide ranges of flow conditions were then successfully predicted. 


\section{Methodology}

In this work, the CHF were predicted based on the analytical method as the following procedures using two-phase flow analysis code with given geometries and parameters were calculated from the entrance of through each part of the flow channel. Pressures, velocities of the vapor and liquid phases, void fraction and the quality are also calculated at the exit of the flow channel. Then, the two-phase flow behavior was evaluated at the exit of the flow channel and the occurrence of dryout and DNB model were judged based on it. In this case, observations were made such as, the error range of the prediction result and the given heat flux and finally the heat flux at the predicted burnout occurrence is considered as the CHF of the particular geometry and flow condition.

\section{A. Analysis of Burnout Phenomenon}

In low quality region (bubbly, slug, chum flow), CHF mechanism is the transition from nucleate boiling to film boiling (Departure form Nucleate Boiling, DNB) which will not discuss further in this report. In short, for comparatively low mass flux and low wall heat flux, the liquid is considered to flow up with changing its flow pattern, continuously from the single phase flow, bubbly flow, churn flow and to annular dispersed flow, where dryout occurs when the liquid film disappear from the heated wall at the exit of the flow channel.

\section{B. Two-phase Flow Models}

In order to predict the CHF, two-phase flow behavior in the flow boiling channel should be predicted accurately. Appropriate basic and constitutive equations of mass, momentum and energy of twophase flow are needed to make this feasible. In the current work, concentration are mainly for larger void fraction where flow regime changes to annular dispersed flow with liquid film flow on the pipe wall, gas flow in the core region and the existence of liquid droplet flow in the gas flow. Therefore, three-fluid model [9, 10] was used in order to predict the dryout phenomena, where the liquid film thickness on the heated wall should be evaluated appropriately.

One dimensional steady state subcooled flow boiling was analyzed in this work, since this is of practical importance and the effects of two-phase flow model and constitutive equation are easy to evaluate under this condition. Furthermore, for simplicity of the analysis, the conservation equation of energy was reduced to the relation between mass generation rate of vapor, $M_{g}$, and wall heat flux $q_{w}$ which is given as the following equation

$$
M_{g}=\frac{4}{D_{e}} \frac{q_{w}}{i_{f g}}
$$

where $i_{f g}$ is the latent heat of vaporization and $D_{e}$ is the flow diameter. Therefore, the conservation equations of mass and momentum were solved for the prediction of two-phase flow behavior.

\section{Three-Fluid Model}

The CHF in high quality liquid-vapor flow in a heated channel is closely related to the thermal-hydrodynamic characteristics of annular two-phase flow. Mechanisms that may lead to the critical heat flux condition in the annular two-phase flow regime and the following possibilities:

a) Film boiling transition underneath the liquid film

b) Film disruption by hydrodynamic instability of liquid

c) Spontaneous breakdown of the liquid film

d) Breakdown due to thermal capillarity effects

e) Equality between droplet deposition and evaporation

f) Dryout between waves

g) Liquid entrainment with no subsequent deposition h) Film disruption due to nucleate boiling within the film

i) Integral entrainment-deposition effect leading to zero film rate at the point of onset of $\mathrm{CHF}$

Several investigators have linked CHF with liquid film dryout [case (i)], and many independent investigators have confirmed that CHF occurs when the liquid films becomes too thin and breaks down into rivulets around dry patches. In general, the condition of zero, or near zero, liquid film flow can be used as a criterion of CHF.

Since at high qualities, the flow is annular and CHF is caused by dryout of the liquid film on the heated surface, the three-fluid model is considered as the best tool to evaluate the dryout CHF. Hence in this case, the working fluid is considered as a combination of liquid film, droplet and vapor, and therefore the conservation equations are considered separately for each of the fluid component.

\section{Basic equation of three-fluid model}

The continuity or mass conservation equations are

Vapor: $\quad \frac{\partial}{\partial z}\left(\alpha_{g} \rho_{g} u_{g}\right)=M_{g}$,

Liquid film: $\quad \frac{\partial}{\partial_{z}}\left(\alpha_{l} \rho_{l} u_{l}\right)=M_{l}-M_{e}+M_{d}$

Droplet: $\frac{\partial}{\partial_{z}}\left(\alpha_{d} \rho_{d} u_{d}\right)=M_{e}-M_{d}$,

Hence, the volume fraction must sum to unity so that

$$
\alpha_{d}+\alpha_{l}+\alpha_{g}=1
$$

which can also be written

$$
\frac{d \alpha_{d}}{d z}+\frac{d \alpha_{l}}{d z}+\frac{\alpha_{g}}{d z}=1
$$

The axial momentum equations are given by

Vapor:

$$
\frac{\partial}{\partial z}\left(\alpha_{g} \rho_{g} u_{g}^{2}\right)=-\alpha_{g} \frac{\partial P}{\partial z}-F_{\mathrm{lg}}-F_{e g}+M_{b} u_{l}-\alpha_{g} \rho_{g} g
$$

Liquid film:

$$
\frac{\partial}{\partial z}\left(\alpha_{l} \rho_{l} u_{l}^{2}\right)=-\alpha_{l} \frac{\partial P}{\partial z}+F_{\mathrm{lg}}-F_{e l}-F_{w l}+M_{d} u_{e}-\left(M_{e}+M_{b}\right) u_{l}-\alpha_{l} \rho_{l} g
$$

Droplets:

$\frac{\partial}{\partial z}\left(\alpha_{d} \rho_{d} u_{d}^{2}\right)=-\alpha_{d} \frac{\partial P}{\partial z}+F_{e l}+F_{e g}-M_{d} u_{e}+\left(M_{e}+M_{b}\right) u_{l}-\alpha_{d} \rho_{d} g$

The suffix ' $l$ ', ' $d$ ' and ' $g$ ' denote the liquid film, liquid droplets and vapor respectively. The terms of the above equation are as below

$M_{b}$ : mass generation rate of vapor phase,

$M_{d}:$ total droplet deposition rate,

$M_{e}:$ total droplet entrainment rate,

$u_{l}, u_{e}$ : intrinsic velocity for mass transfer for liquid film and droplet, respectively,

$F_{l g}$ :liquid film-vapor interfacial momentum transfer force,

$F_{e g}$ : droplet-vapor momentum transfer force,

$F_{e l}:$ droplet-liquid film momentum transfer force,

$F_{w l}$ : wall-liquid film momentum transfer force (wall friction).

\section{Evaluation of Droplet Deposition Coefficient and Entrainment Rates}

A number of correlations on droplet deposition coefficient and entrainment rate which can help the calculation of deposition quantities of entrained liquid droplets onto the liquid film and 
entrainment quantities of liquid from the wave on the liquid film, were developed $[9,11-25]$ based on various experimental data and theoretical models. Since no assurance can be made for the validity of these correlations, systematic comparisonsas the effects of these correlations on the dryout-type CHF were carried out.

The droplet deposition rate, $m_{d}$ is generally given in the form of the relationship of droplet deposition coefficient, $k_{d}(\mathrm{~m} / \mathrm{s}\}$, and the average mass density of droplets in the gas core, $C\left(\mathrm{~kg} / \mathrm{m}^{3}\right)$ as given by,

$$
m_{d}=k_{d} C
$$

The correlations of deposition correlations $k_{d}$, and entrainment rate, $m_{e}$, will be discussed in detail in the next section. Since the flow conditions used in these comparisons are considerably near to the dryout occurrence in typical nuclear reactor, several correlations with good comparisons were tested in order to evaluate the dryout-type CHF.

\section{Analytical Prediction of CHF using Three-Fluid Model}

For the convenience of comparison, the standard flow condition was set as listed below, which is close to the condition of typical steam plant operation.

$$
\begin{aligned}
& \text { Pressure, } P=7 \mathrm{MPa} \\
& \text { Mass flux, } G=500-3000 \mathrm{~kg} / \mathrm{m}^{2} \mathrm{~s} \\
& \text { Heated length, } L=4.03 \mathrm{~m} \\
& \text { Flow diameter, } D_{\mathrm{h}}=0.01 \mathrm{~m} \\
& \text { Subcooling, } H_{\mathrm{sub}}=50-470 \mathrm{~kJ} / \mathrm{kg}
\end{aligned}
$$

The experimental CHF data from Risø National Laboratory database were used for these analyses.

\section{RESULTS AND DisCUSSIONS}

\section{A. Effects of the deposition coefficient and entrainment rate correlations on the $\mathrm{CHF}$ prediction}

Dryout is a phenomenon that occurs when the liquid film on the heated surface disappears due to evaporation and droplets formation. Therefore, in order to predict the dryout CHF, accurate knowledge on the liquid film thickness is needed. It is therefore, the deposition of entrained liquid droplets onto the liquid film and the entrainment rate of liquid droplet formation from the liquid film must be appropriately evaluated.

Since the focus in this work to predict the CHF in the annular flow, a generalize prediction method based on the three-fluid model (for dryout heat fluxes) were suggested at the end of analyses. For this purpose, CHF were predicted and compared with experimental data for a number of combinations of deposition and entrainment rate correlations. This is done using the developed two-phase flow analysis code as well as different combinations of droplet deposition and entrainment rate correlations, which had discussed in detail in the next section.

Under the above condition, the experimental dryout heat flux was obtained at $943 \mathrm{~kW} / \mathrm{m}^{2}$. The results of the predicted dryout heat flux are shown in Table 1. Based on these results, it is found that the predicted dryout heat fluxes considerably differ from each other depending upon the correlations of droplet deposition and entrainment rates. The blank columns in this table indicate that unreasonably high or low dryout heat fluxes are predicted. This result indicates that the constitutive equations of droplet deposition and entrainment rates are quite important in predicting the dryout heat flux by three-fluid model and more detailed researches of these parameters are needed for the prediction of dryout heat flux.

From the Table 1, with detailed analysis, it is found that the predicted dryout heat flux is well conform to the experimental heat flux by using the entrainment correlation by Sugawara [11], Whalley et al. [21], and Wurtz [24], as well as the deposition coefficient by Sugawara [11], Whalley et al. [21] and Saito et al. [19]. Any combination of these correlations (highlighted in Table
1) gives reasonable predictions of dryout heat flux tested in this analysis. There are total of nine combinations that can be treated as the reliable correlations of droplet deposition and entrainment rates for detail evaluation of dryout CHF. The calculated CHF were further evaluated and shown in figures $1(\mathrm{a} \sim \mathrm{d})$ with different variables effects.

Table 1. CHF prediction based on Combination of $M_{d}$ and $M_{E}$ proposed by different scholars

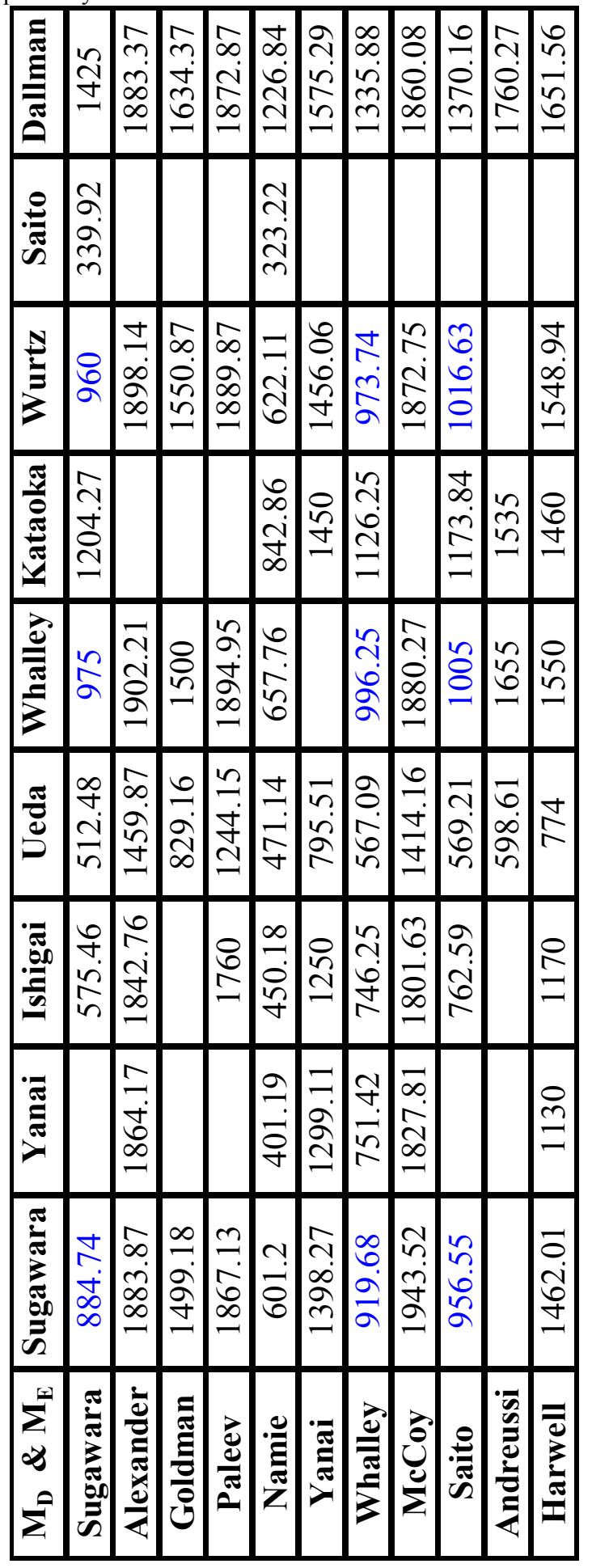

As a target of the work, a generalize method of predicting CHF for boiling for dryout case is suggested by using the developed three-fluid model associated with appropriate combination droplet and entrainment rate. Those combination for different variables are listed in tables $2 \sim 4$. 

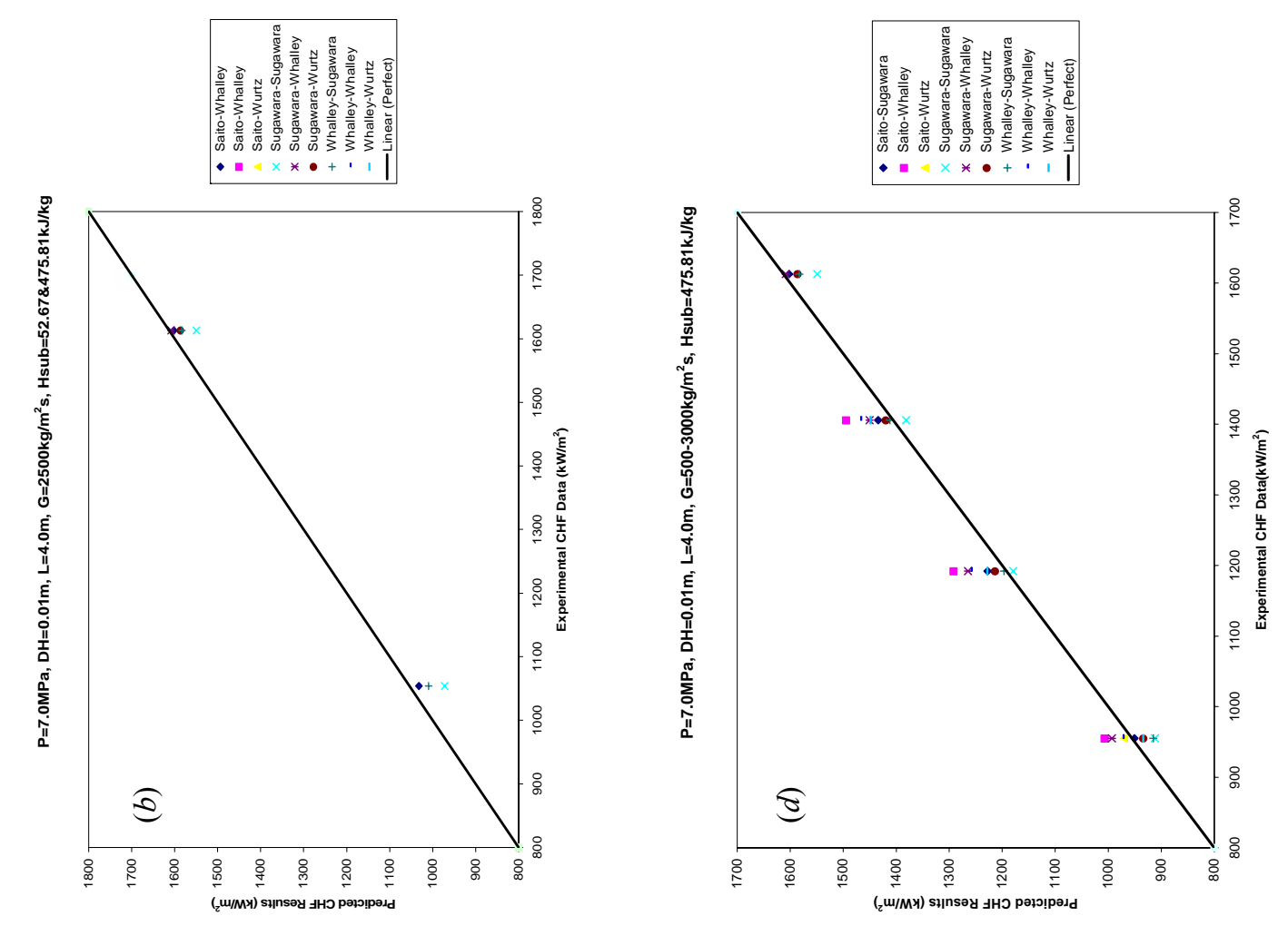

苞
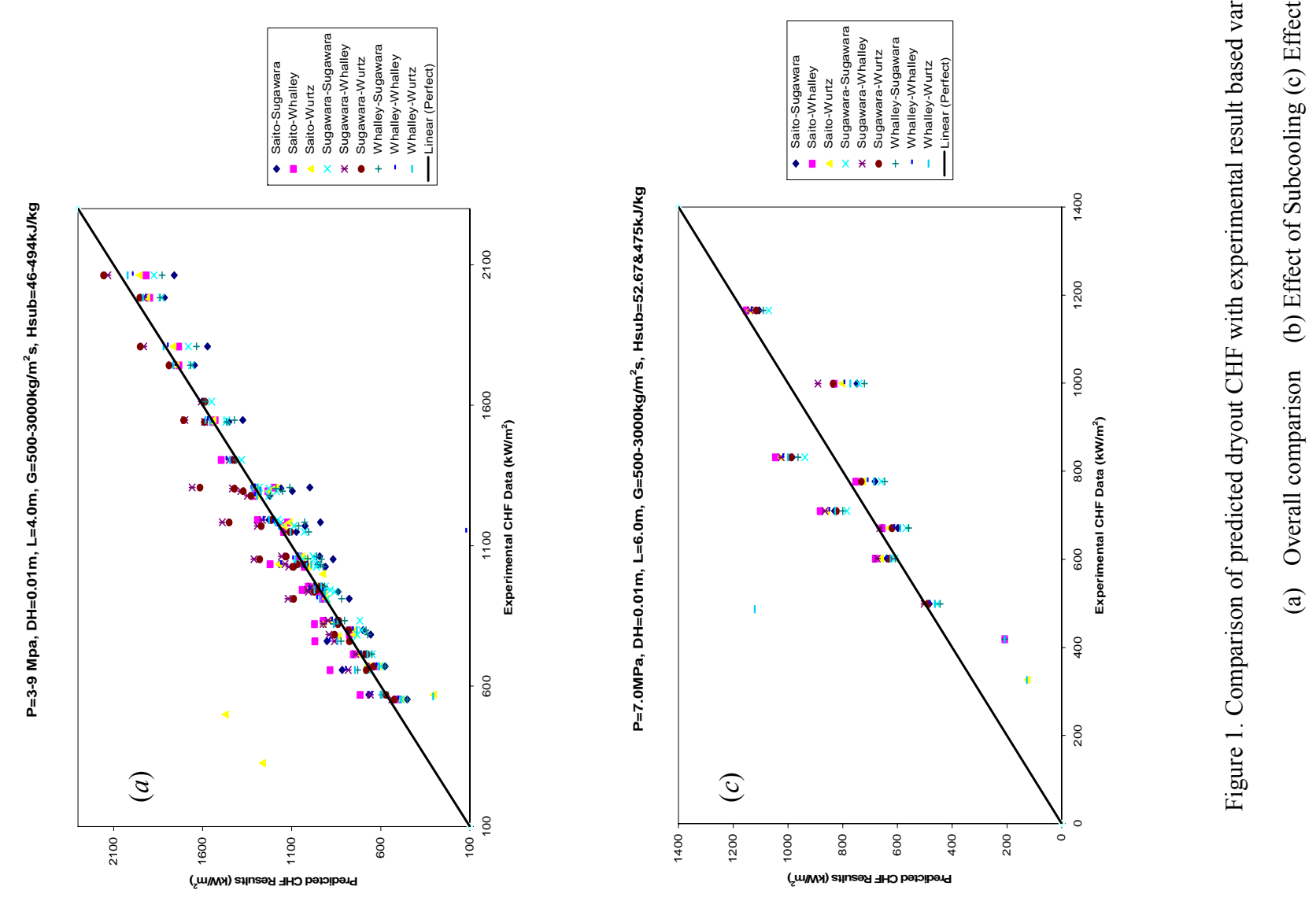
From results presented here, the effects of the correlation of droplet deposition and entrainment rates on dryout heat flux found to be predominant in the CHF prediction for dryout model in annular dispersed flow. The present models are based on the dimensional analyses of two-phase behavior. For more general prediction of $\mathrm{CHF}$ in flow boiling, the detailed modeling and analyses including multidimensional behavior and turbulence behaviors of two-phase flow will be strongly desirable.

For droplet and entrainment rate, since those correlations are expressed in various dimensionless and dimensional parameters, comparisons were therefore carried out under various dimensional flow conditions.

Table 2. Recommended combination of $\mathrm{M}_{\mathrm{d}}$ and $\mathrm{M}_{\mathrm{E}}$ for various Pressures

\begin{tabular}{|c|c|}
\hline $\begin{array}{c}\text { Pressure, } \boldsymbol{P} \\
\text { (MPa) }\end{array}$ & $\begin{array}{c}\text { Recommended combination } \\
\text { of } \boldsymbol{M}_{\boldsymbol{d}} \text { and } \boldsymbol{M}_{\boldsymbol{E}}\end{array}$ \\
\hline 3 & Whalley [21] - Wurtz [24] \\
\hline 5 & Whalley [21] - Whalley [21] \\
\hline 7 & Sugawara [11] - Wurtz [24] \\
\hline 9 & Sugawara [11] - Wurtz [24] \\
\hline
\end{tabular}

Table 3. Recommended combination of $\mathrm{M}_{\mathrm{d}}$ and $\mathrm{M}_{\mathrm{E}}$ for various Mass Flux

\begin{tabular}{|c|c|}
\hline $\begin{array}{c}\text { Mass Flux, } \boldsymbol{G} \\
\left(\mathbf{~ k g} / \mathbf{m}^{2} \mathbf{s}\right)\end{array}$ & $\begin{array}{c}\text { Recommended combination for } \\
\boldsymbol{M}_{\boldsymbol{d}} \text { and } \boldsymbol{M}_{\boldsymbol{E}}\end{array}$ \\
\hline 1000 & $\begin{array}{c}\text { Whalley [21] - Wurtz [24] } \\
\text { Whalley [21] - Whalley [21] }\end{array}$ \\
\hline 1500 & $\begin{array}{c}\text { Sugawara [11] - Wurtz [24] } \\
\text { Whalley [21] -Wurtz [24] }\end{array}$ \\
\hline 2000 & $\begin{array}{c}\text { Sugawara [11] - Wurtz [24] } \\
\text { Whalley [21] - Sugawara [11] }\end{array}$ \\
\hline 2500 & $\begin{array}{c}\text { Saito [19] - Sugawara [11] } \\
\text { Whalley [21] - Sugawara [11] }\end{array}$ \\
\hline
\end{tabular}

Table 4. Recommended combination of $\mathrm{M}_{\mathrm{d}}$ and $\mathrm{M}_{\mathrm{E}}$ for various Heating Length

\begin{tabular}{|c|c|}
\hline $\begin{array}{c}\text { Heated Length, } \\
\boldsymbol{L}(\mathbf{m})\end{array}$ & $\begin{array}{c}\text { Recommended combination for } \boldsymbol{M}_{\boldsymbol{d}} \\
\text { and } \boldsymbol{M}_{\boldsymbol{E}}\end{array}$ \\
\hline 2 & $\begin{array}{c}\text { Sugawara [11] - Wurtz [24] } \\
\text { Whalley [21] - Sugawara [11] }\end{array}$ \\
\hline 4 & $\begin{array}{r}\text { Sugawara [11] - Wurtz [24] } \\
\text { Whalley [21]- Wurtz [24] }\end{array}$ \\
\hline 6 & $\begin{array}{r}\text { Sugawara [11] - Whalley [21] } \\
\text { Whalley [21] - Wurtz [24] }\end{array}$ \\
\hline 8 & $\begin{array}{c}\text { Saito [19] -Wurtz [24] } \\
\text { Whalley [21]- Whalley [21] }\end{array}$ \\
\hline
\end{tabular}

\section{CONCLUSION}

Historically, we have seen that the problem in loss-of-coolant accidents (LOCAs) safety studies is to show that fuel limits, especially clad temperature, are not exceeded for all credible circuit breaches. The analysis involves a wide range of hydrodynamic and heat-transfer phenomena. The accuracies normally expected of steady-state predictions will not be attainable. However, such accuracies are not required in a safety case, and uncertainties are covered by built-in pessimisms.

In view of this, the annular flow which is a particularly important flow pattern since for a wide range of pressure and flow conditions, it occurs over the major part of the mass quality range. In other words, in a vertical tube evaporator as much as 90 per cent of the tube length may be in annular flow. It is as a consequence to develop a generalize method of predicting CHF for flow boiling in this important region. A three-fluid model has been chosen to predict systematically the dryout-type CHF for wide range of geometrical and flow conditions.
For the current work, a generalized method to predict the critical heat fluxes was suggested based on the prediction result versus the experimental data. An appropriate flow condition was set and the effect of the droplet deposition and entrainment rate were discussed. For dryout model in annular dispersed flow, the effects of the correlation of droplet deposition and entrainment rates on dryout heat flux were found to be predominant. It is therefore, an effort to produce some appropriate set of correlation of droplet deposition and entrainment rates must be carried out further for more generalization especially for wide range of flow condition, type of fluid and applications.

\section{REFERENCES}

[1] P. Saha and N. Zuber, $5^{\text {th }}$ International Heat Transfer Conference, Tokyo, Volume 4, pp 174(1974)

[2] E.L.Bibeau and M. Salcudean, Subcooled void growth mechanism and prediction at low pressure and low velocity, International Journal of Multiphase Flow, Vol.20, no.5, pp 837-\&63, 1994

[3] G.P.Celata et al., Burnout in highly subcooled water flow boiling in small diameter tubes, International Journal of Heat Transfer, Vol.36, no.6, pp 1269-1285,1993

[4] Y. Katto, A prediction model of subcooled water flow boiling CHF for pressure in the range 0.l-20MPa, International Journal of Heat Transfer, Vol.35, no.5, pp 115-1123, 1992

[5] Goon-Cherl Park and Byong-Jo Jun, Experimental study of interfacial area concentration in a boiling loop, Nuclear Engineering Design, Vol.456, 1995

[6] R. Dowlati et al., Two-phase cross flow and boiling heat transfer in horizontal tubes, Transaction of ASME, Vol.118, pp 124-131, February 1996

[7] M. Z. Zainon, Master of Engineering Thesis, Kyoto University, 1997

[8] A.W. Bennett et al., Studies on burnout in boiling heat transfer to water in round tubes with none-uniform heating, $A E E W-R, 5076,1996$

[9] I. Kataoka and M, Ishii, Mechanism and correlation of droplet entrainment and deposition in annular two-phase flow, Argonne National Laboratory Report, ANL-82-44, 1982

[10] An advance study on constitutive equations of three-fluid model, Japan Society of Multiphase Flow Report, 1994

[11] Sugawara, A study on flow behaviour and dryout phenomena in forced convective two-phase flow boiling, $\mathrm{Ph} D$ Thesis, Tokyo Institute of Technology, 1990

[12] L G. Alexander and C. L. Coldren, Droplet transfer from suspending air to duct wall, Industrial Engineering Chemistry, Vo1. 43, pp 1325,1951

[13] K. Goldman et al, Burnout in turbulence flow- A droplet deposition model Transition of ASME, , International Journal of Heat Transfer, Vol. 83, pp 159, 1961

[14] I. I. Paleev and B. S. Fillipovichm Phenomena of liquid transfer in two-phase annular dispersed flow. , International Journal of Heat and Mass Transfer, 9, pp

$$
\text { a. } 1089,1966
$$

[15] S. Namie and T. Ueda, Aerosol transport in annular two-phase flow. Transition of JSME, series B, Vol.38, pp2126, 1972

[16] M. Yanai, A study on heat conduction in a heated flow channel, $P h$. $D$ Thesis, Kyoto University, 1971

[17] P. Huchinson and P. B. Whalley, A possible characteristic of entrainment in annular flow, Chemical Engineering Science, Vol.28, 1973

[18] D. D. McCoy and T .J. Hanratty, Rates of deposition of droplets in annular two-phase flow, International Journal of Multiphase Flow, Vol. 3, pp 319,1977

[19] T. Saito et al, Multi-fluid modelling of annular two-phase flow, Nuclear Engineering Design, 50, 1978

[20] P. Andreussi and B.J.Azzopardim, Droplet deposition and interchange in annular gas-liquid flow, International Journal of Multiphase Flow, Vol.9, pp 697, 1983

[21] P.B Whalley and G. F. Hewitt, The correlation of liquid entrainment fraction and entrainment rate in annular two-phase flow, AERE-R, 9187. Harwell

$$
\text { a. Laboratory, } 1978
$$

[22] S. Ishigai and T. Takagi, A study on critical heat flux in flow bulling of a horizontal flow channel, Transition of JSME, series B, Vol.36, pp 629, 1970

[23] T. Ueda, Droplet diameter and entrainment rate of the annular gasliquid two-phase flow, Transition of JSME, series B, Vol. 45, pp 127, 1979 
[24] J.Wurtz, An experimental and theoretical investigation of annular steam-water flow in tubes annuli at 30-90 bar, RISO Report, no. 372, 1978

[25] J. C. Dallman and T. J Hanratty, Interpretation of entrainment measurement in annular gas-liquid flow, Momentum, Heat and Mass Transfer in Chemical Process and Energy Engineering System, Volume II, Hemisphere McGraw Hill, Washington, pp 681, 1979

\section{APPENDIX}

\section{Droplet Deposition Coefficient Correlations}

- Sugawara's equation [11] $\frac{k}{u_{g}}=0.009 \mathrm{Re}_{g}^{-0.2}\left(\frac{C}{\rho_{g}}\right)^{-0.5} \operatorname{Pr}_{g}^{-2 / 3}$

- Alexander \& Coldren's equation [12]

$$
k=0.0041 u_{g}^{1.17} \quad \text { at } u_{g}: 24 \mathrm{~m} / \mathrm{s} \sim 91 \mathrm{~m} / \mathrm{s}
$$

- Goldman's equation [13] $\frac{k}{u_{g}}=0.0023 \operatorname{Re}_{g}^{-0.2}$

- Paleev's equation [14].

$$
\frac{k}{u_{g}}=0.022 \operatorname{Re}_{g}^{-0.25}\left(\frac{C}{\rho_{f}}\right)^{-0.26}\left(\frac{\rho_{f}}{\rho_{g}}\right)^{0.26} \quad C=\frac{G_{e}}{u_{g}},
$$

- Namie’s equation [15] $\frac{k}{u_{g}}=\operatorname{Re}_{g}^{-0.3} f(a) 10^{-0.012 G_{e}}$

$f(a)$ : a theoretical by derived function of droplet diameter in turbulent flow.

- Yanai's equation [16].

$$
\frac{k}{u_{g}}=\operatorname{Re}_{g}^{-0.5}
$$

- Harwell's equation [17] $\frac{k}{u_{c}^{\bullet}}=87\left(\frac{\mu_{f}^{2}}{D \sigma \rho_{f}}\right)^{0.5}$

- McCoy \& Hanratty’s equation [18].

$$
\frac{k}{u_{f}}=3.25 \times 10^{-4} \tau^{+^{2}} \quad\left(\tau^{+^{2}}<22.9\right)
$$

- Saito's equation [19].

$k=\left(1.698 \times 10^{2}\right)-\left(2.117 \times 10^{-4} \mathrm{C}\right)+\left(2.896 \times 10^{-4} \mathrm{C}^{2}\right)-\left(2.192 \times 10^{-8} \mathrm{C}^{3}\right)$ $+\left(7.778 \times 10^{-11} C^{4}\right)-\left(1.027 \times 10^{-13} C^{5}\right)$

- Andreussi's equation [20].

$$
\frac{k}{u_{f}}=\frac{0.115}{1+2.3\left(\frac{C}{\rho_{g}}\right)}
$$

- Whalley \& Hewitt's equation [21].

$$
\begin{array}{rlrl}
k & =0.405 \sigma^{0.915} & \sigma<0.0383 \\
k & =9.48 \times 10^{4} \sigma^{4.7} & \sigma & >0.0383
\end{array}
$$

\section{Droplet Entrainment Rate Correlations}

- Yanai's equation [16]. $m_{e}=3.5 \times 10^{-5}\left(\frac{\tau_{i}}{\sigma}\right)^{3} \sqrt{\frac{\tau_{i}}{\sigma_{f}}} \Delta h^{2} \rho_{f}$

- Ishigai's equation [22]

$m_{e}=0.084 \frac{\lambda_{g}}{8}\left(\frac{u_{f} \mu_{f}}{\sigma}\right) \rho_{g} u_{g} \delta^{+} \Phi_{g}^{2}$

- Ueda's equation ${ }^{[23]} m_{e}=3.54 \times 10^{-3}\left\{\frac{\tau_{i}}{\sigma}\left(\frac{u_{l}}{\sigma}\right)^{0.6}\right\}^{0.57}$

- Whalley's equation [17]. $m_{e}=k C_{e} \quad k=0.405 \sigma^{0.915}$

- Kataoka and Ishii’s equation [9].

$\frac{m_{e} D}{\mu_{f}}=1.2 \times 10^{3} \operatorname{Re}_{f}^{-0.5} \operatorname{Re}_{f l}^{-0.5} W e^{-1.5}\left(\operatorname{Re}_{f l}-\operatorname{Re}_{f l o o}\right)^{2}$

$+6.6 \times 10^{-7} \operatorname{Re}_{f}^{-0.74} \operatorname{Re}_{f l}^{0.185} W e^{0.925}\left(\frac{\mu_{g}}{\mu_{f}}\right)^{0.26} \quad\left(\mathrm{E} \leq E_{\infty}\right)$

where, $E_{\infty}=\tanh \left(7.25 \times 10^{-7} W e^{1.25} \operatorname{Re}_{f}^{0.25}\right)$

- Wurtz's equation [24] $m_{e}=2\left(\frac{k_{s} \tau_{i}}{\sigma}\right)\left(\frac{u_{f} \mu_{f}}{\sigma}\right)$

- Sugawara's equation [11]

$m_{e}=1.07\left(\frac{\Delta h_{e q} \tau_{i}}{\sigma}\right)\left(\frac{u_{f} \mu_{f}}{\sigma}\right)\left(\frac{\rho_{f}}{\rho_{g}}\right)^{0.4}$

- Saito's equation [19]

$m_{e}=0.001 \rho_{f}\left[1-\exp \left\{-4605\left(\frac{\tau_{i} \delta}{\sigma}-10\right)\right\}\right]\left(\frac{\tau_{i} \delta}{\sigma}\right) \geq 10$

- Dallman's equation [25] $m_{e}=k_{A} \sqrt{\rho_{g} \rho_{g} u_{g}^{2}} \frac{m_{f l}-m_{f l c}}{\pi D}$

Nomenclatures

Re : Renolds number We : Webber number

$\tau$ : Shear stress $\quad \delta$ : Liquid film thickness

$\lambda_{g}:$ Friction factor $\quad \sigma$ : Surface tension

$\mu$ : Viscosity $\quad \rho:$ Density

$m$ : mass flux $\quad C$ : Mass density of droplet 


\title{
Performance Evaluation of Constant Frequency Torque Controller-Based and SVM-Based of Direct Torque Control of Induction Motor Drives
}

\author{
M. Zarafi, N. R. N. Idris, Z. H. Ismail, S. Aizam and H. Rohaiza
}

\begin{abstract}
Direct torque control is a control technique used in $\mathrm{AC}$ drive system to obtain high performance torque control. The conventional DTC drive contains a pair of hysteresis comparators, a flux and torque estimator and voltage look-up table. The torque and flux are controlled simultaneously by applying suitable voltage vectors, and limiting these quantities within their hytaresis band. At the same time, de-coupled control of torque and flux were achieved. However, the DTC drives utilizing hysteresis comparators suffer from high torque ripple and variable switching frequency. The most common solution to this problem is to use the space vector based which depends on the reference torque and flux. The reference voltage vector is then realized using a voltage space vector modulator (SVM). Nevertheless, by applying this method, the basic structure of DTC is lost and needs for high performance of processor. On the other hand, constant frequency torque controller also has managed to solve the problem. The proposed controller has retained the basic structure of DTC drive as in hysteresis-based. This paper present the comparison and evaluation of the performances of those techniques of DTC drive that applied to induction machine through simulation using MATLAB/SIMULINK. The evaluation was made based on the drive performances, which include dynamic torque, feasibility, and the complexity of the system. The results obtained showed that the constant frequency torque controllerbased gives better performance in terms of dynamic torque and at the same time retain the simple structure of DTC drive system.
\end{abstract}

Keywords: induction motor, space vector modulation (SVM), constant switching, direct torque control (DTC).

\section{INTRODUCTION}

Direct torque control (DTC) of induction motor drives becomes popular and widely used in industrial applications due to a fast and good dynamic torque response as well as

M. Zarafi, S. Aizam and H. Rohaiza are with the Depatment of Electrical Power Engineering, Faculty of Electrical and Electronics Engineering, Universiti Tun Hussein Onn Malaysia, 86400 Pt. Raja Bt. Pahat, Johore Malaysia. (phone: +607-4537698; e-mail: zarafi@uthm.edu.my; aizam@uthm.edu.my and rohaiza@uthm.edu.my).

N. R. N. Idris and Z. H. Ismail are with the Department of Energy Conversion, Faculty of Electrical Engineering, Universiti Teknologi Malaysia, 81310 UTM Skudai, Johore, Malaysia.(e-mail: nikrumzi@ieee.org and zool@fke.utm.my). provides a simple control structure. Since it was introduced in the middle of 1980's [1], [2] many researchers have been working in this area and several modifications and improvements have been made in order to overcome the two major disadvantages of the hysteresis-based of DTC scheme, namely the high torque ripple and variable switching frequency of the inverter. Previous proposed techniques to overcome these problems include the use of variable hysteresis band, controlled duty cycle technique and use of space vector modulation (DTC-SVM) based. All these techniques have managed to improve the performance of DTC, in the expense of loosing the simple structure of DTC. In [3]-[5], a simple approach to solve the problems and at the same time retaining the simple structure of DTC was introduced. In this approach, a constant frequency torque controller was used to replace the hysteresis torque controller.

For DTC-SVM based, in order to reduce the torque ripple and to fix the switching frequency, the control system should be able to generate a desired voltage vector by means of space vector modulator. Therefore, using this technique it is require for more complex control structure and present motor parameters dependence. The increased number of voltage vectors allows the definition of more accurate switching tables in which the selection of the voltage vectors is operated according to the rotor speed, the flux error and the torque error. Due to all of these requirements and to obtain a small torque ripple, the DTC-SVM drive is requiring for fast processor.

In the case of constant frequency torque controller based, the method used in obtaining a constant switching frequency and reducing the torque ripple is much simple. It is accomplished by introducing a new torque controller to replace the torque hysteresis-based controller. This means that the simple structure of DTC as initially proposed by I. Takahashi is retained.

This paper investigates the performance of the direct torque control of space vector modulation-based and constant frequency torque controller-based. The evaluation is made on the performance of dynamic torque response, torque ripple and the structure of both schemes. The rest of 
the paper is organized as follows. Section II presents the basic concepts of DTC drives. Section III describes the principles of SVM-based and constant frequency torque controller-based of DTC. Section IV discusses the simulation results of both schemes. Finally, conclusions are given in Section $\mathrm{V}$.

\section{BASIC CONCEPTS OF DTC}

AC motors are the most common motors used in industrial motion control system, as well as in main powered home appliances. Simple and rugged design, low cost, low maintenance and direct connection to an $\mathrm{AC}$ source are the main advantages of $\mathrm{AC}$ induction motors. Due to the advantages, it always has a competitor in the DCmotor or universal-motor. At present, induction motor drives are dominating the world market.

Since two decades ago, DTC of induction motor drives based on vector control have becomes popular and widely used due to its advantages. The first DTC scheme was introduced in 1980's as an alternative to field-oriented control (FOC) scheme. The configuration of DTC is very simple and gives fast and good dynamic torque response. If compared to the field-oriented control technique, in DTC scheme, there is no need for co-ordinate transformation, pulse-width modulator, and speed sensor. Nowadays, many applications especially in industrial have switched to this control technique in order to get better performances of the induction motor used.

In order to analyze the DTC in terms of its switching frequency and torque ripples, the induction motor equations written in general reference frame are used:

$$
\begin{aligned}
& \underset{v_{S}}{-g}=R_{S} i_{S}^{-g}+\frac{d \psi_{S}^{-g}}{d t}+j \omega_{g} \psi_{S}^{-g} \\
& 0=R_{r}{ }_{i r}^{-g}+\frac{d \psi_{r}^{-g}}{d t}+j\left(\omega_{g}-\omega_{r}\right) \psi_{r}^{-g}
\end{aligned}
$$

Where

$-g$
$\psi_{S}$ and $\psi_{r}^{-g}$ are the stator and rotor flux leakages respectively and given by

$$
\begin{aligned}
& -g \\
& \psi_{S}=L_{s} i_{S}^{-g}+L_{m} i_{r}^{-g} \\
& -g \\
& \psi_{r}=L_{r}{ }^{-g} i_{r}+L_{m}{ }^{-g} i_{s}
\end{aligned}
$$

In the above equations, $\bar{v}_{s}^{g}, \bar{i}_{s}^{g}$, and $\bar{i}_{r}^{g}$ are the stator voltage, stator current and rotor current space vectors respectively, whereas $L_{s}, L_{r}$, and $L_{m}$ are the stator self inductance, rotor self inductance and mutual self inductance, respectively. All of the equation are referred to the rotating general reference frame which represented by superscript "g". $\omega_{g}$ and $\omega_{r}$ are the speed of the general reference frame and rotor respectively.

In a symmetrical three phase induction motor, the instantaneous electromagnetic torque is proportional to the cross vectorial product of the stator flux linkage space vector and the stator current space vector,

$t_{e}=\frac{3}{2} p \bar{\psi}_{s} \times \bar{i}_{s}$

Where $\overline{\psi_{s}}$ and $\overline{i_{s}}$ is the vector of stator flux linkage and stator current respectively, while $p$ is the number of polepairs. Equation (5) also can be written in the following form:

$t_{e}=\frac{3}{2} P\left|\overline{\psi_{s}}\right|\left|\overline{i_{s}}\right| \sin \left(\alpha_{s}-\rho_{s}\right)$

Where $\alpha_{s}$ is the stator current angle and $\rho_{\mathrm{s}}$ is the stator flux angle space vector in the horizontal axis of the stationary frame as shown in 'figure 1'.

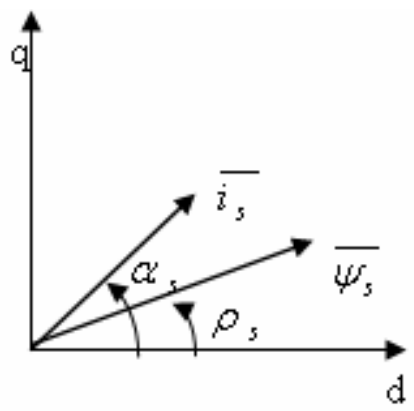

Figure 1. Stator flux leakage and stator current space vector

By manipulating equations (3) and (4), the electromagnetic torque in the stationary reference frame in equation (6) becomes:

$t_{e}=\frac{3}{2} P \frac{L_{m}}{L_{s} L_{r}} \bar{\psi}_{s} \times \overline{\psi_{r}}$

$$
\begin{aligned}
& =\frac{3}{2} P \frac{L_{m}}{L_{s} L_{r}} \psi_{s} \psi_{r} \sin \left(\rho_{s}-\rho_{r}\right) \\
& =\frac{3}{2} P \frac{L_{m}}{L_{s} L_{r}} \psi_{s} \psi_{r} \sin \delta_{s r}
\end{aligned}
$$

where $\rho_{r}$ is the angle of the rotor flux and $\delta_{s r}=\rho_{s}-\rho_{r}$ is the angle between the stator and rotor flux space vector with respect to the real axis of the stationary reference frame.

In DTC, rapid changes of the torque can be obtained by rotating the stator flux in the forward direction or by rotating in the reverse direction or by stopping it according to the demanded torque [6]. In other words, the direct torque control can be achieved by quickly changing the position of the stator flux space vector (change in $\delta_{s r}$ ). For simplicity, it is assumed that the stator resistance is small and can be neglected, thus the stator voltage equation can be expressed as below, 
$\frac{d \bar{\psi}_{s}}{d t}=\overline{v_{s}}$

or

$$
\Delta \bar{\psi}_{s}=\bar{v}_{s} \Delta t
$$

If the induction motor is supplied by three phase voltage source inverter (VSI), it consists of eight possible voltage switching space vectors $\left(v_{1} \ldots \ldots v_{8}\right)$ by means of six active switching vectors $\left(v_{1}\right.$ to $\left.v_{6}\right)$ and the rest is zero switching vectors $\left(v_{7}\right.$ and $\left.v_{8}\right)$. Figure 2 shows the corresponding of eight switching vectors and the voltage space vector, hence the dc link voltage $V_{d c}$ can be expressed as follows:

$\bar{v}_{s}(t)=\frac{2}{3} V_{d c}\left(v_{s A}(t)+a v_{s B}(t)+a^{2} v_{s C}(t)\right)$

where

$a=e^{j 2 \pi / 3}$

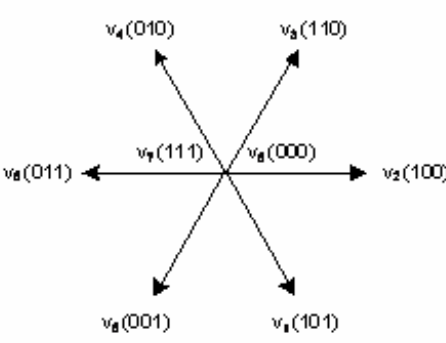

(a)

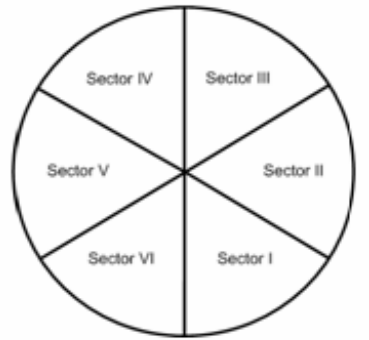

(b)
Figure 2. (a) Voltage vectors of three-phase VSI; (b) Six sectors of stator flux plane

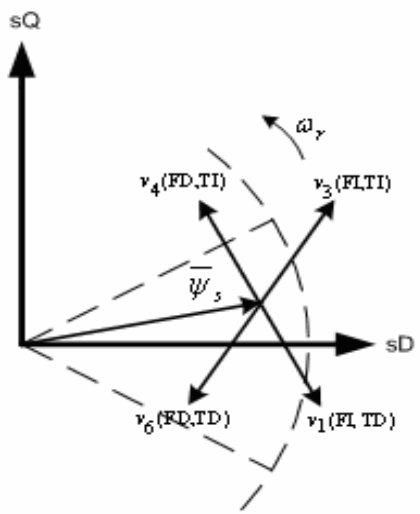

Figure 3. Position of stator flux space vector (FI: flux increase; FD: flux decrease; TI: torque increase; TD: torque decrease)

From equation (11), the stator flux vector can be moved with small change in time. Hence, the stator flux can be controlled and forced to follow the reference flux given by selecting the appropriate switching of voltage vectors. In order to select the appropriate voltage vectors of the inverter, (either to increase or reduce stator flux and either to increase torque or to reduce torque) it is depends on the position of stator flux vector on the stator flux plane as shown in 'figure 2(b)'. Let the stator flux is in sector II and rotates in counter-clockwise direction as illustrated in 'figure 3'. If an increase in the electromagnetic torque is required, the stator flux can be increased and decreased by selecting voltage vector $v_{3}$ and $v_{4}$ respectively. Whereas, if decrease torque is required (stator flux moving in clockwise direction), switching vector $v_{l}$ and $v_{6}$ will cause the stator flux to increase and decease respectively. Stopping the rotation of the stator flux corresponds to the case when the electromagnetic torque does not have to be changed. This condition is defined when the reference value of the torque is equal to its actual value and to stop the rotation, zero switching vector $\left(v_{7}\right.$ or $\left.v_{8}\right)$ is applied.

According to the stator flux plane, a set of switching voltage vectors or rules of which voltage vector should be chosen in a particular sector can be constructed as illustrated in 'table 1 '.

Table 1. Voltage vectors selection table

\begin{tabular}{|c|l|c|c|c|c|c|c|}
\hline \multicolumn{2}{|c|}{ Counter clockwise } & Sec I & Sec II & Sec III & Sec IV & Sec V & Sec VI \\
\hline \multirow{2}{*}{$\begin{array}{c}\text { Inc } \\
\text { Flux }\end{array}$} & Inc Toque & 100 & 110 & 010 & 011 & 001 & 101 \\
\cline { 2 - 8 } & Dec Toque & 000 & 111 & 000 & 111 & 000 & 111 \\
\hline \multirow{2}{*}{$\begin{array}{c}\text { Dec } \\
\text { Flux }\end{array}$} & Inc Toque & 110 & 010 & 011 & 001 & 101 & 100 \\
\cline { 2 - 8 } & Dec Toque & 111 & 000 & 111 & 000 & 111 & 000 \\
\hline \multicolumn{2}{|c|}{ Clockwise } & Sec I & Sec II & Sec III & Sec IV & Sec V & Sec VI \\
\hline \multirow{2}{*}{\begin{tabular}{c|l|c|c|c|} 
Inc \\
Flux
\end{tabular}} & Inc Toque & 001 & 101 & 100 & 110 & 010 & 011 \\
\cline { 2 - 8 } & Dec Toque & 000 & 111 & 000 & 111 & 000 & 111 \\
\hline \multirow{2}{*}{$\begin{array}{c}\text { Dec } \\
\text { Flux }\end{array}$} & Inc Toque & 011 & 001 & 101 & 100 & 110 & 010 \\
\cline { 2 - 8 } & Dec Toque & 111 & 000 & 111 & 000 & 111 & 000 \\
\hline
\end{tabular}

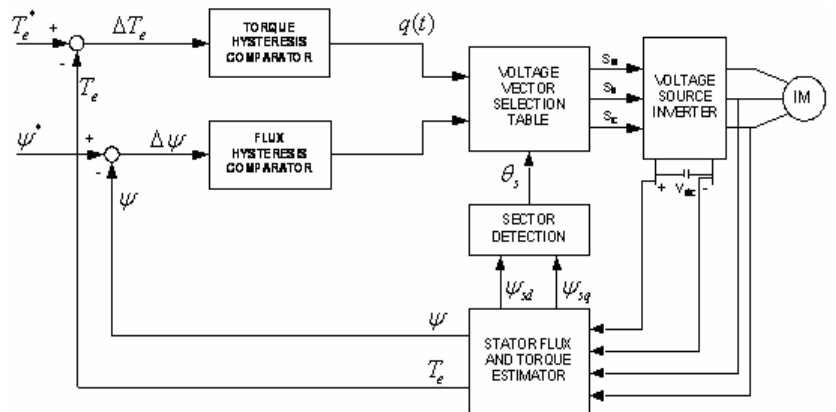

Figure 4. Basic configuration of DTC (hysteresis-based)

The basic system configuration of DTC scheme is depicted in 'figure 4'. The reference values of torque and flux are compared with the measured value of torque and stator flux respectively, which are calculated in the estimator block. The error values from the comparison process are fed into three-level and two-level hysteresis comparator respectively. According to the hysteresis comparators, it is used to restrict the flux and torque errors within the hysteresis band in order to obtain a good torque response. The restricted values in digital signal are used to select the voltage vectors of the inverter listed in Table 1 . It is also depends on the position of stator flux on its stator flux plane as illustrated in 'figure 2(b)'. 


\section{PRINCIPLes Of DTC-SVM Based AND Constant FREQUENCY TORQUe CONTROLler BASED}

\section{A. DTC-SVM Based}

There are various types of direct torque control-space vector modulation schemes that have been proposed by many researchers. Each scheme will perform the different control technique but its aims are still similar which is to attain the constant switching frequency and to reduce the torque ripple. The differences between various DTC-SVM are on how the reference voltage is generated - the reference voltage is then implemented using space vector modulator. Space vector modulation is used to define the inverter switching state or voltage vector positions different from six standard positions.

In this paper, the focus in only on the DTC-SVM scheme with closed-loop torque control. For this technique, it is based on load angle control strategy and significantly overcomes the most important drawbacks of conventional DTC hysteresis-based [7], [8]. A single space vector that represent the usual space vector transformation apply to a three-phase voltage system is define as

$v_{s}=\frac{3}{2}\left(v_{a}+a v_{b}+a^{2} v_{c}\right)$

where $a=-\frac{1}{2}+j \frac{\sqrt{3}}{2}$ and it is possible to obtain a simple equation set that describe the $\mathrm{AC}$ machine dynamic behavior in a stator fixed coordinate system as in equation (1) - (4). As stated in equation (9), it is representing the basic relation between torque and machine fluxes. Based on equation (7), it is possible to achieve machine speed and torque control directly by actuating over the load angle.

Due to slow rotor flux dynamics, the easiest way to change the load angle is to force a change in the stator flux by application of the appropriate stator voltage vector, $v_{s}[9]$. By assuming the voltage drop on the stator resistance in equation (1) is small which can be neglected, the stator flux vector is the time integral of the stator voltage vector.

In DTC-SVM with closed-loop torque control, the aim is to select the exact stator voltage vector, $v_{s}$ that change $\psi_{s}$ to meet the load angle reference, and the desired torque while keeping the flux amplitude constant. A space vector modulation algorithm is used to apply the required stator voltage vector. It is expected that torque ripple is almost eliminated. By additional of proportional-integral (PI) regulator, a simple flux calculation block is applied and at the same time departs from the rotating coordinate transformation. This strategy is straightforward by using of equation (7) [10]. The block diagram of DTC-SVM based is illustrated in 'figure 5'. Even this technique has managed to overcome the two main drawback of conventional DTC, it is leads to increase the complexity of DTC configuration and needs for fast processor to be implemented in digital system.

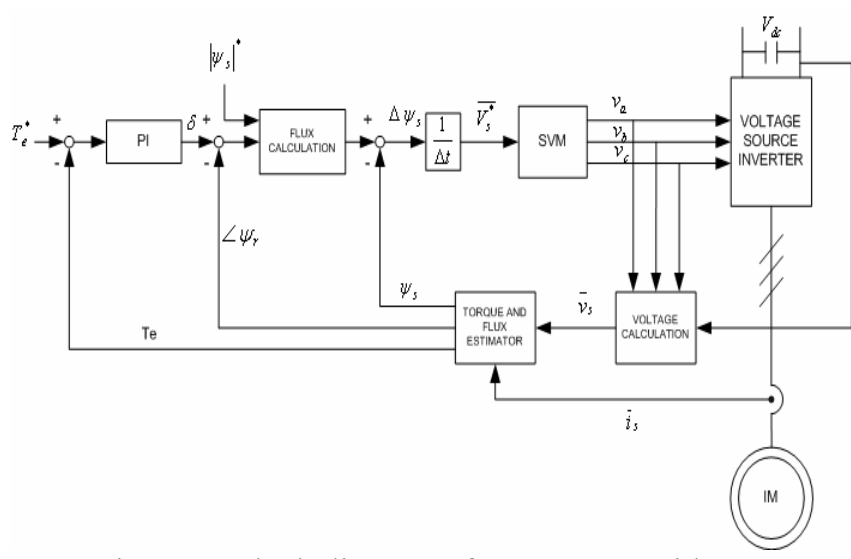

Figure 5. Block diagram of DTC-SVM with torque controller

\section{B. Constant Frequency Torque Controller-Based of DTC}

A constant frequency torque controller for DTC of an induction motor drive has been proposed as in reference [3][5] to overcome the same problem described previously. The controller is capable of producing constant switching frequency in the drive operating condition and at the same time reduced for high torque ripples. In advance, the implementation of the controller is simple and the basic drive control structure is retained as in hysteresis DTC drive. A simple structure of torque controller has been introduced to replace the conventional three-level torque hysteresis comparator that used in conventional DTC scheme. A constant switching frequency is obtained by comparing a fixed frequency of triangular carrier signals with the compensated torque error signals from the PI controller. 'Figure 6' shows the structure of the constant frequency torque controller used in this strategy.

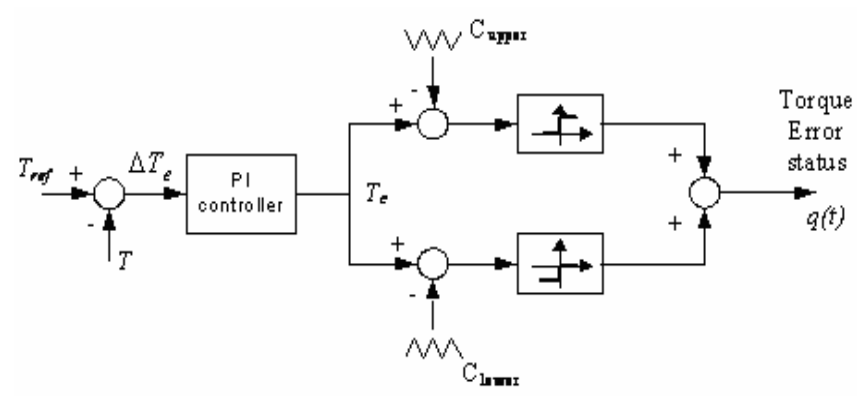

Figure 6. The structure of the constant frequency torque controller

The controller consists of two triangular waveform generators, two comparators and a PI controller as shown in 'figure 6'. The two triangular waveforms $\mathrm{C}_{\text {upper }}$ and $\mathrm{C}_{\text {lower, }}$, are $180^{\circ}$ out of phase from each other. The working principle of the controller is similar to the three-level torque hysteresis comparator as in conventional DTC [1]. The output of the controller can be either of three level which is 1,0 or -1 . The designated value of the instantaneous output of the controller and the averaged triangular period is given by equation (14) and (15) respectively. The overall configuration of DTC utilizing a constant frequency torque controller is shown in 'figure 7'. 


$$
\begin{aligned}
& q(t)= \begin{cases}1 & ; \text { for } \mathrm{T}_{\mathrm{c}} \geq \mathrm{C}_{\text {upper }} \\
0 & ; \text { for } \mathrm{C}_{\text {lower }}<\mathrm{T}_{\mathrm{c}}<\mathrm{C}_{\text {upper }} \\
-1 & ; \text { for } \mathrm{T}_{\mathrm{c}} \leq \mathrm{C}_{\text {lower }}\end{cases} \\
& d(t)=\frac{1}{T_{t r i}} \int_{t}^{t+T_{\text {tri }}} q(t) d t
\end{aligned}
$$

The dynamics of the motor is modeled by the following

$$
J \frac{d \omega_{m}}{d t}=J \frac{2}{p} \frac{d \omega_{r}}{d t}=T_{e}-T_{l o a d}
$$

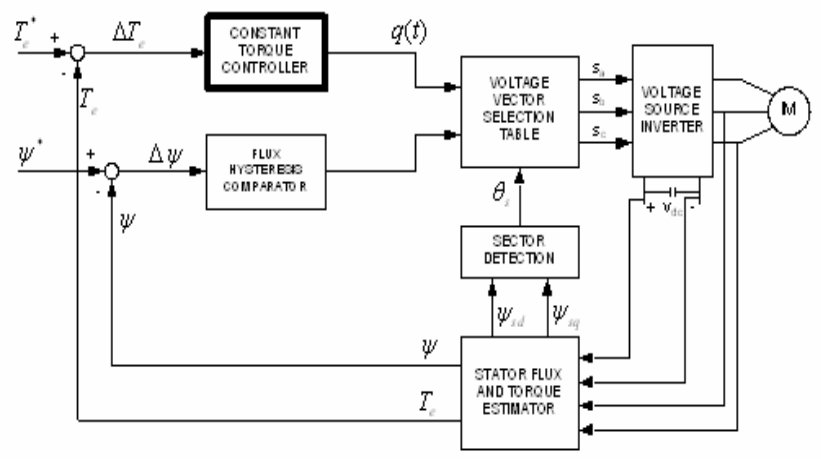

Figure 7. The configuration of constant frequency torque controller in DTC drive

Using (1) - (6) and, (16) in the stationary reference frame, the positive and negative torque slopes are given by

$$
\begin{aligned}
& \frac{d T_{e}^{+}}{d t}=-T_{e}\left(\frac{1}{\sigma \tau_{s}}+\frac{1}{\sigma \tau_{r}}\right)+\frac{3}{2} \frac{p}{2} \frac{L_{m}}{\sigma L_{s} L_{r}}\left(\begin{array}{l}
- \\
v_{s}-j \omega_{r} \bar{\psi}_{s}
\end{array}\right) \cdot j \psi_{r} \\
& \frac{d T_{e}^{-}}{d t}=-T_{e}\left(\frac{1}{\sigma \tau_{s}}+\frac{1}{\sigma \tau_{r}}\right)-\frac{3}{2} \frac{p}{2} \frac{L_{m}}{\sigma L_{s} L_{r}} j \omega_{r} \bar{\psi}_{s} \bullet j \psi_{r}
\end{aligned}
$$

where $\sigma$ is the total flux leakage factor, and $\tau_{s}$ and $\tau_{r}$ are the stator and rotor time constant respectively.

These are simplified by assuming that the stator and rotor fluxes are constant and the q components of the selected voltage vectors are zero, which is the voltage vectors are tangential to the circular stator flux locus. The average and simplified torque loop equation is written as

$$
\frac{d T_{e}}{d t}=-A_{t} T_{e}+B_{t} v_{s}^{\psi s} d+K_{t}\left(\omega_{s l i p}\right)
$$

Where

$$
A_{t}=\frac{1}{\sigma \tau_{s r}} \quad B_{t}=\frac{3 p}{4} \frac{L_{m}}{\sigma L_{s} L_{r}} \psi_{s} \quad K_{t}=\frac{3 p}{4} \frac{L_{m}}{\sigma L_{s} L_{r}}\left(\psi{ }_{s}{ }_{r}{ }^{\psi s}\right)
$$

Finally, the torque equation in s-domain can be written as
$T_{e}(s)=\frac{B_{t} v_{s}^{\psi s} d(s)+K_{t} \omega_{s l i p}(s)}{s+A_{t}}$

According to (20) and linearized it, the torque loop as shown in 'figure 8' can be constructed.

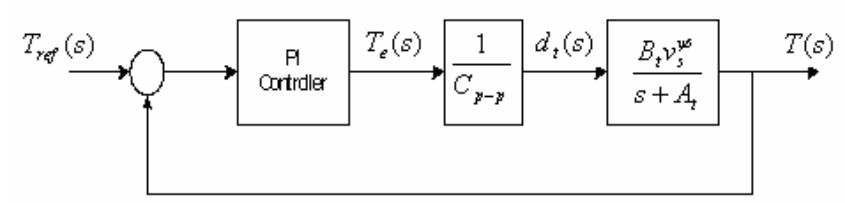

Figure 8 Linearized torque loop.

\section{Simulation Results}

The simulation of DTC-SVM based and constant frequency torque controller based of DTC induction motor drive was performed using MATLAB/SIMULINK simulation package. The parameters of induction machine as tabulated in 'table 1' were used in the simulations.

Table 2: Induction machine parameters.

\begin{tabular}{|ll|ll|}
\hline $\begin{array}{l}\text { Stator } \\
\text { resistance } \\
\text { Rotor reistance }\end{array}$ & $5.51 \Omega$ & $\begin{array}{l}\text { Moment of } \\
\text { inertia } \\
\text { Number of } \\
\text { poles }\end{array}$ & $0.01 \mathrm{kgm}^{2}$ \\
$\begin{array}{l}\text { Stator self } \\
\text { inductance }\end{array}$ & $306.5 \mathrm{mH}$ & Rated speed & $1410 \mathrm{rpm}$ \\
$\begin{array}{l}\text { Rotor self } \\
\text { inductance } \\
\begin{array}{l}\text { Mutual } \\
\text { inductance }\end{array}\end{array}$ & $2906.5 \mathrm{mH}$ & $\mathrm{Vdc}$ & $654 \mathrm{~V}$ \\
\hline
\end{tabular}

For constant frequency torque controller based, the frequency of upper and lower triangular waveforms employed for constant torque controller is set at $20 \mathrm{kHz}$ with a peak-to-peak of 100 units. While the gain $K p$ and $K i$ of PI controller are set to 57 and 3630 respectively [5]. The magnitude of stator flux reference is set at its rated value which is $1.2 \mathrm{~Wb}$ for both schemes and the stator flux is restricted within its hysteresis band of $0.05 \mathrm{~Wb}$.

The results from the simulation show that the stator flux locus trajectoriesis circular when the voltage vectors switched between two active and zero vectors as depicted in 'figure 9'. 'Figure 10' shows the torque response of both scheme with $\pm 4 \mathrm{Nm}$ square-wave torque reference. Both scheme have manage in reducing the torque ripples as discuss in [3]-[5] and [7]-[10], the simulation results of torque ripple demonstrate that DTC-SVM based and constant frequency torque controller based have almost same performance $(\Delta \mathrm{T} \approx 0.15 \mathrm{Nm})$. However, in terms of dynamic torque response, DTC-SVM based has slow dynamic compared to the constant frequency torque controller based. 'Figure 11' shows the constant switching of both DTC schemes obtained from the simulation. For DTC-SVM, the switching signal is determined by mathematical equation whereas for constant frequency torque controller based is determined by comparing the triangular waveform with the output of PI controller. 


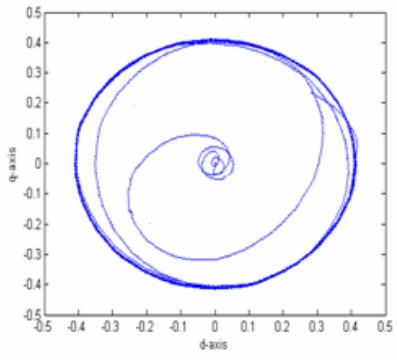

(a)

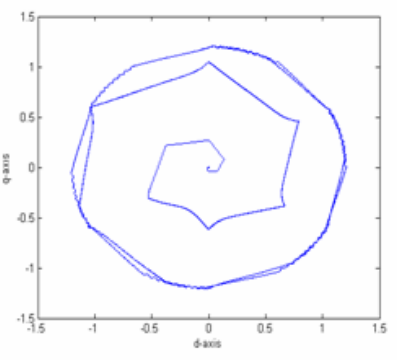

(b)
Figure 9 Flux locus. (a) DTC-SVM based, (b) Constant frequency torque controller based $(\Delta \psi=0.03 \mathrm{~Wb})$.

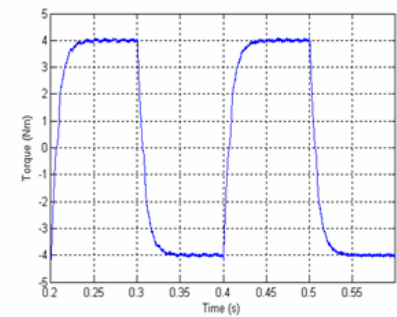

(a)

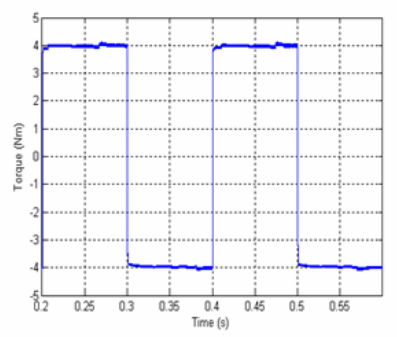

(b)
Figure 10 Torque response. (a) DTC-SVM based, (b) Constant frequency torque controller based.

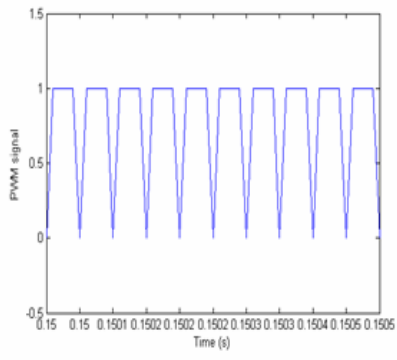

(a)

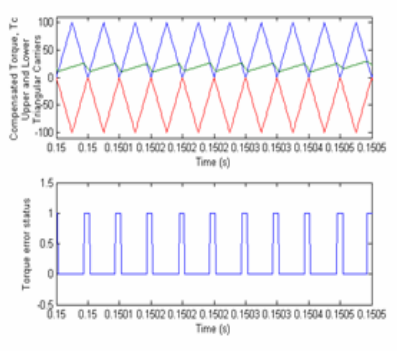

(b)
Figure 11. Switching signal. (a) DTC-SVM based, (b) Constant frequency torque controller based

\section{CONCLUSION}

This paper has presented two different approaches of DTC drives in order to fix the switching frequency and reduce high torque ripple addressed in conventional DTC scheme (hysteresis-based). From the simulation results obtained, the torque ripple of DTC-SVM with closed-loop torque control is much less than constant frequency torque controller-based. However, the dynamic torque response during transient condition is slower than constant frequency torque controller-based to achieve the steady-state condition. In the case of constant frequency torque controller-based, the structure of DTC drive is simple and retain as conventional DTC (hysteresis-based) but not for DTC-SVM. Even though, both scheme has manage to fix the switching frequency.

\section{REFERENCES}

[1] I. Takahashi and T. Noguchi, "A new quick-response and highefficiency control strategy of an induction motor", IEEE Trans. Ind. Appl. Vol. IA-22, No. 5, pp. 820-827. Sept. /Oct. 1986.

[2] M. Depenbrock, "Direct Self Control of inverter-fed of induction machine", IEEE Trans. Power Electro., vol3, pp. 420-429. Oct. 1998.
[3] N. N. R. Idris and A. H. M. Yatim, "Reduced torque ripple and constant torque switching frequency strategy for direct torque control of induction machine" $15^{\text {th }}$ IEEE-APEC 2000, pp. 154-161.

[4] N. R. N. Idris; A. H. M. Yatim; N. A. Azli, "Direct torque control of induction machines with constant switching frequency and improved stator flux estimation"; 27th Annual Conference of the IEEE ,Industrial Electronics Society, 2001. IECON '01. Vol. 2 , pp.1285 1291 Dec. 2001.

[5] N. R .N. Idris, A. H. M. Yatim, N. D. Muhamad and T. C. Ling, "Constant frequency torque and flux controllers for direct torque control of induction machines", Accepted for the $34^{\text {th }}$ IEEE Power Electronics Specialist Conference PESC03, Acapulco, Mexico June 2003.

[6] P. Vas, "Sensorless Vector and Direct Torque Control," Oxford, U.K, Oxford University Press, 1998.

[7] J. Rodriquez, Jorge Pontt, C Selva and H. Miranda, “A Novel Direct Torque Control Scheme for Induction Machines with Space Vector Modulation," IEEE Trans Power Electronic pp 1392-1397, 2004.

[8] Giuseppe S. Buja, and Marian P. Kazmierkowski, "Direct Torque Control of PWM Inverter-Fed AC Motors - A Survey," IEEE Trans On Ins Electronics, 50(4) 744-757 August 2004.

[9] T. G. Habetler, F. Profumo, M. Patorelli, and L. M. Tolbert, Direct Torque Control of Induction Motor using Space Vector Modulation," IEEE Trans. Ind. Application, vol. 28, pp. 1045-1053, Sept/Oct 1992.

[10] Domenico Casadei, Giovanni Serra, and Angelo Tani, "Implementation of a Direct Torque Control Algorithm for Induction Motors Based on Discrete Space Vector Modulation," IEEE Transaction on Power Electronics, vol. 15, no. 4, Julay 2000. 


\title{
Incompressible Finite Difference Double- Population Thermal Lattice Boltzmann Model
}

\author{
Nor A. C. Sidik
}

\begin{abstract}
In this paper, the lattice Boltzmann method (LBM), a numerical tool based on the particle distribution function is used to simulate thermal fluid flow problems. A well-known finite difference technique is combined with the double-population thermal lattice Boltzmann model to solve two-dimensional thermal fluid flow phenomena. A small number of macroscopic velocity components are applied for the calculation of temperature field. The simulations of natural convection in a square cavity were carried out in order to validate this approach. The combination of finite difference with double-population thermal lattice Boltzmann model is found to be an efficient and stable numerical approach for high Rayleigh number calculations.
\end{abstract}

Keywords: Lattice Boltzmann, thermal LBM, natural convection

\section{INTRODUCTION}

For more than a decade, lattice Boltzmann method (LBM) has been demonstrated to be a very effective numerical tool for a broad variety of complex fluid flow phenomena that are problematic for conventional method [1][2][3][4]. Compared with traditional computational fluid dynamics, LBM algorithms are much easier to be implemented especially in complex geometries and multicomponent flows [5].

Historically, LBM was derived from the lattice gas (LG) automata [6]. It utilizes the particle distribution function to describe collective behaviors of fluid molecules. The macroscopic quantities such as density and velocity are then obtained through moment integrations of the distribution function.

Although promising, the current LBM still have a few shortcomings that limit its general application as a practical computational fluid dynamics tool. One of these shortcomings, which is specifically addressed in this paper, is the low order accuracy of the current LBM model.

Generally, there are three types of thermal lattice Boltzmann models have been proposed; multi-speed model [7], passive scalar model [8] and double-distribution function (DDF) model [9]. Among these models, the DDF model is reported to be the most stable[10] and widely used in simulating thermal fluid flow problems[11][12][13].

This work was supported by Universiti Teknologi Malaysia.

Nor A. C. Sidik is with the Faculty of Mechanical Engineering, Department of Themofluid, Universiti Teknologi Malaysia, 81310 UTM Skudai, Johor, Malaysia( phone: 607-5534718; fax: 607-5566169; e-mail: azwadi@fkm.utm.my).
The DDF model, used in this research, is based on the work of He [9] and Luo [14]. This model introduces an internal energy density distribution function in order to simulate the temperature field. It has been shown that this model is simple and applicable to problems with different Prandtl numbers. More importantly, this model requires low order moment and thus provides higher numerical stability than the multi-speed and passive-scalar models.

The conventional thermal LBM, however, only gives second order accuracy in space and time. Since He and Luo [14],[15] and Abe [16] demonstrated that the lattice Boltzmann equation is a discretized form of the continuous Boltzmann equation and the discretization of physical space is not coupled with the discretization of momentum space, any standard numerical technique can serve the purpose of solving the discrete Boltzmann equation. It is not surprising that the well-known finite difference has being introduced in order to improve the accuracy of thermal LBM.

In this paper, the DDF thermal LBM is combined with the finite difference technique. Finite difference technique is applied to solve the advection term in the governing equations of DDF thermal LBM. This combination contributes in allowing us to increase the accuracy both in time and space. In order to verify the proposed model, a natural convection of air, rectangular enclosure with localized heating from below and symmetrical cooling from the sides is considered.

\section{DOUble Distribution FunCtion Thermal LBM}

The governing equation of the DDF thermal LBM are

$$
\begin{aligned}
& \frac{\partial f}{\partial t}+\mathbf{c} \frac{\partial f}{\partial \mathbf{x}}=\Omega_{f}+F_{f} \\
& \frac{\partial g}{\partial t}+\mathbf{c} \frac{\partial g}{\partial \mathbf{x}}=\Omega_{g}
\end{aligned}
$$

where the density distribution function $f=f(\boldsymbol{x}, \boldsymbol{c}, t)$ is used to simulate the density and velocity fields and the internal energy density distribution function $g=g(\boldsymbol{x}, \boldsymbol{c}, t)$ is used to simulate the macroscopic temperature field. $\mathbf{c}$ in (1) and (2) is the microscopic velocity, $\Omega$ is the collision term and $F_{f}$ is the external force. The original version of collision term was very complicated. One needs to apply the probability function in order to predict the direction of each particle distribution function after undergo the collision process at each node. In 1954, Bhatnagar-Gross-Krook [17] proposed a simplified version the collision function where part of the 
particle distribution function tends to the equilibrium condition after the collision process and another part of distribution function (non-equilibrium part) stream to the neighbor nodes. This idea was applied into the lattice Boltzmann collision model and known as the BGK collision model. The equation describe this collision process is given by

$\Omega=\frac{1}{\tau}\left(f^{e q}-f\right)$

where $\tau$ is time to reach equilibrium condition during collision process. However, the relaxation time of energy carried by the particles to its equilibrium is different to that of momentum. Therefore we need to use a different two relaxation times to characterize the momentum and energy

$$
\begin{aligned}
& \Omega_{f}=\frac{1}{\tau_{f}}\left(f^{e q}-f\right) \\
& \Omega_{g}=\frac{1}{\tau_{g}}\left(g^{e q}-g\right)
\end{aligned}
$$

Equations (1) and (2) can be rewritten as follow

$$
\begin{aligned}
& \frac{\partial f}{\partial t}+\mathbf{c} \frac{\partial f}{\partial \mathbf{x}}=\frac{1}{\tau_{f}}\left(f^{e q}-f\right)+F_{f} \\
& \frac{\partial g}{\partial t}+\mathbf{c} \frac{\partial g}{\partial \mathbf{x}}=\frac{1}{\tau_{g}}\left(g^{e q}-g\right)
\end{aligned}
$$

Equation (6) and (7) are known as the evolution equation of the density and internal energy density distribution function respectively.

The $f^{e q}$ and $g^{e q}$ in (6) and (7) are the equilibrium distribution function for density and temperature respectively defined as below.

$$
\begin{aligned}
f^{e q}= & \rho\left(\frac{1}{2 \pi R T}\right)^{D / 2} \exp \left(-\frac{\mathbf{c}^{2}}{2 R T}\right)\left[1+\left(\frac{\mathbf{c} \cdot \mathbf{u}}{R T}\right)+\right. \\
& \left.\frac{(\mathbf{c} \cdot \mathbf{u})^{2}}{2(R T)^{2}}-\frac{\mathbf{u}^{2}}{2 R T}\right] \\
g^{e q}= & T\left(\frac{1}{2 \pi R T}\right)^{D / 2} \exp \left(-\frac{\mathbf{c}^{2}}{2 R T}\right)\left[1+\left(\frac{\mathbf{c} \cdot \mathbf{u}}{R T}\right)\right]
\end{aligned}
$$

In order to apply the lattice Boltzmann scheme into the digital computer, the evolution equations of $(6 \sim 9)$ need to be discretised in the velocity space. Details of the discretization procedure can be found in Azwadi [11]. As a result, the expression for the discretised equilibrium density and internal energy density distribution function are

$$
f_{i}^{e q}=\rho \omega_{i}\left[1+3 \mathbf{c}_{i} \cdot \mathbf{u}+\frac{9}{2}\left(\mathbf{c}_{i} \cdot \mathbf{u}\right)^{2}-\frac{3}{2} \mathbf{u}^{2}\right]
$$

and

$$
g_{i}^{e q}=T \omega_{i}\left[1+3 \mathbf{c}_{i} \cdot \mathbf{u}\right]
$$

respectively. Whereas the discretised form of the evolution equation for both distribution functions are

$$
\begin{aligned}
& \frac{\partial f_{i}}{\partial t}+\mathbf{c}_{i} \frac{\partial f_{i}}{\partial \mathbf{x}}=\frac{1}{\tau_{f}}\left(f_{i}^{e q}-f_{i}\right)+F_{f} \\
& \frac{\partial g_{i}}{\partial t}+\mathbf{c}_{i} \frac{\partial g_{i}}{\partial \mathbf{x}}=\frac{1}{\tau_{g}}\left(g_{i}^{e q}-g_{i}\right)
\end{aligned}
$$

The value of $\omega$ in (10) and (11) depends on the direction of microscopic velocity, $\mathbf{c}_{\mathrm{i}}$ of particle distribution function. In this paper, the nine-microscopic-velocity model for density distribution and four-microscopic-velocity model for internal energy distribution function are chosen. The respective lattice geometries are shown in figure 1.
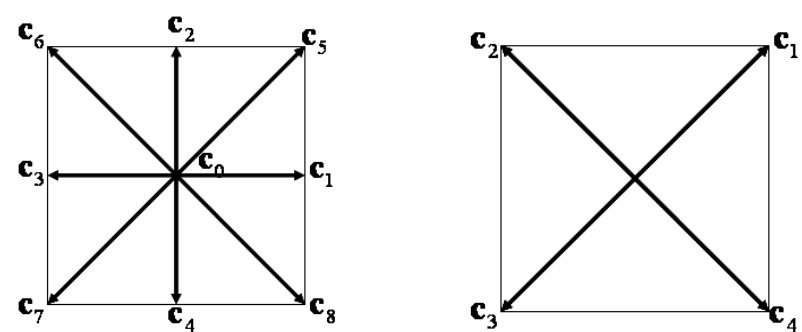

Figure 1. Nine-microscopic-velocity-model, $\omega_{0}=4 / 9, \omega_{1 \sim 4}=$ $1 / 9$ and $\omega_{5 \sim 8}=1 / 36$ (left); Four-microscopic velocity-model,

$$
\omega_{1 \sim 4}=1 / 4 \text { (right) }
$$

The macroscopic variables, such as the density $\rho$, velocity $\mathbf{u}$, and temperature $T$ can be evaluated as the moment to the distribution function

$\rho=\int f d \mathbf{c}, \rho \mathbf{u}=\int \mathbf{c} f d \mathbf{c}, T=\int g d \mathbf{c}$

The macroscopic equations, continuity, momentum and energy equations can be obtained from (12) and (13) using multi-scaling expansion (Chapmann-Enskog expansion). Detail derivation can be found in Luo [18] and Azwadi [19] and will not be shown here.

$\nabla \cdot \mathbf{u}=0$

$\frac{\partial \mathbf{u}}{\partial t}+\mathbf{u} \nabla \cdot \mathbf{u}=\frac{1}{\rho} \nabla p+v \nabla^{2} \mathbf{u}$

$\frac{\partial T}{\partial t}+\nabla \cdot(\mathbf{u} T)=\chi \nabla^{2} T$

The viscosity and thermal diffusivity in these models are related to the time relaxations as below

$v=\frac{\tau_{f}}{3}$

$\chi=\tau_{g}$

\section{FINITE DIFFERENCE DDF THERMAL LBM}

In the finite difference formulation of LBM, equations 
(12) and (13) are again discretized in phase space and time and solved using one of the several available finite difference numerical schemes. This provides us the opportunity to increase the spatial and temporal accuracy up to the desired level whereas only second order accuracy in space and time is available in the original LBM.

In this paper, the temporal discretization is obtained using second order Rungge-Kutta (modified) Euler method. The time evolution of particle distributions is then derived by

$$
\begin{aligned}
& f_{i}^{n+\frac{1}{2}}=f_{i}^{n}+\frac{\Delta t}{2}\left[-\mathbf{c}_{i} \cdot \nabla f_{i}^{n}+\frac{\left(f_{i}^{e q, n}-f_{i}^{n}\right)}{\tau_{f}}\right] \\
& f_{i}^{n+1}=f_{i}^{n}+\Delta t\left[-\mathbf{c}_{i} \cdot \nabla f_{i}^{n+\frac{1}{2}}+\frac{\left(f_{i}^{e q, n+\frac{1}{2}}-f_{i}^{n+\frac{1}{2}}\right)}{\tau_{f}}\right]
\end{aligned}
$$

The third order upwind scheme, was applied to calculate the advection term in (12) and (13). The same procedures were carried out for the evolution of temperature equation. From this combination, the accuracy of the finite difference DDF thermal LBM is second order in time and third order in space.

\section{NUMERICAL SIMULATION}

In the previous section, the combination of finite difference DDF thermal LBM was proposed as an alternative approach in simulating thermal fluid flow problem. In this section, the proposed model is applied to simulate natural convection in a square cavity with localized heating from below and symmetrical cooling from the sides. Symmetrical cooling from the sides is expected to be an efficient cooling option, while partial heating at the lower surface simulates the electronic components such as chips. The temperature difference between the partial bottom wall and other walls introduces a temperature gradient in a fluid, and the consequent density difference induces a fluid motion, that is, convection.

Figure 2 shows a schematic diagram of the setup in the simulation. No-slip boundary conditions [20] are imposed on all the faces of the square with height, $H$. The thermal conditions applied on the walls are depicted in figure 2 . The lower wall has a centrally located heat source, I $=H / 5$, which is assumed to be isothermally heated at a constant temperature, $T_{H}$.

The Boussinesq approximation is applied to the buoyancy force term.

$$
\mathbf{G}=\beta g\left(T-T_{m}\right) \mathbf{j}
$$

where $\beta$ is the thermal expansion coefficient, $g$ is the acceleration due to gravity, $T_{m}$ is the average temperature and $\mathbf{j}$ is the vertical direction opposite to that of gravity. So the external force in (12) is

$$
F_{f}=3 \mathbf{G}(\mathbf{c}-\mathbf{u}) f_{i}^{e q}
$$

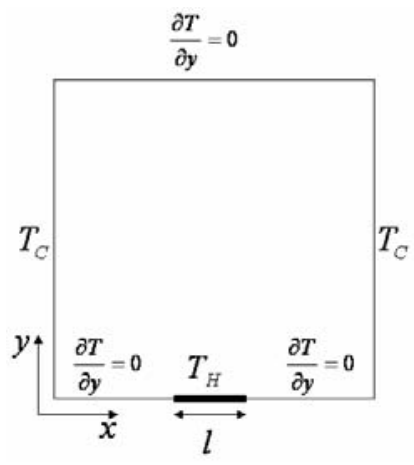

Figure 2. Geometry and boundary condition of the problem.

The dynamical similarity depends on two dimensionless parameters: the Prandtl number $P r$ and the Rayleigh number $R a$,

$\operatorname{Pr}=\frac{v}{\chi}$

$R a=\frac{g \beta \Delta T H^{3}}{v \chi}$

The characteristic speed $v_{c}=(g H T)^{1 / 2}$ is carefully chosen, so that the incompressible flow approximation holds. In all simulations, $\operatorname{Pr}$ is set to be 0.71 in order to simulate air cooling of electronic components. Through grid dependence study, the grid sizes of $101 \times 101,151 \times 151,201 \times 201$ and $251 \times 251$ for $R a=10^{3}, 10^{4}, 10^{5}$ and $10^{6}$ are found to be sufficient respectively.

The following criterion is employed to check for steady state solution

$\max \left|\left(\left(u^{2}+v^{2}\right)^{n+1}\right)^{\frac{1}{2}}-\left(\left(u^{2}+v^{2}\right)^{n}\right)^{\frac{1}{2}}\right| \leq 10^{-7}$

$\max \left|T^{n+1}-T^{n}\right| \leq 10^{-7}$

where the calculation is carried out over the entire system.

The main characteristic of natural convection flow are shown in terms of streamlines, isotherms, horizontal and vertical velocity components in figures $3 \sim 6$. For each case, 30 equally spaced contours have been used to define the corresponding structure.

Owing to the symmetrical boundary condition, the flow reaches an asymptotic steady state exhibiting a symmetric motion about the vertical centerline of the cavity for all values of $R a$ considered. The flow pattern is characterized by primary flows that rotate clockwise in the right half and counter-clockwise in the left half of the cavity as shown in figure 3. Owing to the symmetry, the flow in the left and right halves of the enclosure is identical except for the sense of rotation. For all cases or $R a$ considered, the hot fluid rises above the source until it reaches the top wall, then moves outwards along the horizontal wall before moving downwards along the sidewalls under the effect of cooling. 


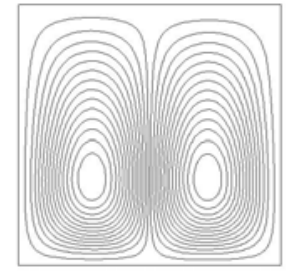

(a)

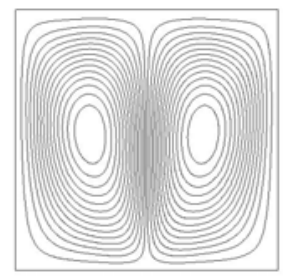

(c)

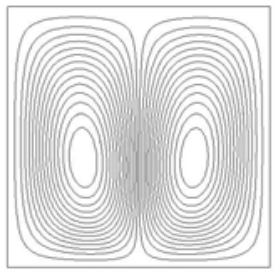

(b)

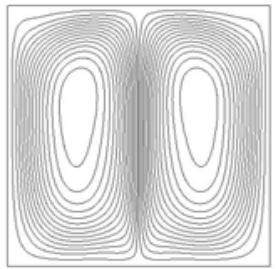

(d)

Figure 3. Streamlines for (a) $R a=10^{3}$, (b) $R a=10^{4}$, (c) $R a$ $=10^{5}$, and (d) $R a=10^{6}$

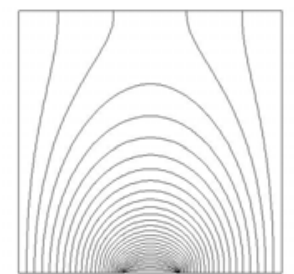

(a)

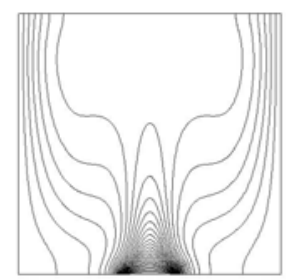

(c)

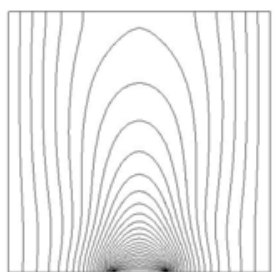

(b)

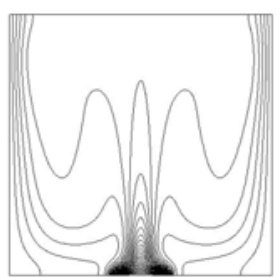

(d)
Figure 4. Isotherms for (a) $R a=10^{3}$, (b) $R a=10^{4}$, (c) $R a=$ $10^{5}$, and (d) $R a=10^{6}$

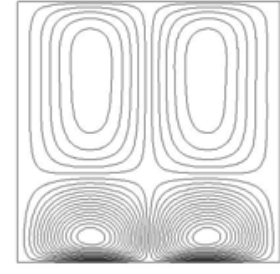

(a)

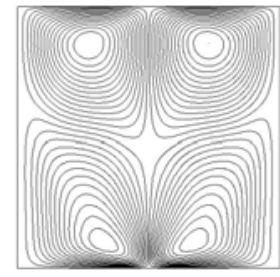

(c)

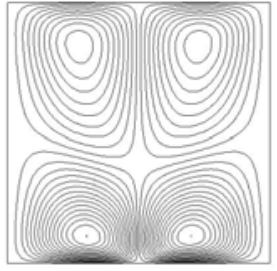

(b)

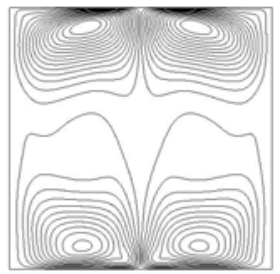

(d)
Figure 5. Horizontal velocity components for (a) $R a=10^{3}$, (b) $R a=10^{4}$, (c) $R a=10^{5}$, and (d) $R a=10^{6}$

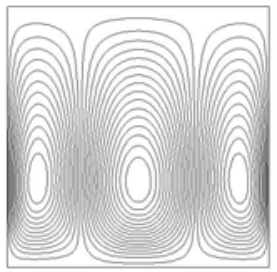

(a)

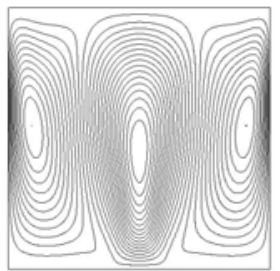

(c)

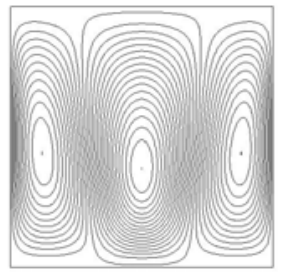

(b)

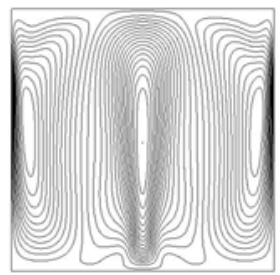

(d)
Figure 6 Vertical velocity component for (a) $R a=10^{3}$, (b) $R a=10^{4}$, (c) $R a=10^{5}$, and (d) $R a=10^{6}$

Figure 4 shows the isotherm lines for each case considered. At low Rayleigh number, $R a=10^{3}$, the isotherms deviate slightly from a diagonally symmetric structure indicates that the convection intensity is very weak and the main heat transfer mechanism is by conduction. As the Rayliegh number increased, $R a=10^{4}$, the intensity of the recirculation inside the cavity increase, and the cores of the cells move upwards. The effect of convection can also be seen from isotherms lines. At $R a=10^{5}$, the formation of thermal boundary layers can be observed, because of increased recirculation intensity. At $R a=10^{6}$, the thermal boundary layers become thinner causing increased energy transport and the isotherms become stratified due to high value of Rayleigh number.

\section{CONCLUSION}

In this paper, the natural convection in a square cavity with localized heating from below and symmetrical cooling from the sides has been studied using finite difference double-distribution function thermal lattice Boltzmann model. The evolution of lattice Boltzmann equation has been disrcetised using the third order accuracy finite difference upwind scheme. The flow pattern including vortices and thermal boundary layer can be seen clearly. The results obtained demonstrate that this approach is very efficient procedure to study flow and heat transfer in a differentially heated cavity flow.

\section{ACKNOWLEDGMENT}

The author wishes to thank Universiti Teknologi Malaysia and the Malaysian Government for supporting this research.

\section{REFERENCES}

[1] J. Bernsdorf, G. Brenner and F. Durst, "Numerical analysis of the pressure drop in porous media with lattice Boltzmann (BGK) automata," Comp. Physics, vol. 129, pp. 247-255, 2000.

[2] C. S. Nor Azwadi and T. Tanahasi, "Laminar flow over a backward facing step geometry for various expansion ratios with lattice Boltzmann method", in Proc. 11th Asean Congress in Fluid Mechanics, Kuala Lumpur, 2006, pp. 25-29.

[3] S. Chen, H. Chen, D. Martinez and W. H. Matthaeus, "Lattice Boltzmann model for simulation of magnetohydrodynamics," Phys. Rev. Letters, vol. 67, pp. 3776-3779, 1991. 
[4] C. S. Nor Azwadi and T. Tanahashi, "Simplified thermal lattice Boltzmann model in incompressible limit", in Proc. 11th Asean Congress in Fluid Mechanics, Kuala Lumpur, 2006, pp. 681-686.

[5] N. S. Martys and H. Chen, "Simulation of multicomponent fluid in complex three-dimensional geometries by the lattice Boltzmann method," Physics Rev. A, vol. 53, pp. 743-750, 1996.

[6] U. Frish, B. Hasslacher and Y. Pomeau, "Lattice gas automata for the Navier-Stokes equation," Physics Rev. Letters, vol. 56, pp. 1505$1508,1986$.

[7] G. McNamara and B. Alder, "Analysis of the lattice Boltzmann treatment of hydrodynamics," Physica A, vol. 194, pp. 218-228, 1993.

[8] X. Shan, "Simulation of Rayleigh-Bernard convection using lattice Boltzmann method," Physics Rev. E, vol. 55, pp. 2780-2788, 1997.

[9] X. He, S. Chen and G. D. Doolen, "A novel thermal lattice Boltzmann model for the lattice Boltzmann method in incompressible limit," $J$. Comp. Physics, vol. 146, pp. 282-300, 1998.

[10] Y. Peng, C. Shu and Y. T. Chew, "Simplified thermal lattice Boltzmann model for incompressible thermal flows," Physics Rev. E, vol. 68, pp. 026701-026708, 2003.

[11] C. S. Nor Azwadi and T. Tanahashi, "Simplified thermal lattice Boltzmann model incompressible limit," Intl. J. Modern Physics B, vol. 20, pp. 2437-2449, 2006.

[12] J. Onishi, Y. Chen and H. Ohashi, "Lattice Boltzmann simulation of natural convection in a square cavity," JSME Intl. J.Ser. B, vol. 44, pp. 53-62, 2001.

[13] C. Shu, Y. Peng and Y. T. Chew, "Simulation of natural convection in a square cavity by Taylor series expansion and least square based lattice Boltzmann method," Intl. J. Modern Physics C, vol. 13, pp. 1399-1414, 2002.

[14] X. He and L. S. Luo, "A priori derivation of the lattice Boltzmann equation,” Phys. Rev. E, vol. 55, pp. 6333-6336, 1997.

[15] X. He and L. S. Luo, "Theory of the lattice Boltzmann method: From the Boltzmann equation to the lattice Boltzmann equation," Phys. Rev. E, vol. 56, pp. 6811-6817, 1997.

[16] T. Abe, "Derivation of the lattice Boltzmann method by means of the discrete ordinate method for the Boltzmann equation," J. Comp. Physics, vol. 131, pp. 241-246, 1997.

[17] P. L. Bhatnagar, E. P. Gross and M. Krook, "A model for collision processes in gasses; 1. Small amplitude processes in charged and neutral one component," Physics Rev, vol. 94, pp. 511-525, 1954.

[18] L. S. Luo and X. He, "Lattice Boltzmann model for the incompressible Navier-Stokes equation," J. Stat. Phys. vol, 88, pp. 927-944 1997.

[19] C. S. Nor Azwadi and T. Tanahashi, "Three-dimensional thermal lattice Boltzmann simulation of natural convection in a cubic cavity," Intl. J. Modern Physics B, vol. 21, pp. 87-96, 2007.

[20] L. S. Luo and X. He, "Lattice Boltzmann model for the incompressible Navier-Stokes equation," J. Stat. Phys. vol, 88, pp. 927-944 1997.

[21] J. Hardy, Y. Pomeau and D. Pazzis, "Time evolution of a two dimensional model system. 1. Invariant state and time correlation functions," J. Math. Phys., vol, 14, pp. 1746-1759 1973. 


\title{
Performance Analysis of High Efficiency Crystalline Silicon Solar Cells by AMPS -1D
}

\author{
Nowshad Amin and Kamaruzzaman Sopian
}

\begin{abstract}
This paper presents the theoretical analysis of crystalline silicon thin film solar cells by numerical modeling simulator named AMPS to design and investigate the higher efficiency cells. The $\mathrm{x}$-Si thin-film solar cells with a structure of $\mathbf{n - S i} / \mathbf{n}-\mathrm{Si} / \mathbf{p}-\mathrm{Si} / \mathbf{p}^{+}-\mathrm{Si}$ (alias PERL structure) are mainly discussed. Effects of various parameters such as carrier concentration of different layers as well as the thickness variation effect are investigated to find out the optimum device structure that may help to discover the latent efficiency of $\mathrm{x}-\mathrm{Si}$ solar cell by minimizing the loss factors and cost. However, the current-voltage (I-V) characteristics of these $\mathrm{x}$-Si thin films are mainly investigated to fetch the optimum conditions for achieving the best performance $x-S i$ thin solar cells. Recommendation can be made based on the investigated results, with some limitations in the numerical analysis of AMPS.
\end{abstract}

Keywords: photovoltaics, crystalline Si thin film solar cells, theoretical analysis, AMPS, PERL structure

\section{INTRODUCTION}

Most commercially available solar cells are made from wafers of highly pure monocrystalline (also simply called as crystalline) or polycrystalline silicon. Such solar cells can attain efficiencies of up to $18 \%$ in commercial manufacture and over $20 \%$ in the laboratory. However, the silicon wafers used to make them are relatively expensive, making up 20$40 \%$ of the final module cost. Although silicon is highly abundant (comprising one quarter of the earth's crust), making a very pure wafer suitable for solar cell manufacture requires much energy and is therefore relatively costly. Moreover, a solar cell made using a 300-400 $\mu \mathrm{m}$ thick wafer generates $90 \%$ of its energy from the top $15-20 \mu \mathrm{m}$. The rest of the wafer is required simply to hold the cell together [1][3].

The alternative to these "bulk silicon" technologies is to deposit a thin layer of semiconductor onto a supporting material such as glass, metal etc. Various materials can be used such as cadmium telluride, copper indium diselenide and amorphous silicon. Thin film solar cells made from cadmium telluride or copper indium diselenide have yet to

Nowshad Amin is with the Dept. of Electrical, Electronic and System Engineering of the Faculty of Engineering, National University of Malaysia (UKM), Bangi, Selangor 43600 Malaysia (phone: 03-8921-6325; fax: 038921-6146; e-mail: nowshad@eng.ukm.my).

Kamaruzzaman Sopian is with the Solar Energy Research Institute of the National University of Malaysia (UKM), Bangi, Selangor 43600 Malaysia (e-mail: ksopian@eng.ukm.my). be fully commercialized, but offer some promise of achieving low costs with reasonably good performance.

In numerous industrial and public institute laboratories, work on thin film solar cells made of crystalline silicon is at the centre of research and development. The thickness of this type of thin film solar cell should total around 10-50 $\mu \mathrm{m}$. This is much larger than solar cells made of compound semiconductors (e.g. CdTe, CIS). Since silicon, unlike other semiconductors, is an indirect bandgap semiconductor, its suitability for this type of thin film solar cell is limited, due to the low absorption of photovoltaically useful sunlight. There are, however, strong arguments in favor of silicon. As we know, silicon will never present any resource problems. Besides, the material is non-toxic both in the operation of solar cells and for disposal purposes. Moreover, solar cells made from crystalline silicon do not show any degradation of efficiency. The manufacturing technologies are closely related and linked with the technologies for the manufacture of semiconductor devices, both integrated circuits and large area high performance semiconductor devices. Therefore, the technologies for the production of solar cells from crystalline silicon can participate in the large pool of experience relating to the manufacture of extremely pure starting material and process technologies. In addition to the advantages of low material usage, calculations show that the efficiency of such a thin film solar cell can be increased [4].

\section{OBJeCtives \& Methodology}

Crystalline silicon ( $\mathrm{Si}$ ) forms the backbone of photovoltaic (PV) technology both in research and volume manufacturing. Since, the cost of a Si wafer accounts for approximately $50 \%$ of a typical photovoltaic module cost, highly economic use of $\mathrm{Si}$ in thin, flexible film format has been identified as the most effective approach aimed at reducing cost consistent with performance enhancement. The experience with thin (100-200 $\mu \mathrm{m})$ Si wafers in highthroughput environment has determined that wafer breakage due to its reduced wafer mechanical strength offsets potential commercial benefits. Therefore, an alternate approach is needed to manufacture thin $(\sim 5-50 \mu \mathrm{m})$, flexible Si films on inexpensive substrates using low-cost, high throughput method. From the above overview, the short to medium term future for industrial solar cells appears to be based on crystalline and multicrystalline silicon wafers. For costs reasons the substrates will have to be progressively thinner over time while simultaneously maintaining, but preferably increasing cell efficiency. In 
summary, the most important challenge for solar cell is the design of crystalline silicon $(\mathrm{x}-\mathrm{Si})$ based solar cell structures with high performance of characteristics, as well as ease of fabrication. In order to address this challenge, this study has investigated and optimized crystalline silicon based solar cell structures by Analysis of Microstructure and Photonics Structures (hereafter AMPS) simulator from various perspectives.

AMPS is a one-dimensional device simulator which stands for Analysis of Microelectronics and Photonics Structures. It was engineered to be a very general and versatile computer simulation tool for the analysis of device physics and for device design. AMPS-1D is currently capable of generating I-V characteristics under illumination and without illumination. Besides, it can generate quantum efficiency (QE) and spectral response (SR) for voltage bias and light bias situation. It mainly treats devices composed of single crystal, polycrystalline, amorphous material or combinations thereof.

From the solution provided by an AMPS simulation, output such as current-voltage characteristics in the dark and, if desired, under illumination can be obtained. These may be computed as a function of temperature. For solar cell and detector structures, collection efficiencies as a function of voltage, light bias, and temperature can also be obtained. In addition, important information such as electric field distributions, free and trapped carrier populations, recombination profiles, and individual carrier current densities as a function of position can be extracted from the AMPS. As stated earlier, AMPS versatility can be used to analyze transport in a wide variety of device structures that can contain combination of crystalline, polycrystalline, or amorphous layers. AMPS is formulated to analyze, design and optimize structures intended for microelectronics, photovoltaic or opto-electronic applications [5].

\section{DEVICE FABRICATION AND CHARACTERIZATION}

This section discusses the set-up of the crystalline silicon (x-Si) solar cell model with AMPS. Different types of input parameters are explained in the context of the conventional case, which was the baseline for subsequent assorted investigations. Input parameters have been selected based on experimental data, literature values, theory, or in some cases, reasonable estimate. The conventional case is partially selected so that no single loss mechanism, i.e. a high number of defects in the $\mathrm{x}-\mathrm{Si}$, significantly limits the performance. This however, is often not the case in real devices and therefore, the conventional case will out perform most experimental devices.

\section{A. Modeling Setup}

In AMPS, any user has the choices of two different approaches. One is termed as density of states (DOS) mode; the other is the carrier lifetime mode.

In the DOS mode, the details of recombination traffics, trapping and the charge state of the defects (and the effects of this charge on the electric field variation across a surface) are fully accounted due to all the recombination traffics, trapping, re-admission and their effects on the electric field. This approach requires capture cross section information to quantify the attractiveness of the various defects to electrons and holes. The Shockly-Read-Hall (S-R-H) model is used for the capture and emission process in this DOS approach.

Whereas, the lifetime mode accepts inputs in the form of carrier lifetimes, which are assumed constant, independent of light and voltage bias, and does not address the underlying recombination processes. All work done here is based on lifetime mode.

Three types of parameters are needed in pursuing the AMPS numerical analysis for any structure. These are:

- Material properties for every single layer, including the front and back contact.

- Environmental conditions.

- Model settings for instance model type, grid spacing for the numerical calculations, bias voltage for which $\mathrm{J}-\mathrm{V}$ and QE output should be generated.

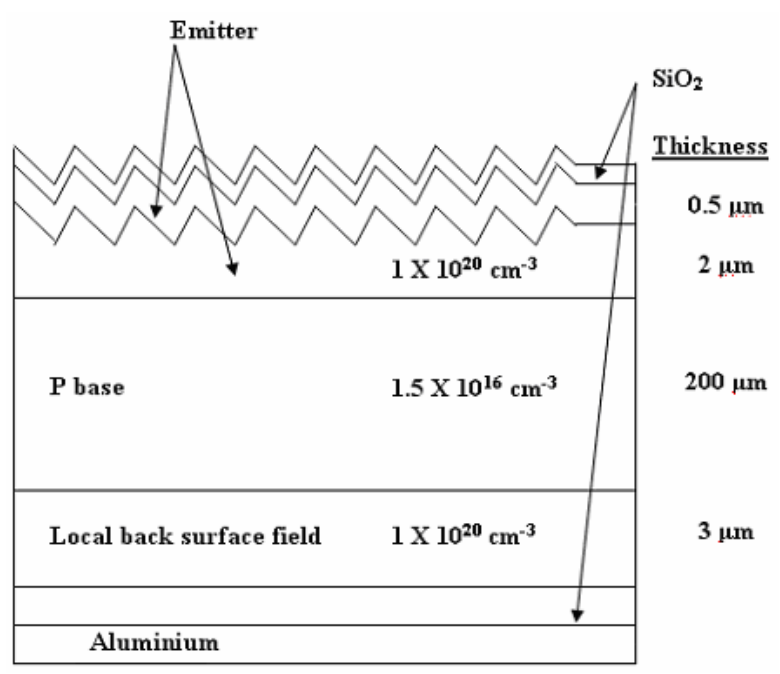

Figure 1. The structure of high efficiency solar cell made of monocrystalline silicon (PERL Cell)

\section{B. Material Properties of PERL Cell}

Here, a structure as known as passivated emitter, rearlocally diffused (PERL), which claims the highest laboratory efficiency to date will be introduced and examined from various perspectives to obtain the optimum structure together with the device physics. The PERL structure is illustrated in figure 1 and all the related parameters are shown in table 5 and table 6 , respectively. The parameters are based on practical values as extracted from related resources.

\section{1) $\mathrm{SiO}_{2}$ layer}

The $\mathrm{SiO}_{2}$ passivation layer has a thickness of approximately $100 \mathrm{~nm}$ to achieve a high antireflection effect on the emitter side (light entry). The $\mathrm{SiO}_{2}$ layer had played a decisive role in almost all silicon semiconductor devices. Its relatively simple manufacturing process by high temperature treatment under oxygen, but predominantly the masking behavior of the $\mathrm{SiO}_{2}$ toward dopants and its passivating effect, contributed decisively to making the silicon the basic material for the most semiconductor devices. The effect of the $\mathrm{SiO}_{2}$ layer can be described as follows. The abrupt ending of the crystal at the surface leads to a density of defects of approximately $10^{15} \mathrm{~cm}^{-2}$, which are traps in the forbidden band, thus giving rise to a high recombination rate. These traps are largely saturated by the $\mathrm{SiO}_{2}$ layer and 
thus become ineffective.

Table 1. General device properties of PERL cell

\begin{tabular}{|l|l|l|}
\hline & $\begin{array}{l}\text { Front Surface } \\
(\mathrm{x}=0 \mu \mathrm{m})\end{array}$ & $\begin{array}{l}\text { Back Surface } \\
(\mathrm{x}=205.7 \mu \mathrm{m})\end{array}$ \\
\hline$\Phi_{\mathrm{b} 0 / \mathrm{L}}=\mathrm{E}_{\mathrm{c}}-\mathrm{E}_{\mathrm{F}}$ & $0.00 \mathrm{eV}$ & $0.90 \mathrm{eV}$ \\
\hline $\begin{array}{l}\text { Surface recombination } \\
\text { electrons }\end{array}$ & $51.6 \mathrm{~cm} / \mathrm{s}$ & $51.6 \mathrm{~cm} / \mathrm{s}$ \\
\hline Surface recombination holes & $97.2 \mathrm{~cm} / \mathrm{s}$ & $97.2 \mathrm{~cm} / \mathrm{s}$ \\
\hline Reflectivity & 0.03 & 0.8 \\
\hline
\end{tabular}

Table 2. Layer properties of PERL cell

\begin{tabular}{|c|c|c|c|c|}
\hline & $\begin{array}{l}\mathrm{n}-\mathrm{Si} \\
\text { emitter }\end{array}$ & $\begin{array}{l}\text { n-Si } \\
\text { emitter }\end{array}$ & p-Si base & $\begin{array}{l}\text { Local back } \\
\text { surface } \\
\text { field }\end{array}$ \\
\hline Width & $500 \mathrm{~nm}$ & $2000 \mathrm{~nm}$ & $\begin{array}{l}200000 \\
\mathrm{~nm}\end{array}$ & $3000 \mathrm{~nm}$ \\
\hline $\begin{array}{l}\text { Dielectric } \\
\text { constant }\end{array}$ & 11.9 & 11.9 & 11.9 & 11.9 \\
\hline $\begin{array}{l}\text { Electron } \\
\text { mobility }\end{array}$ & $\begin{array}{l}1500 \\
\mathrm{~cm}^{2} / \mathrm{Vs}\end{array}$ & $\begin{array}{l}1500 \\
\mathrm{~cm}^{2} / \mathrm{Vs}\end{array}$ & $\begin{array}{l}1500 \\
\mathrm{~cm}^{2} / \mathrm{Vs}\end{array}$ & $\begin{array}{l}1500 \\
\mathrm{~cm}^{2} / \mathrm{Vs}\end{array}$ \\
\hline $\begin{array}{l}\text { Hole } \\
\text { mobility }\end{array}$ & $450 \mathrm{~cm}^{2} / \mathrm{Vs}$ & $450 \mathrm{~cm}^{2} / \mathrm{Vs}$ & $\begin{array}{l}450 \\
\mathrm{~cm}^{2} / \mathrm{Vs}\end{array}$ & $450 \mathrm{~cm}^{2} / \mathrm{Vs}$ \\
\hline $\begin{array}{l}\text { Carrier } \\
\text { Density }\end{array}$ & $\begin{array}{l}\mathrm{ND}=10^{19} \\
\mathrm{~cm}^{-3}\end{array}$ & $\begin{array}{l}\mathrm{ND}=10^{20} \\
\mathrm{~cm}^{-3}\end{array}$ & $\begin{array}{l}\mathrm{NA}=1.5 \\
\times 10^{16} \mathrm{~cm}^{-3}\end{array}$ & $\begin{array}{l}\mathrm{NA}=10^{20} \\
\mathrm{~cm}^{-3}\end{array}$ \\
\hline Bandgap & $1.12 \mathrm{eV}$ & $1.12 \mathrm{eV}$ & $1.12 \mathrm{eV}$ & $1.12 \mathrm{eV}$ \\
\hline $\begin{array}{l}\text { Effective } \\
\text { dens. NC }\end{array}$ & $\begin{array}{ll}2.80 & \times 10^{19} \\
\mathrm{~cm}^{-3} & \\
\end{array}$ & $\begin{array}{ll}2.80 & \times 10^{19} \\
\mathrm{~cm}^{-3} & \\
\end{array}$ & $\begin{array}{l}2.80 \times 10^{19} \\
\mathrm{~cm}^{-3}\end{array}$ & $\begin{array}{ll}2.80 & \times 10^{19} \\
\mathrm{~cm}^{-3} & \\
\end{array}$ \\
\hline $\begin{array}{l}\text { Effective } \\
\text { dens. NV }\end{array}$ & $\begin{array}{ll}1.04 & \times 10^{19} \\
\mathrm{~cm}^{-3} & \\
\end{array}$ & $\begin{array}{ll}1.04 & \times 10^{19} \\
\mathrm{~cm}^{-3} & \\
\end{array}$ & $\begin{array}{l}1.04 \times 10^{19} \\
\mathrm{~cm}^{-3}\end{array}$ & $\begin{array}{ll}1.04 & \times 10^{19} \\
\mathrm{~cm}^{-3} & \\
\end{array}$ \\
\hline $\begin{array}{l}\text { Electron } \\
\text { affinity }\end{array}$ & $4.05 \mathrm{eV}$ & $4.05 \mathrm{eV}$ & $4.05 \mathrm{eV}$ & $4.05 \mathrm{eV}$ \\
\hline
\end{tabular}

\section{2) Emitter layer}

The emitter is in two stages and has a surface doping concentration of approximately $1 \times 10^{19} \mathrm{~cm}^{-3}$ and a penetration depth of $0.5 \mu \mathrm{m}$ under the $\mathrm{SiO}_{2}$ layer, the surface concentration is approximately $1 \times 10^{20} \mathrm{~cm}^{-3}$ and the penetration depth is approximately $2 \mu \mathrm{m}$ under the metal grid structure.

\section{3) Base layer}

The base material is monocrystalline, float zone pulled and p-doped (approximately $1.5 \times 10^{16} \mathrm{~cm}^{-3}$ ), with a resistivity of approximately $1 \Omega-\mathrm{cm}$. The base thickness is approximately $200 \mu \mathrm{m}$, primarily due to technological manufacturing and handling processes.

\section{4) Textured surfaces}

The front surface facing the light is textured, preferably with inverted pyramids. The front surface, apart from the grid structure, is coated with a thermally grown layer of silicon dioxide for passivation.

\section{5) Back surface field (BSF)}

The back surface of the cell is coated with a thermally grown $\mathrm{SiO}_{2}$ layer for passivation except for the point contacts (approximately 1\%-2\% of the total area). The local $\mathrm{p}^{+}$doped BSF has a surface carrier concentration of around $10^{20} \mathrm{~cm}^{-3}$ and a penetration depth of $3 \mu \mathrm{m}$. The back surface contact has reflective characteristics and consists, for example, vapor-deposited aluminum.

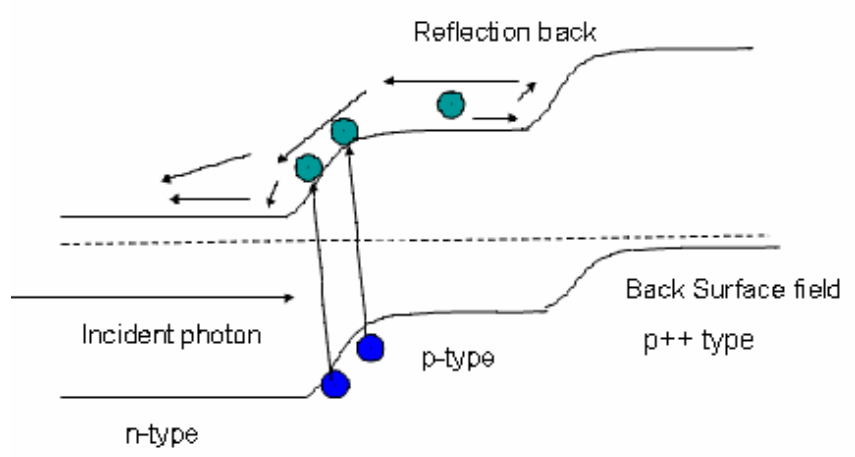

Figure 2. Energy band diagram of a BSF solar cell

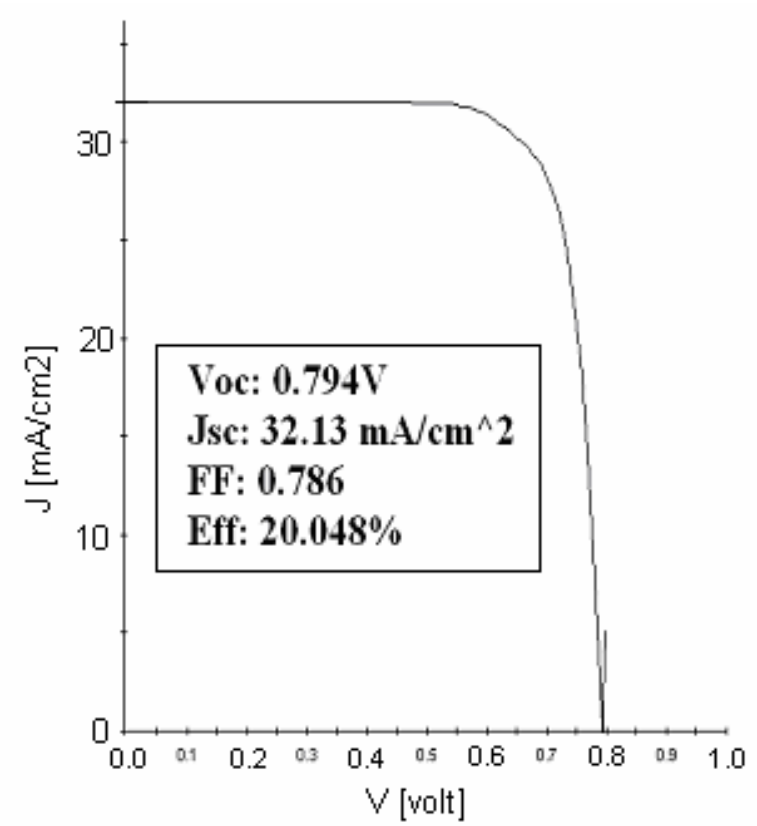

Figure 3. I-V characteristics of the PERL solar cell

\section{C.Theoretical Analysis for Passivated Emitter, Rear-locally Diffused (PERL) Cell Structure}

To simplify the analysis, a very simple structure of n-Si/n$\mathrm{Si} / \mathrm{p}-\mathrm{Si} / \mathrm{p}^{+} \mathrm{Si}$ has been taken into account as shown in figure 1. The light illuminated side is the $\mathrm{n}-\mathrm{Si}$ emitter window layer as same as the usual fabricated cells.

The conversion efficiency of $24 \%$ (open-circuit voltage $\left(\mathrm{V}_{\mathrm{oc}}\right): 0.708 \mathrm{~V}$, short circuit current $\left(\mathrm{J}_{\mathrm{sc}}\right): 40.8 \mathrm{~mA} / \mathrm{cm}^{2}$, fill factor (FF): 0.831) was achieved for this cell in laboratory [6]. The initial modeling results for the PERL cell using AMPS are shown in figure 3. It shows light I-V characteristics of the cell with parameters of $\mathrm{Voc}_{\mathrm{oc}}=0.794 \mathrm{~V}$, $\mathrm{J}_{\mathrm{sc}}=32.13 \mathrm{~mA} / \mathrm{cm}^{2}, \mathrm{FF}=0.786$, Efficiency $=20.048 \%$. The efficiency is similar (but not same) to the best cells that have been made to date. There are some difference in efficiency between the result achieved in laboratory cells and AMPS. It is generally agreed that by fully optimizing all design parameters, the PERL structure is able to demonstrate $24 \%$ efficiency under one sun illumination. However, this is not possible to achieve in AMPS simulation since it is considering only one dimensional structure but in real case the solar cell module is made in three dimensional with all light trapping effect. 


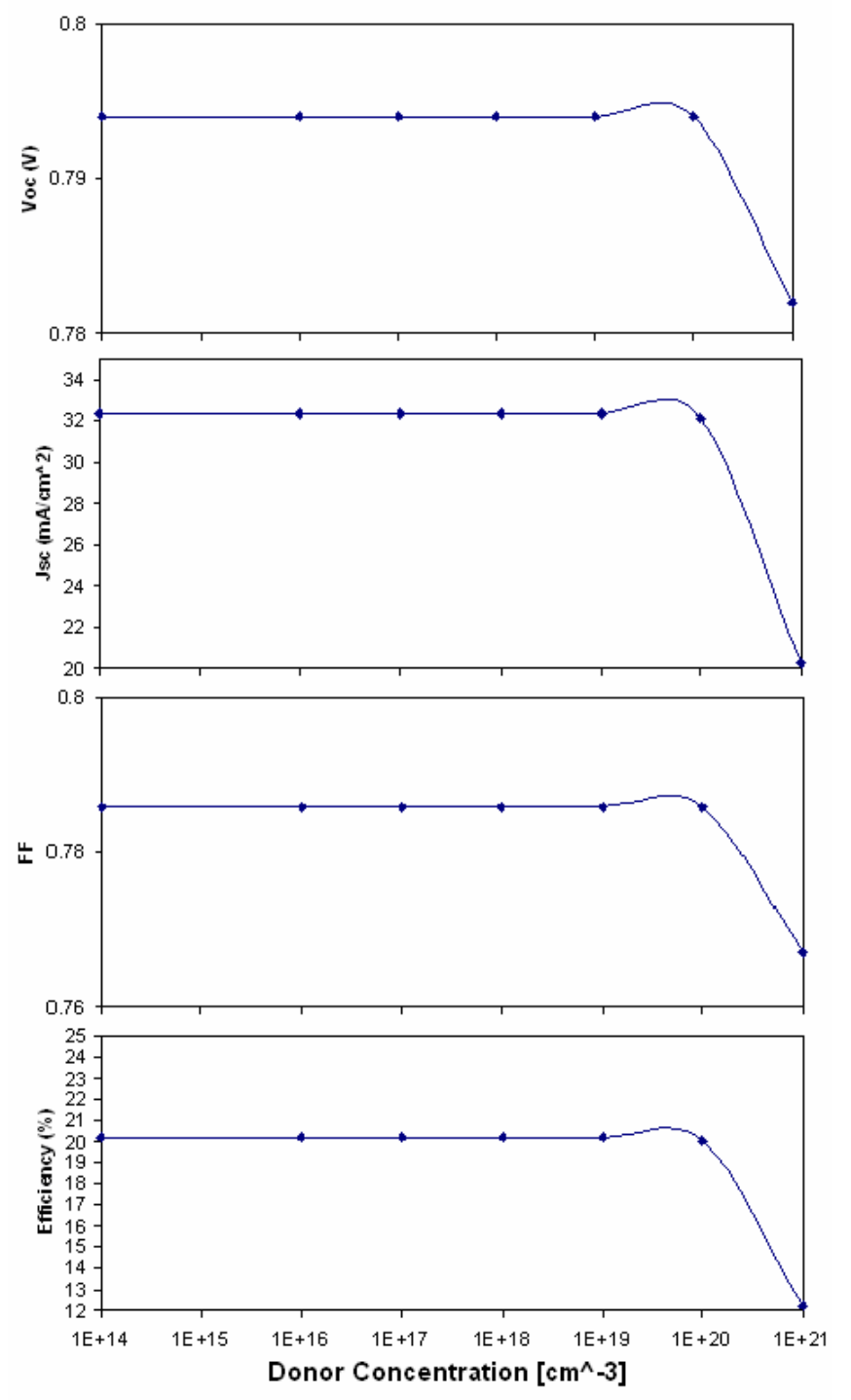

Figure 4. The effect of bottom emitter layer donor concentration on PERL cell characteristics

\section{D.Effect of the carrier concentration of bottom emitter layer on cell performance}

At first the top emitter layer has been investigated with different carrier concentration, where almost similar trend as the bottom emitter layer has been observed. Therefore, only the bottom emitter layer effect will be discussed next. In the following analysis, all parameters have been kept the same except the concentration at the bottom emitter layer with the varying range from $10^{14} \mathrm{~cm}^{-3}$ to $10^{21} \mathrm{~cm}^{-3}$. Results for various solar cell parameters are shown in figure 4. As can be seen, there has not much significant changes in all the cell parameters. Up to a density of $10^{20} \mathrm{~cm}^{-3}$ open circuit voltage and FF remain approximately constant, but the values for the concentration over $10^{20} \mathrm{~cm}^{-3}, \mathrm{~V}_{\mathrm{oc}}, \mathrm{J}_{\mathrm{sc}}$ and FF decrease drastically.
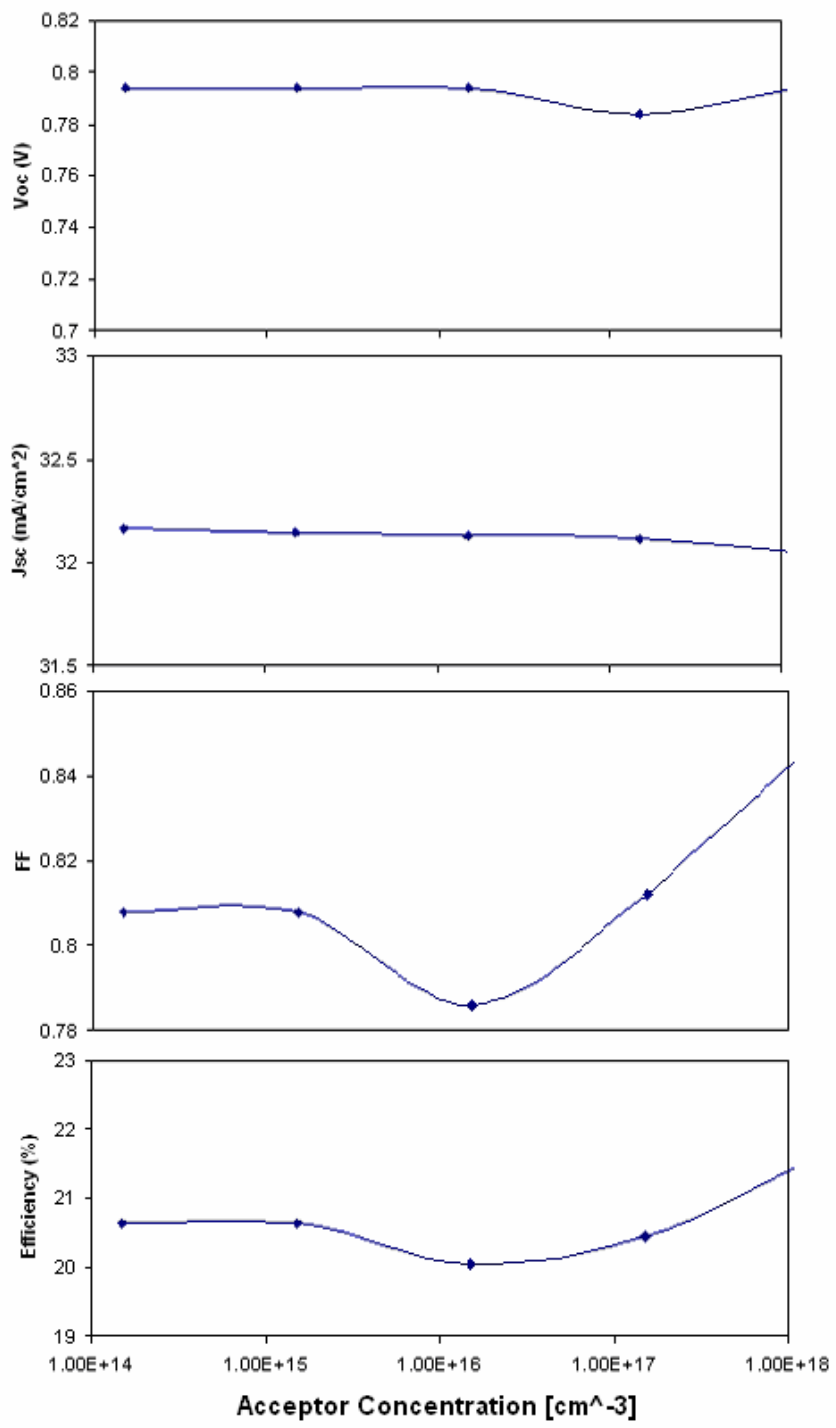

Figure 5. The effect of the acceptor concentration in base layer on the PERL cell characteristics

\section{E.Effect of the Carrier Concentration of Base Layer on Cell Performance}

In the following analysis, all parameters remain the same as well, except the acceptor concentration at the base layer varied from $1.50 \times 10^{14} \mathrm{~cm}^{-3}$ to $1.50 \times 10^{18} \mathrm{~cm}^{-3}$.

A plot on calculated solar cell parameters versus base doping is shown in figure 5. For low base resistivities, the device operates in low injection conditions at the maximum power point, and a one-exponential model with a dependence of the exp $(q V m / k T)$ type is to be used for all conditions of operation, resulting in a fill factor dependency only on short circuit current and open circuit voltage. Therefore, the conversion efficiency is controlled by the behavior of Jsc and Voc. As far as superposition applies, front-illuminated cells present higher Jsc and Voc and therefore higher efficiency..

Increasing the value of acceptor concentration introduces additional recombination centers and additional space charge. Output for various defect densities is shown in figure 5. Light I-V shows insignificant changes in voltage and fill factor. Up to a density of $10^{16} \mathrm{~cm}^{-3}$, voltage and FF remain approximately constant, but beyond $10^{16} \mathrm{~cm}^{-3}$ Voc does not follow a constant pattern and FF increases rapidly. 
As the acceptor concentration increases, the efficiency increases too. It reaches the highest efficiency when the concentration is $1.50 \times 10^{18} \mathrm{~cm}^{-3}$, the limit value for this simulation.
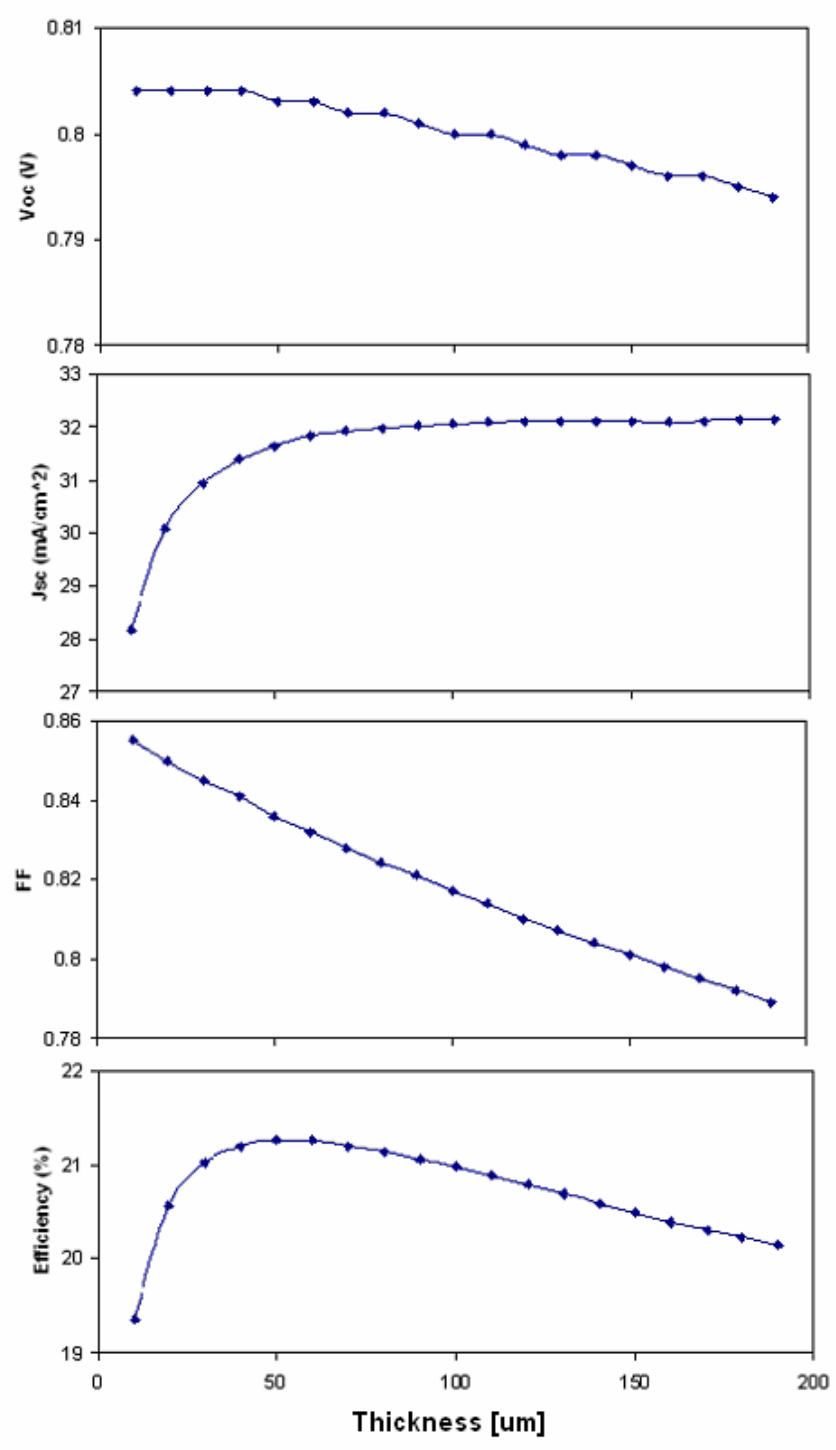

Figure 6 . The effect of the base thickness reduction on the solar cell characteristics

\section{F. Base Layer Thickness Reduction Effect}

The next analysis is the most important part of this study that examines the thickness reduction effect. By reducing the thickness of any growing layer or from sawing the silicon wafer, one can save the energy, materials as well as the unnecessary bulk that limits the efficiency by being resistive loss of the device. It is therefore important to fabricate the thin film solar cells to the extreme permissible thickness to reduce all sorts of losses. A trial has been carried out with the thickness variation of the base layer by decreasing it from the usual practical thickness. Here, all other parameters remained the same except the thickness of the base layer has been varied from $190 \mu \mathrm{m}-10 \mu \mathrm{m}$. Thus, the influence of the base thickness of silicon solar cells has been investigated using silicon base layer with thickness ranging from $190 \mu \mathrm{m}$ to $10 \mu \mathrm{m}$. For silicon solar cell structures with a high minority carrier diffusion length, it can be noticed from figure 6 that the Jsc remains quite unaffected till the thickness of around 50-70 $\mu \mathrm{m}$ and then decreases as the layer becomes thinner, probably due to a shorter optical path length. However, Voc and FF gain much as can be attributed to series resistance reduction by thinning the base layer. The highest efficiency can be achieved at the thickness of around $50 \mu \mathrm{m}$ as can be found from this numerical analysis.

\section{CONCLUSIONS}

This study has demonstrated the capabilities of numerical analysis to investigate and optimize the crystalline silicon (x-Si) solar cells by Analysis of Microelectronics and Photonic Structures (AMPS) simulator. Comprehensive studies on the influence of various device parameters have been performed to confirm the validity of the model as well as to improve the physical understanding of $\mathrm{x}-\mathrm{Si}$ cells. Numerical models regarding the conventional $\mathrm{x}-\mathrm{Si}$ solar cells have been used to examine the potentially attainable efficiency under several assumptions. The basic issue of this part is to find out the consequences of varying the thickness and concentration in each layer of mainly the PERL cell structure, which has been expected to upgrade the efficiency. The trends of the emitter as well as base carrier concentration effect on the overall solar cell performance have been revealed. Moreover, the thickness reduction of the base region, which is mostly thick in practical cases, has been investigated and a thickness of around $50 \mu \mathrm{m}$ has been found to be optimum for PERL structured crystalline silicon solar cells. Therefore, all these case studies hint to achieve low-cost and high-efficiency $\mathrm{x}-\mathrm{Si}$ solar cells with fabrication possibilities.

\section{REFERENCES}

[1] S. Reber, A.Hurrle, "Crystalline silicon thin film solar cells-recent results at Fraunhofer ISE," Elsevier Ltd., 2004.

[2] Yoshihiro Hamakawa, "Solar PV energy conversion and the $21^{\text {st }}$ century's civilization," Elsevier Science B. V., 2002.

[3] M. A. Green, J. Zhao, "Progress and outlook for high efficiency crystalline silicon solar cells," Elsevier Science B. V., 2001

[4] Adolf Goetzberger, Joachim Knobloch and Bernard Voss, Crystalline Silicon Solar Cells, John Wiley \& Sons Ltd.,1998.

[5] AMPS-1D Manual for Windows '95/NT, The Electronic Materials and Processing Research Laboratory at the Pennsylvania State University, University Park, PA 16802.

[6] Jozef Szlufcik, S. Sivoththamn, Low-Cost Industrial technologies of Crystalline Silicon Solar Cells, Proceedings of The IEEE, vol. 85 


\title{
Analysis of Philippine Industrial Carbon Dioxide Emissions from Combustion Processes Using Input-Output Models
}

\author{
Raymond R. Tan and Joel Q. Tanchuco
}

\begin{abstract}
Climate change is now considered as a major environmental issue in the international community. Many initiatives to address require accounting of carbon dioxide emissions, which result from many human activities. Life cycle based approaches are especially useful to account for the interconnectivity of industrial processes in modern economies. This paper describes carbon emissions from the Philippine economy, as estimated using a low-resolution input-output model. Results show that the highest carbon intensities are found in power generation and transportation; these two sectors also are significant contributors of indirect emissions to downstream sectors of the economy.
\end{abstract}

Keywords: climate change, carbon emissions, life cycle analysis, input-output modeling

\section{INTRODUCTION}

Climate change is considered by the international community as one of the major environmental issues facing the world today. By far the greatest contributor to climate change are carbon dioxide emissions, which result from human activities such as the combustion of fossil fuels. Annual global carbon dioxide emissions now amount to roughly 25 billion tons. In response to growing concerns about this threat, there has been increased research in various mitigation techniques, including:

- Use of inherently low-carbon, non-combustion based energy sources such as nuclear, wind, solar, and hydroelectric power, or carbon-neutral fuels based on biomass.

- Use of carbon sequestration technologies in conjunction with fossil fuel combustion.

- Energy conservation and efficiency enhancement.

Accurate accounting of system carbon emissions is of course essential to measuring the effectiveness of any such measures. The global nature of the impacts of these greenhouse gases makes it necessary to account for total

Raymond R. Tan is associate professor of the Chemical Engineering Department and researcher of the Center for Engineering and Sustainable Development Research at De La Salle University-Manila, 2401 Taft Avenue, 1004 Manila, Philippines (telephone/fax 632-536-0260; e-mail: tanra@dlsu.edu.ph).

Joel Q. Tanchuco is assistant professor of the Economics Department of De La Salle University-Manila, 2401 Taft Avenue, 1004 Manila, Philippines (e-mail: tanchucoj@dlsu.edu.ph). releases generated, both directly and indirectly, using a life cycle-based framework. This approach is essential since carbon emissions may be generated (or absorbed) by different activities. For example, combustion of fuel wood generates carbon dioxide emissions when the carbon in the fuel is oxidized; however, from a systems standpoint, these emissions are offset by carbon fixation during photosynthesis. Thus, provided the fuel wood is replenished continuously by replanting, a biomass combustion system can operate with nearly zero net carbon dioxide emissions. In more complex systems, there is often a high degree of interconnectedness among different industrial sectors, which then requires systematic modeling tools to determine the actual level of emissions generated. One such approach, based on input-output techniques, is described in the next section.

\section{INPUT-OUTPUT MODELS}

In 1973, Leontief received the Nobel Prize in economics for his work on input-output analysis (IOA). IOA is a technique for studying the network structure of economies based on transactions between industrial sectors. The basic model can be readily extended to environmental problems $[1,2]$. The general model is:

$g=B(I-A)^{-1} y$

where $\mathrm{g}$ is the inventory vector of emissions per unit of net output; $\mathrm{B}$ is the environmental intervention matrix that summarizes industry sector emission intensities; I is the identity matrix; $A$ is the transaction matrix summarizing the exchange of goods between sectors of the economy; and $\mathbf{y}$ is the net or final output vector of the system. Equation (1) is generic in nature, and the flow of goods given by A can be expresses in terms either of physical quantity or economic value. Note that if the unit costs of the different commodities or products in the system are given, then physical flows are easily converted to economic equivalents, and vice-versa. A simple example is given in the next section to illustrate how the model works.

\section{A Simple Illustrative CASE Study}

Consider the case of a hypothetical two-sector economy whose major flows are summarized in Table 1 . The 
columns labeled as Sectors I and II indicate the flows of goods (measured in monetary terms) and pollutants (given in physical units), with negative signs denoting inputs. For example, production of each $\$ 1$ of output of Sector I requires $\$ 0.2$ of inputs purchased from Sector II, and generates $0.2 \mathrm{~kg}$ of emissions. Note that this ratio is preserved throughout the entire sector's output, such that $\$ 1000$ of output from Sector I requires $\$ 200$ of goods from Sector II and also gives $200 \mathrm{~kg}$ of emissions. Furthermore, it is noted that each row, excluding the final column, shows the destinations of the output of the sectors. That is, out of every $\$ 1$ of output of Sector I, $\$ 0.2$ is sold to Sector II, while the residual $\$ 0.8$ is sold to the final consumer. Similarly, for each $\$ 1$ of output of Sector II, $\$ 0.2$ is sold to Sector I, another $\$ 0.2$ is consumed within Sector II itself, and the remaining $\$ 0.6$ reaches the consumer.

Table 1. Extended IO table for illustrative example

\begin{tabular}{lccc}
\hline & Sector I & Sector II & Net Output \\
\hline Sector I & 0 & 0.2 & 40 \\
Sector II & 0.2 & 0.2 & 30 \\
Pollutant $(\mathrm{kg})$ & 0.2 & 0.4 & $?$ \\
\hline
\end{tabular}

It can be seen even in simple systems that feedback loops may exist in the flow of goods. For example, Sector I requires inputs from Sector II, but Sector II also requires inputs from Sector I. This situation easily arises in real systems; for instance, coal may be used to generate electricity, but the processing of the coal itself may need electricity inputs as well. Another point is that a sector may use part of its own output as a raw material or input into itself. For instance, in an agricultural system, part of the crops produced may be consumed to feed livestock. The last column of Table 1 shows the net system output. The entries for Sectors I and II denote the net output that reaches the consumer or end-user, say in millions of $\$$. The entries of 40 and 30 correspond to $\mathbf{y}$ in (1). The total system emissions is unknown and denoted by "?" in the table. This value can be found by solving (1). Figure 1a shows the balanced economy, with the economic commodity flows in millions of $\$$ and the emissions in millions of $\mathrm{kg}$. Note that the ratios specified in Table 1 are satisfied here, and that a total of 30 million $\mathrm{kg}$ of emissions are generated.

Suppose that it is necessary to find the emissions associated with each of the two final output streams of the economy. This is not simply a matter of determining that Sectors I and II generate 10 and 20 million $\mathrm{kg}$ of emissions each, since the two sectors are mutually dependent. Neither can exist without the other. The correct approach is to split the entire system into two "layers," each dedicated to producing the net outputs of each of the two sectors. For example, if the net output of Sector II in (1) is set to zero, the resulting solution gives the system in Figure 1b, which produces a net output from Sector I, and just enough output from Sector II to meet the needs of Sector I for raw materials. Likewise, if the net output of Sector I in (1) is set to zero, the result is shown in Figure 1c. Note that if the flows of Figures $1 \mathrm{~b}$ and $1 \mathrm{c}$ are added, the entire economic system of Figure 1a is reconstituted.

Figures $1 \mathrm{~b}$ indicates that, to produce $\$ 40$ million of output from Sector I generates 12.6 million $\mathrm{kg}$ of emissions, of which $67 \%$ (8.4 million $\mathrm{kg}$ ) are generated directly within the sector, and 33\% (4.2 million $\mathrm{kg}$ ) are from indirect emissions through linkages within the economic system. The emissions intensity of Sector I is $0.315 \mathrm{~kg}$ per $\$$, of which about one-third is indirect in nature. A similar analysis can then be done for Sector II. The same general modeling principle can then be applied for much larger systems; the next section shows results for the analysis of carbon dioxide emissions from the 11 major sectors of the Philippine economy.

\section{PhilipPine CARbon EMissions}

For this study, matrix A in (1) is derived from Philippine national accounts [3] while B comes from emissions statistics [4]. Note that, since there is only one gas listed in $\mathrm{B}$, this matrix actually reduces to a vector, and the final result $\mathrm{g}$ becomes a scalar quantity. The carbon dioxide emission intensities of the eleven sectors per 1000 pesos (approximately US\$20) are shown in Figure 2. With the exception of agriculture, which has the lowest carbon intensity, and the utilities and transportation sectors, eight sectors generate about $10-15 \mathrm{~kg} \mathrm{CO}_{2}$ per thousand pesos of output. Agricultural activities generate the least carbon emissions among all the sectors, although it must be noted that releases of other greenhouse gases such as methane and nitrous oxide are not included in these figures, and may in fact be significant. Utilities and transportation generate disproportionately high emission levels per unit output, at roughly 140 and $55 \mathrm{~kg} \quad \mathrm{CO}_{2}$ per thousand pesos, respectively. This is not surprising, as the activities in these sectors of the economy make extensive use of fossil fuel combustion. It is also notable that in most of the sectors, other than utilities, transportation and real estate, indirect emissions arising from linkages within the economy account for large proportion of the emissions. Table 2 shows the major indirect contributors of carbon dioxide emissions to each sector of the Philippine economy; manufacturing, utilities and transportation appear as major contributors (more than 5\%of subtotal) in most of the sectors.

Table 2. Major contributors to indirect $\mathrm{CO}_{2}$ emissions

\begin{tabular}{lc}
\hline \multicolumn{1}{c}{ Sector } & Major Indirect Contributors $(>5 \%)$ \\
\hline 1. Agriculture & $3,5,6$ \\
2. Mining & 5,6 \\
3. Manufacturing & 5,6 \\
4. Construction & $3,5,6$ \\
5. Utilities & --- \\
6. Transportation & 5 \\
7. Trade & $3,5,6$ \\
8. Finance & $3,5,6$ \\
9. Real Estate & 5 \\
10. Private Services & $3,5,6$ \\
11. Government & $3,5,6$ \\
\hline
\end{tabular}




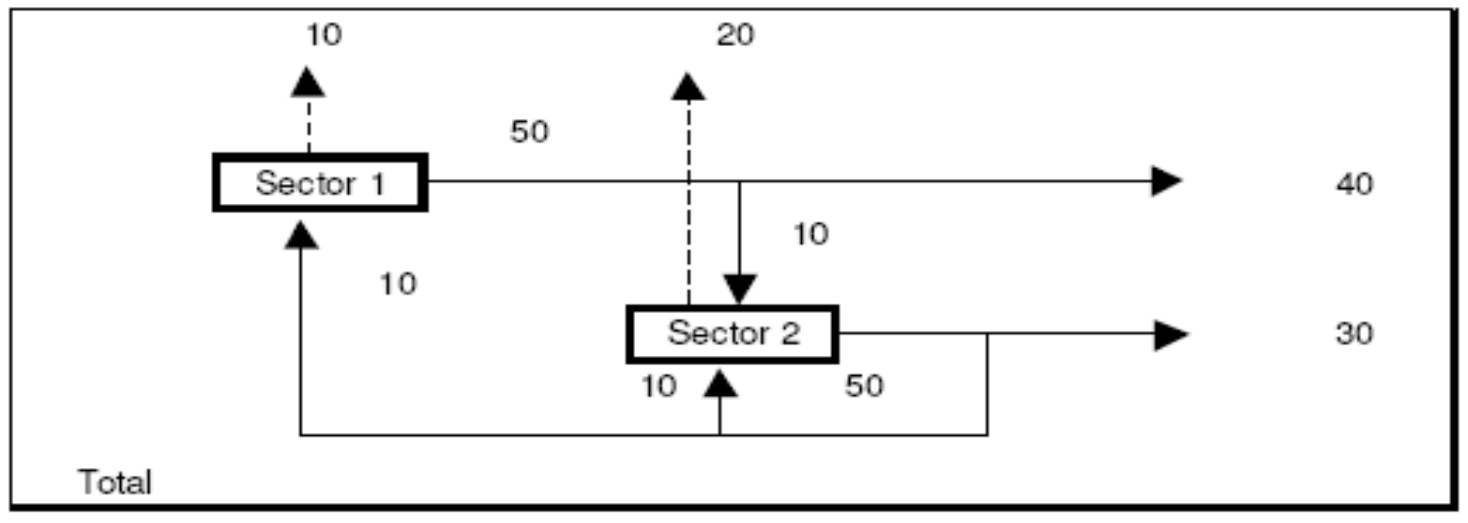

(a)

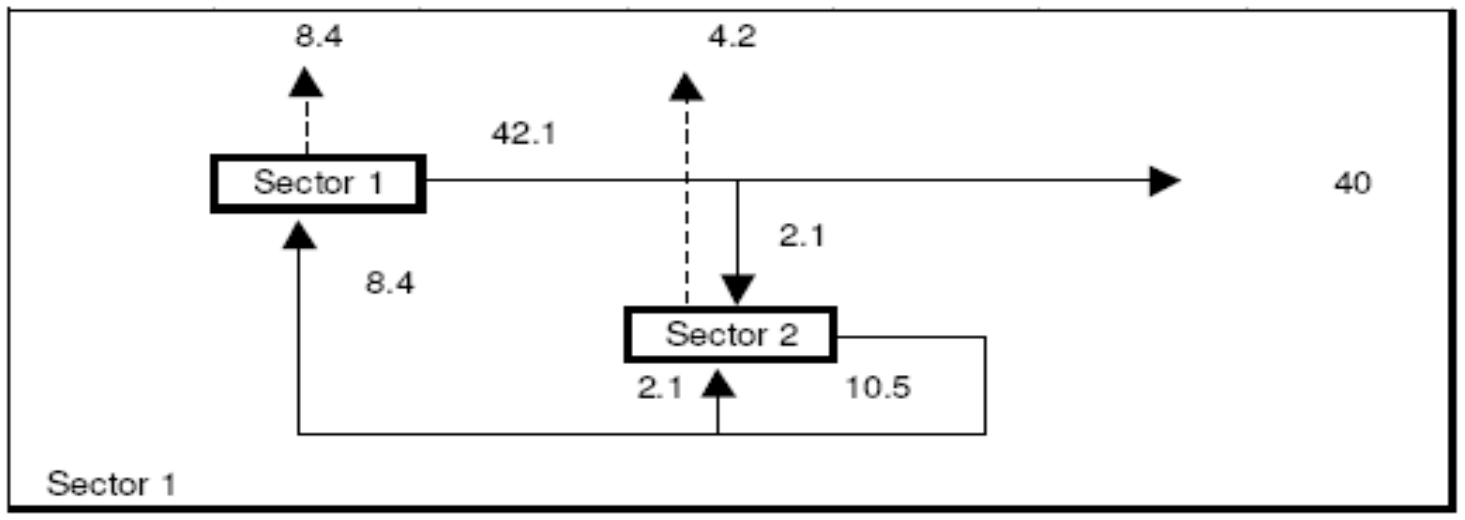

(b)

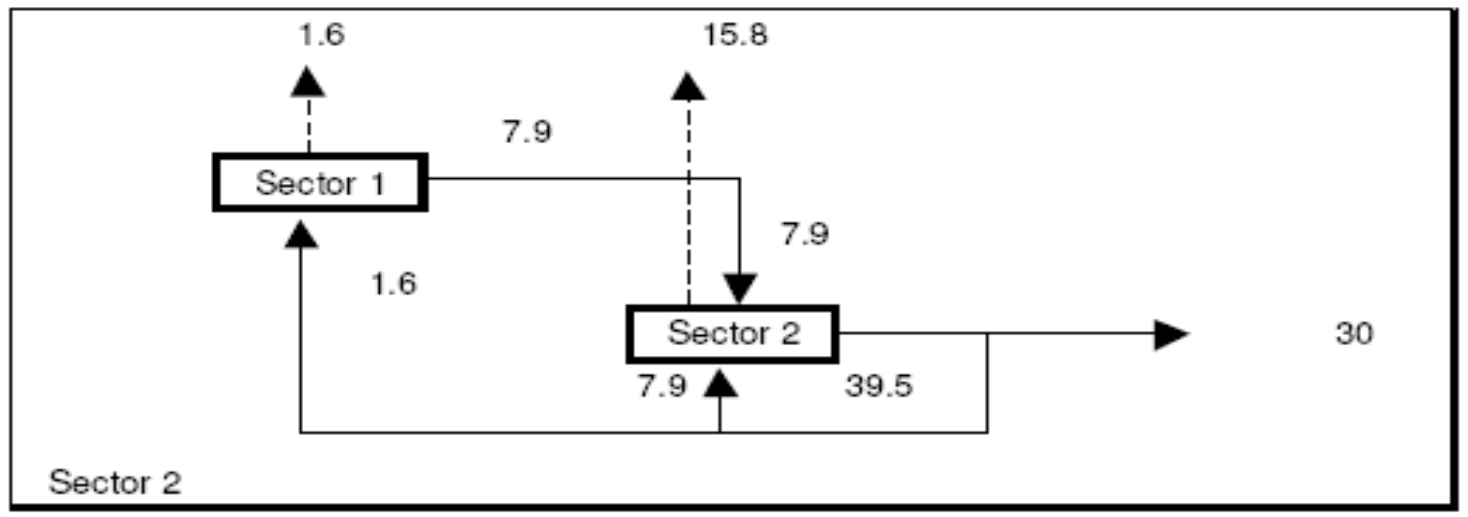

(c)

Figure 1. Emissions distribution in a hypothetical two-sector economy 


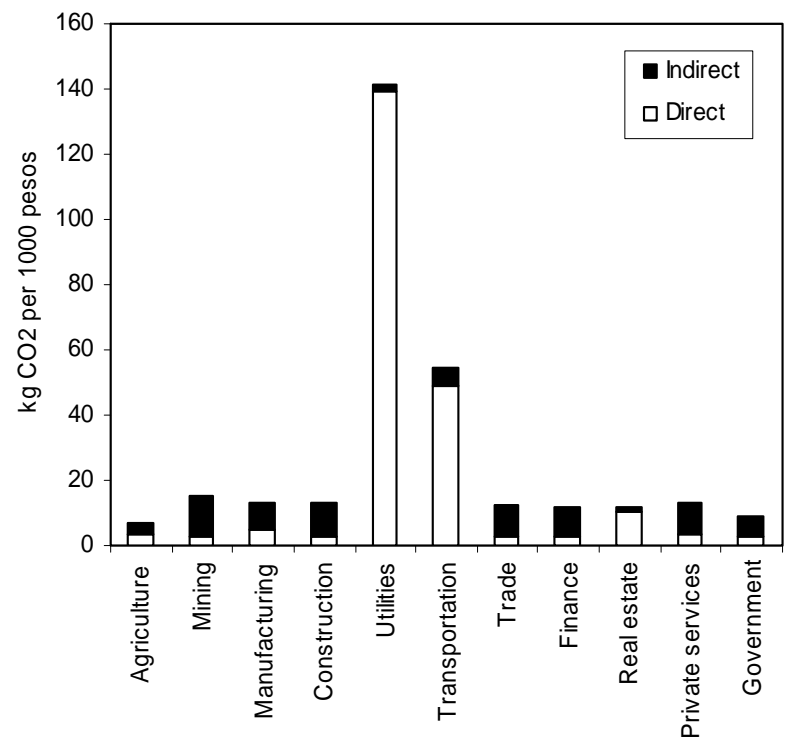

Figure 2. Direct and indirect $\mathrm{CO}_{2}$ emissions of eleven major sectors of the Philippine economy

\section{CONCLUSIONS}

Environmentally extended input-output analysis has been used to quantify carbon dioxide intensities of the eleven major sectors of the Philippine economy. Other than utilities and transportation, most of the sectors generate 15 $\mathrm{kg}$ of $\mathrm{CO}_{2}$ or less per thousand pesos (or approximately US\$20) of economic output. These two sectors have disproportionately high carbon intensities, and also appear as major indirect contributors to other sectors' emissions.

At the moment, further work is being done on more detailed analysis using higher-resolution IO tables with 60 sectors. More work is needed on accounting for other emissions as well, although the availability of data is a major impediment. Furthermore, there is some need to integrate this approach with a dynamic optimization framework to determine desirable technological and structural trajectories over the coming decades. Finally, a comparison among different countries in the Southeast Asian region will be useful for benchmarking and dissemination of best practices for mitigation of greenhouse gas releases.

\section{REFERENCES}

[1] Heijungs, R and Suh, S. 2002. The computational structure of life cycle assessment. Kluwer, Dordrecht.

[2] Hendrickson, C. T., Lave, L. B. and Matthews, H. S. 2006. Environmental life cycle assessment of goods and services. An inputoutput approach. RFF Press, Washington, DC.

[3] http://www.nscb.gov.ph/io/default.asp. Accessed April 2007.

[4] http://earthtrends.wri.org/pdf_library/country_profiles/cli_cou_608.pd f. Accessed on April 2007. 


\title{
Harmonic Effect on the Three-Phase System Due to Unbalanced Harmonic Current Source Using Load Flow Method
}

\author{
I. Daut, R. Chan Bahaudin, C.M. Hadzer, S.F. Siraj, S. Hardi and N. Hashim
}

\begin{abstract}
In power system, the continuity and reliability of power flow is very important. Power quality used to be to the ability of the electric utilities to provide electric power without interruption. Now a day, power quality encompasses any deviation from a perfect sinusoidal form. One distortion to the sinusoidal waveform which has risky consequences is harmonic. The increased used of nonlinear equipment have caused harmonics to become more common. In this paper we discuss the harmonic effect on the three-phase power system due to unbalanced in magnitude, and in phase of harmonic current source, and analyze based on harmonic power flow. The results show the Total Harmonic Distortion(THD) and Telephone Influence Factor(TIF) at each bus by means of MiPower Software. higher in bus 4 at current source harmonic is low, but it is below $3.0 \%$ therefore we do not need filter to eliminate.
\end{abstract}

Keywords: unbalanced harmonic current source, harmonic effect, load flow method, MiPower

\section{INTRODUCTION}

Harmonics are different from the other noise signals that they have frequencies that are multiples of the fundamental $(60 \mathrm{~Hz})$. A signal at $120 \mathrm{~Hz}$ is referred to as the $2^{\text {nd }}$ harmonic, the $3^{\text {rd }}$ harmonic is $180 \mathrm{~Hz}$, etc[1]. Waveform distortions are: a) Odd harmonics gives half wave symmetric distortion, b) Even harmonics gives half wave unsymmetrical distortion (Note: DC-components give the same result), and c) Interharmonics gives unsymmetrical distortion between period [2]. Harmonic currents generated by non-linear electronic loads produce penalty losses in every element of an electrical distribution system. Harmonics are a natural consequence of electronics and modern day electronic supplies. These harmonic related losses reduce system efficiency, cause apparatus overheating. Three-phase loads do not generate triplen harmonics. As a result, harmonic problems in industrial facilities dominated by three-phase loads will most often result from currents flowing at the $5^{\text {th }}$,

I. Daut, R. Chan Bahaudin, C.M. Hadzer, S.F. Siraj, S. Hardi and N. Hashim are with the Electrical Engineering Department University Malaysia Perlis (UniMAP) Pusat Pengajian Kejuruteraan Sistem Elektrik Kompleks Pusat Pengajian UniMAP(Blok A) Jl. Kangar - Arau02600 Jejawi, Arau, Perlis, Malaysia (Tel: +604-9798390, Faks: +604-979830, emails: ismaildaut@unimap.edu.my,_risnidar@unimap.edu.my, chemat@unimap.edu.my, siti_siraj@yahoo.com, surya@unimap.edu.my, haidar@unimap.edu.my). $7^{\text {th }}, 11^{\text {th }}$, or even higher order harmonics. Good harmonic prediction requires clear understanding of two different but closely related topics. One is the non-linear voltage/current characteristics of some power system components and its related effect, and the second is the derivation of suitable harmonic models of the predominantly linear network components, and of the harmonic flow resulting from their interconnection.[3] An electrical system supplies power to loads by delivering current at the fundamental frequency. Only fundamental frequency current can provide real power. Current delivered at harmonic frequencies does not deliver any power to the load. When current of a single frequency is present in a system, we can use the measured values in Ohm's law and power calculations. However, when currents of more than one frequency are present, direct addition of the current values leads to a summed value that does not correctly represent the total effect of the multiple currents. In three-phase system balanced conditions, system and loads, fundamental voltages and currents are shifted onethird of a cycle compared to each other. For a three-phase non-linear load, the phase angles are shifted 180 degrees, both for the $5^{\text {th }}$ and $7^{\text {th }}$ harmonic currents. The sequence systems do however not change. Zero sequence third harmonic currents exist only for single-phase non-linear loads, during balanced conditions. For unbalanced conditions, during load unbalanced or unbalanced of the power system all harmonics and the fundamental can consist of any sequence component [2]. In this paper we discussed about harmonic effect due to unbalanced in magnitude, in phase, and in magnitude and phase current source and shows the THD and TIF at all buses each phase.

To describe power quality of power system, THD (Total harmonic Distortion) is an important index widely used. It considers the contribution of every individual harmonic component on the signal. Total Harmonic Distortion in $69 \mathrm{kV}$ and below for individual Voltage Distortion 3\% and $5.0 \%$ for THD. It's defined for voltage signal

$T H D_{V}=\frac{\sqrt{\sum_{h=2}^{\infty} V_{h}^{2}}}{V_{1}}$

and for current signal: 


$$
T H D_{i}=\frac{\sqrt{\sum_{h=2}^{\infty} I_{h}^{2}}}{I_{1}}
$$

where $\mathrm{THD}_{\mathrm{V}}$ is Total Harmonic Distortion for voltage, $\mathrm{V}_{\mathrm{h}}$ is voltage at harmonic order, $\mathrm{h}$ is harmonic order, $\mathrm{THD}_{\mathrm{i}}$ is Total Harmonic Current and $\mathrm{I}_{\mathrm{h}}$ is current at harmonic order.

\section{Power System Modelling}

In general, the network may contain several harmonic sources and may be unbalanced. The derivation of the harmonic voltages and currents will, therefore, require a three-phase harmonic flow solution. The detail of components representation depends on their relative position with respect to the harmonic source, as well as their size in comparison with harmonic source. [3-5]

\section{A. Transmission Line}

For three-phase system, the elements of admittance matrix are themselves $3 \times 3$ matrices consisting of self and transfer admittances between phases. Usually, for long transmission line is used the equivalent PI model.

\section{B. Generator and Transformer}

The generators and transformers can be modeled as a series combination of resistance and inductive reactance, i.e.

$Y_{g h}=\frac{1}{R \sqrt{h}+j X_{d}^{\prime \prime} h}$ for the generator

$$
Y_{t h}=\frac{1}{R \sqrt{h}+j X_{t} h} \text { for the transformer }
$$

where $\mathrm{R}$ is derived from the machine power losses, $\mathrm{X}_{\mathrm{d}}{ }^{\prime}$ is the generator subtransient reactance, and $X_{t}$ is the transformer's short circuit reactance[3].

A frequency dependent multiplying factor can be added to the reactance terms to account for skin effect.

\section{Loads}

The three-phase group is used mainly in industry applications and in the power system. For example: Adjustable speed drive, Large UPS's, Arc furnaces, HVDClinks, SVC's [2]. The load is modeled as: a) loads are not considered in the power flow at harmonic frequency, b) parallel combination of resistance and inductive reactance, c) reactance is assumed to be frequency dependent while parallel resistance is kept constant, d) derived by measurements on medium voltage load using audio frequency ripple control generators, e) the load impedance calculated at $50 \mathrm{~Hz}$ remains constant for all frequencies and f) series combination of resistance and inductive reactance [4]. In this paper the load model as parallel combination of resistance and reactance.

\section{D.Series Capacitors}

Series capacitors can be modeled as series element consisting of resistance (usually zero or negligible value) in series with reactance.

\section{E. Series Capacitors}

Each current source data consist of the bus number at which the current source is provided. This followed by injected current at the bus for harmonic orders.

\section{HARMONIC ANALYSIS}

A fundamental frequency load flow solution is needed to extend to accurately model multiple harmonic sources. Most of power system non-linearities manifest themselves as harmonic current sources, but sometimes, harmonic voltage sources are used to represent the distortion background present in the network prior to the installation of the new non-linear load, moreover, some power electronic applications apply voltage rather then current distortion.

The harmonic analysis steps are:

1. Determine the bus admittance matrix $Y_{h}$ at harmonic frequency

2. Invert $Y_{h}$ to $Z_{h}$ (bus impedance matrix) at harmonic frequency

3. Using $[V]_{h}=[Z]_{h}[I]_{h}$, determine the harmonic voltage at all buses.

4. Determine the harmonic current flow in all branches.

5. If the harmonic distortion is above the prescribed value, filters are introduced at desired locations

6. Perform the harmonic power flow to determine the reduction in THD [4].

\section{DATA AND RESUlts}

Figure 1 shows the single line diagram of a 8-buses system with 1 generator $10 \mathrm{MVA}, 13.8 \mathrm{KV}, \mathrm{z}_{1}=\mathrm{z}_{2}=\mathrm{z}_{0}=$ j0.02857, 2 current sources and 2 unbalanced loads in MW and MVA where load $I$ at bus $2 Z_{A}=1+j 0.5, Z_{B}=1.2+j 0.7$, $\mathrm{Z}_{\mathrm{C}}=0.9+\mathrm{j} 0.35$ and load II at bus $3 \mathrm{Z}_{\mathrm{A}}=0.5+\mathrm{j} 0.025$, $Z_{B}=1+j 0.5, \quad Z_{C}=0.7+j 0.35,3$ series capacitor 10 MVA, $13.8 \mathrm{kV}$ and $\mathrm{x}=0.00476$ in bus $1-3, \mathrm{x}=0.0238$ in bus $3-5$ and $\mathrm{x}=0.0226$ in bus 5-7, 4 transformers 10MVA, $13.8 / 0.48 \mathrm{kV}, \mathrm{x}=0.22 \mathrm{p}$.u in bus $1-2,13.8 / 4.16 \mathrm{kV}, \mathrm{x}=0.11 \mathrm{p} . \mathrm{u}$ in bus $3-4,13.8 / 4.16 \mathrm{kV}, \mathrm{x}=0.055 \mathrm{p}$.u in bus $5-6$, and $13.8 / 4.16 \mathrm{kV}, \mathrm{x}=0.11 \mathrm{p} . \mathrm{u}$ in bus $7-8$. Base $100 \mathrm{MVA}, 60 \mathrm{~Hz}$.

Table 1. shows the RMS Current Source Data at bus 2 and bus 4 Table 2 Harmonic Distortion Factor (HDF) in phase A, B, C at all buses and Table 3 the result of Telephone Influence Factors (TIF) for all bus.. Figure 2 shows Total Voltage Distortion Factor (TVDF) and TVDF average at all buses in phase A, B and C. with Unbalanced Magnitude harmonic Current Source. And from Table 6 until Table 8 and Fig 3, for case Unbalanced Phase Angle Harmonic Current Source. We see, that from both cases, THD for case 1, at bus 4 in phase A, B and C are differ, while for case 2 rather same. But, THD in all cases are below $3.0 \%$. 
Table 1. Series reactor data

\begin{tabular}{|c|c|c|c|}
\hline $\begin{array}{c}\text { Bus No } \\
\text { p-q }\end{array}$ & MVA rating & kV rating & $\begin{array}{c}\text { Reactance in } \\
\text { p.u }\end{array}$ \\
\hline $1-3$ & 10 & 13.8 & 0.00476 \\
\hline $3-5$ & 10 & 13.8 & 0.0238 \\
\hline $5-7$ & 10 & 13.8 & 0.02286 \\
\hline
\end{tabular}

Table 2. Two winding transformer data

\begin{tabular}{|c|c|c|c|c|c|}
\hline $\begin{array}{c}\text { Bus No } \\
\mathrm{p}-\mathrm{q}\end{array}$ & $\begin{array}{c}\text { MVA } \\
\text { rating }\end{array}$ & $\begin{array}{c}\text { Primary } \\
\mathrm{kV}\end{array}$ & $\begin{array}{c}\text { Second } \\
\mathrm{kV}\end{array}$ & $\begin{array}{c}\mathrm{Z} \text { in } \\
\mathrm{p} . \mathrm{u}\end{array}$ & $\begin{array}{c}\mathrm{X} / \mathrm{R} \text { in } \\
\mathrm{p} . \mathrm{u}\end{array}$ \\
\hline $1-2$ & 10 & 13.8 & 0.48 & 0.22 & 0.9999 \\
\hline $3-4$ & 10 & 13.8 & 4.16 & 0.11 & 0.9999 \\
\hline $5-6$ & 10 & 13.8 & 4.16 & 0.055 & 0.9999 \\
\hline $7-8$ & 10 & 13.8 & 4.16 & 0.11 & 0.9999 \\
\hline
\end{tabular}

Table 3. RMS current source data at bus 2 and bus 4 with unbalanced magnitude current sources

\begin{tabular}{|c|c|c|c|c|c|c|c|c|c|c|c|c|}
\hline & \multicolumn{6}{|c|}{ Current Source at bus 2} & \multicolumn{6}{|c|}{ Current Source at bus 4} \\
\hline Hno & $\begin{array}{l}\text { ph A } \\
\text { amp }\end{array}$ & Angle & $\begin{array}{l}\text { ph B } \\
\text { amp }\end{array}$ & Angle & $\begin{array}{l}\text { ph C } \\
\text { amp }\end{array}$ & Angle & $\begin{array}{l}\text { ph A } \\
\text { amp }\end{array}$ & Angle & $\begin{array}{l}\text { ph B } \\
\text { amp }\end{array}$ & Angle & $\begin{array}{l}\text { ph C } \\
\text { amp }\end{array}$ & Angle \\
\hline 5 & $\begin{array}{l}145.00 \\
0\end{array}$ & 0.00 & $\begin{array}{l}142.00 \\
0\end{array}$ & -120.00 & $\begin{array}{l}147.00 \\
0\end{array}$ & 120.00 & 68.000 & 0.00 & $\begin{array}{l}6.5 .000 \\
5 \\
\end{array}$ & -120.00 & $\begin{array}{l}66.87 \\
7 \\
\end{array}$ & 120.00 \\
\hline 7 & 98.000 & 0.00 & 99.000 & -120.00 & 97.500 & 120.00 & 47.000 & 0.00 & 45.000 & -120.00 & $\begin{array}{l}45.50 \\
0 \\
\end{array}$ & 120.00 \\
\hline 11 & 55.000 & 0.00 & 54.500 & -120.00 & 57.000 & 120.00 & 27.000 & 0.00 & 24.000 & -120.00 & $\begin{array}{l}28.00 \\
0\end{array}$ & 120.00 \\
\hline 13 & 43.500 & 0.00 & 42.000 & -120.00 & 40.000 & 120.00 & 22.000 & 0.00 & 19.00 & -120.00 & $\begin{array}{l}18.50 \\
0\end{array}$ & 120.00 \\
\hline 17 & 27.000 & 0.00 & 26.500 & -120.00 & 25.000 & 120.00 & 15.000 & 0.00 & 13.500 & -120.00 & $\begin{array}{l}14.80 \\
0\end{array}$ & 120.00 \\
\hline 19 & 22.000 & 0.00 & 21.000 & -120.00 & 20.500 & 120.00 & 10.500 & 0.00 & 9.500 & -120.00 & 9.400 & 120.00 \\
\hline 23 & 15.000 & 0.00 & 15.500 & -120.00 & 14.900 & 120.00 & 7.500 & 0.00 & 7.000 & -120.00 & 6.850 & 120.00 \\
\hline 25 & 12.500 & 0.00 & 11.500 & -120.00 & 11.000 & 120.00 & 6.200 & 0.00 & 5.750 & -120.00 & 5.500 & 120.00 \\
\hline 29 & $10 . .500$ & 0.00 & 10.800 & -120.00 & 10.100 & 120.00 & 4.900 & 0.00 & 4.750 & -120.00 & 4.600 & 120.00 \\
\hline 31 & 9.000 & 8.500 & 8.750 & -120.00 & 8.970 & 120.00 & 4.300 & 0.00 & 4.180 & -120.00 & 4.000 & 120.00 \\
\hline 35 & 8.200 & 0.00 & 8.000 & -120.00 & 7.900 & 120.00 & 3.832 & 0.00 & 3.900 & -120.00 & 3.750 & 120.00 \\
\hline
\end{tabular}

Table 4. Harmonic distortion factors

\begin{tabular}{|l|l|l|l|l|l|}
\hline Node & Name & $\%$ HDF-A & \% HDF-B & \% HDF-C & $\%$ HDF AVR \\
\hline 1 & Gen 1 & 0.0128 & 0.0120 & 0.0123 & 0.0124 \\
\hline 2 & User 1 & 0.0057 & 0.0056 & 0.0057 & 0.0057 \\
\hline 3 & Supply 2 & 0.0150 & 0.0142 & 0.0145 & 0.0146 \\
\hline 4 & User 2 & 0.6251 & 0.5790 & 0.5958 & 0.6003 \\
\hline 5 & Supply 3 & 0.0150 & 0.0142 & 0.0145 & 0.0146 \\
\hline 6 & User 3 & 0.0147 & 0.0138 & 0.0142 & 0.0142 \\
\hline 7 & Supply 4 & 0.0150 & 0.0142 & 0.0145 & 0.0146 \\
\hline 8 & User 4 & 0.0147 & 0.0138 & 0.0142 & 0.0142 \\
\hline
\end{tabular}

Table 5. Telephone influence factors (TIF)

\begin{tabular}{|l|l|l|l|l|}
\hline Node & \% TIF - A & $\begin{array}{l}\text { \% TIF- } \\
\text { B }\end{array}$ & \%TIF-C & $\begin{array}{l}\text { \% TIF- } \\
\text { AVR }\end{array}$ \\
\hline 1 & 33.04 & 30.6 & 31.47 & 31.72 \\
\hline 2 & 13.94 & 13.72 & 13.65 & 13.77 \\
\hline 3 & 38.94 & 36.04 & 37.07 & 37.37 \\
\hline 4 & 2955 & 2775 & 2774 & 2836 \\
\hline 5 & 38.94 & 36.04 & 37.07 & 37.37 \\
\hline 6 & 37.99 & 35.16 & 36.17 & 36.46 \\
\hline 7 & 38.94 & 36.04 & 37.07 & 37.37 \\
\hline 8 & 37.99 & 35.16 & 36.17 & 36.46 \\
\hline
\end{tabular}

Table 6. RMS current source data at bus 2 and bus 4 unbalanced phase angle harmonic source

\begin{tabular}{|c|c|c|c|c|c|c|c|c|c|c|c|c|}
\hline & \multicolumn{6}{|c|}{ Current Source at bus 2} & \multicolumn{6}{|c|}{ Current Source at bus 4} \\
\hline Hno & $\begin{array}{l}\text { ph A } \\
\text { amp }\end{array}$ & Angle & $\begin{array}{l}\text { ph B } \\
\text { amp }\end{array}$ & Angle & $\begin{array}{l}\text { ph C } \\
\text { amp }\end{array}$ & Angle & $\begin{array}{l}\text { ph A } \\
\text { amp }\end{array}$ & Angle & $\begin{array}{l}\text { ph B } \\
\text { amp }\end{array}$ & Angle & $\begin{array}{l}\text { ph C } \\
\text { amp }\end{array}$ & Angle \\
\hline 5 & 143.52 & 0.00 & 143.52 & -120.00 & 143.52 & 135.00 & 66.877 & 0.00 & 66.877 & -120.00 & 66.877 & 125.00 \\
\hline 7 & 98.73 & 0.00 & 98.73 & -120.00 & 98.73 & 135.00 & 45.978 & 0.00 & 45.978 & -120.00 & 45.978 & 125.00 \\
\hline 11 & 54.57 & 0.00 & 54.57 & -120.00 & 54.57 & 135.00 & 25.427 & 0.00 & 25.27 & -120.00 & 25.427 & 125.00 \\
\hline 13 & 42.61 & 0.00 & 42.61 & -120.00 & 42.61 & 135.00 & 19.854 & 0.00 & 19.854 & -120.00 & 19.854 & 125.00 \\
\hline 17 & 26.163 & 0.00 & 26.163 & -120.00 & 26.163 & 135.00 & 12.191 & 0.00 & 12.191 & -120.00 & 12.191 & 125.00 \\
\hline 19 & 20.183 & 0.00 & 20.183 & -120.00 & 20.183 & 135.00 & 9.405 & 0.00 & 9.405 & -120.00 & 9.405 & 125.00 \\
\hline 23 & 14.95 & 0.00 & 14.95 & -120.00 & 14.95 & 135.00 & 6.966 & 0.00 & 6.966 & -120.00 & 6.966 & 125.00 \\
\hline 25 & 11.96 & 0.00 & 11.96 & -120.00 & 11.96 & 135.00 & 5.573 & 0.00 & 5.573 & -120.00 & 5.573 & 125.00 \\
\hline 29 & 10.465 & 0.00 & 10.465 & -120.00 & 10.465 & 135.00 & 4.876 & 0.00 & 4.876 & -120.00 & 4.876 & 125.00 \\
\hline 31 & 8.97 & 0.00 & 8.97 & -120.00 & 8.97 & 135.00 & 4.180 & 0.00 & 4.180 & -120.00 & 4.180 & 125.00 \\
\hline
\end{tabular}




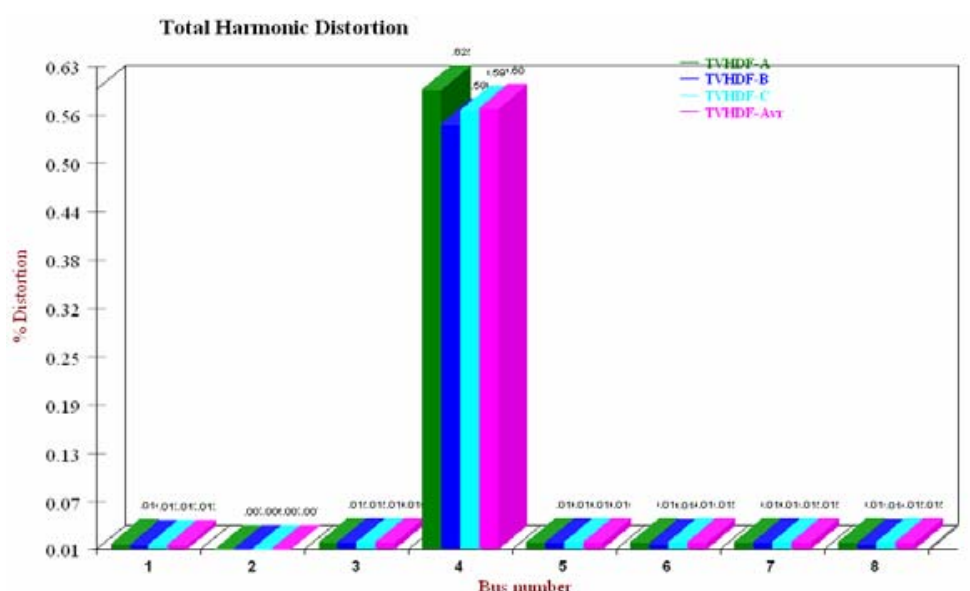

Figure. 2. THD for unbalanced magnitude Harmonic current sources

Table 7. Harmonic distortion factor

\begin{tabular}{|l|l|l|l|l|l|}
\hline Node & Name & \% HDF-A & \% HDF-B & \% HDF-C & \% HDF AVR \\
\hline 1 & Gen 1 & 0.0123 & 0.0123 & 0.0124 & 0.0123 \\
\hline 2 & User 1 & 0.0056 & 0.0056 & 0.0056 & 0.0056 \\
\hline 3 & Supply 2 & 0.0145 & 0.0145 & 0.0145 & 0.0145 \\
\hline 4 & User 2 & 0.5864 & 0.5864 & 0.5865 & 0.5865 \\
\hline 5 & Supply 3 & 0.0145 & 0.0145 & 0.0145 & 0.0145 \\
\hline 6 & User 3 & 0.0142 & 0.0142 & 0.0142 & 0.0142 \\
\hline 7 & Supply 4 & 0.01545 & 0.0145 & 0.0145 & 0.0145 \\
\hline 8 & User 4 & 0.0142 & 0.0142 & 0.0142 & 0.0142 \\
\hline
\end{tabular}

Table 8. Telephone influence factors (TIF)

\begin{tabular}{|l|l|l|l|l|}
\hline $\begin{array}{l}\text { Nod } \\
\mathrm{e}\end{array}$ & $\%$ TIF - A & $\%$ TIF- B & \%TIF-C & $\%$ TIF-AVR \\
\hline 1 & 30.96 & 30.96 & 30.97 & 30.96 \\
\hline 2 & 13.74 & 13.74 & 13.66 & 13.71 \\
\hline 3 & 36.46 & 36.46 & 36.48 & 36.47 \\
\hline 4 & 2955 & 2760 & 2760 & 2760 \\
\hline 5 & 36.46 & 36.46 & 36.47 & 36.47 \\
\hline 6 & 35.57 & 35.57 & 35.59 & 35.58 \\
\hline 7 & 36.46 & 36.46 & 36.48 & 36.47 \\
\hline 8 & 35.57 & 35.57 & 36.59 & 35.58 \\
\hline
\end{tabular}

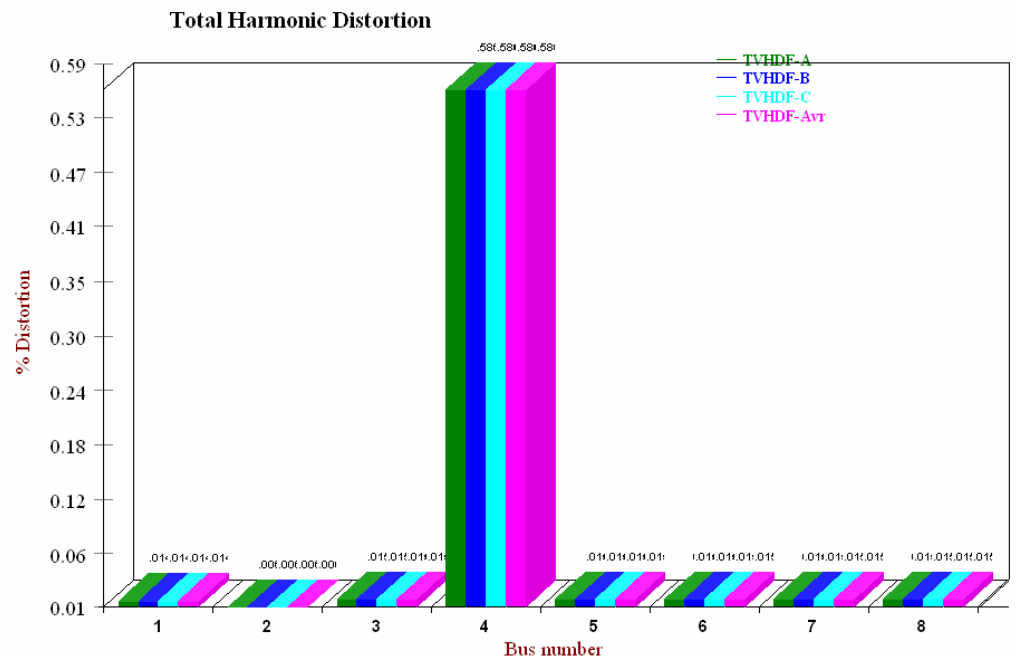

Figure 3. THD for unbalanced phase angle harmonic current source 


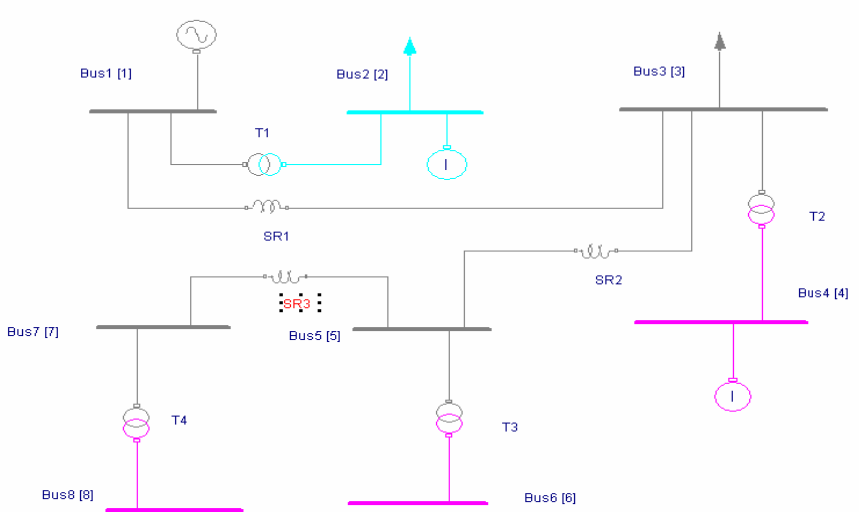

Figure 1. Single line diagram for example

\section{CONCLUSIONS}

Total harmonic Distortion (THD) and Total Voltage Harmonic Distortion (TVHD) is higher in bus 4 at current source harmonic is low, but it is below $3.0 \%$ so, do not need filter to eliminate harmonic and Telephone Influence Factor (TIF) is under standard value too.

\section{REFERENCES}

[1] Patricia Irwin, PE, Power Quality, Dealing with harmonic distortion, necdigest $^{\mathrm{TM}}$, Fall 2003.

[2] Johan Lundquist, "On harmonic Distortion in Power Systems", Dissertation, Dept of Electric Power Engineering, Chalmers University of Technology Göteborg, Sweden, 2001, pp 6-23.

[3] J. Arrrilaga Bruce C Smith, Neville R Watson, Alan R Wood, Power System Harmonic Analysis, John Wiley \& Sons, Singapore, 2000,pp 98-107.

[4] Power Research \& Development Consultants Pvt, MiPower, How To Solve, Bangalore-560010.

[5] J. Arrilaga, N.R. Watson, S.Chen, Power System Quality Assesment, John Wiley \& Sons, Buffins lane. Chichester,2001, pp 177-202. 


\title{
Simulation of Diesel Engine Fueled by Compressed Natural Gas Performance Using GT-Power
}

\author{
Rosli A. Bakar, Semin and Abdul R. Ismail
}

\begin{abstract}
The purpose of the present study is to investigate effects of diesel engine fueled by compressed natural gas performance using simulation. CNG engines are different from diesel engines. A GT-Power computational model and simulation test for the evaluation of four-stroke single-cylinder diesel engine using natural gas fuel is currently not available. In this research the computational modeling using commercial computational dynamics of GT-SUITE 6.2 software was development to simulate the engine performance. This paper describes the development of a CNG engine modeling and simulation for the evaluation of natural gas engine fuel performance in diesel engine applications. The simulation uses a four-stroke single-cylinder diesel engine $407 \mathrm{cc}$. The developed of CNG engine simulation is capable of discriminating engine with different fuels. Output of the model simulation shown the effect of diesel engine fueled by CNG performance with simulate in any parameters.
\end{abstract}

Keywords: compressed natural gas, diesel engine, simulation

\section{INTRODUCTION}

In the diesel engines, the details of the diesel engine design vary significantly over the engine performance and size range. In particular, different combustion chamber geometries and fuel injection characteristics are required to deal effectively with major diesel engine design problem achieving sufficiently rapid fuel-air mixing rates to complete the fuel-burning process in the time available. A wide variety of inlet port geometries, cylinder head and piston shapes, and fuel-injection patterns are used to accomplish this over the diesel size range. Heywood [7] written that the engine ratings usually indicate the highest power at which manufacturer expect their products to give satisfactory of power, economy, reliability and durability under service conditions. Maximum torque and the speed at which it is achieved, is usually given also.

Rosli A. Bakar, Automotive Focus Group, Faculty of Mechanical Engineering, University Malaysia Pahang, Locked Bag 12, 25000 Kuantan, Pahang, Malaysia.

Semin, Automotive Focus Group, Faculty of Mechanical Engineering, University Malaysia Pahang, Locked Bag 12, 25000 Kuantan, Pahang, Malaysia. (phone: 609-5492217; fax: 609-5492244; e-mail: semin utec@yahoo.com).

Abdul R. Ismail, Automotive Focus Group, Faculty of Mechanical Engineering, University Malaysia Pahang, Locked Bag 12, 25000 Kuantan, Pahang, Malaysia.
The Compressed Natural Gas (CNG) has long been used in industries and stationary engines, but the application of $\mathrm{CNG}$ as a transport fuel has been considerably advanced over the last decade by the development of lightweight high-pressure storage cylinders. Most existing of natural gas for vehicles engines use in the petrol engines with modified by after-market retrofit conversions and retain bi-fuel capability. Bi-fuelled vehicle conversions generally suffer from a power loss and can encounter driveability problems, due to the design and/or installation of the retrofit packages. Significant improvements in power and driveability can be realized with more sophisticated, vehicle-specific retrofit kits, or in factory-built bi-fuelled vehicles. Engine and vehicle manufacturers are increasingly involved in the development of original engine equipment, especially for heavy-duty diesel replacement applications, although many still regard the demand as too limited and dispersed to warrant large-scale manufacture. This paper is to explore the application of dedicated or mono fuel compressed natural gas $(\mathrm{CNG})$ as an alternative fuel for diesel engines and its performance effect in the diesel engines using simulation. Dedicated natural gas engines are optimized for the natural gas fuel only. They can be derived from petrol engines or may be designed for the purpose. Until manufacturer original equipment (OE) engines are more readily available, however, the practice of converting diesel engines to spark ignition will continue, which involves the replacement of diesel fuelling equipment by a gas carburetor and the addition of an ignition system and spark plugs.

GT-POWER is the leading engine simulation tool used by engine and vehicle makers and suppliers and is suitable for analysis of a wide range of engine issues [8]. GT-POWER is designed applicable to all type of internal combustion engines and provides the user with many components to model any advanced concept. GT-POWER is based on onedimensional gas dynamics, representing the flow and heat transfer in the piping and in the other component of an engine system. GT-POWER is one model from GT-SUITE software applications [8]. A four-stroke direct-injection diesel engine typical was measured manually and modeled using GT-POWER in this paper to investigate the engine performance. 
The investigation in this research is the performance effect of diesel fuel substituted to compressed natural gas $(\mathrm{CNG})$ fuel as an alternative fuel for the same diesel engine specification. Its known that the properties of diesel fuel is difference with the properties of compressed natural gas (CNG) or methane. The diesel and CNG properties have shown in 'table 1'. The objects were investigated in this reseach are brake power, brake torque, brake specific fuel consumption, volumetric efficiency and pressure in cylinder.

Table 1. CNG properties at $25^{\circ} \mathrm{C}$ and $1 \mathrm{~atm}[10]$.

\begin{tabular}{|c|c|}
\hline Property & $\mathrm{CNG}$ \\
\hline Density $\left(\mathrm{kg} / \mathrm{m}^{3}\right)$ & 0.72 \\
\hline Flammability limits (volume $\%$ in air) & $4.3-15$ \\
\hline Flammability limits $(\phi)$ & $0.4-1.6$ \\
\hline Autoignition temperature in air $(\mathrm{K})$ & 723 \\
\hline Minimum ignition energy $(\mathrm{mJ})^{\mathrm{b}}$ & 0.28 \\
\hline Flame velocity $\left(\mathrm{m} \mathrm{s}^{-1}\right)^{\mathrm{b}}$ & 0.38 \\
\hline Adiabatic flame temperature $(\mathrm{K})^{\mathrm{b}}$ & 2214 \\
\hline Quenching distance $(\mathrm{mm})^{\mathrm{b}}$ & $2.1^{\mathrm{c}}$ \\
\hline Stoichiometric fuel/air mass ratio & 0.069 \\
\hline Stoichiometric volume fraction $\%$ & 9.48 \\
\hline Lower heating value $(\mathrm{MJ} / \mathrm{kg})$ & 45.8 \\
\hline Heat of combustion $\left(\mathrm{MJ} / \mathrm{kg}_{\text {air }}\right)^{\mathrm{b}}$ & 2.9 \\
\hline
\end{tabular}

\section{Simulation Methodology}

The single cylinder direct injection diesel engines have been modeled and simulated using diesel fuel and compressed natural gas fuel in this paper. The specification of the diesel engine model was presented in 'table 2' below:

Table 2. Diesel engine specification

\begin{tabular}{ll}
\hline Engine Specification & Value \\
\hline Bore $(\mathrm{mm})$ & 86 \\
Stroke $(\mathrm{mm})$ & 70 \\
Displacement $(\mathrm{L})$ & 0.406 \\
Initial angle of fuel delivery & $-22^{\circ} \pm 1^{\circ} \mathrm{BTDC}$ \\
$(\mathrm{CA})$ & \\
Intake valve clearance $(\mathrm{mm})$ & $0.1 \sim 0.15$ (cold state) \\
Exhaust valve clearance $(\mathrm{mm})$ & $0.1 \sim 0.15($ cold state) \\
Exhaust temperature $\left({ }^{\circ} \mathrm{C}\right)$ & $\leq 480$ \\
Pressure of injection $(\mathrm{MPa})$ & $\leq 19.6 \pm 0.49$ \\
Mean effective pressure $(\mathrm{kPa})$ & $561.6 @ 3000 \mathrm{rpm}$ \\
& $543.5 @ 3600 \mathrm{rpm}$ \\
Consumption rate of fuel $(\mathrm{g} / \mathrm{kW})$ & $\leq 273.5 @ 3000 \mathrm{rpm}$ \\
& $\leq 285.7 @ 3600 \mathrm{rpm}$ \\
\hline
\end{tabular}

To simulate the engine performance in this paper, the research start on developed the GT-POWER of singlecylinder four-stroke direct-injection diesel engine modeling. The model is done step by step, the first step is open all of the selected diesel engine components to measure the engine components part size. Then, the engine components size data will be input to the GT-POWER library of the all engine components data. To create the GT-POWER model, select Window and then Tile with Template Library from the menu. This will place the GT-POWER template library on the left hand side of the screen. The template library contains all of the available templates that can be used in GT-POWER. Some of these templates those that will be needed in the project need to be copied into the project before they can be used to create objects and parts. For the purpose of this model, click on the icons listed and drag them from the template library into the project library. Some of these are templates and some are objects that have already been defined and included in the GT-POWER template library [8]. In this model the engine according to Bakar [9] was breakdown to the tree system, there are intake system, engine cylinder and fuel injection system, and exhaust system. In the selected diesel engine, the intake system its have any component, size and different data. The system was started from environment till the intake valve. The engine cylinder and fuel injection system is focused in engine cylinder performance were support diesel fuel from fuel injection system, fresh air intake system and exhaust gas to exhaust system. There are any components in the engine cylinder and fuel injection system in the diesel engine. The components, size and data must be record and inserted to the GT-POWER form. The components are injector, cylinder and engine. The last system in the diesel engine is the exhaust system. In this system was started from exhaust valve and finished in the environment. All of this diesel engine components connected by orifficeconn. Then, the modeling the diesel engine model using GTPOWER software in this research can be developed. The modeling focuses on fuel injection shown in 'figure 1'. Data component and fuel nozzle hole needed for building an engine model. A list of information that is needed to build a model in GT-POWER is included in library. Not every item will be needed for all models. If the model is being built at an early design stage, determining optimal values for some of the items listed may be the purpose of the simulation. If this is the case, those particular attributes should be defined as parameters and run for a series of cases to determine an optimal value.

Data in engine characteristics are compression ratio, firing order, inline or V configuration, V-angle (optional), 2 or 4 stroke. Data in cylinder geometry are bore, stroke, connecting rod length, pin offset, piston TDC clearance height, head bowl geometry, piston area and head area. Data in intake and exhaust system is geometry of all components. Data in throttles are throttle location and discharge coefficients versus throttle angle in both flow directions. Data in fuel injectors are location and number of injectors, number of nozzle holes and nozzle diameter, injection rate, fuel type and LHV. Data in intake and exhaust valves are valve diameter, lift profile, discharge coefficient, valve lash. Data in ambient state are pressure, temperature and humidity. Performance data can be very useful when tuning a model after it has been built. This research is focuses in diesel engine fuel changed to compressed natural gas simulation. The GT-POWER model development of single cylinder direct injection diesel engine is shows in 'figure 1'.

In the GT-POWER model data entries, air with compositions of $76.7 \% \mathrm{~N}_{2}$ and $23.3 \% \mathrm{O}_{2}$ at pressure 1 bar and temperature $26.85^{\circ} \mathrm{C}$ is being input to env-01. The properties for env-02 are similar with env-01. The intake system consists of intrunner airfilter or intake runner for air 
filter, airfilter-01 and intrunner-01. These diesel engine parts represent the intake system pipes with specific parameters as shown in 'table 3'. The diesel engine exhaust system of GT-POWER model consists of exhport-01, exhrunner-01, muffler-01 and exhrunner-exit-01. These diesel engine parts also represent the exhaust system pipes with specific parameters as shown in 'table 4'. In the diesel engine model using GT-POWER, intvalve-01 represents intake valve and exhvalve- 01 represents exhaust valve. The diesel engine component parameters of these valves are shown in 'table 5 '.

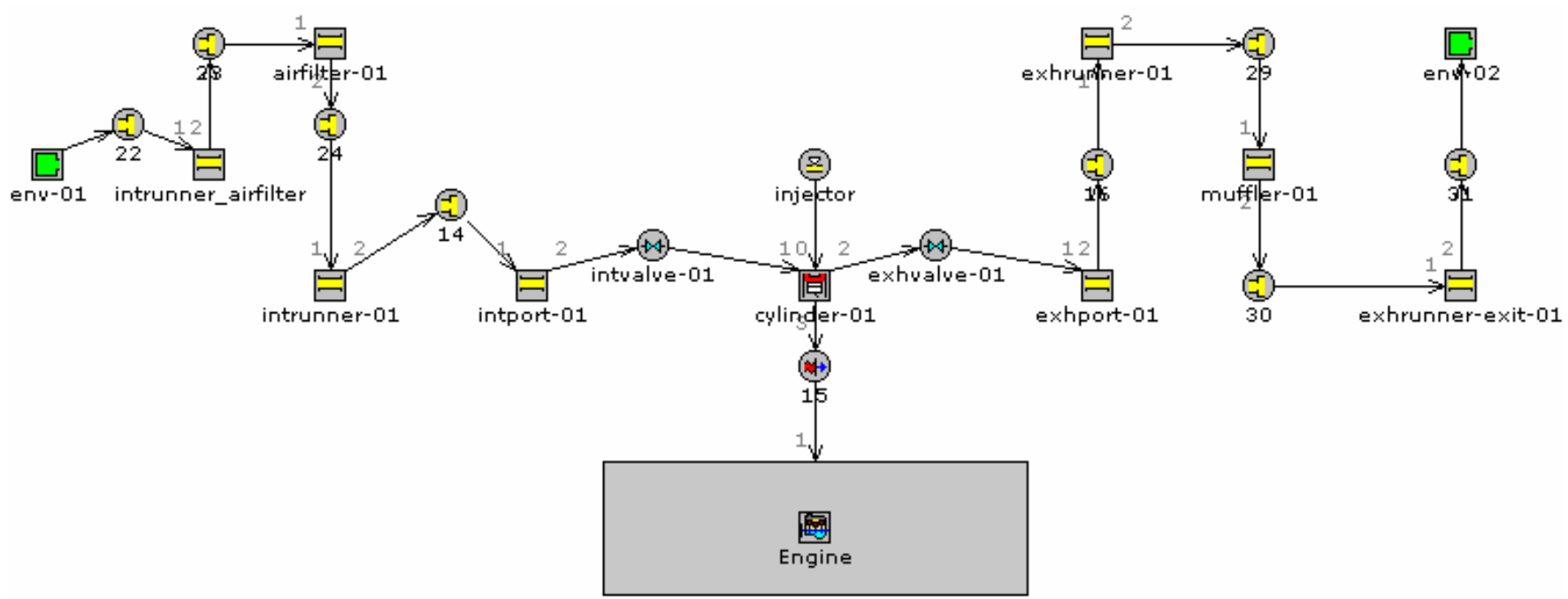

Figure 1. Single cylinder direct injection diesel engine model using GT-POWER

Table 3. Diesel engine parameters for intake system data

\begin{tabular}{lllll}
\hline & $\begin{array}{l}\text { Intrunner } \\
\text { airfilter }\end{array}$ & $\begin{array}{l}\text { Airfilter- } \\
01\end{array}$ & $\begin{array}{l}\text { Intrunner- } \\
01\end{array}$ & $\begin{array}{l}\text { Intport- } \\
01\end{array}$ \\
\hline Dia. inlet end (mm) & 44.8800 & 159.632 & 40.44 & 40.6973 \\
Dia. outlet end (mm) & 62.13 & 159.632 & 40.1 & 32.78 \\
Length (mm) & 80 & 69.64 & 59.7 & 55.2 \\
Disc. length (mm) & 34.4 & 34.4 & 34.4 & 34.4 \\
Wall temperat. $\left({ }^{\circ} \mathrm{C}\right)$ & 28.85 & 28.85 & 76.85 & 176.85 \\
\hline
\end{tabular}

Table 4. Diesel engine Parameters for exhaust system data

\begin{tabular}{lllll}
\hline & exhport- & exhrunner & Muffler- & $\begin{array}{l}\text { exhrunner } \\
\text {-exit-01 }\end{array}$ \\
\hline Dia. inlet end $(\mathrm{mm})$ & 26.38 & 27.86 & 138.88 & 34.6 \\
Dia. outlet end $(\mathrm{mm})$ & 29.82 & 27.86 & 138.88 & 34.6 \\
Length $(\mathrm{mm})$ & 40.4 & 98 & 283.4 & 25.6 \\
Disc. length $(\mathrm{mm})$ & 47.3 & 47.3 & 47.3 & 47.3 \\
Wall temperat. $\left({ }^{\circ} \mathrm{C}\right)$ & 480 & 480 & 480 & 480 \\
\hline
\end{tabular}

Table 5. Diesel engine parameters for intake and exhaust valves data

\begin{tabular}{lll}
\hline & Intvalve-01 & Exhvalve-01 \\
\hline Valve diameter $(\mathrm{mm})$ & 35.54 & 29.04 \\
CAM timing angle $\left({ }^{\circ} \mathrm{CA}\right)$ & 462.5 & 214.6 \\
Valve lash $(\mathrm{mm})$ & 0.125 & 0.125 \\
\hline
\end{tabular}

\section{RESULTS AND DISCUSSION}

The GT-POWER simulation results for performance of diesel engine fuelled using diesel fuel and $\mathrm{CNG}$ fuel are shown in 'figure 2', 'figure 3' and 'figure 4'.

'Figure 2' shows that when diesel fuel is being used, the brake power changes from $0.09 \mathrm{~kW}$ at $200 \mathrm{rpm}$ to $2 \mathrm{~kW}$ at $4000 \mathrm{rpm}$. The peak value is $4.3 \mathrm{~kW}$ at $3000 \mathrm{rpm}$ which is the maximum brake power for diesel fuel. Meanwhile when $\mathrm{CNG}$ fuel is being used the brake power changes from 0.04 $\mathrm{kW}$ at $200 \mathrm{rpm}$ to $2.4 \mathrm{~kW}$ at $4000 \mathrm{rpm}$. The maximum brake power for CNG fuel is $2.4 \mathrm{~kW}$ at $4000 \mathrm{rpm}$. By comparison, the maximum brake power of engine will reduce $44 \%$ when using CNG as fuel. The brake power performance of engine fuelled by diesel fuel and CNG fuel is shown in 'figure 2' below:

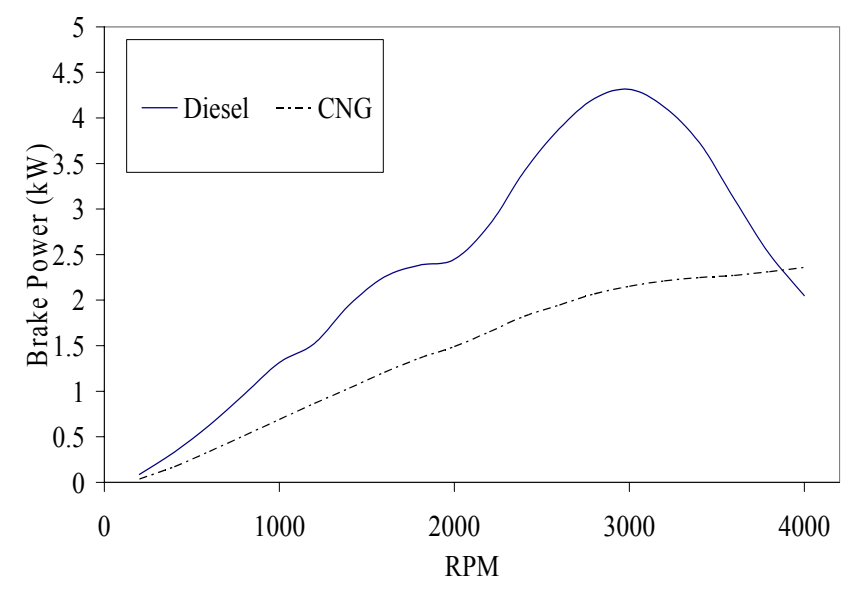

Figure 2. Brake power performance of engine fuelled by diesel fuel and CNG fuel 


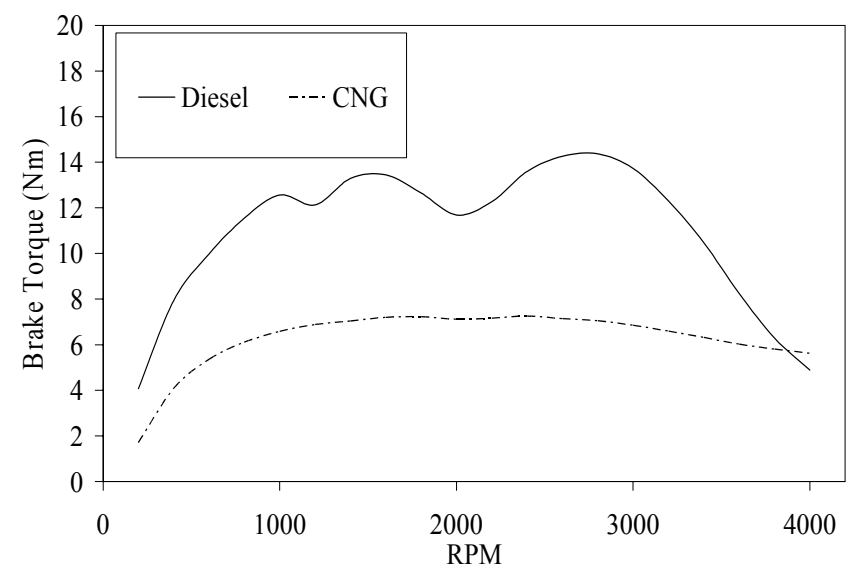

Figure 3. Brake torque performance of engine fuelled by diesel fuel and CNG fuel

The brake torque versus rpm performance of engine fuelled by diesel fuel and CNG fuel is shown in 'figure 3'. For diesel fuel, the brake torque changes from $4 \mathrm{Nm}$ at 200 $\mathrm{rpm}$ to $4.9 \mathrm{Nm}$ at $4000 \mathrm{rpm}$. There are three peak values; $12.6 \mathrm{Nm}$ at $1000 \mathrm{rpm}, 13.5 \mathrm{Nm}$ at $1500 \mathrm{rpm}$ and $14.4 \mathrm{Nm}$ at $2700 \mathrm{rpm}$. The maximum brake torque is $14.4 \mathrm{Nm}$ at 2700 rpm. Meanwhile, for $\mathrm{CNG}$ fuel, the brake torque changes from $1.7 \mathrm{Nm}$ at $200 \mathrm{rpm}$ to $5.6 \mathrm{Nm}$ at $4000 \mathrm{rpm}$. The maximum brake torque for this fuel is $7.3 \mathrm{Nm}$ at $2400 \mathrm{rpm}$. By comparison, the maximum brake torque will reduce by $49 \%$ when using $\mathrm{CNG}$ as fuel.

The brake specific fuel consumption of engine fuelled by diesel fuel and CNG fuel is shown in figure 4. For diesel fuel, the brake specific fuel consumption changes from $1232 \mathrm{~g} / \mathrm{kWh}$ at $200 \mathrm{rpm}$ to $1025 \mathrm{~g} / \mathrm{kWh}$ at $4000 \mathrm{rpm}$. The minimum brake specific fuel for diesel fuel is $349 \mathrm{~g} / \mathrm{kWh}$ at $2800 \mathrm{rpm}$. Meanwhile, for CNG fuel, the brake specific fuel consumption changes from $2940 \mathrm{~g} / \mathrm{kWh}$ at $200 \mathrm{rpm}$ to 891 $\mathrm{g} / \mathrm{kWh}$ at $4000 \mathrm{rpm}$. The minimum value for this fuel is 691 $\mathrm{g} / \mathrm{kWh}$ at $2400 \mathrm{rpm}$. By comparison, the minimum brake specific fuel consumption will increase $49 \%$ when using $\mathrm{CNG}$ as fuel.

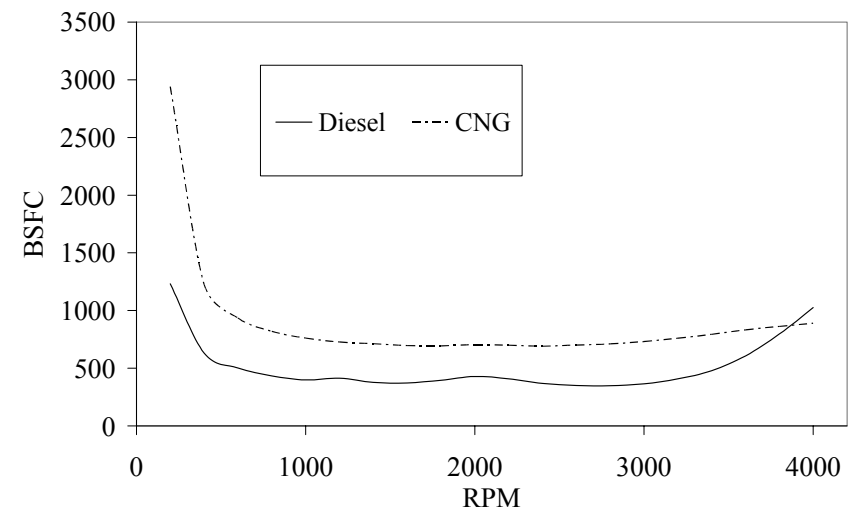

Figure 4. Brake specific fuel consumption of engine fuelled by diesel fuel and CNG fuel

The usage of $\mathrm{CNG}$ in the small diesel engine will increase brake specific fuel consumption and reduction in terms of brake power and brake torque. This is because of gas $(\mathrm{CNG})$ fuel will reduce volumetric efficiency compared to liquid (diesel) fuel as shown in 'figure 5 '.

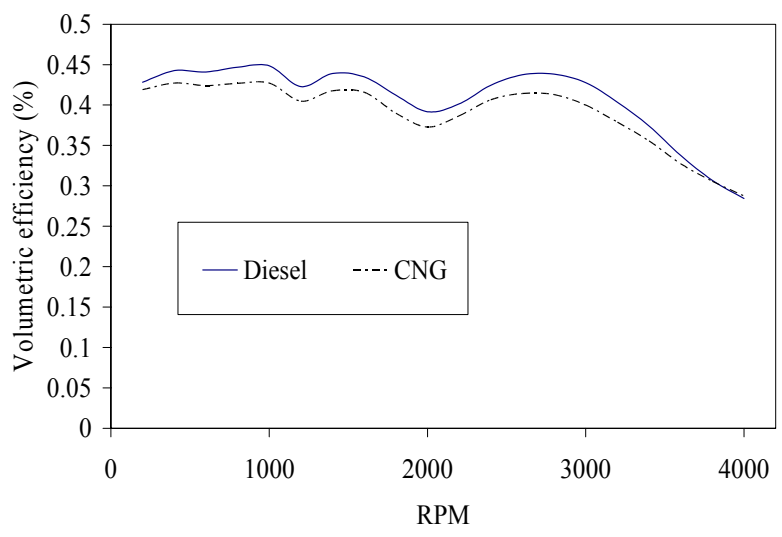

Figure 5. Volumetric efficiency of engine fuelled by diesel fuel and CNG fuel

Another factor that must be considered is composition of hydrocarbon for each fuel. Diesel fuel which have higher hydrocarbon than $\mathrm{CNG}$ fuel, will give more energy to the engine as shown in 'figure 6', 'figure 7' through 'figure 8'. At $1000 \mathrm{rpm}$ ignition process will occur at 5.7 and 4.5 degree crank angle for diesel and $\mathrm{CNG}$ respectively. The effect from this combustion will cause diesel fuel generates pressure 81 bar compare to $\mathrm{CNG}$ that will generates 70 bar. The pressure reduction is $8 \%$ from diesel fuel pressure.

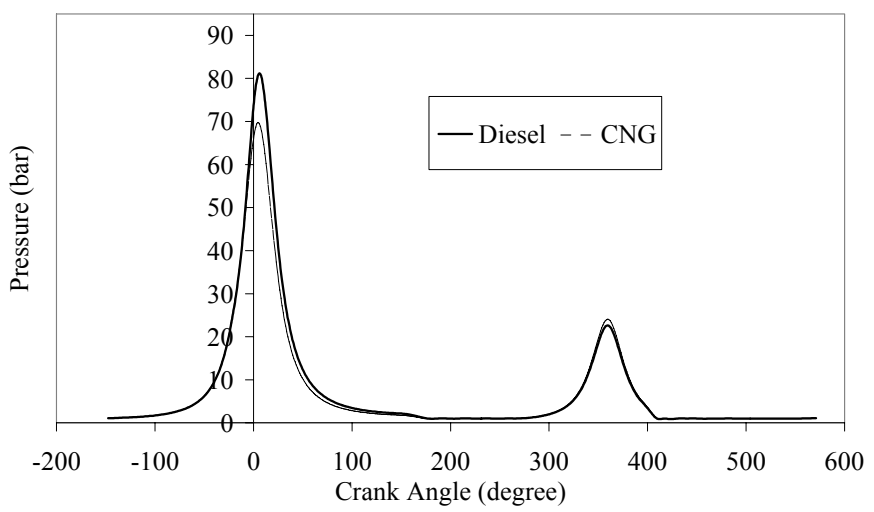

Figure 6. Variation of pressure engine fuelled by diesel fuel and $\mathrm{CNG}$ fuel with crank angle at $1000 \mathrm{rpm}$

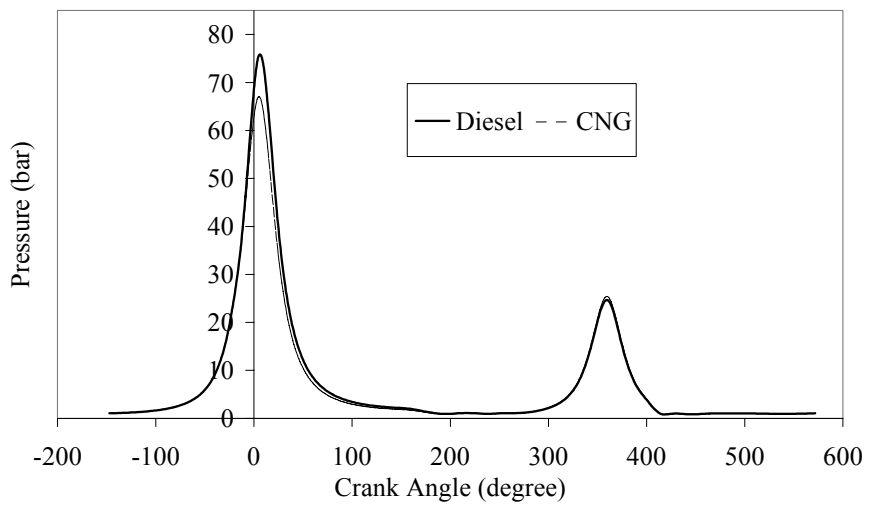

Figure 7. Variation of pressure engine fuelled by diesel fuel and CNG fuel with crank angle at $2000 \mathrm{rpm}$ 


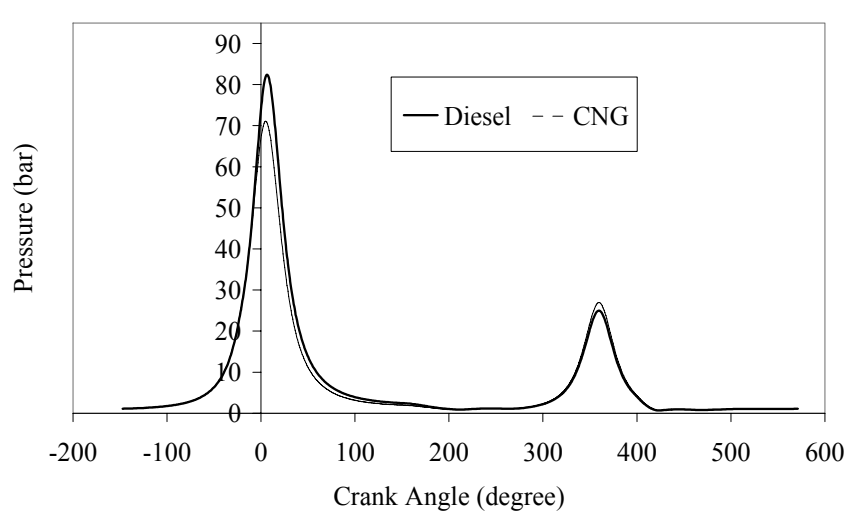

Figure 8. Variation of pressure engine fuelled by diesel fuel and $\mathrm{CNG}$ fuel with crank angle at $3000 \mathrm{rpm}$

At $2000 \mathrm{rpm}$ ignition process will occur at 5.7 and 5.5 degree crank angle for diesel and CNG respectively. The pressures that will be generated are 76 bar and 67 bar for diesel fuel and CNG fuel respectively as shown in figure 7. The pressure reduction is $12 \%$ from diesel fuel pressure. Meanwhile at $3000 \mathrm{rpm}$, ignition process will occur at 6.2 and 5.1 degree crank angle for diesel fuel and $\mathrm{CNG}$ fuel respectively. The pressures that will be generated are 82 bar and 71 bar as shown in 'figure 8 '. The pressure reduction is $13 \%$ from diesel fuel pressure.

\section{CONCLUSION}

From these simulations of diesel engine fueled by compressed natural gas performance using GT-POWER simulation studies, it is observed that $\mathrm{CNG}$ can be used as an alternative fuel into small diesel engine. The studied parameters show that there are reduction by $44 \%$ in brake power, $49 \%$ in brake torque and addition of $49 \%$ in brake specific fuel consumption. To ensure that the percentage can be reduced, the engine needs some modification. For further research, experimental study will be conducted to validate the simulation results and to reduce the percentages for the mentioned parameters.

\section{ACKNOWLEDGMENT}

We would like to acknowledge University Malaysia Pahang for providing the funding and facilities to support this research project.

\section{REFERENCES}

[1] Kato, T., Saeki, K., Nishide, H. \& Yamada, T., "Development of CNG fueled engine with lean burn for small size commercial van," JSAE, Review 22, pp. 365-368, 2001.

[2] Bakar, Rosli. A., Mardani, A. S., \& Wong, H. M. , "Heat Transfer Analysis in Cooling System of a CNG Fuelled SI Engine," BICET, Brunei, October 9-11, 2001.

[3] Johansson, B., Olsson, K., "Combustion Chambers for Natural Gas SI Engine Part 1: Fluid Flow and Combustion," SAE Transaction Paper 950469, 1995

[4] Dam, W. D., Graham, J. P., Stockwell, R. T. \& Montez, A. M., “A New CNG Engine Test for the Evaluation of Natural Gas Engine Oils," SAE Technical Paper Series, 981370, 1998

[5] Dyntar, D. C., Onder and Guzzella, L., "Modelling and Control of CNG Engines," SAE Technical Paper Series, 2002-01-1295, 2002.

[6] Riegler, U. G. \& Bargende, M., "Direct Coupled 1D/3D-CFDComputation (GT-Power/Star-CD) of the Flow in the Switch-Over Intake System of an 8-Cylinder SI Engine with External Exhaust Gas Recirculation," SAE Technical Paper Series, 2002-01-0901, 2002.
[7] Heywood, J. B., "Internal Combustion Engine Fundamentals", McGraw-Hill, Singapore, 1998.

[8] Gamma Technologies, “GT-POWER User's Manual 6.1”, Gamma Technologies Inc, 2004.

[9] Bakar, Rosli. A., Semin., Ismail, Abdul. R., "Effect Of Engine Performance For Four-Stroke Diesel Engine Using Simulation", Proceeding of The $5^{\text {th }}$ International Conference On Numerical Analysis in Engineering, Padang, Indonesia, May 18-19, 2007. 12.

[10] Sera, Mardani. A., Bakar, Rosli. A., Leong, Kwan. S., "CNG Engine Performance Improvement Strategy through Advanced Intake System", SAE Paper 2003-01-1937, 2003. 


\title{
Voltage Sag and the Sensitive Load
}

\author{
I. Daut, C.M. Hadzer, S. Hardi, R. Chan Bahaudin, S.F. Siraj and N. Hashim
}

\begin{abstract}
Voltage sag is the important factor in power quality problem faced by many industrial customers. The most frequently reported among various types of power quality disturbance are voltage sags. The disturbance will cause the sensitive load/equipment (adjustable speed drive, contactor starter in motor, etc) to drop out.

The main source of voltage sags are faults on the utility system and motor starting. Voltage sag caused by faults are generally more severe than those caused by starting. Voltage sag is normally characterized by a magnitude and duration. The load sensitivity is generally determined by the both values. The sag magnitude is depending on fault location and fault type. The sag duration depends on the clearing time the protective device used.

This paper discusses estimation of voltage sag caused by fault in distribution systems that supplied to the sensitive load and sag duration can be controlled using protective device. The result shows that the faults on the adjacent sensitive load are more severe and sensitive load protected by protective device characteristics with longer operating time will most likely to drop out.
\end{abstract}

Keywords: voltage sag, clearing time, sag severity, sensitive load

\section{INTRODUCTION}

A voltage sag according to IEEE std. 1159-1998, in [1] is defined as an rms reduction in the ac voltage between (10$90) \%$, at power frequency, for duration form a half cycle to one minute. If duration is greater than 1 minute it is considered under voltage. Voltage sag is classified as instantaneous when its duration ranges from 0.5 cycles to 30 cycles, momentary lasting between 30 cycles and 3 seconds, and temporary extending from 3 to 60 seconds.

The measurement of voltage sag is stated as a percentage of the nominal voltage it is a measurement of the remaining voltage and stated as a sag to a percentage value. A voltage sag of $70 \%$ means that the nominal voltage reduce to $70 \%$. An example voltage sag is shown Figure 1 where the voltage decrease to $70 \%$ of nominal for 3 cycles.

Voltage sag are most frequent among various types of power quality disturbance (e.g,. voltage sag, voltage sweels, over voltages, interruption, transient, flicker, and harmonics) [2]. Therefore the sags are most important

I. Daut, C.M. Hadzer, S. Hardi, R. Chan Bahaudin, S.F. Siraj and N. Hashim are with the Department of Electrical Electrical, University Malaysia Perlis (UniMAP), Pusat Pengajian Kejuruteraan Sistem Elektrik Kompleks, Pusat Pengajian UniMAP (Blok A), Jl. Kangar Arau, 02600 Jejawi (Tel: +604-9798390, Faks: +604979830, emails: daut@unimap.edu.my, chemat@unimap.edu.my, surya@unimap.edu.my, risnidar@unimap.edu.my, sity_siraj@yahoo.com, norhaidar@unimap.edu.my). power quality problems faced by many industrial customers. The equipment used in modern industrial processes such as programmable logic controllers (PLCs), adjustable speed drives (ASDs), computers, relays and contactors in motors starters are highly sensitive to voltage sag, resulting in process shutdown when they drop out.

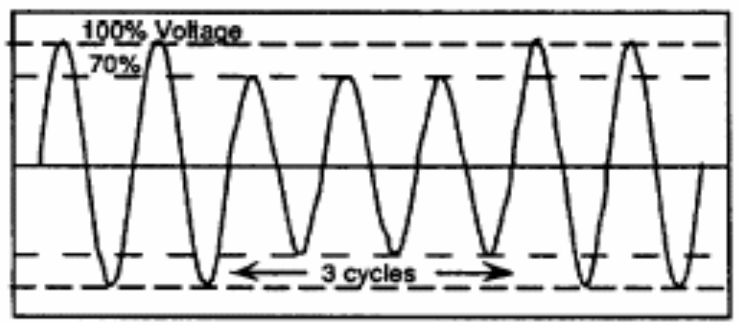

Figure 1. Voltage sag (70\% depth, 3 cycles duration)

Voltage sag is generally originated by the utility network or inside industrial plant faults and motor starting [4]. Voltage sags caused by faults (short circuits) on the network system are generally more severe than those caused by starting. Although the starting of large loads will result flicker, drop out contactors and interrupting sensitive equipment, but the duration of disturbance is not long typically (30 cycles) and also not as deep as that caused by faults.

Faults on the transmission systems can affect even more customers. The customers hundreds of miles from the fault location can still experience voltage sag resulting in equipment mis-operation when the fault is on the transmission system [3]. Voltage sag is normally characterized by a magnitude and duration. Voltage sag magnitude and duration are factors that can directly shutdown or mal-function of customer load.

\section{Assessment of Voltage SAG}

Voltage sags can be characterized by their magnitude (voltage during fault) and their duration. The magnitude is determined by fault location and fault types whereas duration is determined by the fault clearing time. Magnitude and duration are two essential and important sag characteristics which determine the equipment behavior. The value both can directly affect shutdown or malfunction of sensitive load.

\section{A. Magnitude of Voltage Sag}


The magnitude of sag is determined by fault location and fault types. The faults in transmission and distributions systems in power system are often the source of the severe sags. Three phases and single line to ground faults are commonly considered in the analysis. The three phase fault causes deepest sag, where as the single line to ground faults are most frequent occurrence. This study focused on the three phase fault.

A power systems configuration is shown in Figure 2 . The transformer data, the transmission and distribution feeder lines data and loads data are in Table 1, 2 and 3, respectively.

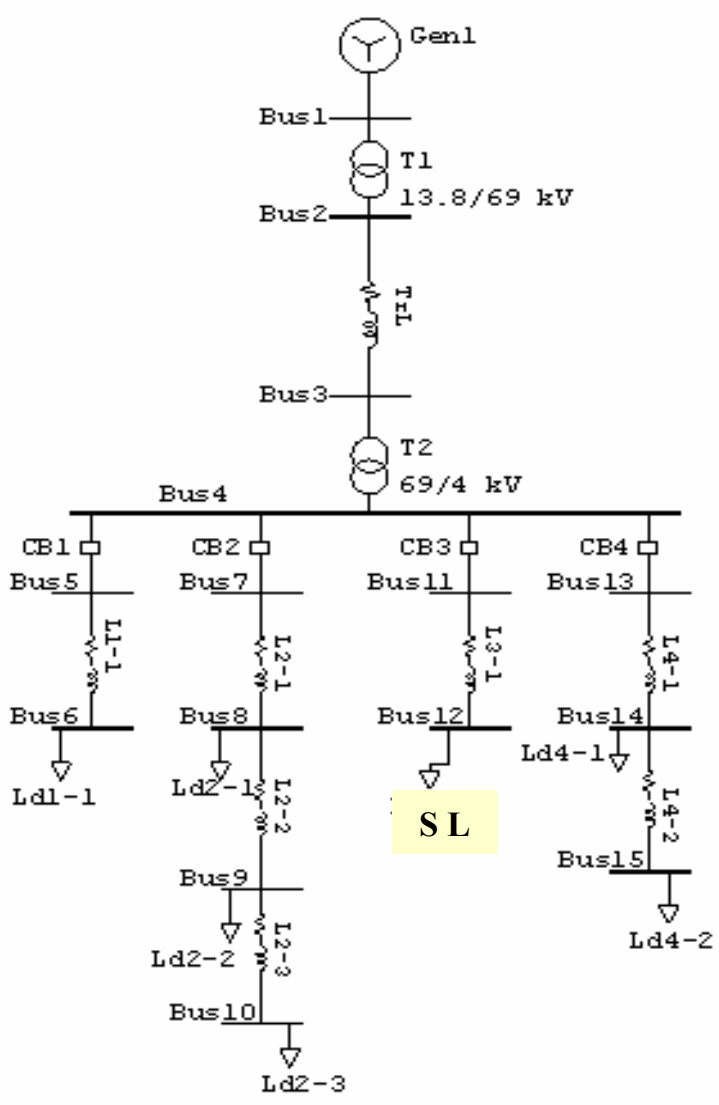

Figure 2. Power system under study

The three phase generator of $13,8 \mathrm{kV}, 35 \mathrm{MW}, 50 \mathrm{~Hz}$, solidly grounded as in Figure 1, are supplying four feeders through transmission line and two transformer delta-wye connection solidly grounded. The sensitive load (SL) located on feeder \#3. The simulation study using ETAP program presented in this paper concentrated on assessing the impact of distribution three faults on the voltage level at sensitive load. The result of simulate as in Table 4 .

\section{Table 1. Transformer data}

\begin{tabular}{|l|c|c|}
\hline \multirow{2}{*}{ Characteristic } & \multicolumn{2}{|c|}{$\begin{array}{c}\text { Substation } \\
\text { Transformer }\end{array}$} \\
\cline { 2 - 3 } & $\mathrm{T} 1(\mathrm{kV})$ & $\mathrm{T} 2(\mathrm{kV})$ \\
\hline Voltage Rating (kV) & $13.8 / 69$ & $69 / 4$ \\
\hline MVA Rating & 30 & 25 \\
\hline Winding Connection & D-Y Grounded & D-Y Grounded \\
\hline Reactance (Ohm) & $8 \%$ & $6.5 \%$ \\
\hline
\end{tabular}

Table 2. The Trans. and Distr. Feeder lines data

\begin{tabular}{|c|c|c|c|}
\hline Lines & $\begin{array}{c}\text { Length } \\
(\mathrm{km})\end{array}$ & $\begin{array}{c}\text { Pos/Neg. } \\
\text { Impedance } \\
(\mathrm{ohm})\end{array}$ & $\begin{array}{c}\text { Zero } \\
\text { Impedance } \\
\text { (ohm) }\end{array}$ \\
\hline TrL & 20 & $12.2+\mathrm{j} .7 .8$ & $15.2+\mathrm{j} 31.6$ \\
\hline L1-1 & 05. & $0.31+\mathrm{j} 0.2$ & $0.26+\mathrm{j} 0.6$ \\
\hline L2-1 & 1 & $0.42+\mathrm{j} 0.65$ & $0.53+\mathrm{j} 1.2$ \\
\hline L2-2 & 0.5 & $0.31+\mathrm{j} 0.2$ & $0.26+\mathrm{j} 0.6$ \\
\hline L2-3 & 1.5 & $0.63+\mathrm{j} 0.98$ & $0.8+\mathrm{j} 1.8$ \\
\hline L3-1 & 2 & $0.84+\mathrm{j} 1.3$ & $2.12+4.8$ \\
\hline L4-1 & 6 & $2.52+\mathrm{j} 3.9$ & $3.18+\mathrm{j} \mathrm{7.2}$ \\
\hline L4-2 & 3 & $1.26+\mathrm{j} 1.95$ & $1.59+\mathrm{j} 3.6$ \\
\hline
\end{tabular}

Table 3. Distribution feeder load data

\begin{tabular}{|l|c|c|}
\hline \multirow{2}{*}{ Load Bus } & \multicolumn{2}{|c|}{ Load } \\
\cline { 2 - 3 } & S (MVA) & P (MW) \\
\hline Ld1-1 & 3 & 2.7 \\
\hline Ld2-1 & 3 & 2.7 \\
\hline Ld2-2 & 1 & 0.85 \\
\hline Ld2-3 & 2.5 & 2.25 \\
\hline S L & 3 & 2.7 \\
\hline Ld4-1 & 2.5 & 2.38 \\
\hline Ld4-2 & 5 & 4.5 \\
\hline
\end{tabular}

Table 4. Voltage level at sensitive load

\begin{tabular}{|l|l|}
\hline Faulted Distribution Bus & $\begin{array}{l}\text { Voltage level at } \\
\text { Sensitive Load ,kV }\end{array}$ \\
\hline Bus 6 & $2.65(66.25 \%)$ \\
\hline Bus 8 & $3.20(80.0 \%)$ \\
\hline Bus 14 & $3.84(96.5 \%)$ \\
\hline Bus 9 & $3.43(85.67)$ \\
\hline Bus 15 & $3.89(97.0 \%)$ \\
\hline Bus 10 & $3.54(88.5 \%)$ \\
\hline
\end{tabular}

From Table 4, the magnitude of the voltage sag at the load bus sensitive load is dependent upon the location of the fault along distribution feeder from Bus 4. The faults located on the feeders which are remote from the bus where is reduce sag level seen at Bus 12 (SL). Distribution feeder fault close to Bus 4 (e.g., fault on feeder bus 6) causes a significant voltage sag to appear at the sensitive load.

\section{B. Duration of Voltage Sag}

Most of the sags are caused by faults in the supplying network and are mainly initiated by lightning strokes. When a faulted systems, voltage sag occurred until a protective device (circuit breaker) opens to interrupt the flow of the fault current. The duration voltage sag is determined by clearing time of the protective device. The fault clearing times depend on setting of protection relays, the protection scheme and the time delay of the breakers. In other word voltage sag duration is similar to time to trip circuit breaker. The duration of voltage sag is total of the operation time of relay and a circuit breaker,

$t_{C}=t_{R}+t_{C B}$

where, $t_{C}, t_{R}$ and $t_{C B}$ are total duration of voltage sag, the operation time of relay and circuit breaker, respectively. Usually require 5-6 cycles for the breaker to operate. 
There are many types of fault clearing devices and each characteristic usually has an absolute minimum time that it takes to clear the fault. To simplify the illustration, in this study the over-current protective relays are usually used in feeder distribution. The time-current characteristic of very inverse type relay according to IEC60255-3 type B can be represented by following expression operation time in [4],

$$
t_{R}=\frac{13.5}{(I / I p)-1} T p
$$

where, $t_{R}, T p, I$ and $I p$ are relay operating time, time dial setting, fault current through in relay and value of selected tapping current, respectively.

The formulation of $t_{R}$ can be changed depending on the relay characteristic used. For example, if the fault current and CT ratio are $1800 \mathrm{~A}$ and 500/5, respectively. The tapping current and time dial setting are $1 \mathrm{~A}$ and 4.5, respectively, then $t_{R}$ is about $450 \mathrm{~ms}$. We set $t c_{B}$ to 5 cycles (about $100 \mathrm{~ms}$ ), so duration of voltage sag is $550 \mathrm{~ms}$. We can also predict the voltage duration of the systems for time dial setting, $T p$ and value of selected tapping current, $I p$ varies and the result can be shown in Table 5 .

Table 5 OCR Relay Parameter

\begin{tabular}{|c|c|c|}
\hline$T_{P}$ & $I_{P}$ & $t_{R}(\mathrm{~ms})$ \\
\hline 0.1 & 4.5 & 450 \\
\hline 0.1 & 5 & 519 \\
\hline 0.2 & 4.5 & 900 \\
\hline 0.2 & 5 & 1024 \\
\hline
\end{tabular}

\section{SENSITIVE LOAD}

Generally, the sensitivity of the equipment to voltages sag can be expressed by tolerance curve. Two popular equipment tolerance curves namely the Information Technology Industry Council (ITIC) curve and the SEMI F-47 curve are shown in Figure 3. Each point on the curve indicates how long this piece of equipment is able to ride through voltage sags level without dropout.

ITIC curve was formerly called the Computer and Business Equipment Manufacturer Association (CBEMA) curve. It represents the voltage variation tolerance requirements of information technology industry equipment. For examples: computers, computer peripherals, and other types of information technology equipment such as copier, fax machine [5].

SEMI F47 curve represent immunity of semiconductor manufacturing equipment to voltage sag. It is widely used by semiconductor vendor in evaluating their needs for protection against voltage sag [6]. In some cases, the SEMI curve is more strict than the ITI curve. The curve has a deeper voltage sag characteristic. The most severe point on the SEMI F-47 curve is the 0.2 seconds sag for a voltage to $50 \%$ of nominal. However, some equipment could meet the SEMI requirement but not pass the ITI curve points

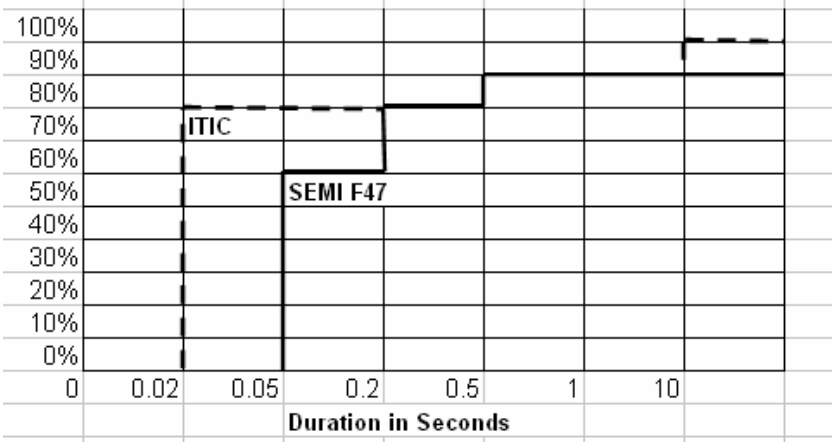

Figure 3. ITIC and SEMI F-47 curves

Voltage sag can easily disrupt the operation of sensitive loads such as electronic adjustable speed drive (ASDs). If the sag is short, it could cause the ASD to introduce speed fluctuations, and damage the end product [7]. ASDs could trip either because the power supply of control electronics of drive also experience a voltage sag, or because some processes can not tolerate the loss of precise speed or torque control, even for a small time interval.

Examples of other equipment, sensitive to voltage variation, are briefly described below [8-10]:

1. Process controllers can be very sensitive to voltage sags. They can cause the equipment they control, to trip off-line during voltage sag conditions.

2. Electronic chip testers are also very sensitive to voltage variations and because of their complexity, they require a large a mount of time to restart. In addition the chips involved in the testing process can be damaged.

3. Programmable logic controllers are very important because any industrial process is often under the control of these devices. The sensitivity to voltage sags varies greatly but portions of an overall PLC system have been found to be very sensitive to trip for voltages as much as $90 \%$ for a few cycles.

4. DC drives are used in many industrial processes; including printing presses and plastics manufacturing. During voltage sag, the controls to the de drives and winders may trip.

5. Machine tools are can be very sensitive too. Often, robots or complicated machine are used for cutting, drilling and metal processing. Any variation in voltage can affect the quality of machined part.

\section{Test Result of SEVERAl LoAds}

Information about sensitivity of individual equipment can be obtained either from the equipment manufacturer or through laboratory test. Different equipment type and different brands of equipment (e.g., two different models of adjustable speed drives) have significantly different sensitivities to voltage sag. Testing result of several loads/equipments had been done by SEMI F47 shown in Figure 4 [9]. The curves are average value result of testing for PLC, 5HP ASD and motor starter coil.

In Figure 4, can be shown that PLC will drop out for 53 $\%$ of voltage in 40 millisecond, ASD and motor starter will be drop out for same time but the different voltage ie; $50 \%$ of voltage and $78 \%$ of voltage for each. Motor starter is more sensitive from the three equipments. 


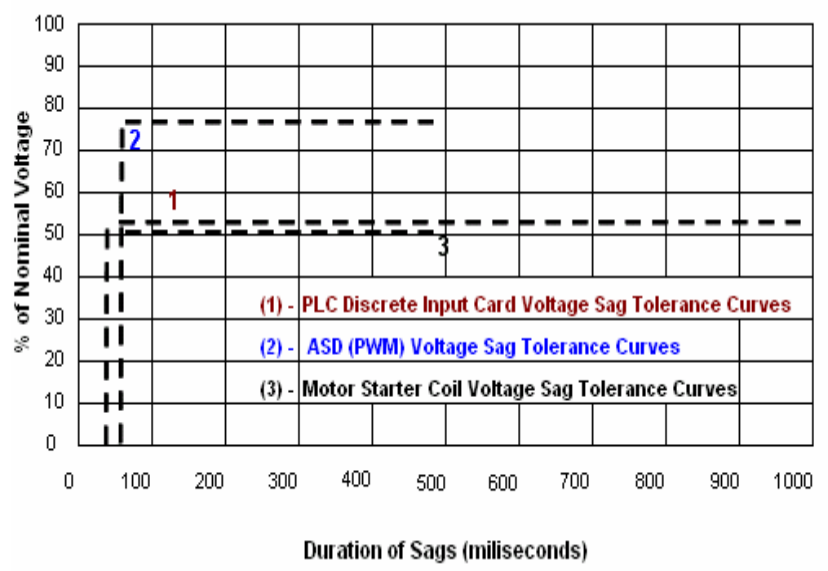

Figure 4. Testing result of several loads/equipments [9]

\section{Conclusions}

Voltage sags are one of the most important power quality problems affecting industrial processes and commercial customers. Adjustable speed drive, programmable logic controllers and motor starter are susceptible to drop out caused by voltage sag.

Susceptibility of load to sag is different. The mostly equipment will drop out after sag $80 \%$ of nominal in 40 milliseconds.

Fault location is significantly to determine magnitude of sag. The fault located where is close to the bus result in severe voltage sag.

Protection systems used have quickly response to prevent subjected equipment to sag for a long time with open circuit breaker.

\section{REFERENCES}

[1] IEEE Std. 1159-1995.," IEEE Recommended Practice for Monitoring Electric Power Quality.

[2] Barry Kenedy.,"Power Quality Primer" Mc-Graw Hill Publication company, U.K, 1999

[3] Mark F. McGranaghan, David R. Muller and Marek J. Samotyj.,"Voltage Sag in Industrial Systems. IEEE Transaction On Industry Applications, Vol. 29. N0.2, March/April 1993.

[4] Siemens.,'Numerical Time Over current Protection," Instruction manual.

[5] ITI (CBEMA) Curve Application Note. Information Technology Industry Council (ITI). Avaiable:http://www.itic.org

[6] SEMI F47-0200 Specification for semiconductor Processing Equipment Voltage Sag Immunity" Avaiable:http://www.semi.org/pubs/semipubs.nsf

[7] Alexis Polycarpou and Hasan Nouri" An overview of voltage sag theory, effect and equipment compability

[8] Christopher J. Merhorn, Timothy D. Davis, and George E. Beam.,"Voltage Sags: Their Impact on the utility and industrial customers. IEEE Trans. Industry application. Vol.34.no.3 May-jun 1998.

[9] IEEE Std. 1346-1998.," IEEE Recommended Practice for Evaluating Electric Power system Compatibility With Electronic Process Equipment

[10] Jeff Lamoree, Dave Mueller, Paull vinett and William Jones and Marek Samotyj.,"Voltage Sag Analysis Case studies" IEEE Transaction on Industry applications, vol.30, no.4, July/August 1994. 


\title{
The Performances of RBD Palm Stearin as Cold Metal Working Lubricant by Plane Strain Extrusion Test
}

\author{
S. Syahrullail, S. Kamitani and K. Nakanishi
}

\begin{abstract}
In this paper, RBD palm stearin, which is one of the refined products of palm oil, was tested as cold metal working lubricant by plane strain extrusion.

Whole experiments were carried out at room temperature $20^{\circ} \mathrm{C}$. Pure aluminum A1050 was used as a workpiece. In these experiments, the extrusion loads were measured and the surface conditions of workpieces were observed. The distribution of relative velocity was calculated in the deformation zone of workpiece by applying the visioplasticity method. Similar experimental and analytical investigation were carried out for a conventional lubricant, paraffinic mineral oil, and the results were compared to those of the RBD palm stearin.

Present investigation confirms that lubrication effect of RBD palm stearin reducing the frictional constraint in the cold metal forming is similar to that of mineral base lubricating oil. However, the existence of RBD palm stearin in solid state at around room temperature affects the surface condition of workpiece, and coarser roughness condition is observed in cold extrusion.
\end{abstract}

Keywords: RBD palm olein, RBD palm stearin, extrusion load, surface roughness, relative velocity

\section{INTRODUCTION}

Today, development of renewable energy base on vegetable oil becomes one of the most urgent research projects in modern society. In Malaysia, much attention has been paid to apply palm oil as lubricant in the metal forming processes. Palm oil is renewable and has high biodegrability compared to mineral oil. Palm oil also has high production rate of oil for every hectare compared to the other vegetable oils, and can fulfills the demand of vegetable base lubricating oil [1].

The usage of vegetable oil and animal fats as industrial lubricant is not a new idea. One of the earliest vegetable / animal fats base lubricating oil was recorded to be used as

S. Syahrullail is with the Faculty of Mechanical Engineering, Universiti Teknologi Malaysia, 81310 UTM Skudai, Johor, MALAYSIA (phone: +607-5534661, fax: +607-5566159; e-mail: syahruls@fkm.utm.my).

S. Kamitani is with the Department of Mechanical Engineering, Kagoshima University, Korimoto 1-21-40, Kagoshima, JAPAN (e-mail: kamitani@mech.kagoshima-u.ac.jp).

K. Nakanishi is with the Department of Mechanical Engineering, Kagoshima University, Korimoto 1-21-40, Kagoshima, JAPAN (e-mail: nakanisi@mech.kagoshima-u.ac.jp). lubricant when huge stone blocks were slide for building monuments in ancient Egypt. A wall painting from ElBersheh, circa $1880 \mathrm{BC}$ also shows a man in front of the statue pouring liquid from a jar, probably vegetable oil to assist the dragging [2].

In modern history, vegetable oil and animal fat are used as lubricating oil, fuel and wax in early $19^{\text {th }}$ century, before the development of mineral base lubricating oil. Palm oil was used for greasing the axle boxes of railway carriages. Palm oil also used in tin-plates industry to prevent oxidation of the iron and as flux before tinning [3]. However, people inclined to utilize mineral base lubricating oil because of the major limitation of vegetable oil in thermal and oxidation stability [4].

Palm oil has been widely tested for engineering application. The potential of palm oil as fuels for diesel engines [5], [6], hydraulic fluid [7] and lubricants [8] were confirmed by the previous researches.

Palm olein was tested as lubricant in metal forming process by extrusion in the previous investigation. The results showed that palm olein could reduce the extrusion load just as paraffinic mineral oil could do so [9]. The other aspect of palm oil is that palm oil contains palm stearin, solid fraction, obtained by fractionation of palm oil after crystallization at a controlled temperature. Therefore, palm stearin is a co-product of palm olein.

In the present investigation, the performances of RBD palm stearin as lubricating oil in the metal forming process were tested by plane strain extrusion. The performances of RBD palm stearin were compared with those of paraffinic mineral oil. The values of extrusion load were measured and the distributions of the relative velocity of material flow in steady state extrusion were measured by applying the visioplasticity method. The surface roughness of the workpiece was also measured at the contact plane with the plane plate tool.

\section{EXPERIMENTAL APPARATUS}

The schematic sketch of the experimental apparatus is shown in Figure 1. The main components are the taper die and the container, plane plate tool and billet as workpiece. The taper die has 45-degrees die angle. The taper die and container is a unit construction part. Whole extrusion apparatus was made with tool steel JIS-SKD11, and necessary heat treatment was done. The billet is extruded 
with local shearing deformation due to frictional constraint by the tool surfaces and large bulk shearing deformation when the taper die changes the direction of plastic flow in the extrusion process by using the above apparatus. The latter bulk shearing deformation is most remarkable at the taper die side and almost zero at the plane plate side. Only the frictional constraint is applied along on the contact surface of a billet with the plane plate tool.

The billets were prepared from the rolled $5 \mathrm{~mm}$ aluminum sheets, JIS-A1050, by the NC wire cut electric discharge machining device. The leading edges of the billets were shaped to coincide with the tool shape of the extrusion apparatus in order to reduce the punch stroke achieving the steady state extrusion condition. Two similar billets were stacked, and used one unit billet as shown in Figure 1. One side of the contact surfaces of the combined billets was the observation plane of plastic flow in plane strain extrusion. Then, the observation plane was not affected with the frictional constraint by the parallel sidewalls. A square grid line pattern measuring material flow in extrusion process was scribed by $\mathrm{NC}$ milling machine on the observation plane of the billet. The lines were $\mathrm{V}$-shaped grooves with $0.5 \mathrm{~mm}$ deep, $0.2 \mathrm{~mm}$ wide and $1.0 \mathrm{~mm}$ spacing.

The billets were annealed by furnace cooling after heating 30 minutes at $350^{\circ} \mathrm{C}$, and the rolling texture with anisotropy of mechanical properties was changed to recrystallized structure with isotropic mechanics properties. The workpiece has $23 \mathrm{HV}$ Vickers hardness after the heat treatment.

\section{EXPERIMENTAL CONDITIONS}

The lubricants used in the present experiments are RBD palm stearin (written as PS) and additive free paraffinic mineral oil VG7 (P1) and VG460 (P3).

Test lubricant was applied on the surface of the plane plate tool on which the billet was contact. The above surface was called the experimental surface of plane plate tool. Specifications of the test lubricants and quantities of application in the tests are listed in Table 1.

Palm stearin used in the present experiments is refined, bleached and deodorized palm stearin. The physical and chemical characteristics of palm stearin were reported by PORIM (Palm Oil Research Institute of Malaysia) and the data incorporated in Malaysian Standard MS 815:1991. RBD palm stearin has high melting point, about $50^{\circ} \mathrm{C}$.

A series of experiments were carried out at room temperature $20^{\circ} \mathrm{C}$, and RBD palm stearin was stay in semisolid condition. Because of this reason, the viscosity of $\mathrm{RBD}$ palm stearin at $20^{\circ} \mathrm{C}$ was not measured.

A specified amount of additive free paraffinic mineral oil VG460 was applied on the surfaces of the smooth sidewall and the taper die with container.

\section{EXPERIMENTAL PROCEDURE}

The plane strain extrusion apparatus was assembled into the confinement fixture and placed on the bolster of a hydraulic press machine. The experiments were carried out at room temperature $20^{\circ} \mathrm{C}$. Extrusion was stopped at a punch stroke in the steady state extrusion condition where the extrusion load and extrusion speed held constant values.

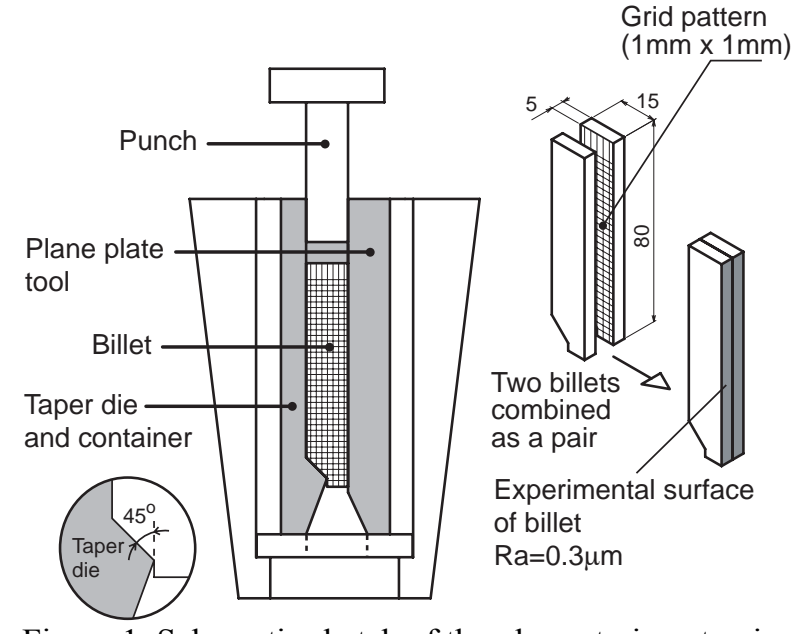

Figure 1. Schematic sketch of the plane strain extrusion apparatus and billets

Table 1.Specification of the lubricants and quantities of application.

\begin{tabular}{|l|c|c|c|}
\hline \multicolumn{1}{|c|}{ Lubricants } & P1 & P3 & PS \\
\hline Viscosity grade & VG7 & VG460 & - \\
\hline $\begin{array}{l}\text { Viscosity at } 20^{\circ} \mathrm{C} \\
\left(\mathrm{mm}^{2} / \mathrm{s}\right)\end{array}$ & 22 & 1900 & - \\
\hline Specific gravity & 0.8840 & 0.9019 & - \\
\hline Flash point $\left({ }^{\circ} \mathrm{C}\right)$ & 138 & 304 & - \\
\hline Pour point $\left({ }^{\circ} \mathrm{C}\right)$ & -20 & -12.5 & $50-54$ \\
\hline Free fatty acid & - & - & Max $2 \%$ \\
\hline Iodine number & - & - & Max 48 \\
\hline $\begin{array}{l}\text { Quantity of } \\
\text { application } \\
\left.\text { (mg/cm }{ }^{2}\right)\end{array}$ & 0.48 & 0.48 & 0.48 \\
\hline
\end{tabular}

P1 : Paraffinic mineral oil VG7

P3 : Paraffinic mineral oil VG460

PS : RBD palm stearin

Partially extruded billets were taken out from the extrusion apparatus and the combined billets were separated. The parallel lines to the direction of extrusion in grid lines on the observation plane of plastic flow of a billet become curved lines and represent the plastic flow lines in the steady state extrusion condition. Figure 2 shows the schematic diagram of the $x-y$ orthogonal coordinates system and equations used in the analyses of the deformation condition.

Since the analytical calculation procedure was explained in earlier publications [10], it is omitted here. 


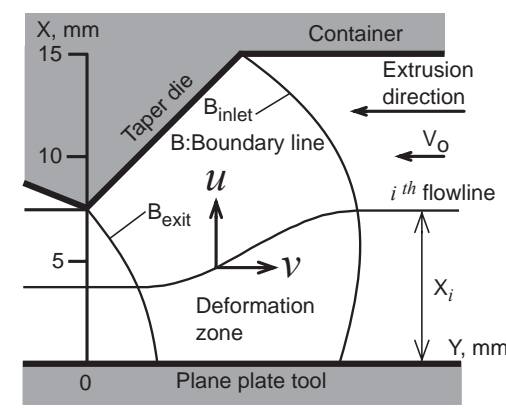

Flow function;

$\psi_{i}=X_{i}\left|V_{0}\right|$

Velocity component ;

$u=\frac{\partial \psi}{\partial Y}, \quad v=-\frac{\partial \psi}{\partial X}$

The strain rate component $\left(\mathrm{s}^{-1}\right)$;

$\dot{\varepsilon}_{X}=\frac{\partial u}{\partial X}, \quad \dot{\varepsilon}_{Y}=\frac{\partial v}{\partial Y}, \quad \dot{\gamma}_{X Y}=\frac{\partial u}{\partial Y}+\frac{\partial v}{\partial X}$

The effective strain rate $\left(\mathrm{s}^{-1}\right)$;

$\dot{\varepsilon}=\frac{2}{3} \sqrt{3 \dot{\varepsilon}_{X}^{2}+\frac{3}{4} \dot{\gamma}_{X Y}^{2}}$

The effective strain (time integration value of the effective strain rate along the flow line) ;

$\varepsilon=\int \dot{\varepsilon} d t$

Figure 2. Coordinates system and equation of visioplasticity method used in the analyses

\section{RESUltS AND Discussion}

\section{A. Extrusion Load}

The extrusion load - piston stroke curves are shown in Figure 3. The steady state extrusion condition starts at around the piston stroke $\mathrm{Y}=22 \mathrm{~mm}$ in all experimental conditions.

The values of extrusion load at punch stroke $Y=30 \mathrm{~mm}$ in steady state condition were almost the similar value ranging from $28.9 \mathrm{kN}$ to $30.8 \mathrm{kN}$.

\section{B. Surface Roughness}

The surface condition of the experimental plane of the billet was observed with CCD camera, and photograph was taken at $\mathrm{Y}=-3 \mathrm{~mm}$ section. The photographs representing the surface conditions of the billet are shown in Figure 4. Comparing the lubrication conditions applying paraffinic mineral oil P3 or RBD palm stearin, the surface conditions of the experimental plane of the billet showed coarser in application of RBD palm stearin lubricant.

The average value of arithmetical mean deviation (Ra) measured in the direction perpendicular to the direction of extrusion at the area (section, $\mathrm{Y}=-3 \mathrm{~mm}$ ) shows lower surface roughness can be achieved by applying paraffinic mineral oil P1. However, almost similar surface condition achieved by applying high viscosity paraffinic mineral oil P3 can be obtained by RBD palm stearin lubricant, which is semi-liquid at room temperature $20^{\circ} \mathrm{C}$.

The arithmetical mean deviation $\mathrm{Ra}$ along on the experimental surface of the billet, measured at all experimental conditions are shown in Figure 5. Result shows that RBD palm stearin creates thick lubricant layer between billet and tool in the extrusion process due to its semi-solid condition. The result is similar to that applied paraffinic mineral oil P3 lubricant. While, thin lubricant layer was created by applying low viscosity paraffinic mineral oil P1, and low value of Ra is achieved in the case.

\section{Observation of the Grid Patterns of the Billet}

Comparisons of the grid patterns of the partially extruded billet at product side were shown in Figure 6. Figure 6(a) explains the definition of grid slope angle. The grid slope angle was measured at product area of the billet. It is represented as $\alpha$ (alfa).

Figure 6(b) shows the photographs of grid lines near the experimental surface of billet at product side $(\mathrm{Y}=0 \mathrm{~mm})$. High frictional constraint on the sliding plane of plane plate tool causes distortion of the vertical grid lines more remarkable than that in the condition with low frictional constraint. We could assume that the ratio of metal contact between billet and tool became high due to the thin lubricant layer created by paraffinic mineral oil P1, and frictional constraint was increased along on the sliding plane (experimental surface of plane plate tool). Comparing to the results applying RBD palm stearin lubricant, that high frictional constraint in extrusion applying paraffinic mineral oil P1 makes the grid patterns much distorted ones. However, comparison of the results applying paraffinic mineral oil P3 or RBD palm stearin shows that the similar distorted grid pattern could be obtained by applying paraffinic mineral oil P3 or RBD palm stearin.

Figure 6(c) shows the mutual comparisons of the vertical lines. Sloping angle of the vertical lines in extrusion with paraffinic mineral oil $\mathrm{P} 1$ is $50^{\circ}$, while those in extrusions with paraffinic mineral oil P3 and RBD palm stearin are around $76^{\circ}$. The above results represents that low frictional constraint can be achieved by applying paraffinic mineral oil P3 and RBD palm stearin.

\section{Relative Velocity}

The relative velocities measured at experimental surface of billet at all experimental conditions were shown in Figure 7. The relative velocity becomes higher in extrusion applying paraffinic mineral oil P3 or RBD palm stearin compared to the results applying paraffinic mineral oil P1. Then, we could confirm that paraffinic mineral oil P3 or RBD palm stearin could reduce frictional constraint.

\section{E. Effective Strain}

Comparisons of the effective strain in the deformation zone are shown in Figure 8. The effective strain distribution in extrusion applying paraffinic mineral oil P1 and that applying RBD palm stearin differ slightly at sliding plane, while the effective strain distribution in extrusion applying paraffinic mineral oil P3 and that applying RBD palm stearin shows similar patterns. 


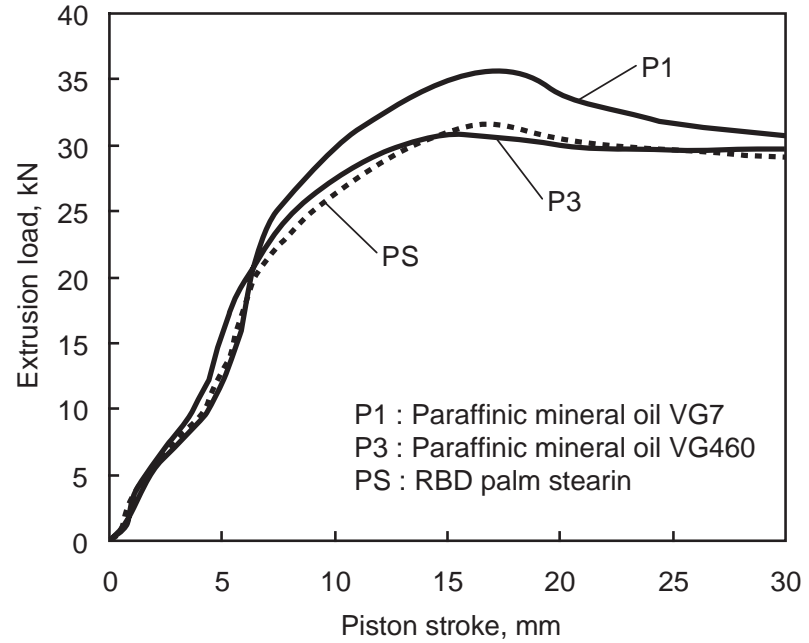

Figure 3.Extrusion load - piston stroke curves

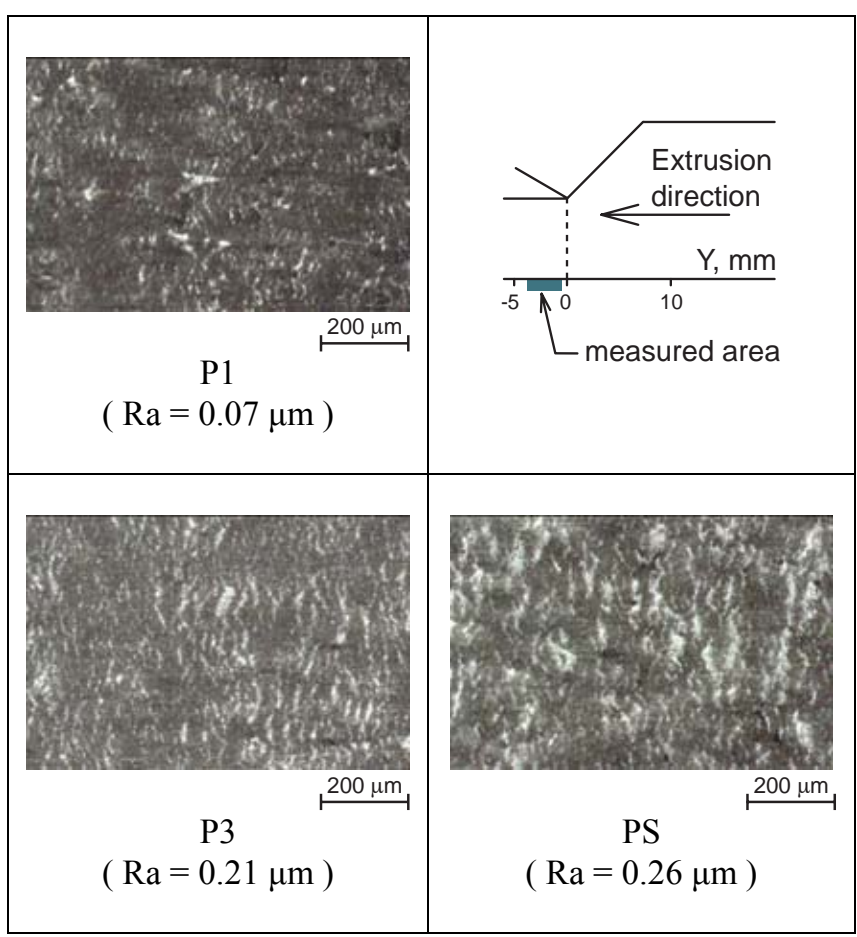

Figure 4. Photographs of the experimental surface of billet at product side $(\mathrm{Y}=-3 \mathrm{~mm})$

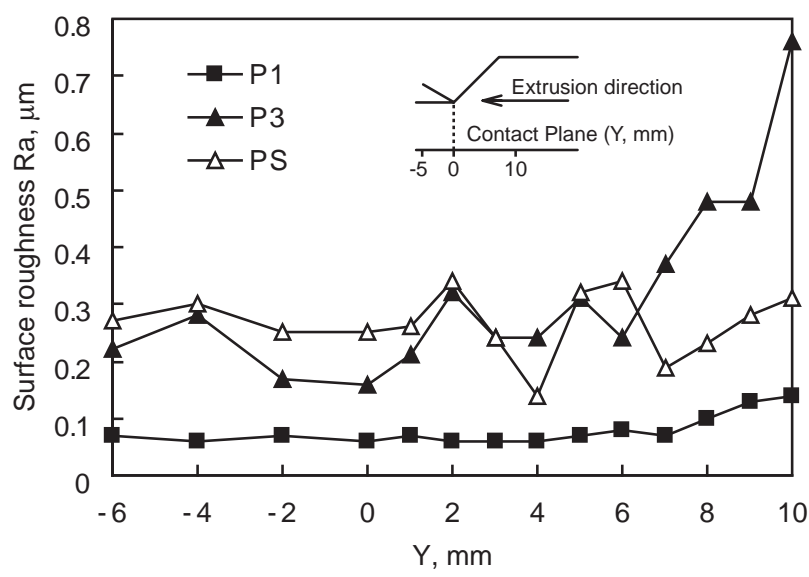

Figure 5. Surface roughnesses Ra of billet along on the plane plate tool

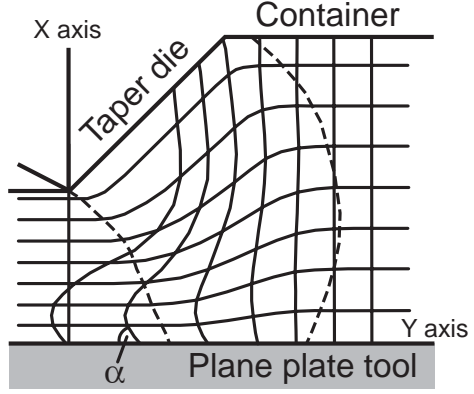

( $\alpha$ : Grid slope angle )

(a) Measurement method of grid slope angle

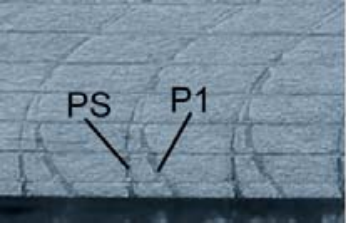

P1 and PS

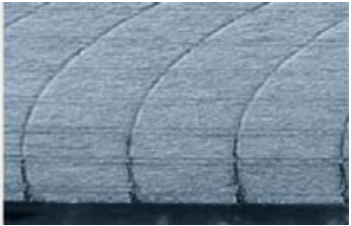

P3 and PS

(b) Close-up photographs of grid lines near the experimental surface of billet at product side.

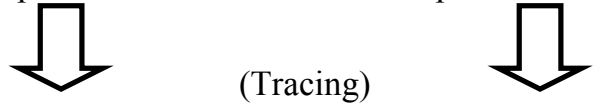

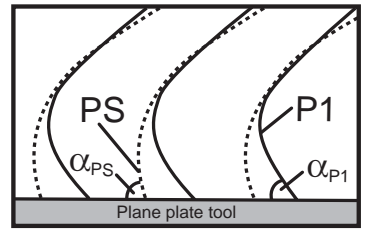

P1 and PS

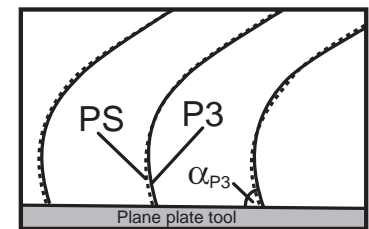

P3 and PS (c) Mutual comparisons of vertical grid lines patterns traced from photographs taken in (a).

$$
\begin{array}{ll}
\alpha_{\mathrm{P} 1}=50^{\circ} & \alpha_{\mathrm{P} 3}=76^{\circ} \\
\alpha_{\mathrm{PS}}=76^{\circ} &
\end{array}
$$

Figure 6. Mutual comparisons of grid slope angle conditions

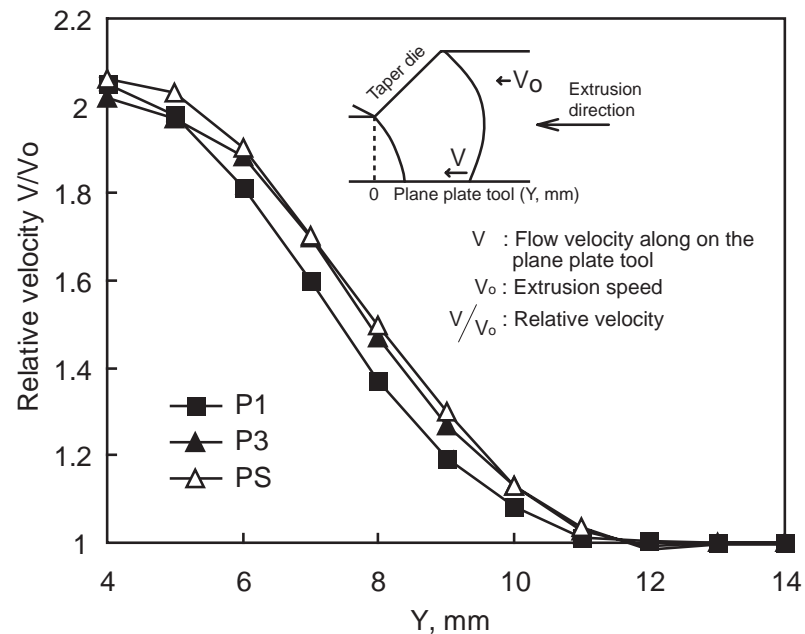

Figure 7. Distributions of relative velocity in deformation area of billet 


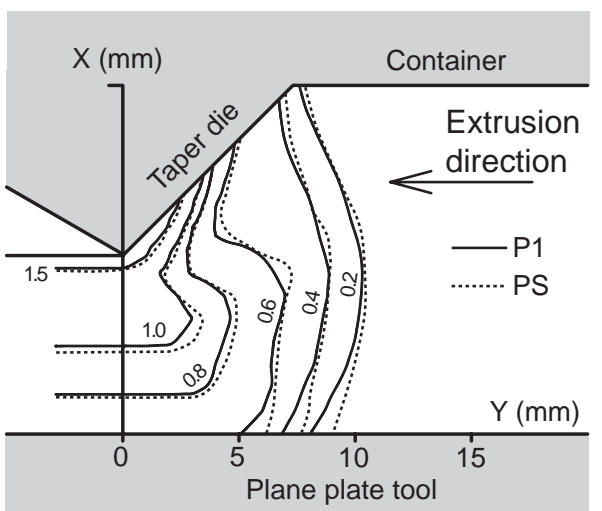

(a) Effective strain of P1 and PS.

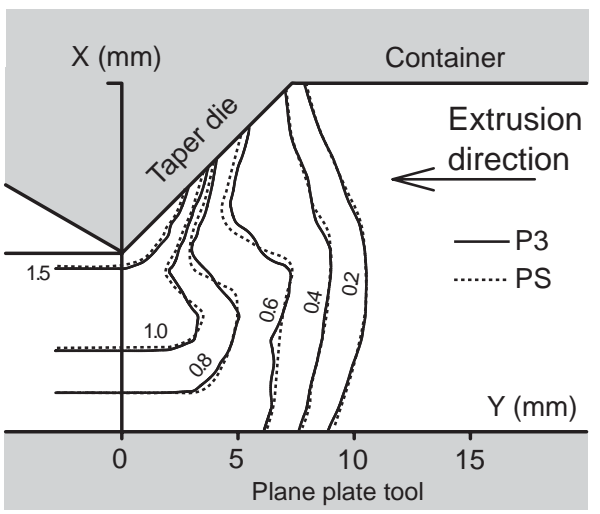

(b) Effective strain of P3 and PS.

Figure 8. Mutual comparisons of effective strain in deformation area of billet

\section{CONCLUSIONS}

The performances of RBD palm stearin as cold metal working lubricant were investigated by plane strain extrusion test. We could confirm from the experimental and analytical results that RBD palm stearin can be applied for cold metal forming process as lubricant. Thick lubricant layer between the workpiece and tool surface could be formed by applying RBD palm stearin because of its high pour point. However, this characteristic becomes an advantage of RBD palm stearin as lubricant due to its performance to reduce fictional constraint between the workpiece and the tool.

\section{ACKNOWLEDGEMENT}

The author would like to thank Faculty of Mechanical Engineering, Universiti Teknologi Malaysia and Production System Engineering Group of Faculty of Engineering, Kagoshima University for their cooperation on this research.

\section{REFERENCES}

[1] K. K. Ming and D. Chandramohan, "Malaysian Palm Oil Industry at Crossroad and its Future Direction," Oil Palm Industry Economic Journal, vol. 2, no.2, pp. 10-15, 2002

[2] M. Nosonovsky, "Oil as a Lubricant in the Ancient Middle East," Tribology Online, vol. 2, no.2, pp. 44-47, 2007.

[3] J. Henderson and D. J. Osborne, "The Palm Oil in our Lives: How This Came About," Endeavour, vol. 24, no. 2, pp. 63-68, 2000.

[4] U. S. Choi, B. G. Ahn, O. K. Kwon and Y. J. Chun, "Tribological behavior of some antiwear additives in vegetable oils," Tribology International, vol. 30, no. 9, pp. 667-683, 1997.

[5] E. Kinoshita, K. Hamasaki and C. Jaqin, "Diesel Combustion of Palm Oil Methyl Ester," SAE paper, no. 2003-01-1929, 2003.
[6] S. Bari, T. H. Lim and C. W. Yu, "Effect of Preheating of Crude Palm Oil (CPO) on Injection System, Performance and Emission of a Diesel Engine," Renewable Energy, vol. 27, pp. 339-351, 2002.

[7] W. B. Wan Nik, F. N. Ani and H. H. Masjuki, "Thermal Performances of Bio-Fluid as Energy Transport Media," in $6^{\text {th }}$ Asia Pacific International Symposium on Combustion and Energy Utilization, Kuala Lumpur, 2002, pp. 558-563.

[8] A. I. Obi and A. K. Oyinlola, "Frictional Characteristic of Fatty-based Oils in Wire Drawing," Wear, vol. 194, pp. 30-37, 1996.

[9] S. Syahrullail, K. Nakanishi and S. Kamitani, "Investigation of the Effect of Frictional Constraint with Application of Palm Olein Oil Lubricant and Paraffin Mineral Oil Lubricant on Plastic Deformation by Plane Strain Extrusion," Japanese Journal of Tribology, vol. 50, no. 6 , pp. $727-738,2005$.

[10] K. Nakanishi, S. Okamura and T. Nakamura, "Analysis of Axisymmetric Hot Extrusion of Aluminum by the Visioplasticity Method Combined with the Numerical Calculation Method for Predicting the Flow Curves," Journal of the Japan Society for Technology of Plasticity, vol. 18, no.203, pp. 990-998, 1977. 


\title{
Refrigeration Efficiency Improvement via Heat Transfer Enhancement
}

\author{
R. Thiruselvam, Mohammad Zainal Yusof, Vijay R. Raghavan
}

\begin{abstract}
The criticality in the improvement of refrigeration system efficiency has now shifted to heat exchangers that need to work across the least possible temperature difference. With smaller temperature differences across the evaporator and condenser, the compressor work is reduced and energy efficiency is improved. To reduce these temperature differences, techniques of heat transfer enhancement are needed. This forms the basis of the present study in which heat transfer characteristics of enhanced annuli are studied experimentally in a water-cooled condenser. Normally superheated refrigerant vapour enters the condenser in turbulent flow conditions, and exits as subcooled liquid which is in the transition region of flow. The annulus-side coefficients are determined using the Wilson Plot technique. The results for the augmented surface are presented graphically and compared with the non-augmented case. For turbulent flow of vapour in plain annuli, the form of the correlation by Monrad and Pelton was satisfactory. For vapour in enhanced annuli, the correlation of Knudsen and Katz determined the basic form. The enhanced annulus outperforms the plain tube by about $25 \%-30 \%$ for superheated vapour heat transfer coefficient. The new empirical constants resulted in good prediction for turbulent superheated vapour flow in the annulus for both plain and enhanced annuli. For the single phase heat transfer correlation proposed, all predicted data are within $\pm 5 \%$ of experimental values. Though R-22 has been chosen for the present study, the dimensionless form of presentation of results enables the correlations to be applied to other refrigerants.
\end{abstract}

Keywords: heat transfer enhancement, refrigeration systems, energy efficiency improvement

\section{INTRODUCTION}

Condensation heat transfer, both inside and outside horizontal tubes, plays a key role in refrigeration, airconditioning and heat pump applications. In recent years the science of condensation heat transfer has been severely challenged by adoption of substitute working fluids and new enhanced surface of heat exchangers. Well-known and

R. Thiruselvam is with the University Tun Hussein Onn Malaysia, Batu Pahat, 86400 Parit Raja, Johor, (+60125698389, +6074536080, tiruselvam_ramahlingam@yahoo.com)

Mohammad Zainal Yusof is with University Tun Hussein Onn Malaysia, Batu Pahat, 86400 Parit Raja, Johor, (+60197239002, +6074536080,zainal@uthm.edu.my)

Vijay R. Raghavan is with the University Tun Hussein Onn Malaysia, Batu Pahat, 86400 Parit Raja, Johor, (+60177168255, +6074536080, vijay@uthm.edu.my) widely established correlations to predict heat transfer during condensation may seem to be quite inaccurate in some new applications, and consequently a renewed effort is now being dedicated to the characterization of flow conditions and associated predictive procedures of heat transfer with condensing vapour. Tremendous research efforts have been made to miniaturize thermal systems and identify innovative technologies for heat transfer enhancement. Heat transfer enhancement techniques are classified as: passive enhancement techniques- not involving any direct application of external source of power, and active enhancement techniques- which require direct application of external power. Passive techniques, in general, have lower energy cost, lower heat transfer augmentation, and large pressure drops when compared to active techniques. Depending on the application and economic feasibility, the designer has to decide on the type of augmentation techniques used. Among such applications, the use of Double-Tube heat exchanger demonstrates an impressive potential for enhanced condensation heat exchangers. The WSHP unit is a reversible-cycle heat pump unit which uses water as the heat source when running in the heating mode and as the heat sink when in the cooling mode. For this purpose, a water-to-refrigerant heat exchanger is used. Generally, this could be a double tube or plate heat exchanger, figure 1 .

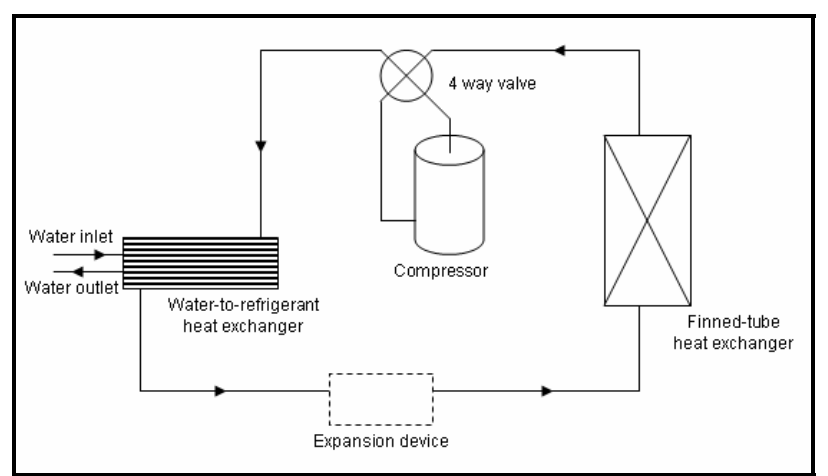

Figure 1. Refrigerant circuit for WSHP unit

The refrigerant circuit is completed with a compressor, expansion device and a finned-tube heat exchanger. The Double Tube heat exchanger is a very efficient design. The water and refrigerant are in direct thermal contact through the walls of the inner tubes. The water and the heat-transfer fluid are in counterflow. This is of particular advantage when desuperheating or subcooling occurs in the heat 
exchanger along with phase change. This type of heat exchanger has two loops similar to those described in the shell-and-tube heat exchanger.

For the given arrangement of double tube, the refrigerant flows in the annulus whereas the cooling water flows in the inner tube, shown in figure 2.

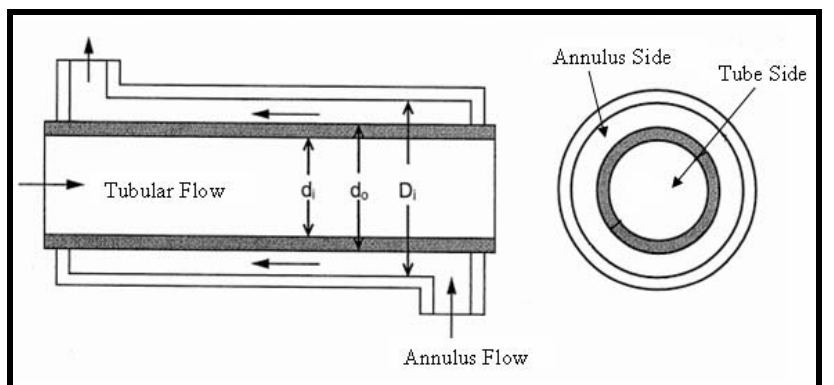

Figure 2. Concentric tube configuration (double tube heat exchanger)

\section{OBJECTIVES AND SCOPE}

The purpose of the study is to obtain the single phase heat transfer coefficient for the superheated vapor refrigerant flow in the annulus of the double tube exchanger. This research is conducted because the inner tube used in the current double-tube design is a $3 \mathrm{D}$ corrugated outer surface enhancement for copper tube (Turbo-C Copper Top Cross); it is difficult to generate a mathematical model for such complex geometries, no standard equation for the heat transfer coefficient is available for the Turbo-C copper top cross, and modification of equations from previous literature will require extensive measurements of pressure, temperature of the refrigerant and water side, and the surface temperature of the fin and fin root surface. Thus, with the above mentioned motivation, the objective of the current research is to characterize the heat transfer performance of the Turbo-C Copper Top Cross tube for superheated vapour. The enhancement effect of the Turbo-C Copper Top Cross tube was also studied for comparison with a plain tube. Experiments were conducted to obtain the necessary data which will be used for parametric analysis of the following independent parameters.

Table 1. Parametric Range for Experiments

\begin{tabular}{|c|l|}
\hline No. & \multicolumn{1}{c|}{ Experimental Condition } \\
\hline 1 & Test Fluid - R22 Chlodifluoromethane \\
\hline 2 & $\begin{array}{l}\text { Test Section } \\
\text { (a) Plain Tube } \\
\text { (b) Copper Top Cross }\end{array}$ \\
\hline
\end{tabular}

\section{WiLson Plot METHOD}

A useful technique for determining individual resistance from an overall resistance was devised by Wilson (Briggs et al [1]) in 1915. Wilson was interested in determining the effects of water temperature and velocity on the overall coefficient for a steam condenser. In most heat exchanger condensing processes, the primary measurements taken connect the energy transferred to the temperature difference between two fluid streams. The overall rate of heat transfer can be calculated from:
$Q=U A_{O} \Delta T$

The objective of the condensation test is directed at determining the condensate heat transfer resistance from the overall thermal balance. When a vapour condenses on the outside of a tube which is internally cooled by flowing liquid, figure 3 , the component thermal resistances are coupled to the overall resistance by:

$\frac{1}{A_{O} U}=R_{O}+R_{W}+R_{i}=\frac{1}{A_{O} h_{O}}+R_{W}+\frac{1}{A_{i} h_{i}}$

It is noted that there will be some heat loss through the outer steel tube as the outer surface of the condenser coil will not be insulated during use in actual unit applications. This heat loss is an added advantage to the entire heat rejection process by the condenser coil. Since the thermal conductivity of steel is less compared to the thermal conductivity of copper $\left(\mathrm{k}_{\text {steel }}<<\mathrm{k}_{\text {copper }}\right)$, the outer surface is assumed to be adiabatic. Additional precaution is taken by insulating the outer steel pipe with 1" thick Superloon insulation material to create an adiabatic outer surface. Equation (2) can then be transformed to:

$\frac{1}{U}=\frac{A_{O}}{A_{i}} \frac{1}{h_{i}}+\left[A_{O} R_{W}+\frac{1}{h_{O}}\right]$

Equation (3) can than be interpreted as a linear function which has the linear form $y=m x+b$, if $R_{w}$ and $h_{o}$ are constant, and it is the basis of the Wilson Plot method. Given such a test series, a line can be plotted as shown in Fig. 3.

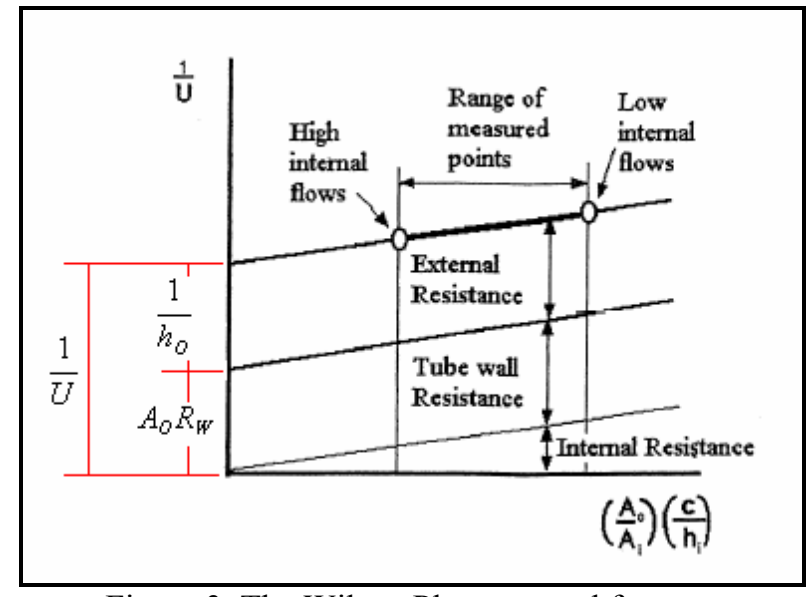

Figure 3. The Wilson Plot - general features

The experimental points extend from a high internal flow rate, with low overall heat transfer resistance, to a low internal flow rate and high overall heat transfer resistance. A line drawn through these points intersects the $\mathrm{U}^{-1}-$ axis at a point where the internal heat transfer resistance approaches zero, and thus the overall heat transfer resistance at this point is simply the sum of wall resistance and the external heat transfer resistance. The internal heat transfer coefficient, at any flow rate, can than be determined independently using (3). It should be noted that the accuracy and reliability of the Wilson Plot Method depends on the 
validity of the correlation used to describe the internal and external heat transfer coefficient.

\section{Test Facility and Method of Data Analysis}

The commissioning of the test section was carried out using HCFC R-22 as refrigeration medium in the annulus and water as coolant in the inner tube. The primary objective of these tests is to determine the correlation for overall heat transfer of the condensation process. The overall energy flow in the test section can be determined using three independent routes. These routes use:

- the temperature increase in the coolant flow

- the mass of condensate collected under the test section

- the circumferentially integrated temperature drop across the tube wall

Deans et al [2] has reported that the maximum difference in the calculated overall energy flows using these three routes to analyze the condensation process was less than $5 \%$. The temperature increase in the coolant flow is chosen due to its ease in experimental measurements and the use of this method with respect to the Wilson Plot method. The test facility was designed and assembled to cater for this need. The test facility is capable of testing either plain or enhanced straight Double-Tube condenser at conditions typical of a vapour compression refrigeration system.

\section{Test FACILITy CONFIgURATION}

The Double-Tube heat exchanger configuration used in this study is a one-pass, single-track system. The singletrack system means that that only one test section and one refrigerant compressor may be installed in the system for individual testing, which simply means that the refrigerant is not circulated within the test facility during the data run. The refrigerant passes through the Double-Tube condenser only once as it travels from the high-side (compressor discharge line) to the low-side (compressor suction line). The single-track and single-pass system makes it possible to obtain one successful data point every time the facility is operated. If the operating conditions such as refrigerant mass flow rate or compressor discharge pressure are varied (non-geometric variables), it is possible to obtain additional data points without changing the test tube or compressor. The presence of the Electronic Expansion Valve (EXV) allows us to do this. Use of conventional expansion device such as the capillary tube here will require additional work repetition where the refrigerant circuit will have to be vacuumed, leak tested and recharged for the individual length of capillary tube needed to throttle the refrigerant flow. The two main variable components in this test facility are the test section and the refrigerant compressor. The facility is designed and installed with valves and fittings for both the refrigerant medium and the cooling water. This is to allow for quick and easy replacement of the test section and/or refrigerant compressor. Each refrigerant has an individual range of refrigerant flow rate, depending on the amount of refrigerant charge and compressor suction and discharge pressure. Three types of refrigerant compressor were chosen (1 HP, $2 \mathrm{HP}$, and $3 \mathrm{HP}$ ) to provide a wide range of refrigerant mass flow rates. Straight horizontal test section with two types of inner tube was used in this study, i.e. (1) Plain Tube (Plain Annulus) and (2) Turbo-C (Enhanced Annulus).

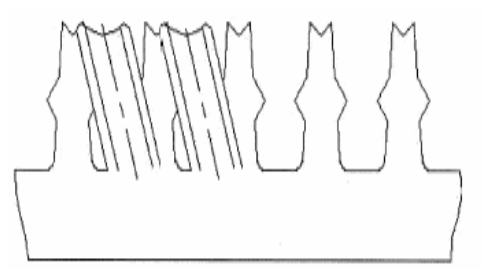

Figure 4. Illustration of enhanced surface (Turbo-C)

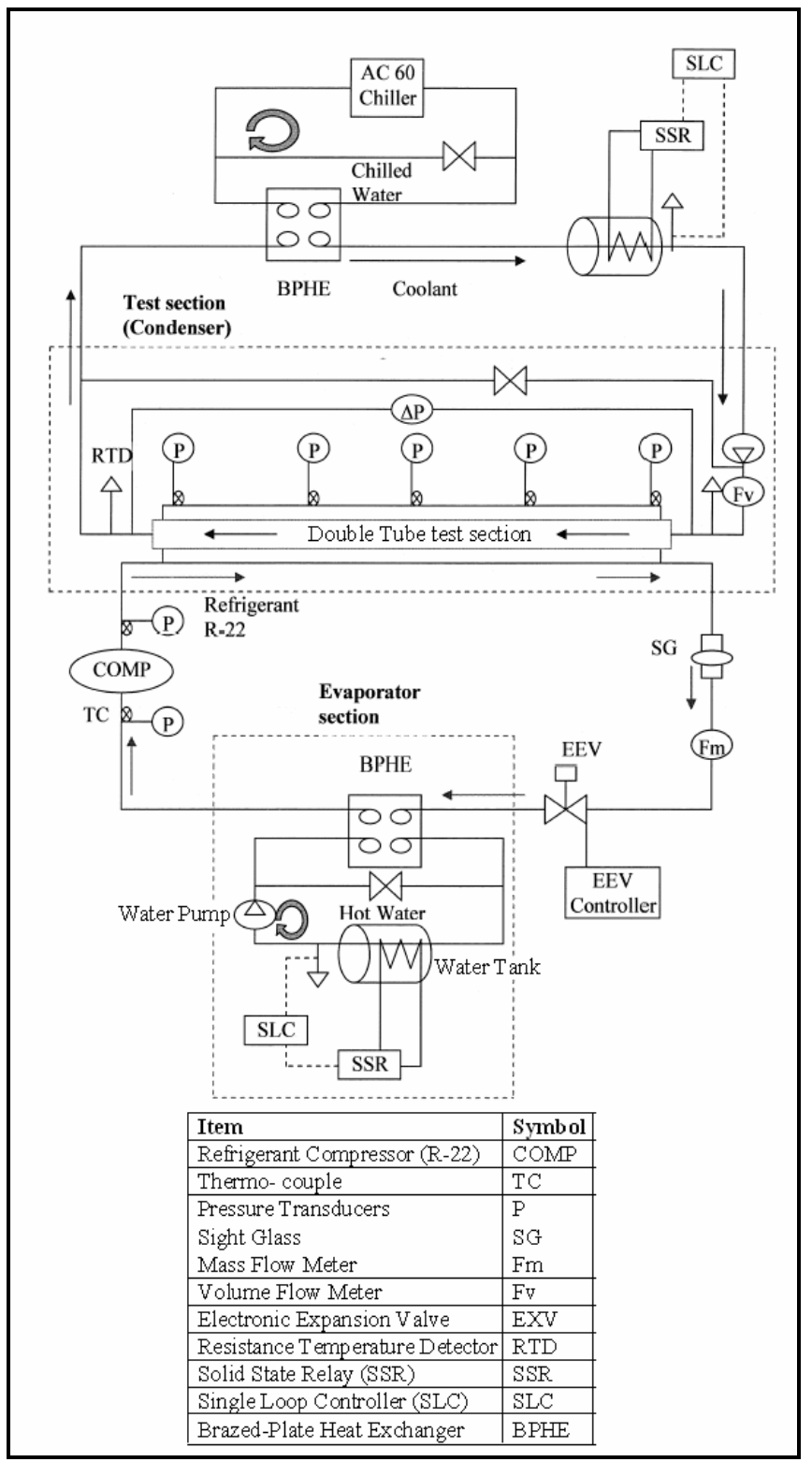

Figure 5: Schematic Diagram of Experiment

Table 2: Description of the Test Section Tubes Used

\begin{tabular}{|l|l|l|}
\hline Description & Plain Tube & Turbo-C \\
\hline OD & $22.22 \mathrm{~mm}$ & $22.22 \mathrm{~mm}$ \\
\hline ID & $17.82 \mathrm{~mm}$ & $17.82 \mathrm{~mm}$ \\
\hline Length & $2980 \mathrm{~mm}$ & $2980 \mathrm{~mm}$ \\
\hline $\begin{array}{l}\text { Outer } \\
\text { Surface }\end{array}$ & $\begin{array}{l}\text { Smooth } \\
\text { Surface }\end{array}$ & 3-D Integral Fin \\
\hline $\begin{array}{l}\text { Inner } \\
\text { Surface }\end{array}$ & $\begin{array}{l}\text { Smooth } \\
\text { Surface }\end{array}$ & Smooth Surface \\
\hline $\begin{array}{l}\text { Other } \\
\text { Information }\end{array}$ & N.A. & $\begin{array}{l}\text { Pitch of Fin }=0.75 \mathrm{~mm} \\
\text { Pitch of Corrugation }= \\
5 \mathrm{~mm}\end{array}$ \\
\hline
\end{tabular}




\section{DATA REDUCTION \& EXPERIMENTAL UNCERTAINTIES}

In the Dry Run Test the compressed vapour enters the test section as superheated vapour and exits in the same phase. The heat transferred to the cooling water is kept sufficiently low to allow the vapour temperature at exit to be above the saturation temperature; hence no phase change occurs. This will allow us to obtain the single phase heat transfer coefficient for superheated vapour.

$Q_{S H}=\dot{m}_{O}(\Delta h)_{S H}=\bar{U}_{S H} A_{O}(L M T D)$

$\frac{1}{\bar{U}_{S H} A_{O}}=\frac{1}{\overline{h_{S H}} A_{O}}+R_{W}+\frac{1}{\overline{h_{i}} A_{i}}$

Uncertainties in the experimental data were calculated based on the propagation of error method, described by Kline and McClintock [3]. Accuracy for various measurement devices, refrigerant properties and water properties are given in Table 3, 4 and 5.

Table 3. Uncertainties of various measurement devices

\begin{tabular}{|l|l|}
\hline Parameter (Make, Type) & Uncertainties \\
\hline $\begin{array}{l}\text { Water Volume Flow (YOKOAWA, } \\
\text { Magnetic Flow Meter) }\end{array}$ & $\begin{array}{l} \pm 0.08 \% \\
\text { of reading }\end{array}$ \\
\hline $\begin{array}{l}\text { Refrigerant Mass Flow Meter } \\
\text { (YOKOGAWA, Coriolis) }\end{array}$ & $\begin{array}{l} \pm 0.05 \% \\
\text { of reading }\end{array}$ \\
\hline $\begin{array}{l}\text { Refrigerant Pressure (HAWK, } \\
\text { Pressure Transducer) }\end{array}$ & $\pm 0.18 \mathrm{psig}$ \\
\hline $\begin{array}{l}\text { Water temperature (CHINO, Pt-100 } \\
\text { RTD) }\end{array}$ & $\pm 0.01^{0} \mathrm{C}$ \\
\hline $\begin{array}{l}\text { Refrigerant Temperature (VANCO, } \\
\text { Type-T Thermocouple) }\end{array}$ & $\pm 0.06^{0} \mathrm{C}$ \\
\hline
\end{tabular}

Data provided by OYL R\&D Center (M) Sdn. Bhd.

Table 4. Uncertainties of refrigerant R-22 properties

\begin{tabular}{|l|l|l|}
\hline $\begin{array}{l}\text { Predicted } \\
\text { Properties }\end{array}$ & Uncertainties & Source \\
\hline Density & $\pm 0.1 \%$ & Kamei et al. [4] \\
\hline $\begin{array}{l}\text { Isobaric Heat } \\
\text { Capacity }\end{array}$ & $\pm 1.0 \%$ & \\
\hline Viscosity & $\pm 2.1 \%$ & Klein et al. [5] \\
\hline $\begin{array}{l}\text { Thermal } \\
\text { Conductivity }\end{array}$ & $\pm 3.7 \%$ & McLinden et al. [6] \\
\hline
\end{tabular}

Properties data obtained from ASHRAE Fundamentals Handbook 2001 [7]

Table 5. Uncertainties of predicted water properties

\begin{tabular}{|l|l|l|}
\hline Predicted Properties & Uncertainties & Source \\
\hline Density & $\pm 0.02 \%$ & $\begin{array}{l}\text { Wagner and } \\
\text { Pru } B \text { [8] }\end{array}$ \\
\hline Isobaric Heat Capacity & $\pm 0.3 \%$ & Kestin et al. \\
Viscosity & $\pm 0.5 \%$ & {$[9]$} \\
\hline Thermal Conductivity & $\pm 0.5 \%$ & \\
\hline
\end{tabular}

Properties data obtained from ASHRAE Fundamentals Handbook 2001 [37]

Uncertainties in the single phase heat transfer coefficient (superheated vapour and subcooled liquid) and the condensation heat transfer coefficient were calculated for various test runs in the smooth and enhanced annulus as a root-sum-square (RSS) method. Experimental results and the associated uncertainties are listed in Table 6. The uncertainties are dominated by the uncertainties associated with the refrigerant and water properties. Higher uncertainties were found at higher refrigerant mass flow rate.

Table 6. Uncertainty analysis for experimental data

\begin{tabular}{|c|c|c|c|c|}
\hline $\begin{array}{l}\text { Test } \\
\text { Sequence }\end{array}$ & \multicolumn{2}{|c|}{$\begin{array}{l}\text { Plain Tube } \\
\mathrm{h}\left(\mathrm{W} / \mathbf{m}^{2} . K\right)\end{array}$} & \multicolumn{2}{|c|}{$\begin{array}{c}\text { Turbo-C } \\
h\left(W / \mathbf{m}^{2} . K\right)\end{array}$} \\
\hline $\begin{array}{l}\text { Compresso } \\
\text { r }\end{array}$ & Highest & Lowest & Highest & Lowest \\
\hline $1 \mathrm{HP}$ & $\begin{array}{l}345.57 \\
+5.48 \%\end{array}$ & $\begin{array}{l}284.80 \\
+4.80 \%\end{array}$ & $\begin{array}{l}456.70 \\
+4.96 \%\end{array}$ & $\begin{array}{l}391.32 \\
+5.10 \%\end{array}$ \\
\hline $2 \mathrm{HP}$ & $\begin{array}{l}688.13 \\
+4.90 \%\end{array}$ & $\begin{array}{l}508.12 \\
+3.88 \%\end{array}$ & $\begin{array}{l}779.12 \\
+4.23 \%\end{array}$ & $\begin{array}{l}731.46 \\
+3.89 \%\end{array}$ \\
\hline $3 \mathrm{HP}$ & $\begin{array}{l}979.44 \\
+4.42 \%\end{array}$ & $\begin{array}{l}817.18 \\
+3.72 \%\end{array}$ & $\begin{array}{r}1076.89 \\
+3.87 \%\end{array}$ & $\begin{array}{l}991.88 \\
+3.86 \%\end{array}$ \\
\hline
\end{tabular}

\section{TEST RESULTS}

The test results discussed above accomplished the objective of this study whereby the single phase heat transfer coefficient and the condensation heat transfer coefficient are obtained. The literature search failed to identify a condensation correlation for the current Turbo-C tubes which makes it difficult to compare and verify our results. Hence the comparisons are made with the plain annulus result which will be a base case to illustrate the effect of the 3-D Integral Fin Surface Enhancement. Equation (6) is used to obtain the single phase heat transfer coefficient from experimental results for superheated vapour.

$\bar{h}_{S H}=\left[\left(\frac{A_{i} \Delta T_{(L M T D) S H}}{Q_{S H}}\right)-\left(A_{O} R_{W}\right)-\left(\frac{A_{O}}{A_{i} \bar{h}_{i}}\right)\right]^{-1}$

When the resulting heat transfer coefficient obtained from the experiment was plotted against the changes in $\mathrm{Re}, \mathrm{Pr}$ and $k$ (due to the change in properties of refrigerant for each individual data run), a coherent curve was obtained. The results of the single phase heat transfer coefficients for superheated vapour, for enhanced and plain annuli, are illustrated in figure 6. Both tests show a consistent trend, as expected, where the heat transfer coefficient increases with increase of mass flux.

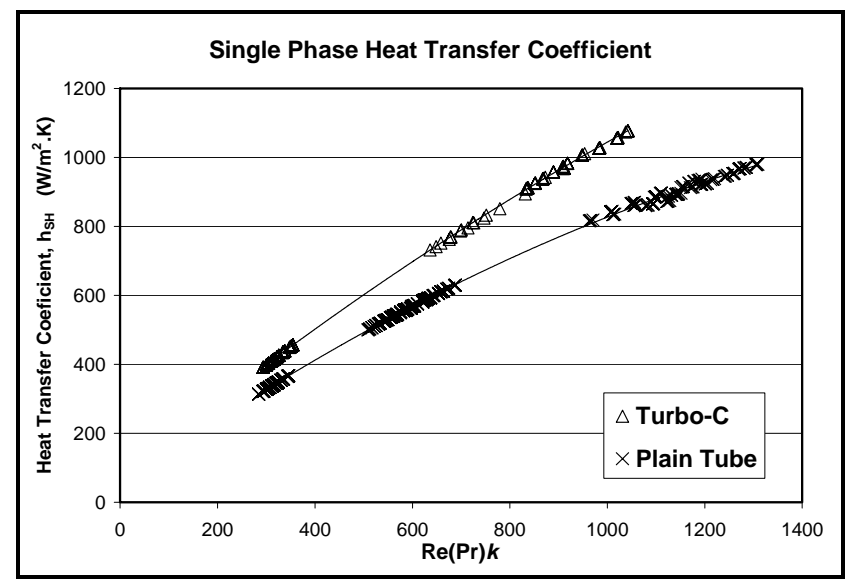

Figure 6. Comparison of single phase heat transfer coefficient 
The 3-Dimensional integral fin present for the Turbo-C annulus acts as an extended surface. The higher heat transfer coefficient for superheated vapour, when compared to plain annulus is attributed to the effective surface area. This condition explains the trend illustrated in figure 6 which may be summarized as:

a) The Turbo-C tube outperforms the plain tube by about $25 \%-30 \%$ for superheated vapour.

b) The single phase heat transfer coefficient increases with increasing mass flux.

\section{VIII.CORRELATIONS FOR PLAIN AND ENHANCED ANNULI}

This experimental work was conducted to develop design correlations so that the results of the study could be directly used by the HVAC community for design and development of double tube condenser systems. The first step in this effort was comparison of the current data with available correlations. The correlations available in the literature range from purely theoretical ones to those purely empirical in nature. All fluid properties required in the data reduction and the correlations were evaluated using property tables in ASHRAE Handbook of Fundamentals 2001 [7]. All subsequent analysis of correlations is given in nondimensional form. Comprehensive reviews of literature led to selection of a few correlations which best represent the annulus geometries and flow characteristics of the fluids in the test section. The selected correlation was compared with experimental work on plain tube. This is done to assess the applicability of the selected correlations. The one that most accurately represents the range and conditions of interest was used as the starting point. These correlations give the basic representation of the average heat transfer coefficients for a given set of parametric conditions. Next, it was assumed that the presence of the fluid inlet and exit fittings (both refrigerant and cooling water) and surface enhancement did not alter the form of the heat transfer coefficient substantially and that any difference present can be handled by adjusting the empirical constants.

Table 5.1 Individual correlations for test conditions

Smooth annulus [10]
$(\overline{N u})=\frac{(\bar{h}) D_{h}}{k}=0.020\left(\operatorname{Re}_{v}\right)^{0.8}(\operatorname{Pr})^{1 / 3}\left(\frac{D_{i}}{d_{O}}\right)^{0.53}$
for $\operatorname{Re}_{v}=>10,000$
Enhanced annulus [11]
$\left.(\overline{N u} v)=\frac{(\bar{h}) D_{h}}{k}=0.04 \operatorname{Re}_{v}\right)^{0.87}(\operatorname{Pr})^{0.4}\left(\frac{s}{D_{h}}\right)^{0.4}\left(\frac{e}{D_{h}}\right)^{-0.19}$
for $\quad \operatorname{Re}_{v}=>10,000$

Considering the current case of condensation of pure vapour (R-22) flowing on a horizontal water-cooled tube, this process can be divided into three flow regions, namely, superheated vapour, condensation and subcooled liquid. Correlation development can generally lead to a deeper understanding of the heat transfer phenomenon that occurs in the annulus side. However, it is optimistic to expect such explanation in the present case in which one fluid is investigated for one diameter ratio. The correlation for forced convection in tubes is given in the form of:
$N u=\frac{h d}{k}=C\left(\operatorname{Re}^{m}\right) \operatorname{Pr}^{1 / 3}$

This common correlation due to Sieder-Tate is a simple approach which reveals the physical aspect of the experimental theory, but this is not the form of correlation which gives the best result. Another available correlation that has a similar form gives a lower scatter:

$N u=\frac{h d}{k}=C\left(\operatorname{Re}^{m}\right) \operatorname{Pr}^{n}$

where $\mathrm{m}$ is a function of Pr. Considering the conditions of our test, it is safe to assume that the changes in the Pr value of the superheated vapour and subcooled liquid of the condensation process have a minor influence on the way $\mathrm{Re}$ affects the $\mathrm{Nu}$ correlation. Hence if the index $\mathrm{m}$, as yet to be found, is constant, a simple linear regression will reveal the value of $\mathrm{C}$ and $\mathrm{m}$ in Eq. (9). In the Dry Run Test for superheated vapour flow, the compressed vapour enters the test section in superheated state and exits at the same phase. The heat transferred to the cooling water is kept sufficiently low to allow the vapour exit temperature to be above the saturation temperature and to avoid phase change

$Q_{S H}=\dot{m}_{O}(\Delta h)_{S H}=\bar{h}_{S H} A_{O}(L M T D)$

$\bar{h}_{S H}=\frac{\dot{m}_{O}(\Delta h)_{S H}}{A_{O}(L M T D)}$

$\overline{N u_{S H}}=\frac{\overline{h_{S H}} d_{h}}{k}=C\left(\operatorname{Re}^{m}\right) \operatorname{Pr}^{n}$

$\ln \left(\overline{N u_{S H}}\right)=\ln (C)+m \ln (\mathrm{Re})+\left(\frac{1}{n}\right) \ln (\operatorname{Pr})$

By plotting $\ln (\mathrm{Nu})$ against $\ln (\mathrm{Re})$ for the results of Dry Run Test for both plain tube and Turbo-C, the single phase heat transfer coefficient for superheated vapour and subcooled liquid was obtained. Figure 7 shows the relationship between heat transfer coefficient and superheated vapour flow in the annulus of plain and enhanced annulus in non-dimensional numbers. Considering the Reynolds Number for the test results is $>10000$, the flow in the plain annulus is taken as turbulent region. Equation (7) was used to evaluate the Nusselt type correlation which is given by Monrad and Pelton [10]. Thus, the single phase heat transfer coefficient for superheated vapour for the plain annulus is:

$(\overline{N u})_{\text {VAPOUR }}^{\text {PLAIN TUBE }}=0.0146(\mathrm{Re})^{0.8293}(\operatorname{Pr})^{1 / 3}\left(\frac{D_{i}}{d_{O}}\right)^{0.53}$

Equation (8) was used to evaluate the Nusselt type correlation which is given by Knudsen and Katz [11]. Thus, the single phase heat transfer coefficient for superheated vapour flow for enhanced annulus is:

$(\overline{N u})_{\text {VAPOUR }}^{\text {TURO-C }}=0.0248(\mathrm{Re})^{0.8412}(\mathrm{Pr})^{0.4}\left(\frac{s}{D_{h}}\right)^{0.4}\left(\frac{e}{D_{h}}\right)^{-0.19}$

Comparison of experimental Nusselt value using (15) and (16) against predicted Nusselt value is illustrated in figure 8 . 


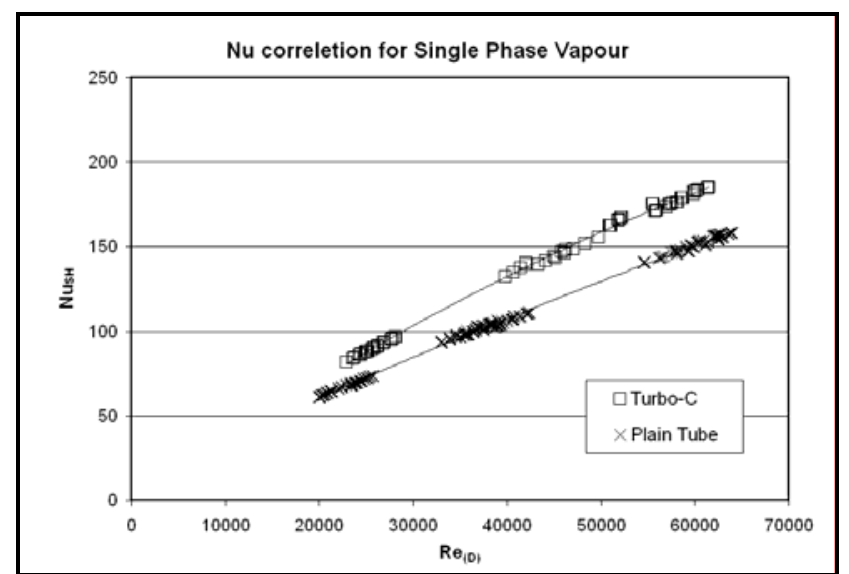

Figure 7. Comparison of single phase heat transfer coefficient non-dimensional number

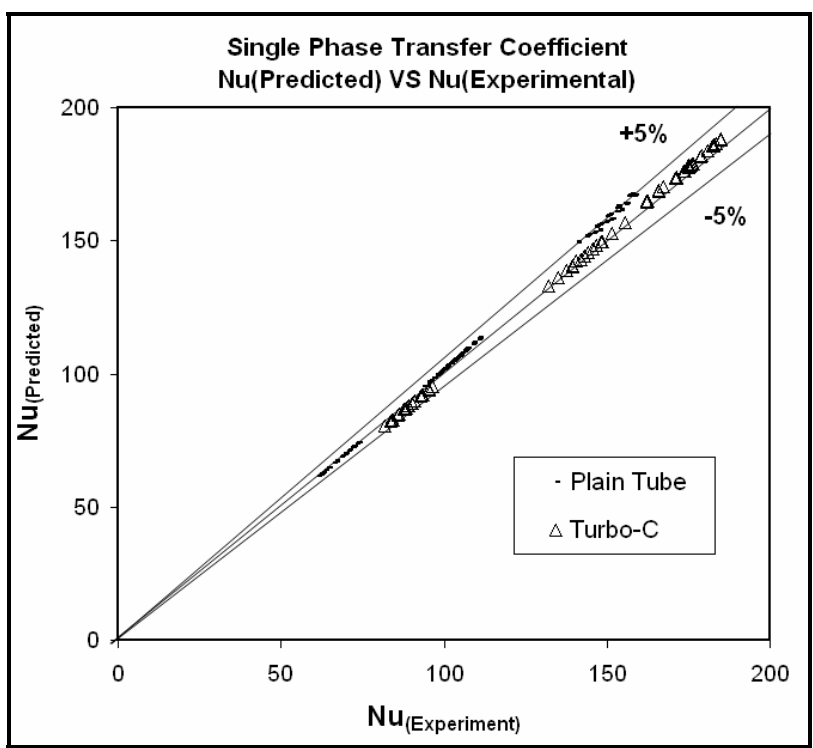

Figure 8. Comparison of experimental Nusselt against number predicted Nusselt number for superheated vapour

\section{OVERVIEW REMARKS}

The overall objective of the present study was to develop the single phase heat transfer coefficient for superheated vapour. The correlation by Monrad and Pelton [10] was used for plain annuli. For heat transfer in enhanced annuli, the correlation by Knudsen and Katz [11] determined the basic form. The new empirical constants resulted in good prediction for turbulent superheated vapour flow in the annulus for both plain annulus and enhanced annulus. On examining the accuracy of the single phase heat transfer correlation proposed, all predicted data is within the $\pm 5 \%$ of experimental value. The superheated vapour flow of plain annulus has an absolute deviation of $\pm 3.12 \%$ and $\pm 2.52 \%$ respectively. Similar results are observed for the superheated vapour and subcooled liquid flow for enhanced annulus with $+3.91 \%$ and $+2.46 \%$ receptively.

\section{ACKNOWLEDGMENT}

The authors would like to acknowledge with appreciation, the help and support from OYL R\&D CENTER, in providing all facilities for this research work.

\section{REFERENCES}

[1] Briggs, D.E., Young, E.H., "Modified Wilson plot techniques for obtaining heat transfer correlations for shell and tube heat exchangers," Chemical Engineering Progress Symposium Series, No. 92, Vol. 65, 1969, pp. 35-45.

[2] Deans, J., Sinderman, A., Morrison, J.N.A., "Use of the Wilson plot method to design and commission a condensation heat transfer test facility," Two-Phase Flow Modelling and Experimentation, Edizioni ETS, 1999.

[3] Kline, S., and McClintok, F., "Describing uncertainties in singlesample experiments," Mechanical Engineering, Vol. 75, Jan 1953, pp. 3-8.

[4] Kamei, A., Beyerlein, S.W., and Jacobsen, R.T., "Application of nonlinear regression in the development of a wide range formulation for HCFC-22," International Journal of Thermophysics, Vol. 16, No. 5, 1995, pp. 1155-1164.

[5] Klein, S.A., McLinden, M.O., “An improved extended corresponding states method for estimation of viscosity of pure refrigerants and mixtures," International Journal of Refrigeration, Vol. 20, No. 3, 1997, pp. 208-217.

[6] Mclinden, M.O., Klein, S.A., Perkins, R.A., “An improved extended corresponding states model of thermal conductivity refrigerants and refrigerant mixtures," International Journal of Refrigeration, Vol. 20, No. 3, 1997, pp. 208-217.

[7] ASHRAE Fundamentals Handbook, Appendix E Thermophysics Properties, 2001.

[8] Wagner, W., and $\operatorname{Pru} B$, A., "New international formulation for the thermodymanic properties of ordinary water substance for general and scientific use," Journal of Physical and Chemical Reference Data, Vol. 31, No. 2, 2002.

[9] Kestin, J., Sengers, J.V., Kamgar-Parsi, B., and Levelt Sengers, J.M.H., "Thermophysical properties of fluid $\mathrm{H}_{2} \mathrm{O}$," Journal of Physical and Chemical Reference Data, Vol. 13, No. 1, 1984.

[10] Monrad, C.C., and Pelton, J.F., Transection AIChE, Vol. 38, 1942, pp. 593.

[11] Knudsen, J.G., and Katz, D.L., Chemical Engineering Progress, Vol. 46, 1950, pp. 490.

\section{NOMENCLATURE}

area $\left(\mathrm{m}^{2}\right)$

Coefficient of the heat transfer correlation

diameter $(\mathrm{m})$

convective heat transfer coefficient $\left(\mathrm{W} / \mathrm{m}^{2} \mathrm{~K}\right)$

latent heat of vaporization $(\mathrm{J} / \mathrm{kg})$

thermal conductivity $(\mathrm{W} / \mathrm{mK})$

Prandtl Number

rate of heat transfer (W)

thermal resistance $(\mathrm{K} / \mathrm{W})$

Reynolds Number

temperature $(\mathrm{K})$

overall heat transfer coefficient $\left(\mathrm{W} / \mathrm{m}^{2} \mathrm{~K}\right)$

change in temperature $(\mathrm{K})$

density $\left(\mathrm{kg} / \mathrm{m}^{3}\right)$

viscosity $(\mathrm{kg} / \mathrm{ms})$

Log mean temperature difference

mass flow rate $(\mathrm{kg} / \mathrm{hr})$

Subscripts:

c coolant

cw coolant wall

i internal

liquid

outside

tube surface

saturation

superheated vapour

wall 


\title{
Exergy Analysis of a Cogeneration and Cooling Plant
}

\author{
C. Rangkuti, A. L.Tamiru, S. I. Gilani, and M. Amin
}

\begin{abstract}
First law equation and performance indicators other than exergy are often applied in the performance analysis of cogeneration and cooling plants. However, this approach treats heat and work with minor distinction and was found inappropriate for the analysis of such plants. Instead, the identification of those units or systems responsible for higher losses, exergetic analysis is the preferred method for it takes the quality difference into account. In this paper, exergetic approach is used to estimate the efficiencies of a cogenerator, steam absorption chillers (SACs), and electric chillers (ECs), The case considered is UTP cogeneration and cooling plant that has a capacity of 8.4 MW electric power, 12 ton/hr of steam and 3800RT of chilled water $(2 \times 1250 R T$ from SACs and $4 \times 325 R T$ from ECs). Hourly data for one week $\left(1^{\text {st }}-7^{\text {th }}\right.$ August 2005 , a typical week in a dry season with high load) have been considered to estimate the maximum exergetic loses. The result showed that exergetic efficiency of the plant is decreased by $20-30 \%$ during peak hours attributed to running the ECs in the stated period. The report includes description of the system and alternative solutions to enhance the performance of the plant.
\end{abstract}

Keywords: gas turbine generator, heat recovery steam generators, steam absorption chiller, electric chiller, Exergy

\section{INTRODUCTION}

A cogeneration and cooling plant delivers three outputs from a single source of energy. The first output - electric power - is generated in the gas turbine generator (GTG) part of the cogeneration system while the second output steam - is produced in the heat recovery steam generator (HRSG), which is also part of the cogeneration system. The third output - chilled water - though, can be produced using the steam from the HRSG in the steam absorption chillers (SACs) or employing electric chillers (ECs) that are driven by the electricity generated by the GTG. The GTG is featured by an air-compressor, combustor, and gas turbine.

In conventional plants the byproduct of electricity production (heat), which can be as high as 60 to $75 \%$, is

C. Rangkuti is now with the Mechanical Engineering Department Universiti Teknologi PETRONAS, Tronoh, Perak, Malaysia. (phone: 6053687004; fax: 605-365-6461; e-mail: chalilullah@petronas.com.my).

A. L. Tamiru, is now with the Universiti Teknologi PETRONAS, Tronoh, Perak, Malaysia. (e-mail: tamiru_alemu@utp.edu.my).

S. I. Gilani is with the Universiti Teknologi PETRONAS, Tronoh, Perak, Malaysia. (e-mail:syedihtsham@petronas.com.my).

M. Amin, is now with the Universiti Teknologi PETRONAS, Tronoh, Perak, Malaysia. (e-mail: mamin_amajid@petronas.com.my). directly discharged to the environment. Unlike this however, with cogeneration and cooling plants, because of the synchronization between the GTG and HRSG, and also between the HRSG and SACs, energies as high as $25-48 \%$, calculated based on first-law of thermodynamic equation, can be saved [1], [2].

Cogeneration has been in use for more than 100 years in large-scale centralized power plants and industrial applications [3]. But in recent years the concept has evolved to the combined use of cogeneration and cooling plants, especially to the ones that are equipped with SACs, attributed to the growing concern on global warming and high electricity costs. Commercial, institutional, residential and small industrial sections are typical areas of application for cooling and power. In this report, we shall consider Universiti Teknologi PETRONAS (UTP) cogeneration and cooling plant. In previous studies, analysis on electricity and chilled water production - Amin et al [4] based on two weeks data $\left(1^{\text {st }}\right.$ to $7^{\text {th }}$ October of 2004 and $11^{\text {th }}$ to $17^{\text {th }}$ April of 2005), Gilani et al [5] using three weeks data $\left(10^{\text {th }}\right.$ to $16^{\text {th }}$ January $2005,11^{\text {th }}$ to $17^{\text {th }}$ April 2005 , and $11^{\text {th }}$ to $17^{\text {th }}$ July 2005) - and estimation of first law efficiencies [6] (relying on one year data, 2005) for cogeneration part of the whole plant, have been conducted.

The first result revealed assumed working strategies violated while the latter elaborated the relative difference in the efficiency of electricity generation and efficiency of steam production in the cogeneration part of the plant. In addition to that, it has also recommended energy optimization to be applied to effect in better energy conservation. The drawback of the second approach however is that, it treats heat and power with minor distinction and was found inappropriate for the analysis of such plants. In the identification of those units or systems responsible for higher losses, exergetic analysis is the preferred method for it takes the quality difference into account.

In this paper, exergetic approach is used to estimate the efficiencies of a cogenerator, steam absorption chiller (SAC), and electric chiller (EC). Hourly data for one week $\left(1^{\text {st }}-7^{\text {th }}\right.$ August 2005 , a typical week in a dry season with high load) will be considered to estimate the maximum exergetic loses.

In the sections that follow, first physical characteristics of the plant would be discussed followed by the kind and form of equations used in the exergetic performance evaluation of the plant. After that analysis results associated with peak 
and off-peak hour operations would be discussed for the cogenerators, SACs, ECs and whole plant. In the final part of the paper, conclusions together with some recommendations would be outlined.

\section{System CONFiguration}

UTP cogeneration and cooling plant is characterized by two GTG, two units of HRSG, two units of SACs and four units of ECs. The plant is designed and put in service to produce electric power and deliver it to the customers (UTP) and inplant usage. HRSG produced steam is supplied to the steam absorption chillers (SACs) making the chillers produce chilled water. The plant has a capacity of $8.4 \mathrm{MW}$ electric power, $12 \mathrm{ton} / \mathrm{hr}$ of steam and 3800RT of chilled water (2500RT from SACs and 1300RT from ECs). Figure 1 shows process diagram for part of the plant.

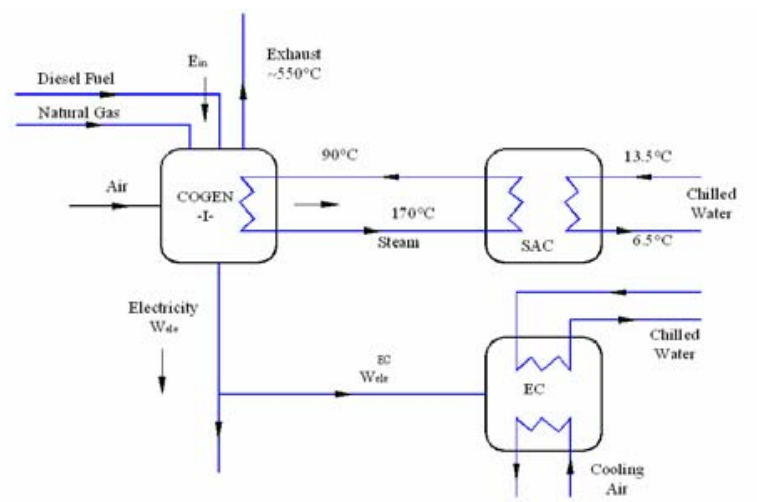

Figure 1. Simplified process diagram of UTP cogeneration and cooling plant

Operating strategy that has put in place is such that the GTGs would run during peak hours (8:00-21:00) and one GTG operates during off-peak hours (0:00-8:00 and 21:0023:00). To keep high reliability of electricity supply, additional or replacement electricity would be obtained from Tenaga Nasional Berhad (TNB). The two HRSG would be used to recover the waste heat. During peak hours or in normal operation mode, both SACs would run concurrently with the HRSGs while one would be run during off-peak hours.

\section{Calculation of EFFiciencies}

Sub-system $1^{\text {st }}$ law efficiencies based on the input and output power for the cogeneration portion of a cogeneration and cooling plant are known by the following expressions, with the first equation representing electrical efficiency and the second indicating efficiency of steam production [7].

$$
\begin{gathered}
\eta_{\text {ele }}=\frac{\dot{W}_{\text {ele }}}{\dot{E}_{\text {in }}} \\
\eta_{\text {heat }}=\frac{\dot{Q}_{S T}}{\dot{E}_{\text {in }}}
\end{gathered}
$$

The terms $\dot{E}_{\text {in }}, \dot{W}_{\text {ele }}$ and $\dot{Q}_{S T}$ stand for rate of input energy supply, rate of electric power generation and heat production respectively. For the sequential production of both electricity and heat, the combined efficiency then equals the sum of the two efficiencies, i.e.

$$
\begin{aligned}
\eta_{\text {combined }} & =\frac{\dot{W}_{\text {ele }}+\dot{Q}_{S T}}{\dot{E}_{\text {in }}} \\
& =\eta_{\text {ele }}+\eta_{S T}
\end{aligned}
$$

For the cooling section of the plant, however, coefficient of performance, which is the ratio between the cooling effect and the input energy, are frequently used to quantify the performance of the units. For the steam absorption chiller only, one can put it as

$C O P^{S A C}=\frac{\dot{Q}_{C}^{S A C}}{\dot{Q}_{S T}}$

While for that of the electric chiller, it takes the form

$C O P^{E C}=\frac{\dot{Q}_{C}^{E C}}{\dot{W}_{\text {ele }}^{E C}}$

Equations (1)-(5) work well only for the separate analysis of cogeneration and cooling systems, respectively. For the combined evaluation, hence, we need to use the concept of figure-of-merit [2] which is given by

$f_{S Y S}=\frac{\left(\dot{W}_{e l e}^{C O G E N}-\dot{W}_{e l e}^{E C}\right)+\dot{Q}_{C}^{S A C}+\dot{Q}_{C}^{E C}}{\dot{E}_{\text {in }}}$

\section{A. Exergetic Efficiencies}

In thermodynamic analysis, exergetic approach outweighs first law approach as it assumes quality difference between work and heat [8]. For the main units of the cogeneration and cooling plant,

$$
\begin{aligned}
& \psi^{C O G E N}=\frac{\dot{W}_{e l e}^{C O G E N}+\tau_{H R S G} \dot{Q}_{S T}}{R_{N G} \times \dot{E}_{N G}+R_{D F} \times \dot{E}_{D F}} \\
& \psi^{S A C}=\frac{-\tau_{S A C} \times \dot{Q}_{C}^{S A C}}{\tau_{H R S G} \times \dot{Q}_{S T}}
\end{aligned}
$$

$\psi^{E C}=\frac{-\tau_{E C} \times \dot{Q}_{C}^{E C}}{\dot{W}_{\text {ele }}^{E C}}$

and for a cogeneration system having the three units together,

$$
\begin{gathered}
\psi^{S Y S}=\frac{\left(\dot{W}_{e l e}^{C O G E N}-\dot{W}_{e l e}^{E C}\right)+\tau_{H R S G} \times \dot{Q}_{S T}}{R_{N G} \times \dot{E}_{N G}+R_{D F} \times \dot{E}_{D F}} \\
-\frac{\tau_{S A C} \times Q_{C}^{S A C}+\tau_{E C} \dot{Q}_{C}^{E C}}{R_{N G} \times \dot{E}_{N G}+R_{D F} \times \dot{E}_{D F}}
\end{gathered}
$$

where $R_{N G}=0.99$ and $R_{D F}=0.93$ stand for values of energy grade functions for natural gas and diesel fuel respectively [10] while $\psi^{\text {COGEN }}, \psi^{S A C}, \psi^{E C}$ and $\psi^{s y s}$ are exergetic efficiencies of a cogenerator, a steam absorption chiller, an electric chiller and the combined system, respectively. In the 
same set of equations, the constants $\tau_{H R S G}, \tau_{S A C}$ and $\tau_{E C}$ signify exergetic temperature factors for a cogenerator, a steam absorption chiller and an electric chiller, respectively. They can be calculated based on a reference temperature of $T_{o}$ and an average temperature between supply and return temperature to the respective units as:

$\tau_{i}=1-\frac{2 T_{0}+546}{\left(T_{I N}+T_{\text {OUT }}\right)_{i}+546}, \quad i=H R S G, S A C, E C$

The value of electrical output from a cogenerator $\dot{W}_{\text {ele }}^{\text {COGEN }}$ is directly obtainable from the plant operation data [9] while $\dot{Q}_{S T}$ and $\dot{E}_{i n}$ are not directly measurable. Hence, with $\dot{m}_{N G}$ and $\dot{m}_{D F}$ signifying mass flow rate of natural gas and diesel fuel respectively, the following equations are applied to compute their respective values.

$\dot{E}_{\text {in }}=\dot{m}_{N G} E_{N G}+\dot{m}_{D F} E_{D F}$

$\dot{Q}_{S T}=\dot{m}_{S T}\left(h_{\text {in }}-h_{\text {out }}\right)$

In the second equation $h_{i n}$ represents specific enthalpy of the steam at a pressure $P_{H R S G}$ of $0.78 \mathrm{MPa}$ and temperature $T_{H R S G}$ of $170^{\circ} \mathrm{C}$ while $h_{\text {out }}$ signifies enthalpy of the condensate at the absorption chiller temperature $T_{S A C}$ of $90^{\circ} \mathrm{C}$.

\section{RESULTS AND DISCUSSION}

During the period of $1^{\text {st }}-7^{\text {th }}$ August 2005, the plant (cf. figure 1) has generated $7 \times 10^{5} \mathrm{KWh}$ of electricity, $5.93 \times 10^{2}$ Ton of steam and $2.20 \times 10^{5} \mathrm{RTH}$ of chilled water. The average supply and return temperatures, respectively, of the steam to and from the SACs are $170^{\circ} \mathrm{C}$ and $90^{\circ} \mathrm{C}$. Besides, the average supply and return temperatures, respectively, of the chilled water to and from the SACs and ECs are assumed to be $13.5^{\circ} \mathrm{C}$ and $6.5^{\circ} \mathrm{C}$.

Figure 2 shows the number of running hours over the week. Tuesday and Thursday, HRSG-A supplied steam to SAC-A and SAC-B. But on Wednesday the two chillers obtained steam from HRSG-B that lasted for 4 hours. On Friday, SACs were getting supply from the steam header.

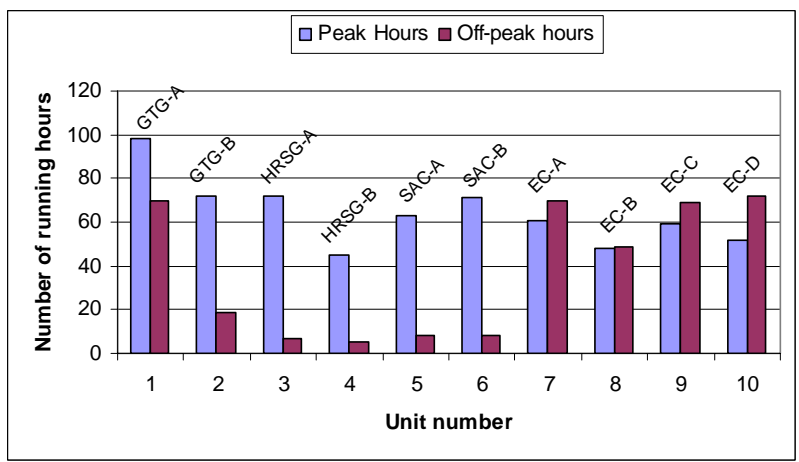

Figure 2. Number of running hours for main units of the cogeneration and cooling plant, $1^{\text {st }}-7^{\text {th }}$ August 2005

\section{A. Performance of COGEN-I and COGEN-II}

Exergetic efficiencies calculated based on equations (7), (11), and (12) are shown in figure 3 and figure 4. During peak hours, COGEN-I and COGEN-II demonstrated an exergetic efficiency of about 30\% except for slight difference on Monday and Sunday. On Monday the efficiency of COGEN-I has reached a value of $37 \%$ with the main reason being HRSG-A worked for 14 hours in synchronous with GTG-A. Sunday, HRSG-A was not run and the exergetic efficiency appears to be equal to the exergetic efficiency of GTG-A only, $20 \%$. And, the same is true for COGEN-II when it was operated on saturday.

For the off-peak period, COGEN-I was featured by an exergetic efficiency of about $24 \%$ while COGEN-II experienced an efficiency of about $20 \%$. The reduced efficiencies are caused by limited run of HRSGs - one to two hours only. Sunday, COGEN-II did not work at all.

Comparing these efficiencies with $1^{\text {st }}$ law efficiencies, the latter shows a value as high as $68 \%$ for COGEN-I and 55\% for COGEN-II (cf. table 1).

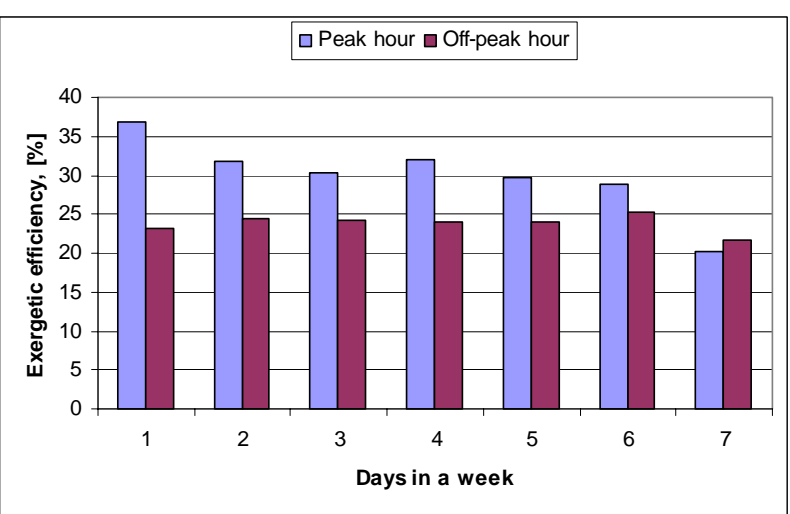

Figure 3. Exergetic efficiency variation of COGEN-I during peak and off-peak hours

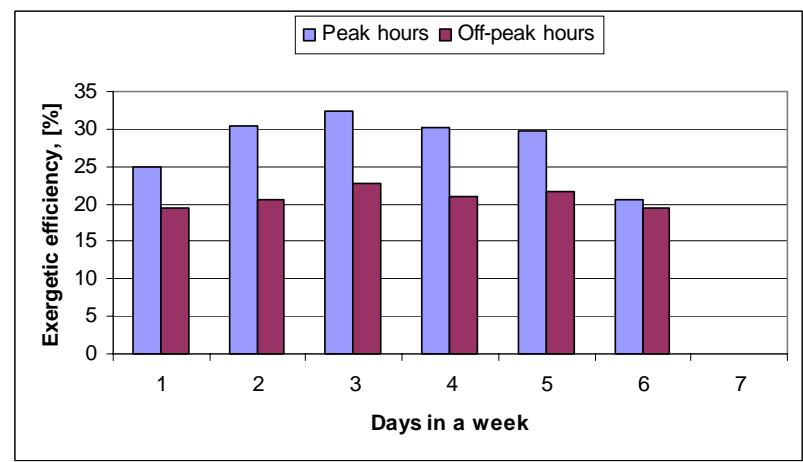

Figure 4. Exergetic efficiency variation of COGEN-II during peak and off-peak hours

\section{B. Performance of SACs}

Exergetic efficiencies of steam absorption chillers rely on the supply and return temperatures of the steam and chilled water. The chilled water supply temperature is controlled to be $6.5^{\circ} \mathrm{C}$ while the return temperature assumes a value in the range of $10^{\circ} \mathrm{C}$ and $13.5^{\circ} \mathrm{C}$. Based on the result form equation (8), both SACs reached an exergetic efficiency of $27.8 \%$ during peak hours except Sunday where both were at stand still (cf. figure 5). When we compare the number of running hours (cf. figure 2), SAC-B worked eight hours more than SAC-A has worked. During off-peak hours, SAC-A and 
SAC-B operated only for eight hours with calculated exergetic efficiency of $22.5 \%$.

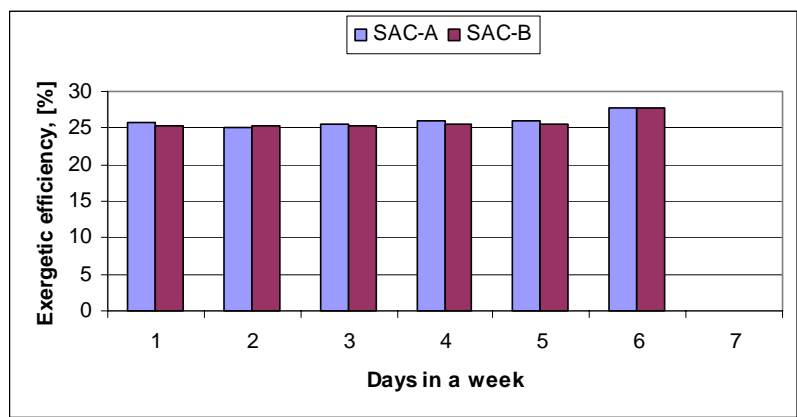

Figure 5. Exergetic efficiency variation of SACs during peak hours

\section{C.Performance of ECs}

The electric chillers function taking electric power from Distributed Control System (DCS) which is connected to the two cogenerators. To calculate exergetic efficiencies, therefore, the amount of electricity going to each EC, the cooling effect from each chiller, and the chilled water supply and return temperatures have to be known. From the plant operation data the chilled water supply and return temperatures vary around $6.5^{\circ} \mathrm{C}$ and $13^{\circ} \mathrm{C}$, respectively.

Calculated values indicate that (cf. figure 6 and figure 7) the electric chillers have experienced exergetic efficiencies of $14 \%$ to $19 \%$ during peak and off-peak hours, respectively. Considering the number of hours each chiller has run during peak period, the record shows $61,48,59$ and 52 hours for EC-A, EC-B, EC-C and EC-D respectively. The maximum operation hour difference between peak and off-peak duty were about 20 hours.

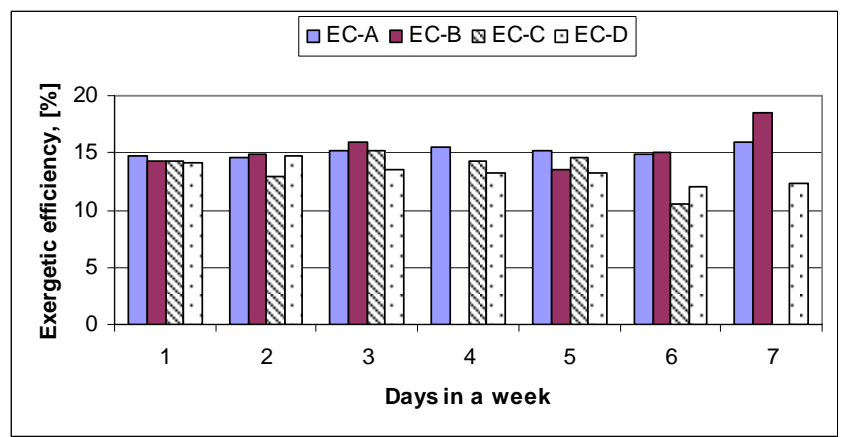

Figure 6. Exergetic efficiency variation of ECs during peak hours

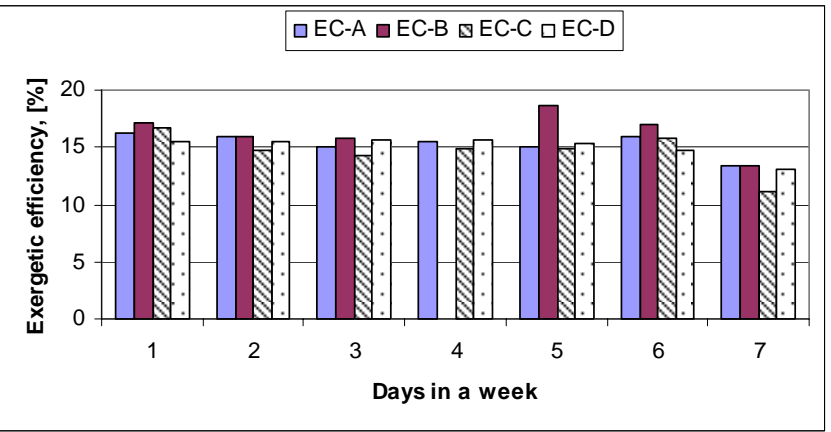

Figure 7. Exergetic efficiency variation of ECs during offpeak hours

\section{D.Performance of Overall plant}

Figure 8 shows exergetic efficiency variation of the overall plant during peak and off-peak hours. As indicated, the plant has reached an efficiency of $24 \%$ during peak hours and an efficiency of $17 \%$ during off-peak hours. But, Sunday the efficiency during peak hours was dropped to $17 \%$ attributed to running GTG-A and electric chillers only. Same day and regarding the number of running hours, GTG-A was operating for 24 hours while the electric chillers were run 10 to 16 hours. The $1^{\text {st }}$ law efficiency obtained using (6) is about $49 \%$ (cf. table 1).

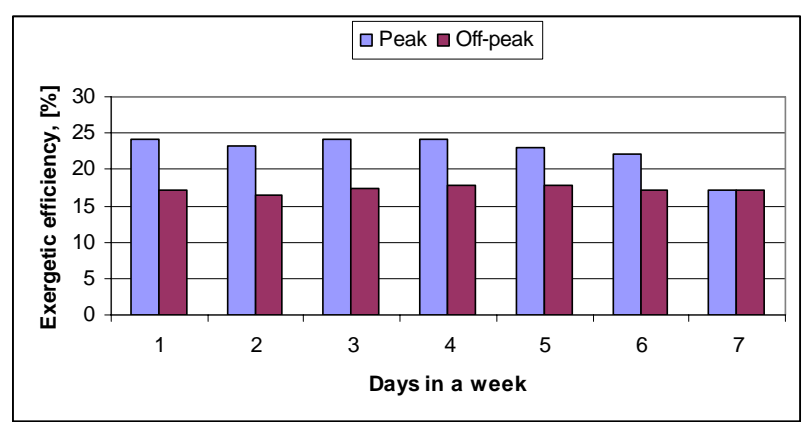

Figure 8. Exergetic efficiency variation of the whole plant during peak and off-peak hours

Table 1. Maximum energy and exergy efficiencies, during peak hours, for main units of the cogeneration and cooling plant

\begin{tabular}{|c|c|c|}
\hline Unit & $\begin{array}{c}\text { Energy } \\
\text { efficiency, [\%] }\end{array}$ & $\begin{array}{c}\text { Exergy } \\
\text { efficiency, [\%] }\end{array}$ \\
\hline \multicolumn{3}{|l|}{ COGEN-I } \\
\hline GTG-A & 25 & 25 \\
\hline HRSG-A & 43 & 12 \\
\hline \multicolumn{3}{|l|}{ COGEN-II } \\
\hline GTG-B & 25 & 25 \\
\hline HRSG-B & 25 & 7 \\
\hline \multicolumn{3}{|l|}{ SACs } \\
\hline SAC-A & 134 & 28 \\
\hline SAC-B & 109 & 28 \\
\hline \multicolumn{3}{|l|}{$\mathrm{ECs}$} \\
\hline EC-A & 280 & 15 \\
\hline EC-B & 281 & 16 \\
\hline EC-C & 279 & 15 \\
\hline EC-D & 270 & 15 \\
\hline Overall plant & 49 & 24 \\
\hline
\end{tabular}




\section{CONCLUSIONS}

It was demonstrated that exergetic efficiency of the plant was about $23 \%$ during peak hours and about $17 \%$ during off-peak hours - about $20 \%$ lower than the maximum exergetic efficiency obtained for the cogenerators (cf. section 4.1). Main reasons for the reduced efficiency are

a. Reduced performance of the GTGs.

Part load operation has made the GTGs to generate only about 3 to 3.2 MWatt each. Had they been running at design point, we could have had higher contribution to the overall exergetic efficiency. Full load capacity for each GTG is $4.2 \mathrm{MW}$.

b. Large portion of the energy wasted to the environment.

It was observed that the HRSGs were run for lower periods as compared to the GTGs - the HRSGs were operated 20 to 30 hours short of GTGs during peak hours and 14 to 63 hours short of GTGs during offpeak hours.

c. Limited run and lower capacity of SACs.

HRSGs have more steam production capacity than required to cover the demand by SACs - there were days where one HRSG was found producing steam adequate enough to supply for both SACs. But attributed limited operation of the SACs and lower steam demand the HRSGs were forced to run at reduced flow rate of the hot exhaust gas.

d. Part of the electricity generated used to drive the electric chillers during peak hours.

It was noticed that all electric chillers were running during peak hours. Surprisingly, the number of hours they functioned was only 13 to 20 hours short of the SACs - a situation in odd with the operating strategies assumed at the design stage (cf. section 2).

HRGSs and ECs are the subsystems with the lower exergetic efficiencies (cf. table 1). To improve the situation therefore i) the HRSGs should be run whenever the GTGs are running; ii) ECs must not be run unless the SACs are under maintenance or there are more demand than can be delivered by the SACs.

Finally, all the $1^{\text {st }}$ law efficiencies (cf. table 1) are higher than exergetic efficiencies and are thus found misleading the analysis. Besides, the case considered here revealed that they are not suitable to analyze power generation and cooling all together. In the future we propose to proceed with the component level exergetic and thermoeconomic analysis of the cogeneration and cooling plant as that leads us to the decision making stage.

\section{ABBRIVIATIONS}

$\begin{array}{ll}\text { COGEN } & \text { Cogenerator } \\ \text { GTG } & \text { Gas Turbine Generator } \\ \text { HRSG } & \text { Heat Recovery Steam Generator } \\ \text { SAC } & \text { Steam Absorption Chiller } \\ \text { EC } & \text { Electric Chiller }\end{array}$

\section{Nomenclature}

$\begin{array}{llll}\dot{m} & \text { Mass flow rate } & E & \text { Energy } \\ W & \text { Work } & Q & \text { Heat } \\ h & \text { Enthalpy } & T & \text { Temperature }\end{array}$

\section{Greek symbols}

$\eta \quad 1^{\text {st }}$ law efficiency

$\psi$ Exergetic efficiency

$\tau$ Exergetic temperature factor

$\begin{array}{llll}\text { Subscripts } & & \\ D F & \text { Diesel Fuel } & \text { out } & \text { Output } \\ \text { in } & \text { Input } & \text { ele } & \text { Electricity } \\ N G & \text { Natural Gas } & \text { ST } & \text { Steam } \\ C & \text { Cooling } & & \end{array}$

\section{ACKNOWLEDGMENT}

The authors acknowledge the support of Universiti Teknologi Petronas (UTP) in conducting this study.

\section{REFERENCES}

[1] M. Krarti(2000): "The Engineering Handbook", CRC press

[2] A.R. Marc, Minnh N. Le, and Ibrahim Diner(2005): "Efficiency analysis of a cogeneration and district energy system", International Journal of Applied Thermal Engineering.

[3] R.Z. Wang, \& Wu, D.W. (2006): "Combined cooling, heating and power: A review", Journal of Progress in Energy and Combustion Science.

[4] M. Amin A.M., S. I. Gilani and C. Rangkuti(2006): "Chillers operating strategy of a thermal energy storage system" International Conference on Energy and Environment, Organized by UniTen, Malaysia.

[5] S. I. Gilani, A. M. Amin, and C. Rangkuti(2006): "A case study on electricity and chilled water production of a Gas District Cooling Plant", Bulletin of Institute of Engineers, Malaysia.

[6] S. I. Gilani, C. Rangkuti, A.L.Tamiru, and B. Ariwhjoedi(2007): "Efficiency analysis of a small cogeneration facility", Conference on Applications and Design in Mechanical Engineering, Kangar, Perlis, Malaysia.

[7] J.H. Horlock(1992): "Combined Power Plants: Including Combined Cycle Gas Turbine (CCGT) Plants", Pergamon Press.

[8] T.Y. Kotas(1985): "The exergetic method of thermal plant analysis", Anchor Brendon Ltd., London.

[9] GDC Daily Plant Reports, January to December 2005.

[10] I. Dincer, M.M. Hussain, and I. Al-Zaharnah(2004): "Energy and exergy utilization in transportation sector of Saudi Arabia", International Journal of Applied Thermal Engineering. 


\title{
Identification of Microorganisms in an Anaerobic Baffled Reactor (ABR) for Methane Production
}

\author{
A. Malakahmad, S.M. Zain, N.E. Basri \& F. Suja’
}

\begin{abstract}
Anaerobic digestion process is one of the alternative methods to convert organic waste into methane gas which is a fuel and energy source. Activities of various kinds of microorganisms are the main factor for anaerobic digestion in the Anaerobic Baffled Reactor (ABR) which produces methane gas. Observations on microorganisms in the ABR showed that, there exist a small amount of protozoa (5\%) and fungi (2\%) in the system, but almost $93 \%$ of the microorganism population consists of bacteria. It is definitely clear that bacteria are responsible for anaerobic biodegradation of organic fraction of municipal solid waste. Three groups of bacteria are involved in the anaerobic digestion: the hydrolytic and fermentative bacteria, the acetogenic bacteria and the methanogenic bacteria.
\end{abstract}

Keywords: anaerobic baffle reactor (ABR), methane, bacteria

\section{INTRODUCTION}

The use of oil and other fossil resources as transportation fuels and commodity chemicals is deeply engrained in today's society, but use of these resources is unsustainable. The unsustainable nature of fossil fuels stems from their finite reserves and their negative environmental impact: combustion of fuels releases carbon dioxide and various pollutants, such as sulfur and nitrogen oxides. The promotion of waste minimization and recycling are important components of modern waste management strategies. Nevertheless, even when the minimization and recycling potentials are fully exploited, there is still a residual fraction, which has to be disposed of. The burdens resulting from landfilling can be minimized by pre-treating the waste and thus limiting its emission potential [1]. Anaerobic treatment processes could reach to an average of $50-55 \%$ reduction of organic content in the treatment of residual waste. Practical tests have shown that threshold values can be achieved at the above-mentioned reduction of organic content with a post-decomposition duration of

A. Malakahmad is with the Department of Civil Engineering, Universiti Teknologi PETRONAS (UTP), 31750 Tronoh, Perak, Malaysia (phone: 6053687349; fax: 6053656716; e-mail: amirhossein@petronas.com.my).

S.M. Zain is with the Department of Civil \& Structural Engineering, Universiti Kebangsaan Malaysia (email: smz@vlsi.eng.ukm.my).

N.E. Basri is with the Department of Civil \& Structural Engineering, Universiti Kebangsaan Malaysia (email: ezlin@vlsi.eng.ukm.my)

F. Suja' is with the Department of Civil \& Structural Engineering, Universiti Kebangsaan Malaysia (email: fati@vlsi.eng.ukm.my). approximately 4-6 weeks. Thus, there is a need for alternative processes to produce energy and chemicals [2]. The transition towards a sustainable energy supply will take considerable time. In the meantime, short-term solutions will aim to lessen the environmental impact of fossil fuels [3].

\section{ANAEROBIC Digestion}

Anaerobic digestion is the classical example of a process that combines the objectives of elimination of organic compounds from a waste stream with the generation of a valuable product in the form of methane-containing biogas. Different bioreactor configurations have been developed for the treatment of liquid and solid waste streams [1]-[3]. For wastewater treatment, the application potential of anaerobic digestion has been extended from medium to highly concentrated wastewaters of agro-industrial origin, to more complex applications like those generated in petrochemical industries [4] and [5], paper industries [5] and [6] and even sewage [7]. There are three clear advantages of the anaerobic treatment over aerobic degradation of organic substrates: the high product and low biomass yield resulting in a limited generation of waste sludge as an unwanted side product; the in situ separation of the product as biogas, limiting costs for product separation; and the use of simple technology, as mixing by the biogas produced circumvents the need for other mixing requirements.

An anaerobic baffled reactor operates with a combination of several anaerobic process principles, the three basic steps involved are: (a) hydrolysis, (b) fermentation, and (c) methanogenesis. The ABR is a fluidized bed reactor similarly to the up flow anaerobic sludge bed (UASB) process. Equal inflow distribution, and the wide spread contact between new and old substrate are important process features. It is known that a three-chamber reactor, together with physical modifications, provided a longer solid retention time and superior performance than the reactor with only two compartments [8].

The selective pressure required to induce methane production from a waste stream containing organic compounds is very simple that avoids the presence of an electron acceptor (e.g. oxygen, nitrate or sulfate) or an external energy source (e.g. light). In the absence of an external electron acceptor, organic substrates can only be fermented; a process where the organic substrate is both the 
electron donor and acceptor. The final end products of the organic substrate fermentation are methane, carbon dioxide and ammonia. Methane is the organic compound with the lowest free energy content per electron upon oxidation to carbon dioxide (Figure 1). This indicates that in a thermodynamically closed system substrates will eventually be converted to methane and carbon dioxide. Microorganisms can obtain the energy required for growth by (stepwise) catalyzing the conversion of organic substrates to methane and carbon dioxide.

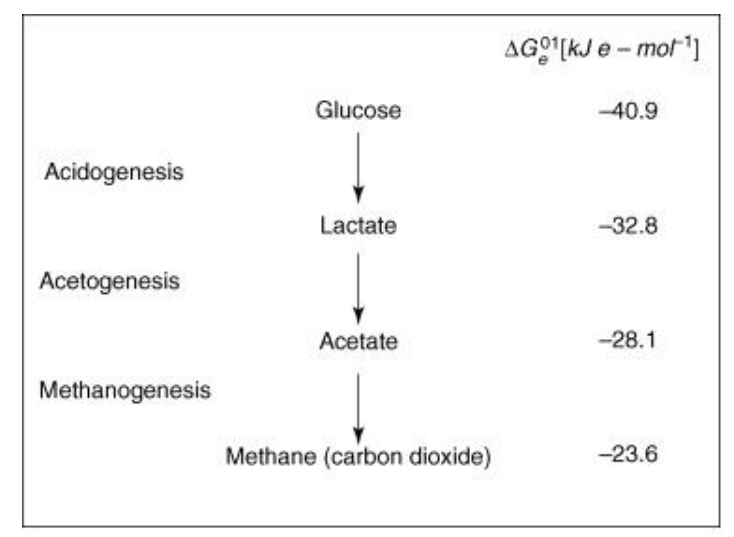

Figure 1. Fermentation of glucose to methane

\section{Methane Generation by Anaerobic Digestion}

Ideally the production of methane and carbon dioxide can be calculated using the following equation [9]:

$\mathrm{C}_{\mathrm{a}} \mathrm{H}_{\mathrm{b}} \mathrm{O}_{\mathrm{c}} \mathrm{N}_{\mathrm{d}}+(4 \mathrm{a}-\mathrm{b}-2 \mathrm{c}+3 \mathrm{~d} / 4) \mathrm{H}_{2} \mathrm{O} \rightarrow$

$(4 a+b-2 c-3 d / 8) \mathrm{CH}_{4}+(4 a-b-2 c+3 d / 4) \mathrm{CO}_{2}$ $+\mathrm{d} \mathrm{NH}_{3}$

In fact, the chemical composition of Municipal Solid Waste (MSW) is difficult, if not impossible to be determined, although some attempts have been made to do so. The best approximation is that the organic fraction of refuse can be described by the chemical formula $\mathrm{C}_{99} \mathrm{H}_{149} \mathrm{O}_{59} \mathrm{~N}$. In using this equation note that the only carbon that can participate in the production of gas is from decomposable materials such as food waste and paper. Other organics, most importantly plastics, do not decompose to produce gas. The two ways of generation methane are to capture the gases produced in landfills, or to pre-treat the refuse and digest it in tanks similar to those used in wastewater treatment plants [10].

\section{Methodology}

Microorganism observations were done to identify the most important and active species in the $\mathrm{ABR}$ as an anaerobic biological reactor. All the experiments for identifying microorganism in the anaerobic process were done according to the standard methods for the examination of water and wastewater (APHA 1992) and are listed in Table 1.
Table 1. Methods for microorganism observations

\begin{tabular}{lc}
\hline \multicolumn{1}{c}{ Parameters } & Method Number \\
\hline Macrofauna & $9211 \mathrm{D}$ \\
Microflora and fauna & $9212 \mathrm{~B}$ \\
Determination of unknowns & $9213 \mathrm{D}$ \\
organism & \\
Total bacteria count & $9221 \mathrm{~B}$ \\
Anaerobic colony count & $9222 \mathrm{E}$ \\
\hline
\end{tabular}

\section{MiCROORGANISMS IN THE ABR SYSTEM}

Observations on microorganisms showed that, there exist small amount of protozoa (5\%) and fungi (2\%) in the system, but almost $93 \%$ of the microorganisms' population consists of bacteria.

The anaerobic digestion of organic material is biochemically and very complicated process, involving hundreds of possible intermediate compounds and reactions, each of which is catalyzed by specific enzymes or catalysts. In general, anaerobic digestion is considered to occur in three stages; Many organic wastes consist of complex organic polymers such as proteins, fats, carbohydrates, cellulose, lignin, etc., some of which are in the form of insoluble solids. In the first stage this organic polymers are broken down by extracellular enzymes produced by hydrolytic bacteria (Figure 2), and dissolves in water.

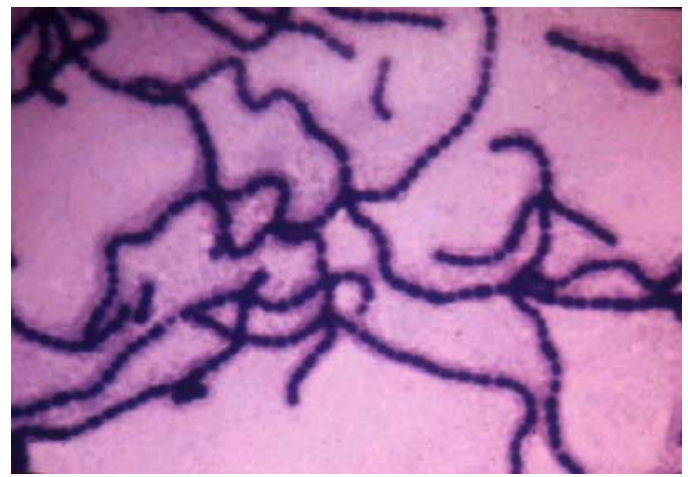

Figure 2.. Streptococci as Hydrolytic Bacteria during Anaerobic Process

In stage 2 the monomeric compounds released by the hydrolytic break down due to bacterial action in stage 1 are further converted to volatile fatty acids, $\mathrm{H}_{2}$ and $\mathrm{CO}_{2}$ by the acetogenic bacteria (Figure 3).

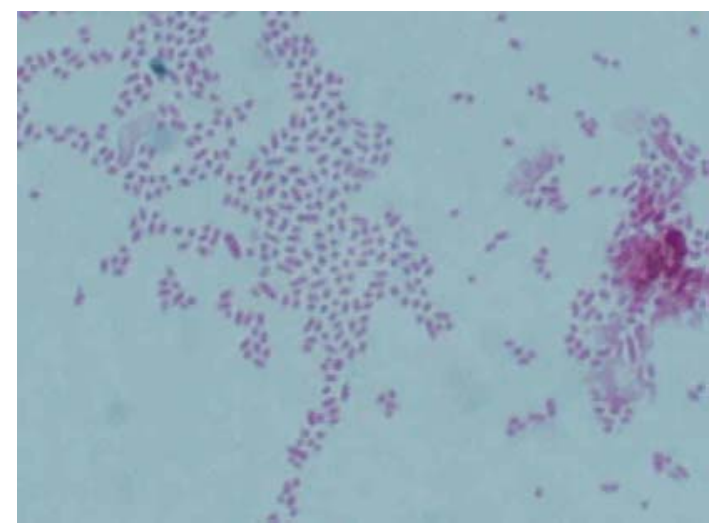

Figure 3. Acetogenic Bacteria During Biogasification

The products of the stage 2 are finally converted $\mathrm{CH}_{4}$ and other end products by a group of bacteria called 
methanogens (Figure 4). Methanogenic bacteria obligate anaerobes whose growth rate is generally slower than the bacteria in stage 1 and 2 . Figure 5 illustrates the quantitative content of all bacteria during the anaerobic degradation in the ABR system.

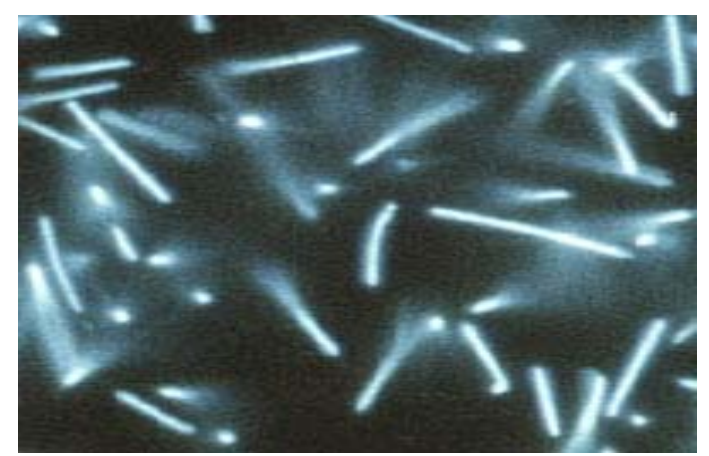

Figure 4. Methanotrix as Active Methane Formers in ABR System

\section{CONCLUSION}

The environmentally acceptable management of municipal solid waste has become a global challenge due to limited resources, ever increasing population, rapid urbanization and industrialization worldwide. The anaerobic digestion of organic material is biochemically and very complicated process, involving hundreds of possible intermediate compounds and reactions, each of which is catalyzed by specific enzymes or catalysts. With the unique construction of the ABR various profiles of microbial communities developed within the reactor. Observations of microorganisms showed that, there exist small amount of protozoa and fungi in the system, but almost $93 \%$ of the microorganisms' population consists of bacteria.

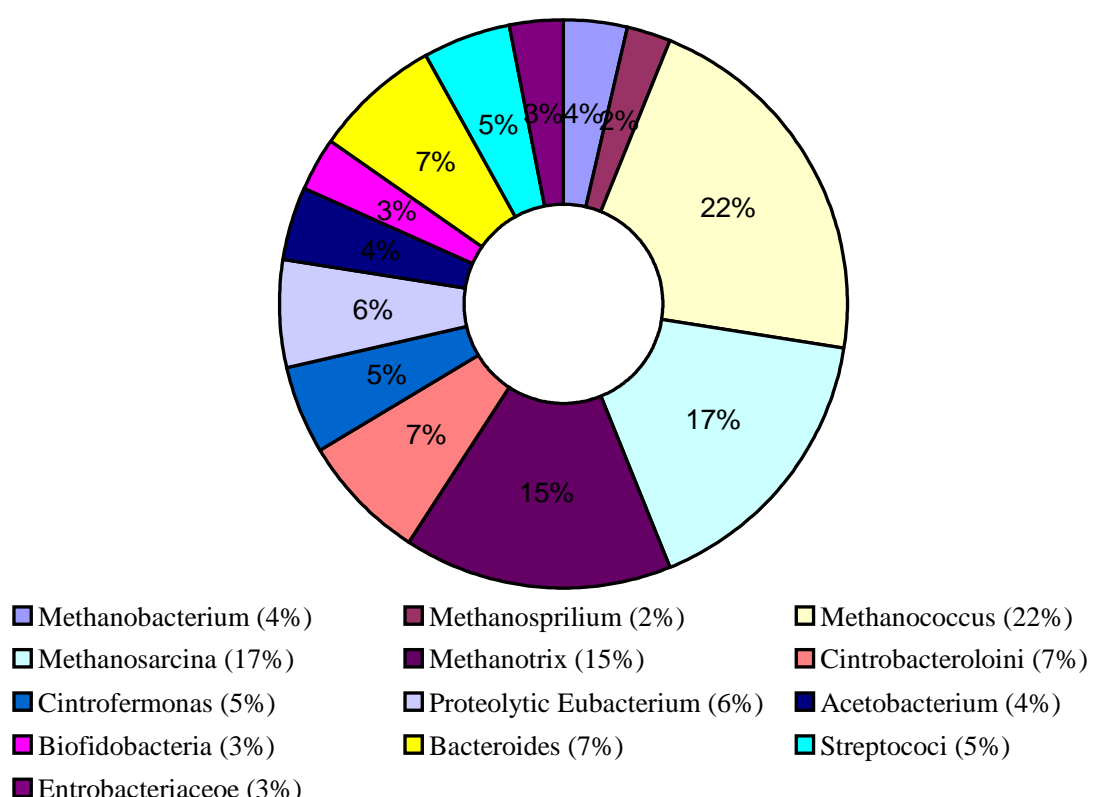

Figure 5. The Quantitative Content of Bacteria during the Anaerobic Degradation

\section{ACKNOWLEDGEMENT}

This paper was supported by Universiti Kebangsaan Malaysia (project code: KK008/2004) and the Universiti Teknologi PETRONAS.

\section{REFERENCES}

[1] A.J. Ragauskas, C.K. Williams, B.H. Davison, G. Britovsek, J. Cairney, C.A. Eckert, W.J. Frederick Jr., J.P. Hallett, D.J. Leak and C.L. Liotta et al., The path forward for biofuels and biomaterials, Science 311 (2006), pp. 484-489.

[2] G.W. Huber, S. Iborra and A. Corma, Synthesis of transportation fuels from biomass: chemistry, catalysts, and engineering, Chem Rev 106 (2006), pp. 4044-4098.

[3] J.W. Gosselink, Pathways to a more sustainable production of energy: sustainable hydrogen — a research objective for Shell, Int J Hydrogen Energy 27 (2002), pp. 1125-1129.

[4] G. Gonzalez-Gil, R. Kleerebezem, A. van Aelst, G.R. Zoutberg, A.I Versprille and G. Lettinga, Toxicity effects of formaldehyde on methanol degrading sludge and its anaerobic conversion in Biobed ${ }^{\circledR}$ expanded granular sludge bed (EGSB) reactors, Water Sci Technol 40 (1999), pp. 195-202.
[5] R. Kleerebezem and H. Macarie, Treating industrial wastewater: Anaerobic digestion comes of age, Chem Eng 110 (2003), pp. 56-64.

[6] J.B. van Lier, P.N.L. Lens and L.W.H. Pol, Anaerobic treatment for C and S removal in 'zero-discharge' paper mills: effects of process design on S removal efficiencies, Water Sci Technol 44 (2001), pp. 189-195.

[7] S. Kortekaas, G. Vidal, Y.L. He, G. Lettinga and J.A. Field, Anaerobic-aerobic treatment of toxic pulping black liquor with upfront effluent recirculation, J Ferment Bioeng 86 (1998), pp. 97110.

[8] L. Seghezzo, C.M. Cuevas, A.P. Trupiano, R.G. Guerra, S.M. Gonzalez, G. Zeeman and G. Lettinga, Stability and activity of anaerobic sludge from UASB reactors treating sewage in subtropical regions, Water Sci Technol 54 (2006), pp. 223-229.

[9] Vesilind, P., A., Worrell, W. \& Renihart, D.2002. Solid waste engineering. CA, USA: Thomson learning.

[10] Barber, W.P. \& Stuckey, D.C. 1999. The use of the anaerobic baffled reactor (ABR) for wastewater treatment: a review. Water Resource 33: $1559-1578$. 


\title{
Power Consumption Pattern of Consumer in Kuching Sarawak
}

\author{
S.S.Ngu, L.C.Kho, J. Annie, C.J. Chai and Leslie K.P. Chai
}

\begin{abstract}
A methodology to find out the power consumption of key electric appliances is presented in this paper. A set of questionnaire is designed to find out the key electric appliances and their usage time period to determine consumer behavior in Kuching, Sarawak. The sampling theory has been applied to calculate the proper sample size to determine the consumer behavior with sufficient confidence level. The proposed method can provide us with better understanding of power consumption behavior for different classes of consumers.
\end{abstract}

Keywords: power consumption pattern, consumer behavior

\section{INTRODUCTION}

The discovery of electrical power is one of the most important discoveries in the history of mankind, rivaled only in importance by the invention of wheels and paper. This is because without electrical power, the life as we know and now take for granted will grind to a halt. Electrical power is generated on a large scale using renewable energy sources such as hydroelectricity, wind as well as solar power, while non-renewable energy sources include the usage of fossil fuels, such as coal and oil. Besides that, there are other types of sources, such as nuclear energy.

Due to increasing population, there is an increase in demand of power. Consumer behaviors play an important role in this phenomenon. Table 1 shows type of consumer that will investigate in this research.

Syarikat SESCO Berhad is responsible for the generation, transmission and distribution of electricity in the state of Sarawak. Estimate total number of power consumers has been increase every year from 260214 in 1996 to 414767 in 2005. The growth rate of electricity consumers within 10

This work was supported in part by Universiti Malaysia Sarawak (UNIMAS) and Syarikat SESCO Berhad.

S. S. Ngu is with Electronics Engineering Department, Universiti Malaysia Sarawak, 94300 Kota Samarahan, Sarawak (e-mail: ssngu@ feng.unimas.my).

L. C. Kho is with Electronics Engineering Department, Universiti Malaysia Sarawak, 94300 Kota Samarahan, Sarawak (e-mail: 1ckho@ feng.unimas.my).

J. Annie is with Electronics Engineering Department, Universiti Malaysia Sarawak, 94300 Kota Samarahan, Sarawak (e-mail: jannie@ feng.unimas.my).

C. J. Chai is with Electronics Engineering Department, Universiti Malaysia Sarawak, 94300 Kota Samarahan, Sarawak (e-mail: cjchai@ feng.unimas.my).

Leslie K.P. Chai is with Sarawak Energy Berhad, Wisma SESCO, Petra Jaya, 93673 Kuching, Sarawak. years is $59.39 \%$. Estimate total electricity sold is $1.94 \mathrm{TWH}$ in 1996 and increase to $3.94 \mathrm{TWH}$ in 2005 . The growth rate of total electricity sold within 10 years is $103.09 \%$. Due to the number of electricity consumption and total electricity sold increasing dramatically as shown in figure 1 and 2, the power consumption pattern, consumer behavior and load characteristic became a critical issue to be investigate so that an more efficiency system planning, operation \& maintenance can be design to meet the peak demand that increase dramatically from year to year.

Table 1: Class of consumer that will investigate

\begin{tabular}{|l|c|}
\hline Class of Consumer & $\begin{array}{c}\text { Type of Consumer by Tariff } \\
\text { Class }\end{array}$ \\
\hline Detached House & Domestic \\
\hline Semi Detached House & Domestic \\
\hline Terrace House & Domestic \\
\hline Residential Unit Floor & Domestic \\
\hline Commercial Unit Floor & Commercial \\
\hline
\end{tabular}

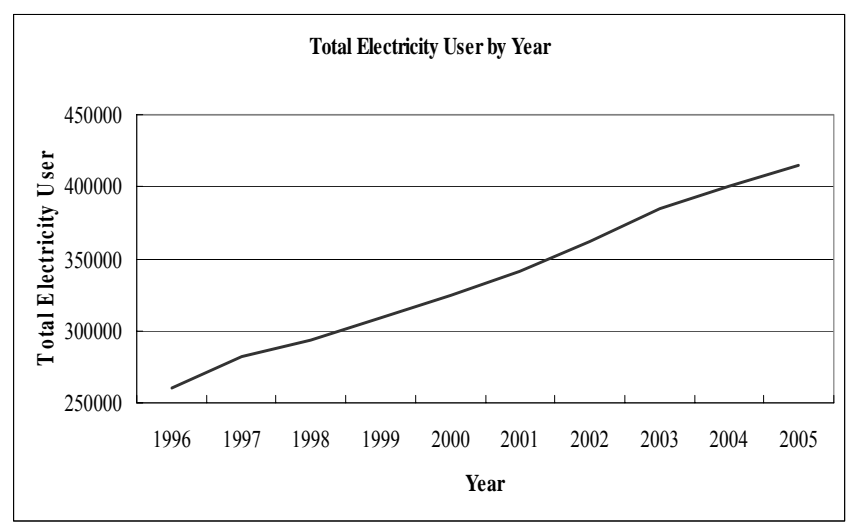

Figure 1. Estimate total electricity consumers Sarawak from year 1996 to 2005

Instead of investigate consumer behavior according to tariff class, this paper will divide each tariff class into more detail consumer class. Since in this paper, domestic and commercial $\mathrm{C} 1$ according to tariff class will be investigate. Domestic had been divided into detached house, semi detached house, terrace house and residential unit floor consumers. In commercial, only commercial unit floor consumers will be investigated. 


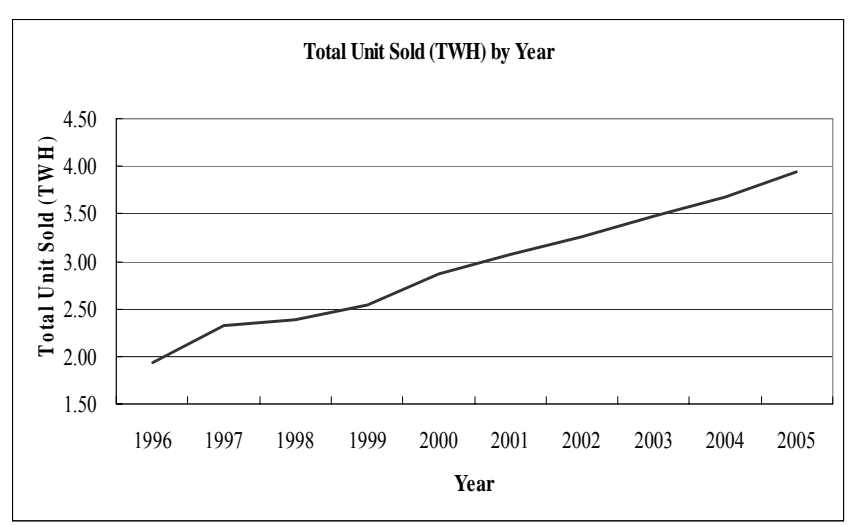

Figure 2: Estimate total unit sold (TWH) by SESCo from year 1996 to 2005

\section{Methodology of Consumer Behavior Research}

In this paper, there are five classes of consumers identified which includes detached house, semi-detached house, terrace house, residential unit floor and commercial unit floor. All these types of consumer classes are to be investigated and analyzed. Questionnaires were designed to determine the key electric appliances and their usage time period. The methodology of this research is illustrated as shown in figure 3.

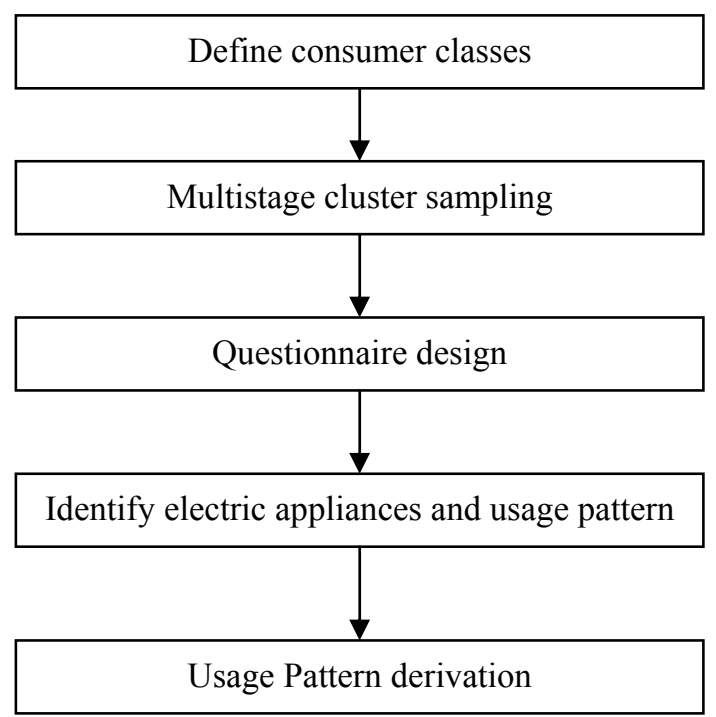

Figure 3. Methodology on consumer behavior studies.

\section{SAMPLE DESIGN}

Due to the large number of consumers, it is impossible to do the survey for every consumer. To make sure that reliable data is colleted with the reliable sample size, it is necessary to use the sampling theory to determine the questionnaire that need to distributed [1].

Table 2. Sample Size

\begin{tabular}{|l|c|}
\hline Type & Sample Size \\
\hline Commercial Unit Floor & 36 \\
\hline Detached House & 34 \\
\hline Semi-Detached House & 47 \\
\hline Terrace House & 68 \\
\hline Residential Unit Floor & 41 \\
\hline
\end{tabular}

The sampling theory is applying base on amount of different class of consumers within the selected area. In this paper, multistage cluster sampling is used to design the sample size. To design the proper sample size, population proportion $\mathrm{p}$ and size of the population $\mathrm{N}$ of total unit for each class of consumer is needed. The sample size, $\mathrm{S}$ with $90 \%$ confidential level [2] for each class of the consumer will be determined by equation (1) and (2) [3]. Table 2 shows the minimum sampling size of commercial unit floor, detached house, semi-detached house, terrace house and residential unit floor consumers.

$$
\begin{aligned}
& s=\frac{s_{o}}{1+\frac{s_{o}-1}{\mathrm{~N}}} \\
& s_{o}=\frac{z^{2} \mathrm{pq}}{\mathrm{e}^{2}}
\end{aligned}
$$

Where,

N Size of the population

$\mathrm{p}$ Population proportion

q (1-p)

e Proportion of sampling error

z Standard Score Corresponding to a given confidence

level

$\mathrm{S}_{\mathrm{o}} \quad$ Sample size

$\mathrm{S}$ Minimum sample size

\section{Design AND ANALysis of Questionaire}

A set of questionnaire had been designed to find the key electric appliances, amount and usage time for electric equipment that use by class of consumer. Table 3 shows the number of questionnaire that had been do and questionnaire that had been filling properly.

Table 3. Percentage of Useable Questionnaire Return Rate

\begin{tabular}{|l|c|c|c|}
\hline Type & $\begin{array}{l}\text { Questionna } \\
\text { ire } \\
\text { Distributed }\end{array}$ & $\begin{array}{c}\text { Useable } \\
\text { Questionnaire }\end{array}$ & $\begin{array}{c}\text { Useable } \\
\text { Questionnaire } \\
\text { Return Rate (\%) }\end{array}$ \\
\hline $\begin{array}{l}\text { Commercial } \\
\text { Unit Floor }\end{array}$ & 110 & 97 & 88 \\
\hline $\begin{array}{l}\text { Detached } \\
\text { House }\end{array}$ & 70 & 46 & 66 \\
\hline $\begin{array}{l}\text { Semi-Detached } \\
\text { House }\end{array}$ & 90 & 61 & 68 \\
\hline Terrace House & 150 & 126 & 84 \\
\hline $\begin{array}{l}\text { Residential Unit } \\
\text { Floor }\end{array}$ & 80 & 44 & 55 \\
\hline
\end{tabular}

Data that were collected from questionnaire will be interpreted by using graphs. There are five types of consumers' usage pattern that needs to be represented by graph.

Through questionnaires that have been made, the key electric appliances and their usage time period can be identified. Figure 4, 5, 6 and 7 show that usage pattern of air conditioners by detached house, semi-detached house, terrace house and residential unit floor consumers which were categorized as residential consumers. Since they were categorize as residential consumers class, usage pattern of air conditioners among them have similar characteristics. Most of the air conditioners will be operated during night time when people stay at home and fewer air conditioners will operate during daytime when people leave their home for work. Usage pattern of air conditioners by residential consumers are shown in figure 8. Figure 9 shows the 
composite commercial unit floor consumers and their usage pattern of air conditioners. Most of the air conditioners will be

operated during daytime when people go for work.

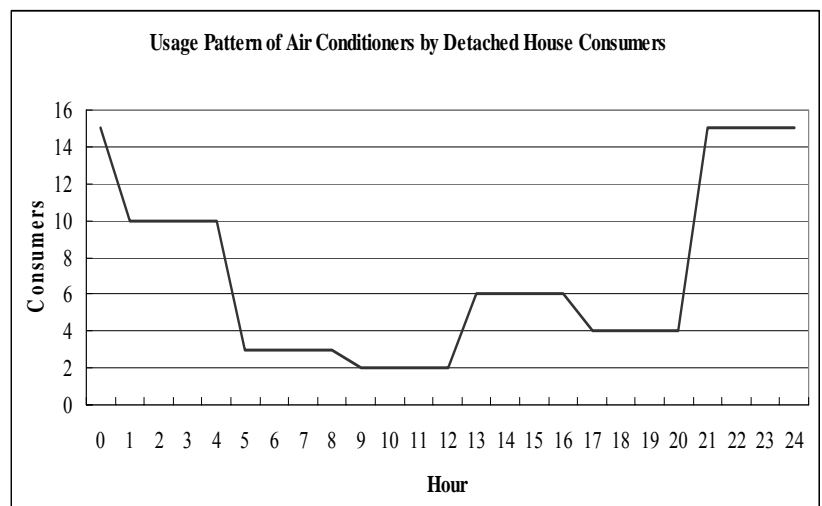

Figure 4. Usage pattern of air conditioners by detached house consumers (Sample Size: 46)

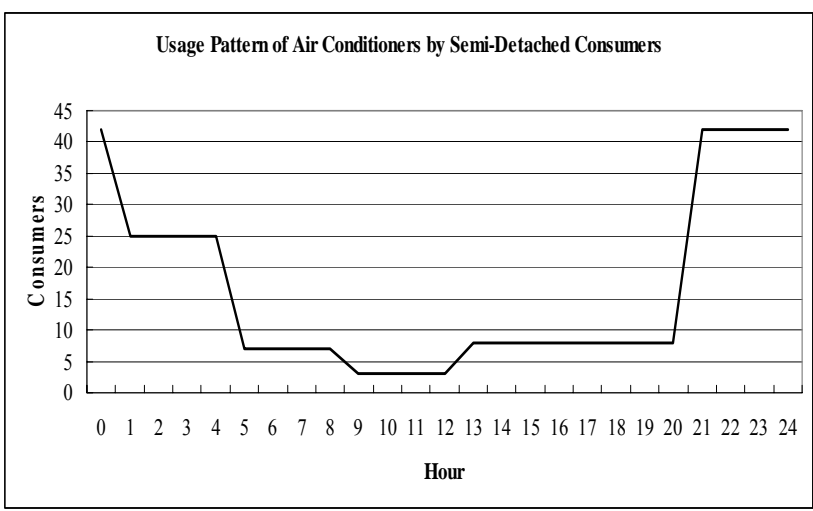

Figure 5. Usage pattern of air conditioners by semi-detached house consumers (Sample Size: 61)

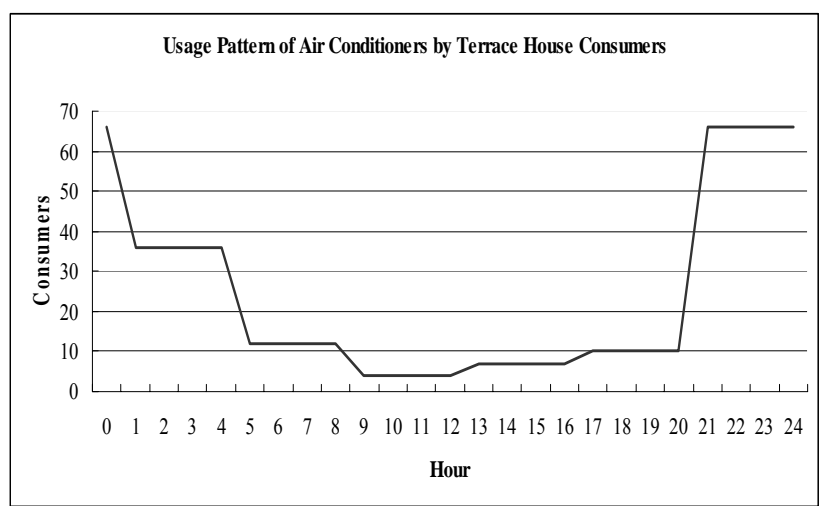

Figure 6. Usage pattern of air conditioners by terrace house consumers (Sample Size: 126)

\section{CONCLUSION}

In this paper, questionnaire is applied to find the key electric appliances, amount and usage time for electric equipment that use by class of consumer. Sample theory is use to design a proper sample size. Usage pattern of air conditioners is derived to show the consumer behavior. By this study, key electric appliances and their usage time period of each consumer class are identified.

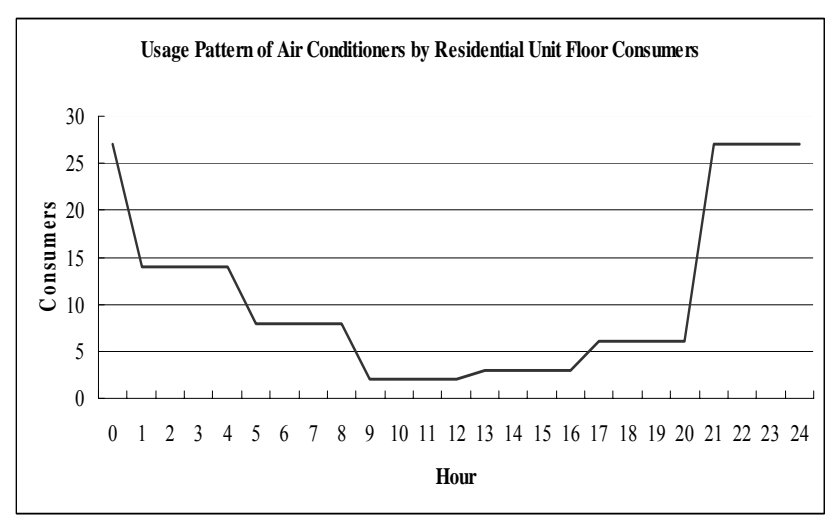

Figure 7. Usage pattern of air conditioners by residential unit floor consumers (Sample Size: 44)

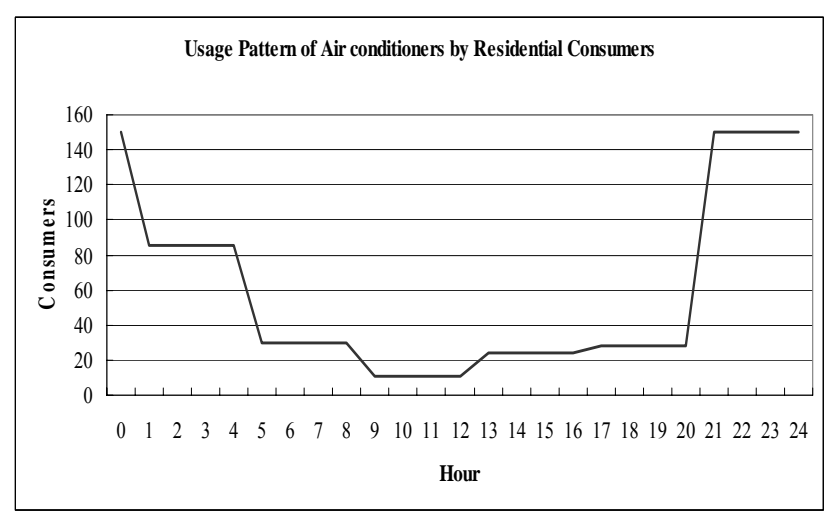

Figure 8. Usage pattern of air Conditioners in residential consumers (Sample Size: 277)

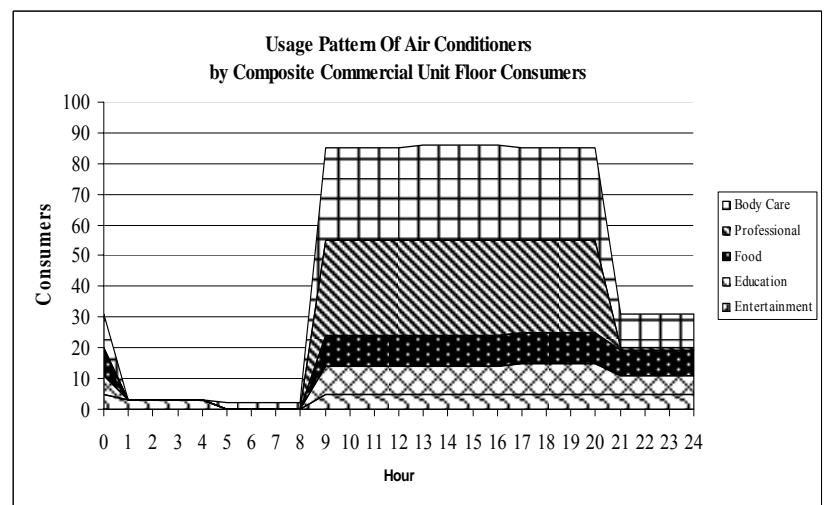

Figure 9. Usage Pattern of Air Conditioners by composite commercial unit floor consumers (Sample Size: 97)

\section{ACKNOWLEDGMENT}

This work was supported by the Universiti Malaysia Sarawak (UNIMAS) of Malaysia and Syarikat SESCO Berhad. The author would like to express sincere appreciation and gratitude to Mr. Leslie Chai (SESCO), UNIMAS's technicians and SESCO's staffs who have provided the tremendous assistance throughout the project.

\section{REFERENCES}

[1] Chen, C.S et. al. "Determination of customer load characteristics by load survey system at Taipower" IEEE Transactions on Power Delivery (1996) Page(s): $1430-1436$.

[2] [2] John Keenan Taylor "Statistical Techniques for Data Analysis", Lewis, 1990.

[3] [3] Luck, DJ Taylor, W.G \& Robin "Marketing Research Eaglewood”, Prentice Hall, 1987. 


\title{
Water Impact Analysis over Distribution Pipelines Network
}

\author{
Abdurazzag Ali Aburas, Manal I Alfellah and Salem Ali Reheil
}

\begin{abstract}
The role of the renewable energy such as solar energy is one of the main issue concern the human life on the plant at present time. Therefore, it is important to generate energy from existing common resources such as oil, wind, nuclear and others that occur naturally and repeatedly in the environmental and can be harnessed for human benefit. In this research paper introduce comprehensive analysis of water impact for producing the electricity power over distribution water pipeline networks. Most research work done before based on the natural water energy power such as rivers, and waterfall. No researcher have looked to powered pumped water pipeline which is a man made power and could be reused for produce electrical power. This paper examines the different type of water pipelines and its sizes, water storage and pressure systems required for produce electricity. The complete mathematical equations and its analysis have been described. The suitable dynamo generator specification is highlighted. The Estimate Additional Components System Losses are given.
\end{abstract}

Keywords: renewable energy, water storage, water pipeline, man made power, electrical power

\section{INTRODUCTION}

The overall energy efficiency of a water system is affected by the efficiency of individual components, in addition to system sizing, load, and system resistance (pressure drop) [1] . A number of key renewable energy technologies have been identified the development priorities, including the improvement of biomass energy utilization (forestry and agriculture), development of wind farms and generators, and expansion of solar energy (thermal and PV), small hydro and geothermal applications [2]. An interesting example of international cooperation for promoting these technologies is wind energy which is being emphasized and supported by the other industrialized countries. The wind resources with an exploitable capacity of about $250 \mathrm{GW}$, is suited for both remote village power development and for large-scale, grid-connected electricity production. With the experience gained from some demonstration sites set up with the US and European manufacturers, China is about to develop wind turbines of size over $200 \mathrm{~kW}$. Development of renewable energy

Abdurazzag Ali Aburas, Manal I Alfellah and Salem Ali Reheil are with the Electrical and Computer Engineering Department, International Islamic University Malaysia, P O Box 10, 50728, 53100 Kuala Lumpur, Malaysia (e-mails: aburas06@iiu.edu.my, manalalfallah@yahoo.co.uk \& smartoplib@yahoo.com). resources can reduce growing dependence on imported oil and the need for additional thermal power plants. Renewable energy can also provide least-cost electricity to remote areas not currently served by existing energy supply systems and has competitive advantage over conventional electrification approaches. The purpose of this research work is to assist designers of small water pipelines to use it for generating electricity power and protect the environment [3]. The design basis for urban or municipal water supply systems in the prairies has evolved over time [4][5][6]. For cities, towns and villages, we have established records and a clear understanding of safety requirements, historic population statistics, water consumption and demands. Over the years, many municipal design guidelines and design standards were developed for larger communities. The design basis for water pipelines has a much more recent history. About $6 \%$ of energy has been produced by hydroelectric power motors [7][8]. Water pipeline means all or a portion of a waterworks, distribution system or extended network of pipes that is owned by a person or association other than a municipality; or it is intended or used to provide water for human consumptive use or hygienic use; and serves one or more of, or any combination of, permanent residences, seasonal residences, acreages, farmsteads, trailer courts, commercial buildings, industrial buildings or other, similar facilities. Pipeline systems that treat and distribute their own source of water are classified as waterworks and are subject to the same design requirements and features as pipelines that do not own their own water source works. Applications for approvals are required to contain information as outlined below. Information should be in a concise form and logical order. Drawings and plans should conform to good engineering practices. Previously submitted information need not be resubmitted unless it is affected by the construction, extension or alteration or updating is appropriate. The following section summarizes the estimating required for the water pipeline. In section three, descriptions of the pipeline with its water storage to produce pressure are given. Section four, the flow rate calculation formula is defined. In section five, conclusion and future works are described, facilitate the review and processing of applications.

\section{Estimating WATER IMPACT IN THE PIPELINE}

Pipeline water demands are to be based on evaluation of the number of people living in the household, number and 
types of livestock, extent of lawn and garden irrigation, existing wells and/or dugouts, tank filling and other yard water use. Local plans for future water use and other related information. Systems with two days storage typically have a design flow based on peak day water demand. Systems without storage typically have a design flow based on the much higher instant water demand. The design flow must be increased for systems with less than two days storage. Design flow is to include allowance for future water demand growth. Many average connections have a design peak day water demand of approximately $6.5 \mathrm{~m} 3 /$ day $(1.0$ igpm) if no other design information is available. Many operating water pipelines show average day water demands as low as $0.65 \mathrm{~m} 3 /$ day $(0.1$ igpm $)$ for domestic demands [11][12].

\section{A. Modeling Water Impact Flow in Pipes}

For empirical purposes, the flow capacity of a water pipeline is commonly modeled by the Hazen-Williams equation "(1)" [12]:

$\mathrm{V}=0.849 \mathrm{C}_{\mathrm{hw}} \mathrm{R}^{0.63} \mathrm{~S}^{0.54}$

Where: $\mathrm{V}$ is Velocity

$\mathrm{C}_{\mathrm{hw}}$ is the Hazen-Williams friction coefficient

$\mathrm{R}$ is the hydraulic radius $\mathrm{S}$ is the slope of the total head line

Many flow calculation equations and associated computer simulation packages operate under derivations of the HazenWilliams equation. Design velocity should be a maximum of $1.5 \mathrm{~m} / \mathrm{s}(5 \mathrm{ft} / \mathrm{s})$, a minimum of $0.6 \mathrm{~m} / \mathrm{s}(2 \mathrm{ft} / \mathrm{s})$. Typical friction coefficients $C_{h w}$ are shown in the (Table 1) below. $\mathrm{C}_{\mathrm{hw}}$ must be conservatively estimated.

The $\mathrm{C}_{\mathrm{hw}}$ coefficients shown below are designed to represent those that could be experienced in the long-term and include allowances for typical fittings and changes in fluid flow direction. The friction coefficients are conservative and should be used only when more sitespecific information is not available.

Table 1. Derivations of the Hazen-Williams equation

\begin{tabular}{|c|c|}
\hline Pipe Description & $\begin{array}{c}\text { Long-Term H-W Friction } \\
\text { Coefficient Chw }\end{array}$ \\
\hline Polyethylene (PE) & 130 \\
Nom dia $<=150 \mathrm{~mm}$ & 132 \\
200 to $250 \mathrm{~mm}$ & 135 \\
300 to $600 \mathrm{~mm}$ & \\
\hline Polyvinyl chloride (PVC) & Same as above \\
\hline
\end{tabular}

\section{WATER PIPELINE WITH STORAGE}

All water pipelines should have water storage. If customers have proper storage, the water pipeline has a greater economy and safety. Storage adds reliability to the water supply in the event of reduced water pipeline flow due to accident or normal maintenance. Properly designed storage facilities can also reduce the instant demand on the water source and thereby reduce the size and cost of pipes, pumps and related infrastructure.

For water pipelines with storage, each residential connection should have a storage tank such as a cistern that is sized for at least two days of future average day water demand. The typical size of water storage for in-house use with a family of three persons is $1.4 \mathrm{~m} 3$ (300 imperial gallons).

\section{A. Pressure Requirements}

In water pipelines, water demands are mainly for domestic and stock-watering requirements. There is typically no fire-fighting flow. The normal operating pressure range for water pipelines is $350 \mathrm{kPa}(50 \mathrm{psi})$ to 550 $\mathrm{kpa}(80 \mathrm{psi})$. The maximum pressure should not exceed 700 $\mathrm{kPa}$ (100 psi) to protect household piping. Under special circumstances where the main lines are allowed to exceed $700 \mathrm{kPa}(100 \mathrm{psi})$, the adjacent lateral pipelines or service lines must be protected with pressure reducing valves. The minimal acceptable water pressure at water pipeline withdrawal points is $98 \mathrm{kPa}$ (14 psi) if all serviced connection withdrawal points are protected by an air gap backflow prevented or equivalent. The minimal acceptable water pressure at all other withdrawal points is $138 \mathrm{kPa}(20$ psi).

\section{Calculating the Water Flow Rate (WFR)}

Finding the flow rate (in liters per second) is required in order to calculate items such as friction loss in the pipeline and the capacity of the dynamo pump to be selected. For example use 4300 liters as storage capacity. The topography conditions at the site are is an important factor in a good system design, and should be obtained as accurately as possible. The total head includes components in addition to the height that the water needs to be pumped. For example the system in the Figure 1 below shows a dynamo generator from a tank above ground level. The topography conditions at the site are a very important factor in a good system design, and should be obtained as accurately as possible. For this application, the topography can include such information as distances between system components and variation in terrain heights.

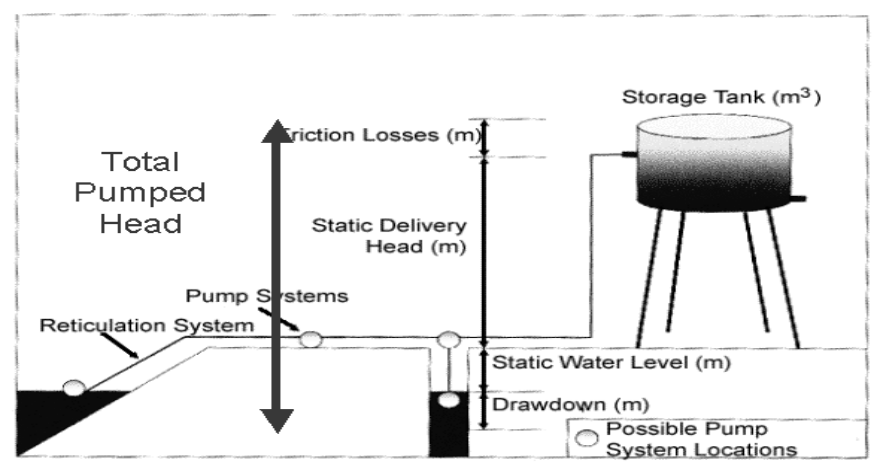

Figure 1. Structure design for dynamo generator

\section{A. Total Calculations}

The total head for dynamo equal the static head plus the dynamic head (all in meters).

The static head $=8+5+2$ or 15 meters. The dynamic head is calculated for a pipeline of length $1200 \mathrm{~m}$ and $25 \mathrm{~mm}$ diameter, class 9. At the flow rate of 0.2 liters per second. Using an appropriate pipeline friction loss chart* for these conditions, a figure of $0.41 \mathrm{~m}$ head to add per $100 \mathrm{~m}$ of pipeline (i.e. for $1200 \mathrm{~m}$ of pipeline, add $12 \times 0.41 \mathrm{~m}=\mathrm{K}$ 
Value $4.92 \mathrm{~m}$.). Pipe frictions for Class 9, PVC pipe (meters head/100 m length of pipe) as illustrated in table 2 .

Table 2. Water flow calculations

\begin{tabular}{|c|c|c|c|c|c|c|}
\hline $\begin{array}{c}\text { Water Flow } \\
\text { (liters per } \\
\text { second) }\end{array}$ & \multicolumn{5}{|c|}{ Nominal pipe size (mm) } \\
\hline & 25 & 32 & 40 & 50 & 65 & 80 \\
\hline \hline 0.10 & 0.12 & & & & & \\
\hline 0.15 & 0.25 & & & & & \\
\hline 0.20 & 0.41 & 0.17 & & & & \\
\hline 0.30 & 0.83 & 0.27 & 0.15 & & & \\
\hline 0.40 & 1.40 & 0.46 & 0.24 & & & \\
\hline 0.50 & 2.10 & 0.66 & 0.35 & 0.12 & & \\
\hline 0.75 & 5.00 & 1.06 & 0.85 & 0.29 & 0.10 & \\
\hline 1.00 & 7.00 & 2.25 & 1.20 & 0.41 & 0.14 & \\
\hline \hline 1.25 & 10.20 & 3.40 & 1.77 & 0.61 & 0.22 & 0.10 \\
\hline 1.50 & 14.30 & 4.78 & 2.50 & 0.85 & 0.29 & 0.13 \\
\hline 1.75 & 19.40 & 6.28 & 3.40 & 1.02 & 0.38 & 0.17 \\
\hline 2.00 & 24.50 & 7.75 & 4.12 & 1.40 & 0.48 & 0.22 \\
\hline \hline 3.00 & & 16.20 & 8.50 & 2.85 & 1.00 & 0.45 \\
\hline 5.00 & & & 21.80 & 7.30 & 2.50 & 1.13 \\
\hline \hline 10.00 & & & & 26.00 & 8.60 & 3.85 \\
\hline \hline
\end{tabular}

From the table above a value of $0.41 \mathrm{~m}$ dynamic head per $100 \mathrm{~m}$ of pipeline is determined for the example system. As there is $1200 \mathrm{~m}$ of pipeline in the example system it is simple to calculate a value for dynamic head. This is mean the $\mathrm{K}=[(1200 \mathrm{~m} * 0.41) / 100 \mathrm{~m}]=4.92 \mathrm{~m}$. The total head for pumping is now $15 \mathrm{~m}+4.92 \mathrm{~m}$ or $20 \mathrm{~m}$ with rounding off. If rounding off is used always increase the value. This gives the design some "reserve". The flow rate is 0.2 liters per second. Changing the type or length of pipe will affect the dynamic head - if for the example system we chose 32 $\mathrm{mm}$ pipe the dynamic head would be approximately $2 \mathrm{~m}$. This is an important system design factor that needs to be considered.

\section{B. The Required Dynamo Generator Power}

The given above example is only a design calculation. There are many other considerations that could be added. A total professional dynamo water generator design would include items such as water temperature, pipeline pressure based on class type of pipe. More accuracy in details is given such as pipeline pressure allowance and temperature variation with the PV array. Addition to that are possible module soiling, shading, electrical and heat losses, as well as motor characteristics. The final design achieved by using this method (above). If done correctly should be relatively close (around 15\%) to a professional design. However, it should be used as a reference guide only for those wishing to understand the principles of hydropower electricity generator system. The given formula "(2)" (the "hydraulic" formula) could be now used to calculate the required power into the hydropower motor drive. This is not the true array power needed, as other considerations still have to be included for instant type of module, temperature effect, losses in the MPPT and controls etc.
Formula: $\mathrm{P}=10 \times \mathrm{FR} \times \mathrm{TH} / \mathrm{SE}$

where $\mathrm{P}=$ (the power into the hydropower motor drive); FR $=$ flow rate in $1 / \mathrm{s} ; \mathrm{TH}=$ the total system in meters; $\mathrm{SE}=$ the hydropower efficiency as a decimal. For our example, the power generates in watts is $\mathrm{P}=10 \times 0.2 \times 20 / 0.4=100$ Watts.

\section{Estimate Additional Components System Losses}

These estimated additional variables for losses including electrical losses; maximize losses, typical PV de-ratings for temperature and other system design variables. There are may also be some additional vector losses after the hydropower motor due to poor topography detail being used. This is could be occur even with a "professional design".

\section{CONCLUSION AND FUTURE WORK}

The components of pipelines distributed network have been described to be used for hydropower electricity based on water impact in pipeline. An analysis of different type water pipelines over distributed network and pressure systems has been explained. The pipeline size is one key of estimating the pressure required for hydraulic dynamo. The falling rain is the possible of energy power generator which is a dark energy until our present time. It would be very powerful source of energy if we have advanced analysis and accurate design system for this future reusable source of energy.

\section{REFERENCES}

[1] Tengfang $\mathrm{Xu}$ "Best Practices for Energy Efficient Cleanrooms: Variable Speed Pumping”, June 15, 2005 the Public Interest Energy Research (PIER).

[2] Thorpe, Th. W.: A review of wave energy, vol. 1: Main report, ETSUR-72: Renewable Energy Enquiries Bureau. Harwell (OX 11 ORA, U.K.): ETSU 1992.

[3] De O. Falcao, A. F. (ed.): Proc. of the 1993 European Wave Energy Symp., Edinburgh, July 21-24, 1993. NEL, East Kilbride, Glasgow (G75 OQU): The Conference Centre.

[4] Beckman W. A. (1996) The teaching of solar energy technology with access to computers. Progress in Solar Energy Education 4(2), 37-40.

[5] Berkovski B. and Gottschalk C. M. (1997) Strengthening human resources for new and renewable energy technologies of the $21 \mathrm{st}$ century UNESCO Engineering Education and Training Programme. Renewable Energy 10(2-3), 441-450.

[6] Bhattacharya S. C. (1996) Formal renewable energy education: a review. Progress in Solar Energy Education 4(1), 12-15.

[7] Charters W. W. S. (1996) The solar challenge: promoting effective education and training. Progress in Solar Energy the user to input information and then simulates Education 4(1), 2-3.

[8] CREST (1999). Solstice: Sustainable Energy and Development Online. Center for Renewable Energy and Sustainable web-based tools which provide cross-platform Technology (CREST), http: / / solstice.crest.org /.

[9] Chen, Wenqi (1997). Investment market and its development in the new energy and renewable energy of China. Energy of China, No. 3, 1997, 45-48.

[10] Cheung, S. P. and Yiu, K. C. (1995). Current status and future development of new energy in Mainland China. Energy Quaterly, 25 (3), 63-75.

[11] Hui, S. C. M. (1997). From renewable energy to sustainability: the challenge for Hong Kong. In: Proc. of thePOLMET '97 Conference, 25-27 November 1997, Hong Kong Institution of Engineers, 351-358.

[12] Guide to Waterworks Design EPB 201, April 2004. 


\title{
A Study on Electric Energy Usage at the Residential Area
}

\author{
Asmarashid Ponniran, Erwan Sulaiman, Siti Amely Jumaat, Maizul Ishak, Megat Azahari Chulan and \\ Suhaimi Saiman
}

\begin{abstract}
Electricity is energy that very important in daily human life. Lack of electricity causes not only inconvenience to domestic consumers due to affect of electrical appliances operation, but also introduces economic losses for industrial and commercial sectors whereby affecting their productions and services. For domestic sector, it is very important for each consumer to manage their daily usage of electricity in proper way. This paper is about electric energy management investigation among the consumers at residential area of Taman Pintas Jaya, Parit Raja, Batu Pahat, Johor. The main reasons of this study are to find out the characteristic of electric energy usage in daily life and investigating the habits of consumers due to usage of common electrical appliances. Questionnaire is the tool that being used for data collection and for analysis purposes. The questionnaire consists of several questions that provided information of the electric energy usage among consumers in their daily life. The finding of this study shows that most of the consumers do not alert how to use electric energy efficiently in their daily life. In other word, the consumers uses electric energy inefficiently in term of the usage of inefficient electrical appliances and the lack of knowledge to use electric energy in daily life efficiently. Therefore this paper reveals the habits of electric energy usage from the consumers' side and the common characteristic of electrical appliances usage in common domestic sector. From the finding obtained, many good advices can be suggested in order to alert consumers to use electric energy efficiently in their daily life.
\end{abstract}

Keywords: electric energy efficient

\section{INTRODUCTION}

Electric energy is very important in daily life. It is indispensable to factories, commercial establishments, homes, and even most recreational facilities. Lack of electricity causes not only inconvenience, but also economic loss due to reduce of industrial production. It is very important for each consumer to manage the daily use of their electricity usage.

The observation of the electric energy usage is carried out among consumers at Taman Pintas Jaya, Parit Raja, Johor. The residential sector include private households where energy is consumed primarily for water heating, air

Asmarashid Ponniran, Erwan Sulaiman, Siti Amely Jumaat, Maizul Ishak, Megat Azahari Chulan and Suhaimi Saiman are with the Faculty of Electrical and Electronic Engineering, Universiti Tun Hussein Onn Malaysia, 86400 Parit Raja, Batu Pahat, Johor, Malaysia (e-mail: asmar@kuittho.edu.my).

conditioning, lighting, refrigeration, cooking, clothes drying and etc.

Most of consumers are unaware of their electric power management. These situations bring to the wasted of electric energy in their daily life. Some focus of this study is to teach the consumers to be smart using electricity in daily life, and also to find alternative ways in reducing the wasted of electric energy consumption.

Anthony J. Pansini in his book, "Guide to Electrical Power Distribution System, Sixth Edition" mentioned previous study about the personal habits of people and the routines of households, offices, stores, and factories, to determine what probable load the utility company will have to supply at any one time. This research is done based on reason if all the electrical appliances in all the consumers' premises in a country were to be turned on at the same time, it would be impossible for the utility company to supply enough necessary energy to run all these appliances. For a utility company to provide energy for this total connected load would be extremely uneconomical since all this appliances actually will not be operating at the same time as illustrate in Figure 1.1.

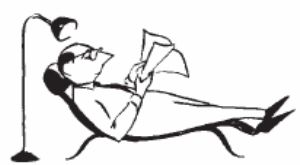

(a)

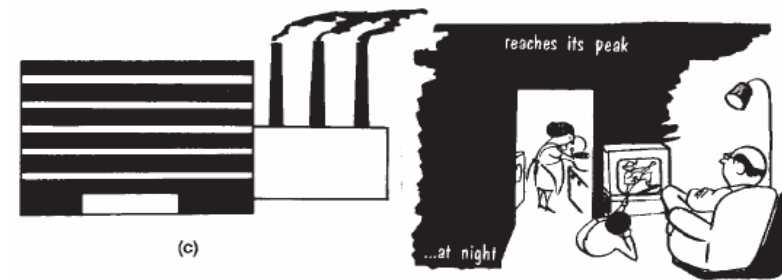

(d)

Figure 1.1. (a) Very low demand, consumer only uses one lamp; (b) Higher demand, consumer is cooking, washing, and running a TV; (c) The factory reaches its maximum demand during the day; (d) At night the domestic demand reaches its peak 


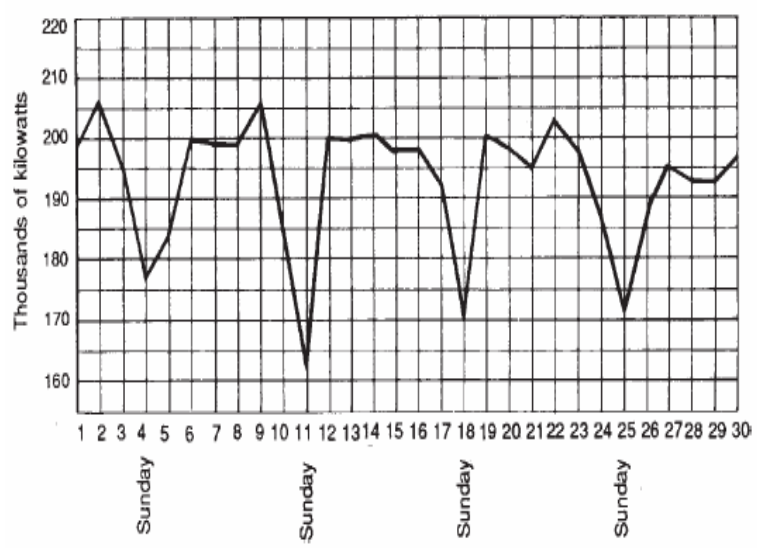

Figure 1.2. Daily peak load for a typical month, that is, how many kilowatts typical utility company supplies consumers in an average month

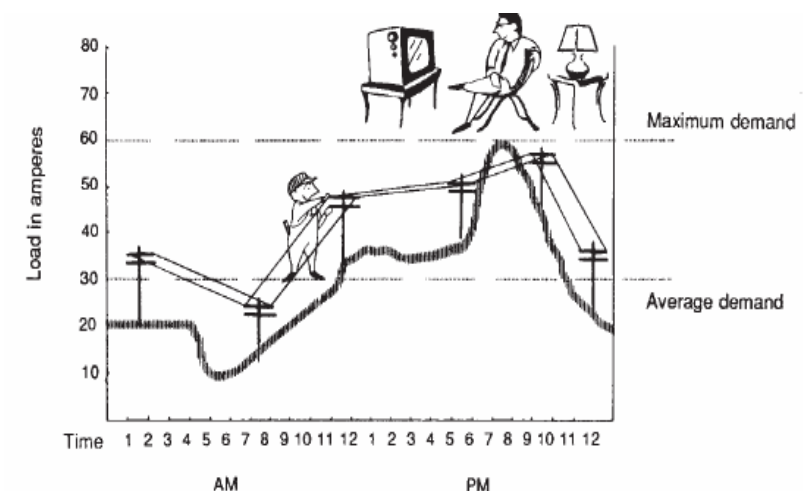

Figure 1.3. The utility company must be equipped to meet the consumer's maximum demand

This study is more focus on how to provide electrical energy to the consumers in effective way according to their habits in daily life. Consequently, consumers become more alert and appreciate the electric energy usage.

\section{A. Power Factor}

Power Factor is a parameter that to measure on how efficiently electrical power is consumed. The ideal Power Factor would be unity or 1. Unfortunately in the real world, Power Factor is reduced by highly inductive loads to 0.7 or less. This induction is caused by equipment such as lightly loaded electric motors, induction motor, power transformers, fluorescent lighting ballasts, welding sets, and induction furnace. Capacitive Power Factor correction (PFC) is applied to electric circuits as a means of minimizing the inductive component of the current and thereby reducing the losses in the supply. Reactive power charges can be made significantly smaller by the Power Factor Correction Capacitors which is a widely recognized method of reducing an electrical load and minimizing wasted energy, improving the efficiency of a plant and reducing the electricity bill. It is not always necessary to reach a power factor of 1 .

\section{MethodOLOGY}

\section{A. Subject}

This study is about power management at demand side among the consumers at residential areas of Taman Pintas Jaya, Parit Raja, Johor. The survey is conducted and involving 50 houses live include categories of family, student, and bachelor. The chosen of the houses have been picked randomly.

\section{B. Tool/Equipment}

The method that is used to obtain samples of data is by using questionnaire. From the questionnaire, the data is analyzed to calculate the power usage of each house. It consists of questions that provide information of electric energy usage among consumers and also their habits in daily usage of electric energy.

\section{Data Finding}

Surveys have been conducted by distributing 50 questionnaires at Taman Pintas Jaya residential area. In each house, each electric appliance used by every house and average total of electricity bill in every month are recorded. The data collection only focused on the appliances that are often used by the consumers. The average total of real power for each house is calculated by summing the individual appliance. The knowledge of each consumer about their knowledge of consumption electricity in efficient also recorded.

\section{ANALYSIS}

Figure 1.4 shows that the majority of respondents are students and bachelor.

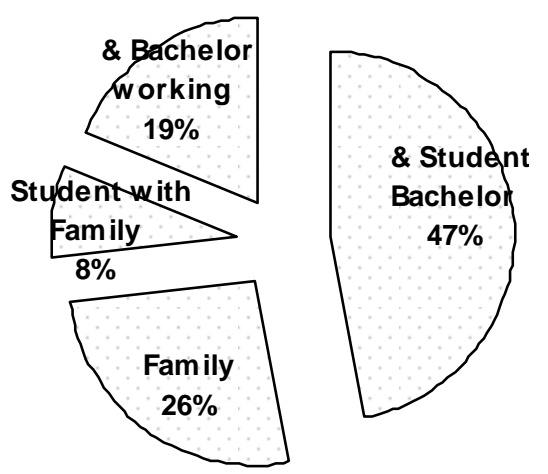

Figure 1.4. The categories of respondent

There are about $275 \mathrm{~kW}$ electric power consumed by these 50 residents during morning (7.00 am to $12.00 \mathrm{pm}$ ), Refer Figure 1.5. At the evening (12.01 pm to $6.00 \mathrm{pm})$, the power consumed are about $184 \mathrm{~kW}$. The highest power consumed during night, about $365 \mathrm{~kW}$.

From this result, the power that consumed by respondents high at the morning because they used a lot of heating appliances during this time like iron, electric kettle, shower heater. Then electric power usages drop at the evening because they are not at home. The highest electric power consumed at night because majority of houses' member stayed at home and used most of the electric appliances.

Table1.1 shows common of electric appliances that being 
used by the respondents. Electric appliances can be categorized into three types; resistive, inductive and capacitive loads.

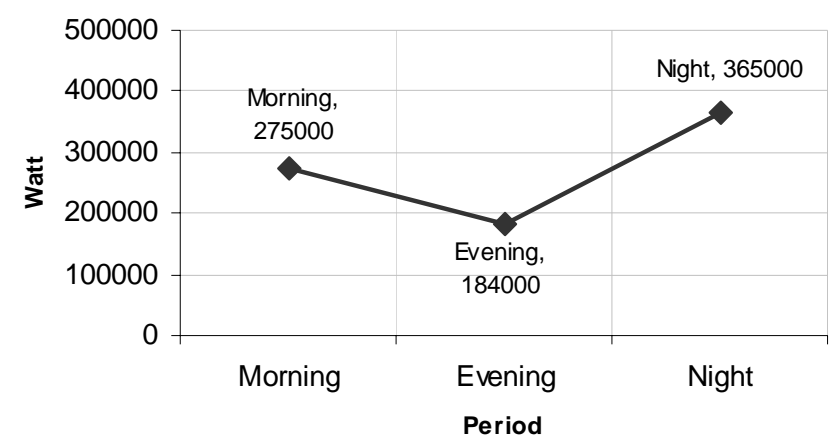

Figure 1.5. Total power usage per day by the respondents

Electric appliances based on resistive load usually consist of heating elements such as electric kettle, shower heater, rice cooker and toaster. From this survey, the heating element loads requires high power compared to other electrical appliances.

The load that consists of inductive or capacitive elements are said to be reactive load. In this survey, the appliances that categorized to be reactive load such as refrigerator, washing machine, air conditioning, fan and vacuum cleaner. These type of appliances consumed lower power as compared to the heating elements load.

Nevertheless, electric appliances that containing heating elements (resistive load) have power factor of 1.0 meanwhile reactive element loads often provide power factor below than 1.0. If very low power factor introduce, the load needs more current in order to meet the real power requirement of the load. The consumers did not really notice this phenomenon because the electricity bills are measured in Watt.

Table 1.1. Common appliances used by the respondents

\begin{tabular}{|l|l|l|}
\hline $\begin{array}{c}\text { N } \\
\text { o }\end{array}$ & \multicolumn{1}{|c|}{ Appliances } & \multicolumn{1}{c|}{ Power (W) } \\
\hline 1 & Pendaflour Lamp & 36 \\
\hline 2 & Refrigerator (220 L) & 500 \\
\hline 3 & Iron & $1200-1400$ \\
\hline 4 & Television & 75 \\
\hline 5 & Washing Machine (5kg) & $240-380$ \\
& Washing Machine (7kg) & $480-700$ \\
\hline 6 & Air-Conditioner & 1200 \\
\hline 7 & Microwave Oven & $1100-1200$ \\
\hline 8 & Personal Computer (CRT) & 750 \\
\hline 9 & Laptop & 65 \\
\hline 10 & Electric Kettle (4L) & $2000-2200$ \\
\hline 11 & Shower Heater (4kg) & 3600 \\
\hline 12 & Rice Cooker (2.8L -3.3L) & $600-700$ \\
\hline 13 & Fan (Ceiling) & 100 \\
& Fan (Stand / Wall) & $50-60$ \\
& Fan (Table) & 35 \\
\hline 14 & Vacuum Cleaner & $1200-1600$ \\
\hline 15 & Hair Dryer & 1200 \\
\hline 16 & Toaster & 700 \\
\hline & & \\
\hline
\end{tabular}

The respondents that have normal family consumed more electric power as compared to the other categories.
According to the study, this type of respondents using more electric appliances at their house like shower heater, air conditional and other appliances as compared to other respondents.

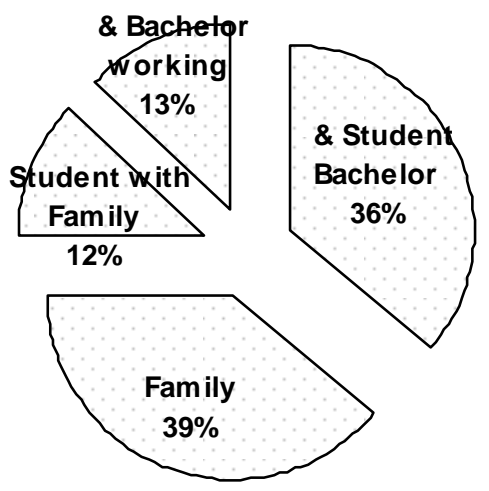

Figure 1.6. Power usage by the respondents according to the categories

From the survey, it is founded that most of the respondents paying their monthly bill from RM 100 to RM 150, refer Figure 1.7.

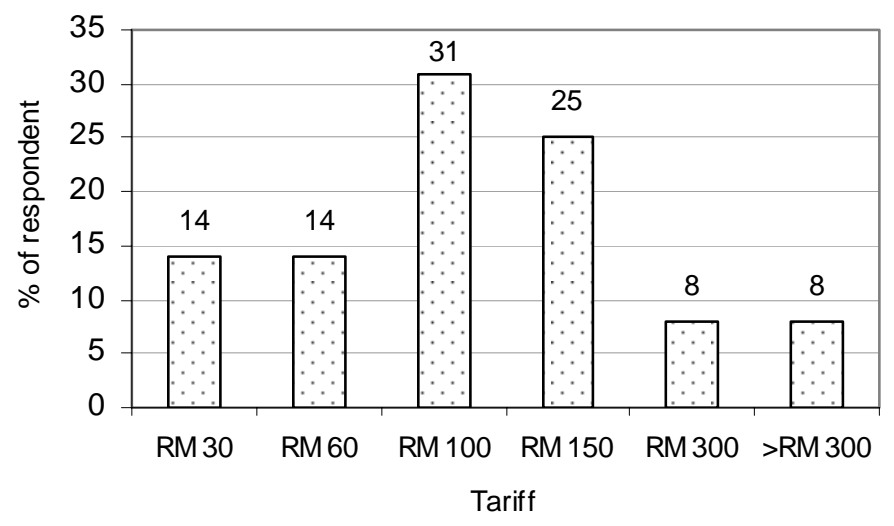

Figure 1.7. Monthly electric tariff in average

The Figure 1.8 showed that most of the respondents alert of energy waste in their house. The initiative taken to save electric energy is by switch off all of unused electric appliances.

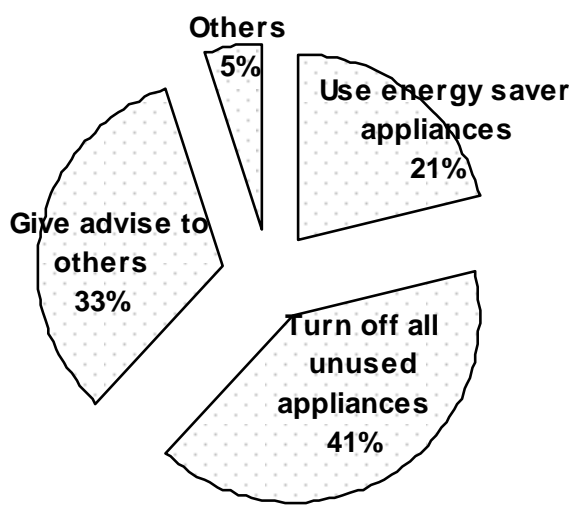

Figure 1.8. Initiative that taken by the respondents in order to save the electric power 


\section{CONCLUSION}

From this study, characteristic of electric power usage among the consumers in their daily life are obtained and analyzed. Some of them know how to save in usage of electric energy in term of individual behavior. But most of respondents do not know the available efficient electric appliances in the market. They only buy the electric appliances if the price is very cheap without alert about the efficiency of the appliances. Therefore several programme about the usage of efficient electric appliances and how to use electric appliances efficiently need to be organized and disseminated to the society. Consequently, society will alert and becomes smart in usage of electric energy in their daily life.

Power factor is always being monitor by the TNB in demand side because it extremely important. Power Factor is a measure of how efficiently electrical power is consumed. The ideal Power Factor would be unity or one. High inductive loads cause reducing power factor. This induction element is introduced by equipment such as electric appliances consists of electric motor, transformer, fluorescent lighting ballast, welding set, fan, and etc. The lower of power factor the more power need to be generated.

\section{Suggestions For SMart Usage of Typical Electric APPLIANCES}

\section{A. Refrigerator}

Refrigerator is used throughout the year and is considered as the most expensive item. Therefore, the energy saving potential is high for these appliances. The lower energy costs of the more efficient, newer refrigerators can often justify replacement even if existing ones are still in good service. Suggestions for smart usage:

- Keep the condenser coils at the rear of the refrigerator clean. Dust and dirt on the condenser coils will reduce the working efficiency of the refrigerator.

- The compartments should be kept full to avoid unnecessary cold air loss on door opening.

- Arrange foods in the refrigerator slightly apart to allow free air circulation. Always cool hot food before storing it in the refrigerator.

- Avoid frequent opening of the refrigerator, or leaving the door open unnecessarily.

\section{B. Washing Machine}

It is a waste of electricity and water to use the washing machine to do the part loads of, say, a pair of trousers or few shirts. Gather the laundry, so that the machine can be filled up. The electricity consumption is almost the same. Suggestions for smart usage:

- Wash only with full loads. The washing machine uses the same amount of energy for full load as for a part load.

- Do not overload the washing machine.

- Choose the correct wash cycle.

\section{Water Heater}

Electric water heaters are of two types, "instant water heaters" (very common now) which heat the water as it is used, and "storage water heaters" (less common), which store heated water for later use. The storage type of heaters tend to waste energy as they attempt to keep the stored water hot all the time, even when the water is not in used. It is encourage those quick showers instead of baths; for less hot water is used in taking a shower.

\section{D.Air Conditioning}

Home air conditioning, which was once a luxury, is now becoming a necessity, or an affordable convenience to the middle income population, as air conditioners become cheaper and more energy efficient. The cheaper, window type air conditioners are now losing out to the "split" type, which have the advantage of separating the compressor from the cooling unit, thus reducing the noise level in the cooled room. The lower energy costs of the more efficient, newer air conditioners can often justify replacement even if existing ones are still in good service. Suggestions for smart usage:

- Do not block air conditioner vents with drapes or furniture.

- Roof, or ceiling insulation, and minimization of air leaks from under doors and through windows can help to reduce the cooling energy consumption.

- Glaze your window with heat reflective glass - some glazing has selective reflective coatings where heat is reflected but light is allowed in.

- On a hot day, close your curtains and blinds. This helps to keep sunshine heat away.

- Landscaping - trees and shrubs next to the house help in reducing the cooling load.

- Shade screens, glass tints, and window/wall awnings are another way of cutting down on heat gain.

\section{E. Lighting}

Suggestions for smart usage:

- Dust your light bulbs and lamp shades once every 1 or 2 months. Keeping them clean increases the amount of light emitted.

- Use the compact fluorescent lamps because:

i. Incandescent lamps

Generate a high amount of heat and are typically used with a rating of $60 \mathrm{~W}$. They are inefficient artificial light source and their light intensity efficiency is about 18 lumens / watt.

ii. Fluorescent lamps

Generate bright light and are most often used with a rating of $32 \mathrm{~W} / 36 \mathrm{~W}$. These lamps come together with ballasts, normally rated at $8 \mathrm{~W}$ loss, although low loss ballasts (up to $6 \mathrm{~W}$ ) or high frequency electronic ballasts (nominally less than $2 \mathrm{~W}$ ) are also available.

\section{REFERENCES}

[1] Joel N. Swisher, Gilberto de Martino Jannuzzi, Robert Y. RedlingerUNEP. "Tools and Method for Integreted Resource Planning: Improveing Energy Efficiency and Protecting the Environment",United Nations Environment Programme,National Laboraory, Denmark. 1997.

[2] CETREE. "Your guide to energy efficiency at home", Center For Education And Training In Renewable Energy And Energy Efficiency, 2002.

[3] Suruhanjaya Tenaga. "Statistics of Electricity Supply Industry in Malaysia, 2002.

[4] HarryVreuls, Novem, INDEEPPRES 1998."The Global Demand Side Management Map-a tool for DSM strategy and planning" Paper 
presented at the 12. CEPSI conference on Electricity the Challenge for Sustainable Development,2-6 Novem 1998 Pattaya,Thailand.

[5] MAP 2000, Environmental Action Plan of the Energy Distribution Sector, Netherlands Association of Energy Distribution Companies, 1997.

[6] International Energy Agency, Implementing Agreement on DemandSide Management Technologies and Programmes, 1997 Annual Report, NUTEK, 1998.

[7] International Programme experience in Providing Energy Efficiency Services Comparing Cost Effectiveness, Report INDEEPAN.1998.1 for Annex 1 of the IEA-DSM Agreement, Novem, 1998.

[8] Lessons learned by the INDEEP database, a task of the IEA-DSM Agreement, paper presented at DS/DSM Europe 1997 Conference by Harry Vreuls, Novem, Operating Agent for Annex 1 IEA DSM Agreement, 1997.

[9] European ex-post evaluation guidebook for DSM and EE services Programmes, final phase 1 Report, SRC Internationals APS Denmark, NUTEK Sweden, DEFU Denmark, 1998. 


\title{
Measurement of Insulation Resistance of Line Insulator under Adverse Conditions
}

\author{
M. A. Salam, Nurul Fadzlinah, Shaera and Zuharni
}

\begin{abstract}
Insulator is one of the essential components of the transmission and distribution systems. The resistance of the insulator normally decreases due to the combine effect of rain and pollutants. This paper investigates the insulation resistance variations of the insulators due to slat-solutions. The insulation resistances at the top and bottom surfaces of the disc type glass and porcelain insulators have been measured with the variation of salt-solutions. The measured values of insulation resistance are compared for both types of insulators. It is found that the insulation resistances at the bottom surface are lower than that on the top surface.
\end{abstract}

Keywords: pollutants, leakage current, disc type porcelain and glass insulators

\section{INTRODUCTION}

The performance of the insulator in an outdoor environment is very important for high reliability of power transmission and distribution networks.

The sources of pollutions can be classified as marine, industrial, and desert types. Sodium Chloride $(\mathrm{NaCl})$ and small rocks are originating from the marine sources. Fumes and related dust are coming from the nearby industry. Sand and its related dust are coming from the desert. Pollutions from different sources are brought by the natural wind and deposited on the insulator surface and forms a dry pollution layer. Dry pollution layer has no effect on the insulator surface. This dry layer offers a conductive layer in the presence of light rain or morning dews. In the presence of high voltage, leakage current flows through the conductive pollution layer and produce a joule-heat. As a result of joule-heat, an uneven dry-band forms on the insulator surface. A small arc appears across these un-even dry bands in the presence of high voltage and forms a closed path

M. A. Salam, Senior Lecturer in the Department of Electrical and Communication Engineering, Institute of Technology Brunei, Jalan Tungku Link, BE 1410, Brunei Darussalam, (corresponding author to provide phone: 673-8919560; fax: 673-2461035/6; e-mail: masalam@itb.edu.bn).

Nurul Fadzlinah, final year student in the Department of Electrical and Communication Engineering, Institute of Technology Brunei, Jalan Tungku Link, BE 1410, Brunei Darussalam (e-mail: author@lamar. colostate.edu).

Shaera and Zuharni, final year students in the Department of Electrical and Communication Engineering, Institute of Technology Brunei, Jalan Tungku Link, BE 1410, Brunei Darussalam (e-mail: author@lamar. colostate.edu). through an atmospheric air. As a result, corona discharge appears on the insulator surface and surrounding the high voltage line. All small arcs merge and form a large arc. Later on, this large arc can trigger the flashover on the insulator surface. The pollution may reduce the flashover voltage of porcelain insulation to half or even to a quarter [1]. Ultimately, reliable power supply is interrupted. Many researches have been done to resist the insulator flashover under adverse environmental condition. Water washing, greasing, semi-conducting glazes can be used as an antipollution measures [2]. One of the latest practices is to use room temperature vulcanizing (RTV) silicone rubber on the high voltage ceramic/glass insulators for imparting better antipollution properties [3]. Silicone rubber has good dielectric properties over a wide range of temperature and offers an excellent insulation resistance to thermal degradation and corona discharge activities [4]. Silicone rubber has also other additional advantages. It can reduce maintenance costs, sustain hydrophobicity, resulting in lower leakage currents, reduce dry band arcing, less risk of flashover, resistance to atmospheric and chemical degradation, good property and colour retention under all weathering conditions [5]. This type of insulator is widely used in the different countries like Middle East, United States, and India. Kim, Cherney and Hackam [6] have applied RTV coatings to porcelain suspension and line post insulators for a comparative study of the performance of coated and uncoated insulators.

The present research is undertaken to understand the behaviour of the disc type porcelain and glass insulators when they are subjected to varying pollution and other environmental parameters. The results obtained from the bottom and top surfaces of those types of insulators are compared.

\section{EXPERIMENT AND RESULTS}

In this research, disc type of porcelain and glass insulators are used for carrying out the measurement of insulation resistance. As pollution, tap water and sodium chloride $(\mathrm{NaCl})$ with different amounts are used to prepare the saltsolution. Initially, a $5 \mathrm{gm}$ of $\mathrm{Nacl}$ is used to mix with 100$\mathrm{ml}$ tap water to get the salt-solution. Later on, this amount of $\mathrm{NaCl}$ salt is increased to $10 \mathrm{gm}, 15 \mathrm{gm}, 20 \mathrm{gm}, 25 \mathrm{gm}$ and $30 \mathrm{gm}$. At first glass insulator is hanged at the wooden insulating frame and polluted the bottom surface by a $5 \mathrm{gm}$ salt-solution with the help of a spray gun. The polluted insulator is then energized by the $100 \mathrm{~V} \mathrm{DC}$ as shown in the 
Figure 1. The current through the insulator surface is measured by the micro-ampere range ammeter. This procedure is repeated for $10 \mathrm{gm}, 15 \mathrm{gm}, 20 \mathrm{gm}, 25 \mathrm{gm}$ and $30 \mathrm{gm}$ salt-solutions and the corresponding currents are also measured. Again, the top surface of the glass insulator is polluted by the above-mentioned salt-solutions and its corresponding currents are measured and recorded in the Table 1. According to Ohm's law, insulation resistances have been calculated from the measured currents and energized voltage. The measured insulation resistance at the upper and bottom surfaces are plotted with the variation of salt-solutions as shown in the Figure 2.

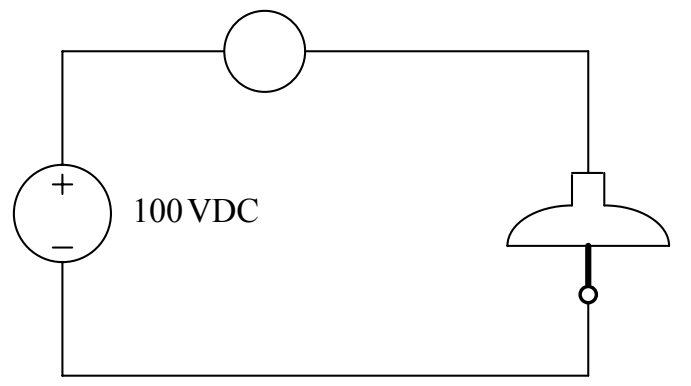

Figure 1. Experimental setup

Table 1. Salt-solution with currents in the bottom and top surfaces of disc type glass insulator

\begin{tabular}{|c|c|c|}
\hline $\begin{array}{c}\text { Salt-solution } \\
(\mathrm{gm} / \mathrm{ml})\end{array}$ & $\begin{array}{c}I_{b} \\
(\mu \mathrm{A})\end{array}$ & $\begin{array}{c}I_{t} \\
(\mu \mathrm{A})\end{array}$ \\
\hline 5 & 0.25 & 0.12 \\
\hline 10 & 0.30 & 0.18 \\
\hline 15 & 0.44 & 0.25 \\
\hline 20 & 0.77 & 0.30 \\
\hline 25 & 1.00 & 0.87 \\
\hline 30 & 1.03 & 1.12 \\
\hline
\end{tabular}

From Figure 2, it is observed that the overall insulation resistance is higher at the top surface than the bottom surface. At $5 \mathrm{gm}$ salt-solution, the difference of insulation resistance between the top and bottom surfaces is around $433 \mathrm{M} \Omega$. At $10 \mathrm{gm}$ salt-solution, this difference is around $223 \mathrm{M} \Omega$ and at $30 \mathrm{gm}$ salt-solution, it is less than that of the 5 gm salt-solution. The experimental measurement of insulation resistances are also continued for the disc type of porcelain insulator with the same salt-solutions as done for the disc type of glass insulator. The measured currents corresponding to different salt-solutions are recorded in the Table 2. The insulation resistances at the top and bottom surfaces of disc type porcelain insulator are plotted against the variation of salt-solutions as shown in the Figure 3. From this Figure 3, it is seen that the difference between the insulation resistances at the top and bottom surfaces for 5 gm salt-solution is higher than the glass insulator. For 30 gm salt-solution, this difference is also remarkable. In the transmission line, the disc type insulator is normally hanged either in vertical or horizontal position. Normally, more pollutants are deposited on the insulator in the vertical position. In its vertical position, the pollution layer on the top surface can be washed away with the help of natural rain. At the bottom, insulator contains many skirts. Pollutants adhere on these skirts and form a thick pollution layer. This thick pollution layer offers a good conducting path in the presence of light rain or mist. Here, in bottom surface, magnitude of leakage current is higher than that on the top surface. As a result, the insulation resistance at the bottom surface is lower than the top surface. This thick pollution layer at the bottom surface cannot be removed by the natural rain. It can be washed with a high-pressure water spray. In the Middle East, this type of routine maintenance is normally done at a regular time interval. Many flashovers have been counted during the high pressure water washing of the energized insulator. To avoid such kind of insulator flashover, we have to follow the standard rule provided by IEEE as well as IEC.

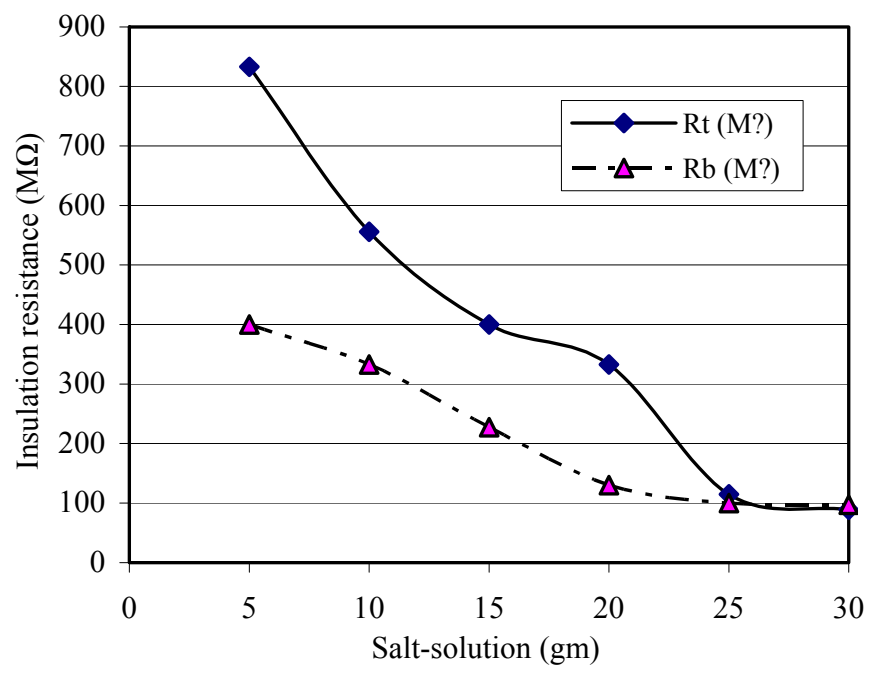

Figure 2. Variation of insulation resistance with saltsolution of glass insulator

Table 2 Salt-solution with currents in the bottom and top surfaces of disc type porcelain insulator.

\begin{tabular}{|c|c|c|}
\hline $\begin{array}{c}\text { Salt-solution } \\
(\mathrm{gm} / \mathrm{ml})\end{array}$ & $\begin{array}{c}I_{b} \\
(\mu \mathrm{A})\end{array}$ & $\begin{array}{c}I_{t} \\
(\mu \mathrm{A})\end{array}$ \\
\hline 5 & 0.81 & 0.11 \\
\hline 10 & 1.59 & 0.18 \\
\hline 15 & 1.79 & 0.26 \\
\hline 20 & 1.90 & 0.35 \\
\hline 25 & 2.21 & 0.40 \\
\hline 30 & 2.86 & 0.52 \\
\hline
\end{tabular}

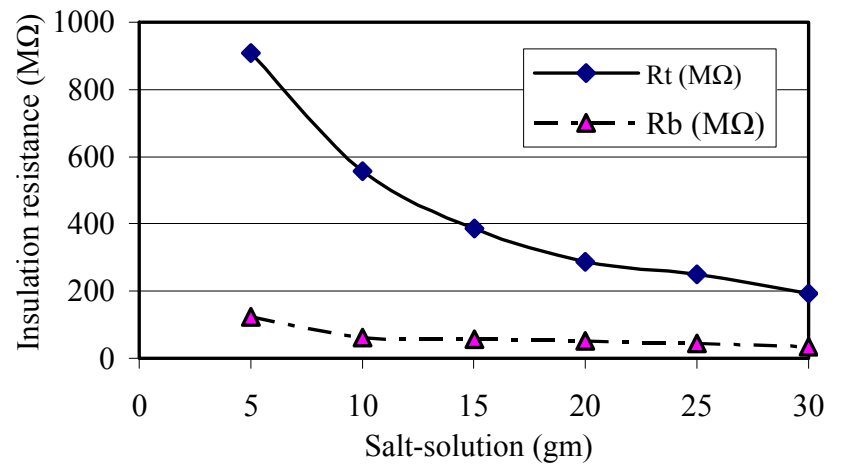

Figure 3. Variation of insulation resistance with saltsolution of porcelain insulator

\section{CONCLUSION}

The measurement of insulation resistance is carried out 
using disc type glass and porcelain insulators. The insulation resistance is measured on the polluted top and bottom surfaces separately for both types of insulators. It is found that the insulation resistance at the top surface is higher than the bottom surface for both glass and porcelain insulators. This type of study can be undertaken for newly developed silicone rubber insulator.

\section{ACKNOWLEDGMENT}

The authors wish to express their appreciation to Engineer Robinson Goh, Department of Electrical Services (DES), Brunei Darussalam for providing disc type glass and porcelain insulators. Special thanks are extended to $\mathrm{Dr}$. $\mathrm{Hj}$ Kassim bin Hj Daud, Director, Dr. Hjh Naemah, Deputy Director, and $\mathrm{Hj}$ Morsidi bin Hj Kassim, Head, Department of Electrical and Communication Engineering, Institute of Technology Brunei, for their financial support.

\section{REFERENCES}

[1] D. Devendranath, Channakeshava, A. D. Rajkumar, "Leakage current and charge in RTV coated insulators under pollution conditions", IEEE Transactions on Dielectrics and Electrical Insulation, Vol. 9 , No. 2, pp. 294-299, April 2002.

[2] J. S. Forrest, T. J. Lambeth and D. F. Oakeshott, "Research in the performance of high voltage insulators in polluted atmospheres", Proce. IEE, 107 A, pp. 172-175, 1960.

[3] IEEE Dielectrics and Electrical Insulation Society's Outdoor Service Environment Committee S-32-3, "Protective coatings for improving contamination performance of outdoor high voltage ceramic insulator", paper 94, WM 096-8 PWRD, New York, January 30 February 3, 1994.

[4] J. W. Chang and R. S. Gorur, "Surface recovery of silicone rubber used for HV outdoor insulation", IEEE Transactions on Dielectrics and Electrical Insulation, Vol. 1, pp. 1039-1046, 1994.

[5] Ibrahim A. Metwally, M. A. Salam, Ali Al-Maqrashi, Saif AR Sumry and Saif Sh Al-Harthy", International Journal of Emerging Electric Power Systems, Vol. 7, Issue 3, Article 3, pp. 1-25, 2006.

[6] S. H. Kim, A. E. Cherney and R. Hackam, "Effects of filler level in RTV silicone rubber coatings in HV insulators", IEEE Transactions on Electrical Insulation, Vol. 27, pp. 1065-1072, 1992. 


\title{
Evaluation of UTHM Administrative Office Block Cooling Load through Dynamic Simulation
}

\author{
Ismail Abdul Rahman, Kamarul Aini Mohd Sari, and Nur Hanisah Mat Tasir
}

\begin{abstract}
The energy requirements for a building have a direct impact on the cost of operating a building and an indirect impact on the environment. The energy estimation can be used as a guide in design, for standards compliance, and for economic optimization. Energy estimation can be done in several ways. The methods vary from steady state/static to dynamic estimation. Building energy simulation is an analysis of energy performance of building dynamically using computer modelling and simulation techniques. This study compares the building cooling load derives from audit report and through energy model with the dynamic simulation result. The building cooling loads were determined by extracting SIRIM energy audit report on the building and also by using energy model of Malaysia reference office building. The building cooling load was estimated using IES software, which simulates cooling load demand for the building dynamically. The study found that the simulated cooling load varies in the range of $6 \%$ from other methods cooling load values. The difference is considered small for estimation purposes.
\end{abstract}

Keywords: cooling load, energy estimation, simulation, energy model

\section{INTRODUCTION}

Buildings are well known to be major energy consumers and so any reduction in their energy usage is welcome. The consequences of an energy inefficient building are great and cause not only high running costs, the depletion of natural fuel resources that are unsustainable, but to excessive global pollution. Based on the application and the level of detail required, different methods could be used to estimate building energy requirements. In general, the methods can be divided into three categories:

- Steady-state methods - they include degree-day method, variable-base degree-day method and bin method [1]. Based on steady-state assumptions, these methods only require simple inputs, but their accuracy and capability are also limited.

- Dynamic methods - the methods estimate and analyse the building energy consumption by modelling the dynamic behaviour of buildings. The most popular one

Ismail Abdul Rahman, Kamarul Aini Mohd Sari, and Nur Hanisah Mat Tasir are with the Department of Building and Construction Engineering, Faculty of Civil Engineering and Environmental, Universiti Tun Hussein On Malaysia (UTHM) is generally known as building energy simulation which models building energy performance over the time domain[2].

- Measurement-based methods — these methods focus on the actual building operation and behaviour rather than on a prediction based on physical properties and performance specifications [3].

This study is to compare cooling loads determined through various methods that are by simulation, extraction from energy audit report and using energy model developed from reference office building [4]. The selected building is located in the University Tun Hussein Onn Malaysia (UTHM) campus, Batu Pahat, Johor. The building for this study is an administrative office block.

\section{ENERGY AUDIT REPORT}

Energy audit from SIRIM [5] involved 49 buildings consisting of administrative office, lecture halls, lecture rooms and others. Not all the buildings are air-conditioned. The air conditioning used in some of the buildings are classified as; central air distribution system, central circulating water system and multiple unit system (split/window type). Certain buildings use a combination of centralised air conditioning system and multiple unit system where the multiple units act as standby and occasionally used in the evening when staffs work overtime. A timer control system is applied to the central air conditioning systems. The energy was audited for one-year period from September 2001 to October 2002. The report given by SIRIM was used to extract the cooling load of the administrative block.

The coefficient of performance (COP) of an air conditioning is the ratio of the energy removed to the energy supplied [6]. The annual average COP of the airconditioning units given by the report varies from 1.1 to 2.6. Some of these are lower than some of the recommended values that range from 2.0 to 3.3 [7].

\section{A The Building}

The building in this study is parts of the buildings that were audited by SIRIM. It is an administrative and office building, which was been occupied since the year 2000. The 
total floor area for this building is $3,666 \mathrm{~m}^{2}$. In term of construction, this building is considered as a lightweight building because the wall is made-up of half brick plastered both sides.

The building is fully enclosed and air-conditioned. Only the staircase and toilet are not air-conditioned. The indoor set point temperature of the building varies from room to room with the temperature range from 18 to 27 degree Celsius. The numbers and types of air-conditioning system used in this building are seven multiple unit (split) and five central air-cooled systems (packages). The capacities of the seven split type units are 2HPeach. The package types have two different capacities, two units with 20HP each and three units with 50HP each (HP denotes horse power of the compressor, which is the usual way of rating air conditioning system in Malaysia).

The audit report found that the electricity consumption of the building was more than it should have been. It argued that high consumption could be the faulty of air distribution system, operating the central and the split unit of air conditioning systems simultaneously and the uncontrollable infiltration of outside air.

\section{B Extraction of Cooling Load}

Two approaches were used to estimate cooling load adopted by the report are by meter logging on main switch board (MSB) and by rated power as specified on the nameplates of equipment.

Logging approach is where the meter was logged at the Main Switch Board (MSB) of the building. From the report the meter was logged for a day. The annual estimation was by multiplying numbers of days the equipment run. The cooling load index using this method is as in table 4.0. The logging for a day may not so accurate to present for the whole year. As the logging was done at MSB, cable energy losses were not considered in the report.

Rated power technique used the rated power of the system and equipment. This approach is subjected to errors because the actual usages of the electricity fluctuate along the time. The cooling load index from this technique is as in table 5.0.

\section{SimUlation}

The energy consumption of the building was simulated using the IES software together with Malaysia typical weather year hourly data. The simulation on the building was based on APSim idealised room control. The idealised temperature and humidity control is modelled by APSim operating in standalone mode with an assumption that the HVAC system and HVAC plant performed as required in the input data of the simulation.

Weather data used in the simulation is important because it contributes to external heat gains to the building. Ideally, the weather data used should be at the location of the building to be simulated, but such data is not available. This simulation used the Malaysia typical weather year hourly data [8] generated from Subang weather station data. Since the weather data used are not from the actual site and period, we have to expect the discrepancy of the simulation result. The distance between the building location and Subang is quite far, but Malaysia which is located around the equator, experiences weather that does not vary much from place to place[9].

\section{A Model Construction}

Before a simulation can be carried out, the model of building has to be constructed or imported from other file. To construct the building model, we can use IES application tool known as Model IT. This tool uses a drag and pulldown menu bar to construct the building model. For this work, the author constructed the building model using the tool based on the available building plan.

\section{B Input Data}

Once the shape of the building has been constructed, the construction types of the building and the room thermal attributes data were then assigned. Each construction type consists of a layer-by-layer description of the element's thermo-physical properties, together with other data such as surface solar absorptivity and emmissivity. The construction attributes of the building are as in table 1.0. The room thermal data is a set of attributes that describe conditions and events in the room. Room thermal attributes data assigned to the building are as in table 2.0.

\section{Simulated Cooling Load}

The annual simulated cooling load of the building is $806,729 \mathrm{kWh} / \mathrm{yr}$ as in table 3.0. This figure is converted into building cooling load index by dividing with the floor area. Thus, simulated building cooling load index becomes 220.0 $\mathrm{kWh} / \mathrm{m}^{2} / \mathrm{yr}$. This value is compared with values generated from other methods as in table 7.0. From this table, the index generated from the simulation approach is lower either from the logged or the rated methods.

\section{ENERGY MODEL}

Energy index is the consumption of energy for one metre square area for a period of one year. The simulated index is compared with the index generated from reference office energy model [4] below:

\section{Cooling Load $=$}

$[464+57 S C+121 W W R-17 A T+2 E Q+2 L L+170 O C]$

$k W h / m^{2} / y r$

The values of the parameters of the model above are in table 6.0. The generated building cooling index of the building is $216.7 \mathrm{~kW} / \mathrm{m}^{2} / \mathbf{y r}$.

The percentage difference of this cooling load index with other is as in table 7.0. The difference is probably because the model is relatively rough as only six parameters were considered. The model would be much better if other parameters are considered for example the ventilation rate, building form, thermal insulation, HVAC system and others. However, this model is practical, quick and effective in estimating the building load of Malaysia office building. 


\section{CONCLUSION}

Before a comparison can be made between the methods, it is better to consider the shortcomings each of the approach.

Logging approach gives the actual power usage of the equipment. If the logging is done at the MSB, then consideration should be given to the cable loss from the MSB to the equipment. Unfortunately, in for the report the logging was done for only one day and the cable loss was not considered.

Rated approach is a static method, which estimates the energy index in a rough way. This method refers to the rated power nameplate of the equipment. The rated power on equipment is usually based on design values with some safety factor. Therefore, using this method should gives higher energy index compare to other methods.

Model method is relatively rough as only six parameters were considered but is better than OTTV technique. The application of the developed energy equation on the actual building shows that the estimated cooling load is approximately to the simulated value. This means that the equation can be used as early energy estimation for the office building in Malaysia.

Simulation method gives reliable value if all the input data are similar to the actual case. Since in this case, the input data are not very definite especially the weather data used are not from the location, only one set-point temperature for the simulation but in actual case several setpoint temperatures, using default values where the information is not available example few of the building features, operating hours and others.

Simulated value is lower either from the logged or the rated methods However, this value is higher than that by model method. The differences are shown as in figure 1.0 and in table 7.0. Difference variations are not that significant, about $5 \%$. The simulated value is much accepted as the report claimed that the building consumed more energy than expected and the model is still rough as it considered six parameters.

more. Use a space after authors' initials. Papers that have not been published should be cited as "unpublished" [4]. Papers that have been submitted for publication should be cited as "submitted for publication" [5]. Papers that have been accepted for publication, but not yet specified for an issue should be cited as "to be published" [6]. Please give affiliations and addresses for private communications [7].

Capitalize only the first word in a paper title, except for proper nouns and element symbols.

\section{REFERENCES}

[1] ASHRAE, Handbook - Fundamentals, American Society of Heating Refrigerating and Air-Conditioning Engineers, Atlanta, GA. 1997.

[2] Clarke, J.A. and A.D. Irving, Building energy simulation: An introduction. Energy and Buildings, 1988. 10(3): p. 157-159.

[3] Busch, J.F. and J.J. Deringer. A building envelope energy standard for Malaysia. in Proc. of the Far East Conference on Air Conditioning in Hot Climates, September 3-5, Singapore, pp. 302-320. 1987.

[4] I.A.Rahman. Sensitive Analysis of Energy Performance and Cooling Load Model for Malaysia Reference Office Building. in World Housing Congress 2007 on Affordable Quality Housing Proceedings. 1st -5th July 2007. Primula Beach Resort Hotel Kuala Terengganu.

[5] SIRIM, Energy Audit in Government Building Project Consultancy Report for KUiTTHO, Batu Pahat, Johor. Feb 2003, Environmental \& Bioprocess Technology Centre, SIRIM Berhad. Kuala Lumpur.
[6] Jones, W.P., Air Conditioning Engineering. Fifth ed. 2001: Butterworth-Heinemann.UK.

[7] CEE, High-Efficiency Commercial Air Conditioning \& Heat Pumps. 2004, Consortium For Energy Efficiency, http://www.cee1.org/com/hecac/hecac-main.php3.

[8] Rahman, I.A. and J. Dewsbury, Test Reference Year (TRY) for Subang Malaysia. Building and Environment, 2007. 42(10): p. 3636-3641.

[9] W.W.P., Map of Malaysia, World Wide Portals, Developing Virtual Real EstateGreenwich2000 Ltd. UK, http://wwp.greenwichmeantime.com/timezone/asia/malaysia/map.htm. 


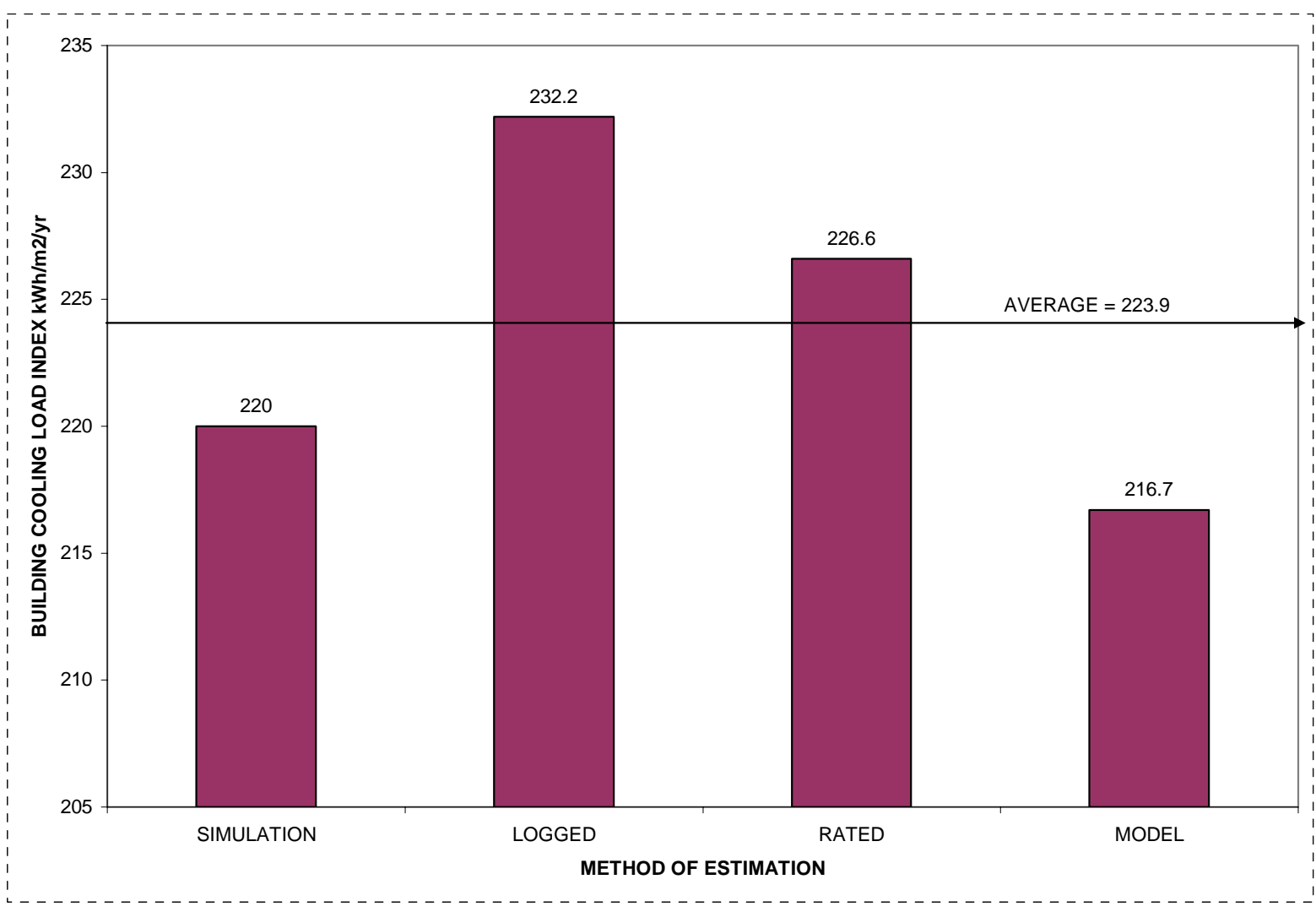

Figure 1.0 Estimated building cooling load index

Table 1.0Construction attributes input to the building

\begin{tabular}{|c|c|c|}
\hline Item & Descriptions & $\begin{array}{l}\text { U values } \\
\text { W/m². K }\end{array}$ \\
\hline Roof & Sloped clay tiles and insulated with glass wool & 0.45 \\
\hline \multirow{2}{*}{$\begin{array}{l}\text { Ceiling or } \\
\text { intermediate } \\
\text { floor }\end{array}$} & Concrete with ceiling and finishes & 1.24 \\
\hline & Timber with ceiling and finishes & 0.67 \\
\hline External Wall & $\begin{array}{l}115 \mathrm{~mm} \text { brickwork with } \\
12 \mathrm{~mm} \text { dense cement render both faces }\end{array}$ & 2.76 \\
\hline \multirow[t]{2}{*}{ Internal Partition } & $\begin{array}{l}115 \mathrm{~mm} \text { brickwork with } \\
12 \mathrm{~mm} \text { dense cement render both faces }\end{array}$ & 2.14 \\
\hline & $2 \times 12 \mathrm{~mm}$ gypsum plaster board and $50 \mathrm{~mm}$ air gap & 1.77 \\
\hline \multirow{2}{*}{ Door } & Wooden & 2.07 \\
\hline & Single glazing $6 \mathrm{~mm}$ & 5.65 \\
\hline External Glazing & Single glazing $6 \mathrm{~mm}$ & 5.65 \\
\hline Internal Glazing & Single glazing $6 \mathrm{~mm}$ & 4.27 \\
\hline
\end{tabular}

Table 2.0 Room thermal attributes input to the building

\begin{tabular}{|l|l|c|}
\hline \multicolumn{1}{|c|}{ Items } & \multicolumn{1}{|c|}{ Descriptions } & Values \\
\hline \multirow{2}{*}{ Cooling Simulation set-point } & Room & $22^{0} \mathrm{C}$ \\
\cline { 2 - 3 } & Corridor & $26^{0} \mathrm{C}$ \\
\hline \multirow{2}{*}{ Relative Humidity } & Maximum & $65 \%$ \\
\cline { 2 - 3 } & Minimum & $55 \%$ \\
\hline \multirow{3}{*}{ Profile of working hours } & Mon to Fri full working day & Oam to $5.00 \mathrm{pm}$ \\
\cline { 2 - 3 } & Sat. half working day & Off \\
\cline { 2 - 3 } & Sun is not a working day & $15 \mathrm{w} / \mathrm{m}^{2}$ \\
\hline \multirow{3}{*}{ Casual gains } & Computers & $0.1 \mathrm{persons} / \mathrm{m}^{2}$ \\
\cline { 2 - 3 } & People & $16 \mathrm{w} / \mathrm{m}^{2}$ \\
\cline { 2 - 3 } & Lighting & $3 \mathrm{ach}$ \\
\hline \multirow{2}{*}{ Ventilation } & Mechanical & $0.5 \mathrm{ach}$ \\
\cline { 2 - 3 } & Infiltration & $\mathrm{ach}$ \\
\hline
\end{tabular}


Table 3.0 Output of the simulation

\begin{tabular}{|l|c|}
\hline \multicolumn{1}{|c|}{ Months } & $\begin{array}{c}\text { Simulated cooling load } \\
\text { kWh }\end{array}$ \\
\hline JAN & 58,834 \\
\hline FEB & 61,706 \\
\hline MAR & 67,873 \\
\hline APR & 75,344 \\
\hline MAY & 68,338 \\
\hline JUN & 73,941 \\
\hline JUL & 71,071 \\
\hline AUG & 68,066 \\
\hline SEP & 66,495 \\
\hline OCT & 62,361 \\
\hline NOV & 67,102 \\
\hline DEC & 65,600 \\
\hline Annual $(\mathrm{kWh} / \mathrm{yr})$ & 806,729 \\
\hline Floor area $\left(\mathrm{m}^{2}\right)$ & 3666 \\
\hline Building cooling load & $\mathbf{2 2 0 . 0}$ \\
\hline index $\mathbf{k W h} / \mathbf{m}^{2} / \mathbf{y r}$ & \\
\hline
\end{tabular}

Table 4.0Energy index based on MSB logged data from SIRIM report[5]

\begin{tabular}{|l|c|}
\hline \multicolumn{1}{|c|}{ Item } & Values \\
\hline Electricity consumption (logging-kWh/day) & 1548 \\
\hline Operating time (days/yr) & 275 \\
\hline $\begin{array}{l}\text { Total air conditioning electricity consumption } \\
(\mathrm{kWh} / \mathrm{yr})\end{array}$ & 425,700 \\
\hline COP $($ assumed) & 2.0 \\
\hline Building cooling load $(\mathrm{kWh} / \mathrm{yr})$ & 851,400 \\
\hline Floor area $\left(\mathrm{m}^{2}\right)$ & 3,666 \\
\hline Building cooling load index $\left(\mathrm{kWh} / \mathrm{m}^{2} / \mathrm{yr}\right)$ & $\mathbf{2 3 2 . 2}$ \\
\hline
\end{tabular}

Table 5.0Energy index based on rated power from SIRIM report [5]

\begin{tabular}{|l|c|}
\hline \multicolumn{1}{|c|}{ Item } & Values \\
\hline Electrical load $(\mathrm{kW})$ & 152.2 \\
\hline Operating Hours $(\mathrm{hr} / \mathrm{yr})$ & 2730 \\
\hline Total air conditioning electricity consumption $(\mathrm{kWh} / \mathrm{yr})$ & 415,506 \\
\hline COP $($ assumed) & 2.0 \\
\hline Building cooling load $(\mathrm{kWh} / \mathrm{yr})$ & 831,012 \\
\hline Floor area $\left(\mathrm{m}^{2}\right)$ & 3,666 \\
\hline Building cooling load index $\left(\mathrm{kWh} / \mathrm{m}^{2} / \mathrm{yr}\right)$ & $\mathbf{2 2 6 . 6}$ \\
\hline
\end{tabular}

Table 6.0Energy estimation using energy model

\begin{tabular}{|l|l|}
\hline \multicolumn{1}{|c|}{ Item } & \multicolumn{1}{c|}{ Values } \\
\hline Shading coefficient(SC) & 0.69 \\
\hline Window-wall-ratio(WWR) & 0.40 \\
\hline Set-point temperature(AT) & $22^{0} \mathrm{C}$ \\
\hline Equipment load(EQ) & $15 \mathrm{~W} / \mathrm{m}^{2}$ \\
\hline Lighting Load(LL) & $16 \mathrm{~W} / \mathrm{m}^{2}$ \\
\hline Occupancy density(OC) & $0.1 \mathrm{person} / \mathrm{m}^{2}$ \\
\hline $\begin{array}{l}\text { Estimated building cooling load index using this energy model: } \\
\text { Cooling Load }=[464+57 S C+121 W W R-17 A T\end{array}$ & $216.7 \mathrm{kWh} / \mathrm{m} 2 / \mathrm{yr}$ \\
$+2 E Q+2 L L+170 O C] \quad \mathrm{kWh} / \mathrm{m}^{2} / y r$ & \\
\hline
\end{tabular}


Table 7.0Energy index with different methods of estimation

\begin{tabular}{|c|c|c|}
\hline Methods & $\begin{array}{c}\text { Building cooling index } \\
\left(\mathbf{k W h} / \mathbf{m}^{2} / \mathbf{y r}\right)\end{array}$ & $\begin{array}{l} \pm \text { percentage above or } \\
\text { below simulated value }\end{array}$ \\
\hline Simulation & 220.0 & - \\
\hline MSB logged data & 232.2 & $+5.5 \%$ \\
\hline Rated power & 226.6 & $+3.0 \%$ \\
\hline Energy model & 216.7 & $-1.5 \%$ \\
\hline
\end{tabular}




\title{
Gasification Behaviour of Malaysian Biomass: Sugarcane Waste
}

\author{
Barranco, R., Cooper, M., Hanson, S., Lee, K. U., Low, K. S. and Teo, C. H.
}

\begin{abstract}
With the unrelenting rise in world population and continuing efforts to improve living standards in 'developing countries', energy demand is set to carry on increasing for the foreseeable future. Even if it is assumed that there is no immediate risk of fossil fuels running short, it is debatable whether exploiting those resources at an increasing rate is advisable with mounting evidence pointing towards anthropogenic causes for global warming. In this scenario all available renewable and $\mathrm{CO}_{2}$-neutral routes for energy provision must be explored fully.

The work reported in this paper contributes to a project which explores the use of biomass gasification as one of the sources to provide hydrogen for the 'hydrogen economy', a much favoured alternative to the fossil fuel economy.

This paper reports the results from characterising sugar cane waste sourced in Malaysia. Apart from standard analysis, its behaviour during slow pyrolysis and its reactivity with $\mathrm{CO}_{2}$ were investigated. This was carried out to identify its gasification characteristics and to propose a suitable gasification method. The results lead to the assumption that sugarcane waste is indeed a suitable feedstock for hydrogen production via gasification. It devoltilises extensively at low temperatures, with most of its high hydrogen content belonging to the volatiles fraction. The resulting char has high enough an energy content to count as a valuable fuel and it is reactive to $\mathrm{CO}_{2}$ at high temperatures, and therefore suited to gasification.
\end{abstract}

Keywords: biomass, gasification, fuel characterisation, $\mathrm{CO}_{2}$ reactivity

\section{INTRODUCTION}

Energy demand is predicted to continue to increase as the world population grows and efforts to supply sufficient energy to all people are sustained. Even conservative estimates expect a 12200 mtoe energy demand in 2030 [1], an increase of over $10 \%$ on the 2004 level. A more dramatic increase would be required to meet the goal of delivering the same standard of living enjoyed in a few industrialised countries to all regions by 2050 . For this to happen energy demand would increase to eight times that of the year 2000 [2].

Hanson, S., Lee, K. U., Low, K. S., and Teo, C. H. are in the Faculty of Engineering and Computer Science, University of Nottingham, Malaysia Campus, Jalan Broga, 43500 Semenyih (corresponding author S. Hanson: tel: $03 \quad 8924$ 8128, fax: $03 \quad 8924$ 8017, e-mail: svenja.hanson@nottingham.edu.my).

Barranco, R., and Cooper, F. A. are at the School of Chemical and Environmental Engineering, University Park, Nottingham, UK.
Currently $80 \%$ of the primary energy demand is met by fossil fuels [1], with renewable sources not even touching the $15 \%$ mark. It is now widely accepted that climate change is a reality and that anthropogenic greenhouse gas emissions, mainly from fossil fuel use, are to blame [3], [4]. Any possible contribution to the energy mix by $\mathrm{CO}_{2}$ free or $\mathrm{CO}_{2}$ neutral sources must therefore be considered seriously. Whereas growing crops for fuel remains controversial [5] because of its competition with food production and the preservation of natural habitats, there are no objections against using agricultural wastes for energy generation. A problem could, however, be posed by the fact that these wastes tend to be low-grade fuels. Gasification has been identified as a technology applicable to low-grade fuels such as biomass, which are technically or economically unfeasible using conventional technology [6].

Furthermore, it has been suggested that biomass may be a good feedstock for renewable hydrogen production, and may be able to contribute to the transition to hydrogen as the main energy carrier (the 'hydrogen economy'). Biomass from agricultural waste in other regions have been investigated for their hydrogen producing potential [7]. In this project it is planned to do so for agricultural wastes typically found in Malaysia. Sugarcane waste was chosen as the first sample to be characterised for its suitability as a fuel, for gasification and for its potential as a hydrogen feedstock.

\section{CHARACTERISATION OF MALAYSIAN SUGARCANE}

Sugarcane waste was obtained from local market stalls selling cane juice. The waste was air dried until no further significant weight loss was observed, and ground to $<3 \mathrm{~mm}$ particle size. The fraction $<1 \mathrm{~mm}$ was sieved out and is referred to as 'fines'. Sugarcane char was obtained from the coarse $(1-3 \mathrm{~mm})$ sugarcane waste by carbonisation in nitrogen at $700^{\circ} \mathrm{C}$.

\section{A. Manual Proximate Analysis}

This procedure was based on the standard method for the proximate analysis of coal. The sample size used was as close to $1 \mathrm{~g}$ as the low sample density permitted. The moisture content of the air-dry samples was determined by drying in an oven at $110^{\circ} \mathrm{C}$ for one hour. The moisture-free sample was then ashed at $825^{\circ} \mathrm{C}$ in a muffle furnace. A fresh sample was tested for volatile matter at $900^{\circ} \mathrm{C}$ by 7 minutes exposure to the temperature in a tall crucible with a lid. The results are summarised in 'table 1'. Fixed carbon 
was determined by difference.

Table 1. Results from Manual Proximate Analysis of Sugarcane Waste

\begin{tabular}{|l|c|c|c|c|}
\hline & $\begin{array}{l}\text { Moisture } \\
/ \% \mathrm{wt}\end{array}$ & $\begin{array}{l}\text { Ash } \\
/ \% \mathrm{wt} \mathrm{db}\end{array}$ & $\begin{array}{l}\mathrm{VM} \\
/ \% \mathrm{wt} \\
\mathrm{daf}\end{array}$ & $\begin{array}{l}\text { Fixed C } \\
/ \% \mathrm{wt}\end{array}$ \\
\hline $\begin{array}{l}\text { Sugar Cane } \\
\text { (coarse) }\end{array}$ & 7.4 & 2.1 & 84.8 & 13.8 \\
\hline $\begin{array}{l}\text { Sugar Cane } \\
\text { (fine) }\end{array}$ & 7.9 & 1.9 & 87.3 & 11.5 \\
\hline $\begin{array}{l}\text { Char from } \\
\text { coarse } \\
\text { Sugar Cane }\end{array}$ & 6.1 & 24.6 & 7.3 & 81.3 \\
\hline
\end{tabular}

Proximate Analysis by Thermo-gravimetric Analysis (TGA)

Samples sizes of typically $3-4 \mathrm{mg}$ were heated in nitrogen. The protocol written for TGA heated the sample rapidly to $110^{\circ} \mathrm{C}$ and held it there for 5 minutes. The weight loss at this time was taken as the moisture content. It was then further heated to $920^{\circ} \mathrm{C}$ at $3{ }^{\circ} \mathrm{C} / \mathrm{min}$ and held at that temperature for 20 minutes. The weight loss at that point was taken as the volatile mater loss. The sample was then cooled to $820^{\circ} \mathrm{C}$ and the gas flow changed to air. The weight percent remaining after 10 minutes in air at $820^{\circ} \mathrm{C}$ was taken as the ash content. The results are shown in 'table 2'.

Table 2. Results from TGA Proximate Analysis of Sugarcane Waste

\begin{tabular}{|l|c|c|c|c|}
\hline & $\begin{array}{l}\text { Moisture } \\
/ \% \mathrm{wt}\end{array}$ & $\begin{array}{l}\text { Ash } \\
/ \% \mathrm{wt} \mathrm{db}\end{array}$ & $\begin{array}{l}\mathrm{VM} \\
/ \% \mathrm{wt} \\
\mathrm{daf}\end{array}$ & $\begin{array}{l}\text { Fixed C } \\
/ \% \mathrm{wt}\end{array}$ \\
\hline $\begin{array}{l}\text { Sugar Cane } \\
\text { (coarse) }\end{array}$ & 5.7 & 1.8 & 100.0 & 0.0 \\
\hline $\begin{array}{l}\text { Sugar Cane } \\
\text { (fine) }\end{array}$ & 5.0 & 2.6 & 100.0 & 0.0 \\
\hline $\begin{array}{l}\text { Char from } \\
\text { coarse } \\
\text { sugar cane }\end{array}$ & 5.8 & 17.5 & 87.1 & 10.0 \\
\hline
\end{tabular}

The advantage of TGA over manual proximate analysis is that the weight is recorded every second, so that it also identifies over which temperature ranges the weight loss occurs at the highest rate. 'Figure 1' shows this as a percentage weight loss versus temperature plot. A second graph was added from the $\mathrm{CO}_{2}$ reactivity tests, where the samples were heated at $50^{\circ} \mathrm{C} / \mathrm{min}$ in nitrogen until they reached the target temperature. The sugarcane waste looses weight most rapidly between 200 and $350 / 400^{\circ} \mathrm{C}$. Rapid weight loss ceases earlier at the higher heating rate of $50^{\circ} \mathrm{C} / \mathrm{min}$, but at a similar level of weight loss of around $70 \%$ wt. At the slower heating rate there is another range of rapid weight loss around $800^{\circ} \mathrm{C}$, which was not seen at the higher heating rate, and appears to account for the additional weight loss at the slow rate. The sugarcane char looses weight slowly at both heating rates up to $800^{\circ} \mathrm{C}$. For the slow heating rate it then undergoes rapid weight loss. It is plausible that devolatilisation weight loss for the char recommences once it is heated beyond its original carbonisation temperature, but the extent was unexpected and results need to be re-confirmed before drawing any definite conclusions. This is especially necessary as the rapid weight loss was not observed at the faster heating rate and conflicts with the much lower weight loss determined with the manual method, which relies on fast heating. The fast heating rate, as could be expected, matches the manually determined volatile matter content for both, the sugarcane waste and its char, much better.

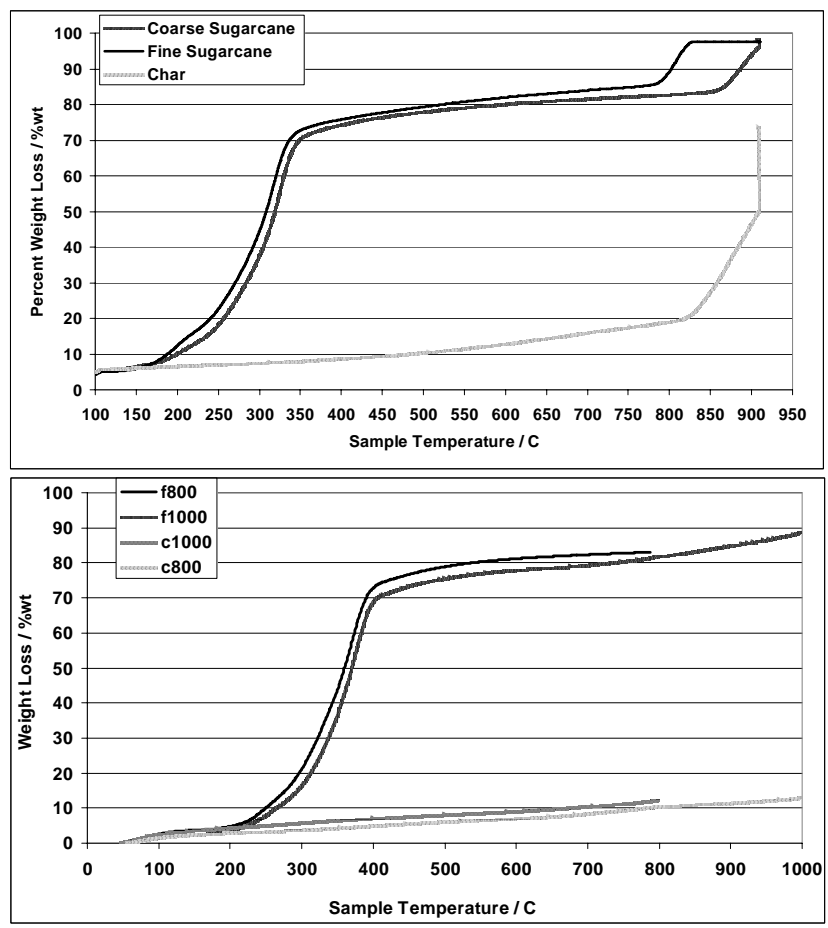

Figure 1. Devolatilisation of Sugarcane Waste and its Char in Nitrogen between at $3^{\circ} \mathrm{C} / \mathrm{min}$ (top) and at $50^{\circ} \mathrm{C} / \mathrm{min}$ (bottom)

\section{B. Char Production in a Tubular Furnace}

Char was produced from $1 \mathrm{~g}$ samples of the coarse sugarcane waste in nitrogen at $500,600,700$ and $800{ }^{\circ} \mathrm{C}$ in a tubular furnace. In Table 3 the yields are compared with those from the TGA predictions taken from 'figure 1'. They were found to be slightly higher than those predicted by TGA, but followed the expected downward trend with increasing temperature. From the tubular furnace carbonisations it is expected that roughly a quarter of the sugarcane waste mass remains when it is heated in an inert atmosphere to temperatures between 500 and $800^{\circ} \mathrm{C}$.

Table 3. Char yield predicted from TGA Proximate Analysis

\begin{tabular}{|c|c|c|c|c|}
\hline $\begin{array}{c}\mathrm{T} \\
/{ }^{\circ} \mathrm{C}\end{array}$ & $\begin{array}{l}\text { Coarse } \\
\text { Sugarcane }\end{array}$ & $\begin{array}{l}\text { Fine } \\
\text { Sugarcane }\end{array}$ & $\begin{array}{l}\text { Coarse } \\
\text { Sugarcane } \\
(50 / \mathrm{min})\end{array}$ & $\begin{array}{l}\text { Coarse } \\
\text { Sugarcane } \\
\text { Retort }\end{array}$ \\
\hline 400 & 25.5 & 24.0 & 29.0 & \\
\hline 500 & 22.0 & 20.5 & 23.0 & 25.8 \\
\hline 600 & 20.0 & 18.0 & 20.5 & 24.4 \\
\hline 700 & 18.5 & 16.0 & 19.0 & 24.6 \\
\hline 800 & 17.0 & 10.5 & 17.5 & 23.4 \\
\hline
\end{tabular}

Fine sugarcane waste $(<1 \mathrm{~mm})$ and the higher heating rate $\left(50^{\circ} \mathrm{C} / \mathrm{min}\right)$ were included in 'table 3 ' to illustrate that temperature is only one of the factors determining char yield, albeit probably the most important one. Some variations must be expected with particle size (lower yield 
for smaller particles) and heating rate (slightly higher yield for higher heating rates).
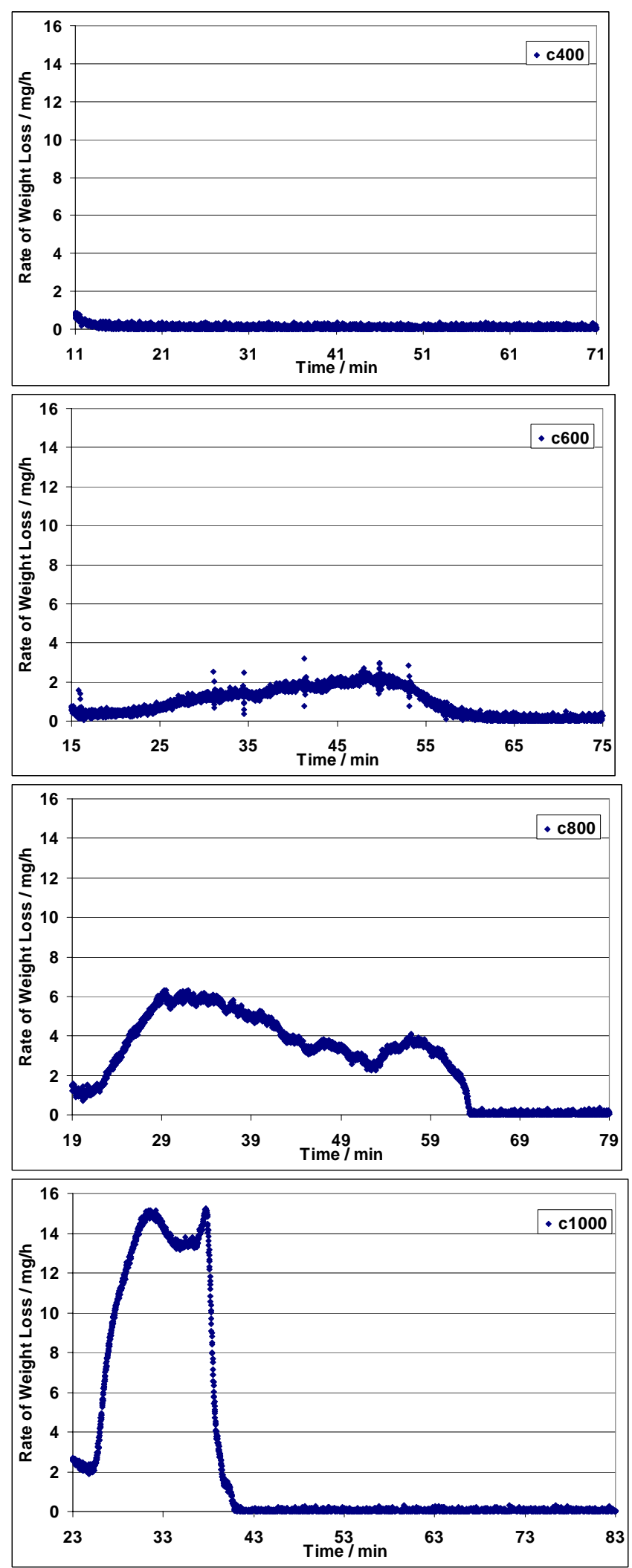

Figure 2. Rate of Weight Loss from Char (in $\mathrm{mg} / \mathrm{h}$ ) during one hour exposure to $\mathrm{CO}_{2}$ at $400,600,800$ and $1000^{\circ} \mathrm{C}$

\section{Char Reactivity in $\mathrm{CO}_{2}$}

The extent of gasification will depend on how reactive is the residue after devolatilisation, the char, in the presence of gasifying agents. In this case only $\mathrm{CO}_{2}$ is considered, as it is the most compatible with TGA. Larger scale trials with more realistic gas mixtures are planned for a later stage of the project. The char samples were heated in nitrogen at $50^{\circ} \mathrm{C} / \mathrm{min}$ until the target temperatures were reached. The gas mixture was then switched to $\mathrm{CO}_{2}$ and the target temperature held for an hour. 'Figure 2' shows the rate of weight loss (in $\mathrm{mg} / \mathrm{h}$ ) over that hour at 400, 600, 800 and $1000{ }^{\circ} \mathrm{C}$. At $400^{\circ} \mathrm{C}$, and presumably below, the rate of weight loss is very small. Between 400 and $600^{\circ} \mathrm{C}$ it increases to the extent that all the available reactive portion of the sample is depleted before the end of the hour of $\mathrm{CO}_{2}$ exposure.

'Table 4' summarises the percentage of the sample lost under $\mathrm{CO}_{2}$ exposure, and also the devolatilisation losses incurred before reaching the target temperature. The data for non-carbonised sugarcane waste is included to show that the weight loss is dominated by the devolatilisation step, leaving little material to take part in carbonisation reactions. For char some devolatilisation still occurs, but to a limited extent leaving over $80 \%$ wt of the sample for gasification. The weight loss recorded in Table 4 very clearly confirms the existence of the step change in weight loss, and thus reaction rates between 400 and $600^{\circ} \mathrm{C}$. The exact location of this step change needs to be investigated further.

Table 4. Percent Weight Loss during one hour of exposure to $\mathrm{CO}_{2}$.

\begin{tabular}{|c|c|c|c|c|c|c|}
\hline & \multicolumn{3}{|l|}{ Sugarcane } & \multicolumn{3}{l|}{ Sugarcane Char } \\
\hline $\mathrm{T} /{ }^{\circ} \mathrm{C}$ & Start & End & $\%$ lost & $\begin{array}{l}\text { Star } \\
\mathrm{t}\end{array}$ & End & $\%$ lost \\
\hline 200 & 7.6 & 14.8 & 7.2 & 4.4 & 6.1 & 1.7 \\
\hline 300 & 31.9 & 94.2 & 62.3 & 13.9 & 16.4 & 2.5 \\
\hline 400 & 87.8 & 95.2 & 7.4 & 6.7 & 8.8 & 2.1 \\
\hline 600 & 82.1 & 96.5 & 14.4 & 18.8 & 94.8 & 76 \\
\hline 800 & 83.2 & 100 & 16.8 & 10.6 & 99.1 & 88.5 \\
\hline 1000 & 89.3 & 98.7 & 9.4 & 18 & 99.5 & 81.5 \\
\hline
\end{tabular}

By adjusting the times it took for the reactive part of the samples to deplete for sample sizes and the extent of prior devolatilisation, the weight loss rates, rates of reactions with $\mathrm{CO}_{2}$, were estimated and plotted against temperature in 'figure 3 '.

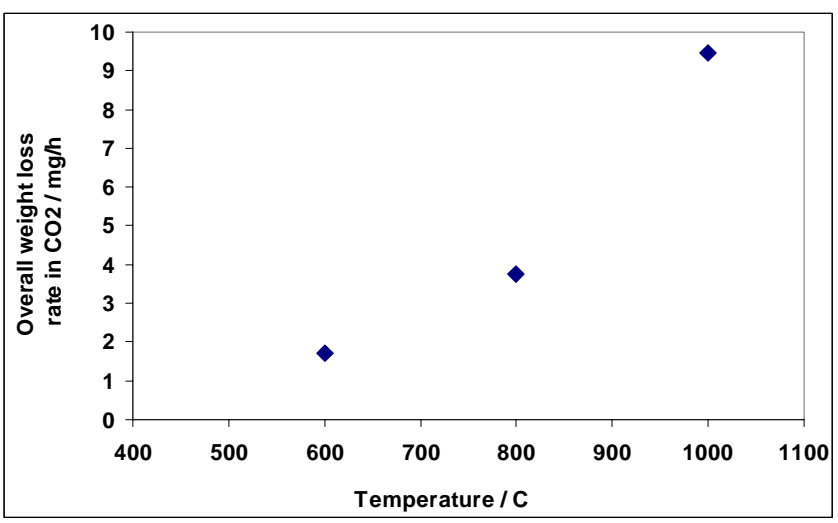

Figure 3. Weight Loss Rate of Char in $\mathrm{CO}_{2}$ adjusted for Devolatilisation Losses and Sample Size

Further work is needed to confirm this trend with larger samples sizes, but if the TGA observations hold, an exponential relationship between char reaction rate with $\mathrm{CO}_{2}$ and temperature is expected. 


\section{Ultimate Analysis and Calorific Values}

Ultimate analysis of the sugarcane waste and its char was carried out using an CHNS-O elemental analyser. The calorific values (CV's) were determined by bomb calorimetry and are reported as gross values in 'table 5' alongside the elemental composition.

Table 5. Ultimate Analysis and Calorific Values

\begin{tabular}{|l|c|c|c|c|c|}
\hline \multicolumn{1}{|c|}{$\%$ weight } & $\mathrm{N}$ & $\mathrm{C}$ & $\mathrm{H}$ & $\begin{array}{c}\mathrm{O} \\
\text { (diff.) }\end{array}$ & $\begin{array}{c}\mathrm{CV} / \\
\mathrm{MJ} / \mathrm{kg}\end{array}$ \\
\hline $\begin{array}{l}\text { Sugar Cane } \\
\text { Waste (coarse) }\end{array}$ & 1.8 & 43.6 & 6.5 & 48.8 & 17.1 \\
\hline $\begin{array}{l}\text { Sugar Cane } \\
\text { Waste (fine) }\end{array}$ & 0.6 & 42.3 & 5.8 & 51.3 & n.a. \\
\hline $\begin{array}{l}\text { Sugarcane } \\
\text { Char, 700C, } \mathrm{N}_{2}\end{array}$ & 2.0 & 77.4 & 1.0 & 19.6 & 27.3 \\
\hline
\end{tabular}

\section{DISCUSSION}

From the results it can be seen that air-dried sugarcane waste has a low carbon content and low calorific value, and thus cannot be regarded as valuable fuel, especially as it also has a low bulk density. If it is to be used other than for on-site combustion, some form of upgrading is required. Carbon content and calorific value have been shown to improve considerably by carbonisation at $700^{\circ} \mathrm{C}$. The resulting char matches the energy content of a low rank coal, but with a much lower moisture and, in the case of some coals, sulphur content.

Char yield for carbonisation at temperatures between 500 and $800^{\circ} \mathrm{C}$ was shown to be of the order of a quarter of the sample, so that char making alone without using the volatiles which are driven off, would waste three-quarters of the sample mass, including an estimated $56 \%$ wt of the carbon and $96 \%$ wt of the hydrogen. As the hydrogen content of the sugarcane waste, as determined by elemental analysis, is high by comparison with other solid fuels, it is suspected that the devolatilisation gas from it may be high in hydrogen, and further investigation of its potential as a renewable source of hydrogen seems definitely worthwhile.

The TGA analysis showed that around $70 \%$ of weight of the sugarcane has been driven off as volatile matter when it was heated to $350-400^{\circ} \mathrm{C}$ in an inert atmosphere. But to attain a reasonable rate of reaction of the residue with $\mathrm{CO}_{2}$, temperatures well above $600^{\circ} \mathrm{C}$ were needed. The high volatile matter content and the low temperature by which the majority of it has been released, means that the occurrence of actual gasification reactions is not necessary to achieve a good gas yield. A tailor-made gasifier for sugarcane waste might use char for combustion, rather than gasify it. The combustion gas would provide the carrier gas and heat for a carbonisation unit. Obviously such a set-up would need to be studied both in theory and by laboratory experiment. Specifically it is foreseen that considerable optimisation work is needed to specify the conditions, such as temperature, particle size, heating rate and thre air-tochar ratio. The sugarcane waste needs to be carbonised to just the right extent to yield enough char, so that the char combustion supplies sufficient energy to maintain the process.

An interesting side issue is the comparison between manual and TGA proximate analysis. Whereas the moisture and ash mostly agree between manual and TGA proximate analysis, the volatile matter, especially for the char, differs significantly. It is much higher for the TGA method, leaving no fixed carbon for in the fresh sample, and only 10 $\%$ wt in the char. This might partly be accounted for by the smaller sample size and free gas flow, which favours vapour leaving rather than re-condensing. But the main reason is proposed to be heating rate and time dependence of the devolatilisation process. When the cold sample is placed in the hot furnace, rapid heating takes place and the time the sample is exposed to temperatures at which devolatilisation occurs may be insufficient to expel the maximum amount of volatiles possible at those temperatures. At the slow heating rate in the TGA sufficient time is available for complete devolatilisation. This result is significant for gasification, in that the volatile release prediction will have to take the gasification method into account. If the sugarcane is slowly moving through a fixed bed it is likely that more complete devolatilisation occurs, matching the TGA prediction. Whereas, if it is injected into the hot zone of a fluidised bed, rapid heating and lower volatile release in line with the manual method may be expected. Obviously, if the alternative method of relatively low temperature devolatilisation and char combustion is adopted, the behaviour of the sugarcane waste and its char at temperatures around $800^{\circ} \mathrm{C}$ becomes irrelevant.

\section{CONCLUSIONS}

Sugarcane waste is a low-grade fuel that would benefit greatly from upgrading. At carbonisation temperatures between 500 and $800^{\circ} \mathrm{C}$ a char yield of around $25 \%$ wt is obtained. The char posseses a reasonable calorific value of $27.3 \mathrm{MJ} / \mathrm{kg}$, matching that of low rank coals. This char was also found to gasify well in $\mathrm{CO}_{2}$ at temperatures above $600^{\circ} \mathrm{C}$. Devolatilisation of the sugarcane waste is extensive, with about $70 \% \mathrm{wt}$, including half of the carbon and over nine-tenths of the hydrogen, being released by $350-400^{\circ} \mathrm{C}$. Sugarcane waste has a high hydrogen content, aound $6 \%$ by weight, which mostly belongs to the volatiles fraction. Therefore it may well be a good feedstock for hydrogen production from biomass. Low temperature carbonisation using combustion of the residual char as a source of energy and the carrier gas may be a better option for sugarcane waste than conventional high temperature gasification.

\section{ACKNOWLEDGMENT}

The University of Nottingham, Malaysia Campus, authors would like to thank Prof C.E. Snape for permission to use the analytical laboratories of the Fuel, Energy and $\mathrm{CO}_{2}$ Mitigation Research Group at the University Park Campus, UK, and all the staff in the research group for their help. Also, we would like to thank the Staff of the Environmental Technology Center, University of Nottingham, UK, especially Dr G. Busca, for the calorific value determinations.

\section{REFERENCES}

[1] International Energy Agency (IEA), 'Key World Energy Statistics', 2006, (http://www.iea.org).

[2] Kirby, A, 'Energy: Meeting soaring demand', BBC news website, 2004, (http://news.bbc.co.uk/2/hi/science/nature/3995135.stm). 
[3] Intergovernmental Panel on Climate Change (IPCC), 'Climate Change 2007 - The Physical Basis’, 2007 (http://ipcc-wg1.ucar.edu/wg1/wg1report.html).

[4] Stern, N, 'Stern Review on the Economics of Climate Change', 2007 (http://www.hm-

treasury.gov.uk/independent_reviews/stern_review_economics_climat e_change/sternreview_index.cfm).

[5] Stewart, K., Webster, P, 'Solution or stopgap?', tce, 795, 2007, p.40.

[6] Li, C-Z., 'Special Issue - Gasification: a Route to Clean Energy', Trans IchemE, Part B, 2006, 84(B6), p.407

[7] Demirbas, A., 'Yields of hydrogen-rich gaseous products via pyrolysis from selected biomass samples', Fuel, 80, 2001, p. 1885. 


\title{
Estimation of Solar Energy Potential in East Malaysia by Using Satellite Images
}

\author{
Ayu Wazira Azhari, Kamaruzzaman Sopian, Abdul Haqi Ibrahim
}

\begin{abstract}
Solar energy has been identified as one of the most potential alternative energy resources as Malaysia receives abundant sunshine all year long. Even though there are many solar instruments that can be used in measuring solar radiation intensity, the high cost has limited its usage to rich countries. As an alternative, a cheaper method in measuring solar intensity includes use of satellite images.

In this study, satellite images are used and compared with the actual readings from solar instruments. The results are then used to estimate solar intensity for other places where solar instrument is not available and finally a map that shows the solar radiation for East Malaysia is developed by using GIS application.

The findings show that on average, East Malaysia receive from $2.961 \mathrm{kWj} / \mathrm{m}^{2}$ to $6.157 \mathrm{kWj} / \mathrm{m}^{2}$ in a year. The minimum radiation received is about $0.612 \mathrm{kWj} / \mathrm{m}^{2}$ which occurs in December where else the maximum value is $6.838 \mathrm{kWj} / \mathrm{m}^{2}$ which falls in November. Mapping of solar radiation can indirectly provide us with information on potential areas for solar application and suitable for developing countries which cannot afford to have a complete solar instrument system.
\end{abstract}

Keywords: solar radiation modeling, solar radiation mapping, satellite images, renewable energy, solar energy

\section{INTRODUCTION}

Solar energy has been identified as one of the most potential energy resources in Malaysia besides the usage of hydropower and other renewable energy resources. Although there are many solar instruments that can be used in measuring solar radiation intensity, most developing and under develop countries are unable to fully utilized these instrument as the cost is very high.

As an alternative, a cheaper method in measuring solar intensity has been identified by means of certain parameters such as sunshine duration [1], [2] the use of meteorological parameters [3] and many more. In addition, the use of

This work was supported in part by Universiti Malaysia Perlis (UniMAP) and Universiti Kebangsaan Malaysia (UKM).

Ayu Wazira Azhari is with the School of Environmental Engineering, Universiti Malaysia Perlis, Kompleks Pusat Pengajian Jejawi 3, 02600, Arau Perlis, Malaysia. (Phone: +6 049796993 ; fax: +6 979 8636; e-mail: ayuwazira@unimap.edu.my).

Kamaruzzaman Sopian is the Director of Solar Energy Research Institutes, Universiti Kebangsaan Malaysia, 43600 Bangi Selangor, Malaysia. (e-mail: ksopian@vlsi.eng.ukm.my).

Abdul Haqi Ibrahim is with the School of Environmental Engineering, Universiti Malaysia Perlis, Kompleks Pusat Pengajian Jejawi 3, 02600, Arau Perlis, Malaysia. (e-mail: abdulhaqi@unimap.edu.my). mathematical modeling and satellite images is also applicable and can be use to get information on solar radiation intensity for many places [4] - [6].

Solar radiation mapping is very important in solar energy application as it can provide information on potential area suitable for placement of solar power station [6]. There have been a lot of studies that use meteorological satellite data for estimation of solar radiation. Different methodologies developed for this purpose used different types of images from satellites.

In 1996, Islam and Exell have developed a low cost system called RADMAP that uses low resolution APT images transmitted from NOAA polar orbiting satellites. This system can be use to estimates solar radiation at low cost but it requires information on cloud cover and cloudy sky albedo to obtain the effective cloudiness of the sky [4]. As an alternative, this study will only use information from satellite images and data collected from several ground measuring station of solar radiation available in Malaysia. The cloud cover from satellite images are calculated and compared with the actual readings from ground measuring station. The results are then used to estimate solar intensity for other places where solar instruments are not available and finally maps that shows the solar radiation for East Malaysia is developed by using GIS application.

\section{RESEARCH METHODOLOGY}

In this research, MTSAT-1R geostationary satellite images are use covering the whole of East Malaysia. This grey scale satellite images are taken hourly from 0700 until 1900 for the period of one year starting from January until December 2006. Solar radiation data are collected from several grounds measuring station of solar radiation available at Meteorological Department.

In determining the cloud cover, the satellite images use are divided into smaller target areas of size $50 \times 50 \mathrm{~km}$. In this case, there are possibilities of having more than one type of cloud cover in a target area. As a solution, a pseudo cloud is form in replace of the original cloud by taking into consideration the thickness and area of the cloud in a target area.

The thickness of clouds are determine by dividing it into five cloud types ranging from very low to very high cloud cover. This can be identified by studying the different color scale where dark color indicates low cloud cover and bright indicates high cloud cover. A grey scale from dark to bright is used to help in measuring the thickness of cloud cover. In 
addition, the area of cloud cover is also taken into consideration.

When $T$ is the thickness of cloud cover, the fraction of cloud cover in a target area $C$ can be calculated as in (1) where $a$ is the area of cloud cover in a target area.

$C=\frac{\sum T \times a}{4} \times 100$

By taking the average readings from the two ground measuring station, comparisons are made with the percentage of cloud cover calculated from the satellite images. The solar intensity for every hour is needed as percentage of cloud cover from every hour will give different solar intensity. The solar intensity for time $t$ can be written as in (2) where $I_{t}$ is the solar intensity at $t$ hour, $i_{n 90}$ is the average $90 \%$ solar intensity from the ground measuring station and $C_{t}$ is the cloud cover for hour $t$.

$I_{t}=i_{n 90}-\left(\frac{i_{n 90}}{100} \times C_{t}\right)$

Equation (3) shows the daily solar radiation $I_{\text {total }}$ where $t$ is taken from 0700 until 1900.

$I_{\text {total }}=\sum_{t_{0}}^{t_{n}} I_{t}$

The monthly average daily global solar radiation $I$ can be calculated by taking the average of daily solar radiation for each month and can be written as in (4) where $n$ is either 28 , 29,30 or 31 days depending on the month.

$I=\frac{\sum_{1}^{n} I_{\text {total }}}{n}$

\section{RESULTS AND DISCUSSION}

Mapping of solar radiation gives the best initial impression on solar intensity of East Malaysia for every different month including the average solar radiations receive in a year. This is because, in East Malaysia, there are only four solar radiation station available which are Kuching and Miri for Sarawak and Kota Kinabalu and Tawau for Sabah. This will cause difficulties and inaccurate estimation for other places as the area is very big. Estimation of solar radiation by using satellite images will give better and more accurate readings because it covers the whole of East Malaysia and information on specific division is more accurate.

Figure 1 shows the yearly average daily solar radiation for East Malaysia. As expected, the yearly average daily solar radiation map shows that there is no significant different solar radiation intensity for both Sabah and Sarawak. It is estimated that on average East Malaysia receives about $5.026 \mathrm{kWh} / \mathrm{m}^{2}$ of solar radiation in a year. The average maximum solar radiation receives is $5.555 \mathrm{kWh} / \mathrm{m}^{2}$, while the lowest average solar radiation receives is $4.518 \mathrm{kWh} / \mathrm{m}^{2}$. Some places like the West Coast Division and Interior Division of Sabah receive higher solar radiation compared to Kudat and Tawau while in Sarawak, Kapit, Sarikei,
Betong, Sri Aman and part of Miri received higher solar radiation compared to others.

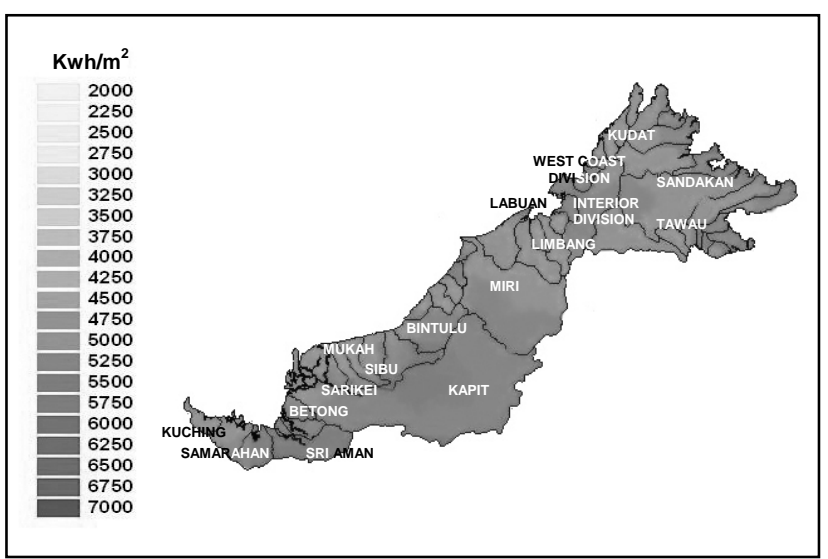

Figure 1 Yearly average daily solar radiation For East Malaysia

On the other hand, by looking closely at the monthly average solar radiation for each month, we can see that there are significant different in solar radiation intensity for each month. Figure 2 to 13 shows the monthly average daily solar radiations for East Malaysia for the month of January until December. In January, the amount of solar radiation receive are on average ranging from $3.007 \mathrm{kWh} / \mathrm{m}^{2}$ to $5.544 \mathrm{kWh} / \mathrm{m}^{2}$. Place like the West Coast Division and Interior division of Sabah and Miri and Limbang in Sarawak received minimum solar radiation.

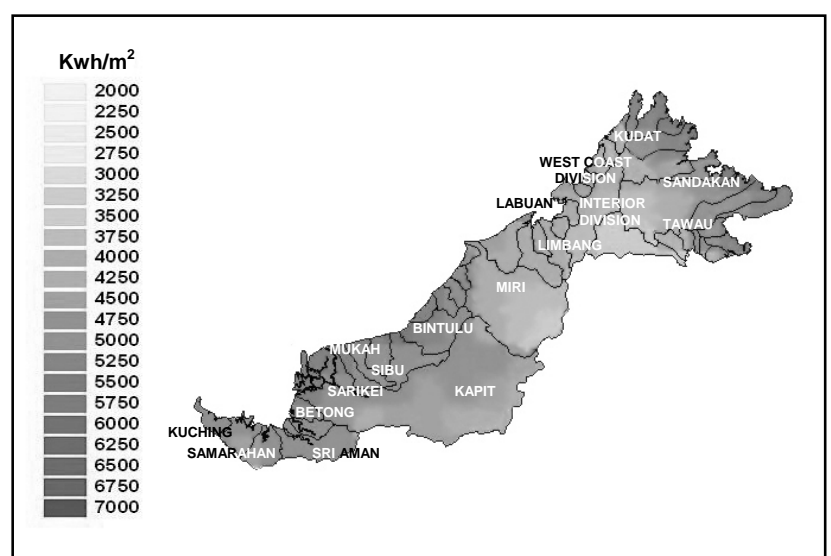

Figure 2. Monthly average daily solar radiations for East Malaysia in the month of January

As for the month of February, most places receive average of $6.165 \mathrm{kWh} / \mathrm{m}^{2}$ of solar radiation daily with the maximum of $6.621 \mathrm{kWh} / \mathrm{m}^{2}$ and minimum solar radiation of $4.448 \mathrm{kWh} / \mathrm{m}^{2}$. On the other hand, in March, the amount of solar radiation receive in a day is lower with the average of $4.955 \mathrm{kWh} / \mathrm{m}^{2}$. Only a few places such as Labuan, East Sandakan and Mukah receive higher solar radiation up to $6.041 \mathrm{kWh} / \mathrm{m}^{2}$. Most places in Sabah received higher solar radiation of $5.109 \mathrm{kWh} / \mathrm{m}^{2}$ compared to Sarawak. Mukah and Kuching in Sarawak receive the lowest solar radiation of $3.070 \mathrm{kWh} / \mathrm{m}^{2}$ compared to average of $4.088 \mathrm{kWh} / \mathrm{m}^{2}$. 


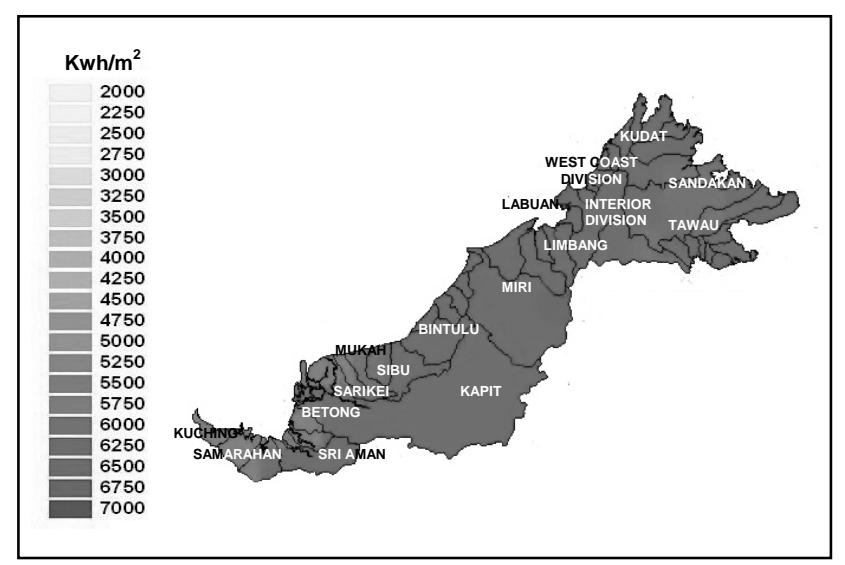

Figure 3. Monthly average daily solar radiations for East Malaysia in the month of February

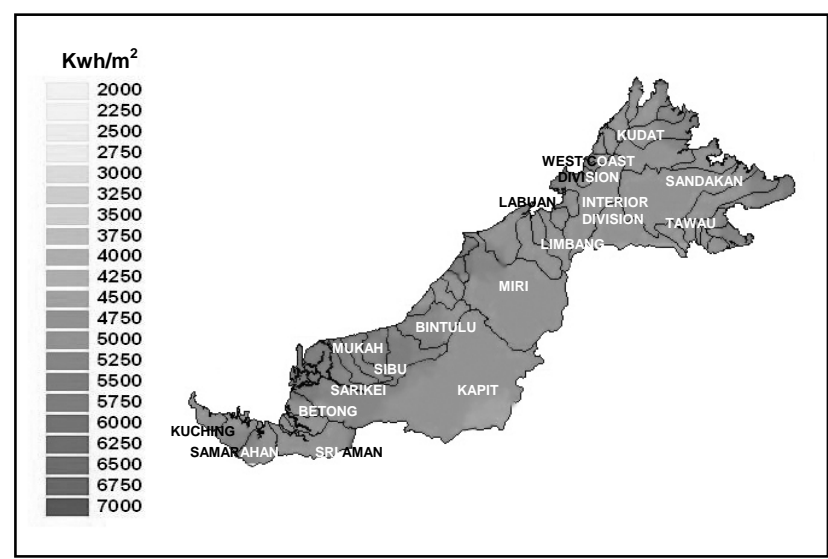

Figure 4. Monthly average daily solar radiations for East Malaysia in the month of March

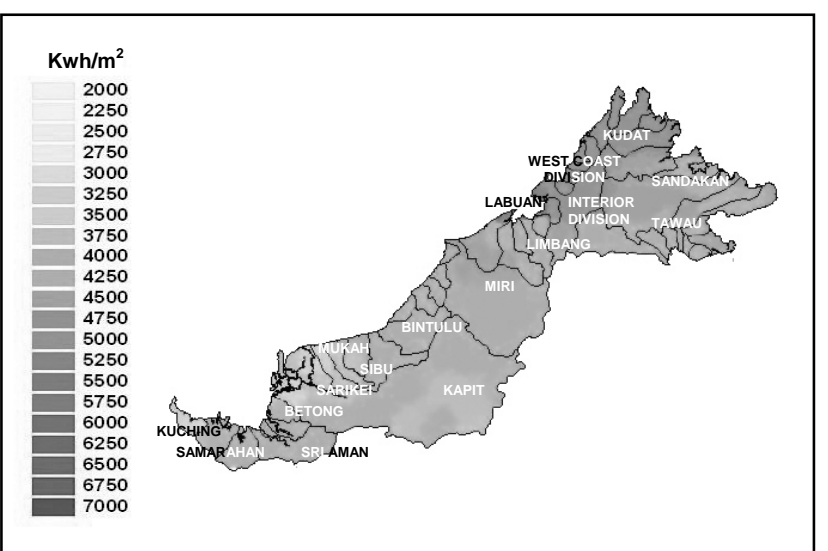

Figure 5. Monthly average daily solar radiations for East Malaysia in the month of April

Solar radiation receive in May is almost the same as in February, but the average solar radiation receive is lower which is $5.053 \mathrm{kWh} / \mathrm{m}^{2}$. Sandakan and Kapit receive the highest solar radiation of $6.602 \mathrm{kWh} / \mathrm{m}^{2}$ while Mukah and Kuching receive the lowest of $4.309 \mathrm{kWh} / \mathrm{m}^{2}$. On the contrary, most division of Sarawak which is Kuching up to Bintulu receives very high solar radiation in June which is $6.707 \mathrm{kWh} / \mathrm{m}^{2}$. On the other hand, Kudat received the lowest solar radiation of $2.975 \mathrm{kWh} / \mathrm{m}^{2}$. Other divisions receive average of $5.084 \mathrm{kWh} / \mathrm{m}^{2}$ of solar radiation daily. There are no significant different in the amount of solar radiation receive for the month of July where the average reading is estimated to be at $5.283 \mathrm{kWh} / \mathrm{m}^{2}$.

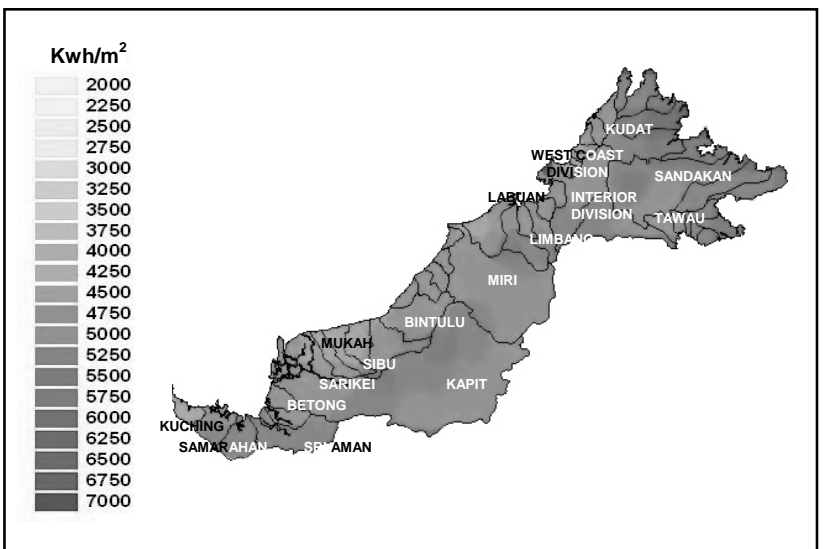

Figure 6. Monthly average daily solar radiations for East Malaysia in the month of May

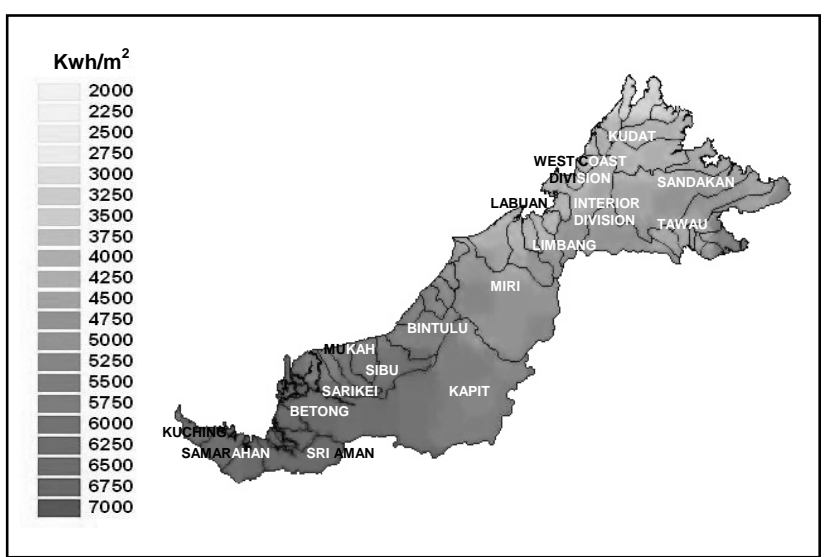

Figure 7. Monthly average daily solar radiations for East Malaysia in the month of June

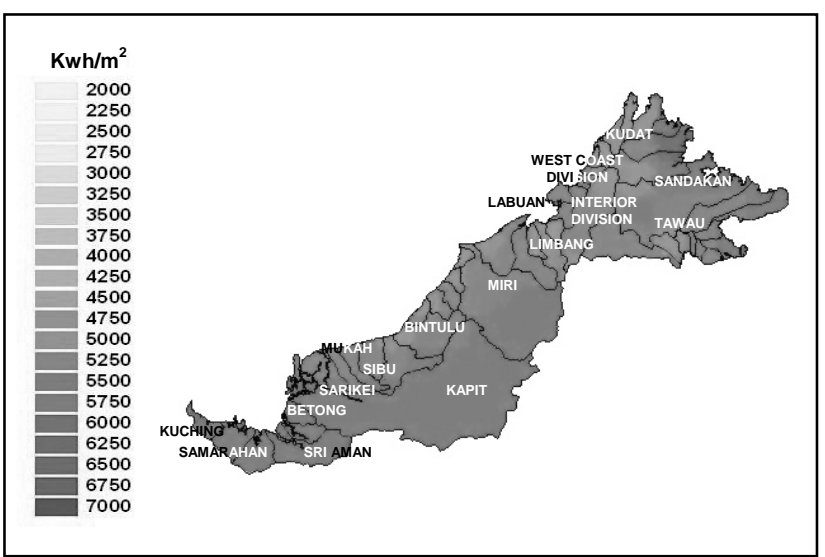

Figure 8. Monthly average daily solar radiations for East Malaysia in the month of July

In August, a few places like Kuching, Samarahan, Sri Aman, Tawau and part of Sandakan receive high solar radiation of $6.809 \mathrm{kWh} / \mathrm{m}^{2}$ while Kudat and Betong receive very low solar radiation of $2.902 \mathrm{kWh} / \mathrm{m}^{2}$. On average, other places receive about $5.798 \mathrm{kWh} / \mathrm{m}^{2}$ per day. Solar radiations receive in September and October show almost the same patent where Kapit receive the highest solar radiation compared to other places. As for in September, the solar radiation receive ranging from $3.953 \mathrm{kWh} / \mathrm{m}^{2}$ to $5.530 \mathrm{kWh} / \mathrm{m}^{2}$ with average of $4.991 \mathrm{kWh} / \mathrm{m}^{2}$ while in 
October, the amount of solar radiation receive daily ranging from $4.678 \mathrm{kWh} / \mathrm{m}^{2}$ to $6.428 \mathrm{kWh} / \mathrm{m}^{2}$ with average of 5.462 $\mathrm{kWh} / \mathrm{m}^{2}$.

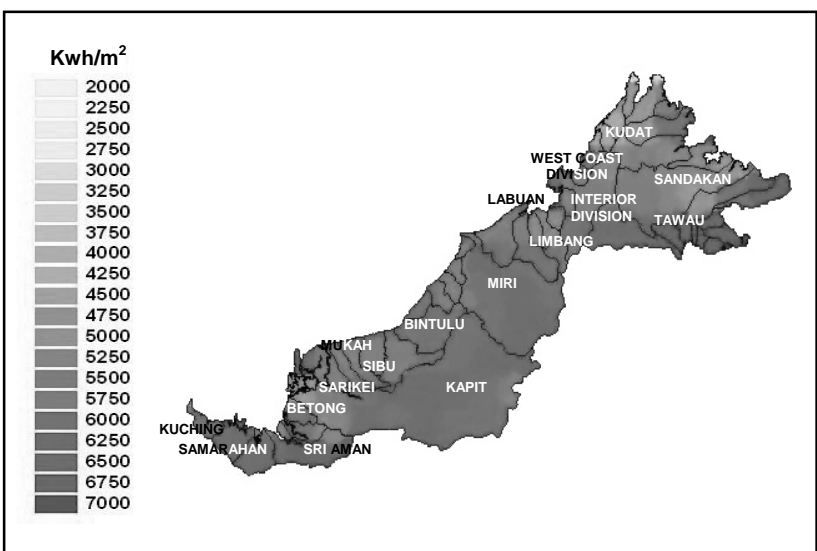

Figure 9. Monthly average daily solar radiations for East Malaysia in the month of August

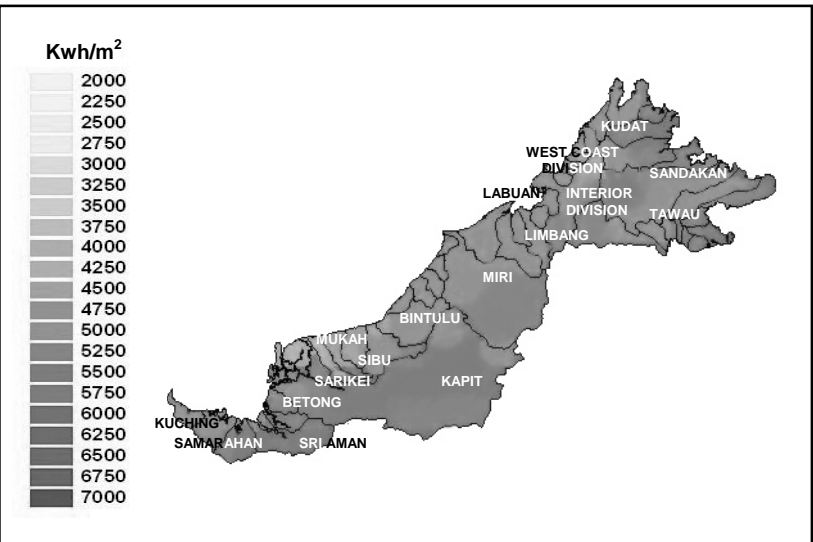

Figure 10. Monthly average daily solar radiations for East Malaysia in the month of September

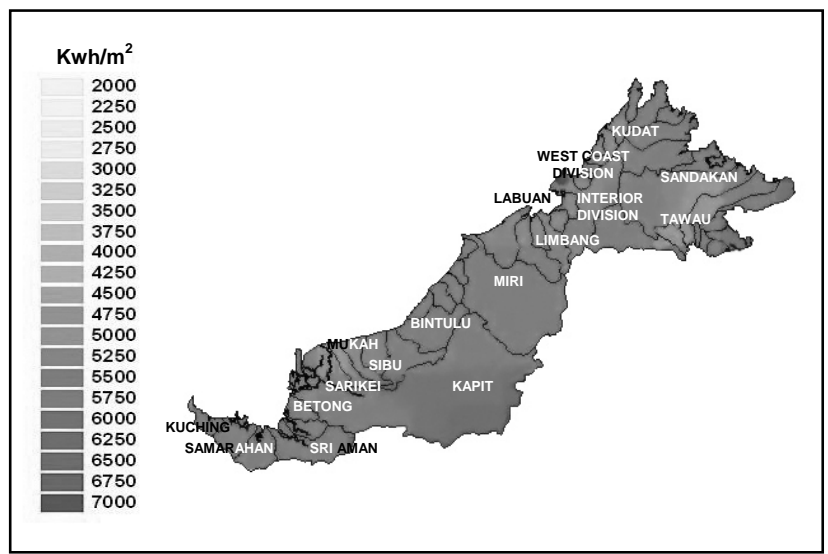

Figure 11. Monthly average daily solar radiations for East Malaysia in the month of October

There is a significant difference in solar radiation receive in November in comparison to solar radiation receive in December. In November, average of $5.985 \mathrm{kWh} / \mathrm{m}^{2}$ solar radiation receive with Labuan, Interior Division and Sandakan receive the highest solar radiation of 6.838 $\mathrm{kWh} / \mathrm{m}^{2}$. On the other hand, in December, the average solar radiation receive is only $2.951 \mathrm{kWh} / \mathrm{m}^{2}$ with the lowest of $0.612 \mathrm{kWh} / \mathrm{m}^{2}$.

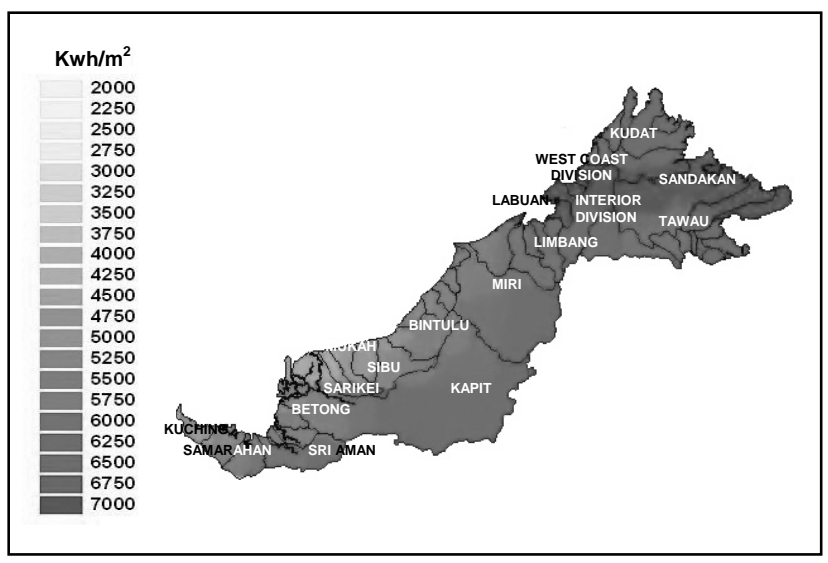

Figure 12 . Monthly average daily solar radiations for East Malaysia in the month of November

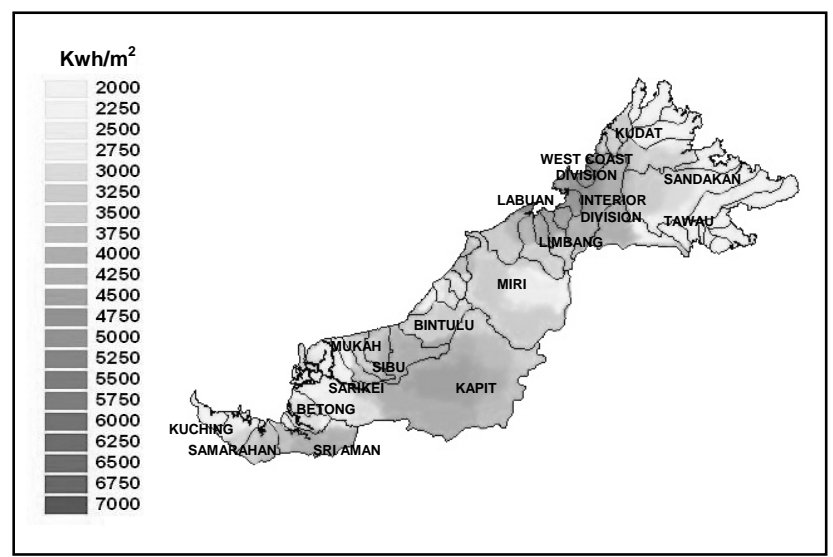

Figure 13. Monthly average daily solar radiations for East Malaysia in the month of December

Previous studies on Peninsular Malaysia shows that there seems to be an increasement in solar radiation recorded from 1982 [7] to 1992 [8] and the recent study in 2006. The average minimum solar radiation is increasing from 3.07 $\mathrm{kWh} / \mathrm{m}^{2}$ in 1982 to $3.373 \mathrm{kWh} / \mathrm{m}^{2}$ in 1992 and finally in 2006 the average minimum solar radiation for Malaysia is estimated to be $4.518 \mathrm{kWh} / \mathrm{m}^{2}$. The increase in average minimum solar radiation indicates that the solar radiation for most places is increasing. This might be an effect from the incensement of pollution rate that leads to global warming. It is also supported by the increase in average solar radiation from $4.800 \mathrm{kWh} / \mathrm{m}^{2}$ in 1982 to $5.024 \mathrm{kWh} / \mathrm{m}^{2}$ in 2006 . The average maximum solar radiation also shows a slight increase from $5.47 \mathrm{kWh} / \mathrm{m}^{2}$ in 1982 to $5.572 \mathrm{kWh} / \mathrm{m}^{2}$ in 1992.

\section{CONCLUSION}

Estimation of solar radiation by using satellite images can give results at acceptable accuracy and lower cost. This method is applicable especially for developed and under developed countries in recognizing the potential of solar energy application. On average, East Malaysia receives from $4.518 \mathrm{kWh} / \mathrm{m}^{2}$ to $5.555 \mathrm{kWh} / \mathrm{m}^{2}$ of solar radiation a year. The highest solar radiation is estimated at 6.838 $\mathrm{kWh} / \mathrm{m}^{2}$ in November while the lowest is $0.612 \mathrm{kWh} / \mathrm{m}^{2}$ in December. Kapit, Samarahan, Sri Aman, Sibu, Limbang, West Coast Division, Interior Division and Labuan have the 
highest potential for solar energy application due to its high solar radiation throughout the year.

\section{ACKNOWLEDGMENT}

A million thanks to the Ministry of Higher Education and Universiti Malaysia Perlis who had sponsor my Master studies. Most thank you to National University of Malaysia for providing me with the equipment needed for my research. Not to forget the Malaysia Meteorological Department who had provide me with the data for my research.

\section{REFERENCES}

[1] Tadros, M. T. Y. 2000. Uses of sunshine duration to estimate the global solar radiation over eight meteorological stations in Egypt. Renewable Energy 21(2000): 231 - 246.

[2] Nguyen, B. T. \& Pryor, T. L. 1996. A computer model to estimate solar radiation in Vietnam. WREC 1996:1274 - 1278.

[3] Trabea, A. A. \& Mosalam Shaltout, M. A. 2000. Correlation of global solar radiation with meteorological parameters over Egypt. Renewable Energy 21(2000): 297 - 308.

[4] Islam, MD. Rafiqul \& Exell, R. H. B. 1996. Solar radiation mapping from satellite image using a low cost system. Solar Energy 56(3): 225-237.

[5] Malik, A. Q., Mufti, A., Hiser, H. W. \& Veziroglu, N. T. 1998. Solar mapping of Pakistan using visible images from geostationary satellites. Renewable Energy 13(1): 1-16.

[6] Janjai, S., Laksanaboonsong, J., Nunez, M. \& Thongsathitya, A. 2005. Development of a method for generating operational solar radiation maps from satellite data for a tropical environment. Solar Energy 78: 739-751.

[7] Chuah, D. G. S. \& Lee, S. L. 1984. Solar radiation in Malaysia. Singapura: Oxford University Press.

[8] Kamaruzzaman Sopian \& Mohd Yusof Hj. Othman. 1992. Estimates of monthly average daily global solar radiation in Malaysia. Renewable Energy 2(3): 319 - 325. 


\title{
Mesoporous Solid Base Catalyst for the Heterogeneous Transesterification of Palm Oil to Biodiesel
}

\author{
A. Z. Abdullah*, N. Razali, K. T. Lee, B. Salamatinia and H. Mootabadi
}

\begin{abstract}
Mesoporous silica supports, SBA-15 was successfully synthesized with the template of nonionic triblock copolymers (TCP) and tetraethylorthosilicate (TEOS) as silica source using one pot synthesis method at a temperature of 40 ${ }^{\circ} \mathrm{C}$ and a reaction time of $3 \mathrm{~h}$. Mesoporous catalyst of different sodium hydroxide $(\mathrm{NaOH})$ loadings $(5-10 \%)$ supported on mesoporous silica were prepared by impregnation technique. The activity of the sodium impregnated mesoporous material in the transesterification process of palm oil for biodiesel production was investigated. The transesterification process was carried out in batch mode using two necked round bottom flask, $250 \mathrm{ml}$ of reactant at various methanol to oil ratios at $\mathbf{7 0}$ ${ }^{\circ} \mathrm{C}$, and reaction time of up to $4.5 \mathrm{~h}$ and $1 \mathrm{~g}$ of the catalyst amount. Results showed that the highest conversion of palm oil to biodiesel was achieved using 10 wt \% of sodium loading on mesoporous silica. A conversion of aver $70 \%$ was achieved. The catalyst also showed high stability in this reaction with minimal changes to its surface characteristics. Therefore, mesoporous silica catalyst was proven to be potential novel catalyst for heterogeneous biodiesel production.
\end{abstract}

Keywords: Mesoporous, base catalyst, heterogeneous, SBA-15, transesterification, biodiesel

\section{INTRODUCTION}

There is increasing worldwide concern for environmental protection and for the conservation of non-renewable natural resources. Fatty acid methyl esters (FAME) show great potential as diesel substitutes and they are known to be sources of biodiesel [1]. Biodiesel is synthesized from direct transesterification of vegetable oils where the corresponding triglycerides react with a short-chain alcohol in the presence of a catalyst [2]-[5].

Transesterification of vegetable oils to biodiesel with methanol can be carried out using both homogeneous (acid or base) and heterogeneous (acid or base) catalysts. Homogeneous base catalysts provide much faster reaction rates than heterogeneous catalysts in transesterification [3]. However, large amount of water is required to transfer the catalysts from the organic phase to a water phase. Therefore, it is considerable costly to separate homogeneous catalysts [4]. Heterogeneous is an economically and

A. Z. Abdullah, N. Razali, K. T. Lee, B. Salamatinia and H. Mootabadi are with School of Chemical Engineering, Engineering Campus, Universiti Sains Malaysia, 14300 Nibong Tebal, Penang, Malaysia (Tel: +604-599 6411, fax: +604-594 1013, e-mail: chzuhairi@eng.usm.my). ecologically important field in catalysis research because these catalysts have many advantages: they are noncorrosive, environmentally benign and present fewer disposal problems. They are also much easier to separate from liquid products and they can be designed to give higher activity, selectivity and longer catalyst lifetimes [4]. Many types of heterogeneous catalysts such as alkaline earth metal oxides, various alkaline metal compounds supported on alumina, silica or zeolites can catalyze the transesterification reactions.

Conventional catalyst supports for the transesterification reaction usually have broad pore size distribution, low surface area and low thermal stability [6]. Micropores present in those materials usually limit the diffusion of large molecule reactants to cause lower reaction rate [2]. Recently developed ordered mesoporous silica namely MCM-41, SBA-15 and meso-cellular foam (MCF) have large surface area $\left(500-1200 \mathrm{~m}^{2} / \mathrm{g}\right)$, uniform sized pores and thermal stability up to $823 \mathrm{~K}$ and would be interesting solids to act as catalyst supports [6]. In this study, mesoporous SBA-15 was used to be impregnated with $\mathrm{NaOH}$ as the catalyst for transesterification of palm oil with methanol to produce FAME. The characteristics of the catalysts were elucidated and correlated to their observed behavior in the transesterification reaction.

\section{EXPERIMENTAL}

Pluronic p123 triblock copolymer was used as the surfactant. The molar composition of the synthesis gel was 1 $\mathrm{SiO}_{2}$ :0.017 P123:2.9 HCl:202.6 $\mathrm{H}_{2} \mathrm{O}$. After mixing, the gel was heated at $40{ }^{\circ} \mathrm{C}$ for $24 \mathrm{~h}$. the white precipitate was then filtered, washed with distilled water and oven dried. Then, the dry precipitate was calcined at $500{ }^{\circ} \mathrm{C}$ for $6 \mathrm{~h}$. $\mathrm{NaOH} / \mathrm{SBA}-15$ catalysts (5-15 wt. \%) were prepared using an impregnation method and vacuum dried. The catalysts formed were characterized using SEM, TEM and surface analysis.

The transesterification of palm oil with methanol was conducted in a two-necked flask with a reflux condenser and a magnetic stirrer. $50 \mathrm{ml}$ of palm oil was used and the methanol:oil ratio was $6: 1$. After the reaction, the catalyst was separated by centrifugation and two liquid phases appeared: FAME at the upper layer and glycerol in the bottom layer. After separating, the biodiesel was washed with distilled water to remove contaminate. The analysis of 
the transesterification products was carried out using a Clarus 500 GC equipped with Innowax capillary column and a flame ionizing detector (FID).

\section{RESULTS AND DISCUSSION}

The SBA-15 mesoporous material synthesized showed macroporous structure with irregular form of silica pellets as observed under SEM ('figure 1(a)'). The characteristics were desirable as far as the catalyst is concerned as it introduced open structure of the silica mass to facilitate diffusion. By observing under TEM, it was noted that the silica pellets were actually made of an array of straight channels of meso size range. Thus, it was concluded that the mesoporous SBA-15 was successfully synthesized.

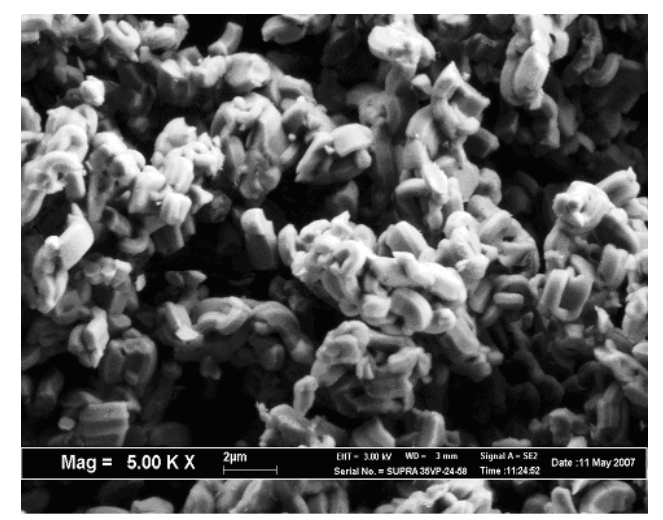

(a)

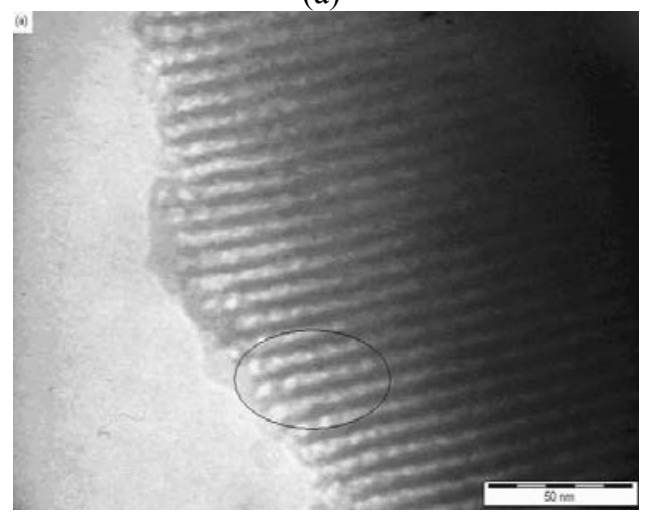

(b)

Figure 1. Microstructure of the SBA-15 synthesized as seen under a) SEM and, b) TEM
The surface and pore characteristics of the catalysts are summarized in 'table 1'. The BET surface area was 455 $\mathrm{m}^{2} / \mathrm{g}$ and upon increasing $\mathrm{NaOH}$ impregnation, the surface area decreased. The average pore diameters were in meso size range with size also showed a decreasing trend with higher $\mathrm{NaOH}$ loading.

Table 1. Surface characteristics of the $\mathrm{NaOH} / \mathrm{SBA}-15$ catalysts at various loadings.

\begin{tabular}{lcc}
\hline \multicolumn{1}{c}{ Catalyst } & $\begin{array}{c}\mathrm{S}_{\mathrm{BET}} \\
\left(\mathrm{m}^{2} / \mathrm{g}\right)\end{array}$ & $\begin{array}{c}\mathrm{d}_{\text {pore }} \\
(\mathrm{nm})\end{array}$ \\
\hline SBA-15 & 455 & 5.9 \\
$5 \mathrm{wt} \% \mathrm{NaOH} / \mathrm{SBA}-15$ & 418 & 5.2 \\
$10 \mathrm{wt} \% \mathrm{NaOH} / \mathrm{SBA}-15$ & 390 & 4.7 \\
$15 \mathrm{wt} \% \mathrm{NaOH} / \mathrm{SBA}-15$ & 210 & 3.1 \\
\hline
\end{tabular}

An experiment was conducted to determine the effect of reaction time on the transesterification reaction. In this study, $0.9 \mathrm{~g}$ of 5 wt. $\% \mathrm{NaOH}$ was used with the molar ratio of oil to methanol of 1:6. The reaction temperature was kept constant at $70{ }^{\circ} \mathrm{C}$ and the reaction time was tested up to 4.5 $\mathrm{h}$. Based on the data in 'table 2', the conversion was found to increase with reaction time. As the system was a heterogeneous system, the mass transfer was slow to cause low conversion at shorter reaction time. The conversion in the first $1.5 \mathrm{~h}$ was only $2.4 \%$ and increased to $12.7 \%$ at 4.5 h. The reactants need longer time to diffuse into the SBA-15 mesoporous channels that hosted active base sites to catalyze the reaction. It should be highlighted that longer reaction time resulted in the appearance of a white gel in the product causing the increase in its viscosity. This would certainly affect the product separation.

One of the most important variables affecting the yield of ester is the molar ratio of alcohol to triglyceride. The stoichiometric ratio for the transesterification requires three moles of alcohol and one mole of glyceride to yield three moles of fatty acid methyl ester and one mole of glycerol. The biodiesel yield could be increased by introducing an excess amount of methanol to shift the equilibrium to the forward reaction [1]. The molar ratio is associated with the type of catalyst used. An alkali-catalyzed reaction generally requires only a 6:1 ratio to achieve good ester yield for a given reaction time [2].

Table 2. Summary of the experimental conditions and conversion obtained

\begin{tabular}{ccccc}
\hline $\mathrm{NaOH}$ wt. $\%$ & Oil:MeOH & $\mathrm{T}\left({ }^{\circ} \mathrm{C}\right)$ & $\mathrm{t}(\mathrm{h})$ & Conversion $(\%)$ \\
\hline 5 & $1: 6$ & 70 & 1.5 & 2.4 \\
5 & $1: 6$ & 70 & 2.5 & 8.3 \\
5 & $1: 6$ & 70 & 4.5 & 12.7 \\
5 & $1: 6$ & 70 & 2.5 & 13.6 \\
5 & $1: 9$ & 70 & 2.5 & 14.5 \\
5 & $1: 12$ & 70 & 2.5 & 17.6 \\
5 & $1: 6$ & 70 & 2.5 & 8.7 \\
10 & $1: 6$ & 70 & 2.5 & 70.7 \\
15 & $1: 6$ & 70 & 2.5 & 40.6 \\
\hline
\end{tabular}

An experiment was conducted to study the effect of the molar ratio on the transesterification. $0.9 \mathrm{~g}$ of $5 \mathrm{wt}$. \% was used to catalyze the reaction. The molar ratio of oil to methanol was varied from 1:6, 1:9 and 1:12. The reaction temperature was kept constant at $70{ }^{\circ} \mathrm{C}$ and the reaction was continued for $2.5 \mathrm{~h}$. The results obtained are also given in 'table 2'. As noted, the conversion increased with increasing methanol ratio. As the transesterification is a reversible reaction, more methanol was required to shift the equilibrium to the forward reaction. When mass transfer was limited due to problems of mixing, it seemed to be significantly slower than the reaction rate. Therefore, the 
production yield could be increased by introducing an excess amount of this reactant to the favor of the forward reaction. However, when the amount of methanol was over $15: 1$, it was not favorable for the purification of biodiesel. Glycerol separation became more difficult thus decreasing the biodiesel yield [1]. In addition, more energy was needed to recover large amount of unreacted methanol. Moreover, methanol could increase the dissolution of palm oil, intermediates and biodiesel resulting in the wastage of the materials. Based on the result, the optimum molar ratio was $12: 1$.

The effect of catalyst loading was studied using the molar ratio of oil to methanol at 1:6, a reaction temperature of 70 ${ }^{\circ} \mathrm{C}$. The reaction was carried out with $\mathrm{NaOH} / \mathrm{SBA}-15$ at 5 $\%, 10 \%$ and $15 \%$ based on the oil weight for $2.5 \mathrm{~h}$.

Based on the result obtained, the catalyst loading was found to significantly affect the reaction. The conversion increased from $8.7 \%$ to $70.7 \%$ as the loading was increased from 5 $\%$ to $10 \%$. Undoubtedly, the activity of the $\mathrm{NaOH} / \mathrm{SBA}-15$ was dependent not only the strength of basic sites but also upon their amount. The increasing catalyst loading provided more active sites to catalyze the reaction. However, increasing catalyst loading also caused the slurry became too viscous giving rise to the problem of mixing. At low loading of $\mathrm{NaOH}$, the active basic sites were more dispersed on the SBA-15 surface and strong adsorption of reactant might occur at unreactive sites. However, if the SBA-15 was loaded with too much of $\mathrm{NaOH}$, the dispersion of the base could be very poor leading to lower catalytic activity. Thus, some of the basic sites were inaccessible to the reactants. This was consistent with a report by Xie et al. [5].

\section{CONCLUSIONS}

Mesoporous silica supports, SBA-15 was successfully synthesized with the template of nonionic triblock copolymers (TCP) and tetraethylorthosilicate (TEOS) act silica source using one pot synthesis method at a temperature of $40{ }^{\circ} \mathrm{C}$ and a reaction time of $3 \mathrm{~h}$. Mesoporous catalyst of different sodium (Na) loadings (5-10\%) supported on mesoporous silica were successfully prepared by impregnation technique. The activity of the sodium impregnated mesoporous material in the transesterification process of palm oil for biodiesel production was investigated. Results showed that the highest conversion of palm oil to biodiesel was achieved using $10 \mathrm{wt} \%$ of sodiumhydroxyde loading on mesoporous silica. A conversion of over $70 \%$ was achieved. The catalyst also showed high stability in this reaction with minimal changes to its surface characteristics. Therefore, mesoporous silica catalyst was proven to be potential novel catalyst for heterogeneous biodiesel production.

\section{ACKNOWLEDGEMENT}

The financial support from the Ministry of Higher Education of Malaysia in the form of FRGS grant (6027007) is gratefully acknowledged.

\section{REFERENCES}

[1] X. Liu, H. He, Y. Wang, S. Zhu, "Transesterification of soybean oil to biodiesel using $\mathrm{SrO}$ as a solid base catalyst", Catalysis Communications, 8, 2007, pp. 1107-1111.
[2] [2] F. Satoshi, H. matsuhashi, K. Arata, "Biodiesel fuel production with solid amorphous-zirconia catalysis in fixed bed reactor", Biomass and Bioenergy, 30, 2006, pp. 870-873.

[3] [3] H.J. Kim, B.S. Kang, M.J. Kim, Y.M. Park, D.K. Kim, J.S. Lee, K.Y. Lee, "Transesterification of vegetable oil to biodiesel using heterogeneous base catalyst", Catalysis Today, 93-95, 2004, pp. 315320 .

[4] [4] J.M. Marchetti, V.U. Miguel, A.F. Errazu, "Possible methods for biodiesel production", Renewable and Sustainable Energy Reviews, 11, 2007, pp. 1300-1311.

[5] [5] W. Xie, H. Peng, L. Chen, "transesterification of soybean oil catalyzed by potassium loaded on alumina as a solid-base catalyst", Applied Catalysis A: General, 300, 2006, pp. 67-74.

[6] [6] P.H. Pandya, R.V. Jasra, B.l. Newalkar, P.N. Bhatt, "Studies on the activity and stability of immobilized $\alpha$-amylase in ordered mesoporous silicas", Microporous and Mesoporous Materials, 77, 2005, pp. 67-77. 


\title{
The Rheology Properties of Sabah Bentonite at High Temperature Environment
}

\author{
Sonny Irawan, Ismail B Mohd Saaid, Norrulhuda Bt Hj Mohd Taib and Sultan Mohammed Al- \\ Rawhani
}

\begin{abstract}
Drilling fluid property changes due to elevated temperature and aging time frequently cause problems in drilling deep wells. This study was designed to investigate the effect of high temperature on the rheological properties of some important Malaysian Sabah bentonite (especially from Andrassy area) and their activation potential for use in deep well drilling fluid. The experiment method includes three different equipments, namely Roller Oven, to roll the mud and to allow the chemical reaction taking place in the mud, and Dynamic Filtration loss, to measure the filtrate of properties of drilling fluid at high temperature and dynamic condition, and Fann Viscometer, to measure the rheological properties of drilling fluids. Drilling fluid viscosities and filtration in a programmed temperature range of $25-250{ }^{\circ} \mathrm{C}$ (at constant reservoir pressure and a shear rate of $3448.2 \mathrm{kPa}$ and $170 \mathrm{~s}^{-1}$ ) were measured. Mud formulation consists of Sabah bentonite, Hydro-gell bentonite, carboxyl metyl cellulose (CMC), Tannathin, Ben-Ex and SP-101 in fresh water. Laboratory observation showed that The effective viscosity, plastic viscosity, yield point and gel strength (10 $\mathrm{sec}$ and $10 \mathrm{~min}$ ) of Sabah bentonite decrease with the increase in the temperature. Furthermore, compensatory materials used to counteract adverse reaction of the thermal decay of various materials, along which new product which enhanced fluid thermal stability are recommended.
\end{abstract}

Keywords: bentonite, drilling fluid, rheology, additives, high temperature

\section{INTRODUCTION}

Successful deep drilling and hot wells critically depends on the rheological properties of the drilling fluids design for specific down hole conditions. Rheological properties of water-base drilling fluids under down hole condition may be very different from those measured at the surface conditions, because elevated temperature affects the drilling fluids properties [1]-[5]. An increase in temperature reduces the effectiveness of most drilling fluids additives that would maintain the rheological, fluid loss and electro-chemical properties. Problems of elevated temperature are accelerated when high chemical contaminants, such as salt of sodium, calcium and magnesium are encountered [6]. Elevated

Sonny Irawan, Ismail B Mohd Saaid, Norrulhuda Bt Hj Mohd Taib and Sultan Mohammed Al-Rawhani are now with Department of Geoscience and Petroleum Engineering, Universiti Teknologi PETRONAS, Bandar Seri Iskandar, 31750 Tronoh, Perak Darul Ridzuan, Malaysia, : +605-368 7085, Fax:+605 3655670 (email:drsonny_irawan@petronas.com.my). temperature itself is considered as once of the drilling fluid contaminant which can not be treated with any additives [8]. Drilling fluids in the lower portion of the become excessively thick when it is not circulated. Prolonged heating may cause solidification of drilling fluids. In that case of stuck pipe, circulation may continue for long time at elevated temperature and thus aging comes under consideration, aging effects the rheology of drilling fluid a long with temperature and investigation shows that the effect of dynamic aging is greater than static aging. This study was designed to investigate the effect of high temperature on the rheological properties of some important Malaysian Sabah bentonite (especially from Andrassy area) and their activation potential for use in deep well drilling fluid. The bentonite study was formed by hydrothermal alteration of volcanic tuff. Their related rock type is andesitic tuff and the geological age is Miocene.

\section{RHEOLOGICAL PROPERTIES INSTRUMENT}

The experimental apparatus used in this study consists of a Fann model 50C viscometer and Baroid roller oven [10]. The Fann model 50C viscometer is rotational coaxialcylinder type. This device allows us to calculate the plastic viscosity and yield point of drilling fluids from shear stress readings at 300 and $600 \mathrm{rpm}$. Although, they can use through six rotation speeds are $600,300,200,100,6$ and 3 $\mathrm{rpm}$ when their rotor is driven by an electric motor [7]. The shear stress or scale reading is determined as a function of the shear rate (from the speed of rotation). Even though, these viscometers provide a good approximation of the drilling mud rheological properties, the applicability of their measurement is some limited because they are carried out at one given temperature (usually at ambient temperature) which constitutes a serious advantage. This technical limitation seems to be very important for actual well drilling applications, when the drilling fluid transport properties were assumed to be dependent on temperature [5], [7]. The upper temperature and pressure operating limits are $280^{\circ} \mathrm{C}$ and $6896 \mathrm{kPa}$, respectively. The Fann 50C has infinitely variable rotor speeds from 1 to $625 \mathrm{rpm}$ with viscosity range of $0.001-300$ Pa.s ( $1-300 \times 10^{3} \mathrm{mPa} . \mathrm{s}$ or centipoises). The main advantage of this equipment offers other than viscometers is that effective viscosity measurements can be carried out directly at dynamic temperature conditions. Such a capability enables the drilling mud viscosity variability at deep well well drilling conditions to be simulated. Baroid 
roller oven is insulated, thermostatically controlled oven with stainless steel exterior and interior for heating and agitating sample in suitable container (aging cell). Roller oven is primary designed for laboratory use to simulate the heating and agitation that the drilling mud is subjected to while being circulated down the hole and back to the mud pit. Agitation is particularly important when investigating mud $\mathrm{s}$ in which the base exchange reactions occur and in the determination of thermal stabilities of both mud and additive. The roller along with the aging at an approximate speed of $50 \mathrm{rpm}$.

\section{MATERIAL AND EXPERIMENT METHOD}

In this study, the Sabah bentonite samples was collected, namely SA5 (N4 ${ }^{0} 18.97^{\prime}$ - E $\left.117^{0} 57.37^{\prime}\right)$ from Andrassy area in Tawau district. The SA5 sample was collected exactly at $0.5 \mathrm{~m}$ depths. The Andrassy area is underlain mainly by the high level alluvium and volcanic rock, and occurs in a bed underlying of Pleistocene to Holocene in age [1]. The Sabah bentonite was dried at $65^{\circ} \mathrm{C}$ for 24 hours, ground in a Tema mill and pass through a $75 \mu \mathrm{m}$ sieve. The performance test of Sabah bentonite then compared with Drill-Gal bentonite as a standard bentonite. The chemical additives are shown in 'table 1', with strength varying between $0.05-5 \mathrm{ppb}$ and at varying temperature between $25-250^{\circ} \mathrm{C}$. The rheological tests were conducted according to American Petroleum Institute specifications [9].

Table 1. Chemical additives used in the experiment

\begin{tabular}{|c|c|c|c|c|}
\hline \multirow{2}{*}{ Product } & \multirow{2}{*}{$\begin{array}{c}\text { Concen tration } \\
\text { (ppb) }\end{array}$} & \multirow{2}{*}{$\begin{array}{c}\text { Material } \\
\text { Description }\end{array}$} & \multicolumn{2}{|c|}{ Functions } \\
\hline & & & Main & Secondary \\
\hline CMC & $0.2-4$ & $\begin{array}{c}\text { Carboxi methyl } \\
\text { Cellulose }\end{array}$ & $\begin{array}{l}\text { Filtration } \\
\text { reducer }\end{array}$ & $\begin{array}{l}\text { Viscosity } \\
\text { control } \\
\text { agents }\end{array}$ \\
\hline TANNATHIN & $1-5$ & $\begin{array}{l}\text { Groud } \\
\text { Lignite }\end{array}$ & $\begin{array}{c}\text { Thinner } \\
\text { dispersant }\end{array}$ & $\begin{array}{l}\text { Filtration } \\
\text { reducer }\end{array}$ \\
\hline BEN-EX & $0.05-2$ & $\begin{array}{l}\text { Bentonite } \\
\text { Extender }\end{array}$ & Flocculants & Viscosifier \\
\hline SP-101 & $0.5-2$ & $\begin{array}{l}\text { Sodium } \\
\text { Polyocrylate } \\
\text { fluid loss } \\
\text { Polymer }\end{array}$ & $\begin{array}{l}\text { Filtration } \\
\text { loss reducer }\end{array}$ & $\begin{array}{c}\text { Shale control } \\
\text { agents }\end{array}$ \\
\hline
\end{tabular}

The viscosities were measured using a Fann 50C viscometer. A $50 \mathrm{ml}$ accurately measured volume of mud was placed in the sample cup. This sample volume must be precise measurement, because an excess of mud could contaminate the bearings and seals of the system, while an insufficient mud volume could cause mixing of the sample and pressurizing oil at the interface. After introducing the sample the dynamic test was initiated using operating conditions as shown in 'table 2 '.

The shear stress, shear rate and temperature of the drilling fluid were continuously and automatically logged on a strip chart using a two channel potentiometric servo recorder. These channels are used separately to record the temperature, shear stress and shear rate. Shear rate measurement are logged by momentarily interrupting the shear stress signal each 4 minute of time period. Thus, viscosity and temperature variations with time are separately given in a rheogram for each fluid analyzed. The dynamic rheological data were then transferred to a personal computer to be subsequently analyzed. The sample can be heated through $250^{\circ} \mathrm{C}$ by an oil bath which is also used for cooling the sample. The temperature range of $25-250^{\circ} \mathrm{C}$ is programmable. Pressures in the sample cup can reach through $6896 \mathrm{kPa}$ and generated either by a nitrogen gas cylinder or by a compressed air which can be controlled with a pressure regulator.

Table 2. Fann 50C operating conditions for dynamics rheological tests

\begin{tabular}{|l|l|}
\hline Operating Parameter & Setting Values \\
\hline Initial temperature $\left(\mathrm{T}_{\mathrm{i}}\right)$ & $25^{\circ} \mathrm{C}$ \\
Final temperature $\left(\mathrm{T}_{\mathrm{f}}\right)$ & $260^{\circ} \mathrm{C}$ \\
Reservoir pressure & $3448 \mathrm{kpa}$ \\
Time to attain $\left(\mathrm{T}_{\mathrm{f}}\right)$ & $1-24$ hours \\
Chart speed & $0.25 \mathrm{~cm} / \mathrm{min}$ \\
Shear rate & $170 \mathrm{~s}^{-1}$ \\
Viscosity range & $55 \%(55 \mathrm{mPa} . \mathrm{s})$ \\
Total time of test & 3 hours \\
\hline
\end{tabular}

\section{RESUlTS AND DisCUSSION}

Apparent viscosity (AV), Viscosity Plastic (YP) and Yield Point (YP) are increase with temperature increasing. VP decreased because of decreasing solid concentration or by decreasing of surface area. As the water viscosity decreases with temperature the VP decrease proportional. Therefore, controlling PV of mud in practical term involves controlling size, concentration and shape of solid. For the YP which is the initial resistance to flow caused by electrochemical forces between the particles. This electrochemical force is due to charges on the surface of particles dispersed in fluid phase, and because the distance between the particles is decrease, the attraction between particles is greatly increased and if the distance between particles is increase, the attraction between particles is greatly decrease. At $250^{\circ} \mathrm{C}$ in temperature, Sabah bentonite was indicative of the flocculation phenomena as shown in 'table 3'; furthermore, activation treatments are required by adding chemical additives to improve its rheological performance.

Table 3. Sabah bentonite rheological test un-treatment (original condition) comparing with Drill-Gal bentonite at temperature through $250^{\circ} \mathrm{C}$

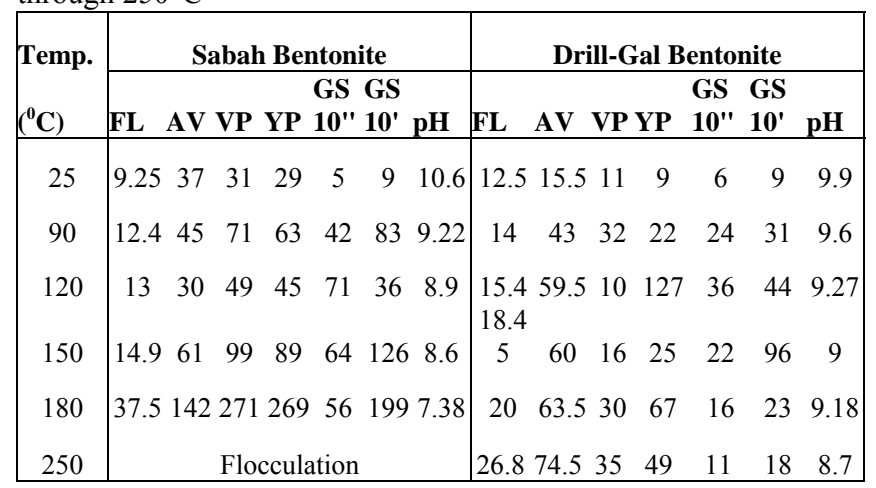

Additional of CMC additive (1 ppb) only in Sabah bentonite has decreasing filtration loss from $37.5 \mathrm{ml} / 30$ minutes to $16 \mathrm{ml} / 30$ minutes at temperature $180^{\circ} \mathrm{C}$. The 
summaries of test results are shown in 'table 4' through 'table 7', respectively.

Table 4. Sabah bentonite rheological test treatment by CMC (1 ppb) comparing with Drill-Gal bentonite at temperature through $250^{\circ} \mathrm{C}$

\begin{tabular}{|c|c|c|c|c|c|c|c|c|c|c|c|c|c|}
\hline \multirow{2}{*}{$\begin{array}{l}\text { Temp. } \\
\left({ }^{0} \mathrm{C}\right)\end{array}$} & \multicolumn{6}{|c|}{ Sabah Bentonite } & \multicolumn{7}{|c|}{ Drill-Gal Bentonite } \\
\hline & FL & AV & VPYP & $\begin{array}{c}\text { GS } \\
10 " \\
\end{array}$ & $\begin{array}{l}\text { GS } \\
10 ' \\
\end{array}$ & $\mathbf{p H}$ & FL & AV & VP & $\mathbf{Y P}$ & $\begin{array}{c}\text { GS } \\
10^{\prime \prime}\end{array}$ & $\begin{array}{l}\text { GS } \\
10^{\prime} \\
\end{array}$ & pH \\
\hline & 8.3 & & & & & 9.2 & & & & & & & 9.0 \\
\hline 25 & 5 & $\begin{array}{l}34 \\
40 .\end{array}$ & 2237 & 3 & 25 & $\begin{array}{c}8 \\
8.5\end{array}$ & $\begin{array}{l}6.5 \\
12 .\end{array}$ & $\begin{array}{l}9.5 \\
15 .\end{array}$ & 5 & $\begin{array}{c}9 \\
10 .\end{array}$ & 4 & 4 & $\begin{array}{c}0 \\
8.5\end{array}$ \\
\hline 90 & 9.5 & 5 & 1160 & 46 & 80 & $\begin{array}{c}3 \\
8.2\end{array}$ & 5 & $\begin{array}{c}3 \\
16 .\end{array}$ & 10 & 5 & 12 & 45 & $\begin{array}{c}0 \\
8.5\end{array}$ \\
\hline 120 & $\begin{array}{l}8.6 \\
13 .\end{array}$ & 39 & $\begin{array}{ll}1 & 69 \\
& 10\end{array}$ & 72 & 110 & $\begin{array}{c}4 \\
7.3\end{array}$ & 14 & $\begin{array}{c}5 \\
13 .\end{array}$ & $\begin{array}{c}11 \\
11 .\end{array}$ & $\begin{array}{l}11 \\
11 .\end{array}$ & 22 & 20 & $\begin{array}{c}0 \\
7.2\end{array}$ \\
\hline 150 & 8 & $\begin{array}{l}53 \\
67 .\end{array}$ & $\begin{array}{cc}2 & 2 \\
& 13\end{array}$ & 97 & 98 & $\begin{array}{c}1 \\
7.1\end{array}$ & 16 & 5 & 5 & 5 & 22 & 60 & $\begin{array}{c}5 \\
7.5\end{array}$ \\
\hline 180 & 16 & 5 & 20 & 52 & 62 & 4 & 20 & 9 & 17 & 17 & 20 & 70 & $\begin{array}{c}3 \\
7.5\end{array}$ \\
\hline 250 & & & Floce & ulation & & & 35 & 32 & 37 & 32 & 39 & 50 & 0 \\
\hline
\end{tabular}

Table 5. Sabah bentonite rheological test treatment by Tannathin $(2 \mathrm{ppb})$ comparing with Drill-Gal bentonite at temperature through $250^{\circ} \mathrm{C}$

\begin{tabular}{|c|c|c|c|c|c|c|c|c|c|c|c|c|c|c|}
\hline \multirow{2}{*}{$\begin{array}{c}\text { Temp. } \\
\left({ }^{0} \mathrm{C}\right)\end{array}$} & \multicolumn{7}{|c|}{ Sabah Bentonite } & \multicolumn{7}{|c|}{ Drill-Gal Bentonite } \\
\hline & FL & AV & VP & YP & $\begin{array}{c}\text { GS } \\
10 "\end{array}$ & $\begin{array}{l}\text { GS } \\
10^{\prime}\end{array}$ & pH & FL & AV & VP & YP & $\begin{array}{c}\text { GS } \\
10^{\prime \prime}\end{array}$ & $\begin{array}{l}\text { GS } \\
10^{\prime} \\
\end{array}$ & pH \\
\hline 25 & 1 & 20 & 10 & 20 & 9 & 37 & 9 & 6.5 & 17 & 10 & 22 & 9 & 10 & 9 \\
\hline 90 & 6.50 & 7 & 6 & 2 & 11 & 70 & 8 & 6.75 & 13.5 & r & 20 & 12 & 27 & 8.50 \\
\hline 120 & 11.5 & 7.5 & 3 & 9 & 12 & 72 & 7.93 & 9.5 & 15.5 & 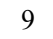 & 20 & 23 & 25 & 8.25 \\
\hline 150 & 12.5 & 10 & 5 & 10 & 16 & 54 & 7.25 & 10.5 & 19 & 11 & 24.5 & 17 & 68 & 8.00 \\
\hline 180 & 15.25 & 10.5 & 7 & 7 & 19 & 69 & 7.50 & 15 & 20.5 & 12 & 53 & 18 & 78 & 7.85 \\
\hline 250 & & & loce & ulat & tion & & & 17.5 & 22.5 & 12.5 & 28.7 & 7 & 88 & 7.5 \\
\hline
\end{tabular}

Table 6. Sabah bentonite rheological test treatment by SP$101(0.5 \mathrm{ppb})$ comparing with Drill-Gal bentonite at temperature through $250^{\circ} \mathrm{C}$

\begin{tabular}{|c|c|c|c|c|c|c|c|c|c|c|c|c|c|c|}
\hline \multirow{2}{*}{$\begin{array}{l}\text { Temp. } \\
\left({ }^{0} \mathrm{C}\right)\end{array}$} & \multicolumn{7}{|c|}{ Sabah Bentonite } & \multicolumn{7}{|c|}{ Drill-Gal Bentonite } \\
\hline & FL & AV & VP & YP & $\begin{array}{l}\text { GS } \\
10^{\prime}\end{array}$ & $\begin{array}{l}\text { GS } \\
10 \\
\end{array}$ & $\mathrm{pH}$ & FL & AV & VF & YP & $\begin{array}{l}\text { GS } \\
10 "\end{array}$ & $\begin{array}{l}\text { GS } \\
10^{\prime}\end{array}$ & pH \\
\hline 25 & 12. & 11 & 5 & 12 & 14 & 12 & 9 & 12 & 11 & 7 & 14.5 & 4 & 9 & 9.5 \\
\hline 90 & 11 & 8 & 6 & 4 & 8 & 16 & 8.60 & 14.5 & 11 & 8 & 15 & 8 & 24 & 8.7 \\
\hline 120 & 17 & 12.5 & 7 & 9 & 8 & 20 & 8.50 & 16 & 12.5 & 6 & 15.5 & 9 & 38 & 8.5 \\
\hline 150 & 15 & 27.5 & 20 & 15 & 6 & 12 & 8.25 & 19.5 & 20 & 12 & 26.5 & 9 & 49 & 8.40 \\
\hline 180 & 18 & 33 & 24 & 18 & 4 & 9 & 8 & 23.5 & 22 & 15 & 29.5 & 14 & 85 & 8.25 \\
\hline 210 & Flo & culat & ion & & & & & 24 & 23.5 & 25 & 36 & 20 & 110 & 8 \\
\hline
\end{tabular}

Addition of activating agent (additive) such as CMC (1 ppb), Tannathin (2 ppb), and SP-101 (0.50 ppb), could be decreasing filtration loss (close to API standard) at range optimum temperature between $27^{\circ} \mathrm{C}$ through $180^{\circ} \mathrm{C}$. Additive Ben-ex $(0.50 \mathrm{ppb})$ is the best activating agents that have temperature resistance through $260{ }^{\circ} \mathrm{C}$ ('Table 7').
Table 7. Sabah bentonite rheological test treatment by Ben-ex $(0.5 \mathrm{ppb})$ comparing with Drill-Gal bentonite at temperature through $250^{\circ} \mathrm{C}$

\begin{tabular}{|c|c|c|c|c|c|c|c|c|c|c|c|c|c|c|}
\hline \multirow{2}{*}{$\begin{array}{l}\text { Temp. } \\
\left({ }^{0} \mathrm{C}\right)\end{array}$} & \multicolumn{7}{|c|}{ Sabah Bentonite } & \multicolumn{7}{|c|}{ Drill-Gal Bentonite } \\
\hline & FL & AV & VP & YP & $\begin{array}{l}\text { GS } \\
10 " \\
\end{array}$ & $\begin{array}{l}\text { GS } \\
10^{\prime} \\
\end{array}$ & $\mathrm{pH}$ & FL & AV & VP & YP & $\begin{array}{l}\text { GS } \\
10^{\prime} \\
\end{array}$ & $\begin{array}{l}\text { GS } \\
10^{\prime} \\
\end{array}$ & $\mathrm{H}$ \\
\hline 25 & 11 & 32 & 23.5 & 40.5 & & 33 & 9 & 9 & 17 & 13 & 24 & 4 & 6 & 9.90 \\
\hline 90 & 14 & 28 & 17 & 34.5 & & 47 & 8.75 & 10 & 15 & 12.5 & 21.8 & 11 & 21 & 9.70 \\
\hline 120 & 15 & 21.5 & 13 & 21.8 & 11 & 59 & 8.66 & 12.5 & 16 & 10 & 21.5 & 16 & 22 & 9.40 \\
\hline 150 & 15 & 19 & 28 & 21 & 14 & 82 & 8.2 & 13 & 22 & 12 & 28.5 & 19 & 41 & 9.30 \\
\hline 180 & 16 & 13.5 & 31.5 & 15 & 9 & 26 & 8.02 & 17.5 & 23.5 & 14 & 21 & 24 & 50 & 9.20 \\
\hline 210 & 18 & 12 & 38 & 19.5 & & 17 & 8.8 & & - & - & - & - & - & - \\
\hline 260 & 19.5 & 18 & 43 & 22 & 11 & 58 & 8 & 28 & 28 & 21 & 40 & 32 & 98 & 9 \\
\hline
\end{tabular}

\section{CONCLUSIONS}

In this study, Sabah bentonite was test and activated with addition many additives with variation in temperature for usage as deep well drilling-mud viscosifier. Based on the investigation, it is shown that additives CMC (1 ppb), Tannathin (2 ppb), and SP-101 (0.50 ppb) could decreasing filtration loss (close to API standard) at range optimum temperature between $27^{\circ} \mathrm{C}$ through $180^{\circ} \mathrm{C}$. Ben-ex $(0.50$ $\mathrm{ppb})$ is the best activating agents that have temperature resistance through $260{ }^{\circ} \mathrm{C}$.

\section{REFERENCES}

[1] Kelsey, J.R., and Carson, C.C.: Deep well Drilling - Drilling for Deep well Energy, Deep well Sciences and Technology, 1, (1987), p.39-61.

[2] Beirute, R.M.: A Circulating and Shut-in Well Temperature Profile Simulator, Journal of Petroleum Technology, 9, 1991, p.1140-1146.

[3] Arnold, F.C.: Temperature Variation in A Circulating Wellbore Fluid, Journal of Energy Resources Technology, 112, 1990, p.79-83.

[4] Wisian, K.W. et al.: Field Comparison of Conventional and New Technology of Temperature Logging System, Geothermics, 27, 1988, p. 131 .

[5] Fisk, J.V., and Jamison, D.E.: Physical Properties of Drilling Fluids at High Temperature and Pressure, Proceedings, IADC/SPE 17200 Drilling Conference, Dallas, Texas, USA, (1988), p.257-265.

[6] Annis, M.R.: High-Temperature Flow Properties of Water Base Drilling Fluids, Journal of Petroleum Technology, 1967, p.10741080 .

[7] Monicard, R.: Drilling Mud and Cement Slurry Rheology Manual, Technique 27 Rue Ginoux 75737, Paris, France, (1982).

[8] Ravi, K.M., and Sutton, D.L.: New Rheological Correlation for Cement Slurries as Function of Temperature, Proceedings, $65^{\text {th }}$ Annual Technical Conference and Exhibition of the SPE, New Orleans, USA, (1990), p.455-462.

[9] The Rheology of Oil-Well Drilling Fluids, American Petroleum Institute (API) Bulletin 13D, (1990), p.28.

[10] Fann Instrument: Operating Instructions Model 50C Fann Viscometer, Fann Instrument Corporation.

\section{NOMENCLATURES}

CMC : Carboxy-methyl-cellulose

FL : Filtration loss, $\mathrm{ml} / 30 \mathrm{~min}$

AV : Apparent viscosity, cp

VP : Viscosity plastic, $\mathrm{cp}$

YP : Yield point, $\mathrm{lb} / 100 \mathrm{sq}-\mathrm{ft}$

GS10" : Gel strength at 10 second, lb/100 sq-ft

GS10' : Gel strength at 10 minutes, $1 \mathrm{~b} / 100 \mathrm{sq}-\mathrm{ft}$

API : American Petroleum Institute Houston Texas, USA, (1998). 


\title{
Analysis of Field Distribution of a Cylindrical Permanent Magnet Linear Generator
}

\author{
Wijono and Hamzah Arof
}

\begin{abstract}
This paper deals with the analytical calculation of the magnetic field of a cylindrical permanent magnet linear generator. It offers an alternative solution to the magnetic field problem. A new approach to solve Poisson equation using Struve function is introduced and it offers a simple but accurate analytical estimation to the calculation of the open circuit flux density and induced voltage. The finite element analysis simulation is performed to validate the analytical calculation.
\end{abstract}

Keywords: linear generator, permanent magnet, analytical method, Struve function, Halbach, electromagnetic field, finite element analysis

\section{INTRODUCTION}

A linear generator is a machine that converts linear mechanical energy into electrical energy. The machine is directly driven by a linear prime mover. It is different from its rotary counterpart in a way that the design of a linear machine mostly follows the characteristic of the prime mover.

The linear generator provides electrical power as a stand alone or as an alternator of a power generation system. A wide range of output power can be had from linear generators from a few watts for a flashlight to a few megawatts from a tidal wave power for normal utilities.

Unlike rotary machines, limited publications on the linear generator show that research on linear generators has not reached the saturation level and therefore there is still a lot of space for research and development.

This paper presents a calculation of the flux distribution of a linear generator. Maxwell and Poisson equations are used to compute magnetic field quantities in all parts of the machine.

A comprehensive analysis of the field distribution of linear machines is presented in [7]. Treatment of linear machines equipped with Halbach and quasi or discrete Halbach is outlined as a special case in [5] in detail. Several types of winding construction are also offered. The analysis can also be adapted from the rotary machine studies presented in [14]-[16].

Wang et. al. [5], [7] solve the field equations by integrating of the permanent magnet geometry. The exponential form can also be used to solve the Maxwell and Poisson equation in both rotary [12]-[16] and linear machines [2]. In this paper a direct solution involving a Struve function is proposed. The direct solution takes less time since there is no integral form in the matrix of simultaneous equations, and the whole field source is taken into calculation instead of its surface only. The only requirement needed for the direct solution is just some experience in handling differential equations.

The validity of the direct analytic approach presented in this paper is verified by comparing its solutions to those of finite element method. The calculation of the unknown parameters is made easy by taking advantage of the symmetry of the cylindrical machine. A 2D axisymmetry model is set up to give a simpler machine representation of the real $3 \mathrm{D}$ object.

\section{MACHINE CONSTRUCTION}

The machine is a long-translator type and constructed of a stator and a translator as shown in the 'figure 1' and 'figure 2'. Six windings are located in the stator core. Seven pieces of permanent magnets are mounted on a non-permeable translator shaft to produce the magnetic field. Those permanent magnets are arranged in a discrete Halbach series, i.e. radially magnetized permanent magnets (RMPMs) and axially magnetized permanent magnets (AMPMs) are stacked alternately. 


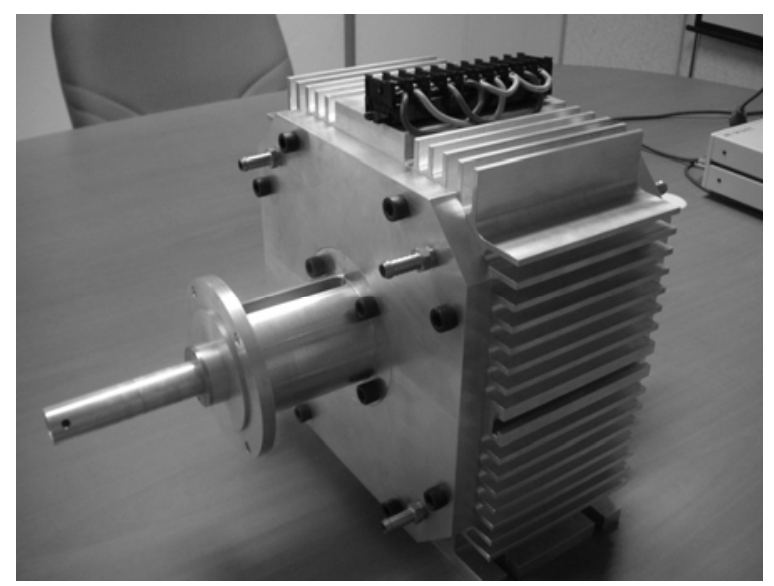

Fig.1. Linear generator prototype.

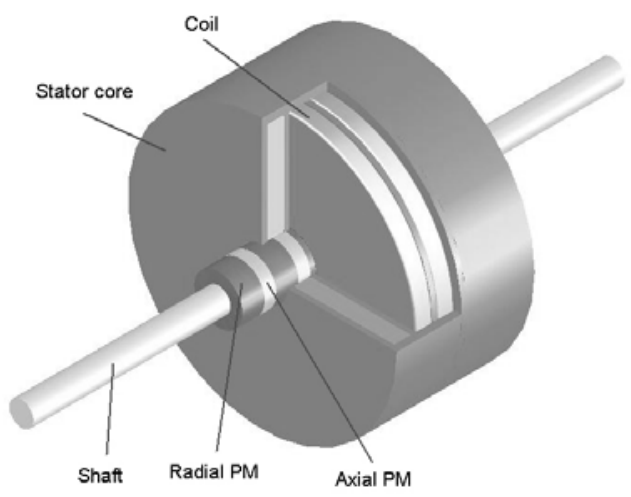

Fig.2. Linear generator construction.

The machine is run in a reciprocating sinusoidal motion. The system produces a linear three phase ac output. At every translator motion, all windings are always active since the translator is longer than the stator.

\section{MODELING AND ANALAYSIS}

'Figure 3' shows the cross section of the upper half of the linear generator. As can be seen, the machine can be divided into three regions where region 1 includes the air gap and the winding; region 2 includes the permanent magnet set and region 3 includes the shaft. The region number is noted as a subscript in all equations. The machine specification is listed in 'table 1 '.

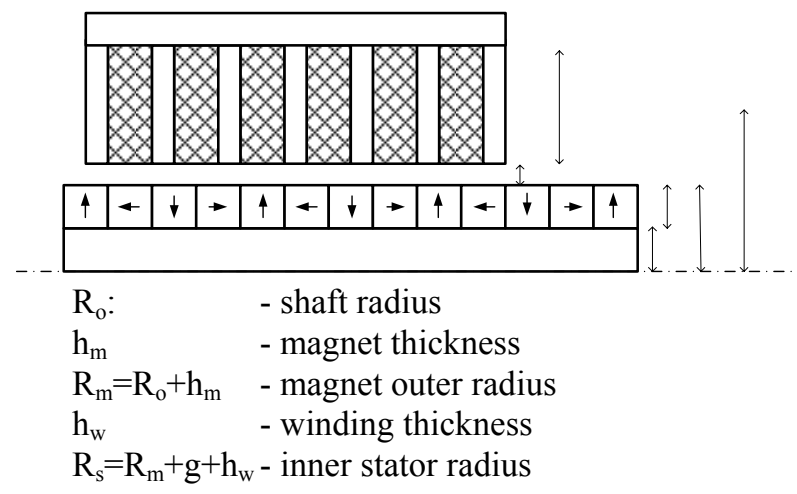

Fig. 3. Cross section of the upper half of the linear generator.
Table.1. Generator specification.

\begin{tabular}{|l|r|l|}
\hline Speed & 3000 & $\mathrm{rpm}$ \\
\hline Stroke & 69 & $\mathrm{~mm}$ \\
\hline $\mathrm{R}_{0}$ & 12.5 & $\mathrm{~mm}$ \\
\hline $\mathrm{h}_{\mathrm{m}}$ & 12 & $\mathrm{~mm}$ \\
\hline $\mathrm{h}_{\mathrm{w}}$ & 102 & $\mathrm{~mm}$ \\
\hline AMPM length & 12 & $\mathrm{~mm}$ \\
\hline RMPM length & 22.5 & $\mathrm{~mm}$ \\
\hline $\mathrm{B}_{\mathrm{r}}$ & 1.12 & $\mathrm{~T}$ \\
\hline$\mu_{\mathrm{r}}$, shaft & 1 & \\
\hline$\mu_{\mathrm{r}}$, core & infinity & \\
\hline
\end{tabular}

In the first step of the analysis, the governing equations are developed while ignoring the existence of stator teeth. The machine is assumed to be infinitively long and has a periodic construction. The permeability of the core is assumed to be infinity while the permeability of the winding, air gap and permanent magnet as well as the shaft is assumed to be one. Next, the effect of teeth is represented by the Carter coefficient. Some other assumptions are made to simplify the calculation.

\section{A. Flux Distribution}

The flux distribution is calculated at any point in all zones. The flux density in the air gap, the winding and the shaft is given by,

$$
\mathbf{B}=\mu_{0} \mathbf{H}
$$

where $\mu_{0}$ is permeability of free space and $\mathbf{H}$ is the field intensity. The flux density in the permanent magnet is given by the combination of the remanent magnetization of permanent magnet and the external field intensity. It can be written as:

$\mathbf{B}=\mu_{0} \mu_{r} \mathbf{H}+\mu_{0} \mathbf{M}$

where $\mu_{r}$ is the relative recoil permeability of the magnets and $\mathbf{M}$ is the remanent magnetization vector. It is assumed that the permanent magnet has a linear demagnetization characteristic; so that the magnetization vector is directly related to the field density as:

$\mathbf{M}=\frac{\mathbf{B}_{r e m}}{\mu_{0}}$

The flux density $\mathbf{B}$ can be expressed as the curl of the magnetic vector potential A

$\mathbf{B}=\nabla \times \mathbf{A}$

Maxwell's and Poisson's equations for the air gap/winding, permanent magnets and shaft are respectively given as, 


$$
\begin{aligned}
& \nabla^{2} \mathbf{A}_{1}=0 \\
& \nabla^{2} \mathbf{A}_{2}=-\mu_{0} \nabla \times \mathbf{M} \\
& \nabla^{2} \mathbf{A}_{3}=0
\end{aligned}
$$

The vector potential A has only one nonzero component, $A_{\theta}$, which is independent of $\theta$ in the cylindrical system.

The boundary conditions of the system exist at the interfaces of different zones. Six boundary conditions are needed to solve the simultaneous field equations.

$$
\begin{aligned}
& B_{z 1} \mid r=R_{S}=0 ; \\
& \left.B_{r 1}\right|_{r=R_{m}}=B_{r 2} \mid r=R_{m} ; \\
& \left.B_{r 2}\right|_{r=R_{0}}=B_{r 3} \mid r=R_{0} ; \\
& \left.A_{\theta 3}\right|_{r=0}=0 ; \\
& \left.H_{z 1}\right|_{r=R_{m}}=\left.H_{z 2}\right|_{r=R_{m}} ; \\
& \left.H_{z 2}\right|_{r=R_{0}}=H_{z 3} \mid r=R_{0}
\end{aligned}
$$

Then the flux distributions are derived for all zones. It yields:

$$
\begin{aligned}
& B_{r 1}=\sum_{\eta=1,2, \ldots}^{\infty} m_{n}\left(\begin{array}{l}
A_{\eta 1} \operatorname{BesselI}_{1}\left(m_{\eta} r\right)+ \\
B_{\eta 1} \operatorname{BesselK}_{1}\left(m_{\eta} r\right)
\end{array}\right) \sin \left(m_{\eta} z\right) \\
& B_{z 1}=\sum_{\eta=1,2, \ldots}^{\infty} m_{\eta}\left(\begin{array}{l}
A_{\eta 1} \operatorname{BesselI}_{0}\left(m_{\eta^{r}}\right)^{-} \\
B_{\eta 1} \operatorname{BesselK}_{0}\left(m_{\eta^{r}}\right)
\end{array}\right) \cos \left(m_{n} z\right) \\
& B_{r 2}=\sum_{\eta=1,2, \ldots}^{\infty}\left(\begin{array}{l}
\frac{1}{2} \frac{\operatorname{StruveL}_{1}\left(m_{\eta} r\right) \cdot P_{\eta} \sin \left(m_{\eta^{z}}\right)}{m_{\eta}}+ \\
m_{n}\left(\begin{array}{c}
A_{\eta 1} \operatorname{BesselI}_{1}\left(m_{\eta^{r}}\right)+ \\
B_{\eta 1} \operatorname{BesselK}_{1}\left(m_{\eta^{r}}\right)
\end{array}\right) \sin \left(m_{\eta} z\right)
\end{array}\right) \\
& B_{z 2}=\sum_{n=1,2, \ldots}^{\infty}\left(\begin{array}{l}
\frac{1}{2} \frac{\pi \operatorname{StruveL}_{0}\left(m_{\eta} r\right) P_{\eta} \cos \left(m_{\eta} z\right)}{m_{\eta}}+ \\
m_{\eta}\left(\begin{array}{c}
A_{n 2} \operatorname{BesselI}_{0}\left(m_{\eta} r\right)- \\
B_{n 2} \operatorname{BesselK}_{0}\left(m_{\eta} r\right)
\end{array}\right) \cos \left(m_{\eta^{z}}\right)
\end{array}\right) \\
& B_{r 3}=\sum_{\eta=1,2, \ldots}^{\infty} m_{\eta}\left(\begin{array}{l}
A_{\eta 3} \operatorname{BesselI}_{1}\left(m_{\eta^{r}}\right)+ \\
B_{\eta 3} \operatorname{BesselK}_{1}\left(m_{\eta^{r}}\right)
\end{array}\right) \sin \left(m_{\eta^{z}}\right) \\
& B_{z 3}=\sum_{\eta=1,2, \ldots}^{\infty} m_{\eta}\left(\begin{array}{l}
A_{\eta 3} \operatorname{BesselI}_{0}\left(m_{\eta} r\right)- \\
B_{\eta 3} \operatorname{BesselK}_{0}\left(m_{\eta} r\right)
\end{array}\right) \cos \left(m_{\eta^{z}}\right)
\end{aligned}
$$

where BesselI $I_{1}$ and BesselI $_{0}$ are modified Bessel functions of the first kind of order 1 and of order 0 , respectively, and BesselK $_{I}$ and BesselK $K_{0}$ are modified Bessel functions of the second kind of order 1 and of order 0 , respectively. The function Struve is proposed here to solve the field equation. Functions StruveL $L_{1}$ and StruveL $L_{0}$ are modified Struve functions of the first kind of order 1 and of order 0 , respectively.

\section{B. Induced Voltage}

The flux linkage in the winding is computed by adding a coefficient, known as the Carter coefficient, to represent the existence of the stator teeth [5], [7]. This coefficient is given by

$$
K_{C}=\frac{\tau_{s p}}{\tau_{s p}-\gamma g^{\prime}}
$$

where $\tau_{s p}$ is the stator slot pitch, and $g^{\prime}=g+h_{m} / \mu_{r}$. The slotting factor $\gamma$ is given by

$\gamma=\frac{4}{\pi}\left[\frac{b_{0}}{2 g^{\prime}} \tan ^{-1}\left(\frac{b_{0}}{2 g^{\prime}}\right)-\ln \sqrt{1+\left(\frac{b_{0}}{2 g^{\prime}}\right)^{2}}\right]$

where $b_{0}$ is the width of the stator slot openings.

Instead of the air gap distance $g$, an effective air-gap $g_{e}$ is used. This parameter is given by,

$g_{e}=g+\left(K_{C}-1\right) g^{\prime}$

Therefore, the stator bore radius should also be recalculated with the effect of slotting incorporated. The equivalent stator bore radius $R_{s e}$ is given by

$R_{i e}=R_{m}+g_{e}$

where $R_{m}$ is the outer radius of the magnets.

The flux linkage of a stator winding can be obtained by integrating the vector potential in the region 1 over the winding pitch. The total flux linkage of a phase winding is the sum of the flux linkages in all the windings which are connected in series.

$\Psi_{w}=\frac{N_{w}}{\tau_{w} \cdot\left(R_{S}-R_{i}\right)} \int_{z-\frac{\tau_{w}}{2}}^{z+\frac{\tau_{w}}{2}} \int_{R_{i}}^{R_{S}} 2 \cdot \pi \cdot r \cdot A_{I \theta}(r, z) d r d z$

The induced EMF in each phase winding is given by

$e_{w}=-\frac{d \Psi_{w}(z)}{d t}=-\frac{d \Psi_{w}(z)}{d z} v(t)$

where $v(t)$ is the velocity of the translator, in general, as a function of time.

\section{RESUlTS}

The FEA is performed for the static and parametric simulation. The static simulation produces the flux distribution in the machine as shown in 'figure 4'. The 
parametric simulation is proposed to calculate the induced voltage.

The flux distribution in the air gap is calculated using equations (9) and (10). The radius of location is setup to $r=$ $R_{m}+g / 2$. The radial flux density $B_{r}$ and the tangential flux density $B_{z}$ are plotted in 'figure 5 ' together with the flux density resulted from the FEA simulation.

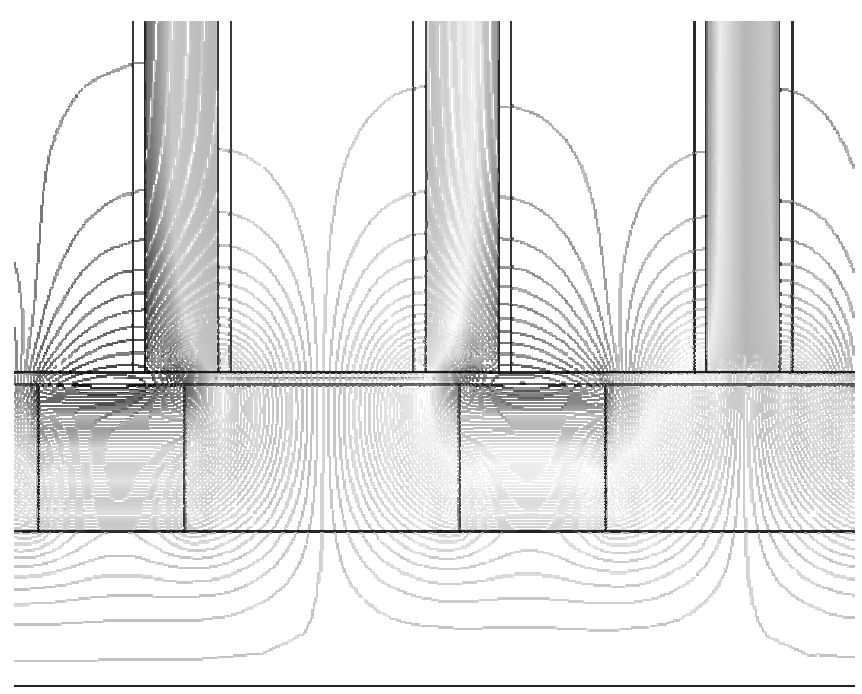

Fig.4. Flux distribution.

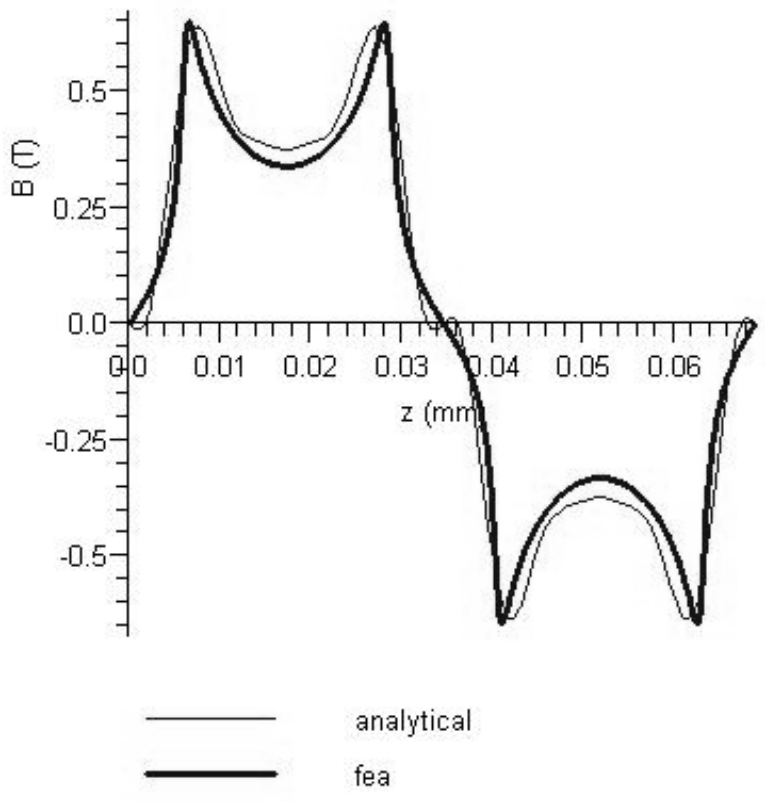

(a) $B_{r}$

Fig. 5. Air gap flux density $B_{r}$ and $B_{z}$

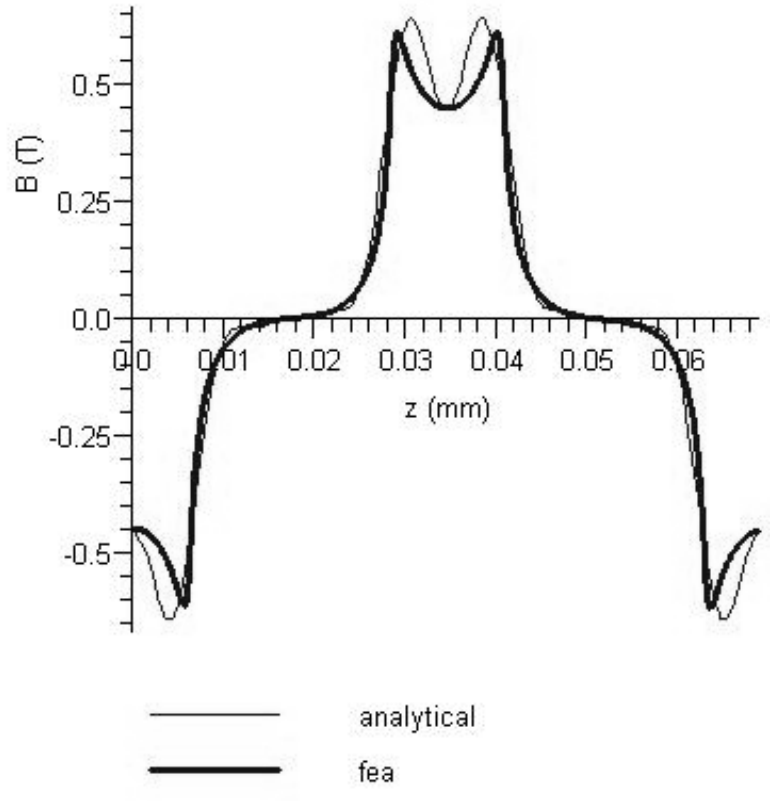

(b) $\mathrm{Bz}$

Fig. 5. Continued

The open circuit induced voltage $e_{w}$ calculated using equation (20) and using the FEA simulation are shown the 'figure 6'.

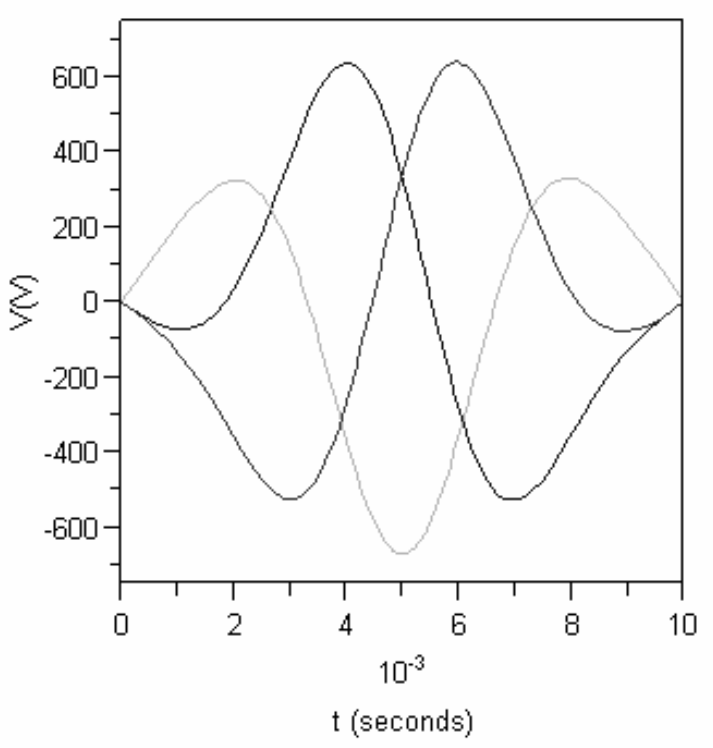

(a) Analytical

Fig. 6. Three Phase Induced Voltage 


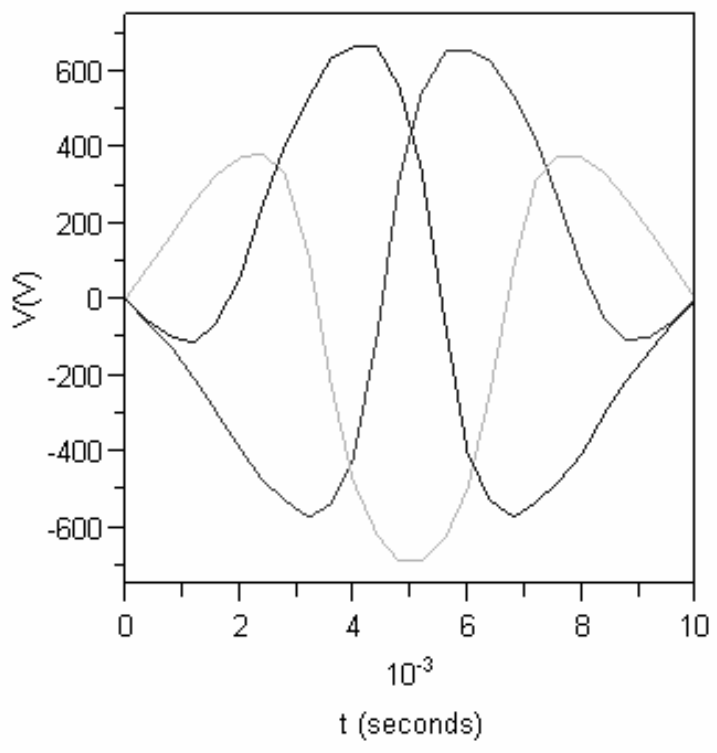

(b) Finite Element Analysis

Fig. 6. Continued

\section{DISCUSSION}

The flux distribution produced by FEA simulation as shown in 'figure 4' is calculated using actual machine parameters. It is shown that almost all flux lines generated by the permanent magnet flows through teeth to reach coils. Some leakage presents in coils and in the shaft.

'Figure 5' shows that the analytical results confirm to the simulation except. In the 'figure 6', the analytical results seem to agree well with the finite element analysis simulation. Consequently, other electrical parameters of the machine can be derived deductively from these parameters.

\section{CONCLUSIONS}

The analytical calculation of the open circuit magnetic field of a cylindrical permanent magnet linear generator is presented in this paper. The governing equation to the field problem is derived from the Laplace and Poisson equations. A special function known as Struve function is proposed to be used in solving the field equations. The solution is obtained without considering the geometrical condition of the permanent magnet as the field source.

The flux density in the air gap and open circuit induced voltage calculated using the analytical method are then compared to the ones produced by the finite element simulation. It can be seen that the results from the two methods match very well and therefore vindicate the validity of the analytical approach. It has been shown that the simple analytical method presented in this paper can be used as a tool for field estimation and design optimization in machine design.

\section{ACKNOWLEDGMENT}

The authors gratefully appreciate and thank the Ministry Of Science, Technology and Environment, Malaysia, for the funding of this research project under IRPA Grant No.3302-03-3013.

\section{REFERENCES}

[1] H. Arof, Wijono and K. M. Nor (2003) Linear Generator: Design and Simulation. In: Proceedings of National Power Engineering Conference (PECon 2003) Bangi, Malaysia. pp. 306-311.

[2] Ion Boldea, S.A. Nasar, Zhenxing Fu (1988) Fields, Forces, and Performance Equations of Air-Core Linear Self-Synchronous Motor with Rectangular Current Control. IEEE Trans Magn, Vol. 24, No. 5. September, pp. 2194-2203.

[3] Jiabin Wang, and David Howe (2004) Design Optimization of Radially Magnetized, Iron-Cored, Tubular Permanent-Magnet Machines and Drive Systems. IEEE Trans Magn, Vol. 40, No. 5, pp. 3262-3277.

[4] Jiabin Wang, and David Howe (2005) Tubular Modular PermanentMagnet Machines Equipped With Quasi-Halbach Magnetized Magnets-Part I: Magnetic Field Distribution, EMF, and Thrust Force, IEEE Trans Magn, Vol. 41, No. 9, pp. 2470-2478.

[5] Jiabin Wang, David Howe, and Geraint W. Jewell (2003) Fringing in Tubular Permanent-Magnet Machines: Part I. Magnetic Field Distribution, Flux Linkage, and Thrust Force, IEEE Trans Magn, Vol. 39, No. 6, pp. 3507-3516.

[6] Jiabin Wang, David Howe, and Geraint W. Jewell (2004) Analysis and Design Optimization of an Improved Axially Magnetized Tubular Permanent-Magnet Machine. IEEE Trans Energy Conver. Vol. 19, No. 2, pp. 289-295.

[7] Jiabin Wang, Geraint W. Jewell, and David Howe (1999) A General Framework for the Analysis and Design of Tubular Linear Permanent Magnet Machines, IEEE Trans Magn, Vol. 35, No. 3, pp. 1986-2000.

[8] Jiabin Wang; Howe, D. (2005) A Linear Permanent Magnet Generator for a Free-Piston Energy Converter, In: Proceeding of IEEE International Conference Electric Machines and Drives, pp. 1521 1528 .

[9] K. Ng, Z Q. Zhu and D Howe (1996) Open-Circuit Field Distribution in a Brushless Motor with Diametrically Magnetized PM Motor, Accounting for Eddy Current Effects, IEEE Trans Magn, Vol 32, No 5 , pp. 5070-5072.

[10] Khalid Mohamed Nor, Hamzah Arof, and Wijono (2004) Design of a $5 \mathrm{~kW}$ Tubular Permanent Magnet Linear Generator. In: Proceedings of 39th International Universities Power Engineering Conference (UPEC 2004) University of the West of England (UWE) Bristol, UK., pp. 528-532.

[11] Khalid Mohamed Nor, Hamzah Arof, and Wijono (2005) Design of a Three Phase Tubular Permanent Magnet Linear Generator. In: Proceedings of The 5th Iasted International Conference Power And Energy Systems, EuroPes 2005, Benalmádena, Spain, pp. N/A.

[12] Wijono, Hamzah Arof, 2007, Open Circuit Field Distribution and Induced Voltage of a Cylindrical Permanent Magnet Linear Generator, in: American Journal of Applied Sciences 4 (11), pp. 912917.

[13] Z. J. Liu. C. Bi, H. C. Tan and T-S. Low (1995) A Combined Numerical and Analytical Approach for Magnetic Field Analysis of Permanent Magnet Machines, IEEE Trans Magn. Vol. 31, No. 3., pp. 1372-1375.

[14] Z. P. Xia, Z. Q. Zhu, and D. Howe (2004) Analytical Magnetic Field Analysis of Halbach Magnetized Permanent-Magnet Machines, IEEE Trans Magn, Vol. 40, No. 4, pp.1864-1872.

[15] Z. Q. Zhu, D. Howe. and Z. P. Xia (1994) Prediction of Open-circuit Air gap Field Distribution Magnet Rotor Topology in Brushless Machines Having an Inset Permanent, IEEE Trans Magn, Vol. 30, No. I , pp.98-107.

[16] Z. Q. Zhu, David Howe, Ekkehard Bolte, and Bemd Ackermann (1993) Instantaneous Magnetic Field Distribution in Brushless Permanent Magnet dc Motors, Part I: Open-circuit Field, IEEE Trans Magn, Vol. 29, No. 1, pp. 124-135.

[17] Z. Q. Zhu, Member, ZEEE, David Howe, Ekkehard Bolte, and Bemd Ackermann (1993) Instantaneous Magnetic Field Distribution in Brushless Permanent Magnet dc Motors, Part III: Effect of Armature Slotting, IEEE Trans Magn, Vol. 29, No. 1, pp. 143-151. 


\title{
Study of Roof Evaporative Cooling by Flooding, Misting and Sprinkler Spraying Systems
}

\author{
Chu Chi Ming, Ong Kein Kok and Tan Liang Harn
}

\begin{abstract}
For years, metal roofing has been installed in the construction of buildings for housing and factories. It gains popularity owing to its lightness for easy installation, flexibility in bending, long lasting and most of all compatible to typical tile, concrete or clay roof. However, with its high thermal conductivity and low capacity, it gains and disseminates solar radiation quickly and heats up the space under it. In the tropics this creates a very uncomfortable condition to the daily activities and may affect working performance. In this study there were three systems tested: flooding, misting and sprinkler systems. Water was flooded or sprayed to the roof surface to create a water film. This thin layer of fine water would absorb heat from the surroundings and evaporate, thus, preventing the roof surface from being heated up. The temperature of the roof was recorded and the performance of these three cooling systems evaluated. From this research, it was found that the sprinkler system managed to maintain the roof temperature to below $40^{\circ} \mathrm{C}$ even when it was at the hour of peak solar radiation, in agreement with prior analysis. The flooding system was also able to reduce the roof temperature by the same amount, about $20 \mathrm{~K}$. Factors such as wind velocity, direction and hourly solar radiation rate had been taken into consideration during the analysis. The outcome of this experiment shows great potential for evaporative cooling system to be commercialized. The sprinkler system is recommended to be developed and implemented to augment air-conditioning cooling systems in order to minimize the consumption of energy.
\end{abstract}

Keywords: roof cooling, evaporative, water

\section{INTRODUCTION}

The demand on metal roofing has been rising from year to year due to the growth of population and construction of buildings. The most widely used material for metal roofing is zinc coated steel sheet. This material has a very high flexibility in bending and shaping features. Besides that,

This work was supported wholely by Universiti Malaysia Sabah with a grant No. B-02-02-02 ER/UR/U065.

Chu Chi Ming is with School of Engineering and IT, Universiti Malaysia Sabah, Sepanggar Bay Campus, 88502 Kota Kinabalu, Sabah, Malaysia (Tel.: +6088 320000 Ext. 3135 fax: +6088 320348; email:chrischu@ums.edu).

Ong Kein Kok and Tan Liang Harn were undergraduates of Chemical Engineering Programme, School of Engineering and IT, Universiti Malaysia Sabah, Sepanggar Bay Campus, 88502 Kota Kinabalu, Sabah, Malaysia. with a special coated paint, it is virtually weather proof.

More than $50 \%$ of a building's heat come from the roof [1], owing to the large area that the roof occupies for a building and most of all, the roof has a longer exposure time to the sun compared to other parts of building [2].

By absorbing the solar energy from the sun, the water will evaporate from the roof surface and hence reduce the penetration of heat through the roof [3], [4]. Studies by several workers on mist-spraying systems on roof for cooling load purpose have been carried out both theoretically and experimentally [5], [6]. In a preliminary analysis of a possible scheme using evaporation on a roof, the calculation showed that the roof temperature was cooled to around $40^{\circ} \mathrm{C}$ [7]. This experimental study is on metal roof cooling through passive evaporation of water.

Three systems have been put to test throughout the whole month of February 2005, namely, flooding, mist-spraying and sprinkler systems. The roof being studied for cooling was located at the School of Engineering and Information Technology in Universiti Malaysia Sabah, where the roofs were faced to the south in order to maximise exposure to the sun.

\section{A. Aim of Project}

Malaysia is hot and high in humidity throughout the year. Thus it is worth investigating evaporation cooling methods. In this project, our aim is to find an economic roof cooling system that saves electricity consumption by observing, comparing and monitoring the evaporation roof cooling through 3 different systems.

\section{B. Objectives}

i) Compare the effectiveness of these systems by measuring the roof temperatures at various times and solar irradiation rates.

ii) Monitoring and comparing the efficiency of the cooling systems in attaining the effectiveness required. 


\section{INSTRUMENTATION AND MEASUREMENT}

\section{A. Outline of the Studied Roof}

Three ocean-blue coloured roofs were separately constructed for this research: Mists roof, Sprinkler / flooding roof and control roof. Their areas are $2.25 \mathrm{~m}^{2}, 2.25$ $\mathrm{m}^{2}$ and $0.625 \mathrm{~m}^{2}$ respectively. The set up of the test loop including the roofs, pump, piping and water storage tank are shown in 'figure 1'.

The chosen site has the most hours of direct irradiation on a sunny day. For measuring temperatures, Type-K thermocouples were used. To ensure these thermocouple wires are firmly attached to the roof, the thermocouple attachment pad is used to stick the weld-end point of thermocouple wire to the roof and covered with silicon gel. To check that the contacts of thermocouple plugs were intact, the temperatures read by these wires were compared with the temperatures measured by a surface probe thermometer. The monitoring equipment used are listed in Table 1 .

Table 1. Monitoring equipment list

\begin{tabular}{|l|l|}
\hline Equipment & Function \\
\hline Thermocouple wires & Sense temperature \\
\hline $\begin{array}{l}4 \text { channel data logger } \\
\text { thermometer }\end{array}$ & Read and record temperatures \\
\hline $\begin{array}{l}\text { Surface probe } \\
\text { thermometer }\end{array}$ & Counter check temperature \\
\hline Anemometer & $\begin{array}{l}\text { Detect wind speed and } \\
\text { direction }\end{array}$ \\
\hline Hygrometer & $\begin{array}{l}\text { Measure relative humidity } \\
\text { and air temperature }\end{array}$ \\
\hline
\end{tabular}

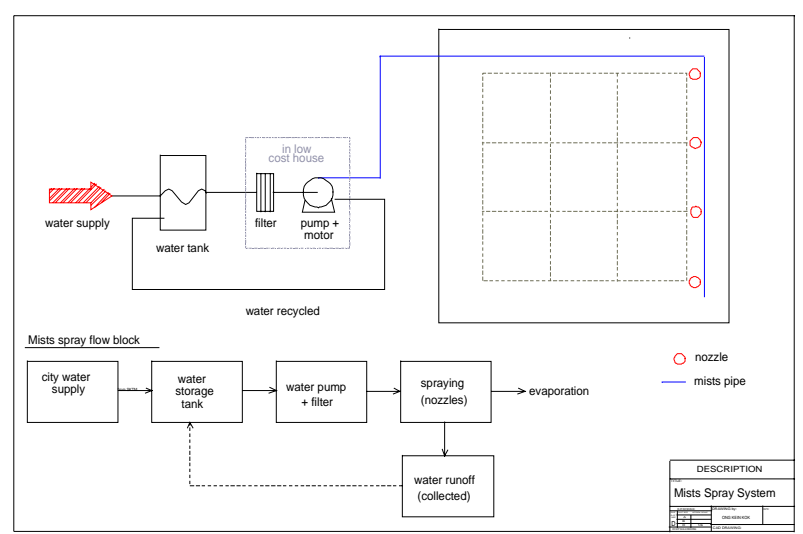

(a) The arrangement of equipment for tests in Mist-Spraying and Sprinkler modes

Figure 1. Mode of testing

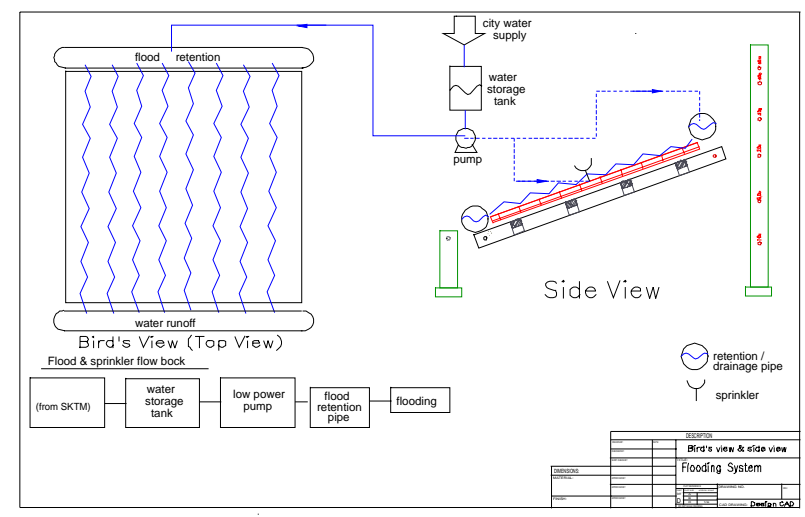

(b) The arrangement of equipment for tests in Flooding mode

Figure 1. Continued

\section{B. Equipment Components}

In the mist system, water was being forced out under pressure, of between 5 to 20 bar from 0.8 micrometer nozzles ('figure $2 \mathrm{a}$ '). These nozzles are placed parallel to the roof surface facing upward so that the mist would float above the roof acting as a barrier before falling onto the surface ('figure 2b'). When it sprays, a thin water film covers the roof surface. This thin layer of fine water would absorb heat from the surrounding and evaporate, thus preventing the roof surface from being heated up. The same principle applies to the sprinkler system ('figure 2c'). Their main difference is that in sprinkling, the number of sprinkler head was only one and that it sprinkles horizontally (Figure $2 \mathrm{~d}$ ), requiring much less pressure to pump water flow over the roof and its flow was continuous.

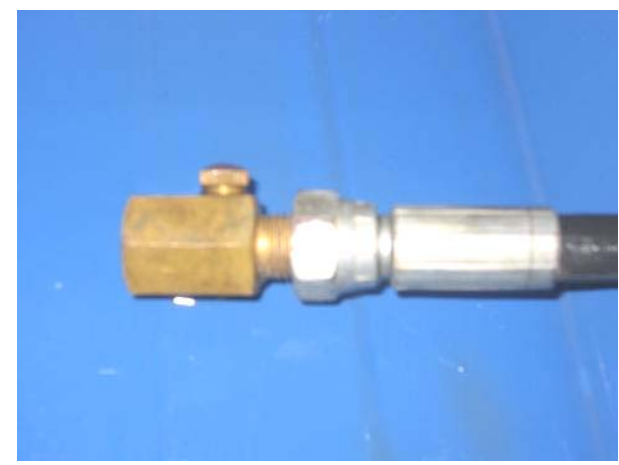

(a) Mist nozzle

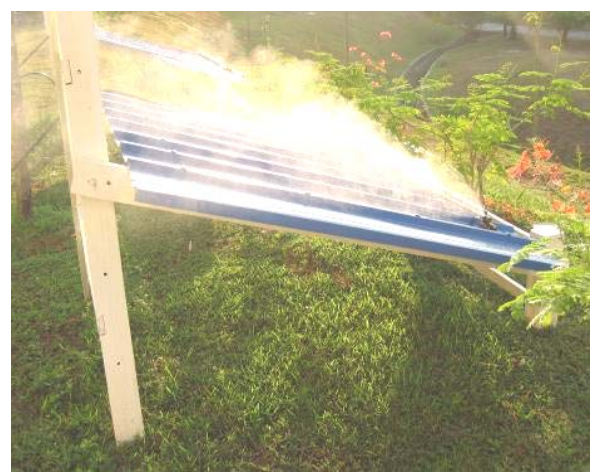

(b) Mist-spraying system

Figure 2. Mist-Spraying, Sprinkler and Flooding systems in operation 


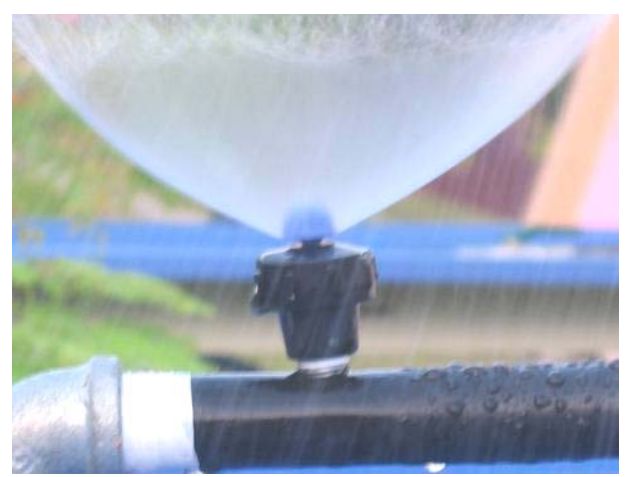

(c) Sprinkler head

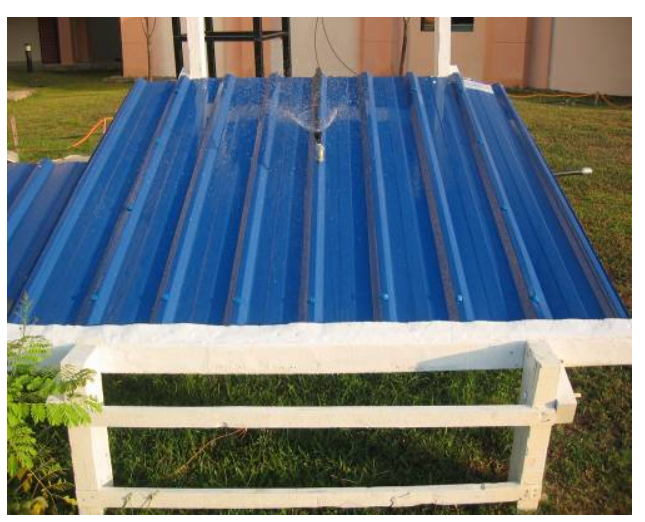

(d) Sprinkler system

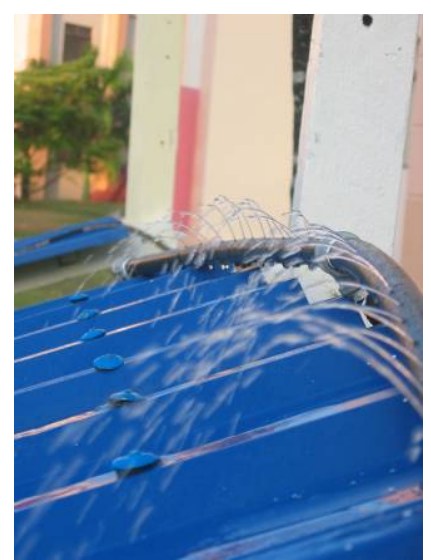

(e) Flooding Distributor Pipe

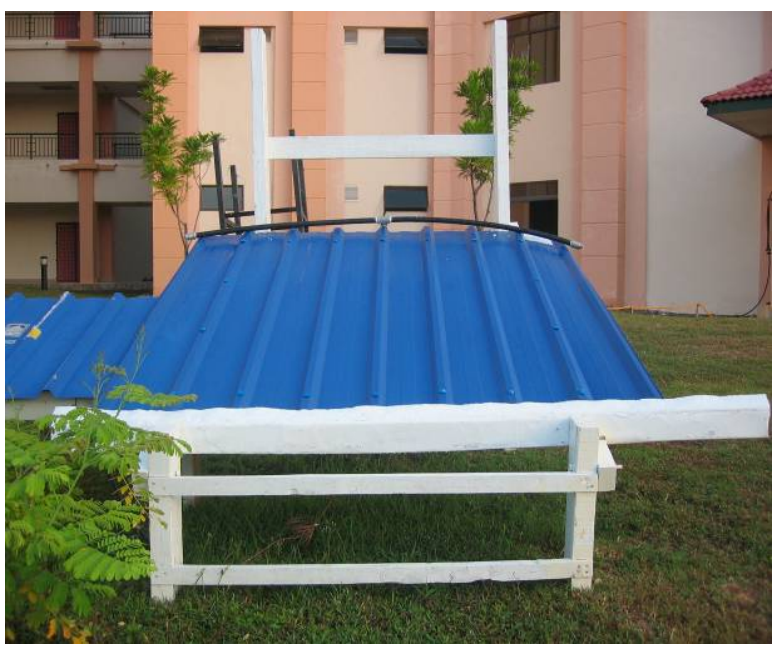

(f) Flooding Roof

Figure 2. Continued

'Figure 3 ' is the block diagram of the mist system and sprinkler system. In the flooding system, water was simply allowed to flow freely down the roof from an elevated tank. It may be observed from the photographs that the water was distributed over most of the roof section.

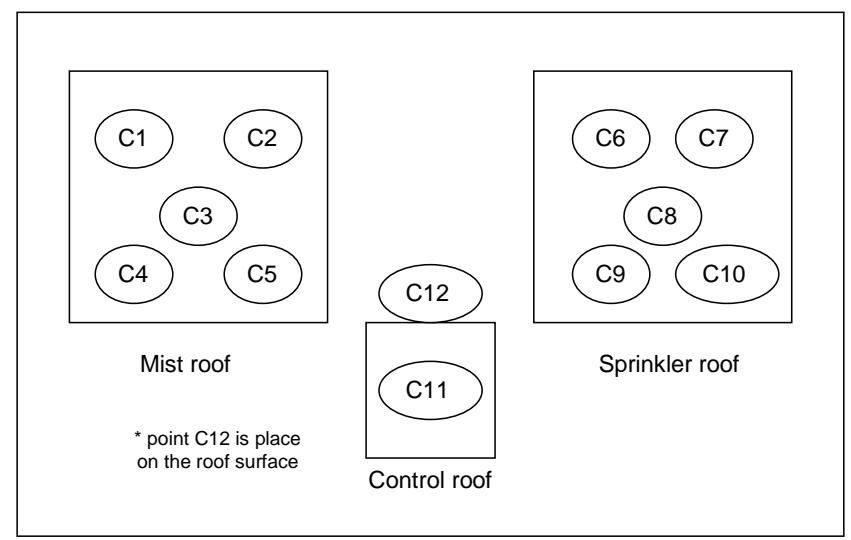

Figure 3. Configuration of thermocouples for temperature monitoring on mist spraying roof,sprinkler roof and control roof

\section{Equipment Components}

The type- $\mathrm{K}$ thermocouples applied were calibrated to within $\pm 0.33 \mathrm{~K}$ before they were attached to the roof. Temperature measurement (in ${ }^{\circ} \mathrm{F}$ ) were taken for all twelve points simultaneously at every minute. In 'figure 3 ' is the configuration of the monitoring points.

The maximum temperature of the roof surface achieved during this experiment was recorded at $60.7{ }^{\circ} \mathrm{C}\left(141^{\circ} \mathrm{F}\right)$ at 12:30 p.m. The heat capacity of a typical zinc roof at such high temperature is $393.15 \mathrm{~J} / \mathrm{kg} \mathrm{K}$ and with a thermo conductivity value of $110 \mathrm{~W} / \mathrm{m} \mathrm{K}$.

Typically the sun would rise at 6:30 a.m., the solar irradiation peaking at $1 \mathrm{pm}$, and the sun setting at $6: 25 \mathrm{p} . \mathrm{m}$. The roof would become hot to the touch when its temperature exceeded $37^{\circ} \mathrm{C}\left(100^{\circ} \mathrm{F}\right)$. Thus, for an ideal roof cooling design, it is most desirable to keep the roof temperature below about $37^{\circ} \mathrm{C}$.

The relative humidity ranged from 64 to $94 \%$ throughout the day. Wind speed ranged from 0 to $1.2 \mathrm{~m} / \mathrm{s}$ with the average at $0.3 \mathrm{~m} / \mathrm{s}$, i.e. reasonably calm.

The flow rate of the mist-spraying system was measured to be about 155 litre $/ \mathrm{hr}$, about the same as the flooding system at the flow rate of around 150 litre/hr.; in contrast the flow rate of the sprinkler system were measured to be at 83 litre $/ \mathrm{hr}$ for the $2.25 \mathrm{~m}^{2}$ roof. By scaling up to an area of $300 \mathrm{~m}^{2}$, the equivalent flow rate of the sprinkler system would be about 11000 litre $/ \mathrm{hr}$.

\section{RESUlt AND Discussion OF MEASUREMENT}

The following discussion is based on experiment dated $15^{\text {th }}$ Feb 2005 with the mist system operated at the 5 bar of pumping pressure, 15 min running with 5 min intermittent stop; for comparing with the flooding system, the mist system was operated at intervals of 10 minutes on and 5 minutes off, collected on $14^{\text {th }}$ February 2005. Roofs were at $20^{\circ}$ gradient from ground level. Only one day's data need be shown for discussion as the equatorial weather is highly consistent for sunny days by examining the data obtained from the local meteorological service [8]. 


\section{A. Hourly Analysis}

In the hourly analysis, the hourly average temperature of roof was plotted against the time of day in 'figure 4' for the mist-spraying and the sprinkler systems, and 'figure 5' for the flooding and mist-spraying systems.

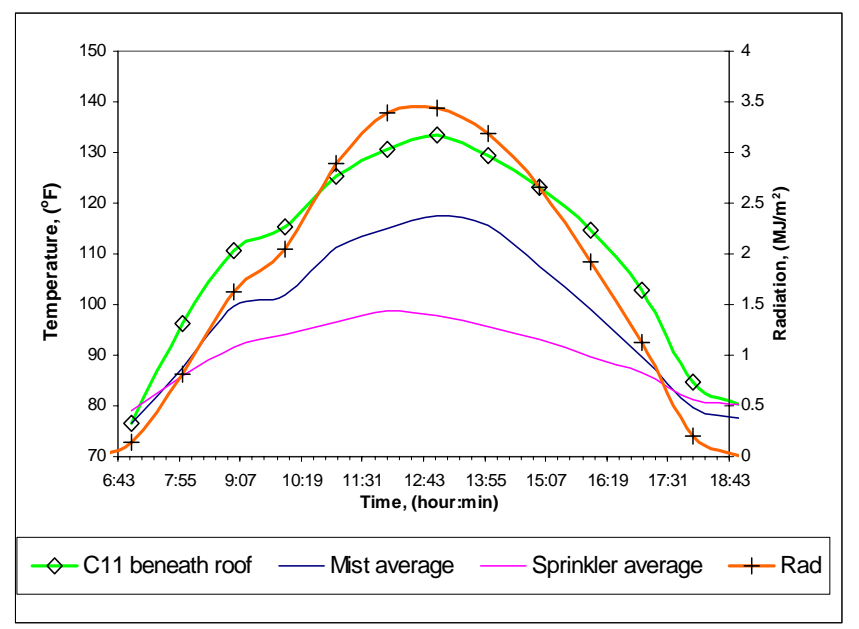

Figure 4. Temperature profile versus time comparison between the Mist-spraying and Sprinkler Systems

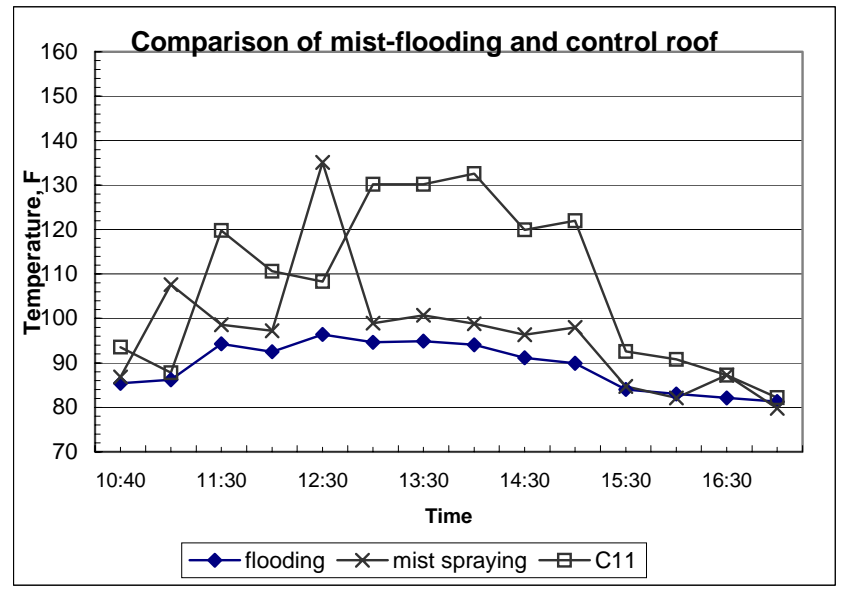

Figure 5. Temperature profile versus time comparison between the Mist-spraying and the Flooding Systems

The plot in 'figure 4' shows the temperature change of a typical metal roof during the radiation hours a) without any cooling b) mist cooling and c) sprinkler cooling. In this work, temperature $\mathrm{C} 11$ beneath roof is being used as the control set for reference. The roof surface temperature (C12) and temperature beneath the roof surface $(\mathrm{C} 11)$ are very close to each other, their difference is below $3 \mathrm{~K}\left(5^{\circ} \mathrm{F}\right)$. This is understandable as the roof is very thin, at $0.39 \mathrm{~mm}$. The hourly solar irradiation rate was obtained from the local weather service [5] and shows a direct relationship with the control roof temperature which is as expected. The mist spray, flooding and sprinkler systems, while showing a peak in the roof temperature during the peak radiation hour, exhibited lower temperatures than the control roof in the decreasing order of mist, sprinkler and flooding systems. It would appear therefore, that the cooling was due more to convection of heat from the roof to the flowing water than by the evaporation of water droplets as was intended for the mist spraying system.
Overall, the mist and sprinkler systems do reduce the roof temperature, but the sprinkler managed to maintain the roof temperature to below $40^{\circ} \mathrm{C}\left(105^{\circ} \mathrm{F}\right)$ throughout the day even at the peak sunshine hour. The mist system was however less stable showing fluctuations due to the mists being easily affected by the wind gusts, averaging $0.3 \mathrm{~m} / \mathrm{s}$ $(0-1.2 \mathrm{~m} / \mathrm{s})$. The nozzles of the mist system were also located at the bottom of the roof section and the mist sprayed upward. It might not be the best configuration for cooling purpose as the small mist droplets fell onto the roof by gravity only. The sprinkler nozzles on the other hand were designed for maximum impact of droplets to the ground and in this case, the roof. This is reflected by the fact that the maximum deviation in the sprinkler system was $5 \mathrm{~K}$ whereas the mist spraying system was $23 \mathrm{~K}$.

The flooding system also performs better than the mistspraying system and on par in cooling effect with the sprinkler system but the flow rate is much higher than the latter. The size of pump required to sprinkle a $300 \mathrm{~m}^{2}$ area of roof has been estimated to be at less than $1 / 4 \mathrm{HP}$ for 3000 $\mathrm{gal} / \mathrm{hr}$.

\section{B. Minute-wise Analysis}

The raw data plotted by the minute basis in 'figure 6' showed that an unexpected phenomenon was observed in the mist cooling system. At some points, the roof temperature can be seen to rise and exceed the control roof temperature, C11. Alternative measurement using surface probe and by swapping the thermocouples showed no change in the phenomenon. The cause of this behaviour might be that during the resting interval the droplets formed on the roof began to collect heat from the sun by their convex shape mimicking convex glass lens thus concentrating the radiation to the droplet area. This would continue until the temperature of the droplets on the roof was raised high enough to overcome the surface tension before allowing water vapour to escape by evaporation. However, to vindicate this hypothesis a microscopic study will have to be carried out which is beyond the scope of this paper.

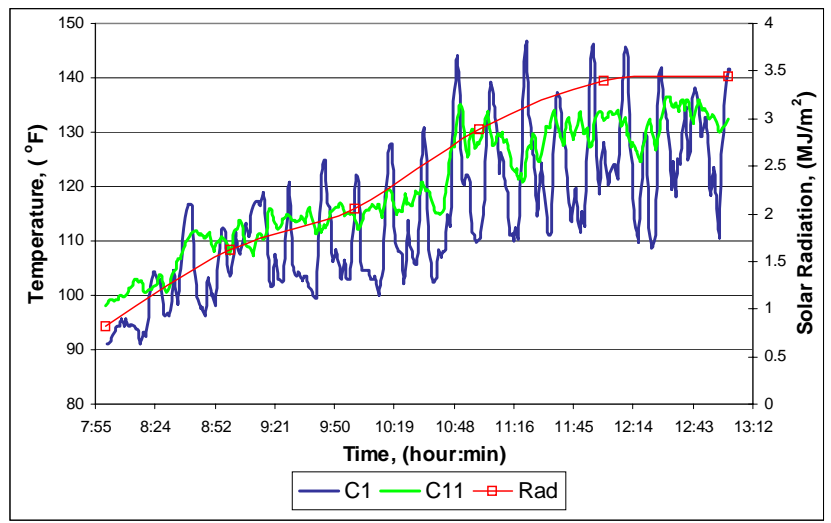

Figure 6. Temperature profile and solar radiation versus time comparison between the Mist-Spraying System (a) and the Control Roof (b)

The hourly and minute-by-minute analyses of the data collected show that the sprinkler type of roof cooling is the most effective and probably also most efficient of the three cooling systems. 


\section{Effect of Relative Humidity}

'Figure 7' is a plot of the roof surface temperature and relative humidity over 72 hours. The relative humidity data were obtained courtesy of Malaysian Meteorological Services [8]. Generally, both of the curves are out of phase from each other. Concurring with the theory of evaporative cooling, the greatest amount of evaporation cooling is found at the lowest surrounding humidity and vice versa as shown in 'figures 4 and 5 '.

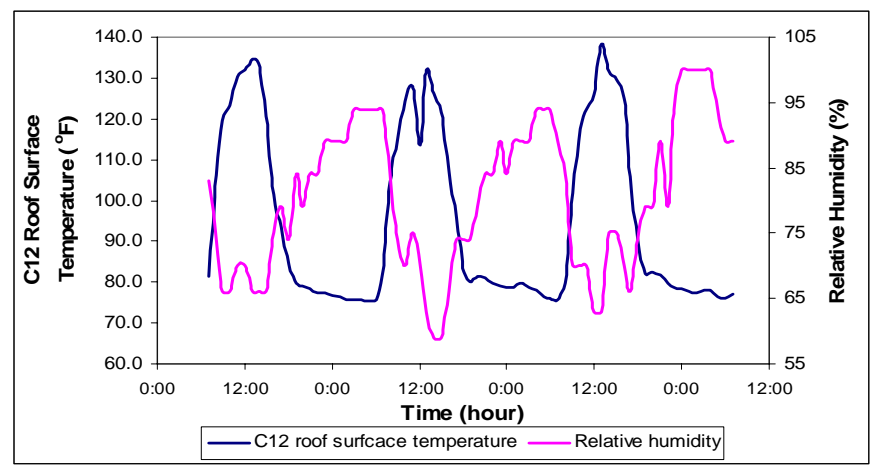

Figure 7. Typical roof surface temperature and relative humidity patterns (72 hours; from 7:00 a.m. 25 Feb 2005 to 7:00 a.m. 28 Feb 2005)

\section{CONCLUSIONS}

Three systems of roof cooling using water have been tested under identical conditions on hourly and minute-byminute basis using metal roof sections.

The most effective and efficient system is the sprinkler type as used for lawn watering. The temperature of the metal roof $\left(2.25 \mathrm{~m}^{2}\right)$ could be maintained below $40^{\circ} \mathrm{C}$ $\left(105^{\circ} \mathrm{F}\right)$.

The tests show that substantial reduction in consumption of electricity by mechanical air-conditioning is feasible. A series of tests on a complete loop of sprinkler cooling system should be carried out at a pilot-scale in order to design a cooling system which can be commercialized.

\section{REFERENCES}

[1] Nahar NM, Sharma P, Puurohit MM. 1999. Studies on solar passive cooling techniques for arid areas. Energy Conversion and Management. 40:89-95.

[2] Givoni, B. 1994. Passive and Low Energy Cooling of Buildings. New York: Van Nostrand Reinhold.

[3] Nayak, J. K.; Srivastava, A.; Singh, U.; Sodha, M.S. 1982. Building and Environment:The Relative Performance of Different Approaches to the Passive Cooling of Roofs. 17(2): 145-161.

[4] Sodha, M. S.; Govind; Bansal, P. K.; Kaushik, S. C. 1980. Building and Environment: Reduction of heat Flux by a Flowing Water Layer Over an Insulated Roof. 15: 133-140.

[5] White, C. R., Hanley, T. R. and Mathiasmeier, K.J., 1987, Heat Transport Model for Roof Mist Spraying Systems, ASHRAE Journal, V.29, No. 10, Oct, ISSN:0001-2491.

[6] Gandhidasan, P., 1989, Simplified Model for the behaviour of a roofspray cooling system, Applied Energy, v.34, No. 1, pp.69-77.

[7] Chu, C.M., A. Bono and A. Prabhakar, 2003, Possible Schemes for Solar-Powered Air-Conditioning in 2-Storey Terrace Houses, pp. 633-640, Environment Protection \& Energy Solution for Sustainable Development, Proceedings of the International Symposium on Renewable Energy, 14-17 September 2003, Kuala Lumpur, Malaysia.

[8] Malaysian Meteorological Services, $7^{\text {th }}$ Floor, Dang Bandang Building, 88000 Kota Kinabalu, Sabah, Malaysia. 


\title{
Burner Flame Observation by Using High Speed Video Image
}

\author{
A.K. Amirruddin, M.M. Rashidi and S.M. Sapuan
}

\begin{abstract}
The aim of this paper is to present the development of the high speed video observations of utility boiler flames. The main interest was to obtain close up video images of the root of the burner flame. An assessment of commercially available high speed cameras and endoscopes was carried out, and a high speed camera and a 1.5 meter long rigid endoscope were purchased. There were two types of looking probes being designed and built; a short forward looking probe and a long reach probe. The short forward looking probe was used for looking at a utility boiler flame by using the endoscope. It was tested at Combustion Test Facility (CTF) with the high speed camera. The high speed video footage shows the flow patterns of the main body of the flame. The long reach probe was to look at the root of the burner flame from close up by using a high speed camera placed at the end of the probe. This was tested at CTF and worked well, although the images of coal flames were not very clear. Through these findings, the technology required to build a long endoscope probe equipped with a high speed camera has been successfully tested.
\end{abstract}

Keywords: PF flame, burner flame image, high speed video image

\section{INTRODUCTION}

The behaviour of flames in large utility boiler plant is therefore of considerable interest both to operators and to researchers in improving burner technology. Flame behaviour can best be investigated using video cameras to record the flame. Video cameras are now widely used to provide operators with an overview of flame behaviour during running of plant. Researchers analyse the detailed video images of flames to determine temperature and stability patterns. The flow patterns of the flame, particularly in the near burner region, are also of interest to researchers, to enable them to compare Computer Fluid Dynamics (CFD) analysis with actual results, and to improve flameholder designs, also to achieve more stable

A.K. Amirruddin is with Automotive Focus Group, Faculty of Mechanical Engineering, Universiti Malaysia Pahang (UMP), Locked Bag 12, 25000 Kuantan, Pahang, Malaysia (Phone/Fax: +60-09-5942208/ 095942244, Email: amirruddin@ump.edu.my)

M.M Rashidi is with Faculty of Mechanical Engineering, Universiti Malaysia Pahang (UMP), Locked Bag 12, 25000 Kuantan, Pahang, Malaysia (Phone/Fax: +60-09-5942208/ 09-5942244, Email:mrashidi@ump.edu.my)

S.M. Sapuan is with Faculty of Engineering, Universiti Putra Malaysia, 43400 Serdang, Selangor, Malaysia (Phone/fax: +60-03-89466318, Email: sapuan@eng.upm.edu.my) burner flames. Due to the high velocity of a utility burner flame, a high speed video camera (in the order of 1000 frames per second) is needed to record flow patterns.

The aim of this paper is to present the development of the high speed video observation of utility burner flames. This involves building probes incorporating commercially available high speed cameras and endoscopes, and also probes using miniature charge-coupled device (CCD) cameras to prove long reach probe technology. The probes have to be designed to be safely introduced to the boiler, and be capable of withstanding the heat within the boiler. A 1.5 metre long forward looking endoscope probe was developed and tested before being inserted from a viewing port in the side wall of the boiler has been developed and tested. The advantage of this long reach probe is that a close up view of the flameholder and the quarl can be obtained. Once it is established that it is possible to get a good quality useful image, then an endoscope can be used in a similar probe, enabling the near burner region of the flame to be recorded using a high speed camera and be assessed in detail.

\section{BURNER FLAME}

Pulverised coal is transported to the burner in an air stream called the primary air. The extra air required for combustion (the secondary air) is supplied through a windbox and mixed at the throat of the burner. This allows the coal to ignite in the region known as the quarl. The combustion process continues as the coal burns in the flame, and as the gases and unburned coal flow through the boiler. Heat from the flame radiates back to the incoming pulverised coal, igniting it and sustaining the flame which is stabilised by a device called a flame holder.

$\mathrm{NO}_{\mathrm{x}}$ is one of the undesirable products of the combustion of fossil fuels, causing environmental damage. In pulverised coal combustion more than $75 \%$ of the $\mathrm{NO}_{\mathrm{x}}$ formed is fuel $\mathrm{NO}_{\mathrm{x}}[1]$. Fuel $\mathrm{NO}_{\mathrm{x}}$ is the $\mathrm{NO}_{\mathrm{x}}$ formed by the oxidation of the nitrogen present in the fuel. High oxygen availability and high flame temperatures during devolatisation encourage the conversion of volatile released nitrogen to $\mathrm{NO}_{\mathrm{x}}$. The most cost effective way to tackle the problem of $\mathrm{NO}_{\mathrm{x}}$ is to suppress the formation of fuel $\mathrm{NO}_{\mathrm{x}}$, by reducing the availability of air during the devolatisation stage is known as air staging. It can be achieved by adding some of the combustion air at a different location in the boiler, thereby reducing the amount of oxygen available while the devolatisation step is taking place. Careful burner design 
can also achieve a type of air mixing, where only some of the combustion air is allowed to mix with the fuel during the devolatisation stage. In low $\mathrm{NO}_{\mathrm{x}}$ burners, the secondary air is split into a secondary and a tertiary stream.

Due to extreme temperature inside a utility boiler, it is difficult to operate image recording equipment within the boiler. It is possible to have a camera looking through a viewing port window, but the angle of the view is restricted as the window may become dirty quickly and impossible to get a close up view. New plant is now being built with a video camera unit embedded into the boiler wall, primarily to provide the operator with more information to help control the boiler. Researchers have also installed large video monitoring systems into utility boilers to record flame images [2]. Most video probes use an endoscope to keep the camera remote from the intense heat of the boiler [3], [4], [5] but there have also been video probes using miniature cameras in a water cooled protective housing [3]. Most probes use a compressed air flow to help with cooling and to keep the objective lens clean.

Most of the published research about image detection and processing are from universities in China. This may well be a reflection of the fact that most western countries are moving away from coal as a fuel for power generation. In analysing the flame images, some researchers have used colorimetric temperature measurement techniques to establish temperature distributions across the boiler [6]. The stated aim of this research is to develop automatic control systems that use pattern recognition of analysed images, to obtain a more efficient operation of plant. A 2-D temperature distribution from a 2-D image of the burner flame has been generated using a two-colour pyrometer, based on Wein's law of radiation, again with the intention of improving combustion control systems [2]. Another algorithm has been developed for visualisation of flame temperature, using a ratio pyrometer, based on similar principles to the above methods [4]. These researchers are hoping to identify relationships between the visualised characteristics of the flame and $\mathrm{NO}_{\mathrm{x}}$ emissions and flame stability.

In other research the relative luminous intensities of the flame image have been used to calculate a uniformity function based on intensity deviation across the flame [5]. This has been shown to be capable of characterising the flame and showing correlation of calculated image parameters with fuel/air flow rates and emissions of $\mathrm{CO}_{2}$ and NO.

Research at Imperial College has based on image analysis on intensity and colour values [3]. Statistical treatment of this data has lead to approximate temperature distribution information, and also patterns of temperature variation. The usefulness of high speed video images has been highlighted, as normal speed video appears as a collection of random images, with no discernible link between them. It is hoped that high speed video will allow flow mechanisms to be recognised and calculated. No published research on high speed video monitoring of utility boilers has been found. Therefore, the work on high speed video monitoring of utility boiler is presented in this paper.

\section{High SPEEd High Resolution CMOS}

The High-Speed High Resolution CMOS Camera is shown in Figure 1. An image at rate up to 500 frame/sec. can be recorded at a resolution of 1024 x 1024 effective pixels. The flow pattern of the flame can be recorded and studied.

The HCC-1000 camera was mounted on the left side viewing port window, facing $45^{\circ}$ towards the burner quarl. The HCC-1000 camera offers superior resolution that would be important when analysing the images to determine flow patterns. The specified requirement for this project is to use a camera capable of recording 1000 frames per second able to provide a video image of adequate quality. The main disadvantage with this camera is that it does not have a colour image. However, it will still be possible to compare the relative brightness of the flame, and it is expected that colour information can be recorded by using different colour filters and its high resolution.

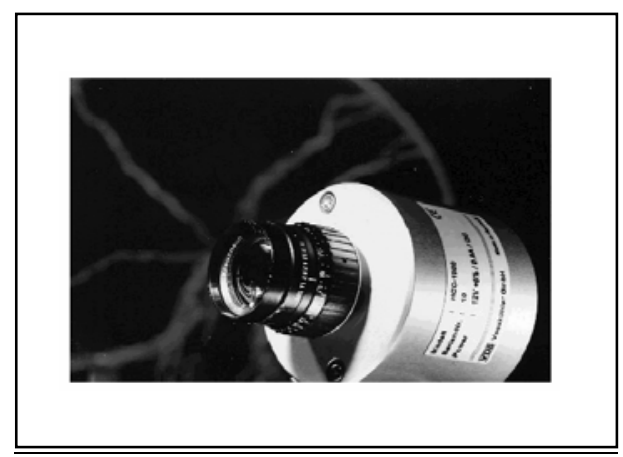

Figure 1. The high-speed high resolution CMOS camera

\section{ENDOSCOPE CRITERIA CHOSEN}

It is not practical to have the high speed camera inside the furnace, as it is too large to fit through most probing ports. Also the risk of destroying the camera through a loss of the cooling system is too great to allow the camera to be placed inside the furnace. However, it is possible to set the camera up looking through a viewing port window at the Combustion Test Facility (CTF). Despite the simplicity of this solution, it is not the best option because the view of the flame is restricted and the inside of the window may get dirty, with no way to clean it properly while the burner is in operation. The best way to get an overall view of the flame, with a system that is easily transferable to other boilers, is to use an endoscope probe. This is also the method currently used for achieving ordinary video images of boiler flames.

For the purpose of recording high speed video footage of the entire flame, a forward looking endoscope of about 1.5 metres in length was required. In order to get close up high speed video footage of the root of the flame, a sideways looking endoscope of about 5 metres in length was inserted from the back of the burner. The types of endoscope used in each application were too rigid; endoscope, based on a series of relay lenses, or a flexible endoscope (fiberscope), and made from bundled flexible optical fibres.

The endoscopes of immediate interest to this project were rigid endoscopes of $8 \mathrm{~mm}$ or $10 \mathrm{~mm}$ diameter, available in lengths of approx. $820 \mathrm{~mm}$ with extensions in multiples of approx. $300 \mathrm{~mm}$. In order that the best quality of image is obtained, the endoscope should have the largest possible diameter, to ensure that as much light as possible is 
transmitted, and also the shortest length necessary, so that there is minimal image degradation. From tests with an ordinary CCD camera it was clear that the rigid endoscope provided a higher quality image at short lengths. They can easily make a 5 metre long flexible endoscope. It would be made from either 30,000 strands or 50,000 strand fibre optic cable. This equates to a square pixel resolution of $173 \times 173$ or $224 \times 224$. This is less than the high speed camera resolution, and it was decided that a further demonstration was necessary once we had acquired the high speed camera.

The endoscope required for a long reach probe has to be a flexible fiberscope. It was possible to focus the endoscope so that the individual pixels formed by individual fibre optic strands could be seen, but the actual image was not very clear. It did not seem that a fiberscope could provide an adequate quality of image. The endoscope can be provided in $14 \mathrm{~mm}$ and $18 \mathrm{~mm}$ diameter sizes, and with 0.5 metre or 1 metre extension tubes. It is possible to have a rigid endoscope of overall length of 5 metres or even more, but that breaks down easily into short lengths. These include forward looking, $45^{\circ}, 90^{\circ}$ (sideways looking) and $110^{\circ}$ (looking slightly backwards). There is also an adjustable lens, which can be angled between $60^{\circ}$ and $110^{\circ}$. It was decided that an extendable rigid endoscope would be the best option for the long reach video probe for use with the high speed camera.

\section{Design Consideration OF Short Endoscope Probe}

The design of the short (1.5 metre long) endoscope probe requires that it has a cooling system, so that it would not overheat when exposed to the inside of the boiler during operation. Only the tip of the endoscope with the objective lens was exposed to the radiation in the boiler and the main body of the endoscope needed a heat shield to protect it. The cooling system used compressed air, as it was generally easily available on site compared to cooling water. The main advantage of using air was that the cooling system need not be closed. It was possible to let the cooling air exhaust into the boiler. If the air flow can be properly arranged, it could keep the lens clean and free from dust. The port where the probe was mounted was sealed around the heat shield of the endoscope, so that the camera is always protected from the boiler radiation. The probing port used at CTF was fitted with a ball valve, so that the port can be opened and closed, and also a flange, so that a mounting plate can be fixed to the port. The method of fixing the probe allowed it to be quickly removed from the boiler when there was a problem such as the loss of air supply.

The design of the probe used a double layer of stainless steel tube as the heat shield around the endoscope. Which $15 \mathrm{~mm}$ outer diameter and provided a close surround to the endoscope. The internal diameter of the tube was $13 \mathrm{~mm}$ with the external diameter of the endoscope was $10 \mathrm{~mm}$. That left a $1.5 \mathrm{~mm}$ thick annulus for cooling air. There was an excellent heat transfer in that space, so that the endoscope was well cooled. The outer tube was larger, one of the standard available sizes of $18,22,28,35,42$, or $54 \mathrm{~mm}$ outer diameter. The size chosen was determined by the best size for protecting the tip of the endoscope while still allowing a full viewing angle. Cooling air was passed inside the outer tube.

At the open end of the probe, where the tip of the endoscope was exposed, it is necessary to have a reducing area for the air flow. This was to ensure that there was a convergent air flow, giving a favourable pressure gradient, so that there would not be any flow separation and recirculation, which could bring fly ash into the probe. Ash could result a build-up of slag around the probe, or the lens becoming dirty. For a reducing area, it was necessary to have a reducing cone at the end of the outer tube. It was also necessary to ensure that there was a reduction in area from the pinch point between the inner and outer tubes and the hole in the cone on the end of the outer tube. This geometry is shown schematically below in Figure 2.

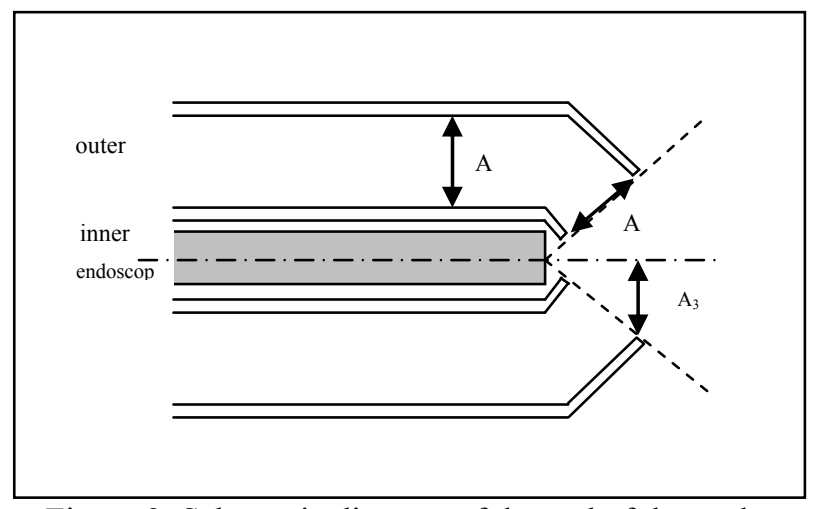

Figure 2. Schematic diagram of the end of the probe geometry

The end of the inner tube was formed into a slight cone at the end as to help in keeping recirculated eddies from forming in the air flow which would bring dirt directly onto the lens. The variation in the three areas indicated in Figure 1 , gave the preferable size of the outer tube and the suitable size for the hole in the end cone. It was decided that a 54 $\mathrm{mm}$ outer diameter tube would give the best air flow reduction while providing an adequate radiation shield. The size of the hole in the end cone was made to be $30 \mathrm{~mm}$ diameter, allowing a viewing angle of $80^{\circ}$. Finished probe gave the following areas and area ratios as shown in Table 1.

Table 1. Probe size

\begin{tabular}{l|l|l}
\hline & Area & Are ratio \\
\hline Pipe; $\mathrm{A}_{1}=\pi \times\left(25.5^{2}-7.5^{2}\right)$ & $1866 \mathrm{~mm}^{2}$ & $\mathrm{~A}_{2} / \mathrm{A}_{1}=0.523$ \\
$\begin{array}{l}\text { Pinch Point; } \mathrm{A}_{2}=\pi \times\left(15^{2}-5^{2}\right) \\
/ \text { sin40 }\end{array}$ & $975 \mathrm{~mm}^{\circ}$ & $\mathrm{A}_{3} / \mathrm{A}_{2}=0.725$ \\
Exit; $\mathrm{A}_{3}=\pi \times 15^{2}$ & $707 \mathrm{~mm}^{2}$ & $\mathrm{~A}_{3} / \mathrm{A}_{1}=0.379$ \\
\hline
\end{tabular}

\section{THE ENDOSCOPE FABRICATION}

The end cone of the outer tube was manufactured by initially turning the cone piece on a lathe and then welding it onto the $54 \mathrm{~mm}$ diameter tube. The small end cone of the inner tube was made by cutting short (5 $\mathrm{mm}$ long) axial slots at the end of the $15 \mathrm{~mm}$ diameter tube and bending the end of the tube slightly inwards. The back end of the $15 \mathrm{~mm}$ diameter tube was fitted with an equal $\mathrm{T}$ fitting, so that air could be supplied into the tube. Behind the equal $\mathrm{T}$ fitting a $1 / 2$ " BSP female thread to $15 \mathrm{~mm}$ fitting was attached. This 
was done so that the endoscope could be connected to the probe using its own $1 / 2$ " BSP thread.

In order to seal the back end of the $54 \mathrm{~mm}$ diameter tube, a rubber bung was used. This provided an airtight seal and was remote enough from the boiler radiation not to be damaged. A hole was drilled in the centre of the bung for the $15 \mathrm{~mm}$ inner tube to pass through, again providing an airtight seal around the $15 \mathrm{~mm}$ tube. Another hole was drilled in the bung for an air supply pipe, allowing compressed air to be delivered between the inner and outer tubes. A smaller third hole was drilled in the bung so that a thermocouple could be passed through the probe and fixed to measure the temperature at the hot end of the probe. The rubber bung was pressed into the $54 \mathrm{~mm}$ tube so that it had a tight fit, and was then secured with a retaining wire fixed to the outside of the $54 \mathrm{~mm}$ tube with a jubilee clip.

The air supply was connected to the probe using $15 \mathrm{~mm}$ diameter reinforced PVC hose. The air line was split using a three way $\mathrm{T}$ piece and connected with $15 \mathrm{~mm}$ hose to the connection tubes for both the inner tube and outer tube air supply. All the hose connections were fastened with jubilee clips. The method of keeping the inner tube aligned axially with the outer tube was an important issue. The first option considered was to have rigid spacers attached to the inner tube. However the concern was that with thermal expansion of the outer tube, the spacers would lose contact with the outer tube and the inner tube would be able to move around inside it. With a fast air flow around it, the inner tube could start to shake about, and it then would not be possible to record a steady image. This would present problems for image analysis software. Therefore it was decided that the positioning system needed to be sprung. The danger of this approach of course being that if it was not sufficiently rigid there would still be movement and an unsteady image produced. To correctly align the inner tube with a spring system, three thin lengths of thin stainless steel sheet were each bent into a hump and attached to the inner tube at $120^{\circ}$ to each other with jubilee clips. These were adjusted so that when the inner tube was inserted in the $54 \mathrm{~mm}$ tube, it was properly aligned and held tightly in place. The completed probe is shown schematically in Figure 3.

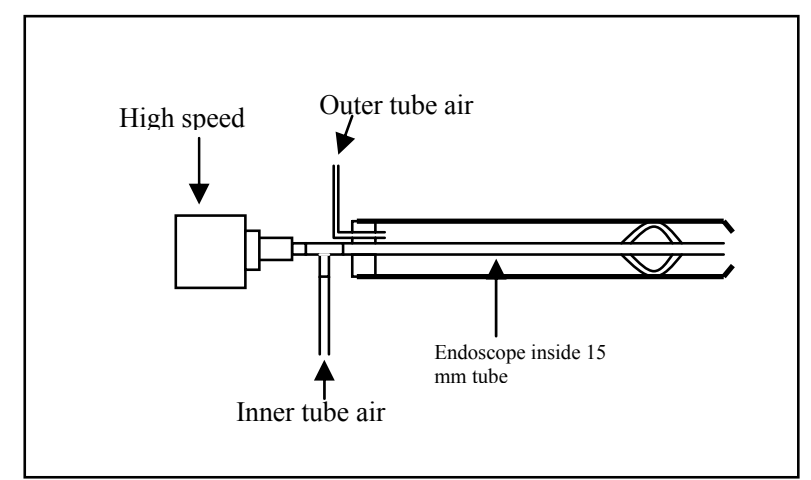

Figure 3. Schematic diagram of the short forward looking endoscope probe

\section{High SpeEd CAMERA Probe IMAge Trial OBSERVATIONS}

All the equipment was set up on site for testing the probe with the high speed camera. This included a computer, as the high speed camera was controlled by computer, and all the video sequences were downloaded directly to the computer. The probing port to be used for the probe was prepared with a ball valve and a flange plate with a $54 \mathrm{~mm}$ diameter compression tank fitting to hold the probe securely in the positive pressure furnace.

The port used was in the side wall of the furnace, looking at a right angle directly at the flame. The position of the port was close to the burner wall, so that the view included the flame leaving the quarl and the main body of the flame. The procedure followed for inserting the probe into the furnace was initially all line up the probe with the port closed. Then the air supply was turned half on, and the port was opened. Then the probe was inserted halfway into the furnace. The air supply was turned fully on and then the probe was finally fully inserted into the furnace and the tank fitting was tightened. The procedure followed for removing the probe was the reverse of the above procedure.

The tip of the thermocouple in the probe was bent round the end of the endoscope to monitor the temperature of the objective lens. The initial temperature reading was $15^{\circ} \mathrm{C}$. This slowly increased to $20^{\circ} \mathrm{C}$ after about 30 minutes and had reached $25^{\circ} \mathrm{C}$ after about 1 hour. This may not be the actual temperature of the objective lens, but it gives confidence that the tip region of the endoscope was kept reasonably cool despite being exposed to the furnace. When the probe was removed from the furnace after it had been in place for more than 1 hour, the outer tube was very hot, but the inner tube was cold. It could be seen that the outer tube had run red hot because the stainless steel at the end of the probe has changed colour.

The initial results from the probe were disappointing because of difficulty in getting the image focused well. However, on the second day of testing the endoscope was able to focus better. Three high speed video sequences of a pulverized coal flame were recorded. The quality of these video images was encouraging. When played as a slowed down video sequence the results of this test were good enough to give an idea of the flow pattern of the flame. Shown in Figure 4 is a video image recorded at 923 frames per second.

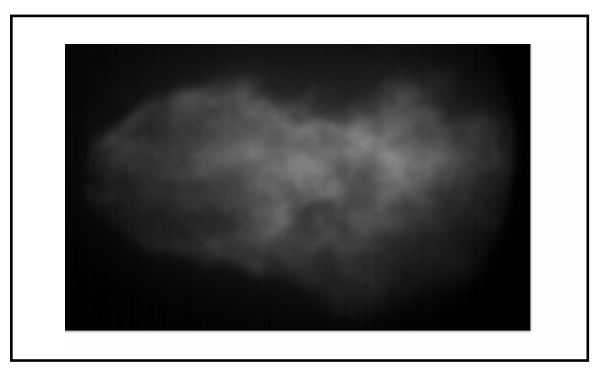

Figure 4. A high speed video images of a PRB coal flame

With those features mentioned in the above section, the flame taken during the start up of oil flame showed clearly the burner and quarl components by using long reach video probe. The image is as shown in Figure 5.

The image of the coal flame root, immediately downstream of the flameholder was captured when the oil fuel was switched to coal. A number of video sequences (each approximately 1000 frames per second) of this coal flame were collected. When the high-speed cameras operate at such speed, images with small enough relative motion 
between the frames would show the true flame flow pattern. From the sequences, it could be viewed for the first time that there was an existence of a strong reverse recirculation zone in the outer layer of the anchored flame. The swirling motion was found along with a forward flowing secondary air immediately outside the burning flame. One of the frame examples is as shown in Figure 6.

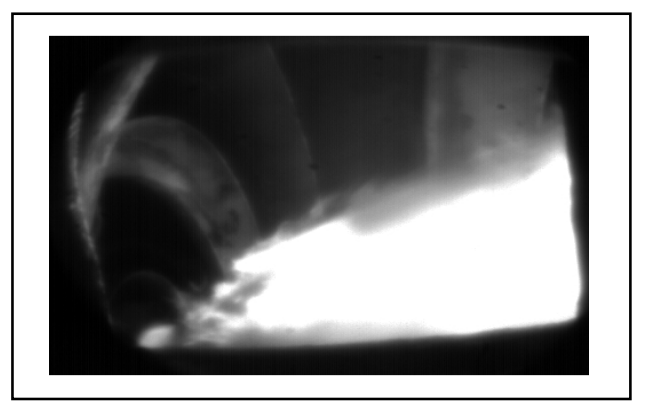

Figure 5. View of burner flame holder and quarl region obtained using high speed long reach video probe (trial on oil)

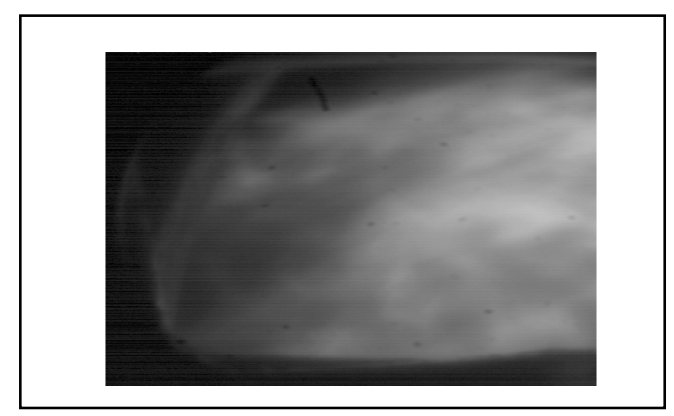

Figure 6 . View of the flame when the burner was on coal running trial obtained using high speed long reach video probe

\section{CONCLUSIONS}

The conclusion of the test was that the probe had worked correctly, allowing the endoscope to be exposed to a full size burner flame and record high speed video footage of it. However the quality of the images, while sufficient for a qualitative analysis, may not be good enough for a detailed quantitative computer software based analysis.

An assessment of high speed video cameras and an assessment of available endoscopes were carried out at the start of the project. Then a short forward looking endoscope probe was designed and tested at CTF. The quality of the image was good enough for a qualitative analysis of the flow pattern of the flame to be carried out, but was not as sharp as had been hoped. However, when compared to an industrially made endoscope probe, it was found that the sharpness of the image was comparable.

The video trials were considered satisfactory because the camera probe could be inserted and function correctly in allowing the endoscope to be exposed to a full size burner flame and recorded the high-speed video footage of the flame. The quality of the images was sufficient for a qualitative analysis but inadequate for a detailed quantitative computer software based analysis. However, when compared to an industrially made endoscope probe, it was found that the sharpness of the image was comparable.

\section{ACKNOWLEDGEMENTS}

I would like to thanks Dr. Jon Gibbins, Thermofluids Group, Imperial College London for the ideas and opinion for this research.

\section{REFERENCES}

[1] Stultz, S.C., ed. (1992) Steam / its generation and use, $40^{\text {th }}$ edition, The Babcock \& Wilcox Company, Barberton, Ohio, U.S.A.

[2] Zhou, H.-C.; Zhang, S.-S.; Huang, Y.-L.; Zheng, C.-G. (2000) Monitoring of 2-D combustion temperature images in a $670 \mathrm{t} / \mathrm{h}$ utility boiler and simulation of its application in combustion control. Developments in Chemical Engineering and Mineral Processing, vol. 8, n 3, pp. 311-322.

[3] Gibbins, J.; Lin, Y.-M.; Bowden, S.; Cameron, S. (2001) Video observations of full-size pulverised coal flames. Combustion Science \& Technology, n 162, pp. 263-280

[4] He, W.; Yu, Y.; Xu, W.; Ma, L. (1999) Development of video processing based on coal flame detector system. American Society of Mechanical Engineers, Fuels and Combustion Technologies Division, vol. 23, n 1, pp. 349-353.

[5] Lu, G.; Yan, Y; Ward D.D. (2000) Advanced monitoring, characterisation and evaluation of gas-fired flames in a utility boiler. Journal of the Institute of Energy, vol. 73, n 494, pp. 43-49.

[6] Huang, Y.; Qu, T.; Zhou, H.; Yuan, P.; Han, S. (2000) Application of $\mathrm{CCD}$ images and colorimetry temperature measure for combustion monitoring and control. Proceedings of SPIE - The International Society for Optical Engineering, vol. 4077, pp. 184-188. 


\title{
Physical Properties of Local Palm Oil Clinker and Fly Ash
}

\author{
Mohd Hilton Ahmad and Nurazuwa Mohd Noor
}

\begin{abstract}
As Malaysia will focus on biotechnology field as indicated in Ninth Malaysian Plan (RMK9) will bring tremendous effects to the local palm oil industry which also produces palm oil clinker as its waste by-product. This paper will studied the suitability of palm oil clinker to be used as coarse and fine aggregate for palm oil clinker concrete production. The parameters studied includes sieve analysis, specific gravity, moisture content, water adsorption, bulk density, aggregate impact value, crushing aggregate value, etc. Another local waste material from coal combustion, pulverized fuel ash (pfa) which react as pozzolanic material which have been proved by previous researchers as cement replacement in concrete mix proportioning to enhance the concrete properties also been studied for its physical properties. This study includes consistency of cement-fly ash paste, initial and final setting of different proportion of cement-fly ash paste as well as the specific gravity of fly ash. All parameters for local palm oil clinker and pulverized fly ash were conducted and measured according to British Standard. All parameters were analyzed and compared to the values that been proposed by British Standard. The results given by the palm oil clinker and fly ash were found to be in good agreement with the concrete testing values for structural use.
\end{abstract}

Keywords: sieve analysis; consistency of binders; specific gravity; palm oil clinker; pulverized fly ash

\section{INTRODUCTION}

As a major manufacturer of palm oil, Malaysia produces lots of palm oil products. Palm oil industry is also an establish business in Malaysia which is still developing and growing. In the palm oil mill, after it is being processed, the palm oil shells are used as burning fuel and as a result, the waste produced by this process is called the clinker. The clinker is normally treated as waste and has no economical value. Therefore, it will be very useful if the palm oil clinker can be recycled as a form of industrial material, thus eliminating waste and providing a cheap option to manufacture concrete. It will be of much benefit to the construction industry as to minimize cost at the same time preserve natural aggregates.
Mohd Hilton Ahmad is with the Faculty of Civil and Environmental Engineering, Universiti Tun Hussein Onn Malaysia, 86400 Parit Raja, Batu Pahat, Johor, Malaysia. (Corresponding author. Phone: 607-4537320; fax: 607-4536070; e-mail: hilton@uthm.edu.my).

Nurazuwa Mohd Noor is with the Faculty of Civil and Environmental Engineering, Universiti Tun Hussein Onn Malaysia, 86400 Parit Raja, Batu Pahat, Johor, Malaysia. (e-mail: nurazuwa@uthm.edu.my).
Due the limitation and difficulties in finding strong natural aggregate in certain region, artificial aggregate has been used widely to replace the natural aggregates. Current technologies and discoveries has made it possible to create a strong artificial aggregate that out performs natural aggregates. Many studies and research has been carried out to improve and refine on the aggregate itself. In many cases, natural aggregate has been substituted with artificial aggregate to provide that extra strength that natural aggregate are difficult to provide.

This paper reports the results of an investigation into the physical properties of palm oil clinker and fly ash. Both components are essential to produce stronger and durable lightweight palm oil clinker concrete. Studies by Neville shows that $10 \%$ replacement was selected as it gave the optimum reaction.

\section{MATERIALS AND EXPERIMENTAL PROCEDURES}

\section{A. Palm Oil Clinker as Aggregate}

The Palm Oil Clinker (POC) was taken from the palm oil mill factory from Dengkil. The factory is Sri Ulu Langat Palm Oil Mill. The by-product that is collected from inside the boiler is called clinker. The clinker looks like a porous stone which is gray in color. The process of producing palm oil clinker in palm oil mill is shown in Table 1. All the clinkers are prepared to be crushed into required size. Clinker with nominal size of $20 \mathrm{~mm}$ is used as coarse aggregate and size below $5 \mathrm{~mm}$ is used as fine aggregate. The clinkers were flaky and irregular shaped. The broken edges were rough and spiky.

The physical properties such as water adsorption, moisture content and bulk density are shown in Table 2. Palm oil clinker and conventional coarse aggregate, i.e., gravel used in saturated surface dry condition. 
Table 1. Production process of palm oil clinker

\begin{tabular}{|c|c|c|}
\hline Process & Activity & Remarks \\
\hline 1 & Sterilize & $\begin{array}{l}\text { - The raw palm oil is } \\
\text { transferred from the farm to } \\
\text { the factory and is boiled in } \\
\text { hot water to sterilize the } \\
\text { palm oil. } \\
\text { - This sterilization process } \\
\text { also softens the palm oil to } \\
\text { make it easier for stripping } \\
\text { later on. }\end{array}$ \\
\hline 2 & Stripping & $\begin{array}{l}\text { - The trunk of the palm oil is } \\
\text { stripped in a rotating drum } \\
\text { "stripper" and the palm oil } \\
\text { fruits are separated from the } \\
\text { tusk. } \\
\text { - The tusk is thrown away } \\
\text { and regarded as waste. }\end{array}$ \\
\hline 3 & Extraction & $\begin{array}{l}\text { - A process where the palm oil } \\
\text { is crushed so that the palm } \\
\text { oil in the fruit section will be } \\
\text { extracted. } \\
\text { - This process will extract the } \\
\text { juice (palm oil) and leaves } \\
\text { the skin, seed and the } \\
\text { remaining fibrous material. }\end{array}$ \\
\hline 4 & Separation & $\begin{array}{l}\text { - } \text { This separation process } \\
\text { separates the seed with the } \\
\text { skin and the fibrous material } \\
\text { because the seed is useless. } \\
\text { - The fibrous material still } \\
\text { contains some palm oil and } \\
\text { is ideal for burning to } \\
\text { regenerate power. }\end{array}$ \\
\hline 5 & Burning & $\begin{array}{l}\text { - The remaining fibrous } \\
\text { material is sent to a furnace } \\
\text { where it is burnt in } \\
\text { temperature more than } 500 \\
\text { degrees Celsius to power the } \\
\text { steam engine which in turns } \\
\text { generates power. } \\
\text { The burning process can last } \\
\text { for more than } 24 \text { hours to } \\
\text { give a complete burn. }\end{array}$ \\
\hline 6 & Collection & $\begin{array}{l}\text { After the burning process, } \\
\text { the fibrous material is now } \\
\text { transformed into a hard and } \\
\text { solid compound which we } \\
\text { called the "Clinker". } \\
\text { The Clinker is then collected } \\
\text { at the base of the furnace } \\
\text { where it is either disposed of } \\
\text { as landfill or being recycled } \\
\text { for better usage. }\end{array}$ \\
\hline
\end{tabular}

Table 2. Physical properties of POC and gravel

\begin{tabular}{lll}
\hline Physical properties & $\begin{array}{l}\text { Coarse } \\
\text { aggregat } \\
\text { e POC }\end{array}$ & $\begin{array}{l}\text { Crushed } \\
\text { stone }\end{array}$ \\
\hline Specific gravity (SSD condition) & 2.17 & 2.60 \\
Moisture content (\%) & 0.08 & 0.05 \\
Water adsorption (\%) & 4.65 & 1.79 \\
Bulk density (kg/m $\left./ \mathrm{m}^{3}\right)$ & 863.65 & 1815.23 \\
Fineness Modulus (Fine aggregate) & 2.84 & 2.65 \\
\hline
\end{tabular}

\section{B. Fly Ash}

Fly Ash was taken from TNB Kapar in Selangor. It is byproduct from power generator which broadly used as pozzolans in concrete technology. The fly ash used for this experiment belongs to type "F". It is grey in color and can be directly used upon collection.

\section{Other Concrete Mix Components}

River sand was used as fine aggregate which has a fineness modulus of 2.56. Fly ash and Malaysian Ordinary Portland Cement was used as binders. Superplasticizers also been used as an admixtures for water-reducing agent.

\section{Standard Code of Practice}

For palm oil clinker test, there is a few test was taken; i.e., sieve analysis, moisture content, water adsorption, specific gravity, bulk density (bulk and loose), aggregate crushing value (ACV) and aggregate impact value (AIV). On the other hand, fly ash which used as partial binder was undergoing few tests, i.e., specific gravity, consistency of binders paste, initial and final setting of binders paste and Blaine surface area.

There is 2 major code of practice was implemented; i.e.; British Code of Practice which is mostly similar to Malaysian code of Practice and American Standard Testing Material (ASTM). Table 3 shows the code of practice been done for each type of test.

Table 3. Code of Practice for Aggregates Testing

\begin{tabular}{|l|l|l|}
\hline $\begin{array}{l}\text { No } \\
1\end{array}$ & Testing & Code of Practice taken \\
\hline 2 & Sieve Analysis & BS 812: Part 1: 1975 \\
\hline 3 & Waisture Content & BS 812: Part 109: 1990 \\
\hline 4 & Specific Gravity & $\begin{array}{l}\text { BS 812: Part 107 } \\
\text { (Draft) }\end{array}$ \\
\hline 5 & Bulk Density & $\begin{array}{l}\text { BS 812: Part 107 } \\
\text { (Draft) }\end{array}$ \\
\hline 6 & $\begin{array}{l}\text { Aggregate Crushing } \\
\text { Value }\end{array}$ & BS 812: Part 2: 1975 \\
\hline 7 & Aggregate Impact Value & BS 812: Part 112: 1990 \\
\hline 8 & $\begin{array}{l}\text { Aggregate Abrasion } \\
\text { Value }\end{array}$ & $\begin{array}{l}\text { BS 812: Part 113 } \\
\text { (Draft) }\end{array}$ \\
\hline
\end{tabular}


Table 4. Code of Practice for Binders Testing

\begin{tabular}{|l|l|l|}
\hline $\begin{array}{l}\text { No } \\
\cdot\end{array}$ & Testing & Code of Practice taken \\
\hline 1 & $\begin{array}{l}\text { Specific surface } \\
\text { (Fineness) }\end{array}$ & ASTM C 115-93 \\
\hline 2 & Initial and Final setting & ASTM C 191-92 \\
\hline 3 & Specific Gravity & BS 4550: Part 3: 1978 \\
\hline
\end{tabular}

\section{RESULTS AND DISCUSSION}

\section{A. Palm Oil Clinker Material Test}

\section{Visual Inspection}

Particle shape and surface texture classification of aggregates is given by BS 812: Part 1: 1975. Since palm oil clinker come in a big size and it is crushed to a maximum size of $20 \mathrm{~mm}$ to be used as coarse aggregates. We can see a possessing of well-defined edges at the intersection of roughly plane faces and rough fracture of medium-grained rock containing no easily visible crystallium constituents, we can classify that palm oil clinker aggregate is angular and rough.

Visual inspection of both properties follows to BS812: Part 1: 1975 to reduce the misunderstanding the classification. External features of the aggregates, especially particle shape and surface texture are essential of fresh and hardened concrete properties. Rough plane faces and rough textures of palm oil clinker aggregates can contribute to good bonding with the mortar constituents.

\section{Size Classification}

Palm oil clinker (POC) aggregates has a range of size up to a maximum size of $20 \mathrm{~mm}$. The massive size of POC taken from palm oil mill is crushed to the desired shape with a maximum size of $20 \mathrm{~mm}$. To determine the range of sizes particle aggregate used, sieve analysis is carried out using vibrator shaker which the results are shown in Figure 1. From the graph in Figure 1, coarse aggregate has beyond its lower upper case for $10 \mathrm{~mm}$ and $14 \mathrm{~mm}$ sieve size. However, the fall is not significant and acceptable. The fine aggregates show the well grading as its entire particle sizes falling within the specified grading.

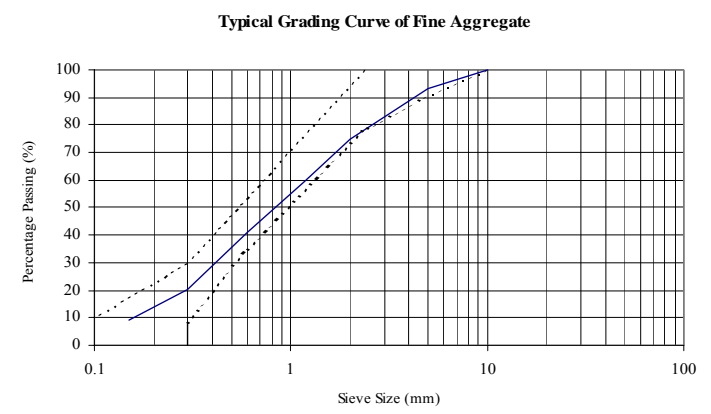

Figure 1. Typical grading of Palm oil clinker (top) fine aggregates (bottom) coarse aggregate

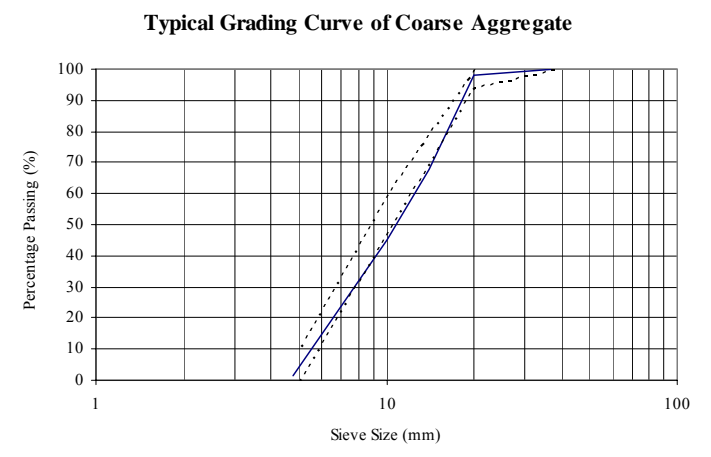

Figure 1. Continued

Fineness modulus is calculated for fine aggregates to detect a slight variation in the aggregates from the same sources, which might affect the workability of the fresh concrete. In this case, the fineness modulus for palm oil clinker is 2.84. Typical values ranging from 2.2 (very fine type of aggregates) and 3.2 (very coarse type), showing the palm oil clinker has coarse grading. This might due to the crushing work by lab crusher produce more coarse rather than fine particles.

By right, the bigger size of aggregates requires less water requirement of the wetted per unit mass. In this case, watercement ratio can be reduced and this will increase the strength of the concrete. However, bigger size gave lesser bond area hence gives lesser strength. Normally, the nominal size used is 10,20 and $40 \mathrm{~mm}$. $20 \mathrm{~mm}$ is chosen as it is the average size and commonly used in construction practice.

\section{Specific Gravity}

BS 812: Part 107 expressed specific gravity as "particle density" in $\mathrm{kg} / \mathrm{m}^{3}$. There are few types of specific gravity, namely bulk specific gravity (saturated surface dry which filled the pores with water) and absolute specific gravity. Conventional aggregates have an apparent specific gravity between 2.6 and 2.7. Since lightweight aggregates introduce air in its density, it will fall below this range.

POC shows its values of specific gravity as shown in Table 5. This value gave the requirement for designing the mixture of lightweight concrete. Minimum density always applies especially in designing structures required less load like dams, bridges as well as embankment for stability purposes.

Table 5. Specific gravity of palm oil clinker

\begin{tabular}{|c|c|c|c|}
\hline \multicolumn{2}{|l|}{$\begin{array}{l}\text { Physical } \\
\text { Properties }\end{array}$} & $\begin{array}{l}\text { Coarse palm } \\
\text { oil clinker }\end{array}$ & $\begin{array}{l}\text { Fine palm } \\
\text { oil clinker }\end{array}$ \\
\hline \multirow{3}{*}{$\begin{array}{l}\text { Specific } \\
\text { Gravity } \\
(\mathrm{SG})\end{array}$} & Apparent SG & 1.78 & 2.17 \\
\hline & $\begin{array}{l}\text { Bulk } \\
\text { (Dry) }\end{array} \quad$ SG & - & 1.61 \\
\hline & $\begin{array}{l}\text { Bulk } \\
\text { (SSD) }\end{array}$ & 1.64 & 1.87 \\
\hline
\end{tabular}

\section{Bulk Density}

The bulk density shows how dense the aggregates are packed and roughly show size distributions and shape of particles. As specified in BS812: Part 2: 1975 recognize two 
types of bulk density, loose and compacted. The values of bulk density and void ratio for palm oil clinker sample are shown in Table 6 . For structurally satisfactory, density of aggregates lies between $700-1400 \mathrm{~kg} / \mathrm{m}^{3}$.

Table 6. Bulk Density for palm oil clinker aggregates

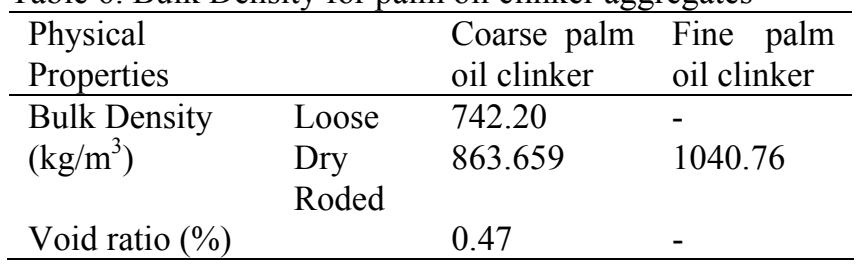

As we know coarse aggregates constituent filled about $60 \%$ of concrete matrix. Void ratio indicates volume of mortar required to fill the space between the coarse aggregate particles. Bulk density shows roughly the information for the design purposes as commonly used in concrete mix design. Void ratio shows the required mortar to bind together. Within the concrete matrix, ratio between loose and compacted bulk density is 0.87 which prescribed by ASTM C29-91a.

\section{Adsorption and Moisture Content}

Since POC is a porous aggregate, it will allow high amount of water to fulfill the pores compared to solid aggregates. For design purposes, this value is important to determine how much water is required to assure the aggregates are in SSD state when in use. This value is quite critical because if POC in used, the water in design mix will be adsorbed by the aggregates and there is not sufficient water for workability and consistency and therefore, insufficient the hardened concrete properties especially strength and durability.

Moisture content of an aggregate depends on the humidity of the environment where the aggregate is placed. As the aggregates stored in the lab, there will no exposure to rain which collects moisture not only on the surface but also inside the aggregates as the pores will adsorbed water inside the particles. This amount gave a considerable important since it will be calculated in water requirement in concrete mix design as it is impossible to oven-dry huge amount of aggregates on-site.

Table 7. Moisture content and water Adsorption of palm oil clinker aggregates.

\begin{tabular}{|l|l|l|l|}
\hline $\begin{array}{l}\text { Physical } \\
\text { Properties }\end{array}$ & $\begin{array}{l}\text { Coarse palm } \\
\text { oil clinker }\end{array}$ & $\begin{array}{l}\text { Fine palm } \\
\text { oil clinker }\end{array}$ \\
\hline \multicolumn{2}{|l|}{ Moisture Content $(\%)$} & 0.084 & 0.383 \\
\hline \multirow{2}{*}{$\begin{array}{l}\text { Water } \\
\text { Adsorption } \\
(\%)\end{array}$} & 5 minutes & 2.97 & - \\
\cline { 2 - 4 } & 1 hour & 3.38 & - \\
\cline { 2 - 4 } & 24 hours & 4.65 & 16.17 \\
\hline
\end{tabular}

\section{Aggregate Crushing Value (ACV)}

Since coarse aggregates comprises of $60 \%$ of concrete constituents, strength of the aggregates will imply on the strength of the hardened concrete. The measuring principle for this test is the fines produced by slowly increasing load to a specified maximum. The selection of a suitably high characteristic concrete strength alone is normally taken to assure a sufficient potential resistance to abrasion and attrition.

Table 8. Aggregate crushing value

\begin{tabular}{|c|c|}
\hline Physical Properties & Palm oil clinker \\
\hline Aggregate & 18.44 \\
\hline $\begin{array}{ll}\text { Crushing } & \text { Value } \\
(\mathrm{ACV})(\%) & \end{array}$ & \\
\hline
\end{tabular}

Generally, the lower compressive strength, the higher is the crushing values. However, there is no explicit relationship and test on the relationship need to be obtained for verification.

\section{Aggregate impact value (AIV)}

Impact value will determine the toughness of the aggregates which is the resistance of aggregate to failure by impact. The value and BS882 classifications are summarized in Table 9.

Table 9. Aggregate impact values

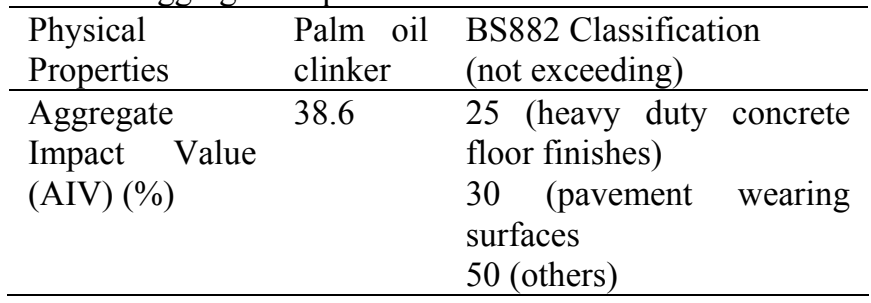

Palm oil clinker is not a solid particle. It is a porous material which has air within its particles to make it lightweight. In aggregate crushing value which the aggregate is subjected to impact, it gave quite a high value which is $38.6 \%$. Based on classification by BS 882 , this type of aggregates cannot be applied on heavy duty concrete floor finishes and pavement wearing surfaces. But it passes for other applications.

\section{Deleterious substances}

Since POC is made from well-burnt residue of palm oil high-temperature furnaces, fused or sintered into lumps, there will be some amount of carbon appears on the surface of the POC. The carbon should be cleaned from the surface of the aggregates which might interference the hydration process of cement, preventing the good coating between aggregates and cement paste; and carbon will make the aggregates unsound and weak.

\section{Fineness of binders}

The fineness of 'binders' give the total surface area of cement ready for hydration rate. The finer the cement particles, the better for hydration process, hence the strength will be increased. However, there is limitation, which too fines will increase the cost of production and made the low workability of fresh concrete. As more fines, the rapid hydration will not produces from the concrete matrix to be developed properly, leaving pores behind. The fineness of OPC and Fly ash is shown in Table 10. 
Table 10. Fineness of binders

\begin{tabular}{llll}
\hline $\begin{array}{l}\text { Physical } \\
\text { Properties }\end{array}$ & $\begin{array}{l}\text { Ordinary } \\
\text { Portland Cement } \\
\text { (OPC) }\end{array}$ & $\begin{array}{l}\text { Pulverized } \\
\text { Ash (FA) }\end{array}$ & Fly \\
\hline $\begin{array}{l}\text { Blaine Specific } \\
\begin{array}{l}\text { Surface } \\
\left(\mathrm{cm}^{2} / \mathrm{g}\right)\end{array}\end{array}$ & 3200 & 1800 \\
\hline
\end{tabular}

From Table 10, the fineness of fly ash is higher than OPC. The more fineness of fly ash makes the rapid process of hydration rather than OPC.

\section{Initial and Final Setting}

Before the initial and final setting times can be done, consistency of standard paste has to determine first which gave the amount of water required for any given cement to produce a paste of standard consistence. Water content required which expressed as a percentage by mass of dry cement lies within 26-33 for conventional Ordinary Portland Cement (OPC). Table 11 shows the results of initial and final setting mixed of water content that give the consistency of cement paste.

Table 11. Initial and Final setting of cement

\begin{tabular}{|c|c|c|c|c|}
\hline $\begin{array}{l}\text { Physical } \\
\text { Properties }\end{array}$ & & $\begin{array}{l}\text { Water } \\
\text { conten } \\
\mathrm{t}(\%) \\
\end{array}$ & $\begin{array}{l}\text { Initial } \\
\text { Setting } \\
\text { (h:m) } \\
\end{array}$ & $\begin{array}{l}\text { Final } \\
\text { Setting } \\
(\mathrm{h}: \mathrm{m}) \\
\end{array}$ \\
\hline \multirow[t]{4}{*}{$\begin{array}{l}\text { Binders } \\
\operatorname{mix}\end{array}$} & $\begin{array}{l}100 \% \\
\text { OPC }\end{array}$ & 25.6 & $2: 15$ & $4: 05$ \\
\hline & $\begin{array}{l}90 \% \text { OPC } \\
+5 \% \text { fly } \\
\text { ash }\end{array}$ & 28.3 & $2: 45$ & $4: 22$ \\
\hline & $\begin{array}{l}90 \% \text { OPC } \\
+10 \% \text { fly } \\
\text { ash }\end{array}$ & 28.1 & $3: 14$ & $4: 25$ \\
\hline & $\begin{array}{l}90 \% \text { OPC } \\
+15 \% \text { fly } \\
\text { ash }\end{array}$ & 29.0 & $2: 17$ & $3: 08$ \\
\hline
\end{tabular}

Basically, water percentage increased when the ratio of pozzolans was increased. When the ratio of substituted materials increased, the specific surface of the mixtures also increased. As specified in Table10, value for Blaine specific surface for fly ash is around $3200 \mathrm{~cm}^{2} / \mathrm{g}$ and OPC is 1800 $\mathrm{cm}^{2} / \mathrm{g}$.

Setting times decreased when water percentage increased. When the ratio of substituted materials was increased, setting time decreased. When fly ash is ground with OPC, the fineness of the cement increases; hence, the hydration process becomes faster, and reduces setting time. ASTM C191-92 specifies that initial setting of binders should be more than 45 minutes and final setting time less than 10 hours.

This however, different from observing the mixture of $10 \%$ fly ash replacement. It requires less water content from $5 \%$ replacement of fly ash. As the requirement of water increased, the setting times increased as well. We know that increasing the setting times will increase the time thus increasing the cost of production. However, research by Neville shows that optimum strength was obtained by using fly ash replacement by $10 \%$.

\section{CONCLUSIONS}

The following conclusions can be drawn from this investigation:-

1. Palm oil clinker is a lightweight material that can save up to $40 \%$ when batched. This can reduce the cost of production due to lesser dead load.

2. High water adsorption should be taken a serious place when designing palm oil clinker mixtures.

3. High specific surface of fly ash than OPC can enhanced the hydration rate; thus produced a stronger and durable concrete.

4. Both palm oil clinker and fly ash have a great potential to be developed in future.

\section{ACKNOWLEDGMENT}

The authors would like to express his gratitude to Universiti Tun Hussein Onn Malaysia for rewarding a short grant to produce this paper.

\section{REFERENCES}

[1] A. M. Neville. 1995. Properties of Concrete-Fourth Edition.Longman.

[2] BS 812. Testing Aggregates

[3] C. H. Goodspeed, S. Vanikar, and R. A. Cook. 1996. HighPerformance Concrete Defined for Highway Structures. Concrete International, Feb, Vol. 18, No. 2, pp. 62-67.

[4] Joao A. Rossignolo, Marcos V.C. Agnesini 2002 Mechanical properties of polymer-modified lightweight aggregate concrete Cement and Concrete Research Vol 32, pp.329-324.

[5] Ken W. Day. 1999. Concrete Mix Design, Quality Control and Specification. E \& FN Spon.

[6] Peter C. Hewlett. 1998. Lea's Chemistry of Cement and ConcreteFourth Edition.Arnold.

[7] S. W. Forster. 1994. High-Performance Concrete - Stretching the Paradigm. Concrete International, Oct, Vol. 16, No. 10, pp. 33-34.

[8] T.S. Nagaraj and Zahida banu. 1996 Efficient utilization of rock dust and peebbles as aggregates in Portland cement concrete. Jan 96. The Indian Concrete Journal pp. 53-56. 


\title{
Design, Development and Performance Evaluation of Parabolic Trough Solar Collector in Tropical Regions
}

\author{
Syed Ihtsham-ul-Haq Gilani, Chalilullah Rangkuti, Aklilu Tesfamichael, and Tsen Wee Yew
}

\begin{abstract}
The design, development and test of a parabolic trough collector (PTC) was conducted under local climatological condition for water heating system. The design parameters of the PTC developed were geometrically optimized with due consideration to material availability. The optimized design parameters were focal length $0.735 \mathrm{~m}$, and concentration ratio 21.4. The PTC was examined at outdoor environment for $2.4 \mathrm{l} / \mathrm{min}$ flow rate in order to evaluate its performance. The maximum instantaneous efficiency and the collection efficiency at an average solar flux of $469 \mathrm{~W} / \mathrm{m}^{2}$ were found $16.5 \%$ and $14.2 \%$ respectively. The effect of magnitude of flow rate was also examined for the flow rates $1.2 \mathrm{l} / \mathrm{min}, 2.4$ $\mathrm{l} / \mathrm{min}$ and $6 \mathrm{l} / \mathrm{min}$. Design specifications, description of the prototype, data measuring instruments, performance analysis with conclusions and recommendations to further enhance the performance of the concentrator are presented in this paper.
\end{abstract}

Keywords: parabolic trough collector, design and development, solar water heater, performance evaluation

\section{INTRODUCTION}

Many developing countries with high levels of solar radiation are well placed to implement solar thermal technologies for bulk power and process heat generation [1]. In the past decades, researchers and engineers tried to find the best solution for utilizing solar energy, which has a lot of parameters to be considered such as collector geometry, latitude, solar time, solar collecting types and etc. Parabolic trough collectors (PTCs) can deliver thermal energy over a wide rang of temperatures. They can be used for a variety of applications such as electricity generation, hot water, space heating, air-conditioning, steam generation, industrial process heating, and desalination, etc. To investigate the

Syed Ihtsham-ul-Haq Gilani Senior Lecturer, University Technology Petronas (UTP), Department of Mechanical Engineering, Tronoh (Perak), Malaysia (Tel: 0060 (5) 3687029, Fax: 006053656461, e-mail syedihtsham@petronas.com.my).

Chalilullah Rangkuti, Associate Proffesor, University Technology Petronas (UTP), Department of Mechanical Engineering, Tronoh (Perak), Malaysia (Tel: 0060 (5) 3687004, Fax: 0060 (5) 3656461, e-mail: chalilullah@petronas.com.my).

Aklilu Tesfamichael, PhD student, University Technology Petronas (UTP), Department of Mechanical Engineering, Tronoh (Perak), Malaysia (Tel: 0060175655752, Fax: 006053654090, email: aklilu_tesfamichael@utp.edu.my).

Tsen Wee Yaw, Final year student in Mechanical Engineering Department, UTP, Tronoh (Perak), Malaysia. performance of PTCs numerous studies were performed. Examples are a parabolic trough solar collector similar in size to small-scale commercial modules, was developed to serve as a test-bed for collector components in an ongoing research program at Mangosuthu Technikon, South African University of Technology. [1]. An appropriate mix of advanced and less sophisticated technologies was employed during construction. The Australian National University, together with commercial partners, has developed a Photovoltaic / Trough concentration system. It comprises two-axis sun-tracking parabolic glass mirrors and a receiver with solar cells placed along the focal line [2]. The performance of a new parabolic trough collector for hot water generation system was investigated [3]. An experimental study was also performed to test the effectiveness of design modifications terms of efficiency and power production in an existing parabolic solar concentrator [4].

This study also focuses on the design, development and performance test of the PTC under local climatological condition for water heating system.

\section{Design And DeVelopment of The PTC}

The parabolic reflector is defined by its (i) aperture width $\mathrm{w}$, (ii) the half angle subtended by the arc of the parabola (rim angle) $\theta_{r}$, and (iii) absorber shape and size. The procedure followed to determine the geometric size in the design phase of the PTC is indicated in figure 1.

\section{A. Optical Design}

For the design of the parabolic trough collector first a relation between the geometrical concentration ratio, rim angle, depth and the focal length is formulated. The system is configured as shown in figure 2.

The equation for the geometrical concentration ratio (CR) of the PTC is given by the following equation.

$$
C R=\frac{\sin \theta_{r}}{\pi \sin \phi}
$$

where $\phi\left(=16^{\prime}\right)$ is the half of the angular subtends of the solar disc on any point on earth [5]. 


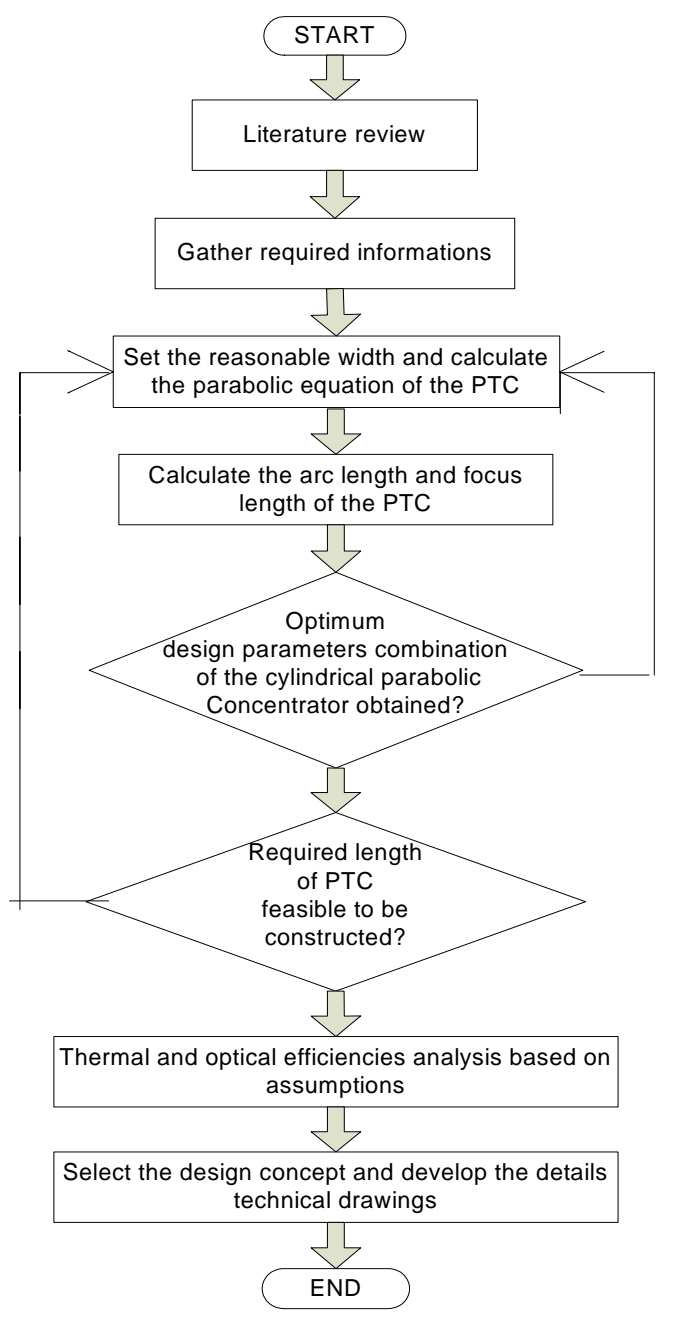

Figure 1. Flow chart used for the design of the parabolic trough collector (PTC).

The equation for the surface of the parabola in Cartesian co-ordinates is [6]:

$y=\frac{1}{4 f} \cdot x^{2}$

where $\mathrm{x}$ is an axis to place the curve and $\mathrm{y}$ is the center line of focal and $f$ its focal length. The focal length $\mathrm{f}$ from a point on the rim of the parabola to the focal point is given by the surface equation:

$f=\frac{w^{2}}{16 d}$

Equation (4) is used to calculate the rim angle, based on the focal length, width and depth [7];

$\cos \theta_{r}=\frac{2 f}{\sqrt{(0.5 w)^{2}+(d-f)^{2}}}-1$

The aim here is to improve the performance of the system by appropriate selection of design parameters. One of these is the required concentration ratio and the optimization of the geometry of the PTC. The optimization geometric is made based on the theoretical equations (1), (3) and (4) for $1.5 \mathrm{~m}$ width. The summery of the variation of the design parameters for different depths is indicated in Table 1. Moreover, figure 3 indicates maximum concentration ratio occurs when the rim angle is $90^{\circ}$ and this happens when the focal length is equal to the depth.

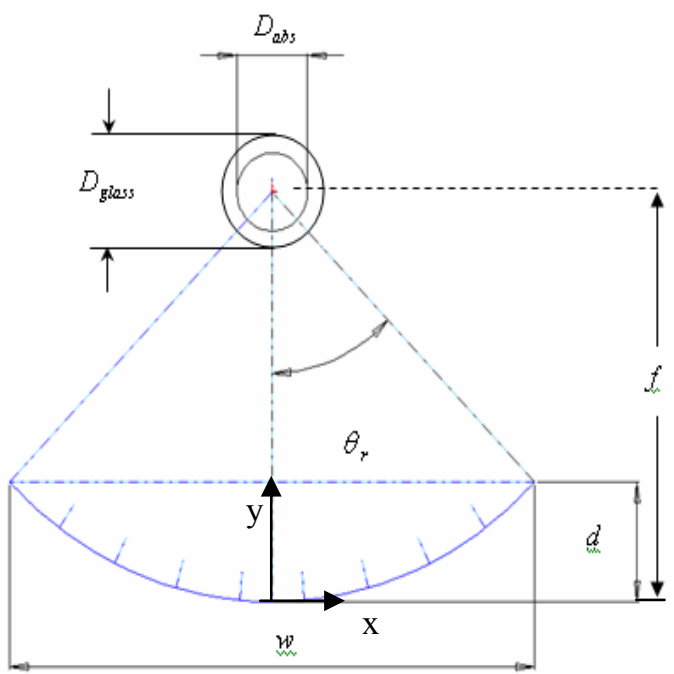

Figure 2. Parabolic trough concentrator including the collector

Table 1: The table shows the variation of design parameters with respect to the depth of PTC

\begin{tabular}{|c|c|c|c|c|}
\hline Depth & Rimangle & Foouslength & Arclength & $\begin{array}{c}\text { Concentration } \\
\text { Ratio }\end{array}$ \\
\hline 0.200 & 56.000 & 0.703 & 1570 & 178.119 \\
\hline 0.355 & 90.000 & 0.315 & 1720 & 214.832 \\
\hline 0.600 & 116.000 & 0.234 & 2000 & 193065 \\
\hline
\end{tabular}

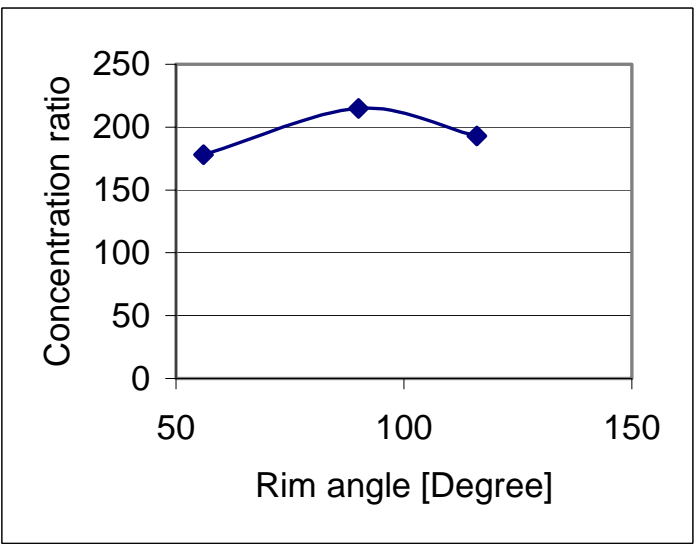

Figure 3. Graph showing the maximum concentration ratios at various rim angles

Figure 4 shows the rim angle of $90^{\circ}$ not only gives maximum concentration ratio but also give an optimum intercept factor and this will contribute to the highest concentration ratio and a proper support structure can be easily designed.

Once the optimum geometric size fixed the PTC is constructed and figure 5 shows the procedure followed during the development and the performance evaluation phase, with due consideration to other practical constraints like method of fabrication, cost, and material availability. 


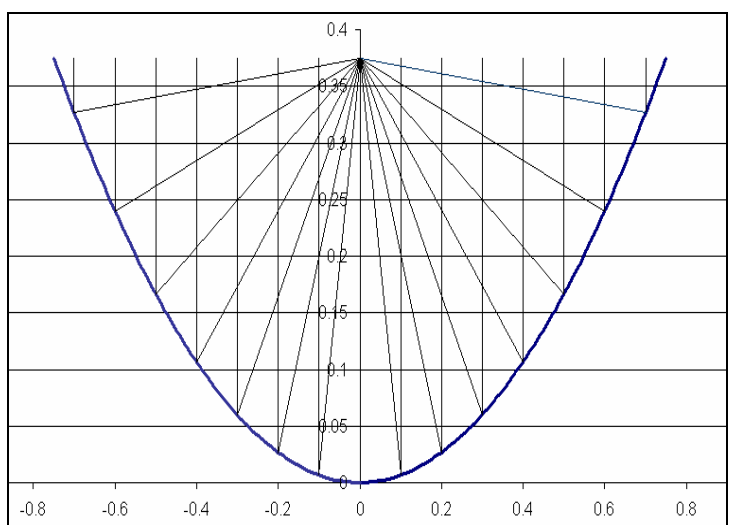

Figure 4. The graph shows the cross section of the designed PTC, depth and the focus point $(d=f)$ - half portion of the absorber receives the solar heat flux

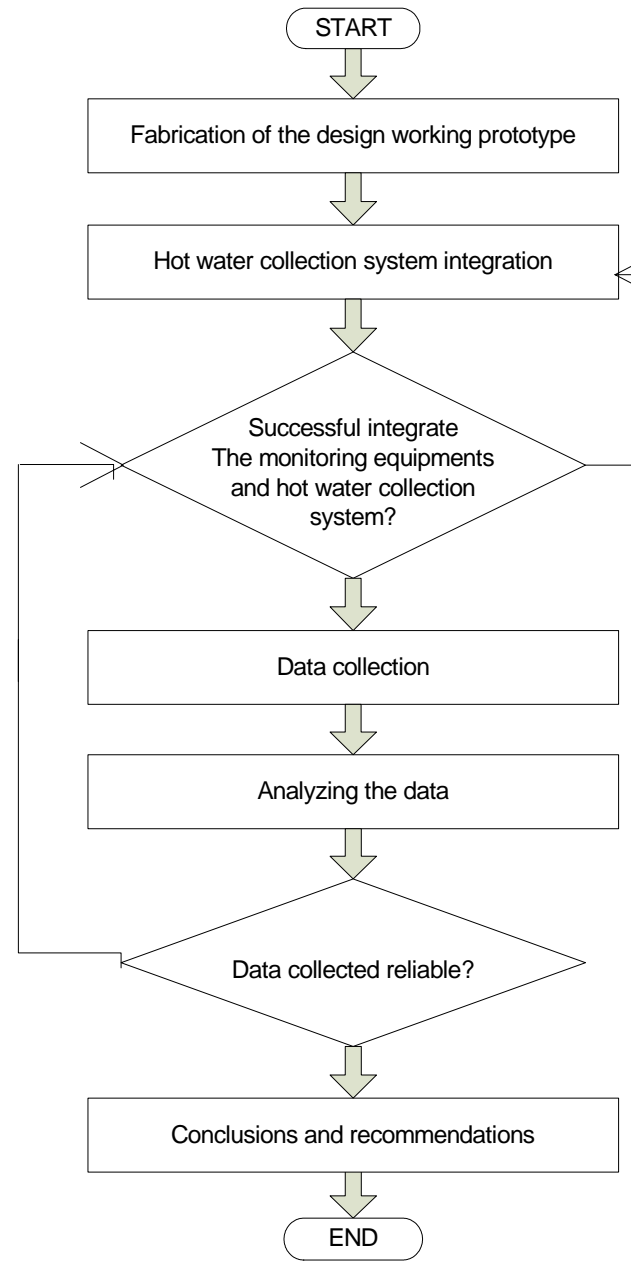

Figure 5. Flow chart used to develop the PTC and performance evaluation procedure

Figure 6 shows the picture of the developed prototype PTC. The figure illustrates the major components of a solar trough collector. It consists of the parabolic trough reflector, steel support structure, and copper absorber pipe in glass tube. The absorber has inside diameter of $20.2 \mathrm{~mm}$, and outside diameter $22 \mathrm{~mm}$ and its length is $1500 \mathrm{~mm}$. The inside and outside diameters of the glass tube are $40 \mathrm{~mm}$ and $50 \mathrm{~mm}$ respectively. The glass envelope protects the absorber from degradation and reduces heat losses. The trough reflector has an aperture area $2.175 \mathrm{~m}^{2}$ (length $1450 \mathrm{~mm}$ and width $1500 \mathrm{~mm}$ ), focal length $735 \mathrm{~mm}$, and rim angle $90^{\circ}$. For the above selected collector and absorber dimensions the actual concentration ratio using (5) is 21.4.

$C R=\frac{\left(w-D_{a b s}\right) L}{\pi D_{a b s} L}$

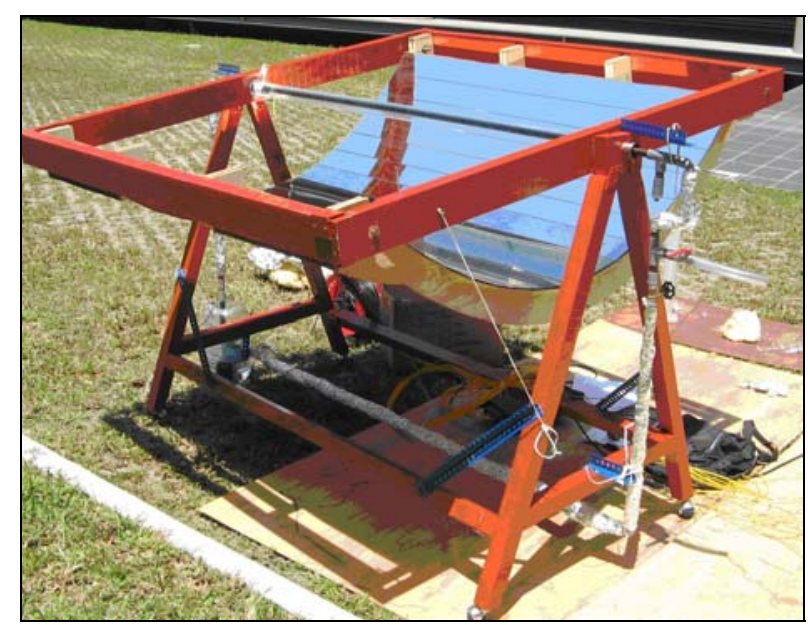

Figure 6 . The picture shows the actual out let fitting during testing at the outdoor environment

\section{PERFORMANCE ANALYSIS}

The performance of the developed PTC water heating system is determined by obtaining values of collector instantaneous efficiency, the system efficiency and collection efficiency for different combinations of incident radiation, ambient temperature and inlet water temperature. The collector water out let temperature $\left(T_{f o}\right)$, ambient temperature $\left(T_{a}\right)$ and storage tank water temperature $\left(T_{s t}\right)$ were recorded with the help of thermocouples. The solar beam radiation intensity was measured by a Solarimeter and the mass flow rate of water was measured by stopwatch and bucket method. The wind speed was measured by a vane type anemometer. The instruments used to collect the data are listed down in Table 2. All parameters were measured as a function of time.

The instantaneous thermal efficiency, $\eta_{i}$ of collector operating under steady-state conditions can be described by (6), [6]:

$\eta_{i}=\frac{Q_{u}}{A_{s} I}=\frac{\dot{m} C_{p}\left(T_{f o}-T_{f i}\right)}{A_{a} I}$

In the present work, the collector system is operated under closed loop mode. Hence, $T_{f i}$ is taken as $T_{s t}$. The rate of energy gained by the water in the storage tank for a time interval of 1 minute is given by:

$Q_{s t}=m_{w} C_{p}\left(T_{s t}{ }^{t}-T_{s t}{ }^{t-1}\right)$

The minutely efficiency of the PTC hot water storage system is estimated by the following equation:

$\eta_{s}=\frac{Q_{s t}}{A_{a} I_{h}}=\frac{m C_{p}\left(T_{s t}{ }^{t}-T_{s t}{ }^{t-1}\right)}{A_{a} I_{h}}$ 
Table 2. Details of the equipment used in the solar water heating system

\begin{tabular}{|c|l|l|}
\hline No & $\begin{array}{l}\text { Tool/ } \\
\text { Equipment }\end{array}$ & Purpose \\
\hline 1 & $\begin{array}{l}\text { Thermometer } \\
\left(0^{\circ} \mathrm{C}-100^{\circ} \mathrm{C}\right)\end{array}$ & $\begin{array}{l}\text { To measure the water } \\
\text { temperature in the } \\
\text { water storage tank. }\end{array}$ \\
\hline 2 & $\begin{array}{l}\text { Wet bulb } \\
\text { thermocouple }\end{array}$ & $\begin{array}{l}\text { To measure the inlet } \\
\text { and outlet temperature } \\
\text { of the heat transfer } \\
\text { fluid (HTF). }\end{array}$ \\
\hline 3 & $\begin{array}{l}\text { Dry bulb } \\
\text { thermocouple }\end{array}$ & $\begin{array}{l}\text { To measure the surface } \\
\text { temperatures of the } \\
\text { copper tube, glass tube } \\
\text { and the ambient } \\
\text { temperature. }\end{array}$ \\
\hline 4 & Data logger & $\begin{array}{l}\text { To indicate and store } \\
\text { the temperatures } \\
\text { monitored. }\end{array}$ \\
\hline 5 & $\begin{array}{l}\text { Digital } \\
\text { Solarimeter }\end{array}$ & $\begin{array}{l}\text { To measure the solar } \\
\text { irradiance }\end{array}$ \\
\hline 6 & Stop watch & $\begin{array}{l}\text { To measure the time } \\
\text { taken of the water } \\
\text { collected for the flow } \\
\text { rate calibration } \\
\text { purpose. }\end{array}$ \\
\hline
\end{tabular}

Another measure of collector performance is the collection efficiency, defined as the ratio of the useful gain over some specified time period to the incident solar energy over the same time period:

$\eta=\frac{\int Q_{u} d t}{A_{a} \int I d t}$

\section{RESUlTS AND DisCUSSIONS}

The results obtained are based on the stated size of the developed collector and absorber dimensions. The average ambient temperature during the experiment was $32^{\circ} \mathrm{C}$. The variation of collector water out let temperature, $T_{f o}$, and storage tank water temperature, $T_{s t}$, within 19 minutes time interval, in April 19, 2006 is shown in figure 7. The collector water temperature increases progressively with time, which varies from $12: 19$ to $12: 37 \mathrm{~h}$, local time, as the water is re-circulated through a hot water storage tank. The mass flow rate of water through the collector is $2.4 \mathrm{l} / \mathrm{min}$. The storage tank water temperature increases steadily from an initial temperature of $41^{\circ} \mathrm{C}$ at $12: 19 \mathrm{~h}$ and touches a maximum value of $48^{\circ} \mathrm{C}$ at $12: 37 \mathrm{~h}$, as no energy is withdrawn from the storage tank during the collection period. At any instant, the storage tank water temperature is less than the collector water temperature.

Figure 8 shows the variation of beam radiation, $I$, and useful heat gain, $Q_{u}$, with time. It is seen that nearly a constant beam radiation with the maximum $\left(482 \mathrm{~W} / \mathrm{m}^{2}=\right.$ $1048 \mathrm{~W}$ ) occurs around noon. Similarly the useful heat is was almost constant. Reason for being constant might be the data collection duration time is small. Moreover, due to the fact that the useful heat gain is strongly influenced by the incident beam radiation and therefore the useful energy follow the solar radiation variation.

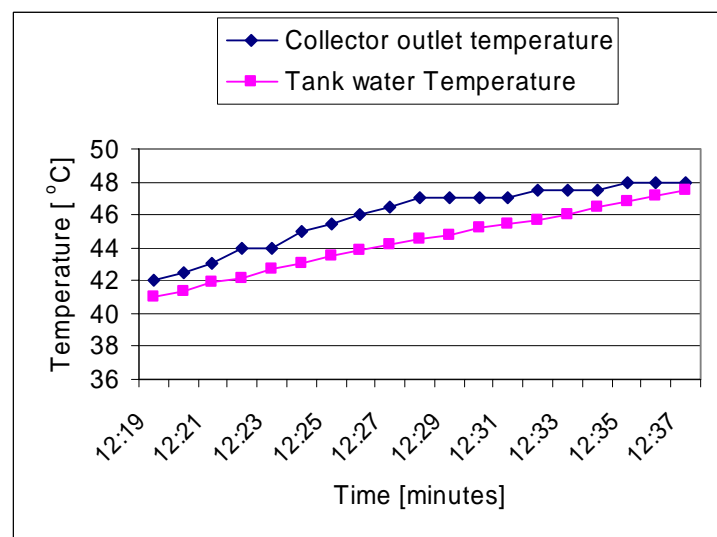

Figure 7. Variation of collector water temperature, $T_{f o}$, and storage tank water temperature, $T_{s t}$, with time

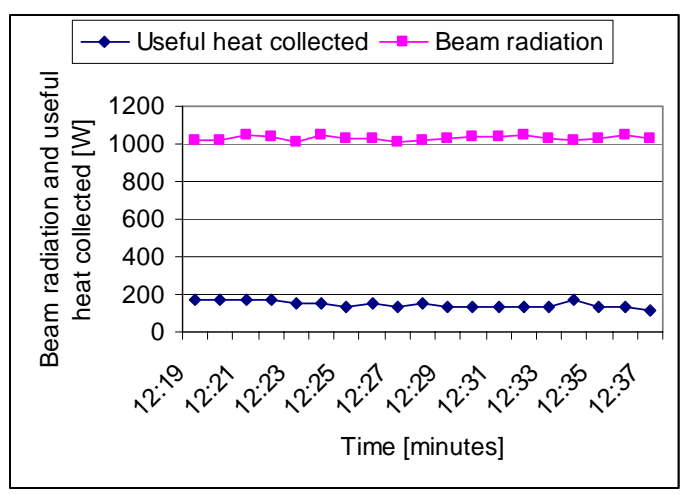

Figure 8. Variation of beam radiation, $I$, and useful heat gain, $Q_{u}$, with time

The collector instantaneous efficiency is computed using (6). Plot showing the variation of the collector instantaneous efficiency with time is given in figure 9. It is noted that the general pattern of variation of efficiency in 19 minutes is the same as that of the useful heat gain because the value of efficiency depends on both the incident beam radiation and the useful heat gain.

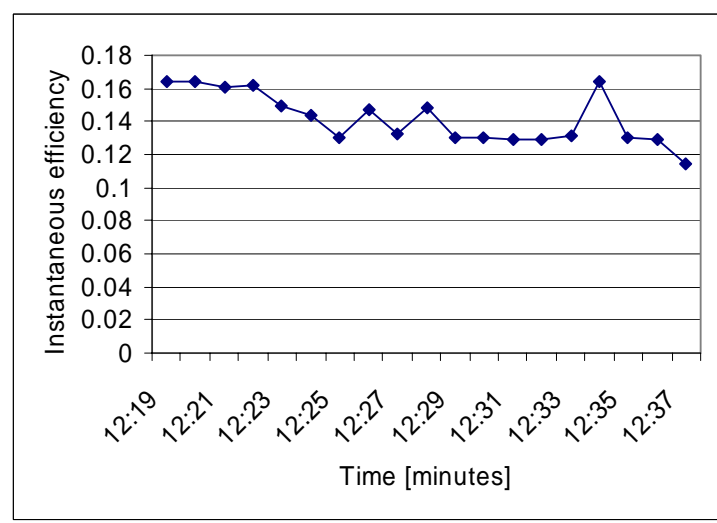

Figure 9. Variation of instantaneous efficiency

The variation of minutely beam radiation and energy gained by the storage tank water per minute with time is presented in figure 10. The figure show the minutely beam radiation is almost constant at noon. The energy gained per minute, $Q_{s t}$, by the storage tank water was increasing 
steadily and then becomes nearly constant. This effect is due to the fact that the energy gained by the storage tank water is directly proportional to the useful heat gain across the collector with time.

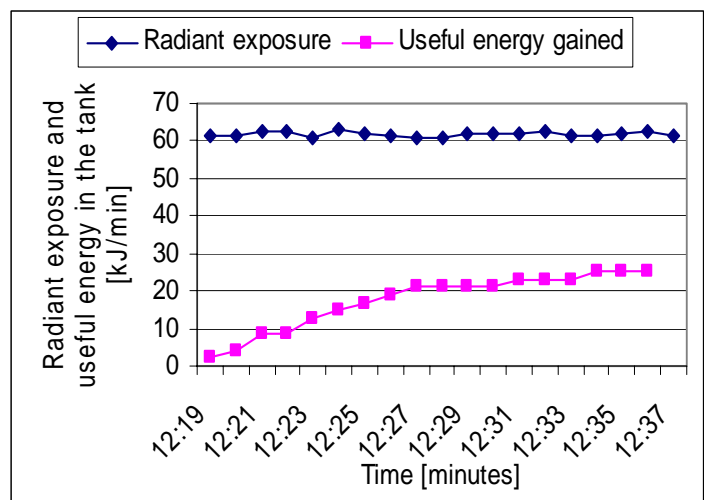

Figure 10. Variation of radiant exposure and useful energy in the tank with time

Figure 11 shows the effect of flow rate on the water temperature gain as it passes through the absorber pipe. The $1.2 \mathrm{l} / \mathrm{min}$ flow rate has highest temperature gain and $6 \mathrm{l} / \mathrm{min}$ has the lowest temperature gain while $2.41 / \mathrm{min}$ sandwiched between them.

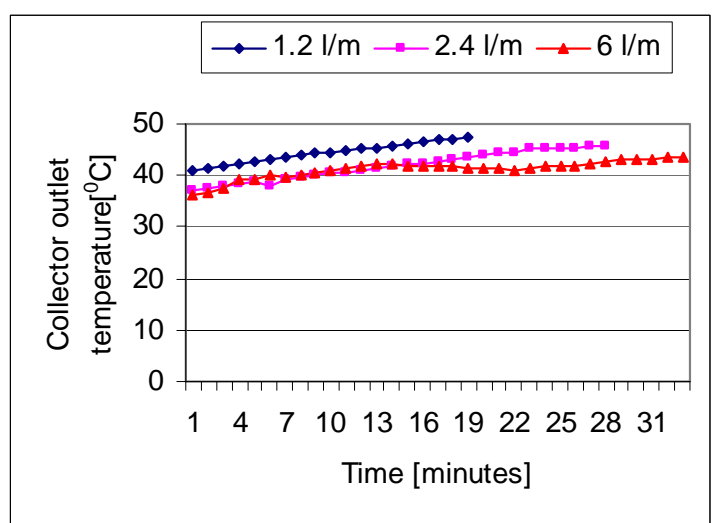

Figure 11. Variation of the out let temperature for various mass flow rates

On the basis of the above discussion and to further enhance the performance of the PTC, the following improvements to the existing PTC are suggested. 1) Painting of the absorber surface with a selective coating material, 2) use of vacuum tube, and 3) installing mechanisms that allow the parabolic concentrator to follow the sun (tracking system).

Further studies require, the system analytical model development and comparing with the experimental result. The precision of the concentrator shape and alignment of collector may also affect the performance.

\section{CONCLUSIONS}

A PTC was designed and developed having a concentration ratio of 21.4 and the focal length of $0.735 \mathrm{~m}$. A test set-up was established to evaluate the performance of the PTC. The PTC was tested experimentally under local climatological conditions, for water heating system and at a flow rate of $2.4 \mathrm{l} / \mathrm{min}$. At an average irradiance of 469 $\mathrm{W} / \mathrm{m}^{2}$, the collector's efficiency was observed $14.2 \%$, whereas its instantaneous efficiency was $16.5 \%$. Effect of water mass flow rate was also investigated and it was observed that the water temperature gain decreases as the mass flow rate increases.

\section{REFERENCES}

[1] Michael Brooks and Thomas Harms, "Design, construction and testing of a parabolic trough solar collector for a developing-country application"

[2] Blakers, A. W. (2000) : "Efficient 20-50 Sun concentrator cells," $16^{\text {th }}$ EC PVSEC, Glasgow, May 2000

[3] A. V. Arasu and S. T. Sornakumar, "Performance characteristics of the Solar parabolic trough collector with hot water generation system," Thermal Science: Vol. 10, No. 2, pp. 167-174, 2006

[4] Toni Ferreira, "Some modifications to the design of a parabolic solar concentrator for construction in Lesotho and their effects on power production," Massachusetts Institute of Technology, FYP, May 15, 2005

[5] Garg, H. P. and Prakash, J. (2000): "Solar Energy Fundamentals and Applications", Tata McGraw Hill.

[6] Duffie, John A. and William A. Beckman, "Solar energy thermal processes," New York: Jon Wiley, 1980.

[7] Balbir Signh, M.S, Sulaiman, F. (2003), Designing a Solar Thermal Cylindrical Parabolic Trough Concentrator by Simulation. 


\title{
Mechanical Properties of Palm Oil Clinker Concrete
}

\author{
Mohd Hilton Ahmad, Sofian Mohd and Nurazuwa Md Noor
}

\begin{abstract}
Palm oil clinker is a by-product of palm oil industry which normally being dumped abundantly as waste which caused to the undesirable effects to our environment sustainability. This paper studied the mechanical properties of local palm oil clinker concrete including compressive strength, three point bending strength and tensile splitting test which is the most important parameters in engineering properties of concrete as the primary measurement to its suitability for the appliance for the structural use. Five series of palm oil clinker concrete with same mix proportioning were batched and tested. The series includes control mix, palm oil clinker as coarse aggregate, palm oil clinker as coarse and fine aggregate and addition of fly ash to the mix proportion. The mechanical properties of this study were conducted and measured accordance to the British Standard. The effects of another local waste material from coal combustion i.e., pulverized fly ash (pfa) which replace the cement content in mix proportion on mechanical properties of palm oil clinker concrete were compared and the mechanism were been analyzed. The results given by the palm oil clinker was found to be in good agreement with the structural use which been indicated by the structural concrete Code of Practice BS8110. Addition of pulverized fly ash as pozzolanic material produces more strong and durable concrete compared to palm oil clinker without fly ash.
\end{abstract}

Keywords: compressive strength; flexural strength; tensile splitting test; palm oil clinker concrete; pulverized fly ash

\section{INTRODUCTION}

One of the major factor that affects the behavior and properties of a Lightweight Concrete is the aggregate itself. The strength, shape, size and gradation of the aggregate contribute directly to the strength of the lightweight concrete. Hence, many studies and research has been carried out to improve and refine on the aggregate itself. In many cases, natural aggregate has been substituted with artificial aggregate to provide that extra strength that natural aggregate are difficult to provide. Due the limitation and difficulties in finding strong natural aggregate in certain

Mohd Hilton Ahmad currently is with the Faculty of Civil and Environmental Engineering, Universiti Tun Hussein Onn Malaysia, 86400 Parit Raja, Batu Pahat, Johor, Malaysia. (Corresponding author. Phone: 607-4537320; fax: 607-4536070; e-mail: hilton@ uthm.edu.my).

Sofian Mohd is with the Faculty of Mechanical and Manufacturing Engineering, Universiti Tun Hussein Onn Malaysia, 86400 Parit Raja, Batu Pahat, Johor, Malaysia. (e-mail: sofian@uthm.edu.my).

Nurazuwa Mohd Noor is with the Faculty of Civil and Environmental Engineering, Universiti Tun Hussein Onn Malaysia, 86400 Parit Raja, Batu Pahat, Johor, Malaysia. (e-mail: nurazuwa@uthm.edu.my). region, artificial aggregate has been used widely to replace the natural aggregates. Current technologies and discoveries have made it possible to create a strong artificial aggregate that out performs natural aggregates.

Malaysia, being one of the largest producer and manufacturer of palm oil products, generates large amount of palm oil by-products, which can be recycled into Palm Oil Clinker. If this palm oil clinker is put into good use, in this case as a main material in high performance concrete mix, then it will largely reduce the cost of high performance concrete. At the same time, it will also reduce the amount of waste generated by the palm oil industry thus achieving a global aim of sustainable development. Not only it reduces the waste, it also preserves the nature by eliminating the need to harvest natural aggregates from natural sources.

Pozzolans were commonly used in concrete technology to enhance the concrete properties. Silica dioxide, $\mathrm{SiO}_{2}$ which the main constituents in pozzolans will react with calcium hydroxide, $\mathrm{Ca}(\mathrm{OH})_{2}$ which is deleterious to concrete strength and product from process of hydration, $\left(\mathrm{C}_{3} \mathrm{~S}+\mathrm{H}_{2} \mathrm{O} \underset{\text { hydration }}{\longrightarrow} \mathrm{C}-\mathrm{S}-\mathrm{H}+\mathrm{Ca}(\mathrm{OH})_{2}\right)$ to produce more $C-S-H$ compound which contribute to its strength. The equilibrium equation of the pozzolanic reaction is given as $\left(\mathrm{Ca}(\mathrm{OH})_{2}+\mathrm{SiO}_{2} \rightarrow \mathrm{C}-\mathrm{S}-\mathrm{H}\right.$.

This paper reports the results of an investigation into the mechanical properties of palm oil clinker concrete. The influence of fly ash at $10 \%$ replacement upon the cement content on the concrete strength also been investigated. $10 \%$ replacement was selected as it gave the optimum reaction as reported by Neville. The target strength for palm oil clinker concrete was at least achieving compressive strength of 30 $\mathrm{N} / \mathrm{mm}^{2}$ so that it can be used as structural concrete members as prescribed in BS 8110 .

\section{MATERIALS AND EXPERIMENTAL PROCEDURES}

\section{A. Palm Oil Clinker as Aggregate}

The Palm Oil Clinker (POC) was taken from the palm oil mill factory from Dengkil. The factory is Sri Ulu Langat Palm Oil Mill. The by-product that is collected from inside the boiler is called clinker. The clinker looks like a porous stone which is gray in color. All the clinkers are prepared to be crushed into required size. Clinker with nominal size of $20 \mathrm{~mm}$ is used as coarse aggregate and size below $4.75 \mathrm{~mm}$ is used as fine aggregate. The clinkers were flaky and irregular shaped. The broken edges were rough and spiky. 
The physical properties such as water adsorption, moisture content and bulk density are shown in Table 1. Palm oil clinker and conventional coarse aggregate, i.e., gravel used in saturated surface dry condition.

Table 1. Physical properties of fine POC and coarse POC

\begin{tabular}{lll}
\hline Physical properties & Fine & $\begin{array}{l}\text { Crushed } \\
\text { stone }\end{array}$ \\
\hline Specific gravity (SSD condition) & 2.17 & 2.60 \\
Moisture content $(\%)$ & 0.08 & 0.05 \\
Water adsorption $(\%)$ & 4.65 & 1.79 \\
Bulk density $\left(\mathrm{kg} / \mathrm{m}^{3}\right)$ & 863.65 & 1815.23 \\
Fineness Modulus & 2.84 & 2.65 \\
\hline
\end{tabular}

\section{B. Other Concrete Mix Components}

River sand was used as fine aggregate which has a fineness modulus of 2.56. Fly ash and Malaysian Ordinary Portland Cement with specific gravity of 2.66 and 3.15 respectively was used as binders. Fly ash was taken from TNB Kapar in Selangor. Superplasticizers also been used as an admixtures for water-reducing agent.

\section{Test Samples}

Basically, the test can be divided into 2 major part; one part by using palm oil clinker (POC) as both coarse and fine aggregate and latter only using POC as coarse aggregate while river sand as fine aggregate. There are five series of test been conducted, namely as shown in Table 2 .

Table 2. Series of palm oil clinker concrete mix

\begin{tabular}{|l|l|l|}
\hline No. & Series & Description \\
\hline 1 & S1 & $\begin{array}{l}\text { control mix (conventional mix OPC) } \\
\text { as binders }\end{array}$ \\
\hline 2 & S2 & $\begin{array}{l}\text { POC coarse and fine aggregates -and } \\
100 \% \text { OPC as binders }\end{array}$ \\
\hline 3 & S3 & $\begin{array}{l}\text { POC coarse aggregate and 90\% OPC + } \\
10 \% \text { fly ash as binders }\end{array}$ \\
\hline 4 & S4 & $\begin{array}{l}\text { POC coarse and fine aggregates and } \\
90 \% \text { OPC }+10 \% \text { fly ash as binders }\end{array}$ \\
\hline
\end{tabular}

Control mix was designed using Department of Environmental (DOE Method) while lightweight series mixtures were design by using FIP Method which was designed as $35 \mathrm{~N} / \mathrm{mm}^{2}$. The water-cement ratio (w/c) was fixed at 0.55 . As this mix falling under lightweight concrete, the cement content must be within $285-510 \mathrm{~kg} / \mathrm{m}^{3}$. Fly ash replaced the Ordinary Portland Cement (OPC) by $10 \%$.

The water content in this mix includes the water adsorption percentage by palm oil clinker. Because of it is porous in nature; palm oil clinker adsorbed a lot of water especially for series 3 and 5 which using palm oil clinker as fine aggregates. We know that higher water cement ratio will produce lower strength of concrete. However, low w/c ratio will reduce workability of the concrete and in worst case, hard in compaction and honeycombing will occur.

Table 3. Mix proportion of concrete samples

\begin{tabular}{|l|l|l|l|l|l|}
\hline \multirow{2}{*}{ Series } & \multicolumn{4}{l|}{ Proportion by weight } \\
\cline { 2 - 5 } & Water & OPC & Fly & Fine & Coarse \\
\hline
\end{tabular}

\begin{tabular}{|l|c|c|c|c|c|}
\hline & & & ash & $\begin{array}{l}\text { aggregat } \\
\text { e }\end{array}$ & $\begin{array}{l}\text { aggregat } \\
\text { e }\end{array}$ \\
\hline S1 & 210 & 382 & - & 668 & 1090 \\
\hline S2 & 231 & 420 & - & $\begin{array}{c}621 \\
\text { (sand) }\end{array}$ & $\begin{array}{c}729 \\
\text { (POC) }\end{array}$ \\
\hline S3 & 231 & 420 & - & $\begin{array}{c}621 \\
\text { (POC) }\end{array}$ & $\begin{array}{c}729 \\
\text { (POC) }\end{array}$ \\
\hline S4 & 231 & 378 & 42 & $\begin{array}{c}621 \\
\text { (sand) }\end{array}$ & $\begin{array}{c}729 \\
\text { (POC) }\end{array}$ \\
\hline S5 & 231 & 378 & 42 & $\begin{array}{c}621 \\
\text { (POC) }\end{array}$ & $\begin{array}{c}729 \\
\text { (POC) }\end{array}$ \\
\hline
\end{tabular}

Hence, we must introduce water-reducing admixture called superplasticizers (sulfonated, naphthaleneformaldehyde condensate type) in the mixture so that it can compensate the water requirement to produce stronger and durable concrete. Its usage depends on water content in the mix design. For this samples, superplasticizers mix proportions was fixed at $1.60 / 100 \mathrm{~kg}$ of cement. As reported by Chen, the superplasticizer dosage went up to 1.75 litre per $100 \mathrm{~kg}$ binders, the one day strength of concrete decrease to a certain extent.

Table 4. Code of Practice for concrete testing

\begin{tabular}{|c|c|c|}
\hline No & Type of testing & Code of Practice Used \\
\hline 1 & $\begin{array}{l}\text { Compressive } \\
\text { strength }\end{array}$ & $\begin{array}{l}\text { BS 1881: Part 115: } 1986 . \\
\text { Specification for Compression } \\
\text { Testing Machines for Concrete }\end{array}$ \\
\hline 2 & $\begin{array}{ll}\text { Flexural } & \text { Test } \\
\text { (3-point } & \\
\text { loading) } & \\
\end{array}$ & $\begin{array}{l}\text { BS 1881: Part 118: } 1983 . \\
\text { Method for Determination of } \\
\text { Flexural Strength. }\end{array}$ \\
\hline 3 & $\begin{array}{l}\text { Tensile } \\
\text { Splitting Test }\end{array}$ & $\begin{array}{l}\text { BS 1881: Part 117: } 1983 \text {. } \\
\text { Method for Determination of } \\
\text { Tensile Splitting Strength. }\end{array}$ \\
\hline
\end{tabular}

All the mixes are done after series of trial mixes is conducted to establish the mix with optimum strength. For compressive test sample, it was cast into a $100 \mathrm{~mm} \times 100 \mathrm{~mm}$ $\mathrm{x} 100 \mathrm{~mm}$ cube. Flexural test sample were cast into a 100x100x500mm prism. Meanwhile, for tensile splitting test were cast into a $150 \mathrm{~mm}$ diameter $\mathrm{x} 300 \mathrm{~mm}$ length cylinder.

\section{D.Testing}

The physical properties of palm oil clinker, river sand, gravel, cement and fly ash were conducted for mix design data purposes. Properties of fresh concrete; i.e., workability were measured throughout all the series mixes. The fresh concrete properties are shown in Table 3.

All the mechanical properties; compressive strength, flexural strength and tensile splitting were performed by using Universal Testing Machine located in Material Lab, Universiti Tun Hussein Onn Malaysia. All the samples were cured in water curing upon 24 hours casting.

Basically, there are 2 types of strength been conducted; first is strength due to compression load which is performed in compression concrete test and the latter case is tension test; which is performed by flexural test (direct tensile test) and tensile splitting test (indirect tensile stress). All the test procedures follow the code of practice as mentioned in Table 4. Compressive test, flexural test (modulus of 
rupture), and tensile Splitting Test is carried out on the $7^{\text {th }}$ and $28^{\text {th }}$ day upon casting.

Table 3. Fresh concrete properties

\begin{tabular}{lll}
\hline Series & $\begin{array}{l}\text { Slump } \\
(\mathrm{mm})\end{array}$ & $\begin{array}{l}\text { Compaction } \\
\text { factor }\end{array}$ \\
\hline S1- Control mix & 190 & 0.98 \\
S2 - POC coarse + OPC & 105 & 0.94 \\
S3 - POC coarse \& fine + OPC & 85 & 0.92 \\
S4 - POC coarse + FLY ASH & 125 & 0.96 \\
S5 - POC coarse \& fine + FLY & 100 & 0.95 \\
ASH & & \\
\hline
\end{tabular}

\section{RESULTS AND DISCUSSION}

\section{A. Cube compressive strength}

The compressive strength for palm oil clinker concrete series and control concrete are tabulated in the form of graphs as shown in Figure 1. Basically all series are showing almost the same patterns of development at $3^{\text {rd }}, 7^{\text {th }}$ and $28^{\text {th }}$ days of testing. Control concrete gave the highest value of compressive strength. Compared with mixture of using 100\% OPC, series with fly ash replacement gave better compressive strength. This is due to the help of pozzolanic reaction that produced more $\mathrm{C}-\mathrm{S}-\mathrm{H}$ which is a substantial compound that contributes to the strength of the concrete.

Series of using POC as coarse aggregate and natural sand gave better results than series using POC aggregates as coarse and fine aggregates. This is due to the fine aggregates which are higher specific surface of porous particles that contribute to the less strength. This, however can produced $33.76 \mathrm{~N} / \mathrm{mm}^{2}$ at 28 days which exceeding the concrete strength that can be used as structural concrete, 30 $\mathrm{N} / \mathrm{mm}^{2}$. Since this mixture using normal OPC, the concrete strength expected to increase after the $28^{\text {th }}$ days due to the matrix strength.

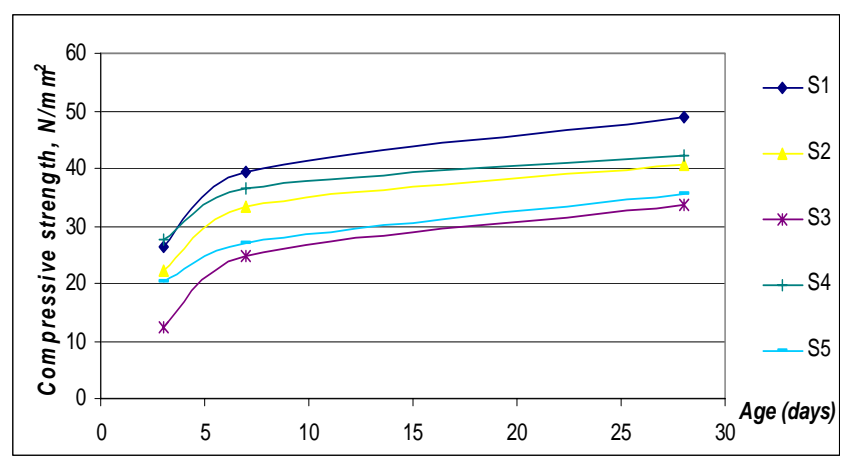

Figure 1. Development of compressive strength for all the series

The density of in saturated surface dry condition is shown in Table 4. Series using only coarse POC aggregates and natural sand (Series 2 and 4) gave around $2000 \mathrm{~kg} / \mathrm{m}^{3}$ and series using POC aggregates as coarse and fine aggregates (Series 3 and 5) gave around $1850 \mathrm{~kg} / \mathrm{m}^{3}$. As density of normal-weight concrete density is $2486 \mathrm{~kg} / \mathrm{m}^{3}$, there is a saving in the self-weight $19 \%$ and $26 \%$ respectively. This savings can produced lighter structures in design stage which will reduced the cost of construction as less dead load can be obtained.

The 7-day compressive strength for palm oil clinker series vary from $24 \mathrm{~N} / \mathrm{mm}^{2}$ (Series 3) to $37 \mathrm{~N} / \mathrm{mm}^{2}$ (series 4) and 28-days compressive strength ranging from $34 \mathrm{~N} / \mathrm{mm}^{2}$ (Series 3) to $42 \mathrm{~N} / \mathrm{mm}^{2}$ (series 4). Comparison with control concrete mix, palm oil clinker concrete series gave less strength from $13 \%-31 \%$ of compressive strength. The aggregates had in comparison with concrete, relatively high strength and their full potential strength was not used.

Table 4. Compressive strength development

\begin{tabular}{llll}
\hline Series & $\begin{array}{l}28^{\text {th }} \text { days / } \\
7^{\text {th }} \text { days } \\
\text { ratio }\end{array}$ & $\begin{array}{l}\text { Relative less } \\
\text { strength } \\
\text { compared to } \\
\text { control concrete }\end{array}$ & $\begin{array}{l}\text { Density in } \\
\text { SSD } \\
\text { conditions } \\
\left(\mathrm{kg} / \mathrm{m}^{3}\right)\end{array}$ \\
\hline S1 & 1.24 & - & 2486 \\
S2 & 1.22 & $16.67 \%$ & 2018 \\
S3 & 1.36 & $30.86 \%$ & 1846 \\
S4 & 1.15 & $13.43 \%$ & 2026 \\
S5 & 1.31 & $27.13 \%$ & 1878 \\
\hline
\end{tabular}

From the comparison above, it can be concluded that conventional mixes are still stronger than lightweight mixes series with POC. However, based on $30 \mathrm{~N} / \mathrm{mm}^{2}$ compressive strength criteria, the lightweight concrete using POC as an artificial lightweight aggregate has performed up to the par and has exceeded $30 \mathrm{~N} / \mathrm{mm}^{2}$ on the $28^{\text {th }}$ day test by achieving an average of $38 \mathrm{~N} / \mathrm{mm}^{2}$. This proves that the POC is satisfactory in its application as a substitute for coarse aggregate in the lightweight concrete. The cube compressive strength is satisfactory with respect to the $7^{\text {th }}$ and $28^{\text {th }}$ day strength.

\section{B. Flexural Test}

When concrete is subjected to bending, tensile and compressive stresses, and in many cases direct shearing stresses developed. The most common plain-concrete structure subjected to flexure is in highway pavement, and the strength of concrete for pavements is commonly evaluated by means of bending tests on beam specimens. The results of the flexural test are shown in Figure 2.

The tensile strength of palm oil clinker lightweight aggregates varies from $5.4-6.4 \mathrm{MPa}$ as seen in Figure 2 . The porosity characteristic of POC aggregates will reduce the flexural strength of POC concrete; this however will be offset by the geometry of the POC aggregates. The angular and sharp edges will increase the tensile strength of the aggregate. Although this effect cannot surpass the control concrete strength, this however will compensate the reduction of strength of the coarse aggregates.

From the results in Figure 2, series with POC lightweight produced lower flexural strength than concrete mix. The reduction of strength compared to control concrete ranging from $19.8 \%$ (series 4 ) to $32.3 \%$ (series 3). However, structurally the flexural strength can be enhanced by using reinforced bars commonly at the bottom zone of the concrete cross section that exposed to tensile cracks. 


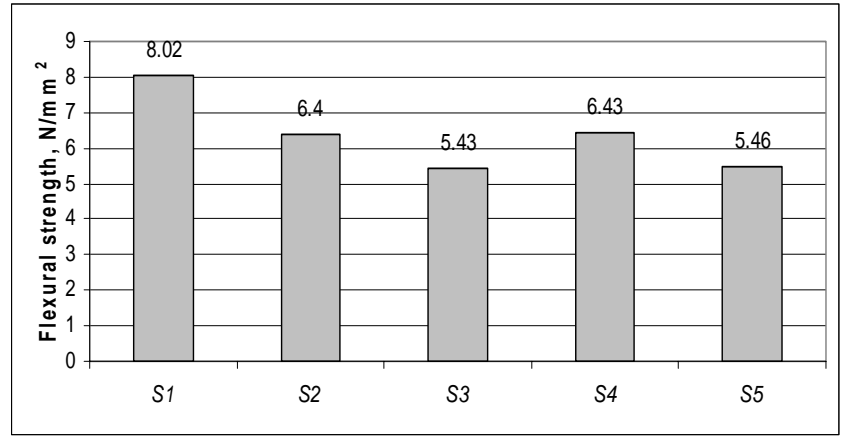

Figure 2. Flexural strength of the series taken

\section{Tensile Splitting Test}

All the series mixes were cast into cylinder mould for the tensile splitting test. Two specimens for each concrete were tested at 7 and 28 days. From the result obtained, control concrete has higher tensile splitting strengths than concrete with palm oil clinker aggregates. This is well understood that the POC aggregates having lower strength than natural aggregates. POC aggregates which are porous might get crushed through the process of mixing and compaction. As reported by $\mathrm{Wu}$, the results of the splitting tensile tests show that the splitting tensile strength of concrete is influenced by the splitting tensile strength of aggregates to a small extent.

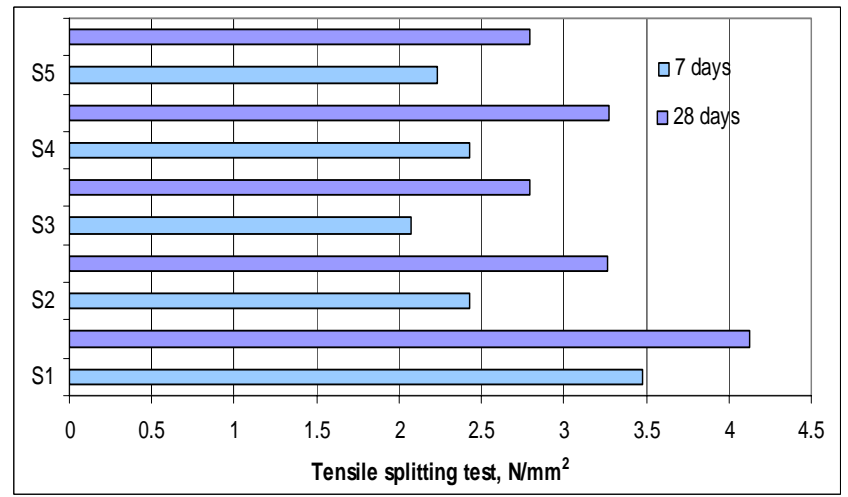

Figure 3. Development of tensile splitting tests all the series

A range of $16 \%$ - 32\% reduction in tensile splitting strength at 28-days was observed for concrete with POC aggregates compared with control concrete, as shown in Table 5. Findings by Zia who reports that at most $35 \%$ lower indirect tensile strength for lightweight concrete compared to control concrete.

Table 5. Tensile splitting test development

\begin{tabular}{|c|c|c|}
\hline Series & $\begin{array}{l}28^{\text {th }} \text { days } / 7^{\text {th }} \text { days } \\
\text { ratio }\end{array}$ & $\begin{array}{lcc}\begin{array}{l}\text { Relative } \\
\text { compared } \\
\text { concrete }\end{array} & \text { to } & \begin{array}{c}\text { strength } \\
\text { control }\end{array} \\
\end{array}$ \\
\hline S1 & 1.18 & - \\
\hline S2 & 1.34 & $21.07 \%$ \\
\hline S3 & 1.28 & $32.45 \%$ \\
\hline S4 & 1.34 & $20.82 \%$ \\
\hline S5 & 1.25 & $16.36 \%$ \\
\hline
\end{tabular}

Compressive, flexural and tensile splitting strength of palm oil clinker series and control concrete are related to data in Figure 1 to Figure 3. From the graphs, flexural and tensile splitting strength can be estimated from known compressive strength. Therefore, it is important to determine the relationship between compressive, flexural and tensile splitting strength of concrete.

Table 6. Relationship of development of flexural and tensile strengths to compressive strength for all series

\begin{tabular}{|c|c|c|c|c|}
\hline & \multicolumn{2}{|c|}{$\begin{array}{l}\text { Flexural strength to } \\
\text { compressive strength }\end{array}$} & \multicolumn{2}{|c|}{$\begin{array}{l}\text { Tensile strength to } \\
\text { compressive strength }\end{array}$} \\
\hline & $7^{\text {th }}$ days & $28^{\text {th }}$ days & $7^{\text {th }}$ days & $28^{\text {th }}$ days \\
\hline S1 & $15 \%$ & $17 \%$ & $8 \%$ & $9 \%$ \\
\hline $\mathrm{S} 2$ & $14 \%$ & $16 \%$ & $7 \%$ & $8 \%$ \\
\hline S3 & $14 \%$ & $16 \%$ & $8 \%$ & $8 \%$ \\
\hline S4 & $14 \%$ & $15 \%$ & $7 \%$ & $8 \%$ \\
\hline S5 & $14 \%$ & $15 \%$ & $8 \%$ & $8 \%$ \\
\hline
\end{tabular}

For palm oil clinker concrete, tensile splitting strength is only $7-8 \%$ (7 days) and $8 \%$ (28 days) of the compressive strength. Meanwhile, the flexural strength is $14 \%$ (7 days) and between $15-16 \%$ (28 days) of its compressive strength. On the other hand, tensile splitting strength of control concrete is from $8 \%$ (7 days) to $9 \%$ (28 days) of its compressive strength.

From Gonnerman and Shuman, the flexural strength ranges from $11-23 \%$ of the compressive strength and the tensile strength ranges from $7-11 \%$ and average about $10 \%$ of the compressive strength. With proper design and research, palm oil clinker has great potential to be used in the construction industry in the future.

\section{COnClusions}

The following conclusions can be drawn from this investigation:-

1. The compressive strength of palm oil clinker concrete series is lower than control concrete. This is attributed to the air content of palm oil clinker aggregates.

2. POC series with $10 \%$ fly ash replacement in cement content produce higher compressive and tensile strengths than POC series with $100 \%$ POC.

3. POC series with using as coarse aggregates only produces higher compressive and tensile strengths than POC series with using POC as both coarse and fine aggregates.

4. However, all the POC series can produce compressive strength more than $30 \mathrm{~N} / \mathrm{mm}^{2}$ to be used as structural members.

\section{ACKNOWLEDGMENT}

The authors would like to express his gratitude to Ministry of Higher Education for financial support.

\section{REFERENCES}

[1] A. M. Neville. 1995. Properties of Concrete - Fourth Edition. Longman.

[2] BS 1881: Part 115: 1986. Specification for Compression Testing Machines for Concrete

[3] BS 1881: Part 116: 1983. Method for Determination of Compressive Strength of Concrete Cubes.

[4] BS 1881: Part 117: 1983. Method for Determination of Tensile Splitting Strength.

[5] BS 1881: Part 118: 1983. Method for Determination of Flexural Strength.

[6] C. H. Goodspeed, S. Vanikar, and R. A. Cook. 1996. HighPerformance Concrete Defined for Highway Structures. Concrete International, Feb, Vol. 18, No. 2, pp. 62-67. 
[7] Chen, Lianrong (Ian) and Ng, Hon Song. 997 High Performance Concrete and its Strength Development $5^{\text {th }}$ International Conference on Concrete Engineering and Technology pp. 47-56.

[8] Concrete Technology Laboratory, School of Civil Engineering, Institute of Industrial Technology, Suranaree University of Technology. Splitting Tensile Strength of Concrete from $1-28$ Days.

[9] FIP Manual of Lightweight of Lightweight Aggregate Concrete: Second Edition. Surrey University Press 1983.

[10] Gerardo G. Gepulango and Mary Earl Daryl A. Grio. Compressive Strength of Concrete from 1 - 28 Days.

[11] Ken W. Day. 1999. Concrete Mix Design, Quality Control and Specification. E \& FN Spon.

[12] M. L. Leming, S. H. Ahmad, P. Zia, J. J. Schemmel, R. P. Elliott, and A. E. Naaman. 1990. High-Performance Concretes: An Annotated Bibliography 1974-1989.

[13] P.-C. Aitcin and A. Neville. 1993. High-Performance Concrete Demystified. Concrete International, Jan, Vol. 15, No. 1, pp. 21-26.

[14] Peter C. Hewlett. 1998. Lea's Chemistry of Cement and ConcreteFourth Edition.Arnold.

[15] P. Zia, M. L. Leming, and S. H. Ahmad. 1991. High-Performance Concretes: A State-of-the-Art Report.

[16] P. Zia, M. L. Leming, S. H. Ahmad, J. J. Schemmel, R. P. Elliott, and A. E. Naaman. 1993. Mechanical Behavior of High-Performance Concretes, Volume 1: Summary Report.

[17] P. Zia, S. H. Ahmad, and M. L. Leming. 1996. High-Performance Concretes: An Annotated Bibliography (1989-1994).

[18] Scientists Celik Ozyildirim and Jose Gomez. Achieving high strength in high performance concrete.

[19] S. W. Forster. 1994. High-Performance Concrete - Stretching the Paradigm. Concrete International, Oct, Vol. 16, No. 10, pp. 33-34.

[20] Wu Ke-Ru, Bing Chen, Wu Yao, Dong Zhang 2001 Effect of coarse aggregate type on mechanical properties of high-performance concrete, Concrete and Cement Research. Vol. 31 pp 1421-1425. 


\title{
Development of Methodology to Establish the Sludge Volume to Power Generated in Utilising Sewage Sludge as a Biofuel
}

\author{
Abdullah, N.A. ${ }^{1}$, Ramli, S. ${ }^{1}$, Ayub, M.A. ${ }^{2}$, Abdul-Talib, S. ${ }^{1}$, Mohd Tajuddin, R. ${ }^{1}$, Atan, I. ${ }^{1}$, Jaafar, J. $^{1}$ \\ and Baki, A. ${ }^{1}$
}

\begin{abstract}
Biogas is a product of decomposition of organic matter during the process of anaerobic digestion (AD). The main components are methane and carbon dioxide. The methane content in the biogas enables it to be used as fuel which can be converted to heat and electricity. The biogas generated by the anaerobic digesters has the potential to be redirected from the flaring facilities to renewable energy (RE) facilities. The biogas may then be used to generate electricity, which in turn can operate the sewage treatment plant (STP) itself. However, feedbacks from the sewerage industry indicated that heavy investments are needed for any RE initiatives on biogas generated by sewage sludge. In order to find the cost effective way of generating energy from biogas, fundamental relationships are necessary to enable development of prototype in the future. Thus, this paper presents a methodology to establish the relationship between the volume of sludge and the amount of power and energy that can be generated.
\end{abstract}

Keywords: anaerobic digestion, biogas, methane, renewable energy, sewage sludge, sludge volume to power ratio

\section{INTRODUCTION}

In response to the rapid development and industrialisation that had taken place over the last 15 years, the energy consumption in this country has increase drastically. To cope with the increase in demand much of the natural hydrocarbon fuel had been utilised, resulting in pollution of the environment and increase in hydrocarbon fuel prices. To overcome this, alternate fuels are being sought worldwide. In Malaysia, work has already begun to identify potential source of RE since 1999. The Government had embarked on efforts to raise awareness on the benefits of RE and the viability of such projects [1]. In October 2002, Pusat Tenaga Malaysia (PTM) was appointed as the agency to implement the Biomass-based Power Generation and Cogeneration in the Malaysian Palm Oil Mills Industry project (BioGen), where empty fruit bunches that is usually disposed as waste will be used to generate power [1]. Following this

1. Abdullah,N.A., Ramli,S., Abdul-Talib,S., Mohd Tajuddin,R., Atan,I., Jaafar,J. and Baki,A., Faculty of Civil Engineering, Universiti Teknologi MARA, 40450 Shah Alam, Selangor, Malaysia (Tel:03-55436179, Fax:55435275, Email: aminbaki2@gmail.com).

2. Ayub,M.A., Faculty of Mechanical Engineering, Universiti Teknologi MARA, 40450 Shah Alam, Selangor, Malaysia. initiatives, the government announced the Fifth Fuel Policy to include RE as alternative fuel resources. The Eight Malaysia Plan contains provisions on the use of biomass for power generation with a specific target that by 2005 five percent of the power supplied to the national grid should come from RE [1].

Biogas is created during the process of AD. The main components of the biogas are methane and carbon dioxide. Methane content in the biogas enables it to be used as fuel which can be converted to heat and electricity. The biogas generated within the anaerobic digesters has the potential to be redirected from the flaring facilities to RE facilities. The biogas may then be used to generate electricity, which in turn can operate the STP itself. The use of biogas generated during the $\mathrm{AD}$ of sewage sludge has been reported in many parts of the world.

Biogas production from sewage sludge treatment is already a well-established means of generating energy in the UK. Over 10 billion litres of sewage are produced everyday in England and Wales. AD of sludge produces a mixture of methane $(60-65 \%), \mathrm{CO} 2(35-40 \%)$ and trace gases. Impurities, such as hydrogen sulphide and water, are removed and the resulting biogas is then used in boilers or combined heat and power (CHP) systems. Biogas may also be used for other applications, such as vehicle fuel, if $\mathrm{CO} 2$ is also removed [2]. In addition to this, reports on studies in Yorkshire and Humberside region have been made [3]. There are 610 STP with a total equivalent population of over $6,000,000$. The total biogas resource from this population, assuming $0.028 \mathrm{~m} 3$ per head per day is approximately $390 \mathrm{GWh}$ per year.

In China, biogas is used by about 25 million people for cooking and lighting for 8-10 months a year. China also has reliable experience of running diesel and gasoline engines with biogas [4]. Bangkok, Thailand, with about 10 million people, is expected to produce up to 63,000 tons/year of sewage sludge by 2010 [4]. The amount of biogas generated during $\mathrm{AD}$ of sewage and brewery sludge at different mixing ratios can be determined [4]. It was found that the maximum quantity of biogas was generated at a mixing ratio of 25:75 (optimum). Table 1 shows that electricity generation using biogas is quite common around the world. 
Table 1. Use of Biogas Generated for Electricity or Heating [5]

\begin{tabular}{|c|c|c|c|c|}
\hline Plant & $\begin{array}{c}\text { Capacity } \\
\text { Tons of Dry } \\
\text { Solids/ year }\end{array}$ & $\begin{array}{l}\text { Sludge } \\
\text { Type }\end{array}$ & $\begin{array}{c}\text { Biogas } \\
\text { Utilisation }\end{array}$ & $\begin{array}{c}\text { Biosolids } \\
\text { Utilisation }\end{array}$ \\
\hline $\begin{array}{c}\text { Oxley } \\
\text { Creek } \\
\text { WWTP, } \\
\text { Brisbane, } \\
\text { Australia }\end{array}$ & 10,800 & $\begin{array}{c}\text { Municipal, } \\
\text { mixture of } \\
\text { primary and } \\
\text { secondary }\end{array}$ & $\begin{array}{c}\text { Electricity } \\
\text { generation } \\
\text { and plant } \\
\text { heating }\end{array}$ & $\begin{array}{l}\text { Dewatered } \\
\text { cake for } \\
\text { agriculture }\end{array}$ \\
\hline $\begin{array}{l}\text { Kapusciska } \\
\text { WWTP, } \\
\text { Poland }\end{array}$ & 7,650 & $\begin{array}{c}\text { Municipal } \\
\text { mixture of } \\
\text { primary and } \\
\text { secondary }\end{array}$ & $\begin{array}{c}\text { Electricity } \\
\text { generation, } \\
\text { plant heating } \\
\text { and } \\
\text { distributed } \\
\text { heating }\end{array}$ & $\begin{array}{l}\text { Dewatered } \\
\text { cake for } \\
\text { agriculture }\end{array}$ \\
\hline $\begin{array}{l}\text { Bruxelles } \\
\text { Nord } \\
\text { WWTP, } \\
\text { Belgium }\end{array}$ & 18,800 & $\begin{array}{c}\text { Municipal, } \\
\text { mixture of } \\
\text { primary and } \\
\text { secondary }\end{array}$ & $\begin{array}{c}\text { Stream } \\
\text { production } \\
\text { (thermal } \\
\text { hydrolysis \& } \\
\text { ATHOS) }\end{array}$ & $\begin{array}{l}\text { Biosolids } \\
\text { destroyed in } \\
\text { ATHOS }\end{array}$ \\
\hline $\begin{array}{c}\text { Niigata } \\
\text { WWTP, } \\
\text { Japan }\end{array}$ & 1,200 & $\begin{array}{c}\text { Municipal, } \\
\text { mixture of } \\
\text { primary and } \\
\text { secondary }\end{array}$ & Test Plant & Test Plant \\
\hline $\begin{array}{l}\text { Frederica } \\
\text { WWTP, } \\
\text { Denmark }\end{array}$ & 8,000 & $\begin{array}{l}\text { Municipal } \\
\text { and } \\
\text { industrial } \\
\text { waste } \\
\text { activated } \\
\text { sludge }\end{array}$ & $\begin{array}{c}\text { Electricity } \\
\text { generation } \\
\text { and plant } \\
\text { heating }\end{array}$ & $\begin{array}{l}\text { Dewatered } \\
\text { cake for } \\
\text { agriculture }\end{array}$ \\
\hline $\begin{array}{l}\text { Dublin } \\
\text { WWTP, } \\
\text { Ireland }\end{array}$ & 36,000 & $\begin{array}{c}\text { Municipal, } \\
\text { mixture of } \\
\text { primary and } \\
\text { secondary }\end{array}$ & $\begin{array}{c}\text { Electricity } \\
\text { generation } \\
\text { and plant } \\
\text { heating }\end{array}$ & $\begin{array}{c}\text { Dried granules } \\
\text { and pasteurised } \\
\text { cake for } \\
\text { agriculture }\end{array}$ \\
\hline $\begin{array}{l}\text { Aberdeen } \\
\text { WWTP, } \\
\text { Scotland }\end{array}$ & 16,500 & $\begin{array}{c}\text { Municipal, } \\
\text { mixture of } \\
\text { primary and } \\
\text { secondary }\end{array}$ & $\begin{array}{c}\text { Electricity } \\
\text { generation } \\
\text { and plant } \\
\text { heating }\end{array}$ & $\begin{array}{l}\text { Dewatered } \\
\text { cake for } \\
\text { agriculture }\end{array}$ \\
\hline $\begin{array}{l}\text { Naestved } \\
\text { WWTP, } \\
\text { Denmark }\end{array}$ & 1,600 & $\begin{array}{l}\text { Municipal } \\
\text { waste } \\
\text { activated } \\
\text { sludge }\end{array}$ & $\begin{array}{c}\text { Electricity } \\
\text { generation } \\
\text { and plant } \\
\text { heating }\end{array}$ & $\begin{array}{l}\text { Dewatered } \\
\text { cake to } \\
\text { composting }\end{array}$ \\
\hline $\begin{array}{l}\text { Chertsey } \\
\text { WWTP, } \\
\text { England }\end{array}$ & 8,000 & $\begin{array}{c}\text { Municipal, } \\
\text { mixture of } \\
\text { primary and } \\
\text { secondary }\end{array}$ & $\begin{array}{l}\text { Process } \\
\text { heating }\end{array}$ & $\begin{array}{l}\text { Wet product to } \\
\text { agriculture }\end{array}$ \\
\hline $\begin{array}{l}\text { Hamar } \\
\text { WWTP, } \\
\text { Norway }\end{array}$ & 3,600 & $\begin{array}{c}\text { Municipal, } \\
\text { mixture of } \\
\text { primary, } \\
\text { secondary \& } \\
\text { chemical }\end{array}$ & $\begin{array}{l}\text { Process and } \\
\text { plant heating }\end{array}$ & $\begin{array}{l}\text { Dewatered } \\
\text { cake to } \\
\text { agriculture }\end{array}$ \\
\hline
\end{tabular}

There are about 9,500 STP (public and private) and over 1 millions individual septic tanks in Malaysia. These sewerage facilities generated about 6.5 millions tons of sewage sludge annually [6]. Reviews were made on the need for RE, such as those obtained through the use of biogas from sewage sludge to generate energy in terms of electricity or heating [6]. It was found that evaluation of potential use of biogas from sewage sludge in Malaysia shows that it is technically feasible but there are logistics factors that may cause limitations.
Based on success of biogas utilisation around the world, the total volume of sewage generated in Malaysia is more than sufficient for RE generation. Reviews were made on four regional STP equipped with anaerobic digesters, which are currently operating in Malaysia. It was found that these four plants are viable for any RE initiatives. However, there was no great effort for the renewable energy project due to lack of expertise and technology locally. Feedbacks from the sewerage industry also indicate that substantial cost and effort are needed for any RE initiatives on biogas generated by sewage sludge. Therefore, this paper will study the potential of implementing an effective method of RE generation from biogas from sludge by developing the relationships between the critical parameters. One of the most basic and important relationships is the ratio between sludge volume to power generated. This ratio enables both the economic and technical feasibility of a RE facility constructed to be evaluated.

\section{DeVelopment of Methodology}

A laboratory based study, utilising bench-scale reactors will be designed as the first stage of the study. The experimental set-up to establish the sludge volume to power ratio will be made. At the end of the study, the sludge volume to power ratio can be applied for utilising biogas for energy generation.

\section{A. Sludge Sample and Sampling Location}

Sludge samples will be collected from the wastewater treatment plant (WWTP) located near to the Mawar College, Universiti Teknologi MARA (UiTM), Selangor. The WWTP was designed for a capacity of 7000 population equivalent $(\mathrm{PE})$. It operates based on the activated sludgeextended aeration processes.

\section{B. Batch Digestion Tests}

Digestion tests will be performed in a reactor at ambient temperature. The total volume of reactors used is $1 \mathrm{~L}$. $500 \mathrm{~mL}$ of sampled sludge will be fermented in the reactor for a few days. The produced biogas will be collected in a cylinder (gas collector), which is equipped with a gas regulator.

Figure 1 shows the apparatus for biogas collection and measurement. The apparatus consists of a reactor which has tubes connected to the thermometer and cylinder. The thermometer will monitor the temperature of sludge in the reactor. The biogas generated will be collected in a cylinder. The cylinder then will be equipped with a gas regulator.

\section{Electrical Energy Transformation}

The resulting biogas normally consists of 50 to 60 percent methane, 30 to 40 percent carbon dioxide and small quantities of residues. The gas is compressed and purified if it contains larger amounts of contaminants, and stored temporarily in a gasometer from which it is fed to a CHP unit at constant pressure. A gas engine transforms the energy stored in the biogas into mechanical and thermal energy. It also powers a synchronous generator, which in turn generates electrical energy for the operation of the STP. The field transformation of sewage sludge into biogas, 
which in turns generates electrical energy, is illustrated in Figure 2 [8].

\section{Potential STUDY LOCATION}

Large WWTP will be equipped with centralised sludge treatment facilities. Apart from these dedicated centralised sludge treatment facilities, there are STP that have anaerobic digesters as part of its sludge treatment units [7]. There are only four STP with anaerobic digestors in Malaysia; Shah Alam STP, Port Dickson STP, Padang Matsirat STP and Sg. Besi STP. The design PE, the estimated sludge volume, the potential energy that can be generated and the estimated ultimate power consumption for these plants are shown in Table 2. Currently, these four facilities are the only plants where the potential for RE generation may be technically and economically possible [7].

Table 2. Potential for RE generation at Four STP in Malaysia [7]

\begin{tabular}{|c|c|c|c|}
\hline Plant & Design PE & $\begin{array}{c}\text { Estimated } \\
\text { Sludge Volume } \\
\left(\mathrm{m}^{3} / \text { day }\right)\end{array}$ & $\begin{array}{c}\text { Potential } \\
\text { Electrical } \\
\text { Energy }(\mathbf{k W})\end{array}$ \\
\hline $\begin{array}{l}\text { Shah } \\
\text { Alam } \\
\text { STP }\end{array}$ & $\begin{array}{l}380,000 \\
\text { (sewage) }\end{array}$ & $\begin{array}{c}520 \\
\text { (at } 1.5 \% \text { dry } \\
\text { solids) } \\
\end{array}$ & 1,700 \\
\hline $\begin{array}{l}\text { Port } \\
\text { Dickson } \\
\text { Town } \\
\text { STP }\end{array}$ & $\begin{array}{c}30,000 \\
\text { (sewage) } \\
98,000 \\
\text { (plant \& tankered } \\
\text { sludge) } \\
\end{array}$ & $\begin{array}{c}336 \\
\text { (at } 4 \% \text { dry } \\
\text { solids) }\end{array}$ & 430 \\
\hline $\begin{array}{l}\text { Padang } \\
\text { Matsirat } \\
\text { STP }\end{array}$ & $\begin{array}{c}22,000 \\
\text { (sewage) } \\
105,000 \\
\text { (plant \& tankered } \\
\text { sludge) } \\
\end{array}$ & $\begin{array}{c}276 \\
\text { (at } 4 \% \text { dry } \\
\text { solids) }\end{array}$ & 360 \\
\hline $\begin{array}{l}\text { Sg Besi } \\
\text { STP }\end{array}$ & $\begin{array}{c}82,500 \\
\text { (sewage) }\end{array}$ & $\begin{array}{c}120 \\
\text { (at } 4 \% \text { dry } \\
\text { solids) } \\
\end{array}$ & 150 \\
\hline
\end{tabular}

\section{CONCLUDING REMARKS}

In conclusion, the use of biogas to generate electricity has been proven worldwide with many successful implementations. In Malaysia, evaluation of the four STP with digesters facilities shows that there is good prospect of utilising biogas for energy generation. There is a potential of implementing cost effective method of RE generation from biogas. Hence, the fundamental relationship on the ratio of sludge volume to power generated will enable future applied research in the development of a prototype plant.

\section{ACKNOWLEDGMENT}

The research team would like to acknowledge the Ministry of Higher Education Malaysia for the fundamental research grant (FRGS-1193) for this study.

\section{REFERENCES}

[1] PTM, (2004), Energysmart, Available: http://www.ptm.org.my/division/download/Energy_Smart/es\%20(14). pdf, accessed on 13 September 2007.

[2] Parliamentary Offices, 2007, Energy And Sewage, Available: http://www.parliamentary.uk/parliamentary_offices/post/ pubd2007.cfm/, accessed on 15 August 2007.

[3] Hawley R.J (2001), Energy From Sewage Sludge In The Yorkshire And Humberside Region, Available: http://crestdl.lboro.ac.uk/support/dissertations/2001/richardhawley.pd f, accessed on 15 August 2007.

[4] Babel S. , Parkpian P. and Sae-Tang J. (2005), "Alternative Energy Generation From Waste Sludge By Anaerobic Co-Digestion”, Journal of Environmental Management, 69, pp.15-24.

[5] Cambi, 2000, Sludge Treatment, Available: http://www.cambi.no/References/sludge/, accessed on 16 September 2007.

[6] Baki A., Abdul-Talib S., Abdul Hamid M.H. and Khor B.C. (2005), "Energy From Sewage Sludge: Potential Application In Malaysia", Proceedings Of $3^{\text {rd }}$ Workshop on Regional Network Formation for Enhancing Research and Education on Green Energy Technologies, Batu Ferringhi, Penang, Malaysia, 7-10 August 2005.

[7] Baki A., Abdul-Talib S. , Abdul Hamid M.H. , Khor B.C. and Salleh M.T. (2006), "The Prospect Of Energy From Sewage Sludge In Malaysia", Proceedings Of Conference On Energy For Sustainable Development: Issues And Prospect For Asia, Phuket, Thailand, 1-3 March 2006

[8] General Electric Company, 1997, Biogases, Available: http://www.geenergy.com/prod_serv/products/recip_engines/en/gas_types/biogas landfill.html, accessed on 15 August 2007.

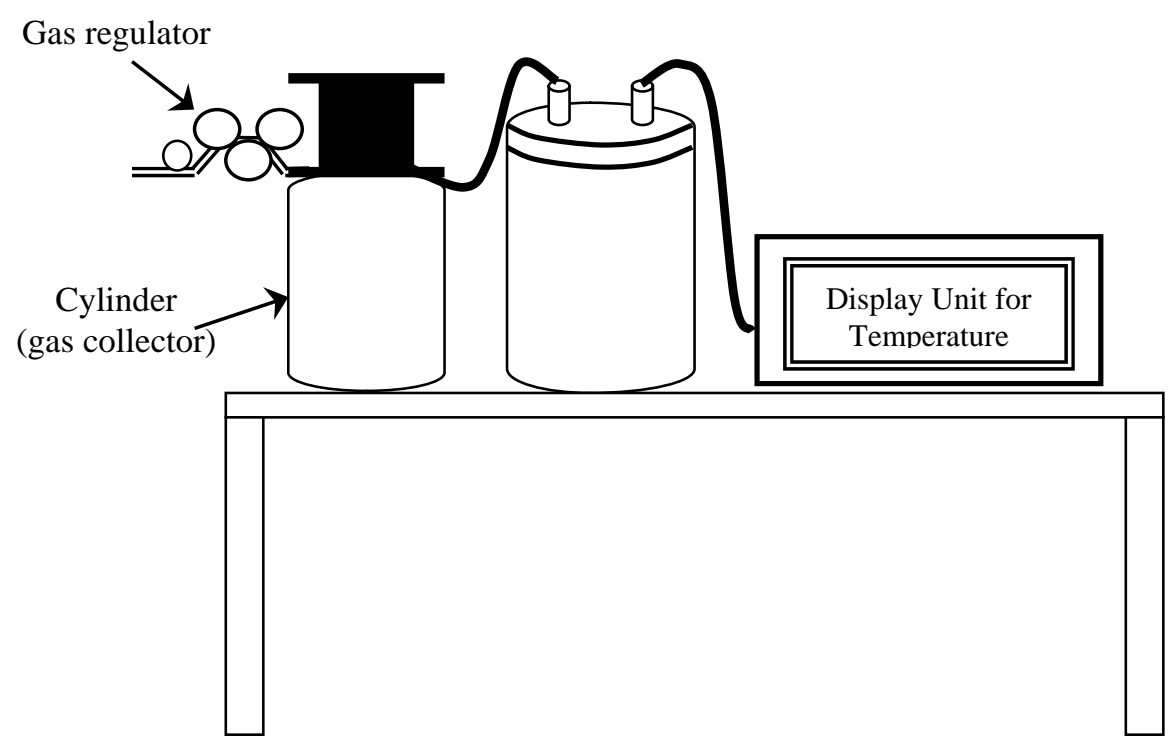

Figure 1. Experimental apparatus for biogas collection and measurement 


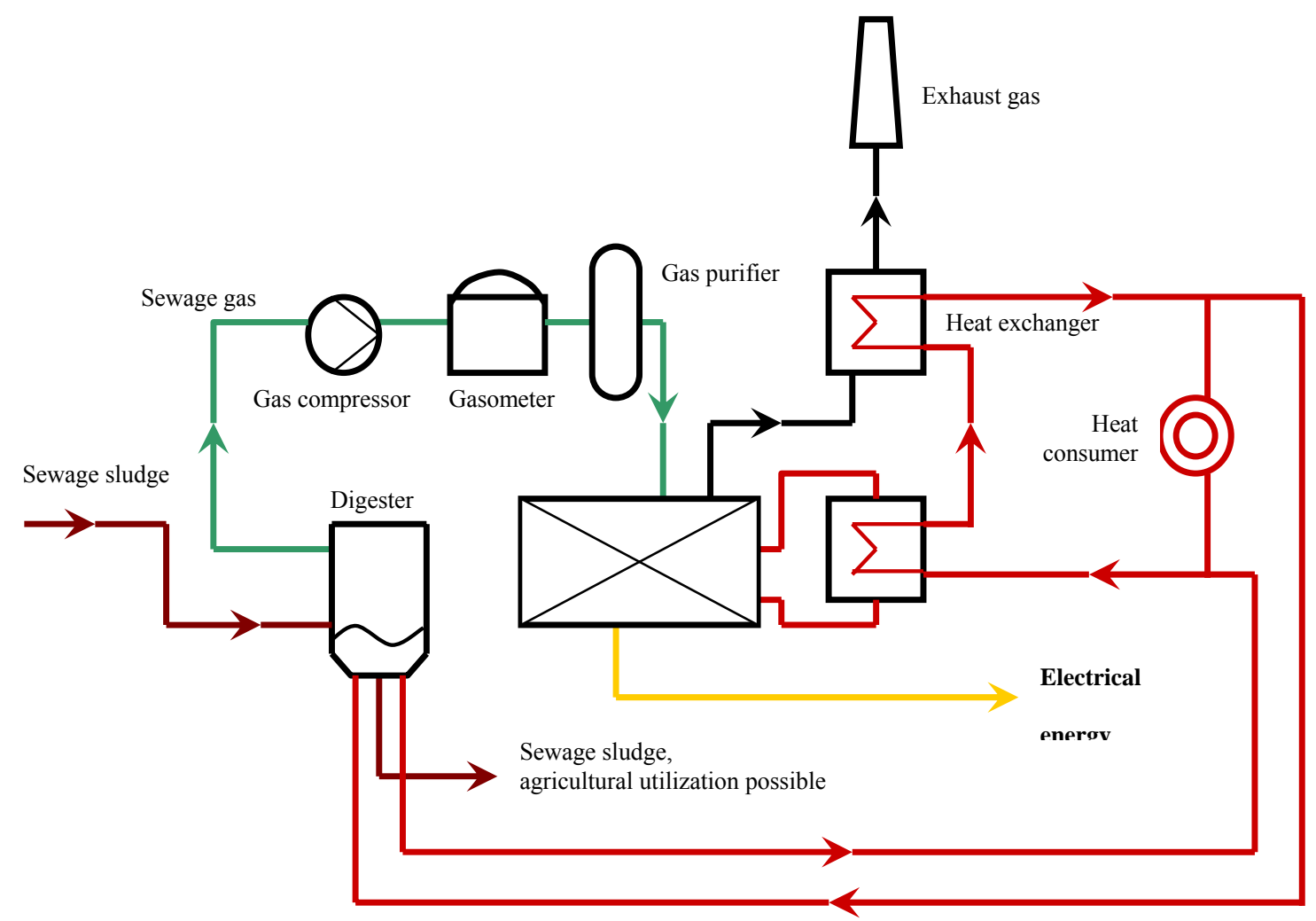

Figure 2. The field transformation of sewage sludge into biogas, which in turn generates electrical energy (adapted from [8]) 


\title{
Development of a Cost-Effective Op-Amp Based Digital Power Factor Meter
}

\author{
B.C. Kok, C.S. Tan, and E. Sulaiman
}

\begin{abstract}
Conventional power factor meter uses analogue system to read the power factor of an AC electric power system which is defined as the ratio of the real power to the apparent power. In the modern life, an easy-captured data yet cost effective power factor measurement device is vital in the electrical power engineering studies. The unstable reading of the analogue based power factor meter especially in the disturbance situations emerged the development of digital based power factor meter (PFM) to replace the conventional electro-dynamic types. The major advantage of using the digital based PFM is the data read by the meter can be stored easily for other applications or studies. This paper presents the development of a cost effective digital PFM which is based on an analogue-to-digital converter (ADC) provided with ratiometric facilities. Generally, the digital power factor meter consists of four main parts which are power supply, inputs circuit, signal processing, and the display part. A signal proportional to the peak voltage $(\mathrm{Vm})$ is applied to the reference input voltage of the ADC, while another signal proportional to the product of the peak voltage $(\mathrm{Vm})$ and power factor is applied to the analogue input. Both signals are processed through various operational amplifier based circuits such as averager, zero detector, and differentiator. The developed digital PFM has been tested and performed for single phase laboratory measurements with three decimal digits display on a liquid crystal display (LCD) unit. The results achieved validate the functionality of the developed digital PFM.
\end{abstract}

Keywords: power factor, phase detection, op-amp, ADC.

\section{INTRODUCTION}

The conventional electrodynamometers are based on the principle of two movable coils set at right angle to each other. These meters suffer from only moderate accuracy which has less sensitivity to line current change. An analogue circuit is where the signals may take on a continuous range of value between some minimum and maximum levels; whereas on digital circuit i.e. logic circuits

This work was supported in part by UTHM short grant scheme (Vote number: 0302).

B. C. Kok is with Universiti Tun Hussein Onn Malaysia, 86400 Batu Pahat, Johor, Malaysia (phone: 607-4537548; fax: 607-4536060; e-mail: bckok@uthm.edu.my).

C. S. Tan, is currently doing her undergraduate study at Universiti Tun Hussein Onn, 86400 Batu Pahat, Johor, Malaysia (e-mail: sying_tan@yahoo.com).

E. Sulaiman is with Universiti Tun Hussein Onn Malaysia, 86400 Batu Pahat, Johor, Malaysia (e-mail: erwan@uthm.edu.my). where each signal value is naturally represented by a digit. As technology growing, digital equipments and instruments are used widely to make the measurement more accurate and easier analyzable.

Bombi and Cisato (1971) have developed a high precision digital power factor meter which suites for accurate laboratory measurements even in the presence of significant voltage, current, and frequency variation [1]. The instrument is in the $0.1 \%$ accuracy class and used the voltage to frequency conversion (VFC) technique to perform the digital integration. This method experiences the difficulty as being dependent on the linearity to the VFC and binary rate error. This method is also sensitive to the accuracy of the component used.

Daniel Slomivitz and Carlos Faverio proposed the power standard based on a high precision power factor meter [2]. There are instruments available to measure the power factor directly, which is based on the measurement of the phase angle between the current and the voltage, through zerocrossing detectors. If the input waveforms have small distortion, large errors will be appeared. Method to reduce the influence of harmonic distortion has been proposed. Main advantage of this proposed meter is less error during capturing the input signal which uses the low-pass filter at inputs of the zero-crossing phase meter. The output of the zero-crossing is a square waveform. The other method to reduce the noise is by using an inverter to measure the phase angle waveform.

This paper presents the development of a high precision power factor meter using operational amplifiers (Op-amps). Op-amps have been utilized almost in all parts of the circuit and it feeds the output signals into the analogue-to-digital converter (ADC). Basic electrical connection of the power factor meter to a single-phase power system is depicted in Figure 1.The various output waveforms at several points from the PFM has been shown in Figure 2. Waveform $A$ represents the voltage output from the secondary winding of the potential transformer (PT). This waveform is then applied into the rectifier and averager circuits to produce DC voltage signal $(B)$. The DC signal acts as the reference input to the ADC circuit. Waveform $A$ is also applied to an analogue gate where the $\mathrm{AC}$ voltage waveform is varied in accordance with the switching from the current signal. The resulting voltage waveform $(F)$ is thus been averaged to provide DC voltage $V_{2}(G)$, which is comparative to $V_{m} \cos \theta$. 


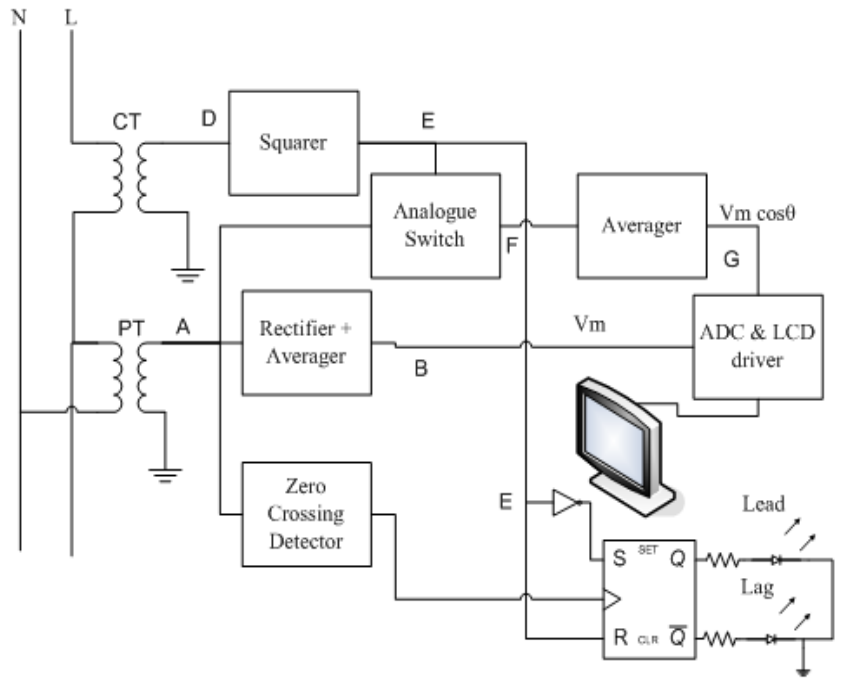

Figure 1. The connection between the digital power factor meter and a single-phase power system

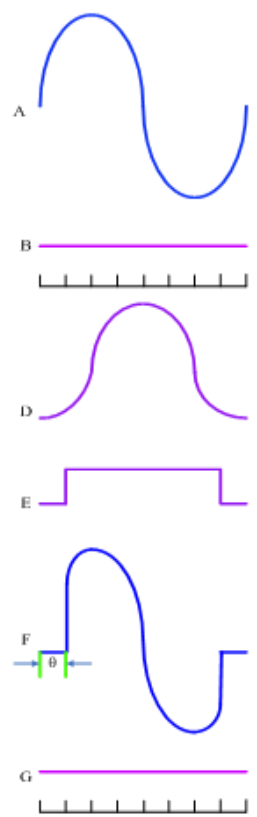

Figure 2. The digital PFM measurement waveforms

The current transformer (CT) produces an AC waveform (D). Waveform $D$ is applied into the voltage squaring amplifier circuit in order to be converted into a square wave $(E)$. The signal from $E$ is then used to control the analogue switch.

The present design for the display is based on the (ICL7126) type dual-slope integrating ADC with all the necessity circuit to drive the digits liquid crystal display (LCD).

The lead-lag indicating signal is obtained by using the zero crossing detector as well as the SR flip-flop. The particular waveforms have been presented in Figure 3.

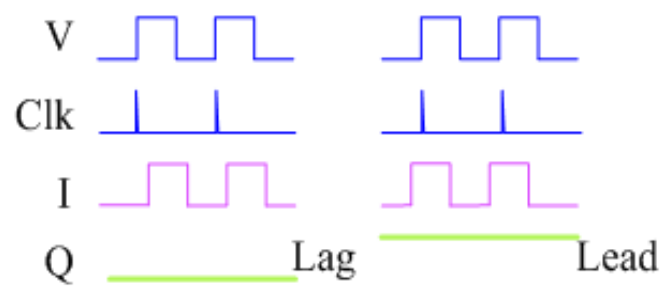

Figure 3. The waveform arrangement of lead-lag indicator
The clock signal produced by the zero crossing detector is the same as the data signal produced by the square wave $E$. Whenever the clock detected the zero current signal, it will gives 0 volt output at $Q$ and vise versa. The overview of the complete circuit diagram for the proposed digital PFM is shown in Figure 4.

\section{DeVELopMENT OF Digital PFM}

This project is adopting a cost-effective method approaches in the design and development of a digital PFM. The concerns of the design are give focuses on the received input signal waveforms ( $V$ and $I$ ) until the displaying value of the measured power factor. Generally, it consists of four main units which are the input circuit unit, power supply unit, signal processing unit, and the display unit. The input circuit unit is deals with the signals received by current transformer and potential transformer while the power supply unit produces the supplied voltage to the remaining components. The signal processing unit consists of rectifier, squarer, differentiator, averager, and flip-flop. Meanwhile, the display unit is concerning on the ADC, LCD and its driver. The measured power factor value will be displayed on LCD and the lead-lag indication signal will be displayed via LED. The basic block diagram is given in Figure 5.

\section{A. Input Circuit Unit}

Measurement part based on the instrument transformers is primarily used to provide isolation between the main primary power system and the secondary control and measuring devices [3]. The current transformer and the potential transformer are the two types transducer used in the design. The locations of the transducers are between the power source and signal processing unit. The purpose of using the transducers is to reduce the input voltage and current before reaching the signal processing circuit as all the components afterwards are IC based which cannot be exposed to high voltage or current applications.

\section{B. Power Supply Unit}

The power supply unit provides a certain level of supplied signal to the rest of the components. The power supply circuit consists of half-wave rectifier and a simple regulator. The input of the power supply circuit is taken from the potential transformer. The output of the power supply unit is a smooth DC waveform. Three capacitors are connected in parallel and feed into the regulator as shown in Figure 6.

\section{Signal Processing Unit}

The signal processing unit processes the input signal from the transducers which is then output the processed signal to the ADC. The signal processing unit consists of the rectifier, squarer, differentiator, averager, and flip-flop. The averager is designed by using the integrator op-amp circuit together with a diode. The integrator op-amp uses two resistors of $100 \mathrm{k} \Omega$, one capacitor of $1 \mu \mathrm{F}$ and an op-amp of 741 . 


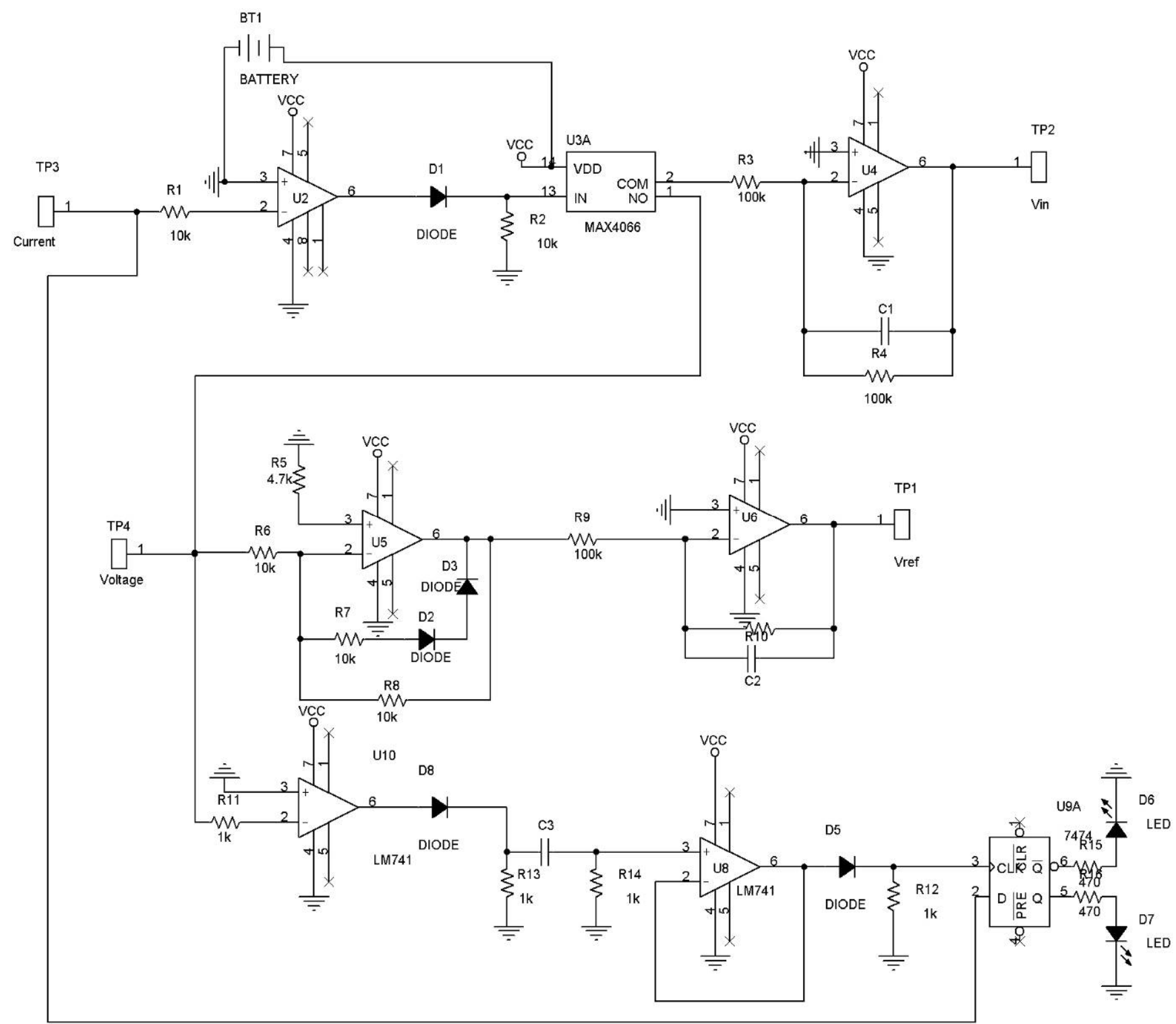

Figure 4. Overview of the digital PFM circuit

The input for the signal processing unit is the sinusoidal waveform and the output is a constant voltage when the resistor value of the $R_{3}$ is equal to the $R_{I}$. The circuit of the averager is shown in Figure 7.

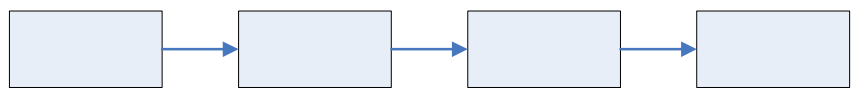

Figure 5: The basic block diagram of the digital PFM.

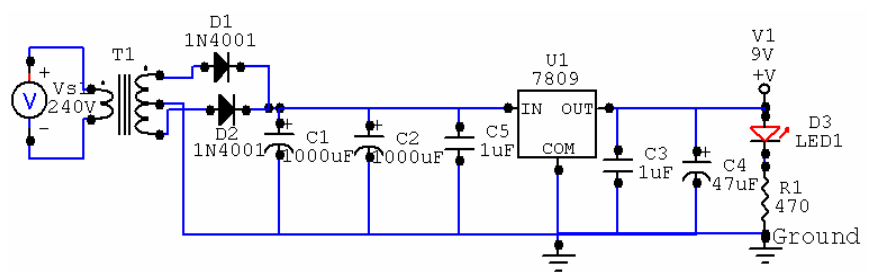

Figure 6 . The power supply circuit

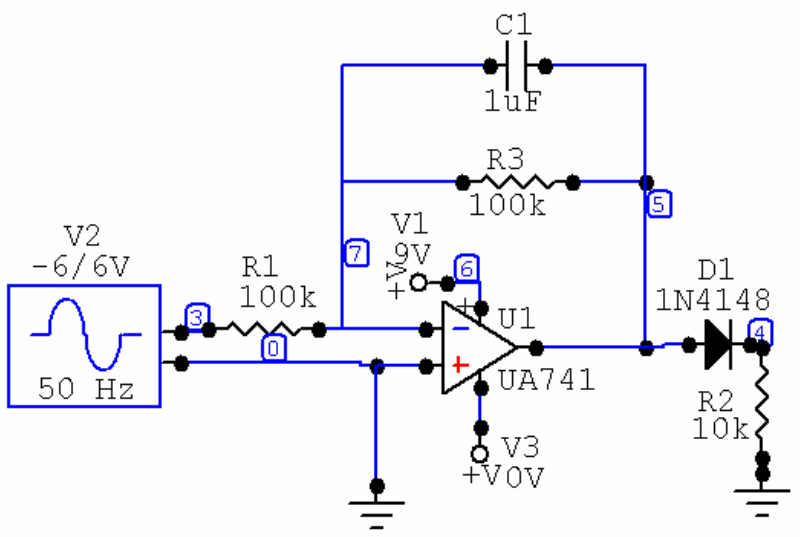

Figure 7. The design circuit of an averager

The rectifier is designed by using the half-wave op-amp rectifier circuit with an active filter. The output waveform from the filter will be a smooth and constant value when the value of the resistor $R_{4}$ equals to $R_{7}$. The half-wave op-amp rectifier is using two resistors of $10 \mathrm{k} \Omega$, one resistor of 4.7 $\mathrm{k} \Omega$, two diodes of $1 \mathrm{~N} 4148$ and an op-amp of 741. The circuit of the rectifier is shown in Figure 8. 


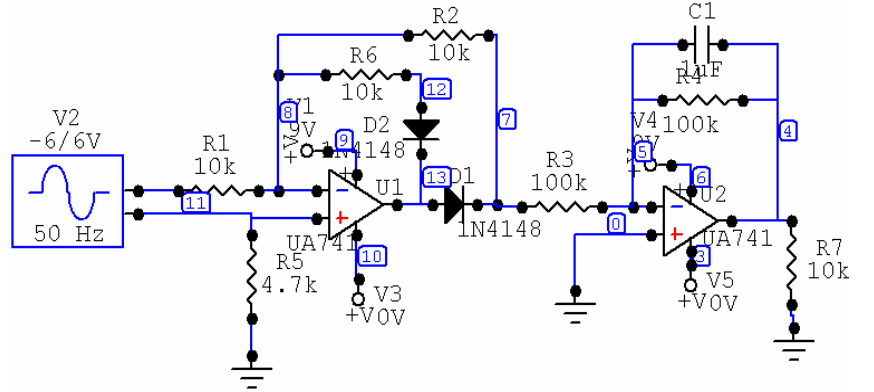

Figure 8 . The complete half-wave rectifier circuit

The squarer is designed by using the op-amp comparator circuit with one diode. The circuit of the squarer is shown in Figure 9.

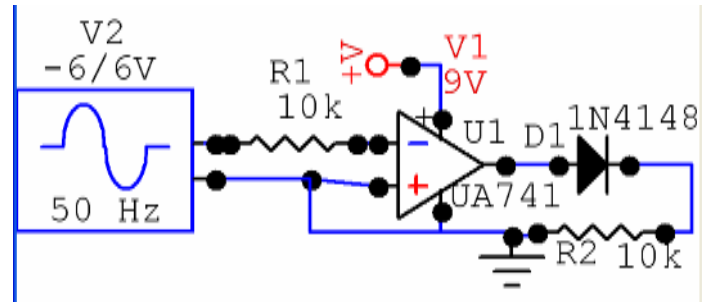

Figure 9. The complete circuit of the squarer

The zero crossing circuit is designed by using the buffer op-amp circuit, passive RC filter, and diodes. The input signal for the zero crossing is a square wave which is output from the squarer. The passive $\mathrm{RC}$ filter uses a resistor of 10 $\mathrm{k} \Omega$ and a capacitor of $0.01 \mu \mathrm{F}$. The main components for the buffer op-amp are one op-amp 741, one resistor of 10 $\mathrm{k} \Omega\left(R_{4}\right)$, and one resistor of $50 \mathrm{k} \Omega\left(R_{3}\right)$. The circuit of the zero crossing is given in Figure 10.

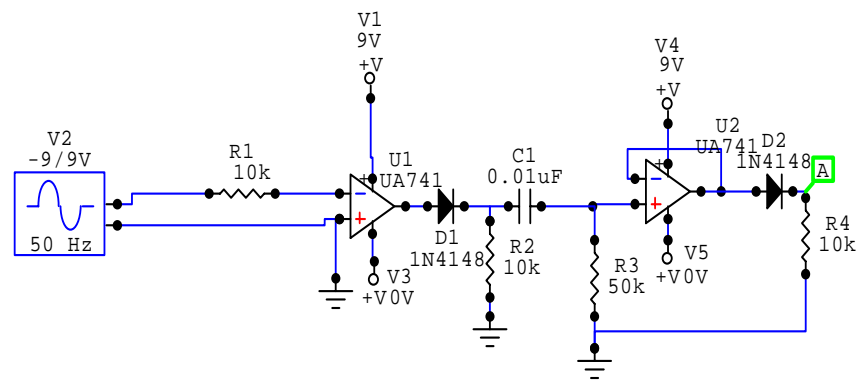

Figure 10. The complete circuit of zero crossing

\section{D.Display Unit}

The display unit has two main components which are $\mathrm{ADC}$ and LCD. ADC is used to convert analogue signal to the digital and it can be directly interfaced with LCD without driver. ADC also provides the ratio metric function which is able to compare the reference and input voltages. The reference input voltage, $V_{R E F}$ is taken from the rectifier voltage, $V_{m}$. The input voltage, $V_{I N}$ is taken from the averager output, $V_{m} \cos \theta$. The digital reading is displayed as:

Display Count $=1000\left(\frac{V_{I N}}{V_{R E F}}\right)$
A $3 \frac{1}{2}$ digits LCD will be used to display the value of the measured power factor. It gives four digits display on the LCD. The measurement range of the power factor is within 0 to 1 .

\section{Simulation AND TeSt Results}

The main focus of the simulation and test results are on the signal processing unit. The simulation results obtained are then verified with the measured results by oscilloscope.

The input waveform is a sinusoidal wave with the frequency of $50 \mathrm{~Hz}$.

The squarer circuit received linear voltage as its input signal and then produced a digital output indicating one input is less than or greater than the other. Op-amp is used to compare the inverting input $(V-)$ with the non-inverting input $(V+)$.

High voltage output will be provided when the $\mathrm{V}+$ input is greater than the $\mathrm{V}$ - input and vice versa. The simulation and measured results of the squarer circuit is shows in Figure 11.

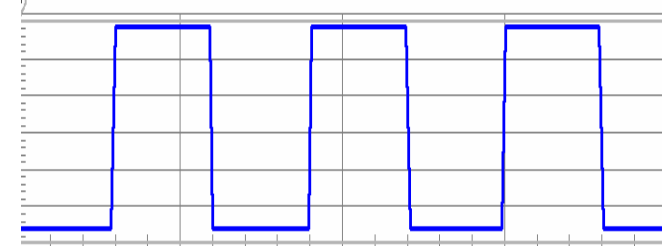

(a)

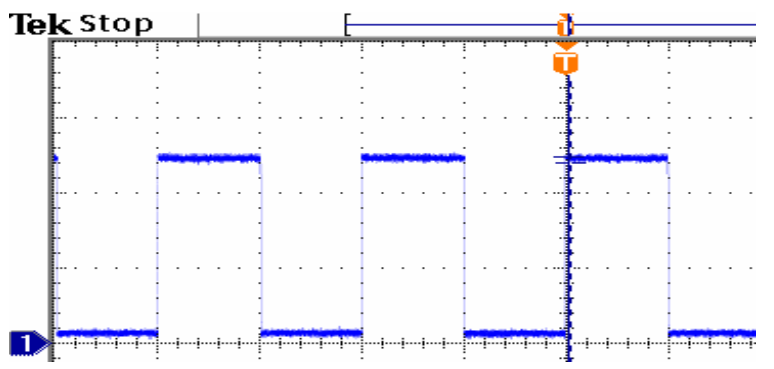

(b)

Figure 11. (a) Simulation result of the squarer circuit; (b) Measured result of the squarer circuit

The main factor that influences the waveform shape is the slew rate of the Op-amp. The higher the slew rate the sharper edge will be obtained. Unfortunately, higher slew rate will be more expensive.

The averager is also known as integrator. The integrator produces a voltage output proportional to the product (multiplication) of the input voltage and time. The op-amp circuit would generate an output voltage proportional to the magnitude and duration that an input voltage signal has deviated from 0 volts.

Stated differently, a constant input signal would generate a certain rate of change in the output voltage. The simulation and measured results of the average is shown in Figure12. 


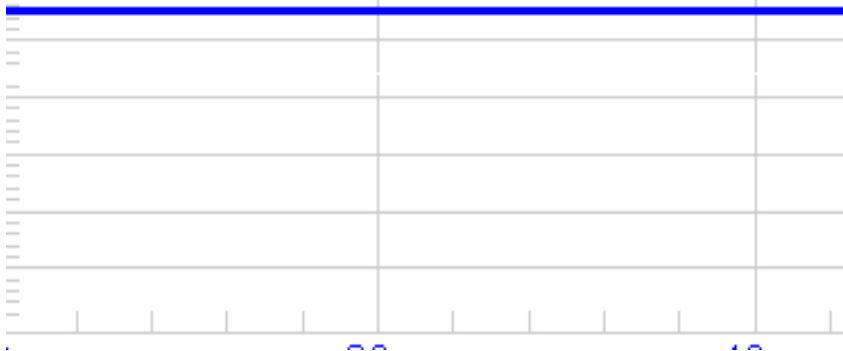

(a)

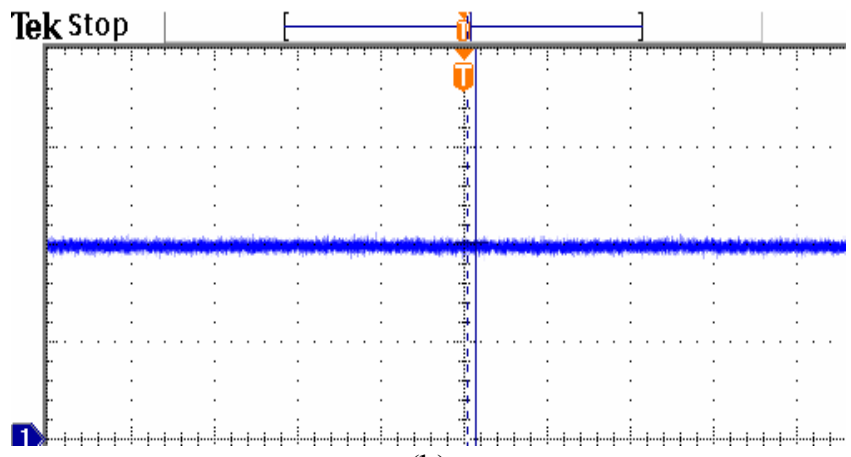

(b)

Figure 12. (a) Simulation result of the averager circuit; (b) Measured result of the averager circuit

Since both of the resistor values are same, a constant output value is produced. As the comparison, the simulation result has perfect constant value but there is some noise and distortion happened in the measured result.

The rectifier circuit shown in Figure 8 uses an inverting amplifier configuration. Diode $D_{1}$ is reverse biased and $D_{2}$ is forward biased during the op-amp output terminal is positive. This occurs when the input signal is negative. Since $D_{2}$ is forward biased, the circuit output is given as follows:

$V_{o}=-V_{i} \frac{R_{2}}{R_{1}}$

In this case, when $R_{2}$ equals to $R_{l}, V_{o}$ will equals to $-V_{i}$ during the negative half-cycle of the input. During the positive half-cycle of the input, the op-amp output terminal goes negative, causing $D_{2}$ to be reverse biased. The negative voltage at the op-amp output forward biases $D_{l}$.

This tends to pull the op-amp inverting input terminal to a negative direction. But, such a move would cause the opamp output to go positive. The sole purpose of $D_{l}$ is to keep the op-amp output from going into saturation and thus maintaining the best possible circuit frequency response. The simulation and measured results of the rectifier circuit is depicted in Figure 13. As a comparison, the measured waveform is closer to the ideal waveform with a little noise generated.

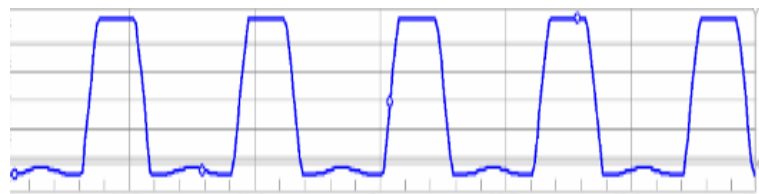

(a)

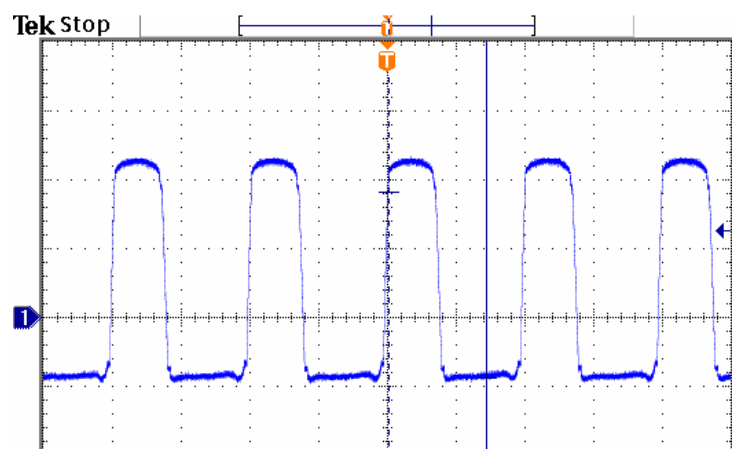

(b)

Figure 13. (a) Simulation result of the rectifier circuit; (b) Measured result of the rectifier circuit

The zero crossing detector constructed by using the comparator, $\mathrm{RC}$ circuit and a buffer. The zero crossing acts as the clock signal to the flip flop. A zero crossing detector is a comparator op-amp circuit that goes into saturation when the sine wave input becomes greater then 0 Volts.

The output of the zero crossing detector op-amp goes into a passive differentiator circuit consisting of a capacitor and a resistor. The diode eliminates the negative part of the differentiated signal. Since a pulse is needed for the positive and negative slopes of the 50 cycles sine wave, a mirror zero crossing detector is constructed that gives a positive pulse when the sine wave input becomes negative.

After passed through the buffer which combined with the diode, it only allows the positive waveform to be passed through and eliminates the negative waveform from the input of the buffer. The simulation and measured results of the squarer circuit is shown in Figure 14.

Both waveforms achieved the ideal waveforms and it has been proven that the design is successfully acted as the clock to the flip-flop.

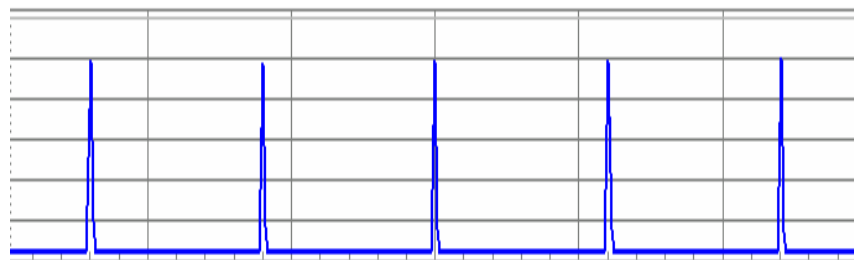

(a)

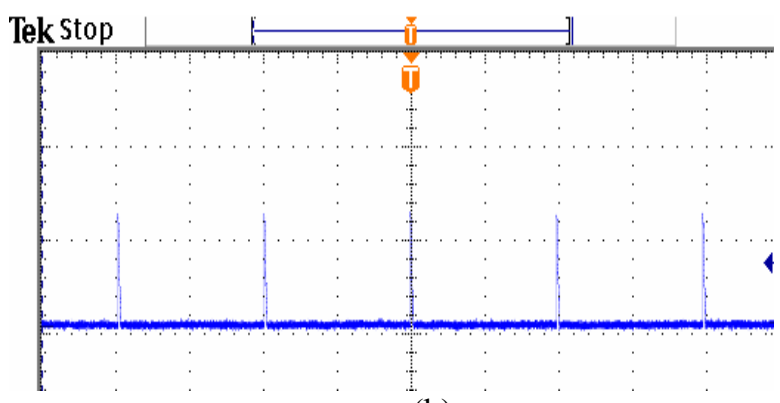

(b)

Figure 14. (a) Simulation result of the zero crossing circuit; (b) Measured result of the zero crossing circuit 


\section{CONCLUSION}

The digital PFM has the ability to capture leading or lagging power factor with the resolution of 0.001 . Lead-lag indication and bidirectional operation are provided. The operation of the proposed digital PFM will not affected by the normal variations of the line current, voltage, and frequency.

Low-offset voltage operational amplifiers with high slew rate are required to improve the performance of the meter and to increase the frequency range. The dynamic response of the system is mainly dominated by the sampling period of the ADC which is 3 readings per second for optimum rejection of $50 \mathrm{~Hz}$ hum pick-up. However, speed limitations are inherent in integrating converters and if a greater response is required, successive approximation converters are suitable.

\section{ACKNOWLEDGMENT}

The authors gratefully acknowledge the staffs in the Laboratory of Electric Machines and Drives as well as Laboratory of Electric Power, UTHM for their valuable contributions towards the succeed of this work.

\section{REFERENCES}

[1] Bombi,F and Ciscato,D (Febuary 1971). "Digital power-factor meter has high order of accuracy." University of Padova, Italy.

[2] Slomovits,D and Faverio,C "Power Standard Based on a High Precision Power-Factor Meter." UTE LABORATORIO, Uruguay.

[3] Harlow,J.N (2003). "Electric Power Transformer Engineering." $1^{\text {st }}$ edition, CRC PRESS, New York. 


\title{
Development of a Microcontroller-Based Power Factor Corrector
}

\author{
C. Uttraphan, B.C. Kok, K.H. Lai, and S.A. Zulkifli
}

\begin{abstract}
Power factor corrector (PFC) has been widely used in the industrial power systems to restore the system power factor to as close to unity as is economically viable. Most of the power factor correctors are of static type in order to deal with the inductive loads such as the electric motors, compressors, and even the fluorescent lightings. The static type PFC however, facing inadequate application whenever the inductive load changes. This paper presents a microcontrollerbased self-adaptive power factor corrector for poor power factor (linear or nonlinear) loads applications. The design intention of this auto-adjustable power factor corrector is to ensure the power system always preserving almost unity power factor and thus optimizing the current consumption. Power factor value is initially measured via microcontroller and it is then compared with the predetermined reference value. Accordingly, the system poor power factor will then be adjusted by the aid of the microcontroller to as close the predetermined value as possible. The proposed power factor corrector has an adjustable sensitivity of $\mathbf{5 0}$ microseconds or 0.9 degree interval step setting. The proposed power factor corrector is demonstrated on a range of laboratory testing by using the induction motors and the fluorescent lightings as the system test loads. From the validation tests, it can be concluded that the proposed power factor corrector has great capability to reduce the wasted energy in distribution system.
\end{abstract}

Keywords: power factor, firing angle, PIC, reactive load

\section{INTRODUCTION}

The low power factor in the power distribution system causes the energy crisis in the supply of energy resources. Most of industrial electric loads have a low power factor not exceeding 0.8 and thus contributes to the distribution system losses [1-4]. There are different methods of power factor correction implemented with large lagging or leading nonlinear loads [1]. One of the new approaches is to use a variable inductor in parallel with a fixed capacitor as a reactive power compensating circuit [5-6]. The inductor current is controlled by adjusting the firing angle of two anti-parallel connected thyristors or using TRIAC [4]. The adjustment of the thyristors' firing angle is made in accordance to the result of a comparison between the measured value of a certain system parameter with its reference value [7-10]. This approach is more reliable because it involves the counts of the leading and lagging current in the power factor with a very accurate and precise step setting, in terms of calculating the phase angle, in the power factor corrector. In spite of giving pre-calculated relations between the system power factor and the static
VAR compensator firing angle, the suggested power-factor correction scheme do not give a real-time solution to the problem of low power factor in nonlinear loads.

This paper proposes a real-time microcontroller-based power factor correction scheme for low power factor loads. The software and hardware required to implement the suggested adaptive power factor correction scheme are explained, and its operation is described.

\section{BLOCK DiAGRAMS AND DESCRIPTIONS}

The block diagram of the microcontroller based power factor corrector is shown in Figure 1.

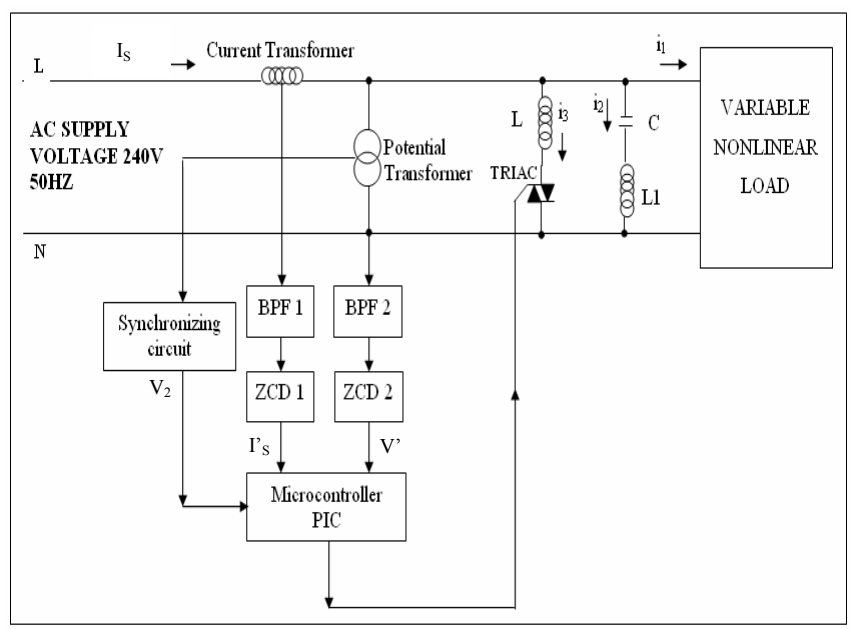

Figure 1. Overview of the block diagram for the microcontroller-based power factor corrector

A microcontroller of PIC16F84A with a crystal of $4 \mathrm{MHz}$ has been utilised in the proposed scheme. The static compensator employed in the system is a parallel combination of a fixed capacitor $C$ and a TRIAC controlled reactor (inductor $L$ ). A small inductance $L_{l}$ is connected in series with $C$ to prevent parallel resonance. Two back-toback thyristors are used to control the current flow through the reactor. The supplied voltage and current signals, taken through a potential transformer and a current transformer, respectively, are applied to band pass filters (BPF 1 and BPF 2). The detail of the filter design is depicted in Figure 2. The outputs of these two filters are the fundamental complex waveforms of the supplied voltage and current, respectively. Subsequently, the two sinusoidal waveforms are being changed to square waves through the two zerocrossing detectors (ZCD 1 and ZCD 2) as the 
microcontroller can only detects the digital signal input, or known as 'pulse'. Figure 3 shows the particular system voltage and current waveforms.

The synchronizing circuit shown in Figure 4 produces a pulse at each zero-crossing of the supplied voltage sine wave. The rising edge of the synchronizing circuit output pulse is synchronized with the zero-crossing of the input sine wave voltage. The output pulses obtained from the synchronizing circuit are then applied to the input of microcontroller as a reference in order to trigger the TRIAC firing angle which is to make sure the pulse is synchronised to the input sine wave voltage.

Afterwards, the pulse signal from the microcontroller drives the gates of the two back-to-back thyristors or TRIAC so as to control the reactor current. The phase angle, $\phi_{m}$ between the fundamental components of the supplied voltage and current ( $V$ and $I$ respectively) is measured by the microcontroller. The details of the source code developed to achieve the above task are explained afterwards. The measured phase angle, $\phi_{m}$ is then compared with a reference value, $\phi_{r}$ that gives the required power factor (displacement factor). The resultant error signal is used to adjust the number that to be loaded into the programmable interval timer in order to change the firing angle, $\alpha$ of the thyristors in the static compensator circuit. These changes should be in a direction that reduces the difference between $\phi_{m}$ and $\phi_{r}$ to a certain acceptable tolerance value. Figure 5 shows the phasor diagram of the system currents shown in Figure 1. This phasor diagram may helps to explain the operation principle of the proposed adaptive power factor corrector and to distinguish between the following three cases, assuming $\phi_{r}$ is always lagging:

(1) $\phi_{r}$ and $\phi_{m}$ have different signs.

(2) $\phi_{r}$ and $\phi_{m}$ have the same sign and $\left|\phi_{r}\right|>\left|\phi_{m}\right|$.

(3) $\phi_{r}$ and $\phi_{m}$ have the same sign and $\left|\phi_{r}\right|<\left|\phi_{m}\right|$.

In the cases (1) and (2), the reactor (inductor $L$ ) current must be increased, and consequently, $\alpha$ must be decreased to get the required power factor $\left(\phi_{m}=\phi_{r}\right)$. In the case (3), the reactor current must be decreased, and consequently, $\alpha$ must be increased to have $\phi_{m}=\phi_{r}$. The above cases are taken into consideration in the main control program to be discussed later. In the proposed scheme, the system power factor is improved by adjusting the displacement factor only. The distortion factor may be improved by using filters adjusted to remove the dominating harmonics.

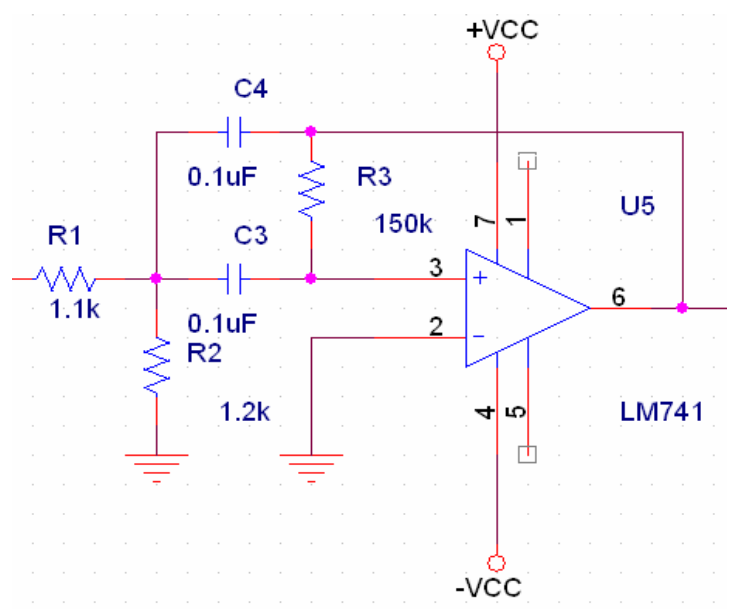

Figure 2. Bandpass filter

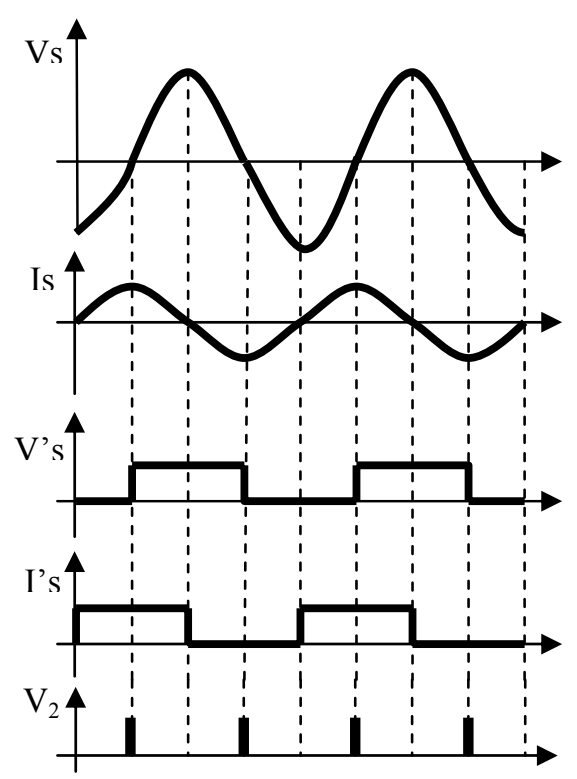

a) Leading current

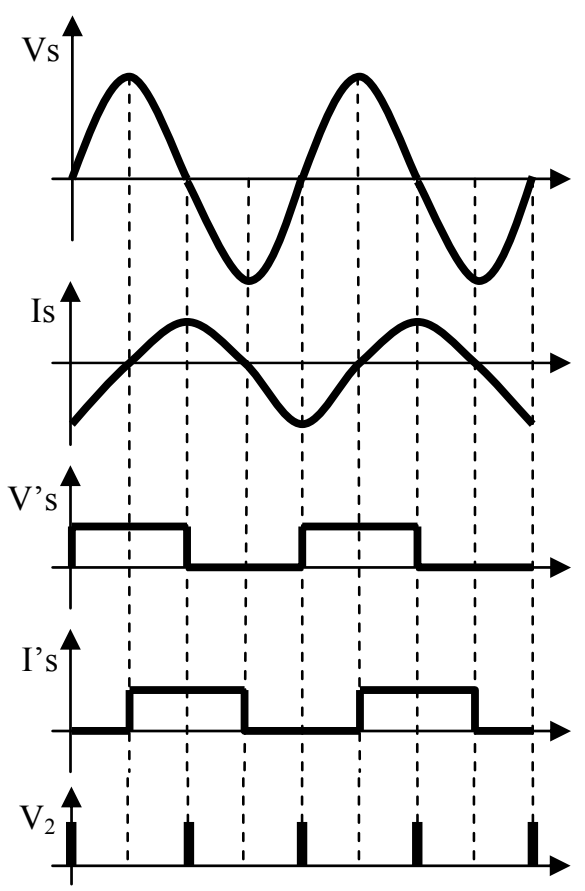

b) Lagging current

Figure 3. System current and voltage waveforms 


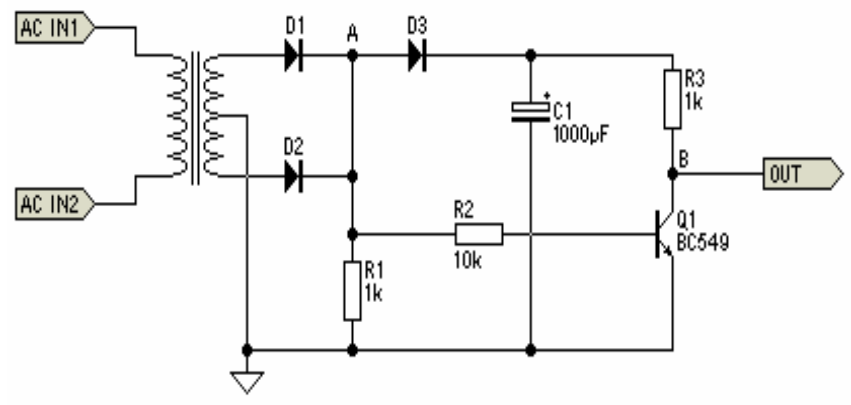

Figure 4. Synchronizing circuit
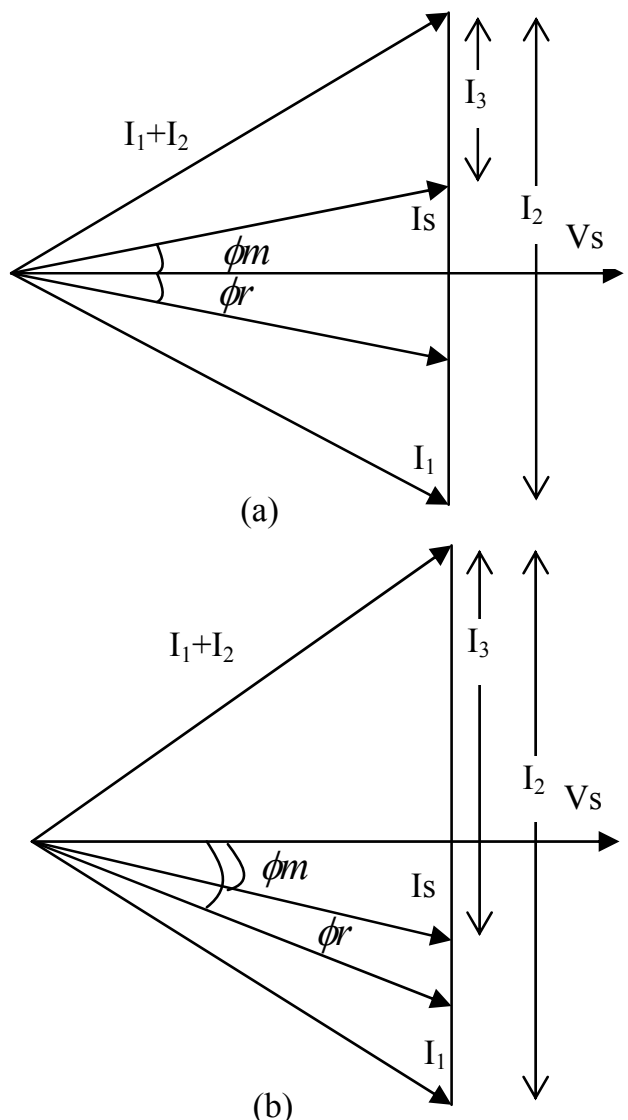

(b)

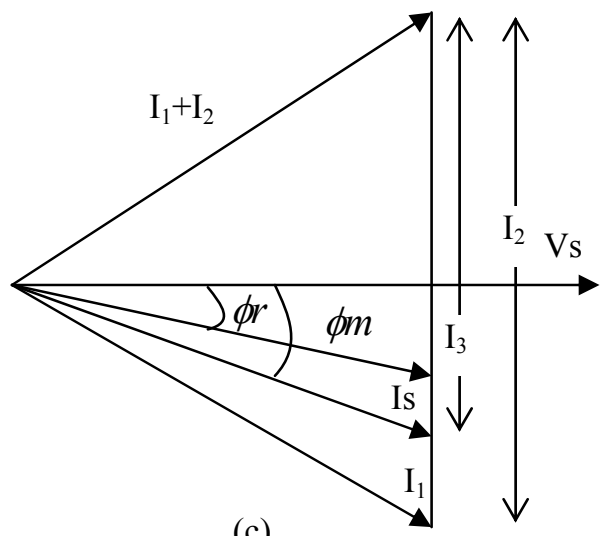

(c)

Figure 5. Phasor diagram of the system currents: (a) $\phi_{r}$ and $\phi_{m}$ have different signs; (b) $\phi_{r}$ and $\phi_{m}$ have the same sign and $\left|\phi_{r}\right|>\left|\phi_{m}\right|$; (c) $\phi_{r}$ and $\phi_{m}$ have the same sign and $\left|\phi_{r}\right|<$

$$
\left|\phi_{m}\right|
$$

\section{SOFTWARE DESCRIPTION}

The microcontroller PIC16F84A has two I/O ports, namely PORTA and PORTB. PORTB has eight pins. RB0, $\mathrm{RB} 1$ and $\mathrm{RB} 2$ are programmed to operate as input pins, in which the RB1 representing voltage, RB2 representing current and RB0 representing synchronizing pulse. Meanwhile, the pin RB3 operates as output pin.

In the first stage, the system is initialized, and the TRIAC triggering angle, $\alpha$ is set to the chosen initial value, says $\alpha_{\text {initial }}$. The value of $\alpha$ is loaded into one file register on the microcontroller and named as $r A L P H A$. The initial pulse signals are shown in Figure 6. The flowchart of the program has been developed and is given in Figure 7.

The Counter register function is to count the number of the steps which is lagging or leading of the current (RB2) for determining the phase angle $\phi_{m}$. A low input test followed by a high input one is first performed on input RB1, which represents the main value of the complex supplied voltage waveform. This is to ensure that $\phi_{m}$, is measured accurately irrespective of the instant of switching the system on.

When input RB1 goes low at the end of the high input test, the microcontroller starts reading and testing input $\mathrm{RB} 2$. If this input is high, this denotes that the main supplied current lags the main supplied voltage and that can be referred to the case of $\left|\phi_{r}\right|>\left|\phi_{m}\right|$ or the $\left|\phi_{r}\right|<\left|\phi_{m}\right|$ where $\phi_{r}$ is the reference value. As long as input RB2 is high, the microcontroller keeps looping and testing that input.

The Counter is incremented after each test. The loop time may be adjusted to any suitable value $D(\mu \mathrm{s})$ by adding the required delay time, since there is a delay time in looping period. When the input RB2 goes low, the contents of Counter are tested. If the contents of Counter is not equals to 0 , the main supplied current is lagging the main supplied voltage by an angle $\phi_{m}$ (equals to the contents of Counter $\times$ $D(\mu \mathrm{s})$.

The microcontroller will then distinguishes whether it is the case of $\left|\phi_{r}\right|>\left|\phi_{m}\right|$ or the case of $\left|\phi_{r}\right|<\left|\phi_{m}\right|$ and thus compares the measured phase angle $\phi_{m}$ with the required or reference value $\phi_{r}$, and accordingly chooses the change $\Delta \alpha$ in the TRIAC firing angle $\alpha$, as shown in the flowchart of Figure 7.

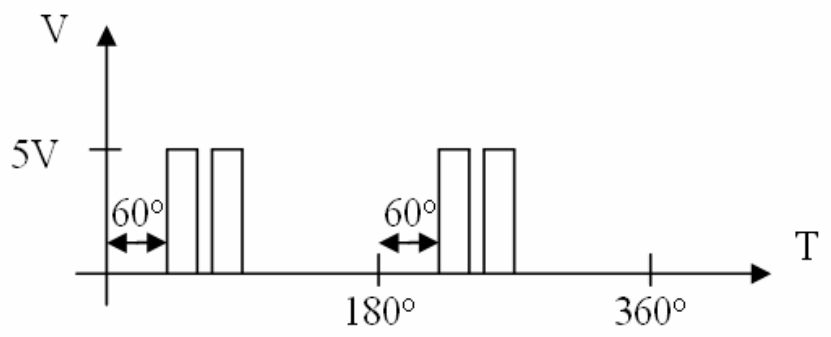

Figure 6. The initial pulse signals from PIC 

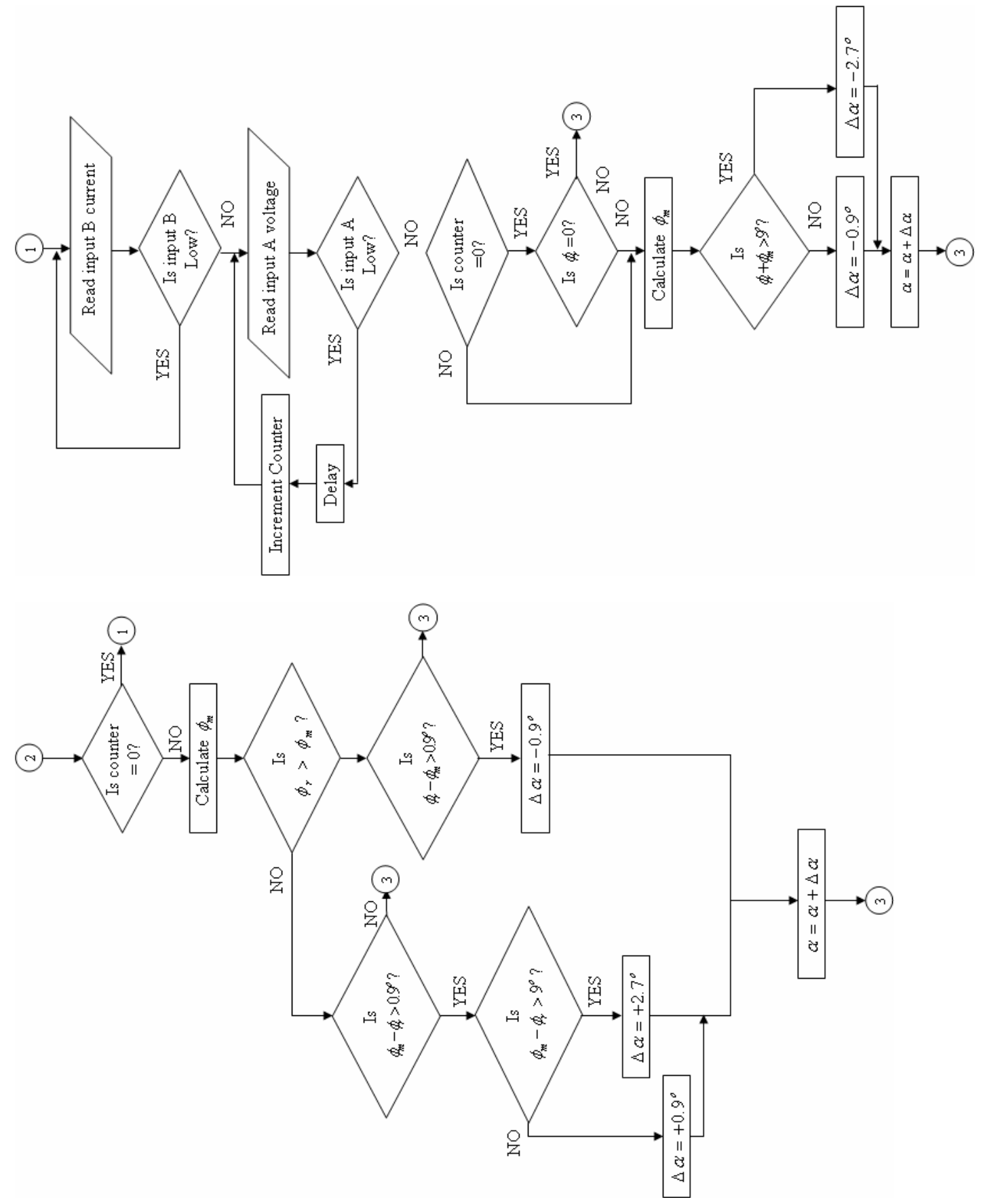

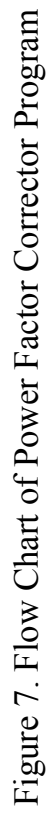

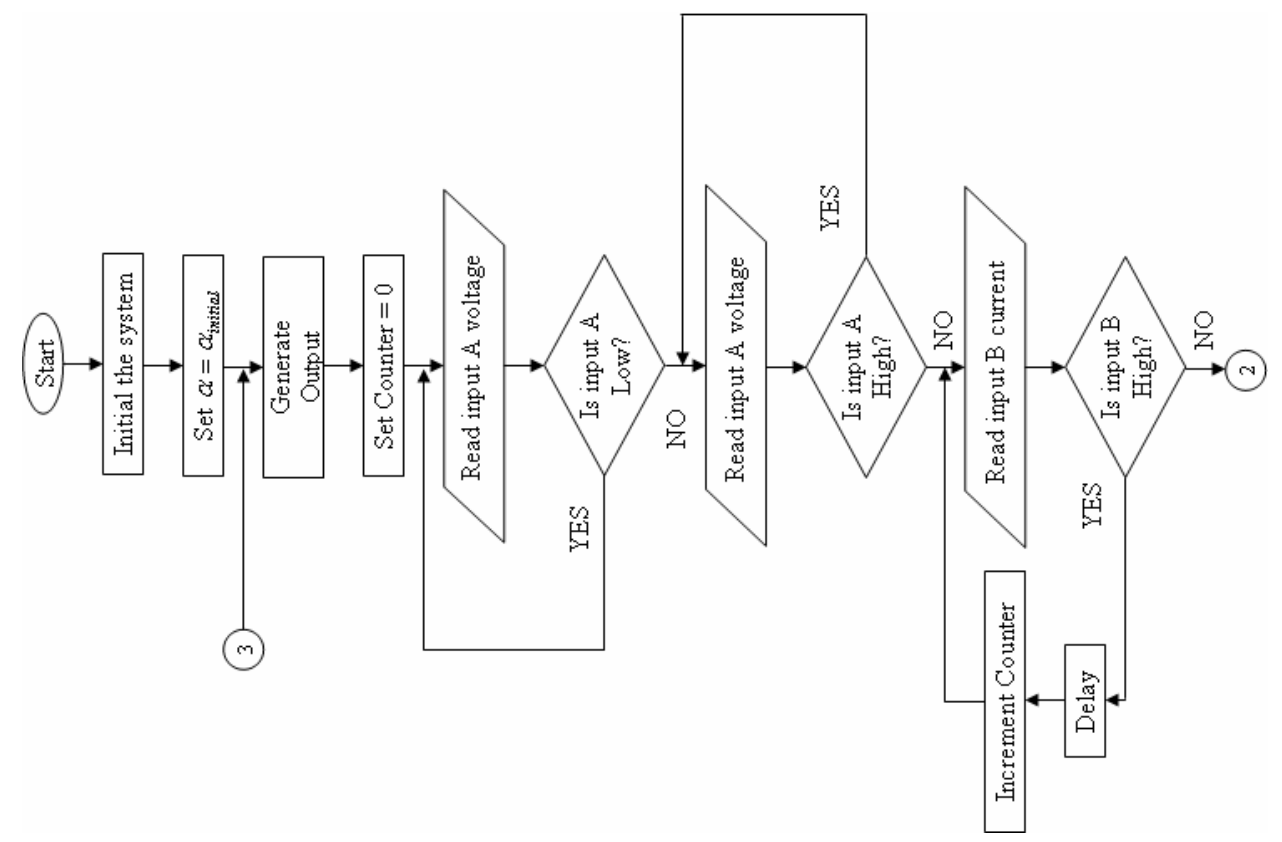


Afterwards, the microcontroller jumps back to reload the $r A L P H A$ with the new value of $\alpha$ and then tests RB1 after clearing the Counter, and the above sequence is repeated. If the contents of Counter are found to be zero, this means that input RB1 is low throughout the test, which in turn means that it is in case 1 in which the fundamental supplied current is leading the fundamental supplied voltage (see Figure 3). In such a case, the microcontroller branches (Figure 6) read and test the input RB2.

As long as the RB2 is low, the microcontroller continues looping and testing that port. When RB2 goes high, port $\mathrm{RB} 1$ is read and tested. As long as RB1 is remaining low, the microcontroller will continues looping and testing RB1. Register Counter is incremented after each test. If the contents of Counter are found to be zero, this means that $\phi_{m}$ is equals to 0 , which in turn means that the fundamental supplied voltage and current are in phase. In such a case, the value of $\alpha$ is kept constant, and the microcontroller jumps back to reload the $r A L P H A$ with $\alpha$, and a new cycle of the testing procedure is started.

If the contents of Counter are not zero, the program starts calculating $\phi_{m}$. In this case, $\phi_{r}$ and $\phi_{m}$ have different signs (since $\phi_{r}$ is always lagging), and hence, the value $\phi_{r}+\phi_{m}$ is tested to find the actual deviation of $\phi_{m}$ from $\phi_{r}$. A large deviation needs a large step change $\Delta \alpha$ in the thyristor firing angle $\alpha$, whereas a small deviation needs a smaller $\Delta \alpha$. After the $r A L P H A$ is reloaded with the new value of $\alpha$, the microcontroller jumps back to read and test port RB1 after clearing the Counter, and the above sequence is repeated.

Referring to the calculated values, all actual measurements and comparison of $\phi_{m}$ and $\phi_{r}$ are made in microseconds rather than in degrees. The loop time, $D$ is adjusted to be $50 \mu \mathrm{s}$, which corresponds to an angle of $0.9^{\circ}$ at a supplied frequency of $50 \mathrm{~Hz}$. Since the LSB in Counter represents a delay angle of $0.9^{\circ}$, a change $(\Delta \alpha)$ of $0.9^{\circ}$ corresponds to a change of a decimal number of 1 , whereas a change of $\Delta \alpha$ equals to $2.7^{\circ}$ corresponds to a decimal number of 3 . When the differences between $\phi_{m}$ and $\phi_{r}$ is decreased to a value less than or equal to $0.9^{\circ}, \phi_{m}$ is considered to be equal to $\phi_{r}$ and thus $\alpha$ is no longer changed. However, one may change this difference to any other value that achieves the required accuracy.

\section{EXPERIMENTAL WORKS}

This active power factor has been undergone many times of tests, simulations and troubleshooting. The simulation software that used is the Electronics Workbench Multisim and Proteus. The particular part in the circuit has been tested for a few times to prove its functionality.

The potential transformer circuit has been tested for the input and the output which denotes that the main supplied voltage has been stepped down to the desired voltage. Figure 8 shows the result of the voltage input and output waveforms. Similarly to the current transformer (CT), the same test and the simulation has also been conducted.

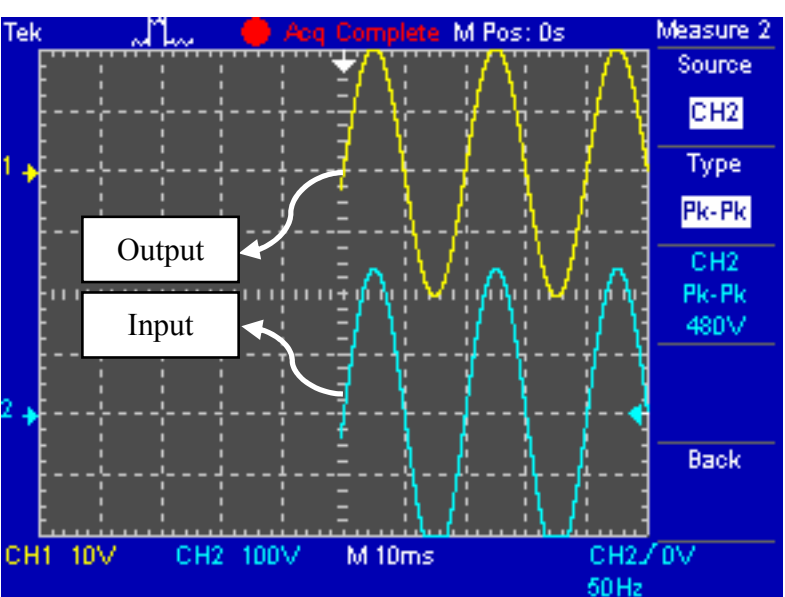

Figure 8. The input of $240 \mathrm{~V}$ AC shown in the channel 2 and the output of $20 \mathrm{~V}$ AC shown in channel 1

The band pass filter circuit has also been tested and simulated for the input and the output waveforms. The input is in the sinusoidal waveform, as shown in Figure 9. The resultant output signal is in the form of digital signal and it is also been shown in Figure 9.

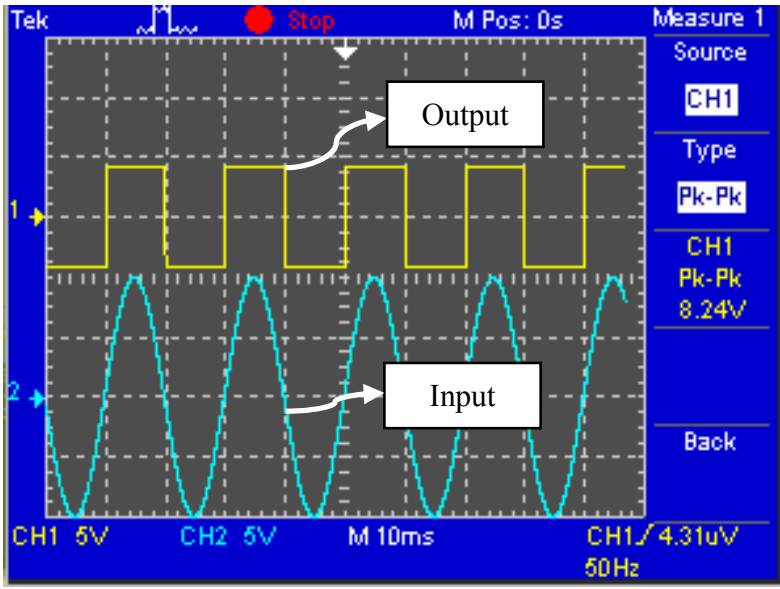

Figure 9. The simulation result of band pass filter circuit

The testing and simulation of the zero-crossing detector circuit has also been done in this works. The simulation is to ensure the voltage and the current signals are converted into digital signal as shown in the Figure 9. The digital signals need to be limited at $5 \mathrm{~V}$ and $0 \mathrm{~V}$, suits to the microcontroller needs. Figure 10 shows the simulation result for this circuit.

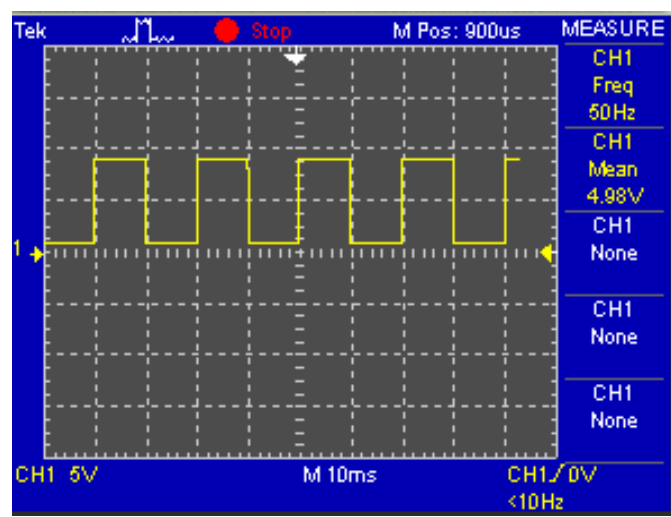

Figure 10. Output signal from zero-crossing detector circuit

In the programming part, the programming source code of the microcontroller circuit is simulated by using the Proteus. 
Figure 11 shows the output from the PIC circuit. The output signal is utilised to control the firing angle of the TRIAC.

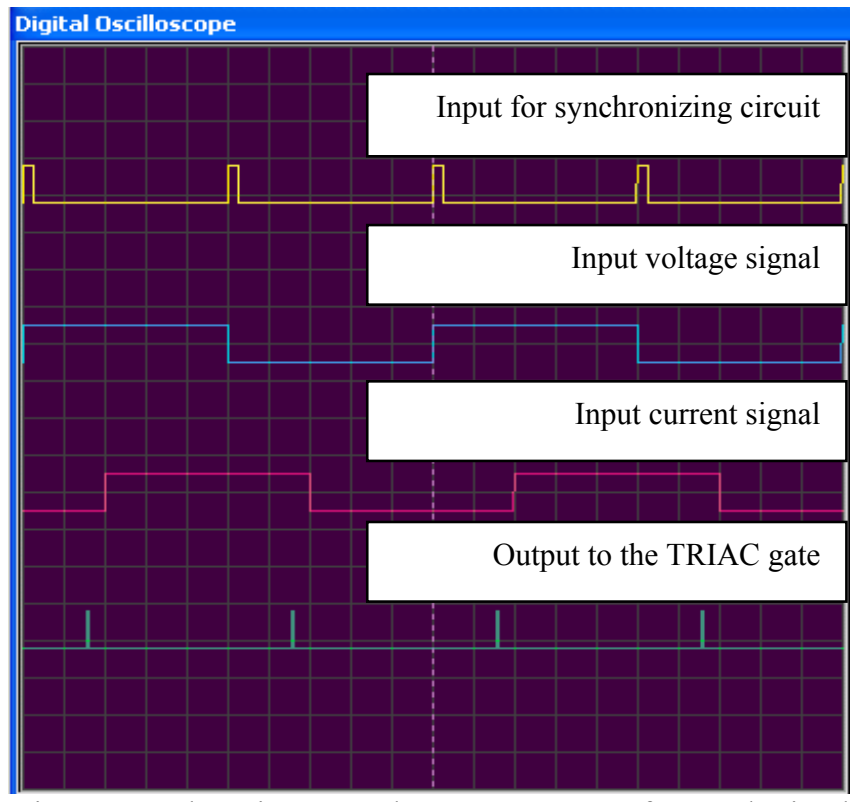

Figure 11. Three inputs and one output waveforms obtained from the PIC circuit

As the input for this active power factor corrector, the supplied voltage of $240 \mathrm{~V} A C$ and $50 \mathrm{~Hz}$ is applied to a capacitance load in series to the circuit. Then, the voltage and current waveforms are measured using digital oscilloscope and the results are shown in Figure 12.

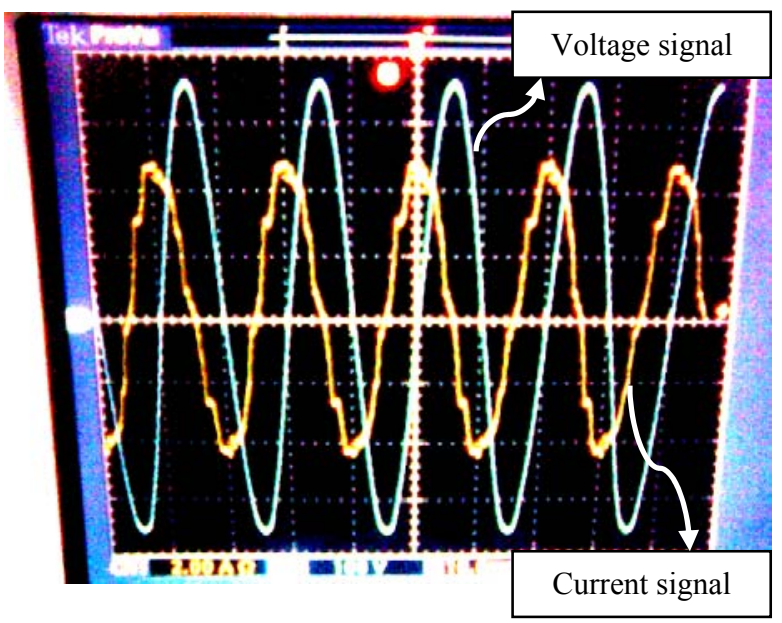

Figure 12. Voltage and current sinus waveforms in the condition of leading power factor

The $R L$ load that comprises of an inductance $(L)$ ranging from $1.37 \mathrm{mH}$ to $6.68 \mathrm{H}$ in series with a resistance $(R)$ ranging from 10 to $30 \Omega$ has been used. The following values (Figure 1) have been used:

$L$ - ranging from $23.2 \mathrm{mH}$ to $1.18 \mathrm{H}$;

$L_{1}=10 \mathrm{mH}$;

$C$ - ranging from 10.6 to $61.21 \mu \mathrm{F}$.

The main supplied voltage used is the single-phase $240 \mathrm{~V}$, $50 \mathrm{~Hz}$. The initial value $\alpha_{\text {initial }}$ for the TRIAC firing angle is chosen to be $60^{\circ}$, and the loop $D$ is adjusted to $50 \mu$ s. The reference phase angle $\phi_{r}$, is chosen to be zero, since that it will exhibit the power factor of unity. The results obtained gave a measured improved power factor ranging from 0.960.98 . Figure 13 shows the square waves representing the fundamental voltage and current signals before and after compensation.

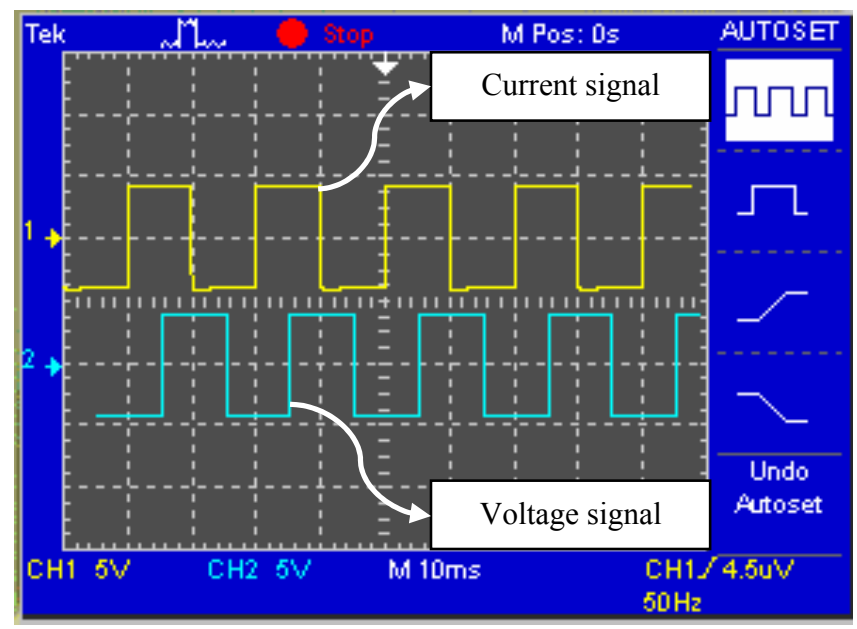

(a) Before compensation

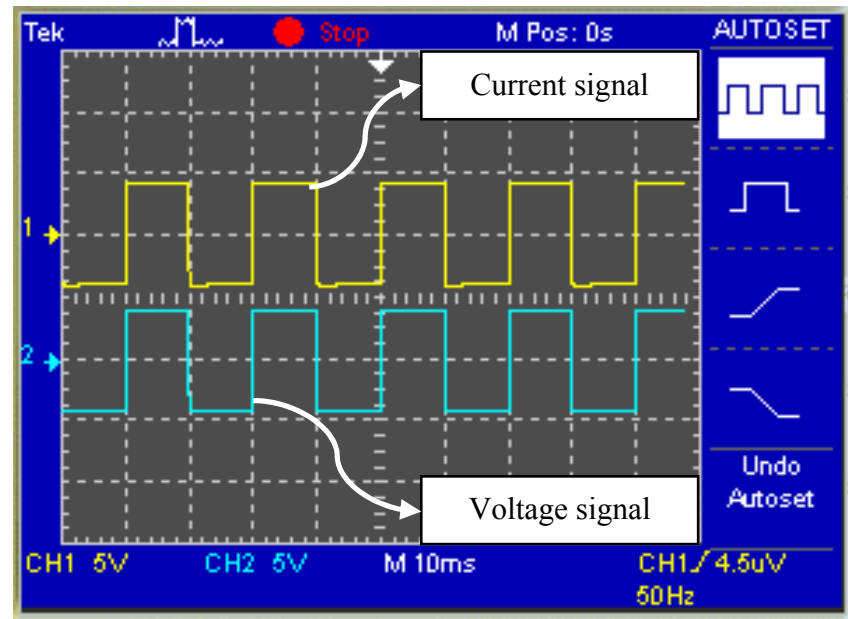

(b) After compensation

Figure 13. Fundamental supply voltage and current signals before and after compensation; (a) Before compensation; (b) after compensation

\section{CONCLUSION}

A microcontroller-based power factor corrector for low power factor loads has been presented in terms of hardware and software development. The system can adjust the supplied system power factor to almost any required reference value. The proposed power factor correction scheme is designed to operate with most possible lagging or leading values of the load power factor; this shows the adaptability of this power factor corrector. The system design allows for more accurate in measuring and compensating power factors than the passive power factor designs. This is because its operation principles' is based on the measurement of the displacement angle between the fundamental components of the supplied voltage and current, rather than measuring the system voltage signal. The principle of operation adopted in this paper may be applied further in designing for the power factor correction schemes for loads driven by 3-phase voltage supplies. 


\section{ACKNOWLEDGMENT}

The authors gratefully acknowledge the staffs in the Laboratory of Electric Machines and Drives as well as Laboratory of Electric Power, UTHM for their valuable contributions towards the success of this work.

\section{REFERENCES}

[1] Alexander, C.K. and Sadiku, M.N.O. (2000). "Fundamentals of Electric Circuit" United States of America: McGraw-Hill Companies, Inc.

[2] Stephen, J. C. (1999). "Electric Machinery and Power System Fundamentals." $3^{\text {rd }}$.ed. United State of America: McGraw-Hill Companies, Inc.

[3] John J. Grainger, William D. Stevenson (1994). "Power System Analysis.” New York: McGraw-Hill.

[4] Jos Arrillaga, Neville R. Watson (2003). "Power System Harmonics" $2^{\text {nd }}$.ed. Chichester: John Wiley.

[5] J. E. Miller (1982). "Reactive Power Control in Electric System." New York: Wiley

[6] Roger C. Dugan, Mark F. McGranaghan, H. Wayne Beaty (1996) "Electrical Power Systems Quality" $1^{\text {st }}$.ed. New York: McGraw Hill.

[7] Paul Gill (1998). "Electrical Power Equipment Maintenance and Testing." Boca Raton, FL: CRC Press.

[8] Keith Harker (1998). "Power System Commissioning and Maintenance practice." London: Institution of Electrical Engineers.

[9] Ramasamy Natarajan (2005). "Power System Capacitors." Boca Raton, FL: Taylor \& Francis.

[10] H.M. El-Bolok, M.E. Masoud, And M.M. Mahmoud (1990). "A Microprocessor -Based Adaptive Power Factor Corrector for Nonlinear Loads" Faculty of Engineering and Technology, University of Helwan, Cairo, Egypt. 


\title{
The Effects of Linear and Non Linear Loads to the Three Phase Four Wire Distribution Systems
}

\author{
Mohd Izhar A.Bakar
}

\begin{abstract}
In this paper described the effects of linear and non linear loads to the three phase four wire distribution systems. The various range of nonlinear loads which are harmonics generated into the electric power line is measured. The experimental results show that the power factor in each power line is decreased and the return current in neutral line increases abruptly even though the system is balanced system. The solutions to reduce the harmonic problems as well as to improve the power quality in the electric power line systems are proposed.
\end{abstract}

Keywords: nonlinear load, harmonic and power factor

\section{INTRODUCTION}

In a three phase four wire distribution systems have been widely employed to deliver electric power at low voltage levels. A typical low voltage of three phase four wire system in the Malaysian had operated at $415 / 240 \mathrm{~V}$ to distribute electric energy to several office buildings and manufacturing plants. Due to the operating conditions have changed dramatically because of the rapid growth of advanced power conversion devices, electronics equipments, computers, office automation, air-conditioning systems, adjustable speed heating ventilation can be caused the current distorted are due to harmonic is increased drastically [1]. According to the Electric Power Research (EPR) in 1995, 35-40\% of all electric power flows through electronic converters. This is expected to increase to $85 \%$ by the year 2010 [2]. All these devices are namely as nonlinear loads and become sources of harmonics. Hence, harmonics is considered as one of the most essential problems in electrical power systems. The simple block diagram in 'figure 1' illustrates the current distortion problem due to harmonic at low voltage levels.

'Figure 1' shows that the voltage waveform at the Point Common Coupling (PCC) is distorted due to harmonic load. This result will provide the line currents distortion is increased, increased losses and contributes to inefficient use of electric energy [3]-[4].

Mohd Izhar A. Bakar, Electrical Engineering Department Universiti Kuala Lumpur - British Malaysian Institute, Batu 8, Jalan Sungai Pusu 53100, Gombak, Selangor (phone: 03-61841000 ext 256; fax: 0361864040; e-mail: izhar_bmi@yahoo.com.my).

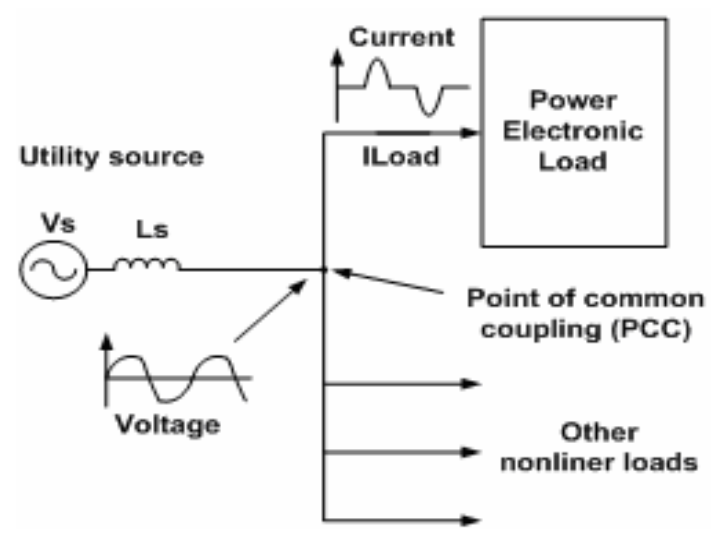

Figure 1

From the measurement results on the several completed set of nonlinear which are taken based on 'figure 1' is tabulated in 'table 1'.

Table 1. The measuring results for various nonlinear loads

\begin{tabular}{|l|c|c|l|c|c|}
\hline Equipment - & Power & THDi & $3^{\text {rd }}$ & $5^{\text {th }}$ & $7^{\text {th }}$ \\
\cline { 4 - 7 } Nonlinear load & Factor (Pf) & $(\%)$ & $(\%)$ & $(\%)$ & $(\%)$ \\
\hline Fluo. lamp & 0.57 & 11.1 & 10.7 & 2.0 & 1.8 \\
\hline Freezer & 0.44 & 61.8 & 11.0 & 4.7 & 11.0 \\
\hline Amplifier & 0.71 & 48.1 & 32.1 & 30.7 & 14.2 \\
\hline Television & 0.66 & 72.5 & 55.1 & 36.8 & 20.3 \\
\hline Photostat m/c. & 0.68 & 69.9 & 37.7 & 40.2 & 30.7 \\
\hline
\end{tabular}

For a three phase four wire distribution systems, under normal operating conditions with the loads are balanced, the current in the neutral current conductor is expected to be small, typically is not to exceed $20 \%$ of the normal load current in the phase [5]. However, the problem of excessive neutral current in three phase four wire systems is arise due to rapid growth of advanced nonlinear loads. These neutral currents which are mostly consist of harmonic orders $3^{\text {rd }}, 9^{\text {th }}$, $15^{\text {th }}, 21^{\text {st }}, 27^{\text {th }}$ and etc. These harmonics is called triplen harmonics. All this harmonic current will be accumulated in the neutral wire, thus results in overloading of the neutral conductor and the distribution transformer.

Effects of harmonics in a power system will boost the cost control, quality control and consumption of electricity as well as decrease the power quality will be caused [6]-[8].

i. Overheating of transformers and power cables

ii. Tripping of circuit breakers and blowing fuses

iii. Capacitor overloading and failures

iv. Signal interference in converter equipment 
v. Interference with telecommunication circuits and ripple control system

vi. Increased transmission losses

vii. Excessive neutral current in neutral wire

\section{METHOD}

The aim of this study is to evaluate the effects of linear and nonlinear load to the three phase four wire distribution systems as shown in 'figure 2'. This prototype is consisted with high current at rating 50A of full bridge rectifier, high capacitive and inductive loads so that it can become as sources of harmonics.

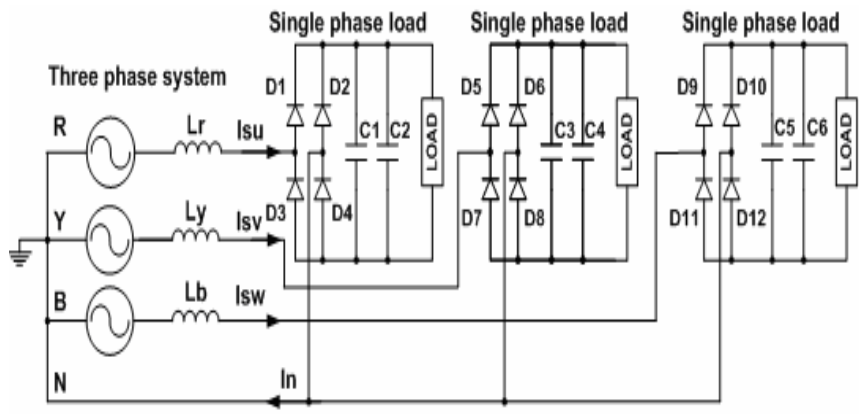

Figure 2

The measurement result at each line such as $\operatorname{Red}(\mathrm{R})$, Yellow(Y), Blue(B) and Neutral(N) lines which are related to the experimental work such as voltage, current, Total Harmonic Distortion (THD) include Power factor (Pf) are properly recorded. Next, all the experimental results are described in the next sections.

\section{THE EXPERIMENTAL AND SIMULATION RESULTS}

The experimental and simulation work is divided into two groups which are linear and nonlinear loads are tested.

\section{A.The Experimental Results for Linear Load}

The linear load use in this experimental work is resistive load $78.1 \Omega$. The results are shown in 'tables 2 and 3' and 'figures 3, 4 and 5'.

Table 2. The results for current, voltage, THDv, THDi, power factor (Pf) and power

\begin{tabular}{|c|c|c|c|c|}
\hline \multirow{2}{*}{ Description } & \multicolumn{4}{|c|}{ Line } \\
\cline { 2 - 5 } & $\begin{array}{c}\text { Red } \\
(\mathrm{R})\end{array}$ & $\begin{array}{c}\text { Yellow } \\
(\mathrm{Y})\end{array}$ & $\begin{array}{c}\text { Blue } \\
(\mathrm{B})\end{array}$ & $\begin{array}{c}\text { Neutral } \\
(\mathrm{N})\end{array}$ \\
\hline VLN(V-rms) & 250.0 & 251.0 & 251.0 & - \\
\hline THDv (\%) & 1.2 & 1.1 & 1.3 & - \\
\hline Iph (A-rms) & 3.07 & 3.03 & 3.06 & 0.12 \\
\hline THDi (\%) & 1.60 & 1.50 & 1.60 & - \\
\hline Pf & 1.00 & 1.00 & 1.00 & - \\
\hline Watt (W) & 770 & 770 & 770 & - \\
\hline Load (R, $\Omega)$ & 78.1 & 78.1 & 78.1 & - \\
\hline
\end{tabular}

Table 3. The results for harmonic currents

\begin{tabular}{|c|c|c|c|c|}
\hline \multirow{2}{*}{$\begin{array}{c}\text { Hurmonic } \\
\text { Number }\end{array}$} & \multicolumn{4}{|c|}{ Harmonic currents (Amp.rms) } \\
\cline { 2 - 5 } & $\begin{array}{c}\text { Red } \\
(\mathrm{R})\end{array}$ & $\begin{array}{c}\text { Yellow } \\
(\mathrm{Y})\end{array}$ & $\begin{array}{c}\text { Blue } \\
(\mathrm{B})\end{array}$ & $\begin{array}{c}\text { Neutral } \\
(\mathrm{N})\end{array}$ \\
\hline 1 & 3.07 & 3.03 & 3.06 & 0.09 \\
\hline 3 & 0.02 & 0.01 & 0.00 & 0.05 \\
\hline 5 & 0.03 & 0.03 & 0.03 & 0.01 \\
\hline 7 & 0.03 & 0.03 & 0.04 & 0.00 \\
\hline 9 & 0.01 & 0.01 & 0.01 & 0.01 \\
\hline 11 & 0.01 & 0.00 & 0.01 & 0.00 \\
\hline 13 & 0.00 & 0.00 & 0.00 & 0.01 \\
\hline 15 & 0.00 & 0.00 & 0.01 & 0.01 \\
\hline 17 & 0.00 & 0.00 & 0.14 & 0.01 \\
\hline 19 & 0.00 & 0.01 & 0.12 & 0.00 \\
\hline 21 & 0.01 & 0.00 & 0.00 & 0.01 \\
\hline 23 & 0.00 & 0.00 & 0.00 & 0.00 \\
\hline 25 & 0.00 & 0.00 & 0.00 & 0.00 \\
\hline 27 & 0.00 & 0.00 & 0.00 & 0.00 \\
\hline 29 & 0.00 & 0.00 & 0.00 & 0.01 \\
\hline 31 & 0.00 & 0.00 & 0.00 & 0.00 \\
\hline & & & & \\
\hline
\end{tabular}

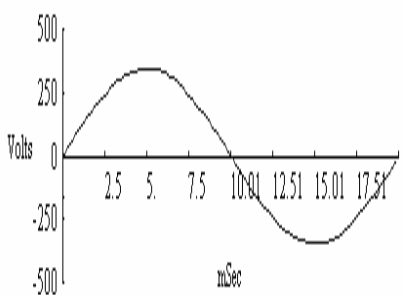

(a)

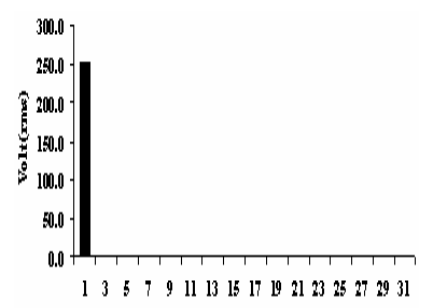

Hammin: Khinhe

(b)
Figure 3. The results for resistive load, $78.1 \Omega$ at R, Y and B lines: (a) Phase voltage waveform; and (b) Harmonic voltage spectrum

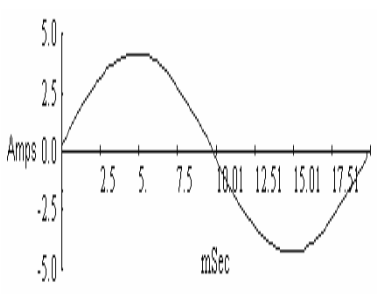

(a)

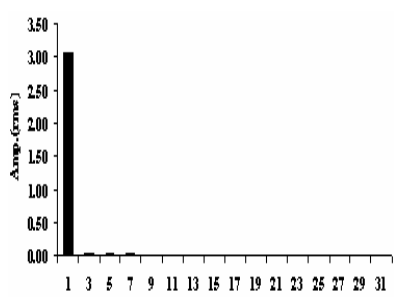

(b)
Figure 4. The results for resistive load, $78.1 \Omega$ at $\mathrm{R}, \mathrm{Y}$ and $\mathrm{B}$ lines: (a) Phase current waveform; and (b) Harmonic current spectrum

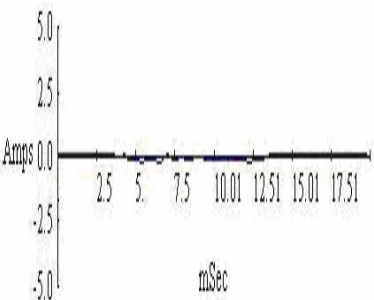

(a)

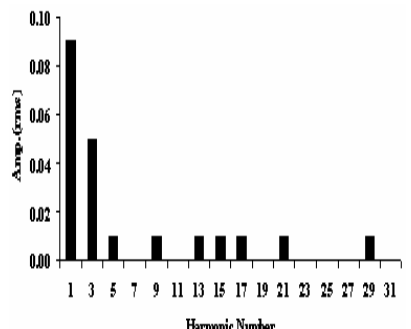

(b)
Figure 5. The results for resistive load, $78.1 \Omega$ at neutral $(\mathrm{N})$ line: (a) Phase current waveform (b) Harmonic current spectrum 


\section{B. The Experimental Results for Nonlinear Load}

The high value of capacitor $2420 \mu \mathrm{F}$ and inductor $195.5 \mathrm{mH}$ as sources of harmonics were used in testing. Table 4, 5, figure 6, 7 and 8 shows the experimental results for voltage, current, THD, waveforms and harmonics for capacitive load $2420 \mu \mathrm{F}$.

Table 4. The results for current, voltage, THDv, THDi, power factor (Pf) and power

\begin{tabular}{|l|c|c|c|c|}
\hline \multirow{2}{*}{ Description } & \multicolumn{4}{|c|}{ Line } \\
\cline { 2 - 5 } & $\begin{array}{c}\text { Red } \\
(\mathrm{R})\end{array}$ & $\begin{array}{c}\text { Yellow } \\
(\mathrm{Y})\end{array}$ & $\begin{array}{c}\text { Blue } \\
(\mathrm{B})\end{array}$ & $\begin{array}{c}\text { Neutral } \\
(\mathrm{N})\end{array}$ \\
\hline VLN(V-rms) & 243.0 & 244.0 & 243.0 & - \\
\hline THDv (\%) & 1.1 & 1.0 & 1.0 & - \\
\hline Iph (A-rms) & 3.51 & 3.67 & 3.63 & 5.81 \\
\hline THDi (\%) & 81.85 & 82.89 & 82.93 & 100.0 \\
\hline Pf & 0.57 & 0.55 & 0.55 & - \\
\hline Watt $(\mathrm{W})$ & 460 & 460 & 460 & - \\
\hline Load $(\mathrm{C}, \mu \mathrm{F})$ & 2420 & 2420 & 2420 & - \\
\hline
\end{tabular}

Table 5. The results for harmonic currents

\begin{tabular}{|c|c|c|c|c|}
\hline \multirow{2}{*}{$\begin{array}{c}\text { Harmonic } \\
\text { Number }\end{array}$} & \multicolumn{4}{|c|}{ Harmonic currents (Amp.rms) } \\
\cline { 2 - 5 } & $\begin{array}{c}\text { Red } \\
(\mathrm{R})\end{array}$ & $\begin{array}{c}\text { Yellow } \\
(\mathrm{Y})\end{array}$ & $\begin{array}{c}\text { Blue } \\
(\mathrm{B})\end{array}$ & $\begin{array}{c}\text { Neutral } \\
(\mathrm{N})\end{array}$ \\
\hline 1 & 1.90 & 1.89 & 1.94 & 0.01 \\
\hline 3 & 1.75 & 1.85 & 1.87 & 5.27 \\
\hline 5 & 1.55 & 1.60 & 1.53 & 0.01 \\
\hline 7 & 1.27 & 1.32 & 1.26 & 0.01 \\
\hline 9 & 0.95 & 1.09 & 1.09 & 2.36 \\
\hline 11 & 0.65 & 0.76 & 0.68 & 0.01 \\
\hline 13 & 0.38 & 0.48 & 0.40 & 0.01 \\
\hline 15 & 0.16 & 0.25 & 0.19 & 0.10 \\
\hline 17 & 0.06 & 0.06 & 0.03 & 0.00 \\
\hline 19 & 0.13 & 0.06 & 0.10 & 0.01 \\
\hline 21 & 0.16 & 0.14 & 0.17 & 0.40 \\
\hline 23 & 0.14 & 0.14 & 0.14 & 0.01 \\
\hline 25 & 0.10 & 0.12 & 0.11 & 0.01 \\
\hline 27 & 0.05 & 0.08 & 0.07 & 0.10 \\
\hline 29 & 0.03 & 0.03 & 0.02 & 0.00 \\
\hline 31 & 0.05 & 0.03 & 0.04 & 0.01 \\
\hline & & & & \\
\hline
\end{tabular}

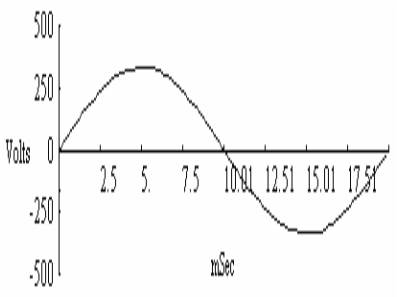

(a)

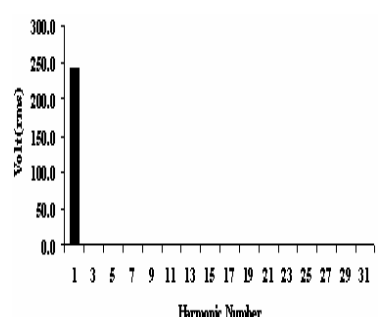

(b)
Figure 6. The results for capacitive load, $2420 \mu \mathrm{F}$ at R, Y and $B$ lines: (a) Phase voltage waveform; and (b) Harmonic voltage spectrum

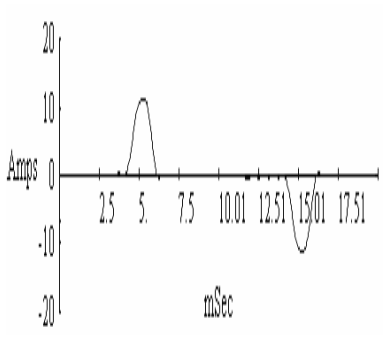

(a)

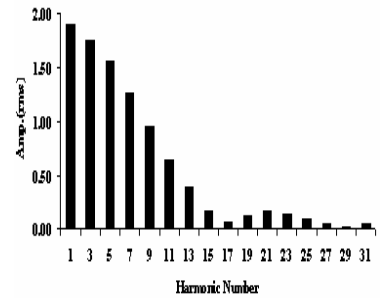

(b)
Figure 7. The results for capacitive load, $2420 \mu \mathrm{F}$ at R, Y and B lines: (a) Phase current waveform; and (b) Harmonic current spectrum

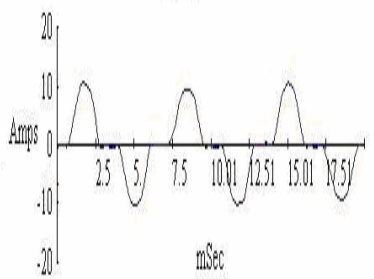

(a)

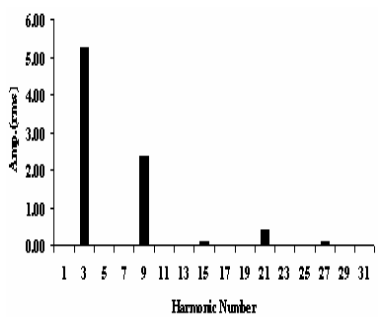

(b)
Figure 8. The results for capacitive load, $2420 \mu \mathrm{F}$ at neutral (N) line: (a) Phase current waveform; and (b) Harmonic current spectrum

The experimental results for voltage, current, THD, waveforms and harmonics for inductive load $195.5 \mathrm{mH}$ as tabulated in 'tables 6 and 7', 'figures 9, 10 and 11'.

Table 6. The results for current, voltage, THDv, THDi, power factor (Pf) and power

\begin{tabular}{|l|c|c|c|c|}
\hline \multirow{2}{*}{ Description } & \multicolumn{5}{|c|}{ Line } \\
\cline { 2 - 5 } & $\begin{array}{c}\text { Red } \\
\circledR\end{array}$ & $\begin{array}{c}\text { Yellow } \\
(Y)\end{array}$ & $\begin{array}{c}\text { Blue } \\
(\mathrm{B})\end{array}$ & $\begin{array}{c}\text { Neutral } \\
(\mathrm{N})\end{array}$ \\
\hline VLN(V-rms) & 239.0 & 243.0 & 239.0 & - \\
\hline THDv (\%) & 0.8 & 0.8 & 0.8 & - \\
\hline Iph (A-rms) & 2.51 & 2.53 & 2.51 & 3.48 \\
\hline THDi (\%) & 92.68 & 92.55 & 92.2 & 100.0 \\
\hline Pf & 0.77 & 0.77 & 0.77 & - \\
\hline Watt (W) & 560 & 570 & 560 & - \\
\hline Load (L, mH) & 195.5 & 195.5 & 195.5 & - \\
\hline
\end{tabular}

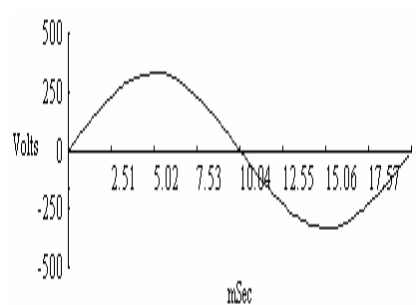

(a)

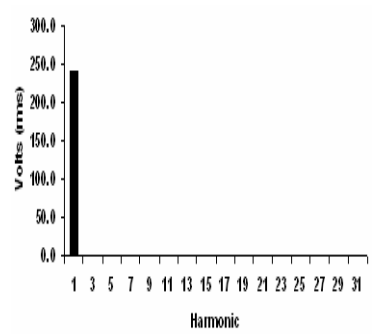

(b)
Figure 9. The results for inductive load, $195.5 \mathrm{mH}$ at $\mathrm{R}, \mathrm{Y}$ and B lines: (a) Phase voltage waveform; and (b) Harmonic voltage spectrum 
Table 7. The results for harmonic currents

\begin{tabular}{|c|c|c|c|c|}
\hline \multirow{2}{*}{$\begin{array}{c}\text { Harmonic } \\
\text { Number }\end{array}$} & \multicolumn{4}{|c|}{ Harmonic currents (Amp.rms) } \\
\cline { 2 - 5 } & $\begin{array}{c}\text { Red } \\
(\mathrm{R})\end{array}$ & $\begin{array}{c}\text { Yellow } \\
(\mathrm{Y})\end{array}$ & $\begin{array}{c}\text { Blue } \\
(\mathrm{B})\end{array}$ & $\begin{array}{c}\text { Neutral } \\
(\mathrm{N})\end{array}$ \\
\hline 1 & 1.38 & 1.39 & 1.38 & 0.01 \\
\hline 3 & 1.25 & 1.24 & 1.25 & 3.64 \\
\hline 5 & 0.37 & 0.38 & 0.36 & 0.01 \\
\hline 7 & 0.27 & 0.27 & 0.27 & 0.01 \\
\hline 9 & 0.21 & 0.22 & 0.23 & 1.20 \\
\hline 11 & 0.17 & 0.18 & 0.17 & 0.00 \\
\hline 13 & 0.15 & 0.14 & 0.14 & 0.01 \\
\hline 15 & 0.06 & 0.13 & 0.14 & 0.42 \\
\hline 17 & 0.11 & 0.11 & 0.10 & 0.00 \\
\hline 19 & 0.10 & 0.09 & 0.09 & 0.01 \\
\hline 21 & 0.09 & 0.09 & 0.09 & 0.19 \\
\hline 23 & 0.08 & 0.08 & 0.08 & 0.00 \\
\hline 25 & 0.07 & 0.07 & 0.07 & 0.00 \\
\hline 27 & 0.06 & 0.07 & 0.08 & 0.10 \\
\hline 29 & 0.06 & 0.06 & 0.06 & 0.00 \\
\hline 31 & 0.12 & 0.05 & 0.05 & 0.00 \\
\hline
\end{tabular}

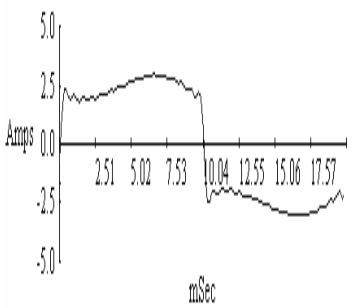

(a)

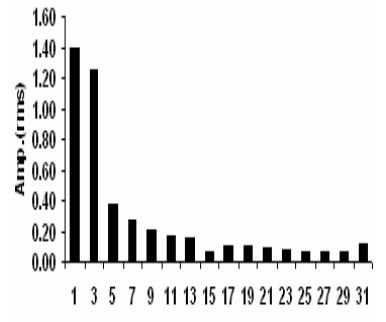

(b)
Figure 10. The results for inductive load, $195.5 \mathrm{mH}$ at $\mathrm{R}, \mathrm{Y}$ and B lines: (a) Phase current waveform; and (b) Harmonic current spectrum

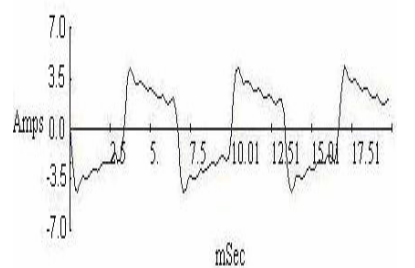

(a)

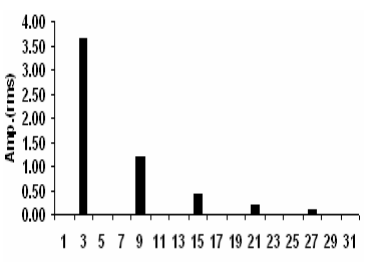

(b)
Figure 11. the results for inductive load, $195.5 \mathrm{mH}$ at neutral (N) line: (a) Phase current waveform; and (b) Harmonic current spectrum

\section{The Simulation Results for Nonlinear Load}

'Tables 8 and 9' and 'figures 12 and 13' show the simulation results of capacitive and inductive loads for current, voltage, THDv, THDi, Power factor, Power, waveforms and harmonic spectrum.
Table 8. The results for current, voltage, THDv, THDi, power factor $(\mathrm{Pf})$ and power

\begin{tabular}{|l|c|c|c|c|}
\hline \multirow{2}{*}{ Description } & \multicolumn{4}{|c|}{ Line } \\
\cline { 2 - 5 } & $\begin{array}{c}\text { Red } \\
(\mathrm{R})\end{array}$ & $\begin{array}{c}\text { Yellow } \\
(\mathrm{Y})\end{array}$ & Blue (B) & Neutral (N) \\
\hline VLN(V-rms) & 239.4 & 239.3 & 239.3 & - \\
\hline THDv (\%) & 0.98 & 0.95 & 0.95 & - \\
\hline Iph (A-rms) & 3.98 & 3.99 & 3.97 & 6.15 \\
\hline THDi (\%) & 82.1 & 82.70 & 82.55 & 100.0 \\
\hline Pf & 0.58 & 0.56 & 0.57 & - \\
\hline Watt $(\mathrm{W})$ & 450 & 448 & 448 & - \\
\hline Load $(\mu \mathrm{F})$ & 2420 & 2420 & 2420 & - \\
\hline
\end{tabular}

Table 9. The results for current, voltage, THDv, THDi, power factor $(\mathrm{Pf})$ and power

\begin{tabular}{|l|c|c|c|c|}
\hline \multirow{2}{*}{ Description } & \multicolumn{4}{|c|}{ Line } \\
\cline { 2 - 5 } & $\begin{array}{c}\text { Red } \\
\circledR\end{array}$ & $\begin{array}{c}\text { Yellow } \\
(\text { Y) }\end{array}$ & $\begin{array}{c}\text { Blue } \\
(\mathrm{B})\end{array}$ & $\begin{array}{c}\text { Neutral } \\
(\mathrm{N})\end{array}$ \\
\hline VLN(V-rms) & 240.1 & 244.2 & 241.2 & - \\
\hline THDv (\%) & 0.8 & 0.8 & 0.8 & - \\
\hline Iph (A-rms) & 2.55 & 2.58 & 2.57 & 3.59 \\
\hline THDi (\%) & 93.24 & 93.33 & 93.34 & 100.0 \\
\hline Pf & 0.78 & 0.76 & 0.76 & - \\
\hline Watt (W) & 568 & 570 & 568 & - \\
\hline Load (L, mH) & 195.5 & 195.5 & 195.5 & - \\
\hline
\end{tabular}

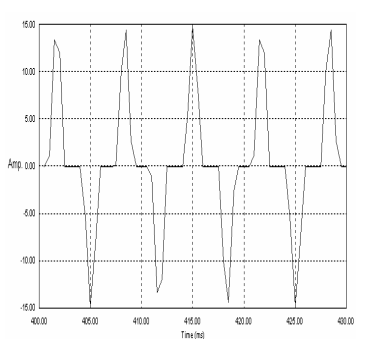

(a)

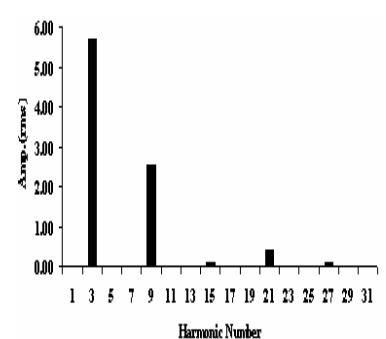

(b)
Figure 12. The results for capacitive load, $2420 \mu \mathrm{F}$ at neutral (N) line: (a) Phase current waveform; and (b) Harmonic current spectrum

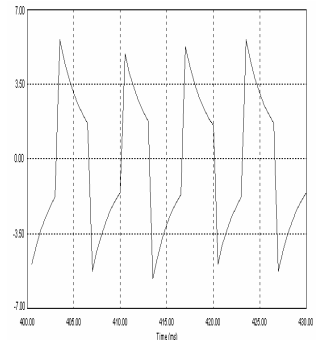

(a)

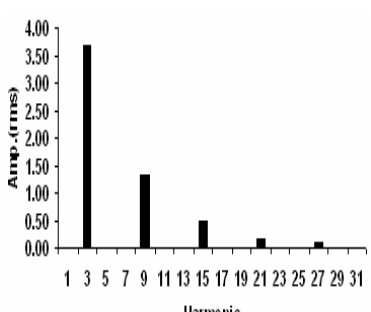

(b)
Figure 13. The results for inductive load, $195.5 \mathrm{mH}$ at neutral (N) line: (a) Phase current waveform (b) Harmonic current spectrum

\section{DISCUSSIONS}

It is clearly shown that the current drawn by resistive load such as resistor is purely sinuswave has no distortion, no harmonics at $\mathrm{R}, \mathrm{Y} \& \mathrm{~B}$ lines and their total rms neutral current in neutral wire is zero Amp. All these results are shown in 'table 2 and 3' and 'figures 3, 4 and 5'. This happens are totally different with nonlinear load such as 
capacitor and inductor. These nonlinear loads drew the current from power supply which is not proportional to the voltage and leads to current distortion and carry many harmonics. These currents distortions waveforms are shown in figures 7, 8, 10 and 11. Meanwhile, the phase current is composite with harmonics order $3^{\text {rd }}, 5^{\text {th }}, 7^{\text {th }}, 9^{\text {th }}, 11^{\text {th }}$ and etc. flowed in each $\mathrm{R}, \mathrm{Y}$ and $\mathrm{B}$ lines as shown in 'tables 5 and 7'. These harmonic currents can caused the THDi is increased and power factor drop abruptly. If the power factor drops, therefore the power used in the system is inefficiently. From the above nonlinear load results such as capacitor and inductor, it is found that the results is similar characteristics to the complete set of measured results for nonlinear load in 'table 1'. The highest harmonic currents are $3^{\text {rd }}$ harmonics comparing with others $5^{\text {th }}, 7^{\text {th }}, 9^{\text {th }}, 11^{\text {th }}$ and etc. either in $\mathrm{R}, \mathrm{Y}, \mathrm{B}$ or $\mathrm{N}$ lines.

The results also shows that the neutral current in neutral line become higher and higher compare with phase current because an accumulated of triplen harmonic currents such as $3^{\text {rd }}, 9^{\text {th }}, 15^{\text {th }}, 21^{\text {th }}$ ant etc in neutral line even though the system is balanced.

According to the limits of harmonic in IEEE 519-1992 the allowable current distortion is less than 20\% [9]. From the above results, it is clearly shown that the current distortion is beyond $20 \%$ compared with IEEE and the neutral current in neutral line also very high, it can become a new issue in the three phase four wire distribution system. In order to reduce THDi, harmonic current, power factor, excessive neutral current as well as power quality, the harmonic filters will be installed to the systems. The harmonic filters can be passive filter or active power filter. The passive filter often uses LC devices to tune the unwanted frequency harmonic. Meanwhile, the power electronic devices includes controller is used in active power filter to sweep the unwanted frequency harmonic. Based on these recommendations the problems such as THDi, power factor, harmonic and excessive neutral current in the three phase four wire systems can be improved.

The experimental and simulation results for their current, voltage, THDv, THDi, Power factor, Power, waveforms and harmonic spectrums are compared. It is found that the result shows a good agreement to each others, consistent and reliable.

\section{CONCLUSIONS}

In this study, the linear and nonlinear load by experimental and simulations were demonstrated. The main effects of nonlinear load to the distribution lines shows that the current drawn by nonlinear load is badly distorted, high total harmonic distortion, power factor decrease and neutral wire will be overloaded with neutral current. Due to this the THDi increase $98 \%$, power factor decrease $50 \%$ and neutral current increase $35 \%$ is compared with phase current. These problems can be caused the systems more exposes to the risk of hazards.

\section{REFERENCES}

[1] Kevin, J.T. and Rich, P., "Eliminating Harmonics from the facility Power System", Cutler-Hammer, Eaton Corp., 1997.

[2] Roger, C. D., "Electrical Power System Quality". McGraw-Hill, New York, 1999.
[3] Takeda, M., Ikeda, K. and Tominaga, Y., "Harmonic Current Compensation with Active Filter", IEEE/IAS., Annual Meeting, 1987, pp. $808-815$.

[4] Juan, C.M. and Samra, A.H., "A New Technique to Reduce LineCurrent Harmonics Generated by a Three-Phase Bridge Rectifier", IEEE Proceeding Southeastcon, April 1998, pp. 354 - 359.

[5] Choy, A.L., "Excessive Neutral Currents in Three-Phase Fluorescent Lighting Circuits", IEEE Transactions On Industry Application", vol.25, 1989, pp. $776-782$.

[6] David, C. (2004). Power Quality Application Guide: Harmonics Causes and Effects. [Online]. Available: http://www.cda.org.uk/megab2/elecapps/pub501/31_lo_R2.pdf.

[7] Richard, P.B. (2004). Harmonics-Understanding the Facts. [Online]. Available: http://www.dranetz-bmi.com/pdf/harmonicspart2.pdf.

[8] Wang, Z., Wang, W., Yao, W. and Liu, J., "Modelling Devices With Nonlinear Voltage - Current Characteristics for Harmonic Studies", IEEE Transactions on Power Delivery, vol.19, 2004, pp. 1802-1811.

[9] IEEE Recommended Practices and Requirements for Harmonic Control in Power System, IEEE Std. $519-1992$. 


\title{
Dynamic Performance Analysis of a Practical PI Regulated AC Variable Speed Drive
}

\author{
Rajendran.S , M.K.A. Ahmed Khan, Gobbi.R, K.Y. Loh and A. Kareem
}

\begin{abstract}
This paper reports the performance of a variable speed drive (VSD) with PI regulator using an experimental approach. The study focuses on the performance of the variable speed drive under step speed response (from standstill to reference speed) and the step load torque change response. The responses are evaluated in terms of the time domain parameters, namely rise time, overshoot (or undershoot), settling time and steady-state error. The experiment was carried out using a variable speed drive with a built-in PI regulator, a servo unit, data acquisition device, $P C$ and other auxiliary devices. The results of the experiment show that the PI regulator provides fast and stable response for the step speed and step load torque, when it is properly tuned. In addition to that, it also eliminates the steady-state error of the system after the disturbance. The results of the step input response prove that a tradeoff among the control performance measures such as rise time, overshoot and settling time must be made if the desired response is to be achieved. The desired response in turn depends on the application as well as the physical limitations of the system. The results of the step load torque on the other hand, show that the selection of PI gains is a tradeoff between maintaining the stability of the system before, and after the load change and, reducing the amount of overshoot and settling time. Experimental results are presented here to verify the performance of the variable speed drive connected to dynamic load.
\end{abstract}

\section{INTRODUCTION}

A common method of speed set-point control in industrial drives is to use a proportional plus integral (PI) controller [1]. The occurrence of steady-state error as a result of load changes in the speed response of variable speed drives has been documented in many papers . In fact, the change of load torque of the motor itself can be considered as a disturbance to the system. The use of PI control is one method employed to eliminate the steady-state error [2]. The different methods of tuning the PI controller has been done by [3-8]. Some of these methods use genetic algorithms while others use the Ziegler-Nichols method and some employ fuzzy logic to obtain the optimum response from the controller. The PI controller can be tuned based on certain evaluation criteria such as rise time, settling time, steadystate error, peak overshoot and maximum undershoot

This work was supported in part by Schneider Electric Malaysia.

Gobbi and K.Y. Loh are with the Multimedia University, Jalan Multimedia, 63100, Cyberjaya, Malaysia

Rajendran.S., M.K.A. Ahamed Khan and A. Kareem are with University Industry Selangor, Malaysia. (e-maill: author@lamar. colostate.edu).
[2],[8]. A large number of studies concerning the tuning and performance of PI-controlled drives particularly by [2], utilize computer software and are mostly simulation studies. A different approach in tuning and studying the performance of the PI-controlled drive is the experimental approach. An experimental approach in the study of PI speed control for a high-performance $\mathrm{AC}$ drive has been done by [1]. The study by [1] however, involves a comparative analysis between fuzzy logic control and PI control in controlling the speed of the AC Drive. In this paper, an experiment was carried out on a variable speed drive (Altivar 18 - Schneider Electric's brand name for a VSD) with built-in PI regulator under various operating loads conditions to study how well the variable speed drive performs. By varying the PI gains, the corresponding response for each test condition will be obtained. The response will then be gauged by its rise time, settling time, and degree of overshoot (or undershoot). In order to gather the physical responses, a data acquisition device and personal computer are used.

\section{MeTHOD OF INVESTIGATION}

The performance of the variable speed drive with PI control is evaluated under the step speed response test and the step load torque change test. The response for both tests is obtained by monitoring the actual speed of the motor with respect to time. In the step speed response test, the motor is started from standstill to a certain reference speed. The test is conducted for reference speeds of 510rpm, 750rpm, 900rpm, 1140rpm, 1450rpm and 1500rpm. For each reference speed, the motor is tested with a load torque (about $50 \%$ of the maximum motor torque) and incremented by $0.5 \mathrm{Nm}$ till $3.5 \mathrm{Nm}$ (which is the maximum motor rated output). The step speed response obtained for each reference speed, at the varying initial load torques are evaluated in terms of its rise time, amount of overshoot and settling time. The proportional and integral gains are varied such that for each case, an under damped response, a critically damped response and an over damped response are obtained. In the step load torque change test, the motor is tested for sudden load changes at the said reference speeds. For each reference speed and initial load torque, a sudden (step) load torque of $1.5 \mathrm{Nm}$ is added to the system. Similarly, a sudden removal of $1.5 \mathrm{Nm}$ load torque from the system is done for each reference speed and initial load torque (except when the initial load torque is zero). The step load torque change response is evaluated in terms of its overshoot or 
undershoots, and its settling time after the load torque change. The proportional and integral gains are varied to observe their effects on the amount of overshoot/undershoot and the settling time of the system.

\section{EXPERIMENTAL SETUP}

The equipments used in the experiment and the way they are connected is shown in figure 1 . The Altivar 18 variable speed drive is powered by the three phase power supply unit. The electrical output of the drive is connected to the three-phase squirrel cage induction motor. The shaft of the motor is mechanically coupled to the shaft of the control unit servo brake, which applies the load torque to the motor. The control unit servo brake also monitors the rotational speed of the motor. The magnitude of the motor's speed in terms of voltage is produced at the analog output of the control unit servo brake. This voltage is used as the feedback for the PI controller inside the Altivar 18 drive. The analog output voltage from the control unit servo brake is also fed to the auxiliary analog input of the data acquisition interface. The data sampled by the data acquisition interface is transferred and displayed on the computer. The data which is recorded in the computer is then transferred to the Microsoft Excel spreadsheet software where the graphs of the responses are plotted and the rise time, overshoot, settling time and steady-state error of the responses are evaluated.

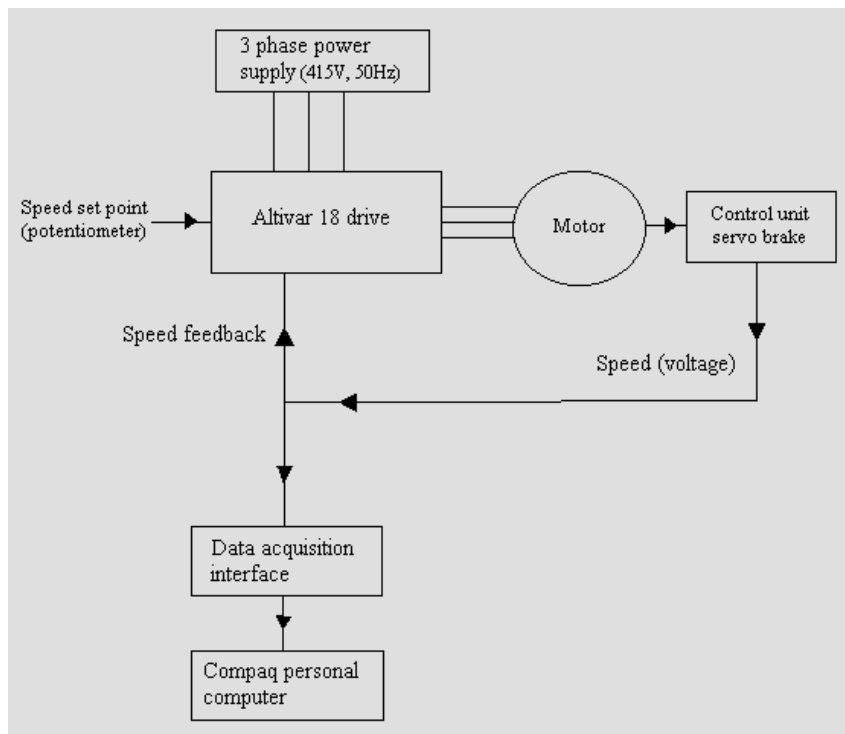

Figure 1. The equipments used in the experiment and the way they are connected (arrows show the flow of signals)

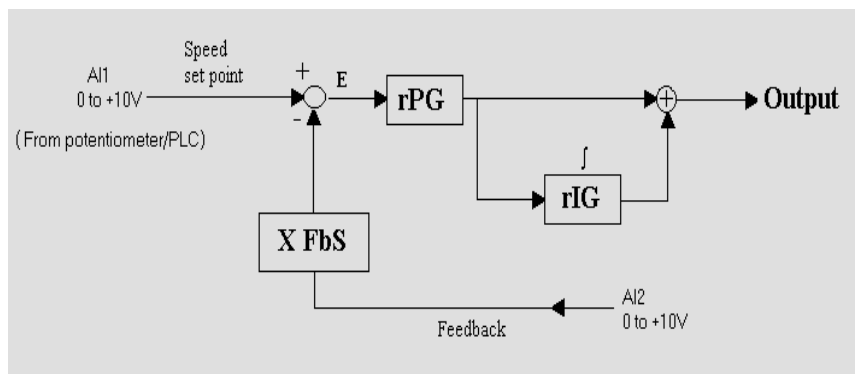

Figure 2. Block diagram of the PI control structure used in the variable speed drive (Altivar 18)

Figure 2 shows the PI control structure used in the variable speed drive. The proportional gain is denoted as "rPG", the integral gain is denoted as " $\mathrm{rGG"}$ and the feedback-scaling factor is denoted as "X FbS". The feedback signal coming from AI2 is multiplied with the feedback-scaling factor in order to bring it up to range with the speed set point or AI1 $(0$ to $10 \mathrm{~V})$. The difference between the speed set point voltage and the feedback voltage after scaling is then multiplied with $\mathrm{rPG}$. The output from $\mathrm{rPG}$ is then multiplied with $\mathrm{rIG}$ and the error is integrated. This value is then added with the output of rPG again. The output of the controller is given in equation (1).

$$
\text { Output }_{\mathrm{PI}}=(\mathrm{rPG} \times \mathrm{E})+\left(\mathrm{rPG} \times \mathrm{rIG} \times \int \mathrm{Edt}\right)
$$

where $\mathrm{E}=$ difference between the speed set point voltage and the feedback voltage after scaling (also called error).

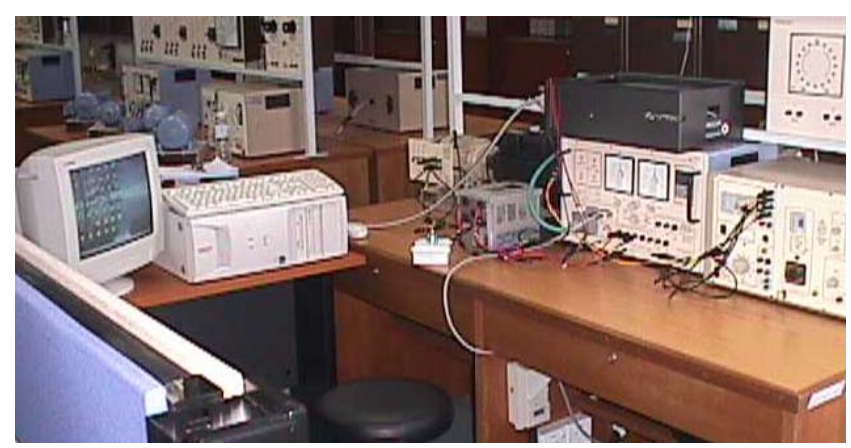

Figure 3. Picture of the equipment setup in the laboratory

\section{EXPERIMENTAL RESULTS}

\section{A. Step Speed Response}

The step speed response of the variable speed drive was evaluated from standstill to reference speeds of $1500 \mathrm{rpm}$, $1350 \mathrm{rpm}, 1140 \mathrm{rpm}, 900 \mathrm{rpm}, 750 \mathrm{rpm}$ and $510 \mathrm{rpm}$. For each reference speed, the initial load torque was set at $0 \mathrm{Nm}$, $1 \mathrm{Nm}, 1.5 \mathrm{Nm}, 2 \mathrm{Nm}, 2.5 \mathrm{Nm}$ and $3.5 \mathrm{Nm}$. The step speed response for each reference speed and their initial load torques were obtained. The proportional gains (rPG) and integral gains (rIG) were adjusted such that the over damped response, critically damped response and under damped response were obtained for each case (see figure 4). It has been found for all reference speeds and initial load torques that, as the rise time is reduced, the tendency for the system to overshoot is increased. The rise time was found to decrease as the proportional and integral gains are increased. The tendency to overshoot also increases if the integral gain is increased. If the PI gains are set too low i.e. $\mathrm{rPG}=0.13$ and $\mathrm{rIG}=0.23$, the system undergoes an over damped response where the rise time and settling times are long. Although this results in slow response, the system does not overshoot and the speed rises smoothly from standstill to the reference speed. The PI gains can also be set so that a critically damped response is obtained. In the critically damped response, the speed rises to the set point in the fastest possible time without overshooting. As a result, the settling time for the critically damped response is significantly less than the over damped system. When the PI gains are set so that an under damped response is obtained, the rise time decreases further and overshoots occur. Due to the overshoot, the settling time of the under damped 
response is slightly more than the critically damped system, but significantly less compared to the over damped system. Comparatively speaking, it can be said that the critically damped response provides better stability than the under damped response, as far as attributes such as the rise time, overshoot and the settling time are concerned.

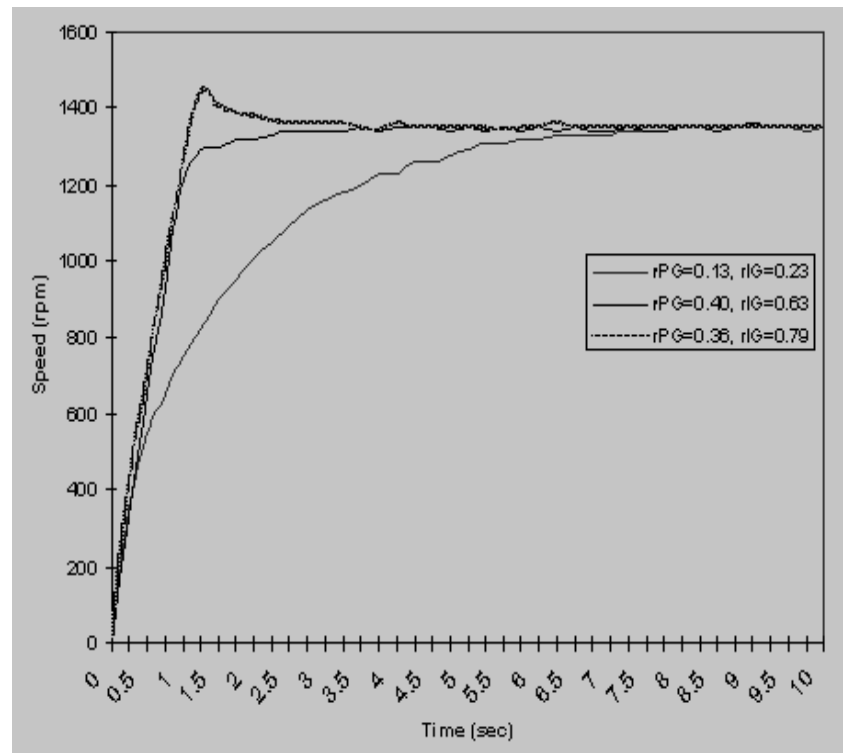

Figure 4. Step speed response from standstill to $1350 \mathrm{rpm}$, with initial load torque of $0 \mathrm{Nm}$. (Grey=under damped response, Black $=$ critically damped response, Dotted black $=$ over damped response)

Table 1. Summary of the step speed response from standstill to reference speed for a critically damped response

\begin{tabular}{|c|c|c|c|c|}
\hline \multirow{2}{*}{$\begin{array}{c}\text { Referenc } \\
\text { e speed } \\
(\mathrm{rpm})\end{array}$} & \multicolumn{2}{|c|}{$\begin{array}{c}\text { Minimum PI } \\
\text { gain }\end{array}$} & \multicolumn{2}{c|}{$\begin{array}{c}\text { Maximum PI } \\
\text { gain }\end{array}$} \\
\cline { 2 - 5 } & $\mathrm{rPG}$ & $\mathrm{rIG}$ & $\mathrm{rPG}$ & $\mathrm{rIG}$ \\
\hline 1500 & 0.37 & 0.58 & 0.40 & 0.61 \\
\hline 1350 & 0.36 & 0.57 & 0.40 & 0.63 \\
\hline 1140 & 0.58 & 0.77 & 0.62 & 0.86 \\
\hline 900 & 0.70 & 0.99 & 0.74 & 1.04 \\
\hline 750 & 0.99 & 1.18 & 1.02 & 1.23 \\
\hline 510 & 1.60 & 1.80 & 1.71 & 1.90 \\
\hline
\end{tabular}

The maximum and minimum values for the PI gains at the reference speeds of $1500 \mathrm{rpm}, 1350 \mathrm{rpm}, 1140 \mathrm{rpm}, 900$ $\mathrm{rpm}, 750 \mathrm{rpm}$ and $510 \mathrm{rpm}$ for the critically damped response of the step speed response test are shown in table 1. The maximum and minimum range of values for the PI gains are valid when the initial load torques ranges from $0 \mathrm{Nm}$ to $3.5 \mathrm{Nm}$. The maximum values of PI gains are used when the initial load torque is $0 \mathrm{Nm}$ while the minimum values of PI gains are used when the initial load torque is $3.5 \mathrm{Nm}$. Therefore, the amount of initial load torque is inversely proportional to the amount of PI gain. In other words, if the initial load torque were to increase, then the PI gains should be reduced to obtain the critically damped response. When the critically damped response is used as a basis for comparison, it can be seen that as the reference speed decreases from $1500 \mathrm{rpm}$ to $510 \mathrm{rpm}$, a gradual increase in the PI gains for both the maximum and minimum values must be made.

\section{B. Step Load Torque Change Response}

The step load torque change response was evaluated for the same reference speeds. For each reference speed, a load torque of $1.5 \mathrm{Nm}$ was added suddenly to the system with initial load torques of $0 \mathrm{Nm}, 1 \mathrm{Nm}$ and $2 \mathrm{Nm}$. Similarly, a load torque of $1.5 \mathrm{Nm}$ was suddenly removed from the system with initial load torques of $1.5 \mathrm{Nm}, 2.5 \mathrm{Nm}$ and $3.5 \mathrm{Nm}$. It has been found that, undershoots in speed occur when the load torque is added suddenly (figure 5), while overshoots in speed occur when the load torque is removed suddenly from the system (figure 6).

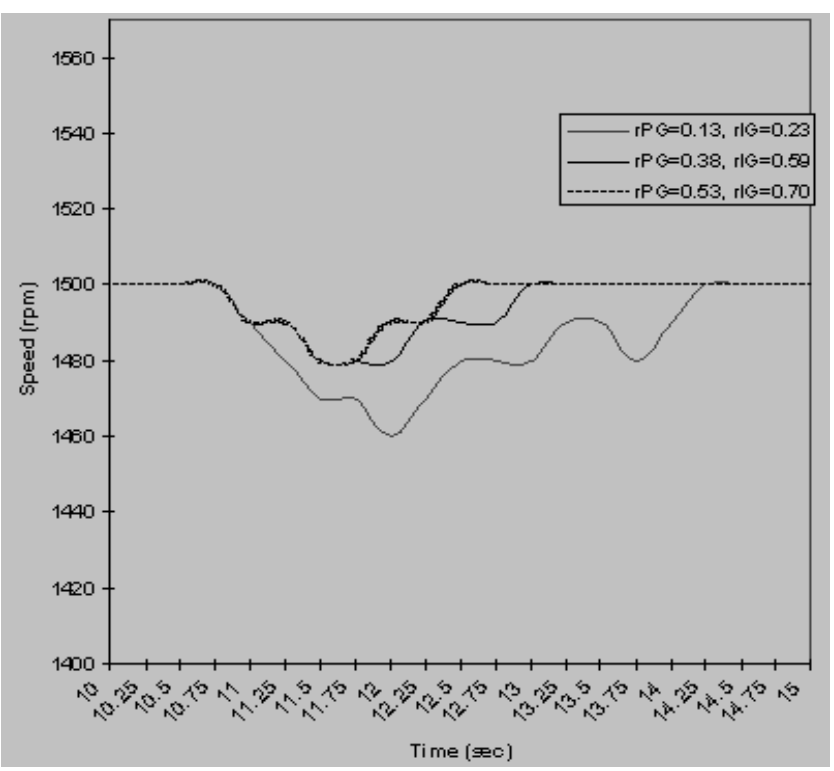

Figure 5. Step load torque change from $1 \mathrm{Nm}$ to $2.5 \mathrm{Nm}$ $(+1.5 \mathrm{Nm})$, at $1500 \mathrm{rpm}$ reference speed

For all reference speeds and their initial load torques, it has been found that increasing the proportional and integral gains reduces the amount of overshoot (or undershoot) and decreases the settling time after the disturbance. In some cases, the amount overshoot or undershoot remains the same despite higher values of PI gain. However in this case, the system returns to the reference speed much faster (shorter settling time). As the values of the PI gains are decreased, the amount of overshoots and undershoots increases, and this results in larger settling times.

Table 2. Average PI gains for minimum undershoot (or overshoot) and settling time at the different reference speeds for a $+/-1.5 \mathrm{Nm}$ step load torque change

\begin{tabular}{|c|c|c|}
\hline $\begin{array}{c}\text { Reference } \\
\text { speed } \\
(\mathrm{rpm})\end{array}$ & \multicolumn{2}{|c|}{ Average PI gains } \\
\cline { 2 - 3 } & $\mathrm{rPG}$ & $\mathrm{rIG}$ \\
\hline 1500 & 0.53 & 0.70 \\
\hline 1350 & 0.40 & 0.79 \\
\hline 1140 & 0.64 & 0.97 \\
\hline 900 & 0.81 & 1.12 \\
\hline 750 & 1.20 & 1.44 \\
\hline 510 & 1.88 & 2.25 \\
\hline
\end{tabular}

The average PI gains for minimum undershoot and minimum settling time at the reference speeds of $1500 \mathrm{rpm}$, $1350 \mathrm{rpm}, 1140 \mathrm{rpm}, 900 \mathrm{rpm}, 750 \mathrm{rpm}$ and $510 \mathrm{rpm}$, for a $+/-1.5 \mathrm{Nm}$ step load torque change are shown in table 2 . The 
average PI gains for each reference speed was obtained by averaging the individual PI gains which produces the smallest undershoot and smallest settling time after being subjected to a $+1.5 \mathrm{Nm}$ load change from the initial load torques values of $0 \mathrm{Nm}, 1 \mathrm{Nm}$ and $2 \mathrm{Nm}$. It can be seen that as the reference speed decreases from $1500 \mathrm{rpm}$ to $510 \mathrm{rpm}$, the average PI gains must be gradually increased in value.

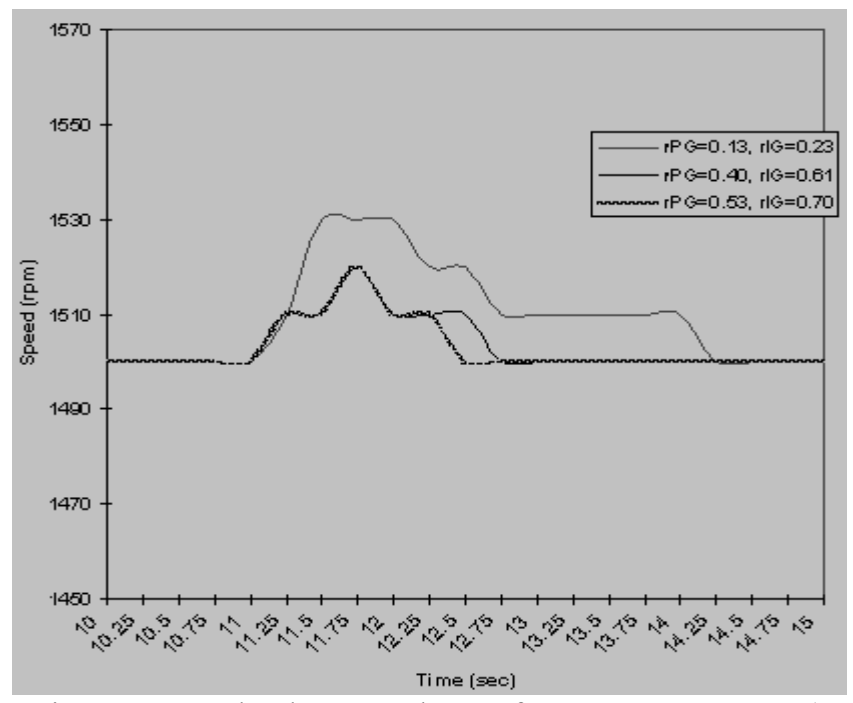

Figure 6. Step load torque change from $1.5 \mathrm{Nm}$ to $0 \mathrm{Nm}$ ($1.5 \mathrm{Nm})$, at $1500 \mathrm{rpm}$ reference speed

\section{DISCUSSION}

\section{A. Step Speed Response}

It has been found that, to reach large reference speeds from standstill such as from 0 to $1500 \mathrm{rpm}$, relatively small values of proportional and integral gains are required, for a critically damped response. As the reference speed is reduced however, such as from 0 to $510 \mathrm{rpm}$, the proportional and integral gains must be increased to maintain a critically damped response. This is due to the smaller amount of error at low reference speeds compared to the larger amount of error at higher reference speeds. Hence, to compensate for the smaller amount of error at low speeds, the proportional and integral gains must be increased to maintain a critically damped response (see table 1).

As the initial load torque increases, the values of the PI gains required to obtain critically damped response was found to decrease slightly. If the PI gains for the previous torque value were maintained, the system would overshoot. The reason for this could be due to increased load on the shaft of the motor at standstill. Consequently, the controller has to provide a larger output so that the shaft can start rotating. In order to do that, the motor's torque must be made larger than the load torque. Once the motor starts rotating, it builds up its speed towards the set point. As the speed of the motor nears the set point however, the additional motor torque is still present due to the large output of the controller (integrating effect of the PI controller). Due to the fact that, the PI controller has no way to slow down the response before it reaches the desired set point, the system overshoots. When the system overshoots, this causes an error in the opposite direction and the PI controller starts regulating the speed again so that it goes back to the set point. Because of this reason, a slight decrease in the values of the proportional and integral gains is necessary to prevent the system from overshooting the set point (for a critically damped response to be obtained), as the initial load torque is increased.

In summary, the following can be said. Increasing the controller's gains can significantly reduce the rise time. However, the tendency to overshoot will increase (under damped condition). This will ultimately lead to a longer settling time. Therefore, a tradeoff for a fast rise time is the increased overshoot and longer settling time. By setting the gains to small values (over damped condition), the system can be made to rise slowly so that overshoots will not occur. But, the rise time and settling time will be very large. Therefore, the tradeoff of having no overshoots (or increased stability) is that rise times and settling times must be made very large.

The gains can also be set in such a way that the speed rises to the set point in the shortest time possible without overshooting. For such a critically damped condition, fast rise times and short settling times can be realized without having overshoots in the system. The critically damped response provides by far, the best response as far as stability and speed of response are concerned. Whether or not a system should be tuned to the condition of under damped, critically damped or over damped, depends on the application as well as the physical limitations of the system. For applications which require very fast response and where overshoots are tolerated, then the under damped response with its corresponding PI gains should be selected. For applications where high stability and smooth acceleration is required, such as conveyor belts, then the over damped response with its corresponding PI gains should be selected. For applications where fast response is required and overshoots are not tolerable, then the critically damped response with its corresponding PI gains should be chosen. The physical limitations on the system such as large rotor inertia for big motors, also places a limit on how fast the system can and should respond such that no damage will occur in the system.

\section{B. Step Load Torque Change Response}

It can be seen from table 2 that the proportional and integral gains at high speed (such as at $1500 \mathrm{rpm}$ ), has been set at lower values compared to the gains at low speed (such as at $510 \mathrm{rpm}$ ), for the given initial load torques. This has been done to take into account the stability of the system.

In most industrial applications, load changes to the system are difficult to anticipated. Most of the time, these systems run without any change in load conditions (steady speed conditions). As such, the system cannot always be made to run under high values of PI gains which ensures the smallest overshoot (or undershoot) and shortest settling time, in the anticipation that a load change were to occur. In fact, the gains must be chosen such that stability of the system is maintained before, and after the load torque change.

If the PI gains were set too high prior to the load change (steady speed conditions), the system's controller becomes overly sensitive to noise as well as other small changes in external variables. Due to the large multiplying factor of the PI gains, small variations in the feedback signal influenced by mechanical and electrical noise gets amplified to very 
large values. This causes a large output from the controller in an attempt to correct the anomaly. As a result, the motor speeds up. Now, that the motor has sped up beyond the reference speed, the controller must correct the error by reducing the speed. Since the gains are set too high, there is a tendency for the controller to overcorrect by reducing the speed below the reference value. Hence, the controller has to correct the error and the whole process repeats itself. The outcome of this process is that, the motor speeds up and slows down alternatively, leading to oscillations in the speed about the set point. Such oscillations may cause the motor to vibrate uncontrollably and become unstable. This effect has been observed experimentally where the motor vibrates and produces loud, high frequency noise whenever the PI gains are set too high. The effect being more pronounced at high speed due to more kinetic energy present in the system. This effect is undesirable especially since the motor is still running at steady speed and not subjected to any load change yet.

In order to maintain stability before, and after the load change, the gains of the system must be as close as possible to the values which yield stable operation at steady speed conditions. These values are dependent on their respective reference speeds. At high reference speeds (1500 rpm), the PI gains are of relatively low values while at low reference speeds (510rpm), the PI gains are significantly higher in value. It can therefore be seen from the results that, at high speeds (1500 rpm to $1140 \mathrm{rpm})$, the amount of overshoot and undershoot as well as settling time are slightly larger compared to at low speeds (750 rpm to $510 \mathrm{rpm})$. This as stated earlier was due to the smaller PI gain at high speeds and the larger PI gain at low speeds. The larger the PI gains, the smaller the overshoot (or undershoot) and the shorter the settling time; and vice versa.

As a summary, it can be said that the selection of PI gains for the step load torque change response is a tradeoff between maintaining the stability of the system (prior to, and after the load change) and, reducing the amount of overshoot and settling time. The tradeoff of maintaining the system stability is that, the PI gains cannot be increased to high values indiscriminately in order to obtain the smallest overshoot or settling time in the event of a load torque change. The tradeoff of reducing the overshoot and settling time (higher PI gains) is that the system stability (before, and after the load torque change) may be affected. Therefore, a compromise must exist such that the PI gains are selected so that the system performs well (stably) before, and after a load torque change and at the same time have overshoots (or undershoot) and settling times which are acceptable to the user.

\section{CONCLUSIONS}

This project studied the performance of the Altivar 18 variable speed drive with PI regulator using the experimental approach. The results of the study show that the PI regulator provided fast and stable response for the step speed response and step load torque change response, when it is properly tuned. In addition to that, it also eliminated the steady-state error of the system after the disturbance. The results of the step speed response indicates that a tradeoff among the control performance measures such as rise time, overshoot and settling time must be made if the desired response is to be achieved. The desired response in turn depends on the application as well as the physical limitations of the system.

The results of the step load torque change response on the other hand, shows that the selection of PI gains is a tradeoff between maintaining the stability of the system (prior to, and after the load change) and, reducing the amount of overshoot and settling time.

The results of this study is limited to time domain analysis to determine the performance of the system. Time domain analysis is used because it provides the most fundamental method of assessing the performance of a control system and the data obtained is also easy and simple to analyze. Also, the results obtained from this study are most applicable to or limited to systems with drives and motors of low horse powers, where the operating speed is between $1500 \mathrm{rpm}$ and $510 \mathrm{rpm}$, and where the load change or disturbance is of the magnitude of $\pm 1.5 \mathrm{Nm}$. For motors and loads of different size, the same method can be easily adapted to determine the PI gains for dynamic performance of a practical variable speed drive.

\section{APPENDIX}

\section{A. Motor Parameters}

The motor parameters are as follows: Output power $=$ $0.37 \mathrm{~kW}$, Voltage $(\Delta / \mathrm{Y})=230 / 400 \mathrm{~V}$, Current $=2 / 1.17 \mathrm{~A}$, Power factor $(\cos \theta)=0.67$, Speed $($ rated $)=1400 \mathrm{rpm}$, Number of stator poles $\left(\mathrm{N}_{\mathrm{s}}\right)=4$.

\section{B. Drive Parameters}

Power supply voltage $=415 \mathrm{~V}$, Power supply frequency $=50 \mathrm{~Hz}$, Maximum output voltage $=415 \mathrm{~V}$, Speed range $=0.5$ to $320 \mathrm{~Hz}$, Maximum output current=7.1A, Output power $=0.75 \mathrm{KW}$.

\section{REFERENCES}

[1] Z. Ibrahim, E. Levi, "A Comparative Analysis of Fuzzy Logic and PI Speed Control in High-Performance AC Drives Using Experimental Approach", IEEE Transactions On Industry Applications, Vol. 38, No. 5, September/October 2002, pp. 1210-1214.

[2] Jamshidi, K., Subrahmanyam, V., "Modified controller for closed loop control of CSI-fed induction motor", Power Electronics and Drive Systems, 1995, Proceedings of 1995 International Conference, Vol. 1, 21-24 Feb. 1995, pp. 135-139.

[3] Ogawa, S., "PI controller tuning for robust performance", Control Applications, 1995, Proceedings of the 4th IEEE Conference, 28-29 Sept. 1995 , pp. 101-106.

[4] Vlachos, C., Evans, J.T., Willimans, D., "PI controller tuning for multivariable processes using genetic algorithms", Genetic Algorithms In Engineering Systems:Innovations And Applications, 1997. GALESIA 97. Second International Conference On (Conf. Publ. No. 446), 2-4 Sept. 1997, pp. 43-49.

[5] Kristiansson, B., Lennartson, B., "Robust and optimal tuning of PI and PID controllers", Control Theory and Applications, IEE Proceedings, Vol. 149, Jan. 2002, pp. 17-25.

[6] Pei-Jin Wang, "PI-tuning methods based on GA", Machine Learning and Cybernetics, 2002, Proceedings 2002 International Conference on Machine Learning and Cybernetics, Vol. 1, 4-5 Nov. 2002, pp. 544547.

[7] Shih-Chang Hsu, Chio-Hao Liu, Chang-Huan Liu, Nai-Jian Wang, "Fuzzy PI controller tuning for a linear permanent magnet synchronous motor drive", Industrial Electronics Society, 2001. IECON '01, The 27th Annual Conference of the IEEE, Vol. 3, 29 Nov.-2 Dec. 2001, pp. 1661-1666.

[8] Kau, G.K., Mukherjee, S.K., Loo, C.K.,, Kwek, L.C., "Evolutionary PID control of non-minimum phase plants", Control, Automation, Robotics and Vision, 2002. ICARCV 2002. 7th International 
Conference on Control, Automation, Robotics and Vision, Vol. 3,2-5 Dec. 2002, pp. 1487-1492.

[9] Thomas M. Jahns, "AC Adjustable-Speed Drives at the Millennium: How Did We Get Here? ”, IEEE Transactions on Power Electronics, Vol. 16, No. 1, January 2001, pp. 17-24.

[10] W. Drury, " Electrical Variable Speed Drives: Mature Consumable or Radical Infant? ”, Power Engineering Journal, April 1999, pp. 65-78.

[11] Kilian, Cristopher T., "Modern Control Technology: Components and Systems", 2nd Edition, Delmar Thomson Learning, New York, 2001, pp. $189-476$

[12] Marlin, Thomas E., "Process Control: Designing Processes and Control Systems for Dynamic Performance", 1st Edition, McGraw Hill, 1995, pp. 1-462.

[13] Gopal, M., Nagrath, I.J., "Control Systems Engineering", 2nd Edition, Wiley Eastern Limited, 1989, pp. 142.

[14] Hunter, Ronald P., "Automated Process Control Systems: Concepts and Hardware", 2nd Edition, Prentice Hall, 1987, pp. 252-271.

[15] Fardo, S., Patrick D., " Industrial Process Control Systems" 1st Edition, Delmar Publishers, 1997, pp. 56-63.

[16] Smuts, J., "PID controllers explained", Canadian Process Equipment \& Control News, February 2002.

[17] Altivar 18 Adjustable Speed Drive Controllers for Asynchronous Motors - User's Manual, Instruction Bulletin 52012-008-01, March 1997, pp. 1-42 


\title{
Modeling of a Three Phase Linear Generator by Using Finite Element Magnetics Method
}

\author{
Rajendran S., H. Arof and M.K.A. Ahmed Khan
}

\begin{abstract}
This dissertation presents the development of a three phase linear generator with the output power of $4.4 \mathrm{~kW}$ and output voltage of $200 \mathrm{~V}$ with minimum cogging force is modeled and simulated. Specific design criteria are employed to meet exclusive requirements related to its stator and translator. A simple model of the electromagnetic analysis of the cogging force and generated emf is presented. Finite element software is used to simulate the three phase linear generator. A radially magnetized magnet is chosen to give a high performance and to solve the cogging force-voltage problem. The design of the AC to DC full-bridge rectifier with capacitor filter is described which acts as a link between the output of the three phase linear generator and the battery. The actual three phase linear generator output voltage waveforms simulated from the FEMM are fed into the full-bridge rectifier. PSPICE simulation results are presented to verify the validity of the model.
\end{abstract}

\section{INTRODUCTION}

The need of remote power generation or via uninterrupted electrical power are being employed and increased dramatically in applications ranging from transportation, manufacturing, and office automation to material processing, health care, and generation systems. The reliability of continuous electrical power supply requires the ability to maintain electrical service in the event of a failure on the utility grid and ability to generate electricity for applications where the utility service is not available.

Standby generators are often used as a backup power supplies in the event of a loss of utility power from the utility failure. In addition, the environment of military field operations requires the ability to generate electricity in areas where connection to the utility is impractical.

Hence, the development of the optimal operation of a power generation unit or stand alone power generation which is reliable, compact and lightweight is necessary for everyday $[1,2]$.

Researches contend that linear electromagnetic machine offers a number of advantages over the conventionally used rotary systems for electrical power generation. It is a better choice in relation with energy efficiency issue. The absence of the crank shaft, rod and rotary parts that contribute to

Rajendran S., and M.K.A. Ahamed Khan are with the Division of Electrical Engineering, University Industry Selangor, Malaysia, (fax: +603 3280 6016, e-mail: rajendran@unsel.edu.my, khan@unisel.edu.my).

Hamzah Arof is with the Department of Electrical Engineering, University of Malaya, Malaysia .(phone: +6037967 52205, fax: +6037967 5316, e-mail: ahamzah@um.edu.my). friction losses, the linear system becomes compact, highly efficient, and lightweight. In addition, gas fuel is recommended to achieve low emission. The linear auxiliary power system is subsequently used and functions alike its rotary counterparts. The linear generator can directly extract the energy from the engine and helps to be more compact with high efficiency and fewer moving parts [3].

In this study, design and simulation aspects of a three phase linear generator intended to be driven by a free-piston internal combustion engine are outlined. Specific design criteria of the three phase linear generator are employed to meet exclusive requirements related to its stator and translator. The optimization of the three phase linear generator is performed to minimize the flux density in the magnetic core and minimize the dimension of the generator while avoiding the saturation. The tooth shoe is introduced to minimize the cogging force. Finite element software is used to simulate the three phase linear generator. The rectification of the three phase linear generator is performed by using PSPICE software.

\section{LINEAR GENERATOR DESIGN}

The generator is designed to have 6 winding slots. It has a stroke length of $60 \mathrm{~mm}$. As the square wire is used, the slot fill factor is 0.75 . The generator is designed to run at 3000 rpm. A non oriented silicon steel lamination is used in the FEMM simulation. The permanent magnet $\mathrm{NdFeB}$ is used to provide a high density magnetic field. The translator consists of a shaft, back iron and permanent magnets separated by aluminum spacers. The material used in shown in Table 1.

Table 1. The three phase linear generator materials

\begin{tabular}{|l|l|}
\hline \multicolumn{1}{|c|}{ Part } & \multicolumn{1}{c|}{ Materials } \\
\hline Permanent magnet & NdFeb 32 MGOe \\
\hline Coil & Copper \\
\hline Back Iron & Steel 1010 \\
\hline Tooth & Steel 1010 \\
\hline Shaft and spacer & Aluminum \\
\hline
\end{tabular}

The schematic cross sectional view of the three phase linear generator is shown in Figure 1. Optimum parameters can be achieved by getting the required output voltage. Same parameters are as constant while others are optimized for best results. Figure 2 shows the flowchart for optimization of the three phase linear generator $[4,5,20]$. 


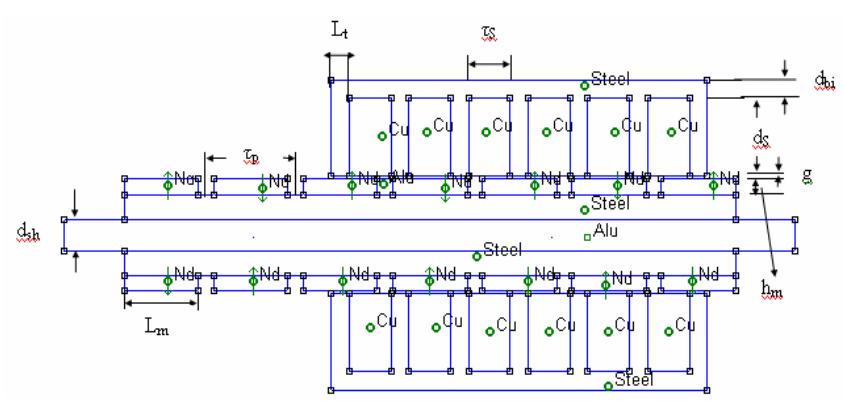

Figure 1. The cross sectional view

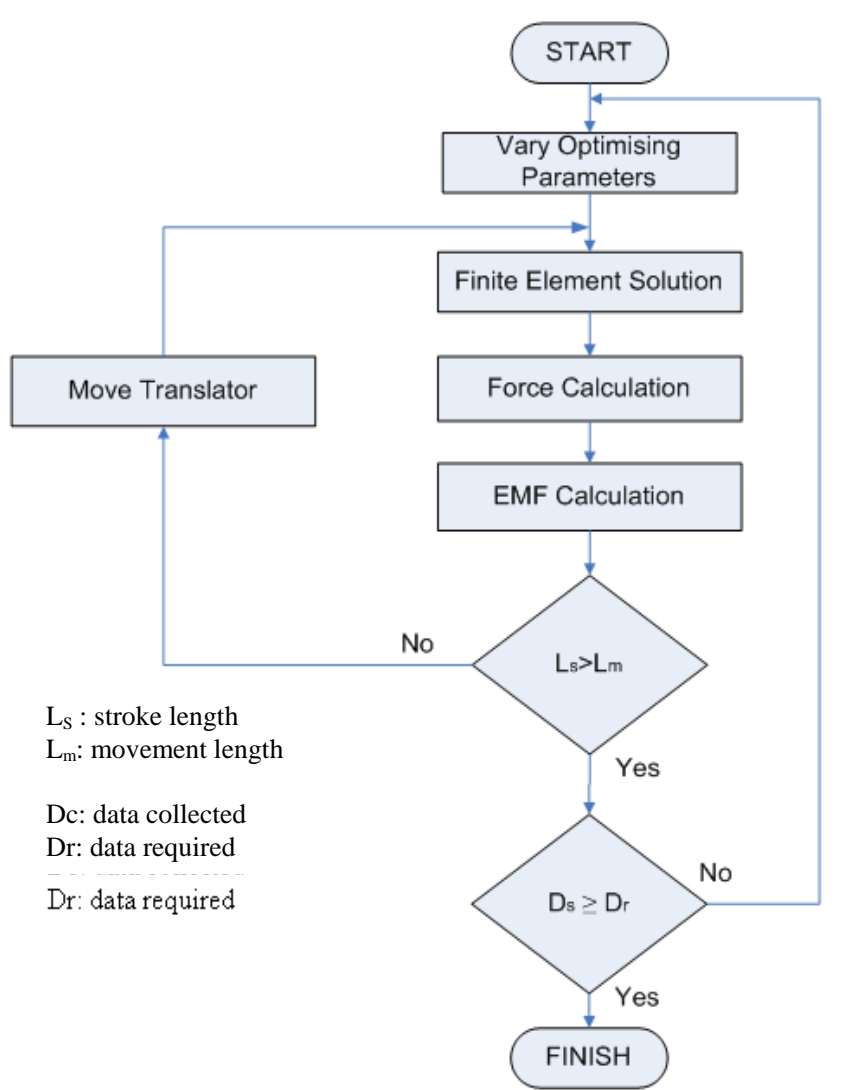

Figure 2. Flowchart of the three phase linear generator optimization

\section{Governing EQUATIONS}

The three phase linear generator is analyzed using the finite element method (FEM) in two dimensions. The permanent magnets are mounted on the translator create a magnetic field around the magnets. When the engine operation takes place, the change of magnetic field exist as the translator move relatively linear to the stationary armature coils. The change in magnetic field then a voltage is induced in the windings as governs by Faraday's law.

The Faraday's law expressed as [4, 5]:

$e_{\text {ind }}=-\frac{d \lambda}{d t}=-N \frac{d \phi(t)}{d t}$

where $\lambda$ is total flux passing through the coil, $\phi$ is flux passing through one turns in the coil, $N$ is number of turns in the coil, $e_{\text {ind }}$ voltage induced on the coil and $\frac{d \phi(t)}{d t}$ is flux derivative with respect to time.

The total output voltage of the linear generator is determined by summing all the coil voltages with polarities such that all of the voltages are additive, as shown in equation (2) [5, 12].

$V=v \sum_{i=1}^{n}(-1)^{i} N_{i} \frac{d \phi}{d x}$

However the equation (2) must be converted from magnitude to an RMS value by using equation (3) as below:

$V_{r m s}=\frac{\sqrt{\sum_{i=1}^{n} N_{i} \frac{d \phi}{d t}}}{n}$

In a one pole pitch translator movement, the quantities of fluxes crossing coils are changed from positive maximum to negative minimum and assumed linearly changed. The total flux linkage of coil having $\mathrm{N}$ turns is thus $N \phi$. Flux calculation for the maximum or minimum flux involving the flux density and the surface area of the magnet and flux reduction factors. [12]. The flux generated by the magnet can be derived from its magnetic remanence and normal surface area.

$\phi_{p m}=B_{s} A_{p m}$

$B_{s}=K_{f} B_{r}$

$A_{p m}=2 \pi r_{\text {inner,magnet. }} l_{\text {magnet }}$

where $B_{s}$ is a flux density on the permanent magnet surface, $B_{r}$ is a magnetic remanence, $K_{f}$ is flux density factor, $A_{p m}$ is a surface area of the permanent magnet, $r_{\text {inner,magnet }}$ is a inner radius of the permanent magnet (the length is same as $r_{\text {shaft }}$ ).

In magnetostatic study or time-invariant analysis, the finite element method is used to determine the linear generator magnetic field as low frequency problems and Maxwell's equations subsets are required [18].

The force acting on a ferromagnetic object can be computed as the sum of forces in the air layer surrounding it. The force of an air material element in the s direction is given by [18]:

$F_{S}=\int_{v o l} \overline{B^{T}} \frac{\partial \bar{H}}{\partial S} d(v o l)+\int_{v o l}\left(\int \overline{B^{T}} d \bar{H}\right) \frac{\partial}{\partial S} d(v o l)$

Where $F_{S}=$ force in element in the s direction; $\frac{\partial \bar{H}}{\partial S}=$ field effect derivative with respect to displacements nodal coordination virtual displacement in the XYZ global direction; and $v o l=$ volume of the element.

The mathematical expressions can be entered into the edit box in a Lua Script in lieu of a numerical value. This is available in the finite element package. The Lua script is used to achieve the translator movement fairly for the linear generator in a prescribed distance and compute the cogging force and electromotive force induced in the linear generator.

The full-wave bridge configuration with diodes as the rectifying devices is commonly used, as it gives an alternating current waveform in the three phase linear 
generator. The three phase linear generator is connected to the battery through diode rectifier system. The three phase ac distorted waveform is distorted with the desired peak value. These voltages are fed into the full bridge diode rectifier, which converts, from $\mathrm{AC}$ to $\mathrm{DC}$ waveform.

The capacitor voltage $v_{c}$ varies between a minimum $\mathrm{V}_{\mathrm{c}(\min )}$ and maximum value $\mathrm{V}_{\mathrm{c}(\max )}$. This is shown in Figure 3 [23].

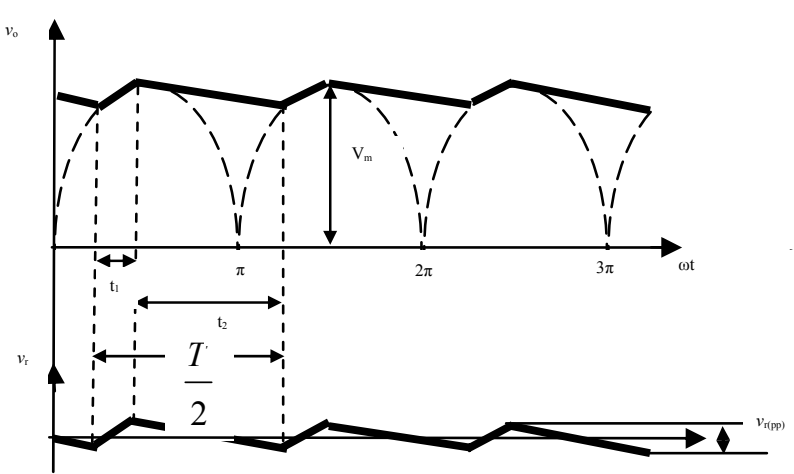

Figure 3. Waveforms for full wave bridge rectifier

The capacitance value of the rectifier can be calculated and is given by equation (8) below:

$$
C=\frac{1}{4 f R}\left(1+\frac{1}{\sqrt{2} R F}\right)
$$

where $f$ is a supply frequency, $R$ is load resistance and $R F$ is ripple factor.

This capacitor value can be minimizing the amount of ripple present in the output waveform of the three phase rectifier system. The total power generated from the three phase linear generator is equal to the total power of the rectifier system and it is given by equation (9).

$P_{a}+P_{b}+P_{c}=V_{d c} \times I_{d c}$

The power $\mathrm{P}_{\mathrm{a}}, \mathrm{P}_{\mathrm{b}}$ and $\mathrm{P}_{\mathrm{c}}$ is the output power in each phases of the three phase linear generator. The $\mathrm{V}_{\mathrm{dc}}$ is an average value from the PSPICE simulation. Hence $\mathrm{I}_{\mathrm{dc}}$ can be estimated from the equation (9).

\section{RESUlTS AND DisCUSSION}

\section{A. Flux Linkage and Induced Voltage}

The translator of the three phase linear generator is aluminum (a standard material in the FEMM library). The translator or the moving portion is $245 \mathrm{~mm}$ long and weighs about $1.485 \mathrm{~kg}$. It travels along the horizontal axis from left to right. Although FEMM only requires that the half of the three phase linear generator model is entered as data, but in this study the illustrative picture of a flux distribution and cross section of the complete coil as shown in Figure 4.

The coils itself is not modeled as individual turns of wire, but rather as a flat copper conductor with a $3.5 \mathrm{~A} / \mathrm{mm}^{2}$ current density. In the final simulation integrated with the Lua Scripting, the number of turns of wire per unit area is used to calculate the actual current flows into the coil. The real case where the actual field is a function of time as the current changes and the translator travels though the $\mathrm{x}$-axis.
Nevertheless, this will give an idea of the coils capability before commit resources to building hardware.

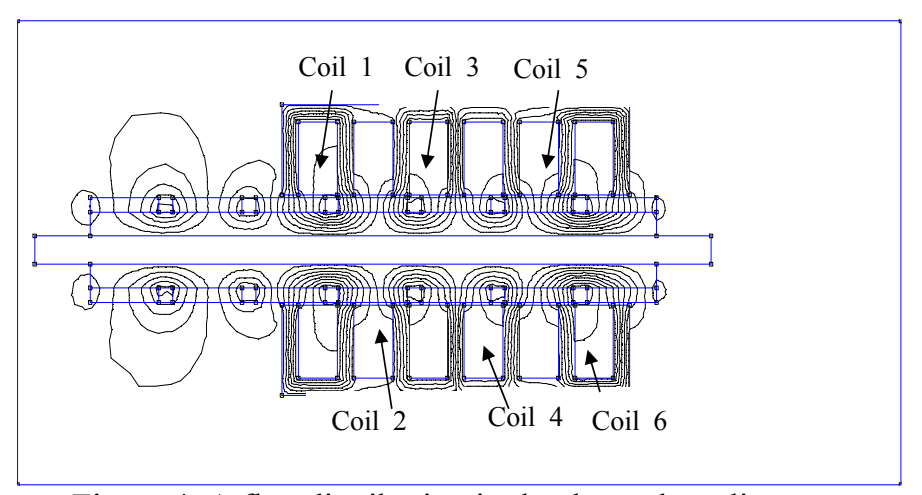

Figure 4. A flux distribution in the three phase linear generator at the starting position

The flux mainly distributes in the stator teeth and the back iron. The flux density in the stator is kept below 1.85 Tesla, the saturated flux density of the material. The fringing fluxes flow in the other teeth through the air in the inner space of stator and in the air gap. These fluxes lead to the presence of ripples in the voltage waveform. The small air gap keeps fringing effect low. The air gap size is $1 \mathrm{~mm}$ length.

The electromotive force (emf) is calculated for each step of movement of the translator of the three phase linear generator by differentiating the flux curve. The Figure 5 shows the magnetic field density vector plot. The blue rectangle is the boundary between the two different regions. The boundary is shown in Red is defined for the force calculation. The exact size and shape of the boundary is not critical although the results may vary a few percent for different boundaries since the accuracy of the finite element analysis is just that, "finite".

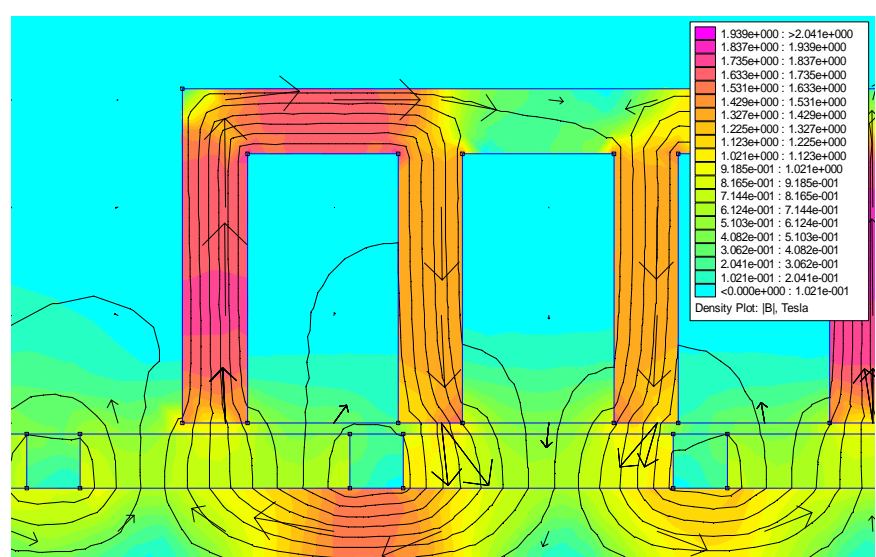

Figure 5. The magnetic field density vector plot

The flux linkage in each coil for one complete revolution is shown in Figure 6.

The resultant back emf simulation three phase output for the final design parameters for one complete revolution is shown in Figure 7.

\section{B. Cogging Force}

The maximum cogging force is expected to be around 533 $\mathrm{N}$. Figure 8 shows the force on the translator as it moves along the axis. Distance is measured between the center of the translator and the center of the coil. Because of 
symmetry, the force is zero when the translator is at the center of the coil. As it moves out of the coil, the force changes sign and acts to pull the translator back. The energy delivered to the translator is equal to the integral of force times the distance.
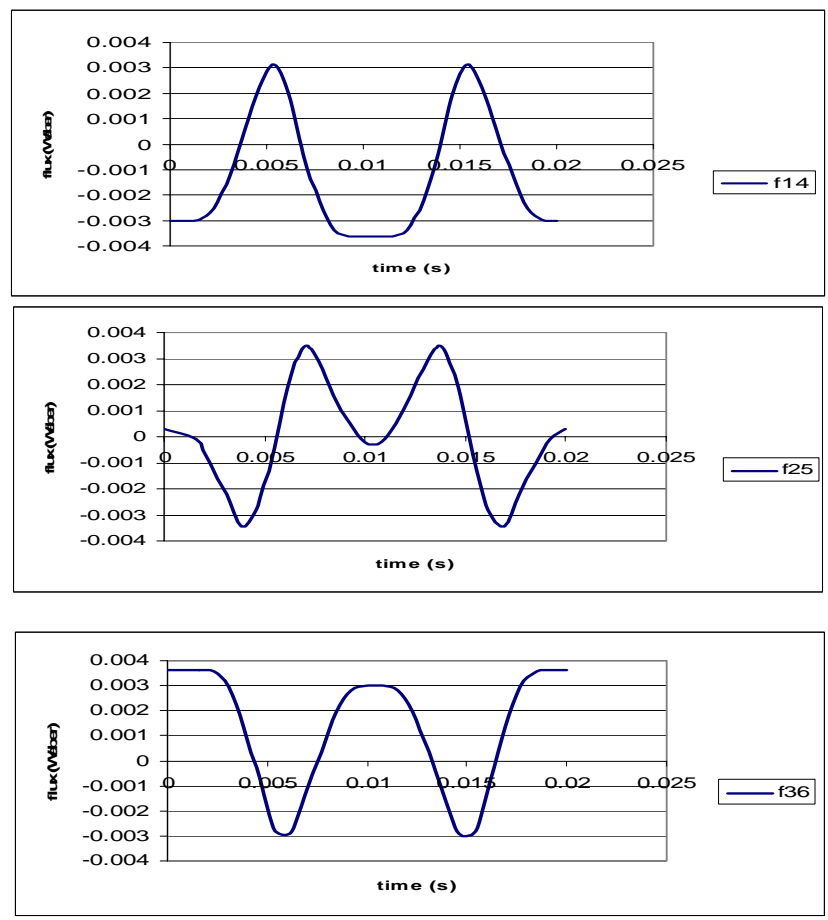

Figure 6. The flux linkage in each coil for one complete revolution

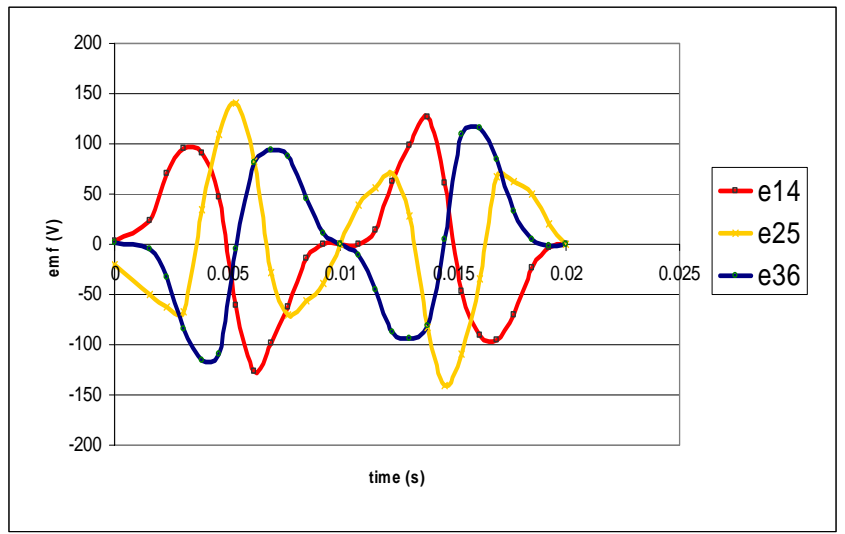

Figure 7. Simulation three phase output voltage

Table 2 shows the overall dimensions of the optimized three phase linear generator. The overall dimension is obtained from the complete optimization process for the above analysis. The stator consists of a steel back iron with the depth of dimensions given in Table 2.

The maximum output power of the three phase linear generator is calculated and is around $4.4 \mathrm{~kW}$. This meant that the three phase linear generator, generates around $70 \mathrm{~V}$ rms (root meant square) voltage with the supply current of $21 \mathrm{~A}$ in each coils.

The weight of the three phase linear generator also performed in this study. Table 3 shows the total weight of the three phase linear generator.

The volume of the stator and translator of the three phase linear generator can be determined in postprocessor of FEMM analysis. The block volume integrals automatically compute the volume of the selected region which appears highlighted in green. The total weight of the three phase linear generator is around $22 \mathrm{~kg}$. The power to weight ratio is calculated and it is equivalent to $200 \mathrm{~W} / \mathrm{kg}$.

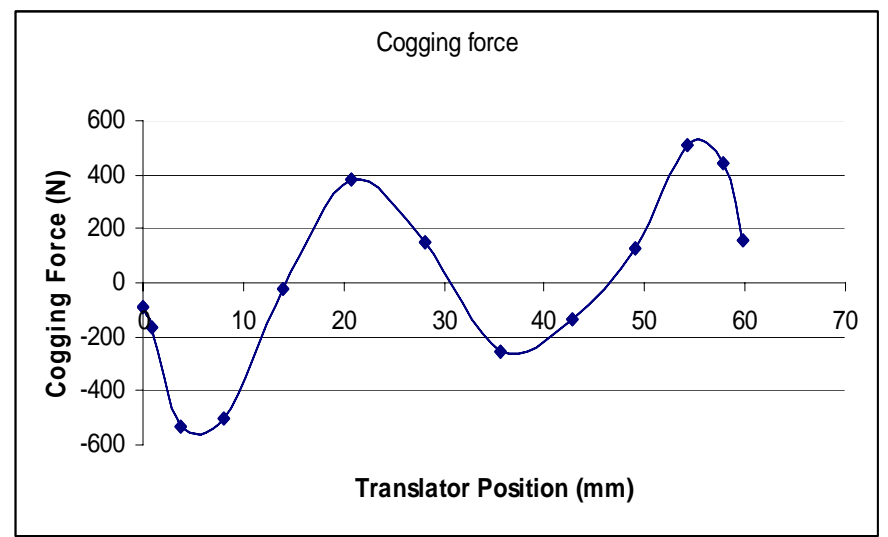

Figure 8. A cogging force

Table 2. Three Phase Linear Generator Dimensions

\begin{tabular}{|c|c|c|c|}
\hline \multirow{7}{*}{ Performance } & $\mathrm{Va}(\mathrm{rms})$ & \multicolumn{2}{|c|}{$70 \mathrm{~V}$} \\
\hline & $\mathrm{Vb}(\mathrm{rms})$ & \multicolumn{2}{|c|}{$72 \mathrm{~V}$} \\
\hline & $\mathrm{Vc}(\mathrm{rms})$ & \multicolumn{2}{|c|}{$70 \mathrm{~V}$} \\
\hline & $\mathrm{Pa}$ & \multicolumn{2}{|c|}{$1461.73 \mathrm{~W}$} \\
\hline & $\mathrm{Pb}$ & \multicolumn{2}{|c|}{$1514.79 \mathrm{~W}$} \\
\hline & $\mathrm{Pc}$ & \multicolumn{2}{|c|}{$1462.34 \mathrm{~W}$} \\
\hline & Cogging force & \multicolumn{2}{|c|}{$532.6 \mathrm{~N}$} \\
\hline Items & Parameter & Symbol & $\begin{array}{l}\text { Value } \\
\text { (mm) }\end{array}$ \\
\hline \multirow{3}{*}{ Translator } & Length of PM & $\mathrm{L}_{\mathrm{m}}$ & 25 \\
\hline & Height of PM & $\mathrm{h}_{\mathrm{m}}$ & 5 \\
\hline & Diameter of shaft & $\mathrm{d}_{\mathrm{sh}}$ & 10 \\
\hline \multirow{6}{*}{ Stator } & Slot pitch & $\tau_{s}$ & 14 \\
\hline & Slot depth & $\mathrm{d}_{\mathrm{s}}$ & 25 \\
\hline & Pole pitch & $\tau_{p}$ & 30 \\
\hline & Tooth Width & $\mathrm{W}_{\mathrm{t}}$ & 200 \\
\hline & Tooth length & $\mathrm{L}_{\mathrm{t}}$ & 6 \\
\hline & $\begin{array}{l}\text { Back iron } \\
\text { thickness }\end{array}$ & $\mathrm{d}_{\mathrm{bi}}$ & 6 \\
\hline \multicolumn{2}{|l|}{ Air gap length } & $\mathrm{g}$ & 1 \\
\hline \multicolumn{2}{|l|}{ Stroke length } & $\mathrm{L}_{\mathrm{s}}$ & 60 \\
\hline \multirow{3}{*}{ Coil } & $\begin{array}{l}\text { Cross section of } \\
\text { wire }\end{array}$ & \multicolumn{2}{|c|}{$1.6 \times 3.8 \mathrm{~mm}^{2}$} \\
\hline & $\begin{array}{l}\text { Number of } \\
\text { windings }\end{array}$ & \multicolumn{2}{|c|}{43} \\
\hline & Current density & \multicolumn{2}{|c|}{$3.5 \mathrm{~A} / \mathrm{mm}^{2}$} \\
\hline
\end{tabular}

\section{Simulation results using PSPICE}

The full-wave bridge rectifier with a capacitor filter has been studied on a simulation model. Figure 9 shows the three phase bridge rectifier with resistive load. The three phase output voltages are fed into the three phase bridge rectifier for rectification purpose. The load resistance can be calculated by using equation (9) and the rated load resistance value is $9 \Omega$.

Evidently, this output waveform has slight variations due to the ripple of the capacitor voltage, which cannot be completely eliminated. From this simulation, the average dc output voltage is found to be around $200 \mathrm{~V}$. 
Table 3. The total weight

\begin{tabular}{|c|c|c|c|c|}
\hline Items & Material & $\begin{array}{c}\text { Volume } \\
\left(\mathrm{m}^{3}\right)\end{array}$ & $\begin{array}{l}\text { Density } \\
\left(\mathrm{kg} / \mathrm{m}^{3}\right)\end{array}$ & $\begin{array}{c}\text { Weight } \\
\text { (kg) }\end{array}$ \\
\hline \multirow{2}{*}{ stator 1} & cupper & 0.00042 & 8890 & 3.7338 \\
\hline & steel & 0.0003612 & 7700 & 2.78124 \\
\hline \multirow{2}{*}{ stator 2} & cupper & 0.00042 & 8890 & 3.7338 \\
\hline & steel & 0.0003612 & 7700 & 2.78124 \\
\hline \multirow{3}{*}{ translator } & steel & 0.000656 & 7700 & 5.0512 \\
\hline & aluminum & 0.00055 & 2700 & 1.485 \\
\hline & $\mathrm{PM}$ & 0.00035 & 7400 & 2.59 \\
\hline Total & & & & 22.15628 \\
\hline
\end{tabular}

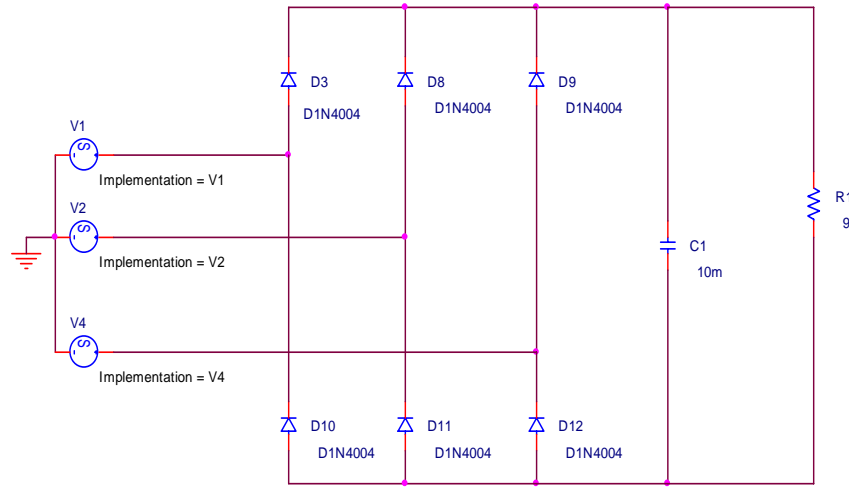

Figure 9. A three phase bridge rectifier with resistive load

Figure 10 illustrates the capacitor voltage waveform, which are connected across the load resistance. This capacitor should be as large as possible to decrease the ripple. The capacitor with $10 \mathrm{mF}$ is used in the simulation, so the voltage is nearly dc. This capacitor is nearly equal to the calculated capacitor value which is $8.41 \mathrm{mF}$. The $\mathrm{C}$ filter is design so that the ripple factor of the output voltage is less than $5 \%$. The capacitor charges almost instantaneously to the supply voltage.

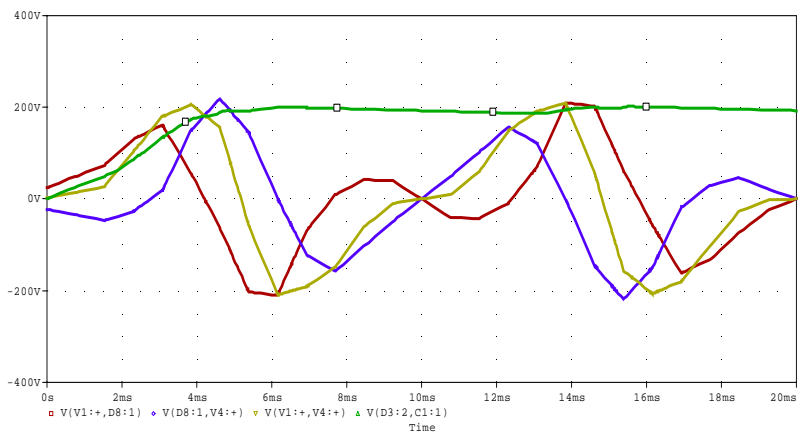

Figure 10. The dc capacitor voltage for resistive load

Figure 11 shows the rated load current waveform for pure resistive load. The voltage and current are rectified to allow the current flow to charge the battery. The value of current and voltage are around $22 \mathrm{~A}$ and $200 \mathrm{~V}$ respectively, hence it's possible that this rectifier is suitable for medium loads around $4.5 \mathrm{~kW}$.

\section{CONCLUSIONS}

The three phase linear generator model of the system has been presented. A Lua scripting program has been developed to integrate the preprocessor and post processor program of the FEMM 2D-software. The effect of slotting and fringing has also been taken into account. The permanent magnet length, air gap length and magnet depth designing factor has been performed in the three phase linear generator optimization. The performance of an $\mathrm{AC}$ to DC rectifier that uses full-wave bridge circuit also has been presented. The ripple factor of the capacitor voltage is design less than $5 \%$ as it gives a smooth output dc voltage. Finally, the three phase linear generator and an AC to DC rectification have been successfully simulated and it gives satisfactory results.

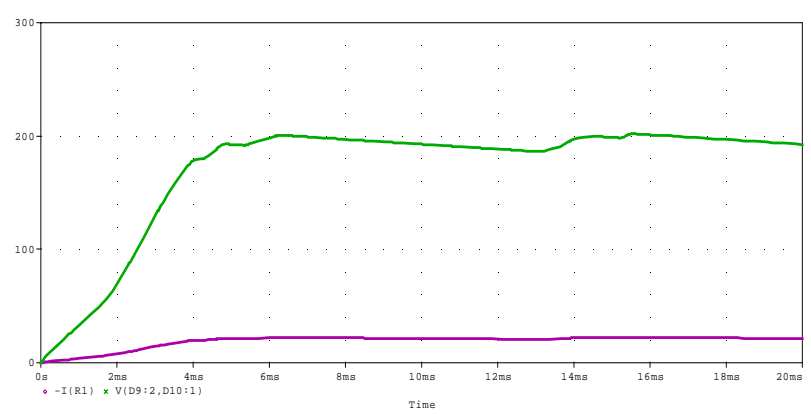

Figure 11. The rated load current waveform for pure resistive load

\section{REFERENCES}

[1] G.W. McLean, "Review of recent progress in linear motors", IEE Proceedings, Vol. 135, Pt. B, No. 6, November 1988.

[2] I. Boldea and S..A Nasar, "Linear Electric Actuators and Generators", IEEE Transactions on Energy Conversion, vol 14, pg 77 - 82, 1999.

[3] I.Boldea and S.A. Nasar, "Permanent Magnet Linear Alternators Part II: Design Guideline", IEEE Transactions on Aerospace Electronic System, Vol AES-23, No 1, pg 79 - 82, 1987.

[4] Oskar Danielsson, Karin Thorburn, Mikael Eriksson and Mats Leijon, "Permanent magnet fixation concepts for linear generator", Division of Electricity and lightning Research, department of Engineering Sciences Uppsala University.

[5] Cawthorne et al: "Development of Linear Alternator - Engine for Hybrid Electric Vehicle Applications". IEEE Translations on vehicular Technology, vol 48, No 6, November 1999.

[6] Arof, H, Wijino, Nor KM, "Linear Generator: Design and Simulation". Power Engineering Conference 2003. PECON 2003, Proceedings. National 15 - 16 Dec 2003 Page(s) : $306-311$.

[7] J.Wang, W. Jewell and D.Huwe, "A general framework for the analysis and design of tubular linear permanent magnet machines". IEEE Trans. Magn., vol. 35, No 3, pp-1986-2000, 1999.

[8] R.E Clark, D.S. Smith, P.H. Mellon, and D. Howe, "Design optimization of moving-magnet actuators for reciprocating electro mechanical systems", IEEE Trans. Magn. vol.31. pp. 3746-48, Nov 1995.

[9] Y. He, Z,Q. Zhu, and D. Howe, "A PWM controlled linear servo system for friction wielding", in Proc. $2^{\text {nd }}$ Int Conf. Elect. Mach. (CICEM'95), Hang zhoo, China, 1995, vol. A2.3, pp. $51-55$.

[10] Z. O. Zhu, Z.P. Xa, D. Howe, and P.H. Mellor", Reduction of Cogging force in slotless linear permanent magnet motors", Proc. Inst. Elect. Eng. vol. B-144, no. 4, pp. 227 - 282, 1997.

[11] Q. Gu and H. Gao, "Effect of Slotting in PM electrical machines", Elect. Mach. Power Syst., vol 10, no . 2, pp 273-284, 1985.

[12] Nor K.M, Arof, H, Wijono, "Design of a $5 \mathrm{~kW}$ tubular permanent magnet linear generator", Universities Power Engineering Conference, 2004, UPEC 2004, $39^{\text {th }}$ International volume 2, 6-8 sept 2004, Page(s): 528-532.

[13] Wang,J, Howe, D; Jewell, G.W, "Power Electronics, Machines and Drives", 2002. International Conferences on (Conf. Publ. No. 487) 47 June 2002, Page(s) 303-308.

[14] Wing nall, A.N., Gilbert, A.H., and Yang, S.J., "Calculation of force on magnetized ferrous cores using Maxwell stress method". IEEE Trans. Magn., 24(1), pp. 459 - 462, 1988.

[15] A. Benhama, A.C. Williamson, and A.J. Reece, "Force and torque computation from 2-D and 3-D finite element field solutions". IEE Proc. Electr. Power Appl., vol 146, No. 1, 1999.

[16] A.Zyl and C. Landy, "Reduction of cogging forces in a tubular linear synchronous motor by optimizing the secondary design", IEEE Africon 2002.

[17] A.Eid, H. Arof, K.M.Nor, "Tubular Permanent Magnet Linear Generator: Performance and Force Calculations", TECHPOS Malaysia, 2003. 
[18] ANSYS Release 7.1, Documentation, 2003.

[19] S.J. Salon, "Finite element analysis of electrical machine", klower Academic Publishers, USA, 1995.

[20] M. Andriollo, G.Martinelli, A.Morini and A.Tortella, "Optimizatio of the on-board Linear Generator in EMS-MAGLEV 'Trains' in IEEE Transactions on magnetics, vol 33, No 5, September 1997.

[21] H.S. Athab, S.M. Bashi, N.Mariun, and S.B. Mohd Noor, "ThreePhase Diode Rectifier with a low Input Current Distortion and Regulated Output Voltage", National Power and Energy Conference (PECon) 2003 Proceeding, Bangi, Malaysia.

[22] N.A Rahim, A. Mahrous, W.P Hew, and K.M. Nor, "Modelling and Simulation of the Linear Generator PWM Multi-Level Converter", National Power and Energy Conference (PECon) 2003 Proceedings, Bangi, Malaysia.

[23] Muhammad H.Rashid, "Power Electronics Circuits, Devices and Application", 2004. 


\title{
Extraction Methodology and Biogas Quality From Palm Oil Mill Effluent
}

\author{
F. Awang, J. Ahmad, A. Saifizul and M. Z. Zainon
}

\begin{abstract}
The main purpose of this research is to study biogas extraction methodology and gas quality from POME. This research was focus on the biogas quality based on methane emission from anaerobic digester. The POME sample properties were determined through a series of experiments in laboratories such as BOD and COD tests. On the other hand, an anaerobic digester tank model with gas trapping system had been constructed to extract the biogas from POME taken from palm oil mill. Experiments conducted are differs in the parameters used (temperature, HRT). The extracted biogas is then analysed to determine the elemental content using gas chromatography and mass spectrometry (GCMS). The overall result such as methane value shows a massive deviation from the existing data value, which is quite lower compare to the existing data. This is not compatible to the general composition of common biogas. This probably occurs due to the some reason. In conclusion, the biogas extraction methodology had been studied and the gas quality produced from POME had been analyzed. However, there is still need for further research and development to improve the existing stage of biogas development to make it more efficient and economical.
\end{abstract}

Keywords: palm oil mill effluent, anaerobic digester, biogas

\section{INTRODUCTION}

Palm oil mill effluent (POME) is one of the waste come out from palm oil milling process. POME is the largest waste generated, as a semi solid and slurry substance. For every tone of oil palm fresh fruit bunch process, it is estimated about $0.5-0.75$ tones of POME will be discharged from the mill. In year 2004, more than 40 million tones of POME was generated from 372 mills in Malaysia [1], due to its highly polluting properties which is high biochemical oxygen demand (BOD) and chemical oxygen demand (COD), is required by law to be treated to acceptable levels before it can be discharged into watercourses or onto land [2]. BOD and COD level must be reduced to a safe range to

Awang. $F$ is a MEngSc candidate at Department of Mechanical Engineering, University of Malaya, 50603 Kuala Lumpur, Malaysia (corresponding author phone: 603-7967 5208; e-mail: fadhilah_awang@yahoo.com).

Ahmad. J, is a MEngSc candidate at Department of Mechanical Engineering, University of Malaya, 50603 Kuala Lumpur, Malaysia (email: houri_julia82@yahoo.com).

Saifizul A, is a Lecturer at Department of Mechanical Engineering, University of Malaya, 50603 Kuala Lumpur, Malaysia (e-mail: saifizul@um.edu.my).

Zainon. M.Z is a Lecturer at Department of Mechanical Engineering, University of Malaya, 50603 Kuala Lumpur, Malaysia (e-mail: zzainon@um.edu.my). prevent water pollution. BOD (biochemical oxygen demand) is the measurement of the concentration of biodegradable organic matter present in a sample of water . It can be used to infer the general quality of the water and its degree of pollution by biodegradable organic matter [3]. The average BOD value of POME is $25000 \mathrm{mg} / \mathrm{L}$ [4]. In environmental chemistry, the chemical oxygen demands (COD) measure the amount of organic compounds in water. Most applications of COD determine the amount of organic pollutants found in water, making COD a useful measurement of water quality [3]. It is expressed in milligrams per liter $(\mathrm{mg} / \mathrm{L})$, which indicates the mass of oxygen consumed per liter of solution. The average COD value of POME is $50000 \mathrm{mg} / \mathrm{L} \mathrm{[4].}$

Basically there are two ways of treating the POME; anaerobic and aerobic digestion. Anaerobic digestion is the process of decomposition of organic matter without the presence of oxygen. Aerobic digestion, on the hand is the process of decomposition of organic matter with the presence of oxygen. In view of the high organic strength of POME, it has been recognized that anaerobic treatment is the best option. It is a versatile biological treatment technology yielding methane as useful bio energy. However more than $85 \%$ of the palm oil mills use solely ponding systems (aerobic treatment) due to their low capital and operating costs since most mills are situated in the plantations, and this situation has more or less been maintained through the years. This is because the industry generally perceives that the installation of waste treatment systems is only intended to satisfy legal effluent discharge requirements. Only a few mills have reported the use and operation of closed-tank anaerobic bioreactors equipped with biogas recovery systems [2].

Two kinds of bacteria are needed for anaerobic digesters to function properly. Fermenting bacteria feed off the organic materials and release organic acids. These acid byproducts then foster the growth of methanogenic (methane producing) bacteria. This second type of bacteria also feeds on the organic matter, and emits methane as a waste [5]. Two commonly bacteria that associated in anaerobic digestion process are mesophile and thermophile, both which operate at different temperatures. Only by working together can these bacteria produce the complete digestion of the organics into biogas. In anaerobic digestion process, there are two conventional operational temperature levels to ensure optimum biological activity of the microorganisms; Mesophilic which takes place optimally around $37^{\circ}-41^{\circ} \mathrm{C}$ or at ambient temperatures between $20^{\circ}-45^{\circ} \mathrm{C}$ with mesophile 
bacteria and thermophilic which takes place optimally around $50^{\circ}-52^{\circ} \mathrm{C}$ at elevated temperatures up to $70^{\circ} \mathrm{C}$ with thermophile bacteria [6].

Anaerobic digestion is the decomposition of complex molecules into simpler substances by micro-organisms in the absence of oxygen. To put this in simpler terms, organic waste is enclosed in an oxygen-free environment (often a man-made digester consisting of a container for the waste and a gas holder for the biogas), allowing naturally occurring bacteria to flourish. These bacteria eat the waste and in doing so give off a methane rich gas. The biochemistry and microbiology of anaerobic digestion are extremely complex and not completely understood. Four stages of anaerobic digestion have been recognized; hydrolysis, acidgenesis, acetogenesis and methanogenesis. Anaerobic digestion, like most biological process, is strongly affected by environmental factors. The following are the most important; temperature, $\mathrm{pH}$ or alkalinity, toxicity, growth factors, and moisture content that must be appropriate to both sets of bacteria to ensure the microbial activity is optimum [7].

The Hydraulic retention time (HRT) is a measure of the average length of time that a certain volume of compound remains in a constructed reactor before it is discharged. The retention time in a digester varies with the amount of feed material, type of material and the temperature. In the case of mesophilic digestion, retention time may be between 15 and 30 days. In the thermophilic phase the process can be faster, requiring only about two weeks to be completed. However, thermophilic digestion is more expensive, requires more energy and is less stable than the mesophilic process. Therefore, the mesophilic process is still widely in use. Alkalinity measure the ability of a solution to neutralize acids to the equivalence points of carbonate or bicarbonate. Alkalinity is a very important parameter in anaerobic digestion process to control the level of acidity in the digester for optimum decomposition of the POME by the bacteria. [6].

Biogas mainly comprising about $65 \%$ methane, $\mathrm{CH}_{4}$ and $35 \%$ carbon dioxide, $\mathrm{CO}_{2}$ [2]. It can be extracted under anaerobic conditions besides two others by-product; stable organic material comprised largely of lignin and chitin and liquid (methanogenic digestate) that is rich in nutrients [4]. Production of biogas by anaerobic digestion provides a convenient way of turning it into renewable energy especially to generate electricity, besides decreasing the amount of POME to be disposed of. The use of biogas is encouraged because it does not increase the amount of $\mathrm{CO}_{2}$ in the atmosphere, since the gas is not released directly into the atmosphere and the carbon dioxide comes from an organic source with a short carbon cycle, biogas does not contribute to increasing atmospheric carbon dioxide concentrations. Thus, it is considered to be an environmentally friendly energy source. The methane in the biogas is also burns relatively cleanly compared to coal [7, $8]$.

The main purpose of the research is to study the biogas extraction methodology and gas quality of palm oil mill effluent (POME) using anaerobic digestion process according to the standard procedure of a palm oil mill. This project could be considered as a re-study case from the previous research on the biogas.

\section{EXPERIMENTAL}

Biogas extractions were performed in a small scale anaerobic digester (50 liters in volume) design for this purpose. It comprised of the (i) tank, (ii) inlet and outlet for POME and (iii) a gas trapping system. The basic idea of designing the tank is taken from the workings and operating of a typical anaerobic digester. There are two types of POME used in this research; digested/treated POME and raw POME. Digested POME, black in color, is the effluent that already being treated in a digester and is only waiting to be disposed. Raw POME which is brownish is the fresh effluent that is discharged directly after oil extraction process in the mill. Even though the digested POME is already treated, it still harbors the bacteria inside which can still be stimulated. The role of raw POME, on the other hand, is to be the 'food' for these bacteria in the digested POME. Thus, the bacteria are stimulated by adding raw POME in the digested one. The ratio of the mixture between the raw and digested POME is 10:90. This is to ensure that the bacteria are not overfed. Once the bacteria are active, they will start to breed and create a microbial population in the mixture. Then, the bacteria will start to digest the raw POME and produce biogas. Three experiments were carried out where each of them has different parameter/condition as shown in table 1.

Table 1. Conditions for 3 experiments

\begin{tabular}{ccc}
\hline $\begin{array}{c}\text { Experiment } \\
\text { No. }\end{array}$ & $\begin{array}{c}\text { Temperature }\left({ }^{\circ} \mathrm{C}\right. \\
\text { HRT(days }\end{array}$ & \begin{tabular}{c} 
HR \\
\hline 1
\end{tabular} \\
2 & 50 & 10 \\
3 & 55 & 10 \\
\hline
\end{tabular}

The parameters that are taken into account are the temperature and HRT of the POME inside the anaerobic tank. Temperature of the POME plays an important role in ensuring high methane yielding. An earlier study revealed that methane production was significantly enhanced in the thermophilic compared with mesophilic digestion of POME [10]. Thus, this fact serves as a basis to the selection of temperature in this experiment. Besides that, the HRT will also determine the quality of the biogas produced from the POME. The first two experiments study on the effect of temperature, the second and third experiments study on the effect of HRT on the biogas produced. Digested POME and raw POME temperature must be maintain at desire temperature and it $\mathrm{pH}$ must greater than 7 until the HTR is achieved. If the $\mathrm{pH}$ dropped below neutral $(<7)$ or acidity range natrium bicarbonate (alkali) is inserted into the sample. It is done until the $\mathrm{pH}$ is above acidity range. It is important to keep the working bacteria under the alkali range because acidogens could kill the methanogens if the acidity is too high. Depending on the HRT, some amount of the mixture will be discharged and be replaced by the same amount of raw POME daily. For safety purpose, the environment surround was ensured to be free from any flame or flammable source. Produced biogas has been trap in tedlar bag and composition of that generated biogas then had been tested using gas chromatography and mass spectrometry (GCMS). The BOD and COD test on raw and digested POME are also conducted in this study. These 
analyses are done based on standard method for the examination of water and wastewater [5].

\section{RESUlTS AND DisCUSSION}

An anaerobic tank does not design exactly like the real digester; it is quite economical compare to the real digester design. Most of the materials used are junk, unused items and leftovers from the labs or anywhere around. Only the working principles are fully taken into account while the operating mechanisms might be different from the ones in the real anaerobic digester. The working principles of an anaerobic digester are:

i. POME input and output

ii. POME mixing/agitating/circulating

iii. Temperature probing

iv. $\mathrm{pH}$ probing

v. Gas capturing/trapping

Figure 1 shows the layout of the tank design. The inlet of the POME is located at the upper part of the tank for easy filling while the outlet of the POME is situated at the bottom part of the tank for discharge. The inlet and outlet of the tank is in the form of pipelines with valves to control the POME going in and out. A water pump is installed inside the tank which acts as a mixing mechanism for the POME inside the tank. It is exactly the same mechanism used for the water circulation in an aquarium. By using this pump, the POME is mixed and agitated by re-circulating the effluent from the bottom to the top of the tank. This is important because the POME being put through the tank is made up of a mixture of solids, suspended solids and liquids. If this mixture is not mixed/agitated periodically, the solids will settle out and cause a scum build-up at the bottom of the tank. In time this scum build-up will cause reduced performance in the tank and it will have to be repaired and cleaned out [6]. Temperature probe measure the temperature of the POME inside. It is important to maintain the temperature within the optimum working range of the bacteria to ensure high methane yielding. Thus, to maintain temperature the tank was insulated. There is also a gas trapping system connected to the tank to capture and store the biogas generated from the POME. Tedlar bags which connected to the gas outlet by a gas tube are used in the trapping system as storage for the biogas. Importantly, as the main objective, 'biogas extraction methodology' includes the generation/production of the biogas from the POME and also the trapping of the biogas in the tedlar bag. However, there is no specific experimental method to determine whether the biogas is generated or not. Through by natural phenomena, the biogas can be predicted to have been released by observing the bubbling of the POME mixture inside the tank. The bubbling occurs when the bacteria is breaking down the organic molecules which releases methane, carbon dioxide and water as by-products. The same phenomenon also occurs to boiled water where bubbling starts to initiates when the boiling point is reached and water vapor (gas) is released. Thus, using this phenomenon as the basis, the biogas production inside the tank is predicted as shown in figure 2(a) and 2(b).

From the table 2, the rate of biogas released was different from one to another. The three experiments use different number of days to collect a full bag of gas each. From the first two experiments, the results show that with the same
HRT, a higher operating temperature will produce more amount of gas. On the other hand, the next two experiments show that with the same operating temperature, a higher HRT will produce more amount of gas.

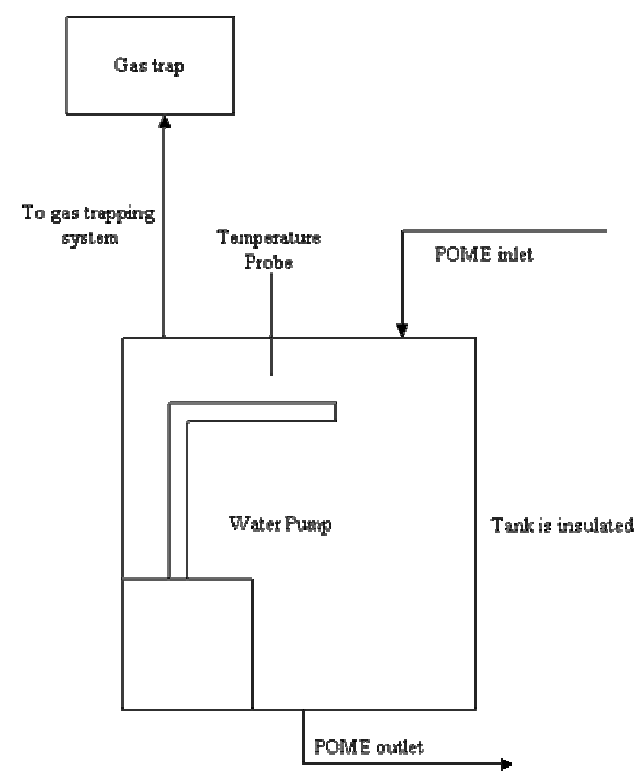

Figure 1. Anaerobic tank design layout

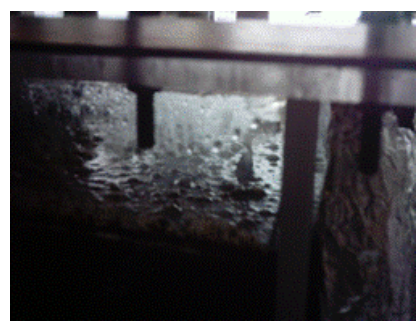

(a)

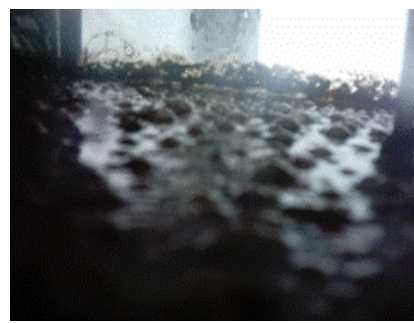

(b)
Figure 2. Biogas generated/produced from observation of POME bubbling

Table 2. Number of days needed to fill a full bag of a 10 liters Tedlar bag for each experiment

\begin{tabular}{cccc}
\hline Experiment No. & $\begin{array}{c}\text { Temperature } \\
\left({ }^{\circ} \mathrm{C}\right)\end{array}$ & $\begin{array}{c}\text { HRT } \\
(\text { days })\end{array}$ & $\begin{array}{c}\text { No. of } \\
\text { days }\end{array}$ \\
\hline 1 & 50 & 10 & 3 \\
2 & 55 & 10 & 2 \\
3 & 55 & 5 & 4 \\
\hline
\end{tabular}

From the BOD and COD test, table 3 shows that raw/fresh POME that comes directly from palm oil mill processing contain high value of BOD and COD compared to digested POME. Removal efficiency of BOD which is $94.17 \%$ and $85.19 \%$ for COD is considered very high. This means that anaerobic digestion is very efficient way in treating the raw POME by creating an active microbial population and thus reduces POME to a less polluted waste. Treated POME has a higher $\mathrm{pH}$ than the raw POME, 7.85 refer to table 3. In other words, the treated POME is less acidic than the raw POME. It shows that microbial activities occur via the increasing of alkalinity of treated POME. During digestion process the acid forming bacteria become active first, reducing the $\mathrm{pH}$ to below acid range (increasing the acid content). The methanogens then start using these acids, increasing the $\mathrm{pH}$ back to neutral. Alkalinity is a very important parameter in anaerobic digestion process for 
optimum decomposition of the POME by the bacteria $(\mathrm{pH}>7)$.

Table 3. BOD, COD and $\mathrm{pH}$ test results

\begin{tabular}{llll}
\hline & BOD $(\mathrm{mg} / \mathrm{L})$ & $\mathrm{COD}(\mathrm{mg} / \mathrm{L})$ & $\mathrm{pH}$ \\
\hline Raw POME & 6000.1 & 20768.4 & 4.46 \\
\hline Digested POME & 350.1 & 3076.8 & 7.85 \\
\hline
\end{tabular}

There are a total of 13 types of elements present in the biogas. From the table 4 , it shows that the ambient air (combination of oxygen and nitrogen) consists almost $60 \%$ of the gas. While the methane and carbon dioxide consists of $7.651 \%$ and $25.17 \%$ of the total amount respectively. This is not compatible to the general composition of common biogas. The main reason is that there is air flow into the tank while POME flown and out from the tank during the experiment. With air occupying most of the volume inside the tedlar bag, the concentration of the biogas is not high which results in low methane content. Another reason for low methane yield is probably due to low quality of POME used (low BOD and COD compare to average value in existing data [6] ) since the BOD and COD test were not carried out soon after the sample collection from the mill where it is kept under storage for quite sometime. Thus under storage, the POME is exposed to the air which might be could promote digestion (pre-digestion) process to occur under aerobic condition before testing. Thus, lower BOD and COD values are obtained. With low BOD and COD level of POME, there is also lower digestion process that can be done by the bacteria to release the biogas.

Table 4. Percentage of each element in the extracted biogas

\begin{tabular}{cc}
\hline Element & Percentage \\
\hline Oxygen, $\mathrm{O}_{2}$ & 11.61 \\
Nitrogen, $\mathrm{N}_{2}$ & 46.08 \\
Methane, $\mathrm{CH}_{4}$ & 7.65 \\
Iso-butane, $\mathrm{C}_{4} \mathrm{H}_{10}$ & 0.14 \\
N-butane, $\mathrm{C}_{4} \mathrm{H}_{10}$ & 0.15 \\
Iso-pentene, $\mathrm{C}_{5} \mathrm{H}_{12}$ & 0.11 \\
N-pentene, $\mathrm{C}_{5} \mathrm{H}_{12}$ & 0.11 \\
Benzene, $\mathrm{C}_{6} \mathrm{H}_{6}$ & 0.04 \\
N-hexane, $\mathrm{C}_{6} \mathrm{H}_{14}$ & 0.004206 \\
N-heptene, $\mathrm{C}_{7} \mathrm{H}_{16}$ & 0.006845 \\
Carbon dioxide, $\mathrm{CO}_{2}$ & 25.18 \\
$\mathrm{C}_{2}$ & 7.72 \\
$\mathrm{C}_{3}$ & 1.24 \\
\hline
\end{tabular}

The water pump is also constantly clogged which resulted in uncirculated POME inside the tank. This is also a factor in the reduced quality of biogas. Temperature and $\mathrm{pH}$ probing is also a flaw. The temperature and $\mathrm{pH}$ inside the tank are not always maintained in the optimum range which also explains why low methane yield. If $\mathrm{pH}$ values lower than 7 (acidic), the rate of methane production is distinctly lower because acidogens could kill the methanogens particularly if the acidity is too high.

\section{CONCLUSION}

The biogas extraction methodology had been studied and the gas quality produced from POME had been analyzed. A 50 liters anaerobic digester tank with gas trapping system had been constructed. The results show that with the same
HRT, a higher operating temperature will produce more amounts of gas and with the same operating temperature; a higher HRT will produce more amount of gas. The extracted biogas which consists of 13 elements had $7.651 \%$ of methane and $25.17 \%$ of $\mathrm{CO}_{2}$.

For future improvements the air inside the tank can be suck out by a vacuum pump. This will reduce the air inside and thus decrease the oxygen and nitrogen composition in the biogas extracted. Tank made other kind of suitable materials (for instance, Perspex) must be strong enough to hold the vacuum condition. A better technique of stirring can be used instead of immerse able rotary pump, since the pump is easily malfunction due to the clogged sludge. Stirring is considered a desirable practice to accelerate gas production. In some design, there is some sort of stirring arrangements. The current heating insulating system can be further improved or replaced by a more efficient insulating system, such as water jacket or electrical elemental heating inside the tank. It is important to maintain the correct temperature. Proper $\mathrm{pH}$ control to optimize the gas production must be taken into account. The types of chemicals which can accelerate the fermentation studied and can also be considered. In the aspect of management, more study can be done on the feeding techniques or device that used to monitor the density of the input materials. There is still need for further research and development to improve the existing stage of biogas development for production efficient result and economical.

\section{ACKNOWLEDGEMENT}

The authors would like to express the gratitude to the Ministry of Science, Technology and Innovation for the financial support from Sciencefund grant: 03-01-03SF0146. Appreciation are also extended to the MPOB in providing equipments for sample tests and the former Manager, Jabor Palm Oil Mill in providing samples and valuable discussion.

\section{REFERENCES}

[1] S. Yacob, Y. Shirai, M. A. Hasan, M. Wakisaka \& S. Subash. (2005). Baseline study of methane emission from anaerobic ponds of palm oil mill effluent treatment. Journal of Science of the Total Environment for Science Direct. 366, 187- 196.

[2] Ma, A. N. (2002). Carbon Credit from Palm: Biomass, Biogas and Biodiesel. Palm Oil Engineering Bulletin, Issue No. 65: 24 - 26.

[3] URL-http://www.environmentalleverage.com/COD\%

[4] S. Yacob, Y. Shirai, M. A. Hasan, M. Wakisaka \& S. Subash. (2006) Start-up operation of semi-commercial closed anaerobic digester for palm oil mill effluent treatment. Journal of Process Biochemistry for ScienceDirect. 4, 962-964.

[5] UR1-

http://www.michigan.gov/documents/anaerobic_digester_FAQs_2005 _137431_7.pdf, 27/03/2007.

[6] URL-http://en.wikipedia.org/wiki/Anaerobic digestion; 04/10/2006

[7] Biogas Production, Energy Victoria, January 1995.

[8] URL-http://en.wikipedia.org/wiki/Anaerobic_digestion; 04/10/2006

[9] URL-

http://www.environmentalleverage.com/COD\%20vs\%20BOD.htm, $30 / 01 / 2007$.

[10] URL-

http://www.cogen3.net/doc/countryinfo/malaysia/TechnicalEconomic AnalysisCHPPalmEffluent_BG.pdf, 21/03/2007. 


\title{
Potentiality of Combustion Enhancement in Co- Firing Palm Oil Biomass \& Fossil Additive
}

\author{
J. Ahmad, F. Awang, A. Saifizul, M. Z. Zainon
}

\begin{abstract}
This report presents the energy conversion from palm oil biomass as an alternative energy source and potentialities of combustion enhancement by coal additive. They were carried out via detail evaluation on the properties and energy data from palm oil biomass where it lead to combustion properties of three types of palm oil mill solid wastes which are the empty fruit bunch (EFB), shell and fiber. These biomasses were studied in various conditions, including the measurement on their calorific values, moisture contents, elemental contents, ashes and volatiles matter contents. Analyses have been divided into various parts; based on the biomass and quantity of blending biomass and coal. Economical Comparison has also been studied. The results revealed that these biomasses are almost half of good quality of coal, and the blended biomass and coal at the current rate shows the improvement of HHV. On top of that, these biomasses and coupled with the right composition of fossil fuels can produce good combustion characteristics as well as positive economical impact to palm oil industry. It is found that, shell have much lower moisture content compared to EFB and fiber. Element wise, these biomasses have lower carbon content compared to coal hence reduce the production of Carbon Dioxide $\left(\mathrm{CO}_{2}\right)$, a gas which is infamous for its effect on global warming. It also discovered that, by adding 30 to 50 per cent of coal into moisture-free biomass, it can produce energy with a high profit margin hourly.
\end{abstract}

Keywords: palm oil biomass, alternative energy, energy conversion, co-firing, combustion

\section{INTRODUCTION}

With the depletion of fossil fuel resources, biomass has been touted as the replacement for it. The term biomass refers to the solar energy that is stored in organic matter such as tress, agricultural crops and other living plant material. However, in general biomass not only considered as plant matter that is grown for use as biofuel, but also includes plant or animal matter used for production of

J. Ahmad is an MEngSc candidate at Department of Mechanical Engineering, University of Malaya, 50603 Kuala Lumpur, Malaysia (phone: 03-79675208; fax: 03-79675317; email: houri_julia82@yahoo.com).

F. Awang is an MEngSc candidate at Department of Mechanical Engineering, University of Malaya, 50603 Kuala Lumpur, Malaysia (email: fadhilah_awang@yahoo.com).

A. Saifizul is a Lecturer, Department of Mechanical Engineering, University of Malaya, 50603 Kuala Lumpur, Malaysia (phone: 03-7967 4495; fax: 03-79675317; email: saifizul@um.edu.my).

M.Z. Zainon is a Lecturer, Department of Mechanical Engineering, University of Malaya, 50603 Kuala Lumpur, Malaysia (phone: 03-7967 6876; fax: 03-79675317; email: zzainon@yahoo.com). fibers, chemicals or heat as well as biodegradable wastes that can be burnt as fuel [1].

A major advantage of using biomass as a source of fuel compared to fossil fuel is its renewability, Malaysia, with palm oil mills running in a cultivation area of 4.1 million hectares in 2006, certainly has a lot of potential for biomass energy utilization. Realizing this, the Malaysian government had announced renewable energy as the fifth fuel in the new Five Fuel Strategy in the energy supply mix in its Eight Malaysian Plan. It was targeted that Renewable energy will contribute $5 \%$ of the country's total electricity demand by the year 2005, that is by the end of the Eight Malaysia Plan period [11].

Currently, the Ministry of Energy, Water and Communication has identified palm oil wastes as the biggest resource that can be easily developed, as Malaysia, being the largest producer of palm oil has an abundance supply of palm oil biomass. Palm oil biomass usually consist shell, fiber, empty fruit bunch (EFB) and palm oil mill effluent (POME). A large fraction of fiber and shell are used as fuel in the palm oil processing mill itself. However, much is wasted by pile burning in the open air with attendant air pollution, dumped in areas adjacent to the mill, or utilized as manure in the palm oil plantation [6].

Owing to environmental and economic consideration coupled with the depletion of fossil fuel, there has been an increased awareness of the use of waste from palm oil. Thus realizing that palm oil could be a world changing opportunity for Malaysia, related government bodies have taken effort to conduct several research and development. Through this effort it was found that palm oil biomass offers a more economical energy source for generating electric power and process steam compared to fossil fuels. This gives strong indication that palm oil biomass has a huge potential as renewable energy source.

Many $R \& D$ efforts are currently being focused to add value and converting this biomass into useful products have been suggested but very little commercial success has been achieved. This problem could be eliminated by blending biomass with coal and firing it through combustion process to produce steam and generate electricity. The blend technology is particularly advantageous for fuel with highly variable properties, and high moisture, and the possibility is high for rapid technology transfer. Other advantages of cofiring coal with biomass are: i) reduction of fossil fuel based $\mathrm{CO}_{2}$, ii) reduction in NOx, iii) reduction in fuel costs since biomass is cheaper than coal, iv) minimization of waste and reduction of soil, water, and air pollution depending on the 
biomass fuel blended with coal and v) potential to use biomass as reburn fuel [7].

In order to demonstrate the performance of boiler burner with co-firing, fuel properties and combustion, and fouling performance data need to be generated. Hence, an interdisciplinary research program was undertaken to obtain the combustor performance when firing coal:palm oil biomass. This report present a database for physical and chemical properties of palm oil biomass and coal:palm oil biomass fuel. The combustion properties concentrated on determination of moisture content, calorific value $(\mathrm{CV})$, volatile matter content (VM), ash content (AC) and elemental content (EC) [8]. The Kumpulan Guthrie Berhad palm oil mill, located in Jabor, Pahang, has been selected for this research. This mill had started the operation since last 30 years. This mill is able to process about 25 tonnes of fresh fruit bunch (FFB) per hour.

\section{Methodology}

All experiments were carried out based on the standard method available. However, slight modifications have made wherever necessary.

\section{A. Protocol for Preparation and Sampling}

The protocol was as follows:

a. Palm oil biomass sources. The materials have been selected are EFB, shell and fiber. They were divided to various portion bases on its type cut to prepare moisture content test. Then, they were grounded and sieved (250 micron) to get powder forms for other test. Before that, it must be completely dried to achieve $0 \%$ moisture.

b. Fossil source. The additive selected is coal. It is ready in powder form but, must be ensured to be moisture free for a complete combustion.

c. Sampling. Finer samples were analyzed in two portion; palm oil biomass and blended coal:palm oil biomass. The mixture are as follow:

[EFB, shelland fiber + coal] with mixing ratio (by weight) of
i) $9: 1$
ii) $7: 3$
iii) $5: 5$

d. Sample analysis. Samples were analyzed for the following parameters: moisture, ash, higher heating value (HHV), volatile matter (VM), elemental content.

e. Laboratory procedures.

i) Moisture Content - Samples has been preheated to $103 \pm 2^{\circ} \mathrm{C}$ then weighed. The procedure was repeated until the difference weight $\leq 0.05 \mathrm{~g}$ and measured by refer (1).

$$
\% \text { Moisture Content }=\frac{\text { Mass of water removed }}{\text { Mass of original sample }}
$$

ii) Higher heating value (HHV) was done using AC-350 semi automatic bomb calorimeter at the Malaysian Palm Oil Board (MPOB).

iii) Ashes content - Samples were burnt into $750 \pm 10 \mathrm{oC}$ (1 hour) and measured by refer (2).

$$
\% \text { Ash Content }=\frac{\text { Mass residueaftercombustion }}{\text { Mass original sample }}
$$

iv) Volatile matter (VM) - Samples heated $\left(950 \pm 10^{\circ} \mathrm{C}\right)$ in the absence of air under specified condition and calculated similar as ash analysis.

v) Elemental Content - The Energy Dispersive Spectrometer (EDS) machine was used to carry out the elemental content. Samples will be dried properly to ensure the good running while used EDS machine.

Table 1. The standard method used for each analysis

\begin{tabular}{lc}
\hline Analysis & Standard method \\
\hline Calorific value & ASTM D 2015-95 \\
Ash Content & ASTM D 3174-93 \\
Volatile matter content & ASTM D 3175-93 \\
Moisture content & BS 4289: Part 3:1978 \\
Elemental content & ASTM D 6349-98 \\
\hline
\end{tabular}

\section{RESULTS AND DISCUSSION}

\section{A. Proximate and Ultimate Analyses}

The analyses in Table 2 show the result of moisture content provided by EFB, shell and fiber. According to results, the low moisture content display in shell show the potential to provide good combustion ability compared other samples.

HHV is a measure of a substance ability to produce energy during combustion of a specified amount. HHV for $\mathrm{EFB}$, shell and fiber shows are almost half of good quality of coal (Table 3). Therefore, palm oil biomass has an ability to produce energy for electricity generation. With blended coal and palm oil biomass HHV shows the increment value proportional to the addition of coal.

$\mathrm{HHV}$ also improve with samples has less ash content. It is because; ash content is insoluble compound act as heat sink in the same way as moisture, lowering combustion efficiency. High ash content makes it less desirable as fuel. Figure 1 shows that the increment of HHV with reducing of ash for EFB sample. The HHV was increased from 15957.5 $\mathrm{kJ} / \mathrm{kg}$ to $22163 \mathrm{~kJ} / \mathrm{kg}$, that was near to the value of coal (27 $486 \mathrm{~kJ} / \mathrm{kg}$ ). Co-firing palm oil biomass with coal help to eliminate the value of ash hence, rise up the combustion process.

VM is generally associated with instability. However in the case of biomass energy, volatiles refer to measure the speed for substances to evaporate. Higher volatility signals a faster evaporation. Hence, it provides a measure of the ability of how easy of biomass can be ignited. The VM is proportional to HHV (Tables 3 and 5) that gives combustibility improvement.

Table 2. Moisture content

\begin{tabular}{ccc}
\hline Sample & $\begin{array}{c}\text { Average moisture } \\
\text { content (\%) }\end{array}$ & Range (\%) \\
\hline EFB & 68 & $67.00-69.00$ \\
Fiber & 42.76 & $35.00-48.00$ \\
Shell & 9.56 & $8.60-10.50$ \\
\hline
\end{tabular}

The highest Carbon content may affect the environment through production of Carbon Dioxide $\left(\mathrm{CO}_{2}\right)$ as reference from coal properties. Although $\mathrm{CO}_{2}$ is considered as a minor gas in the atmosphere, it is very important component as it 
traps infrared radiation. EFB, shell and fiber are more environmental friendly substances as it carbon contents are the least (Table 6).

Table 3. Calorific value

\begin{tabular}{ccc}
\hline Sample & $\begin{array}{c}\text { Average calorific } \\
\text { value (kJ/kg) }\end{array}$ & Range (kJ/kg) \\
\hline EFB & 15957.5 & $15954-15961$ \\
1:9 (Coal:EFB) & 17993 & \\
3:7 (Coal:EFB) & 20149 & \\
5:5 (Coal:EFB) & 22163 & $16737-16729$ \\
LTDS & 16733 & \\
1:9 (Coal:Shell) & 17032 & \\
3:7 (Coal:Shell) & 19597 & \\
5:5 (Coal:Shell) & 21562 & \\
Fiber & 18648 & \\
1:9 (Coal:Fiber) & 18527 & \\
3:7 (Coal:Fiber) & 20720 & \\
5:5 (Coal:Fiber) & 22111 & \\
Coal & 27486 & $27000-28000$ \\
\hline
\end{tabular}

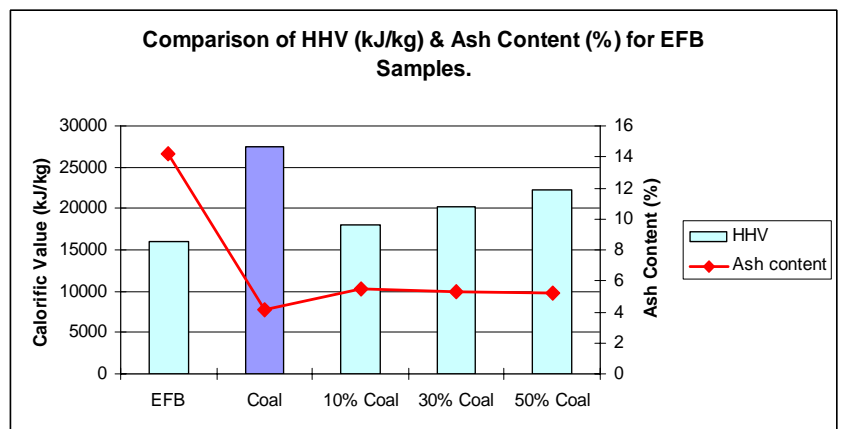

Figure 1. Comparison of HHV and ash value for EFB samples.

\section{B. Economical Analysis}

Economical factor is one of the critical factors in selecting the suitable fuel for electricity generation. Here, some aspect were discussed and calculation were carried out focusing to the profit (money value) of using palm oil biomass which is EFB, shell and fiber as fuel in combustion for electricity generation. Calculation is based on the energy requirement needed in Jabor mill.

Table 4. Ash residue

\begin{tabular}{ccc}
\hline No. & Sample & $\begin{array}{c}\text { Average ash } \\
\text { content (\%) }\end{array}$ \\
\hline 1 & $1: 9$ (Coal:EFB) & 5.46 \\
2 & $3: 7$ (Coal:EFB) & 5.33 \\
3 & $5: 5$ (Coal:EFB) & 5.2 \\
4 & $1: 9$ (Coal:Shell) & 14.8 \\
5 & $3: 7$ (Coal:Shell) & 12.5 \\
6 & $5: 5$ (Coal:Shell) & 11.1 \\
7 & $1: 9$ (Coal:Fiber) & 12.9 \\
8 & $3: 7$ (Coal:Fiber) & 11.1 \\
9 & $5: 5$ (Coal:Fiber) & 9.30 \\
10 & Coal \\
\hline \multicolumn{3}{c}{} \\
\hline
\end{tabular}

Currently, as our case study, Jabor palm oil mill had been selected. It used totally fiber and shell as their fuel to generate steam for mill production. The calculation is based on these two fuels that directly combusted without going any treatment. The shell and fiber can produce 51213780 $\mathrm{kJ} / \mathrm{hr}$ energy and it is enough to produce steam and generate the electricity for mill's operation for processing about 25 tonnes fresh fruit bunch (FFB) per hour.

Table 5. Volatile matter content

\begin{tabular}{ccc}
\hline No. & Sample & $\begin{array}{c}\text { Average VM } \\
\text { content (\%) }\end{array}$ \\
\hline 1 & EFB & 87 \\
2 & $1: 9$ (Coal:EFB) & 88.89 \\
3 & $3: 7$ (Coal:EFB) & 78.19 \\
4 & $5: 5($ Coal:EFB) & 72.82 \\
5 & LTDS & 92.2 \\
6 & $1: 9($ Coal:Shell) & 74.06 \\
7 & $3: 7$ Coal:Shell) & 62.38 \\
8 & $5: 5$ (Coal:Shell) & 72.69 \\
9 & Fiber & 89.82 \\
10 & $1: 9$ (Coal:Fiber) & 99.31 \\
11 & $3: 7$ (Coal:Fiber) & 85.12 \\
12 & $5: 5$ (Coal:Fiber) & 72.14 \\
13 & Coal & 95.35 \\
\hline
\end{tabular}

Table 6. The elemental content

\begin{tabular}{cccccc}
\hline No. & Element & $\begin{array}{c}\text { Coal } \\
(\mathbf{\%})\end{array}$ & $\begin{array}{c}\text { EFB } \\
\mathbf{( \% )}\end{array}$ & $\begin{array}{c}\text { Shell } \\
(\mathbf{\%})\end{array}$ & $\begin{array}{c}\text { Fiber } \\
(\%)\end{array}$ \\
\hline 1 & $\mathrm{C}$ & 79.81 & 57.41 & 54.46 & 59.89 \\
2 & $\mathrm{O}_{2}$ & 20.19 & 34.52 & 28.50 & 37.16 \\
3 & $\mathrm{Mg}$ & - & 0.57 & - & 0.46 \\
4 & $\mathrm{Al}$ & - & 0.47 & 0.86 & - \\
5 & $\mathrm{Si}$ & - & 2.26 & - & 0.36 \\
6 & $\mathrm{~S}$ & - & 1.10 & 14.51 & 0.63 \\
7 & $\mathrm{Cl}$ & - & 0.58 & 0.19 & 0.43 \\
8 & $\mathrm{~K}$ & - & 1.76 & 0.34 & 0.75 \\
9 & $\mathrm{Ca}$ & - & 0.75 & 0.32 & 0.31 \\
10 & $\mathrm{Fe}$ & - & 0.22 & 0.65 & - \\
11 & $\mathrm{Cu}$ & - & 0.35 & - & - \\
\hline
\end{tabular}

Table 7 shows the economical estimation of coal and cofiring palm oil biomass. $100 \%$ coal use in power plant need high production cost. With present of biomass, it helps to reduce the cost with cost-free need for biomass. As for example, HHV for 5:5 (coal:EFB) is $22163 \mathrm{~kJ} / \mathrm{kg}$ and it near to the value of coal $(27486 \mathrm{~kJ} / \mathrm{kg})$ but the cost for this co-fired of EFB is RM288.84 less/half price compared to $100 \%$ coal (RM577.67) used. In this research, it also discovered that, by adding 3:7 to 5:5 into moisture-free substances, it can produce energy which is equivalent to approximately RM 976 to RM 1581 profit margin hourly. Composition 5:5 makes the substances show the highest profit margin (Figures 2 to 4). As conclusion, co-firing gives an advantage in electricity generation for money value compared to coal alone. This means that co-firing is the most practical as well as the most efficient way to implement in the industry.

This value is useful in selecting the suitable fuel for electricity generation because the efficient fuel with the lowest operating cost is the important consideration. However, other properties should be considered too, such as the ash content and volatile matter content.

\section{CONCLUSION}

As the world is coming to a point where the shortage of fossil fuel has become an unavoidable phenomenon, renewable energy has become one of the most sought after 
energy resources globally. Biomass which refers to the solar energy stored in organic material has been seriously touted as the best replacement of fossil fuel.

In Malaysia, the most amount of biomass comes from the palm oil industry accounting for $95 \%$ of the total biomass production. Hence, Malaysia has a great potential into becoming a hub for renewable energy.

Palm oil biomass has the ability to produce energy for power generation through the almost half result of $\mathrm{HHV}$ compared to coal. By co-firing process it shows $\mathrm{HHV}$ increase proportional to the coal added. Then, an increasing this HHV is near the value of coal but still in reasonable cost for fuel.

The ash content is inverse proportional to the HHV, which is less ash content will result an increasing of HHV. Additional of coal in biomass help to reduce the high ash content of this palm oil biomass and improve the VM to reasonable percentage for good combustion achieving.

The presences of biomass in coal make it suitable for fuel with reducing the gas emission instead of fossil fuel alone.

Table 7. Economical estimation for coal and co-firing palm oil biomass

\begin{tabular}{ccccc}
\hline \multirow{6}{*}{ EFB } & & $\begin{array}{c}\text { Productio } \\
\text { n Cost } \\
\text { (RM) }\end{array}$ & $\begin{array}{c}\text { Total } \\
\text { Energy } \\
\text { Value (RM) }\end{array}$ & $\begin{array}{c}\text { Total } \\
\text { Profit } \\
\text { (RM) }\end{array}$ \\
\hline \multirow{5}{*}{ Shell } & coal & 577.67 & & \\
& $(5: 5)$ & 288.84 & 1584.43 & 1295.59 \\
& $(3: 7)$ & 173.3 & 1065.56 & 892.26 \\
& $(1: 9)$ & 57.77 & 510.09 & 452.32 \\
\hline \multirow{4}{*}{ Fiber } & $(5: 5)$ & 186.75 & 1767.82 & 1581.07 \\
& $(3: 7)$ & 112.05 & 1440.5 & 1328.45 \\
& $(1: 9)$ & 37.35 & 1013.23 & 975.88 \\
\hline \multirow{6}{*}{ Coal } & 866.25 & & \\
& $(5: 5)$ & 433.13 & 1249.51 & 816.38 \\
& $(1: 9)$ & 259.88 & 712.11 & 452.23 \\
& & 86.63 & -106.72 & -193.35 \\
\hline
\end{tabular}

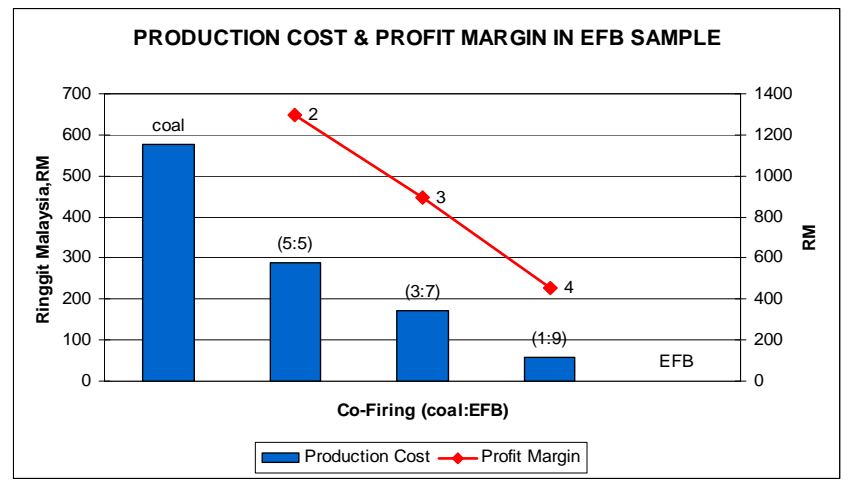

Figure 2. Cost and economy value produced from EFB sample

\section{ACKNOWLEDGMENT}

The authors would like to express their gratitude to Ministry of Sciences, Technology and Innovation (MOSTI) for the financial support under grant Sciencefund 03-01-03SF0146. Appreciations are also extended to MPOB in providing equipment for sample tests and the Jabor Palm Oil Mill former Manager, En. Shahbudin Usop in providing sample and valuable discussions.

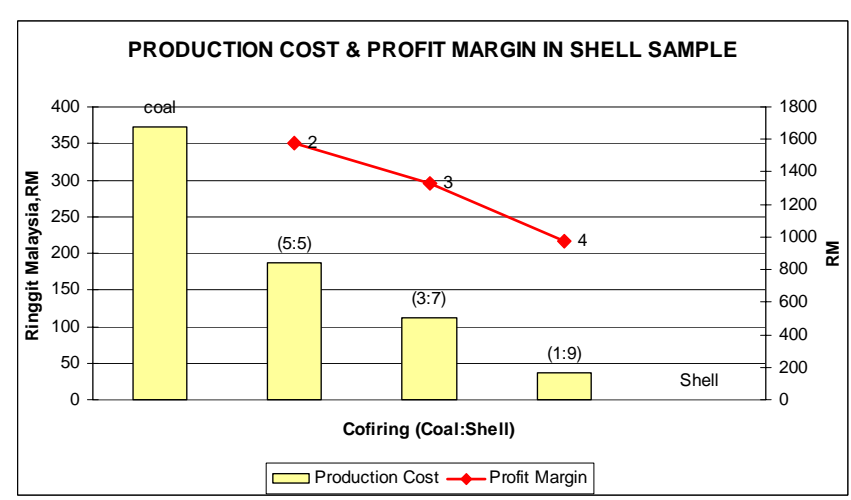

Figure 3. Cost and economy value produced from shell sample

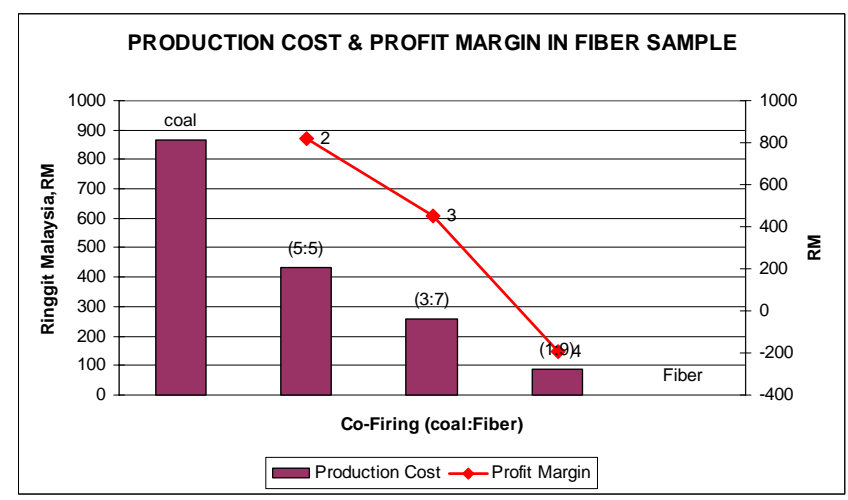

Figure 4. Cost and economy value produced from fiber sample

\section{REFERENCES}

[1] A. Demirbas, (2005). Potential application of renewable energy sources, Biomass combustion problem in boiler power system and combustion related environment issues. Journal of Progress of Energy and Combustion Science. 31.71-192.

[2] P. McKendry, (2002). Energy production from biomass (part 1): Overview of biomass. Journal of Bioresource Technology. 83. 37- 46.

[3] Mohamed Harimi, M.M.H. Megat Ahmad, S.M Sapuan \& Azni Idris. (2005). Numerical analysis of emission component from incineration of palm oil waste. Journal of Biomass and Bioenergy. 28. 339-345.

[4] M.A Ngan,. (2002). Carbon Credit from Palm: Biomass, Biogas and Biodiesel. Palm Oil Engineering Bulletin. 65. 24-26.

[5] T.M.I. Mahlia, M.Z. Abdulmuin, T.M.I. Alamsyah, \& D. Mukhlishien. (2001). An alternative energy resource from palm oil wastes industry for Malaysia and Indonesia. Journal of Energy Cconversion and Management. 42. 2109-2118.

[6] Z. Husain, Z.A. Zainal, \& M.Z. Abdullah, (2003). Analysis of biomass-residue based cogeneration system in palm oil mills. Journal of Biomass and Bioenergy. 24. 117-124.

[7] J.M. Sweeten, K. Annamalai, B. Thien, \& L.A. McDonald (2003). Co-firing of coal \& cattle feedlot biomass (FB) fuels. Part 1. Feedlots biomass (cattle manure) fuel quality \& characteristic. Journal of Fuel. 82. 1167-1182.

[8] V. Subramaniam, C.M Chin, M.A. Ngan, (2004). Energy Database of the oil palm. Palm Oil Bulletin, Kuala Lumpur, Malaysia. 70. 15-22.

[9] M.Y.H. Othman \& K. Sopian. (2005). Renewable energy is the fifth energy sector in Malaysia: Programme and Support. Journal of Renewable Energy.1-10.

[10] S. Prasertsan \& P. Prasertsan (1996). Biomass residue from palm oil mills in Thailand: An overview on quality and potential usage. Journal of Biomass and Bioenergy. 11(5). 387-395.

[11] MPOB: Economics and industry development division. Revision March 2007. Available from http://econ.mpob.gov.my/economy/ EID_Review06.thm. 


\title{
Solar Energy Technologies
}

\author{
Andhe Satyanarayana Murthy
}

\begin{abstract}
Solar radiation is the renewable energy and is abundantly available on the earth. The photovoltaic technology is used to convert this solar energy into electricity. But to make it attractive the present technology has been improved to get more conversion efficiency and the production cost must be reduced. In this paper the various solar energy technologies and mainly the present research and development on photovoltaic technology have been focused. The results of the solar cell characteristics have been plotted and compared with the standard semiconductor diodes.

There are many reasons to develop this technology because there is no pollution and waste material. The fossil fuels pollute the air and water and increase the global warming. Because of the fossils fuels are not renewable energy sources and they will not be available in the near future, the present world looking forward for this alternative and more attractive photovoltaic technology with new inventions.
\end{abstract}

Keywords: solar radiation, photovoltaic, panels, solar cell, efficiency

\section{INTRODUCTION}

In the year 1954 Calvin Fuller, Gerald Pearson and Daryl Chaplin of Bell Laboratories accidentally discovered the solar cell with an efficiency rate of $6 \%$, opening the door to practical electricity generation directly from solar energy.

By the late 1980s silicon cells, as well as those made of gallium arsenide, with efficiencies more than 20 percent had been fabricated. In the year 1989 a concentrator solar cell, achieved an efficiency of 37 percent due to the increased intensity of the collected energy. The earth's remaining fossil fuel reserves can probably provide us with energy for another 100 to 500 years. To raise the contribution to $50 \%$ of world energy use by 2050, as suggested in the Shell Renewable report, would require sweeping changes in our energy infrastructure. These changes can be achieved only through the parallel development of a new, more sophisticated technology.

\section{Solar ENERgy TeChNologies}

There are three solar energy technologies [7], Concentrating solar power, solar electricity (PV) and solar heating and lighting put the sun's energy to work for us in our homes, schools, businesses, and government buildings.

Andhe Satyanarayana Murthy is a Senior Lecturer at KDU COLLEGE, PJ Campus, School of Engineering, SS22/41, Damansara Jaya, Petalling Jaya, Selangor, Malaysia (h/p: 012-9148965, fax: 03-77277096, email: m_andhe@yahoo.com).
They are being developed because they are reliable, they have very few environmental impacts, and they make use of an abundant domestic energy resource: sunlight.

\section{A Concentrating Solar Power}

These technologies - such as dish/engine systems, parabolic troughs, and central power towers - are made of reflective materials that focus or concentrate the sun's considerable heat energy. This concentrated solar energy then drives a generator to produce electricity.

\section{B Photovoltaic}

This solar electric technology makes use of semiconductors to convert sunlight directly to electricity. Photovoltaics can provide a very small amount of electric power for your solar watch or calculator, or a very large amount of power for your local electric utility — and any amount in between.

\section{Solar Heating}

Solar heating technologies make use of low-temperature solar collectors that absorb the sun's heat energy, allowing that heat to be used directly for water or space heating in residential, commercial, and industrial buildings.

\section{Solar Lighting}

Solar lighting technologies rely on roof-mounted solar concentrators to collect sunlight; then, they distribute that light through optical fibers to hybrid lighting fixtures in the building's interior. These fixtures are called hybrids because they combine natural light with electric light to illuminate interior spaces.

\section{E Photovoltaic Technology}

Over $95 \%$ of all the solar cells produced worldwide are composed of the semiconductor material, silicon. As the second most common element in the earth's crust, silicon has the advantage of being available in large quantities. Furthermore while the material is been processed, it does not have an effect on the environment. Another reason for the use of silicon for solar cells is that the energy needed to ionize silicon electrons matches well with the energy of photons coming from the sun. If the photons had less energy (if the solar spectrum were more red), there would not be enough energy to free the electrons, and if the photons had more energy (if the solar spectrum were more blue or 
ultraviolet), then all the energy above what is needed to break the electrons free would be lost as heat.

To produce a solar cell, the semiconductor (silicon) is "doped" or contaminated. "Doping" is the intended introduction of chemical elements, which can obtain excess positive charge carriers (p-conducting semiconductor layer) or negative charge carriers (n-conducting semiconductor layer) from the semiconductor material. If two differently contaminated semiconductor layers are combined, a p-njunction results.

At this junction, an interior electric field is built up, which leads to the separation of the charge carriers that are released by light. Through metal contact, an electric charge can be tapped. If the outer circuit is closed, meaning a user is connected, and then direct current flows. A transparent anti-reflection film protects the cell and decreases the reflective loss on the cell's surface. The generation of electricity using solar panels as shown in figure 1

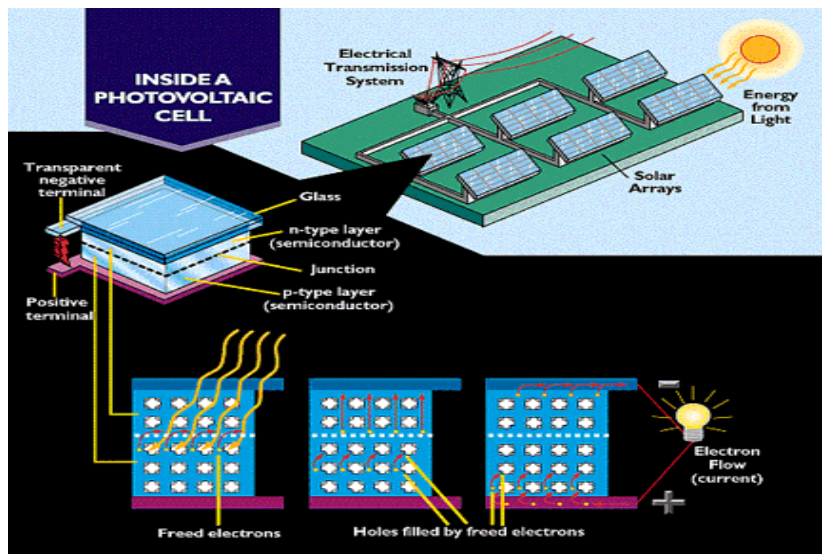

Figure 1: Generation of electricity using solar panels.

\section{F Components Used to Provide Solar Power}

The four primary components (figure 2) for producing electricity using solar power, which provides common 120 volt AC power for daily use are: Solar panels, charge controller, battery and inverter. Solar panels charge the battery, and the charge regulator insures proper charging of the battery. The battery provides DC voltage to the inverter, and the inverter converts the DC voltage to normal AC voltage. If 240 volts $\mathrm{AC}$ is needed, then either a transformer is added or two identical inverters are series-stacked to produce the 240 volts.

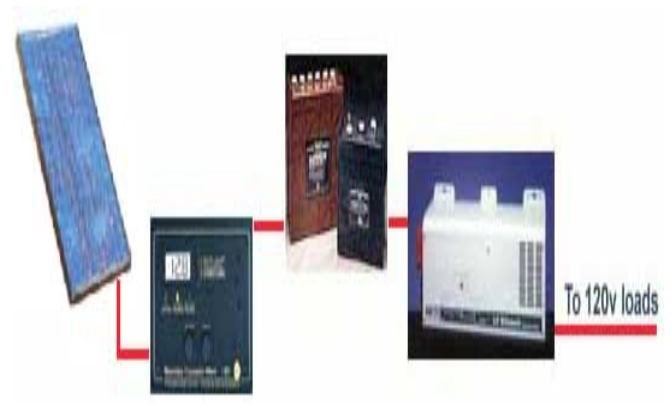

Figure 2: Components of solar electricity.

\section{G Solar Cells}

There are three types of solar cells [19] available: monocrystalline, polycrystalline and amorphous. To produce a monocrystalline silicon cell, absolute pure semi conducting material is necessary. Monocrystalline rods are extracted from melted silicon and sawn into incredibly thin plates. Panels constructed this way are found to provide the highest efficiency, however these are more expensive to produce than other types.

The production of polycrystalline cells is more costefficient. In this process, liquid silicon is poured into blocks that are then sawed into plates. During hardening of the material, crystal structures of varying sizes are formed. At the borders of these crystals, defects begin to emerge. As a result of this crystal defect, the solar cell is less efficient.

When silicon film is deposited on glass or another material, it is an amorphous or thin layer cell. The layer thickness amounts to less than $1 \mu \mathrm{m}$ (a human hair is $50 \mu \mathrm{m}-$ $100 \mu \mathrm{m}$ ), therefore making the production costs lower due to the low material costs. However, the efficiency of amorphous cells is much lowers than that of the other two cell types. Because of this they are mainly used in low power equipment such as calculator.

\section{H Model of Solar Cell}

Consider the model [13] as shown in figure 3 having two levels; the first level has plenty of holes, and assumes most of these with water. Let the rubber balls then fall onto this level, and water splashes from the holes. Although some water splashes back onto the first level, some reaches the second level; it flows from there to a waterwheel that drives a dynamo and generates electricity. On the first level, water flows back into the holes, to be hit again by further rubber balls. In this model the rubber balls represents photons of the sun light, the two levels are n-type and p-type regions, and the water signifies the electrons.

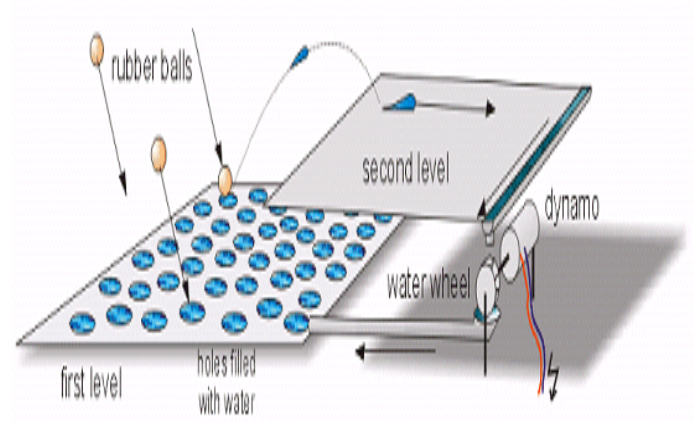

Figure 3: Model of a solar cell.

\section{Solar Panels}

In order to make the appropriate voltages and outputs available for different applications, single solar cells are interconnected to form larger units. Cells connected in series have a higher voltage, while those connected in parallel produce more electric current. The interconnected solar cells are usually embedded in transparent Ethyl-Vinyl-Acetate, 
fitted with an aluminum or stainless steel frame and covered with transparent glass on the front side.

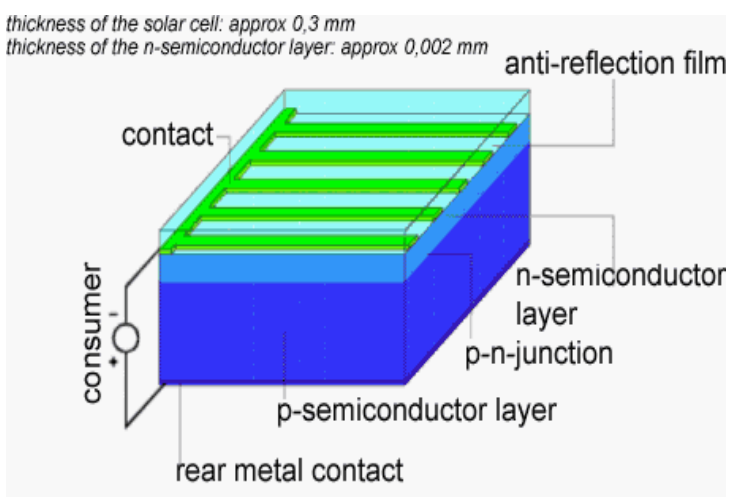

Figure 4: Solar panel.

The working life of a solar panel as shown in figure 4 should be in excess of 20 years. They require virtually no maintenance, but dust or grime on the front of solar panels will substantially reduce the output, so they should be cleaned periodically. Photovoltaic panels also suffer from decreased power output when they heat up, so when it is extremely hot they may not power efficiently.

Silicon cells are approximately $10 \mathrm{~cm}$ by $10 \mathrm{~cm}$ large (recently also $15 \mathrm{~cm}$ by $15 \mathrm{~cm}$ ). A transparent anti-reflection film protects the cell and decreases reflective loss on the cell surface.

\section{$J$ Natural Limits of Efficiency}

In addition to optimizing the production processes, work is also being done to increase the level of efficiency, in order to lower the costs of solar cells. However, different loss mechanisms are setting limits on these plans. Basically, the different semiconductor materials or combinations are suited only for specific spectral ranges. Therefore a specific portion of the radiant energy cannot be used, because the light quanta (photons) do not have enough energy to "activate" the charge carriers.

On the other hand, a certain amount of surplus photon energy is transformed into heat rather than into electrical energy. In addition to that, there are optical losses, such as the shadowing of the cell surface through contact with the glass surface or reflection of incoming rays on the cell surface. Other loss mechanisms are electrical resistance losses in the semiconductor and the connecting cable.

The disrupting influence of material contamination, surface effects and crystal defects, however are also significant. Single loss mechanisms (photons with too little energy are not absorbed, surplus photon energy is transformed into heat) cannot be further improved because of inherent physical limits imposed by the materials themselves. This leads to a theoretical maximum level of efficiency, i.e. approximately $28 \%$ for crystal silicon. Theoretical maximum levels of efficiency of various solar cells at standard conditions are shown in Figure 6.

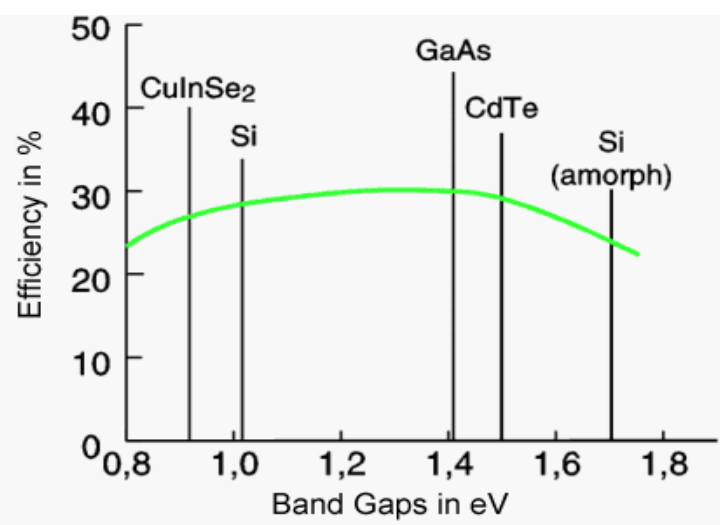

Figure 6: Efficiency of different materials.

\section{MODERN TECHNOLOGIES}

The reflection loss can be reduced by structuring the pyramidal shape, so that incoming light hits the surface several times and the new materials: for example, gallium arsenide (GaAs), cadmium telluride (CdTe) or copper indium selenide $\left(\mathrm{CuInSe}^{2}\right)$. In order to be able to use a wide spectrum of radiation, different semiconductor materials, which are suited for different spectral ranges, will be arranged one on top of the other.

A higher light intensity will be focused on the solar cells by the use of mirror and lens systems. This system tracks the sun, always using direct radiation. The inner electrical fields are not produced by a $\mathrm{p}-\mathrm{n}$ junction, but by the junction of a thin oxide layer to a semiconductor. Electrochemical liquid cells with titanium dioxide are used as electrolytes and dye to improve light absorption.

\section{EXPERIMENTAL RESULTS}

The usable voltage from solar cells depends on the semiconductor material. In silicon it amounts to approximately $0.5 \mathrm{~V}$. Terminal voltages is only weakly dependent on light radiation, while the current intensity increases with higher luminosity. A $100 \mathrm{~cm}^{2}$ silicon cell, for example, reaches a maximum current intensity of approximately 2 A when radiated by $1000 \mathrm{~W} / \mathrm{m}^{2}$. The characteristics of a solar cell as shown in figure 5 at light illuminations. It is observed that if more radiation more current results compared to the low radiations. The characteristics entirely different from the pn junction diode characteristics. 


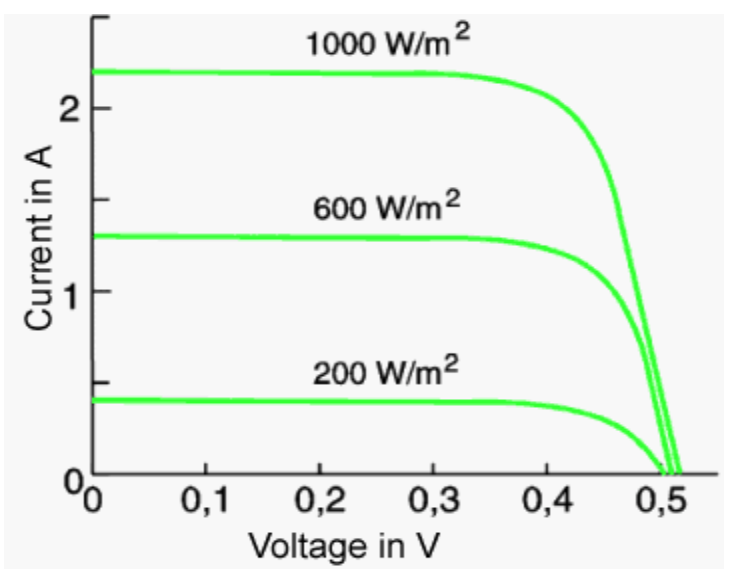

Figure 5: Characteristics of solar cell.

\section{MARKETING}

The highest installed capacity [13] of photovoltaic technology is in Japan followed by Germany and US, these three countries contributing the two thirds of world PV capacity. With the new innovations in PV technology and this may be competitive with the conventional grid connected systems in a few decades. It is one of the most important renewable energy sources for the future.

\section{CONCLUSION}

There will always be materials to produce solar cells whereas fossil fuels may not be available in years to come. A concern is that we are using up fossil fuels at an increasing rate, and that they will run out. In spite of the challenges today facing the manufacturers of solar cells including the higher cost for the materials to gain a greater efficiency, real progress is being made towards supplying a significant and meaningful fraction of the world's energy.

In the future if every roof tops of the houses/apartments fitted with this solar panels covering huge amount of area and produces huge amounts solar electricity. Of course there may be scarcity for the silica material in the future if this technology fully developed.

\section{ACKNOWLEDGEMENT}

I would like to thank the college management for their financial support and encouragement.

\section{REFERENCES}

[1] Practical Photovoltaics: Electricity from Solar Cells. Paperback by Richard J. Komp

[2] Photovoltaic Systems Engineering, Second Edition. Hardcover by Roger A. Messenger

[3] Real Goods Solar Living Sourcebook: Your Complete Guide to Renewable Energy Technologies and Sustainable Living (Real Goods Solar Living Sourcebook) by John Schaeffer

[4] The Solar Electric House: Energy for the EnvironmentallyResponsive, Energy-Independent Home by Steven J. Strong Practical Photovoltaics: Electricity from Solar Cells by Richard J. Komp

[5] http://www.facts-about-solar-energy.com/pictures-of-solarenergy.html

[6] http://en.wikipedia.org/wiki/Sunlight

[7] http://www1.eere.energy.gov/solar/index.htm

[8] http://pkukmweb.ukm.my/ SERI/Introduction.html

[9] http://www.globalwarmingsolutions.co.uk/index.html

[10] http://www.clean-energy-ideas.com/solar_panels.html
[11] http://www.sciencedaily.com/news/matter energy/solar energy/

[12] http://en.wikipedia.org/wiki/Solar_power

[13] http://www.volkerquaschning.de/articles/fundamentals3/index_e.html

[14] http://www.our-energy.com/solar-energy_en.html

[15] http://www.darvill.clara.net/altenerg/solar.htm

[16] http://www.eia.doe.gov/kids/energyfacts/sources/renewable/solar.html \#fromthesun

[17] http://www.powerhousekids.com/stellent2/groups/public/documents/p ub/phk 001537.hcsp

[18] http://www.annesley.sa.edu.au/amep/energyconservation_solarenergy /home.htm

[19] http://www.solarserver.de/wissen/photovoltaik-e.htm 


\title{
Overview on the Infrastructure Planning of Hydrogen Energy in Malaysia
}

\author{
S.K. Kamarudin and W. Anuar
}

\begin{abstract}
The potential future use of hydrogen as an energy carrier in the transportation sector necessitates the need to shift the current energy supply chain from supplying petroleum products such as gasoline and diesel to supply hydrogen. Therefore this research was carried out to determine the hydrogen demand and the most optimum hydrogen network delivered via truck transportation in Peninsular Malaysia. The hydrogen demand are determine using two method which is one by assuming that the hydrogen demand in Peninsular Malaysia as a function of total vehicle numbers, average total distance traveled, and vehicle fuel economy. The second method is by comparing the current supply of gasoline and diesel from the survey that had been done to some PETRONAS stations around Peninsular Malaysia. The estimated demand is assumed to supply fuel cellpowered vehicles including cars and taxies, buses and good vehicles. The final results shows that, the liquefied hydrogen produced by natural gas steam reforming delivered via tanker truck was the most optimum hydrogen supply chain due to its most minimized cost.
\end{abstract}

Keywords: hydrogen energy, fuel, economy, Malaysia

\section{INTRODUCTION}

The world's energy system is at least a 1.5 trillion dollars market dominated by fossil fuels, where small changes can have a large influence on efforts to reach sustainability. Renewable energy sources are key to achieving this goal. Environmental problems at local, regional and global levels, as well as external dependency and security of supply will persist if we rely on an energy future based on fossil fuels. Solutions encompass extending the life of fossil fuel reserves and expanding the share of renewable in the world energy system. (Goldemberg 2006). Environmental and security concerns are stimulating global interest in hydrogen power, renewable energy. By 2030, the world is projected to consume two-thirds more energy than today, with developing countries replacing the industrialized world as the largest group of energy consumers. Renewable energy will increasingly contribute to electricity generation, though hydrogen energy, solar, wind and biomass will expand from an extremely small base (Dorian et al. 2006).

The potential future use of hydrogen as an energy carrier in the transportation sector necessitates the need to shift the

S.K. Kamarudin and W. Anuar are with the Department of Chemical and Process Engineering, Universiti Kebangsaan Malaysia, 43600 UKM Bangi, Selangor, Malaysia (e-mail: ctie@vlsi.eng.ukm.my). current energy supply chain from petroleum products to supplying hydrogen. The future hydrogen supply chain should run from raw materials procurement, through hydrogen production, inventory and warehousing, distribution and delivery of hydrogen to customers or retailers. Furthermore, it should be designed in a way that can serve not only the transportation sector but also commercial domestic demand as well. Of course, tremendous efforts and money are needed to bypass the current energy supply network completely.

The objective of this study is to report hydrogen supply chain network that will determine the number, location and capacity of hydrogen production plant and storage facilities to be set up, the transportation links that are needed to be established in the network, and the flows and production rates of materials.

\section{CURRENT ENERGY DEMAND IN MALAYSIA}

Energy is the key element for economic development. Malaysia is among the important country in world energy market with 75 trillion feet $^{-3}$ natural gas storage and exporting about 300,000 barrel per day. There four types of energy sources in Malaysia, namely, fuel, natural gas, electricity and coal (Energy Information Administration 2005). Figure 1-4 presents the four types of energy sources in Malaysia.

Table 1 and Table 2 present the type of vehicle used in Malaysia for the year 1990-2004. Both tables show an increase in the vehicle usage and demand for petrol as well as diesel. However both fuels contributes to emission of green house pollution.

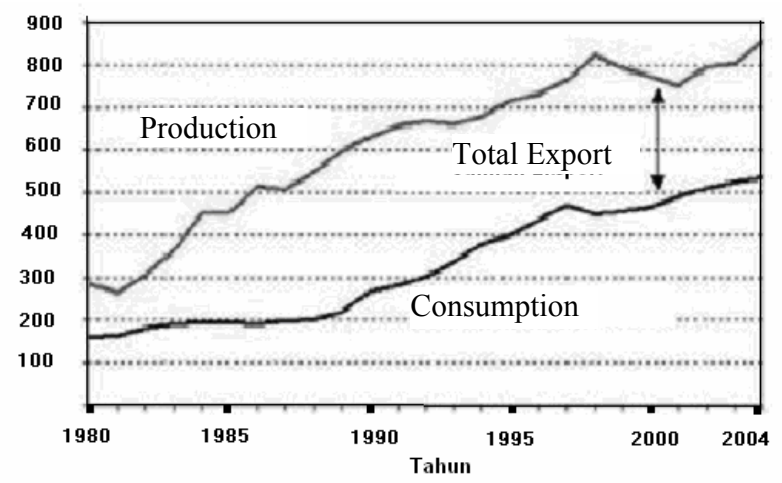

Figure 1. Production and consumption of fuel in Malaysia, 1980-2004 (Energy Information Administration 2005) 


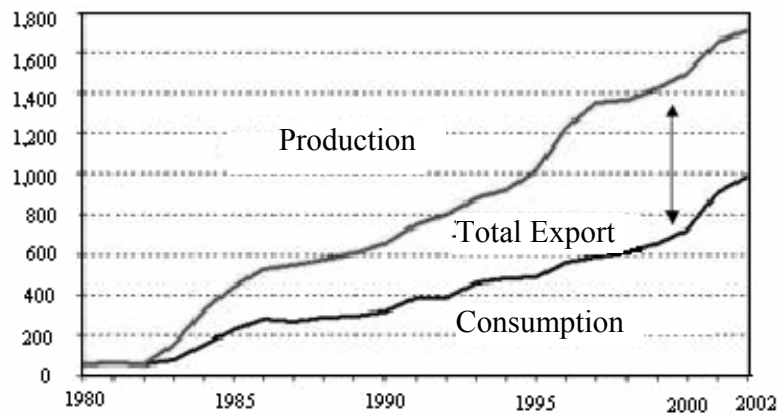

Figure 2. Pproduction and consumption of natural gas in Malaysia, 1980-2002 (Energy Information Administration 2005)

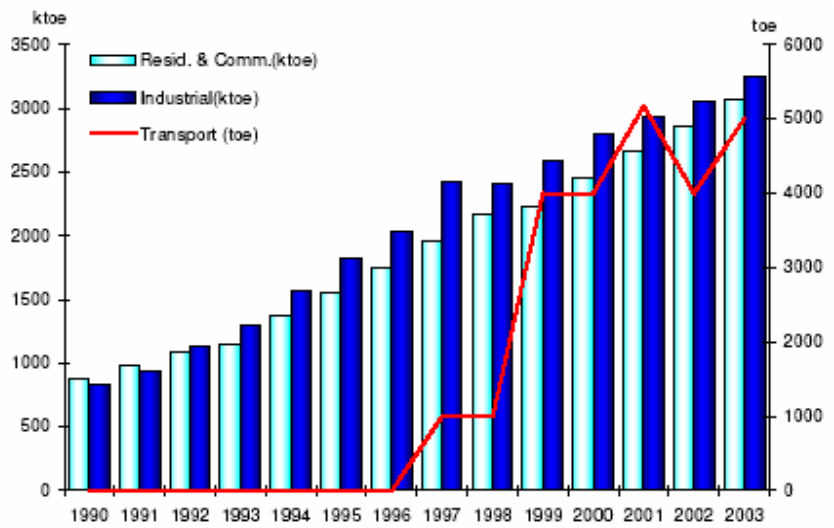

Figure 3. Consumption of electricity in Malaysia, 1990-

2003 (Ministry of Energy, Water and Communication 2003)

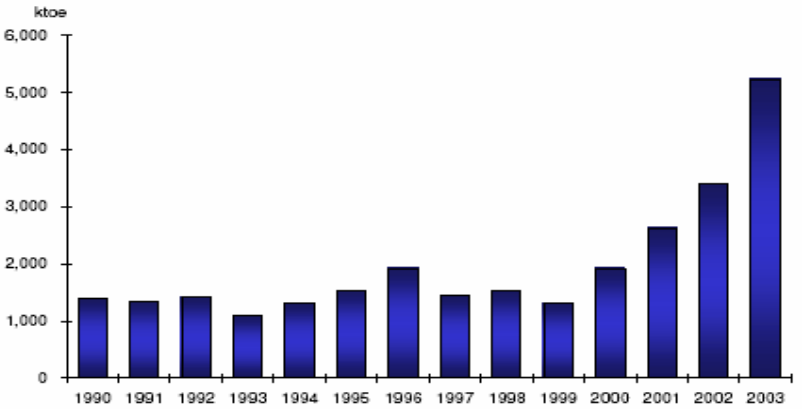

Figure 4. Total import of coal in Malaysia, 1990-2003 (Ministry of Energy, Water and Communication 2003)

\section{Demand of Hydrogen Supply Chain}

The first step in designing a system to deliver hydrogen transportation fuel is characterizing the hydrogen demand to be served. The estimated total hydrogen demand in Peninsular Malaysia is as a function of total vehicle numbers, average total distance traveled and vehicle fuel economy (i.e scf $\mathrm{H}_{2} / \mathrm{mi}$ ). The estimated demand is assumed to supply fuel cell powered vehicle including cars and taxies, buses and light good vehicles. Table 3 shows the parameter that are used for calculating the total hydrogen demand in Peninsular Malaysia.

Another important issue in designing a hydrogen supply chain is identifying the geographical concentration of the demand (i.e number of vehivel per square kilometer). This approach is accomplished by dividing the Peninsular Malaysia into equalized grid squares (see figure 5 and 6). Table 4 predicts the total demand of Hydrogen in Peninsular Malaysia according to States.

Table 1. Type of registered vehicle in Malaysia (Jabatan Pengangkutan Jalan 2005)

\begin{tabular}{|c|c|c|c|c|c|c|c|c|}
\hline Year & Motocycles & Car & Buses & Taxies & Private Cars & Light Good Vehicle & Others & Total \\
\hline 1990 & 188118 & 152737 & 1985 & 2756 & 941 & 30757 & 14926 & 392220 \\
\hline 1991 & 201864 & 148724 & 1337 & 2927 & 480 & 28414 & 10877 & 394623 \\
\hline 1992 & 184171 & 99867 & 1506 & 1710 & 504 & 20355 & 9672 & 317796 \\
\hline 1993 & 226088 & 138203 & 2591 & 2255 & 782 & 20159 & 6123 & 396201 \\
\hline 1994 & 283370 & 180052 & 2213 & 3754 & 2791 & 27482 & 20168 & 519830 \\
\hline 1995 & 307323 & 248398 & 2465 & 4455 & 2722 & 41447 & 29133 & 635943 \\
\hline 1996 & 322145 & 318765 & 2620 & 4358 & 2545 & 69234 & 30844 & 750511 \\
\hline 1997 & 364214 & 372343 & 2947 & 5257 & 1860 & 65160 & 28396 & 840177 \\
\hline 1998 & 237776 & 159642 & 797 & 3569 & 552 & 11786 & 6342 & 420464 \\
\hline 1999 & 236779 & 296716 & 508 & 1925 & 1724 & 19987 & 8102 & 565741 \\
\hline 2000 & 238696 & 344847 & 544 & 2635 & 2883 & 24316 & 11949 & 625869 \\
\hline 2001 & 234751 & 355891 & 652 & 3169 & 1348 & 25612 & 13866 & 675289 \\
\hline 2002 & 222685 & 419713 & 919 & 4446 & 1242 & 25415 & 16768 & 691188 \\
\hline 2003 & 321234 & 424753 & 1014 & 5542 & 1231 & 29975 & 17041 & 800790 \\
\hline 2004 & 397977 & 472116 & 1290 & 7746 & 1797 & 33169 & 18268 & 932363 \\
\hline
\end{tabular}

Table 2. Parameter used for hydrogen demand calculation

\begin{tabular}{lccc}
\hline Parameters & $\begin{array}{c}\text { Car and } \\
\text { Taxies }\end{array}$ & $\begin{array}{c}\text { Light Good } \\
\text { Vehicles }\end{array}$ & Buses \\
\hline Number Of Vehicles & 425128 & 19730 & 1205 \\
\hline $\begin{array}{l}\text { Distance Travelled } \\
\text { (km/veh/yr) }\end{array}$ & $1.53 \mathrm{E}+04$ & $3.80 \mathrm{E}+04$ & $5.51 \mathrm{E}+04$ \\
\hline $\begin{array}{l}\text { Vehicle Fuel } \\
\left.\text { Efficiency (scf/ } \mathrm{H}_{2} / \mathrm{mi}\right)\end{array}$ & 3.60 & 4.59 & 51.91 \\
\hline Case study I $:$ & $\begin{array}{l}\text { Function of total vehicle numbers, average total } \\
\text { distance traveled, and vehicle fuel economy. }\end{array}$ \\
Case Study II : $\begin{array}{l}\text { Current supply of gasoline and diesel from the survey } \\
\text { that had been done to PETRONAS stations around } \\
\text { Peninsular Malaysia. }\end{array}$ \\
\hline
\end{tabular}

\section{A. Overall Objective Function}

The Total cost of the hydrogen supply chain network to be minimized by the optimization subjected to constraints (Wanah Anuar 2006):

$\min \frac{F C C+T C C}{\alpha C C F}+\frac{F O C+T O C}{\alpha}+P C$

where $\mathrm{CCF}=$ capital charge factor; $\mathrm{FCC}=$ Facility capital Costs; FOC $=$ Facility Operating Costs; $\mathrm{PC}=$ Product Costs; $\mathrm{TCC}=$ Transportation capital cost; and $\mathrm{TOC}=$ Tranportation Operating Costs. 
Table 3. Type of registered vehicle In Malaysia and engine power in Malaysia for the year 2005 (source: Department of Transportation 2005)

\begin{tabular}{|c|c|c|c|c|c|c|c|c|c|c|c|c|}
\hline \multirow{3}{*}{$\begin{array}{l}\text { Type Of } \\
\text { Vehicles }\end{array}$} & \multicolumn{12}{|c|}{ Vehicles (C.C) } \\
\hline & \multicolumn{2}{|c|}{$<1000$} & \multicolumn{2}{|c|}{ 1001-1300 } & \multicolumn{2}{|c|}{ 1301-1500 } & \multicolumn{2}{|c|}{ 1501-2000 } & \multicolumn{2}{|c|}{ 2001-2500 } & \multicolumn{2}{|c|}{ 2501-3000 } \\
\hline & Petrol & Diesel & Petrol & Diesel & Petrol & Diesel & Petrol & Diesel & Petrol & Diesel & Petrol & Diesel \\
\hline Motorcycles & 397823 & 33 & 60 & - & 21 & - & 9 & - & 3 & - & - & - \\
\hline Car & 119927 & - & 65151 & 1 & 97842 & 2 & 132054 & 10 & 25519 & 83 & 4583 & 729 \\
\hline Taxi & 2 & - & 1 & - & 5940 & - & 438 & - & 1 & 1 & 1 & 3 \\
\hline Private car & 1 & - & 23 & - & 668 & - & 938 & - & 69 & - & 4 & 11 \\
\hline \multicolumn{13}{|l|}{$\begin{array}{l}\text { Light Good } \\
\text { Vehicles }\end{array}$} \\
\hline$<2500 \mathrm{~kg}$ & 126 & - & 321 & 1 & 3928 & - & 81 & - & - & 14 & 2 & 152 \\
\hline$>2500 \mathrm{~kg}$ & - & 6 & - & - & 5 & - & - & 1 & - & 3 & - & 3 \\
\hline Buses & - & - & - & - & - & - & 19 & - & - & 2 & 11 & 48 \\
\hline Specific & 33 & 3395 & 1 & 13 & 100 & 246 & 23 & 72 & 303 & 1208 & 21 & 717 \\
\hline Others & 1866 & 37 & 135 & - & 192 & 1 & 1985 & 5 & 314 & 464 & 85 & 185 \\
\hline Total & 519778 & 3471 & 65692 & 15 & 108696 & 249 & 135547 & 88 & 26209 & 1775 & 4707 & 1848 \\
\hline \multirow{3}{*}{$\begin{array}{l}\text { Type Of } \\
\text { Vehicles }\end{array}$} & \multicolumn{10}{|c|}{ Vehicles (C.C) } & \multicolumn{2}{|c|}{ Total } \\
\hline & \multicolumn{2}{|c|}{ 3001-3500 } & \multicolumn{2}{|c|}{ 3501-4000 } & \multicolumn{2}{|c|}{ 4001-4500 } & \multicolumn{2}{|c|}{ 4501-5000 } & \multicolumn{2}{|c|}{$>5000$} & & \\
\hline & Petrol & Diesel & Petrol & Diesel & Petrol & Diesel & Petrol & Diesel & Petrol & Diesel & Petrol & Diesel \\
\hline Motorcycles & - & - & - & - & - & - & - & - & 2 & - & 397918 & 33 \\
\hline Car & 507 & 65 & 152 & - & 97 & 500 & 140 & 5 & 37 & - & 446009 & 1395 \\
\hline Taxi & 1 & - & 2 & - & - & 3 & - & 1 & - & 9 & 6386 & 17 \\
\hline Private car & 1 & - & - & - & - & - & - & - & - & - & 1704 & 11 \\
\hline $\begin{array}{l}\text { Light Good } \\
\text { Vehicles }\end{array}$ & & & & & & & & & & & & \\
\hline$>2500 \mathrm{~kg}$ & - & 1 & - & 21 & 1 & 31 & - & 4 & 2 & 2682 & 7 & 2752 \\
\hline Buses & - & - & - & - & - & - & - & 1 & - & 56 & 30 & 107 \\
\hline Specific & 10 & 720 & 17 & 904 & 14 & 488 & 4 & 612 & 42 & 2254 & 568 & 10629 \\
\hline Others & 70 & 14 & 14 & 21 & 7 & 29 & 3 & 6 & 9 & 926 & 4680 & 1688 \\
\hline Total & 589 & 817 & 186 & 1184 & 119 & 1628 & 150 & 752 & 92 & 5936 & 861765 & 17763 \\
\hline
\end{tabular}

Table 4. Demand of hydrogen in Peninsular Malaysia 2004 (case study I)

\begin{tabular}{lccc}
\hline \multicolumn{1}{c}{ State } & $\begin{array}{c}\text { Population/ } \\
\text { State }\end{array}$ & $\begin{array}{c}\text { Population/ } \\
\text { Total } \\
\text { Population }\end{array}$ & $\begin{array}{c}\text { Demand } \\
\text { (kg/day) }\end{array}$ \\
\hline Johor & $3,029,300$ & 0.1487 & 234,261 \\
Kedah & $1,813,100$ & 0.0890 & 140,210 \\
Kelantan & $1,479,700$ & 0.0726 & 114,428 \\
Melaka & 700,100 & 0.0344 & 54,140 \\
Negeri Sembilan & 929,600 & 0.0456 & 71,888 \\
Pahang & $1,399,500$ & 0.0687 & 108,226 \\
Perak & $2,225,000$ & 0.1092 & 172,063 \\
Perlis & 221,200 & 0.0109 & 17,106 \\
Pulau Pinang & $1,442,800$ & 0.0708 & 111,574 \\
Selangor \& Putrajaya & $4,613,900$ & 0.2265 & 356,801 \\
Terengganu & 990,600 & 0.0486 & 76,605 \\
W.P Kuala Lumpur & $1,529,000$ & 0.0750 & 118,240 \\
\hline Total & $\mathbf{2 0 , 3 7 3 , 8 0 0}$ & $\mathbf{1 . 0 0 0 0}$ & $\mathbf{1 , 5 7 5 , 5 4 2}$ \\
\hline
\end{tabular}

\section{RESUlTS AND DISCUSSION}

It results that the hydrogen demand calculated using the second method are much higher than the first method which required to at least 18 production plants to serve the demand compare to 11 plants for the first method. The case study considered the production of hydrogen by four commercially proven technologies, namely steam reforming of natural gas, coal gasification, biomass gasification and water electrolysis. The purified hydrogen produced from each plant type was either liquefied and stored in spherical tanks and transported via cryogenic tanker truck or compressed and stored in tube-type vessels and transported by tube trailers to different customers' site. Unit costs, capital costs and operating costs for different production type, storage and transportation were calculated. Table 5 summarized the result for the hydrogen network demand. 
Table 5. Summarized results of the hydrogen network demand

\begin{tabular}{lcc}
\hline & $\begin{array}{c}\text { Liquid } \\
\text { Hydrogen }\end{array}$ & $\begin{array}{c}\text { Compressed } \\
\text { Hydorgen }\end{array}$ \\
\hline Production Cost (RM/kg) & & \\
\hline $\begin{array}{l}\text { Natural Gas from stim } \\
\text { reformingi }\end{array}$ & 6.08 & 3.20 \\
Biomass gasification & 6.50 & 3.62 \\
$\begin{array}{l}\text { Coal gasification } \\
\text { Water eletrolysis }\end{array}$ & 8.30 & 5.34 \\
\hline Storage Cost (RM/kg) & 26.53 & 23.60 \\
\hline $\begin{array}{l}\text { Cryogenic Tanker } \\
\text { High Pressure Vessel }\end{array}$ & 0.22 & \\
\hline Cost Of Transportation within the grid (RM/kg) & \\
\hline $\begin{array}{l}\text { Truck tanker } \\
\text { Tube Treler }\end{array}$ & 0.16 \\
\hline
\end{tabular}

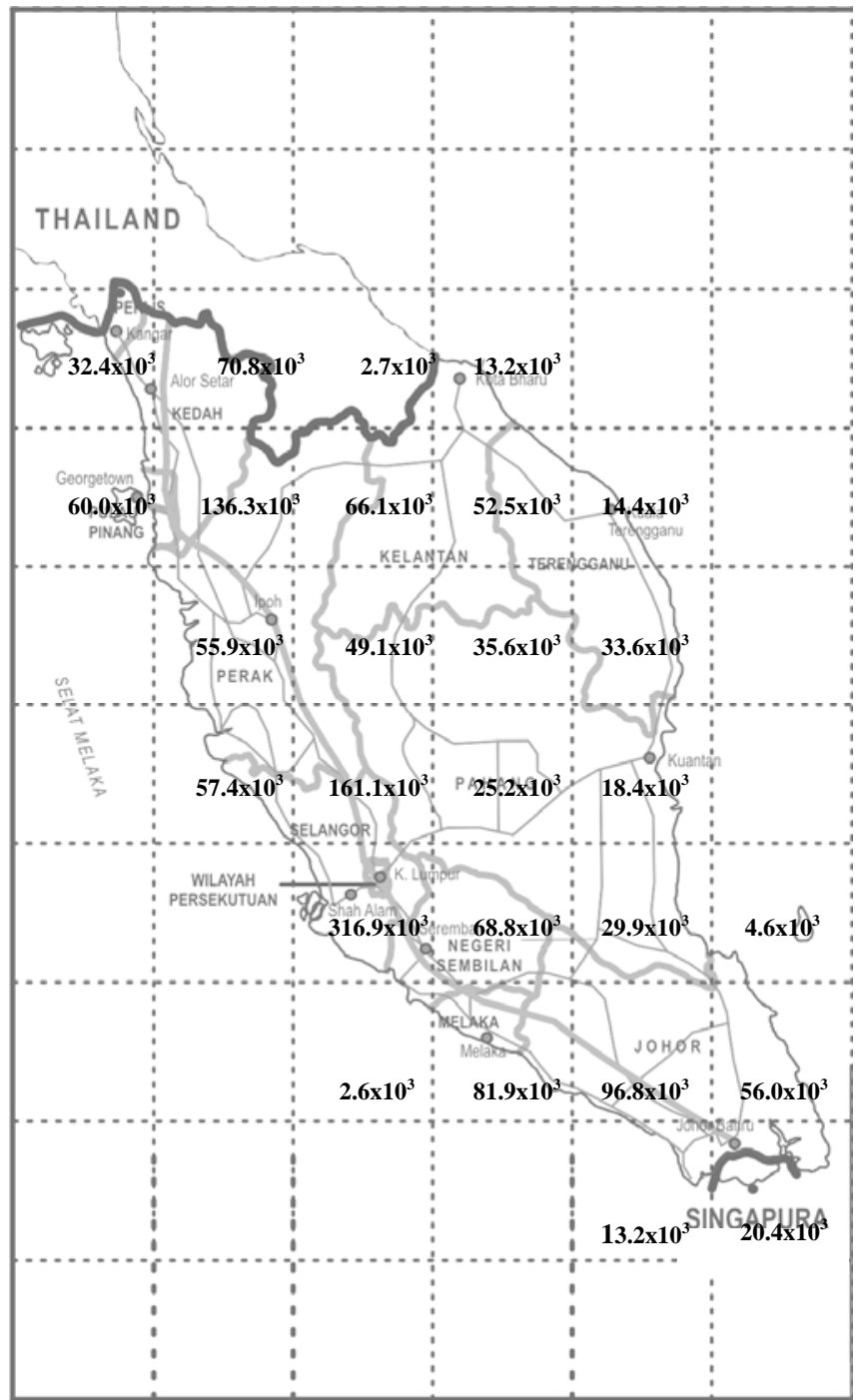

Figure 5. Hydrogen demand for road transportation in Peninsular Malaysia (case study I)

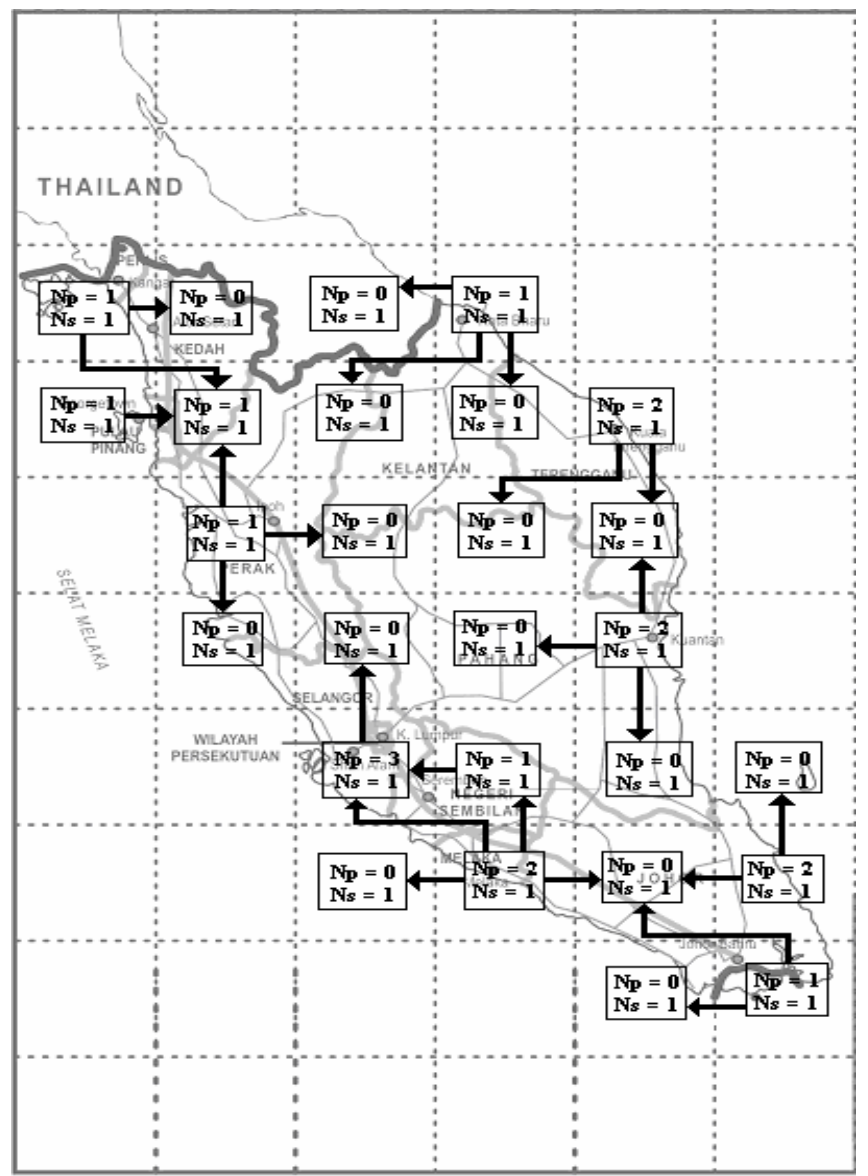

Figure 6. Hydrogen demand for road transportation in Peninsular Malaysia (case study II)

\section{CONCLUSIONS}

Finally the optimum hydrogen demand in peninsular Malaysia predicted as liquefied hydrogen produced by natural gas steam reforming delivered via tanker truck due to its most minimized cost.

\section{ACKNOWLEDGEMENT}

The authors gratefully acknowledge the financial support given for this work by the Malaysian Ministry Of Higher Education under the Fundamental Research Grant Scheme (FRGS) by Grant No: UKM-KK-02-FRGS0017-2006.

\section{REFERENCES}

[1] Department Of Transportation 2006. Official portal of Jabatan Pengangkutan Jalan. (atas talian) http://www.jpj.gov.my (23 Januari 2006).

[2] Dorian, J.P., Franssen, H.T. \& Simbeck MD, D.R. 2006. Global Challenges in energy. Energy Policy. 34(15):1984-1991.

[3] Energy Information Administration 2005 Energy Information Administration. 2005. Malaysia country analysis brief. (atas talian) http://www.eia.doe.gov (23 Januari 2006).

[4] Goldembergy, J. 2006. The promise of clean energy. Energy policy. 34(15):2185-2190

[5] Ministry of Energy, Water and Communication. 2003. National energy balance 2003 Malaysia.

[6] Wanah Anuar. 2006. Pembangunan Model Matematik Untuk Perancangan Infrastruktur Tenaga Hidrogen Di Malaysia. Latihan Ilmiah. Universiti Kebangsaan Malaysia. 


\title{
Profit Optimization of a Refrigerated Gas Plant
}

\author{
Nooryusmiza Yusoff ${ }^{1}$, M. Ramasamy ${ }^{2}$, Suzana Yusup $^{3}$
}

\begin{abstract}
The operation of a gas processing plant with tight profit margin and rigid contractual constraints at both ends poses a challenging problem. The problem is further compounded by continuous fluctuations in the feed compositions and economic conditions of the plant. To widen its profit margin, the plant needs to operate more efficiently. This requires continuous re-evaluation of the operating conditions through an efficient optimization algorithm. The success of solving the optimization problem rests on the accuracy of the plant model used, as well as the inclusion of technical and business aspects of the plant operation.

This paper proposes means for optimizing a refrigerated gas plant (RGP) based on a steady-state simulation model. The RGP is simulated using AspenTech HYSYS and calibrated with the actual plant data. Key optimization variables are prudently identified in order to induce maximization of a profit function. Four case studies namely the base case (1B), one-feed (1F), two-feed (2F) and three-feed (3F) inlet streams are carried out. As compared with Case 1B, the profit margins rise by $9.3 \%, 12.3 \%$ and $13.4 \%$ for Cases $1 F, 2 F$ and $3 F$, respectively. It is observed that the best option for this RGP is to focus on balancing the feed gas supplies while respecting the plant constraints. In addition, it is found that optimizing utility consumption results in insignificant savings. The utilities only contribute less than $2.4 \%$ of the total expenses in all four case studies.
\end{abstract}

Keywords: gas plant, simulation, economics, optimization

\section{INTRODUCTION}

Gas processing plants are considered as midstream operators. Their upstream suppliers are gas producers while downstream customers are mostly power producers, petrochemical industries and residential dwellers. On the supply side, raw gas is delivered by several producers and mixed at the plant inlet. To enhance the performance of gas plants, profit optimization may be carried out on the supplier and/or customer sides. In business sense, the complexity is compounded by the fact that contractual obligations may be different from one customer to another.

The main product of a refrigerated gas plant (RGP) is the sales gas (SG) consisting of mainly methane (C1). The natural gas liquids (NGLs) like ethane $(\mathrm{C} 2)$, propane $(\mathrm{C} 3)$, butane (C4) and condensates ( $\mathrm{C} 5+$ ) are the valuable byproducts. Under the current practices, the RGP profit

This work is supported by the Universiti Teknologi PETRONAS (UTP), at which all authors are affiliated as lecturers of the Chemical Engineering Department.

${ }^{1}$ Nooryusmiza Yusoff (Phone: 05-368-7584; Facsimile: 05-365-6176; Email: nooryus@petronas.com.my).

${ }^{2}$ M. Ramasamy (E-mail: marappagounder@petronas.com.my).

${ }^{3}$ Suzana Yusup (E-mail: drsuzana_yusuf@petronas.com.my). optimization is performed in a two-level process. The top level (L2) optimization, executed by the planners, focuses on overall input-output material balance and economic relations. The outcomes, usually the feed flow rates, are forwarded to the operating personnel who are responsible to perform the lower level (L1) optimization. At L1, major efforts are concentrated on the engineering sides such as energy and loss management (ELM). An example of ELM is the optimization study of heat exchanger network (HEN) integration of a turbo-expander plant (TEP) by [1].

The current practice has two practical drawbacks. First, this two-level optimization process lacks integration. As a result, the direction given by the planners may not be workable on the operational side due to such factors as column flooding, product flow fluctuation and reboiler tube clogging. Second, unplanned/emergency shutdown cases like a major equipment failure may occur during the absence of the planners. The plant may be kept running albeit at suboptimal condition. This situation requires a new direction set by the planners. In most cases, however, the operating personnel perform troubleshooting to quickly fix the problem. This may result in substantial product losses.

In this paper, a new approach based on the previous works of [2]-[3] is discussed. This approach entails the integration of L1 and L2 plant optimization. Economic and technical data are fed into the model optimizer and solved simultaneously. The new approach differs from the previous works as the plant is modeled using a sequential modular process simulator like HYSYS instead of an equationoriented process model to solve the material and energy balances. The latter is flexible, and thus found applications in less complex systems [4]. The former is more userfriendly and robust, and hence more practically utilized in industry.

\section{PROCESS DESCRIPTION}

The refrigerated gas plant (RGP) is simulated using a rigorous process simulator (AspenTech HYSYS). The thermodynamic properties are estimated by the PengRobinson equation of state. The key operating values are calibrated with the data collected from a real gas processing plant ('figure 1'). The feeds to the RGP come from three main gas producers $\mathrm{A}, \mathrm{B}$ and $\mathrm{C}$. The feed gas compositions vary as listed in 'table 1'. The values fall within the approximate range of typical natural gas compositions found across the world [5]. 


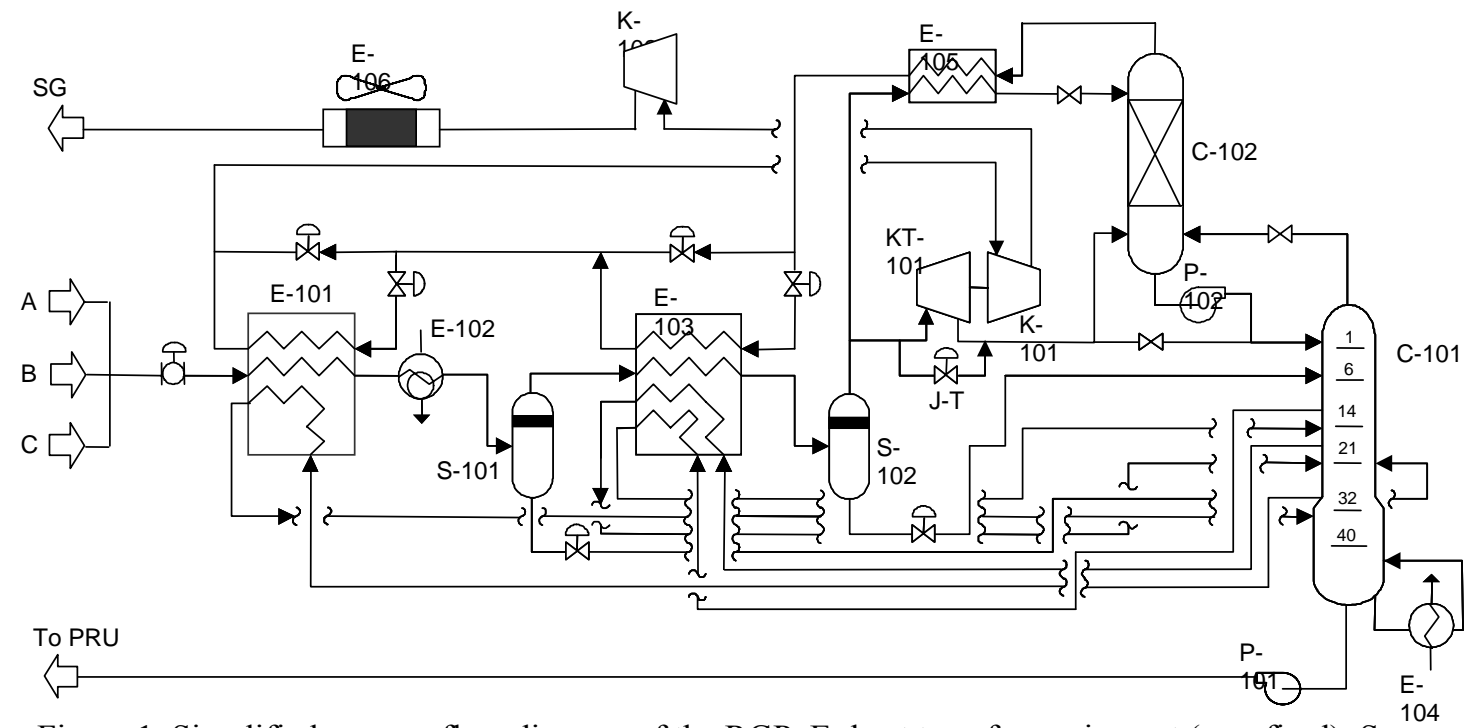

Figure 1. Simplified process flow diagram of the RGP. E=heat transfer equipment (non-fired); $\mathrm{S}=$ separator; $\mathrm{KT}=$ turboexpander; $\mathrm{K}=$ compressor; $\mathrm{P}=$ pump; $\mathrm{C}=$ column; $\mathrm{J}$ - $\mathrm{T}=$ Joule-Thompson valve

Table 1: Compositions (mol fraction) of feed gases.

\begin{tabular}{lccc}
\hline Component & A & B & C \\
\hline Methane & 0.8865 & 0.7587 & 0.6797 \\
Ethane & 0.0622 & 0.0836 & 0.1056 \\
Propane & 0.0287 & 0.0535 & 0.0905 \\
$i$-Butane & 0.0102 & 0.0097 & 0.0302 \\
$n$-Butane & 0.0059 & 0.0194 & 0.0402 \\
$i$-Pentane & 0.0003 & 0.0058 & 0.0121 \\
$n$-Pentane & 0.0002 & 0.0068 & 0.0101 \\
$n$-Hexane & 0.0001 & 0.0002 & 0.0028 \\
Nitrogen & 0.0039 & 0.0043 & 0.0012 \\
Carbon dioxide & 0.0020 & 0.0580 & 0.0276 \\
\hline
\end{tabular}

Water, sulfur and mercury are assumed to be absent when the feed gas (FG) enters the RGP. In other words, all feed gas streams are assumed to be sweet, dry gas with varying richness and carbon dioxide (CO2) levels. At normal operation (base case), the mixed FG flows at 280 metric ton per hour (MT/h), and enters the RGP at $20{ }^{\circ} \mathrm{C}$ and 50 bar. It is cooled by exchanging heat with the residual gas in three cold boxes (a variant of brazed aluminum plate heat exchanger), a C3-refrigeration cooler and an air cooler.

The FG is flashed in two stages in order to enhance vapor-liquid separation. The resulting vapor is expanded in a turboexpander (TE) and/or Joule-Thompson (J-T) valve to improve NGLs recovery. The liquids are fed to different stages of the demethanizer (C-101) column. The bottoms of $\mathrm{C}-101$ are sent to the Product Recovery Unit (PRU) for further processing of the NGLs. The top of C-101 is fed to an absorption unit (C-102) to minimize ethane losses. Recompression of the residual gas is carried out twice to meet the pipeline pressure specification at $30 \mathrm{bar}$.

\section{Plant ECONOMics}

The complexity of the RGP business model depends on both ends of its boundary limits. At the supply side, the three producers supply the feed gas at different rates since the contractual terms vary from one producer to another. In general, the contractual terms between the producers and RGP can be classified as:

1. a fixed processing fee

2. a "keep-whole" NGLs contract

3. retention of a fraction of the NGLs

The RGP enters into agreements for a diverse combination of terms with different producers. This is done to ensure its economic sustainability. For this study, the feed gas prices are fixed on long-term contracts. They are listed in 'table 2' together with the prices of products.

Table 2: Prices of feed gases and products.

\begin{tabular}{crl}
\hline Component & Price & Unit \\
\hline FG-A & 3.50 & RM/MMBtu \\
FG-B & 3.10 & RM/MMBtu \\
FG-C & 2.75 & RM/MMBtu \\
SG & 6.40 & RM/MMBtu \\
C2 & 1.32 & RM/MMBtu \\
C3 & 542.29 & RM/MT \\
C4 & 689.70 & RM/MT \\
\hline
\end{tabular}

Besides the feed gas, the RGP also has to pay for the utilities and consider the maintenance of the expandercompressor (KT/K-101). The former includes low pressure (6 bar) steam, refrigeration and electricity. The maintenance of $\mathrm{KT} / \mathrm{K}-101$ includes the normal deterioration of mechanical parts as well as topping up of seal gas and lubrication oil. The maintenance cost is estimated to be half of the refrigeration cost. 'table 3' summarizes the utilities and maintenance costs.

Table 3: Costs of utilities.

\begin{tabular}{lrl}
\hline Utilities/Maintenance & Price & Unit \\
\hline Steam & 26.0 & $\mathrm{RM} / \mathrm{MWh}$ \\
Refrigeration & 49.0 & $\mathrm{RM} / \mathrm{MWh}$ \\
Electricity & 180.0 & $\mathrm{RM} / \mathrm{MWh}$ \\
Expander-Compressor & 24.5 & $\mathrm{RM} / \mathrm{MWh}$ \\
\hline
\end{tabular}




\section{OPTIMIZATION}

The goal of the optimization process is to maximize RGP profit while respecting certain plant constraints, and contractual obligations to both producers and customers. This is generally carried out through maximization of product throughputs and/or minimization of operating costs. By integrating L1 and L2 optimization processes, the objective function can be written as:

$$
\underset{\mathbf{u}}{\operatorname{Max}} P(\mathbf{u}, \mathbf{x})
$$

where

$$
\begin{aligned}
& P=\text { Revenues }- \text { Expenses } \\
& \text { Revenues }=f_{1}(\mathrm{SG}, \mathrm{C} 2, \mathrm{C} 3, \mathrm{C} 4) \\
& \text { Expenses }=f_{2}(\mathrm{FGs}, \text { utilities, maintenance }) \\
& \mathbf{u}=\text { optimization variables: } \mathbf{u} \chi R^{m}, m=9 \text { as in 'table } 4 \text { ' } \\
& \mathbf{x}=\text { process variables: } \mathbf{x} \chi R^{\mathrm{n}}, n=12 \text { as in 'table } 5 \text { ' }
\end{aligned}
$$

The profit function is maximized subject to the following constraints:

a. FG flow rate $\leq 310 \mathrm{MT} / \mathrm{h}$

b. SG

$$
\begin{array}{ll}
\text { i. } & 35.1 \text { [ GHV }\left[48.1 \mathrm{MJ} / \mathrm{Sm}^{3}\right. \\
\text { ii. } & \text { Specific gravity (relative to air) } \leq 0.75 \\
\text { iii. } & \mathrm{CO} 2 \text { content } \leq 2.0 \mathrm{~mole} \% \\
\text { iv. } & \text { Flow rate } \geq 295 \mathrm{MCM} / \mathrm{h}
\end{array}
$$

c. Equipment
i. 20 [ C-101 flooding [ $90 \%$
ii. E-101 UA $\left[10,000 \mathrm{MJ} /{ }^{\circ} \mathrm{C}-\mathrm{h}\right.$
iii. E-103 UA [ $5000 \mathrm{MJ} /{ }^{\circ} \mathrm{C}-\mathrm{h}$
iv. E-105 UA [ $1000 \mathrm{MJ} /{ }^{\circ} \mathrm{C}-\mathrm{h}$
v. $\quad$ E-101 LMTD $\geq 5{ }^{\circ} \mathrm{C}$
vi. E-103 LMTD $\geq 5{ }^{\circ} \mathrm{C}$
vii. E-105 $\mathrm{LMTD} \geq 5{ }^{\circ} \mathrm{C}$

The significance of the above constraints can be explained as follows. The FG flow rate of $310 \mathrm{MT} / \mathrm{h}$ represents the maximum load that the RGP can take. The constraints on SG are as specified by the customer. Specifically, the constraints on gross heating value (GHV) and specific gravity are to ensure the quality of the SG. The maximum $\mathrm{CO} 2$ content of less than $2.0 \mathrm{~mol} \%$ is to satisfy the environmental regulation imposed by the major customers. The minimum SG flow rate of 295 thousands cubic meters per hour $(\mathrm{MCM} / \mathrm{h})$ is the load demanded by the customers. The flooding constraint on C-101 is to ensure good separation of the $\mathrm{C} 1$ from the heavier NGLs. The maximum capacities (UAs) of cold boxes are taken as per design. The log-mean temperature difference (LMTD) of greater than $5{ }^{\circ} \mathrm{C}$ is to prevent temperature cross violation at the cold boxes.

The maximization of the profit function is performed by manipulating the optimization variables, which are initially normalized with respect to their respective low and high bounds. The optimization variables, as well as their corresponding units and bounds are listed below:
a. 150 [ FG-A[ $310 \mathrm{MT} / \mathrm{h}$
b. $0[\mathrm{FG}-\mathrm{B}[150 \mathrm{MT} / \mathrm{h}$
c. $0[\mathrm{FG}-\mathrm{C}[100 \mathrm{MT} / \mathrm{h}$
d. 0 [ SR-1 [ 1
e. $0[\mathrm{SR}-2[1$
f. $10[$ TE Pout $[30$ bar
g. $\quad 100[\mathrm{E}-102 \mathrm{Q}[5000 \mathrm{~kW}$
h. $100[\mathrm{E}-106 \mathrm{Q}[2000 \mathrm{~kW}$
i. $100[\mathrm{~K}-102 \mathrm{Q}[5000 \mathrm{~kW}$

The optimization problem is solved using the built-in Box, SQP and/or Mixed algorithms in HYSYS [6]. The procedure ('figure 2') is stopped when one of the following necessary conditions for optimality is achieved:

1. The chosen algorithm reaches convergence, or

2. A new state with higher profit value is attained for nonconvergence cases.

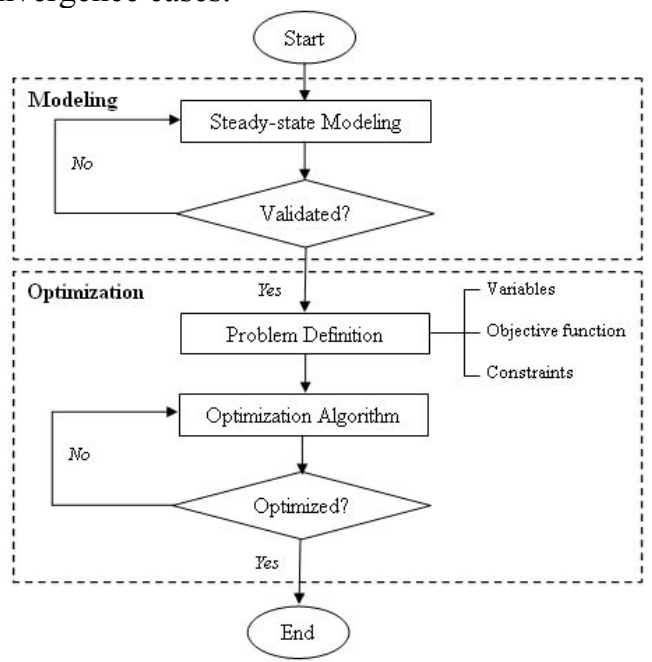

Figure 2. Modeling and optimization process flowchart

\section{Discussion OF RESUlts}

This section is divided into two parts. The first part deals with the operational matters. In the latter part, economic issues are discussed.

\section{a. Process}

Safety is an important aspect in plant operation. For this reason, buffers are often created to ensure reliability and smooth operation of the plant. The buffers are beneficial and can be turned into opportunities for enhancing plant performance. These opportunities may entail some of the plant assets to "sweat" as some variables may have to move closer to constraints. 'table 4' shows the moves of optimization variables in the attempts to shrink these buffers while 'table 5' displays the resulting constraint values of all cases:

For Case $1 \mathrm{~F}$, the changes in the variable values are as expected. The first step is to move the FG-A flow rate closer to the plant limit, which is $310 \mathrm{MT} / \mathrm{h}$. This way, more SG can be produced and delivered to the customer. At C-101, the average flooding only increases by about $3 \%$ from Case $1 \mathrm{~B}(60.22 \%)$. The $\mathrm{CO} 2$ presence in the $\mathrm{SG}$ is at $0.15 \%$, which is far below customer specification $(2 \%)$. This can be explained by the fact that FG-A is a lean gas with low $\mathrm{CO} 2$ content. 
Table 5: The constraints final values.

\begin{tabular}{|l|l|r|r|r|r|}
\hline Constraint & Unit & \multicolumn{1}{l|}{ Base } & 1-Feed & 2-Feed & \multicolumn{1}{l|}{ 3-Feed } \\
\hline FG flow & $\mathrm{MT} / \mathrm{h}$ & 280.00 & 304.00 & 310.00 & 310.00 \\
\hline SG GHV & $\mathrm{MJ} / \mathrm{Sm}^{3}$ & 38.12 & 38.15 & 38.19 & 38.23 \\
\hline SG specific gravity & - & 0.57 & 0.57 & 0.57 & 0.57 \\
\hline SG CO2 & $\mathrm{Mol} \%$ & 0.145 & 0.146 & 0.384 & 0.362 \\
\hline SG flow & $\mathrm{MCM} / \mathrm{h}$ & 325 & 353 & 355 & 354 \\
\hline C-101 flooding & $\%$ & 60.22 & 63.25 & 60.43 & 52.26 \\
\hline E-101 UA & $\mathrm{MJ} /\left(\mathrm{h}^{\circ}{ }^{\circ} \mathrm{C}\right)$ & 3520 & 6020 & 9190 & 5240 \\
\hline E-103 UA & $\mathrm{MJ} /\left(\mathrm{h}^{\circ} \mathrm{C}\right)$ & 885 & 1100 & 1040 & 914 \\
\hline E-105 UA & $\mathrm{MJ} /\left(\mathrm{h}^{\circ}{ }^{\circ} \mathrm{C}\right)$ & 499 & 493 & 352 & 289 \\
\hline E-101 LMTD & ${ }^{\circ} \mathrm{C}$ & 12.66 & 8.04 & 5.47 & 9.62 \\
\hline E-103 LMTD & ${ }^{\circ} \mathrm{C}$ & 17.42 & 17.55 & 18.73 & 20.24 \\
\hline E-105 LMTD & ${ }^{\circ} \mathrm{C}$ & 13.79 & 13.63 & 10.73 & 6.58 \\
\hline
\end{tabular}

Table 4: The final values of optimization variables.

\begin{tabular}{|c|c|c|c|c|c|}
\hline Variable & Unit & Base & 1-Feed & 2-Feed & 3-Feed \\
\hline FG-A & $\mathrm{MT} / \mathrm{h}$ & 280.00 & 304.00 & 290.00 & 288.05 \\
\hline FG-B & $\mathrm{MT} / \mathrm{h}$ & 0.00 & 0.00 & 20.00 & 15.28 \\
\hline FG-C & $\mathrm{MT} / \mathrm{h}$ & 0.00 & 0.00 & 0.00 & 6.67 \\
\hline SR1-1 & - & 0.10 & 0.09 & 0.05 & 0.03 \\
\hline SR2-1 & - & 1.00 & 0.96 & 0.83 & 0.90 \\
\hline TE Pout & Bar & 23.08 & 22.49 & 22.80 & 23.03 \\
\hline E-102Q & $\mathrm{kW}$ & $\begin{array}{r}4600.0 \\
0\end{array}$ & 4247.50 & 4300.00 & 4513.09 \\
\hline K-102Q & $\mathrm{kW}$ & $\begin{array}{r}3000.0 \\
0 \\
\end{array}$ & 2800.00 & 3000.00 & 2800.00 \\
\hline E-106Q & $\mathrm{kW}$ & $\begin{array}{r}2000.0 \\
0 \\
\end{array}$ & 1027.14 & 600.00 & 100.00 \\
\hline
\end{tabular}

The RGP temperature is mostly "controlled" by refrigeration cooler (E-102). The higher the cooler duty (E102Q), the colder the RGP becomes. Specifically, the increase in E-102Q will drop the first flash vessel (S-101) temperature. In turn, this will yield more liquid fraction containing mostly NGLs at the bottom. On the other hand, some of these NGLs will escape to the S-101 vapor outlet and go to E-103 at higher temperature. This condition is undesirable because it may cause temperature cross violations at this cold box and/or indirectly at E-101 and E105. For Case $1 \mathrm{~B}$, the $\mathrm{E}-102 \mathrm{Q}$ is set at $4600 \mathrm{~kW}$ corresponding to S-101 temperature of $-46.6{ }^{\circ} \mathrm{C}$. For Case $1 \mathrm{~F}$, the duty is lowered to $4248 \mathrm{~kW}$ in order to reduce the operating expenditure (OPEX).

The next step is to promote additional FG to pass through the turbo-expander (KT-101) and Joule-Thompson (J/T) valve. In this case, the first split ratio (SR1) decreases by $1 \%$. In terms of mass, it means that more FG is available to induce rotation of the KT-101 blades as opposed to exchanging heat at E-105. The second split ratio (SR2) drops by $4 \%$. At the same time, the turbo-expander outlet pressure is reduced from 23.08 to 22.49 bar. Hence more rotational energy can be supplied by KT-101 to the mechanically linked compressor (K-101). This shows that a right balance in expansion work between KT-101 and J/T valve has been found.

The final optimization steps are to adjust the SG pressure and temperature so that they just meet the customer requirements. In this case, the minimum pressure is 30 bar and the maximum temperature is $50{ }^{\circ} \mathrm{C}$. For Case $1 \mathrm{~B}, 3000$ $\mathrm{kW}$ of compressor duty $(\mathrm{K}-102 \mathrm{Q})$ is used to increase the $\mathrm{SG}$ pressure from 23.67 at K-101 outlet to 31.39 bar. The K$102 \mathrm{Q}$ is reduced to $2800 \mathrm{~kW}$ for Case $1 \mathrm{~F}$ such that the final
SG pressure settles at 30.23 bar. The SG temperature for Case $1 \mathrm{~B}$ is $38.37{ }^{\circ} \mathrm{C}$. This overcooling is obtained at a cooler duty (E-106Q) of $2000 \mathrm{~kW}$. For Case 1F, the E-106Q is decreased by about half to give a final SG temperature of $45.63{ }^{\circ} \mathrm{C}$, which is closer to the constraint.

Similar explanations hold true for Cases $2 \mathrm{~F}$ and $3 \mathrm{~F}$. The only notable difference is that there are more than one FG streams to consider. The two additional streams are rich gases (higher NGLs contents) with medium and high $\mathrm{CO} 2$ levels. FG-B is a typical raw natural gas coming from an older gas field while FG-C is obtained from a refinery or crude oil terminal off-gas. To achieve the economic optimum, the main focus is on varying the FG flow rates. This is carried out with the aim of extracting more SG and NGLs while curtailing CO2 level to the lowest possible at $\mathrm{SG}$ product stream. For Case $2 \mathrm{~F}$, a temperature cross violation occurs at E-101 partly due to the presence of 20 $\mathrm{MT} / \mathrm{h}$ of rich gas (FG-B). To rectify this problem, E-102Q is increased to $4300 \mathrm{~kW}$ from $4247 \mathrm{~kW}$ in Case $1 \mathrm{~F}$. This is done to maintain the S-101 temperature to be around $-44{ }^{\circ} \mathrm{C}$. Similarly for Case $3 \mathrm{~F}$, a temperature cross violation also takes place but this time at E-105. The violation is partly due to adding $6.67 \mathrm{MT} / \mathrm{h}$ of very rich gas (FG-C) to the RGP. Consequently, the E-102Q has to be further increased to $4513 \mathrm{~kW}$.

\section{b. Economics}

Profit is the bottom line of any plant economic consideration. This can be achieved by increasing revenues, decreasing expenses or both at the same time. In this work, economic data are collected from an actual GPP operating in a fixed regime environment. In other words, the FG supplies and product demands are agreed on long-term contract bases. Hence the FGs, SG and NGLs prices do not fluctuate as those in the daily spot market. Even the utility costs, which are due to fuel gas to generate steam, C3 refrigeration cycle, and electricity to run pumps, compressors and expanders, remain unchanged in the foreseeable future. In other words, an optimization project as illustrated in this work will unlikely hit cost overrun since the product values and OPEX are precisely known a priori.

The optimized economics for all case studies of the RGP are shown in 'figure 3'. For the base case (1B), the hourly profit is RM42,839. The revenues are obtained from the sales of SG (78.69\%), C2 (1.16\%), C3 (10.14\%) and C4 $(10.01 \%)$ as illustrated in 'figure 4 '. The expenses are mostly attributed to FG-A (97.58\%) ('figure 5'). 
In Case $1 \mathrm{~F}$, the portions of $\mathrm{SG}, \mathrm{C} 3$ and $\mathrm{C} 4$ in the pie chart may appear to increase only by $0.01 \%$ but that of $\mathrm{C} 2$ decreases by $0.02 \%$ ('figure 4 '). In actual fact, the revenues are up by $8.6 \%$ and exceeding RM100,000/h. This means that the size of the pie chart is enlarged by exactly the same percentage whilst the expenses also increase by $7.9 \%$. The majority is caused by the FG-A cost $(98.16 \%)$. The other $1.84 \%$ of the expenses is due to the major utilities' costs. In the end, the profit jumps to RM $46,835 / \mathrm{h}$ (up by $9.3 \%$ ) amid increments in both revenues and expenses.

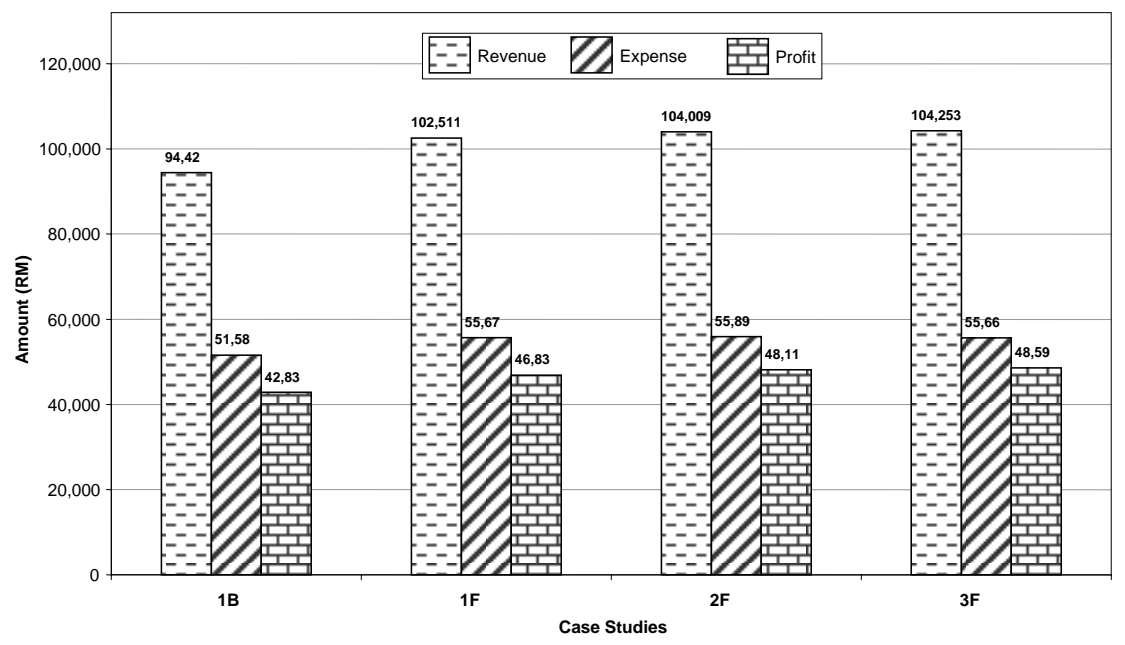

Figure 3. Economics of RGP

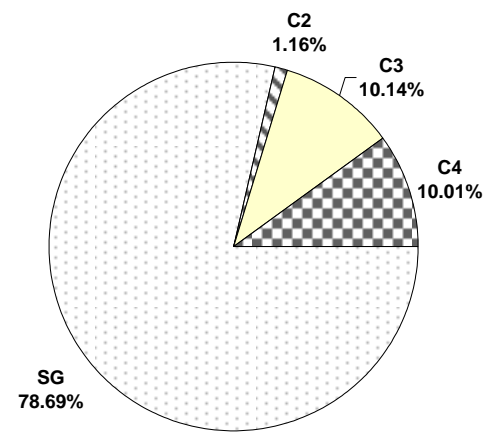

(a) Case 1B

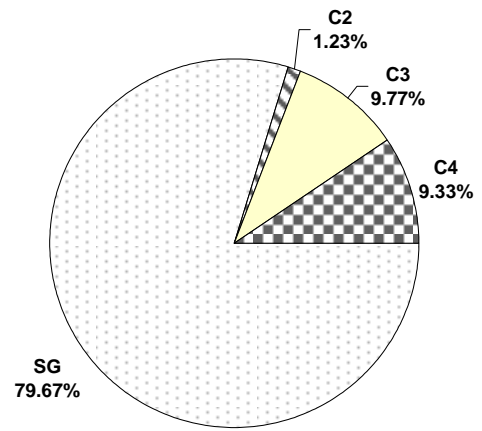

(c) Case $2 \mathrm{~F}$

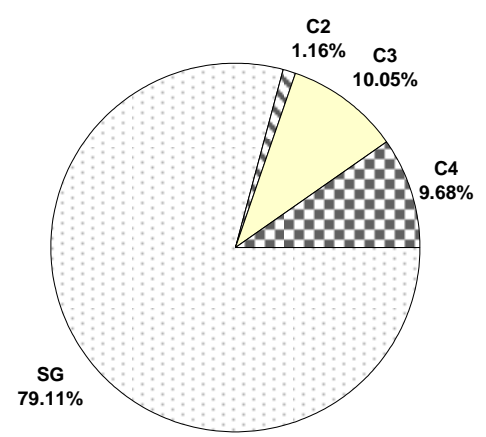

(b) Case $1 \mathrm{~F}$

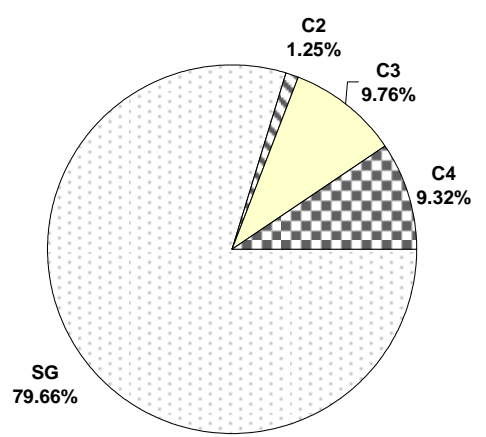

(d) Case 2F

Figure 4. Breakdown of GPP revenues 

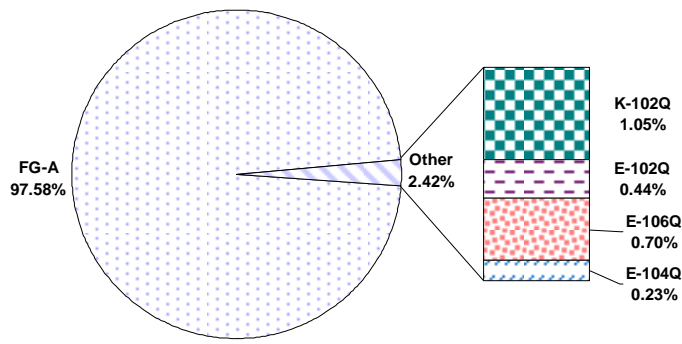

(a) Case 1B
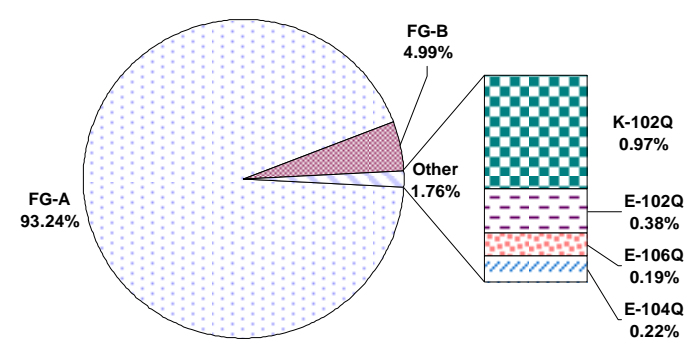

(c) Case $2 \mathrm{~F}$

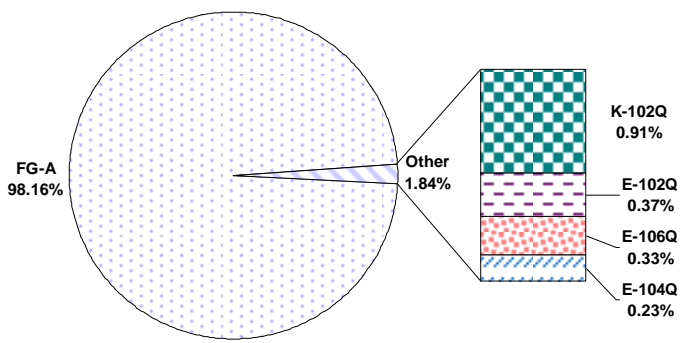

(b) Case $1 \mathrm{~F}$

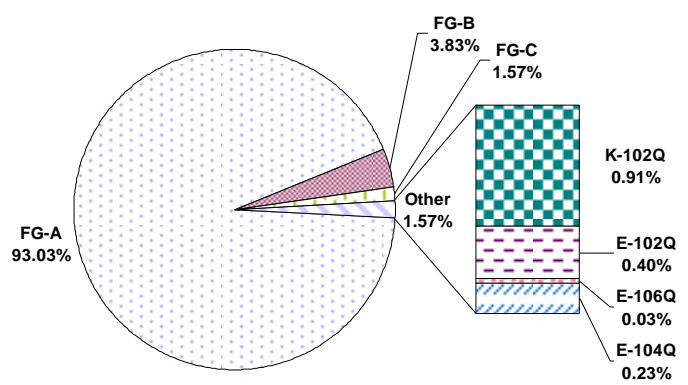

(d) Case 3F

Figure 5. Breakdown of RGP major expenses

For Case $2 \mathrm{~F}$, the revenues are up by $10.2 \%$. Although the expenses also increase by $8.4 \%$, the margin is higher by $12.3 \%$ to yield a profit of RM48,110/h. The major expenses are due to FG-A $(93.24 \%)$ and FG-B (4.99\%). The utilities only take up $1.76 \%$ of the total expenses.

For Case $3 \mathrm{~F}$, the profit is RM48,592/h or up by $13.4 \%$. This comes at the highest plant revenues (RM104,253/h) and moderate expenses (RM55,660/h). The majority of the expenses are again due to the costs of FGs.

\section{CONCLUSIONS}

Four case studies on the RGP are performed by integrating the L1 (operational) and L2 (economic) optimization problems. The RGP profit margin for Case $3 \mathrm{~F}$ is the highest as compared with the other three cases. Specifically, the profit is up by $13.4 \%$ when weighed against Case 1B. For Cases $1 \mathrm{~F}$ and $2 \mathrm{~F}$, the profit increments are $9.3 \%$ and $12.3 \%$, respectively. It is therefore concluded that the RGP should opt for adding two rich FG streams (FG-B and FG-C) to the existing one (FG-A). This can be performed by continuous reevaluation of the plant operating conditions at frequent intervals.

\section{ACKNOWLEDGMENT}

This work was inspired by the late Dr. VR Radhakrishnan, a Professor of Chemical Engineering at the Universiti Teknologi PETRONAS.

\section{REFERENCES}

[1] Konukman and Akman (2005). "Flexibility and operability analysis of a HEN-integrated natural gas expander plant," Chemical Engineering Science, 60, $7057-7074$.

[2] Naysmith, M. R. (1997). "Real-time Optimization of Chemical Processes - a Comparison of Closed versus Open Form Equations using Commercial Simulation Packages," Ph.D. Dissertation. University of Waterloo, Canada.

[3] Bullin, K. A. (1999). "Economic Optimization of Natural Gas Processing Plants Including Business Aspects," Ph.D. Dissertation. Texas A\&M University, USA.

[4] Bullin, K. A. and Hall, K. R. (2000). "Optimization of Natural Gas Processing Plants Including Business Aspects," Proceedings of the Seventy-Ninth Gas Processors Association Annual Convention. Atlanta, GA, USA.

[5] NaturalGas.org. (2007, July 10). [Online]. Available at http://www.naturalgas.org.

[6] Aspen Technology (2006). "Chapter 6: Optimizer Operation," HYSYS 2006 Operations Guide. Cambridge, MA, USA. 


\title{
EnergyPlus ${ }^{\circledR}$ Simulation Program to Evaluate the Performance of Sustainable Buildings?
}

\author{
Ooi Koon Beng and Azhaili Baharun
}

\begin{abstract}
Building Simulation tools can be classified as Knowledge-Based tools, Performance Evaluation Tools or Green Building Rating tools. The EnergyPlus ${ }^{\circledR}$ simulation engine is a performance evaluation tool that has been used to design the New York Times building and the Freedom Tower that will replace the World Trade Center in New York. It is updated every 6 months and Version 2.0, released in April 2007 adds new features that support low-energy buildings. It includes green roofs where research was done at the Portland State University, Phase change materials (PCM) with variable thermal conductivity and a temperature-enthalpy function incorporated in conduction finite difference solution algorithm, evaporative condenser option added to existing refrigerated case compressor racks including availability schedule and basin heater with controls and energy management system. Experiments will be conducted to validate the heat transfer properties of building surfaces with actual temperature measurements and weather data. Additional experiments will be conducted to validate the performance of cooling and heating coils that are used in the calculation of energy consumption of buildings. The capability of EnergyPlus to autosize components will also be verified.
\end{abstract}

Keywords: EnergyPlus ${ }^{\circledR}$, applications, new ideas, comfort, energy savings, sustainable buildings

\section{INTRODUCTION}

The EnergyPlus ${ }^{\circledR}$ simulation software is chosen to evaluate sustainable buildings because it is one of the softwares that has satisifed the Australian Building Greenhouse Rating (ABGR) validation protocol for computer simulation. This is a part of ENE-1, the conditional requirement in the Energy category to obtain a Green Star office rating from the Green Building Council of Australia (GBCA). EnergyPlus ${ }^{\circledR}$ was initiated in 1966, and is being developed under the Building Technologies Program of the U.S. DOE (Department of Energy)'s Energy Efficiency and Renewable Energy Building Technologies Program. It is based on two other successful programs, the Building Loads Analysis and System Thermodynamics (BLAST) tools and DOE-2, which have had histories of over 2 decades.

Since DOE-2 and BLAST were designed in the days of mainframe computers, expanding their capabilities further was becoming difficult, time consuming and expensive

Ooi Koon Beng and Azhaili Baharun are with the Engineering Faculty, University Malaysia Sarawak, 94300 Kota Samarahan, Sarawak, Malaysia.
(Crawley et al 1999). Workshops were also conducted for over 30 developers and 50 users and results from the surveys received from the participants, on many aspects of the requirement for a new generation language (Crawley et al 1997) resulted in the US Department of Energy decided to use Fortran 90/95, an object-oriented programming language to write EnergyPlus ${ }^{\circledR}$ to replace these two programs.

With the work of Michael Witte, Linda Lawrie, Fred Buhl, Brent Griffith and many others in the pioneer research team of EnergyPlus, Version 1.4, which runs on the Windows 98SE and up or Linux operating systems, was released in October 2006. Feedbacks through EnergyPlus_Support@yahoo.com are addressed and new versions are released every six months.

Since the aim of the developers of EnergyPlus ${ }^{\circledR}$ is to produce an accurate simulation engine for use internationally (it is available free from http://www.eere.energy.gov/buildings/energyplus) userfriendly interfaces have been left to others. DesignBuilder of UK is using EnergyPlus ${ }^{\circledR}$ as its simulation engine to make a commercial package for architects and green star professionals who want to have a fast way to evaluate buildings.

While trying to use EnergyPlus ${ }^{\circledR}$, it has been found beneficial to draw diagrams showing the nodes (points at which fluid properties are evaluated and passed on to subsequent equipment) Each zone has three nodes, the inlet node, the outlet node and the zone node. The inlet node matches the air supply node and the zone outlet node matches the return air node. Each node is given a separate number. If more than one zone is supplied by one air fan, then there is a splitter at the air supply and a mixer at the return air.

\section{A What is EnergyPlus $\AA$ ?}

\section{Inputs}

The input to the EnergyPlus ${ }^{\circledR}$ simulation engine is the idf file (with building dimensions, materials, constructions, etc.). The .idf files of the 1.4 .0 version of EnergyPlus ${ }^{\circledR}$ have 49 classes and each class have several objects. The variables (either alpha or numeric and are defined in an input data dictionary, idd file) are entered as fields of these objects. For some variables, where range checks are desired, 
maximum and minimum values are specified in these idd files which are created by the developers.

There is a Location-Climate-Weather File Access class and the DesignDays object which holds the date, the environment and the weather data of a location which is sufficient to run a meaningful simulation. If an ASHRAE weather data for that location exist, the DesignDays data can be extracted from the header of the ASHRAE weather file. Otherwise, one can make up the DesignDays data by taking local weather measurements. The use of DesignDays simulation allows the maximum, minimum and/or extreme conditions over a 24 hours period to be quickly evaluated.

The full year simulation, using the .epw file data and many schedule objects, allows the total annual cost and energy usage to be calculated, taking into account of all the working days, holidays, rainy, cloudy, sunny days, and seasonal variations. The result can also be tabulating in various formats, such as total, average, monthly, etc. using post-processing applications.

Since the weather data format of different countries are not the same, EnergyPlus ${ }^{\circledR}$ provides, as one of its preprocessor, an executable file to do the conversion to the required .epw format.

\section{Simulation algorithms}

Many accurate algorithms-engineering formulas specified using the Fortran engineering language--within the simulation engine act on these inputs to calculate the size (specifications of the power and air flow rates, say) of the air conditioner for heating or cooling the building zone for a particular day (called the design day) in winter or summer. EnergyPlus ${ }^{\circledR}$ is also able (the limit of its ability is the availability of engineering formulae) to calculate for many other parameters required by the designer.

The calculus that describes the engineering relationships for transient and steady state solutions are converted to state space or finite difference equations so that EnergyPlus ${ }^{\circledR}$ can be run by the modern digital computers that run on either the Windows or Linux operating systems.

Innovative methods of air conditioning like chilled beams, displacement ventilation and underfloor air distribution systems can also be simulated by EnergyPlus ${ }^{\circledR}$. The author is informed that three buildings on Hong Kong use displacement ventilation.

Levermore (1992) wrote a whole chapter on Low Energy air conditioning and lighting control. Displacement ventilation (cool air being introduced into the space from the bottom) and its control may be feasible in Malaysia. But because of the high humidity, chilled beams may create drops of water from the ceilings and may only be applicable in Australia.

Preliminary tests in Singapore, a tropical country with high humidity by Cheong et al (2004) show that occupants subjected to displacement ventilation do not feel that the air quality is good enough and experience a sick building syndrome, especially at the higher temperatures of the range of 20 to $26^{\circ} \mathrm{C}$ tested under displacement ventilation.

Outputs
Each simulation run generates many output files, some of which shows the integrity of the simulation. One of these files, the .err file, tells of errors (and warnings) that need corrections in the input files. The others are an audit file, a meter file, an input output file. Since these can be very big text files, which can be very difficult to read, the outputs are also available as spreadsheets (.csv files that can be viewed with Microsoft Excel) or html files. To help the user to visualize the building zones and HVAC, EnergyPlus ${ }^{\circledR}$ has two types of drawing files,.3-D dxf files of the buildings, which can be viewed by a CAD viewer like VoloView ${ }^{\mathrm{TM}}$ and spatial vector graphics (.svg) files which show the HVAC systems.

EnergyPlus's Group Simulation capability can simulate a group of input files with one weather file. The group of files can be files of the same building dimensions but of different construction materials. This would help the user see the effect of change in building parameters in order to optimize them.

\section{Example files}

Over 200 example files are available to learners of EnergyPlus ${ }^{\circledR}$. They are in a Microsoft Excel file with description, highlights of features and links to insert the idf files into EPLaunch, the user friendly input screen of EnergyPlus ${ }^{\circledR}$. Learners can also join the yahoo support group on the internet.

\section{Validation}

The Green Building Council in Australia (not the US Green Building Council) depends on the Australian Building Greenhouse Rating (ABGR) to find the $\mathrm{CO}_{2}$ emissions (based on the energy consumption for the past 12 months) contributed by an existing building. For buildings that are yet to be built, a simulation has to be done and EnergyPlus ${ }^{\circledR}$ is one of the softwares that satisfy the ABGR's Validation Protocol for Computer Simulations.

This Validation Protocol, aimed at ensuring that simulations provide useful and realistic assistance to the design process, stipulates that the simulation package must have passed either (i) the BESTEST validation test or (ii) be certified in accordance with ANSI/ASHRAE 140-2001, Standard Method of Test for Evaluation of Building Energy Analysis Computer Programs, or (iii) European Union draft standard EN13791 July 2000. EnergyPlus ${ }^{\circledR}$ (Oct 2006) released reports of its compliance with (i) and (ii).

Experiments are being conducted at University Malaysia Sarawak to independently validate the building envelope's heat transfer properties and the performance of the cooling coil used by EnergyPlus for air conditioners. Experiments will later be conducted in Melbourne winter months to extend this validation to the performance of heating coils. Wherever possible, current weather data will be used.

\section{USER FRIENDLY INTERFACES}

Because the developers (US DOE, Gard Analytics and Linda Lawrie) of EnergyPlus ${ }^{\circledR}$ concentrate on the accuracy of this simulation engine to make sure that simulation results will match the objectives of simulation as a design 
tool for sustainable buildings (modern and future buildings that will use renewable energy sources like building integrated photovoltaics, rainwater harvesting etc), user friendly interfaces are left to other software makers to add on to EnergyPlus ${ }^{\circledR}$.

DesignBuilder ${ }^{\circledR}$, UK, an architect's tool that quickly draws and shows a building, has incorporated EnergyPlus ${ }^{\circledR}$ as its engine to generate the energy (heat gains/losses, lighting, people occupancy and HVAC etc) of buildings based on the standard climates of the different parts of the world). This commercial software is being developed in tandem with new versions of EnergyPlus ${ }^{\circledR}$. Besides fixing bugs these new versions also have new features like water consumption of buildings etc.

DesignBuilder ${ }^{\circledR}$ also has templates for construction, glazing, lighting etc that follow the Energy Codes of UK.

\section{A Computational Fluid Dynamics (CFD)}

DesignBuilder (v1.2 uses EnergyPlus ${ }^{\circledR} 1.4$ as its engine was released in Dec 2006) uses the pressure coefficients of openings for its natural ventilation modeling. For more detailed analysis, or for buildings having 4 or more stories you should obtain specific pressure coefficient data from CFD analysis of from wind tunnel measurements, then it can be used as the data for pressure coefficients.

Besides the RoomAir Models class (which models several temperature patterns in the room and Mundt and several University of California San Diego model controls, EnergyPlus v1.4 has a new AirFlow Network System class that caters for natural ventilation, multizones and distribution.

The main difficulty to couple CFD softwares to Building simulation softwares lies in the period of the time steps. CFD softwares use time steps in the region of minutes and seconds while building simulation softwares use time steps in hours and minutes.

Nevertheless, Zhiqiang Zhai et al (2002) concluded that the convective heat transfer coefficient calculated by MITCFD is almost twice as large as the one originally determined in EnergyPlus and increased load requirements by about $10 \%$.

\section{COMFORT}

Many criteria of comfort of occupants are available. A Peoples Mean Vote (PMV) with a range from -4 to 4 where occupants of a building feel very hot, hot, warm, slightly warm, neutral, slightly cool, cool, cold or very cold is used in some documentation to define thermal comfort. In some cases the very hot and very cold are omitted and the range is then from -3 to 3. PMV is used in ISO7730 (1984).

Another measure of thermal comfort, which depends not only on room temperature, but of other variables like humidity, clothing etc described below, is PPD, percent people dissatisfied. It is obvious that the higher the PMV, the more people would be dissatisfied. At $0 \mathrm{PMV}, 5 \%$ of people are still dissatisfied because it is not possible to be perfect with about $2.5 \%$ of people uncomfortably cold and $2.5 \%$ uncomfortably hot.

The EnergyPlus ${ }^{\circledR}$ team adopted the findings of three noted researchers or research institutes, Fanger, Pierce and $\mathrm{KSU}$, into their simulation engine. Eleven variables that influence the condition of thermal comfort are common in the formulae of these three models.

Thermal resistance of the clothing $\left(\mathrm{I}_{\mathrm{Cl}}\right)$ and Metabolic Rate $\left(\mathrm{H} / \mathrm{A}_{\mathrm{Du}}\right)$ are two of these variables that are categorised as personal. Air Temperature (Ta), Mean Radiant Temperature (Tr), Relative Air Velocity (v) and Water Vapour Pressure in Ambient Air (Pa) are four environmental variables, and the five physiological variables are Skin Temperature $\left(\mathrm{T}_{\mathrm{sk}}\right)$, Core or Internal Temperature $\left(\mathrm{T}_{\mathrm{cr}}\right)$, Sweat Rate, Skin Wettedness (w) and Thermal Conductance $(\mathrm{K})$ between the core and skin.

Some of these variables are taken into account by EnergyPlus in four schedules, Activity Level Schedule, Work Efficiency Schedule, Clothing Schedule and Air Velocity Schedule. The remaining variables are calculated values that result from the overall simulation of conditions in the zone for each time step.

The Activity Level Schedule field is the name of the schedule that shows the amount of Metabolic Rate. The Metabolic rate for typing is 117 Watts per a male adult. For women adult and children, multiplication factors of 0.85 and 0.75 are used.

The Work Efficiency Schedule field is the name of the schedule with hourly (or less) values of between 0 and 1 . Zero means all the work is converted to heat and one means all the work done is converted to mechanical energy.

The Clothing Schedule field is the name of the schedule shows the amount of clothing (in units of Clo, where 1 Clo $=0.155 \mathrm{Km}^{2} / \mathrm{W}$ and $0 \mathrm{Clo}$ is nude to $1.5 \mathrm{Clo}$ is heavy suit) being worn by a typical occupant during the various times of the simulation.

The Air Velocity Schedule field is the name of the schedule that shows the air flow (in meters per second) in the space.

The EnergyPlus ${ }^{\circledR}$ engine has a separate object to show the (non-discrete) results of the feeling of thermal comfort for each of these three comfort models. They are triggered to calculate (or not) by the data that is entered into the People object. You also need to specify that the simulation output reports the variables associated with the comfort models (e.g. FangerPMV).

Besides the above three models of thermal comfort, EnergyPlus ${ }^{\circledR}$ is also able to report whether a zone is comfortable or not, based on Figure 5.2.1.1 (humidity ratio plotted against operative temperature) of ASHRAE 55-2004 using a simplified average of the air temperature and mean radiant temperature as the operative temperature, described below.

\section{A Simplified ASHRAE 55-2004 Figure 5.2.1.1}

The range of humidity ratio for comfort is from 0.00 to 0.012 . In winter, the occupants are assumed to wear 1.0 clo clothing. The comfortable operative temperature (based on a) at the lower limit of zero humidity ratio ranges from 21.7 to $26.3^{\circ} \mathrm{C}$ and at the upper limit of 0.012 humidity ratio, this comfortable operative temperature ranges from 19.6 to $23.9^{\circ} \mathrm{C}$. In summer the occupants are assumed to wear 0.5 
clo clothing. The comfortable operative temperature at the lower limit of zero humidity ratio ranges from 25.1 to $28.3^{\circ} \mathrm{C}$ and at the upper limit of 0.012 humidity ratio, the comfortable operative temperature ranges from 23.6 to $26.8^{\circ} \mathrm{C}$. On a psychometric chart of humidity ratio over operative temperature, these regions appear as parallelograms that overlap slightly.

Another commercial package, Ecotect ${ }^{\circledR}$ accounts for the activity level of the occupants by moving the ASHRAE comfort region. When the occupants do more active work (move more), the regions are moved left to a lower temperature, and when the occupants are more sedentary, the comfort regions are moved right to a higher temperature.

Malaysian standard MS1525:2001 states that the temperature for comfort should be between $23^{\circ} \mathrm{C}$ and $26^{\circ} \mathrm{C}$ with a $60-70 \%$ relative humidity range. The Singapore Indoor Air Quality's temperatures for comfort are $0.5^{\circ} \mathrm{C}$ less than those for Malaysia with the same upper limit of $70 \%$ for relative humidity. There is no lower limit for relative humidity in the Singapore standard, which is identical to the ASHRAE 55-2004 standard.

The 2004 version of ANSI/ASAHRAE 55 Thermal Environmental Conditions for Human Occupancy has also adopted the PMV method. It has also included an optional method for determining acceptable thermal conditions in naturally conditioned spaces. To use this method, spaces must have operable windows that can be opened by occupants.

"The adaptive model gives engineers a new tool, giving them more flexibility in deciding when and where full HVAC is required in a building and under what circumstances they can incorporate new energy conserving strategies without sacrificing comfort" said Gill Brager the vice chair of ASHRAE 55.

\section{Sustainable BuILDings}

\section{A Natural Ventilation}

When a building is naturally ventilated, no energy is used to condition the air. The energy embodied in the building materials is matter that is not dealt with in this paper. A near perfect sustainable building (perfect sustainability would mean no use of energy at all, since once energy is expended, the future generation will have less energy than the present generation, which is a strict definition of sustainability) should use only energy derived from solar, wind or waves.

This type of air conditioning has been tried on the San Francisco Federal Building (Carilho et al 2003). DesignBuilder's tutorial 2, which can be downloaded free for 30 days, also gives an example of naturally ventilated building and the effect of some mechanical air conditioning when the weather temperature is too hot.

EnergyPlus ${ }^{\circledR}$ provides objects on the Mundt and various UCSD displacement ventilation models.

Wind is the niche area of the Engineering faculty in the Nibong Tebal campus of University Sains Malaysia (USM). The author will work collaboratively with USM when it comes to the effect of wind in tall buildings.

\section{B Basement}

An underground basement in a single storey building makes use of the more even ground temperature to lower the fluctuation in air temperature of the building, but the construction cost is high. Though EnergyPlus ${ }^{\circledR}$ has an accurate program to simulate the effects of basement or buildings that are coupled to the ground, raised houses, on stilts or otherwise, to provide natural drafts have proved to be very comfortable dwellings in the tropics.

\section{Thermal Storage Walls}

Buildings with walls or internal structures that have high thermal storage, known as Trombe (Ellis 2003) walls in the US, absorbs heat on a hot summer day and gives out heat during the cooler night.

This method, (most probably adopted by cave dwellers from experience), can be applied to modern buildings and EnergyPlus ${ }^{\circledR}$ has taken initiatives to determine the location and size of these walls within the building and predict both the air and radiant temperature in the building zone.

\section{Photovoltaic}

Photovoltaics, is treated separately from solar collectors by EnergyPlus ${ }^{\circledR}$. While solar collectors only use the sun's energy to heat up water for the house, BIPV's are able to generate electricity and be sold to the main grid supplier.

In Malaysia, the Energy-10 simulation software, (Danida 2006) which was developed for $10,000 \mathrm{ft}^{2}$ building (Crawley et al 2005) was used to simulate the Low Energy Office (LEO). The LEO of Malaysia complies with MS1525, the Malaysian Code of Practice on Energy Efficiency and use of Renewable Energy for non-residential buildings.

Pusat Tenaga Malaysia (PTM), the Malaysian Center of Energy will move into its Zero Energy Office (ZEO) in mid 2007. The requirement for this building (ISBN 983-40995$7-6,2006)$ is that it must not consume more electricity (shown red in Figure 1 below) than it can produce using renewable energy sources on site. To achieve this, the ZEO has solar collectors on its roof and MBIPV, the Malaysian Building Integrated Photovoltaic Technology, Application Project SURIA, is also under the same authority of the Malaysian government, the PTM.

Building simulation for the LEO was done using Energy10 while the IES Virtual Environment software was used to simulate the ZEO. The PTM has allocated half a year after it occupies the ZEO to validate the results of the IES Virtual Environment.

Figures 1 and 2 respectively show the progress in the reduction of energy consumption of buildings in Malaysia and a picture of the Zero Energy Building.

\section{A Sustainable Building in Australia}

This section will use Australia's Melbourne Council House $2(\mathrm{CH} 2)$ in Victoria, which has achieved a 6 star energy rating from GBCA, as an example of what other features are possible with a sustainable building.

$\mathrm{CH} 2$ started construction in 2004, uses about 26 square metres of photovoltaic cells on the roof to generate about $3.5 \mathrm{~kW}$ of electricity from the sun's energy. This energy will power the movement of the louvers used to shade the west façade. 


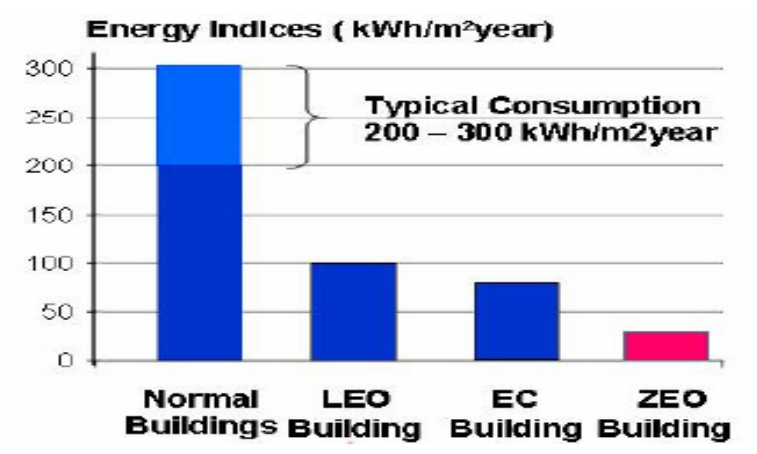

Figure 1. Continuous improvement of energy efficiency of buildings in Malaysia

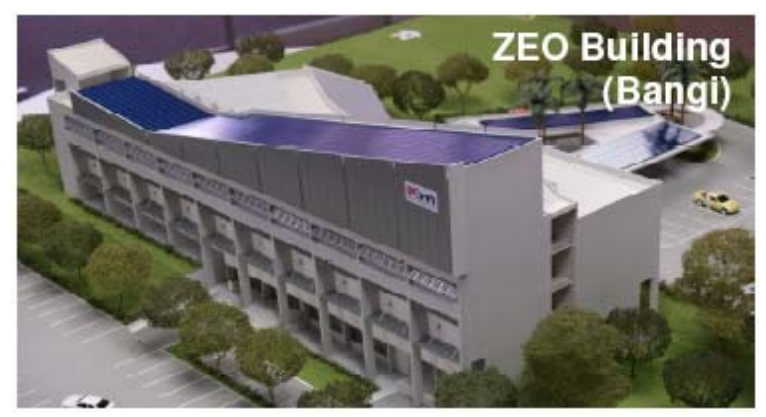

Figure 2. Picture of the Malaysian ZEO with the solar panels on the roofs

\section{A Wind}

$\mathrm{CH}_{2}$, which is rated as six-star building by the Green Building Council of Australia, has six wind turbines that extract air from the offices spaces through ducts on the north façade. The turbines, especially designed for $\mathrm{CH}_{2}$, are $3.5 \mathrm{~m}$ high and replace electric fans that would normally carry out the same function.

\section{B Phase Change Material}

$\mathrm{CH}_{2}$ 's Phase Change Material (PCM) tank is much like a battery that stores coolness, or 'coolth'. Essentially the battery comprises a series of pipes containing the PCM. Each of the PCM pipes has a pipe running through it and another encasing it.

Phase change refers to the process of a material changing from a liquid to a solid or vice versa. Water is a PCM that freezes at $0^{\circ} \mathrm{C}$. The PCM in the $\mathrm{CH}_{2}$ system will freeze at $15^{\circ} \mathrm{C}$.

Water cooled by the shower towers $\left(\mathrm{CH}_{2}\right.$ has five shower towers that shower water down a 3.5 storey enclosure to cool air and water through evaporative cooling) will travel through the tank and freeze the PCM. A separate stream of water will pass through the tank to be chilled, travel through the chilled ceiling panels and chilled beams to cool the building then run back into the tank to begin again.

During short periods in summer the system may have insufficient capacity and will be supplemented by a chiller.

\section{OTHER FEATURES OF $\mathrm{CH}_{2}$}

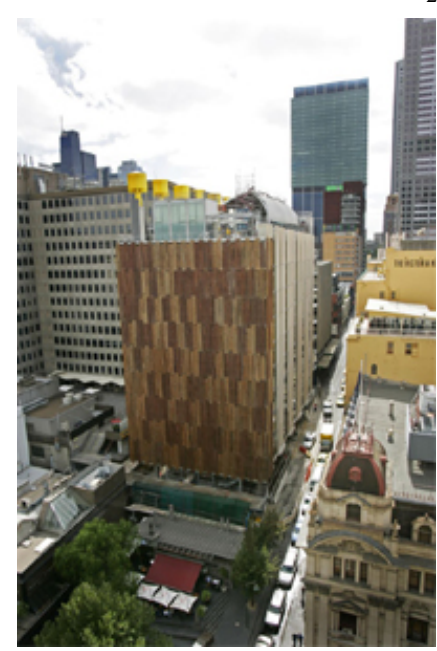

Figure 3. $\mathrm{CH} 2$ on February 2006 showing the timber clad walls

\section{A Low Energy Computing}

$\mathrm{CH}_{2}$ uses LCD monitors and thin client workstations, which consume $77 \%$ less energy than older, bulky CRT monitors and individual personal computers.

Thin client workstations allow office workers access to more centrally located powerful computing resources while reducing the number of computer processors. LCD monitors are energy efficient, anti-glare, do not flicker, do not emit radiation, produce less heat, generate less greenhouse gas in their operation and produce less pollution in their manufacture.

\section{B Low Energy Lighting}

The use of T5 light fittings for ambient lighting and individual task lighting for workstations will consume $65 \%$ less energy than the lighting system in the Council's current building.

\section{Electricity from Co-generation}

A gas-fired co-generation plant on the roof will be used to generate electricity and heat, reducing reliance on the public electricity grid.

The co-generation plant will have much lower $\mathrm{CO} 2$ emissions than coal-fired electrical generation and will provide $60 \mathrm{kVA}$ of electricity, meeting about 30 per cent of $\mathrm{CH}_{2}$ 's electricity needs.

\section{Heat from Co-generation}

Heat from the co-generation plant (about $100 \mathrm{Kw}$ ) will be used to help $\mathrm{CH}_{2}$ 's air conditioning plant. This heat can be used directly for heating or, via an absorption chiller, for cooling. It is estimated the co-generation plant will satisfy $80 \%$ of the building's fresh air heating/cooling requirements just by using waste heat.

\section{E Heat Recovery}

Heat is recovered from the air that gets exhausted out the offices. $\mathrm{CH}_{2}$ 's fresh air system uses no re-circulated air so fresh air from outside needs to be constantly heated or cooled to be supplied at $18^{\circ} \mathrm{C}$. Through a simple heat 
exchange process, the temperature of the air exhausted from the space is used to help heat or cool the fresh supply air.

\section{F Solar Hot Water}

About 60 per cent of the hot water supply will be provided by 48 square metres of solar hot water panels on the roof. On days with little solar heat gain, a gas boiler will heat water instead.

\section{CONCLUSION}

The author will validate some objects of EnergyPlus ${ }^{\circledR}$ using buildings in Kuching, Sarawak, Malaysia and Melbourne, Victoria, Australia with actual measurements of temperature and energy consumption. The author will use the knowledge gained in this exercise to develop building simulation expertise in order to help determine sustainable, energy efficient buildings while monitoring the comfort level of the occupants. The verification of the autosizing features of EnergyPlus will be discussed.

The author will also simulate the effect of innovatively using the lifts of tall buildings as leaky pistons to assist in natural ventilation as a contribution to passive cooling.

\section{ACKNOWLEDGEMENT}

The author would like to acknowledge the help and advice that he got from Azhaili Baharun, Drury B Crawley, John Wilson, Li Yuan Lu, Julia Lamborn and many others he has asked for help from, too many to list here.

\section{NOMENCLATURE}

\section{ABGR: Australian Building Greenhouse Rating}

ASHRAE: American Society of Heating, Refrigeration and Airconditioning Engineers

BLAST: Building Loads Analysis and System Thermodynamics program. It was superseded by iBLAST and now by EnergyPlus.

$\mathrm{CH} 2$ : Council House 2 in Melbourne Victoria, Australia.

DOE-2: Short form of the second version of the Building Simulation program by the US Department of Energy. It has been around for over 2 decades and is being replaced by EnergyPlus.

\section{GBCA: Green Building Council of Australia}

HVAC: Heating Ventilation and Air Conditioning

\section{LEO: Low Energy Building}

Nodes: points at which fluid properties are evaluated and passed on to subsequent equipment

\section{PCM: Phase Change Material}

\section{ZEO: Zero Energy Building}

Zone: A building can be divided into a few thermal zones, and each zone is separately analysed

\section{REFERENCES}

[1] AusIndustry Technical Research Papers 2006. GBCA AusIndustry Project Green Star Diffusion 2005 Mechanical Engineering.

[2] Carrilho da Graca G, Linden PF et al. Design and Testing of a Control Strategy for a Large Naturally Ventilated Office Building.
Proceedings of the Eighth International IBPSA conference in Eindhoven, Netherlands, 2003, Pp 399-406

[3] Cheong KW.D, Yu WJ, Tharn KW, Sekhar SC, Kosonen R. 2006. A study of perceived air quality and sick building syndrome in a field environment chamber served by displacement ventilation system in the tropics. Building and Environment 41 (2006) pp1530-1539

[4] Crawley, DB, Hand JW, Kummert, M, Griffith BT, 2005 'Contrasting the capabilities of building energy performance simulation programs. Version 1.0 .July 2005'. U.S. Department of Energy, Energy Efficiency and Renewable Energy, Building Technologies Program. viewed 18 February 2006, http://www.eere.energy.gov/buildings/energyplus/

[5] Crawley DB, Lawrie L, Winkelmann FC, Buhl WF, Erdem AE, Pederson CO, Liesen RJ, Fisher DE, Srand RK, Taylor RD, What Next for Building Energy Simulation - A Glimse of the Future Building Simulation 19978 pages from 92

[6] Crawley DB, Lawrie L, Pederson CO, Liesen RJ, Fisher DE, Srand RK, Taylor RD, Winkelmann FC, Buhl WF, Erdem AE, Huang YJ. EnergyPlus A New Generation Building Energy Simulation Program. Building Simulation 19998 Pages

[7] Crawley DB, Hand JW, Lawrie L Improving the weather information available to Simulation Programs. Proceedings of Building Simulation 1999. 8 pages

[8] Danida Project in Malaysia RE\&EE Component. Sub Component II, EE in Buildings MONITORING AND EVALUATION OF THE LEO BUILDING Report January 2006

[9] Ellis PG Development and Validation of the Unvented Trombe Wall Model in EnergyPlus. MSc Thesis Graduate College of the University of Illinois 2003

[10] Ellis PG and Torcellini PA Simulating Tall Buildings using EnergyPlus Proceedings of the Ninth International IBPSA conference Montreal Canada, 2005 Pp 279-286.

[11] EnergyPlus Testing with Building Thermal Envelope and Fabric Load Tests from ANSI/ASHRAE Standard 140-2004, EnergyPlus Version 1.4.0.025, October 2006

[12] EnergyPlus Testing with HVAC Equipment Performance Tests E100 to E200 from ANSI/ASHRAE Standard 140-2004, EnergyPlus Version 1.4.0.025, October 2006

[13] EnergyPlus Testing with HVAC BESTEST Part 2 - Tests E300 to E545, EnergyPlus Version 1.4.0.025, October 2006.

[14] EnergyPlus Testing with Fuel-Fired Furnace HVAC BESTEST, EnergyPlus Version 1.4.0.025, October 2006.

[15] EnergyPlus ${ }^{\circledR} \quad$ Documentation Input-Output Reference Manual,Engineering Reference Manual

[16] Erik L. Olsen and Qinyan (Yan) Chen Energy consumption and comfort analysis for different low-energy cooling systems in a mild climate Energy and Buildings Volume 35, Issue 6, July 2003, Pages $560-571$

[17] Green Building Council of Australia, 2005, Technical Manual Green Star - Office Design.

[18] IEN Independent Energy Network Sdn Bhd. http://www.ien.dk/Brochure\%20in\%20English,\%20FINAL.pdf viewed $19^{\text {th }}$ Jan 2007. ISBN 983-40995-7-6 Achieving Industrial Energy Efficiency in Malaysia viewed 15 January 2006, http://www.undp.org.my/uploads/Achieving_Industrial_Energy_Effic iency_2006.pdf

[19] Levermore G.J. Building Energy Management Systems Applications to low-energy HVAC and natural ventilation control 1992

[20] MS 1525:2001 - Code of Practice on Energy Efficiency and use of Renewable Energy for non-residential buildings

[21] Zhai Z, Chen Q, Haves $\mathrm{P}$ and Klems JH, 'On approaches to couple energy simulation and computational fluid dynamics programs' Building and Environment 37 (2002) 857-864.

[22] Zhang LZ and Niu JL 'Indoor humidity behaviours associated with decoupled cooling in hot and humid climates' Building and Environment 38 (2003) 99-107

[23] Zhiqiang Zhai, Qingyan Chen, Philip Haves and Joseph H. Klems On approaches to couple energy simulation and computational fluid dynamics programs Building and Environment Volume 37, Issues 8-9 August-September 2002, Pages 857-864. 


\title{
Biodiesel Production from Different Used Vegetable Cooking Oil as Environmental Recycling Process and Economic Source-A Review
}

\author{
A.B.M. Sharif Hossain, Amru Nasrulhaq Boyce, Abu Saleh Ahmed, Haji Mohamed A. Majid, S. \\ Chandran, and R. Zuliana
}

\begin{abstract}
The use of biomass including their wastes can contribute to mitigate the environmental burden already being faced by our society. Converting waste oils to biodiesel fuel for diesel engines in transportation sector is considered as important step in term of recycling and reusing material, and reducing $\mathrm{CO}_{2}$ emission equivalent to the amount that is produced when petroleum derived diesel fuel is used. This review concerns biodiesiel production techniques and quality assessment of different waste vegetable oils. Biodiesel (fatty acid ethyl ester) is a nontoxic and biodegradable alternative fuel that is obtained from renewable sources. Biodiesel production from different bioresources or bimasses partially developed for diesel engine and has been applied recently to measuring the fuel properties from agricultural commodities. Biodiesel from virgin oil, in comparison to petroleum-based diesel fuel, is expensive cost of manufacturing, primarily the raw material cost. Used cooking oil is one of the economical sources for biodiesel production. However, the products formed during frying, such as free fatty acid and some polymerized triglycerides can affect the transesterification reaction and the biodiesel properties. The biodiesel obtained from waste cooking oil has been reported better engine performance and less emission when tested on commercial diesel engines. Interrelationships between the quality of
\end{abstract}

The financial support for this project funding from Science Fund, MOSTI, Malaysia is gratefully acknowledged.

A.B.M. Sharif Hossain is with the Biofuel Production Project, Department of Biotechnology and Biochemistry, Institute of Biological Science, University of Malaya. Kuala Lumpur, Malaysia (phone: +60379674356; fax: +60379674178; e-mail: sharif@um.edu.my).

Amru Nasrulhaq Boyce is with the Biofuel Production Project, Department of Biotechnology and Biochemistry, Institute of Biological Science, University of Malaya. Kuala Lumpur, Malaysia.

Abu Saleh Ahmed is with the Department of Mechanical and Menufacturing Engineering, Faculty of Engineering, Uiversiti Malaysia Sarawak, 94300 Kota Samarahan, Sarawak, Malaysia (email: aasaleh@feng.unimas.my).

Haji Mohamed A. Majid is with the Biofuel Production Project, Department of Biotechnology and Biochemistry, Institute of Biological Science, University of Malaya. Kuala Lumpur, Malaysia.

S. Chandran is with the Biofuel Production Project, Department of Biotechnology and Biochemistry, Institute of Biological Science, University of Malaya. Kuala Lumpur, Malaysia.

R. Zuliana is with the Biofuel Production Project, Department of Biotechnology and Biochemistry, Institute of Biological Science, University of Malaya. Kuala Lumpur, Malaysia. biodiesel and production resources are described. This paper reviews the current methodology for measuring and characterizing the biodiesel production and engine performance. Emphasis is placed upon production quality and sources of variation in test results due to such factors as viscosity, cloud point and flash point. The present paper attempts to review methods for the transesterification of waste cooking oil and the performance of biodiesel obtained from waste cooking oil in a commercial diesel engine. The paper also examines the basic chemical components (sulphur, lead, carbon residue, free glycerin, phosphorus content) involved during the process on biodiesel quality.

Keywords: biomass, waste oils, biodiesl production, engine performance

\section{INTRODUCTION}

Bioenergy is one of the key option to mitigate greenhouse gas emissions and to substitute fossil fuels [1]. The need of energy is increasing continuously, because of increases in industrialization and population. The basic sources of this energy are petroleum, natural gas, coal, hydro, and nuclear [2]. The United States consumes 178 million tonnes of diesel fuel annually, and the global consumption is 934 million tonnes of diesel fuel per year [3]. Report shows Canada emitted 731 million tonnes of greenhouse gases [4]. Currently, North Americans consume 390 million tonnes of gasoline and 10 million tones ethanol [5]. The major disadvantage of using petroleum-based fuels is atmospheric pollution created by the use of petroleum diesel. Petroleum diesel combustion is a major source of greenhouse gas (GHG). Apart from these emissions, petroleum diesel is also major source of other air contaminants including NOx, SOx, $\mathrm{CO}$, particulate matter, and volatile organic compounds (VOCs) [6]. The decreasing fossil fuel reserves, and the atmospheric pollution created by petroleum-based fuels, have necessitated the need for an alternative source of energy.

Biomass is one of the better sources of energy [2]. Largescale introduction of biomass energy could contribute to sustainable development on several fronts, environmentally, socially and economic [7]. Biomass feedstock originating from agricultural waste or energy crop can be converted 
thermochemically or fermentatively to a variery of biofuels, such as ethanol, $\mathrm{H}_{2}$ and biodiesel. Hydrogen is known as a clean energy carrier that is recyclable, efficient and does not produce greenhouse gases while combustion [8]. Fuels from renewable biomass have the potential to reduce the amount of $\mathrm{CO}_{2}$, particulate matter, and $\mathrm{GHG}$ emissions. This is because the carbon contained in biomass-derived fuel is biogenic and renewable [3]. Therefore, petroleum-based fuels can be complemented by fuels obtained from renewable sources. Many researchers have tried to develop vegetable-oil-based derivatives that approximate the properties and performance of petroleum-based diesel fuel.

Biodiesel (monoalkyl esters) is one of such alternative fuel, which is obtained by the transesterification of triglyceride oil with monohydric alcohols. It has been wellreported that biodiesel obtained from canola and soybean, palm, sunflower oil acts very well as a diesel fuel substitute [9]. Biodiesel is a nontoxic and biodegradable alternative fuel that is obtained from renewable sources. A major hurdle in the commercialization of biodiesel from virgin oil, in comparison to petroleum-based diesel fuel, is its cost of manufacturing, primarily the raw material cost. Used cooking oil is one of the economical sources for biodiesel production as renewable energy resources [2]. The cost of vegetable oil has a crucial role in the economics of the biodiesel. The significant factors that affect the cost of biodiesel are feedstock cost, plant size, and value of the glycerine byproduct [10]. It has been observed that one of the most crucial variables that affects the cost of biodiesel is the cost of the raw materials particular, biomass energy is considered the most promising alternative to fossil fuel [11, 12]. Waste cooking oil, which is much less expensive than pure vegetable oil, is a promising alternative to vegetable oil for biodiesel production. Restaurant waste oils and rendered animal fats are less expensive than food-grade canola and soybean oil [13]. Currently all these waste oils are sold commercially as animal feed. However, since 2002, the European Union (EU) has enforced a ban on feeding these mixtures to animals, because, many harmful compounds are formed during frying that back into the food chain through the animal meat [14]. Hence, the waste cooking oil must be disposed of safely or be used in a way that is not harmful to human beings.

The disposal of waste cooking oil is problematic, because disposal methods may contaminate environmental water [2]. Many developed countries have set policies that penalize the disposal of waste oil through the water drainage [15]. The production of biodiesel from waste cooking oil is one of the better ways to utilize it efficiently and economically. The data on the requirements of diesel fuel and availability of waste cooking oil in any country indicate that the biodiesel obtained from waste cooking oil may not replace diesel fuel completely. However, a substantial amount of diesel fuel can be prepared from waste cooking oil, such as palm, soybean, canola, rice bran, sunflower, coconut and corn oil which would partly decrease the dependency on petroleumbased fuel.

\section{MATERIALS AND METHODS}

Oil frying: Physical and chemical properties of the frying oil has been changed during frying. The oil (lipids) is an integral part of frying. In frying, oil is heated in air and in the presence of light at temperatures of $160-200{ }^{\circ} \mathrm{C}$ for relatively long periods of time. For economical reasons, the same oil/fat is used many times or continuously [2]. Generally, in public restaurants, frying is conducted in the same oil for several days; however, in household frying, fat is exchanged after several weeks [14]. Obviously, the conditions used for frying cause major physical and chemical changes in the oil, which differs from oil to oil, depending on their composition. Some common physical changes observed in vegetable oil after frying are (i) an increase in the viscosity, (ii) an increase in the specific heat, (iii) a change in the surface tension, (iv) a change in color, and (v) an increase in the tendency of fat to foam [14]. During frying, basically three types of reactions occur: thermolytic, oxidative, and hydrolytic $[13,16,17]$.

1) Thermolytic Reactions. A thermolytic reaction occurs in the absence of oxygen at very high temperatures. If the triglycerides that contain saturated fatty acids are heated at very high temperature $\left(180^{\circ} \mathrm{C}\right)$ in the absence of oxygen, then they produce series of normal alkanes, alkenes, lower fatty acids, symmetric ketones, oxopropyl esters, $\mathrm{CO}$, and $\mathrm{CO}_{2}$. In the case of glycerides, this reaction happens between acyl groups within the same molecule [13].

2) Oxidative Reactions. Unsaturated fatty acids may react with molecular oxygen via a free-radical mechanism. Hydroperoxides formed as a primary product during the reaction may further form many other compounds, such as isomeric hydroperoxides that contain conjugated diene groups. Hydroperoxides also produce many chemicals with a significant variation in molecular weight, flavor threshold, and biological significance [13].

3) Hydrolytic Reactions. The steam produced during the preparation of food causes the hydrolysis of triglycerides, resulting in the formation of FFA, glycerol, and monoglycerides and diglycerides (Nawar, 1984). The change in oil composition by the hydrolytic reaction can be quantified by measuring the monoglyceride and diglyceride content and not the FFA content of oil, because some of the FFA are lost during frying [17].

Step in production of Biodiesel:

Filtration, mixing alcohol $(\mathrm{EtOH} / \mathrm{MeOH})$ and catalyst $\left(\mathrm{NaOH}, \mathrm{KOH}, \mathrm{HCl}\right.$ and $\left.\mathrm{H}_{2} \mathrm{SO}_{4}\right)$, transesterification (reaction), Seperation, methyal/ethyal ester washing, drying and storage [18].

\section{A. Transesterification Methods for Waste Cooking Oil}

The transesterification of waste cooking oil can be performed using alkaline, acidic, and enzymatic catalysts. Depending on the undesirable compounds (especially FFA and water), each catalyst has its own advantages and disadvantages. General reaction mechanism (transesterification) of Biodiesel is given below: 

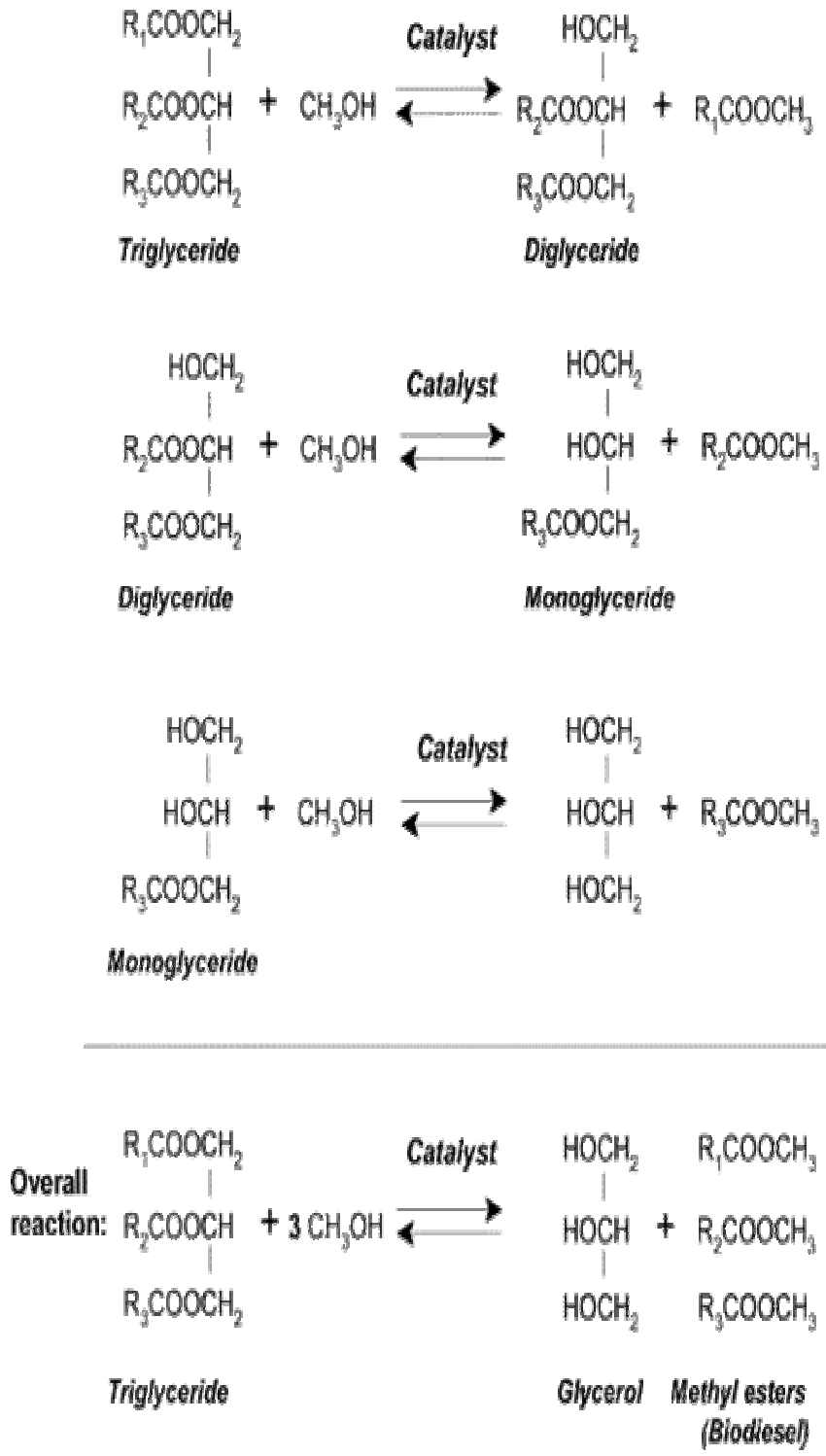

1) Alkali-Catalyzed Transesterification. Common alkaline catalysts (such as $\mathrm{NaOH}, \mathrm{KOH}$, and $\mathrm{NaOCH} 3$ ) are well-known for the transesterification reaction of edible oil [19]. The rate of the alkaline-catalyzed transesterification reaction is fast, compared to that using acids, and it is reported that the rate could be as high as 4000 times, compared to that using an acidic catalyst [19]. The application of an alkaline catalyst in the transesterification of waste cooking oil is somewhat limited, because the FFA in waste cooking oil reacts with the alkaline catalyst $(\mathrm{KOH}, \mathrm{NaOH})$ and forms soap. The soap formed during the reaction prevents the glycerol separation, which drastically reduces the ester yield. The water in waste cooking oil also affects the methyl ester yield by favoring a saponification reaction [20]. Range of $0.42-2.07$ using $\mathrm{NaOH}$ as a catalyst has been reported [21].

2) Acid-Catalyzed Transesterification: [22] It has been reported that an acid catalyst is insensitive to FFA and is better than the alkaline catalyst for vegetable oil with $>1 \%$ FFA. The only disadvantage of an acidic catalyst is a slower reaction rate. The transesterification of edible oil using strong acids such as sulfuric and hydrochloric acid are available in literature reports. The acid-catalyzed transesterification of pure soybean oil was studied by Canckci et al. [23]. The effects of the molar ratio of oil to methanol $(1: 3.3$ to $1: 30)$, reaction temperature $\left(25\right.$ to $\left.60{ }^{\circ} \mathrm{C}\right), \mathrm{H} 2 \mathrm{SO} 4$ concentration $(1 \%$ $5 \%$ ), reaction time (48 and $96 \mathrm{~h})$, water content $(0.1 \%$ $5 \%)$, and FFA amount (0\%-33\%).

3) Acid- and Alkali-Catalyzed Two-Step Transesterification. Acidic and alkaline catalysts have their own merits and demerits in the transesterification of waste cooking oil. A process was developed [23], where the high-FFA feedstock was initially treated using an acidic catalyst $\left(\mathrm{H}_{2} \mathrm{SO}_{4}\right)$ to reduce the FFA level to $<1 \%$. The pretreated feedstock (with $<1 \%$ FFA) was then transesterified with methanol, using an alkaline catalyst $(\mathrm{KOH})$. The effects of the type of alcohol (methanol and ethanol), the amount of acid catalyst $(0,5,15$, and $25 \mathrm{wt} \%)$, and reaction time $(1$, 15,30 , and $60 \mathrm{~min}$ ) on the reduction of the FFA level were studied with a synthetic mixture of $20 \%$ and $40 \%$ palmitic acid in soybean oil, as well with yellow grease $(12 \% \quad$ FFA $)$ and brown grease $(33 \%$ FFA $)$ as a feedstock [23].

4) Enzyme-Catalyzed Transesterification. Chemical (acid or alkali)-catalyzed transesterification of waste cooking oil has problems, such as pretreatment of feedstock, recovery of glycerol, removal of the catalyst, and the energy-intensive nature of the process (high stirring speed, and temperature required for good conversions). Enzyme (such as lipase)-catalyzed reactions have advantages over traditional chemical-catalyzed reactions: the generation of no byproducts, easy product recovery, mild reaction conditions, and catalyst recycling. Also, enzymatic reactions are insensitive to FFA and water content in waste cooking oil [24].

5) Catalyst-Free Technology for Transesterification. Novel methanolysis processes are being developed for the synthesis of biodiesel from vegetable oils using noncatalytic methods. Kinetic analysis of the transesterification reaction of rapeseed oil in subcritical and supercritical methanol revealed that the rate of formation of methyl esters from rapeseed oil increased dramatically in the supercritical state. At subcritical temperatures $\left(<239^{\circ} \mathrm{C}\right)$, the reaction rates are low, but at the supercritical state $\left(\right.$ at $350{ }^{\circ} \mathrm{C}$ ), the rate constant increased by a factor of $\sim 85 .{ }^{\circ} \mathrm{C}$ anda molar ratio of oil to methanol of $1: 42$ [25].

\section{RESULTS AND DisCUSSION}

Many studies have been conducted on production and performance of biodiesel. The performance of biodiesel obtained from waste cooking oil was compared with that of petroleum-based diesel fuel, mainly in terms of emissions, engine performance, and fuel consumption were studied [2].

\section{A. Biodiesel Production}

Steam injection and sedimentation treatment were performed on waste cooking oil, and the effects of such refining on the properties of the oil and the corresponding esters were studied [26]. Effects of these treatments on physical and chemical properties of waste cooking oil are shown in Table 1. Some of the important changes observed in the oil after first and second treatment were (i) a 
reduction in moisture content, (ii) a reduction in FFA, and (iii) a substantial reduction in viscosity and increase in energy value with treatment. The treated raw oil, obtained after treatment 1 (referenced hereafter as T1) and after treatment 2 (referenced hereafter as T2) was transesterified with methanol (6:1 molar ratio of methanol to oil) using $\mathrm{KOH}(1 \%)$ as a catalyst at $60{ }^{\circ} \mathrm{C}$.

Table 1. Effects of various treatments on the physical and chemical properties of waste cooking oil ${ }^{\mathrm{a}}$

\begin{tabular}{|l|l|l|l|l|l|l|}
\hline & \multicolumn{3}{l|}{ Batch 1 } & \multicolumn{3}{l|}{ Batch2 } \\
\hline Property & raw & T1 & T2 & raw & T1 & T2 \\
\hline Moisture content (\%) & 1.1 & 0.5 & 0.4 & 1.4 & 0.6 & 0.4 \\
\hline Density $\left(\mathrm{kg} / \mathrm{m}^{3}\right)$ & 0.937 & 0.925 & 0.921 & 0.39 & 0.929 & 0.922 \\
\hline Kinematic viscosity $\left(\mathrm{mm}^{2} / \mathrm{s}\right)$ & 190.2 & 130.1 & 85.3 & 201.3 & 110.2 & 70.1 \\
\hline Acid value & 5.3 & 4.4 & 3.9 & 6.3 & 4.9 & 4.3 \\
\hline Odine value & 104.3 & 103.7 & 105.2 & 115.3 & 117.2 & 116.2 \\
\hline PV (meq/kg) & 5.6 & 5.3 & 4.6 & 6.3 & 5.7 & 4.4 \\
\hline Saponification number & 204.3 & 194.2 & 184.2 & 195.1 & 194.3 & 193.9 \\
\hline Unsaponifiable matter $(\% \mathrm{w} / \mathrm{w})$ & 3.9 & 2.7 & 1.9 & 4.9 & 3.0 & 2.1 \\
\hline Energy value $(\mathrm{k} / \mathrm{g})$ & 37.2 & 38.8 & 38.6 & 37.9 & 38.3 & 39.1 \\
\hline
\end{tabular}

${ }^{a}$ Data taken from ref 26 . The term "T1" represents the first stage of pretreatment and heating at $65^{\circ} \mathrm{C}$, followed by sedimentation. The term "T2" represents the second stage of pretreatment and heating at $65{ }^{\circ} \mathrm{C}$, followed by sedimentation.

The transesterification of used oils collected from the cafeterias at the University of Guelph, Canada, using an acidic catalyst $\left(\mathrm{H}_{2} \mathrm{SO}_{4}\right)$ and an alkaline catalyst $(\mathrm{KOH})$ and was compared [27]. Two types of used oils (partially hydrogenated soybean oil and margarine) were transesterified with methanol, ethanol, 1-propanol, 2propanol, 1-butanol, and 2-ethoxyethanol [28]. It has been compared between two catalysts such as $\mathrm{KOH}$ and a combination of barium and calcium acetate for the preparation of methyl esters from waste cooking oil. Various reaction parameters and ester yields are shown in Table 2. Catalytic activities of $\mathrm{HCl}$ and $\mathrm{H}_{2} \mathrm{SO}_{4}$ for the transesterification of waste palm oil were compared [28]. Compared to $\mathrm{HCl}, \mathrm{H}_{2} \mathrm{SO}_{4}$ was a better catalyst. A process has been developed to produce esters from feedstocks that have a high FFA content, diglycerides and monoglyerides, using calcium and barium acetate as a catalyst. This catalyst did not form soap with FFA [29]. It has been compared between two catalysts such as $\mathrm{KOH}$ and a combination of barium and calcium acetate for the preparation of methyl esters from waste cooking oil [30]. Various reaction parameters and ester yields are shown in Table 3.
Table 2. Reaction conditions for transesterification of used oils and yields of liquid fuels ${ }^{a}{ }^{a}$ Data taken from ref 27.

\begin{tabular}{|l|l|l|l|l|l|l|}
\hline & & & & & \multicolumn{2}{|c|}{ Ester Yield (wt \%) } \\
\hline alcohol & $\begin{array}{l}\text { molar ratio } \\
\text { (alcohol:oil) }\end{array}$ & $\begin{array}{l}\text { temp } \\
\left({ }^{\circ} \mathrm{C}\right)\end{array}$ & $\begin{array}{l}\text { tim } \\
\mathrm{e}(\mathrm{h})\end{array}$ & catalyst & $\begin{array}{l}2 \text { days at } \\
25^{\circ} \mathrm{C}\end{array}$ & $\begin{array}{l}7 \text { days } \\
\text { at 5 } \\
{ }^{\circ} \mathrm{C}\end{array}$ \\
\hline methanol & $3.6: 1$ & 65 & 40 & $0.1 \% \mathrm{H}_{2} \mathrm{SO}_{4}$ & 79.3 & 64.0 \\
\hline methanol & & 50 & 24 & $0.4 \% \mathrm{KOH}$ & 91.9 & 85.3 \\
\hline ethanol & $3.6: 1$ & 73 & 40 & $0.1 \% \mathrm{H}_{2} \mathrm{SO}_{4}$ & 66.9 & 54.8 \\
\hline ethanol & & 50 & 24 & $0.4 \% \mathrm{KOH}$ & 28.9 & \\
\hline 1-propanol & $3.5: 1$ & 90 & 40 & $0.1 \% \mathrm{H}_{2} \mathrm{SO}_{4}$ & 92.2 & 76.2 \\
\hline 1-propanol & & 50 & 24 & $0.4 \% \mathrm{KOH}$ & 42.7 & \\
\hline 2-propanol & $3.5: 1$ & 80 & 40 & $0.1 \% \mathrm{H}_{2} \mathrm{SO}_{4}$ & 78.7 & 54.4 \\
\hline 2-propanol & & 50 & 24 & $0.4 \% \mathrm{KOH}_{1}$ & 51.2 & \\
\hline 1-butanol & $3.6: 1$ & 105 & 40 & $0.1 \% \mathrm{H}_{2} \mathrm{SO}_{4}$ & 78.1 & 61.9 \\
\hline 1-butanol & & 50 & 24 & $0.4 \% \mathrm{KOH}$ & 59.5 & \\
\hline 2-ethoxyethanol & $4.2: 1$ & 125 & 40 & $0.1 \% \mathrm{H}_{2} \mathrm{SO}_{4}$ & 53.5 & 39.2 \\
\hline 2-ethoxyethanol & & 50 & 24 & $0.4 \% \mathrm{KOH}$ & 37.0 & \\
\hline
\end{tabular}

Table 3. Comparison of two catalysts for the preparation of methyl esters from waste oils ${ }^{a}$

\begin{tabular}{|l|l|l|l|l|}
\hline $\begin{array}{l}\text { methanol } \\
\text { /oil ratio }\end{array}$ & catalyst & $\begin{array}{l}\text { reaction } \\
\text { time (h) }\end{array}$ & conditions & $\begin{array}{l}\text { ester yield } \\
\text { (wt \% of } \\
\text { fresh oil) }\end{array}$ \\
\hline $0.2-0.28$ & $\begin{array}{l}0.01 \%-2 \% \\
\text { KOH }\end{array}$ & $1-2$ & no heat & $83-92$ \\
\hline 0.38 & $\begin{array}{l}0.12 \% \\
\text { barium } \\
\text { acetate and } \\
0.34 \% \\
\text { calcium } \\
\text { acetate }\end{array}$ & $2-3$ & $\begin{array}{l}\text { high } \\
\text { temperature } \\
\text { and high } \\
\text { pressure }\end{array}$ & $87-94$ \\
\hline
\end{tabular}

${ }^{a}$ Data taken from ref 30.

The effect of immobilization on the catalytic activity of $P$. cepacia lipase was examined [31]. P. cepacia lipase (PS-30) was immobilized within a phyllosilicate sol-gel matrix (IM PS-30) and then used for the transesterification of waste fryer grease with primary and secondary alcohols. The reaction was conducted under solvent-free conditions but in the presence of molecular sieves $(0.4 \%)$, to eliminate water using both free and immobilized PS-30 lipase. The solventfree condition did not give good conversions with methanol. This process gave poor conversion (47\%-89\%) when free lipase was used. However, immobilized lipase gave better conversions (84\%-94\%) for both primary and secondary alcohols. The results are shown in Table 4.

Fatty acid esterification reaction was affected by the presence of water. The supercritical methanol method is determined to be more water-tolerant than the conventional method using an alkaline catalyst. The yield of methyl esters obtained from various raw materials that contained different amounts of water and FFA via the supercritical methanol method is shown in Table 5 [32]. A Four-different continuous process has been developed in flow sheets for biodiesel production from virgin oil or waste vegetable oil using alkaline or acidic conditions [33]. Detailed operating 
conditions and equipment designs for each process were obtained shown in Table 6.

Table 4. Yield of esters obtained from restaurant grease using different alcohols and enzymes

\begin{tabular}{|l|l|l|l|l|l|l|}
\hline \multicolumn{7}{|l|}{ Alkyl Ester Yield (\%) } \\
\hline alcohol & Gran-T.I. & Gran-C.a. & SP435 & IM PS-30 & free $^{b}$ IM PS-30 & IM PS-30 $^{b}$ \\
\hline methanol & 4 & 27 & 60 & 88 & 47 & 94 \\
\hline ethanol & 87 & 76 & 70 & 88 & 81 & 88 \\
\hline $\begin{array}{l}\text { ethanol } \\
(95 \%)\end{array}$ & 64 & 41 & 30 & 86 & 81 & 94 \\
\hline propanol & 87 & 79 & 52 & 87 & 87 & 87 \\
\hline 2-propanol & 61 & 59 & 87 & 46 & 75 & 90 \\
\hline butanol & 90 & 56 & 88 & 97 & 89 & 94 \\
\hline isobutanol & 97 & 89 & 94 & 72 & 87 & 84 \\
\hline
\end{tabular}

${ }^{a}$ Data taken from refs 10 and 31 . Reaction conditions were as follows (unless noted otherwise): molar ratio of grease to alcohol, 1:4; enzyme loading, $10 \mathrm{wt} \%$; reaction time, $24 \mathrm{~h}$; reaction temperature, $40{ }^{\circ} \mathrm{C}$; and no solvent. ${ }^{b}$ Reaction conditions were as follows: molar ratio of grease to alcohol, $1: 4 ; 100 \mathrm{mg}$ immobilized lipase or $10 \mathrm{mg}$ free lipase powder; reaction temperature, $50{ }^{\circ} \mathrm{C}$; reaction time, $18 \mathrm{~h}$; and $0.4 \%$ molecular sieves.

Table 5. Comparison of the yields of methyl esters in supercritical methanol and via alkaline-catalyzed and acidcatalyzed methods ${ }^{a}$

\begin{tabular}{|l|l|l|l|l|l|}
\hline & & & \multicolumn{3}{|c|}{ Yield of Methyl Esters (wt \%) } \\
\hline vegetable oil & $\begin{array}{l}\text { FFA content } \\
\left(\text { wt }^{\text {}}\right)\end{array}$ & $\begin{array}{l}\text { water content } \\
(\text { wt } \%)\end{array}$ & $\begin{array}{l}\text { alkaline- } \\
\text { catalyzed }\end{array}$ & $\begin{array}{l}\text { acid- } \\
\text { catalyzed }\end{array}$ & $\begin{array}{l}\text { supercritica } \\
1 \text { methanol }\end{array}$ \\
\hline rapeseed oil & 2.0 & 0.02 & 97.0 & 98.4 & 98.5 \\
\hline palm oil & 5.3 & 2.1 & 94.4 & 97.8 & 98.9 \\
\hline used frying oilc & 5.6 & 0.2 & 94.1 & 97.8 & 96.9 \\
\hline waste palm oil & $>20.0$ & $>61.0$ & no reaction & no reaction & 95.8 \\
\hline
\end{tabular}

${ }^{a}$ Data taken from ref $37 .{ }^{b}$ Given as the weight percentage of free fatty acids, relative to vegetable oil. ${ }^{c}$ Obtained from a household in Kyoto City.

\section{B. Engine Performance for Biodiesel}

Methyl esters has been tested which were prepared using waste cooking oil for their emissions in a vehicle [34]. The vehicle was tested using methyl esters of used frying oil as fuel, under the U.S. Federal Test Procedure (US-FTP) and in the Highway Fuel Economy Test (HWFET). Test results were compared with US-2D reference fuel. The ester fuel showed slightly lower hydrocarbon (HC) and $\mathrm{CO}$ emissions but increased NOx, compared to US-2D fuel, under US-FTP 72 and almost doubled the NOx values. Ethyl ester obtained from used palm oil was tested in a single-cylinder directinjection (DI) diesel engine by blending different proportions of ester with diesel fuel [25]. They reported that the blends included $100 \%$ ester, $3: 1$ ester/diesel (25D), 1:1 ester/diesel (50D), 1:3 ester/diesel (75D), and 100\% diesel fuel (100D). All of the blends, including 100\% ester, were tested in the engine, and their performance was compared with diesel fuel, in terms of engine performance and exhaust emissions. Engine performance of $100 \%$ ester was better than that of the diesel fuel. The blends of ester and diesel fuel burned more efficiently with better fuel economy and generated lower emissions, including lower $\mathrm{CO}$ and unburned hydrocarbons

Waste olive oil methyl ester was used in a diesel Perkins engine to examine their exhaust emissions under several steady-state operating conditions [35]. Results obtained for the ester fuel were compared with those using No. 2 diesel fuel. The use of biodiesel confirmed the lower emissions of $\mathrm{CO}, \mathrm{CO}_{2}, \mathrm{NO}$, and $\mathrm{SO}_{2}$, with an increase in the emissions of $\mathrm{NO}_{2}$. There was a slight increase in brake-specific fuel consumption (BSFC), in the case of biodiesel. For both fuels, the combustion efficiency did not vary. However, the exhaust gases had produced slightly different odors, and a slight fried food smell was detected when biodiesel that had originated from waste cooking oil was used [35]. Overall, the engine performance of biodiesel obtained from waste cooking oil was better than that of diesel fuel, and no change in engine operation was observed during the tests. The emissions produced by the use of biodiesel are less than those using diesel fuels. Only an increase in NOx was observed when biodiesel obtained from waste cooking oil was used. Generally, the biodiesel fuel consumption was somewhat higher, compared to that observed with regular diesel fuel, because of the lower calorific value of the former. However, the blends of the ester fuel with diesel fuel (75:25 and 50:50) maintained a balance for fuel consumption and emissions. The use of biodiesel that originated from waste cooking oil resulted in a slight fried food smell, when used on a large scale with diesel fuel [2].

\section{C.Present Project Designs for Biodiesel Production From Virgin and Waste Cooking Oil}

As discussed previously, there are various methods reported for the production of biodiesel from waste cooking oil. However, the two-step process (acid-catalyzed, followed by alkaline-catalyzed) is one of the better alternatives for the production of biodiesel from waste cooking oil. Our project is being implemented in the laboratory of Bioresource Science, Department of Biotechnology, Institute of Biological Science, Faculty of Science, University of Malaya, Kuala Lumpur, Malaysia using soybean, palm, sunflower, corn, coconut, rice bran and canola oil. Methanol and ethanolester are produced by using base catalyst $(\mathrm{NaOH}$ and $\mathrm{KOH})$ and acid catalyst ( $\mathrm{HCl}$ and $\mathrm{H}_{2} \mathrm{SO}_{4}$ ) shown in Table 7. The developed step for biodiesel production are filtration, mixing of ethanol/methanol and catalyst, transesterification (mixing of ethanol/methanol and catalyst with oil), shaking for 2.5 hours, esterification (fatty acid ester and crude glycerine production), separation after 16 hours (fatty acid ester and crude glycerine), biodiesel washinig by hot water $(5 \%$ by volume of the oil) and drained glycerine (continued until the ester layer become clear), biodiesel drying by dryer. These steps have been maintained by following National Biodiesel Board [36]. Figure 1. Photo is shown the biodiesel (Methyal 
ester after sementation) fuel from waste canola and palm Oil. Photo was taken from present on going project.

Table 6. Conditions for Transesterification of Virgin Oil and Waste Vegetable Oil ${ }^{a}$ Data taken from ref 33

\begin{tabular}{|c|c|c|c|c|c|}
\hline process & oil & $\begin{array}{l}\text { methanol:oil } \\
\text { molar ratio }\end{array}$ & catalyst & temp ${ }^{\circ} \mathrm{C}$ & pressure $(\mathrm{kPa})$ \\
\hline $\begin{array}{l}\text { process } \\
\text { I }\end{array}$ & virgin oil & $6: 1$ & $1 \% \mathrm{NaOH}$ & 60 & 400 \\
\hline $\begin{array}{l}\text { process } \\
\text { II }\end{array}$ & $\begin{array}{l}\text { waste oil } \\
\text { (pretreatment) }\end{array}$ & $6: 1$ & $1 \% \mathrm{H}_{2} \mathrm{SO}_{4}$ & 70 & 400 \\
\hline $\begin{array}{l}\text { process } \\
\text { II }\end{array}$ & $\begin{array}{l}\text { waste oil } \\
\text { (transesterificat } \\
\text { ion) }\end{array}$ & $6: 1$ & $1 \% \mathrm{NaOH}$ & 60 & 400 \\
\hline $\begin{array}{l}\text { process } \\
\text { III }\end{array}$ & waste oil & $50: 1$ & 1.3:1 $\mathrm{H}_{2} \mathrm{SO}_{4}:$ oil & 80 & 400 \\
\hline $\begin{array}{l}\text { process } \\
\text { IV }\end{array}$ & $\begin{array}{l}\text { waste oil } \\
\text { (hexane used } \\
\text { for extraction } \\
\text { of methyl } \\
\text { esters) }\end{array}$ & $50: 1$ & 1.3:1 $\mathrm{H}_{2} \mathrm{SO}_{4}:$ il & 80 & 400 \\
\hline
\end{tabular}

Table7. Different condition and ester yield as affected by methanol, ethanol and catalyst

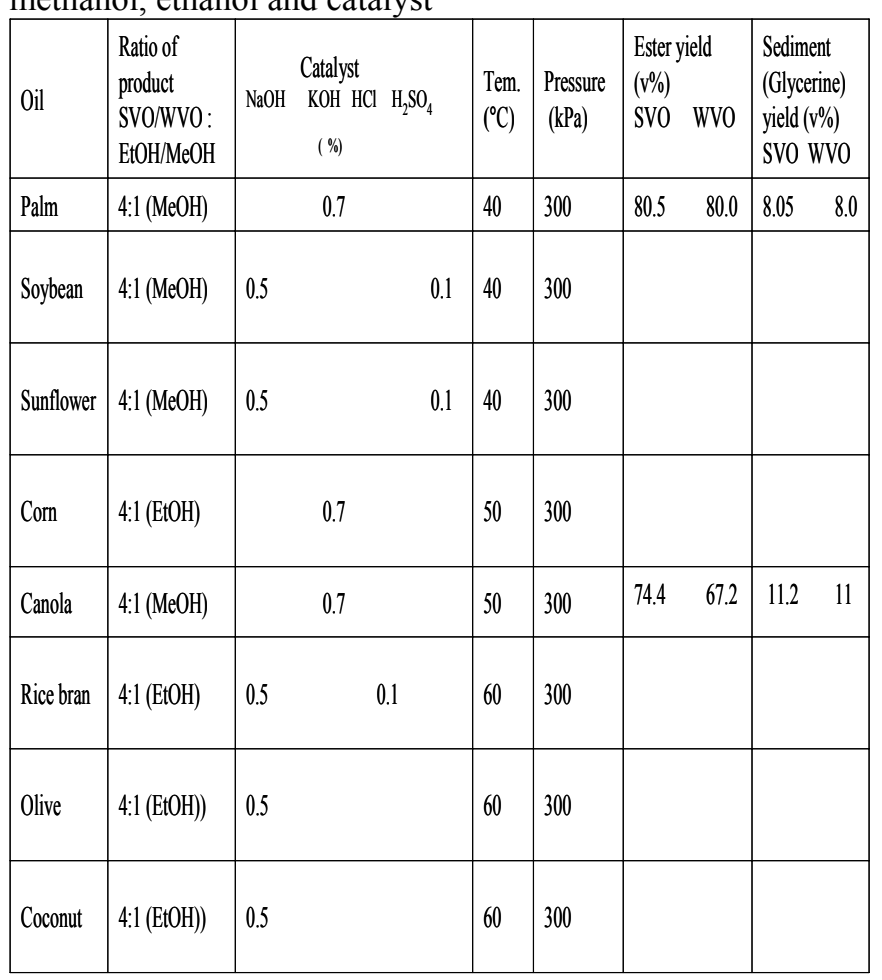

Data taken from present on going project. $\mathrm{SVO}=$ Straight vegetable oil, $\mathrm{WVO}=$ Waste vegetable oil

\section{CONCLUSION}

Waste cooking oil is an economical choice for biodiesel production, because of its availability and low cost. This oil has many undesirable compounds such as polymers, free fatty acid (FFA), and many other chemicals that are formed during frying, which are of major concern during the transesterification reaction. Pretreatment of the waste cooking oil to remove these undesirable chemicals is not practical. Depending on the water and FFA content of the waste cooking oil, a transesterification method should be selected. If the FFA and water contents are $<1 \mathrm{wt} \%$ and $<0.5$ wt $\%$, respectively, then an alkaline catalyst is more suitable for the ester production. If the FFA content of oil is high ( $>1$ wt \%), then an acid catalyst is a good choice. Enzyme-catalyzed transesterification is a very good option to all chemical-catalyzed reactions; however, it must be developed for its commercialization. The catalyst-free supercritical methanol method has great potential for biodiesel production from waste cooking oil however, the requirements of high temperature $\left(350^{\circ} \mathrm{C}\right)$, high pressure (45 $\mathrm{MPa})$, and high molar ratio of oil to alcohol $(1: 42)$ makes the use of this process difficult on an industrial scale. The performances of biodiesel obtained from waste cooking oil as transportation fuel or as an additive are better in all aspects, except for increased NOx emissions and high carbon residue $(\mathrm{CR})$.

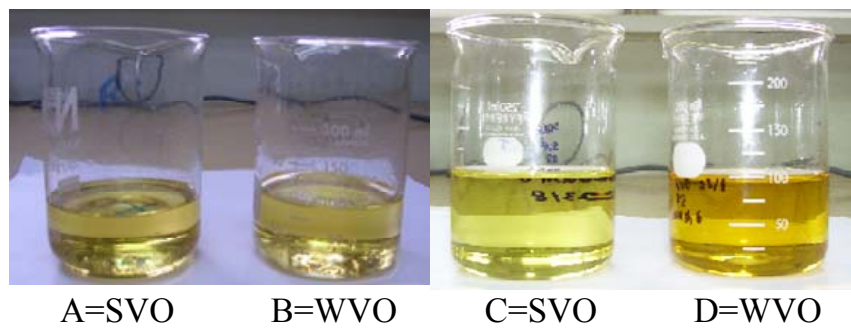

Figure 1. Photos show the biodiesel (Methyal ester after sedimentation) fuel from waste canola $(\mathrm{A}, \mathrm{B})$ and palm $(\mathrm{C}$, D) oil. Photo taken from present on going project, Department of Biotechnology, Institute of Biological Science, University of Malaya, Malayasia

\section{REFERENCES}

[1] Goldemberg, J. World Energy Assessment, Preface.United Nations Development Programme, new York, NY, USA. 2000.

[2] Kulkarni, M. G. and Dalai, A. K. Waste cooking iol-an economical source for biodiesel: a review. Ind.Eng. Chem. Res.45: 2901-2913. 2006.

[3] Holbein, B. E.; Stephen, J. D.; Layzell, D. B. Canadian Biodiesel Initiative. Final Report; Biocap Canada, Kingston, Ontario, Canada, 2004.

[4] Anonymous, CBC news in Debth. Canada-Kyoto Timeline. 2006. Available in www.cbc.ca/news/background/kyoto/timeline.html4

[5] Anonymous, Pulp and paper Canada. 2007a. Available in www.recycle.net/trade/rs191750.html

[6] Klass, L. D. Biomass for Renewable Energy, Fuels and Chemicals; Academic Press: New York, 1998; pp 1-2.

[7] Turkenburg, W. C. Renewable energy technologies. In: Goldemberg, J. (Ed). World Energy Assessment, Preface.United Nations Development Programme, new York, NY, USA. 2000. Pp. 219-272.

[8] Cheong, D. Y., Hasen. C. L. and Stevens. D. K. Production of biohydrogen by mesophilic anaerobic fermentation in an acid base sequencing batchReactor. Biotechnol. Bioeng. 2007, 96, 421.

[9] Lang, X.; Dalai, A. K.; Bakhshi, N. N.; Reaney, M. J. and Hertz, P. B. Preparation and Characterization of Biodiesels from Various Bio-Oils. Bioresour. Technol. 2002, 80, 53-62.

[10] Nelson, R. G.; Hower, S. A. Potential feedstock supply and costs for biodiesel production. In Bioenergy '94, Proceedings of the Sixth National Bioenergy Conference, Reno/Sparks, NV, 1994.

[11] Noordam, M. and R. Withers. Producing biodiesel from canola in the inland northwest: an economic feasibility study. Idaho Agricultural Experiment Station Bulletin No. 785. University of Idaho College of Agricultural, Moscow, ID, 1996, p 12.

[12] Demirbas, A. Protential application of renewable energy source, Biomass combursion problem in Bioler power system and combustion related nvironmental issues. Progr. Energy Combust. Sci. 2005, 31, 171. 
[13] Canackci, M. and Gerpen, J. V. A Pilot Plant to Produce Biodiesel from High Free Fatty Acid Feedstocks. Trans. ASAE 2003, 46 (4), 945-954.

[14] Cvengros, J. and Cvengrosova, Z. Used Frying Oils and Fats and their Utilization in the Production of Methyl Esters of Higher Fatty Acids. Biomass Bioenergy 2004, 27, 173-181.

[15] Dorado, M. P.; Ballesteros, E.; de Almeida, J. A.; Schellert, C.; Lohrelein, H. P. and Krause, R. An Alkali-Catalyzed Transesterification Process for High Free Fatty Acid Waste Oils. Trans. ASAE 2002, 45 (3), 525-529.

[16] Nawar, W. W. Chemical Changes in Lipids Produced by Thermal Processing. J. Chem. Ed. 1984, 61 (4), 299-302.

[17] Mittelbach, M. and Enzelsberger, H. Transesterification of Heated Rapeseed Oil for Extending Diesel Fuel. J. Am. Oil Chem. Soc. 1999 76 (5), 545-550.

[18] Anonymous, National Biodiesel Board. 2002. Available in www.biodiesel.org.

[19] Bastida, S. and Sanchez-Muniz, F. J. Thermal Oxidation of Olive Oil, Sunflower Oil and a Mix of Both Oils during Forty Discontinuous Domestic Fryings of Different Foods. Food Sci. Technol. Int. 2001, 7 (1), 15-21.

[20] Fukuda, H.; Kondo, A. and Noda, H. Biodiesel Fuel Production by Transesterification of Oils. J. Biosci. Bioeng. 2001, 92 (5), 405-416.

[21] Felizardo, P.; Correia, M. J. N.; Raposo, I.; Mendes, J. F.; Berkemeier, R. and Bordado, J. M. Production of Biodiesel from Waste Frying Oils. Waste Manage. 2006. 26: 487-494.

[22] Freedman, B.; Pryde, E. H. and Mounts, T. L. Variables Affecting the Yields of Fatty Esters From Transesterified Vegetable Oils. J. Am. Oil Chem. Soc. 1984, 61 (10), 1638-1643.

[23] Canckci, M. and Gerpen, J. V. The Performance and Emissions of a Diesel Engine Fuelled with Biodiesel from Yellow Grease and Soybean Oil. Presented in the Proceedings of 2001 ASAE International Meeting, Sacramento, CA, July 30-August 1, 2001, Paper No. ASAE 01-6049.

[24] Lepper, H. and Friesenhagen, L. Process for the Production of Fatty Acid Esters of Short-Chain Aliphatic Alcohols from Fats and/or Oils Containing Free Fatty Acids. U.S. Patent No. 4,608,202, August 26, 1986.

[25] Mittelbach, M.; Tritthart, P.; Diesel Fuel Derived from Vegetable Oils. III. Emission Tests Using Methyl Esters of Used Frying Oil. J. Am. Oil Chem. Soc. 1988, 65 (7), 1185-1187.

[26] Supple, B.; Holward-Hildige, R.; Gonzalez-Gomez, E.; Leahy, J. J. The effect of Steam Treating Waste Cooking Oil on the Yield of Methyl Ester. J. Am. Oil Chem. Soc. 2002, 79 (2), 175-178.

[27] Nye, M. J.; Williamson, T. W.; Deshpande, S.; Schrader, J. H.; Snively, W. H.; Yurkewich, T. P. Conversion of Used Frying Oil to Diesel Fuel by Transesterification: Preliminary Tests. J. Am. Oil Chem. Soc. 1983, 60 (8), 1598-1601.

[28] Al-Widyan, M. I.; Al-Shyoukh, A. O. Experimental Evaluation of the Transesterification of Waste Palm Oil into Biodiesel. Bioresour. Technol. 2002, 85, 253-256.

[29] Basu, H. N.; Norris, M. E. Process for Production of Esters for Use as a Diesel Fuel Substitute Using a Non-alkaline Catalyst. U.S. Patent No. 5,525,126, June 11, 1996.

[30] Rose, P.; Norris, M. Evaluate Biodiesel Made from Waste Fats and Oils. Final Report, Agricultural Utilization Research Institute, Crookston, MN, June 2002.

[31] Hsu, A.; Jones, K.; Marmer, W. N. Production of Alkyl Esters from Tallow and Grease Using Lipase Immobilized in a Phyllosilicate SolGel. J. Am. Oil Chem. Soc. 2001, 78 (6), 585-588.

[32] Warabi, Y.; Kusdiana, D.; Saka, S.; Reactivity of Triglycerides and Fatty Acids of Rapeseed Oil in Supercritical Alcohols. Bioresour. Technol. 2004, 91, 283-287.

[33] Zhang, Y.; Dube, M. A.; McLean, D. D.; Kates, M. Biodiesel Production from Waste Cooking Oil: 1. Process Design and Technological Assessment. Bioresour. Technol. 2003, 89, 1-16.

[34] Mittelbach, M. Specifications and Quality Control of Biodiesel. Bioresour. Technol. 1996, 56, 7-11

[35] Dorado, M. P.; Ballesteros, E.; Almeida, J. A.; Schellert, C.; Lohrlein, H. P.; Krause, R. Exhaust Emissions from a Diesel Engine Fuelled with Transesterified Waste Olive Oil. Fuel 2003, 82, 1311-1315.

[36] Anonymous, 2007b. National Biodiesel Board. Available in www.biodiesel.org/

[37] Saka, S.; Kusdiana, D. Biodiesel fuel from rapeseed oil as prepared in supercritical methanol treatment. Fuel 2001.80, 225-231. 


\title{
Development DC Converter for Low Voltage High Current Applications Using CUK DC-DC Converter
}

\author{
W. Salah, and S. Taib and A. Al-Mofleh
}

\begin{abstract}
This paper presents the design of Transformer less DC-DC converter for 100W Low voltage High Current (LVHC) applications. The converter design based on CUK topology which is basically combination of buck and boost converter topologies. The developed converter converts input DC voltage of $18 \mathrm{~V}$ or more to a $5 \mathrm{~V}$ stable and ripple free load current. A fast switching and low ON-resistance power MOSFETs was used to step down voltage. In this paper the concept of basic CUK converter is reviewed. The simulation results and measured data of the power circuit are analyzed and discussed. The supply requirements for TEC will be discussed and the use PV power source to feed the TEC. The practical application of TEC is presented for potential commercialization.
\end{abstract}

Keywords: SMPS, transformerless converter, DC-DC converter, MOSFETs, CUK converter, voltage divider, TEC, PV

\section{INTRODUCTION}

In a basic switching power supply, the regulating elements consist of transistors that act as rapidly opened and closed switches. The resultant pulse train is coupled to an output network which provides smoothing of the dc output. Regulation is accomplished by control circuits that vary the on-off periods (duty cycle) of the switching elements if the output voltage attempts to chance [1].

Unregulated power supplies consist of a rectifier and filter is not capable of providing a ripple free dc output voltage whose value remains reasonably constant. Power processing has always been an essential feature of most electrical equipment. The differences in voltage and current requirements for different applications have led to the design of dedicated power converters to meet their specific requirements.

The subject for energy conservation are consumer electronics devices such as air conditioners, TVs, lighting and office automation machines such as computers, display monitors and printers. The power conversion units for these types of equipment use characteristically high-efficiency

W. Salah \& A. Al-Mofleh is with the School of Electrical \& Electronic Eng USM Engineering Campus, N. Tebal, P. Pinang (email: wael.sal@gmail.com).

$\mathrm{S}$. Taib is the Senior Lecturer at the School of Electrical \& Electronic Engineering, USM Engineering Campus, N. Tebal, P. Pinang and also the Task Manager for CETREE USM Penang (email: soibtaib@eng.usm.my). switched mode power supply (SMPS). But, reflecting the trend toward energy conservation, there are increasing demands for such power supplies with higher efficiency, lower loss and reduced stand-by waiting power [2][5]. The most significant differences between the linear and the switch mode regulators involve their efficiency, size, weight, thermal requirements, response time, and noise characteristics.

Thermoelectric modules (TEC) are solid-state heat pumps that operate on the Peltier effect. A thermoelectric module consists of an array of " $p$ " and " $n$ " type semiconductor elements heavily doped with electrical carriers. The array of elements is soldered so that it is electrically connected in series and thermally connected in parallel. This array is then affixed to two ceramic substrates, one on each side of the elements [7].

\section{Methodology}

Switching converters occupies between two type of converters which are the line regulated and the quiestiant resonant converters, the main problem of line regulator relates to the high power losses that is dissipated as a heat and also low overall efficient which is around $40 \%$. On the other hand this type of regulators are cheep and simple compared to questiant resonant which are complex and costly but provide high efficient [5].

The elimination of magnetic elements and the use of switches and capacitors as a based design elements for the design of DC-DC converters, then light weight converter can be realized [2].

In switching supplies, the regulating elements consist of series connected transistors that act as rapidly opened and closed switches. The input ac is first converted to unregulated de which, in turn, is "chopped" by the switching element operating at a rapid rate. The resultant pulse train is transformer-coupled to an output network which provides final rectification and smoothing of the dc output. Regulation is accomplished by control circuits that vary the on-off periods (duty cycle) of the switching elements if the output voltage attempts to chance.

The idea of switch-capacitor (SC) is to charge capacitors is series, this will provide division of voltage and so wide range of step down achieved, then the charged capacitors have to be discharged in parallel in order to supply the stored energy to the load [4]. 
The CUK converter basically derived from connecting a boost converter followed by a buck stage as shown in Fig. 1. A practical realization of a CUK converter could be as follows, the output polarity is inversed relative to input side [3].

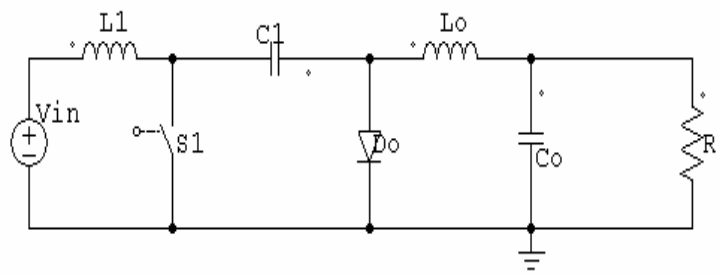

Fig. 1. Inverting CUK converter

The CUK topology is an inverting topology, for the purpose of obtaining same polarity referring to the input, a diode and a switch has to be added to a non-inverting CUK converter. As shown in Fig. 2 the addition of D1 and S2 enable the transfer of energy stored in $\mathrm{C} 1$ with the same polarity from input relative to the output side. And then by combining the common element a single stage noninverting CUK stage is constructed [1].

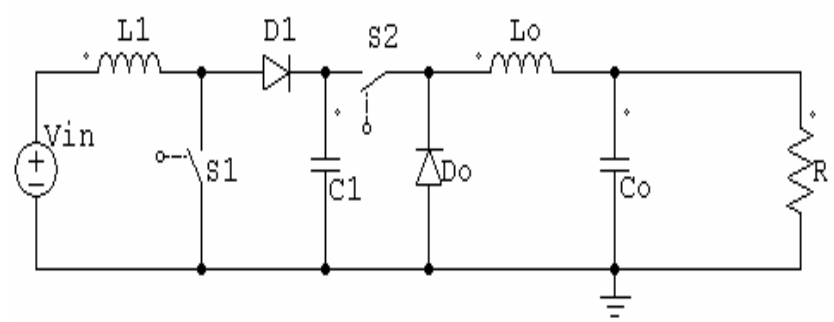

Fig. 2. Non-inverting CUK converter

By considering the topology of the non-inverting CUK converter, then the new topology can be basically principled on dividing input voltage to charge series connected capacitor during switch on-time and the discharge the capacitor during switches off-time as shown in Fig.3.

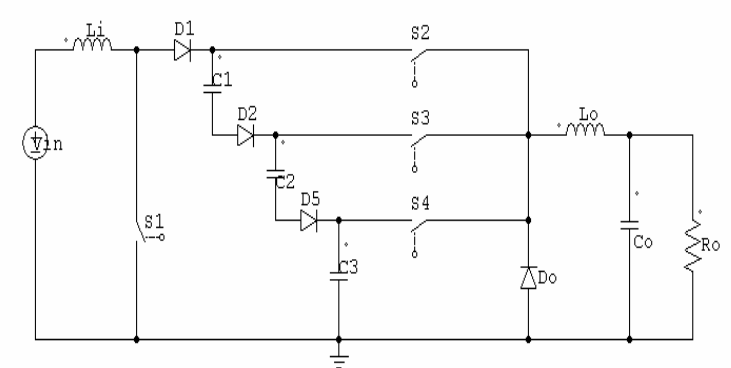

Fig. 3. The 3-Stage non-inverting CUK converter

The DC step down conversion associated with the use of transformer will lead to small value range of duty ration this will make it hard to switch On and Off the static switches. So the use of the proposed topology then large step-down voltage can be achieved without very small duty ratio and without usage of transformer. Therefore the losses related to usage of transformer can be eliminated [2][5].

The other advantage of this topology is that the energy transfer from input to the output is through the capacitors. These capacitors are charged on series during Off-time of the power switches and discharged on parallel during On- time of switches [6].

\section{Voltage REgulation}

An ideal constant voltage power supply would have zero output impedance at all frequencies. Thus the voltage would remain perfectly constant in spite of any changes in output current demanded by the load.

Typical application of power supplies includes the conversion of a raw input voltage to a controlled or stabilized voltage for the operation of electronic equipment.

The use of high-voltage, fast switching power transistors, fast recovery diodes, and new filter capacitors with lower series resistance and inductance, makes the switching supplies in a position of great concern in the power supply industry.

\section{TEC COOLING}

Heat is always absorbed at the cold side of the n- and ptype elements. The electrical charge carriers (holes in the ptype; electrons in the n-type) always travel from the cold side to the hot side, and heat is always released at the hot side of thermoelectric element. The heat pumping capacity of a module is proportional to the current and is dependent on the element geometry, number of couples, and material properties [8].

This cooling arrangement can be easily supplied from a PV panel with a DC-DC converter. The input power to the DC converter will be supplied from an input solar panel, the use of PV as a source of power for the converter make it reliable for outdoor cooling applications where it is hard to find a source for powering the cooling components for the system.

\section{ANALYSIS}

Fig. 4 shows a diagram for the equivalent circuit of three stages with the source of losses in the Off-state of power switch.

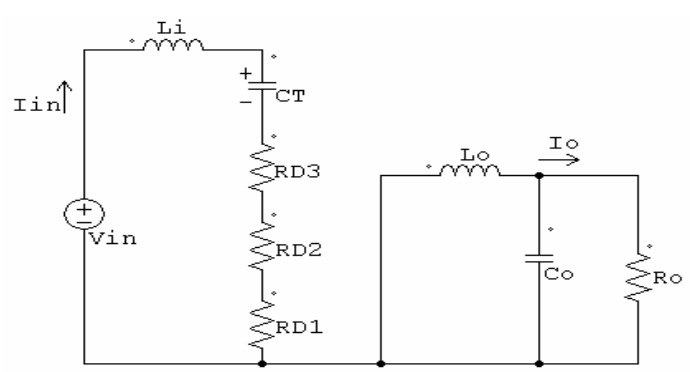

Fig. 4. Losses in the Off-state of power switch

Fig. 5 shows a diagram for the equivalent circuit of three stages with the source of losses in the on state of power switch.

The non-inverting capacitance voltage divider current stress on static switches is quit reliable, whereas during the On-state of switch the boost originally switch handle the input current, and the other switches the current passes through S2,S3 and S4 is Io/3 per switching leg, which leads to reduction of the dissipated power. 


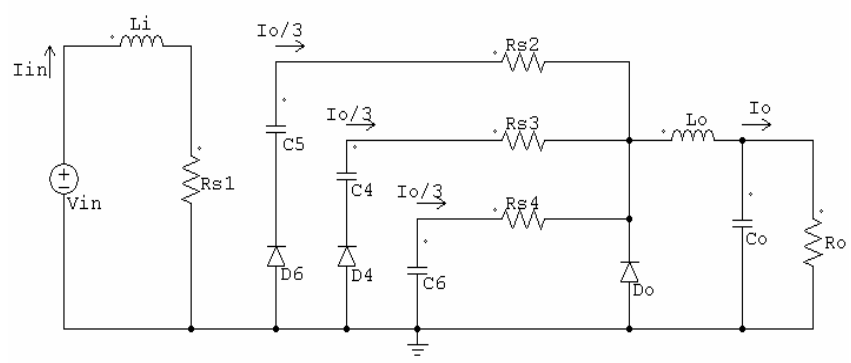

Fig. 5. Losses in the ON-state of power switch

With a similar way of analysis of single stage converter, the input/output voltage relation can be derived as given by:

$V_{o}=\frac{D V_{i}}{3(1-D)}-\frac{D^{2} V_{R S}}{3(1-D)}-D V_{R D}-D V_{R S}$

By neglecting the voltage drop $\left(\mathrm{V}_{\mathrm{RS}}, \mathrm{V}_{\mathrm{RD}}\right)$ then the output voltage is given by

$V_{o}=\frac{D V_{i}}{3(1-D)}$

and

$D=\frac{3 V_{o}}{V_{i}+3 V_{o}}$

where $\mathrm{D}$ is the duty cycle.

\section{AppliCATIONS}

Plate 1 shows a pre-developed prototype for TEC cooling. This prototype uses 4 TECs of $15 \times 15 \times 3 \mathrm{~mm}$ size attached to the four sides. This prototype is tested using the converter and draw a current of 2 to 5 amps.

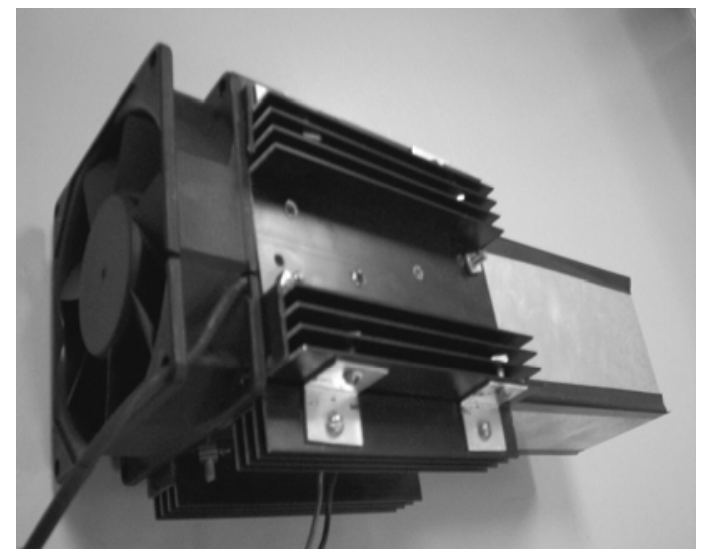

Plate 1. A pre-developed TEC cooling prototypes

A sample of solar fridge prototype unit is developed using 4 TEC as shown in Plate 2. The prototype with a 12 in $x 8$ in $\times 6$ dimension using solar module of $70 \mathrm{~W}$ capable to function in both cooling and heating operation. The unit takes about 30 minutes to reach the minimum temperature of $18^{0} \mathrm{C}$ within the compartment.

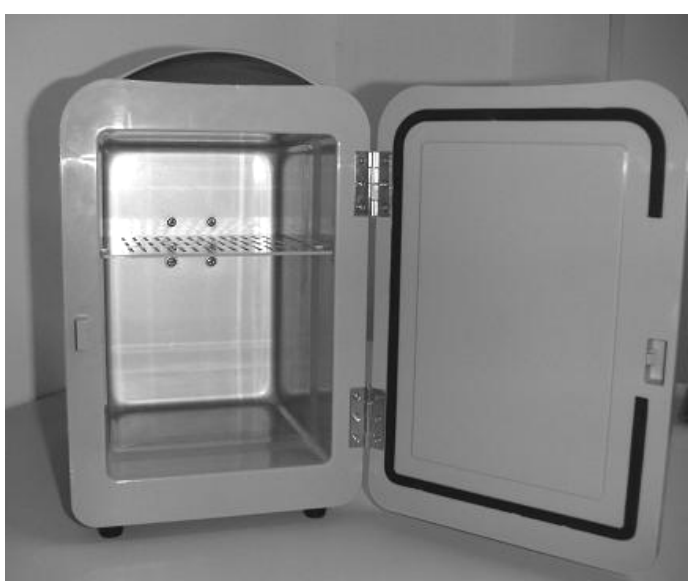

Plate 2. Mini solar and heater

\section{RESULTS}

\section{A. Simulation Software}

The SIMCAD program is used for the evaluation of the proposed circuit. The PWM control driving signal for power MOSFETs can be generated by PWM IC control model using current or voltage mode voltage regulation. The useful of power measurement integrated feature allows easier observation for system dynamic performance. The power switched considered to be ideal thought out circuit simulation, so it will be operated as a traditional switch in $\mathrm{ON}$ and $\mathrm{OFF}$ states.

\section{B. Measured and Simulated Results}

Fig. 6 shows the simulation of input and output current waveforms, the upper is the converter output current and the lower is the input current.

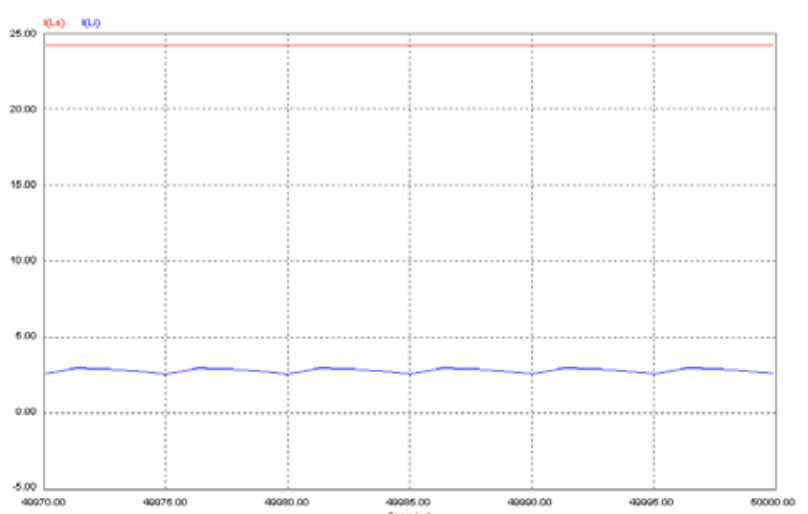

Fig. 6. The input and output current waveforms

Fig. 7 show the measured waveform of the voltage across the power switch S1 was matched with the waveform obtained from simulation that is illustrated in Fig. 8. Also Fig. 8 shows the simulated current through the switch $\mathrm{S} 1$, the maximum current being about 3.5A.

The measured output voltage is shown in Fig. 9. Fig. 10 shows the measured output voltage analyzed buy Fluke power quality analyzer software. The simulation results of output current and voltage are shown in Fig. 11, the upper waveform shows the output current, and the lower one shows the output voltage. 


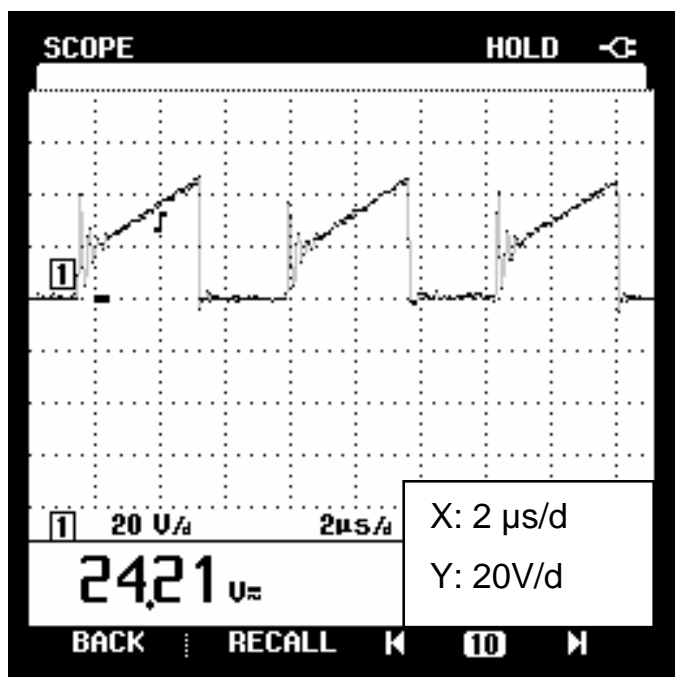

Fig. 7. Measured voltage across MOSFET S1

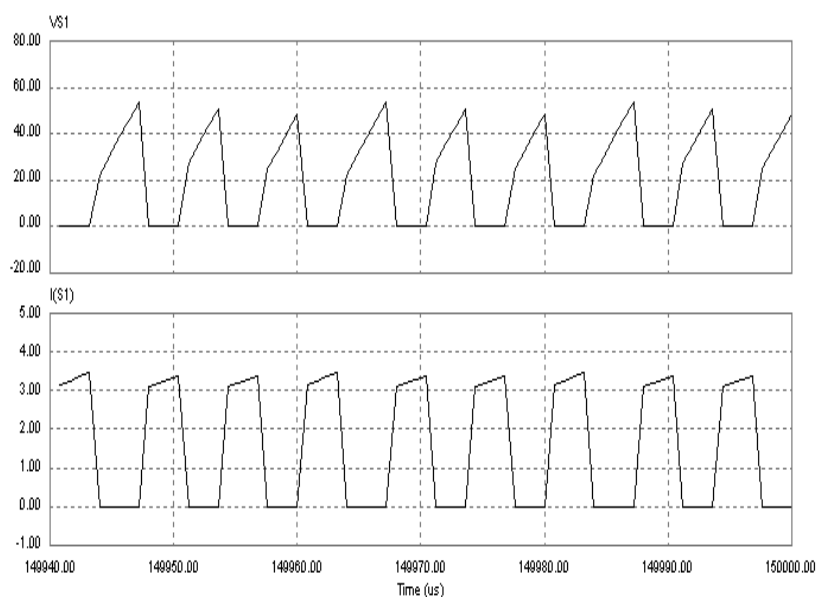

Fig. 8. Simulation waveforms of MOSFET switch S1

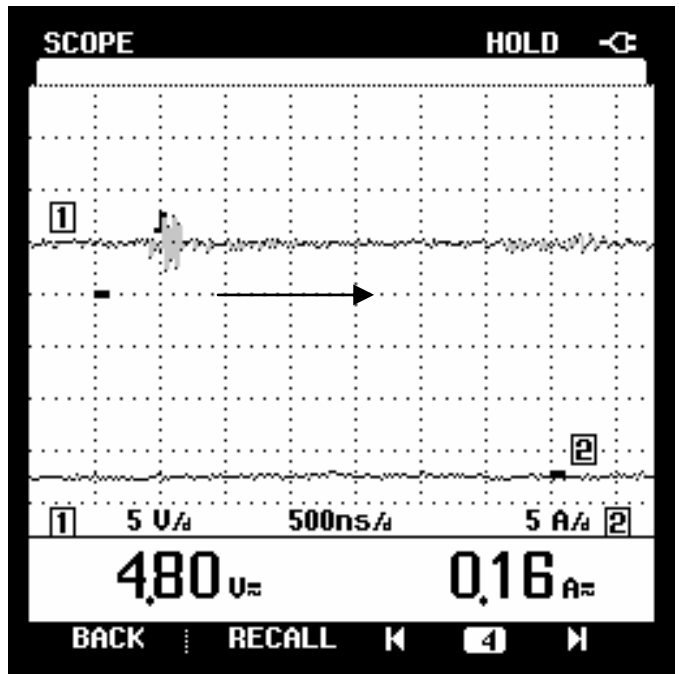

Fig. 9. Measured output voltage $V_{o}$

Fig. 12 shows the converter efficiency versus output power. The figure shows the efficiency at power ranges up to $70 \mathrm{~W}$. The figure also shows the converter ability to supply the rated load with efficiency up to $80 \%$. As illustrated in the figure the converter efficiency varies with load change from $35 \%$ up to $80 \%$ at full load.

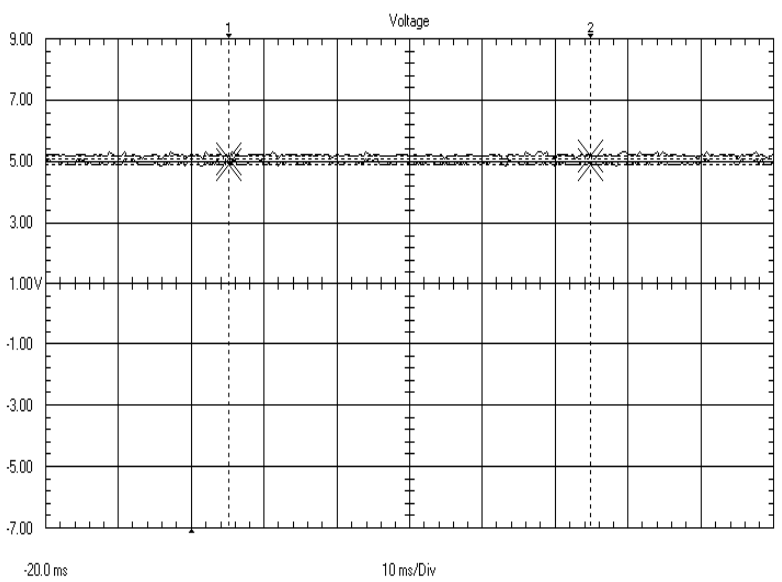

Fig. 10. Measured output voltage Vo with Fluke power quality analyzer

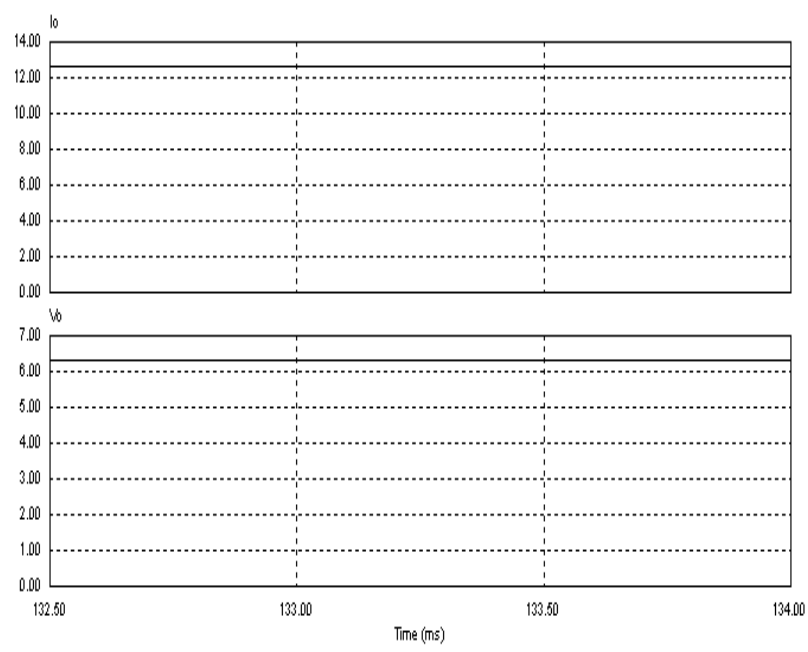

Fig. 11. Simulated output voltage and current

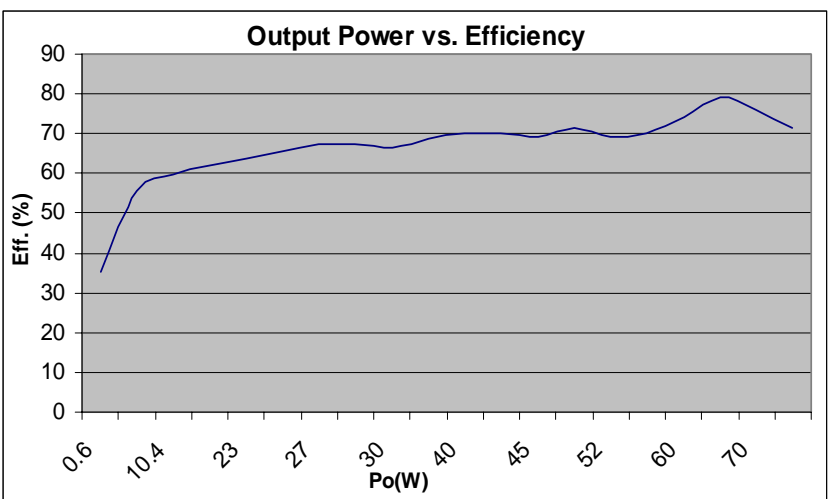

Fig. 12. Output power versus efficiency

The circuit efficiency for the proposed topology is shown in Fig. 13, and the graph shows that the circuit efficiency increased to $95 \%$ after transient time.

The converter was tested at different frequencies. It is found that the circuit draws high efficiency at higher value of switching frequency ie. more than $80 \mathrm{KHz}$, and lower efficiency for lower value of switching frequency, which is less than $80 \mathrm{KHz}$.

The graph shown in Fig. 13 shows the converter efficiency along with different frequency, with fixed input voltage and fixed load at $25 \%$ duty ratio. 


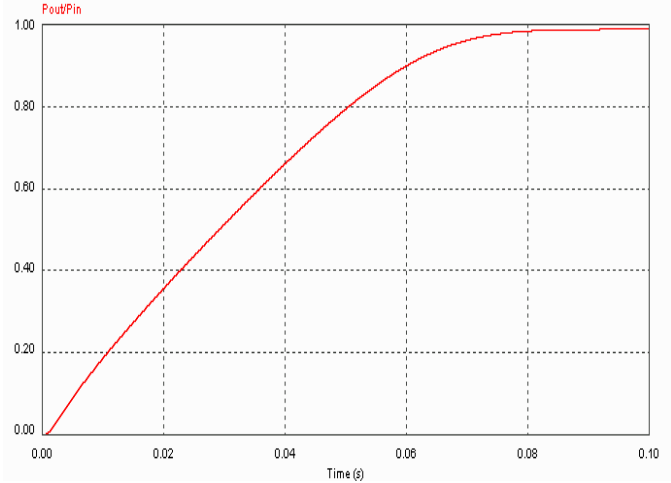

Fig. 12. The circuit efficiency

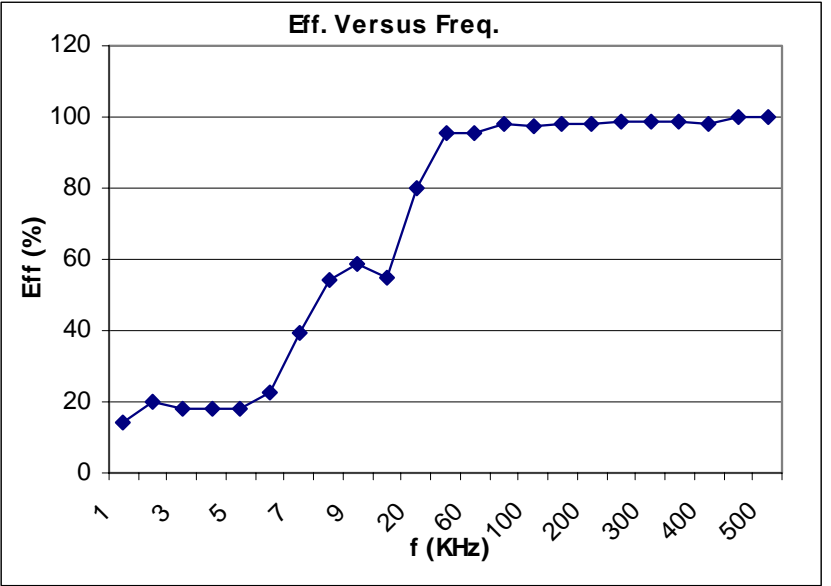

Fig. 13. Efficiency versus frequency at $\mathrm{D}=25 \%$

\section{VIII.CONCLUSIONS}

In comparison to the basic Cuk and Buck boost cascaded topologies efficiency the measured efficiency of $80 \%$ for implemented converter is acceptable referring to $74 \%$ average efficiency for these converters.

The circuit were simulated at frequencies from $1 \mathrm{Khz}$ up to $500 \mathrm{KHz}$ working frequency, It can be seen from the simulation waveforms that the circuit operated properly at high frequency, and the output current is ripple free and the efficiency is more than $90 \%$.

Switching topology when used in power supply is found to be smaller, lighter, and dissipate less power with respect to other types of regulation.

The paper had discussed the use of the TEC-Thermo Electric Coolers in cooling away connected applications with the use solar energy as power source. Also this paper had discussed the possibility of making use of the TECThermo Electric Coolers cooling applications. Such application with small area a away connected with the use solar energy as power source.

\section{REFERENCES}

[1] W. Huang, K. Yen, G. Roig, and E. Lee, "Voltage divided noninverting Cuk converter with large conversion ratios", in IEEE Proc. 1991, pp.II-1005-II-1007.

[2] R. D. Middlebrook, "Transformerless DC-to-DC Converters with Large conversion Ratios", in IEEE Transaction on Power Electronics Vol. 3,NO.4, pp.484-488, October 1988.

[3] B. Bryant, M.K. Kazimierczuk, "Derivation of the Cuk PWM DC-DC converter circuit topology" in Proc. 2003 International Symposium Circuits and Systems, pp. III-292-III-295.

[4] K. Kumabara and E. Miyachika, "Switched-Capacitor DC-DC Converter", Telecommunications Energy Conference, 1988 INTELEC '88., 10th International, pp. 213 - 218 ,1988, Japan.
[5] J. Liu, Z. Chen, Z. Du, "Switched capacitor DC-DC converters enable electronic products to become more compact", in Proc. 1996 IEEE Semiconductor Electronics, Conf., pp. 234-237.

[6] S.V. Cheong, S.H.Chung, and A. Ioinovici, "Development of power electronics converters based on switched-capacitor circuits", in Proc. 1992. IEEE Circuits and Systems, pp 1907-1910.

[7] Optimizing TEC Drive Current Application Note. Available at: www.ilxlightwave.com

[8] Thermoelectric Handbook, Available at: www.melcor.com 


\title{
Computational Fluid Dynamics (CFD) Simulation of a Downdraft Gasifier Using Wood Residue as Biomass Fuel
}

\author{
Mahbub Hasan, Shanti Faridah Salleh and Raymond John
}

\begin{abstract}
Malaysia is one of the major wood processing countries in South-East Asia. The use of wood residue for combustion and gasification process is of increasing interest for renewable energy production especially for small scale industry in Malaysia. Although a downdraft biomass gasifier produces comparatively low energy, it promises lower tar content and easy operation compared to other gasifiers. The development of appropriate design of a downdraft gasifier for wood is rather challenging, time consuming and cost intensive. Computational Fluid Dynamic (CFD) simulation though, is an effective tool for 3D modeling of the complex geometry of a gasifier. Authors developed a 3D model of a downdraft gasifier using STAR-CD, a powerful general purpose CFD code developed by CD-adapco. The gasifier geometry was meshed into thousands of tetrahedral volume cells. Low Reynolds Number $k-\varepsilon$ model was employed during simulation. The software solves the underlying conversion equation of mass, momentum and energy in each discretized control volume. Simulation results provided temperature profile and velocity magnitude at nodal points of different computational grids. Results were analyzed and discussed pertaining to the effect of temperature distribution and air velocity on combustion and gasification process. These results proved the potential of the downdraft gasifier as a renewable energy producer.
\end{abstract}

Keywords: CFD simulation, downdraft gasifier, wood biomass

\section{INTRODUCTION}

Biomass gasification, consisting of pyrolysis and carbolysis thermochemical processes, converts biomass into combustible gasses through the addition of heat [1]. Combustible gases, also known as producer gases, consists of carbon monoxide $(\mathrm{CO})$, hydrogen $\left(\mathrm{H}_{2}\right)$, carbon dioxide $\left(\mathrm{CO}_{2}\right)$, water $\left(\mathrm{H}_{2} \mathrm{O}\right)$, methane $\left(\mathrm{CH}_{4}\right)$, volatile tars and ash particles. Producer gases can be used in order to run internal combustion engines (both compression and spark ignition) and as substitute for furnace oil in direct heat applications.

Mahbub Hasan, Department of Mechanical and Manufacturing Engineering, Faculty of Engineering, Universiti Malaysia Sarawak, 94300 Kota Samarahan, Sarawak, Malaysia (phone: +6082583237; fax: +6082583410; e-mail: hmahbub@feng.unimas.my).

Shanti Faridah Salleh, Department of Mechanical and Manufacturing, Faculty of Engineering, Universiti Malaysia Sarawak, 94300 Kota Samarahan, Sarawak, Malaysia (e-mail: sshanti@feng.unimas.my).

Raymond John, Department of Mechanical and Manufacturing Engineering, Faculty of Engineering, Universiti Malaysia Sarawak, 94300 Kota Samarahan, Sarawak, Malaysia (e-mail: rayy24@yahoo.com).
These gases can also be used for producing, in an economically viable way, methanol-an extremely attractive chemical, which is useful both as fuel for heat engines as well as chemical feedstock for industries [2].

Gasifier is a device in which the pyrolysis and char conversion takes place [1]. Generally there are two main types of gasifiers available; fixed bed gasifier and fluid bed gasifier. The fixed bed gasifier principle supports the fuel in the bottom and is commonly divided into three subgroups; updraft gasifier, downdraft gasifier and crossdraft gasifier. The downdraft gasifier has low sensitivity to charcoal dust and tar content of fuel. Such characteristic is important because it can maintain its efficiency regardless of what biomass fuel is being used. In Malaysia, many rural industries such as local food industries utilize downdraft gasifier as a power generator to run their operation. Fuel consumption in a downdraft gasifier is minimal. Therefore it is more preferable for small scale applications [3].

Current research describes CFD simulation of a downdraft gasifier using wood residue as biomass fuel. The selection of wood is due to their vast availability as a result of big scale wood base manufacturing activities happening in Malaysia. STAR-CD, a powerful general purpose CFD code developed by CD-adapco, was used as the simulation software. The gasifier geometry was meshed into thousands of tetrahedral volume cells. Low Reynolds Number $k-\varepsilon$ model was employed during simulation. The STAR-CD software applies conversion equation of mass, momentum and energy at each discretized control volume. Simulation results provided temperature profile and velocity magnitude at nodal points of different computational grids. These results proved the potential of the downdraft gasifier as a renewable energy producer.

\section{Simulation Methodology}

The total methodology of current research was divided into seven stages, which are shown in 'figure 1' and described below. 


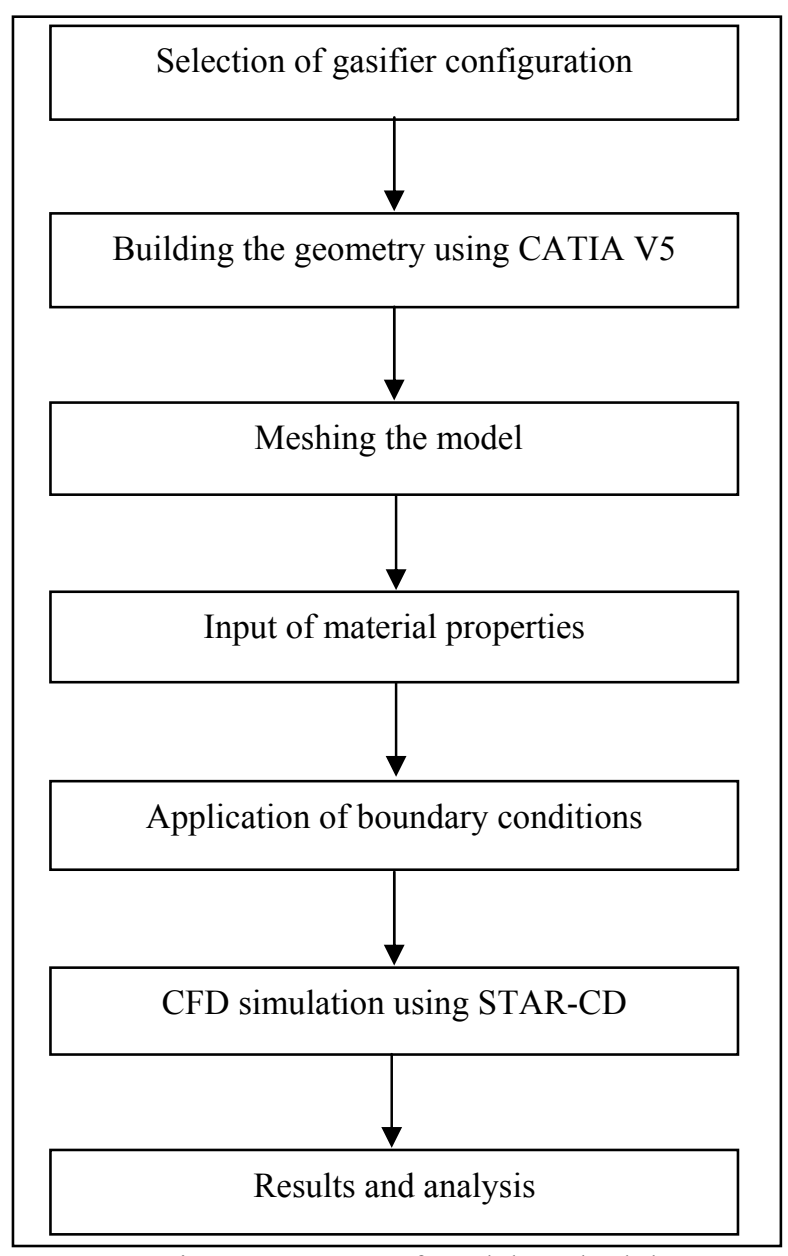

Figure 1. Stages of model methodology

\section{A. Selection of Gasifier Configuration}

Model 110/180 was chosen in current research as the basis in order to build the gasifier model basically due to its highest gas production [4]. Dimension of different sections of the model is shown in 'figure 2'.

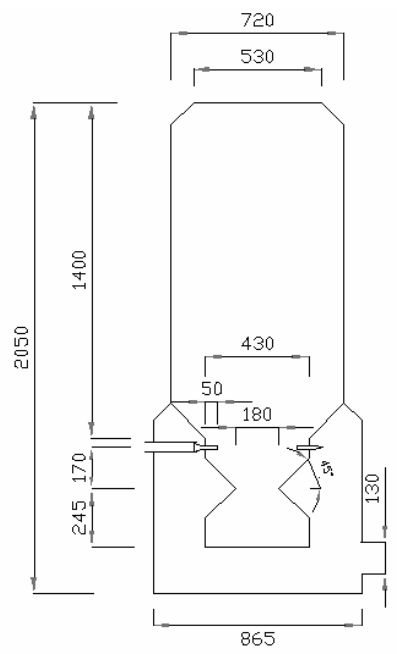

Figure 2. Dimension of different sections of the model in $\mathrm{mm}$

\section{B. Geometry Building}

Computer Aided Design (CAD) software CATIA V5 was used to create the geometry of the gasifier. The main reason for using CATIA V5 was to create the reverse solid for geometry. In other words, the hollow part of the gasifier (internal) was solid whereas the solid baffle and throat were hollow. This is because the internal volume was later discretized into a large number of cells so that fluidization became possible and flow could be modeled. As for the design of current research, the geometry of the gasifier was modeled as a simple cylinder. Therefore the gasifier could be used for any type of biomass fuels. The complete 3D model of the gasifier is shown in 'figure 3'.

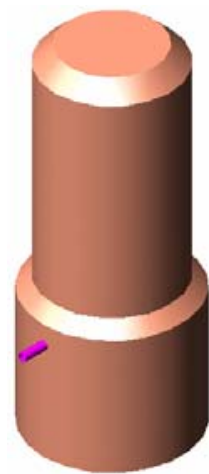

Figure 3. Complete 3D model of the gasifier

\section{C.Model Meshing}

The next step in the methodology is to mesh the domain. The model created in CATIA V5 was imported into proSTAR through IGES file. The tetrahedral meshing scheme was chosen here because of its applicability to any geometrical shapes without necessity of defining individual vertex along the geometry. Picture of the meshed geometry is shown in 'figure 4'.

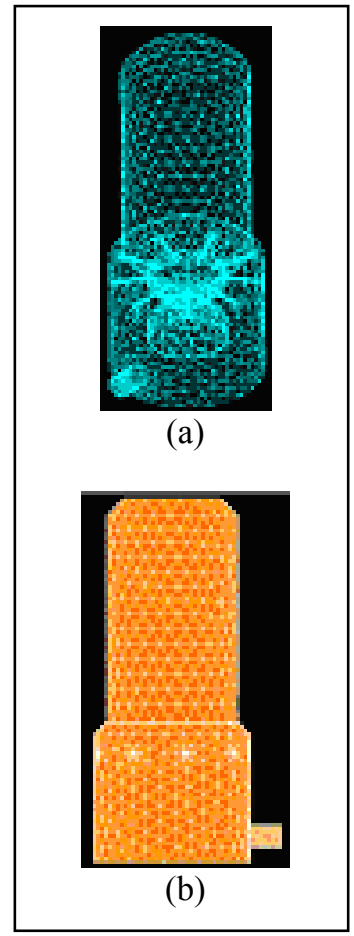

Figure 4. Tetrahedral meshing (a) surface wireframe view and (b) shaded model 


\section{D.Input of Material Properties}

After meshing the cells, the thermophysical properties were set accordingly. Basically the gasifier domain consisted of air and biomass fuel (wood chips). 'Table 1' mention all the properties used in current research.

Table 1. Thermophysical properties of wood and air [4]-[6]

\begin{tabular}{|c|c|c|c|}
\hline \multicolumn{2}{|c|}{ Wood Biomass } & \multicolumn{2}{c|}{ Air } \\
\hline Properties & Value & Properties & Value \\
\hline $\begin{array}{c}\text { Moisture } \\
\text { content }(\%)\end{array}$ & 10 & Density $\left(\mathrm{Kg} / \mathrm{m}^{3}\right)$ & 1.225 \\
\hline $\begin{array}{c}\text { Lower heating } \\
\text { value }(\mathrm{KJ} / \mathrm{Kg})\end{array}$ & 8400 & $\begin{array}{c}\text { Specific heat } \\
(\mathrm{J} / \mathrm{kgK})\end{array}$ & 1006.43 \\
\hline $\begin{array}{c}\text { Bulk density } \\
\left(\mathrm{Kg} / \text { loose } \mathrm{m}^{3}\right)\end{array}$ & 150 & $\begin{array}{c}\text { Thermal } \\
\text { Conductivity } \\
(\mathrm{W} / \mathrm{mK})\end{array}$ & 0.0242 \\
\hline $\begin{array}{c}\text { Energy density } \\
\left(\mathrm{MWh} / \mathrm{m}^{3} \text { of }\right. \\
\text { bulk volume })\end{array}$ & 0.7 & $\begin{array}{c}\text { Viscosity } \\
(\mathrm{Kg} / \mathrm{ms})\end{array}$ & $1.789 \mathrm{e}-05$ \\
\hline $\begin{array}{c}\text { Ash content } \\
(\%)\end{array}$ & 0.4 & $\begin{array}{c}\text { Molecular } \\
\text { Weight } \\
(\mathrm{Kg} / \mathrm{Kgmol})\end{array}$ & 28.966 \\
\hline
\end{tabular}

\section{E. Application of Boundary Conditions}

In current research, boundary conditions were applied to three different sections of the model; the inlet, outlet and wall ('figure 5'). Nine nozzles were set as the inlet where the air was injected into the domain. The input value for this parameter was set at $10 \mathrm{~m} / \mathrm{s}$. The outer round surface of the bottom pipe was selected as the outlet. The outer surface of the gasifier including the baffle was set as the wall.

\section{F. CFD Simulation}

The CFD simulation was run once all the parameters were set. Low Reynolds Number $k-\varepsilon$ model was chosen as the turbulence model during simulation. The model was based on the three-dimensional conservation equations for mass (equation 1), momentum (equation 2) and energy (equation 3 ). Those equations are mentioned below.

$$
\begin{aligned}
& s_{m}=\frac{1}{\sqrt{g}} \frac{\partial}{\partial t}(\sqrt{g} \rho)+\frac{\partial}{\partial x_{j}}\left(\rho \tilde{u}_{j}\right) \\
& \frac{1}{\sqrt{g}} \frac{\partial}{\partial t}\left(\sqrt{g} \rho u_{i}\right)+\frac{\partial}{\partial x_{j}}\left(\rho \tilde{u}_{j} u_{i}-\tau_{i j}\right)=-\frac{\partial p}{\partial x_{i}}+s_{i} \\
& \frac{1}{\sqrt{g}} \frac{\partial}{\partial t}(\sqrt{g} \rho h)+\frac{\partial}{\partial x_{j}}\left(\rho \tilde{u}_{j} h-F_{h, j}\right)= \\
& \frac{1}{\sqrt{g}} \frac{\partial}{\partial t}(\sqrt{g} p)+\frac{\partial}{\partial x_{j}}\left(\tilde{u}_{j} p\right)-p \frac{\partial u_{j}}{\partial x_{j}}+\tau_{i j} \frac{\partial u_{i}}{\partial x_{j}}+s_{h}
\end{aligned}
$$

Where $t$ is time, $x_{i}$ is cartesian coordinate $(i=1,2,3)$, $u_{i}$ is absolute fluid velocity component, $\tilde{\mathrm{u}}_{\mathrm{j}}$ is relative velocity between fluid and local (moving) coordinate frame that moves with velocity $u_{c j}, p$ is piezometric pressure, $\rho$ is density, $\tau_{\mathrm{ij}}$ is stress tensor components, $\mathrm{s}_{\mathrm{m}}$ is mass source, $\mathrm{s}_{\mathrm{i}}$ is momentum components, $\sqrt{g}$ is determinant of metric tensor, $F_{h, j}$ is diffusion energy flux in direction $x_{j}, s_{h}$ is energy source and $h_{t}$ is thermal enthalpy.

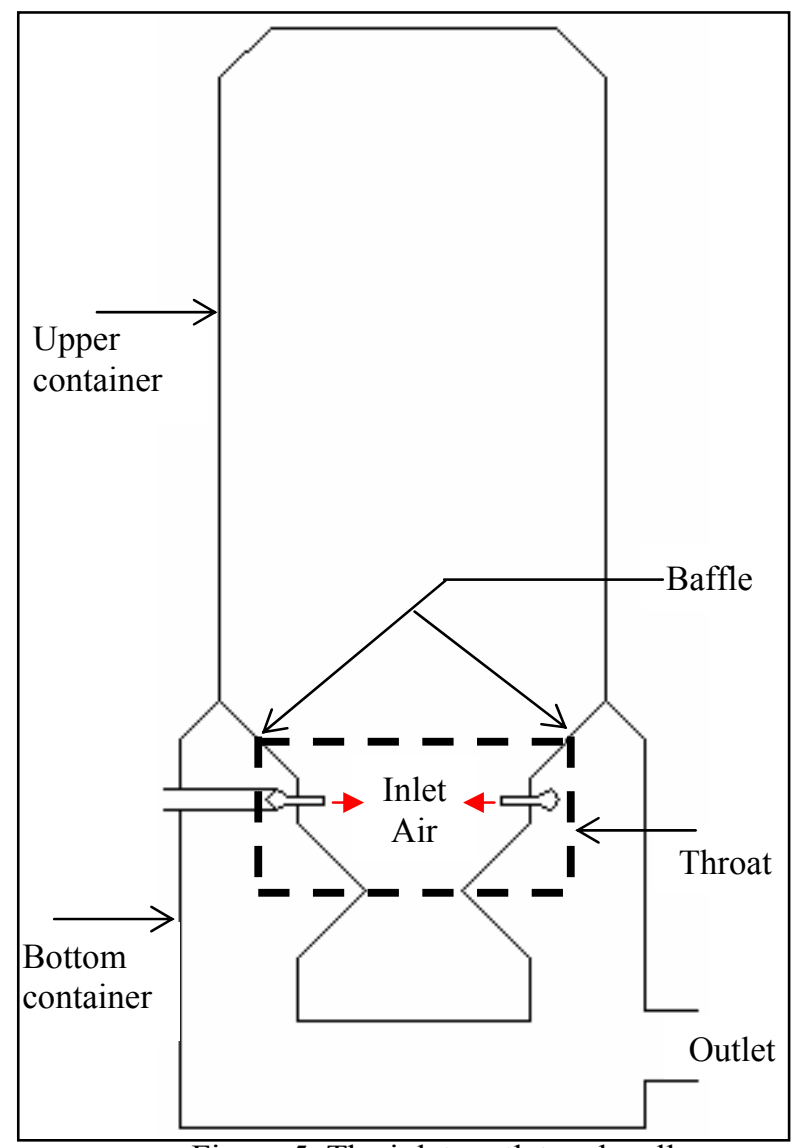

Figure 5. The inlet, outlet and wall

\section{G.Results Analysis}

Finally all the results were evaluated and analyzed. Those results are described in the next section.

\section{RESUlTS AND DisCUSSION}

\section{A. Temperature Profile}

Temperature profile obtained from the simulation is shown 'figure 6'. According to 'figure 6', temperature contour at symmetry plane ranges from $200^{\circ} \mathrm{C}$ to $1000^{\circ} \mathrm{C}$. The high temperature gaseous air descends towards the baffle area and exits through the outlet. As for the upward flow, a gradual decrease in temperature occurs until the flow reaches the top section. The heat is released mainly at the throat area where combustion takes place. As shown in temperature profile, the temperature at this region reaches up to $1000^{\circ} \mathrm{C}$. A highly exothermic process occurs at this area due to reaction with inlet air. Then the heat propagates upward and devolatilizes the wood chips components until the middle part of the gasifier.

Approximately at the middle of the upper container, the profile shows that the temperature ranges from $400^{\circ} \mathrm{C}$ to $700^{\circ} \mathrm{C}$. Theoretical temperature range for pyrolysis is $450^{\circ} \mathrm{C}$ to $600^{\circ} \mathrm{C}$. Therefore pyrolysis occurs at this zone. During pyrolysis the heat vaporizes the volatile components of wood chips. 


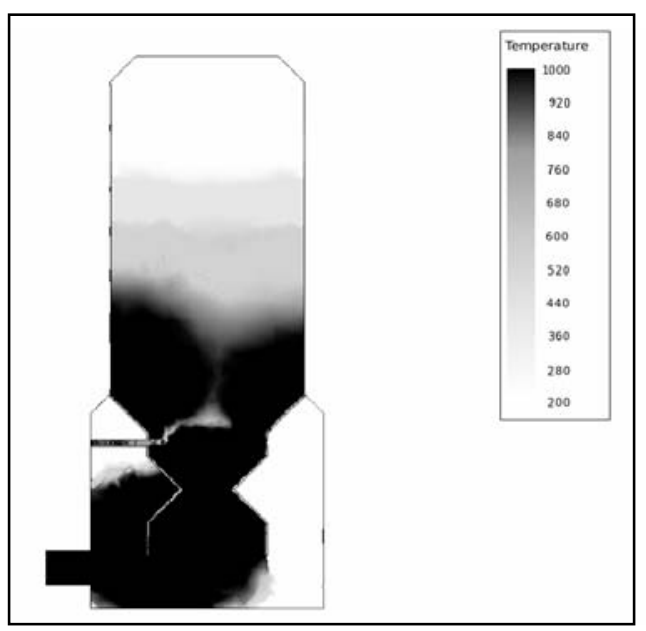

Figure 6. Temperature profile inside the gasifier in ${ }^{\circ} \mathrm{C}$

The top section is for drying and heating of wood chips having temperature around $200^{\circ} \mathrm{C}$. At this stage, moisture is released and some wood chips start to devolatilize. As for the bottom container, the temperature also shows the same profile at the opposite area of the outlet. This is because the hot gaseous air tries to find the way out and hence flows towards the outlet. Therefore there is no hot gaseous air on the other side. In addition, the hot gaseous air does not disperse to the upper level of bottom container since the outlet is located at the lower level and the hot gaseous air tends to find the exit first.

\section{B. Flow Pattern}

'Figure 7' is showing the velocity profile of the fluid inside the gasifier. The air velocity at the nozzle outlet is around $10 \mathrm{~m} / \mathrm{s}$, as the constriction accelerates the air. It implies fully developed turbulence in the flow and recirculation in the combustion zone induced by the nine nozzles. As the air flows towards center, the velocity magnitude reduces to $5 \mathrm{~m} / \mathrm{s}$. At the center of the nozzles horizontal plane, the velocity magnitude is $1 \mathrm{~m} / \mathrm{s}$. This happens because the air flow is coming from the circumferential direction and making the flow stagnant at the center. Nevertheless the air flow collisions create upward and downward flow and maintain flow velocity at 5 $\mathrm{m} / \mathrm{s}$.

The air flows upward with decreasing velocity and ignites the burning process. The oxygen content from the air supply is used during burning process. Hot gaseous air is then released. The hot gaseous air refers to air at high temperature, which contains several combustible gasses. Above the combustion zone, the flow is mainly of hot gaseous air. The hot gaseous air is also produced during pyrolysis process. This gaseous air circulates and moves downwards inside the gasifier with low velocity at around $0.3 \mathrm{~m} / \mathrm{s}$. As for the downward air flow, the air is also used for burning process. The air velocity diminishes as it moves downward towards the bottom. After the combustion zone, the hot gaseous air produced flows further downward through the reduction zone with a velocity magnitude of 1.5 $\mathrm{m} / \mathrm{s}$. After passing through the grate, the velocity reduces to $1 \mathrm{~m} / \mathrm{s}$. Nevertheless the velocity plot shows increment once the hot gaseous air enters the outlet channel. This is due to the constriction of the opening.

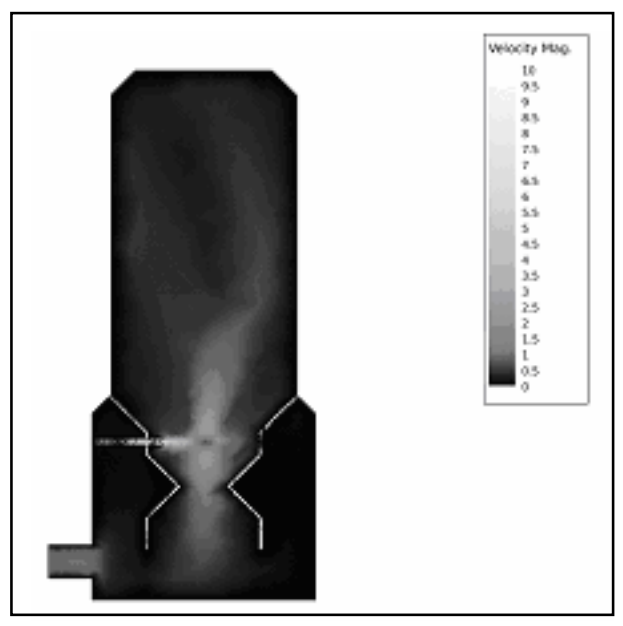

Figure 7. Velocity profile of fluids inside the gasifier in $\mathrm{m} / \mathrm{s}$

\section{Combustion of Wood}

Combustion of wood occurs at the throat zone where nine nozzles are located circumferentially. The combustion occurs once it reacts with air supply. The combustion propagates at the same region with the air flow. The propagation views are quite similar with the propagation view of air flow. This explains that the combustion can occur only with the existence of oxygen. As the air flow collision creates the turbulent mixing flow pointing upward and downward, the combustion also propagates towards the same direction creating high exothermic process at the center of the combustion zone. The isometric surface view ('figure 8') shows that the combustion covers the entire horizontal plane of the combustion zone. This ensures that complete combustion occurs at that region.

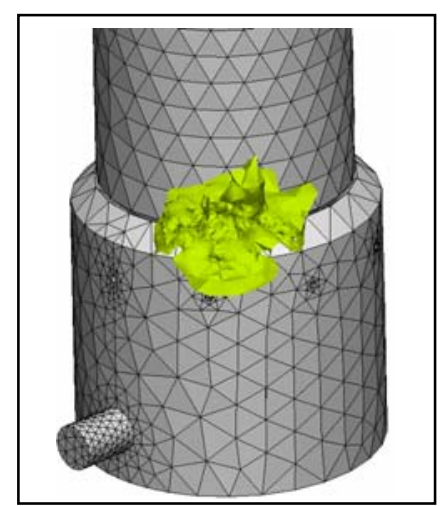

Figure 8. Isometric surface view of wood combustion

\section{Effect of Temperature and Air Flow on Gasification}

One of the important objectives in regulating the gasification process is to produce quality gasses. Nonetheless pyrolysis produces large quantities of tar and gasses including carbon dioxide [2]. Therefore the tar content needs to be eliminated. Obviously the most important factor in thermal cracking is the existent of high temperature. Even a slight increase in temperature above $950^{\circ} \mathrm{C}$ can significantly reduce tar content of producer gas [7]. According to simulation results, the maximum temperature obtained inside the gasifier is $1000^{\circ} \mathrm{C}$. Thus the tar content in wood chips that is in the hot region is theoretically cracked. 
Apart from that, air flow also plays an important role in determining the efficiency of gasification process. Flow pattern is mainly influenced by the air inlet velocity. Injection of air from nine nozzles at $10 \mathrm{~m} / \mathrm{s}$ increases the turbulence level, which is effective for air and pyrolysis gas mixing. As a result, temperature is more homogeneous in the hot zone. However, increasing the air injection velocity also increases the gas velocity in that zone. According to Gerun et al. [8], increase in the injection velocity not only leads to higher turbulence level, but also lowers the reaction rate. This means increment in turbulence level may lead to lower residence time. Nonetheless the air inlet velocity of $10 \mathrm{~m} / \mathrm{s}$ is just enough ('figure 7'), because the turbulence mainly occurs at the combustion zone with a little flow in reduction zone. Therefore as the hot gaseous air passes through the reduction zone, the residence time is sufficient to crack the tar content.

\section{CONCLUSION}

Current study describes computational fluid dynamics (CFD) simulation of a downdraft gasifier using wood as biomass fuel. A 3-D downdraft biomass gasifier modeling was conducted using STAR-CD. The gasifier geometry was discretized into 76,658 tetrahedral volume cells and imported into the finite volume CFD-solver STAR-CD. Low Reynolds Number $k-\varepsilon$ model was employed during simulation. The model used the three-dimensional conservation equations for mass, momentum and energy in solving the problem. These equations are used to solve mass flux, velocity and temperature. The results obtained from the simulation show that eliminating the baffle at the upper container actually extends the hot temperature zone approximately up to the middle of the gasifier. This makes devolatilization of the wood chips faster and at large amount at one time. Moreover hot gaseous air flowing through the throat at $1000^{\circ} \mathrm{C}$ is beneficial to the reduction zone as the high temperature helps breaking the tar component and hence produces cleaner gasses. As for velocity magnitude results, the maximum velocity is around $10 \mathrm{~m} / \mathrm{s}$, which are initiated from the tip of the nozzles. The air flow from nine circumferential nozzles creates a turbulence flow at the middle of combustion zone, helping the combustion process to propagate and burn more wood chips. Altogether the simulation shows the potential of the downdraft gasifier as a renewable energy producer.

\section{REFERENCES}

[1] P. S. Anderson and T. B. Reed, Biomass Gasification: Clean Residential Stoves, Commercial Power Generation and Global Impacts, Available

http://www.repp.org/discussioninggroups/resources/stoves/Anderson/ GasifierLAMNET.pdf, Date of Access: 10/09/06.

[2] A. K. Rajvanshi, Biomass Gasification, Available at http://www.nariphaltan.virtualave.net/gasbook.pdf, Date of Access: $01 / 08 / 06$.

[3] H. H. Jakobsen and T. Helge, Gasification Breakthrough in Biomass, Available at http://www.dbdh.dk/pdf/ren-energy-pdf/side14-17.pdf, Date of Access: 26/08/06.

[4] FAO, Energy and Environment Basics, Regional Wood Energy Development Programme in Asia, 1997.

[5] Woodenergy, Biomass Fuel, Available at http://www.woodenergy.ie/open24/defaultarticle.php?cArticlePath=5 29, Date of Access: 10/10/06

[6] STAR-CD Version 3.22 User Guide Manual, CD adapco Group. Computational Dynamic Limited, 2004.
[7] S. C. Bhattacharya and D. Animesh, Two-Stage Gasification of Wood with Preheated Air Supply: A Promising Technique for Producing Gas of Low Tar Content, Available at http://www.retsasia.ait.ac.th/Publications/TWO-STAGE\%20 GASIFICATION, Date of Access: 26/08/06.

[8] L. Gerun, J. Bellettre and M. Tazerout, Numerical Investigation of Air Injection during Biomass Gasification in a Two-Stage Downdraft Configuration, Available at http://www.sol3d.com/publications/lg/configuration.pdf, Date of access: 18/02/07. 


\title{
Prospects of Biogas Production Utilizing the Sewage Waste Water
}

\author{
Abu Saleh Ahmed, Ehsan Ahmed, Mahbub Hasan and Sinin Hamdan
}

\begin{abstract}
Biomass is considered as one of the major natural resource and contributing around $10-14 \%$ of the world energy supply. The more efficient use of biomass is demanded to solve the global crises such as exhaustion of fossil fuel and global warming. Biomass is a term for all organic material that stems from plants. Biomass is produced by green plants converting sunlight into plant materials through photosynthesis and includes land and water-based vegetation, as well as all organic waste. Therefore, the environmental friendly energy system should be able to construct by controlling the production and utilization of biomass. The biological conversion of biomass to bio-energy has received increasing attention as a means of reducing environmental pollution. Huge amounts of municipal waste have become an environmental issue. The relatively high amounts of organic matter in sewage are suitable substrate for anaerobic digestion. The Aerobic Thermophilic (AT) pretreatment system is one of the best options for maximizing methane gas production from anaerobic digestion of sewage sludge. Through the secretion of hydrolytic protease enzymes, the aerobic thermophilic bacteria can convert the suspended organic solids into soluble organic matter. This paper will describe the process of AT pre-treatment system for the anaerobic digestion of the sewage sludge. It is concluded that addition of small amount of AT sludge in the process enhances the anaerobic digestion and can result in surplus methane production. Finally, further research directions in producing methane gas from the sewage sludge are also highlighted in this paper.
\end{abstract}

Keywords: biomass, bioenergy, biogas, sewage waste water

\section{INTRODUCTION}

Biomass is considered as one of the major natural resource and contributing around $10-14 \%$ of the world energy supply [1]. The biological conversion of biomass into bio-energy has received increasing attention as a means of reducing environmental pollution. The vast amounts of

Abu Saleh Ahmed is with the Department of Mechanical and Manufacturing Engineering, Faculty of Engineering, Universiti Malaysia Sarawak, 94300 Kota Samarahan, Sarawak, Malaysia (phone: +6082583299; fax: +6082583410; e-mail: aasaleh@feng.unimas.my).

Ehsan Ahmed is with the Department of Civil Engineering, Faculty of Engineering, Universiti Malaysia Sarawak, 94300 Kota Samarahan, Sarawak, Malaysia (e-mail: aehsan@feng.unimas.my).

Mahbub Hasan is with the Department of Mechanical and Manufacturing Engineering, Faculty of Engineering, Universiti Malaysia Sarawak, 94300 Kota Samarahan, Sarawak, Malaysia (e-mail: hmahbub@feng.unimas.my).

Sinin Hamdan is with the Department of Mechanical and Manufacturing Engineering, Faculty of Engineering, Universiti Malaysia Sarawak, 94300 Kota Samarahan, Sarawak, Malaysia (e-mail: hsinin@feng.unimas.my). municipal, industrial and agricultural wastes that are released every day create serious environmental problems. Water pollution by solid waste disposal is also becoming a severe problem. To protect the environment, landfilling and ocean dumping are now discouraged. Because of its high content of organic matter, sewage sludge is used as a substrate for anaerobic digestion to recover biogas [2]. Anaerobic digestion of organic wastes is an effective technology for both treatment and energy conversion. Pretreatment processes have been developed to improve the sludge degradation rate and handling. Different pretreatment processes have been studied; in particular heat treatment at $40^{\circ}-180^{\circ} \mathrm{C}$ [3]-[5], chemical treatment [6] and mechanical disintegration and biological hydrolysis with or without enzyme addition [7]. Hasegawa et al. [8] reported that aerobic thermophilic (AT) bacteria have great potential as a cost-effective pretreatment for biological waste for anaerobic digestion and allowing easy management of the sludge. An AT pretreatment system could be useful for the anaerobic digestion of sewage sludge [9], [10]. Thermal pretreatment and excreted enzymes were found to influence hydrolysis in anaerobic conditions [11]-[13]. The treatment of municipal and industrial waste by AT bacteria was also studied [14]. Several researchers have reported that thermal bacteria hydrolyze sludge and make it easily degradable in anaerobic conditions [15]. Several researchers have been studying the aerobic thermophilic pretreatment of whole sewage sludge [8]-[10] and fullscale aerobic and anaerobic thermophilic digestion plants for sewage sludge is operated in some places throughout the world. In this paper, the effect of the addition of a small amount of AT bacterial sludge to methanogenic sludge in order to accelerate anaerobic digestion is described. It will also highlight the isolation of AT bacteria and their enhancement of anaerobic digestion.

\section{BASIC CONCEPT OF ANAEROBIC DIGESTION}

Anaerobic digestion is a fermentative process of organic matter in absence of $\mathrm{O}_{2}$ and the end products are $\mathrm{CH}_{4}$ and $\mathrm{CO}_{2}$. Figure 1 shows the flow chart of anaerobic process mechanisms. 


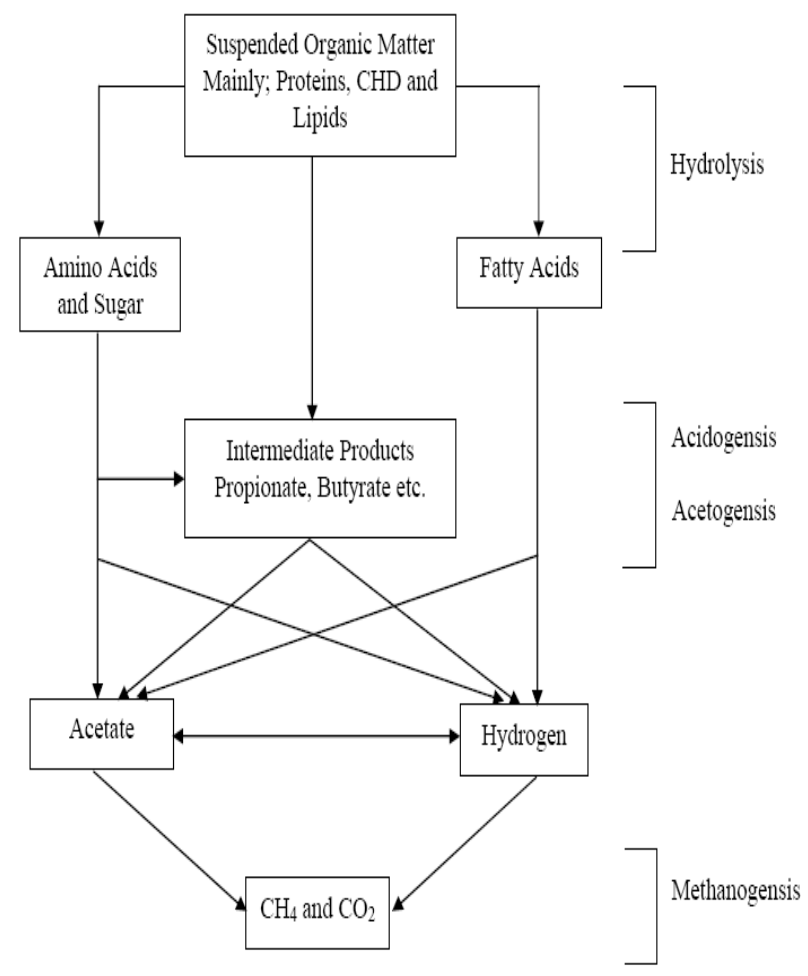

Figure 1. Flow diagram of anaerobic process mechanism

\section{A.The Upward-flow Anaerobic Sludge Bed (UASB) Process}

Anaerobic granular sludge bed technology refers to a special kind of reactor concept for the "high rate" anaerobic treatment of wastewater. The concept was initiated with upward-flow anaerobic sludge blanket (UASB) reactor. A scheme of a UASB is shown in figure 2 below. From a hardware perspective, a UASB reactor at first appearance is nothing more than an empty tank (thus an extremely simple and inexpensive design). Wastewater is distributed into the tank at appropriately spaced inlets. The wastewater passes upwards through an anaerobic sludge bed where the microorganisms in the sludge come into contact with wastewater-substrates. The sludge bed is composed of microorganisms that naturally form granules (pellets) of 0.5 to $2 \mathrm{~mm}$ diameter that have a high sedimentation velocity and thus resist wash-out from the system even at high hydraulic loads. The resulting anaerobic degradation process typically is responsible for the production of gas (e.g. biogas containing $\mathrm{CH}_{4}$ and $\mathrm{CO}_{2}$ ). The upward motion of released gas bubbles causes hydraulic turbulence that provides reactor mixing without any mechanical parts. At the top of the reactor, the water phase is separated from sludge solids and gas in a three-phase separator (also known the gas-liquid-solids separator). The three-phase-separator is commonly a gas cap with a settler situated above it. Below the opening of the gas cap, baffles are used to deflect gas to the gas-cap opening.

\section{B.Isolation Process of AT Bacteria from AT Seed Sludge}

The isolation process of AT bacteria from AT seed sludge is described in this section. At first, the AT seed sludge would be diluted to $1: 10^{4}$ with distilled water. The diluted sample would be spread on an F-JX agar plate. The F-JX agar contained $0.1 \%(\mathrm{w} / \mathrm{v})$ yeast extract, $0.1 \%(\mathrm{w} / \mathrm{v})$ trypticase peptone and $3 \%(\mathrm{w} / \mathrm{v})$ of a special type of agar (Taiyo-agar, GP-700, Shimizu Shokuhin, Japan). The pH of the F-JX medium would be adjusted to 7.0. After the growth of bacterial colonies on the agar plate, an isolated colony would be picked up from the plate and streaked onto another agar plate. This procedure would be repeated at least five times to ensure a pure culture. Autoclaved culture medium $(100 \mathrm{ml})$ would be added to a $250-\mathrm{ml}$ flask. The isolated colony would be transferred from the plate into the flask. After that, the flask culture would be incubated at $65^{\circ} \mathrm{C}$ by shaking at $140 \mathrm{rpm}$ for 5 days [16]. The cell pellets would be collected by centrifugation and added to methanogenic sludge for enhancing the production of biogas from waste water.

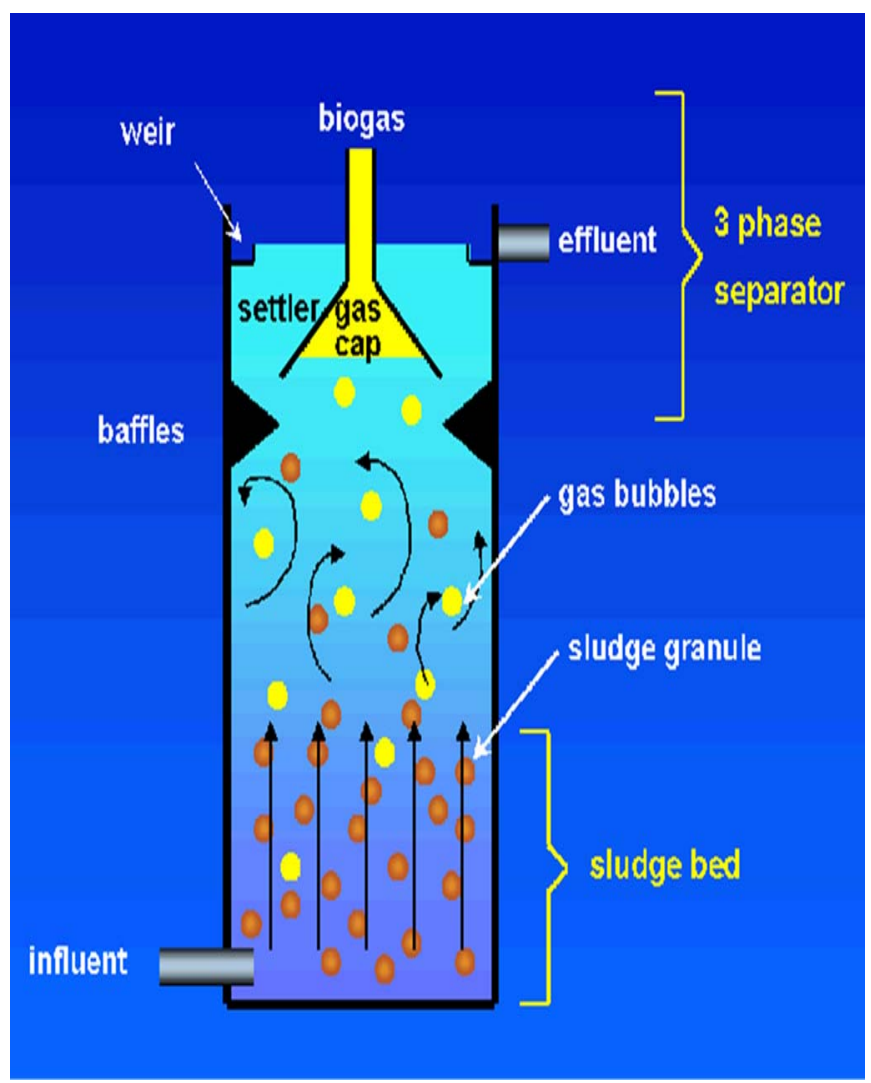

Figure 2. The upward-flow anaerobic sludge bed (UASB) reactor concept

\section{EXPECTED RESULTS}

This paper has described the effect of aerobic thermophillic bacteria in enhancing the biogas production from the sewage sludge. The expected outcomes from this study are as follows:-

- Exploration of biological systems to eliminate environmental harmful materials in order to convert them into energy sources.

- Methanogenic bacteria together with small amount of AT sludge could be used to convert the environmentally clean substances into energy efficiently in the form of methane gas.

- Prediction of the effects of more energy production and its use on the regional and global environment by acquiring data and developing the necessary understanding of environmental processes. 


\section{CONCLUSIONS}

This paper reviews the process of biogas production from the sewage waste water. The basic concept of biogas production is described. The enhancing effect of aerobic thermophilic (AT) bacteria in producing methane gas from the waste are then reported. It was concluded that the weakly production of biogas from anaerobic digestion of waste water can be enhanced significantly using AT seed sludge. Hence, future research work can be carried out to improve the production of biogas from waste water by incorporating thermophilic bacterial activity in the AT sludge.

\section{REFERENCES}

[1] P. McKendry, Energy production from biomass (part 1): overview of biomass, Bioresource Technology, 2002, pp. 83, 37-46.

[2] O. J. Skjelhaugen, Thermophilic aerobic reactor for processing organic liquid wastes, Water Res, 1999, vol. 33, pp. 1593-1602.

[3] R. T. Haug, T. J. LeBrun, and L. D. Tortorici, Thermal pretreatment of sludge - a field demonstration, J. Water Pollut Control Fed, 1983, vol. 55, pp. 23-34.

[4] S. Sawayama, S. Inoue, T. Yagishita, T. Ogi, and S. Y. Yokoyama, Thermochemical liquidization and anaerobic treatment of dewatered sludge. J Ferment Bioeng, 1995, vol. 9, pp. 300-302.

[5] U. Keep, I. Machenbach, N. Weisz, and O. E. Solbeim, Enhanced stabilisation of sewage sludge through thermal hydrolysis - three years experience with a full scale plant, Water Sci Technol, 2000, vol. 42, pp. 89-96.

[6] Y. Sakai, T. Fukas, H. Yasui, and M. Shibata, An activated sludge process without excess sludge production, Water Sci Technol, 1997, vol. 36 , pp. $163-170$

[7] J. Muller, G. Lehne, J. Schwedes, S. Battenberg, R. Naveke, D. N. Kopp, A. Scheminski, R. Krul, and D. C. Hempel, Disintegration of sewage sludges and influences on anaerobic, Water Sci Technol, 1998, vol. 38, pp. 425-433.

[8] S. Hasegawa, N. Shiota, K. Katsura, and A. Akashi, Solubilization of organic sludge by thermophilic aerobic bacteria as a pretreatment for anaerobic digestion, Water Sci Technol, 2000, vol. 41, pp. 163-169.

[9] K. R. Pagilla, K. C. Craney, and W. H. Kido, Aerobic thermophilic pretreatment of mixed sludge for pathogen reduction and Nocardia control, Water Environ Res, 1996, vol. 68, pp. 1093-1098.

[10] K. R. Pagilla, H. Kim, and T. Chainman, Aerobic thermophilic and anaerobic mesophilic treatment of swine waste, Water Res, 2000, vol. 34 , pp. $2747-2753$.

[11] W. Verstraete, L. de Baere, and A. Rozzi, Phase separation in anaerobic digestion: motives and methods, Tri Cebedeau, 1981, vol. 34, pp. 367-375.

[12] R. Schieder, R. Schneider, and F. Bischof, Thermal hydrolysis (TDH) as a pretreatment method for the digestion of organic waste, Water Sci Technol, 2000, vol. 41, pp. 181-187.

[13] V. Blonskaji and T. Vaalu, Single- and two-stage anaerobic mesophilic sludge digestion with thermal pretreatment. Appropriate environmental solid waste management and technologies for developing countries, World Environment Congress and Exhibition, Istanbul, Turky, July 8-11, 2002, pp. 1463-1470.

[14] M. Bomio, B. Sonnleitner, and A. Fiechter, Growth and biocatalytic activities of aerobic thermophilic populations in sewage sludge, Appl Microbiol Biotechnol, 1989, vol. 32, pp. 356-362.

[15] S. Kume and Y. Fujio, Digestion of municipal sewage sludge by a mixture of thermophilic Bacilli and their culture extract, J Gen Appl Microbiol, 1990, vol. 36, pp. 189-194.

[16] M. S. Miah, C. Tada, Y. Yang, and S. Sawayama, Aerobic thermophillic bacteria enhance biogas production, J Mater Cycles Waste Manag, 2005, vol. 7, pp. 48-54. 


\title{
Finite Element Analysis of Thermal Gradient of Transformer Windings
}

\author{
Chong Siew Wei, Mohammad Shahril Osman, and Martin Anyi
}

\begin{abstract}
An ideal transformer is one which has no losses. In practice, it is impossible to realize such a transformer with purely inductive coils wound on a loss-less core. Transformer losses are the electrical energy that converts to heat energy and heat up the transformer. At higher operating temperature, transformer insulating materials deteriorate faster. Transformer will damage if there is breakdown of its insulation system. Transformer losses can be reduced by proper design, use of suitable materials, coupled with good workmanship in production. Nevertheless these losses are inevitable. In order to reduce temperature rise of a transformer, efficient heat dissipation is vital. JL Analyzer, a finite element analysis software, has been used for simulation of thermal gradient of transformer windings with various designs such as windings with no cooling duct, windings with one cooling duct, windings with more than one cooling ducts, etc. From the simulations, it appears that thermal gradient is not high when cooling ducts are placed within windings for the purpose of oil ventilation. The result also shows that temperature is almost constant in each layer of copper conductor and temperature drops mainly across the insulating paper.
\end{abstract}

Keywords: transformer, heat losses, finite element method

\section{INTRODUCTION}

An ideal transformer is one which has no losses. In practice, it is impossible to realize such a transformer with purely inductive coils wound on a loss-less core. Transformer losses are the electrical energy that converts to heat energy and heat up the transformer. At higher operating temperature, transformer insulating materials deteriorate faster. If there is a breakdown of the transformer insulation system, there will be short-circuit between turns and therefore transformer is damaged.

Transformer losses can be reduced by proper design, use of suitable materials, coupled with good workmanship in production. Nevertheless these losses are inevitable. In order to reduce temperature rise of a transformer, efficient heat dissipation is vital.

The International Electrotechnical Commission (IEC) Standard specifies that limits for increase in top oil and average winding temperature of an oil-immersed transformer (under steady state and continuous rated power) are $60^{\circ} \mathrm{C}$ and $65^{\circ} \mathrm{C}$ respectively. If the temperature condition

Chong Siew Wei, Mohammad Shahril Osman and Martin Anyi are with the Faculty of Engineering, Universiti Malaysia Sarawak, 94300 Kota Samarahan, Sarawak, Malaysia. at the intended installation site exceeds $20^{\circ} \mathrm{C}$, the specified temperature rise limits for the transformer shall be reduced by the same amount as the excess. Assuming an average ambient temperature of $30^{\circ} \mathrm{C}$ in Malaysia, the limits for top oil temperature rise and average winding temperature rise are therefore adjusted by $10^{\circ} \mathrm{C}$ lower to $50^{\circ} \mathrm{C}$ and $55^{\circ} \mathrm{C}$ respectively.

\section{METHOD}

JL Analyzer, a finite element analysis software, is used for simulation of thermal gradient of transformer windings with different arrangements and number of cooling duct. The purpose is to find out the design that gives the most optimal heat dissipation capability.

Finite element analysis is the simulation of a physical system (geometry and loading environment) by a mathematical approximation of the real system. Using simple, interrelated building blocks called elements; a real system with infinite unknowns is approximated with a finite number of unknowns.

The first step involves Geometry Modeling where the winding is drawn to scale, followed by mesh generation. Mesh generation is the process of dividing the analysis continuum into a number of discrete parts or finite elements. Therefore, the finer the mesh, the better the result is. The smallest mesh size (which is 1.0 in this version) is used for higher accuracy, at the drawback of longer analysis time.

Properties of materials, which are the thermal conductivities of conductor (copper) and insulator (kraft insulating paper) are then assigned. Thermal conductivity for copper is $401 \mathrm{~W} /(\mathrm{m} . \mathrm{K})$ [2] and for kraft insulating paper is $0.1815 \mathrm{~W} /(\mathrm{m} . \mathrm{K})$ [3] as shown in Table 1.

Table 1. Thermal conductivities of copper and paper

\begin{tabular}{|c|c|}
\hline Materials & Thermal conductivities \\
\hline Copper & $401 \mathrm{~W} /(\mathrm{m} . \mathrm{K})$ \\
\hline Kraft insulating paper & $0.1815 \mathrm{~W} /(\mathrm{m} . \mathrm{K})$ \\
\hline
\end{tabular}

Cooling duct of $4 \mathrm{~mm}$ thick within the winding enables oil to flow freely [3], and therefore heat could be carried away from the internal part of the winding efficiently. As such, the oil temperature in the duct is the same as the average oil temperature of $75^{\circ} \mathrm{C}$ (which is the ambient temperature of $30^{\circ} \mathrm{C}$ plus the targeted oil temperature rise of $45^{\circ} \mathrm{C}$ ). This forms the boundary condition of the model, which is needed to stop the software from calculating infinitely through the computer's virtual ether (or 
mathematically known as a zero pivot). Load, in this context, is the heat generated due to copper and iron losses. The proportionate volumetric heat generated is entered.

Steady state analysis is then performed as the heat flow of a transformer which is in continual operation will not vary with time when it reaches thermal equilibrium state. The last step involves reviewing of result, where the temperature contour is plotted.

\section{RESULTS AND DISCUSSION}

The results of various winding designs are as follows:

\section{A. Design 1}

2 cooling ducts are placed between primary and secondary windings, and there is no cooling duct within primary winding or within secondary winding. It is as shown in Figure 1 below.

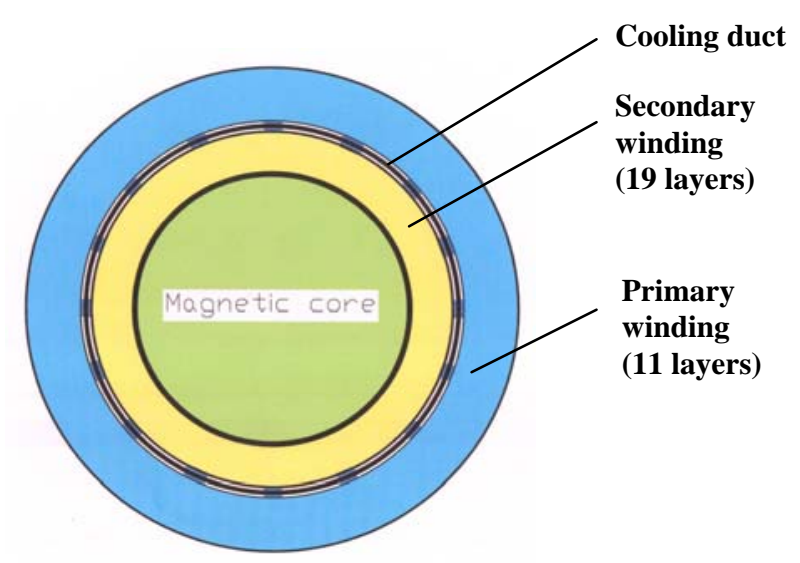

Figure 1. Top view of the winding (Design 1)

The simulation result of secondary winding is shown in Figure 2. It shows that the temperatures of this part of the winding vary from $75.0{ }^{\circ} \mathrm{C}$ to $104.0{ }^{\circ} \mathrm{C}$, or a thermal gradient of $29.0^{\circ} \mathrm{C}$. The temperature is almost constant in each layer of copper conductor and temperature drops mainly across the kraft insulating paper. This is because the thermal conductivity for copper is very high (about 401 $\mathrm{W} / \mathrm{m} . \mathrm{K}$ ) as compared to that of kraft insulating paper (about $0.1815 \mathrm{~W} / \mathrm{m} . \mathrm{K})$.

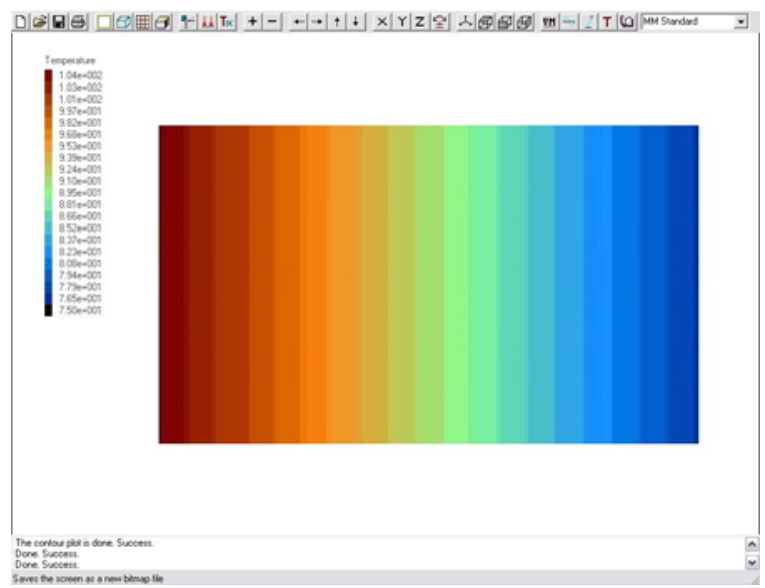

Figure 2. Thermal gradient of the secondary winding

The simulation result of primary winding is shown in Figure 3 . The temperature in the centre of primary winding is the highest, which is $88.5^{\circ} \mathrm{C}$. The temperature gradually lowers towards both left and right sides of the windings where the winding is in direct contact with oil, reaching $75.0{ }^{\circ} \mathrm{C}$ at the inmost and outmost layers of the winding. Thermal gradient of this block is $13.5^{\circ} \mathrm{C}$.

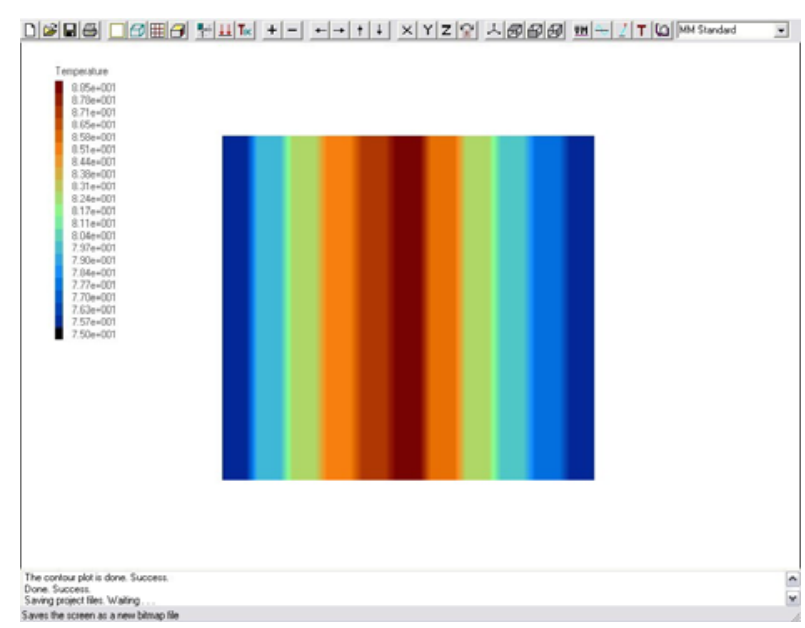

Figure 3. Thermal gradient of the primary winding

The results of simulation are summarized in Table 2. The thermal gradients for both secondary and primary windings are too high.

Table 2. Summary of simulation results for Design 1

\begin{tabular}{|l|c|c|}
\hline $\begin{array}{l}\text { Section of transformer } \\
\text { winding }\end{array}$ & $\begin{array}{c}\text { Range of } \\
\text { Temperature } \\
\left({ }^{\mathbf{0}} \mathbf{C}\right)\end{array}$ & $\begin{array}{c}\text { Thermal } \\
\text { Gradient } \\
\left({ }^{\mathbf{0}} \mathbf{C}\right)\end{array}$ \\
\hline Secondary Winding & $75.0-104 . .0$ & 29.0 \\
\hline Primary Winding & $75.0-88.5$ & 13.5 \\
\hline
\end{tabular}

\section{B. Design 2}

A sketch of the winding (top view) is shown in Figure 4. Secondary winding is subdivided into block A of 6 layers and block B of 13 layers. Between block A and block B of secondary winding, there is a cooling duct of $4 \mathrm{~mm}$ thick (cooling duct 2A). As for primary winding, it is subdivided into block A of 5 layers and block B of 6 layers, with a cooling duct of $4 \mathrm{~mm}$ thick between block A and block B (cooling duct 1B). There are also two cooling ducts of $4 \mathrm{~mm}$ each between secondary and primary windings (cooling ducts $2 \mathrm{~B}$ and $1 \mathrm{~A}$ ).

The simulation result of block A of secondary winding is shown in Figure 5. It shows that the temperatures of this part of the winding vary from $75.0{ }^{\circ} \mathrm{C}$ to $81.0{ }^{\circ} \mathrm{C}$, or a thermal gradient of $6.0^{\circ} \mathrm{C}$.

The simulation result of block B of secondary winding is shown in Figure 6. The temperatures in the centre of block $\mathrm{B}$ of secondary winding is the highest, that is $81.5^{\circ} \mathrm{C}$. The temperature gradually lowers towards both left and right sides of the windings where the cooling ducts are located, reaching $75.0{ }^{\circ} \mathrm{C}$ at the inmost and outmost layers of this block. Thermal gradient of this block is $6.5^{\circ} \mathrm{C}$. 


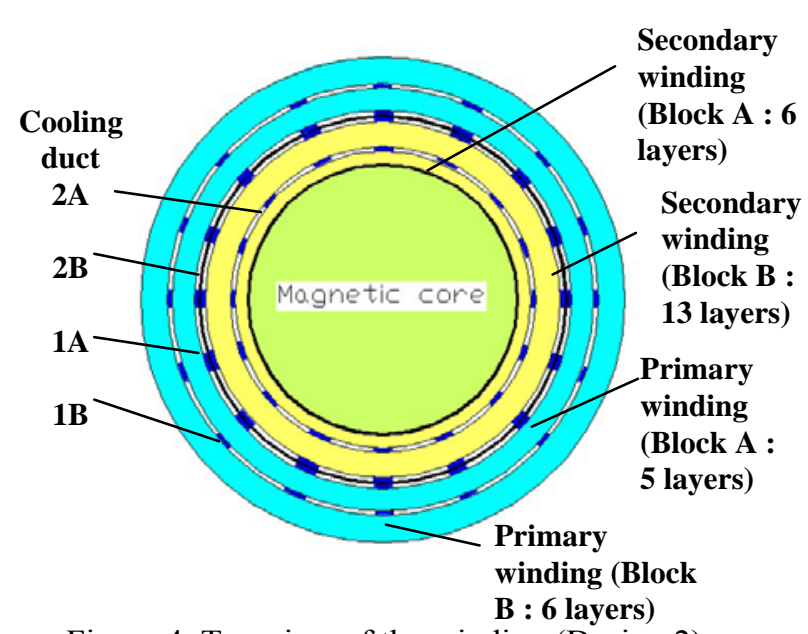

Figure 4. Top view of the winding (Design 2)

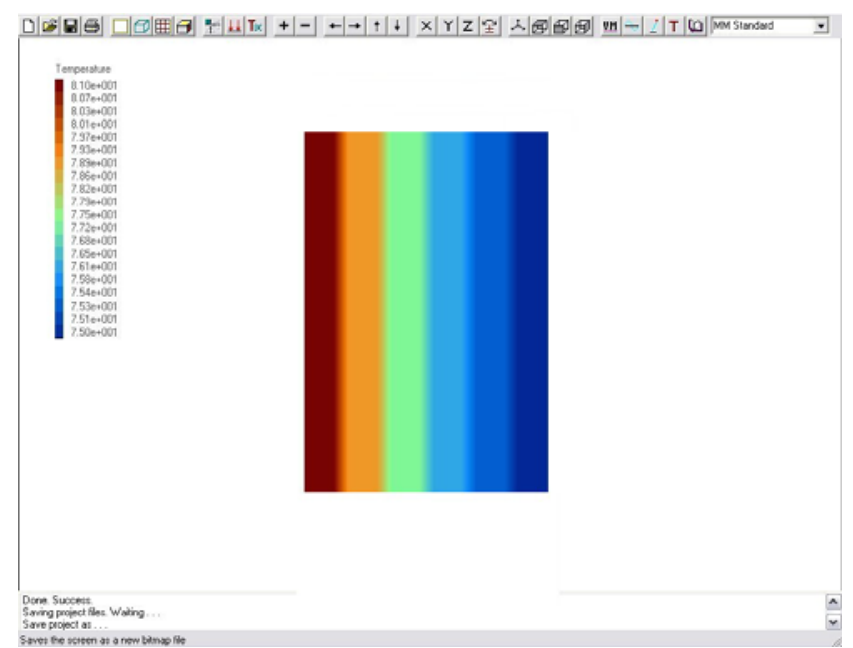

Figure 5. Result of simulation for block A of secondary winding
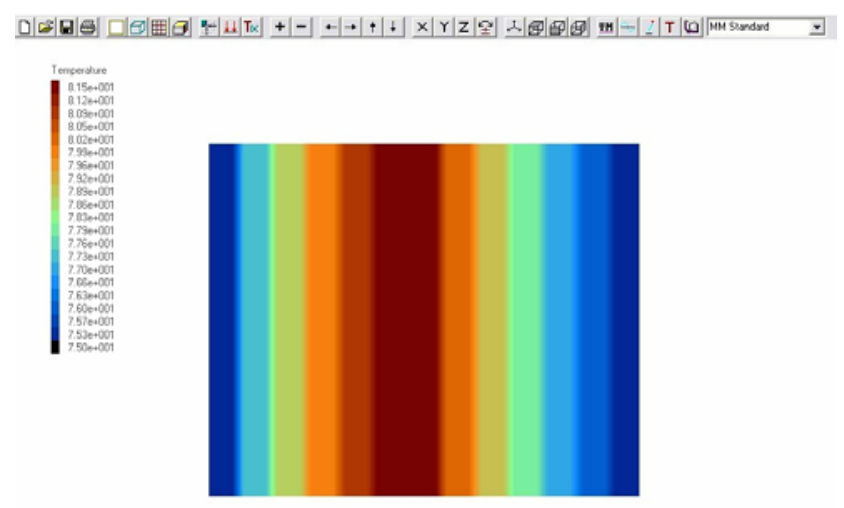

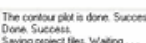

Figure 6. Result of simulation for block B of secondary winding

For block A of primary winding, the simulation result shows that pattern of heat dissipation is similar to that of block $\mathrm{B}$ of secondary winding, that is temperature in the centre of the block is the highest $\left(80.0^{\circ} \mathrm{C}\right)$ and drops across both left and right sides of the winding $\left(75.0{ }^{\circ} \mathrm{C}\right.$ at both ends). This is as shown in Figure 7, with a thermal gradient of $5.0^{\circ} \mathrm{C}$.

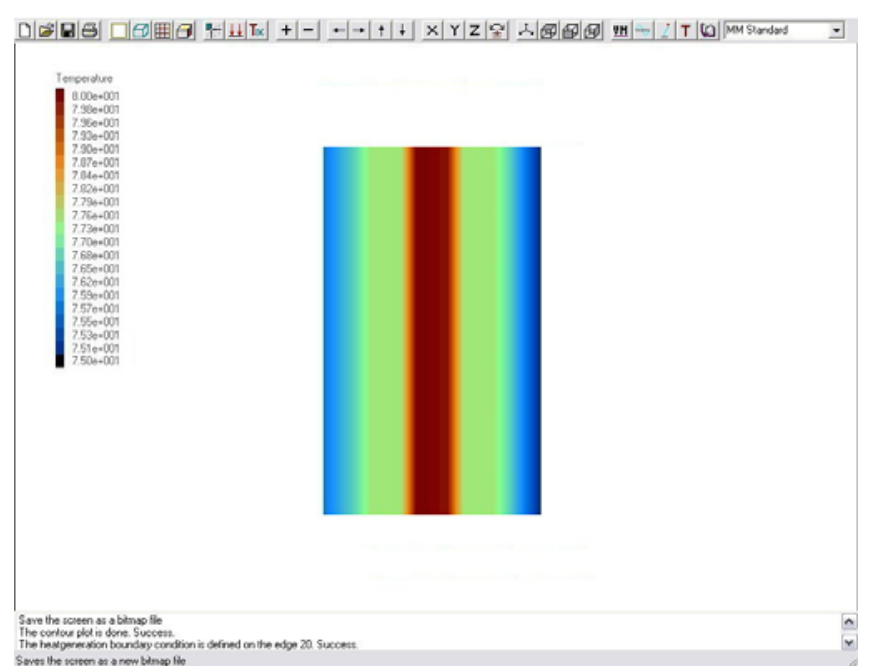

Figure 7. Result of simulation for block A of primary winding

Result of simulation for block B of primary winding is shown in Figure 8. The highest temperature is $81.3{ }^{\circ} \mathrm{C}$ in the middle of the block and it is $75.0^{\circ} \mathrm{C}$ at both ends. Thermal gradient of this block is $6.3{ }^{\circ} \mathrm{C}$.

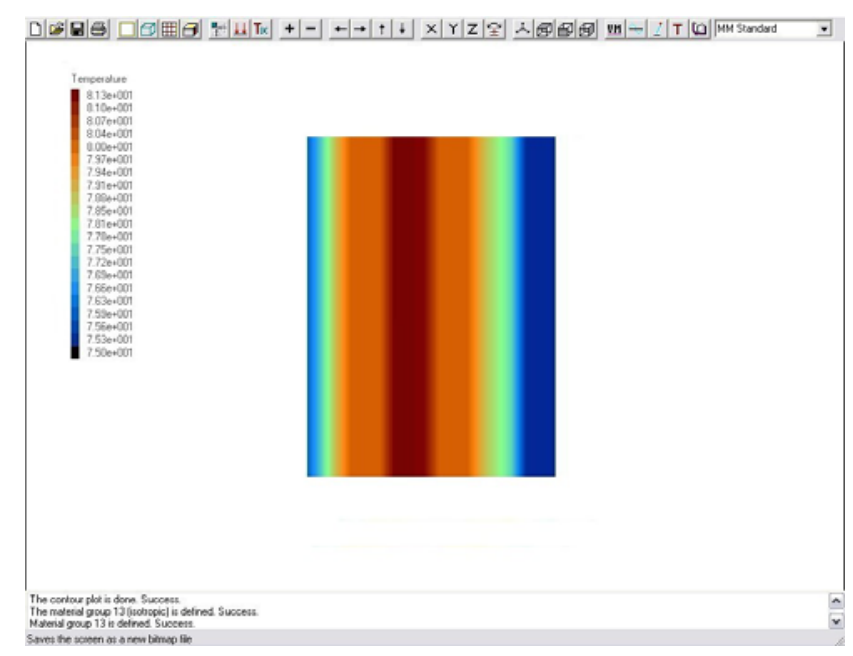

Figure 8. Result of simulation for block B of primary winding

Table 3 summarizes the thermal gradients of the various sections of the windings. Thermal gradient for block A of secondary winding is $6.0{ }^{\circ} \mathrm{C}$ whereas for block $\mathrm{B}$ of secondary winding is $6.5{ }^{\circ} \mathrm{C}$. This means the highest temperature of the entire secondary winding is $6.5^{\circ} \mathrm{C}$ higher than the oil temperature. Similarly, for primary winding, the highest temperature of the entire primary winding is $6.3^{\circ} \mathrm{C}$ higher than the oil temperature. It can be deduced that such arrangement of cooling ducts within the windings enable good ventilation of oil, and thus reduces the thermal gradient a lot as compared to Design 1.

\section{C.Design 3}

Secondary winding is subdivided into block A of 3 layers, block B of 8 layers and block $\mathrm{C}$ of 8 layers. There are cooling duct of $4 \mathrm{~mm}$ thick between blocks. As for primary winding, it is subdivided into block A of 3 layers, block B of 4 layers and block $\mathrm{C}$ of 4 layers. Similarly, there are cooling duct of $4 \mathrm{~mm}$ thick between blocks. There are also 
two cooling ducts of $4 \mathrm{~mm}$ each between secondary and primary windings. A sketch of the winding (top view) is shown in Figure 9.

Table 3. Summary of thermal gradients of the various sections of the windings

\begin{tabular}{|c|c|c|}
\hline $\begin{array}{c}\text { Section of } \\
\text { transformer } \\
\text { winding }\end{array}$ & $\begin{array}{c}\text { Range of } \\
\text { Temperature } \\
\left.\mathbf{(}^{\mathbf{0}} \mathbf{C}\right)\end{array}$ & $\begin{array}{c}\text { Thermal } \\
\text { Gradient } \\
\left({ }^{\mathbf{0}} \mathbf{C}\right)\end{array}$ \\
\hline $\begin{array}{c}\text { Block A of } \\
\text { Secondary Winding }\end{array}$ & $75.0-81.0$ & 6.0 \\
\hline $\begin{array}{c}\text { Block B of } \\
\text { Secondary Winding }\end{array}$ & $75.0-81.5$ & 6.5 \\
\hline $\begin{array}{c}\text { Block A of Primary } \\
\text { Winding }\end{array}$ & $75.0-80.0$ & 5.0 \\
\hline $\begin{array}{c}\text { Block B of Primary } \\
\text { Winding }\end{array}$ & $75.0-81.3$ & 6.3 \\
\hline
\end{tabular}

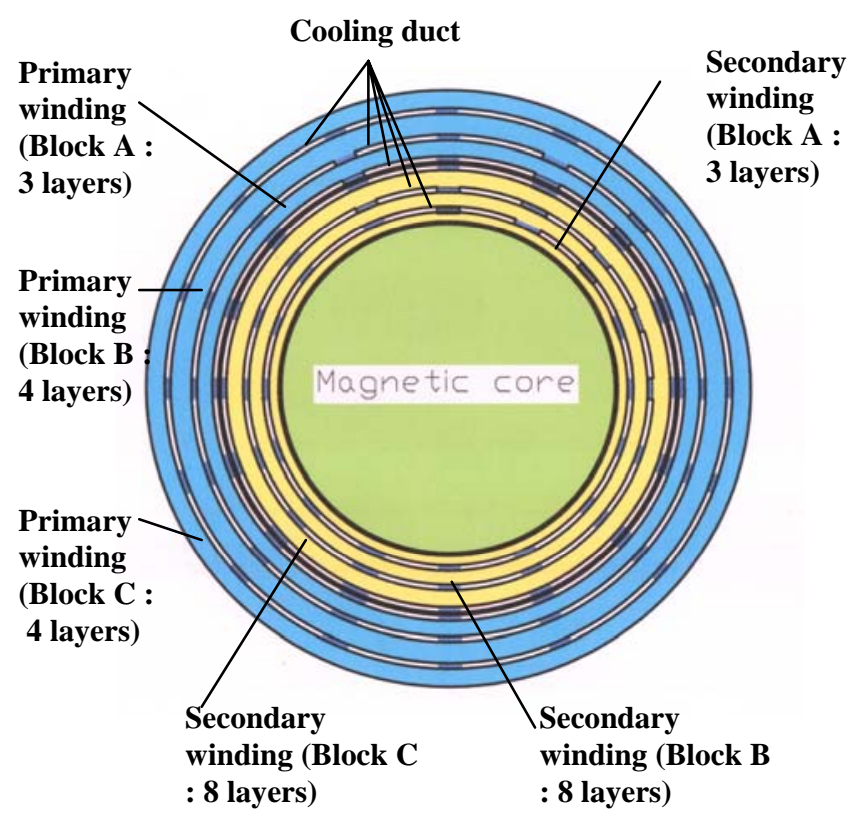

Figure 9. Top view of the winding (Design 3)

Results of simulation are as shown in Figures 10, 11, 12, 13,14 and 15 below.

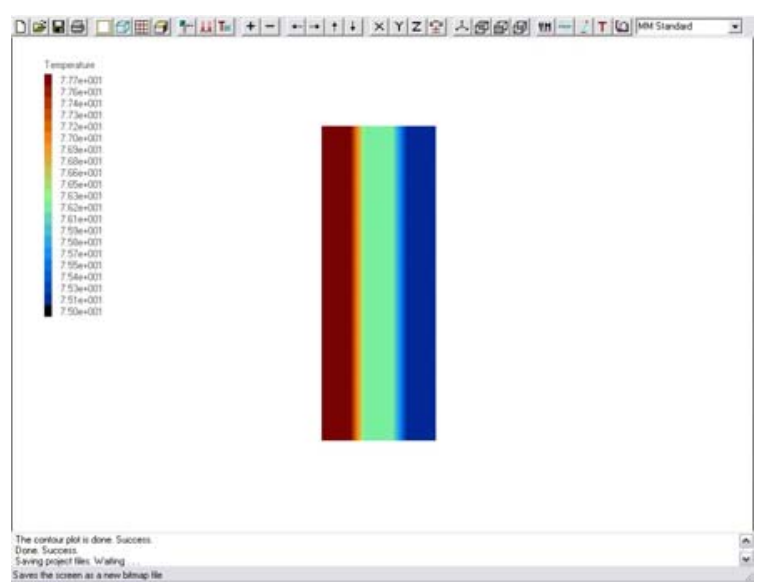

Figure 10. Thermal gradient of secondary winding (Block A)

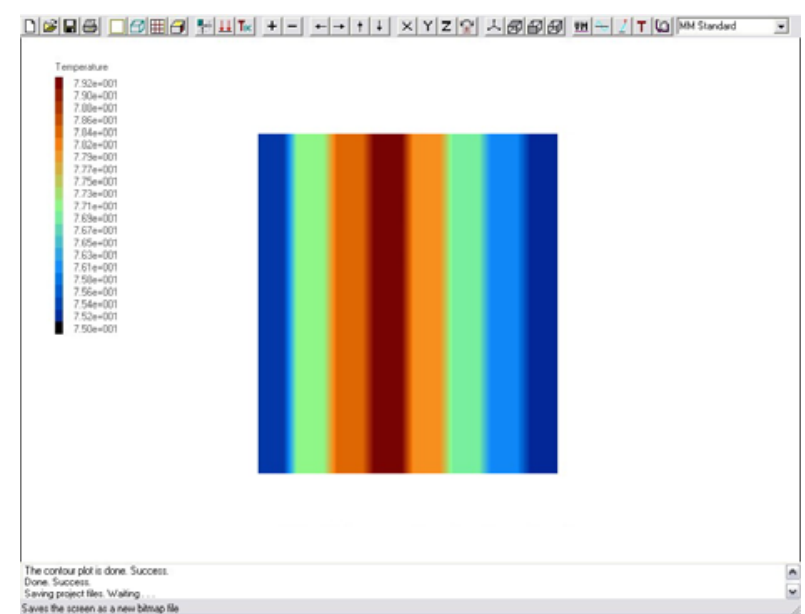

Figure 11. Thermal gradient of secondary winding (Block B)

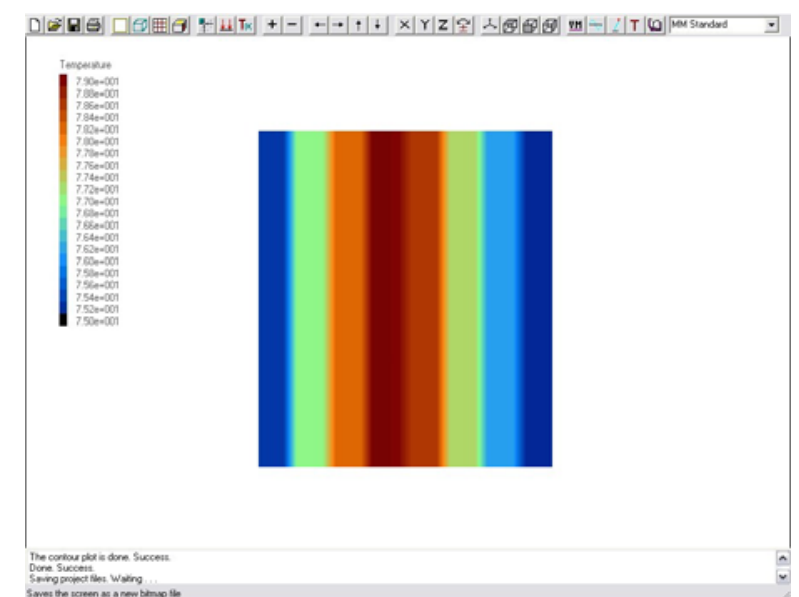

Figure 12. Thermal gradient of secondary winding (Block C)

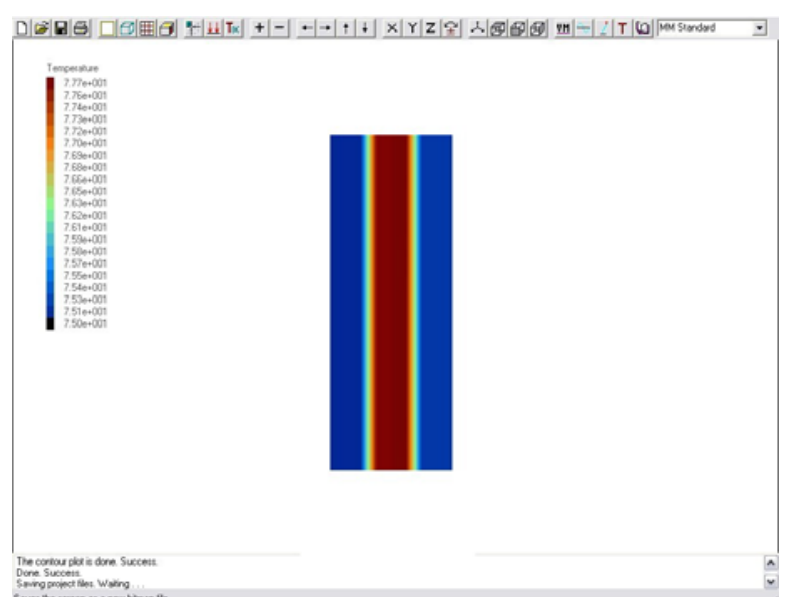

Figure 13. Thermal gradient of primary winding (Block A)

The results of simulation are summarized in Table 4 . The thermal gradients for various sections of secondary winding range from $2.7^{\circ} \mathrm{C}$ to $4.2^{\circ} \mathrm{C}$, whereas for primary winding is from $2.7^{\circ} \mathrm{C}$ and $4.4^{\circ} \mathrm{C}$. These thermal gradients are even lower than those of Design 2. 


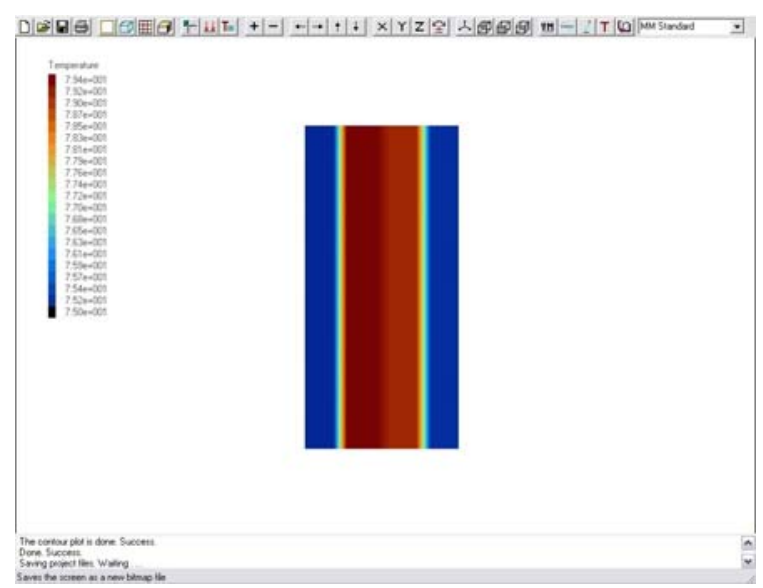

Figure 14. Thermal gradient of primary winding (Block B)

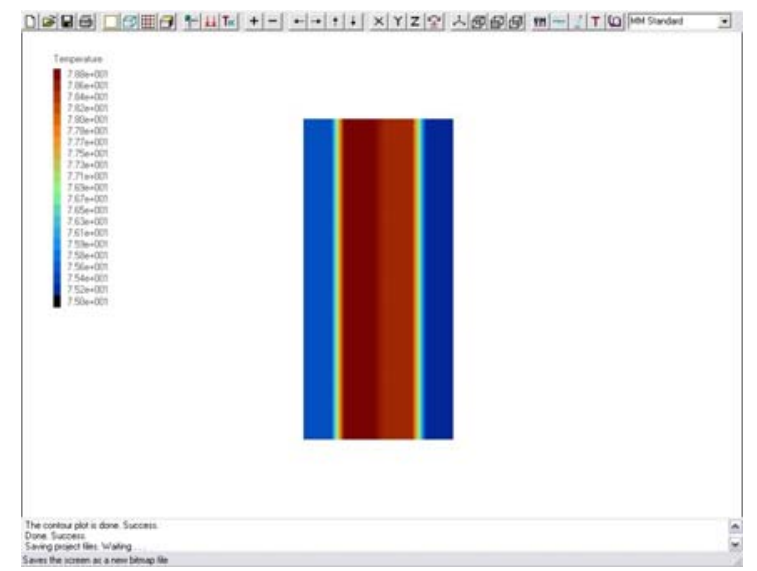

Figure 15. Thermal gradient of primary winding (Block C)

Table 4. Summary of simulation results for design 3

\begin{tabular}{|l|c|c|}
\hline $\begin{array}{l}\text { Section of } \\
\text { transformer winding }\end{array}$ & $\begin{array}{c}\text { Range of } \\
\text { Temperature } \\
\left(\mathbf{(}^{\mathbf{}} \mathbf{C}\right)\end{array}$ & $\begin{array}{c}\text { Thermal } \\
\text { Gradient } \\
\left({ }^{\mathbf{0}} \mathbf{C}\right)\end{array}$ \\
\hline $\begin{array}{l}\text { Block A of Secondary } \\
\text { Winding }\end{array}$ & $75.0-77.7$ & 2.7 \\
\hline $\begin{array}{l}\text { Block B of Secondary } \\
\text { Winding }\end{array}$ & $75.0-79.2$ & 4.2 \\
\hline $\begin{array}{l}\text { Block C of Secondary } \\
\text { Winding }\end{array}$ & $75.0-79.0$ & 4.0 \\
\hline $\begin{array}{l}\text { Block A of Primary } \\
\text { Winding }\end{array}$ & $75.0-77.7$ & 2.7 \\
\hline $\begin{array}{l}\text { Block B of Primary } \\
\text { Winding }\end{array}$ & $75.0-79.4$ & 4.4 \\
\hline $\begin{array}{l}\text { Block C of Primary } \\
\text { Winding }\end{array}$ & $75.0-78.8$ & 3.8 \\
\hline
\end{tabular}

\section{CONCLUSION}

Provided that the transformer tank and radiator have sufficient surfaces for dissipation of heat to the surrounding and are able to limit the oil temperature rise not exceeding $45^{\circ} \mathrm{C}$, low thermal gradient of transformer windings will ensure that the winding temperature rise will not exceed $55^{\circ} \mathrm{C}$ as per requirement of IEC 60076 standard.

Design 1 apparently does not meet this requirement as the thermal gradient of secondary winding is $29^{\circ} \mathrm{C}$. For Design 2 , thermal gradients for both primary and secondary windings are acceptably low. Cooling ducts enables ventilation of oil for good heat dissipation. For design 3, thermal gradients for both primary and secondary windings are very low. However, more cooling duct inserted within windings entails higher construction cost due to enlarged winding diameter.

The research was done with assumption that the transformer is loaded with the rated power of $1000 \mathrm{kVA}$. Its temperature rise is a conventional reference basis for continuous loading duty under normal ambient temperature and rated operating conditions. However, in actual case, temperature rise of a transformer depends to a high degree on extraordinary events, such as over-voltages, shortcircuits in the system, and emergency overloading [4]. Studies should be made in future on the effect of different type of loading duty (i.e. normal cyclic undisturbed loading, temporary emergency loading or long-time emergency loading) to the operating temperature of a transformer, particularly the temperature of the hottest part of the winding. This hot-spot temperature is, in turn, used for evaluation of the rate of thermal ageing to provide recommendations for limitations of permissible loading.

\section{REFERENCES}

[1] IEC (2000). IEC60076-2 Power Transformer - Temperature Rise. International Electrotechnical Commission, p.13-29, 51-55.

[2] Thermal Conductivity of Some Common Materials, www.EngineeringToolBox.com.

[3] Efacec Energia R\&D Department (1995). EDTE31002 Gradient Calculation for Liquid Dielectric Transformer. Efacec Energia Transformer Design Manual, p.3-7.

[4] IEC (1991). IEC354 Loading guide for Oil-immersed Power Transformer. International Electrotechnical Commission, p.11, 37. 


\title{
Drying Improvement via Application of CFD
}

\author{
A. Chai, H.U. Ha and A. Rigit
}

\begin{abstract}
The computational fluid-dynamics (CFD) simulation results performed on an industrial roller-kiln are presented in this paper. The results are obtained through implementation of user mathematical-models into a simulation solver, Star-CD. This CFD solver was used to perform the modelling and simulation of the roller-kiln drying process. Tetrahedral meshes were generated with boundary conditions according to the actual roller-kiln. Improvements on this initial boundary conditions were also incorporated into the solver, with altered burner-temperature configurations. The CFD simulation results on both initial and improved boundaries were obtained and presented as contour-plots on temperature profiles. The results demonstrated homogeneous heat transfer within the air-flow throughout the roller-kiln. An experimental prototype was also fabricated to replicate a scaled-model of the actual roller-kiln. Results obtained from the experiment also showed consistent and uniform temperature distribution, when compared to the CFD results. This means that a similar drying profile can be achieved despite the change in burner-temperature. Hence, improvement on drying can be realized.
\end{abstract}

Keywords: CFD, drying, mathematical-models, prototype

\section{INTRODUCTION}

The drying process in the ceramic-tiles manufacturing industry utilizes the roller-kiln, which operates at extreme conditions to meet with specific product requirement [1]. The roller-kiln can be operating simply to meet the final product output regardless of the wasteful consumption of resources such as gas. Without appropriate observation and improvement, the burners on the roller-kiln will only operate continuously despite the combustion of vast quantity of gas. This results in excessive depletion of energy in the form of work heat.

Much works have been performed throughout the past decade, especially on rotary-kilns, melters and calciners, with studies on the heat transfer and moisture transportation [2][3]. Other similar works have been carried out on industrial systems such as multi-staged dryers [4]. CFD was also applied to obtain visualization on mass and heat transfer and identification of energy inefficiency, such as through the use of temperature plots [5][6]. Nevertheless,

A. Chai is with the School of Engineering, Swinburne University of Technology, Kuching, Sarawak, Malaysia. (phone: +6082-416353 ext.8309; e-mail: achai@swinburne.edu.my).

H.U. Ha is with the School of Engineering, Swinburne University of Technology, Kuching, Sarawak, Malaysia. (e-mail: hha@swinburne.edu.my).

A. Rigit is with the Faculty of Engineering, Universiti Malaysia Sarawak, Sarawak, Malaysia. (e-mail: arigit@feng.unimas.my). there still lacks the specific research on the roller-kiln in the ceramic-tiles manufacturing industry. Through mathematical modelling and CFD simulation, fundamental studies on the drying process were achieved and the heat transportation phenomena were distinguished. The areas of inefficiency were also identified from the CFD results, and different parameters were investigated and defined.

\section{MATHEMATICAL MODELLING}

A lumped-parameter model was derived, defining temperature $(T)$ and humidity $(\phi)$ as functions of time $(t)$ and Cartesian-coordinate length $(l)$. Since the roller-kiln has internal air-flow resulting from the combustion of the burners, the overall transfer of heat is considered as forced convection.

The humidity parameter was derived for only the gas- and tile-phase of the drying phenomena occurring within the roller-kiln. The model is shown in (1), and was used as a basis for deriving the drying kinetics of the ceramic-tiles in the roller-kiln,

$$
\frac{\partial \phi(l, t)}{\partial t}+\frac{\partial T(l, t)}{\partial t} \pm v(t)\left[\frac{\partial \phi(l, t)}{\partial l}+\frac{\partial T(l, t)}{\partial l}\right]=f(\phi, T, l, t)
$$

where $\phi$ is the humidity, $T$ the temperature and $v$ the linear velocity in the tile or gas phase, $l$ is the roller-kiln length and $t$ is time. Equation (1) was further simplified to parametric models where the Cartesian-co-ordinate length (l) was replaced by $L$. (1) is now of the general differentiation form shown in (2), (3) and (4).

$$
\begin{aligned}
& \frac{d \phi_{\text {out }}}{d t}+v_{s} \frac{\left(\phi_{s, \text { out }}-\phi_{s, \text { in }}\right)}{L}=-R_{w} \\
& C_{s} \frac{d T_{s, \text { out }}}{d t}+v_{s} C_{s} \frac{\left(T_{s, \text { out }}-T_{s, \text { in }}\right)}{L} \\
& =\frac{U_{v} V_{v}}{\rho_{s}}\left(T_{g, \text { out }}-T_{s, \text { out }}\right)-\lambda R_{w} \\
& C_{g} \frac{d T_{g, \text { out }}}{d t}+v_{g} C_{g} \frac{\left(T_{g} \text {, out }-T_{g, \text { in }}\right)}{L} \\
& =-\frac{U_{v} V_{v}}{\rho_{g}}\left(T_{g, \text { out }}-T_{s, \text { out }}\right)-\lambda \frac{\rho_{s}}{\rho_{g}} R_{w}
\end{aligned}
$$

With $\phi_{\text {s,out }}$ being moisture value. $T_{g \text {,out }}$ and $T_{s, \text { out }}$ describe the temperature values for the air-flow and ceramic-tiles in 
the roller-kiln respectively. $\phi_{s, i n}$ represents the moisture value at the burners, while $T_{s, i n}$ and $T_{g, i n}$ represents the temperature values at the gas-air and tile respectively.

\section{CFC MODELLING AND SIMULATION}

The CFD simulation of the roller-kiln model was performed using a commercial computational fluiddynamics (CFD) solver, Star-CD. The Central Differencing (CD) scheme was selected as the differencing scheme to be applied onto the simulation for heat transfer of the rollerkiln, incorporating equations (2), (3) and (4).

Molecular properties were defined to fit the thermophysical properties of the air-gas ratio of heated air-flow. The specific heat constant is $71.5 \times 10^{3} \mathrm{~W} / \mathrm{mK}$. Temperature solving was chosen to be the total thermal enthalpy. At initial temperature of $300 \mathrm{~K}$, molecular viscosity was $15.89 \times 10^{6} \mathrm{~m}^{2} / \mathrm{s}$, density as $1.1614 \mathrm{~kg} / \mathrm{m}^{3}$ and the specific heat constant at $26.3 \times 10^{3} \mathrm{~W} / \mathrm{mK}$. Inlets, simulating the burners, have air-flow velocity of $15 \mathrm{~m} / \mathrm{s}$. The flow switch of the air-flow was solved by mass-flux, hence solving for both heat and mass transfer. The Semi Implicit Method for Pressure Implicit Splitting of Operators (SIMPISO) algorithm was applied. An under-relaxation factor of 0.8 was chosen, with residual tolerance of 0.001 . The conjugate gradient (CG) method was employed while solving the SIMPISO algorithms. A total of 1,298,634 unstructured tetrahedral cells were meshed for the volume of the geometry.

A secondary simulation was performed on the roller-kiln. The inlets boundary-conditions were altered in a way that ten burners deliver heated air-flow at $15 \mathrm{~m} / \mathrm{s}$ and a temperature of $1100 \mathrm{~K}$, the other ten burners combusting airflow at a lower temperature of $950 \mathrm{~K}$. The burners were allocated in such a way that five high-temperature and five lower-temperature burners are placed at each side of the roller-kiln.

The model grid was simulated and analyzed using the preand post-processing Pro-Star solver in Star-CD. The rollerkiln grid was solved for convergence with an iteration count of 1500 . Figure 1 shows the temperature distribution profile in contour-plot, with temperature values presented at a maximum and minimum of $1073 \mathrm{~K}$ and $1070.5 \mathrm{~K}$ respectively. The contour-plot was chosen to be near the proximity of the horizontal burners plane, allowing visual observation and understanding of the temperature distribution near the burner region. It can be seen the heated air-flow entering the roller-kiln has an unvarying pattern compared to each other, showing steady temperature readings by the patterns at each burner location.

Figure 2 shows the temperature profile with the altered burner inlet temperature configuration in this secondary simulation. It can be seen the overall temperature range in the distribution pattern nonetheless maintains a close range of approximately $1000 \mathrm{~K}$. The monitored values for the $\mathrm{u}, \mathrm{v}$, $\mathrm{w}$ momentum and temperature for the roller-kiln on a specific monitoring point on the plane horizontal to the burner inlets are shown in figure 3(a) and 3(b) respectively. The temperature graph in figure 3 (b) shows a convergence in solutions after iteration counts reach 900, indicating temperature maintains at the required rate.

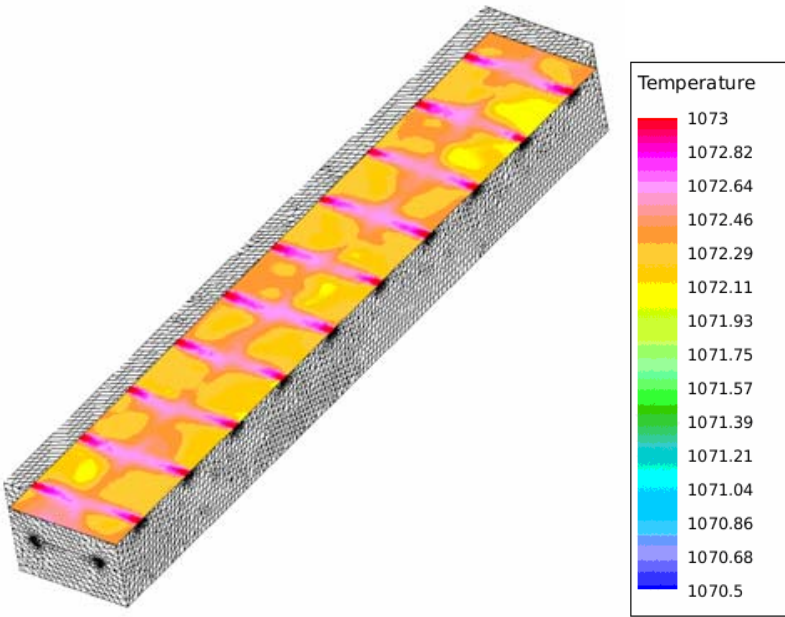

Figure 1. The temperature distribution in contour-plot with plane horizontal to the burner inlets

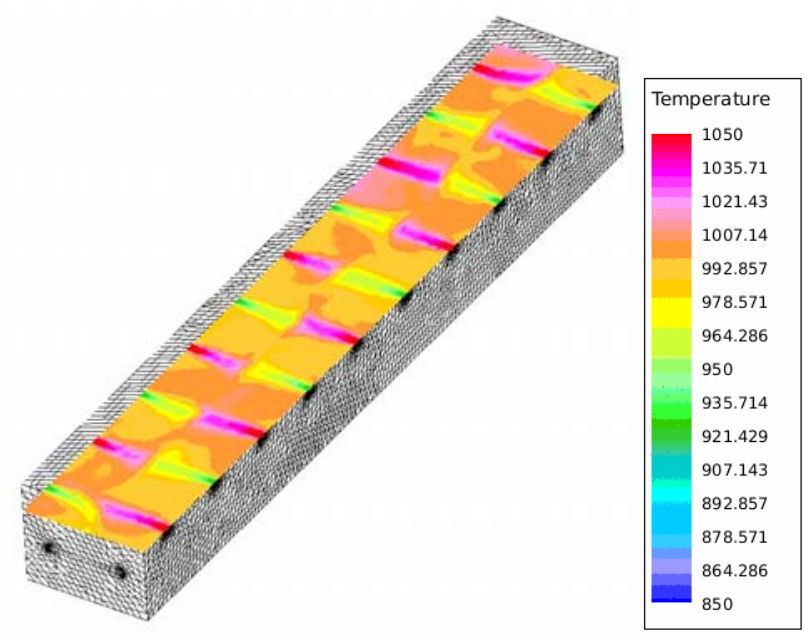

Figure 2. The temperature distribution in contour-plot with plane horizontal to the burner inlets. The colour differences represent the different temperature settings for the individual burner inlets

The monitored values for $\mathrm{u}, \mathrm{v}, \mathrm{w}$ momentum and temperature for the secondary CFD simulation are shown in figure 4(a) and 4(b) respectively. The monitored temperature graph in figure 4(b) shows convergence after iteration counts of 1200 , also similar to the temperature profile in figure $3(\mathrm{~b})$. 


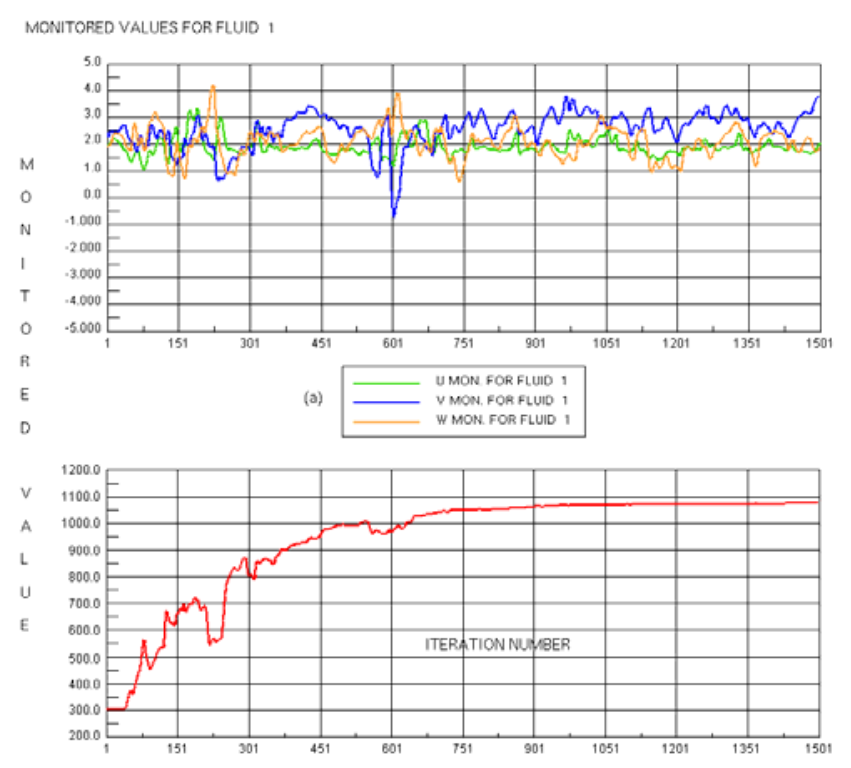

(b)

Figure 3. Graph showing the monitored values for (a) u, v, $\mathrm{W}$ momentum and (b) temperature during initial simulation

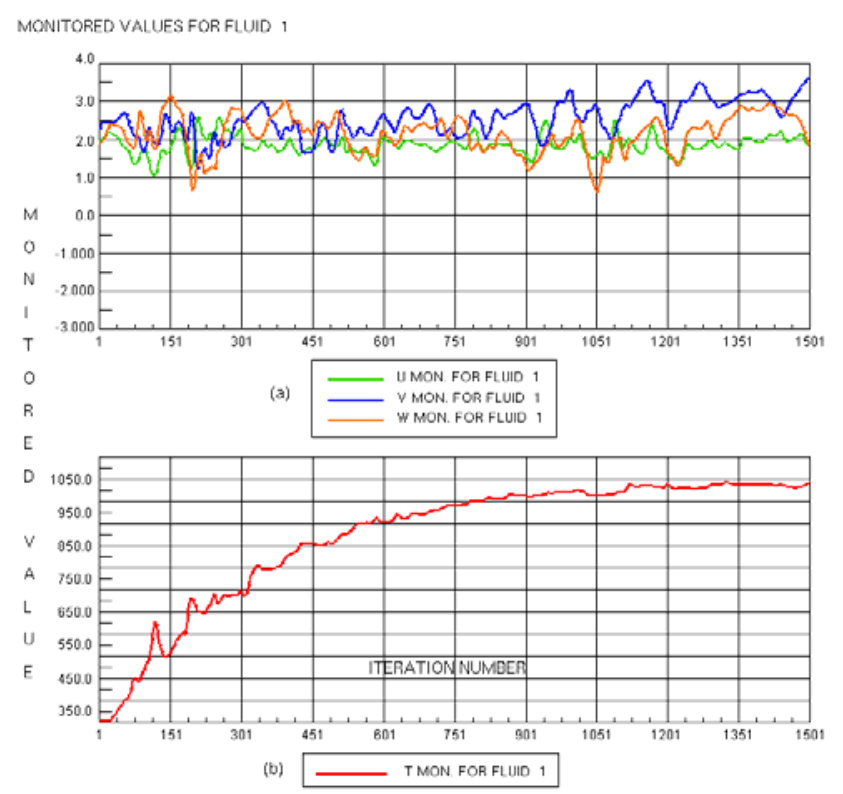

Figure 4. Graph showing the monitored values for (a) u, v, $\mathrm{w}$ momentum and (b) temperature during secondary simulation.

\section{EXPERIMENTAL VALIDATIONS}

A scaled prototype of the actual roller-kiln was fabricated, shown in figure 5, having the exact parameters set-up as the actual roller-kiln. This prototype has a 1/40 scale of the dimensions of the actual roller-kiln. Burner-inlets of the roller-kiln were replicated using heated air-flow supplied by a 1-inlet 10-outlet manifold with tube-heating elements to pre-heat the internal manifold volume air. Figure 6 shows the analysis of the similarity on the temperature profiles between the CFD and experimental results.

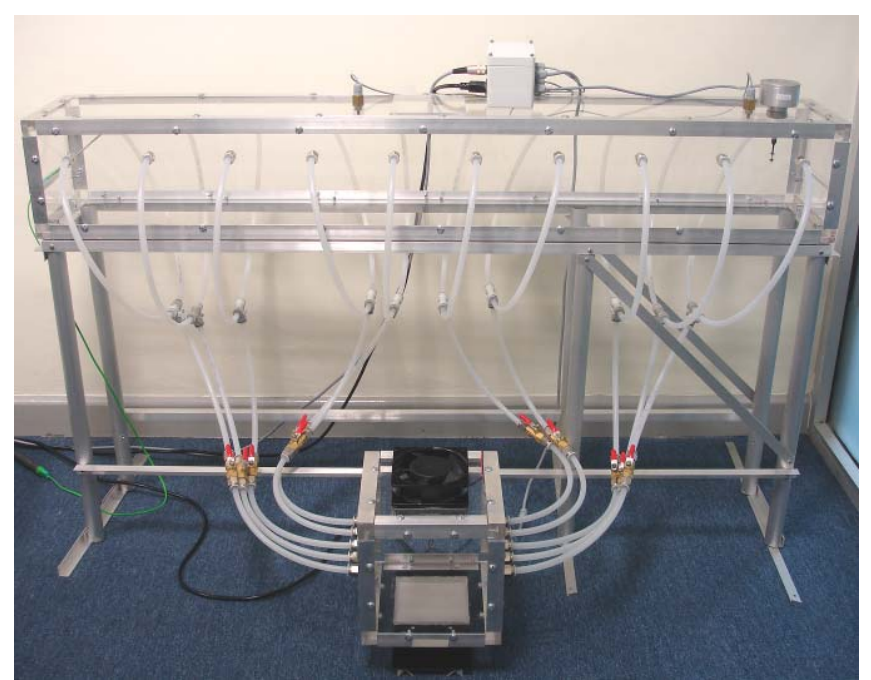

Figure 5. Prototype having 1/40 scale of the actual rollerkiln dimensions

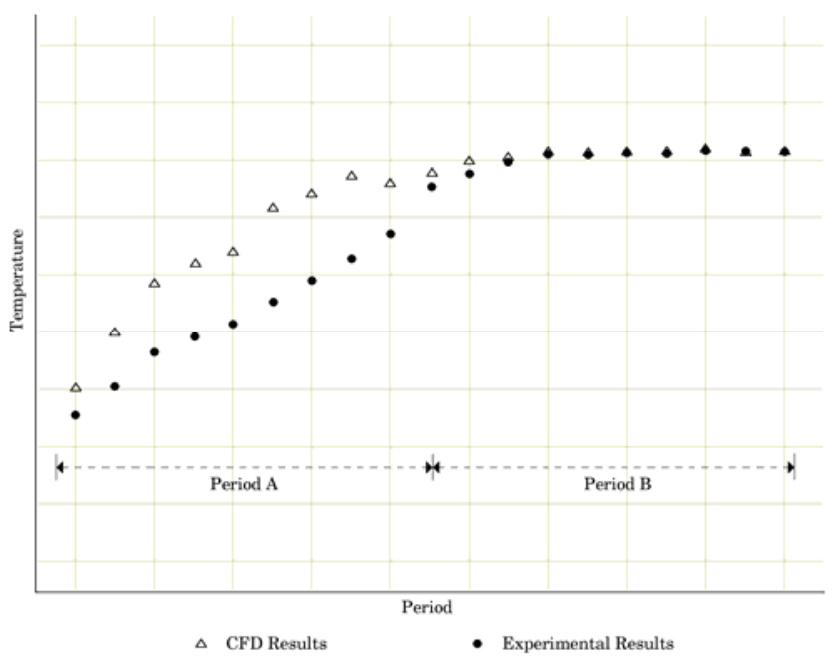

Figure 6. Comparison of temperature profiles of both CFD simulation and experimental result

Period A in figure 6 represents the increase of temperature for both CFD and experimental results during the starting-up of the roller-kiln. The results showed a noticeable difference in the slow increase of temperature for the prototype as opposed to the more rapid temperature ascend in the CFD results. This dissimilarity in period A implies that the transfer of heat within the prototype consumes more time since ambient temperature external to the manifold and prototype is about $294 \mathrm{~K}$. Heated air-flow entering the prototype through the nozzle inlets consumes time to distribute the heat across the entire volume within the prototype. Nonetheless, temperature in the prototype converged to the required temperature of $350 \mathrm{~K}$, as illustrated in period B. The data intersection of the two profiles in period $\mathrm{B}$ also implies that both CFD simulation and the experimental validation model set-ups coincide with each other.

\section{CONCLUSIONS}

From figure 6, it has been established that the experimental results are in concurrence with the CFD simulations on the roller-kiln. This also implies that both experimental and CFD simulation parameters were 
established accurately, and the simulation results obtained using Star-CD have been validated.

Since the thermo-physical properties for the improved burner boundary simulation model were established according to the actual roller-kiln operating characteristics, simulation results have shown that the improvement performed on the burner boundary conditions are viable and realistic. This again implies that heat distribution was nevertheless achieved with less energy, therefore contributing to the improvement of the roller-kiln.

\section{REFERENCES}

[1] J. Brumbaugh, HVAC Fundamentals (Volume1): Heating Systems, Furnaces \& Boilers, $4^{\text {th }}$ Edition, Wiley Publishing, 2004.

[2] G. L. Bai and Garrahan, P. "The temperature and moisture content in lumber during preheating and drying". Journal of Wood Science and Technology, 18, 1984.

[3] P. S. Kuts, V. Y. Sklyar and A. L. Ol'shanskii, "Generalized Equation for Kinetics of Convective Drying of Moist Materials". Journal of Engineering Physics and Thermophysics, 53 (1), 1987.

[4] M. B. Stakic and R. M. Topic, "Numerical Simulation of Multi-staged Dryer". In Proceedings of the $12^{\text {th }}$ International Drying Symposium IDS2000, 2000.

[5] S. V. Patankar, "Computational Modeling of Flow and Heat Transfer in Industrial Applications". International Journal of Heat and Fluid Flow, 23, 2002.

[6] K. Pougatch, M. Salcudean and I. Gartshore, "A Numerical Model of the Reacting Multiphase Flow in a Pulp Digester". Journal of Applied Mathematical Modelling, 30, 2006. 


\title{
Design of a Solar-Based Dryer for Agricultural Product Drying
}

\author{
A. Rigit, A. Chai, E. Junaidi and M. S. Osman
}

\begin{abstract}
Outdoor drying is a common practice and traditional method exercised especially in tropical climates for the drying of agricultural products. Considerable savings can be achieved through this particular method, as source of energy is sustainable and limitless. However, there are limitations to the usage of the solar-dryer, such as the level of humidity at locality and temperature range from the solar energy source. Solar-drying in an enclosed structure is therefore favourable, as this natural-convection driven drying is an attractive way in reducing post-harvest losses. This method can also be used regardless of any weather changes, especially in our Malaysian tropical weather. It is presented in this research paper the design stages of a portable solar-based dryer, which is to be utilized for the drying of the pepperberries. The portable solar-dryer can also be used in rural or sub-rural farms or plantations. Computational simulations stage planning and results are also presented here, illustrating the temperature profile within the dryer during simulated operation.
\end{abstract}

Keywords: solar-dryer, portable, pepper, simulation

\section{INTRODUCTION}

Direct outdoor drying is a common practice where drying is concerned. It is a traditional method exercised especially in tropical climates for the drying of agricultural products. Considerable savings can be achieved through this particular method, since the source of energy is sustainable and limitless. However, the drawback of this solar-drying method is weather dependent, and this method can be exposed to problems such as contamination, microbial attacks, which can all have significant effects on the quality (or quantity) of the end-product [1]. Additionally, the period for the drying time can be long and this results in losses on the post-harvest. Several types of natural convection solardryers have already been investigated globally [2,3], however with limitations on the performance of the dryers based on typical weather where the dryers were operated or utilized. These limitations are usually the level of humidity at locality, temperature range from the solar energy source,

A. Rigit is with the Faculty of Engineering, Universiti Malaysia Sarawak, Sarawak, Malaysia. (e-mail: arigit@feng.unimas.my).

A. Chai is with the School of Engineering, Swinburne University of Technology, Kuching, Sarawak, Malaysia. (phone: +6082-416353 ext 8309; e-mail: achai@swinburne.edu.my).

M. S. Osman is with the Faculty of Engineering, Universiti Malaysia Sarawak, Sarawak, Malaysia. (e-mail: omshahril@feng.unimas.my).

E. Junaidi is with the Faculty of Engineering, Universiti Malaysia Sarawak, Sarawak, Malaysia. (e-mail: jervina@feng.unimas.my). wind velocity and the product being processed through the solar-dryer. Solar-drying in an enclosed structure is therefore required, as this method of drying is an attractive way in reducing post-harvest losses. This method can also be used regardless of any weather changes, especially in our Malaysian tropical weather. The portable solar-dryer can also be used in rural or sub-rural farms or plantations. The solar-dryer can be operated based on the fundamentals of physics and thermodynamics, where air-flow is circulated within the dryer governed by the phenomena of natural convection [4].

\section{Methodology in Design}

The solar-dryer can be designed based on three approaches. Direct, indirect and mixed-mode. Research has already been performed on all three methods [1], showing that the mixed-mode type solar-dryer is the most efficient in performance. This mode of drying is also most effective in tropical humid areas where climatic conditions assist the drying of agricultural products via usage of the natural energy sources originating from the sun. The solar-dryer can be in the form where exposing sections of the outer-layer or surfaces of the dryer is constructed of Perspex (transparent fibre-glass).

It is the main objective that the solar-dryer be based on the laws of natural convection, where radiation of heat is used as the main heating tool for the pepper-berries. Radiation from the sun-rays can penetrate the Perspex (transparent) layer, thus heating the pepper-berries placed within the main drying chamber. The air-flow circulation within the solar-dryer is the second most important factor of the drying performance of the dryer. The geometry design of the dryer can aid the rate of air-flow circulation through to the air outlet. Pepper-berry trays can also be positioned at locations where cross-flow of the air-flow occurs when entering via the air inlet, meaning each berry can be exposed in the circulation path. With the combination of the heat from the radiation and the air-flow, natural convection is then used as the drying tool applied onto the drying of the pepper-berries.

A preliminary design of the solar-dryer has been completed, which is illustrated in figure 1. The solar-dryer can be in the form where the exposing section of the outerlayer or surfaces of the dryer is constructed of Perspex (transparent fibre-glass) [5]. It is the main objective that the solar-dryer be based on the laws of natural convection, where radiation of heat is used as the main heating tool. The 
air-flow circulation within the solar-dryer is the second most important factor of the drying performance of the dryer. The geometry design of the dryer can aid the rate of air-flow circulation through to the air outlet. Cross-flow of the airflow occurs when entering via the air inlet, meaning product to-be-dried can be exposed in the circulation path.

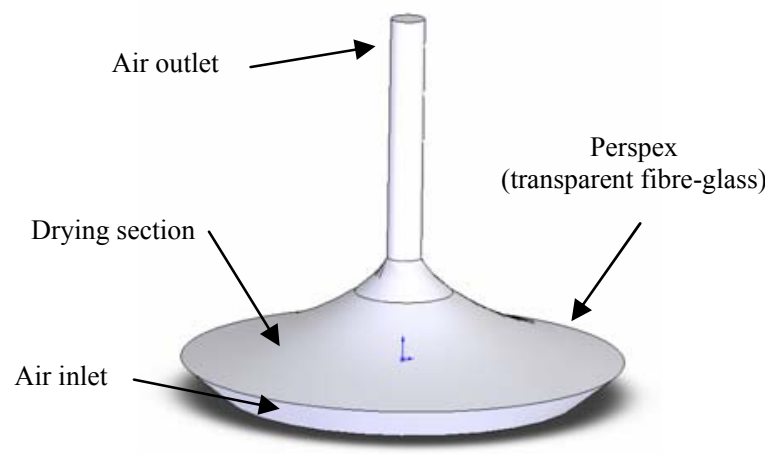

Figure 1. Preliminary solar-dryer design.

\section{Computational Simulation}

The computational fluid-dynamics (CFD) method is most useful when certain designs of the solar-dryer need to be simulated to observe the outcome of results, such as air-flow circulation patterns and heat-transfer phenomenon within the main drying chamber. A typical CFD modelling, simulation and results analysis flowchart is shown in figure 2. Figure 3 illustrates the overall process in the design, fabrication and testing of the solar-based pepper-berries dryer.

The preliminary design of the solar-dryer shown in the previous section was simulated for the air-flow circulation pattern and the results are shown in this section. The CFD results consist of the air-flow velocity-magnitude in contour-plots, streamlines and iso-surf plots. Figure 4 shows the meshing performed on the geometry model of the solardryer before any boundary conditions were set. The modelling meshes are tetrahedral meshes, which occupy the entire volume of the geometry. Figure 5 illustrates the cutsection of the model, showing the meshing at a clearer level. The refinement of the meshes towards the mid-section of the solar-dryer is also visible. This refinement helps in achieving higher levels of accuracy in the CFD simulation results.

With the CFD simulations completed, results based on the air-flow were extracted. Figure 6 shows the contour-plots of the air-flow circulation in velocity-magnitude within the solar-dryer at both $\mathrm{x}$ and $\mathrm{y}$ axis. Areas near the inclined tray surfaces and the Perspex surfaces of the solar-dryer show higher air-flow velocity. This phenomenon shows that airflow circulation exists and is homogeneous throughout the main drying chamber. Consistency in circulation pattern is also observed in this figure.

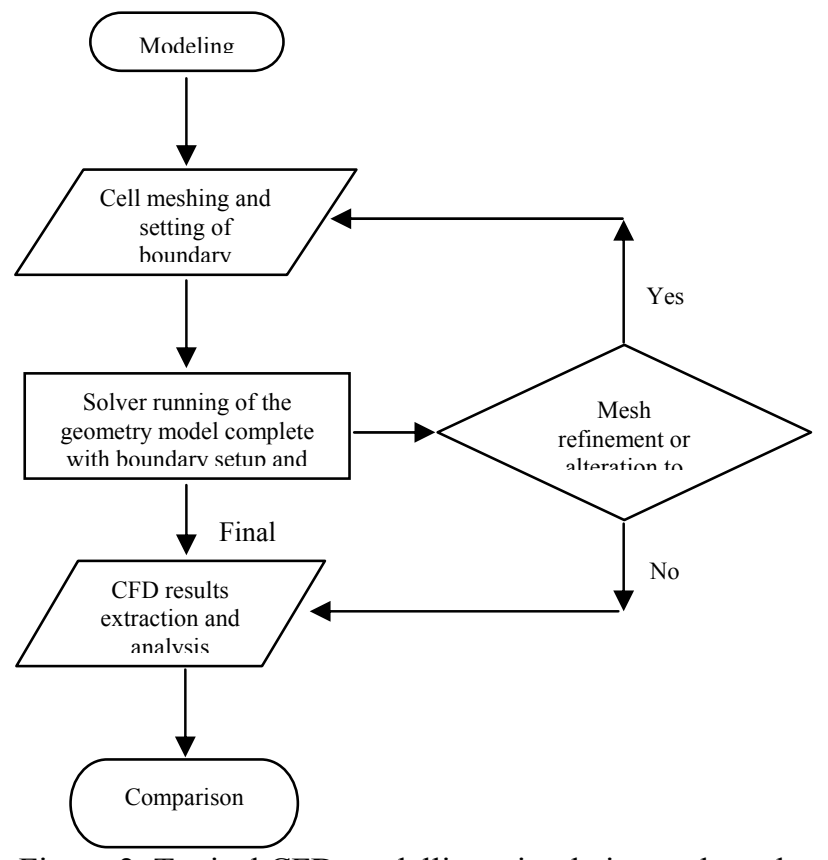

Figure 2. Typical CFD modelling, simulation and results analysis process

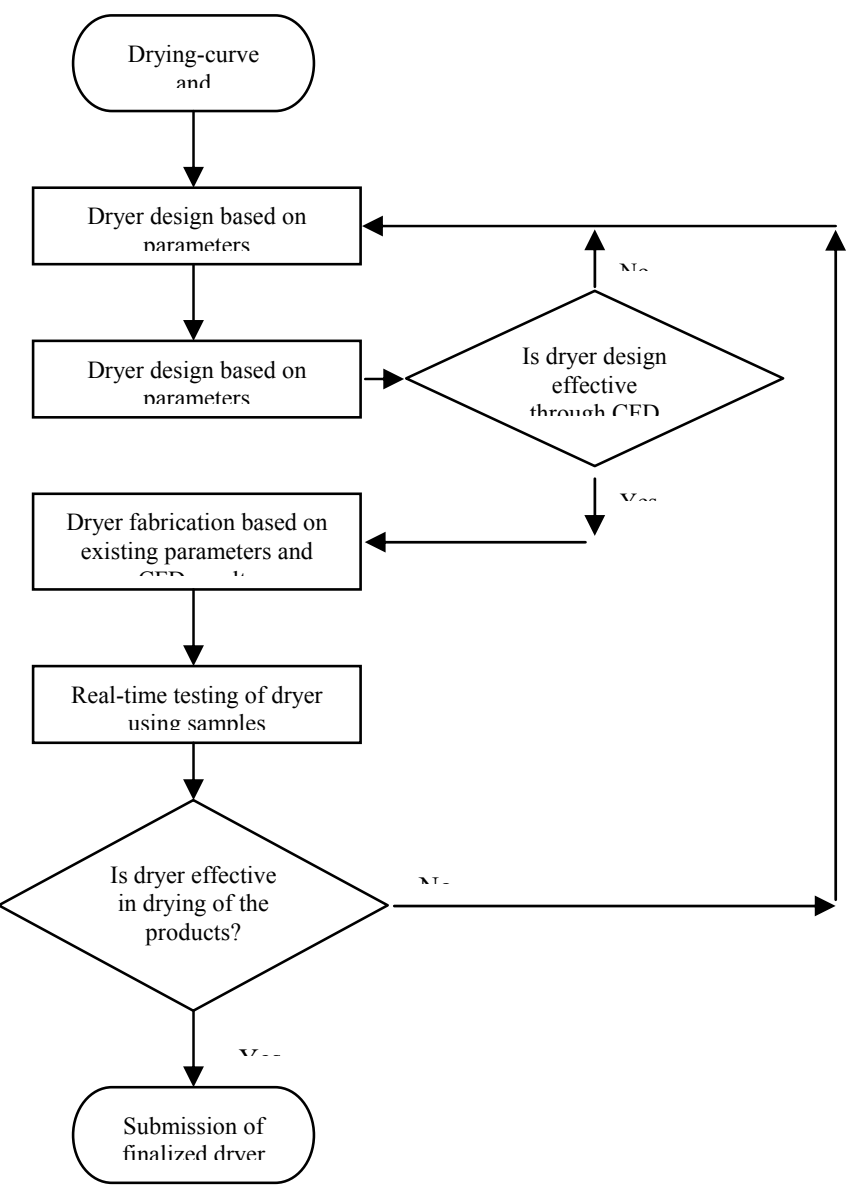

Figure 3. Overall process in the design, fabrication and testing of the solar-based pepper-berries dryer 


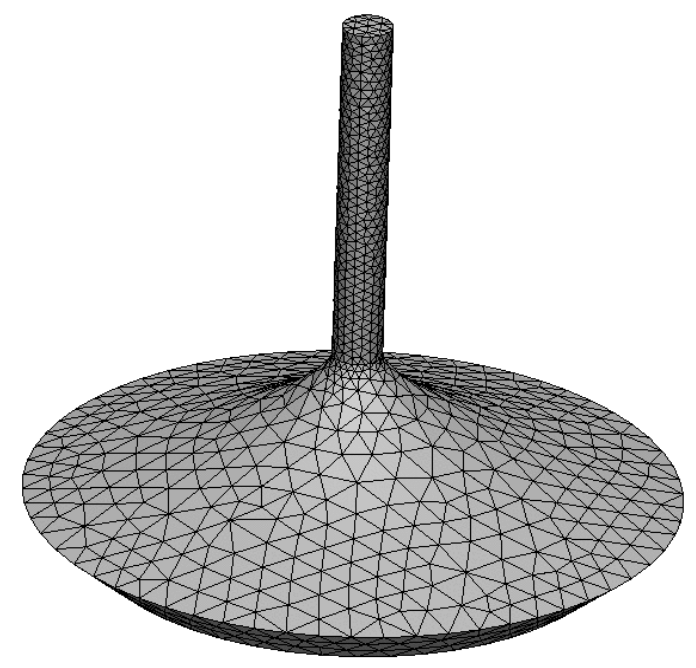

Figure 4. Modeling and tetrahedral meshing of the preliminary solar-based dryer

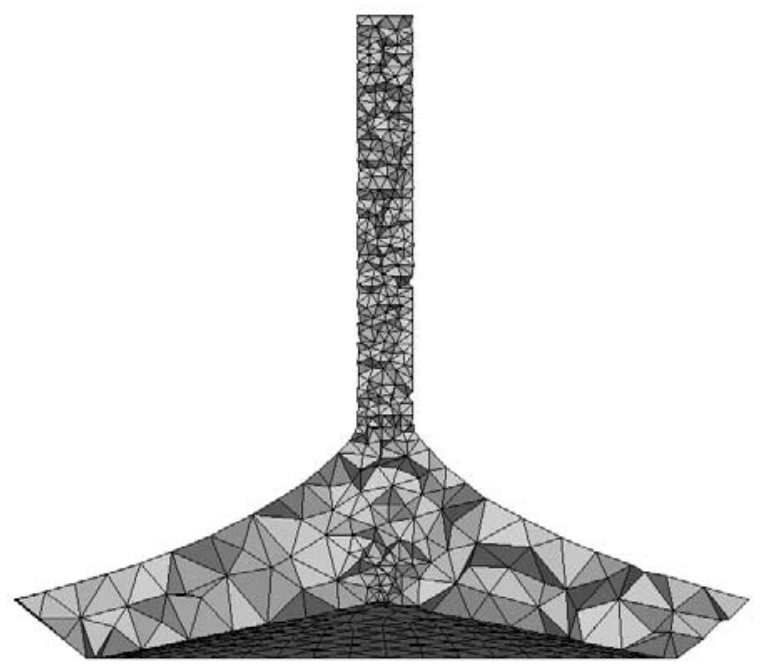

Figure 5. Tetrahedral meshing at mid-section

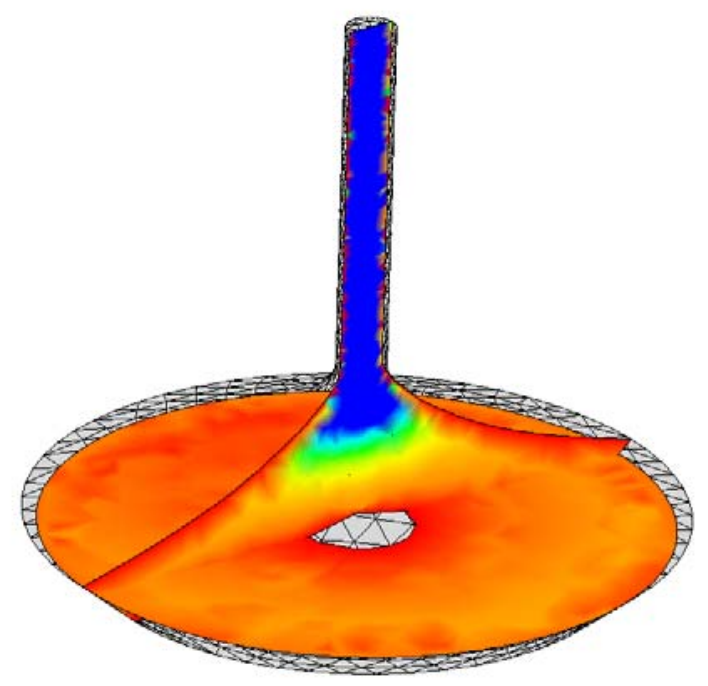

Figure 6. Air-flow velocity-magnitude in contour-plots at $x-$ and $y$-axis

An iso-surf plot is also illustrated in figure 7, showing the volumetric pattern of air-flow entering the solar-dryer via the inlet and exiting through the outlet. This figure shows that with an appropriate design especially on the outlet diameter, air-flow velocity can be controlled, thus regulating the heat transfer via natural convection. The iso-surf plot also demonstrated that velocity-magnitude throughout the dryer is consistent at any location near the inclined peppertrays. This also signifies that this preliminary solar-dryer design is able to perform the drying of the pepper-berries through solar-drying.

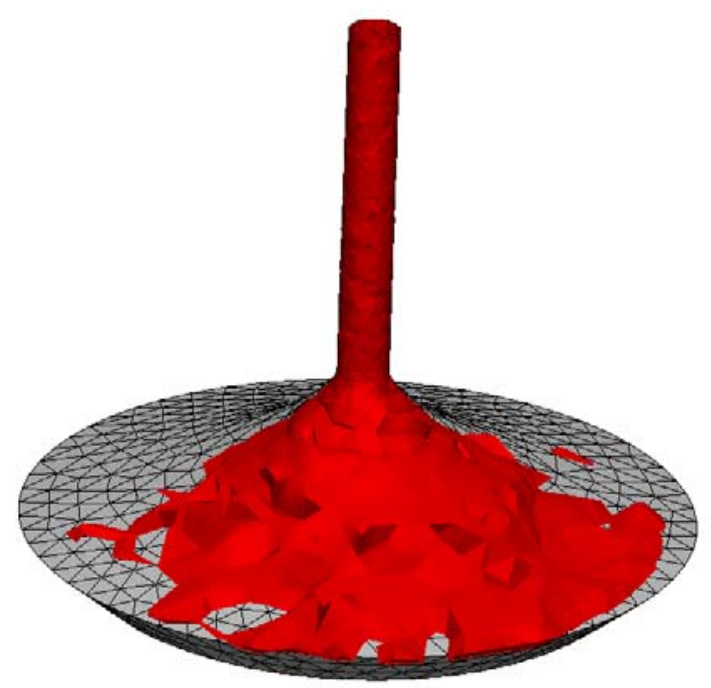

Figure 7. Iso-surf plot of circulation within the solar-dryer.

Figure 8 illustrates the streamlines of the air-flow within the solar-dryer. Consistency in circulation pattern with airflow originating from the entire inlet section is again observed in this figure.

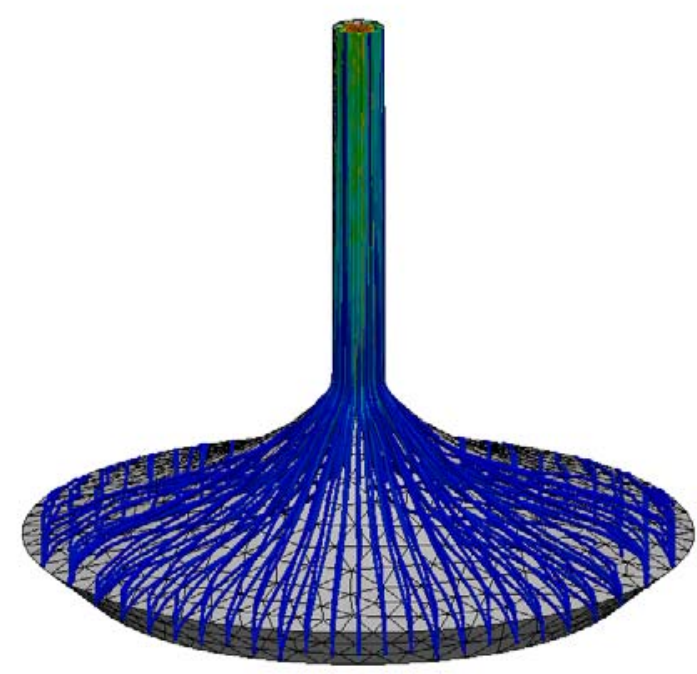

Figure 8. Streamline of air-flow circulation within the solardryer.

\section{SUMMARY}

With the preliminary design of the solar- dryer prototype simulated and the results analyzed, it is obvious that a simple design can be effective. Areas of efficiency and possible enhancement can also be identified. Improvements on both geometry design and CFD modelling can then be 
further implemented. These enhancements can include changes in air-flow inlet and outlet sizes. Meshing techniques and mesh sizes also play important factors in the accuracy of the CFD results. Boundary conditions can also be further investigated to observe the parameters that can affect the outcome of the results and the final design of the solar-dryer. Changes in applied methods, such as algorithm solving and relaxation factors, within Star-CD also create different sets of results in the air-flow or temperature visualization. All possible methods and different boundaryconditions can be investigated.

\section{REFERENCES}

[1] F. K. Forson, M. A. A. Nazha, F. O. Akuffo, and H. Rajakaruna. "Design of mixed-mode natural convection solar crop dryers: Application of principles and rules of thumb". Journal of Renewable Energy, Elsevier, 2007.

[2] B. Bena and R. J. Fuller. "Natural convection solar dryer with biomass back-up heater". International Journal of Solar Energy, 2002 .

[3] M. A. Hossain, J. L. Woods and B.K. Bala. "Simulation of solar drying of chilli in solar tunnel drier". International Journal of Sustainable Energy, 2005.

[4] P. Gbaha, H. Yobouet Andoh and J. Kouassi Saraka. "Experimental investigation of a solar dryer with natural convective heat flow". Journal of Renewable Energy, Elsevier, 2007.

[5] S. Janjai and P. Tung. "Performance of a solar dryer using hot air from roof-integrated solar collectors for drying herbs and spices". Journal of Renewable Energy, Elsevier, 2005. 


\title{
Energy Efficient Nano Coatings for Architectural Flat Glass towards Malaysian Low Energy Buildings
}

\author{
M.M. Hasan, R. Saidur, A.S.M.A. Haseeb, H.H. Masjuki
}

\begin{abstract}
Spectrally selective coatings on architectural windows can play a significant role in energy savings in commercial and residential buildings. A major part of electricity consumption is contributed by air-conditioning systems caused by cooling load in tropical warm climates like in Malaysia. The part of global solar irradiation that penetrates into buildings through windows causes an increase of cooling load. In this paper, the recent trends for optical coatings in glass industries are explored. Different coated glazings are presented with their optical and thermal characteristics. A comparative analysis among different types of coated products is also performed. It has been observed that for warm climate, it is necessary to block near infra red portion of sunlight to reduce solar heat gain through windows. For this purpose $\mathrm{Ag}$ or $\mathrm{Cu}$ metallic thin film meet the requirements but they reduce visible transmittance as well. So for increasing visible transmittance a dielectric layer is required. Here a variety of dielectric layers are studied. Furthermore, for comparing among coated glazing performance, an analysis is done by a computer simulation program. Finally for reaching the goal of Malaysian low energy buildings, appropriate optical coatings for architectural flat glass are recommended.
\end{abstract}

Keywords: solar radiation, near infra red (NIR), nano coating, solar control coating, Low-emittance coating, energy savings.

\section{INTRODUCTION}

The world wide relentless trend to decrease energy consumption necessitates variety of energy saving measures for the future green world. The high consumption of energy for cooling buildings as well as its resulting increase of $\mathrm{CO}_{2}$

This work was supported financially by a project approved by Ministry of science, technology and innovation of Malaysian Government; project no. 03-01-03-SF0158.

M.M. Hasan is with Department of Mechanical Engineering, Faculty of Engineering, University of Malaya, 50603 Kuala Lumpur, Malaysia (Corresponding author: phone: +603-79674448, fax: +603-79675317, email: mmhasan92@yahoo.com).

R. Saidur is with Department of Mechanical Engineering, Faculty of Engineering, University of Malaya, 50603 Kuala Lumpur, Malaysia (email: saidur@um.edu.my).

A.S.M.A. Haseeb is with Department of Mechanical Engineering, Faculty of Engineering, University of Malaya, 50603 Kuala Lumpur, Malaysia (e-mail: haseeb@um.edu.my).

H.H. Masjuki is with Department of Mechanical Engineering, Faculty of Engineering, University of Malaya, 50603 Kuala Lumpur, Malaysia

(e-mail: masjuki@um.edu.my) emission in Malaysia is posing a threat to environment. It also drives architects, energy experts to emphasize more on climate responsive buildings, especially the facades and the right selection of glass/glazing to produce low-energy architecture. Solar energy is an inexhaustible energy source reaching earth in the form of electromagnetic waves. Glazed openings ranging from typical windows to large glazed building surfaces, have become a common feature in architecture, allowing natural light into the building. From Figure 1, it is obvious that a great portion of solar energy passes through windows directly or indirectly resulting in solar heat gain for building envelope.

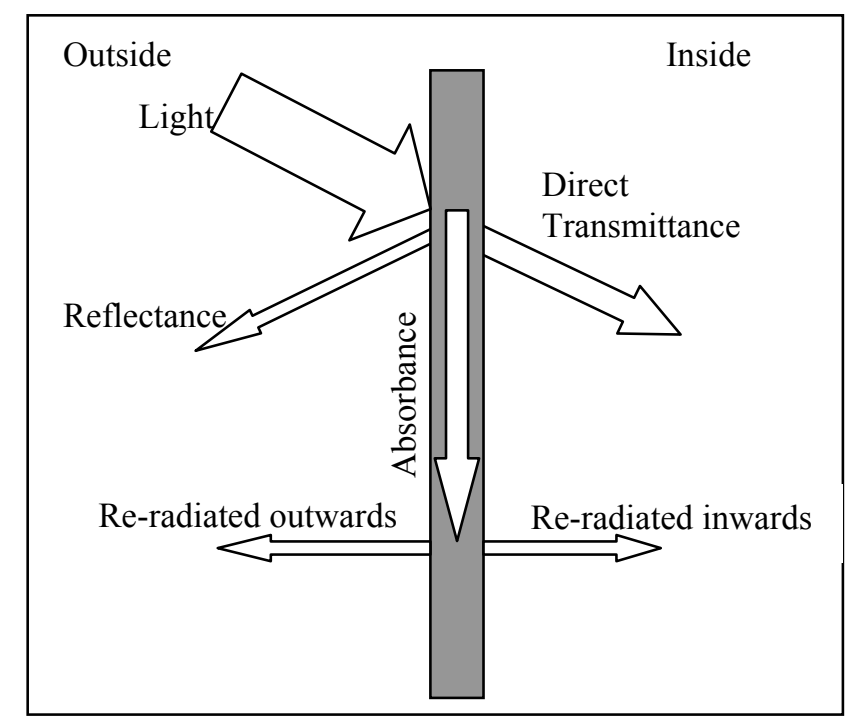

Figure 1. Distribution of sun light through a single glazing

According to the Leadership in Energy and Environmental Design (LEED), one of the most prominently recognized architectural standards in green or sustainable building design, glass selection becomes a main element in this equation to contribute towards achieving a green building [1]. Over the past 40 years, the introduction and development of the use of thin film coatings to enhance the thermal performance of window glazings and to exhibit a new dimension in the aesthetic aspects of building design has occurred. Previously, the glass manufacturers had made 
significant advances to improve the thermal performance through development of sealed insulating glass and tinted or heat absorbing glasses. But there were practical limitations in these developments, and serious thought began to be given in the 1950s to the possibilities of using coatings for additional performance enhancement. Introductory installations of coated glass began in the 1960s with the development of large-scale coating facilities, and these were expanded considerably over the last two decades as the merits of coatings began to be appreciated [2]

In warm climates, an energy-efficient glass window should have spectrally selective coatings such that it transmits nearly all the energy in the visible and reflects all the energy in the infrared. Thus an ideal energy-efficient window for a warm climate would have transmittance (T) and reflectance $(\mathrm{R})$ to be given by: $\mathrm{T}=1$ and $\mathrm{R}=0$ for wavelength 400-700 nm (i.e. visible region), and, $\mathrm{T}=0$ and $\mathrm{R}=1$ for wavelength $>700 \mathrm{~nm}$ (infrared, IR, region) [3]. In other words, an energy-efficient window is a device capable of providing lighting and thermal comfort at minimum demand of paid energy. In a warm climate, when overheating from excessive solar input is a problem, one can obtain energy efficiency by using multilayer thin filmcoated glass windows that are transparent for visible light and reflecting for IR solar radiation [4]. The use of daylighting combined with high performance lighting through a energy-efficient glazing can lead to $30-50 \%$ savings and in some cases up to $70 \%$ [5].

The Solar Heat Gain Coefficient (SHGC) is the fraction of the heat from the sun that enters through a window. The lower a window's solar heat gain coefficient or shading coefficient (SC), the less solar heat it transmits, and the greater is its shading ability. The U-factor measures how well a product prevents heat from escaping. The lower the U-factor, the greater a window's resistance to heat flow and the better its insulating value. For a warm climate like Malaysia, the minimum possible SHGC value will lower the air-conditioning cooling of buildings. In this paper, an introduction to energy-efficient glazings has been presented. Afterwards, three computer tools OPTICS 5.1, WINDOW 5.2, RESFEN-5 developed by Windows and Daylighting Group in Lawrence Berkeley National Laboratory (LBNL), have been used to compare energy performance for a customized house among commercially available glazings available in International Glazing Database IGDB-15.3. Moreover, several coated glazings are selected as energyefficient glazings for warm climates. Few available coating materials in industries have been discussed too.

\section{ENERGY-EFFICIENT GLAZINGS}

In on-line chemical vapor deposition (CVD), multiple coatings less than a micron thick, to reflect visible and infrared wavelengths, can be deposited in the few seconds available as the glass ribbon flows beneath the coaters [6]. Since the end of the seventies vacuum coating technologies for the deposition of optical thin films on large area glass substrates have enjoyed a steady growth. The main applications today are found in the fields of so-called low emissivity and solar control coatings [7].

Low-emittance (Low-E) coatings are microscopically thin, virtually invisible, metal or metallic oxide layers deposited on a window or skylight glazing surface primarily to reduce the U-factor by suppressing radiative heat flow as shown in Figure 2. Coating a glass surface with a lowemittance material and facing that coating into the gap between the glass layers blocks a significant amount of the radiant heat transfer, thus lowering the total heat flow through the window. Different types of Low-E coatings have been designed to allow for high solar gain, moderate solar gain, or low solar gain [8].

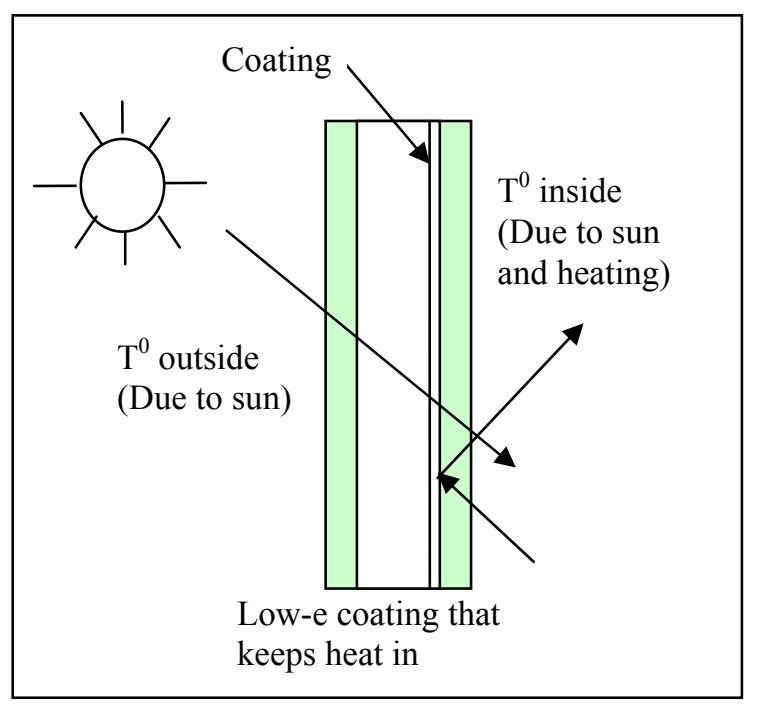

Figure 2. Low-E coating for a double glazed window

High quality low-E coatings deposited by magnetron sputtering consist of ultra thin silver film between two transparent antireflective layers, typically metal oxides like $\mathrm{SnO}_{2}, \mathrm{ZnO}, \mathrm{TiO}_{2}$. Today nearly all low emissivity glazings are based on a thin silver film. To maintain the high transparency of glass in the range of the visible spectrum and to protect the silver from corrosion, additional antireflective and protective layers of high refractive materials (e.g. $\mathrm{SnO}_{2}, \mathrm{ZnO}, \mathrm{Si}_{3} \mathrm{~N}_{4}, \mathrm{TiO}_{2}$ ) have to be employed [7]. Optical transmittance and reflectance spectra of the stackedlayers of $\mathrm{TiO}_{2}$ and $\mathrm{SnO}_{2}: \mathrm{F}$ showed good optical properties of high visible-light transmission and midrange infrared reflection [10].

In solar control glazing, the highly reflective metal film that would otherwise be opaque to the visible light is sandwiched between the two dielectric layers that act as antireflective coatings. Three-layer systems of dielectric/metal/dielectric (D/M/D) on glass substrates have been used for spectrally selective coatings for various purposes including the energy efficiency. By varying the material and thickness of the three layers, the optical properties of the $\mathrm{D} / \mathrm{M} / \mathrm{D}$ films can be tailored to suit different applications. The $\mathrm{D} / \mathrm{M} / \mathrm{D}$ films on a glass substrate were used as a spectrally selective filter that reflects infrared radiation (due to the properties of the metal layer) and transmits most of the visible spectrum (due to the properties of the dielectric layers) [3]. A thin silver material layer is mostly used as a reflective metal film and two same or different dielectric materials $\mathrm{TiO}_{2}, \mathrm{WO}_{3}$, and $\mathrm{ZnS}$ as layers were found to suit the optimized $\mathrm{D} / \mathrm{M} / \mathrm{D}$ films. A typical basic combination used earlier on was $\mathrm{TiO}_{2} / \mathrm{Ag} / \mathrm{TiO}$ but commercial variations gradually evolved. First two pairs of tri-layers, later on an additional thin metal and silicon nitride were put to use replacing some silver, and tin oxide 
was used instead of $\mathrm{TiO}_{2}$. These glazings were proved to be very good in terms of performance, resulting in light gain and a reduction in solar heat [11]. To improve the glazing performance very thin (less than $2 \mathrm{~nm}$ ) Cu block layers are used and it also protects silver against oxidation. Solar control coatings comprising $\mathrm{TiO}_{2}$ and $\mathrm{MgF}_{2}$ films, in which the silver layer beside $\mathrm{MgF}_{2}$ (with thickness more than 10 $\mathrm{nm}$ ) is not surrounded by oxide layers, have transmittance which is higher than $75 \%$ in the visible region and less than $10 \%$ at wavelengths longer than $1000 \mathrm{~nm} \mathrm{[12].} \mathrm{Windows}$ with a single $\mathrm{Cu}_{2} \mathrm{O}$ film blocked only $50 \%$ of the near infrared. It was necessary to improve such windows by adding a second film of $\mathrm{CuS}$ yielding excellent results (NIR transmittance below 30\%). A third kind of film was produced $\left(\mathrm{Cu} / \mathrm{Cu}_{2} \mathrm{O}\right)$ with high visible transmittance of $50 \%$ and good NIR selectivity. Thus copper composite based films are suitable for use as energy-efficient glazings in residential and commercial buildings [13].

\section{Methodology}

Computer modeling and simulation to determine fenestration product energy properties (U-factor, SHGC, Visible Transmittance) has emerged as the most costeffective and accurate means to quantify them. In Lawrence Berkeley National Laboratory (LBNL), Windows and Daylighting group have developed few computer tools for assessing energy performance of commercially available glazings [14].

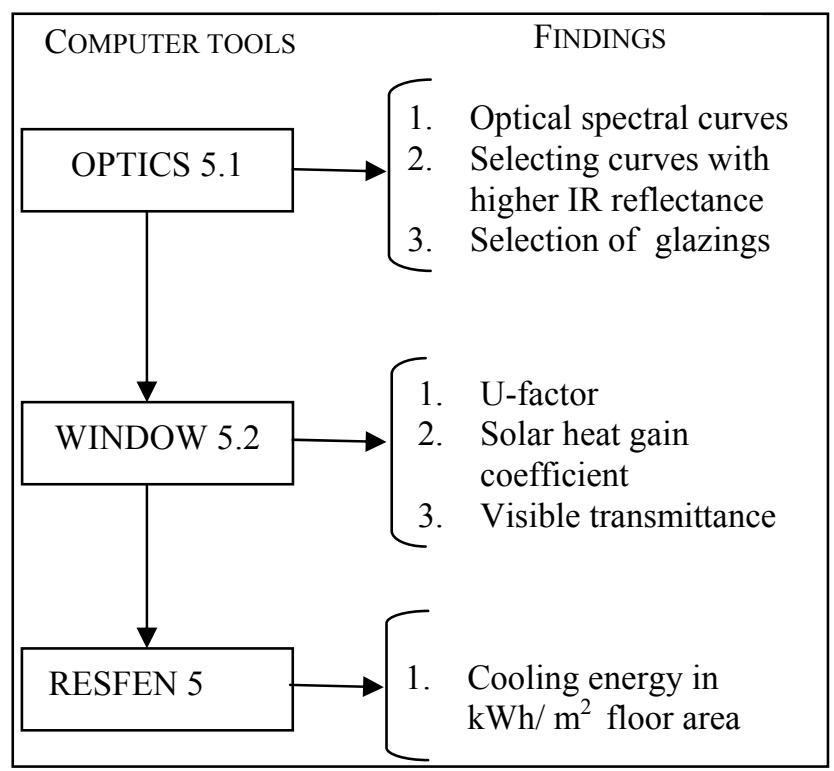

Figure 3. Flow chart of the computer simulation process

In the paper, the computer tools used sequentially are OPTICS 5.1, WINDOW 5.2, and RESFEN-5. The flow chart in Figure 3 shows how different computer tools have been used and their findings. Through OPTICS 5.1, the spectral behavior of 2145 glazing systems from the database IGDB-15.3 have been studied. The glazing systems in the database are of four types, such as monolithic, laminated, coated and applied film. Among those types, first three types have been selected to study taking into consideration their transmittance curves. A number of glazings are found based on their higher visible transmittance and higher IR reflectance.
WINDOW 5.2 has been used to find U-factor, SHGC and visible transmittance (VT) for a customized window shown in Figure 4 assuming different parameters given in Table 1. Here it is clear that the environmental conditions chosen fit with Malaysian climate, because average maximum temperature is about $32^{0} \mathrm{C}$ [15].

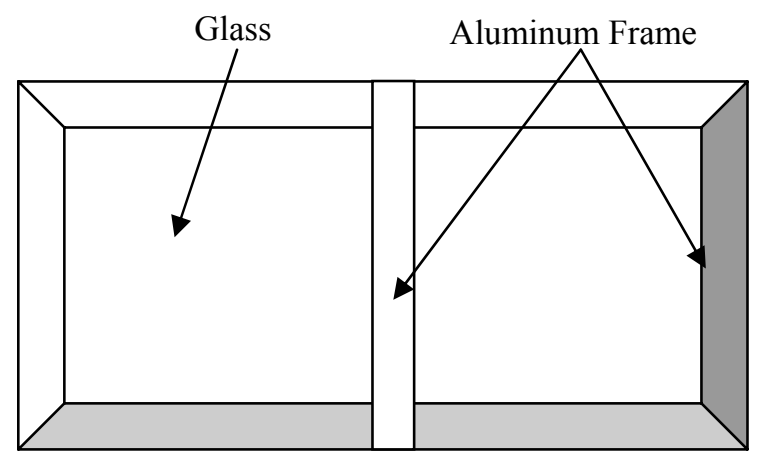

Figure 4 Custom dual vision horizontal window type

Table 1. Assumed parameters for a typical window

\begin{tabular}{|c|c|c|}
\hline Parameter & \multicolumn{2}{|l|}{ Typical Window } \\
\hline \multirow{3}{*}{$\begin{array}{l}\text { Environmental } \\
\text { Condition }\end{array}$} & \multicolumn{2}{|c|}{ NFRC 100-2001 Summer } \\
\hline & $\begin{array}{l}\text { For U-factor } \\
\mathrm{T}(\text { inside })=24^{0} \mathrm{C} \\
\mathrm{T}(\text { outside })=32^{0} \\
\mathrm{C}\end{array}$ & $\begin{array}{l}\text { For SHGC } \\
\mathrm{T}(\text { inside })=24^{0} \mathrm{C} \\
\mathrm{T}(\text { outside })=32^{0} \mathrm{C}\end{array}$ \\
\hline & \multicolumn{2}{|c|}{ Solar irradiation $783 \mathrm{~W} / \mathrm{m}^{2}$} \\
\hline Window Type & \multicolumn{2}{|c|}{ Custom Dual Vision Horizontal } \\
\hline Tilt & \multicolumn{2}{|c|}{$90^{0}$} \\
\hline Width & \multicolumn{2}{|l|}{$3000 \mathrm{~mm}$} \\
\hline Height & \multicolumn{2}{|l|}{$2000 \mathrm{~mm}$} \\
\hline Area & \multicolumn{2}{|l|}{$6.00 \mathrm{~m}^{2}$} \\
\hline
\end{tabular}

Finally RESFEN-5 is used to pick the most energyefficient glazings used in the custom window fitted in a new single-story house. Table 2 shows the fixed parameters for the user defined house. It calculates heating and cooling energy use. The thermal properties that RESFEN-5 requires are: U-factor, Solar Heat Gain Coefficient, and air leakage rate. RESFEN-5 calculates the energy and cost implications of the window compared to an insulated wall. In this paper, only energy usage per $\mathrm{m}^{2}$ of floor area is taken under consideration.

It is noteworthy that the location of the house for simulating energy is a specific state Honolulu, USA because the program has no provision to use Kuala Lumpur as a location. From Table 3 it is observed that the maximum and minimum weather temperature in Kuala Lumpur is close to that of Honolulu. Hence it is assumed that the results can be compared with Malaysia. 
Table 2. House data from FESFEN-5

\begin{tabular}{|l|l|}
\hline Parameters & Typical House \\
\hline Floor Area & Fixed: $300 \mathrm{~m}^{2}$ \\
\hline House Construction: & $\begin{array}{l}\text { New Construction (Frame, 1- } \\
\text { Story). Dependant on location }\end{array}$ \\
\hline Location & HI Honolulu \\
\hline House Type & 1-Story New Frame \\
\hline Foundation Type & Basement \\
\hline Fenestration Type & User Defined \\
\hline $\begin{array}{l}\text { Fenestration Area \& } \\
\text { Distribution }\end{array}$ & $\begin{array}{l}\text { Fixed: } 14 \% \text { of total floor area } \\
\left(300 \mathrm{~m}^{2}\right), \text { two windows } \\
\text { distributed on each east and } \\
\text { west orientations and three } \\
\text { windows in north orientation. }\end{array}$ \\
\hline HVAC System Type & Electric Heat Pump \\
\hline Total Window Area & $12 \mathrm{~m}^{2}$ \\
\hline Total Floor Area & $300 \mathrm{~m}^{2}$ \\
\hline Air Leakage Rate & 1.5 litre/s-m ${ }^{2}$ \\
\hline
\end{tabular}

Table 3. Mean temperature round the year for Honolulu and Kuala Lumpur (Source: World Weather Information Service available in http://www.worldweather.org)

\begin{tabular}{|c|c|c|c|c|}
\hline Place & \multicolumn{2}{|l|}{$\begin{array}{l}\text { Honolulu } \\
\text { (USA) }\end{array}$} & \multicolumn{2}{l|}{$\begin{array}{l}\text { Kuala Lumpur } \\
\text { (Malaysia) }\end{array}$} \\
\hline \multirow{2}{*}{ Month } & \multicolumn{2}{|c|}{\begin{tabular}{c} 
Mean Temperature $\left({ }^{0} \mathrm{C}\right)$ \\
\multicolumn{2}{l|}{ Mean Temperature $\left({ }^{0} \mathrm{C}\right)$}
\end{tabular}} \\
\cline { 2 - 5 } & $\begin{array}{c}\text { Daily } \\
\text { Minimum }\end{array}$ & $\begin{array}{c}\text { Daily } \\
\text { Maximum }\end{array}$ & $\begin{array}{c}\text { Daily } \\
\text { Minimum }\end{array}$ & $\begin{array}{c}\text { Daily } \\
\text { Maximum }\end{array}$ \\
\hline Jan & 18.7 & 26.7 & 22.5 & 32.1 \\
\hline Feb & 18.6 & 26.9 & 22.8 & 32.9 \\
\hline Mar & 19.6 & 27.6 & 23.2 & 33.2 \\
\hline Apr & 20.4 & 28.2 & 23.7 & 33.1 \\
\hline May & 21.3 & 29.3 & 23.9 & 32.9 \\
\hline Jun & 22.3 & 30.3 & 23.6 & 32.7 \\
\hline Jul & 23.1 & 30.8 & 23.2 & 32.3 \\
\hline Aug & 23.4 & 31.5 & 23.1 & 32.3 \\
\hline Sep & 23.1 & 31.4 & 23.2 & 32.1 \\
\hline Oct & 22.4 & 30.5 & 23.2 & 32.1 \\
\hline Nov & 21.3 & 28.9 & 23.2 & 31.6 \\
\hline Dec & 19.4 & 27.3 & 22.9 & 31.5 \\
\hline
\end{tabular}

\section{RESULTS AND DISCUSSION}

OPTICS 5.1 provides optical spectra for all glazing systems of IGDB-15.3 database. Taking into consideration of the transmittance curves of monolithic, laminated and coated glazings with their decreasing transmittance, WINDOW 5.2 has been used to find U-factor, SHGC and VT for each types of glazings in the customized window. In addition, cooling energy consumption has been found through the simulation software RESFEN-5 for each glazing types fitted in a typical user defined house. From each glazing types, thirteen types of glazings have been selected to cover the range from maximum to minimum energy consumption. All the simulated values are shown in Table 4.

From Table 4, it can be concluded that coated glazings show higher VT and least cooling energy consumptions. Besides it is also found that cooling energy consumption values mainly vary with SHGC but its variation with Ufactor is not so dominant. Here, the cooling energy consumption decreases with the decreasing value of SHGC.

Although several glazings reduce cooling energy consumption significantly, they have a lower visible transmittance resulting in an increase of artificial lighting for the interior of the house. As a result, their total energy consumption due to cooling load requirements as well as artificial lighting will be higher than that of the coated glazing. That is why, out of more than 1000 coated glazings in IGDB-15.3, 44 glazings of thickness $6 \mathrm{~mm}$ have been selected with VT higher than 0.60 and least possible cooling energy shown in Table 5 with their NFRC ID.

Further, six types of glazings used in customized window are selected considering minimum energy consumption from Table 5 which is shown in Table 6. Another six types are selected considering higher VT and also maintaining energy usage as less as possible shown in Table 7.

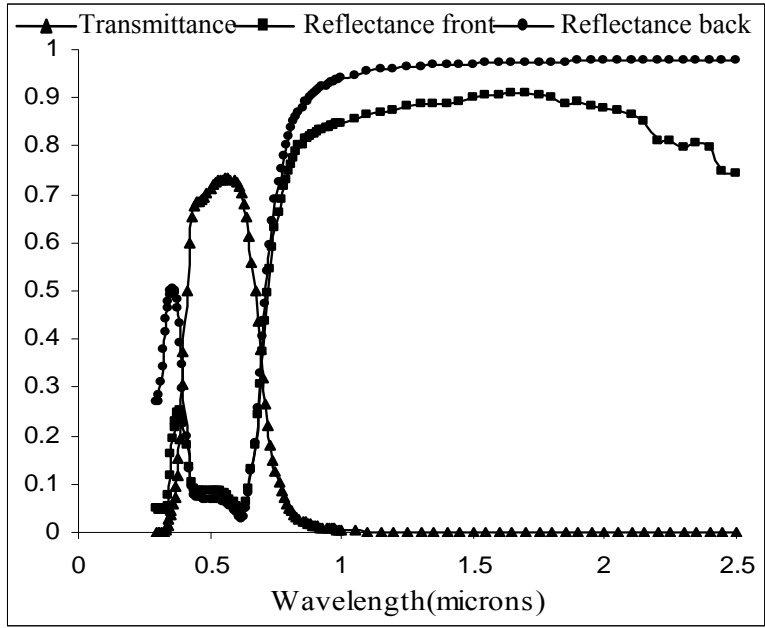

Figure 4. Optical spectra for NFRC_ID 5000 coated glazing

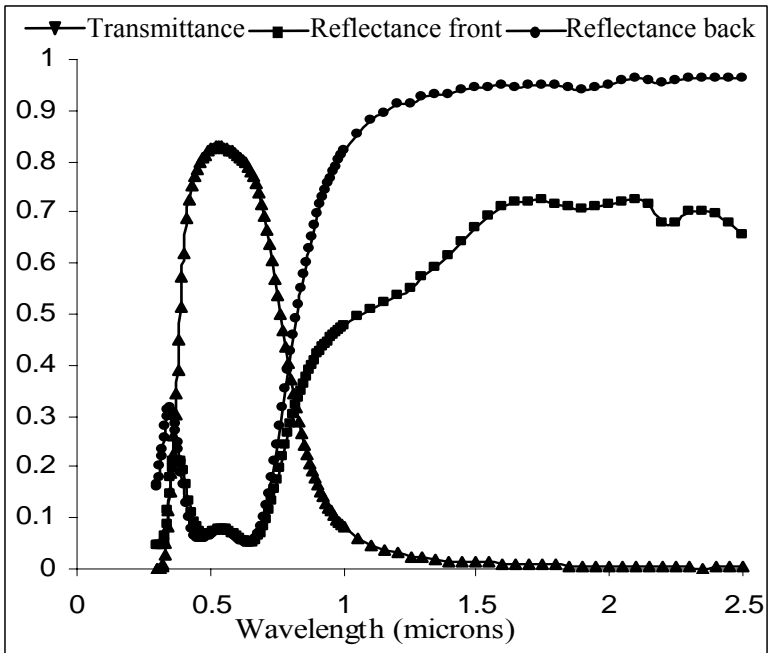

Figure 5. Optical spectra for NFRC_ID 4251 coated glazing 
Table 4. Cooling energy consumption and other parametric values for different types of glazings using the computer tools

\begin{tabular}{|c|c|c|c|c|c|c|c|c|c|c|c|c|c|c|}
\hline $\begin{array}{l}\text { NFRC } \\
\text { ID }\end{array}$ & $\begin{array}{l}\text { U- } \\
\text { factor } \\
(\mathrm{W} / \\
\left.\mathrm{m}^{2} \mathrm{~K}\right)\end{array}$ & $\begin{array}{l}\text { SHG- } \\
\mathrm{C}\end{array}$ & VT & $\begin{array}{l}\text { Cooling } \\
\text { Energy } \\
(\mathrm{kWh} / \\
\left.\mathrm{m}^{2}\right)\end{array}$ & $\begin{array}{l}\text { NFRC } \\
\text { ID }\end{array}$ & $\begin{array}{l}\mathrm{U}- \\
\text { factor } \\
(\mathrm{W} / \\
\left.\mathrm{m}^{2} \mathrm{~K}\right)\end{array}$ & $\begin{array}{l}\text { SHG- } \\
\mathrm{C}\end{array}$ & VT & $\begin{array}{l}\text { Cooling } \\
\text { Energy } \\
(\mathrm{kWh} / \\
\left.\mathrm{m}^{2}\right)\end{array}$ & $\begin{array}{l}\text { NFRC } \\
\text { ID }\end{array}$ & $\begin{array}{l}\mathrm{U}- \\
\text { factor } \\
(\mathrm{W} / \\
\left.\mathrm{m}^{2} \mathrm{~K}\right)\end{array}$ & $\begin{array}{l}\text { SHG- } \\
\mathrm{C}\end{array}$ & VT & $\begin{array}{l}\text { Cooling } \\
\text { Energy } \\
(\mathrm{kWh} / \\
\left.\mathrm{m}^{2}\right)\end{array}$ \\
\hline 9814 & 5.137 & 0.826 & 0.81 & 35.55 & 8104 & 5.028 & 0.782 & 0.81 & 34.49 & 1001 & 5.229 & 0.761 & 0.73 & 33.90 \\
\hline 6703 & 5.138 & 0.773 & 0.79 & 34.20 & 3901 & 5.122 & 0.757 & 0.80 & 33.83 & 1005 & 5.142 & 0.698 & 0.68 & 32.34 \\
\hline 2004 & 5.142 & 0.761 & 0.79 & 33.92 & 1818 & 5.074 & 0.699 & 0.76 & 32.38 & 4230 & 2.924 & 0.648 & 0.72 & 31.87 \\
\hline 1310 & 5.225 & 0.712 & 0.66 & 32.67 & 1833 & 5.074 & 0.679 & 0.72 & 31.87 & 2006 & 2.593 & 0.599 & 0.77 & 30.75 \\
\hline 1204 & 5.186 & 0.674 & 0.55 & 31.71 & 1835 & 5.090 & 0.628 & 0.61 & 30.58 & 7140 & 4.767 & 0.552 & 0.78 & 28.76 \\
\hline 5322 & 5.226 & 0.613 & 0.72 & 30.17 & 1834 & 5.090 & 0.589 & 0.46 & 29.61 & 926 & 4.794 & 0.497 & 0.68 & 27.35 \\
\hline 9862 & 5.184 & 0.555 & 0.58 & 28.72 & 1823 & 5.047 & 0.528 & 0.34 & 28.07 & 4251 & 4.763 & 0.453 & 0.73 & 26.25 \\
\hline 5325 & 5.166 & 0.504 & 0.63 & 27.43 & 1805 & 5.074 & 0.510 & 0.27 & 27.61 & 6229 & 2.396 & 0.403 & 0.70 & 25.75 \\
\hline 1302 & 5.136 & 0.466 & 0.38 & 26.48 & 1808 & 5.074 & 0.479 & 0.25 & 26.81 & 1029 & 2.385 & 0.349 & 0.58 & 24.36 \\
\hline 1212 & 4.993 & 0.428 & 0.33 & 25.55 & 5876 & 4.964 & 0.419 & 0.33 & 25.33 & 5000 & 2.303 & 0.298 & 0.64 & 23.06 \\
\hline 417 & 5.200 & 0.401 & 0.22 & 24.81 & 1807 & 5.074 & 0.372 & 0.08 & 24.10 & 5316 & 2.329 & 0.268 & 0.46 & 22.27 \\
\hline 419 & 5.161 & 0.348 & 0.11 & 23.46 & 2032 & 4.849 & 0.353 & 0.38 & 23.66 & 5807 & 3.846 & 0.215 & 0.06 & 20.39 \\
\hline 1336 & 5.142 & 0.322 & 0.07 & 22.80 & 5904 & 5.098 & 0.336 & 0.12 & 23.17 & 1200 & 2.853 & 0.170 & 0.04 & 19.52 \\
\hline
\end{tabular}

Table 5. Glazings with VT higher than 0.60 and least cooling energy

\begin{tabular}{|l|l|l|l|l|l|l|l|l|l|}
\hline $\begin{array}{l}\text { NFRC } \\
\text { ID }\end{array}$ & $\begin{array}{l}\text { U-factor } \\
\left(\mathrm{W} / \mathrm{m}^{2} \mathrm{~K}\right)\end{array}$ & SHGC & VT & $\begin{array}{l}\text { Cooling } \\
\text { Energy } \\
\left(\mathrm{kWh} / \mathrm{m}^{2}\right)\end{array}$ & $\begin{array}{l}\text { NFRC } \\
\text { ID }\end{array}$ & $\begin{array}{l}\text { U-factor } \\
\left(\mathrm{W} / \mathrm{m}^{2}\right. \\
\mathrm{K})\end{array}$ & SHGC & VT & $\begin{array}{l}\text { Cooling } \\
\text { Energy } \\
\left(\mathrm{kWh} / \mathrm{m}^{2}\right)\end{array}$ \\
\hline 927 & 4.773 & 0.477 & 0.66 & 26.85 & 3110 & 2.393 & 0.400 & 0.67 & 25.67 \\
\hline 4039 & 4.769 & 0.471 & 0.64 & 26.69 & 5346 & 2.435 & 0.397 & 0.68 & 25.58 \\
\hline 6075 & 2.600 & 0.439 & 0.65 & 26.61 & 919 & 4.774 & 0.423 & 0.58 & 25.49 \\
\hline 1025 & 2.389 & 0.428 & 0.60 & 26.41 & 2029 & 2.382 & 0.386 & 0.67 & 25.32 \\
\hline 2014 & 2.406 & 0.427 & 0.69 & 26.38 & 3256 & 2.379 & 0.385 & 0.67 & 25.30 \\
\hline 4251 & 4.763 & 0.453 & 0.73 & 26.25 & 935 & 4.773 & 0.413 & 0.67 & 25.23 \\
\hline 4024 & 4.764 & 0.453 & 0.71 & 26.25 & 968 & 4.777 & 0.413 & 0.65 & 25.23 \\
\hline 6055 & 2.600 & 0.425 & 0.65 & 26.25 & 2024 & 2.423 & 0.382 & 0.60 & 25.20 \\
\hline 6859 & 4.772 & 0.449 & 0.72 & 26.14 & 5600 & 2.430 & 0.377 & 0.63 & 25.07 \\
\hline 3216 & 2.415 & 0.417 & 0.66 & 26.12 & 7117 & 2.328 & 0.369 & 0.66 & 24.91 \\
\hline 7114 & 2.383 & 0.416 & 0.72 & 26.11 & 7115 & 2.332 & 0.366 & 0.67 & 24.83 \\
\hline 5349 & 2.376 & 0.415 & 0.72 & 26.08 & 4043 & 4.757 & 0.393 & 0.65 & 24.72 \\
\hline 786 & 4.795 & 0.444 & 0.61 & 26.01 & 772 & 4.771 & 0.393 & 0.62 & 24.72 \\
\hline 963 & 4.777 & 0.443 & 0.68 & 25.99 & 6858 & 4.772 & 0.389 & 0.69 & 24.61 \\
\hline 6857 & 4.772 & 0.441 & 0.70 & 25.94 & 5254 & 2.376 & 0.357 & 0.61 & 24.58 \\
\hline 5651 & 2.668 & 0.413 & 0.63 & 25.90 & 6071 & 2.396 & 0.355 & 0.60 & 24.52 \\
\hline 5284 & 2.374 & 0.407 & 0.70 & 25.86 & 4319 & 2.384 & 0.351 & 0.60 & 24.42 \\
\hline 3236 & 2.342 & 0.406 & 0.69 & 25.86 & 6051 & 2.396 & 0.351 & 0.60 & 24.41 \\
\hline 6196 & 2.396 & 0.405 & 0.71 & 25.80 & 6850 & 4.765 & 0.380 & 0.60 & 24.39 \\
\hline 6046 & 2.396 & 0.404 & 0.70 & 25.78 & 5424 & 2.376 & 0.349 & 0.51 & 24.37 \\
\hline 6151 & 2.396 & 0.404 & 0.70 & 25.78 & 2157 & 2.320 & 0.307 & 0.63 & 23.30 \\
\hline 6229 & 2.396 & 0.403 & 0.70 & 25.75 & 5000 & 2.303 & 0.298 & 0.64 & 23.06 \\
\hline & & & & & & & & & \\
\hline
\end{tabular}

Table6. Several commercial glazings on the minimum energy consumption basis

\begin{tabular}{|c|c|c|c|c|c|c|}
\hline $\begin{array}{l}\text { NFRC } \\
\text { ID }\end{array}$ & Product Name & Manufacturer & $\begin{array}{l}\text { U-value } \\
\left(\mathrm{W} / \mathrm{m}^{2}-\mathrm{K}\right)\end{array}$ & SHGC & VT & $\begin{array}{l}\text { Cooling } \\
\text { Energy kWh } \\
\text { per } \mathrm{m}^{2} \text { floor } \\
\text { area }\end{array}$ \\
\hline 5000 & Solarban 70XL on Starphire & PPG Industries & 2.303 & 0.298 & 0.64 & 23.06 \\
\hline 2157 & LoE $^{3}-366$ on Clear & Cardinal IG & 2.320 & 0.307 & 0.63 & 23.30 \\
\hline 6858 & Silverstar $®$ & Glas Trösch AG & 4.772 & 0.389 & 0.69 & 24.61 \\
\hline 772 & Sunbelt LowE on Clear & AFG Industries & 4.771 & 0.393 & 0.62 & 24.72 \\
\hline 4043 & Suncool $^{\mathrm{TM}}$ Brilliant $66 / 33$ & Pilkington & 4.757 & 0.393 & 0.65 & 24.72 \\
\hline 7115 & ipasol neutral 68/34 & Interpane Glas Industrie $\mathrm{Ag}$ & 2.332 & 0.366 & 0.67 & 24.83 \\
\hline
\end{tabular}


Table 7. Several commercial glazings on the maximum visible transmittance basis

\begin{tabular}{|l|l|l|l|l|l|l|}
\hline $\begin{array}{l}\text { NFRC_ } \\
\text { ID }\end{array}$ & Product Name & Manufacturer & $\begin{array}{l}\text { U-value } \\
\left(\mathrm{W} / \mathrm{m}^{2}-\mathrm{K}\right)\end{array}$ & SHGC & VT & $\begin{array}{l}\text { Cooling } \\
\text { Energy kWh } \\
\text { per } \mathrm{m}^{2} \text { floor } \\
\text { area }\end{array}$ \\
\hline 4251 & Planibel Energy NT & GLAVERBEL S.A. & 4.763 & 0.453 & 0.73 & 26.25 \\
\hline 6859 & Silverstar & Glas Trösch AG & 4.772 & 0.449 & 0.72 & 26.14 \\
\hline 6229 & VE-2M on UltraWhite & Viracon & 2.396 & 0.403 & 0.70 & 25.75 \\
\hline 5284 & Solarban $60 \AA$ on Clear & PPG Industries & 2.374 & 0.407 & 0.70 & 25.86 \\
\hline 2029 & LoE 270 on Clear & Cardinal IG & 2.382 & 0.386 & 0.67 & 25.32 \\
\hline 935 & Silver Hi\%T LowE on Clear & AFG Industries & 4.773 & 0.413 & 0.67 & 25.23 \\
\hline
\end{tabular}

The optical spectral curves from OPTICS 5.1 have been developed for the glazings with minimum energy, namely NFRC ID 5000 and of the highest visible transmittance, namely NFRC_ID 4251. The optical spectral curves are shown in Figure 4 and in Figure 5 for the glazings NFRC_ID 5000 and NFRC_ID 4251 respectively. From the spectral behavior of the glazings it is clear that for maintaining higher visible transmittance and higher IR reflectance, the ideal energy-efficient glazings necessitates that transmittance in visible portion would be as high as possible and reflectance in IR portion needs to be $100 \%$.

The commercially available optical coatings in different glass or relevant industries could be either pyrolytic (hard coat) or sputter coated (soft coat). Pyrolytic coatings are metallic oxides applied during the float manufacturing process. In soft coat, a thin, multi-layered piece of optically clear film is retrofitted to the inside surface of glass. It is sputter-coated with durable, exotic metals and their oxides such as titanium, copper, gold, silver, chromium, aluminum and other alloys.

\section{CONCLUSION}

It is observed from computer simulation that with the decreasing value of SHGC for a glazing, the cooling energy consumption decreases as well. It also decreases with the lower value of U-factor. With the same SHGC values, the glazing with lower U-factor has low energy consumption because of high insulation. Considering a minimum energy consumption criteria it is found that NFRC 5000 is the best option. It gives visible transmittance $\overline{0.64}$ and 23.06 $\mathrm{kwh} / \mathrm{m}^{2}$. It indicates that it maintains a reasonable visible transmittance as well. And NFRC 4251 is selected as a suitable choice considering high visible transmittance and less energy consumption. Its visible transmittance and cooling energy are 0.73 and $26.25 \mathrm{kwh} / \mathrm{m}^{2}$ respectively. Either of these above commercially available coated glazing could be recommended for achieving the target of Malaysian low energy buildings. On the other hand, from the discussion on energy-efficient coating it can be said that to produce energy efficient coating it is necessary to get a thin film layer or a combination of layers with newly developed materials to increase the visible transmittance and near infrared reflectance.

\section{REFERENCES}

[1] Mohsen M. Aboulnaga, "Towards green buildings: Glass as a building element - the use and misuse in the gulf region," in Renewable Energy J., vol. 31, 2006, pp. 631-653.

[2] P.H. Berning, "Principles of design of architectural coatings," Applied Optics J. vol. 22, 1983, pp. 4127.
[3] S.M.A. Durrani, E.E. Khawaja, A.M. Al-Shukri , M.F. Al-Kuhaili, "Dielectric/Ag/dielectric coated energy-efficient glass windows for warm climates," Energy and Buildings J. vol. 36, 2004, pp. 891-898.

[4] A.M. Al-Shukri, "Thin film coated energy-efficient glass windows for warm climates," Desalination j., vol. 209, 2007, pp. 290-297.

[5] M. Wilson, J.H. Walker, M. Santamouris, S. Jaure, "Design process for energy efficient new and Refurbished Housing," in a University of North London Publication, 2002, pp. 2.

[6] www.britglass.org.uk/Files/form2Float_Process.pdf, 12/09/2007.

[7] G. Brauer, "Large area glass coating," Surface and Coatings Technology J., vol. 112, 1999, pp. 358-365.

[8] http://www.efficientwindows.org/lowe.cfm, 08/09/2007.

[9] Masahisa Okada, Yasusei Yamada, Ping Jin, Masato Tazawa, Kazuki Yoshimura, "Fabrication of multifunctional coating which combines low-e property and visible-light-responsive photocatalytic activity," Thin Solid Films J., vol. 442, 2003, pp. 217-221.

[10] G. Leftheriotis, P. Yianoulis and D. Patrikios , "Deposition and optical properties of optimised $\mathrm{ZnS} / \mathrm{Ag} / \mathrm{ZnS}$ thin films for energy saving applications," Thin Solid Films J., vol. 306, issue 1, 1997, pp. 92-99

[11] G.B. Smitha, A. Ben-Davidb, P.D. Swifta, "A new type of TiN coating combining broad band visible transparency and solar control," Renewable Energy J., vol. 22, 2001, pp. 79-84

[12] J. K. Fu, G. Atanassov, Y. S. Dai, F. H. Tan and Z. Q. Mo, "Single films and heat mirrors produced by plasma ion assisted deposition," Journal of Non-Crystalline Solids, vol. 218, vol. 2, 1997, pp. 403-410.

[13] Genaro Correa, Rafael Almanza, "Copper based thin films to improve glazing for energy-savings in buildings," Solar Energy J. vol.76, 2004, pp.111-115

[14] http://windows.lbl.gov/software/window/window.html, 10/06/2007

[15] http://www.worldweather.org/020/c00082.htm, 07/09/2007 


\title{
Comparative Study of Thermal Properties of Tile and Granite Using Thermography
}

\author{
Sinin Hamdan, Mahbub Hasan and Chin Did Sing
}

\begin{abstract}
Tiles are composed of clay minerals mined from the earth's crust. Tiles are a popular choice nowadays to create patterned floors and walls due to their beauty, versatile and durable characteristics. Granites are crystalline igneous rock composed of magma. Nowadays they are widely used in buildings, sculptures and monuments. Both tiles and granites are being used for a decade for floor covering purposes in houses. Current study presents a comparative study of thermal properties of tiles and granites using thermography. Although all three heat transfer modes (conduction, convection and radiation) were used, results obtained from the conduction experiments were utilized as the main basis for data analysis. A simple heater plate was used as the heat source during steadystate heating and cooling processes. In order to obtain accurate results, a closed chamber was built to retain heat that was transferred from the hot plate to the tiles and granites specimens. Different sets of parameters and conditions were applied during experiment. Surface temperatures were measured and data were extracted from a data logging system in order to obtain thermal performance of the two materials. According to experimental results, tiles were good heat conductor compared to granites. Granites rejected heat at a faster rate at a high temperature compared to tiles, however the heat rejection rate for granites slowed down at room temperature.
\end{abstract}

Keywords: comparative study, tiles \& granites, thermal property, thermography

\section{INTRODUCTION}

Tiles are composed of a mixture of clays, silica, coloring and other substances. Last stage of their manufacturing involves firing at very high temperature. The reaction at high temperature for a period of time strengthens the material results in hard tiles that can sustain high load without deformation. Tiles are suitable for using as floor coverings because of their inertness and unalterable properties. Water and most other chemical substances including fire cannot alter the composition of the tiles. Tiles

Sinin Hamdan, Department of Mechanical and Manufacturing Engineering, Faculty of Engineering, Universiti Malaysia Sarawak, 94300 Kota Samarahan, Sarawak, Malaysia (phone: +6082583232; fax: +6082583410; e-mail: hsinin@feng.unimas.my).

Mahbub Hasan, Department of Mechanical and Manufacturing, Faculty of Engineering, Universiti Malaysia Sarawak, 94300 Kota Samarahan, Sarawak, Malaysia (e-mail: hmahbub@feng.unimas.my).

Chin Did sing, Department of Mechanical and Manufacturing Engineering, Faculty of Engineering, Universiti Malaysia Sarawak, 94300 Kota Samarahan, Sarawak, Malaysia (e-mail: didsingh83@yahoo.com). are considered to be brittle as they can only sustain moderate impact [1].

Granites originate from deep in the earth's molten mantle. As these extremely hot liquid materials rise and cool, they form a crystalline and granular structure, hence forming granites. Granites are typically the hardest among all the dimensional stones. They are available in various colors. The variation in color has added an additional aesthetical value into granites, which eventually become the preference of many users as building materials [2-4]. Granites are resistance to scratching, stain and heat. They are also suitable for flooring or paving, wall cladding and all other types of counter tops and tombstones.

The study of heat transfer through floor coverings is vital as it can give a better illustration how heat is transferred into the house. Heat is transmitted from the ground to the house through the tiles or granites floor covering. It had been a decade since tiles and granites are being used for floor covering purposes in house. Current study presents a comparative study of thermal properties of tiles and granites using thermography [5]. Conduction experiments were utilized as the main basis for data analysis. A simple heater plate was used as the heat source during steady-state heating and cooling processes. A closed chamber was built to retain heat in order to obtain accurate results. Surface temperatures were measured and data were extracted from a data logging system in order to obtain thermal performance of the two materials. Experimental results showed that tiles were good heat conductor compared to granites. Granites were able to reject heat at a faster rate at a high temperature compared to tiles, however the heat rejection rate for granites slowed down at room temperature.

\section{Methodology}

Comparative study of thermal properties of tiles and granites involved all three heat transfer modes (conduction, convection and radiation). However only the results obtained from the conduction experiments were utilized as the main basis for data analysis. Each experiment had different apparatus setup and material parameter and was repeated three times in order to obtain an average value.

During the heat gain experiments, the material was placed directly on top of the heater plate. The heater plate was enclosed in a chamber and heat was conducted to the base of the testing material. Convections occurred naturally to the top surface and held in the open air by 2 retot stands in which the distance between the material base and the heater 
plate surface was $2 \mathrm{~mm}$. For the heat loss experiments, the material was left on the original position after the heater plate was switched off from the maximum temperature achieved. The material experienced natural heat loss without the aid of a fan or other ventilating device until it was cooled to $30^{\circ} \mathrm{C}$.

\section{A. Calibration}

The heater plate used during heat gain and heat loss experiments had six (6) knobs. Calibration tests were initially conducted in order to verify the maximum temperature that each knob could reach. The knobs of the heater plate were numbered from 1 to 6 that could achieve six different temperatures namely 220, 268, 309, 373, 414 and $446^{\circ} \mathrm{C}$ respectively.

\section{RESULTS}

Results obtained from heat gain and heat loss experiments are shown in 'figures 1 and 2' in order to give a better overall view of the thermal performance for the two materials i.e. tiles and granites. Thermograms captured by the infrared thermography are also shown in figures ' 3 to 30'. These thermographs help in investigating the temperature distribution and depicting the thermal performance of the two samples.

\section{A. Results of Heat Gain through Conduction}

Results of conduction heat gain data for both tiles and granites are shown in 'figure 1'. Three different runs were conducted on the conduction heat gain experiments for tiles. At the maximum temperature of $446^{\circ} \mathrm{C}$ supplied to the tiles, it was found that the maximum average temperature that the tiles could absorb was $223^{\circ} \mathrm{C}$. Therefore the data was taken for every temperature increment of approximately $30^{\circ} \mathrm{C}$ starting from the room temperature until $210^{\circ} \mathrm{C}$. At the maximum temperature of $446^{\circ} \mathrm{C}$ supplied to the granites, it was found that the maximum temperature that the granite could absorb is $178.5^{\circ} \mathrm{C}$.

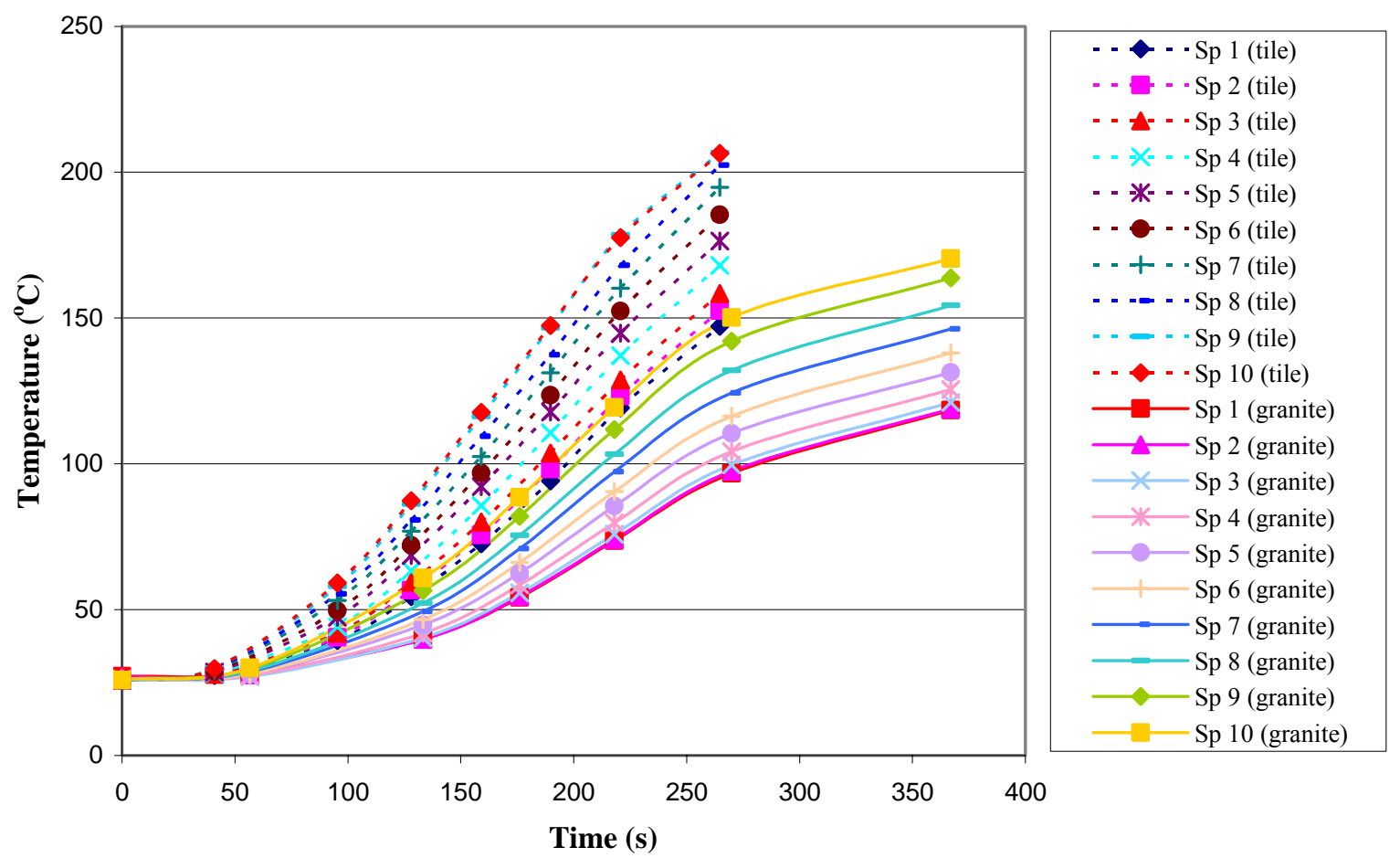

Figure 1. Average maximum temperature gained by tiles and granites during conduction heat gain experiments

\section{B. Results of Heat Loss through Conduction}

'Figure 2' shows the average of three temperature loss with time for tiles and granites. At the maximum temperature of $223^{\circ} \mathrm{C}$ sustained by the tiles, the heat loss experiment commenced at $210^{\circ} \mathrm{C}$. At the maximum temperature of $178.5^{\circ} \mathrm{C}$ sustained of by the granite, the heat loss experiment was started at $170^{\circ} \mathrm{C}$. Due to the capability of the infrared camera which was only able to capture the images every 5 seconds, it was not possible to obtain exactly $30^{\circ} \mathrm{C}$ increment in temperature.

\section{Results from Thermographs}

Thermographs obtained during heat gain and loss experiments are shown in 'figures 3 to 30'. 'Figures 3 to17' provide a clearer picture of how heat gain and loss was distributed along the tiles surface. The images were captured by infrared camera at intervals of $0,41,95,128$, 159, 190, 221 and 265 seconds respectively. At time interval equals to 0 , the temperature of the tile surface was at room temperature. Each of the following time frames was equivalent to approximately $30^{\circ} \mathrm{C}$ temperature increase at the tiles surface.

'Figures 18 to 30 ' provides a clearer picture of how heat gain and loss was distributed along the granites surface. The 
images were captured by infrared camera at intervals of 0 , 57, 133, 176, 218, 270 and 367 seconds respectively. At time equals to 0 , the temperature of the granite surface was at room temperature. Heat loss data were taken starting from $170^{\circ} \mathrm{C}$ and the subsequent data were taken at every temperature drop of approximately $30^{\circ} \mathrm{C}$. The images are captured by infrared camera at an interval of $0,258,683$,
1338, 2413 and 5976 seconds respectively. At time equals to 0 , the temperature of the granite surface is at $170^{\circ} \mathrm{C}$. Each of the following time frames is equivalent to approximately $30^{\circ} \mathrm{C}$ increase for the granite surface. The plates below provide a clearer picture of how heat is being distributed along the granite surface.

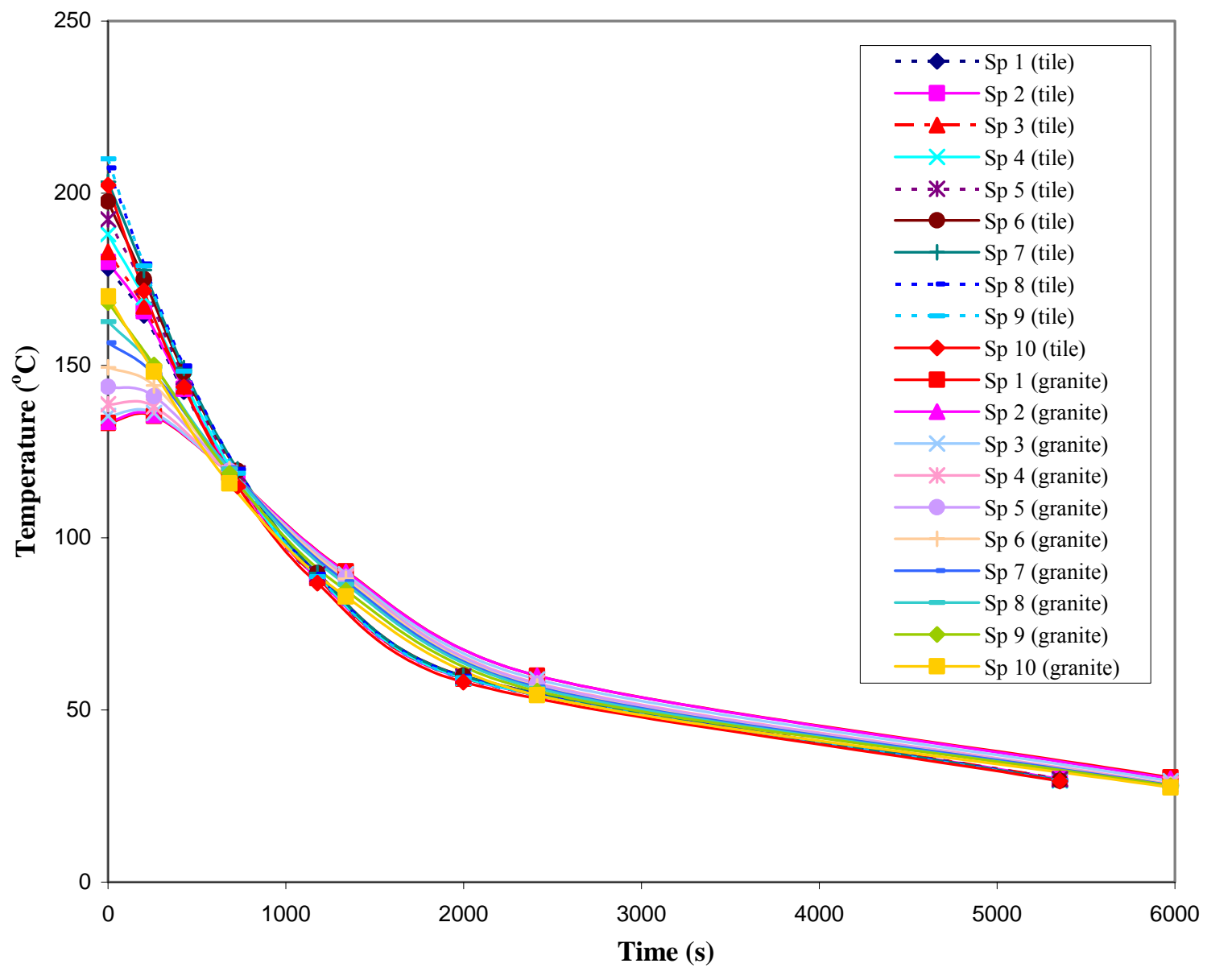

Figure 2. Average conduction temperature loses by tiles and granites

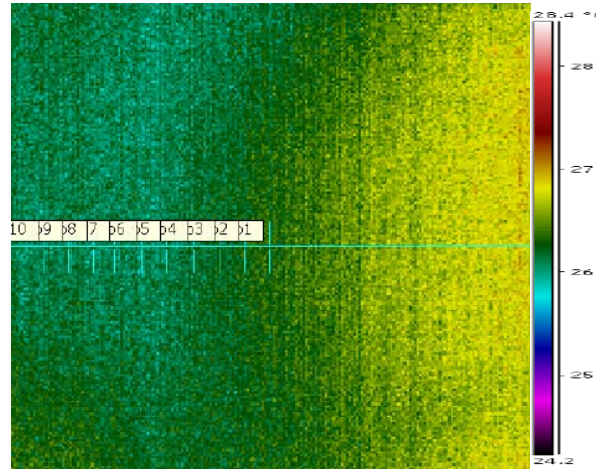

Figure 3. Thermogram for tile at $\mathrm{t}=0 \mathrm{~s}$ and $\mathrm{T}=$ room temperature

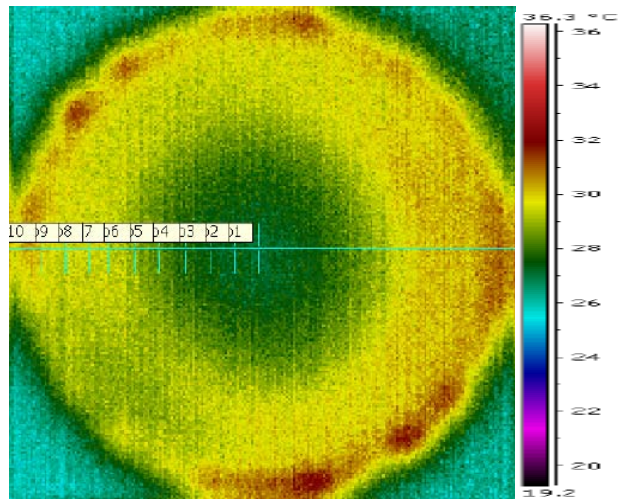

Figure 4. Thermogram for tile at $\mathrm{t}=41 \mathrm{~s}$ and $\mathrm{T} \approx 30^{\circ} \mathrm{C}$ 


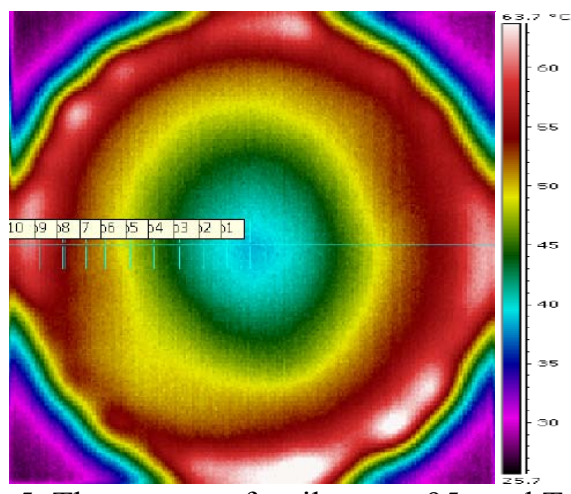

Figure 5. Thermogram for tile at $\mathrm{t}=95 \mathrm{~s}$ and $\mathrm{T} \approx 60^{\circ} \mathrm{C}$

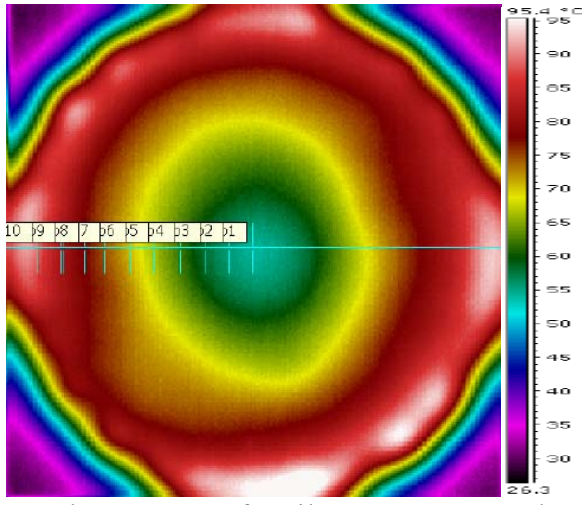

Figure 6 Thermogram for tile at $\mathrm{t}=128 \mathrm{~s}$ and $\mathrm{T} \approx 90^{\circ} \mathrm{C}$

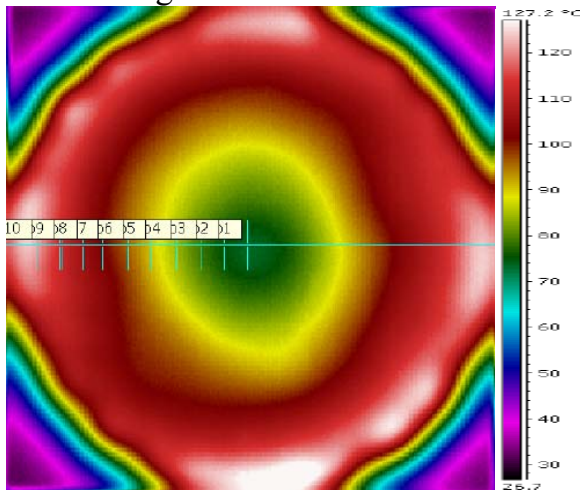

Figure 7. Thermogram for tile at $\mathrm{t}=159 \mathrm{~s}$ and $\mathrm{T} \approx 120^{\circ} \mathrm{C}$

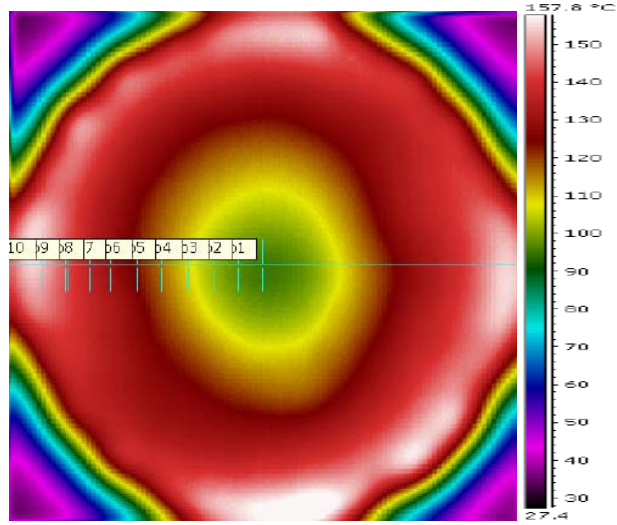

Figure 8 Thermogram for tile at $\mathrm{t}=190 \mathrm{~s}$ and $\mathrm{T} \approx 150^{\circ} \mathrm{C}$

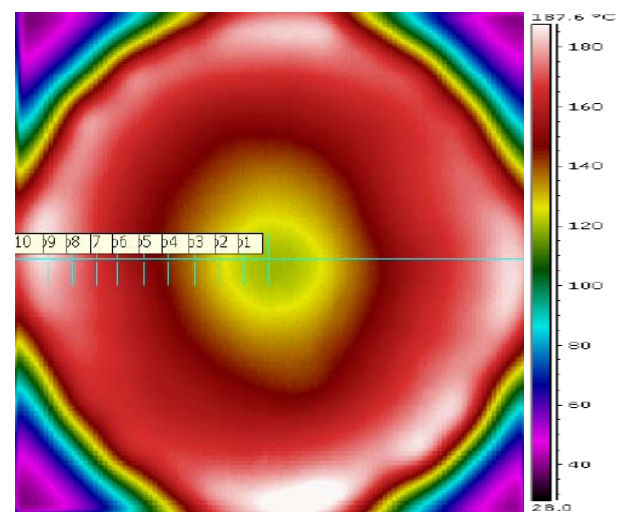

Figure 9 Thermogram for tile at $\mathrm{t}=221 \mathrm{~s}$ and $\mathrm{T} \approx 180^{\circ} \mathrm{C}$

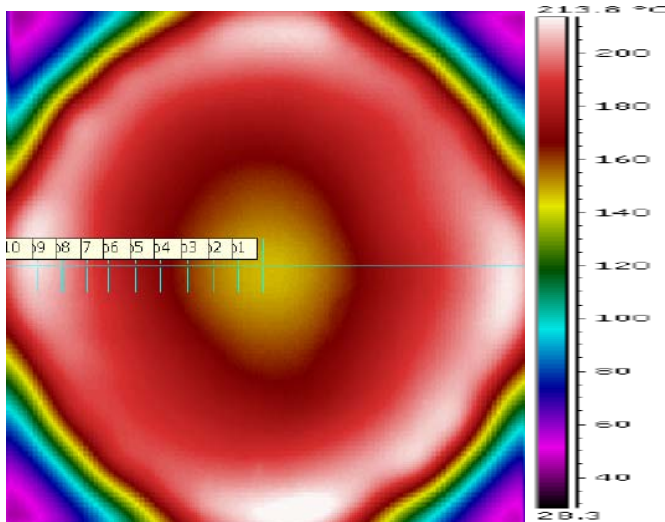

Figure 10 Thermogram for tile at $\mathrm{t}=256 \mathrm{~s}$ and $\mathrm{T} \approx 210^{\circ} \mathrm{C}$

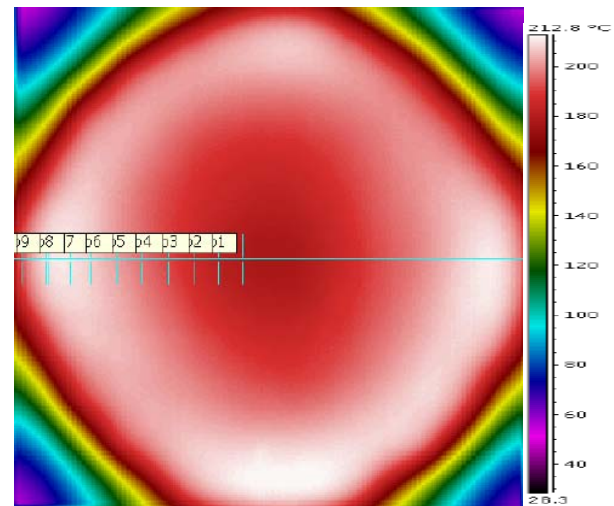

Figure 11 . Thermogram for tile at $\mathrm{t}=0 \mathrm{~s}$ and $\mathrm{T}=210^{\circ} \mathrm{C}$

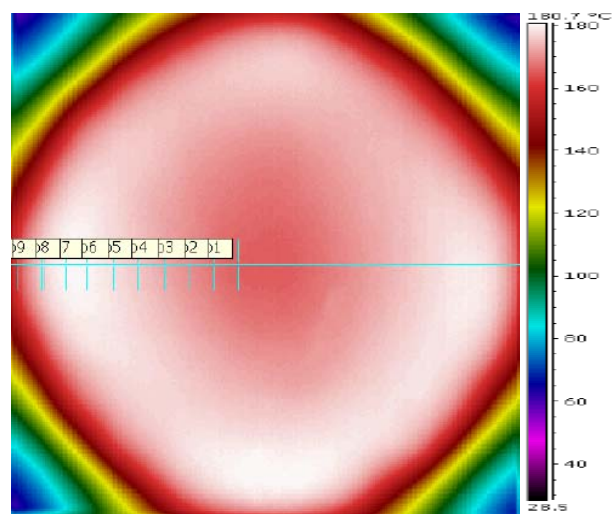

Figure 12. Thermogram for tile at $\mathrm{t}=201 \mathrm{~s}$ and $\mathrm{T} \approx 180^{\circ} \mathrm{C}$ 


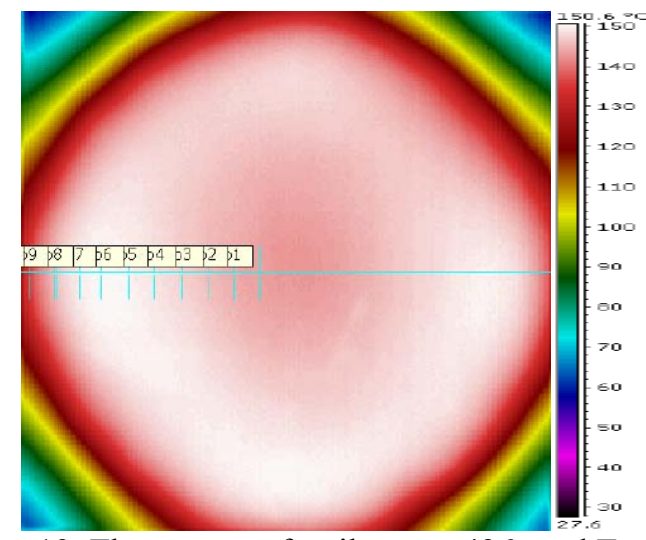

Figure 13. Thermogram for tile at $\mathrm{t}=426 \mathrm{~s}$ and $\mathrm{T} \approx 150^{\circ} \mathrm{C}$

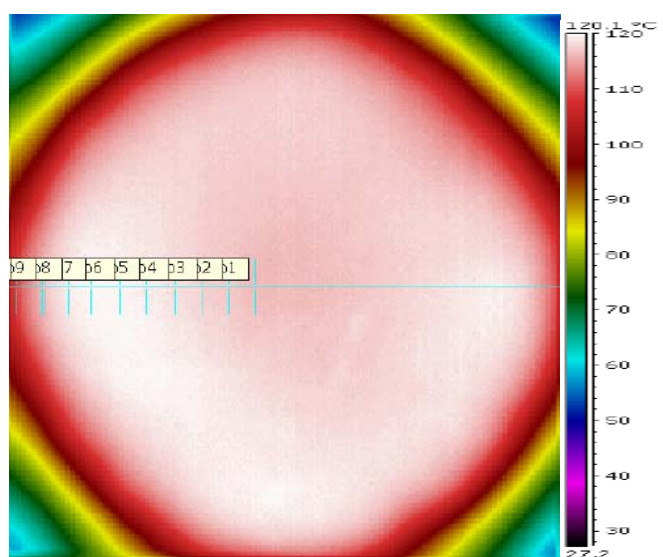

Figure 14. Thermogram for tile at $\mathrm{t}=728 \mathrm{~s}$ and $\mathrm{T} \approx 120^{\circ} \mathrm{C}$

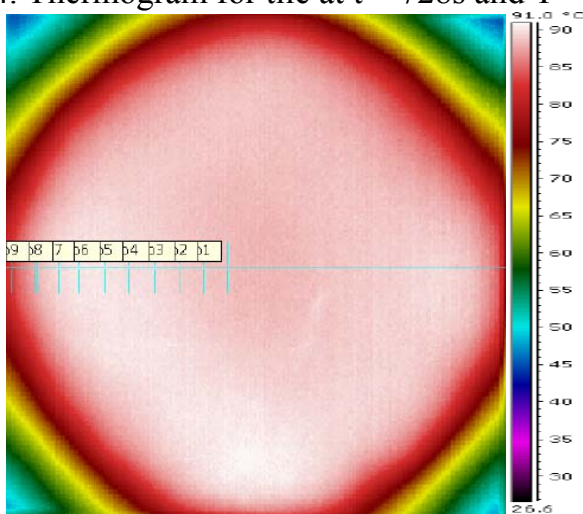

Figure 15. Thermogram for tile at $\mathrm{t}=1177 \mathrm{~s}$ and $\mathrm{T} \approx 90^{\circ} \mathrm{C}$

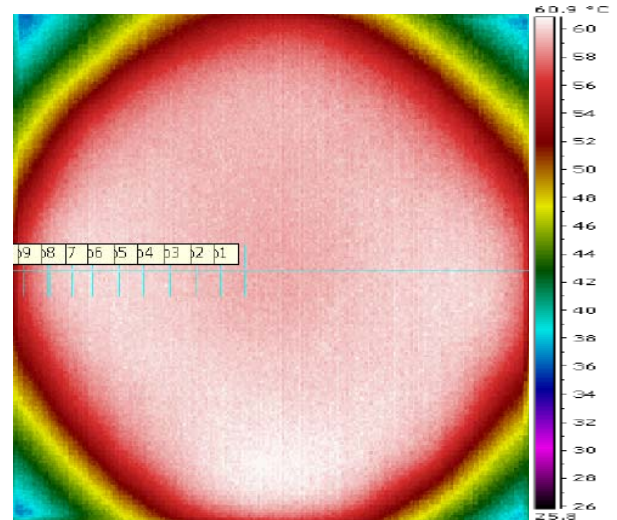

Figure 16. Thermogram for tile at $\mathrm{t}=1999 \mathrm{~s}$ and $\mathrm{T} \approx 60^{\circ} \mathrm{C}$

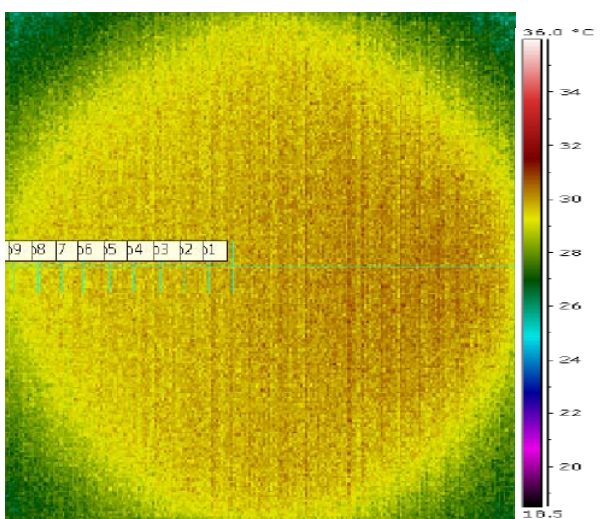

Figure 17. Thermogram for tile at $\mathrm{t}=5352 \mathrm{~s}$ and $\mathrm{T} \approx 30^{\circ} \mathrm{C}$

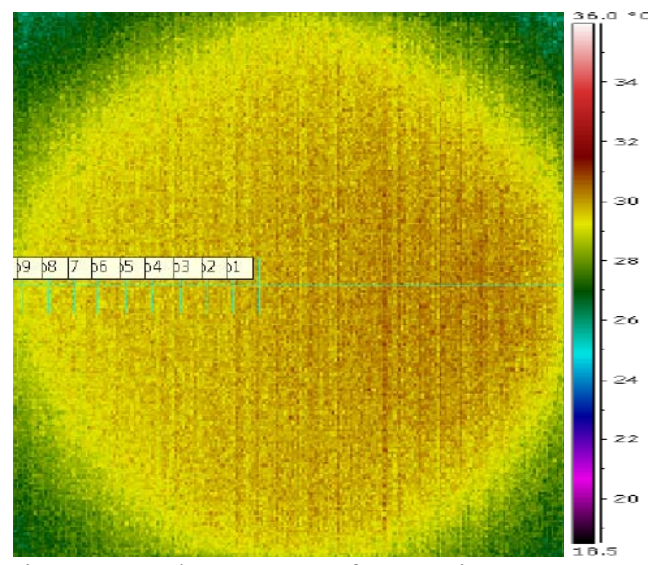

Figure 18. Thermogram for granite at $\mathrm{t}=0 \mathrm{~s}$ and

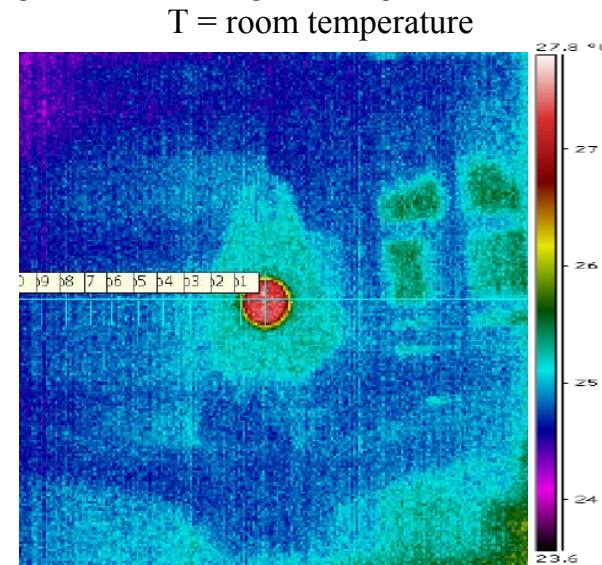

Figure 19. Thermogram for granite at $\mathrm{t}=57 \mathrm{~s}$ and $\mathrm{T} \approx 30^{\circ} \mathrm{C}$

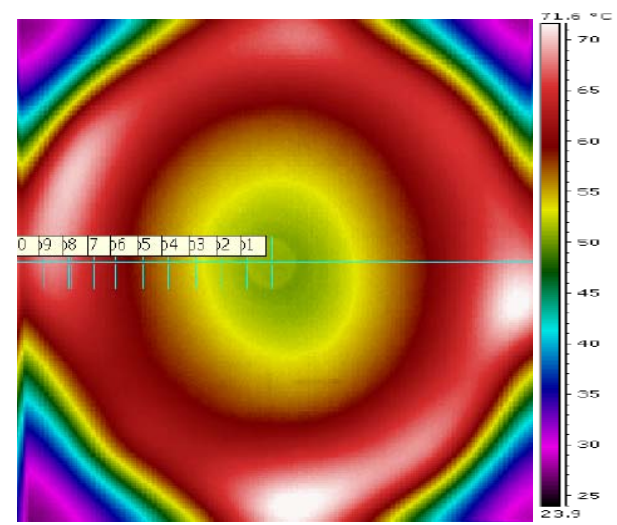

Figure 20. Thermogram for granite at $\mathrm{t}=133 \mathrm{~s}$ and $\mathrm{T} \approx 60^{\circ} \mathrm{C}$ 


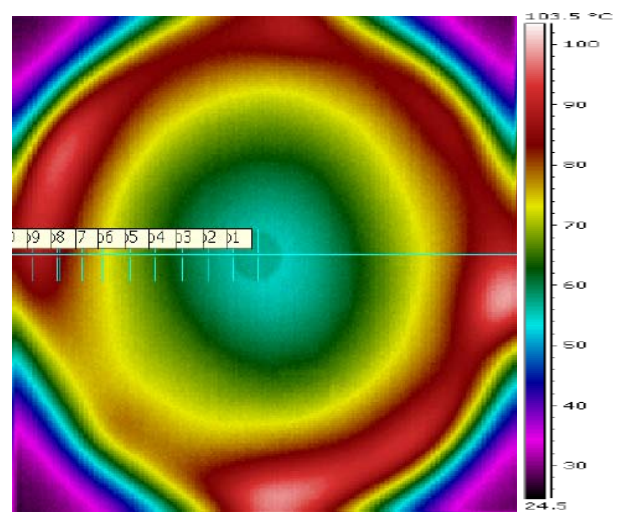

Figure 21. Thermogram for granite at $\mathrm{t}=176 \mathrm{~s}$ and $\mathrm{T} \approx 90{ }^{\circ} \mathrm{C}$

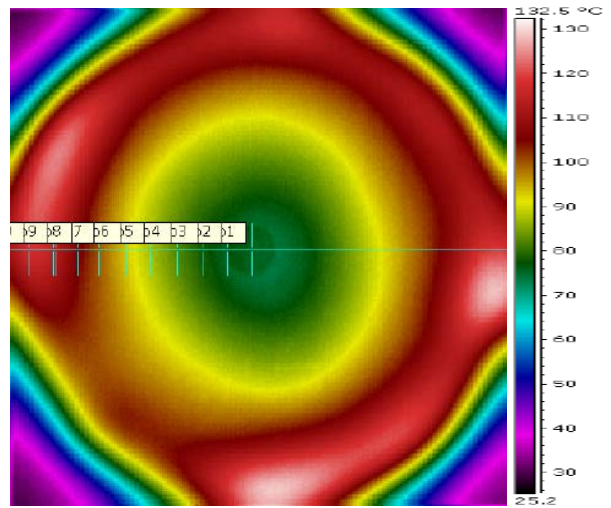

Figure 22. Thermogram for granite at $\mathrm{t}=218 \mathrm{~s}$ and

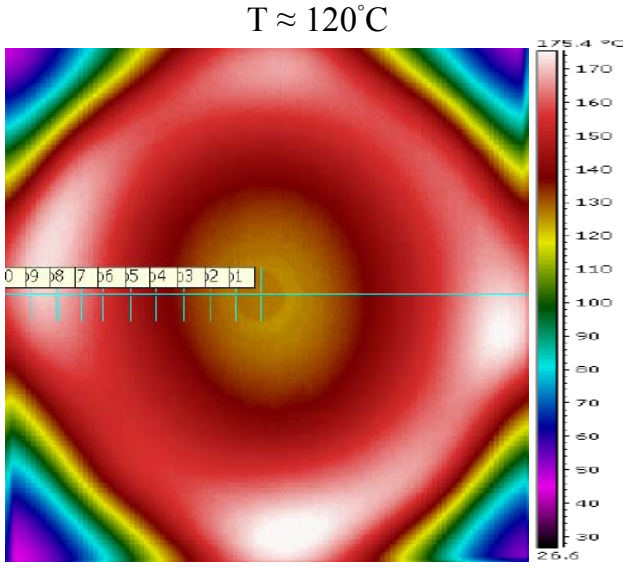

Figure 23, Thermogram for granite at $\mathrm{t}=270 \mathrm{~s}$ and $\mathrm{T} \approx 150^{\circ} \mathrm{C}$

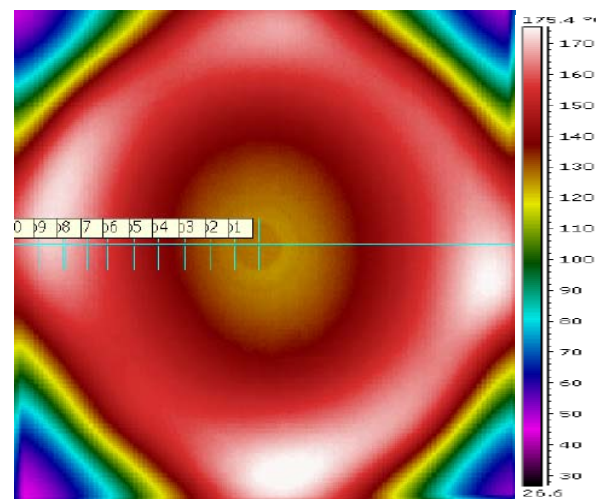

Figure 24. Thermogram for granite at $\mathrm{t}=367 \mathrm{~s}$ and $\mathrm{T} \approx 170^{\circ} \mathrm{C}$

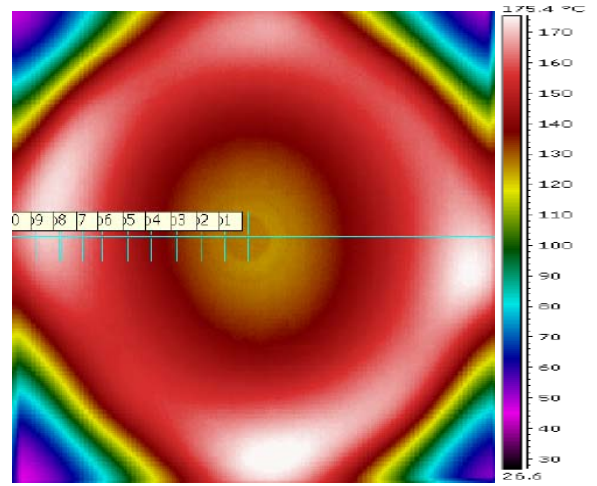

Figure 25. Thermogram for granite at $\mathrm{t}=0 \mathrm{~s}$ and $\mathrm{T} \approx 170^{\circ} \mathrm{C}$

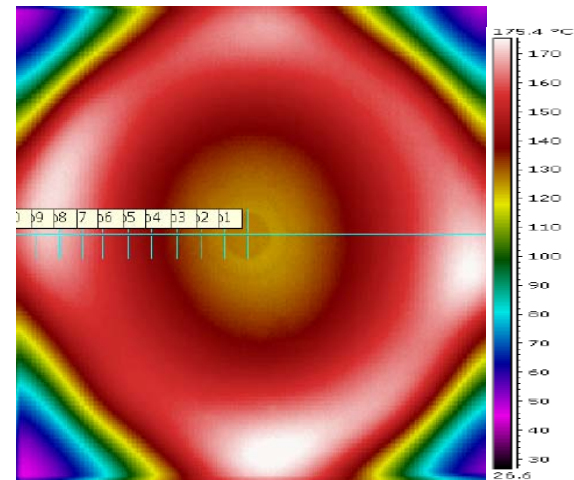

Figure 26. Thermogram for granite at $\mathrm{t}=258 \mathrm{~s}$ and

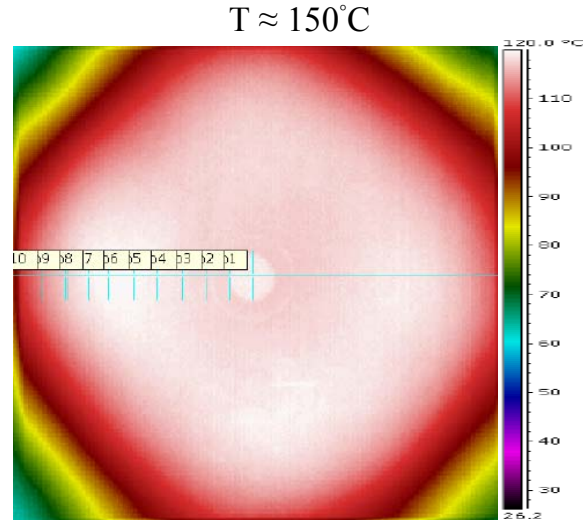

Figure 27. Thermogram for granite at $\mathrm{t}=683 \mathrm{~s}$ and $\mathrm{T} \approx 120^{\circ} \mathrm{C}$

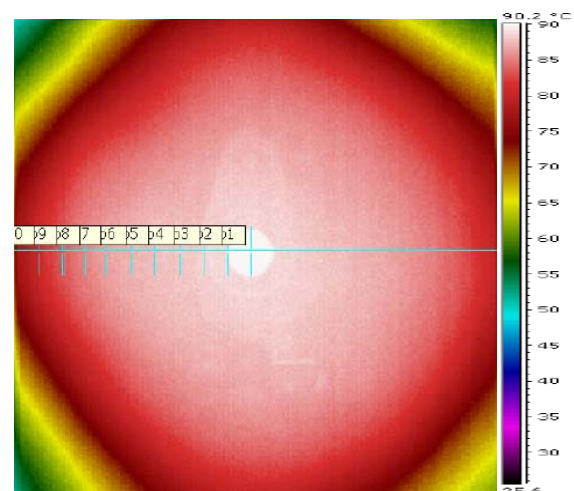

Figure 28. Thermogram for granite at $\mathrm{t}=1338 \mathrm{~s}$ and $\mathrm{T} \approx 90^{\circ} \mathrm{C}$ 


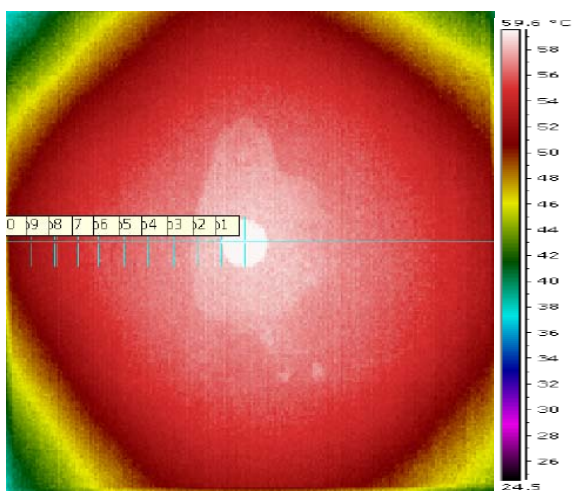

Figure 29. Thermogram for granite at $\mathrm{t}=2413 \mathrm{~s}$ and $\mathrm{T} \approx 60^{\circ} \mathrm{C}$

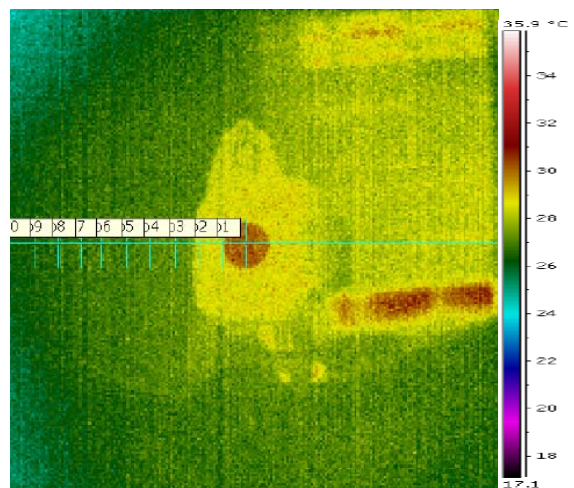

Figure 30. Thermogram for granite at $\mathrm{t}=5976 \mathrm{~s}$ and $\mathrm{T} \approx 30^{\circ} \mathrm{C}$

\section{CONCLUSION}

Current study presents a comparative study of thermal properties of tiles and granites using thermography. All three heat transfer modes (conduction, convection and radiation) were used, however results obtained from the conduction experiments were utilized as the main basis for data analysis. A simple heater plate was utilized as the heat source during heating and cooling processes. In order to obtain accurate results, a closed chamber was built to retain heat. Different sets of parameters and conditions were applied during experiment. Surface temperatures were measured and data were extracted from a data logging system in order to obtain thermal performance of tiles and granites. According to experimental results, granites were poor conductor of heat compared to tiles. Granites rejected heat at a faster rate at a high temperature, however they took longer time to cool down to room temperature.

\section{REFERENCES}

[1] L. Doulas, M. Santamouris and I. Livada, Passive Cooling of Outdoor Urban Spaces, The Role of Material Solar Energy, Vol. 77, pp. 231$249,2004$.

[2] H. Akhbari, M. Pomerantz, H. Taha, A. Rosenfeld and J. Romm, Policies to Reduce Heat Islands: Magnitudes of Benefits and Incentives to Achieve them, Proceedings of ACEEE, Summer Study on Energy Efficiency in Buildings, Vol. 9, pp.177, 1996.

[3] B. Givoni, and M. E. Hoffman, Effect of Building Materials on Internal Temperatures, Research Report, Research Station, Technion Haifa, 1968.

[4] Synnefa, M. Santamouris, and I. Livada, A Comparative Study of the Thermal Performance of Reflective Coatings for the Urban Environment. International Conference, Passive and Low Energy Cooling for the Built Environment, pp. 101-107, 2005.

[5] N. P. Avdelidis, B. C. Hawtin, and D. P. Almond, Transient Thermoography in the Assessment of Defects of Aircraft
Composites." Journal of NDT \& E International, Vol. 43 (6), pp. 433439, 2003. 


\title{
Overview of Fuel Cell Powered Radio Controlled Car
}

\author{
Azizul Mohamad and Norasmadi Abdul Rahim
}

\begin{abstract}
Fuel cell is emerging as one of the most promising renewable energy sources. Various applications of fuel cell had been investigated. In this paper, an overview will be given on the design principles of an ongoing project in utilizing fuel cell to power a radio-controlled (RC) car. One of major issues in radio-controlled car is the time taken in charging the batteries. Normally the time used for charging is greater than the actual time of using the radio-controlled car by several folds. Depleting power of the batteries will also lead to reducing range of radio-controlled car. In order to solve both of these problems, the use of fuel cell as power source was proposed. Fuel cell will be able to be charged at a much faster rate in relative to the conventional batteries, and it able to provide sustainable level of power output throughout the utilization. This project used a standard radio controlled car and the only change made was on its power supply system by replacing conventional batteries with fuel cell. Several components such as fuel cell stack, hydrogen storage tank(s) and pressure regulator have to be installed in the car body in this transformation. Among major concerns are the costs of these components, the optimal specification for each of these parts, the overall size and weight of the system, and the efficiency of fuel cell powered system as compared to the conventional one.
\end{abstract}

Keywords: fuel cell, radio-controlled car

\section{INTRODUCTION}

A radio controlled car is an electro-mechanical device that mainly used as a toy and may performs useful work in field application. It can transport itself under its own power and vary in scale from milliwatts to kilowatts.

Radio-controlled car places stringent demands on the power system. Energetic autonomy, or the ability to function for a useful operation time independent of any tether, refuelling, or recharging, is a driving force in a radiocontrolled car designed for a field application.

The device that enables mobile operation is the power system, defined here as the group of devices that store a quantity of transported energy and transform it at a desired rate into output energy, typically mechanical rotary or translational energy.

Azizul Mohamad is with Mechanical Engineering Program, School of Mechatronic Engineering, Universiti Malaysia Perlis, 01000 Kangar, Perlis, MALAYSIA (phone: 604-979-8679; fax: 604-979-8142; e-mail: azizul@unimap.edu.my).

Norasmadi Abdul Rahim is with Mechatronic Engineering Program, School of Mechatronic Engineering, Universiti Malaysia Perlis, 01000 Kangar, Perlis, MALAYSIA (e-mail: norasmadi@unimap.edu.my).
A power system is made up of two types of generalized components: the energy source, defined as a device or substance energy, and energy converters, defined as devices, which receive energy in one form as input and transform it into another form as output.

For a typical radio-controlled car, a common energy source is a conventional battery, which possesses a need for lengthy charging process prior to utilization. Some other type of radio-controlled car utilizes a small internal combustion engine as its energy converter, which uses fossil fuel as its energy source.

This paper will review the application of fuel cell as the alternative power source and will discourse the comparison of these two systems as well as potential future researches in this field. Focus will be given to various components that made up the fuel cell system.

\section{BACKGROUND}

Regardless of size, radio-controlled car must carry a sufficient amount of energy with it in order to achieve a useful operation time in the field before recharging or refuelling. This concept of energetic autonomy requires two criteria, namely the ability to operate for a useful amount of time, and the ability to operate independent of an external source of power.

Many mobile systems satisfy one criterion of energetic autonomy but fail at the other. For example, a radiocontrolled car designed to navigate a mountainous trail that can only operate for ten minutes in the field before needing recharging fails the first criterion. The same radio-controlled car with a tether connecting it to a large stationary power system providing arbitrarily long operation time fails the second [1].

Weight is of primary concern in radio-controlled car where payload capacity and manoeuvrability are important. Stationary power systems, such as a power plant, can weigh as much as necessary since they are anchored to the ground and need not be moved.

Fuel cell is an energy converter that converts hydrogen or methanol directly into electrical energy. They are not heat engines but rather electrochemical devices and are not limited by the Carnot efficiency [2].

The fuel cell as shown in Figure 1 is an electrochemical energy conversion device that converts chemical energy of hydrogen and oxygen into electricity and heat by electrochemical redox reactions at the anode and the cathode of the cell, respectively, that produces water as the 
only byproduct. It is the chemical engineering way of producing energy [2].

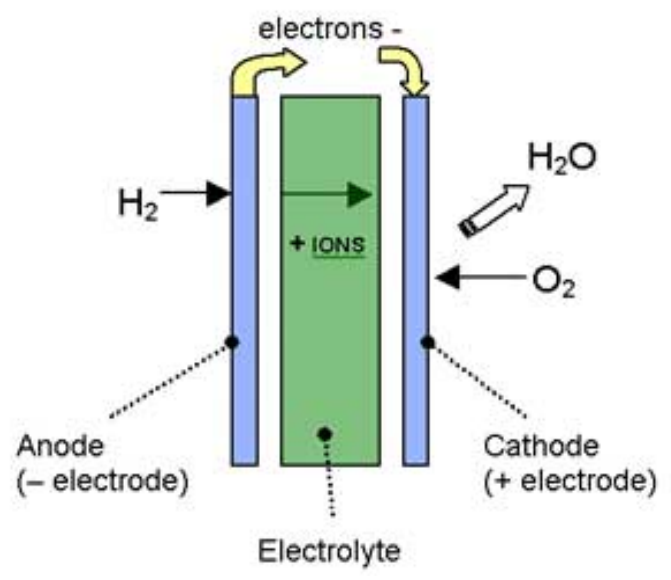

Figure 1. Fuel cell diagram

Sir William Grove invented the first working fuel cell in 1843 by reacting oxygen and hydrogen on separate platinum electrodes that were immersed in dilute sulphuric acid inside five cells of a gas voltaic battery and using the current produced to electrolyze water in another similar cell.

Since then, there have been several attempts to apply it for more than 100 years but none was very successful. In the 1920s, early German fuel cell research developed primitive carbonate and solid oxide fuel cells and from 1932 to 1959, Francis T. Bacon developed a fuel cell that used alkaline electrolyte and nickel electrodes.

It was only after a more efficient design of the fuel cell was made in the 1960s for the Gemini and Apollo space missions that fuel cell technology came of age. General Electric produced the fuel cell powered electrical power system for NASA's Gemini and Apollo space capsules that also provides drinking water for the crew.

There are six main types of fuel cell of commercial importance: alkaline fuel cell (AFC), phosphoric acid fuel cell (PAFC), molten carbonate fuel cell (MCFC), solid oxide fuel cell (SOFC), polymer electrolyte membrane fuel cell (PEMFC) and direct methanol fuel cell (DMFC).

Each of these fuel cell has its own working temperature, efficiency, potential applications, as well as own advantages and disadvantages. The technology for the first three type of fuel cell is already matured, while the bottom three is still undergo major research and development [2].

As far as mobile applications are concerned, two types of fuel cell that exhibit promising prospects are polymer electrolyte membrane fuel cell (PEMFC), also known as proton exchange membrane fuel cell, and direct methanol fuel cell (DMFC). One of the main reasons for their suitability is a relatively low working temperature as compared to other fuel cell types. Through discussion on the suitability of these fuel cells are to be discussed at the later stage of this paper.

Table 1 shows briefly their working temperature, efficiency and potential applications.

Table 2 shows briefly the advantages and disadvantages for each fuel cell types.

Fuel cells generally have a higher conversion efficiency than heat engines, typically ranging from 40-60\%. Their specific power $(20-200 \mathrm{~W} / \mathrm{kg})$ is generally lower than internal combustion engines.
Table 1. Fuel cell working temperature, efficiency and potential applications

\begin{tabular}{|l|c|c|l|}
\hline \multicolumn{1}{|c|}{ Fuel cell } & $\begin{array}{c}\text { Temp. } \\
\left({ }^{\circ} \mathbf{C}\right)\end{array}$ & $\begin{array}{c}\text { Efficiency } \\
\mathbf{( \% )}\end{array}$ & $\begin{array}{c}\text { Potential } \\
\text { applications }\end{array}$ \\
\hline $\begin{array}{l}\text { Alkaline } \\
(\text { AFC) }\end{array}$ & $50-90$ & $50-70$ & $\begin{array}{l}\text { Space } \\
\text { application }\end{array}$ \\
\hline $\begin{array}{l}\text { Phosphoric } \\
\text { acid (PAFC) }\end{array}$ & $\begin{array}{c}175- \\
220\end{array}$ & $40-45$ & $\begin{array}{l}\text { Stand-alone \& } \\
\text { combined heat } \\
\text { \& power }\end{array}$ \\
\hline $\begin{array}{l}\text { Molten } \\
\text { carbonate } \\
\text { (MCFC) }\end{array}$ & $600-$ & $50-60$ & $\begin{array}{l}\text { Central, stand- } \\
\text { alone \& } \\
\text { combined heat } \\
\text { \& power }\end{array}$ \\
\hline $\begin{array}{l}\text { Solid oxide } \\
\text { (SOFC) }\end{array}$ & $800-$ & $50-60$ & $\begin{array}{l}\text { Central, stand- } \\
\text { alone \& } \\
\text { combined heat } \\
\text { \& power }\end{array}$ \\
\hline $\begin{array}{l}\text { Polymer } \\
\text { electrolyte } \\
\text { membrane } \\
\text { (PEMFC) }\end{array}$ & $60-100$ & $40-50$ & $\begin{array}{l}\text { Vehicle \& } \\
\text { portable }\end{array}$ \\
\hline $\begin{array}{l}\text { Direct } \\
\text { methanol } \\
\text { (DMFC) }\end{array}$ & $50-120$ & $25-40$ & $\begin{array}{l}\text { Vehicle \& } \\
\text { small portable }\end{array}$ \\
\hline
\end{tabular}

Table 2. Fuel cell advantages and disadvantages

\begin{tabular}{|l|l|l|}
\hline \multicolumn{1}{|c|}{ Fuel cell } & \multicolumn{1}{|c|}{ Advantages } & \multicolumn{1}{c|}{ Disadvantages } \\
\hline $\begin{array}{l}\text { Alkaline } \\
\text { (AFC) }\end{array}$ & High efficiency & $\begin{array}{l}\text { Intolerant to } \mathrm{CO}_{2} \text { in } \\
\text { impure } \mathrm{H}_{2} \text { and air, } \\
\text { corrosion, expensive }\end{array}$ \\
\hline $\begin{array}{l}\text { Phosphoric } \\
\text { acid } \\
\text { (PAFC) }\end{array}$ & $\begin{array}{l}\text { Tolerant to } \\
\text { impure } \mathrm{H}_{2}, \\
\text { commercial }\end{array}$ & $\begin{array}{l}\text { Low power density, } \\
\text { corrosion \& sulphur } \\
\text { poisoning }\end{array}$ \\
\hline $\begin{array}{l}\text { Molten } \\
\text { carbonate } \\
\text { (MCFC) }\end{array}$ & $\begin{array}{l}\text { High efficiency, } \\
\text { Near } \\
\text { commercial }\end{array}$ & $\begin{array}{l}\text { Electrolyte instability, } \\
\text { corrosion \& sulphur } \\
\text { poisoning }\end{array}$ \\
\hline $\begin{array}{l}\text { Solid oxide } \\
\text { (SOFC) }\end{array}$ & $\begin{array}{l}\text { High efficiency } \\
\text { \& direct fossil } \\
\text { fuel }\end{array}$ & $\begin{array}{l}\text { High temperature, } \\
\text { thermal stress failure, } \\
\text { coking \& sulphur } \\
\text { poisoning }\end{array}$ \\
\hline $\begin{array}{l}\text { Polymer } \\
\text { electrolyte } \\
\text { membrane } \\
\text { (PEMFC) }\end{array}$ & $\begin{array}{l}\text { High power } \\
\text { density, low } \\
\text { temperature }\end{array}$ & $\begin{array}{l}\text { Intolerant to CO in } \\
\text { impure } \mathrm{H}_{2} \text { and } \\
\text { expensive }\end{array}$ \\
\hline $\begin{array}{l}\text { Direct } \\
\text { methanol } \\
\text { (DMFC) }\end{array}$ & $\begin{array}{l}\text { No reforming, } \\
\text { high power } \\
\text { density \& low } \\
\text { temperature }\end{array}$ & $\begin{array}{l}\text { Low efficiency, } \\
\text { methanol cross-over \& } \\
\text { poisonous by-products }\end{array}$ \\
\hline
\end{tabular}

Fuel cells have the advantage of quiet operation and no harmful emissions with pure hydrogen fuel (the only exhaust is water). If methanol or another hydrocarbon is used as a fuel, they produce emissions of carbon dioxide.

Fuel cell's modular compact design enables the consumer to increase or decrease power by simply adding or removing the modules to the required power without having to redesign and reconstruct the whole plant. This feature is important for the sustainability of the overall system in coping with the future needs and demands [1].

It is a clean technology and therefore, has very low chemical pollution. It could use pure hydrogen fuel or a 
variety of primary fuels such as natural gas and methanol that could be used directly or used to produce the hydrogen fuel instead. In a high temperature fuel cell, combined heat and power would increase its efficiency.

Due to their scalability, new applications for fuel cells are being investigated all the time. Some see them replacing batteries in certain portable applications, offering extended run times with simple refuelling rather than the need for a lengthy recharge.

When it comes to the selection of a power source for a particular application, a plot of specific power versus specific energy is sometimes used to help quantify the assessment. Figure 2 provides a general comparison of different technologies on this basis [3]. If one ignores the extremes as provided by engines and supercapacitors, one sees a significant overlap between batteries and fuel cells.

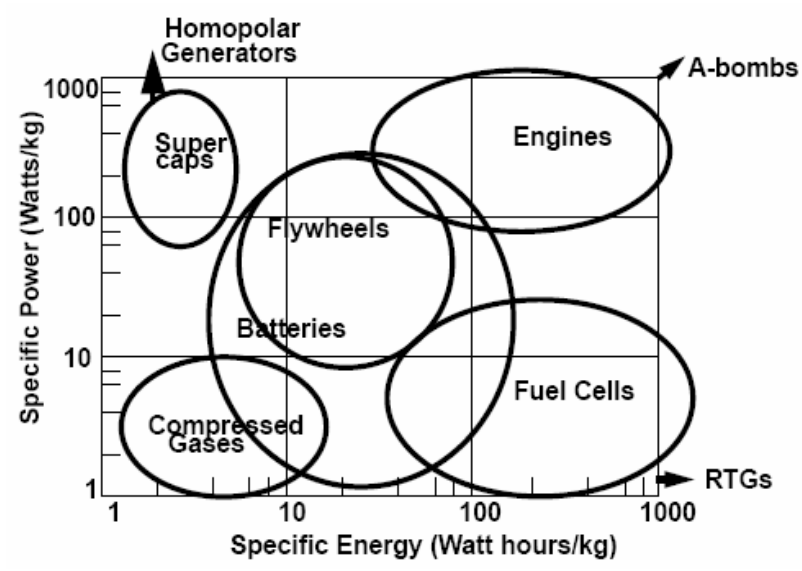

Figure 2. Comparison of different power sources

Most portable fuel cell development is focusing on mainstream uses such as cell phones and laptops, but there are other battery-powered devices where fuel cells could offer benefits.

While the most common application of radio-controlled car is at the hobbyist level as a toy, it can also be used in the educational area, such as in high school and college level courses to teach principles of electronics, robotics and mechatronics[3], as well as for surveillance and rescue purposes.

Radio-controlled car is powered by various sources. Electric cars are powered by small but powerful electric motors and rechargeable nickel cadmium, nickel metal hydride, or lithium polymer cells. There are also brushed or brushless electric motors. Most fuel-powered models use glow plug engines, small internal combustion engines fueled by a special mixture of nitromethane, methanol, and oil (in most cases a blend of castor oil and synthetic oil). These are referred to as "nitro" cars. Recently, exceptionally large models have been introduced that are powered by small gasoline engines, similar to weedwhacker motors, which use a mix of oil and gasoline. [4]

\section{ANALYSIS}

The conversion is performed by replacing the conventional battery system with fuel cell system, which consist of fuel cell stack, fuel storage, DC/DC converter and purging mechanism. Details of these components are discussed next.

\section{A. Fuel Cell Stack}

Direct methanol fuel cell (DMFC) is relatively new compared to fuel cells powered by pure hydrogen such as PEMs. A 2003 survey indicated that only about $12 \%$ of existing portable systems are DMFC, yet there are an equal number of DMFC and PEM systems under development. This is due to the theoretical advantage of methanol and the practical problems that have to be overcome [3].

DMFCs are attractive because they do not have the fuel storage problems associated with PEMs, as methanol has a higher energy density than hydrogen and can be stored as a liquid at room temperature. Methanol management remains both a safety and operational issue.

Polymer electrolyte membrane (PEM) fuel cells are commonly used for mobile applications because they typically have a higher specific power and less balance of plant weight than other fuel cell types.

In order to be a viable mobile prime mover, a PEM fuel cell must have several characteristics [1]:

- The reaction air must be at atmospheric or nearatmospheric pressure. If the air must be pressurized this requires a heavy compressor which also increases the parasitic load on the fuel cell.

- The stack must be air cooled, not liquid cooled. Liquid cooling requires a separate water system, which includes a pump, hoses, fittings, and the water itself, which add weight and complexity.

- The stack must not require external humidification of the reaction gases. The membrane in a fuel cell must be properly humidified to ensure reliable operation. Selfhumidifying fuel cells eliminates the necessity of a separate humidification system.

\section{B. Fuel Storage}

Hydrogen has a very high specific energy, $33 \mathrm{kWh} / \mathrm{kg}$, but is difficult to store in a lightweight. It can be compressed to high pressures and stored in lightweight carbon fibre tanks, but this only achieves a fuel mass fraction of approximately 3\% with commercially available tanks [1].

Storing hydrogen in a metal hydride tank takes up less volume than compressed hydrogen tanks but only achieves a fuel mass fraction of approximately $1 \%$. A pressure regulator that was designed for metal hydrides was used to control the flow of hydrogen from the hydride tank to the stack, and to drop the supply pressure to that required by the stack.

Liquid hydrogen kept at $-253^{\circ} \mathrm{C}$ is compact and produces a fuel mass fraction of $6 \%$ but requires bulky insulated tanks and is inconvenient to use for logistical reasons. Another possibility is physical hydrogen storage in carbon nanofibers, which has been shown to exceed a $60 \%$ hydrogen mass fraction under controlled. Fuel cells powered by methanol are another emerging option and benefit from the high energy density of liquid fuels.

\section{DC/DC Converter}

In order to supply the radio-controlled car with a constant voltage, a DC/DC converter circuit capable of boosting various voltage levels with high efficiency was used. Thus, control of the power system was achieved using the radiocontrolled car's existing processing capability. Power 
sharing between the stack and the capacitors was passive and based on the voltage differences [3].

\section{D.Purging Mechanism}

Current industry practice is to periodically purge the stack to remove the excess moisture from the anode (hydrogen) side. Although the need to purge can be reduced through improved temperature, fuel flow and air flow control, the need to keep the membrane moisturized combined with the production of water on the cathode (air side) means that sooner or later excess water will accumulate on the anode side [3].

\section{DISCUSSION}

One of the advantages of fuel cells is that they decouple energy storage from power production. This makes it easy to provide more energy (in the form of fuel) as needed, and as long as the fuel is supplied, the power available remains the same.

In effect, a fuel cell gives similar benefit as that of an internal combustion engine, but it is also quieter, more efficient, non-polluting, and more easily scaled down.

However, it is more complex than a battery and its $30 \%-$ $50 \%$ fuel efficiency cannot match the over $>90 \%$ cycle efficiencies of batteries if a source of electricity is available anyway.

If a radio-controlled car currently uses lithium batteries, weight is probably a significant factor, and cost a lesser issue. In such cases, fuel cells, probably in tandem with capacitors or a small battery, will become attractive in the future once component weights are optimized.

If long run times are important and power requirements are relatively low-say, for some sort of security surveillance radio-controlled car-a fuel cell becomes even more attractive. However, if a radio-controlled car currently uses a lead acid battery, cost is probably more important than weight.

Conversion from conventional power system to fuel cell based power system will be justifiable if the improvements in performance and reliability are more than additional costs and weight that are contributed by the alternative system. However, the overall cost and weight of fuel cell system will definitely reduce as the system become mature.

\section{CONCLUSION}

Radio-controlled car is converted from a conventional lead acid battery to a fuel cell as the power source. The fuelcell-based power supply system is assembled from commercial components. The needs for energetic autonomy in radio-controlled cars as well as a brief explanation on fuel cell types and characteristics were mentioned. Various components involve in the conversion are discussed. This exercise was served to demonstrate the potential of the fuel cell technology despite its high cost.

\section{REFERENCES}

[1] J. W Raade, Graphical Analysis of Power Systems for Mobile Robotics, PhD Dissertation, University of California at Berkeley, 2006.

[2] R. O'Hayre, S.W. Cha, W. Colella, F.B. Prinz, Fuel Cell Fundamental. John Wiley \& Son, Inc, 2006

[3] A.N. Wilhelm, B.W. Surgenor and J.G. Pharoah, Design and Evaluation of a Micro-Fuel-Cell Based Power System for a Mobile
Robot, IEEE/ASME Transactions on Mechatronics, 11(4), 2006, 471475.

[4] Internet. "Radio-controlled car", http://en.wikipedia.com/wiki/Radiocontrolled_car, Accessed on 20 July 2007 


\title{
Design Enhancement and Testing of a Pepper Solar Tumble Dryer Using a Biomass Back-Up Heater
}

\author{
A. Rahim Md. Amin, Mahbub Hasan and Ong Tean Wee
}

\begin{abstract}
A solar dryer is an equipment that uses solar energy for drying foods. During solar drying, foods are prevented from dust, rain, wind. insects, birds, rodents and other domestic animals unlike open drying process. Thus solar drying produces foods having better quality compared to open drying process. However the traditional solar dryers can only be operated during good weather condition and day time. Current research describes design enhancements of a pepper solar tumble dryer developed in Universiti Malaysia Sarawak using a biomass back-up heater. The scope of design enhancement was constrained by the existing solar tumble dryer as the designed biomass back-up heater had to be compatible with the existing solar dryer. The methodology involved selection of a biomass back-up heater design, fabrication and testing of the resultant heater alongside the existing pepper solar tumble dryer. In order to achieve the goals, variables such as type of fuel, basic material properties and designs were considered. The design of the biomass heater was improved step by step with each set of testing. Drying rate, temperature and humidity were measured during the whole drying process. Testing results suggested that the biomass back-up heater shortened the drying time and the drying process could be carried out in any weather condition.
\end{abstract}

Keywords: design enhancement, testing, pepper solar tumble dryer, biomass back-up heater

\section{INTRODUCTION}

Pepper is a branching vine that is planted in hot climate countries like South-East Asia, India, Brazil and others. Sarawak is the largest pepper producing state in Malaysia (95\% of total production), where 14,000 hectares of land mainly fertile hill slopes are used for pepper plantation. Pepper plants require around 3 years to be matured. Pepper berries are harvested when one or more of them become yellow or orange in colour and hard to tough in character [1].

A. Rahim Md. Amin, Department of Mechanical and Manufacturing Engineering, Faculty of Engineering, Universiti Malaysia Sarawak, 94300 Kota Samarahan, Sarawak, Malaysia (phone: +6082583318; fax: +6082583410; e-mail: arahim@feng.unimas.my).

Mahbub Hasan, Department of Mechanical and Manufacturing, Faculty of Engineering, Universiti Malaysia Sarawak, 94300 Kota Samarahan, Sarawak, Malaysia (e-mail: hmahbub@feng.unimas.my).

Ong Tean Wee, Department of Mechanical and Manufacturing Engineering, Faculty of Engineering, Universiti Malaysia Sarawak, 94300 Kota Samarahan, Sarawak, Malaysia.
After harvesting, certain processes are involved in converting the peppers into finished products for selling. The drying process is the most important section in the preparation cycle of pepper. If the drying process is slow, it will lead to mould growth in the pepper. The sale value of mould pepper can be less than $50 \%$ compared to the normal pepper. In some extreme cases, the whole crop can be lost. Sun drying, the simplest and cheapest method of pepper drying, is usually adequate to dry the product, which is. During sun drying, peppers are just laid on the mats under the sun. However, there are some problems associated with this method. Dust and dirt are blown onto the crop during drying. Sometimes there are unexpected rains that can rewet the crop, especially during the wet season that often coincides with the harvesting season of the spices. Solar drying is an alternate way of replacing the conventional sun drying process. It can be employed effectively to control drying rate and produce good quality products compared to open sun drying. Previous research carried out in UNIMAS developed a pepper solar tumble dryer that can be used to serve the purpose [2]. Malaysia is a tropical country where solar energy is available almost the whole day. Still there is a fare chance of rain during the day all through the year. Thus the developed solar dryer could function only during day time with good weather condition.

Current research describes design enhancement and testing of the pepper solar tumble dryer developed before using a biomass back-up heater in order to overcome the problems associated with the old dryer. The methodology involved selection of a biomass back-up heater design, fabrication and testing of the resultant heater alongside the existing pepper solar tumble dryer. Variables such as type of fuel, basic material properties and designs were considered. Drying rate, temperature and humidity were measured during the whole drying process. Testing results suggested that the biomass back-up heater shortened the drying time and the drying process could be carried out in any weather condition.

\section{Design Methodology}

\section{A. Selection of a Solar Dryer Design}

At present, various types of heater are available for heating air. Thorough analysis of types of heater was 
conducted at the initial stage of this work. Two designs were finally considered. Those designs are described below in brief.

\section{1) Design 1}

First design used direct heat from the combustion chamber. The fire would heat up the metal plate on top of the combustion chamber and then it would transfer the heat to the air which was passing through. The heated air would exhaust into the long pipe. However this design was not considered as the air temperature produced was too high and not suitable for pepper drying. The picture of the first design is shown in 'figure 1'.

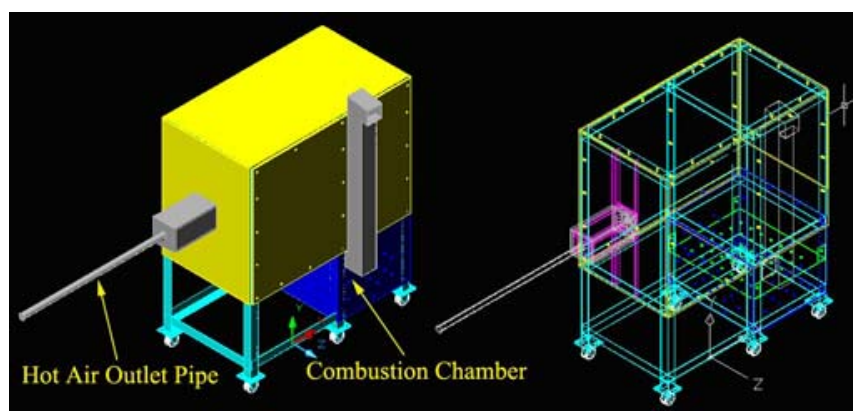

Figure 1. A picture of the first design of back-up heater

\section{2) Design 2}

In order to solve the problem associated with first design, fire was used to produce steam. The steam in turn would heat up the air passing through. In this case, the temperature would decease below $100^{\circ} \mathrm{C}$ and more stable as compared to the first design. Thus authors selected the second design as a basis for manufacturing the back-up heater. A picture of the second design is shown in 'figure 2'. Description of the selected design is given in section 2.4.

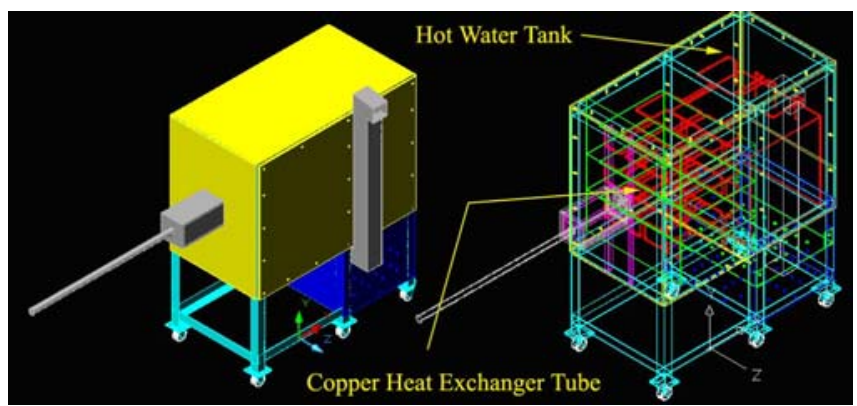

Figure 2. A picture of the second design

\section{B. Selection of Material}

Selecting the right material for a specific design is probably the single most important decision facing a designer. During material selection, a designer needs to think about four criteria; (a) the primary characteristic of the material, such as strength, hardness, thermal conductivity, weight and corrosion, (b) the price comparison between materials having same characteristic, (c) surface finish and (d) availability of the material. Considering all those criteria, the authors selected steel as the material to fabricate main structure. Ply wood was selected to build wall at heat transfer section. Stainless steel was used for the water tank to prevent corrosion. However for the heat exchanger section, copper was chosen as copper has good thermal conduction characteristic. The prototype was equated with wheels so that it was moveable and more flexible. It was painted in dark colour to prevent dirt.

\section{Selection of Biomass Fuel}

Wood and charcoal were initially selected as the biomass fuels for the back-up heater considering their availability in Sarawak. Again wood and charcoal are the cheapest source of energy for the back-up heater besides the free solar energy. Moreover fuel wood can be a greenhouse gas neutral source of energy, if usage is balanced by new plantings [3].

\section{D.Description of Selected Design}

The selected design of the back-up heater consisted of three sections; combustion chamber, water tank and heat exchanger path way. A picture of the selected back-up heater along with the tumble dryer is shown in 'figure 3'. As mentioned earlier, wood and charcoal were used as source of energy to produce steam in order to maintain the temperature below $100^{\circ} \mathrm{C}$. The steam was passed through copper tube. The function of the tube was to act as a heat transfer medium, which maintained the temperature of the heated air. The slides between the copper tube provided a longer pathway and longer time to increase the temperature of heated air. An AC fan was used to draw the heated air into the tumble dryer. A long hot air outlet pipe from the end of the heater was inserted into the tumble dryer in order to provide an equal heat around the tumbler.

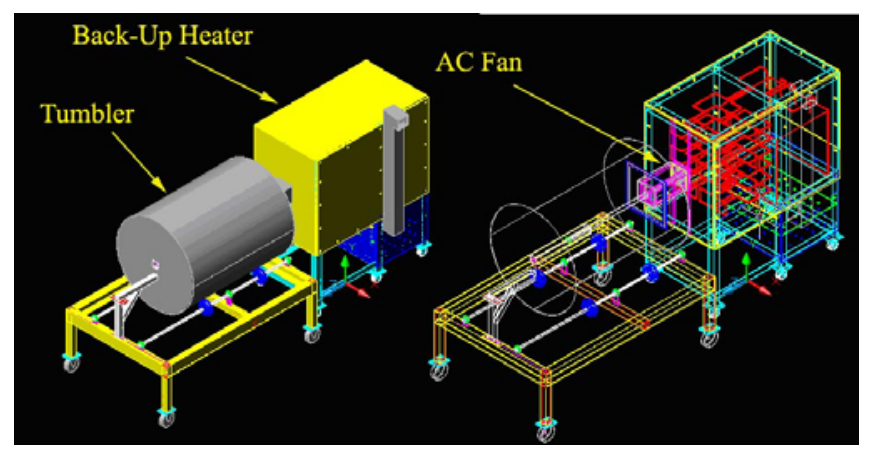

Figure 3. Selected back-up heater along with pepper solar tumble dryer

\section{Testing Procedure}

After detailed design of the biomass back-up heater, a prototype of the heater was fabricated and incorporated with the existing tumble dryer. Testing of the back-up heater was subsequently carried out in order to check its efficiency in terms of supplying e heat to the tumble dryer for pepper drying.

\section{A. Initial Tests}

In order to select one biomass fuel for the back-up heater, both charcoal and wood was burnt inside the combustion chamber. Heat produced was utilized to boil water. The temperature of the boiled water was measured using a thermohygrometer. 


\section{B. Trial Tests}

After selecting the biomass fuel from the initial tests, trial tests of the prototype heater were carried out in order to check its efficiency in terms of producing heat for the tumble dryer. The design configuration of the back-up heater was changed during these tests in order to obtain highest heat producing efficiency. The temperature and humidity were measured using the same thermohygrometer.

\section{C.Actual Tests}

After the trial tests, the back-up heater was incorporated with the tumble dryer. No load trial (without putting pepper insider the dryer) was initially carried out to check whether the back-up heater was efficient enough in supplying heat to the tumble dryer. The tumbler was rotated at a speed of $1 / 2$ rpm during these tests. Again the temperature and humidity were measured using a thermohygrometer.

Finally fresh pepper was put inside the dryer and dried for two days. $1 \mathrm{Kg}$ pepper was used as the starting material. Tests were conducted from 10 am until 12 am (midnight). From 10 am to $5 \mathrm{pm}$, the dryer was rotated at a speed of 3 rpm, while from 5 pm to 12 am the speed was set at $1 / 4 \mathrm{rpm}$. During the day solar heat was utilized to dry pepper, while at night the designed biomass back-up heater was used to supply heat to the dryer. The Pepper was also dried in open air in order compare the solar dryer drying rate with that of the open drying.

\section{RESULTS}

\section{A. Results of Initial Tests}

Results of initial tests using both charcoal and wood as biomass fuel is shown in 'table 1'. According to 'table 1', wood fuel was able to heat water up to a temperature of $100^{\circ} \mathrm{C}$, while the highest water temperature obtained using charcoal was only $86.6^{\circ} \mathrm{C}$. Thus for the rest of the work, wood was used as biomass fuel for the back-up heater.

Table 1. Temperature of water produced using both wood and charcoal fuel

\begin{tabular}{|c|c|c|c|c|}
\hline & \multicolumn{2}{|c|}{ Wood } & \multicolumn{2}{|c|}{ Charcoal } \\
\hline $\begin{array}{c}\text { Duration } \\
\text { (Hour) }\end{array}$ & $\begin{array}{c}\text { Ambient } \\
\text { Air } \\
\text { Temp. }\left({ }^{0} \mathrm{C}\right)\end{array}$ & $\begin{array}{l}\text { Water } \\
\text { Temp. } \\
\left({ }^{0} \mathrm{C}\right)\end{array}$ & $\begin{array}{c}\text { Ambient } \\
\text { Air } \\
\text { Temp. }\left({ }^{0} \mathrm{C}\right)\end{array}$ & $\begin{array}{l}\text { Water } \\
\text { Temp. } \\
\left({ }^{0} \mathrm{C}\right)\end{array}$ \\
\hline 0 & 25.5 & 25.5 & 25.3 & 24.0 \\
\hline 1 & 25.5 & 58.3 & 25.4 & 30.1 \\
\hline 2 & 25.4 & 93.2 & 25.2 & 50.5 \\
\hline 3 & 25.1 & 100.0 & 25.3 & 86.5 \\
\hline 4 & 25.1 & 100.0 & 24.9 & 86.6 \\
\hline
\end{tabular}

\section{B. Results of Trial Tests}

The main function of the biomass back-up heater was to provide dry hot air into the tumble dryer for drying the wet pepper. Previous research carried out by in UNIMAS [4] stated that the suitable temperature for drying pepper is around $40^{\circ} \mathrm{C}$ and the humidity is around $50 \% \mathrm{RH}$. Thus the requirement form the prototype was to produce low humidity and a temperature much higher than $40^{\circ} \mathrm{C}$. Results of initial tests are shown in 'table 2'. The temperature produced from the heater outlet was only $46.5^{\circ} \mathrm{C}$ and the heater outlet humidity was $87.3 \%$. In order to increase the temperature and decrease humidity, slides were dismantled from the heater. It provided shorter pathway of the air, thus increasing heater outlet temperature $\left(69.8^{\circ} \mathrm{C}\right)$ and decreasing humidity $(15.9 \%)$, as shown in 'table 3 '.

Table 2. Results of initial tests

\begin{tabular}{|c|c|c|c|c|}
\hline & \multicolumn{2}{|c|}{ Heater Outlet } & \multicolumn{2}{c|}{ Ambient Air } \\
\hline $\begin{array}{c}\text { Duration } \\
(\text { Min })\end{array}$ & $\begin{array}{c}\text { Temp. } \\
\left({ }^{\circ} \mathrm{C}\right)\end{array}$ & $\begin{array}{c}\text { Humidity } \\
(\% \mathrm{RH})\end{array}$ & $\begin{array}{c}\text { Temp. } \\
\left({ }^{\circ} \mathrm{C}\right)\end{array}$ & $\begin{array}{c}\text { Humidity } \\
(\% \mathrm{RH})\end{array}$ \\
\hline 0 & 28.6 & 96.0 & 27.1 & 94.0 \\
\hline 30 & 32.4 & 89.0 & 28.2 & 90.0 \\
\hline 60 & 39.7 & 75.7 & 28.5 & 87.0 \\
\hline 90 & 42.3 & 72.4 & 28.1 & 89.3 \\
\hline 120 & 45.0 & 70.9 & 27.4 & 88.4 \\
\hline 150 & 46.0 & 71.3 & 27.7 & 87.3 \\
\hline 180 & 46.6 & 68.4 & 27.2 & 90.5 \\
\hline 210 & 46.5 & 70.1 & 27.3 & 92.1 \\
\hline
\end{tabular}

Table 3. Improvement in heater outlet temperature and humidity

\begin{tabular}{|c|c|c|c|c|}
\hline & \multicolumn{2}{|c|}{ Heater Outlet } & \multicolumn{2}{c|}{ Ambient Air } \\
\hline $\begin{array}{c}\text { Duration } \\
(\text { Min })\end{array}$ & $\begin{array}{c}\text { Temp. } \\
\left({ }^{\circ} \mathrm{C}\right)\end{array}$ & $\begin{array}{c}\text { Humidity } \\
(\% \mathrm{RH})\end{array}$ & $\begin{array}{c}\text { Temp. } \\
\left({ }^{\circ} \mathrm{C}\right)\end{array}$ & $\begin{array}{c}\text { Humidity } \\
(\% \mathrm{RH})\end{array}$ \\
\hline 0 & 27.2 & 94.2 & 26.8 & 90.5 \\
\hline 30 & 32.1 & 73.2 & 28.0 & 85.3 \\
\hline 60 & 42.6 & 49.2 & 30.0 & 80.6 \\
\hline 90 & 50.2 & 30.5 & 29.2 & 83.2 \\
\hline 120 & 63.1 & 21.1 & 29.0 & 85.0 \\
\hline 150 & 68.1 & 19.5 & 28.4 & 87.1 \\
\hline 180 & 70.2 & 16.2 & 28.6 & 88.0 \\
\hline 210 & 68.2 & 15.8 & 29.4 & 84.3 \\
\hline 240 & 69.8 & 15.9 & 30.1 & 80.6 \\
\hline
\end{tabular}

\section{Results of Actual Tests}

After incorporating the back-up heater with the existing tumble dryer, testing out carried at night without putting pepper inside the tumbler in order to check whether the back-up heater efficient enough in supplying heat to the tumble dryer. Results are shown in 'table 4'. According to 'table 4', the temperature obtained at the dryer outlet was $40.6^{\circ} \mathrm{C}$, which was sufficient. The relative humidity at the same place was $42 \%$.

Table 4. Results of actual tests without using any pepper

\begin{tabular}{|c|c|c|c|c|}
\hline & \multicolumn{2}{|c|}{ Heater Outlet } & \multicolumn{2}{c|}{ Ambient Air } \\
\hline $\begin{array}{c}\text { Duration } \\
(\text { Min })\end{array}$ & $\begin{array}{c}\text { Temp. } \\
\left({ }^{\circ} \mathrm{C}\right)\end{array}$ & $\begin{array}{c}\text { Humidity } \\
(\% \mathrm{RH})\end{array}$ & $\begin{array}{c}\text { Temp. } \\
\left({ }^{\circ} \mathrm{C}\right)\end{array}$ & $\begin{array}{c}\text { Humidity } \\
(\% \mathrm{RH})\end{array}$ \\
\hline 0 & 60.1 & 15.9 & 28.7 & 76.3 \\
\hline 1 & 59.8 & 15.5 & 36.7 & 61.3 \\
\hline 2 & 59.5 & 16.0 & 38.8 & 53.2 \\
\hline 3 & 61.2 & 14.9 & 39.9 & 47.2 \\
\hline 4 & 62.4 & 15.4 & 41.2 & 46.3 \\
\hline 5 & 60.8 & 15.1 & 40.6 & 42.0 \\
\hline
\end{tabular}

Finally fresh peppers were put inside the dryer and drying tests were conducted for 2 days. Pepper weight was measured after each hour of testing. Peppers were also dried in open air to compare the rate of tumbler drying with open drying. Results are shown in 'figure 4'. According to 'figure 4', tumble drying process was more efficient in drying peppers compared to open drying. Open drying was stopped 
at $5 \mathrm{pm}$, at that time the weight of the pepper was $523.8 \mathrm{gm}$. Tumbler drying was continued until midnight using the back-up heater. The weight measured at midnight was 311.1 gm. Thus $47.1 \%$ of water had been dehydrated during open drying method, while $68.6 \%$ of water had been dehydrated during solar tumble drying method assisted by biomass back-up heater. On the second day, the weight of the pepper was reduced to 288.2 gm after only 3 hours of tumbler drying and the weight remained same for the next 2 hours. So the self weight of the pepper was $288.2 \mathrm{gm}$. At this point, the testing was stopped.

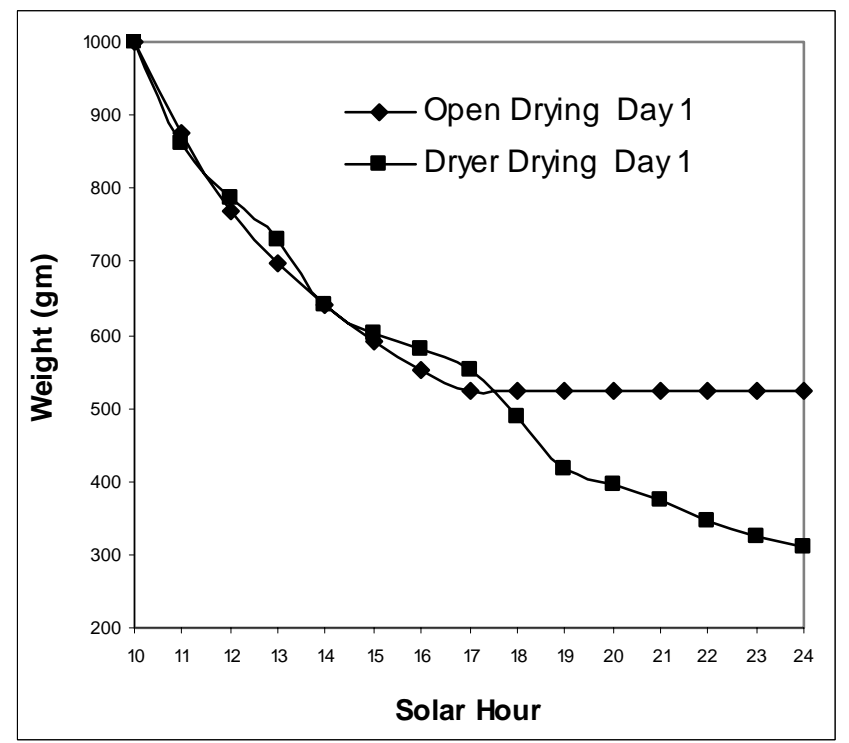

Figure 4. Results of final tests

Normally open sun drying method takes around 4 days to dry fresh pepper berries. Research carried out in UNIMAS [4] showed that tumble pepper dryer itself took 3 days to dry peppers. However using the designed biomass back-up heater, fresh pepper berries were dried only in 2 days. Thus the back-up heater shortened the drying time compared to open drying or tumble dryer drying.

\section{CONCLUSION}

Current study describes design enhancement and testing of a pepper solar tumble dryer using a biomass back-up heater. The methodology involved the selection of a backup heater design, detail design of the heater, fabrication and finally testing of the resultant heater alongside the existing tumble dryer. In order to achieve the goals, variables such as basic material properties, type of biomass fuel and designs were considered. Two designs for the back-up heater were chosen initially. Finally one design was selected for fabrication. The selected design of back-up heater consisted of three sections; combustion chamber, water tank and heat exchanger path way. After fabrication, the prototype was tested to determine its efficiency in terms of supplying heat to the dryer for drying pepper berries. Results suggested that the back-up heater in conjunction with the tumble dryer was able to dry peppers at a faster rate compared to open drying or tumble drying itself. The drying process was continued at night using the designed heater. Thus the new biomass back-up heater can be used along with the tumble dryer at any weather condition in order to dry fresh pepper berries.

\section{REFERENCES}

[1] Malaysian Pepper Marketing Board Homepage, http://61.6.32.133/sarawakpepper/, Date of Access: 03/09/2004.

[2] L. Y. Han, Design and Fabrication of a Pepper Solar Tumble Dryer, Bachelor Thesis, Universiti Malaysia Sarawak, Sarawak, Malaysia, 2004.

[3] B. Bena and R.J. Fuller, Natural Convection Solar Dryer with Biomass Back-Up Heater, Journal of Solar Energy, vol.72, no. 1, pp. 75-83, 2002.

[4] O. C. Tsien, Control and Testing of a Pepper Solar Tumble Dryer, Bachelor Thesis, Universiti Malaysia Sarawak, Sarawak, Malaysia, 2004. 


\title{
GA-Solar: A Simulation of Genetic Algorithm based Solar Tracking System for Photovoltaic Panels
}

\author{
Amirullah M. Noor, Adi Razlan A. Rahman, Syamsiah Mashohor and Khairulmizam Samsudin
}

\begin{abstract}
The maximum power supplied by a Photovoltaic (PV) panels system change over time depending on environmental factors such as the solar irradiation and the temperature of these panels. The average solar energy harvested by the conventional solar panels during the course of the day, is not always maximized. This is due to the static placement of the panel which limits their area of exposure to the sun. In practice, there are three possible approaches for maximizing the solar power extraction in medium and large scale $P V$ systems are sun tracking, maximum power point (MPP) tracking or combination of both. In this paper, a Genetic Algorithm (GA) has been proposed utilizing sun tracking approach to maximize the performance of $\mathrm{PV}$ panels. Literature suggested that the PV panels could achieve maximum performance if the panels are always perpendicular to the sun position. This work evaluates the best combination of GA parameters to optimize a solar tracking system for PV panels in terms of azimuth angle and tilt angle. Results demonstrated the ability of the proposed GA system to search for optimal panel positions in term of consistency and convergence properties.
\end{abstract}

Keywords: genetic algorithm, solar tracking, PV panels

\section{INTRODUCTION}

The photovoltaic (PV) technology is expanding, thanks to growing demand for renewable energy mainly due to the depletion of fossil fuel. Solar panel is the fundamental energy conversion component of photovoltaic (PV) systems [1]. PV technology offers safe and clean energy sources [2]. The abundance of solar energy throughout the whole year in Malaysia due to the geographic location near the Equator line provides strong reason for the implementation of an efficient PV energy system. Studies show that solar panels constitute a large portion (57\%) of the total cost to install PV energy system [3]. As the solar panels are relatively expensive, much research work has been conducted to improve the utilization of solar energy. Physically, the

This work was supported in part by UPM and MOSTI (E-Science) research grant.

Amirullah M. Noor and Adi Razlan A. Rahman are undergraduates in Dept. of Computer and Communication Systems Engineering, UPM, 43400 Serdang, Selangor, Malaysia.

Syamsiah Mashohor and Khairulmizam Samsudin are with Dept. of Computer and Communication Systems Engineering, UPM, 43400 Serdang, Selangor, Malaysia. Tel: (+60)389464348, Fax:(+60)386567127. (email:[syamsiah,kmbs]@eng.upm.edu.my). power supplied by the panels depends on many extrinsic factors, such as insolation levels (incident of solar radiation) , temperature and load condition.

To ensure the economical viability of this energy system, the development of an adaptive system that always obtains the maximum power from these solar panels is essential. Amicroprocessor based automatic sun tracking system using a number of Light Detecting Resistors (LDRs) is proposed in $[4,5]$. The principle idea is to sense the sun light using LDR and the differential signal representing angular error of the panel is used to rotate the panel in such a way that the angular error is minimized. The usage of LDR is to ensure that the solar panel is always aligned towards the direction of the sun. However, a cloudy day and shading condition around the PV panels may reduce the intensity of light and effect the performance of the tracking system. Proper placement of LDR in the system and the correct quantity of LDR are very important in order to produce correct position of solar panel in all time.

Researcher in [6] has presented the design and implementation of a computer-controlled dual-axis sun tracking system to obtain high precision positioning of the cell. The control of dual axis tracking system is not an easy task due to nonlinear dynamics and unavailability of the model parameters. A PC-based fuzzy logic control algorithm utilizing the knowledge of the system behavior is designed in order to achieve the control objectives. Implementation of fuzzy logic reasoning is limited to the predetermined conditions which may not satisfy all conditions in the PV panel environment. Therefore, sun tracking only occurs when these conditions are met, which will not necessarily maximize the solar energy absorption.

In this work, GA based solar tracking is proposed to overcome the limitation of current method. GA is an evolutionary algorithm which can adapt itself to the different conditions, not only suitable for solar farm but also applicable for harvesting energy for satellite, solar powered automotive and robotics.

This paper will discuss the development of GA-based solar tracking system by orienting PV panel on two axis (azimuth and tilt angles) to obtain maximum power. In the following section, the methodology of this system is discussed which comprise of system description, GA architecture and solar tracking simulator. Then, the results 
of the simulation are discussed and finally, the conclusion is presented.

\section{Methodology}

\section{A. System Description}

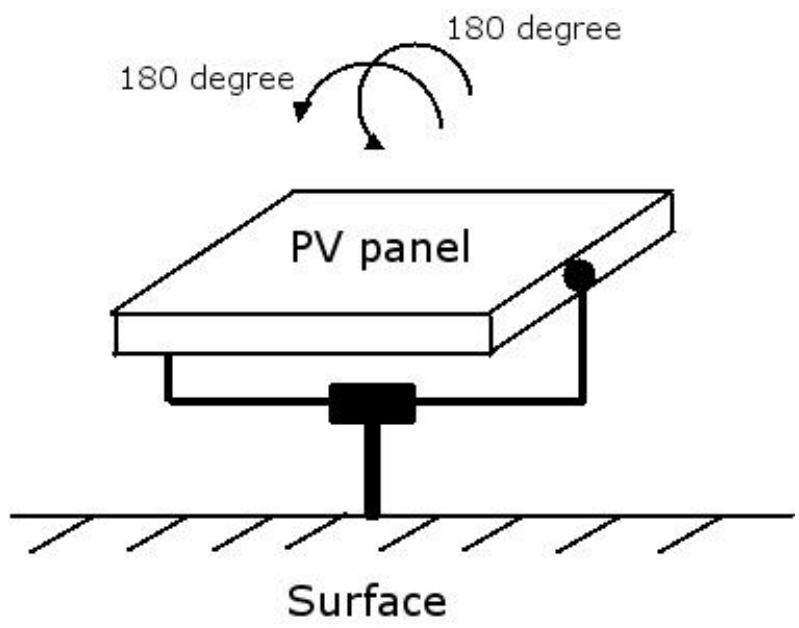

Figure 1. The geometry of the PV panel for GA-Solar system

This paper is a preliminary work of the implementation of sun tracking using our specially-tailored GA for maximizing performance of PV systems. The geometry of the PV panel that will be explored by the GA technique is shown in figure 1. The azimuth and tilt angle can varies from 0 to 180 degrees which can fulfill any location condition either the PV panel is installed on a high building or on the ground.

\section{B. GA Architecture}

In this work, the GA is used to track the best tilt and azimuth angle of the PV panel that generate the maximum power from the sun. The details of GA architecture that is implemented in this simulation is discussed in [7].

For this work, real integer coding has been implemented considering the position of the sun in the sky. The tilt and azimuth angle are both ranged from 0 to 180 degrees. Every individual represents a combination of both position parameters which describes position of the sun. If both tilt and azimuth angle is 90 degrees, the PV panel is normal to the ground surface. The domain of search for these parameters is large $(180 \times 180=32400)$ which is suitable for the GA to explore.

The fitness function in this work is evaluated from the power value measured from the PV panel according to the selected tilt and azimuth angle. There should be only one maximum power value at one time assuming that the panel is perpendicular to the sun, while the minimum value is $0 \mathrm{~W}$.

Iteratively, the whole population for the next generation is formed by selected individuals from the parents and offsprings in current generation. These individuals are ranked based on their fitness performance and only the top fittest individual are selected for a new population. The population will perform

GA activities such as selection, mutation and crossover in every generation will happen until the termination requirements are fulfilled. The GA search will be terminated if an ideal individual which gives the maximum power value has been found or maximum generation has been reached.

Table 1. The values of GA parameter involved in exhaustive search

\begin{tabular}{|l|l|}
\hline GA Parameter & Values \\
\hline Population size & $30,50,100,200$ \\
\hline Maximum generation & $50,100,150,200,250$ \\
\hline Probability of crossover & 0.1 to 1.0 \\
\hline Probability of mutation & 0.001 to 0.01 \\
\hline
\end{tabular}

\section{Solar Tracking Simulator}

In this work, to facilitate the development of the GASolar system a solar tracking simulator has been developed. The simulator assumes that the orientation search is done during the first time calibration, right after a PV system has been installed. Subsequent search would be much faster as the GA would start searching from the best individual. The initial orientation of the panel are generated randomly. The GA is used to search for the best tilt and azimuth angle that gives the maximum power value $(10 \mathrm{~W})$ that has been randomly generated into a Look-Up Table (LUT). As there is only one source of light which is from the sun, we use a bi-variate normal distribution function to initialize the LUT. The position parameters that have the maximum power value are randomly picked and changed every time a new simulation starts.

Figure 2 illustrate four generated LUTs to simulate the power obtained by a PV system oriented to a specific tilt and azimuth angle ([tilt,azimuth]). From figure 2(a), a maximum power from a PV system can be obtained by orienting the panel to $[90,90]$ position, while orienting the panel to $[0,0]$, $[180,90]$ and $[120,80]$ positions would obtain the maximum power from LUT of figure 2(b), figure 2(c) and figure 2(d) respectively.

\section{D.Implementation Setup}

To maximize the capability of GA to position the PV panel to obtain the maximum power, the values of GA parameters must be chosen carefully. Hence, an exhaustive search has been conducted on all combination of GA parameters as listed in table I to find the best combination of GA parameters. The exhaustive search is possible due to the availability of the solar tracking simulator which is able to simulate the 2-axis positioning of PV panel very fast. The total combination of GA parameters are 2000 combinations $(4 \times 10 \times 10 \times 5)$ and each combination is repeated 10 times for consistency evaluation. The effect of every GA parameter in this GA-Solar also can be assessed based on this simulation.

\section{Results AND Discussions}

The goal of the GA-Solar is to obtain the maximum power at all the time and in any condition. Based on that, the selected values of GA parameters that will be used in the future simulation or hardware experiments in realenvironment testing must always give the maximum power to optimize the utilization of the solar energy. The selected GA parameters should also require less generation to 
produce the findings as each measurement of fitness function requires positioning of the 2-axis of the PV panel. To evaluate each combination of the GA parameters, the consistency and convergence properties of GA-Solar to align the PV panel to obtain the maximum power is considered. Consistency is defined as the frequency of finding the maximum power in all 10 sets, while the convergence is defined as the least number of generation required to find the maximum power in all 10 sets.

The goal of the GA-Solar is to obtain the maximum power at all the time and in any condition. Based on that, the selected values of GA parameters that will be used in the future simulation or hardware experiments in realenvironment testing must always give the maximum power to optimize the utilization of the solar energy. The selected GA parameters should also require less generation to produce the findings as each measurement of fitness function requires positioning of the 2-axis of the PV panel. To evaluate each combination of the GA parameters, the consistency and convergence properties of GA-Solar to align the PV panel to obtain the maximum power is considered. Consistency is defined as the frequency of finding the maximum power in all 10 sets, while the convergence is defined as the least number of generation required to find the maximum power in all 10 sets.

The consistency in finding the maximum power is the most important feature of the GA-Solar due to adaptability to different conditions and PV panel designs. In the exhaustive search simulation, the best parameters give consistent best results in finding the maximum power point from the Look-Up-Table for power reading from PV panels that are exposed to the sun. As shown in figure 3,69\% of the simulation results give good consistency in finding the maximum power, which is $10 \mathrm{~W}$ in all 10 sets. With regard to this findings, the consistency of finding maximum power in all 10 sets and convergence (sum of generation) properties of these combination of GA parameters are analyzed in order to choose the best GA parameter values. The GA parameters are analyzed in sequence of population, maximum generation, probability of crossover and probability of mutation.

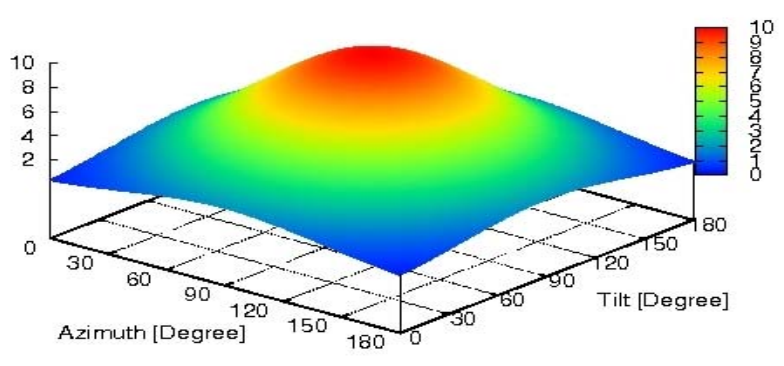

(a)

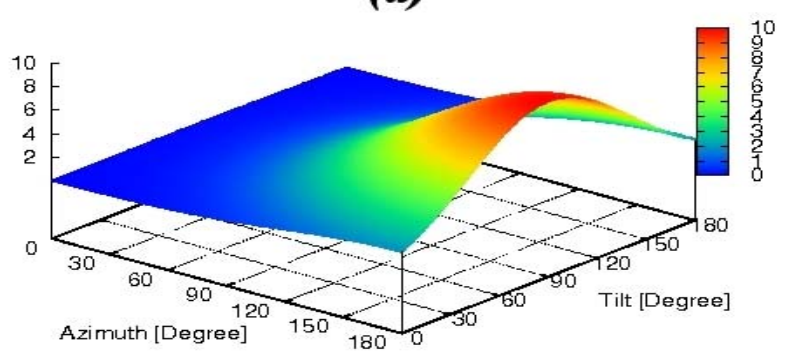

(c)

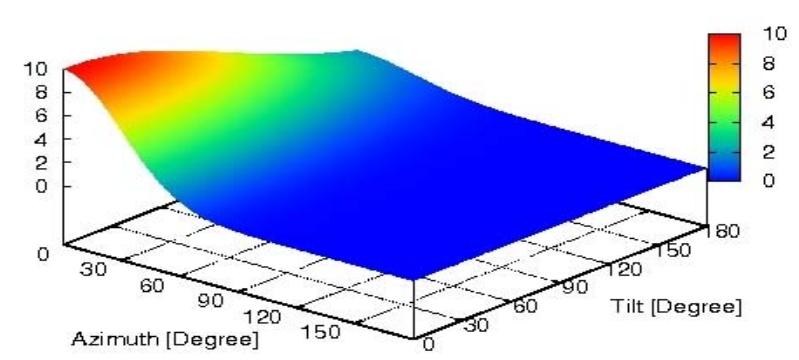

(b)

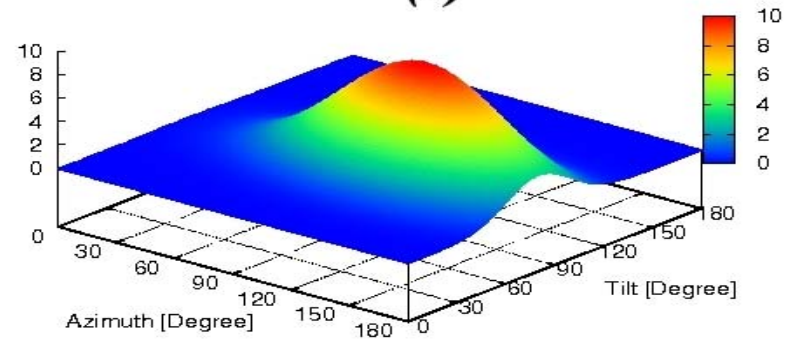

(d)

Figure 2: Generated Look-Up Table for solar tracking simulator using bi-variate distribution function. 


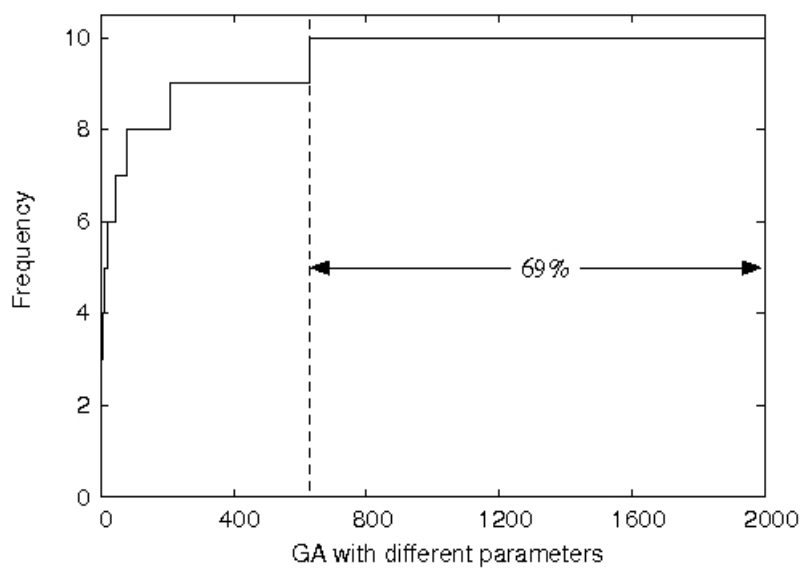

Figure 3. Consistency of GA-Solar in finding the maximum power

The population size have significant impact on computing memory footprint. Table II summarized the consistency and convergence for different population size. Based on the table, the population size of 100 gives the most consistent results in finding the maximum power in every set of simulation and the results are found in the least convergence value.

The maximum generation determines the limit of time that GA will spend to search for the best solution. There is no significant difference of consistency and convergence properties of maximum generation parameter for the population size of 100 . However, a maximum generation of 50 is chosen since the population size of 100 is already large enough to give enough time for the GA to find the solution. It would also ensure that the GA would be terminated if the duration taken to obtain the maximum power is too long. Detail analysis on the probability of crossover shows that the probability of 0.7 has the most consistency and the least convergence values compared to other probability values. With this value of probability, the crossover operation will happen moderately frequent to produce better offsprings for the next generation. For the probability of mutation, the value of 0.001 is selected based on the least convergence required to find the same consistency as the other values. The value of 0.001 gives enough occurrence of mutation for the generation to evolve but excessive probability of mutation may increase the time to compute.

\section{CONCLUSION}

This paper described the simulation of GA-based solar tracking system for PV panels. The solar tracking simulator developed is an important tool to simulate and evaluate the performance of the GA-solar system. The best value for GA parameters have been selected according to the simulation results. The most significant difference of frequency and convergence occur in different population size, followed by different probability of crossover. For various values of probability of mutation, only the convergence yields the significant difference. From the observation, there is no significant difference in consistency neither the convergence properties when the maximum generation is varied. The GA parameter that will be used in future simulation and hardware implementation are population size of 100 , maximum generation of 50 and probability of crossover and mutation are 0.7 and 0.001 respectively. The simulation findings also show that the GA-Solar can be implemented to maximize the power harvested by the PV panel systems.

Future work will concentrate on the hardware implementation to prove that the GA-Solar is capable of maximizing the power obtained by the PV panel in realenvironment testing and posses the ability to adapt to different condition and PV system design.

\section{REFERENCES}

[1] H. S.-H. Chung, K. K. Tse, S. Y. R. Hui, C. M. Mok, and M. T. . Ho, "A novel maximum power point tracking technique for solar panels using a SEPIC or Cuk converter," IEEE Transactions on power electronics, vol. 18, no. 3, pp. 717-724, 2003.

[2] T. H. et al, "Evaluation of neural network based real time maximum power- tracking controller goes PV system," IEEE Transactions on Energy Conversion, vol. 10, no. 3, 1995.

[3] J. Enslin, "Renewable energy as an economic energy source for remote areas," in Renewable Energy, vol. 1, pp. 243-248, 1991.

[4] B. Koyuncu and K. Balasubramanian, "A microprocessor controlled automatic sun tracker," IEEE Transactions on Consumer Electronics, vol. 37, no. 4, pp. 913-917, 1991.

[5] O. Bingol, A. Altinta, and Y. Oner, "Microcontroller based solartracking system and its implementation," Journal of Engineering Sciences, vol. 12, pp. 243-248, 2006.

[6] H. A. Yousef, "Design and implementation of a fuzzy logic computercontrolled sun tracking system," in Proceedings of the IEEE International Symposium on Industrial Electronics, ISIE'99, vol. 3, pp. 1030-1034, 1999.

[7] S. Mashohor, J. R. Evans, and T. Arslan, "Image registration of printed circuit boards using hybrid genetic algorithm," in IEEE Congress on Evolutionary Computation, 2006. 


\title{
Heat Transfer Study of a Progressive Freeze Concentration System for the Treatment of a Simulated Wastewater with Various Type of Insulator
}

\author{
M. Jusoh, R. Mohd. Yunus, M.A. Abu Hassan, and M.I. Abdul Razif
}

\begin{abstract}
Progressive freeze concentration is an operation of separating a single and large ice crystal produced in the solution. This process is carried out at temperature below than $0^{\circ} \mathrm{C}$. With this kind of condition, an effective insulation system is very essential. In this research, three different types of insulator which are polyurethane foams, ethylene propylene rubber and aluminium foils are used in order to minimise the heat transfer between the ambient and the solution in the apparatus. The purpose of this research is to find out the best insulating material for the process and to test the temperature difference by adding a newly designed crystallization chamber to the apparatus. Results are evaluated and observed based on the thermal conductivities for each of the insulators and the quantity of heat flux (equation of heat transfer through one thickness of cylindrical insulation) $Q$ in $\mathrm{W} / \mathrm{m}^{2}$ generated. A new type of progressive freeze concentration experimental apparatus will be used and an analysis regarding the effect of different types of insulation will be carried out. From the experiments conducted, it was proven that polyurethane foam is the best insulating material compared to the other two.
\end{abstract}

Keywords: freeze concentration, progressive freeze concentration, heat transfer, insulation system

\section{INTRODUCTION}

Industrial wastewater can contain various types of pollutant ranging from chemicals to suspended matters and the type of treatment depends on the type of the pollutants. Regardless of the type of treatment that will be applied to the wastewater, it is such an advantage if the volume of the wastewater could be reduced extensively. Reduced volume of wastewater will result in a reduction in operation cost in terms of the utility. Hazardous wastewater is frequently treated by incineration. But to incinerate an aqueous solution, with a solid content of less than $10 \%$, requires tremendous power to 'burn' the water and maintain the high temperature necessary to destroy the hazardous compound

M. Jusoh, M.A. Abu Hassan, and M.I. Abdul Razif are with the Department of Chemical Engineering, Faculty of Chemical \& Natural Resources Engineering, Universiti Teknologi Malaysia, 81310 Skudai, Johor (e-mail: mazura@fkkksa.utm.my).

R. Mohd. Yunus is with the Faculty of Chemical \& Natural Resources Engineering, Universiti Malaysia Pahang, MEC City, 26300 Gambang, Kuantan.
[1]. In addition, the combustion gas produced contributes to the emissions from the process and can rapidly exceed local limits.

In concentration of a solution, there are three methods available: reverse osmosis, evaporation and freeze concentration. Every process has their specific energy and among those three, the energy cost is the highest for evaporation $(2.26 \mathrm{~kJ} / \mathrm{g}$-water $)$, intermediate for freeze concentration $(0.33 \mathrm{~kJ} / \mathrm{g}$-water $)$, and the lowest for reverse osmosis because phase transition is not needed [2]. Evaporation is the simplest method which is worth the energy consumed, but it is very dangerous when hazardous volatile organic compounds (VOCs) are involved [3]. Despite of the low energy consumed in reverse osmosis, it is not a favourable method of concentration because clogging can easily occur, and the high cost involved for the osmotic pressure.

Freeze concentration is an operation to concentrate an aqueous solution by separating ice crystals produced in the solution [4]. There are two methods available for freeze concentration, conventional suspension freeze concentration (SFC) and progressive freeze concentration (PFC). SFC is characterized by the generation of a size distribution of crystal growing isothermally in the mother liquor. However, in this conventional method, the size of ice crystal is still limited [5]. The small ice crystals formed has to be transferred to a ripening vessel to be enlarged, then to a washing column and separated from the mother solution after washing with water [6]. These steps: ice nucleation, ice crystal growth and ice crystal separation make the whole system very expensive, which has made it unfavourable.

In compensating the disadvantages of SFC, a totally different concept of crystallization, PFC is being introduced. In this method, a large single ice crystal instead of small ice crystals in the suspension crystallization method is formed. As only a single crystal is formed, its separation from the mother liquor is much easier to be handled and at a lower cost. However, despite of the easier separation, the productivity of progressive freeze concentration is found to be lower than the conventional suspension freeze concentration.

To improve the productivity of PFC, the operation has to be designed so that the heat transferred into the equipment is 
as minimum possible. It is very important to maintain the system temperature below the freezing point of water. In this research, the effect of different insulator has been investigated on the heat transferred within the system.

\section{Methodology}

The experiment was executed based on the type of system insulation and feed temperature. The investigation will be done through varying the insulation material with and without the crystallization chamber installed.

\section{A. Materials}

A $1.0 \mathrm{wt} \%$ of glucose solutions were used in this study. Glucose solutions are used to replace and at the same time represent the actual wastewater. It is very common that glucose be used in assessing the performance of a wastewater treatment system.

To prepare a $1.0 \mathrm{wt} \%$ of glucose solution, 10 grams of glucose was added to 1 litre of distilled water and stirred for homogeneity.

\section{B. Equipments}

Figure 1 shows the flow diagram of the newly designed progressive freeze concentration experimental apparatus. The apparatus were constructed mainly by pipe lines, valves, a crystallization chamber, a pump, a feed tank, a flow meter and an insulation system.

In this study, three different types of insulator were used which are polyurethane foams, ethylene propylene rubber and aluminium foils. These insulators at first came at bulk size, so alterations have been done to fit them to the rig. All parts of the rig are covered with insulators except a few portions that are not very practical do so, for example the surface area of the valves. Only the bottom part is covered with the insulators, but not the stem and the upper part because if we do so, the insulator will disturb the motion of the valve stem.

To make sure that the insulators are fit to the rig, tapes or ropes are used to attach between the insulators and the pipe lines. It was always reminded that the attachment part was not supposed to change the original form (especially thickness) of the insulators because this will somehow affect the effectiveness of insulation system itself.

\section{Method of Experiment}

The glucose solution was first poured into the feed tank. After the volume of glucose solution was enough for the whole process, valve V2 are opened to circulate the glucose in the system. Next, the peristaltic pump was turned on and set at the speed of $100 \mathrm{rpm}$ and at the same time the stopwatch was started. Valve V2 was closed when there is no more inlet of glucose solution into the system (when there is no more air bubbles coming out from the bottom of the feed tank).

For every 5 minutes, $20 \mathrm{~mL}$ of the glucose solution was drained out of the system through valve V6 to record the temperature. It was made sure that the same volume of glucose solution was drained out each time. The experiment stopped after 30 minutes.

The experiment was repeated with different insulation system and feed temperature.

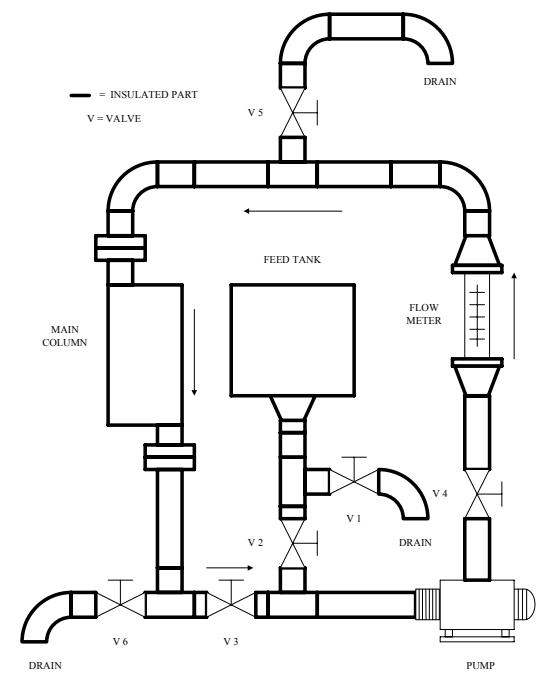

Figure 1. The flow diagram of the PFC system

\section{Determination of Q-value}

The heat transfer between the ambient and the solution in the system can be determined by quantity of heat flux (heat transfer through one thickness of cylindrical insulation):

$$
Q=\frac{t_{1}-t_{2}}{\frac{r_{2} \log _{e} \frac{r_{2}}{r_{1}}}{k}}
$$

where $r_{1}$ is the inner radius of a single layer of cylindrical insulation and $r_{2}$ is the outer radius of a single layer of cylindrical insulation while $t_{2}$ is the outer surface temperature and $t_{1}$ is the inner surface solution. The unit of this equation will be in $\mathrm{W} / \mathrm{m}^{2}$.

The temperature of the glucose solution taken from the system at every 5 minutes was recorded as $t_{1}$. The ambient temperature recorded during the experiment was taken as $t_{2}$. The values of the inner radius of a single layer of cylindrical insulation, $r_{1}$ and the outer radius of a single layer of cylindrical insulation, $\mathrm{r}_{2}$ were recorded earlier.

\section{RESUlTS AND DISCUSSION}

\section{A.Heat Transfer Through The Variations of Insulation System}

The feed temperature is near the freezing point of water, which is at $2^{\circ} \mathrm{C}$. The solution was cooled first in a freezer and in the end almost consists of two phases, mixture of liquid and solution solid in form of ice. The feed tank was design to allow only the inlet of liquid and preventing any solid form to enter the system by placing a permanent metal net at the bottom of the tank.

For the feed temperature at $2{ }^{\circ} \mathrm{C}$ and without the addition of crystallization chamber, polyurethane foams shows the lowest increment of temperature in the solution followed by aluminium foils and ethylene propylene rubbers as been showed in the Table 1. 
Table 1. Average temperatures of solution via different types of insulator at feed $2^{\circ} \mathrm{C}$ without crystallization chamber

\begin{tabular}{|l|l|l|l|}
\hline \multirow{2}{*}{$\begin{array}{l}\text { Time } \\
(\mathrm{min})\end{array}$} & $\begin{array}{l}\text { Average temperatures of the solution with } \\
\text { insulation system of }\end{array}$ \\
\cline { 2 - 4 } & $\begin{array}{l}\text { Polyurethane } \\
\text { foams } \\
\left({ }^{\circ} \mathrm{C}\right)\end{array}$ & $\begin{array}{l}\text { Aluminium } \\
\text { foils } \\
\left({ }^{\circ} \mathrm{C}\right)\end{array}$ & $\begin{array}{l}\text { Ethylene } \\
\text { propylene } \\
\text { rubber } \\
\left({ }^{\circ} \mathrm{C}\right)\end{array}$ \\
\hline 0 & 2.0 & 2.0 & 2.0 \\
\hline 5 & 17.0 & 18.0 & 19.0 \\
\hline 10 & 20.0 & 20.5 & 21.0 \\
\hline 15 & 21.0 & 22.0 & 22.5 \\
\hline 20 & 23.0 & 23.0 & 23.5 \\
\hline 25 & 23.5 & 24.0 & 25.0 \\
\hline 30 & 24.0 & 25.0 & 26.0 \\
\hline
\end{tabular}

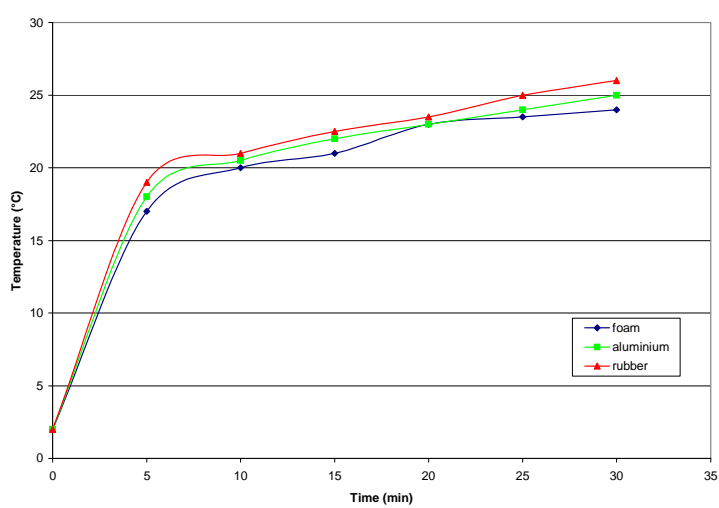

Figure 2. Average temperatures profile at feed $2^{\circ} \mathrm{C}$ with different types of insulator without crystallization chamber

Figure 2 shows the verage temperatures profile at feed $2{ }^{\circ} \mathrm{C}$ with different types of insulator without crystallization chamber. The experimental results followed the theory of thermal conductivity of the insulation materials. Thermal conductivity are the amount of heat transferred through a unit area of a material in a unit time, through a unit thickness, with a unit of temperature difference (energy.length / time.area.temperature) between the surfaces of two opposites sides [7]. Unit of thermal conductivity is $\mathrm{W} /(\mathrm{m} . \mathrm{K})$ in SI unit. This shows that, the lower the value of thermal conductivity for one material, the better it is in conserving the heat by reducing the energy (heat) flow to the other compartment which has a temperature difference.

Thermal conductivities for polyurethane foam is 0.03 $\mathrm{W} /(\mathrm{m} . \mathrm{K})$, aluminium foils $0.06577 \mathrm{~W} /(\mathrm{m} . \mathrm{K})$ and ethylene propylene rubbers $0.18865 \mathrm{~W} /(\mathrm{m} . \mathrm{K})$ [8]. From the results and the theory parts, it shows that polyurethane foams is the best insulating material by minimizing the heat transfer from surroundings to the solution followed by aluminium foils and ethylene propylene rubbers.

In terms of the heat flux generated, the value of $\mathrm{Q}$ for polyurethane foams is $-487.166 \mathrm{~W} / \mathrm{m}^{2}$, aluminium foils $1464.93 \mathrm{~W} / \mathrm{m}^{2}$ and ethylene propylene rubbers - 30,034.3 $\mathrm{W} / \mathrm{m}^{2}$ as shown in Figure 3. The negative sign shows that heat is transferred from the surroundings to the solution. Again, polyurethane foams have the smallest value of heat transfer through that direction. This proves once again that polyurethane foam is the best insulating material compared to aluminium foil and ethylene propylene rubber.

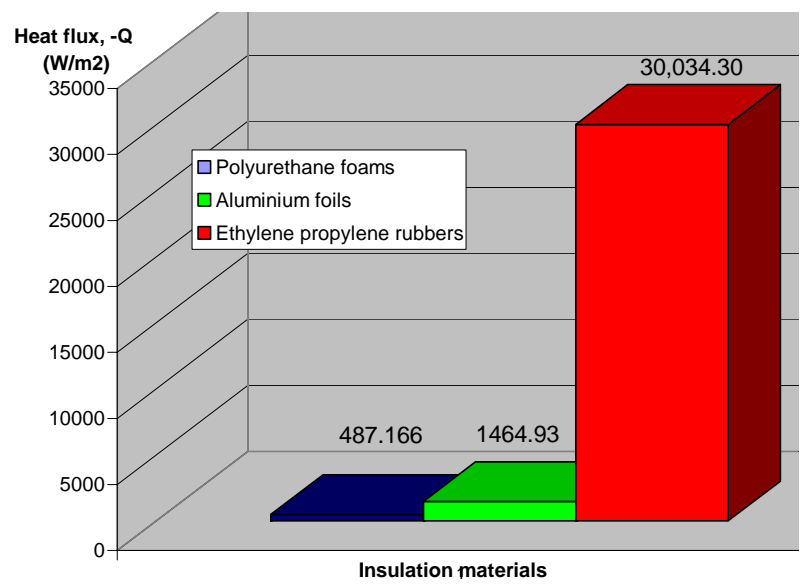

Figure 3. Heat flux generated through different types of insulator at feed temperature $2^{\circ} \mathrm{C}$

\section{B.Heat Transfer with the Addition of Crystallization Chamber}

The purpose of not to install the crystallization chamber in the first experiment is to see the trend of temperature compared to the experiment done by the addition of crystallization chamber with the same condition. At first, it was thought that the crystallization chamber will increase the temperature of solution in the apparatus. But after the experiments have been carried out, it was found that through the addition of the crystallization chamber, the solution temperatures are lower compared to the experiments without the crystallization chamber at the same conditions. This scenario was shown more in detail in Figures 4, 5 and 6 through experimental data for each types of insulation system at feed $2^{\circ} \mathrm{C}$.

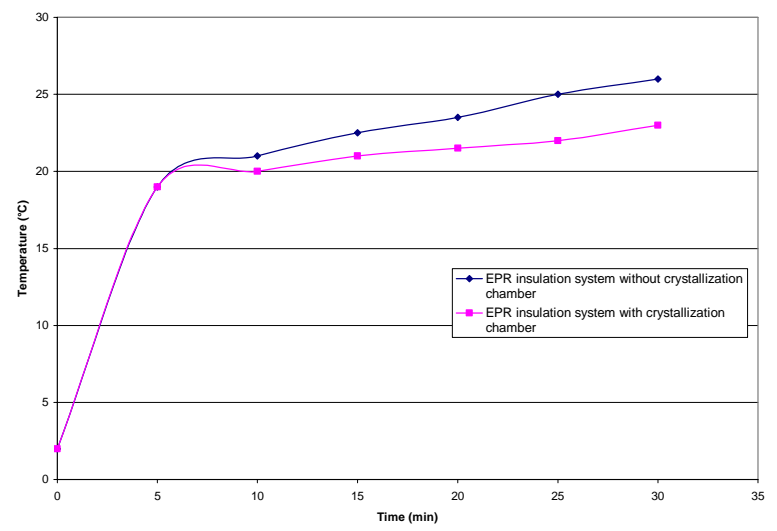

Figure 4. Comparison on temperature profile for EPR insulation system with and without crystallization chamber

Data on solution average temperatures at feed $2^{\circ} \mathrm{C}$ are tabled in Table 2 while the temperature profiles in Figure 7.

This scenario may have been resulted by the ability of the crystallization chamber in retaining the solution temperature. As being mention earlier, the crystallization chamber is made of stainless steel. We should look this through the perspective of heat capacity properties. Heat capacity, $c_{p}$ is defined as the amount of energy required to change the temperature of a unit mass or mole one degree. When the solids heat capacity is higher, the temperature reduction is inversely proportional to the heat capacity [8]. The heat capacity, $\mathrm{c}_{\mathrm{p}}$ of stainless steel is $0.461 \mathrm{~kJ} /(\mathrm{kg} . \mathrm{K})$ [9]. 
This value is quite high compared to a non-metal compound like PVC (polyvinyl chloride). So, those lower temperatures of the solution with the addition of crystallization chamber compared to the solution without the addition of crystallization are because of the ability of the crystallization chamber in temperature reduction compared to the PVC.

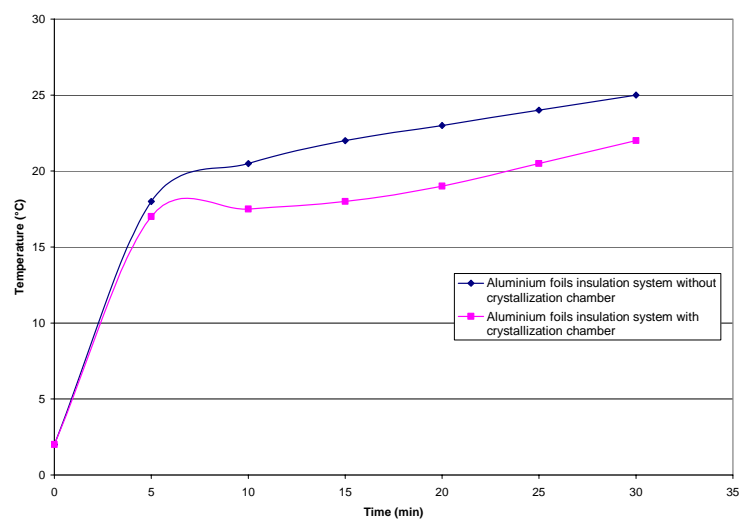

Figure 5. Comparison on temperature profile for aluminium foils insulation system with and without crystallization chamber.

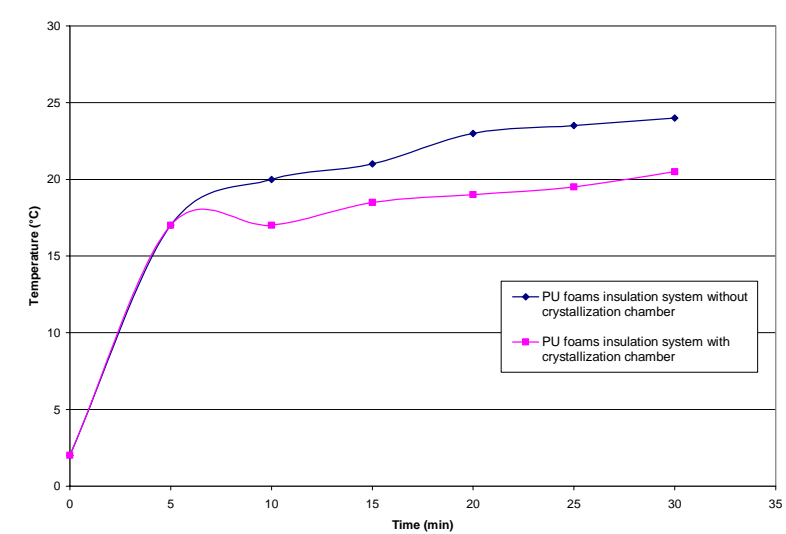

Figure 6. Comparison on temperature profile for PU foams insulation system with and without crystallization chamber

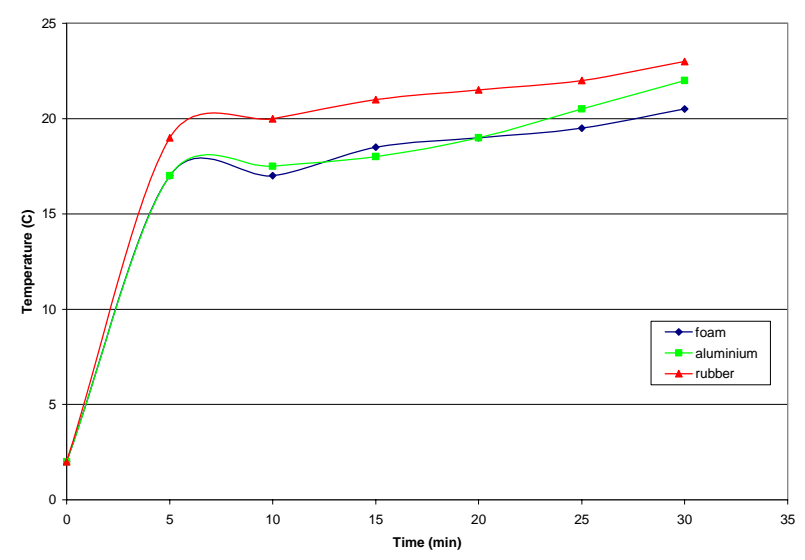

Figure 7. Average temperatures profile at feed $2^{\circ} \mathrm{C}$ via different types of insulator with crystallization chamber

\section{CONCLUSIONS}

The analysis of heat transfer of a progressive freeze concentration design through variation of types of insulator is successfully achieved in this study. From the study, it was found that polyurethane foams is the best insulating material compared to aluminium foils and ethylene propylene rubber due to its minimal heat transfer between the ambient and the sample solution in the progressive freeze concentration apparatus. The reason is that polyurethane foams have the lowest thermal conductivity value compared to the other two.

Along this study, it was also found that the crystallization chamber does not contribute to the increment of the solution temperature in the apparatus. Even without any cooling mechanisms, the chamber was able to reduce the temperature of the solution. This is due to heat capacity of the stainless steel crystallization chamber is quite high compared to a non-metal compound like PVC in the apparatus. So, this crystallization chamber is practical for the usage in progressive freeze concentration process.

Table 2. Average temperatures of solution with different types of insulator at feed $2^{\circ} \mathrm{C}$ with crystallization chamber

\begin{tabular}{|l|l|l|l|}
\hline \multirow{4}{*}{$\begin{array}{l}\text { Time } \\
(\text { min })\end{array}$} & \multicolumn{3}{|l|}{$\begin{array}{l}\text { Average temperatures of the solution } \\
\text { with insulation system of }\end{array}$} \\
\cline { 2 - 4 } & $\begin{array}{l}\text { Polyurethane } \\
\text { foams } \\
\left({ }^{\circ} \mathrm{C}\right)\end{array}$ & $\begin{array}{l}\text { Aluminium } \\
\text { foils } \\
\left({ }^{\circ} \mathrm{C}\right)\end{array}$ & $\begin{array}{l}\text { Ethylene } \\
\text { propylene } \\
\text { rubber } \\
\left({ }^{\circ} \mathrm{C}\right)\end{array}$ \\
\hline 0 & 2.0 & 2.0 & 2.0 \\
\hline 5 & 17.0 & 17.0 & 19.0 \\
\hline 10 & 17.0 & 17.5 & 20.0 \\
\hline 15 & 18.5 & 18.0 & 21.0 \\
\hline 20 & 19.0 & 19.0 & 21.5 \\
\hline 25 & 19.5 & 20.5 & 22.0 \\
\hline 30 & 20.5 & 22.0 & 23.0 \\
\hline
\end{tabular}

\section{ACKNOWLEDGEMENT}

The authors thank University Technology of Malaysia in supporting this research. Thank you for the facility used and human resources involved in conducting this research.

\section{REFERENCES}

[1] Holt, S. (1999), The Role of Freeze Concentration in Waste Water Disposal, Filtration and Separation Journal, $34-35$.

[2] Miyawaki O., Liu L., Shirai Y., Sakashita S. and Kagitani K. (2005). Tubular ice system for scale-up of progressive freeze-concentration. Journal of Food Engineering. Vol (69): 107-113

[3] Rogers, A., 1999, Freeze Concentration in Hazardous Wastewater Management, The Challenge: Establishing the Best Hazardous Wastewater Management Approach, Techapplication Bulletin, Ellectric Power Research Institute, USA.

[4] Muller, M., \& Sekoulov, I. (1992). Waste water reuse by freeze concentration with a falling film reactor. Water Science and Technology, 26, 1475-1482.

[5] Gu, X., Suzuki, T., and Miyawaki, O. (2005). Limiting Partition Coefficient in Progressive Freeze Concentration. Journal of Food Science. Vol (70): 546-551.

[6] Widehem P. and Cochet N. (2003). Pseudomonas syringae as an ice nucleator-application to freeze-concentration. Process Biochemistry. Vol (39): 405-410.

[7] Malloy J. F. (1969). Thermal Insulation. Van Nostrand Reinhold Enviromental Engineering Series, United States of America.

[8] Perry H. R. (1997). Perry's Chemical Engineers' Handbook. $7^{\text {th }}$ Edition. McGraw-Hill, United States of America.

[9] Geankoplis C. J. (2003). Transport Processes and Separation Process Principles (Includes Unit Operations). $4^{\text {th }}$ Edition. Upper Saddle River, New Jersey: Prentice Hall. 


\title{
Hydrodynamics Studies Of Sand/Palm Shells Binary Mixtures In Compartmented Fluidized Bed Gasifier (CFBG)
}

\author{
V.S. Chok, L.F.B. Chin, S.K. Wee, W.W. Tang, A. Gorin, H.B. Chua, H.M. Yan
}

\begin{abstract}
The internally circulating biomass gasification process is under development in Compartmented Fluidized Bed Gasifier (CFBG) following the construction of 0.5 TPD biomass pilot plant most recently. CFBG is capable of producing high calorific value syngas $\left(>11 \mathrm{MJ} / \mathrm{Nm}^{3}\right)$ without the needs of pure oxygen, distinctive to conventional gasifier. Palm shell is chosen as biomass feedstock, which provides potential source of hydrocarbon.

The present work reports studies on the fluidization of palm shell-sand binary mixtures of 2, 5, 10, and 15 wt $\%$. Experiments are carried out in CFBG cold model of $66 \mathrm{~cm}$ ID consisting of two compartments, combustor and gasifier with 2:1 ratio respectively fluidized with air. The minimum fluidization velocity of the palm shell-sand binary mixtures in different wt\% is determined and compared with published $U_{m f}$ correlations developed for small diameter column and different biomasses. Fluidization quality in different diameter, particle size, bed height, superficial velocity and $w \mathrm{t} \%$ of palm shells is studied. The vertical and lateral palm shells distribution in these mixtures is also presented.
\end{abstract}

Keywords: Binary mixtures, fluidization, biomass

\section{INTRODUCTION}

Fluidized bed is selected for gasification process due to its effectiveness of heat and mass transfer between the gas and solid phases [1]. Although this process is considered as one of the oldest technologies and has been made successful in some industrial-scale production of renewable energy from biomass, generally this industry still faces a lot of challenges including the availability of economically viable technology, sophisticated and sustainable natural resources management, and proper market strategies under competitive energy markets [2].

Compartmented Fluidized Bed Gasifier (CFBG) [3]-[11] is a novel gasifier capable of addressing the issues above and is currently under intensive development, following the

This work is supported by MOSTI and MPOB.

V.S. Chok is with Curtin University of Technology, Sarawak Campus, CDT250, 98009 Miri, Sarawak (phone: 608-544-3939; fax: 068-544-3837; e-mail: chok.vui.soon@stud.curtin.edu.my).

L.F.B. Chin, S.K. Wee, W.W. Tang, A.Gorin, H.B. Chua are with Curtin University of Technology, Sarawak Campus, CDT250, 98009 Miri Sarawak (phone: 608-544-3939; fax: 068-544-3837; www.curtin.edu.my)

H.M. Yan was formerly with Curtin University of Technology and the author presently residing in Australia. (E-mail: hm.yan@hotmail.com) construction of 0.5 tones/day (TPD) biomass pilot plant. CFBG is capable of producing high calorific value syngas $\left(>11 \mathrm{MJ} / \mathrm{Nm}^{3}\right)$ with full compatibility for existing power generation and chemical synthesis technologies, without the needs of pure oxygen. Palm shell is selected as gasification feedstock could potentially realize biomass-to-syngas production due to its abundance supply since oil palm is ranked as number 1 fruit crops produced in year 2007 [2].

The objective of this study is to provide practical data and theoretical perspectives for pilot-scale CFBG from the hydrodynamics and mixing behavior of sand/palm shell binary mixtures using a cold flow model. Minimum fluidization velocity $\left(U_{m f}\right)$ obtained experimentally is compared with the published $U_{m f}$ correlations developed for small diameter column and different biomasses.

Wu et al. [12] has reviewed the various $U_{m f}$ correlations for multicomponents system. These correlations were based on two distinct features, either requiring the determination of single component $U_{m f}$ or as a whole. The latter is more likely applicable to biomass as they cannot be solely fluidized [13]. Rao et al. [13] developed correlation from a $5 \mathrm{~cm}$ ID reactor using rice husks, sawdust and groundnut shell powder, up to $15 \mathrm{wt} \%$ with sands. Noda et al. [14] developed correlation using glass beads, wood, marten shot, soybean, small bean, and rubber with sands in $16 \mathrm{~cm}$ ID column. It is found that the $U_{m f}$ for the particles of different sizes and densities strongly depends on the mixing condition of the bed and on the volume fraction of the fluidizing medium. The authors have developed correlation with standard deviation of $\pm 35 \%$. All the above-mentioned correlations have not been tested using the present biomass/sand mixtures and in large reactor where the mixing state is very much scale-dependent.

A bed is said to be well fluidized when all the particles are fully supported by the gas. Fluidization quality $(Q)$ is defined as experimental bed pressure drop / static weight per unit area [10]. Previous work [10] focused in single component system. Therefore, extended study is made on $Q$ using binary components.

Vertical and lateral mixing of palm shells in mixture is of great importance to its reactor performance. In fluidized bed, mixing action is produced by the bubbles bursting and displacement, which provoke particles vertical and horizontal motion. A gas fluidized bed containing a mixture of particles of differing densities will segregate into two 
layers if the density ratio differs significantly from unity [15]. Some previous studies and reviews have been conducted to investigate on mixing/segregating behavior in binary mixtures [12], [16]-[19]. $\mathrm{Wu}$ et al. [12] and Formisani et al. [17] studied equidensity binary system. Nienow et al. [18] studied mixing characteristic of different particle densities and sizes $(\leq 928 \mu \mathrm{m})$. Shen et al. [19] studied binary system but limited to $2 \mathrm{D}$ bed.

The present work studies the fluidization of palm shellsand binary mixtures of 2, 5, 10, and $15 \mathrm{wt} \%$. Experiments are carried out in CFBG cold model of $66 \mathrm{~cm}$ ID consisting of 2 compartments, combustor and gasifier with $2: 1$ ratio respectively fluidized with air. The $U_{m f}$ of these mixtures in different $w t \%$ is determined and compared with published $U_{m f}$ correlations developed for small diameter column and different biomasses. Fluidization quality $(Q)$ in different diameter $(\mathrm{D})$, bed height $(\mathrm{H})$, superficial velocity $\left(U_{o}\right)$, palm shell sizes and $\mathrm{wt} \%$ are studied. The vertical and lateral palm shells distributions in these mixtures are also presented.

\section{EXPERIMENTAL SETUP}

A schematic of the experimental setup is illustrated in figure 1. The cold flow model as shown in figure 2 is divided into 2 compartments by a vertical wall in $65: 35$ ratio, that is combustor and gasifier with $66 \mathrm{~cm}$ and $3.1 \mathrm{~m}$ in diameter and total height respectively, fluidized using ambient air. The flow rates are regulated between 1-2.5 $U_{m f}$ by rotameters to maintain the bubbling mode of fluidization. Pressure drops $(\Delta \mathrm{P})$ are measured using water manometers at 3 different positions to indicate the total $\Delta \mathrm{P}$, across distributor and bed respectively.

The particles used are sand (RS) and palm shell (PS). The sand density is $2620 \mathrm{~kg} / \mathrm{m}^{3}$, with mean diameter $\left(\mathrm{d}_{\mathrm{s}}\right)$ of $272 \mu \mathrm{m}$. Palm shell with moisture less than $10 \mathrm{wt} \%$ and density of $1200 \mathrm{~kg} / \mathrm{m}^{3}$ consists of 3 mean diameter $\left(\mathrm{d}_{\mathrm{ps}}\right)$ of $1.77,3.55$ and $13.95 \mathrm{~mm}$ (with respective size ranges of $1.18-2.36,2.36-4.75$ and $4.75-9.20 \mathrm{~mm}$ ). The palm shell content in the bed ranges from 2, 5, 10 and $15 \mathrm{wt} \%$.

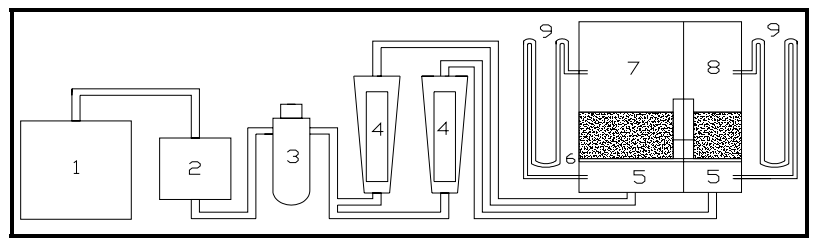

Figure 1. Experimental set up (1: compressor; 2: dryer; 3: pressure regulator; 4: rotameter; 5: plenum; 6: perforated plate distributor; 7: combustor; 8: gasifier; 9: manometers)

\section{METHODOLOGY}

The standard experimental approach in determining monodisperse $U_{m f}$, which is also applied in the present work, is based on defluidization stage of $\Delta \mathrm{P}-U_{o}$ profile. $U_{m f}$ is estimated from the intersection point of the fixed bed line with the constant pressure line. Though the profile and consequently, the determination of binary $U_{m f}$ would generally be affected by different initial conditions [17], the defluidization stage where the components are already mixed is preferred because they are more representative of binary system existing as a "single" bed material.

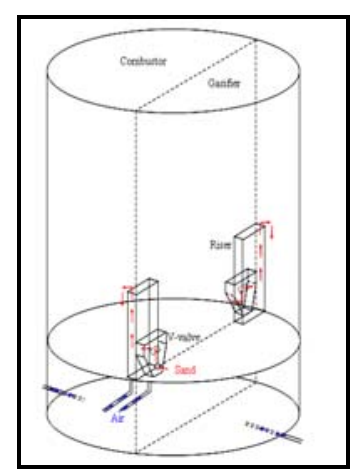

Figure 2. Isometric view of $\mathrm{CFBG}$

For $Q$, bed $\Delta \mathrm{P}$ is computed by subtracting distributor $\Delta \mathrm{P}$ from total $\Delta \mathrm{P}$.

"Thief" probe (diameter $5 \mathrm{~cm}$ ) and sieving method is preferred due to the following advantages: (i) fast response (ii) able to collect sample while the bed is fluidized (iii) able to collect sample at different locations in the bed (iv) able to collect the sample in a number of time under various operating conditions.

"Thief" probe operates by imbedding it vertically in the fluidized bed at a set location. Apertures are thereafter exposed to the bed by opening the enclosure cap. The probe is removed vertically from the bed. The mixtures is then sieved and weighed to determine their component weight fraction.

Prior to the experiments, sands are filled to desired weight/height and packed. Palm shells are then uniformly stacked on the bed surface forming two segregated layers. This approach is selected to track palm shell migration. The $U_{m f}, Q$ and solids mixing behavior are simultaneously measured. Solid sample is collected from 3 different sections (v-valve, center and riser) in 3 different levels (L1L3 indicating top, center, bottom), which constitutes 9 imaginary cells $(n)$ shown in figure 3 .

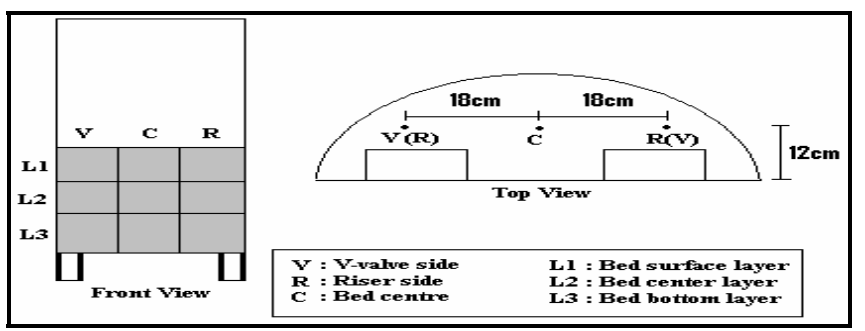

Figure 3. Sampling locations in gasifier. Those in brackets represent combustor side

Data on mixing is presented graphically such that the deviation of the perfect mixing line indicates segregation. Vertical or lateral mixing index $(m)$ is defined as local palm shell $\mathrm{wt} \%$ / load palm shell $\mathrm{wt} \%$ or $(x / X) . m=1$ represent perfect mixing, while segregation may lead to two conditions, either "dilution" $(m<1)$ or accumulation $(m>1)$. Assuming that the 9 locations are able to represent the whole bed composition, the overall mixing index $(M)$ can therefore be computed, in absolute terms according to the equation (1). $M=1$ and $<1$ corresponds to perfect mixing and segregation respectively. 3 repetitive data are taken in each location with standard deviation of $\pm 15 \%$. Each sets is in 15 minutes interval to allow steady state. Shen L.H et al. [19] observed that equilibrium mixing is achieved in 10 seconds. 


$$
M=1-\left(\frac{1}{n}\right)\left(\frac{\mid \text { local PS } w t \%-\text { load PS } w t \% \mid}{\text { load PS wt\% }}\right)
$$

Notwithstanding of the measurement for the study of the effect of bed height to $Q$ and mixing, all the experiments are conducted at constant total bed weight of $77 \mathrm{~kg}$.

\section{IV. $\boldsymbol{U}_{\boldsymbol{m} \boldsymbol{f}}$ DETERMINATION}

Generally, increase in palm shell size or wt $\%$ leads to an increase in $U_{m f}$. In table 2, it is obvious that Noda et al. [14] correlation developed from combination of component $U_{m f}$ is not applicable to these mixtures where the biomass can not be solely fluidized, similarly highlighted by Bilbao et al. [20] in sand/straw binary mixtures. Rao et al. [13] prediction is relative closer but significantly underestimate due to their different bed characteristic e.g. sphericity and bed incipient porosity $\left(\phi=0.86, \varepsilon_{m f}=0.42\right)$ from the present $\operatorname{system}\left(\phi=0.54, \varepsilon_{m f}=0.34\right)$.

Table 2. Experimental (Exp) data, correlations results and percentage error (ER\%) for various palm shell size and $\mathrm{wt} \%$

\begin{tabular}{|c|c|c|c|c|c|}
\hline Palm Shell & \multicolumn{5}{|c|}{$U_{m f}(\mathrm{~m} / \mathrm{s})$} \\
\cline { 2 - 6 } $\mathrm{wt}_{0} ; \mathrm{d}_{\mathrm{ps}}(\mathrm{mm})$ & $\operatorname{Exp}$ & {$[14]$} & $\mathrm{ER} \%$ & {$[13]$} & $\mathrm{ER} \%$ \\
\hline $2 \% ; 3.55$ & 0.045 & 0.555 & 1110 & 0.025 & -44 \\
\hline $5 \% ; 3.55$ & 0.045 & 0.553 & 1130 & 0.031 & -31 \\
\hline $10 \% ; 1.77$ & 0.055 & 0.490 & 791 & 0.038 & -31 \\
\hline $10 \% ; 3.55$ & 0.055 & 0.568 & 930 & 0.044 & -20 \\
\hline $10 \% ; 13.95$ & 0.067 & 0.671 & 901 & 0.060 & -10 \\
\hline $15 \% ; 3.55$ & 0.076 & 0.583 & 670 & 0.066 & -13 \\
\hline
\end{tabular}

\section{FLUIDIZATION QUALITY}

\section{(A) EFFECT IN DIFFERENT DIAMETER}

Figure 4 shows that increase in diameter has no significant changes in $Q$ value for the binary components. However, single component extracted from [10] has higher $Q$ value than binary component in gasifier.

(B) EFFECT IN DIFFERENT PALM SHELL SIZE

It is observed that there is no significant changes on the $Q$ value with the increase of the palm shell sizes in figure 5, contrary to previous single component experiment [10] where through-channeling changes to intermediatechanneling as the particle diameter increases.

\section{(C) EFFECT IN DIFFERENT BED HEIGHT (H)}

In figure 6, no distinctive changes of $Q$ value in different bed aspect ratio, $H / D$ (constant $D$, increasing $H$ ).

\section{(D) EFFECT IN DIFFERENT PALM SHELL WT\%}

In figure $7, Q \approx 0.8$ in single component [10] while $Q$ fluctuates mainly between $0.6-0.7$ in binary mixtures. A through-channeling is observed and the fluctuation becomes more intense with the increase in palm shell $\mathrm{wt} \%$. This is due to the differing shape, size and density of the binary components, leading to more severe local pressure/density variation. Rhodes [21] stated that bubbles formed in group $\mathrm{B}$ and $\mathrm{D}$ particles will continue to grow but never achieving a maximum size associated with large pressure fluctuations. Palm shell used in this study is classified as Geldart D [22] has a tendency to produce spouted beds. The effective particle size and bed voidage will increase while the effective density decreases, when the palm shell wt $\%$ increases in the mixtures. Abdullah et al. [23] stated that bulk density and voidage are the two main factors contributing to bed fluidization quality.

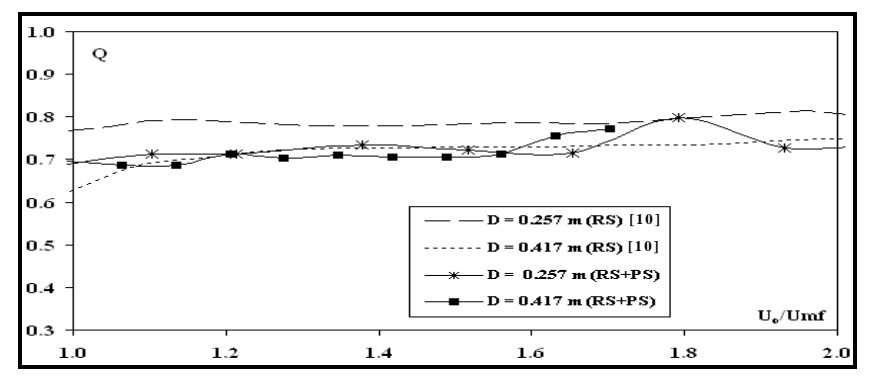

Figure 4. $Q$ versus $U_{d} / U_{m f}$ at different diameter (D) for $10 \mathrm{wt} \%$ palm shell, $\mathrm{d}_{\mathrm{ps}}=3.55 \mathrm{~mm}$ with insertion from [10]

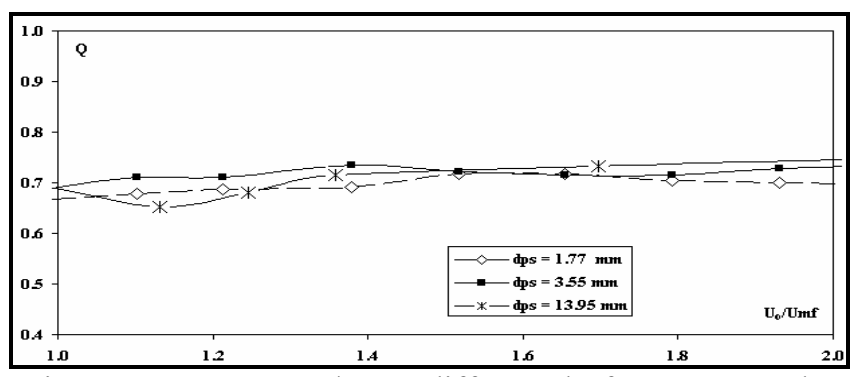

Figure 5. $Q$ versus $U_{o} / U_{\mathrm{mf}}$ at different $\mathrm{d}_{\mathrm{ps}}$ for $10 \mathrm{wt} \%$ palm shell in gasifier

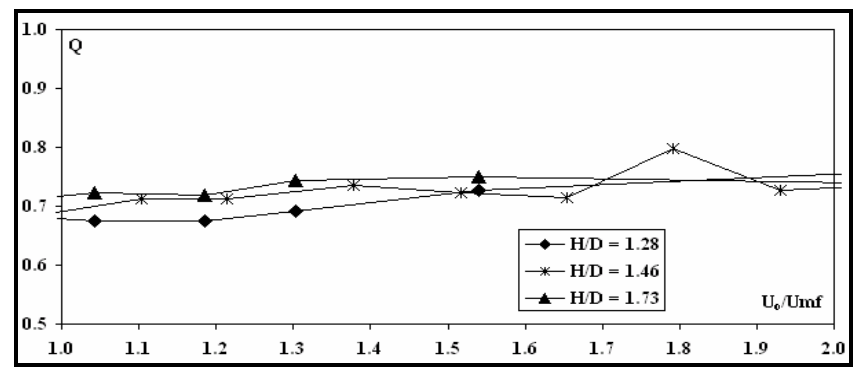

Figure 6. $Q$ versus $U_{d} / U_{m f}$ at different $\mathrm{H} / \mathrm{D}$ for $10 \mathrm{wt} \%$ palm shell with $\mathrm{d}_{\mathrm{ps}}=3.55 \mathrm{~mm}$ in gasifier

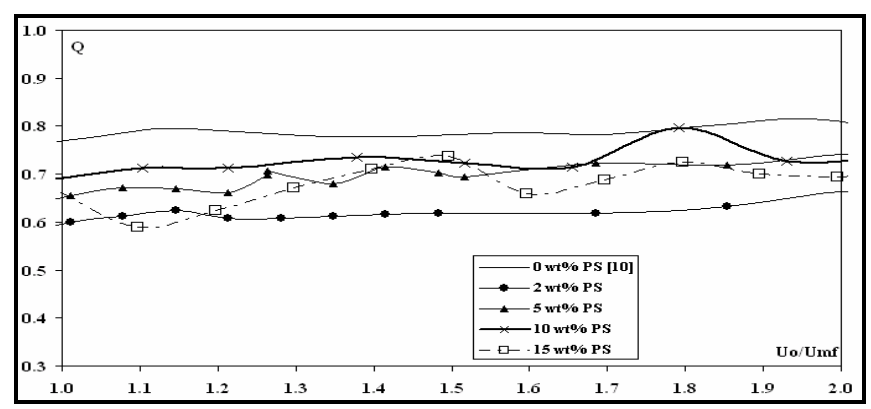

Figure 7. $Q$ versus $U_{o} / U_{m f}$ at different palm shell $\mathrm{wt} \%$ with $\mathrm{d}_{\mathrm{ps}}=3.53 \mathrm{~mm}$ in gasifier with insertion from [10]

\section{Vertical and Lateral MiXing}

EFFECT OF SUPERFICIAL VELOCITY ON VERTITAL MIXING

Figure 8 shows the typical palm shell vertical distribution at various $U_{m f}$. The data is based on $10 \mathrm{wt} \%$ of palm shell and is therefore has a maximum accumulated value of $M=10$, if collected sample is purely palm shell.

Starting from packed bed condition where palm shells initially stacked uniformly at bed surface, with increasing $U_{o}$, they gradually distributed to the lower region of the bed. 
At $1.25 U_{m f}$, by visual observation, bubbles are more likely formed at the bed center compared to the wall side. Consequently, palm shells at the center propagate more rapidly to the lower region of the bed compare to the wall side, initiating the mixing process. Palm shells at the bed surface of center region are also dispersed to wall side by bubbles bursting. At $1.5 U_{m f}$, the concentration gradient on both vertical wall sides getting closer due to the increasing bubbling rate that induces better mixing in the whole bed. However, palm shells with lower density, tends to migrate towards the bed surface due to easiness of being swept away by the air through flow from distributor and carried up by rising bubbles. At higher $U_{o}\left(2.0-2.5 U_{m f}\right)$, palm shells tend to become uniformly mixed, resulting in a smaller palm shells concentration gradient developed along the bed height. Hence, vertical mixing is enhanced.

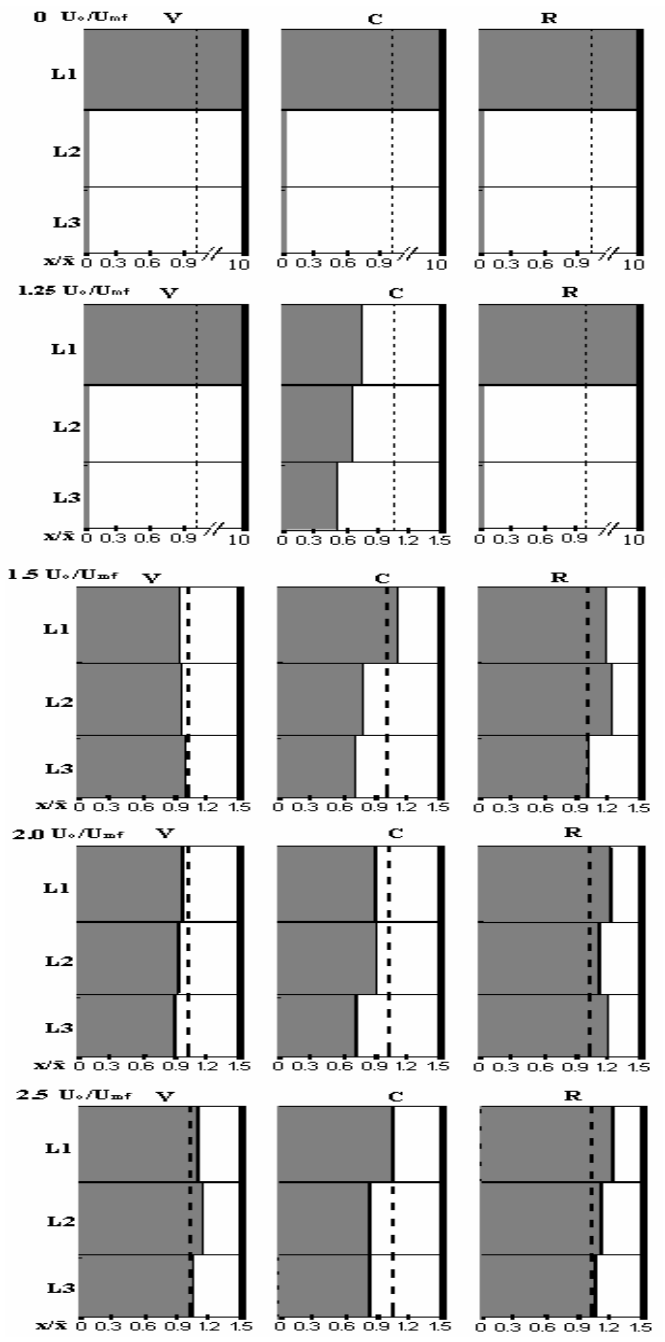

Figure 8. Effect of $U_{o} / U_{m f}$ on vertical mixing for $10 \mathrm{wt} \%$ palm shell with $\mathrm{d}_{\mathrm{ps}}=3.55 \mathrm{~mm}$ in gasifier

EFFECT OF SUPERFICIAL VELOCITY ON LATERAL MIXING

Figure 9 shows the typical palm shell lateral distribution with various $U_{m f}$. Overall, the $M$ indexes of three layers improve with increasing $U_{o}$. Lateral mixing of solids in a gas solid fluidized bed is caused by two mechanisms, which are bursting bubbles at the bed surface and bubbles displacement inside the bed [22]. At the $1.25 U_{m f}$, lateral mixing is initiated by bursting bubbles at the bed surface, where a large fraction of the palm shells at the centre are dispersed. At L2 and L3, local mixing induced by bubbles displacement is insignificant. With increasing $U_{o}$ (1.5$2.5 U_{m f}$ ), lateral palm shells distribution across fluidized bed at L1, L2 and L3 approaching to each other. This is due to increasing bubbling rate, whereby lateral mixing induced by bubbles displacement becomes more significant. Hence, lateral mixing is enhanced.

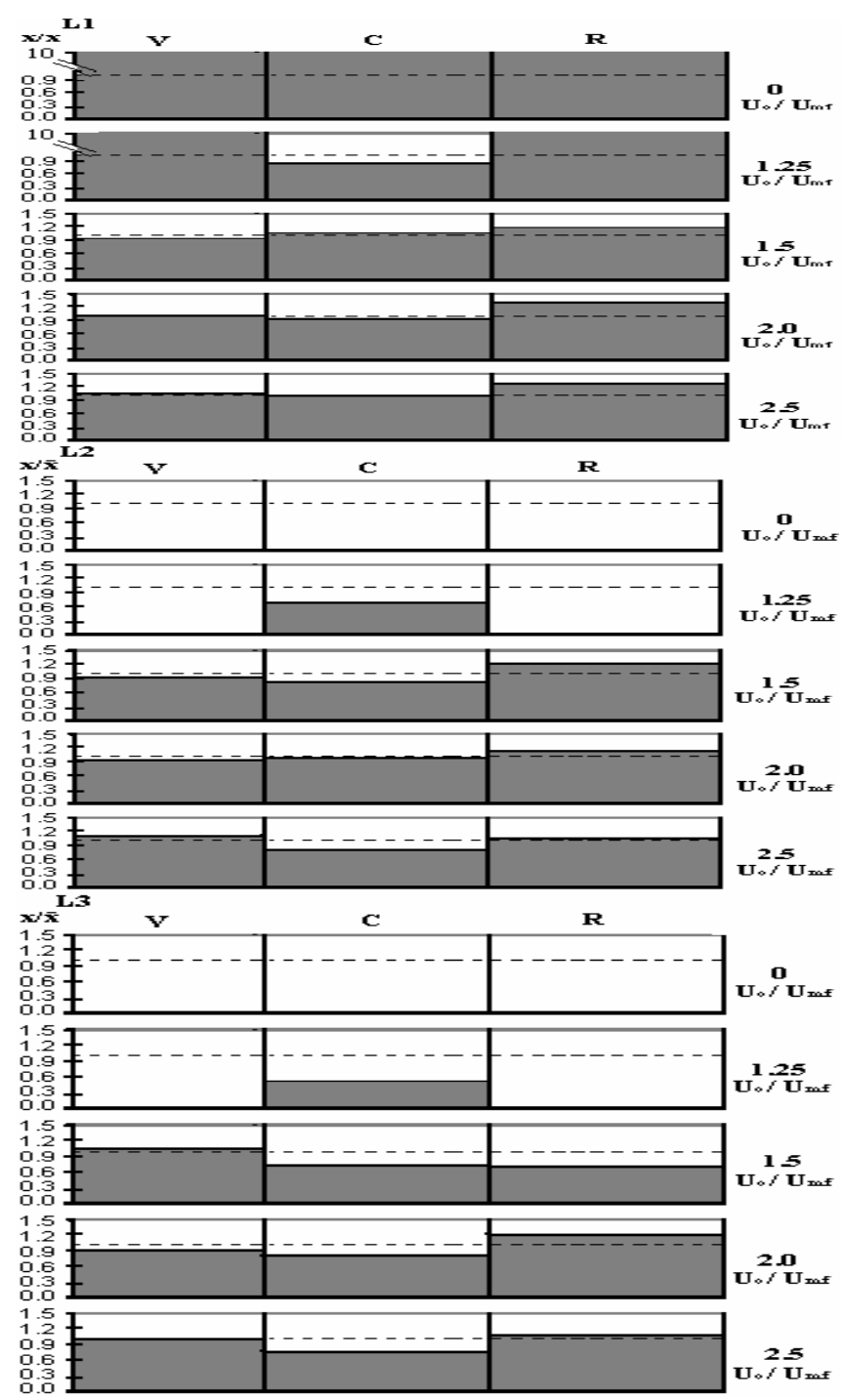

Figure 9 Effect of $U_{o} / U_{m f}$ on lateral mixing for $10 \mathrm{wt} \%$ palm shell with $\mathrm{d}_{\mathrm{ps}}=3.53 \mathrm{~mm}$ in gasifier

\section{EFFECT OF PALM SHELL WT\% ON OVERALL MIXING INDEX}

$M<0$ is possible especially at the onset of fluidization. However, for the ease of interpretation whilst fluidized bed aeration is usually much above $U_{m}$, it is therefore omitted. Generally, increasing $U_{o}$ will improve mixing in segregated systems of mixtures with difference densities/sizes [24]. Shen et al. [25] who reported that wake exchange coefficient, which indicates vertical and lateral solid mixing intensity, reduces with the increase in $U_{m f}$. On the contrary, in figure 10, although $U_{m f}$ increases with palm shell wt $\%, M$ index increases. This is likely due to the increase in $\varepsilon_{m f}$ as shown in table 3 that leads to greater bed expansion, allowing effective vertical and lateral solid mixing.

Table 3: Bed voidage at $U_{m f}$ for various palm shell $\mathrm{wt} \%$.

\begin{tabular}{|c|c|c|c|c|}
\hline Palm Shell wt $\%$ & 2 & 5 & 10 & 15 \\
\hline$\varepsilon_{m f}$ & 0.32 & 0.33 & 0.36 & 0.37 \\
\hline
\end{tabular}




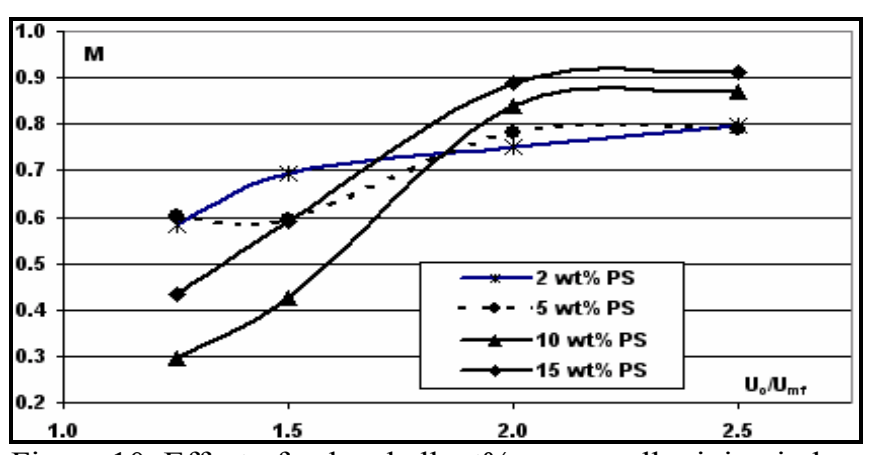

Figure 10. Effect of palm shell $\mathrm{wt} \%$ on overall mixing index $(M)$ for $\mathrm{d}_{\mathrm{ps}}=3.55 \mathrm{~mm}$ in gasifier

EFFect Of Palm Shell Size On OVERALl Mixing INDEX In figure 11 , for smaller palm shell size $(3.37 \mathrm{~mm})$, a good overall mixing index $(M \geq 0.85)$ is achievable at lower $U_{o}$, which is $2 U_{m f}$ compare to larger palm shell size. Wu et al. [12] extensive review on solids mixing concluded that lower degree mixing obtained as the particles size increases due to a decrease of both visible bubbles flow rate and the wake/drift fraction for larger particles. Wirsum et al. [24] reported that smaller "flotsams" (component that tends to float on the fluidized bed surface, as opposed to "jetsam" [26]) lead to better mixing.

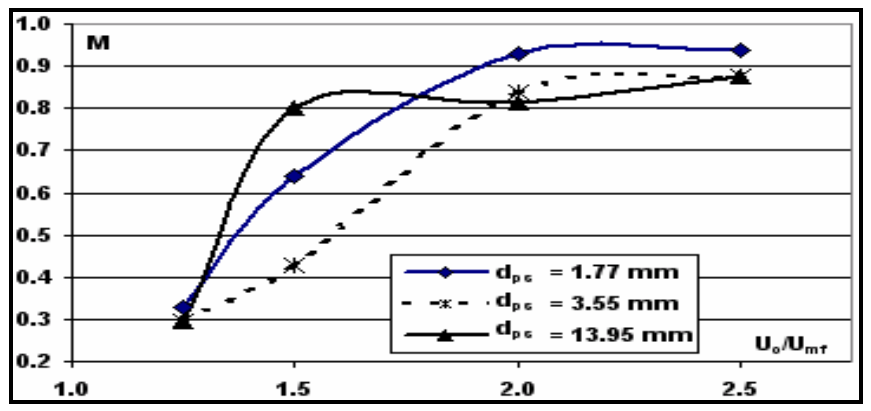

Figure 11. Effect of palm shell sizes on overall mixing index $(M)$ at $10 \mathrm{wt} \%$ palm shell is gasifier

\section{EFFECT OF BED HEIGHT ON OVERALL MIXING INDEX}

In figure 12, good overall mixing index is achieved at $1.5 U_{m f}$ for deep bed, whereas at shallow bed, the same degree of mixing is only achievable at higher $U_{o}\left(\geq 2 U_{m f}\right)$. With increasing bed height, the bed pressure drop increased resulting stable bubbling across bed distributor that uniformly mixed the fluidized bed. Conversely, in shallow beds, where the total bed pressure drop is reduced, then the situation is self-aggravating and the local preferential channeling formed. This contributes to non-uniformity bubbling at the expense of the rest of the bed [27]. Therefore, if $U_{o}$ held constant, poorer mixing attained with a sensitivity to bed height in shallow beds and relatively good mixing in deep beds.

\section{EFFect Of Bed Diameter On Overall Mixing IndeX}

In figure 13, combustor with a larger bed diameter attained good overall mixing index at lower $U_{o}\left(1.5 U_{m f}\right)$. The flow behavior of a gas solid fluidized bed is highly sensitive to scale [27]. In gasifier of smaller bed diameter, the presence $\mathrm{v}$-valve and riser, contributes to greater wall effect as compare to combustor, resulting significant reduction in the overall growth of bubbles [28]. As the diameter increases, the wall effect becomes insignificant, hence enhancing vertical mixing. The lateral mixing is also enhanced with increasing bed width [23]. These combined effects improves overall mixing index.

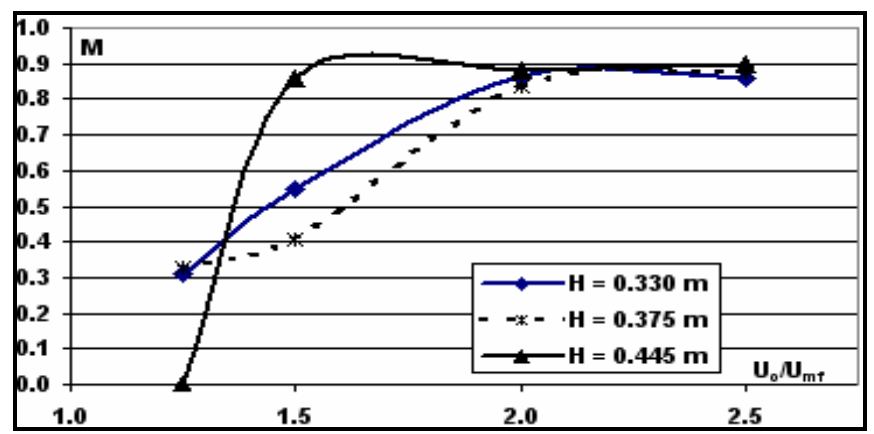

Figure 12. Effect of bed height on overall mixing index $(M)$ of $10 \mathrm{wt} \%$ palm shell with $\mathrm{d}_{\mathrm{ps}}=3.55 \mathrm{~mm}$ in gasifier

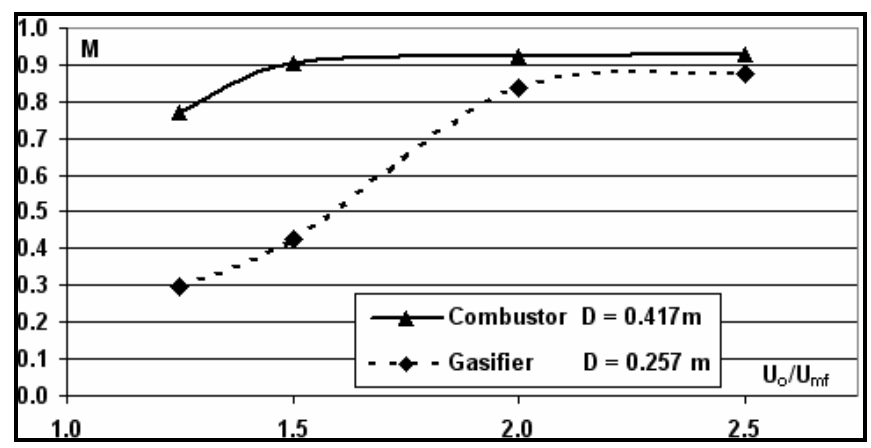

Figure 13. Effect of bed diameter (D) on overall mixing index $(M)$ of $10 \mathrm{wt} \%$ palm shell with $\mathrm{d}_{\mathrm{ps}}=3.55 \mathrm{~mm}$

\section{CONCLUSION}

This study has been carried out to determine the sand/palm shell binary mixtures $U_{m f}, Q$ and the mixing behavior in CFBG. $U_{m f}$ correlations [5] and [6] developed from smallscale laboratory reactor and different biomasses could not be extended for the present system. No significant effect on the $Q$ value with respect of different geometry, particle sizes, bed height, and wt $\%$ of palm shells and throughchanneling is observed throughout the experiments. However, it is found that the single component demonstrates higher $Q$ value compared with the binary mixtures. It is interesting to note that uniform mixing is attainable for these binary system even though the $Q$ value < 0.8. As for mixing/segregation, $m$ and $M$ mixing indexes increase when the $U_{o}$ increases. The bed diameter has more dominant effect as compared to the $\mathrm{wt} \%$ and size of palm shells on overall mixing index. For similar particle sizes and $\mathrm{wt} \%$ of palm shells, larger bed diameter and higher bed height required lower $U_{o}$ to achieve good mixing. There is an optimum $U_{o}$ where uniform mixing $(M \geq 0.85)$ can be established, and in this experiment, it is found to be 1.5 $2.5 U_{m f}$ in all experimental range.

\section{REFERENCES}

[1] W. Ragnar, "Gasification of biomass: comparison of fixed bed and fluidized bed gasifier," Biomass and Bioenergy, vol. 18, 2000, pp. $489-497$.

[2] T.L.K. Yong, K.T. Lee, A.R. Mohamed, S. Bhatia, "Potential of hydrogen from oil palm biomass as a source of renewable energy worldwide," Energy Policy vol. 35, 2007, pp. 5692 - 5710.

[3] D. Sathiyamoorthy, V. Rudolph, "Hydrodynamics of Compartmented Dense Phase Circulating Gas Fluidized Bed," in Proc. of the $3^{\text {rd }}$ Int. Conf. in Circulating Fluidized Beds, Japan, 1990, pp. 505-510. 
[4] Y. He, "Hydrodynamics of a Compartmented Dense Phase Circulating Fluidised Bed," Ph.D. diss., Dept. Chem. Eng., The Univ. of Queensland, Australia, 1993.

[5] H.M. Yan, "Development and Modeling of Coal Combustion and Gasification in a Compartmented Fluidised Bed Gasifier," Ph.D. diss., Dept. Chem. Eng., The Univ. of Queensland, Australia, 1995.

[6] V.S. Chok, S.K. Wee, C. Srinivasakannan, H.B. Chua, H.M. Yan, “A novel compartment fluidized bed gasifier for synthesis gas production and power generation from palm waste," presented at the $2^{\text {nd }}$ Int. Conf. on Chem. and Bioprocessing Eng. in conj. with $19^{\text {th }}$ Symp. Malaysian Chem. Eng. (SOMChE), Malaysia, 2005.

[7] V.S. Chok, S.K. Wee, C. Srinivasakannan, H.B. Chua, H.M. Yan, "Developments in the Fluidized Bed Biomass Gasification Technology: Review," presented at the $1^{\text {st }}$ National Seminar on Palm Oil Milling, Refining Techno., Quality \& Environ., Malaysia, 2006.

[8] S.K. Wee, S.S. Lim, V.S. Chok, C. Srinivasakannan, H.B. Chua, H.M. Yan, "CFD Modeling on Hydrodynamics of Compartmented Fluidized Bed Gasifier (CFBG) Riser using Steam," presented at the $4^{\text {th }}$ National Technical Postgrad. Symp., Malaysia, 2006.

[9] V.S. Chok, S.K. Wee, C. Srinivasakannan, H.B. Chua, H.M. Yan, "Fluidized Bed Biomass Gasification Technology: Review," presented at the Int. Conf. \& Exhib., BIOENERGY Outlook 2007, Singapore.

[10] S.K. Wee, V.S. Chok, C. Srinivasakannan, H.B. Chua, H.M. Yan, "Fluidization Quality Study in Compartmented Fluidized Bed Gasifier," presented at the Int. Conf. \& Exhib., BIOENERGY Outlook 2007, Singapore.

[11] S.K. Wee, V.S. Chok, C. Srinivasakannan, H.B. Chua, H.M. Yan, "Cold Flow Model Hydrodynamics Study of Compartmented Fluidized Bed Gasifier (CFBG)," in proc. Int. Conf. Heat Transfer in Components and Syst. for Sustainable Energy Technologies 2007, France.

[12] S.Y. Wu, J. Baeyans, "Segregation by size difference in gas fluidized beds,", Powd. Tech., vol. 98, 1998, pp. 139- 150.

[13] T.R. Rao, J.V. Ram. Bheemarasetti (2001), "Minimum fluidization velocity of mixtures of biomass and sands," Energy, vol. 26, 2001, pp. $633-644$.

[14] K. Noda, S. Uchida, T. Makino, H. Kamo, "Minimum fluidization velocity of binary mixtures of particles with large size ratio," Powd. Tech., vol. 46, 1986, pp. 149 - 154.

[15] J. M. Beeckmans, B. Stahl, "Mixing and segragation kinetics in a strongly segregated gas-fluidized bed," Powd. Tech., vol. 53(1), 1978, pp. $31-38$.

[16] Q.Q. Sun, H.L. Lu, W.T. Liu, Y.R. He, L.D. Yang, D. Gidaspow, "Simulation and experiment of segregating/mixing of rice husk-sand mixtures in a bubbling fluidized bed," Fuel, vol. 84, 2005, pp. $1739-$ 1748.

[17] B. Formisani, G. De Cristofaro, R. Girimonte, "A fundamental approach to the phenomenology of fluidization of size segregating binary mixtures of solids," Chem. Eng. Sci., vol. 56, 2001, pp. $109-$ 119.

[18] A.W. Nienow, P.N. Rowe, L.Y.L. Cheung, "A quantitative analysis of the mixing of two segregating powders of different density in a gasfluidized bed," Powder Technology, vol. 20, 1978, pp. 89 - 97.

[19] L.H. Shen, J. Xiao, F. Niklasson, F. Johnsson, "Biomass mixing in a fluidized bed biomass gasifier for hydrogen production," Chem. Eng. Sci., vol. 62, 2007, pp. 636-646.

[20] R. Bilbao, J. Lezaun, J.C. Abanades, "Fluidization velocities of sand/straw binary mixtures," Powd. Tech., vol. 52, 1987, pp. $1-6$

[21] M. Rhodes, Introduction to particle technology, John Wiley \& Sons, England, 2002.

[22] D. Kunii and O. Levenspiel, Fluidization Engineering, $2^{\text {nd }}$ Edition, Butterworth-Heinemann, U.S.A, 1991.

[23] M. Z. Abdullah, Z. Husain, S.L. Yin Pong, "Analysis of cold flow fluidization test results for various biomass fuels," Biomass and Bioenergy, vol. 24, 2003, pp. 487 - 494.

[24] M. Wirsum, F. Fett, N. Iwanowa, G. Lukjanow, "Particle mixing in bubbling fluidized beds of binary particle systems," Powd. Tech., vol. 120,2001 , pp. $63-69$

[25] L.H. Shen, M.Y. Zhang, "Effect of particle size on solids mixing in bubbling fluidized bed," Powd. Tech., vol. 97, 1998, pp. 170 - 177.

[26] S. Chiba, A.W. Nienow, T. Chiba, H. Kobayashi, "Fluidized binary mixtures in which the denser components may be flotsam,", Powder Technology, vol. 26, 1980. A. E. Quereshi, D.E. Crease, "Fluidized bed gas distributors," Powd. Tech., vol. 22, 1979, pp. 113 - 119.

[27] P.C. Nicholas, P.C. Paul, Hydrodynamics of Gas-Solids Fluidization, Gulf Pub. Co. Houston, 1984
[28] J.G. Yates, R.S. Ruiz-Martinez, D.J. Cheesman, "Prediction of bubble size in a fluidized bed containing horizontal tubes," Chem. Eng. Sci., vol. 45, 1990, pp. 1105 - 1111 


\title{
$\mathrm{CO}_{2}$ Emissions: Impacts and Forecasting in Malaysia
}

\author{
Z.Z. Noor, N.S. Mohd Safaai, H. Hashim and Z. Ujang
}

\begin{abstract}
C O}_{2}$ has become one of the major greenhouses gaseous which levels in the atmosphere are increasing steadily over recent years. $\mathrm{CO}_{2}$ has caused climate change such as global warming which result in significant impacts on every aspect of human life. Major sources of $\mathrm{CO}_{2}$ emission come from energy generation, transportation and industrial sectors. Amongst these sectors, transportation has been identified to be the main contributor of $\mathrm{CO}_{2}$ emission. Under Kyoto Protocol, all participating nations have agreed to cut down their $\mathrm{CO}_{2}$ emission levels by $20 \%$ as well as to maintain the levels below the agreeable limits. Over the years many studies focusing on modeling projection approach have been carried out to predict $\mathrm{CO}_{2}$ emission from various sectors. These predictions provide an important basis to evaluate the policies designed to reduce the emissions and consequently the associated impacts. The aim of this paper is to study the environmental impacts of $\mathrm{CO}_{2}$ emissions and to conduct a review on the researches, which has been done to forecast future $\mathrm{CO}_{2}$ emission level utilizing modeling approach, taking into considerations the assumptions made to build the model.
\end{abstract}

Keywords: $\mathrm{CO}_{2}$ emission, climate change, global warming, Kyoto Protocol, forecasting

\section{INTRODUCTION}

Over the past few decades, it has been observed that there is an increasing atmospheric concentration of greenhouse gaseous of which as carbon dioxide $\left(\mathrm{CO}_{2}\right)$ is more prominent. $\mathrm{CO}_{2}$ is a colorless, odorless gas, produced when any form of carbon is burned in an excess of oxygen. For this reason, the $\mathrm{CO}_{2}$ greenhouse effect in the world has been enhanced. This means that the atmosphere is trapping more heat that has to escape to space. This enhancement has linked the greenhouse effect to causing climate change such as global warming.

Anticipating the current and future level of $\mathrm{CO}_{2}$ concentration, as well as the impacts will have on the earth's climate, has been the task allotted to the Intergovernmental Panel on Climate Change (IPCC, 1992), a United Nations group of 300 of the world's leading scientists. The IPCC scientists agreed that the earth has warmed by 0.3 to 0.6 degrees celsius over the last century (Hileman, 1992; Chakraborty et. al, 2000). 'Figure 1' shows the average

Z.Z. Noor, N.S. Mohd Safaai and H. Hashim, Department of Chemical Engineering, Faculty of Chemical \& Natural Resources Engineering, Universiti Teknologi Malaysia, 81310 Skudai, Johor, Malaysia (Tel: +6075535534, fax:+607-5581463, e-mail: zainura@fkkksa.utm.my).

Z. Ujang, Deputy Chancellor Office (Development and Innovation), Universiti Teknologi Malaysia, 81310 Skudai, Johor, Malaysia. global temperature in the period of 1880-2005. As indicated by the figure, year 2005 was the hottest on record. The average global surface temperature of 14.77 degrees Celsius was the highest since recordkeeping began in 1880. In fact, the six hottest years on record have all occurred in the last eight years. After 2005, 1998 was the second warmest, with an average global temperature of 14.71 degrees Celsius.

The IPCC also predicts that with the current emission scenario, global mean temperature would rise between 0.9 and $3.5^{\circ} \mathrm{C}$ by the year 2100 . In another global warming study, Kessel (Kessel, 2000) estimated that continued use of fossil energy will lead to an increase of the average global temperature by $1.0-3.58^{\circ} \mathrm{C}$ in the coming $50-100$ years.

Since unabated carbon dioxide emissions are likely to accelerate global warming which could lead to more catastrophic effects, it is generally agreed that countries cannot continue to emit carbon dioxide as they did in the past. Therefore, many countries are playing an active role in reducing $\mathrm{CO}_{2}$ emissions through national mitigation and intergovernmental mechanisms such as the United Nations Framework Convention on Climate Change (UNFCC, 1992). The ultimate objective of this Convention to achieve, in accordance with the relevant provisions of the Convention, stabilisation of greenhouse gas concentrations in the atmosphere at a level that would prevent dangerous anthropogenic interference with the climate system. Such a level should be achieved within a time frame sufficient to allow ecosystems to adapt naturally to climate change, to ensure that food production is not threatened and to enable economic development to proceed in a sustainable manner.

The world's primary international agreement on combating global warming is the Kyoto Protocol, an amendment to the United Nations Framework Convention on Climate Change (UNFCC), negotiated in 1997. The Protocol now covers more than 160 countries globally and over $55 \%$ of global greenhouse gas emissions. In the Protocol, a group of industrialised countries agrees to stabilise or reduce its greenhouse gaseous emissions in the commitment period $2008-2012$ by $5.2 \%$ on average (compared to their 1990 emissions level). The basic concept applied is that industrial entities who take steps to reduce their $\mathrm{CO}_{2}$ emission can make money by selling these credits. Subsequently, those entities which need to expand beyond their limit can purchase credits on the open market. As a member of UNFCC, Malaysia is responsible to meet the requirements of the UNFCC in cutting down $\mathrm{CO}_{2}$ to reduce the risk of global warming. 


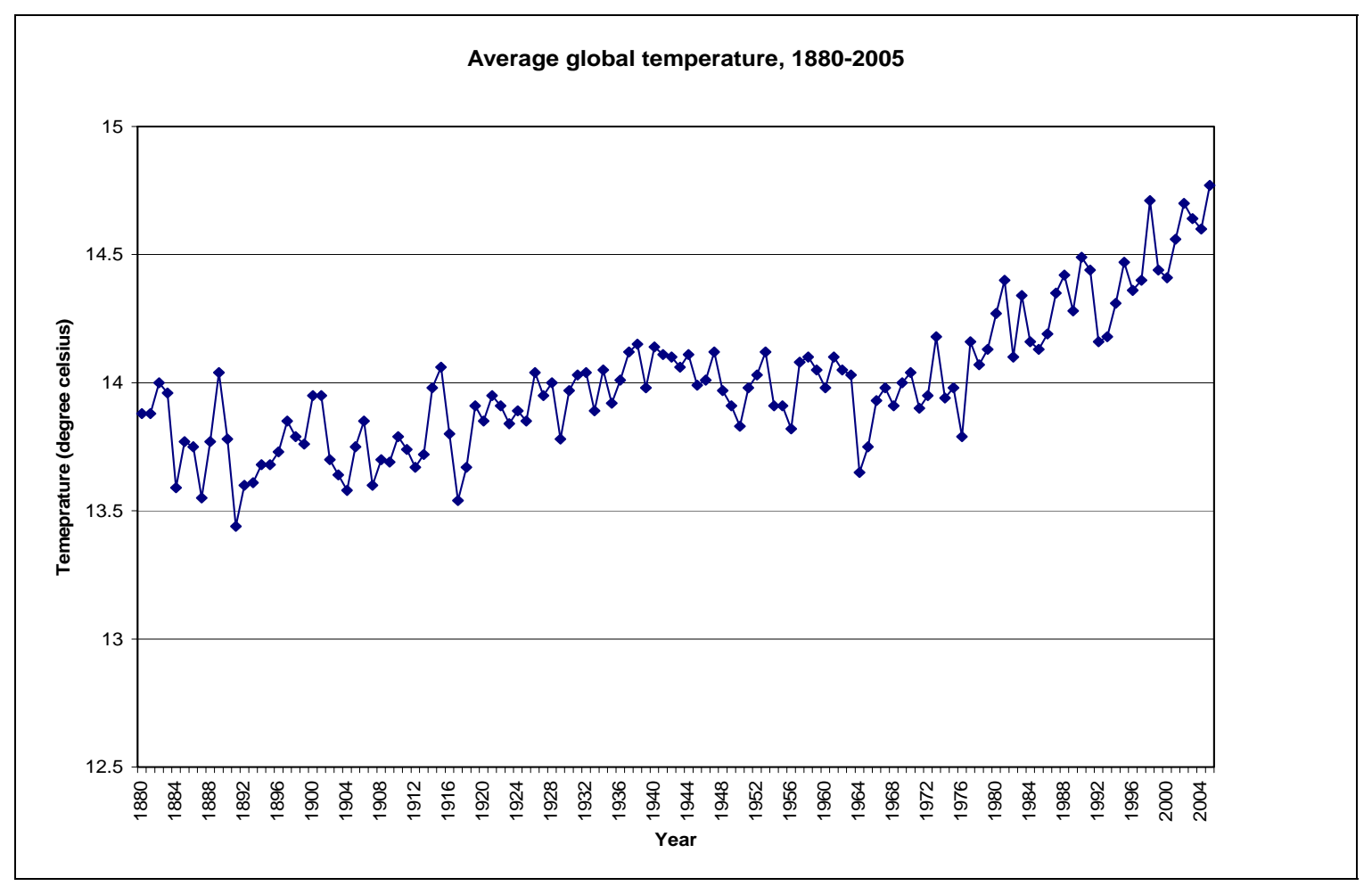

Figure 1. Average global temperature, 1880-2005

(Source: http://www.earthpolicy.org/Indicators/Temp/index.htm)

\section{II. $\mathrm{CO}_{2}$ EMISSIONS: MALAYSIA'S SCENARIO}

In the South East Asia region, Malaysia has the highest per capital emissions after Singapore and Brunei (News Straits Time, 2002). In 1994 alone, Malaysia has emitted 144 million tones of greenhouse gasses, measured in carbon dioxide equivalent. Amongst these emissions, $\mathrm{CO}_{2}$ made up 67.5 per cent, methane $\left(\mathrm{CH}_{4}\right) 32.4$ per cent and $\mathrm{NO}_{x}, 0.1$ per cent.

In this country, there are a few number of sectors contributed to the emissions of $\mathrm{CO}_{2}$. They are electricity and heat production, energy industries, manufacturing and construction, transportation and also residences. Based on data by International Energy Agency (IEA, 2006), transportation had become the main emitter of $\mathrm{CO}_{2}$. Based on the data from Malaysian Automotive Association, numbers of registered vehicles keep increasing from year 1980 until current. The summary of registered vehicles is shown in 'table 1'. With the increased numbers of vehicles on the Malaysian's road, it is expected that the volume of $\mathrm{CO}_{2}$ releases into the air will also increases.

Electricity generation is also a major sector which contributes to the carbon dioxide emission into Malaysia's atmosphere. A study by Mahlia (Mahlia, 2002) has demonstrated that the amount of carbon dioxide emission due to the power generation as illustrated in 'figure 2', will steadily increase as Malaysia is striving towards becoming a developed a country in year 2020 .

Other source by Carbon Dioxide Information Analysis Center had summarised per capita $\mathrm{CO}_{2}$ emissions for Malaysia as shown in 'figure 3'. The estimation values are obtained by dividing total emissions of $\mathrm{CO}_{2}$ by the population for a particular country and year. As demonstrated by the graph, $\mathrm{CO}_{2}$ emission in Malaysia rises steadily during the first 20 years. However, towards year
1990 and beyond, the emission rises dramatically. The particular trend is probably due to the positive growth of industrial and economic sector in Malaysia.

Table 1. Summary of Registered Vehicles in Malaysia, 1980-2007

\begin{tabular}{|c|c|}
\hline Year & Total Vehicles \\
\hline 1980 & 97,262 \\
\hline 1981 & 100,935 \\
\hline 1982 & 102,447 \\
\hline 1983 & 108,314 \\
\hline 1984 & 109,915 \\
\hline 1985 & 94,999 \\
\hline 1986 & 67,847 \\
\hline 1987 & 48,996 \\
\hline 1988 & 71,592 \\
\hline 1989 & 109,357 \\
\hline 1990 & 165,861 \\
\hline 1991 & 181,877 \\
\hline 1992 & 145,084 \\
\hline 1993 & 167,928 \\
\hline 1994 & 200,435 \\
\hline 1995 & 285,792 \\
\hline 1996 & 364,788 \\
\hline 1997 & 404,837 \\
\hline 1998 & 163,851 \\
\hline 1999 & 288,547 \\
\hline 2000 & 343,173 \\
\hline 2001 & 396,381 \\
\hline 2002 & 434,954 \\
\hline 2003 & 405,745 \\
\hline 2004 & 487,605 \\
\hline 2005 & 552,316 \\
\hline 2006 & 490,768 \\
\hline YTD June 2007 & 220,739 \\
\hline
\end{tabular}

(Source: Malaysian Automotive Association) 


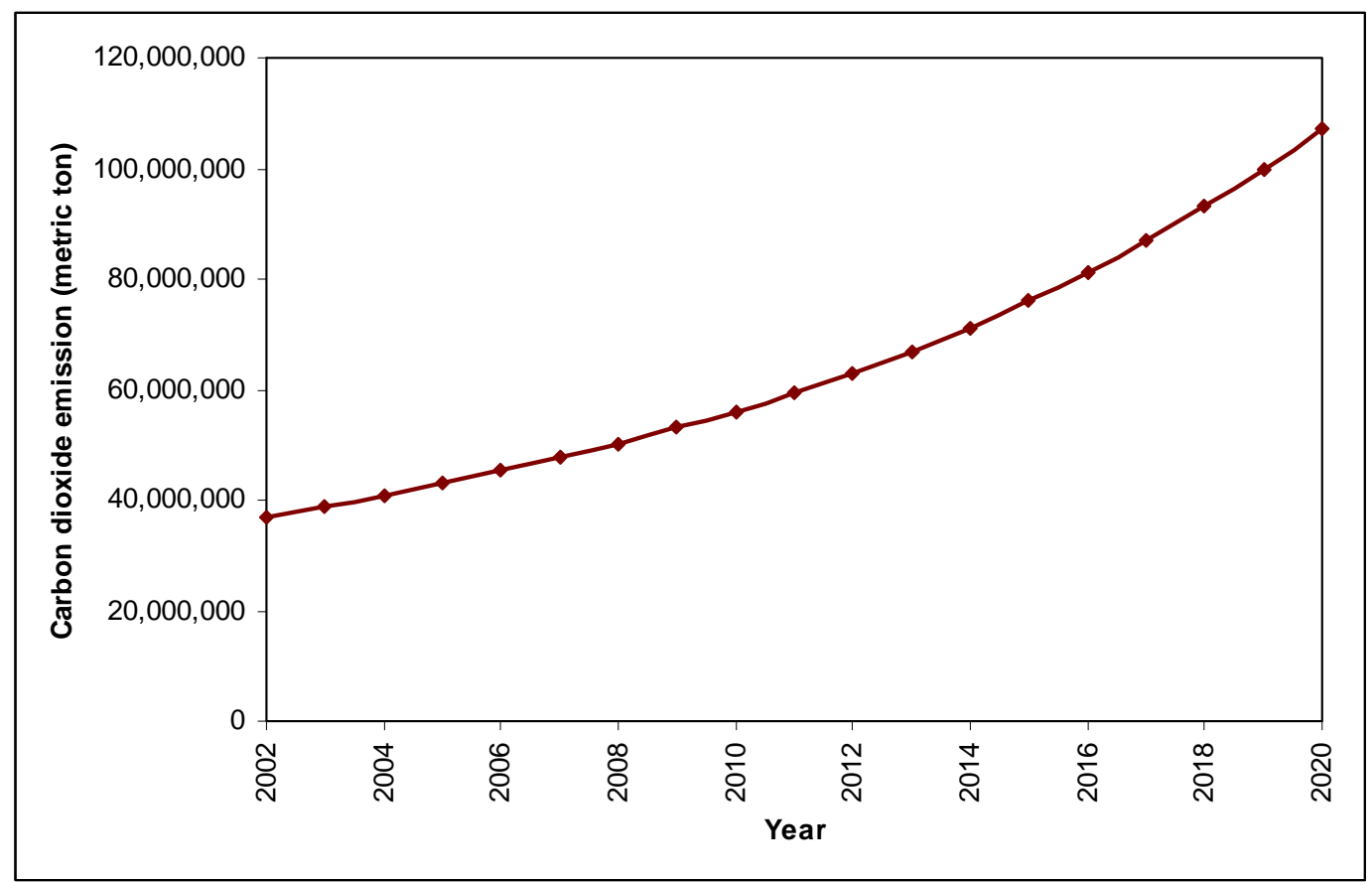

Figure 2. Malaysia's potential $\mathrm{CO}_{2}$ emission by electricity generation (Source data: Mahlia, 2002)

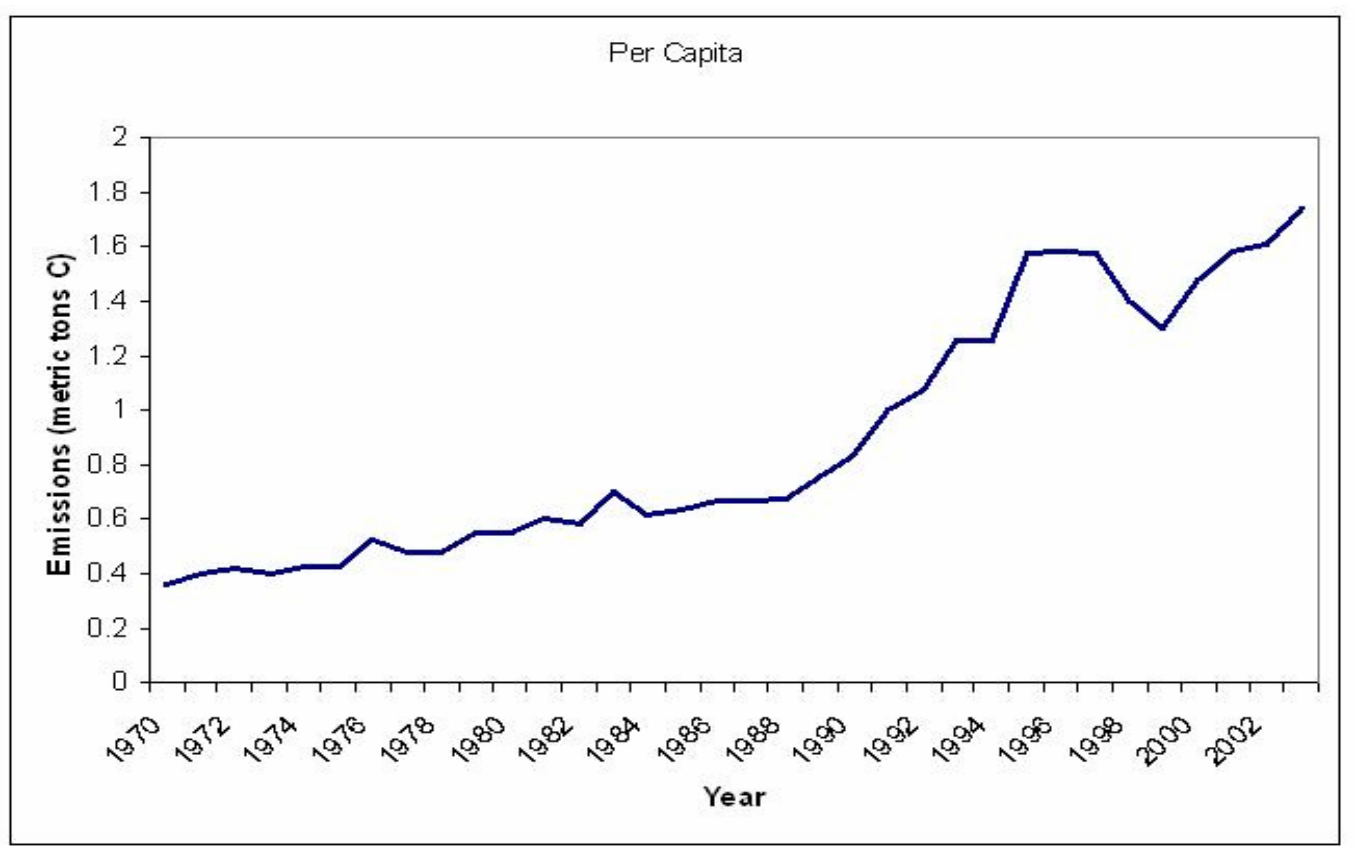

Figure 3. Per capita $\mathrm{CO}_{2}$ emissions estimates for Malaysia

(Source: http://www.cdiac.esd.ornl.gov)

Burning and oxidation of biomass will also exclusively give rise to $\mathrm{CO}_{2}$. Although it is a good alternative as one of the renewable energy sources, the current use of palm oil as biofuel is far from climate neutral. Dependent on several particular assumptions including losses of biogenic carbon associated with ecosystems, estimated emission of $\mathrm{CO}_{2}$ due to the use of fossil fuels and the anaerobic conversion of palm oil mill effluent is approximately 2.8 to $19.7 \mathrm{~kg} \mathrm{CO}_{2}$ equivalent per $\mathrm{kg}$ of palm oil (Reinjnders and Huijbregts, 2006).

Malaysia is still one step behind many countries in having comprehensive and conclusive data regarding the carbon dioxide outlook in the country. This data however, is crucial for Malaysian government in order to come up with appropriate carbon reduction policies. Therefore, further studies should be conducted to obtain such data using the available techniques such as forecasting using fully developed software and/or simulated mathematical models.

\section{III. $\mathrm{CO}_{2}$ EMISSIONS FORECASTING}

Forecasting is defined as an attempt to predict future events. It is also described as a concern of what the future will look like, that act as an aid for effective and efficient planning. There are two categories of forecasting namely qualitative and quantitative. Qualitative forecasting is a very useful technique when there are no historical data. This method usually uses the opinions of experts to predict the future events subjectively via their experiences and knowledge. Quantitative forecasting is a technique that can be applied when there are enough historical data to be 
modeled in making prediction. This technique involves a depth analysis of the historical data in developing a model. There are two types of quantitative techniques that are unvaried and explanatory model. The common time series analysis is normally categorised into both of these types.

Forecasting techniques can be applied to estimate the concentration of $\mathrm{CO}_{2}$ in the atmosphere. To date, different studies have been undertaken to forecast $\mathrm{CO}_{2}$ emissions in future. Kristrom and Lundgren (Kristrom and Lundgren, 2005) projected Sweeden $\mathrm{CO}_{2}$ emission during the period 2000-2010 based on data covering 1900-1999. In their study using Environmental Kuznets Curve, it was reported that $\mathrm{CO}_{2}$ emissions are likely to be lowered in 2010. In Turkey, a linear regression analysis was performed in order to forecast $\mathrm{CO}_{2}$ emissions up to year 2015 (Say and Yucel, 2006). The forecasted emission was modeled by total energy consumption by using the economic and population growth.

Bala (Bala, 2006) studied on computer modeling of the rural energy system and of $\mathrm{CO}_{2}$ emissions for Bangladesh. The software package, "Long-range Energy Alternatives Planning System" (LEAP) has been installed to prepare projection as a tool for planning for sustainable development in Bangladesh. The potential of LEAP model has also been demonstrated by Ghaddar and Mezher (Ghaddar and Mezher, 1999) to compute the final estimates for potential greenhose gaseous emissions in Lebanon.

Other research was done to forecast $\mathrm{CO}_{2}$ emissions in UK transport sector by using DTI Energy Model (UK Energy Research Centre, 2004). It is a partial equilibrium model of the UK energy market and requires a number of assumptions, principally on fossil fuel prices, economic growth and demographics. The relationship between the growth of transport industry and carbon emission has also been study using forecasting technique by Paravanthis and Georgakellos (Paravanthis and Georgakellos, 2007). In their study, these two researchers have developed aggregate car ownership and bus fleet models in order to forecast and compare fuel consumption and $\mathrm{CO}_{2}$ emissions from passenger cars and buses.

A more holistic efficiency index comprising all the relevant indicators simultaneously-population, energy consumption, economic activity and $\mathrm{CO} 2$ emissions-has also been suggested in the literatures (Ramanathan, 2000; 2002). These studies have used a relatively new mathematical programming methodology called Data Envelopment Analysis (DEA) to estimate future level of $\mathrm{CO}_{2}$ emission.

With the use of accurate and validated (current and historical) data, forecasting is a reliable technique to project outlook emission of $\mathrm{CO}_{2}$. The potential $\mathrm{CO}_{2}$ production data is essential especially for policymakers to restrict future emission via appropriate strategies such as economic incentives and more stringent but practical regulations. Looking at the importance of this technique, Malaysia should also move forward and put more concern on forecasting in order to control of future $\mathrm{CO}_{2}$ concentrations in the atmosphere.

\section{CONCLUSIONS}

The recent rapid increase of global carbon dioxide emissions has caused serious concern among policymakers due to its disastrous impacts. Many countries have taken drastic measures to cut down the level of their $\mathrm{CO}_{2}$ emission. Like many countries, Malaysia is also planning to reduce the production of $\mathrm{CO}_{2}$. To do so, current and future concentration of $\mathrm{CO}_{2}$ in Malaysian at atmosphere has to be determined. However, to date, comprehensive data on the production of this gas is still unavailable. Therefore it is imperative to develop a study to estimate the potential production of these gases from variety of sources in Malaysia through forecasting. By having a reliable forecasting technique, the accurate concentration of $\mathrm{CO}_{2}$ in Malaysian at atmosphere can be estimated. This is a prerequisite step to strengthen and assess Malaysia's $\mathrm{CO}_{2}$ baseline data, and to build institutional expertise and adaptation strategies to combat its emission. For instance, the disaster caused by this particular gas can be undermined by reducing the emission from its sources. In addition, control measures and actions can be carried out by the government to curb the $\mathrm{CO}_{2}$ emission.

\section{REFERENCES}

[1] Bala B.K. (1997). "Computer modeling of the rural energy system and of $\mathrm{CO}_{2}$ emissions for Bangladesh.” Energy, 22: 99-1003.

[2] Carbon Dioxide Information Analysis Centre. Available online at the website http://www.cdiac.esd.ornl.gov, accessed on $28^{\text {th }} \mathrm{June}, 2007$.

[3] Chakraborty S., Tiedemann A.V. and Teng P.S. (2000). "Climate change: Potential impact on plant diseases." Environmental Pollution, 108: 317-326.

[4] Earth Policy Institute. Global Temperature Indicator. Available at the website http://www.earth-policy.org/Indicators/Temp/index.htm, accessed on $3^{\text {rd }}$ July 2007.

[5] Ghaddar N. and Mezher. T. (1999). "Modelling of current and future energy intensity and greenhouse gas emissions of the Lebanese industrial sector: assessment of mitigation options." Applied Energy, 63: $53-74$

[6] International Energy Annual (2006), International Energy Agency (IEA), Available online at the website http://www.eia.doe.gov/iea/contents.html, accessed on 28 June 2007.

[7] Kessel D.G. (2000). "Global warming - facts, assessment, countermeasures." Journal of Petroleum Science and Engineering, 26: $157-168$

[8] Krinstrom B. and Lundgren. (2005). "Swedish CO2 emissions 19002010: an exploratory note." Energy Policy, 33: 1223-1230.

[9] Mahlia T. M. I (2002). "Emission from electricity generation in Malaysia, Renewable Energy, 27: 293-300.

[10] Paravanthis G. A. and Georgakellos D. A. (2007). "Trends in energy consumption and carbon dioxide emission of passengers cars and buses", Technological Forecasting \& Social Change, 74: 682-707.

[11] Ramanathan R. (2002). "Combining indicators of energy consumption and $\mathrm{CO}_{2}$ emissions: a cross-country comparison". Int. J.Glob. Energy Issues, 3: 214-227.

[12] Ramanathan R (2000). "A holistic approach to compare energy efficiencies of different transport modes", Energy Policy, 11: 743747.

[13] Reijnders L. and Huijbregts M. A. J. (2006). "Palm oil and the emission of carbon-based greenhouse gases". Journal of Cleaner Production, doi:10.1016/j.jclepro.2006.07.054.

[14] Say N.P. and Yucel M. (2006). Energy Consumption and $\mathrm{CO}_{2}$ emissions in Turkey: empirical analysis and future projection based on an economic growth." Energy Policy, 34: 3870-3876.

[15] United Nations Framework on Climate Change (UNFCC), 1992 Available online at the website http://unfecc.int/resource/docs/convkp/conveng.pdf, accessed on $3^{\text {rd }}$ July 2007.

[16] UK Energy Research Centre (2004) "UK Transport Policy and $\mathrm{CO}_{2}$ Emissions". The DTI updated emissions projections. Available online at the website http://www.ukerc.ac.uk/index.php?option=com_docman\&task=doc_d ownload\&gid=229, accessed on $31^{\text {st }}$ July 2007.

[17] "MALAYSIA: HigHEST PER CAPITAL EMISSIONS AFTER SINGAPORE, BRUNEI.” NEWS STRAits TiMES (2002). 


\title{
The Effect of Ignition and Injection Timings on Compressed Natural Gas Direct Injection (CNGDI) Multi-cylinder Engine
}

\author{
M.K. Hassan, I. Aris, S.Mahmod, R. Sidek, K. N. Zainuddin, N.A. Alina, H.H. Masjuki, \\ M. Redzuan and M.A. Kalam
}

\begin{abstract}
This paper presents the effects of varying the ignition and injection timings of multi-cylinder compressed natural gas direct injection engine (CNGDI). Experimental tests were carried out at Universiti Malaya with computercontrolled dynamometer to measure the CNGDI engine performance. The ignition and injection timings were varied to investigate the effect of these two parameters towards engine performance. The ignition and injection angles were varied with respect to engine speed (rpm), at different combination of ignition and injection angles. The ignition angle was varied in between 23 degrees up to 32 degrees. The injection angle was varied in between 220 degrees until 360 degrees. A closed loop wide band lambda sensor was attached at the exhaust manifold to indicate the oxygen level of the combustion. The engine performance in terms of brake power and torque were monitored and the best combination of timing was identified for each speed. The experimental results indicated that the CNGDI engine requires advanced ignition up to 30 degrees before top dead centre (BTDC) to achieve maximum torque. The injection angle at 300 degree BTDC has shown a good engine performance for each speed.
\end{abstract}

Keywords: compressed natural gas, high compression engine, CNGDI engine, direct injection

\section{INTRODUCTION}

In Malaysia, the number of natural gas vehicles has shown some increments since it was introduced in 1998.

The authors would like to thank the Ministry of Science, Technology and Innovation of Malaysia for giving the research grant to the CNGDI team under IRPA research grant. Special thank also goes to Universiti Malaya, Kuala Lumpur for providing test facilities. The authors also would like to thank the rest of the CNGDI team members for their contributions toward this project.

I. Aris is a Deputy Director of the Institute of Advanced Technology, Universiti Putra Malaysia. He is an Associate Prof. at the Dept. of Electrical and Electronics Engineering, Faculty of Engineering. (phone : 6-0389467531 fax: 6-03-86566061 email : ishak@eng.upm.edu.my)

M. K. Hassan, S. Mahmod, R. Sidek are with the Dept. of Electrical and Electronics Engineering, Faculty of Engineering, UPM.

K. N. Zainuddin and N. A. Alina are research assistants at the Institute of Advanced Technology, UPM.

H. H. Masjuki is a Professor at Dept. of Mechanical Engineering, Faculty of Engneering, Universiti Malaya, Kuala Lumpur, M. Redzuan and M. A. Kalam are research assistants at Tribology and Engine Laboratory, Dept. of Mechanical Engineering, Universiti Malaya.
The exemption of natural gas duty, import and sales duty on the conversion kits and the reduction of road tax up to $25 \%$ are reflected of the government support. However, the number of conversion vehicles until present day are not encouraging. These are due to the feedbacks that conversions vehicles are lack of power, difficult engine cranking in the morning, bulky storage tank and a small number of refueling station available nationwide.

Compressed Natural Gas (CNG) is a substitute for gasoline (petrol) or diesel fuel. It is considered to be an environmentally "clean" alternative to those fuels. It is made by compressing methane $\left(\mathrm{CH}_{4}\right)$ extracted from the natural gas. It is stored and distributed in hard containers, usually cylinders. Malaysia has abundant local natural gas reserve approximately 82.5 trillion standard cubic feet, as at January 1, 2005. PETRONAS, the National Oil Corporation has been championing the development of Natural Gas for Vehicles (NGV) in the country. The key driving factors for pushing NGV are energy utilization and environment. Argentina is leading the pack for NGV user while Malaysia ranked amongst the top 20 worldwide as shown in 'figure 1 '.

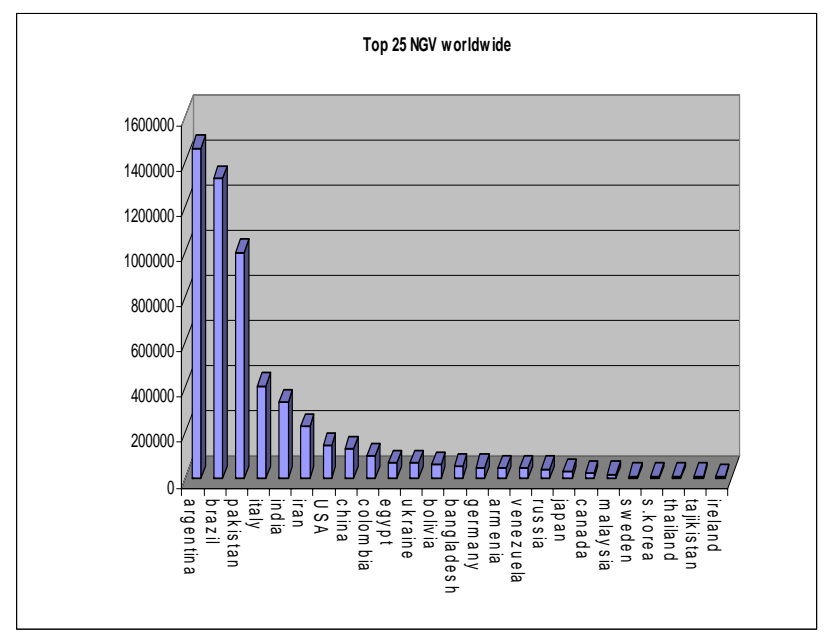

Figure 1. Top $25 \mathrm{NGV}$ worldwide

However, when a refueling station factor is considered, Malaysia ranked sixth place after Japan with a wide gap of refueling station compared to the number of NGV users. In Malaysia, it has been reported that only 46 refueling station 
compared with 19,000 of NGV users however, Japan has 311 refueling stations to serve for 26408 of NGV users. The data illustrated in 'figures 2 and 3', have shown unpleasant situation in Malaysia where the ratio of refueling stations and NGV users is very wide. Thus, the number of conversion vehicles until present day are not encouraging. These are due to the feedbacks that conversions vehicles are lack of power, difficult engine cranking in the morning, bulky storage tank and a small number of refueling station available nationwide.

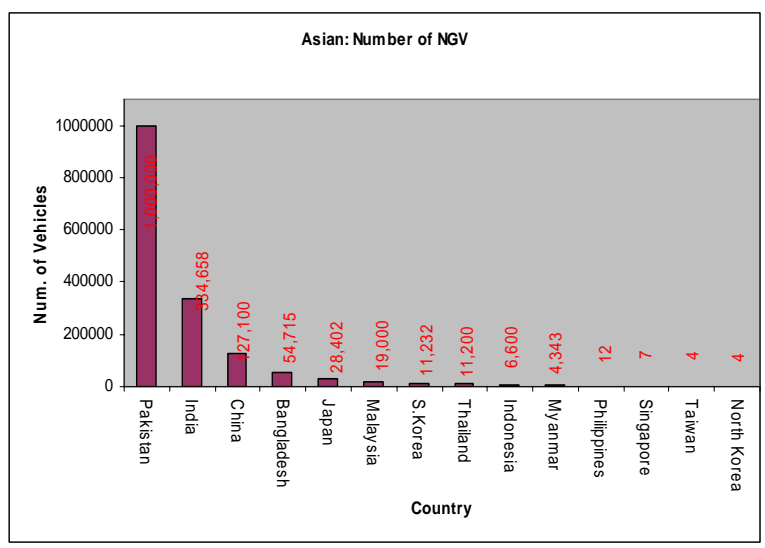

Figure 2. Asian region of NGV users

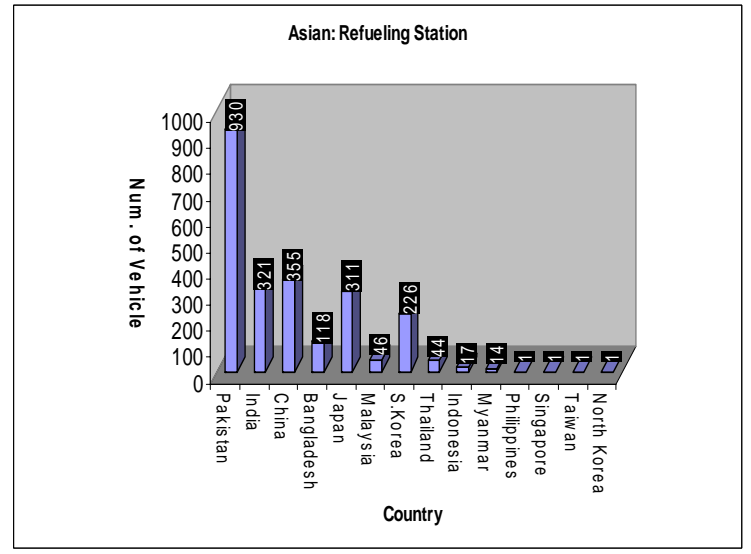

Figure 3. Asian region of NGV refueling stations

Natural gas engine has been acknowledged for having an almost zero emission but lacked of power and torque as reported by researchers worldwide [2]-[7].

Two direct injection methods have been introduced and implemented on gasoline and diesels engines, which are central injection and side injection. That means fuel is injected directly into the cylinder either at the central of the cylinder or at the side of the cylinder. The potential and the different between the two methods are shown in 'figure 4'.

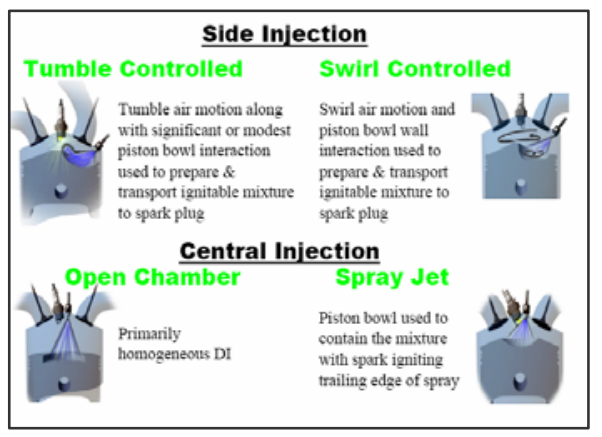

Figure 4. Side injection vs. central injection

However, this particular research would focus on CNGDI central injection system only. As reported by Zuohua Huang [8] who has investigated the CNGDI using rapid compression machine, $\mathrm{CNG}$ direct injection combustion maintains the combustion efficiency more than 0.92 in the range of equivalence ratio from 0.1 to 0.9 . Thus, CNGDI is considered to have much potential to be used in a sparkignition engine on the basis of wide operation range of and high energy conversion efficiency.

The initial result has shown wide spray angle, highpressure injection has given good and stable combustion [9]. The arrangement of CNGDI at multi-cylinder is shown in 'figure 5'.

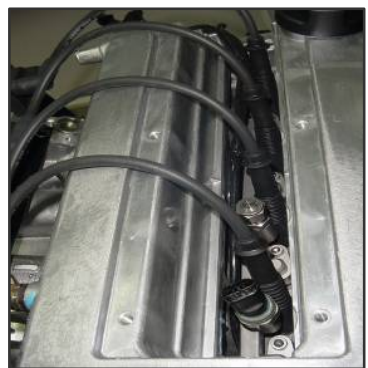

Figure 5. Multi-cylinder engine CNGDI

\section{EXPERIMENTAL SETUP}

The initial result on the single cylinder engine has shown the correlation of torque and power of the engine with the ignition angle. This experiment was carried out at Universiti Teknologi Petronas, Tronoh, Perak. In addition to mechanical approaches used to enhance the engine performance, the engine management system and its calibration technique are other important factors need to be considered. By setting the ignition angle and injection timings properly, the improvement in the engine performance and emission level could be achieved [10], [11].

The experimental test was conducted at the Laboratory of Tribology and Engine, University of Malaya, Kuala Lumpur.

The CNGDI engine was tested in every engine speed with 1000 speed interval, starting from 1000rpm up to $6200 \mathrm{rpm}$. The dynamometer being used is an eddy current typed rated $10,000 \mathrm{rpm}$ and $500 \mathrm{Nm}$. The engine dynamometer controller is calibrated by CP Engineering, United Kingdom. The dynamometer being used is an eddy current typed rated 10,000 rpm and $500 \mathrm{Nm}$. Engine dyno was setup to run the engine from $1000 \mathrm{rpm}$ to $6200 \mathrm{rpm}$ while the throttle opening is $100 \%$. Three tests were conducted continuously and the results of the overall tests were averaged. 'Figures 6 and 7', show the dyno set-up with intake temperature was controlled at 27 degrees Celsius. 


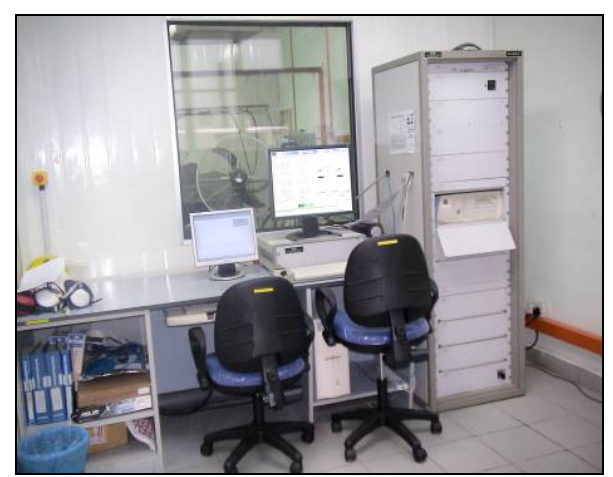

Figure 6. Dynamometer and control room

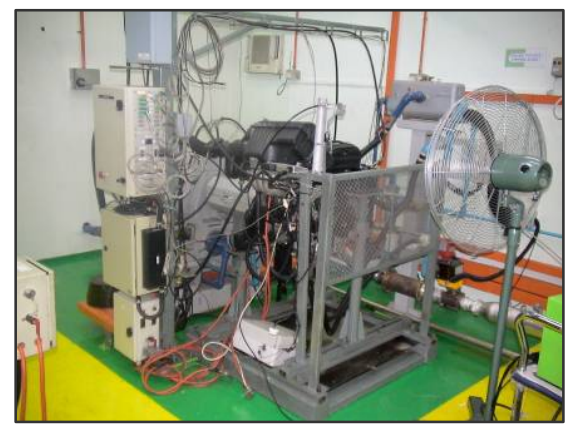

Figure 7: Dynamometer setup

Table 1. CNGDI Engine Specification.

\begin{tabular}{|l|c|}
\hline \multicolumn{1}{|c|}{ Description } & CNGDI \\
\hline Bore x Stroke (mm) & $76 \times 88$ \\
\hline Displacement (cc) & 1597 \\
\hline $\begin{array}{l}\text { Number of } \\
\text { Cylinder (s) }\end{array}$ & 4 \\
\hline Compression Ratio & 14 \\
\hline Combustion Chamber & Bowl \\
\hline IVO ( bTDC) & $12^{0}$ \\
\hline IVC (aBTD) & $48^{0}$ \\
\hline EVO (bBDC) & $45^{0}$ \\
\hline EVC (aTDC) & $10^{0}$ \\
\hline Fuel System & CNGDI \\
\hline Fuel Pressure (bar) & 20 \\
\hline Valve Train & $\begin{array}{l}\text { DOHC 16V \& 4 } \\
\text { cylinders in-line }\end{array}$ \\
\hline
\end{tabular}

The experimental results of the single cylinder CNGDI engine have shown that the homogeneous piston crown as in 'figure 8', produces a better performance of torque and power. Therefore, for multi-cylinder engine homogeneous piston crown was used to investigate the performance of CNGDI engine.

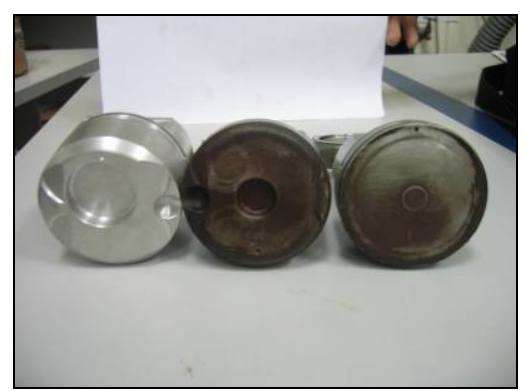

Figure 8 . Homogeneous piston is at the middle

\section{EXPERIMENTAL RESULTS}

A comparative study was conducted to investigate the potential of CNGDI engine fueled with natural gas and CamPro 1.6L fueled with gasoline. The results shown that the CNGDI engine performance is only about 5\% lower than the CamPro 1.6L engine. 'figures 9 and 10' show the comparison of the potential of CNGDI engine and CamPro 1.6L engine.

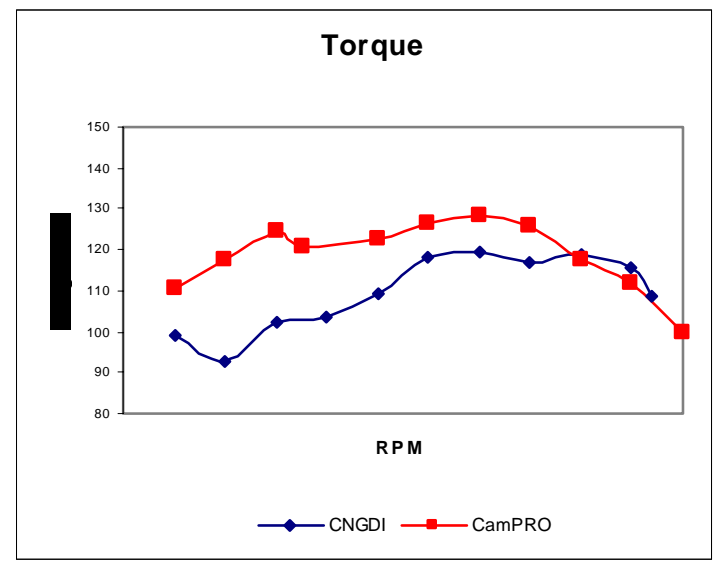

Figure 9. Torque comparison

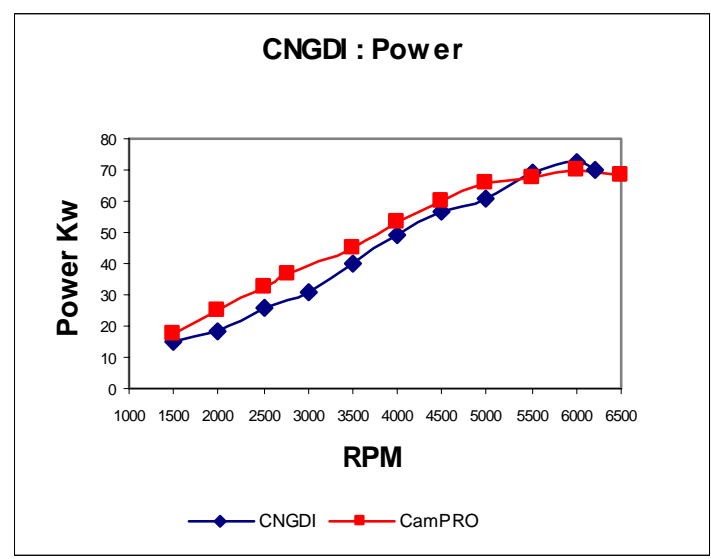

Figure 10. Power comparison

The CNGDI engine produced maximum torque of 142.86 $\mathrm{Nm}$ at $4500 \mathrm{rpm}$ compared to $136 \mathrm{Nm}$ for CamPro $1.6 \mathrm{~L}$ at the same engine speed. It shows that an average of $5 \%$ torque increment at all range of engine speed. The power produced by CNGDI engine is also has shown an increment of about $4.3 \%$ at high $\mathrm{rpm}$. The maximum power for CNGDI engine is $81.59 \mathrm{KW}$ at $6000 \mathrm{rpm}$ and $78 \mathrm{KW}$ for CamPro $1.6 \mathrm{~L}$ at the same speed.

The effect of ignition and injection were investigated to obtain a good engine performance at $3000 \mathrm{rpm}$. In this particular example, only two parameters will be discussed, which is torque and power.

'Figure 1' shows the effect of ignition timing on CNGDI engine. The ignition timing is varied from $23^{\circ} \mathrm{bTDC}$ to $33^{\circ}$ bTDC and the brake torque and brake power is monitored. The best ignitions for $3000 \mathrm{rpm}$ lie between $26^{\circ}$ to $30^{\circ}$ bTDC that would produce an average torque at $109.38 \mathrm{Nm}$. The maximum torque occurs at $28^{0}$, which produces $110.13 \mathrm{Nm}$. The brake power maximum is $34.93 \mathrm{KW}$ at $28^{0}$ and an average of $34.68 \mathrm{KW}$ if the ignition angles lie between $26^{\circ}$ to $30^{\circ} \mathrm{bTDC}$. 


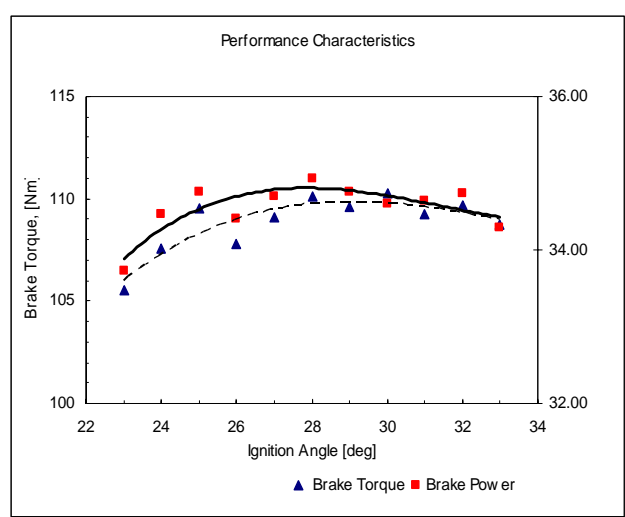

Figure 11. Ignition timing at $3000 \mathrm{rpm}$

The injection timing is also varied between $230^{\circ} \mathrm{bTDC}$ until $350^{\circ}$ bTDC and the engine performance is shown in 'figure 12'. The best end-of-injection timing lies between $280^{\circ} \mathrm{bTDC}$ and $320^{\circ} \mathrm{bTDC}$. An average torque is $96.8 \mathrm{Nm}$ and $30.7 \mathrm{KW}$ between $280^{\circ}$ bTDC and $320^{\circ}$ bTDC.

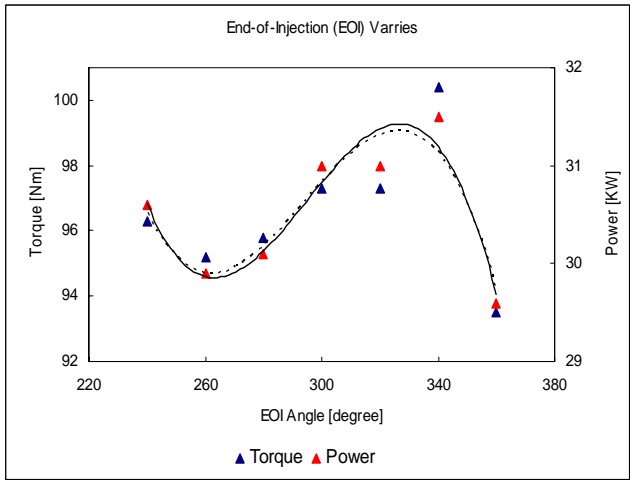

Figure 12. Injection timing at $3000 \mathrm{rpm}$

The right combination of ignition and injection timing would determine the engine potential. For calibration purposes, each ignition and injection timing would be calibrated to find maximum brake torque for the engine at each engine speed.

\section{SUMMARY}

The CNGDI engine has produced good engine performance compared to CamPro1.6L engine, which runs on gasoline. Furthermore, there are rooms for improvement to enhance the engine performance. The CNGDI is best suited with high-pressure injection. The best ignition timing at $3000 \mathrm{rpm}$ for CNGDI engine is between $26^{\circ}$ to $30^{\circ} \mathrm{bTDC}$. And best start of injection timing is between $280^{\circ}$ to $320^{\circ}$ bTDC.

\section{ACKNOWLEDGMENT}

The author would like to thank Ministry of Science, Technology and Innovation of Malaysia for the grant for this research under IRPA research grant. Special thank also goes to Universiti Malaya, Kuala Lumpur for providing test facility.

\section{REFERENCES}

[1] International Association for Natural Gas Vehicles, (ANGVA)http://www.iangv.org/content/view/17/35/ visited on 20 June 2007.

[2] Robert W. Weeks, John J. Moskwa, "Transient Air Flow Rate Estimation in a Natural Gas Engine Using a Nonlinear Observer", SAE 940759.

[3] Peter Meck1, Anupam Gangopadhyay, "Modeling and Validation of a Lean Burn Natural Gas Engine", Journal of Dynamic Systems, Measurement and Control SEPTEMBER 2001, Vol. 123.

[4] Nigel N. Clark, Gregory E. Mott, Christopher M. Atkinson, Remco J. deJong, Richard J. Atkinson, Tim Latvakosky, Michael L. Traver, "Effect of Fuel Composition on the Operation of a Lean-Burn Natural Gas Engine”, SAE 952560.

[5] Akinori Miura, Akira Nakamura, Takayuki Tsuchiya, "Development of Stratified Charged Combustion CNG Engine", Fumitaka Honjo, Technical Report Nissan Diesel Motor Co., Ltd.

[6] Tony Middleton, Barry Neumann, "The International Benchmark For Natural Gas Engines - Performance And Emissions Is Achievable", 8th International and 20th National Conference and exhibition on Natural Gas Vehicles.

[7] Haeng Muk Cho, Bang-Quan He, "Spark ignition natural gas engineA review", Energy Conversion and Management 48 (2007) 608-618, Elsevier.

[8] Zuohua Huang, Seiichi Shiga, Takamasa Ueda, Nobuhisa Jingu, Hisao Nakamura,Tsuneaki Ishima, Tomio Obokata, Mitsuhiro Tsue And Michikata Kono, "Feasibility of CNG DI Stratified Combustion Using a Spark-Ignited Rapid Compression Machine", The Fifth International Symposium On Diagnostics And Modeling Of Combustion In Internal Combustion Engines (Comodia 2001), July 14, 2001, Nagoya

[9] Yusoff A, Muthana I. "Design Of High Pressure Fuel System For CNGDI", ATCi 2005, Conference on Automotive Technology, Dec 68, 2005, Putrajaya, Malaysia

[10] I Aris, M.K. Hassan,I.Mat,Nizam,N.A.Alina,R.K.Zakiah,R.Sidek, S.Mahmood, "Model-Driven Electronic Control Unit with Real-Time Embedded Target", Engineering Transactions, Mahanakorn University of Technology, Thailand.,Vol.8,No.1(18),Jan-June 2005.

[11] Mohd Khair Hassan, Ishak Aris, Roslina Sidek, Senan Mahmod, "Model-Based Approach with Real Time Embedded Target for Rapid ECU Development: An Overview", JIEEEC 2005,15-17 Nov,2005. Amman, Jordan. 


\title{
Experimental Tests and Simulations Of Compressed Natural Gas Direct Injection (CNGDI) Single Cylinder Engine
}

\author{
I. Aris, M.K. Hassan, S. Mahmod, R. Sidek, K.N. Zainuddin, N.A. Alina, H.H. Masjuki, \\ M. Redzuan and M.A. Kalam
}

\begin{abstract}
This paper presents the experimental and simulation results of a single cylinder high compression compressed natural gas engine implementing a direct injection technology (CNGDI). This initial experiment on a single cylinder CNGDI engine is to investigate the feasibility of the CNGDI engine. The experimental work was conducted at Universiti Teknologi Petronas, Tronoh, Perak. The objective of this initial experiment is to investigate the interaction of ignition timing and injection timing towards engine performance. The ignition angles were varied while the engine brake power and brake torque were monitored. The injection angles were also varied in between 120 BTDC to 220 BTDC at different engine speeds. Two different types of combustion namely homogeneous and stratified for CNGDI were carried out. The experimental results have shown that the ignition timing has to be advanced and the best injection timing is at 120 BTDC to 180 BTDC would achieve a good performance. For homogeneous charge combustion operation engine, the injection timing is set at early of compression stroke to ensure the better fuel/air mixing. For stratified charge operation combustion engine, the injection timing is at the late compression stroke.
\end{abstract}

Keywords: high compression engine, compressed natural gas, direct injection and single cylinder

\section{INTRODUCTION}

Natural gas engine has been acknowledged for having an

The authors would like to thank the Ministry of Science, Technology and Innovation of Malaysia for giving the research grant to the CNGDI team under IRPA research grant. Special thank also goes to Universiti Malaya, Kuala Lumpur and Universiti Teknologi Petronas, Perak for providing engine dynamometers. The authors also would like to thank the rest of the CNGDI team members for their contributions toward this project.

I. Aris is a Deputy Director of the Institute of Advanced Technology, Universiti Putra Malaysia. He is an Associate Prof. at the Dept. of Electrical and Electronics Engineering, Faculty of Engineering. (phone : 6-0389467531 fax: 6-03-86566061 email : ishak@eng.upm.edu.my)

M.K. Hassan, S. Mahmod, R. Sidek are with the Dept of Electrical and Electronics Engineering, Faculty of Engineering, UPM.

K.N. Zainuddin and N. A. Alina are research assistants at the Institute of Advanced Technology, UPM.

H.H. Masjuki is a Professor at Dept. of Mechanical Engineering, Faculty of Engneering, Universiti Malaya, Kuala Lumpur, M. Redzuan and M.A.Kalam are research assistants at Tribology and Engine Laboratory, Dept. of Mechanical Engineering, Universiti Malaya. almost zero emission but lacked of power and torque as reported by researchers worldwide [1]-[5]. Haeng Muk Cho [6] has reported a comprehensive review of the spark ignition natural gas engine. He has concluded that to keep the output power and torque of natural gas engine comparable to those of their gasoline or diesel counterparts, high boost of pressure should be used. High activity of catalyst for methane oxidation and lean NOx system or three-way catalyst with precise air-fuel ratio control strategies should be developed to meet future stringent emission standards.

For the CNGDI engine, the compression ratio is increased to 14 because the property of $\mathrm{CNG}$ can withstand higher compression ratio. Fuel is injected at the central of the cylinder with 20-bar pressure. Yusoff A and Muthana I. [7] have verified the potential of high-pressure injection of $\mathrm{CNG}$ direct into the cylinder in their paper. The results shown in 'figures 1 and 2 ' indicate that high-pressure injection will produce a higher torque and power compare with low pressure-injection. It also has an advantage of low fuel consumption.

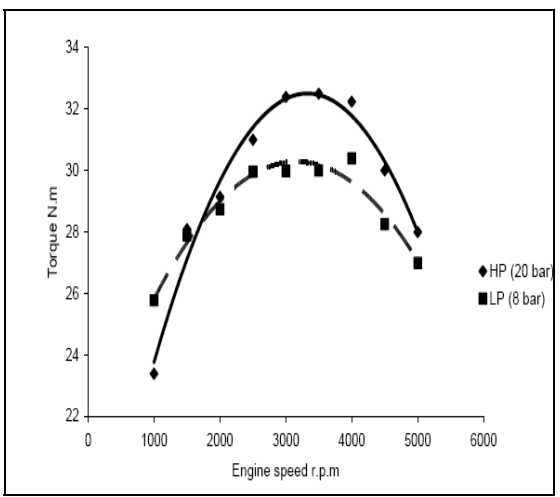

Figure 1. Variation of engine torque versus engine speed for two fuel injection types [7] 


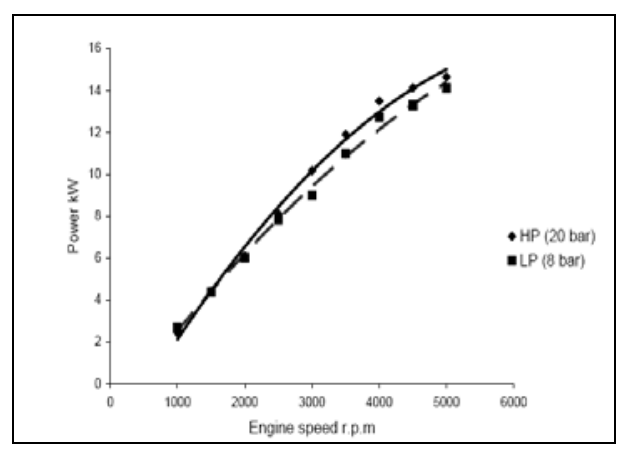

Figure 2. Variation of engine power versus engine speed for two fuel injection types [7]

The initial result has shown wide spray angle has given good and stable combustion. The arrangement of the spark and injector is shown in 'figure 3 ' with $30^{\circ}$ degrees gap.

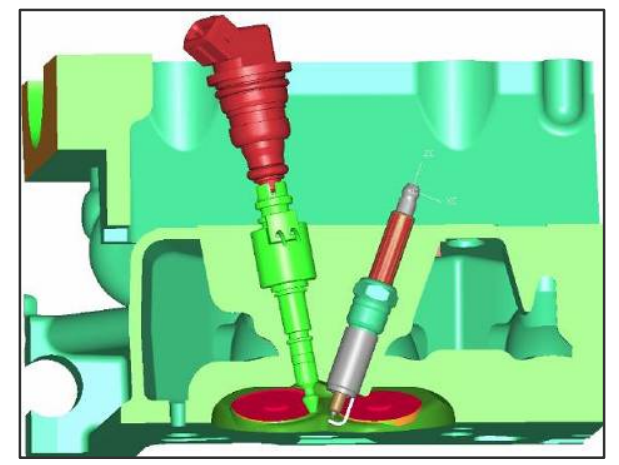

Figure 3. Central injection concept

The initial result on the single cylinder engine has shown the correlation of the torque and power of the engine with the ignition angle. In addition to mechanical approaches used to enhance the engine performance, the engine management system and its calibration technique are other important factors need to be considered. By setting the ignition angle and injection timings properly, the improvement in the engine performance and emission level could be achieved [8], [9].

\section{EXPERIMENTAL SETUP}

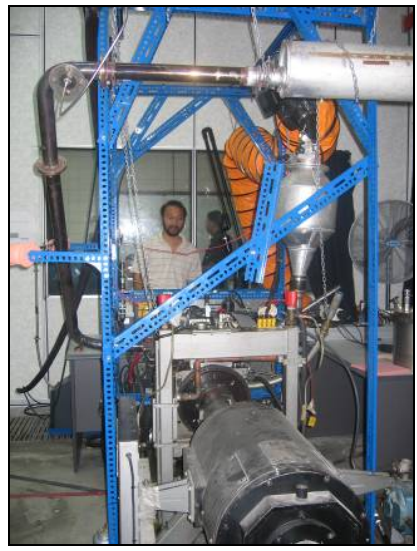

(a)

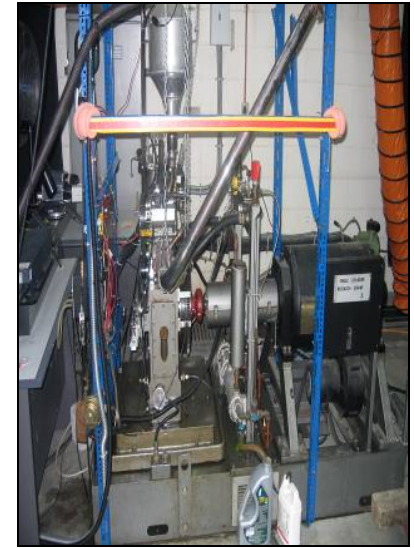

(b)
Figure 4. CNGDI single cylinder engine experimental setup; (a) full view (b) side view

The experimental setup of the single cylinder CNGDI engine is shown in 'figures 4 (a) and (b)'. This experimental work was carried out at Universiti Teknologi Petronas, Tronoh, Perak. The laboratory consists of a control room to monitor the engine operation and one room for the engine under testing. A 20bar pressure is applied to the injector, which is located at the centre of the cylinder. The engine compression ratio is $14: 1$ and the engine specification is depicted in 'table 1 '.

Table 1. Specification of the engine model

\begin{tabular}{|c|c|}
\hline Engine parameters & Value \\
\hline Bore $\times$ Stroke $(\mathrm{mm})$ & $78 \times 84$ \\
\hline Connecting rod length $(\mathrm{mm})$ & 131 \\
\hline Displacement $(\mathrm{cm})$ & $1596(4$ Cylinders - \\
& Inline $)$ \\
\hline Compression ratio & $14: 1$ \\
\hline Engine speed $(\mathrm{rpm})$ & 2000 \\
\hline Intake valve open $\left({ }^{\circ} \mathrm{CA}\right)$ & $12^{\circ}$ before TDC \\
\hline Intake valve close $\left({ }^{\circ} \mathrm{CA}\right)$ & $48^{\circ}$ after BDC \\
\hline Exhaust valve open $\left({ }^{\circ} \mathrm{CA}\right)$ & $45^{\circ}$ before BDC \\
\hline Exhaust valve close $\left({ }^{\circ} \mathrm{CA}\right)$ & $10^{\circ}$ after TDC \\
\hline
\end{tabular}

Two different piston crowns were tested to investigate the combustion effects in the cylinder. The homogeneous and stratified piston crowns that were used in the test are shown in 'figures 5 and 6'.

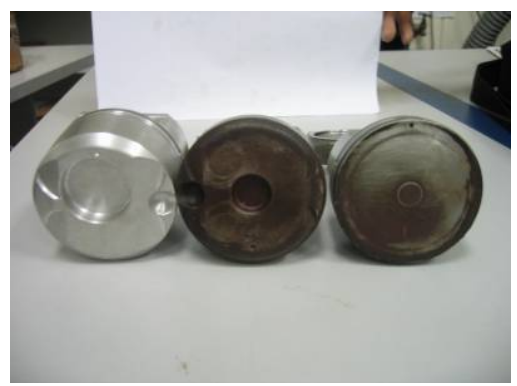

Figure 5. Series of piston crown under test

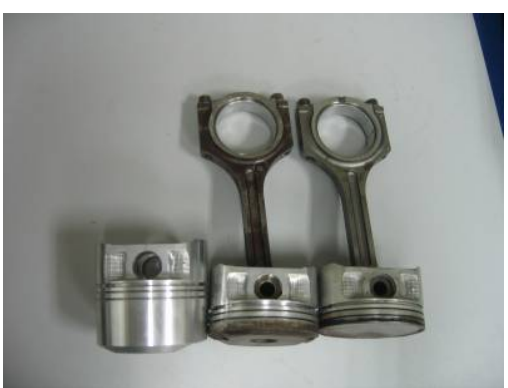

Figure 6 . The length of piston crown under test

\section{EXPERIMENTAL RESULTS}

A comparison of two different piston crowns as shown at 'figure 5', was investigated to identify the potential of each piston crown. The homogeneous crown has produced a better engine performance compared with the stratified piston crown as shown in 'figure 6'. The maximum torque is $31.8 \mathrm{Nm}$ at $3000 \mathrm{rpm}$, compared to $24.65 \mathrm{Nm}$ for the stratified crown. Thus, the torque different is $7.15 \mathrm{Nm}$ for both piston. For stratified crown, the torque is drastically decreased after $3000 \mathrm{rpm}$. 


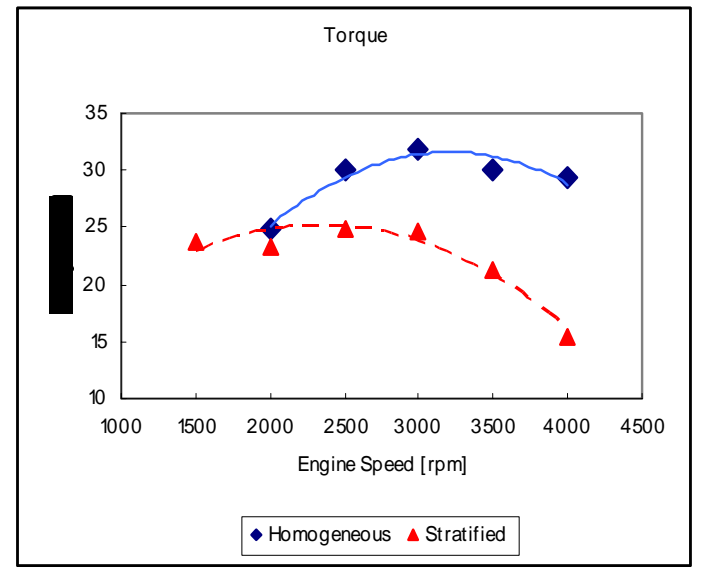

Figure 7: Torque Comparison

In terms of brake power as illustrated in 'figure 8', the maximum power is $12.29 \mathrm{KW}$ at $4000 \mathrm{rpm}$ for homogeneous and $6.5 \mathrm{KW}$ at $2500 \mathrm{rpm}$ for stratified crown. This indicates that the stratified crown produces good engine performance at low speed.

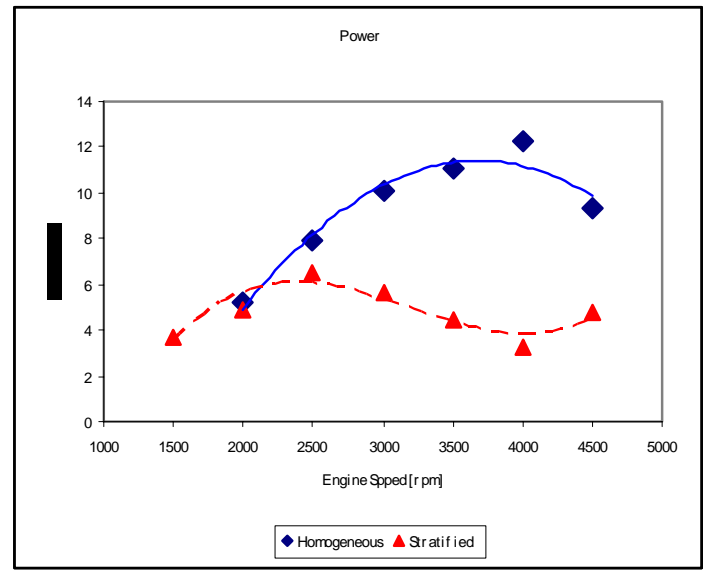

Figure 8. Power Comparison.

'Figures 9 and 10' are another examples of the characteristic of engine performance for each piston crown. Injection timing was varied at $2000 \mathrm{rpm}$ and ignition angle is fixed at $24^{0}$ bTDC. This experiment is to investigate the best injection timing at each engine speed. The test was conducted for all engine speeds starting from $1500 \mathrm{rpm}$ to $4000 \mathrm{rpm}$ and the engine performance is monitored. This particular example is $2000 \mathrm{rpm}$ with fixed $24^{\circ}$ ignition angle. The torque curve for each piston crown at various injection timing is illustrated in 'figure 9'. From the figure, it shows that the torque decreases as the late injection is applied. The best start of injection timing is between $120^{\circ}$ bTDC to $180^{\circ}$ bTDC. However, for the stratified piston crown, the torque different is not so obvious. It seems that the injection timing might not influence the torque curve very much.

The power curve also has shown a decrement of power when late injection is implemented as depicted in 'figure 10 '.

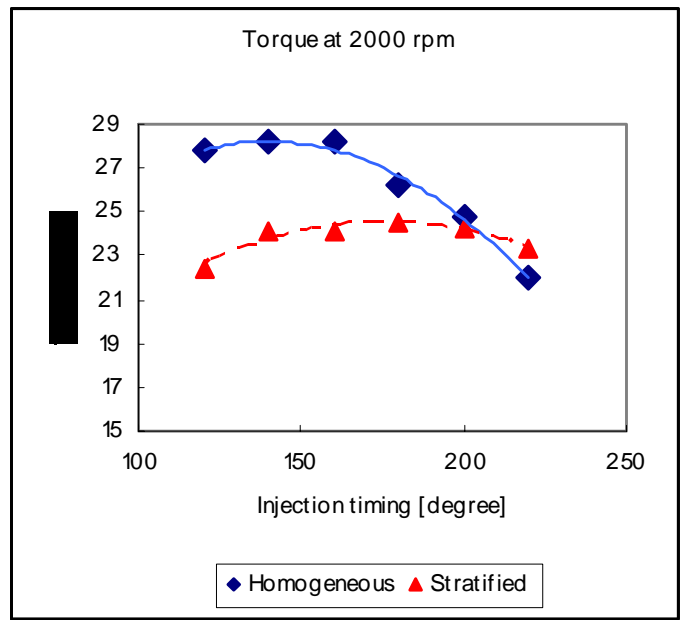

Figure 9. Torque Comparison at $2000 \mathrm{rpm}$

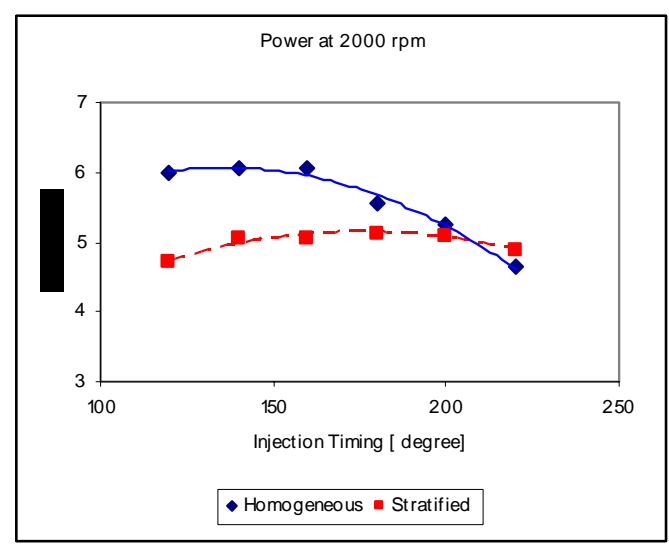

Figure 10: Power Comparison at $2000 \mathrm{rpm}$.

Simulation work was conducted using Lotus Simulation package to verify the CNGDI engine behavior. In this simulation package, the ignition timing is already predefined. The results are shown in 'figures 11 and 12', respectively. The simulation results were used as the guidelines for the experimental work.

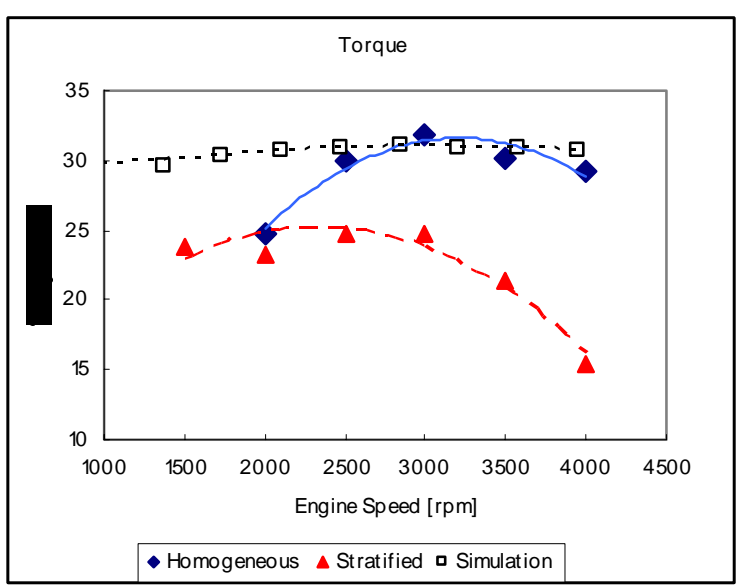

Figure 11. Comparison of torque for homogeneous, stratified and simulation

The results from experimental and simulation works of the single cylinder CNGDI engine were used to design, fabricate and calibrate the multi cylinder CNGDI engine. 


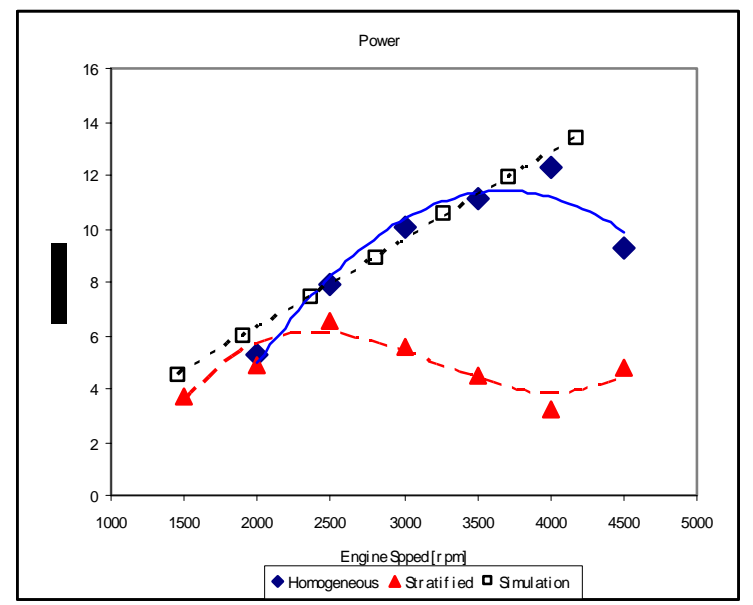

Figure 12. Comparison of power for homogeneous, stratified and simulation

\section{CONCLUSION}

The CNGDI is best suited with high-pressure injection. The experimental and simulation results demonstrate the potential of the homogenous crown compares with the stratified crown. In terms of engine performance, the homogenous crown has shown higher torque and higher power compare to that of the stratified piston crown.

\section{ACKNOWLEDGMENT}

The authors would like to thank the Ministry of Science, Technology and Innovation of Malaysia for the research grant to this project. Special thank also goes to Universiti Malaya, Kuala Lumpur and Universiti Teknologi Petronas, Perak for providing test facilities.

\section{REFERENCES}

[1] Robert W. Weeks, John J. Moskwa, "Transient Air Flow Rate Estimation in a Natural Gas Engine Using a Nonlinear Observer", SAE 940759.

[2] Peter Meckl, Anupam Gangopadhyay, "Modeling and Validation of a Lean Burn Natural Gas Engine", Journal of Dynamic Systems, Measurement and Control SEPTEMBER 2001, Vol. 123.

[3] Nigel N. Clark, Gregory E. Mott, Christopher M. Atkinson, Remco J. deJong, Richard J. Atkinson, Tim Latvakosky, Michael L. Traver, "Effect of Fuel Composition on the Operation of a Lean-Burn Natural Gas Engine”, SAE 952560.

[4] Akinori Miura, Akira Nakamura, Takayuki Tsuchiya, "Development of Stratified Charged Combustion CNG Engine", Fumitaka Honjo, Technical Report Nissan Diesel Motor Co., Ltd.

[5] Tony Middleton, Barry Neumann, "The International Benchmark For Natural Gas Engines - Performance And Emissions Is Achievable", 8th International and 20th National Conference and exhibition on Natural Gas Vehicles.

[6] Haeng Muk Cho, Bang-Quan He, "Spark ignition natural gas engineA review", Energy Conversion and Management 48 (2007) 608-618, Elsevier.

[7] Yusoff A, Muthana I. "Design Of High Pressure Fuel System For CNGDI", ATCi 2005, Conference on Automotive Technology, Dec 68, 2005, Putrajaya, Malaysia

[8] I Aris, M.K. Hassan,I.Mat,Nizam,N.A.Alina,R.K.Zakiah,R.Sidek, S.Mahmood, "Model-Driven Electronic Control Unit with Real-Time Embedded Target", Engineering Transactions, Mahanakorn University of Technology, Thailand.,Vol.8,No.1(18),Jan-June 2005.

[9] Mohd Khair Hassan, Ishak Aris, Roslina Sidek, Senan Mahmod, "Model-Based Approach with Real Time Embedded Target for Rapid ECU Development: An Overview”, JIEEEC 2005,15-17 Nov,2005. Amman, Jordan. 


\title{
Electricity Planning Under $\mathrm{CO}_{2}$ Mitigation: A Canadian Case Study
}

\author{
H. Hashim, Peter Douglas, Ali Elkamel and Eric Croiset
}

\begin{abstract}
In this study, the focus is to investigate a promising way to reduce greenhouse gases (essentially $\mathrm{CO}_{2}$ ) from the electricity generation sector, particularly in Ontario, Canada. An optimization model for Ontario Power Generation (OPG) fleet to meet a given $\mathrm{CO}_{2}$ reduction target by minimizing the cost of electricity (COE) is formulated as a large scale mixed integer linear programme (MILP) and is implemented in General Algebraic Modeling System (GAMS). Three mitigation options were considered in this study: fuel balancing, fuel switching and carbon capture and storage (CCS). The model is able to predict which boiler should be converted from coal to natural gas, which boilers should be retrofitted with $\mathrm{CO}_{2}$ capture technologies, what size of $\mathrm{CO}_{2}$ capture process to put online, what types of new plants need to put on stream in order to supply supplemental energy while minimizing $\mathrm{CO}_{2}$ mitigation costs.
\end{abstract}

Keywords: optimization, $\mathrm{CO}$ reduction, $\mathrm{CO}_{2}$ mitigation, $\mathrm{CO}_{2}$ capture

\section{INTRODUCTION}

In Ontario, Ontario Power Generation (OPG) produces $75 \%$ of Ontario's electricity from mix of energy sources: coal accounts for $35 \%$ of OPG's electricity generating capacity, nuclear supplies $44 \%$, hydroelectric $27 \%$, and the remaining $10 \%$ is renewable energy. As a major electricity production in Ontario, OPG generated $115 \mathrm{TWh}$ electricity and was emitted about 36.7 million tonne of $\mathrm{CO}_{2}$ in 2003, which represents about $31 \%$ increase in $\mathrm{CO}_{2}$ emission compare than 1990 level (OPG, 2003). Therefore, in this paper, three potential $\mathrm{CO}_{2}$ mitigation strategies have been proposed to reduce greenhouse gas (GHG) by $6 \%$ (according to Kyoto target) compared to 1990 levels by 2012. The options that were considered: fuel balancing (optimal adjustment of the operation of existing generating stations to reduce $\mathrm{CO}_{2}$ emissions without making structural changes to the fleet), fuel switching (switching from carbon intensive fuel to less carbon intensive fuel, e.g. coal to natural gas) and carbon capture and storage (CCS). Although fuel switching may be likely possible options,

H. Hashim is with the Faculty of Chemical and Natural Resources Engineering,University Technology Malaysia, 81310, UTM Skudai, Johor, Malaysia (phone: 607-5535478; fax: 607-5581463; e-mail: haslenda@fkkksa.utm.my).

Peter Douglas, Ali Elkamel and Eric Croiset are with the Department of Chemical Engineering, University of Waterloo, Waterloo, Ontario, Canada N2L 3G1. (emails: pdouglas@uwaterloo.ca, aelkamel@uwaterloo.ca, ecroiset@uwaterloo.ca) greater reduction of $\mathrm{CO}_{2}$ from power plant flue gas in a short term is expected to be technically possible using carbon capture and storage (CCS). However, CCS is energy intensive and required large amount of supplemental energy. For this reason, hypothetical new power plants to supply electricity to the grid based on growth rate demand, as well as to eventually supply supplemental energy for the $\mathrm{CO}_{2}$ capture processes, will also be considered. Six categories of electricity generating technologies are considered in order to compensate with energy penalty due to capture process as well as to meet substantial growth in demand includes: subcritical pulverized coal-fired (PC), PC with carbon capture $(\mathrm{PC}+\mathrm{CCS})$, Integrated coal gasification combined cycles (IGCC), IGCC with carbon capture (IGCC+CCS), Natural gas combined cycles (NGCC) and NGCC with carbon capture (NGCC+CCS). For this case study, structural optimization approach can be performed effectively with mixed integer linear programming (MILP) techniques. The optimizer will determine the best strategies to reduce emission at certain target and satisfy substantial growth in demand at the most economical way. The results of the study will show the implication of $\mathrm{CO}_{2}$ emission mitigation in term of generation mix, capacity mix and optimal configuration on OPG's fleet at minimum cost.

\section{Methodology}

\section{A. Superstructure Representation}

Figure 1 shows a superstructure that represents all possible alternative configurations for producing the required electricity demand by the grid considering a $\mathrm{CO}_{2}$ reduction target, fuel balancing or fuel switching, $\mathrm{CO}_{2}$ capture process and sequestration technology containing a large number of feasible alternative configurations. $C_{i}, N G_{i}$, $O_{i}, N_{i}, H_{i}$ and $A_{i}$ represents existing coal, natural gas, oil, nuclear, hydroelectric and alternative energy power plants respectively. Hypothetical new power plants are represented by $N_{i}^{\text {new }}, H_{i}^{\text {new }}, A_{i}^{\text {new }}, P C_{i}^{\text {new }}, N G_{i}^{\text {new }}, I G_{i}^{\text {new }}$ for nuclear, hydroelectric, alternative energy, PC, NGCC and IGCC with and without capture. $C-C_{i}, C-N G_{i}, C-O_{i}, C-N_{i}, C-$ $H_{i}, C-A_{i}, C-N_{i}^{n e w}, C-H_{i}^{\text {new }}, C-A_{i}^{\text {new }}, C-P C_{i}^{\text {new }}, C-N G_{i}^{\text {new }}, C-$ $I G_{i}^{\text {new }}$ represent possible $\mathrm{CO}_{2}$ capture processes, and Sequestration 1 and 2 represent the two potential locations for sequestration in Ontario. 


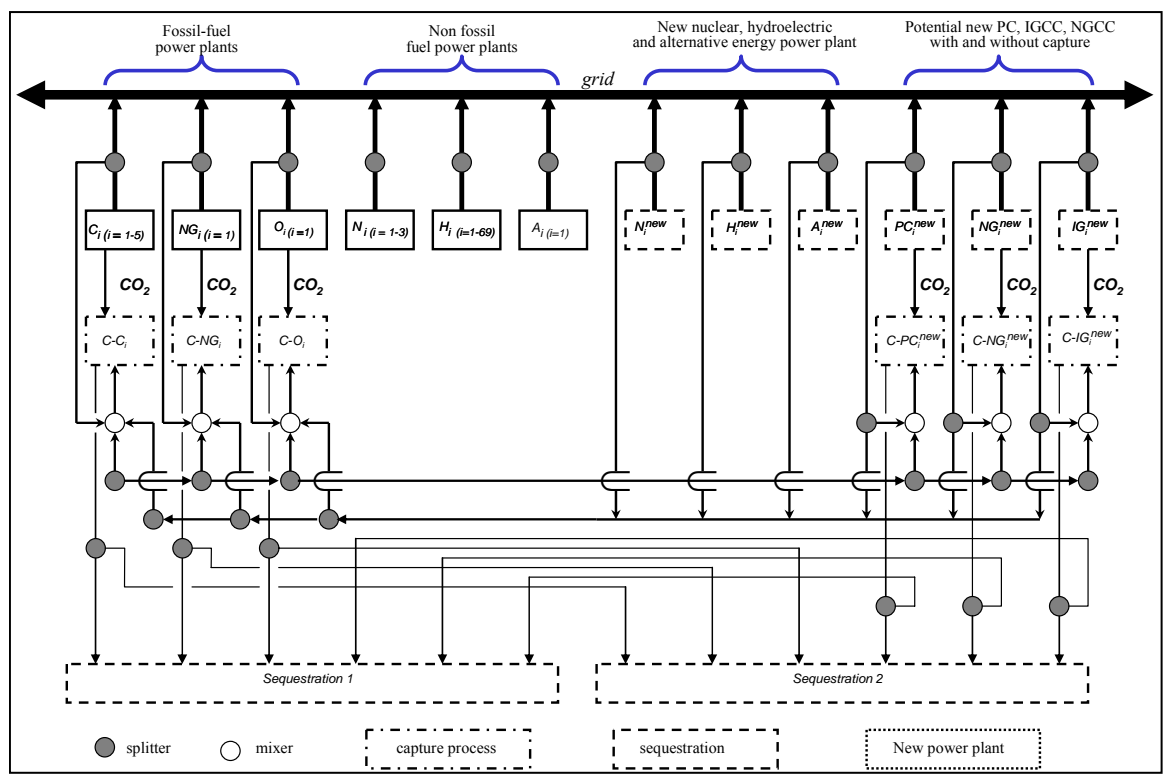

Figure 1. Generic superstructure

\section{B. Model Formulation}

The objective of this study is to synthesize the OPG's existing fleet, incorporating CCS on existing coal-fired power plants and constructing hypothetical new power plants with or without capture to meet a given $\mathrm{CO}_{2}$ reduction target while maintaining or enhancing electricity to the grid at the minimum overall cost. The cost function includes: operating cost of electricity generation for the fleet of generating stations, retrofitting cost associated with fuel switching from coal to natural gas, retrofitting cost for carbon capture retrofit on existing coal-fired power plants and capital and operational cost for hypothetical new power plants and sequestration cost. Note that in the case of a fossil fuel already operating on gas $(j=1)$, there is no retrofitting cost involved (i.e. $R_{i l}=0$ ). The objective function is given by:

$$
\begin{aligned}
& \underbrace{\sum_{i \in F} \sum_{j} C_{i j} E_{i j}+\sum_{i \in N F} C_{i}^{N F} E_{i}}_{\begin{array}{c}
\text { operational cost for } \\
\text { existing plants }
\end{array}}+\underbrace{\sum_{i \in F^{c}} \sum_{j} R_{i j} X_{i j}}_{\text {retrofit cost }} \\
& +\underbrace{\sum_{i \in P^{\text {new }}} S_{i}^{\text {new }} E_{i}^{\max } y_{i}+\sum_{i \in P^{n e w}} C_{i}^{\text {new }} E_{i}^{\text {new }}}_{\begin{array}{c}
\text { capital and operationt cost } \\
\text { for new additional stations }
\end{array}} \\
& +\underbrace{\sum_{i \in F^{c}} \sum_{k} S_{i k}^{c} z_{i k}+\sum_{i \in F^{c}} \sum_{k} C_{k}^{c} E_{i k}}_{\begin{array}{c}
\text { capital and operational cost } \\
\text { of carbon capture retrofit }
\end{array}}+\underbrace{\sum_{i \in F^{c} \cup P^{n e w}} \sum_{s} C_{i s}^{s e q} \alpha_{i} \varepsilon_{i}}_{\text {sequestration cost }} w_{i s}
\end{aligned}
$$

where $C_{i j}$ is the electricity generation cost per MWh if $j^{\text {th }}$ fuel is used in $i^{\text {th }}$ fossil-fuel boiler; $C_{i}\left(C_{i}^{\text {new }}\right)$ is the electricity generation cost per MWh for $i^{\text {th }}$ non-fossil-fuel power plant ( $i^{\text {th }}$ new hypothetical boiler); $C_{k}^{c}$ is operational cost for $k^{\text {th }}$ $\mathrm{CO}_{2}$ capture process $(\$ / \mathrm{MWh}) ; C_{i s}{ }^{\text {seq }}$ is sequestration cost from $i^{\text {th }}$ boiler to $s^{\text {th }}$ storage location ( $\$ /$ tonne $\mathrm{CO}_{2}$ captured); $E_{i}\left(E_{i}^{n e w}\right)$ is the electricity generated $\left(\mathrm{MWh} /\right.$ year) from $i^{\text {th }}$ non-fossil-fuel power plant ( $i^{\text {th }}$ hypothetical boilers); $E_{i j}$ is the electricity generated $\left(\mathrm{MWh} /\right.$ year) from $i^{\text {th }}$ fossil-fuel boilers when using $j^{\text {th }}$ fuel; $E_{i j k}$ is electricity required for $k^{\text {th }}$ $\mathrm{CO}_{2}$ capture process $\left(\mathrm{MWh} /\right.$ year) incorporated in $i^{\text {th }}$ coalfired boiler which running with $j^{\text {th }}$ fuel; $R_{i j}$ is the retrofitting cost for switching $i^{\text {th }}$ coal-fired boiler to $j^{\text {th }}$ fuel (natural gas) express in US\$/year; $S_{i}^{\text {new }}$ is capital cost for new power plant in $\$ / \mathrm{KW} ; S_{i k}^{c}$ is annualized capital cost for $k^{\text {th }}$ capture process in $\$ /$ year; $\varepsilon_{i k}$ is the fractional of $\mathrm{CO}_{2}$ capture; $X_{i j}$ is a binary variable that indicates fuel selection or whether the plant should be shut down for $i^{\text {th }}$ fossil-fuel boiler; binary variable $y_{i}$, is represents existence/non existence of $i^{\text {th }}$ hypothetical new boiler; binary variable $z_{i k}$, is introduced for the selection of $k^{\text {th }}$ retrofit carbon capture on $i^{\text {th }}$ existing coal-fired boiler; $w_{i s}$ is a binary variable that indicates the selection of $s^{\text {th }}$ potential location for $\mathrm{CO}_{2}$ sequestration; $i$ is the set of existing fossil fuel boilers, non-fossil power plants and new hypothetical boilers with and without capture; $j$ is the set of $j^{\text {th }}$ fuel selection that consists of coal or natural gas; $F$ is the set of fossil-fuel boilers including coal $\left(F^{c}\right)$ and natural gas $\left(F^{n g}\right) ; N F$ is the set of non-fossil-fuel power stations, including nuclear, hydroelectric, and wind turbine sources; and $P^{\text {new }}$ is the set of hypothetical new boilers that includes pulverized coal, $P^{P C}\left(P^{P C c a p}\right)$, integrated gas combined cycle, $P^{I G C C}\left(P^{I G C C c a p}\right)$ and natural gas combined cycle, $P^{N G C C}\left(P^{N G C C a p}\right)$ with and (without capture). Note that, in the case of non-fossil-fuel plants, no associated binary variables are defined, as the fuel type for these plants is known a priori.

The objective function represented by equation (1) is subjected to the following constraints:

- Energy balance/demand satisfaction

- Energy balance on capture process

- Capacity constraint on capture process

- Fuel selection and plant shut-down

- Plant capacity constraints

- Upper bound on operational changes

- Lower bound on operational constraints

- Selection of $\mathrm{CO}_{2}$ capture process to be installed in power plant $\mathrm{i}$

- If the existing coal-fired boilers shut down, no capture process will put online

- Selection of hypothetical new power plants

- Selection of $\mathrm{CO}_{2}$ sequestration location

- $\mathrm{CO}_{2}$ sequestration must be determined once capture process is put online

- Emission constraint $/ \mathrm{CO}_{2}$ balance 


\section{CASE STUdY}

Currently, OPG operates 79 electricity generating stations, 5 are coal fired generating stations, $\mathrm{C}(i=1-5), 1$ is a natural gas generating station, $\mathrm{NG}(i=6), 3$ are nuclear generating stations, $\mathrm{N}(i=7-9), 69$ are hydroelectric generating station, $\mathrm{H}(i=10-78)$, and 1 is a small wind turbine, $\mathrm{A}(i=79)$. At nominal levels, OPG generates about 115.8 TWh. and injects it into the grid from a mix of sources i.e. coal, hydroelectric, nuclear and renewable energy. No $\mathrm{CO}_{2}$ capture process currently exists at any OPG power plant; about 36.7 million tonnes of $\mathrm{CO}_{2}$ was emitted in 2002, mainly from fossil fuel power plants. There are 27 fossil fuel boilers at the 6 fossil fuel stations: 4 boilers at Lambton (L1-L4), 8 boilers at Nanticoke (N1-N8), 8 boilers at Lakeview (LV1-LV8), 1 boiler at Atikokan (A1), 4 boilers at Lennox (L1-L4) and Thunder Bay has 2 boilers (TB1-TB2). Currently, 4 (out of 8) boilers operated by Lennox are running on natural gas and the other 4 boilers are running on coal.

The operating cost for nuclear, hydroelectric, and wind turbine was estimated to be US\$21/MWh, US\$3.30/MWh, and US\$2.70/MWh, respectively. Note that, currently, natural gas is the most expensive fuel used by OPG which is US\$47/MWh (OPG, 2002). Since the main objective of this paper is to study $\mathrm{CO}_{2}$ emission reduction through fuel balancing, fuel switching and $\mathrm{CO} 2$ capture, no attempt is made to study the effect of improved technology. An improvement in boiler technology will, in principle, lead to an efficiency higher than our assumed efficiency of $35 \%$.

Index $i(i=1 \sim 79)$ represents all of OPG's power plants. The index $j(j=1 \sim 2)$ represents the fuel selection, $\mathrm{j}=$ 1(coal), 2(natural gas). The retrofit cost was estimated to be US\$20 million/1000 MW with a 30 year lifetime and $15 \%$ annual interest rate. The reserve margin, $r_{i}$ for load distribution for all OPG's fleet power plants is set at $1 \%$ higher than current operational level due to the design constraints and the lower bound was set to be $10 \%$. In other words, the plants have to operate at least with $10 \%$ annual capacity factor; otherwise the plants will be shut down. A summary of OPG's current fossil fuel generating stations is contained in Table 1.

Table 1. Ontario Power Generation fossil fuel power stations

\begin{tabular}{lccccccc}
\hline Station & Fuel & $\begin{array}{c}\text { Heat rate } \\
\text { (GJ/MWh) }\end{array}$ & $\begin{array}{c}\text { Installed } \\
\text { Capacity } \\
\text { (MW) }\end{array}$ & $\begin{array}{c}\text { Number } \\
\text { of units }\end{array}$ & $\begin{array}{c}\text { Annual } \\
\text { capacity } \\
\text { factor }\end{array}$ & $\begin{array}{c}\text { Operational } \\
\text { cost } \\
\text { (\$/MWh) }\end{array}$ & $\begin{array}{c}\mathbf{C O}_{2} \text { emission } \\
\text { (tonne/MWh) }\end{array}$ \\
\hline Nanticoke 1 (N1) & Coal & 9.88 & 500 & 2 & 0.75 & 20 & 0.93 \\
Nanticoke2 (N2) & Coal & 9.88 & 500 & 6 & 0.61 & 20 & 0.93 \\
Lambton1 (L1) & Coal & 9.84 & 500 & 2 & 0.5 & 22 & 0.94 \\
Lambton2 (L2) & Coal & 9.84 & 500 & 2 & 0.75 & 17 & 0.94 \\
Lakeview (LV) & Coal & 10.8 & 142 & 8 & 0.25 & 23 & 0.98 \\
Lennox (LN) & NG & 7.82 & 535 & 4 & 0.15 & 47 & 0.65 \\
Thunder Bay (TB) & Coal & 11.7 & 155 & 2 & 0.55 & 20 & 1.03 \\
Atikokan (A) & Coal & 9.82 & 215 & 1 & 0.44 & 20 & 1.03 \\
\hline
\end{tabular}

According to Rubin et al. (2004), energy penalty associated with $\mathrm{CO}_{2}$ capture process can be calculated using the following equation

$$
E P=1-\frac{\eta_{C C S}}{\eta_{r e f}}
$$

where $E P$ is the energy penalty (fraction reduction in output), $\eta_{C C S}$ and $\eta_{\text {ref }}$ are the net efficiencies of the capture plant and reference plant respectively. This energy penalty also can be defined as the reduction in plant output for a constant fuel input and called "plant derating". For the case of CCS retrofit on existing coal-fired power plant, the energy penalty was assumed at $22 \%$ as suggested by Rubin et al. (2002). The detailed of existing coal fired stations with and without capture characteristics are shown in Table 2. 'ref' is represents existing coal fired power plant characteristics, whereas 'cap' represents the plant performance once CCS is incorporated.

New state-of-the-art PC, IGCC and NGCC with and without capture cost estimation for different sizes are adjusted to the same economic references, which is described in Table 3.

In this study, the performances of power plant were obtained from the literature (Rubin, 2004; Ordorica et al.,
2004; McDaniel, 2002; Parson 1998). Data for new supercritical PC power plants without capture at two different capacities (500 MW, $575 \mathrm{MW})$ and with capture at three different capacities (500 MW, $670 \mathrm{MW}, 710 \mathrm{MW}$ with $90 \% \mathrm{CO}_{2}$ capture) were obtained from Rubin et al. (2004) reported in 2001\$US. On the other hand, plant performance and cost (mid 2001US\$) for a 250 MW IGCC was gathered from real plant data (McDaniel, 2002). Two different plant designs and cost estimation of IGCC with capture (500 MW with $80 \% \mathrm{CO}_{2}$ capture and $513 \mathrm{MW}$ IGCC with $60 \% \mathrm{CO}_{2}$ capture) were based on a study by Ordorica et al. (2004). Plant performance for NGCC without capture of two different plant sizes (326 MW, 395 MW) were obtained from Parson (1998) and for $517 \mathrm{MW}$ from Rubin et al. (2004). Plant characteristics for two different sizes of NGCC with capture (517 MW and $750 \mathrm{MW}$ with $90 \% \mathrm{CO}_{2}$ capture) were obtained from Rubin (2004) and Bechtel (2002), respectively. Finally, cost of all proposed new power plants were adjusted with the same economic assumption and reported in 2004US\$ using Chemical Engineering Plant Cost Indexes (Chemical Engineering Magazine, 1990-2004) with coal price of US\$1.2/GJ and natural gas price of US\$4/GJ. 
Table 2 Parameters and cost comparison of retrofit $\mathrm{CO}_{2}$ capture on existing coal fired power plants

\begin{tabular}{|c|c|c|c|c|c|c|c|c|c|c|c|c|c|c|}
\hline \multirow{2}{*}{ Parameters } & \multicolumn{2}{|c|}{ N1 } & \multicolumn{2}{|c|}{ N2 } & \multicolumn{2}{|c|}{ L1 } & \multicolumn{2}{|c|}{ L2 } & \multicolumn{2}{|c|}{$\mathbf{L V}$} & \multicolumn{2}{|c|}{ TB } & \multicolumn{2}{|c|}{ A } \\
\hline & ref & cap & ref & cap & ref & cap & ref & cap & ref & cap & ref & cap & ref & cap \\
\hline Gross capacity (MW) & 512 & 512 & 512 & 512 & 515 & 515 & 515 & 515 & 150 & 150 & 163 & 163 & 230 & 230 \\
\hline Net power gen. (MW) & 490 & 382 & 490 & 382 & 493 & 385 & 493 & 385 & 142 & 111 & 155 & 120 & 215 & 168 \\
\hline $\begin{array}{l}\text { Heat rate, HHV } \\
(\mathrm{GJ} / \mathrm{MWh})\end{array}$ & 9.88 & 12.7 & 9.88 & 12.7 & 9.84 & 12.6 & 9.84 & 13.1 & 10.8 & 13.8 & 9.82 & 12.5 & 9.82 & 12.5 \\
\hline Capacity factor, $\mathrm{ACF}$ & 0.75 & 0.75 & 0.61 & 0.61 & 0.5 & 0.5 & 0.75 & 0.75 & 0.25 & 0.25 & 0.55 & 0.55 & 0.44 & 0.44 \\
\hline $\mathrm{CO}_{2}$ capture $(\%)$ & - & 90 & . & 90 & - & 90 & - & 90 & . & 90 & - & 90 & - & 90 \\
\hline $\begin{array}{l}\mathrm{CO}_{2} \text { emission } \\
\text { (tonne/MWh) }\end{array}$ & 0.93 & 0.09 & 0.93 & 0.09 & 0.94 & 0.09 & 0.94 & 0.09 & 0.98 & 0.09 & 1.02 & 0.11 & 1.02 & 0.11 \\
\hline $\mathrm{CO}_{2}$ capital cost $(\mathrm{M} \$)^{\mathrm{a}}$ & - & 236 & - & 236 & - & 238 & - & 270 & - & 290 & - & 86 & - & 121 \\
\hline$O \& M \operatorname{cost}(\$ / M W h)^{a}$ & 20 & 13.9 & 20 & 13.9 & 22 & 13.9 & 17 & 13.9 & 23 & 13.9 & 20.0 & 13.9 & 20.0 & 13.9 \\
\hline $\operatorname{COE}(\not / K W h)^{\mathrm{a}}$ & 2.0 & 4.01 & 2.0 & 4.27 & 2.2 & 4.55 & 1.7 & 4.16 & 2.3 & 8.0 & 2.0 & 5.11 & 2.0 & 5.75 \\
\hline $\begin{array}{l}\text { Capture cost }(\$ / \text { tonne } \\
\mathrm{CO} 2 \text { avoided })^{\mathrm{a}}\end{array}$ & - & 24.0 & - & 27.1 & - & 26.6 & - & 27.1 & - & 64.5 & - & 33.8 & - & 40.7 \\
\hline
\end{tabular}

Table 3. Specification of economic parameters (All cost are in 2004 USD)

\begin{tabular}{ll}
\hline Economic life time & 30 year \\
Interest rate of return & $15 \%$ \\
Fuel cost & \\
Coal & $\mathrm{US} \$ 1.2 / \mathrm{GJ}$ \\
$\quad$ Natural gas & $\mathrm{US} \$ 4.0 / \mathrm{GJ}$ \\
\hline
\end{tabular}

\section{RESUlts}

This section will discussed 2 main results: (1) The effect of cost of electricity (COE) by adjusting the $\mathrm{CO}_{2}$ emission reduction by fuel balancing, fuel switching or capturing and (2) The effect of $\mathrm{CO}_{2}$ reduction on distribution of electricity generation for existing and new plants for base load demand.

Figure 2 shows the effect of $\mathrm{CO}_{2}$ reduction on COE. As can be seen, increasing the $\mathrm{CO}_{2}$ emission reduction would result in increase in the cost of generating electricity.

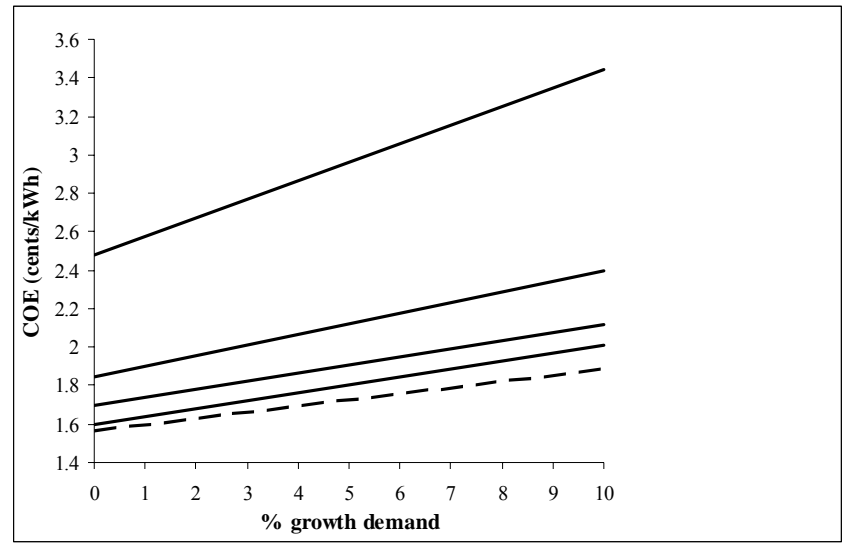

Figure 2. Effect of $\mathrm{CO}_{2}$ reduction to the $\mathrm{COE}$ electricity generation by plants in 2002

Currently, OPG electricity cost is $1.57 \phi / \mathrm{KWh}$ which represents by $0 \% \mathrm{CO}_{2}$ reduction. The optimization results show that fuel balancing can contribute to the reduction of $\mathrm{CO}_{2}$ emissions by only $3 \%$. Fuel balancing also results in a reduction of cost of electricity to $1.54 \phi / \mathrm{KWh}$ by reducing electricity generation from all four natural gas boilers by $32.1 \%$ and two coal fired boilers by $33.4 \%$ and $59.4 \%$, respectively. The electricity generation from other fossil fuel boilers and non fossil fuel power plants were increased by $1 \%$ above the nominal operational level to maintain the electricity to the grid. However, if $\mathrm{CO}_{2}$ emissions are to be reduced beyond than 3\% (e.g. 20\% for Canada Kyoto Target), more stringent measures that include fuel switching, plant retrofitting and $\mathrm{CO}_{2}$ capture will have to be employed. The optimization results show electricity generation from one natural gas boilers (LN4) need to be reduced by $32.1 \%$, one natural gas boiler (LN2) reduced by $4.1 \%$ and nine coal fired boilers (L1,L2,N1,N2, N3, N4,LV7,LV8,TB1) need to be switched to natural gas resulting in an increase of cost of electricity of about $7.1 \%$. Finally, the other coal fired boilers and non-fossil fuel power plants increase the electricity generation by $1 \%$ higher than the nominal operational level to meet the electricity demand. For the case of $60 \% \mathrm{CO}_{2}$ reduction, cost of electricity is increased by $35.6 \%$ since 4 new natural gas boilers are put online to compensate with the reduction in electricity output due to implementation of $\mathrm{CO}_{2}$ capture retrofit on five Nanticoke coal-fired boilers, shut down of all 8 Lakeview boilers, 2 Lambton boilers (L1, L2) and 2 Thunder Bay boilers (TB1, TB2) as well as to achieve greater $\mathrm{CO}_{2}$ reduction.

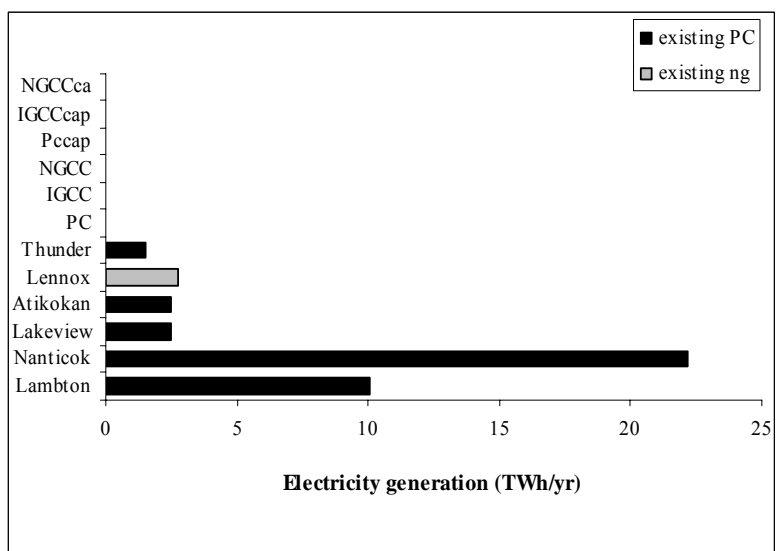

Figure 3. Current OPG's fossil-fuel

Figure 4 illustrate the electricity distribution for base case, $3 \%, 6 \%, 20 \%, 40 \%$ and $60 \% \mathrm{CO}_{2}$ reduction target in order to meet current electricity demand. Figure 3 shows the base case which represents OPG's current operational level (2002). Figure 4 shows the optimization results for the case of $3 \% \mathrm{CO}_{2}$ reduction. For this case, and as is clear from the figure, no fuel switching is needed. In other words, this objective can be obtained by only adjusting operation of current boilers e.g. increasing load from existing non fossil 
power plants and decreasing load from existing fossil power plants (fuel balancing). The results show that electricity generation from all four natural gas boilers has been reduced by $32.1 \%$. The electricity generation from other fossil fuel boilers and non fossil fuel power plants were increased at $1 \%$ higher than the nominal operational level to maintain the electricity to the grid. In order to achieve more than $3 \% \mathrm{CO}_{2}$ reduction (Figure 5), it was found that fuel switching must be implemented. This involves fleet changes from coal to natural gas. The optimization results show, for instance, that in order to achieve $6 \% \mathrm{CO}_{2}$ reduction (Canada's Kyoto target at 1990) while maintaining the electricity to the grid at minimum cost, the electricity generation from 3 natural gas boilers (LN1, LN2 and LN3) need to be reduced by $32.1 \%$, one natural gas boiler (LN4) reduced by $8.2 \%, 2$ coal fired boiler (LV1 and LV2) reduced by $59.4 \%$ and $34.8 \%$ respectively and the other coal fired boilers and non-fossil fuel power plants increase the electricity generation by $1 \%$ higher than the nominal operational level. Finally, one Nanticoke boiler (N8) which is the largest coal-fired boilers for OPG need to be switched to natural gas.

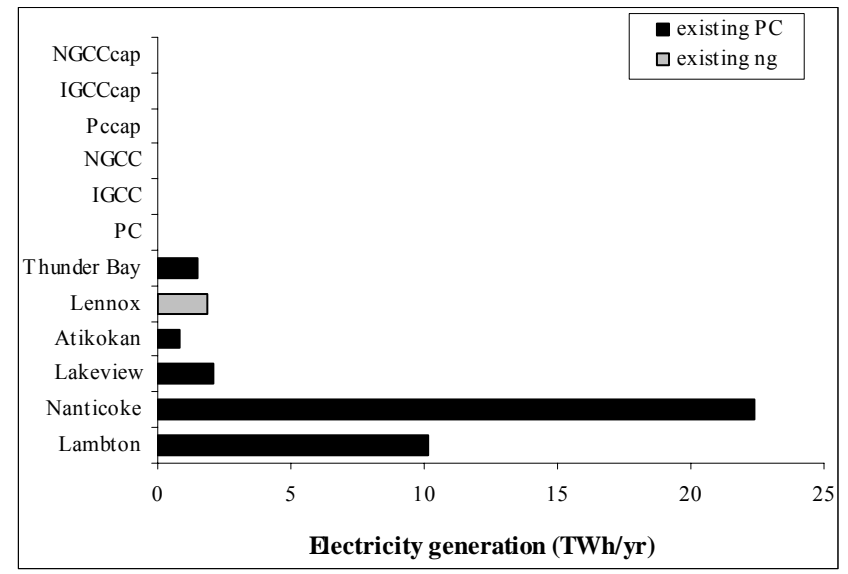

Figure 4. Optimal electricity generation for fossil fuel-plants \& $3 \% \mathrm{CO}_{2}$ reduction

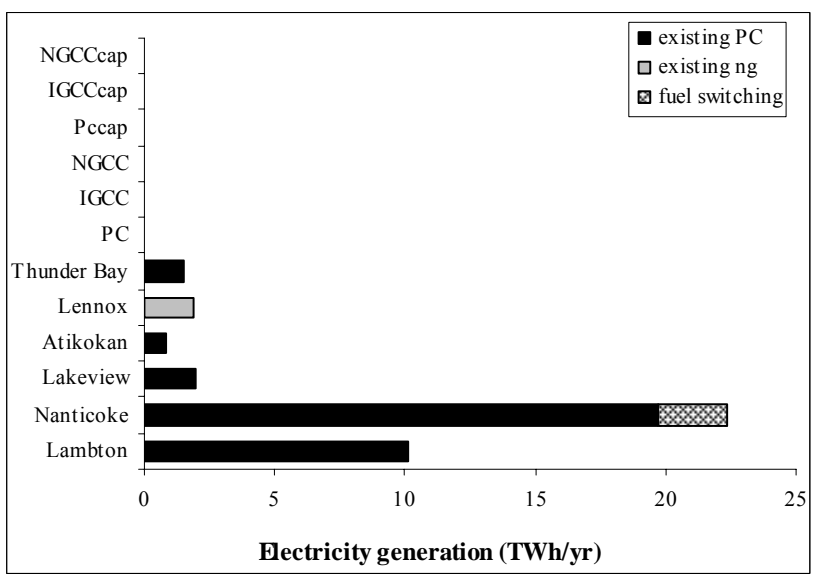

Figure 5. Optimal electricity generation for fossil fuel-plants $\& 6 \% \mathrm{CO}_{2}$ reduction

Figure 6 shows that, $20 \% \mathrm{CO}_{2}$ reduction and meet current electricity demand could be achieved by implementing fuel balancing and switching nine coal-fired boilers to natural gas. For the case of $40 \% \mathrm{CO}_{2}$ reduction as illustrated in Figure 7 , the optimizer choose to switch most of coal fired boilers to natural gas and replacing all 8 Lakeview boilers with a new NGCC.

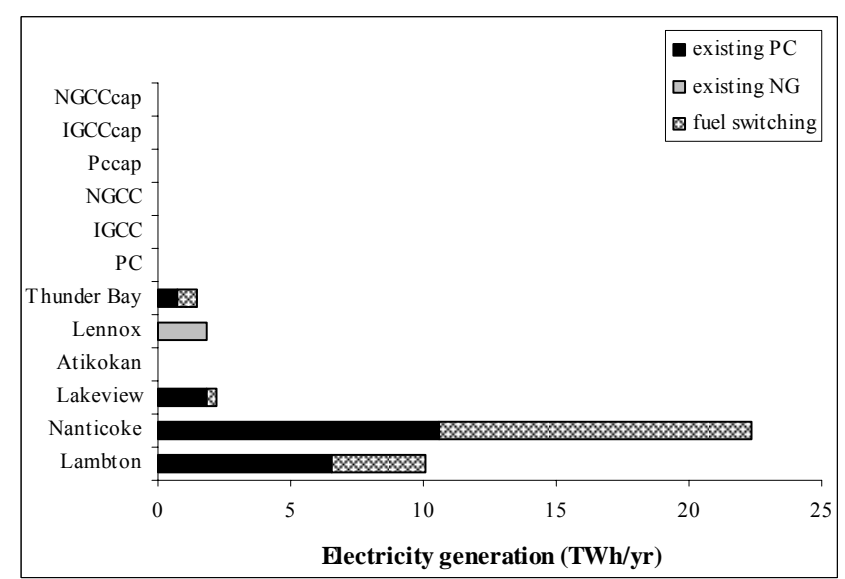

Figure 6. Optimal electricity generation for fossil fuel-plants $\& 20 \% \mathrm{CO}_{2}$ reduction

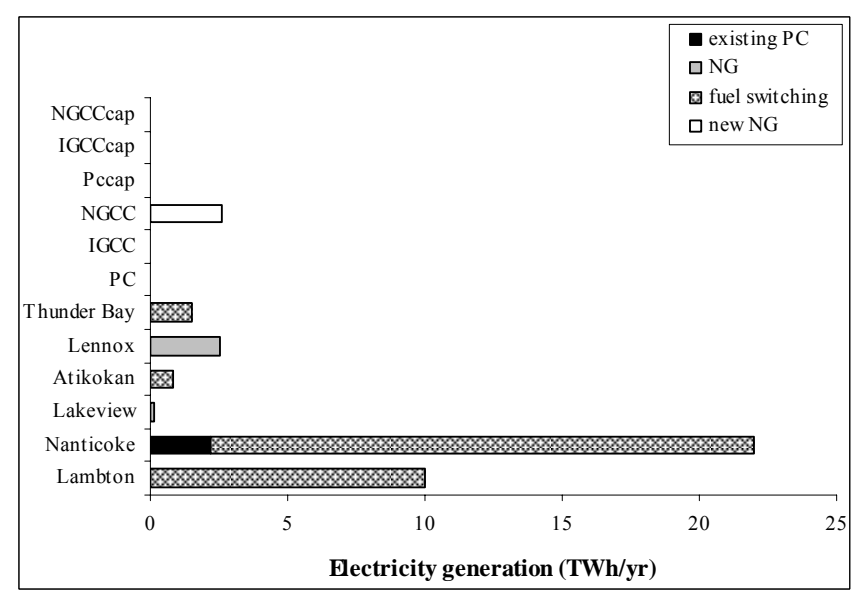

Figure 7. Optimal electricity generation for fossil fuel-plants $\& 40 \% \mathrm{CO}_{2}$ reduction

In order to achieve greater $\mathrm{CO}_{2}$ emission reduction (e.g. $60 \% \mathrm{CO}_{2}$ reduction) and generating current electricity generation, the optimizer choose to implement $\mathrm{CO}_{2}$ capture on five existing Nanticoke boiler (N1, N2, N3, N4, N5) and put on stream four new NGCC (two $326 \mathrm{MW}$, one $395 \mathrm{MW}$, one $517 \mathrm{MW}$ ) resulting in a COE of $2.44 \notin / \mathrm{kWh}$.

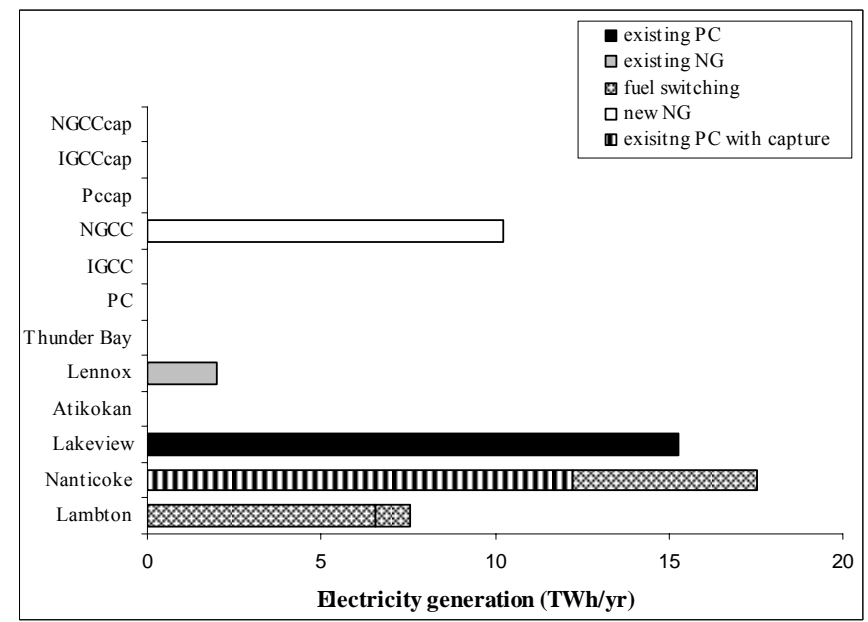

Figure 8. Optimal electricity generation for fossil fuel-plants $\& 60 \% \mathrm{CO}_{2}$ reduction 


\section{CONCLUSIONS}

In this study, the model is formulated as Mixed Integer Non Linear Programme (MINLP) and implemented in GAMS (General Algebraic Modeling System). Exact linearization techniques were employed to facilitate solution development. The computer programme is capable of determining the best mix of fuel, annual capacity factor for existing power plants, location and size of $\mathrm{CO}_{2}$ capture process and finally, construction of new state-of-the-art power plants, such as supercritical pulverized coal (PC), integrated gas combined cycle (IGCC) or natural gas combined cycle (NGCC) with and without $\mathrm{CO}_{2}$ capture. As can be seen, increasing $\mathrm{CO}_{2}$ reduction target leads to switching of more coal-fired boilers to natural gas. For a greater $\mathrm{CO}_{2}$ reduction target, new plants with and without CCS will compete with existing plant that have been paid off but remain competitive due to lower overall cost. Among the option, NGCC is more favorable although average coal prices are projected to fall throughout the forecast due to lower capital costs, higher fuel efficiency and lower $\mathrm{CO}_{2}$ emissions.

Optimization results for maintaining current electricity demand shows that fuel balancing could contribute up to $3 \%$ $\mathrm{CO}_{2}$ reduction by increasing all non fossil fuel power plants by $1 \%$ above the nominal operation level, while reducing electricity generation from all four natural gas boilers (the most expensive fuel) by $32.1 \%$. To meet the electricity demand, the optimization suggests then to increase all coal power plants by $1 \%$ above the nominal annual capacity factor. This result in a decrease of COE by $1.9 \%$ compare to the current situation $(1.57 \notin / \mathrm{kWh})$. However if $\mathrm{CO}_{2}$ emissions are to be reduced by more than $3 \%$, more stringent measures that include fuel switching and retrofitting carbon capture on existing coal-fired boilers have to be employed. For instance, to achieve $6 \% \mathrm{CO}_{2}$ reduction (Canada's Kyoto target), the largest coal fired boilers, Nanticoke (N2), need to be switch to natural gas. In addition, electricity generation from all four existing natural gas boilers is reduced by $32.1 \%$. Finally, electricity generation from the other coal-fired boilers and non fossil fuel power plants need to be increased by $1 \%$ higher than nominal operational level. For this scenario, COE is the same as the base case without optimization, which is $1.57 \phi / \mathrm{KWh}$. In order to achieve greater $\mathrm{CO}_{2}$ emission reduction (e.g. $60 \% \mathrm{CO}_{2}$ reduction) and generating current electricity generation, the optimizer choose to implement a $\mathrm{CO}_{2}$ capture on five existing Nanticoke boiler $(\mathrm{N} 1, \mathrm{~N} 2, \mathrm{~N} 3$, N4, N5) and put on stream four new NGCC (two $326 \mathrm{MW}$, one $395 \mathrm{MW}$, one $517 \mathrm{MW}$ ) resulting in an increase of COE by $35.6 \%$. The optimiser also recommended to store the captured $\mathrm{CO}_{2}$ in Lake Erie.

\section{ACKNOWLEDGEMENT}

The authors wish to thank Ontario Power Generation (OPG) for their technical support towards this opportunity to contribute to the academic study of $\mathrm{CO}_{2}$ capture. The information expressed herein is that of the authors and OPG takes no position on $\mathrm{CO}_{2}$ capture and is simply furthering scientific endeavours.
[2] Genchi, Y., K. Saitoh, N. Arashi, and A. Inaba. 2002. Assessment of $\mathrm{CO}_{2}$ Emissions Reduction Potential by Using an Optimization Model for Regional Energy Supply Systems. Paper B5-6 presented at the Sixth International Conference on Greenhouse Gas Control Technologies, Kyoto International Conference Hall, Japan.

[3] Mavrotas, G. 1999. An energy planning approach based on mixed 0-1 Multiple Objective Linear Programming. International Transactions in Operational Research. 6: 231-244.

[4] Bai, H., and Wei, J. 1996. The $\mathrm{CO}_{2}$ mitigation options for the electric sector. Energy Policy. 24: 221-118.

[5] Climaco J. 1995. A multiple objective linear programming model for power generation expansion planning. International Journal of Energy Research. 19: 419-432.

[6] Noonan F. 1977. Planning electric power generation: A linear mixed integer model employing benders decomposition. Management Science. 23: 946-956.

[7] Brooke A., D. Kendrick, and A. Meeraus. 1988. GAMS: A User's Guide. The Scientific Press, South San Francisco, CA.

[8] Noonan, F., and R. Giglio. 1977. Planning Electric Power Generation: A Nonlinear Mixed Integer Model Employing Benders Decomposition. Management Science. 23: 946-956

[9] Analysis and Modeling Division, Natural Resources Canada. http://climatechange.nrcan.gc.ca/english/Home.asp?x)1

[10] McDaniel, J.E. 2002. Tampa Electric Polk Power Station integrated gasification combined cycle project. Final technical report. Tampa: Tampa Electric Company.

[11] Ordorica-Garcia, G., E. Croiset, P. L. Douglas., and L. Zheng. 2004. Simulation of IGCC power plants with glycol-based $\mathrm{CO}_{2}$ capture using Aspen Plus. Energy Conversion and Management.

[12] Rubin, E.S. et al. September 5-9, 2004. Comparative assessments of fossil fuel power plants with $\mathrm{CO}_{2}$ capture and storage. Proceedings of the 7th International Conference on Greenhouse Gas Control Technologies, Vancouver, BC.

[13] Shafeen, A., E. Croiset, P. L. Douglas, and I. Chatzis. 2004. $\mathrm{CO}_{2}$ sequestration in Ontario, Canada. Part I: storage evaluation of potential reservoirs. Energy Conversion Management. 45: 26452659.

[14] Shafeen, A., E. Croiset, P. L. Douglas, and I. Chatzis. 2004. $\mathrm{CO}_{2}$ sequestration in Ontario, Canada. Part II: cost estimation. Energy Conversion Management. 45: 2645-2659.

[15] Yokoyama, R., Y.Hasegawa, and K.Ito. 2002. A MILP decomposition approach to large scale optimization in structural design of energy supply systems. Energy Conversion and Management. 43: 771-790.

\section{REFERENCES}

[1] Ontario Power Generation (2003): Personal communication 


\title{
A Surge Suppressor Model for Increasing the Energy-Handling Capability in High Voltage Protection
}

\author{
Muhammad Saufi Kamarudin and Erwan Sulaiman
}

\begin{abstract}
High voltage protection for low voltage AC power circuits is very important to minimize the damage of electrical equipment. Unfortunately, review of performance and safety features in commercially-available surge suppressors has shown that sometimes they cannot deliver the proper protection to withstand the high voltage surges. This paper will propose a model for higher capability of discharging high current and providing a constant safer voltage for class $C$ protection system in AC power circuits. Metal oxide varistors (MOVs) are used as surge suppressors. Some configurations of MOVs have been tested to show the difference in protection capabilities. Experiments involving a wide range of high voltage surges revealed that the model provides increased in peak current and energy-handling capabilities for a given application.
\end{abstract}

Keywords: surge suppressor, MOV, high voltage

\section{INTRODUCTION}

Electrical surges generally can be divided into two categories, which are switching surges and lightning surges. Lightning is known to be one of the primary sources of most surges. It is a well-known fact that surge overvoltage is a significant contribution to equipment damage. Direct lightning strokes into high voltage transmission lines cause a severe stress on the electric equipment. The direct stroke in the conductor of an overhead line causes a voltage surge traveling in either direction of the line and to loads.

The switch-mode power supplies used for equipment such as fax machines, printers, elevators, escalators, electronic ballasts, and others, and a great percentage of other loads are becoming nonlinear. Such loads generate current harmonics, leading to distorted voltage. Voltage transients are very brief and unpredictable, making it very difficult in detecting and measuring them. Many works have been done by researchers to better understand these transients.

The destruction potential of transients is defined by their peak voltage, the follow-on current and the time duration of the current flow, that is stated in [1]:

Muhammad Saufi Kamarudin and Erwan Sulaiman are currently with Department of Electrical Power Engineering, Faculty of Electrical and Electronic Engineering, University of Tun Hussein Onn Malaysia, 86400, Batu Pahat, Johor (Phone: 07-4537585; Fax: 07-4536060; email: saufi@uthm.edu.my, erwan@uthm.edu.my).

$$
\mathrm{E}=\int_{0}^{T} V_{C}(t) \bullet i(t) d t
$$

where:

$$
\begin{aligned}
& \mathrm{E}=\text { Transient energy } \\
& \mathrm{i}=\text { Peak transient current } \\
& \mathrm{V}_{\mathrm{C}}=\text { Resulting clamping voltage } \\
& \mathrm{t}=\text { Time } \\
& T=\text { Impulse duration of the transient }
\end{aligned}
$$

Working group of IEEE and IEC Standards have developed different surge waves for testing transient voltage surge suppressor (TVSS) devices meant for outdoor and indoor application locations to the low-voltage power distribution system, as stated in [2]:

1. Outdoor: Combo Wave $-1.2 / 50 \mu$ s voltage wave and $8 / 20 \mu$ s current wave are predominant at the service entrance outdoor location. However, lightning discharges induce oscillations, reflections that ultimately appear as decaying oscillations in a lowvoltage power system.

2. Indoor: Ring Wave - A surge impinging on the system excites the natural resonant frequencies of the conductor system. As a result, not only are the surges typically oscillatory, but also may have different amplitudes and wave shapes at different places in the low-voltage power system. These oscillatory frequencies of surges range from 5 to more than 500 $\mathrm{kHz}$. Based upon such conclusions, a ring wave, $0.5 \mu \mathrm{s}$ with $100 \mathrm{kHz}$, which rises in $0.5 \mu \mathrm{s}$, then decays while oscillating at $100 \mathrm{kHz}$, each peak being $60 \%$ of the preceding peak. Such waves are depicted in 'figure 1'.

\section{Methodology}

Before designing the protection system, some measurements were made to determine the exact components and the quantity of the components to be used in the design. The most important component was of course the varistor. For this project, the authors chose metal-oxide varistor $22.5 \mathrm{~mm}$ disc, manufactured by EPCOS, and the manufacturer's list number is S20K250. The properties of the varistor are as follows:
- Disc diameter
$=22.5 \mathrm{~mm}$
- Disc width
$=5.9 \mathrm{~mm}-6.6 \mathrm{~mm}$
- Disc height
$=27 \mathrm{~mm}$ 
- Lead length

- Lead diameter

$=30 \mathrm{~mm}$

- Peak transient current

$=1.0 \mathrm{~mm}$

- Rated AC voltage

$=8 \mathrm{kA}$

- Rated DC voltage

$=250 \mathrm{~V}$

$=320 \mathrm{~V}$

- Varistor voltage at $1 \mathrm{~mA}$

- Transient energy

$=390 \mathrm{~V}$

- Max. operating temperature

$=140 \mathrm{~J}$

The basic design of the protection system is as in 'figure 2'.

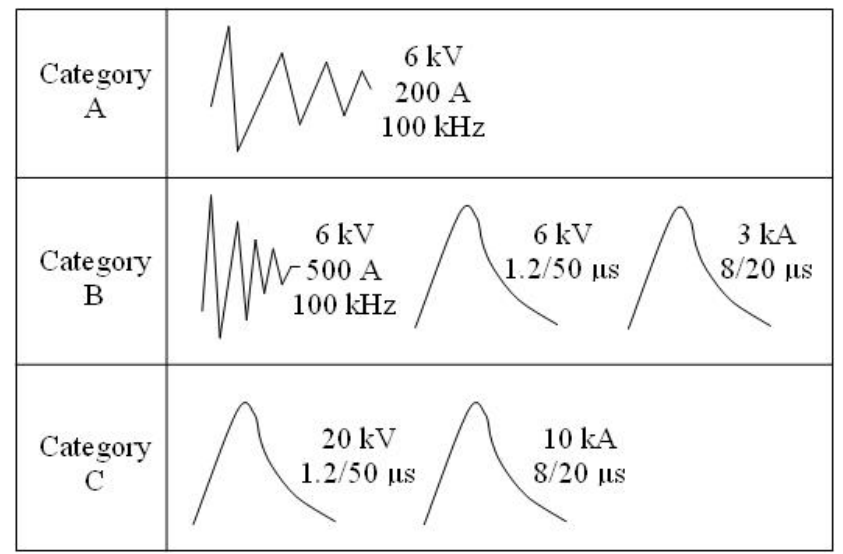

Figure 1. Test Waveforms as described by ANSI/IEEE C62.41-1991 according to [2]

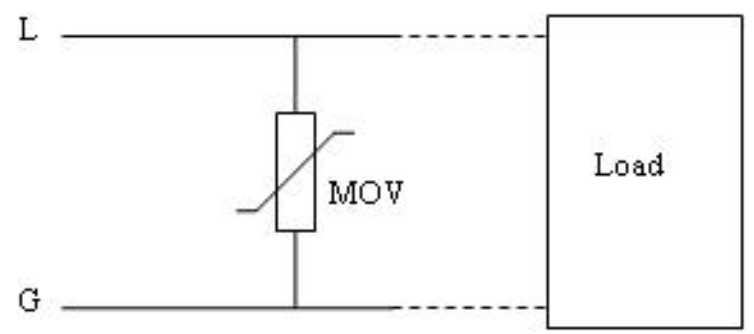

Figure 2. A protection system using single MOV

Tests were then carried out for S20K250 varistors. The tests were conducted in High Voltage and High Current Lab (IVAT) at Universiti Teknologi Malaysia (UTM) Skudai using an impulse generator made by HAEFELY and is known as PSURGE.

The first test was done by using a single MOV. The MOV was put in the PSURGE and connected between line and ground terminals. A surge impulse voltage was then applied to the varistor at a time, from the minimum voltage that can be generated by PSURGE, which is $2.7 \mathrm{kV}$ onwards, with $500 \mathrm{~V}$ increment between steps, until the breakdown of the varistor was reached. The S20K250 varistor was blown into pieces at $17 \mathrm{kV}$, with discharge current of $8.18 \mathrm{kA}$. As the result, the varistor was said to be failed as an open circuit. The varistor was completely destroyed because the applied conditions exceeded the energy rating of the varistor.

For this project, the authors needed to design a class $\mathrm{C}$ protection system, but can withstand surges as much as 20 $\mathrm{kV}$ and $30 \mathrm{kA}$. Referring to 'figure 1', a TVSS device installed for class $\mathrm{C}$ protection system should be able to handle surges up to $20 \mathrm{kV}$ and $10 \mathrm{kA}$. From the first test result, S20K250 varistor alone was not suitable for that particular protection system. The authors tried to make some modifications on the circuit and came out with a protection scheme which consisted of four parallel-connected MOVs. The paralleling of varistors provides increased peak current and energy-handling capabilities for a given application. With this for parallel-connected S20K250 varistors, the protection scheme should be able to withstand surge current of up to at least $30 \mathrm{kA}$, which will be tested. The proposed configuration is shown in 'figure 3 '.

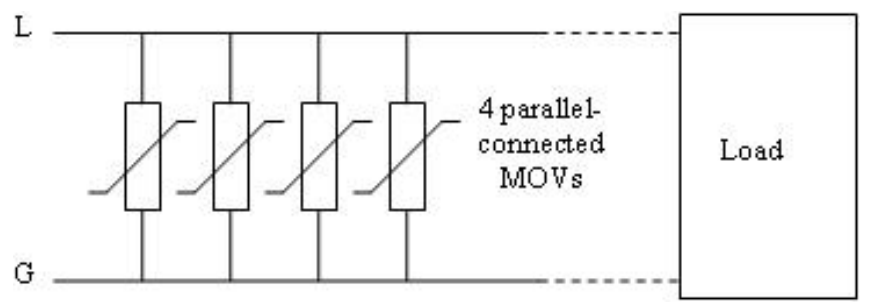

Figure 3. A protection system using four parallel-connected MOVs

The second test was carried out on the four parallelconnected MOVs circuit. Using the same method, the MOVs were connected between line-to-ground terminals in the PSURGE. A surge was then applied at a time, starting with minimum voltage which was $2.7 \mathrm{kV}$. This time, the voltage increment was set to $1 \mathrm{kV}$ between tests. Until the maximum voltage surge which PSURGE can generate, which is $30 \mathrm{kV}$ and discharge current of $14.8 \mathrm{kA}$, the four parallel-connected MOVs were not yet to be blown.

\section{RESUlTS}

'Table 1' and 'table 2' show the result of the first test and the second test, respectively. Due to a quite number of data, the data were summarized to fit in this paper. The tables are showing data for surge voltage applied to the MOVs, residual voltage, discharge current and also voltage at peak current.

Some V-I curves were also plotted to show the graphical difference in terms of the characteristics for both MOV configurations, as shown in these figures.

As stated before, the first test ended up with the MOV blew at surge voltage of $17 \mathrm{kV}$ and discharge current of $8.18 \mathrm{kA}$. That was a good indicator since the manufacturer claimed that the MOV should be able to withstand peak transient current of $8 \mathrm{kA}$.

Table 1. Test Results for Single MOV

\begin{tabular}{cccc}
$\begin{array}{l}\text { Surge } \begin{array}{l}\text { Voltage } \\
(\mathrm{kV})\end{array} \\
2.7\end{array}$ & $\begin{array}{l}\text { Residual } \\
\text { Voltage } \\
(\mathrm{kV})\end{array}$ & $\begin{array}{l}\text { Discharge } \\
\text { Current } \\
(\mathrm{kA})\end{array}$ & $\begin{array}{l}\text { Voltage at } \\
\text { peak current } \\
(\mathrm{kV})\end{array}$ \\
\hline 8.0 & 0.67 & 1.00 & 0.65 \\
12.5 & 0.95 & 3.63 & 0.74 \\
16.0 & 1.18 & 5.86 & 0.82 \\
17.0 & 1.37 & 7.66 & 0.88 \\
\hline
\end{tabular}

As we can see from the figures in the preceding section, there are not much different between single MOV and four parallel-connected MOVs in terms of residual voltage versus discharge current curves ('figure 4 and figure 5'), whether in log scale or in normal scale. Nevertheless, from the comparison between 'table 1' and 'table 2', test results for four parallel-connected MOVs showed a great improvement in the current discharging ability and higher 
strength in withstanding voltage surges. But due to limitation of PSURGE, the authors only did the second test to up to $30 \mathrm{kV}$, which is the maximum voltage surge that PSURGE can generate.

Table 2. Test Results for four parallel-connected MOVs

\begin{tabular}{cccc}
$\begin{array}{l}\text { Surge } \\
\text { Voltage }(\mathrm{kV})\end{array}$ & $\begin{array}{l}\text { Residual } \\
\text { Voltage } \\
(\mathrm{kV})\end{array}$ & $\begin{array}{l}\text { Discharge } \\
\text { Current } \\
(\mathrm{kA})\end{array}$ & $\begin{array}{l}\text { Voltage at } \\
\text { peak current } \\
(\mathrm{kV})\end{array}$ \\
\hline 3 & 0.66 & 1.18 & 0.45 \\
4 & 0.72 & 1.66 & 0.50 \\
5 & 0.78 & 2.17 & 0.55 \\
6 & 0.84 & 2.68 & 0.60 \\
10 & 1.10 & 4.70 & 0.60 \\
16 & 1.44 & 7.70 & 0.60 \\
22 & 1.80 & 10.80 & 0.60 \\
28 & 2.15 & 13.80 & 0.60 \\
30 & 2.27 & 14.80 & 0.60 \\
\hline
\end{tabular}

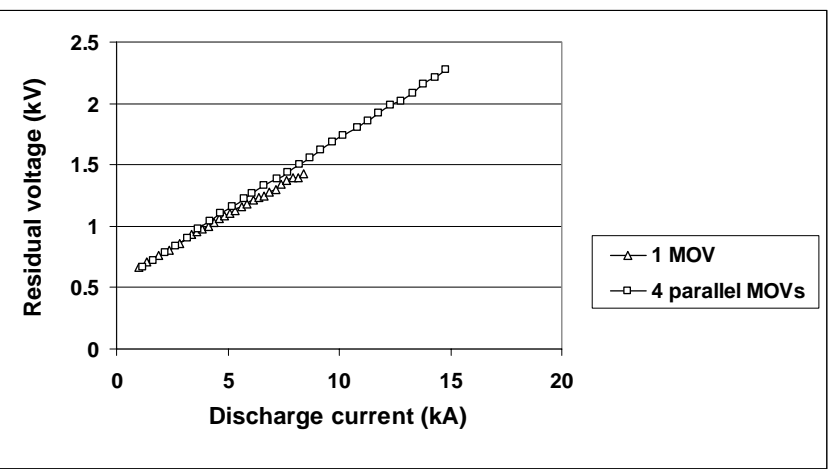

Figure 4. V-I Curve for Single MOV and Four ParallelConnected MOVs

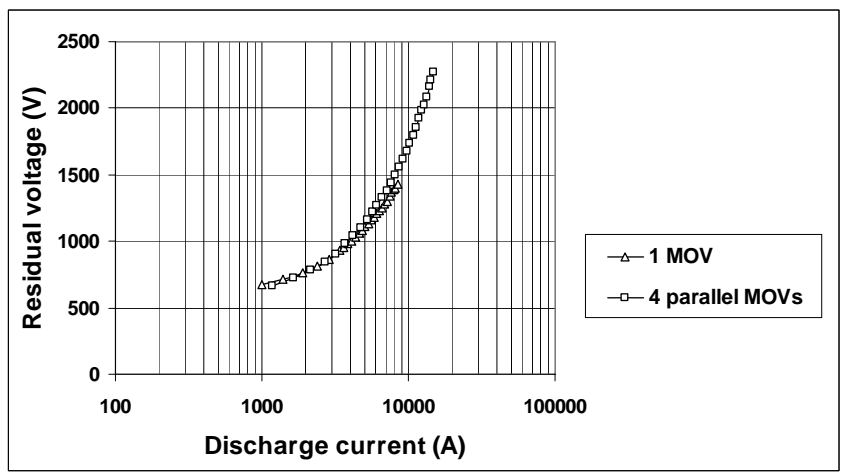

Figure 5. V-I Curve for Single MOV and Four ParallelConnected MOVs (Log Scale)

V-I characteristics curves in 'figure 6 and figure 7' clearly show the difference between single MOV and four parallelconnected MOVs. As we can see, four parallel-connected MOVs gave better results in terms of voltage at peak current, for a particular value of discharge current. While single MOV has a quite linear curve (higher value of voltage at peak current with increasing discharge current), the four parallel-connected MOVs gave a constant voltage at peak current, which is $600 \mathrm{~V}$, starting from $3 \mathrm{kA}$ of discharge current onwards. This fact is really important since calculation in energy handling capability as in "(1)" can include these values.

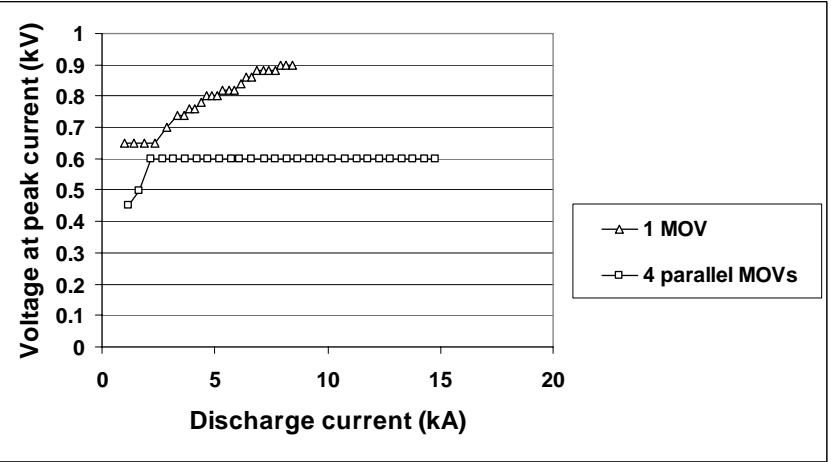

Figure 6. Voltage at peak current versus discharge current for Single MOV and Four Parallel-Connected MOVs

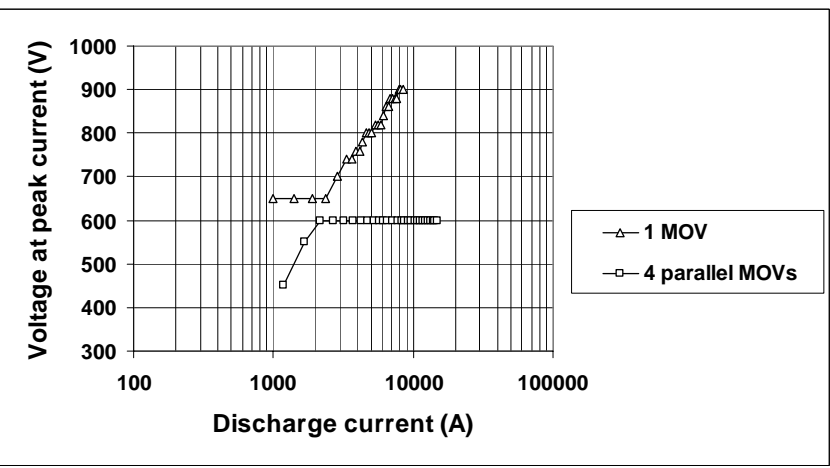

Figure 7. V-I Characteristics for Single MOV and Four Parallel-Connected MOVs (Log Scale)

\section{CONCLUSION}

From this study, it is concluded that higher capability of discharging high current and providing a constant safer voltage for AC power circuits can be achieved by connecting MOVs in parallel. The differences of characteristics regarding the associate voltages and currents between those two connection configurations have been addressed. Experiment results showed that the parallelconnected model provided increased in total peak current and energy-handling capabilities for a given application. Tests can be carried out further to see the breakdown limit of the four parallel-connected MOVs.

\section{ACKNOWLEDGEMENT}

The authors wish to express their gratitude to the staff of High Voltage and High Current Laboratory, Universiti Teknologi Malaysia for their encouragement and assistance in the laboratory works. Credit also given to Universiti Tun Hussen Onn Malaysia.

\section{REFERENCES}

[1] Harris Suppression Products (1998). Application Notes, Littelfuse Inc., Northwest Highway Des Plaines, IL.

[2] Dev Paul (2001). "Low-Voltage Power System Surge Overvoltage Protection", IEEE Transactions on Industry Applications, Vol. 37, No. 1, January/February 2001. 


\title{
Initial Study on the Operation, Energy Usage and Improvement Opportunities of an Ice- Thermal District Cooling System
}

\author{
Mohammad Omar Abdullah, Samsudin Bin Sulaiman, Mohamad Odil Sabri
}

\begin{abstract}
By completion of the Universiti Malaysia Sarawak (UNIMAS) West Campus in the year 2005, total build-up area of this new campus is now approximately 223,619 $\mathrm{m}^{2}$ The high ambient temperature during noon time of about $32{ }^{\circ} \mathrm{C}$ and low rainfall with average annual mean of rainfall around 10 mm or so, causes high temperature build-up in most buildings thus high cooling capacity is required for cooling. The current project aims first to review on the district cooling systems available around the globe and their associated system efficiencies. Subsequently, the various technical aspects and the energy efficiency of the existing district central cooling plants with ice-thermal storage system are discussed. Also, suggestions are made for betterment and corrective operational and maintenance aspect of the central cooling system. It is the objective of the study to present opportunities for energy improvement and hence cost saving.
\end{abstract}

Keywords: district cooling, ice-thermal, energy resources and efficiency, energy saving

\section{INTRODUCTION}

The Universiti Malaysia Sarawak (UNIMAS) is situated in a typical remote area of Kuching, Sarawak. By completion of the UNIMAS West Campus in the year 2005, total build-up area of this new campus is now approximately $223,619 \mathrm{~m}^{2}$ comprise of the academic buildings, colleges and living quarters. The occupants started moving in the first quarter of the year 2006 .

Dr. M. O. Abdullah is currently the Head of Chemical Engineering \& Energy Sustainability Dept, Faculty of Engineering, Universiti Malaysia Sarawak (UNIMAS). He is also a member of ASHRAE, CEng and IMechE (Tel: $\quad+60 \quad 82$ 583338; Fax: $\quad+$ 082-583409; e-mail: amomar@feng.unimas.my).

Samsudin B. Sulaiman is presently working as a Project Coordinator with ASSAR Senari Holdings Sdn Bhd, Petra Jaya, Kuching, Sarawak. He previously worked as a Mechanical Engineer working under the Development and Asset Management Division, UNIMAS (email: sam@assarsenarigp.com).

Mohamad Odil Sabri is a Civil Engineer working under the Development and Asset Management Division, UNIMAS. He is currently working in maintenance management team covering maintenance planning and costing, preparation of the contract document, construction supervision, project and contract management (email: (smodil@bppa.unimas.my).
Of particular interest of the new campus buildings is the cooling system installed in this campus i.e. a district cooling system is selected in favor of the conventional chiller system with the main objectives aimed to reduce development as well as to reduce the operational cost.

A combination system of the individual chiller system, however, was also chosen in view of the remote location of one of the buildings, i.e. the External Laboratory for the Faculty of Resource Science and Technology.

The technology of thermal storage is integrated in the system, in which ice ball is used as energy storage whereby the energy is release in the system when required. The ice ball is made during the off peak or night period where the electrical energy tariff will be reduced by the Electrical Utility company and this is where the savings in energy in terms of cost is achieved.

The current project aims first to review on the district cooling systems available around the globe and their associated system efficiencies. Subsequently, the various technical aspects and the energy efficiency of the existing district central cooling plants with ice-thermal storage system are briefly discussed. Also, suggestions are made for betterment and corrective operational and maintenance aspect of the central cooling system.

\section{REVIEW OF District COOLING Systems EMPLOYING THERMAL STORAGE METHOD}

There are many system advantages of district cooling using thermal storage method compared to the conventional compression cooling system reported in literature. Thermal storage not only dramatically reduces the use of peak-period high cost energy; it can also reduce the total energy usage by as much as $13 \%$ as reported in ref. [1] and [2]. In new construction of multichiller system, initial cost of cool storage system are often comparable to non storage system. The US Department of Energy reported that many ice storage applications can result in lower first cost and/or higher system efficiency compared to non-storage system [3]. The ASHRAE GreenGuide acknowledges that because ice-storage allows downsizing of the refrigeration system, the resulting cost savings may substantially or entirely cover the added incremental cost of the storage system [4].

As pointed out by MacCracken [5], since thermal storage method incorporate AC systems with ICTS operate at full 
load during the night time, the cost of the fuel that is needed for the power plant to produce electricity during the night will be reduced. The two main reasons are: (a) First, in the night, the base load plants are much more energy efficient than day time plants; and (b) Second, line losses are less during the night time because much less power is transmitted at night. Furthermore, results from the study by the California Energy Commission [6] showed that for the two major California utilities, it required $10-30 \%$ less energy to create and deliver power during night periods. [6, 7].

Sebzali \& Rubini [7] had described the method of reducing the peak electrical demand of AC systems during the day period by incorporating an ice cool thermal storage (ICTS) with the AC system in Kuwait. A clinic building has been selected to study the effects of using an ICTS with different operation strategies such as partial (load levelling), partial (demand limiting) and full storage operations on chiller and storage sizes, reduction of peak electrical demand and energy consumption of the chiller for selected charging and discharging hours. It has been found that the full storage operation has the largest chiller and storage capacities, energy consumption and peak electrical reduction. However, partial storage (load levelling) has the smallest chiller and storage capacities and peak electrical reduction. This paper also provides a detailed comparison of using ICTS operating strategies with $\mathrm{AC}$ and AC systems without ICTS. It has been found that incorporating ICTS with a conventional AC system can reduce the peak electrical demand during the peak electrical load period and increase the chiller energy consumption of the AC system. The amount of reduction in electrical power depends on the operation strategy that is selected for the ICTS. Full storage has the highest electrical reduction, however the chiller and storage sizes are high compared with other operation strategies. Full as well as partial load levelling and demand limiting operations consume more energy than a conventional $\mathrm{AC}$ system. It has also been found that the amount of energy consumption can be reduced by shifting the charging time from 6:00 pm to 8:00 pm and 9:00 pm to take the advantage of lower dry bulb temperature and system cooling load.

The system performance can often be improved by the incorporation of a cool-storage system, in that part of the cooling demand is shifted from peak hours to non-peak hours. This brings mutual benefits to the power supplier and the consumers. In order to evaluate the energy performance and cost effectiveness of such an integrated technology, a feasible district cooling plus ice-storage system was developed by Chan et al. [8] for a hypothetical site in Hong Kong. A parametric study making use of the DOE-2 and TRNSYS simulation software was conducted to evaluate the system performance at different partial storage capacities, control strategies, and tariff structures. Other than the basic design factors, the results from 27 cases showed the importance of the tariff structure, the capital and electricity costs in this issue.

Performance of three types of district heating / cooling and hot water supply system with natural and unused energy utilization were examined by using system simulation by Tanaka et al [9]. The first system is the conventional system in which an electric driven turbo chiller and a gas-fired boiler are installed as the heat source. Two alternative systems utilize waste heat from space cooling and heating. One is designed based on short-term heat recovery and the other employs the concept of an annual cycle energy system (i.e. seasonal heat recovery). All of the three systems use solar thermal energy for hot water supply to the residential zone. The index for evaluation is the coefficient of performance of the overall system, based on primary energy. As a result, it was found that the seasonal storage system could decrease the energy consumption by about $26 \%$ and the short-term heat recovery system could decrease it by about $16 \%$ compared with the reference system. In designing the heat recovery system, a balance of cooling / heating demand is an important factor. Therefore a sensitivity analysis of performance of the overall system and the seasonal thermal storage for several load patterns was performed. From these results, it was found that if the amount of heating/cooling demand were well balanced, an improvement of energy performance could be achieved and the utilization factor of the seasonal tank would become higher. Furthermore, the volume of the seasonal storage tank could be reduced.

\section{THE UNIMAS' ICE-ThERMAL DiSTRICT COOLING PLANT}

Figure 1 shows the UNIMAS campus layout plan illustrating the district cooling water pipeline from the Mechanical Electrical Plant (MEP) throughout the university's campus.

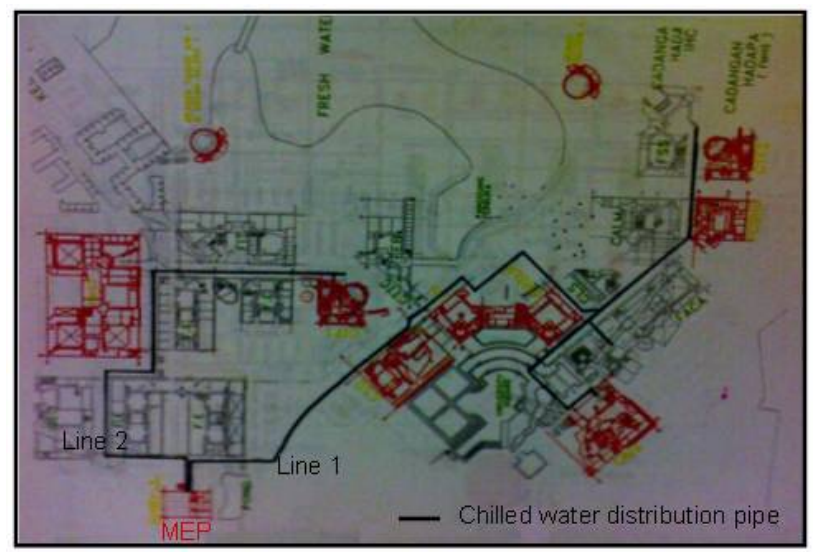

Figure 1. District chilled water distribution pipeline. Note that there are two chilled water lines (Line $1 \&$ Line 2) from the MEP [10]

Figure 2 depicted the simplified schematic block diagram of the cooling system, with plate type heat exchanges for the individual faculty buildings. Table A1 in the Appendix summarize the specifications for the ice-thermal district cooling system.

The ice plant comprises of a brine chiller system, a thermal storage plant, a cooling tower system and a heat exchanger system [10]. It generates chilled water required to meet the cooling load demand for various air-handling units distributed in the buildings. The brine chillers are also used to generate ice for the thermal storage plant. It is divided into the following 5 sections, i.e. (1) brine chiller system, (figure 3 and figure 4) (2) base chiller system; (3) cooling tower system; (4) thermal storage system consists of 1 ice storage tank (figure 5) and associated motorized valves; and (5) heat exchanger system which consists of 3 heat 
exchangers, 3 chilled water pumps and 3 brine pumps (figure 6).

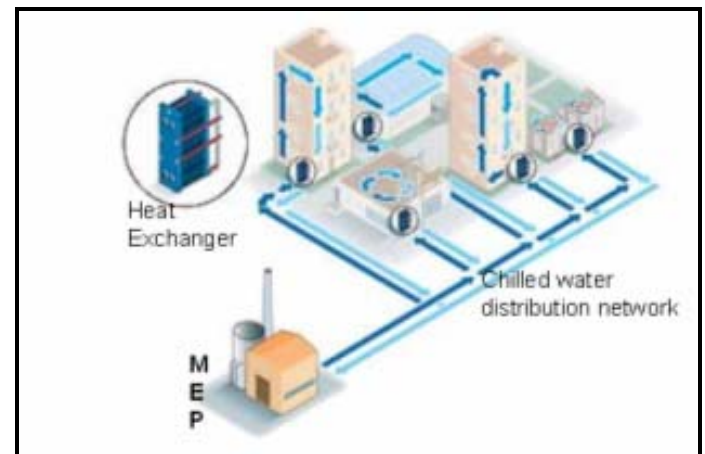

Figure 2. Schematic diagram of the chilled water distribution network [11]

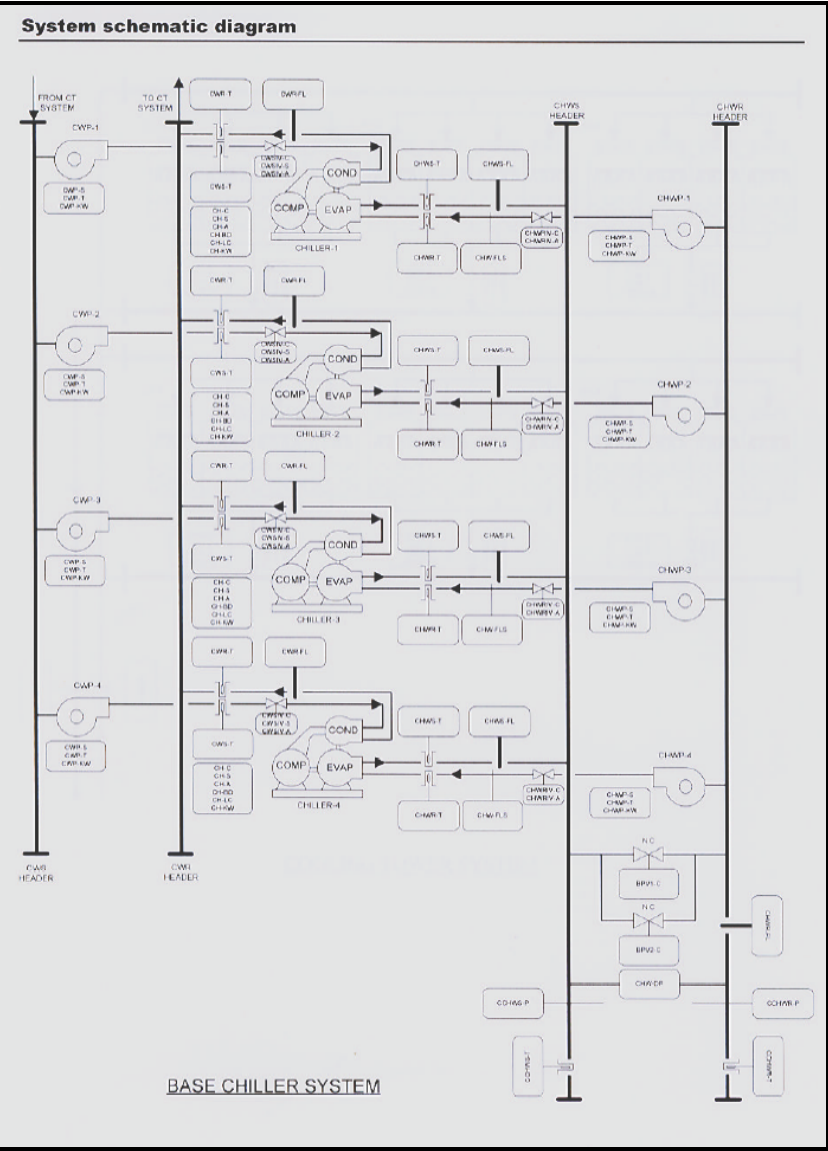

Figure 3. Brine chiller system - schematic diagram

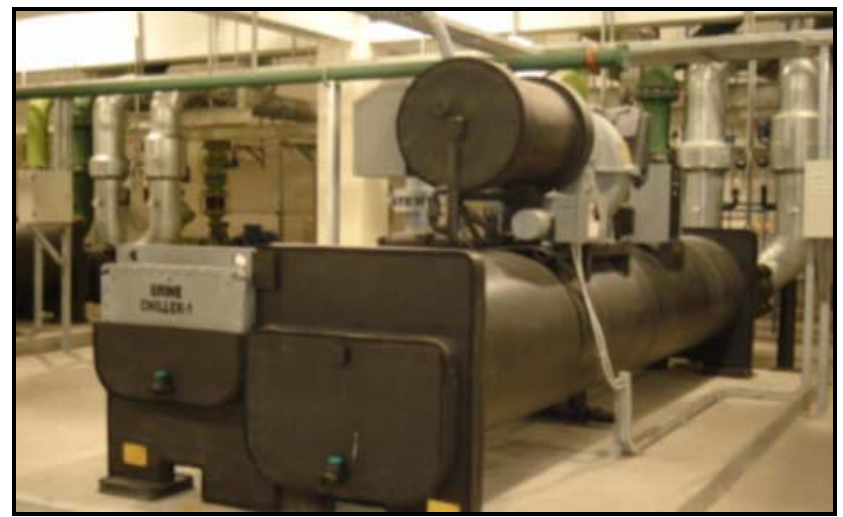

Figure 4. Brine chiller

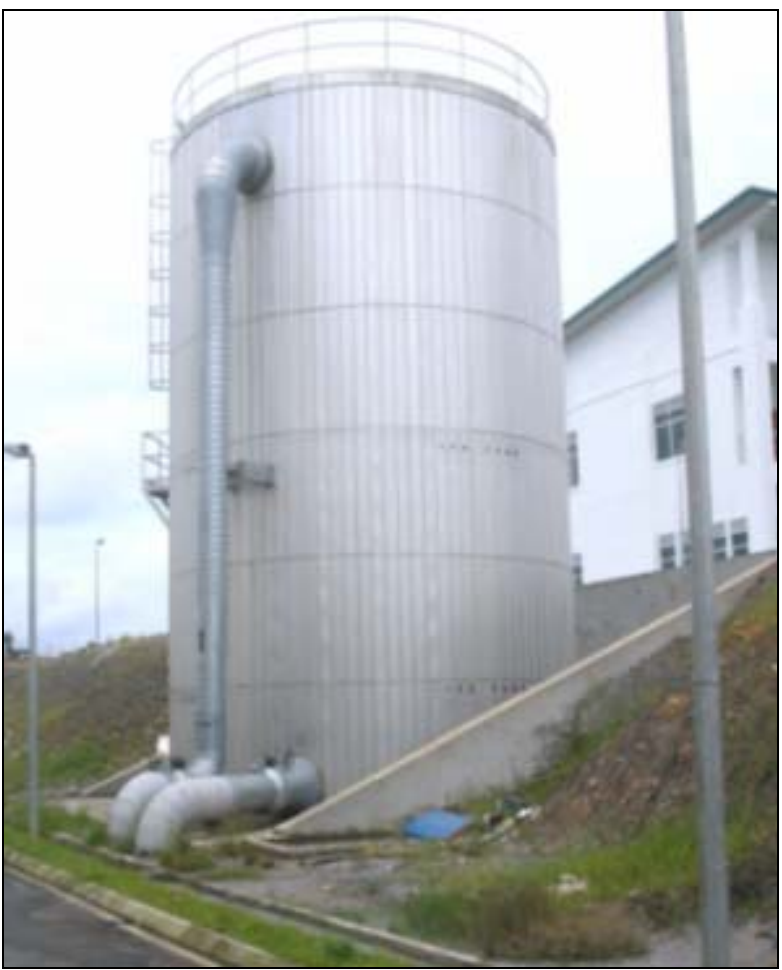

Figure 5. Photo of the ice storage tank

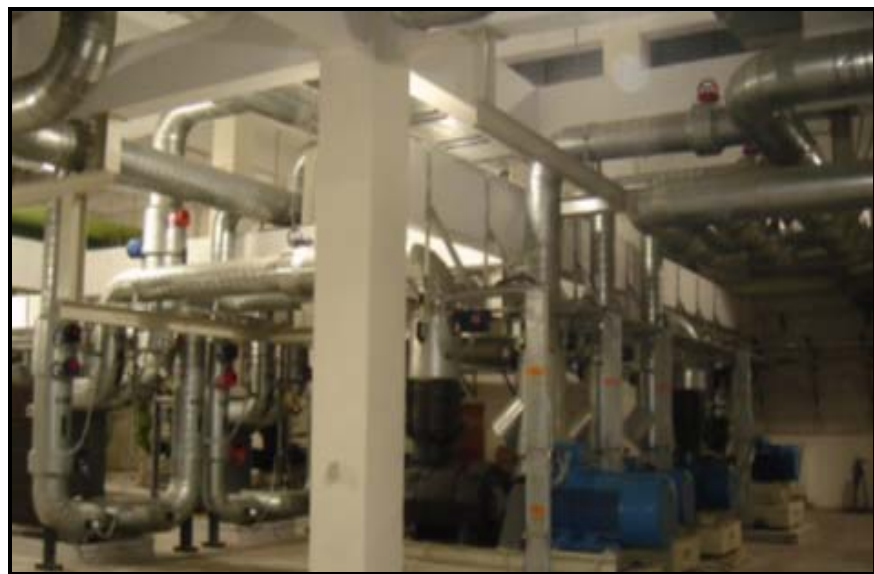

Figure 6. The MEP building with Chiller Pumps and facilities

The main equipment used in the chilled water system is a chiller package that includes:

- A refrigeration compressor (reciprocating, scroll, screw or centrifugal type),

- Shell-and-tube heat exchanger (evaporator) for chilled water production;

- Shell and tube heat exchanger (condenser) for heat rejection in water cooled configuration;

- Copper tube/Aluminum finned condenser coil for heat transfer enhancement, and fan (condensing unit) for air cooled configuration;

- An expansion valve between condenser and the evaporator.

The middle refrigerant loop is connected through a copper piping forming a closed loop. The water circuit on the chilled waterside is connected through an insulated carbon steel pipe and is a closed loop. The condenser water connected through a carbon steel piping is an open loop and 
requires 2 to $3 \%$ make up water as a result of evaporation, drift and blow down losses from the cooling tower.

The chilled water system is also called central air conditioning system. This is because the chilled water system can be networked to have multiple cooling coils distributed through out a large or distributed buildings with the refrigeration equipment (chiller) placed at one base central location.

Chilled water systems are typically applied to the large and/or distributed areas. It is based on a standard rule of thumb that the capacity ranges of 20-2000 TR suitable for an area of 3000 square feet and above.

\section{VARIOUS BUILDINGS AND TYPICAL COOLING LOADS}

The buildings concerned are Faculty of Engineering (FE), Faculty of Research Science and Technology ( FRST), Faculty of Information Technology (FIT), Faculty of Cognitive Science and Human Development (FCSHD), Faculty of Creative Arts (FACA), Centre of Academic Information System (CAIS), Chancellery \& University House, Centre of Teaching Facilities (CTF) \& etc. All these buildings house our academic staffs, support staffs and students depending in the numbers of students for each faculties and buildings. Typical cooling loads estimation is given in Table 1.

Table 1. Cooling load estimation ${ }^{1}$

\begin{tabular}{|l|l|l|r|}
\hline Item & Facility & $\begin{array}{l}\text { Total Net Area( } \\
\mathrm{m}^{2} \text { ) completed } \\
\text { by 2005 }\end{array}$ & $\begin{array}{l}\text { Total } \\
\text { Cooling } \\
\text { Load (TR) }\end{array}$ \\
\hline 1 & FRST $^{2}$ & 45,862 & 2,057 \\
\hline 2 & FIT & 11,792 & 529 \\
\hline 3 & FACA & 13,932 & 625 \\
\hline 4 & FE & 24,808 & 1,113 \\
\hline 5 & FHSD & 4,751 & 213 \\
\hline 6 & CLS & 2,541 & 114 \\
\hline 7 & CTF & 4,632 & 208 \\
\hline 8 & CSD & 8,234 & 370 \\
\hline 9 & CAIS & 15,800 & 709 \\
\hline 10 & CNCL \& UH & 12,595 & 565 \\
\hline & & $\begin{array}{l}\text { TOTAL } \\
\text { COOLING } \\
\text { LOAD }\end{array}$ \\
\hline
\end{tabular}

Source: Hon, Private Communication, Hashim \& NEH (Sept 2000)

\section{INITIAL ENERGY StUdY AND ENERGY SAVING OPPORTUNITIES CONSIDERATIONS}

Recently, a few initial investigations had been done to inspect on the overall district cooling system; to research and identify ways to possibly save cost; thus attempt to solve the high electric bill problem incur on the campus. The total electrical energy consumption for UNIMAS for year 2007 is given in Table A2 [13]. About two-thirds of energy consumption is thought due to air-conditioning applications.

${ }^{1}$ Cooling load estimates (Year 2005) at the assumption of the cooling load as $50 \mathrm{Btu} / \mathrm{hr} / \mathrm{ft}^{2}$

${ }^{2}$ Individual split air-cooling system is use in FRST due to remote location of the building from the MEP and the rest of the buildings.

\section{A. Air-distribution}

One of the greatest concerns is the air-distribution and the associated energy balance. It was found that the distribution ducting system has not been evenly distributed, with some buildings zones having too low temperature whilst others were reported as not having sufficient cooling. Equation (1) could be written to represent the energy balance desired at each $i$ building; indicates that there is a need to scrutinize various quantities and operating parameters of the present system distribution.

$\sum Q_{\text {total }, i}=\left(1.1-\frac{B t u}{h r . c f m . F}\right) \times c f m \times\left(T_{\text {retrun }}-T_{\text {supply }}\right)$

where, $\mathrm{Q}_{\text {total }}$ is heat gain in building; $\mathrm{T}_{\text {supply }}$ is supply temperature fixed at system; and $\mathrm{T}_{\text {return }}$ is return temperature.

Air-lock problems found especially in the FRST building [12] may require air-conditioning pump or other suitable devices for suction lift.

The trouble-shooting and thus remedy of the above distribution problems are well on-going.

\section{B. Existing Building Design with Big Glass Walls Area}

Most buildings were designed with big glass wall which cause temperature buildup in buildings due to unwanted green house effect. Figure 7 showing a typical building with huge glass surface. Whilst it has esthetic design value, the heat loss can be enormous. The use of blinds and other sun shading such as that shown in area B of Figure 8 are suggested for lowering the heat buildup in buildings.

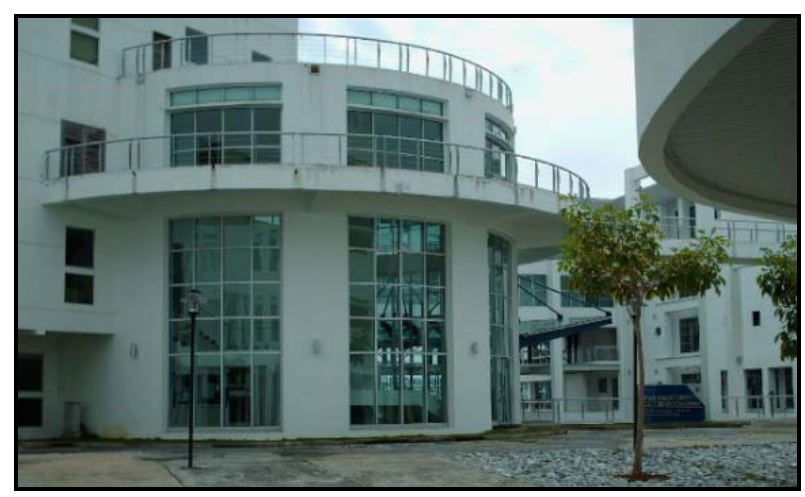

Figure 7. A typical building showing huge glass surface; whilst it has esthetic design value, the heat loss can be enormous

\section{Prolong Door Opening and Bad Practice}

Figure 9 and 10 showing the recent photos of an openingdoor of a glass and a wooden door respectively. Such prolong opening contributes to influx of hot air to the building; whilst replacing the internal cold air from the building; hence unnecessary energy loss.

\section{Operation of a Single Building during Non Office Hours}

The district cooling system is also operated to provide cooling to the CAIS building during non-office hours, and weekends. This operation causes some energy wastage due to transportation loss of the supply chilled water. This is because of the remote of the CAIS building from the MEP 
location.

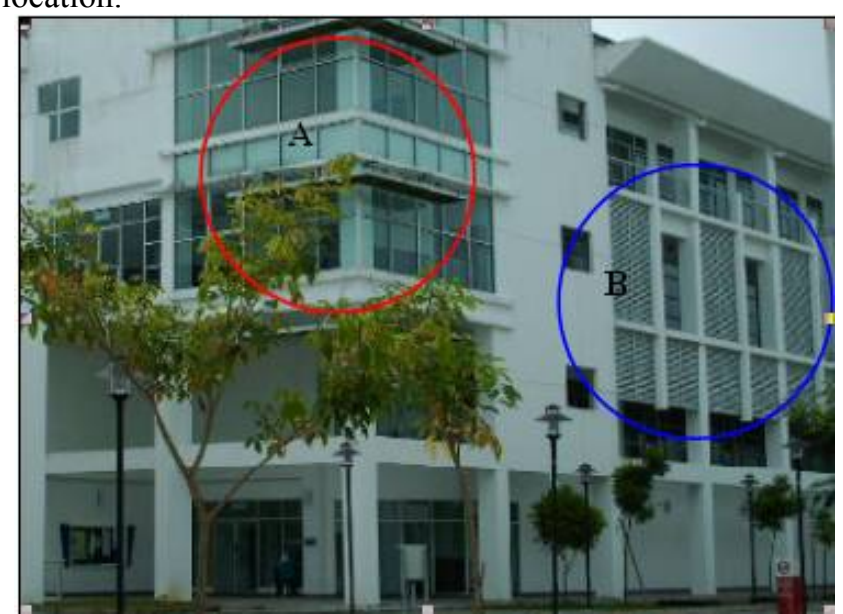

Figure 8. A typical building showing huge glass surface, with (A) exposed glass area and (B) shading structure for minimize heat absorption from the sun

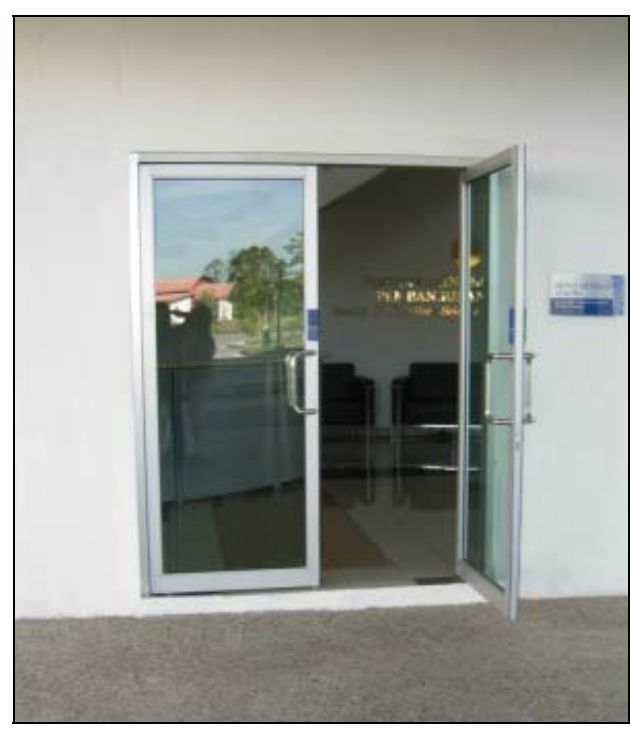

Figure 9. A photo taken recently showing the opening-door of one of the buildings with glass door

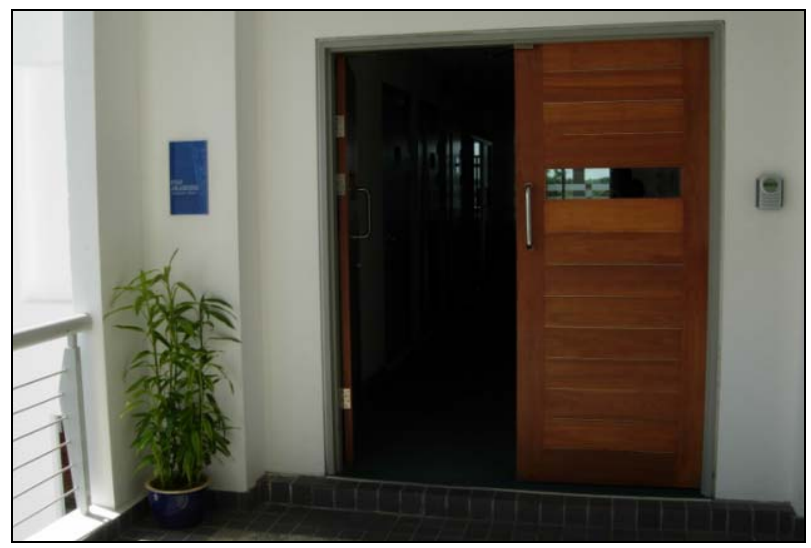

Figure 10. A recent photograph showing the opening-door of one of the buildings with wooden door

\section{CONCLUSIONS}

Various technical aspects and the energy efficiency of the existing district central cooling plants with ice-thermal storage system are discussed. Also, suggestions are made for betterment and corrective operational and maintenance of the central cooling system. Some energy saving opportunities could be summarized and listed as follows:

- Improving building air distribution and networking

- Optimize operating conditions of the total district cooling system including sufficient suction lift for some buildings such as FSTS building.

- Perhaps to suggest an individual cooling system for the CAIS building.

- Insulation improvement of the pipeline

- Tinting on glass walls

- Use of blinds and shading structure to minimize heat buildup

- Good practice and reduction of the duration of door opening.

- Consider using photovoltaic (solar PV) cum shading technology to provide cooling (however initial cost could be high).

\section{RECOMMENDATION FOR FURTHER WORK}

Thermal transient simulation and optimization study of the district cooling system is proposed for further work. Such simulation study carry out by the team members, considering heat and mass transfer of the chilled water and air distributions, is currently only in it's early stage.

\section{APPENDIX}

Table A1. Ice-thermal district cooling specification [13]

\begin{tabular}{|c|c|}
\hline Total capacity & 7,000 refrigerant tones \\
\hline Ice thermal system & $\begin{array}{l}4 \text { chillers (for discharging) } \\
\text { and } 2 \text { brine chillers (for } \\
\text { charging) and } 6 \text { cooling } \\
\text { towers }\end{array}$ \\
\hline Refrigerant & $\begin{array}{l}\text { Ethylene glycol mixed } \\
\text { with water }\end{array}$ \\
\hline $\begin{array}{l}\text { Charging time for two brine } \\
\text { chiller to produce ice }\end{array}$ & $12 \mathrm{pm}$ till 8 am \\
\hline $\begin{array}{l}\text { Temperature in the storage } \\
\text { tank }\end{array}$ & $-5.5 .^{\circ} \mathrm{C}$ \\
\hline $\begin{array}{l}\text { Efficiency of ice-thermal } \\
\text { system }\end{array}$ & $80-90 \%$ at night \\
\hline $\begin{array}{l}\text { Percentage cooling supply } \\
\text { for West Campus }\end{array}$ & Around 25\% \\
\hline
\end{tabular}

Table A2. Total electrical energy consumption and energy cost for UNIMAS for year 2007 [13]

\begin{tabular}{|c|c|c|c|}
\hline Month & $\begin{array}{c}\text { Units Consumed, } \\
\text { KWH }\end{array}$ & $\begin{array}{c}\text { Avg daily } \\
\text { Consumed,KWH }\end{array}$ & Cost,RM \\
\hline January & 452,416 & $48,389,84$ & $900,463.00$ \\
\hline February & 398,392 & $47,864.22$ & $804,530.80$ \\
\hline March & 498,413 & $54,819.47$ & $990,942.40$ \\
\hline April & 482,245 & $49,184.00$ & $915,234.40$ \\
\hline May & 434,634 & $45,348.97$ & $843,902.80$ \\
\hline June & 381,109 & $40,762.93$ & $758,539.60$ \\
\hline July & 464,217 & $50,146.96$ & $933,145.30$ \\
\hline August & 496,089 & $52,376.97$ & $974,623.60$ \\
\hline
\end{tabular}

\section{ACKNOWLEDGMENT}

The support and encouragements from both Mr. Lawrence Abdullah (Electrical engineer of ASET) and Mr. Ngerantar ak Undi (mechanical engineer of ASET) are highly appreciated. The help from the ASET's technicians viz. Abu Bakar and Pelle are also acknowledge herein.

\section{REFERENCES}

[1] O'Neal,E., 1996, Thermal storage system provide comforts and energy efficiency. ASHRAE Journal 38(4). 
[2] MacCracken, M., 2006, California Title 24 \& Cool Storage, ASHRAE Journal October 2006 page 29-33.

[3] DOE, 2007, Department of Energy Federal Technology Alert, Thermal Energy Storage for Space Cooling, DOE/EE-0241 Available: http://www.doe.gov.

[4] Grumman, D.L., 2004, ASHRAE GreenGuid. "ASHRAE GreenTip \#15." Chapter 9. P.86

[5] MacCracken M., 2004, Thermal energy storage in sustainable buildings. ASHRAE Journal 2004;46 (September).

[6] California Energy Commission, 1996, Source energy and environmental impacts of themal energy storage. Report \#500-95-005. Available from: www.energy.ca.gov/reports/reports_500.htm, assessed on 14 November 2006

[7] Sebzali, M.J., Rubini, P.A., 2006, Analysis of ice cool thermal storage for a clinic building in Kuwait, Energy Conversion and Management 47 (2006) 3417-3434.

[8] Chan,A.L.S, Chow, T.T., Square K.F. Fong and John Z. Lin, 2006, Performance evaluation of district cooling plant with ice storage, Energy, Volume 31, Issue 14, November 2006, Pages 2750-2762.

[9] Tanaka, H , Tomita, T. and Okumiya, M., Feasible study of a district energy system with seasonal water thermal storage. Solar Energy Vol. 69 , No. 6, pp. 535-547, 2000

[10] MEP, 2004. MEP, 2004, Facility Management System Project Application/Training Manual, Universiti Malaysia Sarawak.

[11] Samsuddin B Sulaiman, 2007, Technical application and cost implication of district cooling system in UNIMAS new campus, Preliminary MEng thesis.

[12] Abu Bakar, 2007, Tecnician working under the Development and Asset Management Division, UNIMAS. Private communication on 8 Nov 2007.

[13] Pelle Tunggi, 2007, Aset and Management Division, UNIMAS, Private communication 


\title{
The Study of Vortex Formation after a Bluff Body Due To Wind Velocities with the Effect of underneath Wavy Surface
}

\author{
Jamaluddin Md Sheriff, Kahar Osman and Asral
}

\begin{abstract}
The present study observes the effect of wave-like structure on flow after a bluff body. Particle Image Velocimetry (PIV) technique has been employed to measure the flow velocity and to capture the flow pattern in various wavy surface conditions, positions of bluff body of which has height to width ratio of two, and Reynolds numbers. Twodimensional numerical solution for the same test condition has been performed by employing the turbulent model of $k-\varepsilon$. Sinusoidal surfaces were chosen for wavy condition to represent the wavy boundary. Range of Reynolds numbers were 1480 to 3300 and the waves amplitude were 0.13 of the bluff body height (0.13D), 0.33D and 0.67D. Comparisons were made against flow over the flat surface for the same flow condition. For most cases studied, two vortices always present in the region in the vicinity close to the bluff body. Larger size of vortices appears in the upper area than those in the lower area of the flow. For low amplitude wave, the flow tends to follow the wave and resulted in large streamwise negative velocity zone behind the bluff body. As the wave amplitude increases, the size of this negative velocity region will reduce. Low amplitude wave also increases the fluctuation of the flow as the Reynolds number increases. However, higher wave amplitude suppresses the flow to the same order of fluctuations. In general, the numerical solutions were in good agreement with the experimental results.
\end{abstract}

Keywords: PIV, vortex, wave, bluff body

\section{INTRODUCTION}

The profile of flow around bluff bodies such as cubes, rectangular and circular cylinders, and flat plates are relevant to many engineering designs, such as constructions under the action of wind loading like building, chimneys and water tank tower, steel tower suspension bridges or marine structures under the action of water loading. Wind driving forces on infrastructures, located nearby coastal region are considered to be an important parameter in design. The presence of them on the shores is experienced on the wave water in any direction of wind flows. The wind load to the buildings at the coasts can exert from the land and the sea direction. The increase of speed of wind flow magnitudes cause the high amplitude of water wave before

Jamaluddin Md Sheriff, Kahar Osman and Asral are with the Faculty of Mechanical Engineering, Universiti Teknologi Malaysia, 81310 UTM Skudai, Johor, Malaysia (phone: 607-5534744; fax: 607-5566159; e-mail: jamal@fkm.utm.my). exerting the structure. The flow patterns around the structure are different between water wave and over the ground. Since the wind exerting on the structures vortices, this phenomena affects to the structures. It can destructive and risk to the life safety. Because flow vortex detected can create the high energy and cause to collapse the bridges and buildings. So in constructing a structure should be strong enough to decrease the risk caused by the wind. The energy of wind exerting on the structures depend on the direction flow to the cross- area, different direction will give different effect to them (Richard and Hoxey, 2002). The dominant wind loads works as normal to the infrastructure is considered as the main parameter in design process.

To describe the forces exerted to a body in a moving fluid it is common assumption that the forces was depend on the density $\rho$ and dynamic viscosity $\mu$ of the fluid, the relative velocity between the body and wind flow $U$ and a characteristic dimension of body D, Larose and D'Auteil (2006). These four components have effects on each other. By assuming the density $\rho$, viscosity $\mu$ and height of body $\mathrm{D}$ are constant and velocity of flow $\mathrm{U}$ is varied, the effect of Reynolds number to the flow structure after the bluff body was observed. The experimental and numerical study of the velocity profile after a body is reported in this paper. The velocity field was measured using Particle Image Velocimetry (PIV) technique.

The objective of this current study is to investigate the effect of wavy boundaries upon profile of flow after a bluff body for various Reynolds numbers. PIV was used for qualitative visualization, and quantitative of the flow field. These can then be used to analyze the flow using the velocity data determined. This measuring method is a well established quantitative flow visualization technique (Nakagawa and Hanratty (2001), Zhang, Daichin, and Lee (2005), Angele and Klingmann (2006)). The method utilizes techniques of flow visualization whereby the flow is seeded with light scattering particles which are assumed to follow the flow. The plane of interest is illuminated by a sheet of laser light and an image of the in plane movement of the particles recorded. A short duration multiple exposure images of particles are recorded and analyzed to obtain the velocity field.

Experimental study on the characteristics of flow in the wake region of bluff body with the ground effect has been investigated by Kim and Gerops (1998). The author studied 
the effect of various ground clearances on the flow around a variety of bluff bodies. Barlow et al. (2001) presented the ground effect with different aspect ratios, varying ground clearances, and different levels of underbody roughness were performed in wind tunnel experiment. Jefrey et al. (1984) identified the separated region, an attached boundary layer, and a free shear layer formed by the detachment of the boundary layer from the wave surface. Zhang et al. (2005) reported the sinusoidal surface geometry significantly modifies the near-wake structure and strongly controls the three-dimensional vortices formed in the near wake. Larose and Auteuil (2006) demonstrated that the aerodynamics of bluff bodies with sharp edges can be sensitive to Reynolds number effects. Schewe (2001) reported that Reynolds-number effects can be have drastic consequences on the unsteady behaviour and on the fluid forces acting on an aeroelastic system. Tieleman et al. (2003) measured the pressure and area load fluctuations on the top surface of a surface-mounted prism in seven different wind tunnel configurations. Two groups of eight pressure taps placed in critical areas are considered. The characterization is presented in terms of the mean, root mean square, and peak values, probability distributions, peak durations, and correlation coefficients. They conclude that the quality of wind tunnel experiment is depends greatly on the incident turbulence for assessment of wind loads on low-rise structures.

Computational fluid modeling in wake region has been widely used by many researchers in their investigation (Rodi (1997), Bouris and Bergeles (1999), Krajnovic and Davidson (2001). Selvarajan, Tulapurkara, and Ram (1998) reported the amplitude of shear stress along the wall increases and pressure decreases with increase in Reynolds number.

Nowadays, since computing time is not as expensive as it was decades ago, the role of computer modeling has been part and partial of fluid flow research. Taking these advantages, in this study, numerical approach was used to aid predictions and assist observations for flow conditions after the bluff body.

\section{Methodology}

For all test cases Fluent was employed to predict the flow pattern and condition. Two-dimensional model was used to simulate the flow against the bluff body on the wavy surfaces. The flow was simulated in a numerical tunnel. No slip boundary conditions were imposed on all walls. The ratio of the length of the test section to the height of the bluff body is 63 . The ratio of the height of the test section to the height of the bluff body is 14.5. The uniform flow conditions at inlet for Reynolds number 1480, 1850, 2775, and 3300 were used in this study. Air is flowing through the wavy surface from the right and solutions were forced in order to study the characteristic of the vortex in the wake region.

In order to simulate the effect of wavy surface to the flow against the bluff body, sinusoidal surfaces with different amplitude were used. Flat surface was also included for comparison. The $k-\varepsilon$ model was used to capture the turbulent phenomenon. Intensity of the grid was increased within the area of the vortex. Simulations were left to run until steady state conditions were reached.
Figure 1 is a schematic diagram of the experimental setup. The test section of the wind tunnel is $1.89 \mathrm{~m}$ long and has $29 \mathrm{~cm} \mathrm{x} 43.5 \mathrm{~cm}$ cross-sectional area. To ensure the uniformity of the flow at inlet the honeycomb structure were placed. The flow coming out of the honeycomb then flow over the wavy surfaces and against the bluff body. A gate valve was used to control the flow rate of the air in the tunnel. The mean velocity before the bluff body was set varied from $0.8 \mathrm{~m} / \mathrm{s}, 1 \mathrm{~m} / \mathrm{s}, 1.5 \mathrm{~m} / \mathrm{s}$, and $1.8 \mathrm{~m} / \mathrm{s}$. The bluff body wake air velocity was measured during each run. The air flow was seeded with smoke of vaporizing fog oil droplet which produces seeding particles on the order $1 \mu \mathrm{m}$ in diameter.

The experimental technique used in this study for measurement of the velocity is a multiple pulsed photographic system. A simplified diagram of the PIV setup is shown in Figure 1. A DANTEC Neodymium - YAG laser double pulsing at $10 \mathrm{~Hz}$ was used to illuminate the flow field. The Nd-YAG laser source was securely mounted on top of the wind tunnel test section. As shown in the Figure1, the resulting light sheet illuminated a XY-plane, which inline with the centerline of the body. The illuminated section was imaged using NIKON cross correlation CCD camera having $1200 \times 1600$ pixels with time interval of $1 / 1000 \mathrm{sec}$. the camera was set perpendicular to the test section and right angles to the laser sheet as shown in Figure 1.

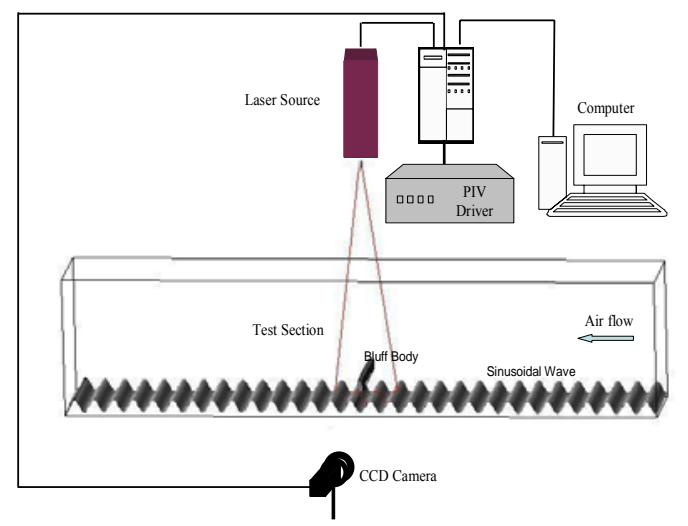

(a)

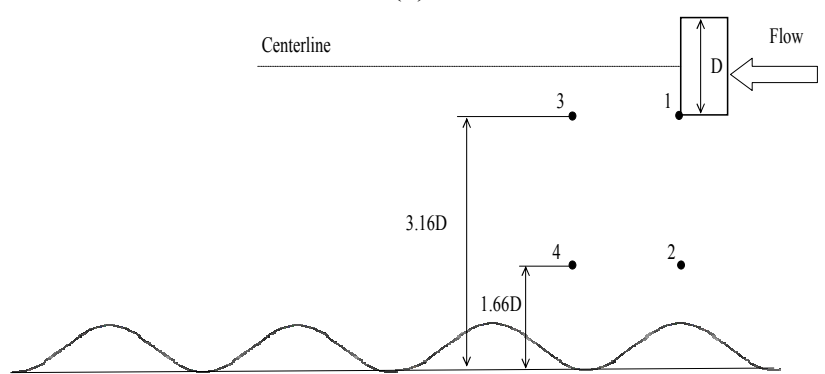

(b)

Figure 1. Schematic diagram of experimental setup and PIV system: (a) the bluff body positions setup, and (b) definition of centerline of flow profile. Not to scale.

The pulsing light sheet and the camera are synchronized so that particle positions at the instant of light pulsed number 1 are registered on frame 1 of the camera and particle positions of light pulse number 2 are registered on frame 2 of the camera. A FlowMap which is dedicated software for DANTEC PIV system was used for data 
acquisition and processing. The experimental data obtained was recorded in the computer and analyzed using FlowManager software which provides fast data capture, evaluation, and display. The meaningful visual representation of velocity field vector map and streamline were also done by this software.

\section{RESULTS AND DISCUSSIONS}

In this section, tabulated data of characteristics of the flow for various Reynolds numbers will be discussed. The characterizations are based on the following definitions.

Region of negative streamwise velocities behind the bluff body- this region is defined as the major activity that occurred behind the bluff body. Similar characterization has been used by Johansen, Wu, and Shyy (2004). Overall bandwidth measured with referenced to peak positive and negative velocities-this bandwidth is defined as measurement of average fluctuations for streamwise and spanwise velocities for all flow Reynolds numbers for the same wave. Fluctuation of velocities with respect to variations of each Reynolds number- this fluctuation is defined as the effect of Reynolds number to the flow. Overall downstream velocities approaching main velocitythis characteristic defines the behavior of the flow at further downstream as it merges into the main stream flow.

Moving the bluff body vertically closer to the flat surface showed region of backflow is in the same order of magnitude. High Reynolds number seemed to produce the smallest region. On the other hand, Johansen, $\mathrm{Wu}$, and Shyy (2004) showed backflow region was reduced in size. Also, the overall flow seemed to be reduced in bandwidth. As the bluff body approached the ground, the flow took longer time to merge with the main flow. Larger fluctuation was observed for low Reynolds number when the body was further away, but reversed phenomena were seen at higher Reynolds number.

These case studies were intended to study the effect of wavy surface on the flow behavior. Wave-1 which was a small amplitude wave with slightly sloped surface, was observed to change the flow behavior. In comparison for four positions where the bluff body located above the peak and the valley of the wave, the size of the region of negative backward flow increased fifty percents as compared to that of flat surface. Again, the size of the region of backward flow was inversely related to Reynolds number, above the peak of wave increased Reynolds number the region becomes smaller for position furthest the wave and larger for position closer to the waves, however for position above the valley is in the contrary. The bandwidth remained to be in the same order. When it is compared to the flat surface, in wave-1 appeared the difference magnitude of the peak of velocity. This result indicated the wavy surface could affect the behavior of flow along the centerline, clearly seen that for the bluff body closer to the wave, the bandwidth becomes smaller and tend to increase compared to the flat surface. High Reynolds number produced high fluctuation for body close and further away from the wave as compared to that of low Reynolds number. Comparing the four positions, position 1 had the larger fluctuation. However, the downstream flow for position 9 seemed to merge into mainstream at earlier time than that of position 1, 4, and 12 .
In the present study the effect of wave amplitude was studied. Wave-2, which had amplitude larger than that of wave-1, was imposed to observe the change of flow behavior. In comparison of four positions where the bluff body was located above the peak and the valley of the wave, the size of the region of negative backward flow showed decreased half in size as compared to that of wave-1, even though the amplitude of wave-2 was double of wave- 1 . When the bluff body was located closest to the wave, the region of negative velocity showed greater region for position 4 . This is due to the fact that the flow after the bluff body was affected by the peak of the wave that it encountered right after the bluff body. On the other hand, flow for position 2 encountered a valley right after the bluff body. This result indicated that in wave-2 the effect of the wavy surface to the flow behavior appeared to be more significant as compared to that in wave-1. As the bluff body moved further vertically upward (position 1 and position 3), the negative region of the flow was seemed to be in the same order of magnitude but both were lower than that of position 4 and higher than that of position 2 . These results show that the effect of wave is diminishing but still present and will eventually reach size of $1 \mathrm{D}$ as discussed by Johansen, Wu and Shyy (2004). According to Nakayama and Sakio (2002), the flow took about twice the amplitude of the wave to reach free stream velocity from the valley. In this study, even though the location of the bluff body was outside the range discussed by Nakamaya and Sakio (2002), the results still showed the effect of the wavy surface. For this case, the bandwidth increased in both locations as it moved further upward (position 2 to position 1 and position 4 to position 3). Comparing the results of wave- 2 with that of wave-1, the latter wave showed smaller bandwidth. This shows that the increase in wave amplitude suppresses the fluctuations of flow for all Reynolds number studied. For location further upward from the wave (position 1 and position 3), the amplitude of the waves showed no significant effect to the fluctuation of the flow.

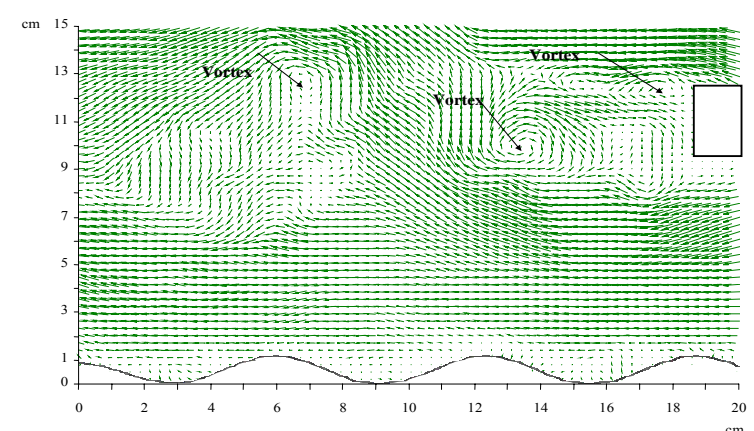

(a)

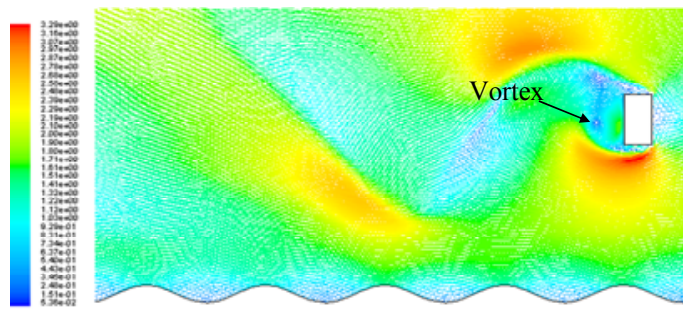

(b)

Figure 2. Velocity vector fields for: position 1 wave-1, $\mathrm{Re}=$ 3300 (a) experimental, (b) numerical. 


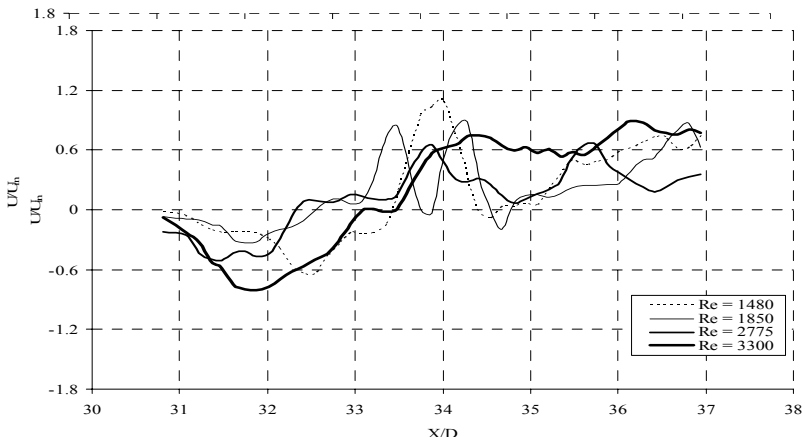

(a)

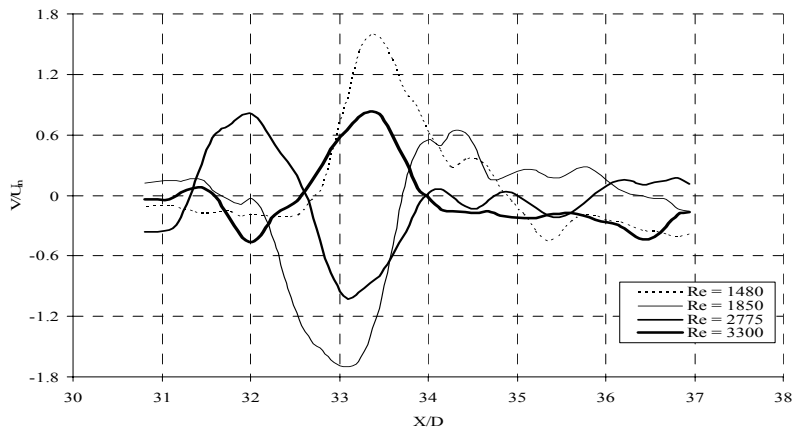

(b)

Figure 3. Velocity components at centerline for position 1 in various Reynolds number for wave-3: (a) Streamwise, (b) Spanwise from experimental.

At position 1, the fluctuation of the flow decreased as the Reynolds number increased. However, as the bluff body moved closer the wave (position 2), the relationship was otherwise. For position 3, the flow again showed decreased in fluctuations as the Reynolds numbers increased but no significant changes in fluctuation for position 4 as the Reynolds numbers were varied. Meanwhile, the downstream flow for position 3 seemed to reach the mainstream velocity the earliest and followed by position 1,4 , and 2 . This means that when the bluff body is located further upward, it seemed to settle down earlier as compared to that of position closer to the wave.

Further increasing the amplitude of wave to approximately $1.3 \mathrm{D}$ showed further changes to the flow behavior. Firstly, the region of negative velocity reduced drastically to approximately 20 percent of its size when moving from position 1 (highest) to position 2 (lowest) when the bluff body is above the peak of the wave. Less drastic reduction was observed when bluff body moved from position 3 (highest) to position 4 (lowest) when the bluff body was placed above the valley. For the locations of bluff body above the peak and the valley of the wave, the region of negative velocity seemed to increase. This shows that, the amplitude of the wave has significant effect on the behavior of the flow right after the bluff body. All results are valid for all Reynolds numbers studied. Secondly, comparing the bandwidths of all flow conditions showed that for the same horizontal position of the bluff body, the bandwidths maintained it magnitude but the magnitude reduced as the bluff body moved vertically closer to the wave, i.e. approximately 70 percent. Larger fluctuation was observed for flow with low Reynolds number when the body was further away, but reversed phenomena were seen at flow with higher Reynolds number. However, no significant effect by Reynolds number to the flow fluctuation as the bluff body moved vertically closer to the wave. Finally, this case study shows no particular trend is observed with respect to the flow approaching main stream velocity. The samples of graphical representations of the discussion are shown in Figure 2 and Figure 3 and the summary of the quantitative criteria are tabulated in Table 1 and Table 2.

Flow field after the bluff body was measured by PIV and calculated using numerical solution. Sample results of timeaverage velocity vector are shown in Figure 1. It can be seen that the vortices were found to shed along the shear layer generated from the edge of bluff body. For position 1 where the bluff body placed further from the surface three vortices were visible for experimental and one for numerical. It is possible that the numerical solution may have not been able to capture all the small vortices present.

For position 2, where the bluff body above the peak of the surface, small vortices emerged behind the bluff body. Approximately four to five small vortices were visible for both numerical and experimental results were observed. It is possible that the numerical solution may have not been able to capture all the small vortices present. The region of formation of vortex took place in a region approximately of 4.3D from behind bluff body. It can be seen that the lower vortex (below centerline at the bluff body) are smaller then the upper vortex. Owing to the wave clearance, which limited the amount of fluid that can be entrained by the lower vortex.

For position 3 where the bluff body placed further above the valley of the wave two vortices displayed in the lower area and one in the upper area of the bluff body. meanwhile, in the middle area backflow clearly seen in this figure. Numerically fail to capture the vector same to the experimental results.

For position 4 where the bluff body placed closer to the valley of the wave, both of numerical and experimental exhibited three vortices after the bluff body. Nonetheless, different in location and size of vortices occurred.

From those figures can be observed closer to the wave, the wavy surface effect clearly seen that the flow fields were significantly affected by the wave due to clearance between the bluff body and the wave becomes narrower.

\section{CONCLUSIONS}

For all cases studied, two vortices appeared in the region in the vicinity close to the bluff body. In some cases only one vortex appeared in this region. Non-symmetrical vertical profiles of the velocities were obtained for flow around bluff body with ground effect as oppose to symmetrical flow for flow around bluff body without ground effect. Flow with tend to follow wave with low amplitude and this results wider size of negative region. As the amplitude increases, the size will reduce. Flow bandwidth was observed to be suppressed as the amplitude of the wave increases. The vertical distance of the bluff body only affect the magnitude of bandwidth. Low amplitude wave was observed to increase the fluctuation of the flow as the Reynolds number increase. However, for higher wave amplitude, variation in Reynolds number yields the same order of fluctuation. 
Table 1. Characteristics of flow for position 1 and position 2 (above the peak of the wave)

\begin{tabular}{|c|c|c|c|}
\hline \multirow[t]{2}{*}{ Surfaces } & \multirow[t]{2}{*}{ Characteristics of flow } & \multicolumn{2}{|c|}{ Finding } \\
\hline & & Position 1 & Position 2 \\
\hline \multirow[t]{7}{*}{ Flat } & $\begin{array}{l}\text { Region negative velocities behind } \\
\text { the bluff body }\end{array}$ & $2.6 \mathrm{D}$ & $2.4 \mathrm{D}$ \\
\hline & $\begin{array}{l}\text { Overall bandwidth measured with } \\
\text { reference to peak positive and } \\
\text { negative velocity }\end{array}$ & $1.54 \mathrm{Uin}$ & 1.4Uin \\
\hline & $\begin{array}{l}\text { Overall downstream velocities } \\
\text { approaching main velocity }\end{array}$ & Early & Late \\
\hline & \multirow{4}{*}{$\begin{array}{l}\text { Fluctuation of velocities with } \\
\text { respect to variation of each } \\
\text { Reynolds number }\end{array}$} & \multicolumn{2}{|c|}{ Low Reynolds number } \\
\hline & & Large & Small \\
\hline & & \multicolumn{2}{|c|}{ High Reynolds number } \\
\hline & & Small & Large \\
\hline \multirow[t]{7}{*}{ Wave-1 } & $\begin{array}{l}\text { Region negative velocities behind } \\
\text { the bluff body }\end{array}$ & $3.7 \mathrm{D}$ & $2.6 \mathrm{D}$ \\
\hline & $\begin{array}{l}\text { Overall bandwidth measured with } \\
\text { reference to peak positive and } \\
\text { negative velocity }\end{array}$ & 1.71Uin & 1.46Uin \\
\hline & $\begin{array}{l}\text { Overall downstream velocities } \\
\text { approaching main velocity }\end{array}$ & Early & Early \\
\hline & \multirow{4}{*}{$\begin{array}{l}\text { Fluctuation of velocities with } \\
\text { respect to variation of each } \\
\text { Reynolds number }\end{array}$} & \multicolumn{2}{|c|}{ Low Reynolds number } \\
\hline & & Large & Large \\
\hline & & \multicolumn{2}{|c|}{ High Reynolds number } \\
\hline & & Large & Large \\
\hline \multirow[t]{7}{*}{ Wave-2 } & $\begin{array}{l}\text { Region negative velocities behind } \\
\text { the bluff body }\end{array}$ & $1.86 \mathrm{D}$ & $1.53 \mathrm{D}$ \\
\hline & $\begin{array}{l}\text { Overall bandwidth measured with } \\
\text { reference to peak positive and } \\
\text { negative velocity }\end{array}$ & 1.5Uin & $0.72 \mathrm{Uin}$ \\
\hline & $\begin{array}{l}\text { Overall downstream velocities } \\
\text { approaching main velocity }\end{array}$ & Early & Late \\
\hline & Fluctuation of velocities with & \multicolumn{2}{|c|}{ Low Reynolds number } \\
\hline & respect to variation of each & Large & Small \\
\hline & & \multicolumn{2}{|c|}{ High Reynolds number } \\
\hline & & Small & Large \\
\hline \multirow[t]{7}{*}{ Wave-3 } & $\begin{array}{l}\text { Region negative velocities behind } \\
\text { the bluff body }\end{array}$ & $2.6 \mathrm{D}$ & $0.53 \mathrm{D}$ \\
\hline & $\begin{array}{l}\text { Overall bandwidth measured with } \\
\text { reference to peak positive and } \\
\text { negative velocity }\end{array}$ & 1.5Uin & $0.48 \mathrm{Uin}$ \\
\hline & $\begin{array}{l}\text { Overall downstream velocities } \\
\text { approaching main velocity }\end{array}$ & Early & Late \\
\hline & Fluctuation of velocities with & \multicolumn{2}{|c|}{ Low Reynolds number } \\
\hline & respect to variation of each & Large & Small \\
\hline & & \multicolumn{2}{|c|}{ High Reynolds number } \\
\hline & & Small & Small \\
\hline
\end{tabular}

Table 2. Characteristics of flow for position 3 and position 4 (above the valley of the wave)

\begin{tabular}{|c|c|c|c|}
\hline \multirow[t]{2}{*}{ Surfaces } & \multirow[t]{2}{*}{ Characteristics of flow } & \multicolumn{2}{|c|}{ Finding } \\
\hline & & Position 3 & Position 4 \\
\hline \multirow[t]{7}{*}{ Wave-1 } & $\begin{array}{l}\text { Region negative velocities behind } \\
\text { the bluff body }\end{array}$ & $3.7 \mathrm{D}$ & $2.5 \mathrm{D}$ \\
\hline & $\begin{array}{l}\text { Overall bandwidth measured with } \\
\text { reference to peak positive and } \\
\text { negative velocity }\end{array}$ & 1.7Uin & $1.58 \mathrm{Uin}$ \\
\hline & $\begin{array}{l}\text { Overall downstream velocities } \\
\text { approaching main velocity }\end{array}$ & Early & Late \\
\hline & \multirow{4}{*}{$\begin{array}{l}\text { Fluctuation of velocities with } \\
\text { respect to variation of each } \\
\text { Reynolds number }\end{array}$} & \multicolumn{2}{|c|}{ Low Reynolds number } \\
\hline & & Small & Small \\
\hline & & \multicolumn{2}{|c|}{ High Reynolds number } \\
\hline & & Small & Small \\
\hline \multirow[t]{7}{*}{ Wave-2 } & $\begin{array}{l}\text { Region negative velocities behind } \\
\text { the bluff body }\end{array}$ & $1.8 \mathrm{D}$ & $2.2 \mathrm{D}$ \\
\hline & $\begin{array}{l}\text { Overall bandwidth measured with } \\
\text { reference to peak positive and } \\
\text { negative velocity }\end{array}$ & 1.91Uin & $1.05 \mathrm{Uin}$ \\
\hline & $\begin{array}{l}\text { Overall downstream velocities } \\
\text { approaching main velocity }\end{array}$ & Early & Late \\
\hline & \multirow{4}{*}{$\begin{array}{l}\text { Fluctuation of velocities with } \\
\text { respect to variation of each } \\
\text { Reynolds number }\end{array}$} & \multicolumn{2}{|c|}{ Low Reynolds number } \\
\hline & & Large & Small \\
\hline & & \multicolumn{2}{|c|}{ High Reynolds number } \\
\hline & & Small & Small \\
\hline \multirow[t]{7}{*}{ Wave-3 } & $\begin{array}{l}\text { Region negative velocities behind } \\
\text { the bluff body }\end{array}$ & $2.5 \mathrm{D}$ & $1 \mathrm{D}$ \\
\hline & $\begin{array}{l}\text { Overall bandwidth measured with } \\
\text { reference to peak positive and } \\
\text { negative velocity }\end{array}$ & 1.46Uin & 0.5 Uin \\
\hline & $\begin{array}{l}\text { Overall downstream velocities } \\
\text { approaching main velocity }\end{array}$ & Early & Late \\
\hline & Fluctuation of velocities with & \multicolumn{2}{|c|}{ Low Reynolds number } \\
\hline & respect to variation of each & Small & Small \\
\hline & Reynolds number & \multicolumn{2}{|c|}{ High Reynolds number } \\
\hline & & Large & Small \\
\hline
\end{tabular}

\section{REFERENCES}

[1] Angele, K.P, and Klingmann, B. Muhammad, (2006),"PIV measurements in a weakly separating and reattaching turbulent boundary layer", European Journal of Mechanics B/Fluids, 25: 204222.

[2] Ahmed, M.R, and Sharma, S.D., (2005), "An investigation on the aerodynamics of a symmetrical airfoil in ground effect", Experimental Thermal and Fluid Science, 29:633-647.

[3] Bentley, J.P., and Mudd, J.W., (2003),"Vortex Shedding Mechanisms in Single and Dual Bluff Bodies", Flow Measurement and Instrumentation, 14: 23-31.

[4] Barlow, Jewel B., Guterres, Rui and Ranzenbach, Robert., (2001), "Experimental parametric study of rectangular bodies with radiused edges in ground effect", Journal of Wind engineering and Indsutrial Aerodynamics, 89:1291-1309.

[5] Bouris, D, and Bergeles, G., (1999), "2D LES of vortex shedding from a square cylinder", Journal of Wind Engineering and Industrial Aerodynamics, 80: 31-46.

[6] Bearmen, P.W., (1997),"Near Wake Flows Behind Two-and ThreeDimensional Bluff Bodies", Journal of Wind Engineering and Industrial Aerodynamics, 69-71: 33-54.

[7] Buckles, Jeffrey, Hanratty, Thomas J., and Adrian, Ronald J., (1984), "Turbulent Flow Over Large-Amplitude Wavy Surfaces", Journal Fluid Mechanics, 140: 27-44.

[8] Jue, Tswen-Chyuan. (2004), "Numerical analysis of vortex shedding behind a porous square cylinder", International Journal of Numerical Methods for Heat \& Fluid Flow, 14: 5, pp. 649-663.

[9] Huang, Rong F., and Lin, Chih L., (2000), "Velocity field of a bluffbody wake", Journal of Wind Engineering and Industrial Aerodynamics, 85:31-45. 
[10] Johansen, Stein T., Wu, Jiongyang, and Shyy, Wei., (2004),"Filterbased unsteady RANS computations", International Journal of Heat and Fluid Flow, 25:10-21.

[11] Kim, M.S, and Geropp. D., (1998), "Experimental investigation of the ground effect on the flow around some two-dimensional bluff bodies with moving-belt technique", Journal of Wind Engineering and Industrial Aerodynamics, 74-76: 511-519.

[12] Nakagawa, Shinji and Hanratty, Thomas J., (2001), "Particle image velocimetry measurements of flow over a wavy wall", Physics of Fluids, 13: 11 .

[13] Rodi, W., (1997), "Comparison of LES and RANS calculations of the flow around bluff bodies", Journal of Wind Engineering and Industrial Aerodynamics, 69-71: 5575.

[14] Saha, A.K, Biswas, G, and Muralidhar, K., (2003), "Threedimensional study of flow past a square cylinder at low Reynolds numbers", International Journal of Heat and Fluid Flow, 24:54-66.

[15] Williamson, C.H.K., (1997), "Advances in Our Understanding of Vortex Dynamics in Bluff Body Wakes, Journal of Wind Engineering and Industrial Aerodynamics, 69-71: 3-32.

[16] Zhang, Wei, Daichin, and Lee, Sang Joon. (2005), "PIV Measurement of The Near-Wake Behind a Sinusoidal Cylinder",SpringerVerlag.10.1007/s00348-005-0981-9.

[17] Larose, G.L., and D'Auteuil, A. (2006). On the Reynolds Number Sensitivity of the Aerodynamics of Bluff Bodies with Sharp Edges. Journal of Wind Engineering and Industrial Aerodynamics. 94:365376.

[18] Schewe, Gunter (2001). Reynolds-number Effects in Flow Around More-or-Less Bluff Bodies. Journal of Wind Engineering and Industrial Aerodynamics. 89: 1267-1289.

[19] Selvarajan, S., Tulapurkara, E.G., and Ram, V.Vasanta (1998). A Numerical Study of Flow Through Wavy-Walled Channels. Int. J. Numer. Meth. Fluids. 26: 519-531.

[20] Tieleman, Henry W., Ge, Zhongfu, Hajj, Muhammad R., and Reinhold, Timothy A. (2003). Pressures on a Surface-Mounted Rectangular Prism Under Varying Incident Turbulence. Journal of Wind Engineering and Industrial Aerodynamics. 91 :1095-1115. 


\title{
The Use of Fly Ash to Reduce the Environmental Impact of Concrete
}

\author{
D. Hardjito
}

\begin{abstract}
The production of cement is increasing about $3 \%$ annually. As the production of one ton of cement liberates about one ton of $\mathrm{CO}_{2}$ to the atmosphere, currently the contribution of Portland cement production worldwide to the greenhouse gas emission is about 1.35 billion tons annually or about $7 \%$ of the total greenhouse gas emissions to the earth's atmosphere. Aside from that, cement is among the most energy-intensive construction materials as well. Due to the environmental concern, the production of ordinary Portland cement attracts critics.

The concrete industry has recognized these issues. For example, the U.S. Concrete Industry has developed plans to address these issues in 'Vision 2030: A Vision for the U.S. Concrete Industry'. In this document, strategies to retain concrete as a construction material of choice for infrastructure development, and at the same time to make it an environmentally friendly material for the future have been outlined.

One strategy to make concrete 'greener' construction material is to utilize fly ash, either as partial or total substitute for Portland cement in concrete. This attempt results in twofold benefits, i.e. to provide a solution with regard to the concern on the carbon dioxide emission from Portland cement production, and to provide way to effectively use fly ash. Fly ash, the byproduct material from burning coal especially in power stations, is available abundantly worldwide. Its availability is increasing and yet its utilization to date is still very low. Without proper plan, the management of fly ash may incur cost, and potentially harm the natural environment as well.

This paper discusses the technology and the current progress of research on utilizing fly ash in concrete.
\end{abstract}

Keywords: cement, concrete, fly ash, environmental concern

\section{INTRODUCTION}

Portland cement is one of the most popular and major construction materials worldwide. In the foreseeable future, this tendency will remain so. The cement production is expected to rise from 1.7 billion tones in 2000 to 2 billion tones in 2010 , whereby the major increase will take place in China and India [1]

However, Portland cement production raises environmental concern, not only due to highly energy intensive, but also due to high amount of carbon dioxide released to the atmosphere. Production of one ton Portland cement will release one ton of carbon dioxide into atmosphere. Moreover, there is also concern on the durability of the concrete structures, especially those built in aggressive environment [2]

On the other hand, fly ash, "the finely divided residue that results from the combustion of ground or powdered coal and that is transported by flue gases from the combustion zone to the particle removal system" [3], is available abundantly worldwide. In 2001, the fly ash production in the USA was in the order of 68 million tons, but only 32 percent was used in various applications, such as in concrete, structural fills, waste stabilisation/solidification, etc. [4]. Worldwide, the estimated production of coal ash in 1998 was more than 390 million tons. The main contributors for this amount were China and India. Only about 14 percent of this fly ash was utilized, while the rest was just disposed in landfills [5]. By the year 2010, the amount of fly ash produced worldwide is estimated to be about 780 million tons annually [6].

The utilization of fly ash and other by-product materials, such as granulated blast furnace slag, rice husk ash, as a substitute or partially substitute of Portland cement in concrete has been studied intensively and extensively. Partial substitution of Portland cement by $20-30 \%$ fly ash has become a common practice. A more substantial development in this area were the development of high volume fly ash concrete with 50-60\% Portland cement replacement by using fly ash, and the development of fly ash-based geopolymer concrete whereby the use of Portland cement is totally replaced by the geopolymer matrix using fly ash as the source material.

This paper discusses the strategy to utilise fly ash in order to make concrete more environmentally friendly construction material for a sustainable development.

\section{Portland CEMENT: Production AND ENVIRONMENTAL ISSUE}

Table 1 shows the Portland cement production till the year of 2010 in million tonnes. By releasing one ton of carbon dioxide for every ton of Portland cement produced, the Portland cement industry contributes around $7 \%$ of the total amount of carbon dioxide worldwide [5]. Significant improvement in the technology to produce Portland cement to make it more environmentally friendly is not expected in the near future. The production of one ton Portland cement also requires approximately 4 GJ energy, to put it into one of the most energy intensive construction materials [7]. 
Table 1. Regional and world cement production to year 2010 (in million tonnes) [5]

\begin{tabular}{|l|c|c|c|c|c|c|}
\hline & $\mathbf{1 9 9 5}$ & $\mathbf{2 0 0 0}$ & $\mathbf{2 0 0 5}$ & $\mathbf{2 0 1 0}$ & $\begin{array}{c}\text { \% of total } \\
\mathbf{1 9 9 5}\end{array}$ & $\begin{array}{c}\text { of total } \\
\mathbf{2 0 1 0}\end{array}$ \\
\hline European Union & & & & & 12.1 & 9.7 \\
\hline Other Europe & 168.1 & 187.9 & 194.1 & 189.3 & 4.7 & 4.9 \\
\hline Former Soviet Union & 65.8 & 80.0 & 90.2 & 94.7 & 4.7 & 6.6 \\
\hline North America & 58.1 & 80.3 & 110.1 & 128.2 & 4.2 & 4.9 \\
\hline C/S America & 92.9 & 94.9 & 94.8 & 94.7 & 6.6 & 7.5 \\
\hline Africa & 89.4 & 106.6 & 127.4 & 145.0 & 6.4 & 4.4 \\
\hline Middle East & 64.8 & 74.3 & 80.7 & 85.5 & 4.6 & 3.8 \\
\hline East Asia & 63.5 & 75.6 & 76.9 & 73.4 & 4.6 & 43.4 \\
\hline S/SE Asia & 623.4 & 732.7 & 798.8 & 844.3 & 44.6 & 14.4 \\
\hline Oceania & 161.2 & 219.1 & 255.0 & 279.2 & 11.6 & 0.6 \\
\hline World totals & 8.0 & 10.6 & 11.1 & 11.8 & 0.6 & 0.0 \\
\hline
\end{tabular}

The need for reducing the environmental impact of concrete has been recognized by the concrete industries. The document entitled "Vision 2030: A vision for the US Concrete Industry" states that "concrete technologist are faced with the challenge of leading future development in a way that protects environmental quality while projecting concrete as a construction material of choice. Public concern will be responsibly addressed regarding climate change resulting from the increased concentration of global warming gas' $[7,8]$.

In order to produce environmentally friendly concrete, Mehta [9] suggested the use of fewer natural resources, less energy, and minimise carbon dioxide emissions. $\mathrm{He}$ categorized these short-term efforts as 'industrial ecology'. The long-term goal of reducing the impact of unwanted byproducts of industry can be attained by lowering the rate of material consumption. Likewise, McCaffrey [10] suggested three alternatives to reduce the amount of carbon dioxide $\left(\mathrm{CO}_{2}\right)$ emissions by the cement industries, i.e. to decrease the amount of calcined material in cement, to decrease the amount of cement in concrete, and to decrease the number of buildings using cement.

In this view, the most feasible alternative to reduce the environmental impact of concrete is to reduce the amount of Portland cement used.

\section{Fly Ash: Availability, Problem And Potential}

Table 2 shows estimation on the fly ash production in 2000 , with the total production worldwide estimated to be 600 million tonnes worldwide, with China, USA and India as the biggest producers. This yearly production should be added with the amount of fly ash that has been stockpiled over the years. In the near future, it is expected that the production of fly ash is steadily increasing, as the power produced by burning coal is remain the cheaper alternative, and as the high quality coal is available abundantly worldwide [1]. So far, only about 14 percent of this fly ash was utilized, while the rest was just disposed in landfills [5]. Without proper attention, disposing fly ash in landfills may cause another threat to the environment.

The most commonly available fly ash is low calcium or class F fly ash with less than $20 \%$ of calcium oxide, which in itself possesses little or even does not posses any cementitious properties. However, with the presence of moisture at ordinary temperature, it will chemically react with calcium hydroxide to form compound having cementitious properties, thus plays a role as an artificial pozzolan. The main constituents of fly ash are silicon and aluminium oxides [3].

In this regard, the incorporation of $20-30 \%$ of fly ash to partially replace the use of Portland cement in concrete has become a common practice in construction work. Other noted advancement in the technology to utilize fly ash in concrete was the development of High Volume Fly Ash (HVFA) concrete and fly ash-based Geopolymer concrete. In HVFA, 50 to $60 \%$ of fly ash is to be used to replace the use of Portland cement in concrete, while for geopolymer concrete it does not require any Portland cement, as its is totally replaced by geopolymer paste with fly ash as the source material.

The use of fly ash in concrete is therefore resulting twofold environmentally benefit, i.e. reduce the use of Portland cement and utilize by-product material.

\section{High Volume Fly Ash (HVFA) CONCRETE}

Malhotra et al $[6,11]$ have initiated in the late of 1980s the development of HVFA concrete using conventional materials and technology. They have shown that it is possible to produce high performance concrete mixtures containing $50-60 \%$ fly ash by mass of the blended cementitious material.

The typical characteristics of the HVFA mixtures are [6]:

- The use of $50-60 \%$ by mass of class $\mathrm{F}$ fly ash of cementitious materials

- The use of low water content, generally less than 130 $\mathrm{kg} / \mathrm{m}^{3}$

- Cement content nor more than $200 \mathrm{~kg} / \mathrm{m}^{3}$ of concrete

- Very low dosage of superplasticiser, if needed.

- Low water to cementitious materials ratio of 0.30

HVFA concrete shows excellent mechanical properties, and it is still gaining significant increase in its strength development at least until 1 year due to the pozzolanic reaction and it shows better durability compared to normal Portland cement concrete [6]. The successful applications of the HVFA concrete into practice have been reported [12, 13].

\section{Fly Ash-BASEd GEOPOLYMER CONCRETE}

Another noted achievement in the use of fly ash in concrete was the development of geopolymer concrete. In this case, no Portland cement is used, as it is totally replaced by the geopolymer paste. Fly ash can be used as the source material for the geopolymer paste. In this case, fly ash, 
which is rich in aluminium and silicon oxide, chemically reacts with the alkaline solutions to form inorganic polymeric binder called geopolymer.

Geopolymer technology has been coined by Davidovits et al [14-16], mainly using calcined kaolin as the source material. Since then, many other possible materials have been investigated its potential to be the source material for geopolymer [17]. Fly ash was found to be one of suitable source material for geopolymer.

The development of fly ash-based geopolymer concrete has gained significant advancement. It is possible to manufacture fly ash-based geopolymer concrete using the common technology to produce normal Portland cement concrete $[18,19]$. Its shows excellent short term and long term properties, as well as its durability in the sulphate environment [20-22].

The application of fly ash-based geopolymer concrete into structural members has been investigated, i.e. as columns, beams, railway sleepers, walls as well as sewerage pipes [23-26]. It was reported that reinforced geopolymer concrete structures behave in similar manner as those of normal Portland cement concrete. Research to further investigate the behaviour and properties of this 'newly' construction material is on going in many different parts of the world.

Table 2. Estimated coal ash production and utilization in 2000 (in million tonnes) [1]

\begin{tabular}{|l|c|c|}
\hline \multicolumn{1}{|c|}{ Country } & Production & Utilization in concrete \\
\hline China & $>200$ & $>15$ \\
\hline India & $>80$ & $5 \%$ \\
\hline USA & $>60$ & $10 \%$ \\
\hline Russia & 60 & 5 \\
\hline Germany & 30 & 12 \\
\hline UK & 10 & 10 \\
\hline
\end{tabular}

\section{CONCLUDING REMARKS}

One most possible way to make concrete 'greener' construction material is to reduce the use of Portland cement. With the abundant availability of fly ash worldwide, it has potential to substantially replace the amount of Portland cement needed for making concrete.

Among the most substantial applications is the use of fly ash into HVFA concrete, which is possible to replace 50$60 \%$ by mass of Portland cement by fly ash, and in fly ashbased geopolymer concrete to totally replace the use of Portland cement in concrete. These two 'newly' construction materials have shown excellent material characteristics and properties, as well as durability. Thus, fly ash may play an important role to make concrete 'greener' construction material.

\section{REFERENCES}

[1] Malhotra, V.M. Role of Supplementary Cementing Materials and Superplasticisers in Reducing Greenhouse Gas Emissions. in ICFRC. 2004. Chennai, India: Allied Publishers Private Ltd.

[2] Mehta, P.K., Durability - Critical Issues for the Future. ACI Concrete International, 1997. 19(7): p. 27-33.

[3] ACI Committee 232, Use of Fly Ash in Concrete. 2004, American Concrete Institute: Farmington Hills, Michigan, USA. p. 41.

[4] ACAA, Fly Ash Facts for Highway Engineers. 2003, American Coal Ash Association: Aurora, USA. p. 74.

[5] Malhotra, V.M., Making Concrete "Greener" With Fly Ash. ACI Concrete International, 1999. 21(5): p. 61-66.
[6] Malhotra, V.M., High-Performance High-Volume Fly Ash Concrete. ACI Concrete International, 2002. 24(7): p. 1-5.

[7] Mehta, P.K., Reducing the Environmental Impact of Concrete. ACI Concrete International, 2001. 23(10): p. 61-66.

[8] Plenge, W.H., Introducing Vision 2030: Our Industry's 30-year Map to the Future. ACI Concrete International, 2001. 23(03): p. 25-34.

[9] Mehta, P.K., Greening of the Concrete Industry for Sustainable Development. ACI Concrete International, 2002. 24(7): p. 23-28.

[10] McCaffrey, R., Climate Change and the Cement Industry. Global Cement and Lime Magazine, 2002(Environmental Special Issue): p. 15-19.

[11] Malhotra, V.M. and P.K. Mehta, High-Performance, High-Volume Fly Ash Concrete: Materials, Mixture Proportioning, Properties, Construction Practice, and Case Histories. 2002, Ottawa, Canada: Supplementary Cementing Materials for Sustainable Development Inc. 101.

[12] Mehta, P.K. and R.W. Burrows, Building Durable Structures in the 21st Century. ACI Concrete International, 2001. 23(03): p. 57-63.

[13] Desai, J.P. Construction and Performance of High-Volume Fly Ash Concrete Roads in India. in Eighth CANMET/ACI International Conference on Fly Ash, Silica Fume, Slag, and Natural Pozzolans in Concrete. 2004. Las Vegas, USA: American Concrete Institute.

[14] Davidovits, J., High-alkali Cements for 21st Century Concretes, in Concrete Technology: Past, Present and Future, P.K. Mehta, Editor. 1994, ACI, Detroit, USA. p. 383-397.

[15] Davidovits, J. Chemistry of Geopolymeric Systems, Terminology. in Geopolymer '99 International Conference. 1999. France.

[16] Davidovits, J. Green-Chemistry and Sustainable Development Granted and False Ideas About Geopolymer Concrete. in GGC 2005 International Workshop on Geopolymers and Geopolymer Concrete. 2005. Perth, Australia.

[17] van Jaarsveld, J.G.S., J.S.J. van Deventer, and G.C. Lukey, The Characterisation of Source Materials in Fly Ash-based Geopolymers. Materials Letters, 2003. 57(7): p. 1272-1280.

[18] Hardjito, D., S.E. Wallah, D.M.J. Sumajouw, and B.V. Rangan. Introducing Fly Ash Geopolymer Concrete: Manufacture and Engineering Properties. in Our World in Concrete '05. 2005. Singapore.

[19] Hardjito, D., S.E. Wallah, D.M.J. Sumajouw, and B.V. Rangan, On The Development of Fly Ash-Based Geopolymer Concrete. ACI Materials Journal, 2004. 101(6): p. 467-472.

[20] Hardjito, D., S.E. Wallah, D.M.J. Sumajouw, and B.V. Rangan, Fly Ash-Based Geopolymer Concrete. Australian Journal of Structural Engineering, 2005. 6(1): p. 77-85.

[21] Wallah, S.E., D. Hardjito, D.M.J. Sumajouw, and B.V. Rangan. Creep and Drying Shrinkage Behaviour of Fly Ash-Based Geopolymer Concrete. in Concrete '05. 2005. Melbourne, Australia: Concrete Institute of Australia.

[22] Wallah, S.E., D. Hardjito, D.M.J. Sumajouw, and B.V. Rangan, Performance of Fly Ash-Based Geopolymer Concrete under Sulfate and Acid Exposure, in Geopolymers, Green Chemistry and Sustainable Development Solutions, J. Davidovits, Editor. 2005, Geopolymer Institute: Saint Quentin, France. p. 153-156.

[23] Sumajouw, D.M.J., D. Hardjito, S.E. Wallah, and B.V. Rangan, Fly Ash-Based Geopolymer Concrete: An Application for Structural Members, in Geopolymers, Green Chemistry and Sustainable Development Solutions, J. Davidovits, Editor. 2005, Geopolymer Institute: Saint Quentin, France. p. 149-152.

[24] Sumajouw, D.M.J., D. Hardjito, S.E. Wallah, and B.V. Rangan, Fly Ash-Based Geopolymer Concrete: Study of Slender Reinforced Columns. Journal of Material Science, 2006. Accepted for publication.

[25] Palomo, A., A. Fernandez-Jimenez, C. Lopez-Hombrados, and J.L. Lleyda. Precast Elements Made of Alkali-Activated Fly Ash Concrete. in Eighth CANMET/ACI International Conference on Fly Ash, Silica Fume, Slag, and Natural Pozzolans in Concrete. 2004. Las Vegas, USA.

[26] Gourley, J.T. and G.B. Johnson, Development in Geopolymer Precast Concrete, in Geopolymer, Green Chemistry and Sustainable Development Solutions, J. Davidovits, Editor. 2005, Geopolymer Institute: Saint Quentin, France. p. 139-143. 


\title{
Environmental Effect Due to the Energy Consumption of Refrigerator-freezer
}

\author{
M. Hasanuzzaman, R. Saidur, H.H. Masjuki and M.M. Hasan
}

\begin{abstract}
Energy is one of the indispensable factors for civilization, economic growth and continuous development. Malaysian economy has grown rapidly in the last two decades. This economic growth has improved the life style as well as increased the usage of electrical appliances in the household. The usage of refrigerator-freezer is increased dramatically with the economic growth; as a result electricity consumption increases. Electricity generation depends on the fossil fuels. According to the fuel mix electricity generation 2000-2010, the fossil fuels is used $64.5 \%$ gas, $27.6 \%$ coal, $5.5 \%$ hydro and $1.4 \%$ petroleum in the year of 2007 . Combustion of these fuels releases greenhouse gases such as carbon dioxide $\left(\mathrm{CO}_{2}\right)$, sulfur dioxide $\left(\mathrm{SO}_{2}\right)$, nitrogen oxide $\left(\mathrm{NO}_{\mathrm{x}}\right)$, carbon monoxide $(\mathrm{CO})$. An experiment is conducted in an environmentally controlled chamber to investigate the effect of ambient temperature, cabinet load, thermostat setting position and open surface water pan area in the cabinet on the energy consumption of the refrigerator-freezer with different operating condition during the closed door condition and found that the average energy consumption is $1042.7 \mathrm{kWh} /$ year. It is a worldwide concern for the environment of the acceleration of global warming and climate change by the emission of gases. The study found that the fossil fuel released about $697.4 \mathrm{~kg} \mathrm{CO}, 5.9 \mathrm{~kg} \mathrm{SO}_{2}, 2.1 \mathrm{~kg}$ $\mathrm{NO}_{\mathrm{x}}$ and $0.4 \mathrm{~kg} \mathrm{CO}$ gases to generate the electricity. The energy saving steps should be taken very seriously which will result in energy savings and emissions reduction for a future green world.
\end{abstract}

Keywords: energy consumption; greenhouse gas emission; refrigerator-freezer

\section{INTRODUCTION}

Energy is one of the indispensable factors for continuous development and economic growth. Energy efficiency improvement is one of the most important functions to reduce energy cost as well as emission reduction. Energy efficiency improvement is the main objective of many national energy policies. Monitoring of the energy consumption and developments in energy efficiency is

M. Hasanuzzaman is with the Department of Mechanical Engineering, University of Malaya, 50603 Kuala Lumpur, Malaysia (Tel:+60379674448, fax: +603-79675317, e-mail: ehasan99@yahoo.com.).

R. Saidur is with the Department of Mechanical Engineering, University of Malaya, 50603 Kuala Lumpur, Malaysia (e-mail: saidur@um.edu.my).

H. H. Masjuki is with the Department of Mechanical Engineering, University of Malaya, 50603 Kuala Lumpur, Malaysia (e-mail: masjuki@um.edu.my).

M. H. Hasan is with the Department of Mechanical Engineering, University of Malaya, 50603 Kuala Lumpur, Malaysia (e-mail: mmhasan92@yahoo.com). necessary in order to check and apply desired policies. Energy is the most important thing for automation and modernization. Automation and modernization is increasing rapidly in our daily life. The energy demands in Malaysia increasing dramatically. The energy demand increased almost 20\% within the last 3 years (from 1999 to 2002) [1]. The energy demand is further expected to increase almost $60 \%$ within 8 years (from 2002 to 2010). To supply the demand energy on need to increase power stations. As a result, emission of greenhouse gases $\left(\mathrm{CO}_{2}, \mathrm{SO}_{2}, \mathrm{NO}_{\mathrm{x}}, \mathrm{CO}\right)$ increase. The energy consumption is investigated and estimated emissions $\left(\mathrm{CO}_{2}, \mathrm{SO}_{2}\right.$ and $\left.\mathrm{NO}_{\mathrm{x}}\right)$ in Asia that is shown in the Table 1 [2]. The global, regional and national $\mathrm{CO}_{2}$ emissions in the year 1996 and estimated that $77.5 \%$ of $\mathrm{CO}_{2}$ emissions are emitted from liquid and solid fuels where as, $18.3 \%$ of $\mathrm{CO}_{2}$ emissions are emitted from gaseous fuels burning [3]. The energy consumption and greenhouse gas emissions of Thailand in the years of 1995, 1998, 2001 and 2006 is investigated and found that electricity is the highest energy intensive sector where fossil fuel is primarily used [4]. The major sources of the emissions are industrial processes and the combustion of fossil fuels. The emission factor was low in the year of 2001and 2006 compare to the year of 1995 and 1998. Emissions are estimated from electricity generation in Malaysia and observed that emissions increase and it will be around 3 times in 2020 than in 2002 [5].

Residential electricity consumption is investigated and predicted that it will increase in $27,053 \mathrm{GWh}$ in 2015 [6]. Refrigerator-freezer is one of the most essential appliances to preserve perishable foods in a household. Keeping fresh foods fresh and freezing the perishable food are the basic functions of household refrigerator-freezer. It is operated all day long and consumes energy as well. A survey is conducted and investigated that in Malaysia the number of refrigerator-freezer increased from 175,842 units in 1970 to $4,196,486$ units in 2000 [7]. From the survey, it is also estimated that electricity consumption has increased from $326 \mathrm{GWh}$ in 1970 to $9,471 \mathrm{GWh}$ in 2000 in its residential sector. The energy consumption of refrigerator-freezers was about $15 \%$ to $20 \%$ of total domestic electric usage [8]. The refrigerator-freezers consumed about $26 \%$ of residential electricity in Malaysia [9]. 
Table 1. $\mathrm{CO}_{2}, \mathrm{SO}_{\mathrm{x}}$ and $\mathrm{NO}_{\mathrm{x}}$ emissions in some Asian countries and total in Asia [2]

\begin{tabular}{|l|c|c|c|c|c|c|c|c|c|}
\hline \multirow{2}{*}{ Country } & \multicolumn{3}{|c|}{$\mathrm{CO}_{2}(\mathrm{kton})$} & \multicolumn{3}{c|}{$\mathrm{SO}_{\mathrm{x}}(\mathrm{kton})$} & \multicolumn{3}{c|}{$\mathrm{NO}_{\mathrm{x}}(\mathrm{kton})$} \\
\cline { 2 - 10 } & 1975 & 1980 & 1987 & 1975 & 1980 & 1987 & 1975 & 1980 & 1987 \\
\hline Bangladesh & 1,290 & 1,919 & 2,839 & 40 & 57 & 49 & 46 & 58 & 66 \\
\hline Indonesia & 11,219 & 21,194 & 29,888 & 201 & 329 & 485 & 331 & 465 & 639 \\
\hline Japan & 271,943 & 281,165 & 271,786 & 2,571 & 1,604 & 1,143 & 2,229 & 2,132 & 1,935 \\
\hline Malaysia & 5,086 & 7,311 & 10,052 & 193 & 272 & 263 & 90 & 126 & 177 \\
\hline Singapore & 3,521 & 5,186 & 6,414 & 85 & 122 & 155 & 43 & 69 & 88 \\
\hline Thailand & 7,397 & 11,034 & 16,679 & 224 & 420 & 612 & 182 & 255 & 384 \\
\hline Asia total & 779,297 & 955,492 & $1,228,676$ & 18,340 & 22,997 & 29,136 & 9,388 & 11,352 & 15,483 \\
\hline
\end{tabular}

The residential sector of India consumed about $39 \%$ of total energy in 2000 [10]. A survey is conducted to investigate household energy patterns and revealed that about $76 \%$ of total residential houses were equipped with one refrigerator-freezer in Malaysia [11]. In some cases, they showed a multiple number of refrigerator-freezers owned by a single house owner. An experiment is performed to observe the effect of usage condition on energy consumption of household refrigerator-freezer. Increasing ambient temperature from $23.9{ }^{\circ} \mathrm{C}$ to $32.2{ }^{\circ} \mathrm{C}$ increased daily energy consumptions from $22 \%$ to $42 \%$ in close door condition [12]. Most of the users keep refrigerator-freezers in the kitchen and some time near the cooker or burner. If the ambient temperature is comparatively high, the temperature different between the ambient and refrigerator-freezer compartment is increased.

The aim of the research is to investigate the effects of operating environmental conditions on the energy consumption and the environmental effect to generate energy that is consumed by the refrigerator-freezer.

\section{Research Methodology}

\section{A. Experimental setup}

The objectives of the experiment are to investigate the effects of ambient temperature, cabinet load, thermostat setting position and open surface water pan area on the energy consumption as well as environmental effect by greenhouse gas emission.

\section{1) Test conditions}

The tests were conducted by varying ambient temperature, cabinet load, thermostat setting position and open surface water pan area independently during the closed door conditions. During the experiment, only one variable was changed and the other variables were kept constant. To investigate the effect of the ambient temperature on moisture transfer and energy consumption, temperature was varied from $18{ }^{\circ} \mathrm{C}$ to $30^{\circ} \mathrm{C}$ in the environmentally controlled chamber. Fresh food and freezer cabinets were loaded to investigate the effects of load and the load was varied from $0 \mathrm{~kg}$ to $8 \mathrm{~kg}$ and $0 \mathrm{~kg}$ to $4 \mathrm{~kg}$ of fresh water in the fresh food and freezer cabinet respectively. The thermostat setting position was changed from $2\left(-13.1^{\circ} \mathrm{C}\right)$ to $6\left(-17.5^{\circ} \mathrm{C}\right)$ to reveal the effects of cabinet temperature. Open surface water pans were used to create wetted surface in the fresh food cabinet. In the work, water pan area was varied from $324 \mathrm{~cm}^{2}$ to $1272 \mathrm{~cm}^{2}$.

\section{2) Test unit}

The refrigerator-freezer used was top mount freezer section, two doors and both doors hinged at right hand side. The specification of the refrigerator-freezer is shown in the following Table.

Table 2. Technical specifications of refrigerator-freezer test unit

\begin{tabular}{|l|c|}
\hline Specifications & \\
\hline Freezer Capacity (liter) & 130 \\
\hline $\begin{array}{l}\text { Fresh Food Compartment Capacity } \\
\text { (liter) }\end{array}$ & 330 \\
\hline Power Rating (W) & 165 \\
\hline Current rating (A) & 1.3 \\
\hline Voltage (V) & 240 \\
\hline Frequency (Hz) & 50 \\
\hline No of door & 2 \\
\hline Refrigerant type & $134 \mathrm{a}\left(\mathrm{CF}_{3} \mathrm{CH}_{2} \mathrm{~F}\right)$ \\
\hline Defrost system & Frost free \\
\hline
\end{tabular}

\section{3) Location of thermocouples and humidity sensor}

Thermocouples were used to measure temperature inside and outside of the walls and doors of refrigerator-freezer, inside the fresh food and freezer cabinet. Five thermocouples were used in freezer cabinets that were placed at both side walls and the cabinet separator. Another six thermocouples were used in fresh food cabinets that were placed at both side walls and the cabinet separators. Six thermocouples are set to monitor the ambient temperature inside the chamber. Humidity sensor was used to measure relative humidity inside fresh food cabinet. The location of thermocouples and humidity sensor are shown in the Figure 1.

\section{4) Location of load during closed door test}

Load was used both in the fresh food and freezer cabinet to make real situation during the closed door test. Both the fresh food and freezer cabinets were divided into two shelves and each shelf contains same load. Open surface water pan was added in the fresh food cabinet that was made the surface wet inside the refrigerator same as moist foods. The location of open surface water pan, load (water bottle) in fresh food and freezer cabinets are shown in the Figure 2. 


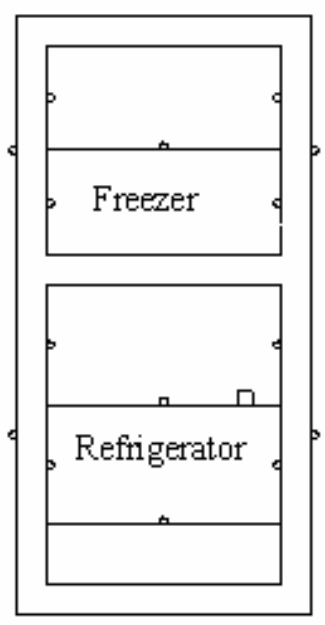

o Themocouple

口 Humidity Sensor

Figure 1. Location of thermocouple and relative humidity sensor

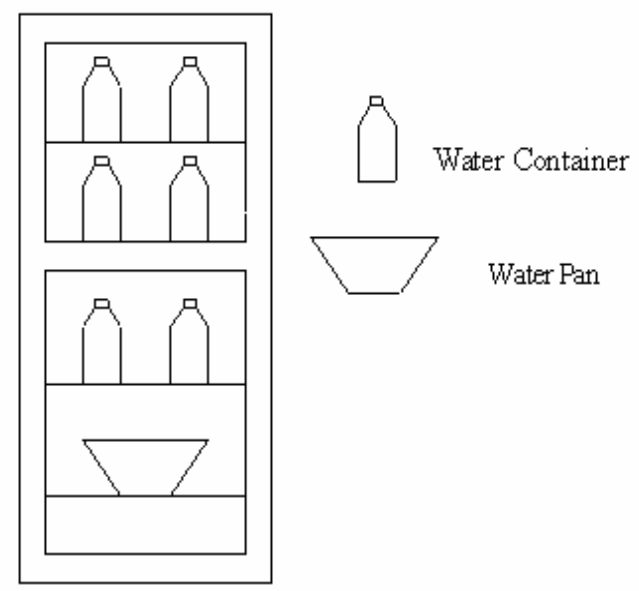

Figure 2. Location of load during close door condition

\section{B. Instrumentation}

The experiment was conducted in an environmentally controlled chamber. Some instruments were used to measure the temperature, load, relative humidity and power consumption for the experiment. To measure the temperature inside as well as outside the test unit, T-type thermocouples were used. Humidity transmitter was used to monitor the relative humidity inside the cabinet of the refrigerator-freezer. Relative humidity measurement range = $3 \%$ to $95 \%$ and Accuracy $= \pm 0.2 \%$. Heat pump was used to maintain the required temperature in the environmentally controlled chamber. The modes of operation of heat pump are heating, cooling and soft dry and the temperature range from $16{ }^{\circ} \mathrm{C}$ to $32{ }^{\circ} \mathrm{C}$. The temperature fluctuation was controlled by using a temperature controller. The controller was interfaced with the heat pump so that the desired temperature could be maintained within the chamber. To maintain relative humidity of the control chamber, dehumidifier was used and the range of dehumidifier from $0 \%$ to $90 \%$ with an accuracy of $\pm 5 \%$. Electronic balance was used to weight the mass of evaporated water from the open surface water pans and cabinet load with weighing capability of $0.01 \mathrm{~g}$ up to $2 \mathrm{~kg}$.
Hewlett Packard data logger was used to store the data from the test unit to personal computer for calculation and analysis. Two additional fused channels were included on the module for making calibrated DC or AC current measurement. The data logger supports direct measurement of temperature, AC voltage, DC voltage, AC current, DC current, resistance, frequency and period. Energy consumption was measured by the YOKOGAWA WT-130 digital power meter, which was interfaced with a PC through RS-232. Lab view software was installed into the PC for data storage and analysis. The accuracy of this power meter is $\pm 0.2 \%$ of reading.

\section{EMISSIONS CALCULATION}

\section{A. Emission Factors of Fossil Fuel}

To estimate the amount of green house gases released for generation of electricity from fossil fuels that consumed refrigerator-freezer, emission factors and fraction of electricity generated by each type of fuel (i.e. coal, gas and oil) have to be considered. Emissions per unit of electricity generation are dependent on the characteristics of the fuel and power plant. Characteristics of a fuel are its contents of energy, carbon, sulfur, nitrogen or their compounds. The power plant characteristic is the amount of heat required to produce one unit of electricity.

Table 3. Emission factors of fossil fuels for electricity generation [5]

\begin{tabular}{|l|c|c|c|c|}
\hline \multirow{2}{*}{ Fuels } & \multicolumn{4}{|c|}{ Emission factor $(\mathrm{kg} / \mathrm{kWh})$} \\
\cline { 2 - 5 } & $\mathrm{CO}_{2}$ & $\mathrm{SO}_{2}$ & $\mathrm{NOx}$ & $\mathrm{CO}$ \\
\hline Coal & 1.1800 & 0.0190 & 0.0052 & 0.0002 \\
\hline Petroleum & 0.8500 & 0.0164 & 0.0025 & 0.0002 \\
\hline Gas & 0.5300 & 0.0005 & 0.0009 & 0.0005 \\
\hline Hydro & 0.0000 & 0.0000 & 0.0000 & 0.0000 \\
\hline
\end{tabular}

\section{B. Electricity Generation}

Coal, natural gas, petroleum and hydro are used to generate electricity in Malaysia. Coal and natural gas base power generation is more comparing to the others. In line with the strategy to ensure security and reliably of the electricity supply, the sources of fuel for power generation was further diversified with the increased the use of coal. In consonance with the growth of economy, peak energy demand is increased from $10,657 \mathrm{MW}$ in 2000 to 13,779 MW in 2005. It is predicted that the peak demand will be $20,087 \mathrm{MW}$ in 2010 [13]. Energy peak demand is increased about $30 \%$ from 2000 to 2005 . Efforts were undertaken to reduce the high dependence on natural gas in the generation mix by increasing the use of coal. As a result, the shares of coal to the total generation mix increased from $8.8 \%$ in 2000 to $21.8 \%$ in 2005 while the natural gas decreased from $77 \%$ to $70.2 \%$.

According to the Table 3, the percentages of electricity generation are estimated based on fuel types in the years of 2007 (coal $27.7 \%$, petroleum 1.4\%, Gas $64.5 \%$ and hydro $5.5 \%)$. 
Table 4. Percentage of mix fuel in electricity generation, 2000-2010 [13]

\begin{tabular}{|c|c|c|c|c|}
\hline Year & $\begin{array}{c}\text { Coal } \\
(\%)\end{array}$ & $\begin{array}{c}\text { Petroleu } \\
\mathrm{m}(\%)\end{array}$ & $\begin{array}{c}\text { Gas } \\
(\%)\end{array}$ & $\begin{array}{c}\text { Hydro } \\
(\%)\end{array}$ \\
\hline 2000 & 8.8 & 4.2 & 77 & 10 \\
\hline 2005 & 21.8 & 2.2 & 70.2 & 5.5 \\
\hline 2010 & 36.5 & 0.2 & 55.9 & 5.6 \\
\hline
\end{tabular}

\section{C.Emission}

Emissions due to electricity use are calculated based on fuel type, percentage of electricity generated by the specific fuel and the emission factor of fuel to produce the electricity in Malaysia. The emissions by electricity used are estimated as:

Emission $=$ ECxPEGFxEFF

where: $E C=$ Electricity consumption (kWh) $P E G F=$ Percentage of electricity generated by the specific fuel

$E F F=$ emission factor of fuel $(\mathrm{kg} / \mathrm{kWh})$.

\section{RESUlTS AND DisCUSSION}

\section{A. Energy Consumption on Closed Door Condition}

The dominant purpose of utilizing household refrigeratorfreezer is to keep food and vegetable fresh and to freeze the perishable food for a longer period of time in days or weeks. During the closed door condition, refrigerator-freezer consumes energy due to moisture transfer by gasket diffusion and cabinet breathing, heat transfer by conduction, convection, radiation, sensible and latent. Refrigeratorfreezer consumes a significant amount of electrical energy of a house continuously.

\section{B. Effect of Different Variables on Energy Consumption}

Refrigerator-freezer energy consumption is influenced by the operating environmental condition. There is a very strong influence of ambient temperature and load on energy consumption compare to the thermostat setting position and open surface water pan area. Thermostat setting position effect on energy consumption is low compared to ambient temperature and load, but there is a strong influence on energy consumption. There is a small influence of water pan area on energy consumption compared to the ambient temperature, load and thermostat setting position. The overall average energy consumption is calculated 2.9 $\mathrm{kWh} /$ day. According to the experiment the refrigeratorfreezer consumes average1042.7 $\mathrm{kWh} /$ year. If the refrigerator-freezer runs at the maximum operating condition, the energy consumption will be about 1227 $\mathrm{kWh} /$ year. On the other hand when the refrigerator-freezer runs at the minimum operating condition, the energy consumption will be about $873 \mathrm{kWh} /$ year. The difference between the maximum and the minimum energy consumption is $354 \mathrm{kWh} /$ year that is very high (about $35 \%$ of the average energy consumption). In the research, it is clear when the refrigerator-freezer runs at the average operating condition, about $20 \%$ energy can be saved. If awareness is increased to the people and be serious to save energy, at least $20 \%$ energy cost reduces from the daily household expenditure. Table 4 shows the maximum, minimum and average energy consumption of the refrigerator-freezer during the closed door condition.

Table 5. Effect of different variables on energy consumption

\begin{tabular}{|l|c|c|c|}
\hline \multicolumn{1}{|c|}{ Variable } & $\begin{array}{c}\text { Maximum } \\
(\mathrm{kWh} / \text { day } \\
)\end{array}$ & $\begin{array}{c}\text { Minimum } \\
(\mathrm{kWh} / \text { day } \\
)\end{array}$ & $\begin{array}{c}\text { Average } \\
(\mathrm{kWh} / \text { day } \\
)\end{array}$ \\
\hline $\begin{array}{l}\text { Ambient } \\
\text { temperature }\end{array}$ & 3.6 & 2.1 & 2.7 \\
\hline Load & 3.5 & 2.2 & 2.8 \\
\hline $\begin{array}{l}\text { Thermostat } \\
\text { setting position }\end{array}$ & 3.2 & 2.3 & 2.8 \\
\hline Water pan area & 3.1 & 2.9 & 3.0 \\
\hline
\end{tabular}

\section{Emission of Green House Gases}

Energy demand in Malaysia is increasing rapidly as well as increasing the greenhouse gases. The installation capacity of energy is increased from 14,291 MW in 2000 to 19,227 MW in 2005. It is predicted that it will be 25,258 MW in 2010 [13]. The installation capacity of energy is increased about $35 \%$ from 2000 to 2005 . Emissions of all greenhouse gases from fuel combustion activities are increasing to fulfill the energy demand. So there is a high impact on the environment as well as the global warming potential. According to the experimental investigation, emissions for the energy generation that is consumed of the refrigeratorfreezer are shown in Table 5. When the energy saving step is taken very seriously, greenhouse gases emission is reduced about $41 \%$. The energy saving step will keep the world green and save household expenditure. If the users of the refrigerator-freezer be serious, at least $20 \%$ greenhouse gas emissions reduce due to the energy consumption of the refrigerator-freezer.

Table 6. Emission due to the mix fuel energy generation

\begin{tabular}{|c|c|c|c|c|}
\hline & $\begin{array}{c}\mathrm{CO}_{2} \\
(\mathrm{~kg})\end{array}$ & $\begin{array}{c}\mathrm{SO}_{2} \\
(\mathrm{~kg})\end{array}$ & $\begin{array}{c}\mathrm{NO}_{\mathrm{x}} \\
(\mathrm{kg})\end{array}$ & $\begin{array}{c}\mathrm{CO} \\
(\mathrm{kg})\end{array}$ \\
\hline Maximum & 835.1 & 7.1 & 2.5 & 0.5 \\
\hline Minimum & 594.1 & 5.1 & 1.8 & 0.3 \\
\hline Average & 697.4 & 5.9 & 2.1 & 0.4 \\
\hline
\end{tabular}

\section{CONCLUSIONS}

It is found that the different environmental operating conditions have a significant influence on the refrigeratorfreezer energy consumption. It has been also observed that the influence of ambient temperature and cabinet load on energy consumption is more dominant compared to thermostat setting position and open surface water pan area. From the experimental investigation, it is found that the refrigerator-freezer consumes $1042.7 \mathrm{kWh} /$ year. To generate the energy, $\mathrm{CO}_{2} 687.42 \mathrm{~kg}, \mathrm{SO}_{2} 5.96 \mathrm{~kg}, \mathrm{NO}_{\mathrm{x}} 2.11$ $\mathrm{kg}$ and $\mathrm{CO} 0.39 \mathrm{~kg}$ are emitted; those have a large impact on the environment. The acceleration of global warming and climate change by the emission of gases is a great issue in the industrial economy base country. In Malaysia coal base power plant is increasing. The emission factor of coal is 1.5 time higher compare to the other fuels. The energy saving 
steps should be taken very seriously which will help to reduce the emissions and keep the country green as well as future world green.

\section{ACKNOWLEDGEMENT}

The authors would like to acknowledge the Ministry of Science, Technology, and Innovation, Malaysia for funding the project. The research has been carried out under the IRPA project no: 03-02-03-1011.

\section{REFERENCES}

[1] Rahman Mohamed. A., Lee, K. T, "Energy for sustainable development in Malaysia: Energy policy and alternative energy," Energy Policy, 34, 2006, pp. 2388-2397.

[2] Nobuo Kato, "Analysis of structure of energy consumption and dynamics of emission of atmospheric species related to the global environment change ( $\mathrm{SOx}, \mathrm{NOx}$ and $\mathrm{CO}_{2}$ ) in Asia," Atmospheric Environment, 30 (5), 1996, pp. 757-785.

[3] Marland G, Boden TA, Andres RJ, Brenkert, Johnston C,"Global, regional $\mathrm{CO}_{2}$ emissions. Trends: A compendium of data on global change. Carbon Dioxide Information Analysis Center," Oak Ridge National Laboratory, Department of Energy, USA, 1999.

[4] Limmeechokchaia, B., Suksuntornsiri, P., "Embedded energy and total greenhouse gas emissions in final consumptions within Thailand," Renewable and Sustainable Energy Reviews, 11, 2007, pp. 259-281.

[5] Mahlia, T.M.I., "Emissions from electricity generation in Malaysia," Renewable Energy, 27, 2002, pp. 293-300.

[6] Varman, M., Masjuki, H.H., Mahlia, T.M.I., "Electricity savings from implementation of minimum energy efficiency standard for TVs in Malaysia," Energy and Buildings, 37, 2005, pp. 685-689.

[7] Mahlia, T.M.I., Masjuki, H.H., Saidur, R., Amalina M.A., "Costbenefit analysis of implementing minimum energy efficiency standards for household refrigerator-freezers in Malaysia," Energy Policy, 32, 2004, pp. 1819-1824.

[8] Der-Yeong Liu, Wen-Ruey Chang, Jian-Yuan Lin, "Performance comparison with effect of door opening on variable and fixed frequency refrigerators/freezers," Applied Thermal Engineering, 24, 2004, pp. 2281-2292.

[9] Mahlia, T.M.I., Masjuki,H.H., Saidur,R., Choudhury, I.A, NoorLeha, A.R., "Projected electricity savings from implementing minimum energy efficiency standard for household refrigerators in Malaysia," Energy, 28, 2003, pp. 751-754.

[10] Reddy, B.S., Balachandra, P., "Integrated energy-environment-policy analysis - a case study of India," Utilities Policy, 11, 2003, pp. 59-73.

[11] Razali, M.A, Othaman, M.N, Sim, O.F. , "Residential and commercial electricity consumers in Subang Jaya and Bandar Baru Bangi," Housing and energy project, Kuala Lumpur, Malaysia: Institute for Advanced Studies, University of Malaya, 1993, pp. 1-111.

[12] Grimes JG, William PEM, Shomaker BL., "Effect of usage conditions on household refrigerator-freezer and freezer energy consumption," ASHRAE Transactions, 83(1), 1977, pp. 818-828.

[13] Sustainable energy development, Chapter $19,9^{\text {th }}$ Malaysian plan 2006-2010, pp. 393-411. 


\title{
Flow Characterization of Gas-Solid Mixture in Fluidized Bed Combustion
}

\author{
Rosyida Permatasari, Mohammad Nazri bin Mohd Jaafar, Tholudin bin Mat Lazim, Mohammad \\ Sayuthy bin Samsudin
}

\begin{abstract}
The fluidization behavior of a non reactive gassolid in the fluidized bed is experimentally and numerically studied. The numerical results are analyzed, and compared with experimental data. Some results on particle image velocimetry (PIV) in 2-D fluidized beds are presented. The PIV applications were used in order to determine the origin of the ejected particles and the particle velocity distribution, using silica sand particles as inert bed material of Fluidized Bed Combustion. The model used commercial CFD software, Fluent, in two-dimensional simulations. Fluent predicted the fluid bed behavior by utilizing its Eulerian Granular Multiphase model where both the gas and solids phases are assumed continuous and fully interpenetrating. The model incorporated the kinetic theory to predict gas-solid interaction. The silica sand (Geldart particle group B) with average size $300 \mu \mathrm{m}$ and $10 \mathrm{~cm}$ height are fluidized with ambient air. Some flow rate of air are introduced i.e. 20 CFM, 30 CFM and 40 CFM. When the sand fluidized in FBC, the results show the formation of a big vortex. It happened at the height $0.25-0.33$ $m$ from the base. Both the experimental and the numerical results show that increasing the fluidization velocity and fluidization height is correlated with airflow rate.
\end{abstract}

Keywords: gas-solid flow, particle image velocimetry (PIV), CFD, fluidized bed combustion, fluidizing velocity

\section{INTRODUCTION}

Fluidized bed combustors are common and important reactors in process engineering because of the good mass and heat transfer rate between the fluid and the particles, and between the particles and the sidewall. It is well known that there are non-uniform flow structures such as bubbles and slugs in fluidization [3]. Large bubbles and slugs are

This work was supported in part by Institutional Grant Universiti Teknologi Malaysia.

Rosyida Permatasari, Mohammad Nazri bin Mohd Jaafar and Tholudin bin Mat Lazim are with the Faculty of Mechanical Engineering, Universiti Teknologi Malaysia, 81310 UTM Skudai, Johor, Malaysia (phone: +6075534851， +6075534589; email: prosyida@yahoo.com, nazri@fkm.utm.my, tholudin@fkm.utm.my).

, is with the Faculty of Mechanical Engineering, Universiti Teknologi Malaysia, 81310 UTM Skudai, Johor, Malaysia (corresponding author to provide; email:).

is with the Faculty of Mechanical Engineering, Universiti Teknologi Malaysia, 81310 UTM Skudai, Johor, Malaysia (corresponding author to provide phone:; email:).

Mohammad Sayuthy bin Samsudin was with the Faculty of Mechanical Engineering, Universiti Teknologi Malaysia, 81310 UTM Skudai, Johor, Malaysia. undesirable for efficient operations, because they can reduce the contact efficiency. In fluidization it is attempted to overcome the problems in handling granular solids and to improve the heat transfer properties.

The flow and mixing of granular materials are important in a wide range of industrial, agricultural, and geophysical situations. Granular flows exhibit a wide range of phenomena including convection, particle size segregation, heaping, waves, fluidization, and pattern formation [4]-[5].

A new technique has introduced for the measurement of average particle displacements in granular flows based on particle image velocimetry (PIV). PIV has been adapted for use in measuring particle displacement and velocity fields in granular flows [6].

CFD modeling of fluidized beds is nowadays commonly performed applying the kinetic theory model of granular flow and a transient description [1]-[2]. And CFD has gained popularity as personal computers are becoming more powerful and capable of solving the governing equations to model the interactive behavior in multiphase systems. The multiphase for gas-solid flow in fluidized beds consist of a vertical cylinder containing particles where gas is introduced through a distributor. The gas rising through the bed suspends the particles. Depending on the gas flow rate, bubbles appear and rise through the bed, intensifying the mixing within the bed.

This work has assessed the multiphase, modeling parameters of the CFD software, Fluent v6.2.16, to model a two-dimensional FBC system. Drag function models found in Fluent's Eulerian Granular Multiphase model and Syamlal O'Brien drag model. CFD simulations of the experiments were carried out in $2 \mathrm{D}$ and after that, the simulation results were compared with the experimental data (PIV data).

However, more validation studies are necessary to test and improve the reliability of the modeling approach especially at higher fluidization velocities.

\section{EXPERIMENTAL AND SIMULATION SETTING}

The experimental set-up is shown in Figure 1. The transparent wall (Perspex) of the turbulent bed cold model is cylinder with $0.144 \mathrm{~m}$ inner diameter and $2 \mathrm{~m}$ height. The thickness of the walls is $0.003 \mathrm{~m}$. To investigate the behavior of sand fluidizing in the FBC, the air flow rates were varied. Therefore, the air flow rates in the experiments were $20 \mathrm{CFM}, 30 \mathrm{CFM}$, and $40 \mathrm{CFM}$, corresponding to 
velocities $0.1926 \mathrm{~m} / \mathrm{s}, \quad 0.2889 \mathrm{~m} / \mathrm{s}$ and $0.385 \mathrm{~m} / \mathrm{s}$, respectively. Bed material density was $2600 \mathrm{~kg} / \mathrm{m} 3$, the mean diameter of the silica sand particles $300 \mu \mathrm{m}$ and its volume fraction approximately 5\%, are showed in Table 1 . The height of sand in the cylinder was $0.1 \mathrm{~m}$. And the images captured with CCD camera using PIV technique were at $0.25 \mathrm{~m}$ view area from the base area, is showed in Figure 2 .

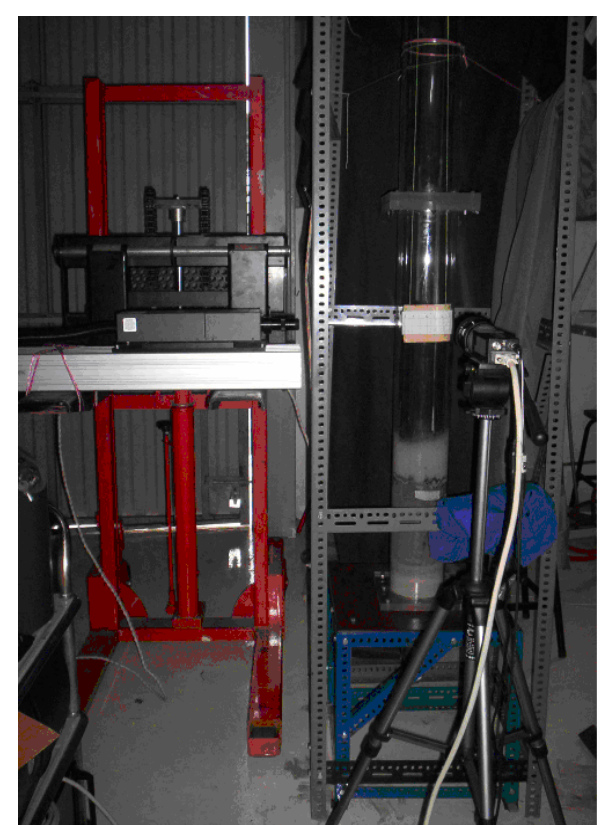

Figure 1. Experimental rig

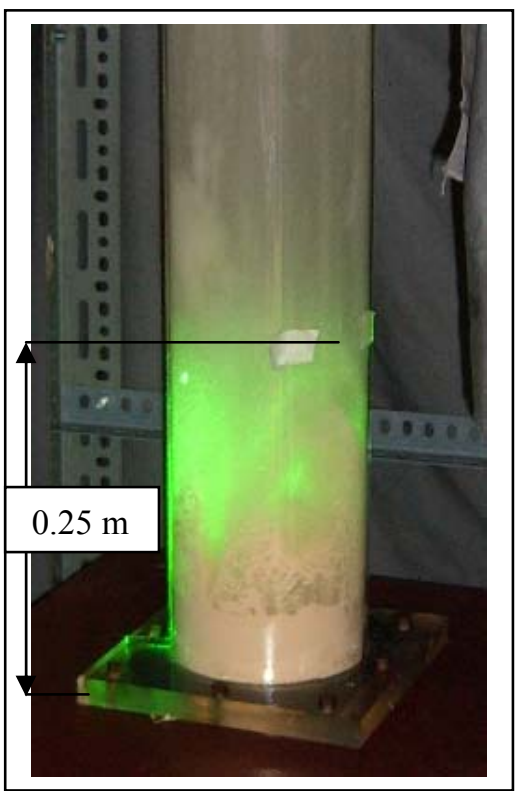

Figure 2. Experimental image
Table 1. List of values of model parameters used in simulations

\begin{tabular}{|c|c|c|}
\hline Description & Value & Comment \\
\hline Particle density & $2600 \mathrm{~kg} / \mathrm{m}^{3}$ & Silica Sand \\
\hline Gas density & $1.2 \mathrm{~kg} / \mathrm{m}^{3}$ & Air \\
\hline Particle diameter & $300 \mu \mathrm{m}$ & $\begin{array}{l}\text { Uniform } \\
\text { distribution }\end{array}$ \\
\hline $\begin{array}{l}\text { Initial solid } \\
\text { packing }\end{array}$ & 0.055 & Fixed value \\
\hline $\begin{array}{l}\text { Superficial gas } \\
\text { velocity }\end{array}$ & $\begin{array}{r}0.1926,0.2889 \\
\quad \text { and } 0.385 \mathrm{~m} / \mathrm{s}\end{array}$ & \\
\hline Bed height & $2 \mathrm{~m}$ & Fixed value \\
\hline $\begin{array}{l}\text { Lateral } \\
\text { Boundary } \\
\text { Condition } \\
\text { type } \\
\end{array}$ & WALL & $\begin{array}{l}\text { No-slip } \\
\text { boundary } \\
\text { condition }\end{array}$ \\
\hline $\begin{array}{l}\text { Inlet Boundary } \\
\text { Condition } \\
\text { type } \\
\end{array}$ & VELOCITY & $\begin{array}{c}\text { Superficial gas } \\
\text { velocity }\end{array}$ \\
\hline $\begin{array}{l}\text { Outlet Boundary } \\
\text { Condition } \\
\text { type }\end{array}$ & OUTFLOW & $\begin{array}{l}\text { Fully developed } \\
\text { flow }\end{array}$ \\
\hline Time steps & $0.001 \mathrm{~s}$ & Fixed value \\
\hline Solids viscosity & Syamlal O'Brien & \\
\hline Iterative steps & 20 & $\begin{array}{c}\text { Default in } \\
\text { Fluent }\end{array}$ \\
\hline $\begin{array}{c}\text { Convergence } \\
\text { criteria }\end{array}$ & $10^{-3}$ & $\begin{array}{c}\text { Default in } \\
\text { Fluent }\end{array}$ \\
\hline
\end{tabular}

\section{Simulation AND EXPERIMENTAL RESUlts}

\section{A. 20 CFM air flow rate}

At 20 CFM air flow rate, CFD result shows that fluidizing height of sand correlated with the time. It takes time to fluidize the sand up, shows in Figure 3. The result shows some vortexes happened between $0.1 \mathrm{~m}$ and $0.5 \mathrm{~m}$ heights.

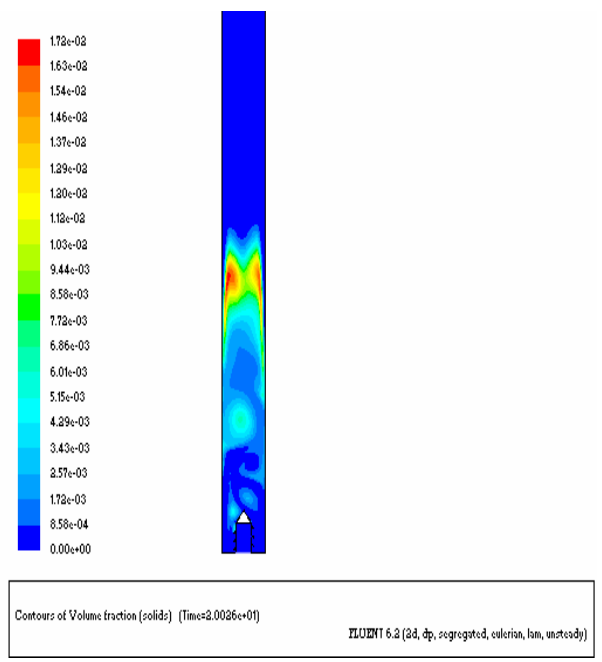

Figure 3. Contours of volume fraction (sand) 


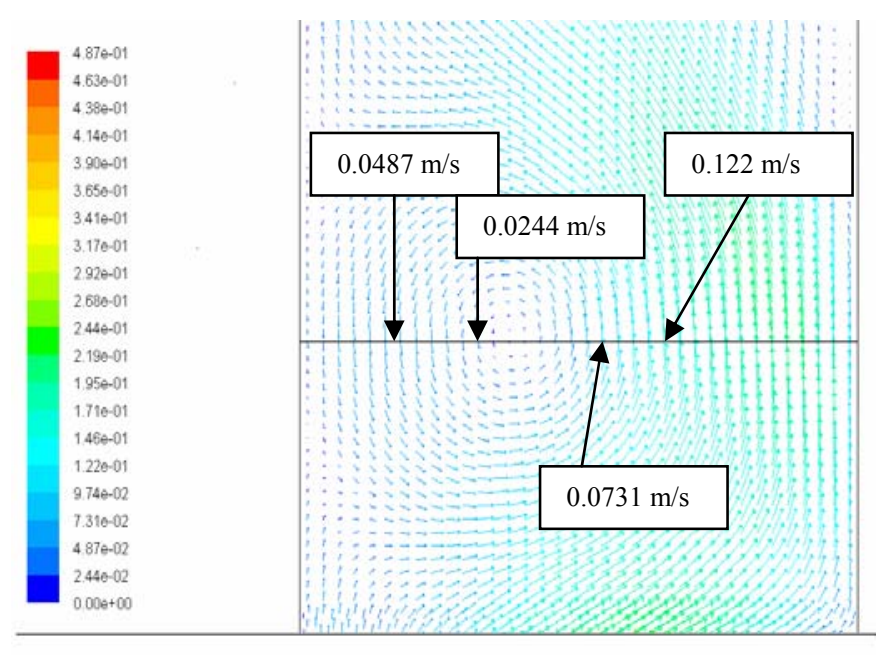

Figure 4. Velocity vectors and velocity values $(\mathrm{m} / \mathrm{s})$ of sand at $0.25 \mathrm{~m}$ height of CFD result

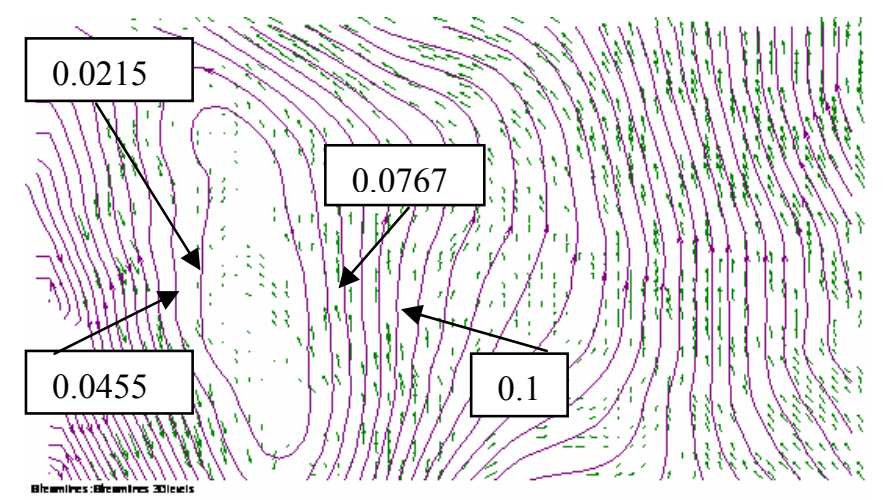

Figure 5 . Velocity vectors and velocity values $(\mathrm{m} / \mathrm{s})$ of sand at $0.25 \mathrm{~m}$ height of PIV result

At the same height i.e. $0.25-0.33 \mathrm{~m}$, results of CFD simulation (Figure 4) and PIV results (Figure 5) show the biggest vortex with its velocity values around 0.1 until $0.0767 \mathrm{~m} / \mathrm{s}$. Therefore, simulation results were in agreement with experimental results.

\section{B. 30 CFM Air Flow Rate}

At $30 \mathrm{CFM}$ air flow rate, CFD result shows that fluidizing height of sand also correlated with the time. And there are some vortexes happened between 0.1 and $0.5 \mathrm{~m}$ heights, shows in Figure 6.

There is a vortex in CFD simulation (Figure 7) at $0.25-$ $0.33 \mathrm{~m}$, same with experimental result in figure 8 . And velocity values are almost alike. Therefore, simulation results were in agreements with experimental results.

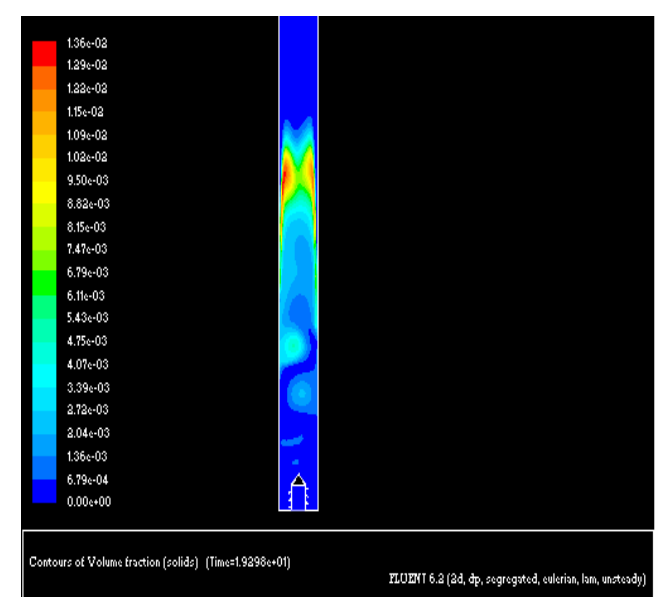

Figure 6. Contours of volume fraction (sand)

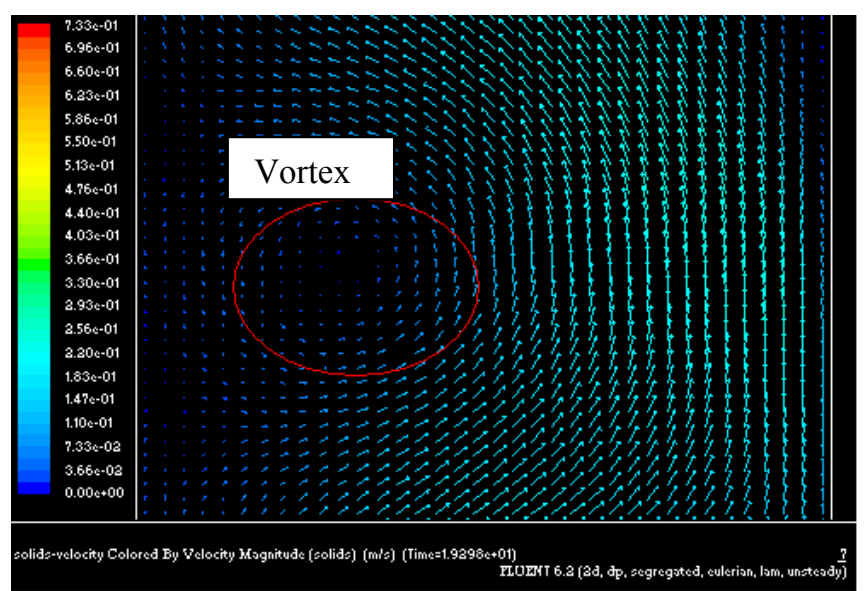

Figure 7 . Velocity vectors and velocity values $(\mathrm{m} / \mathrm{s})$ of sand at $0.25 \mathrm{~m}$ height of CFD result

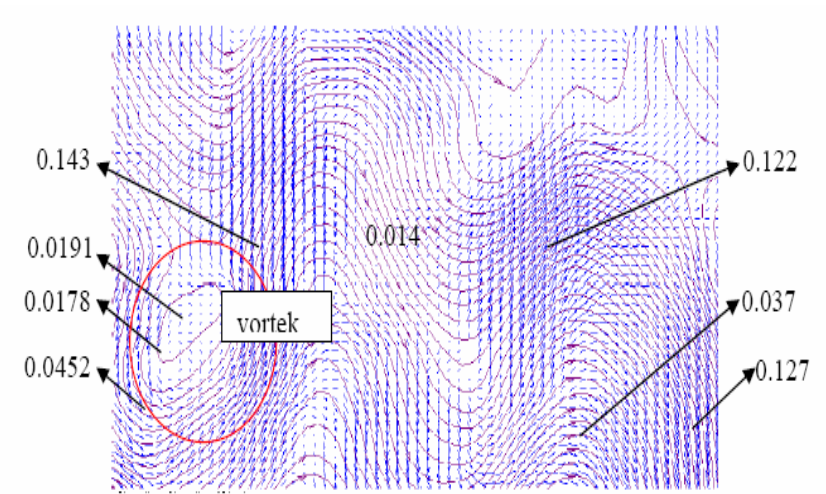

Figure 8 . Velocity vectors and velocity values $(\mathrm{m} / \mathrm{s})$ of sand at $0.25 \mathrm{~m}$ height of PIV result

\section{40 CFM air flow rate}

At 40 CFM air flow rate, CFD result shows that fluidizing height of sand also correlated with the time. And there are some vortexes happened between 0.1 and $0.5 \mathrm{~m}$ heights, shows in Figure 9. 


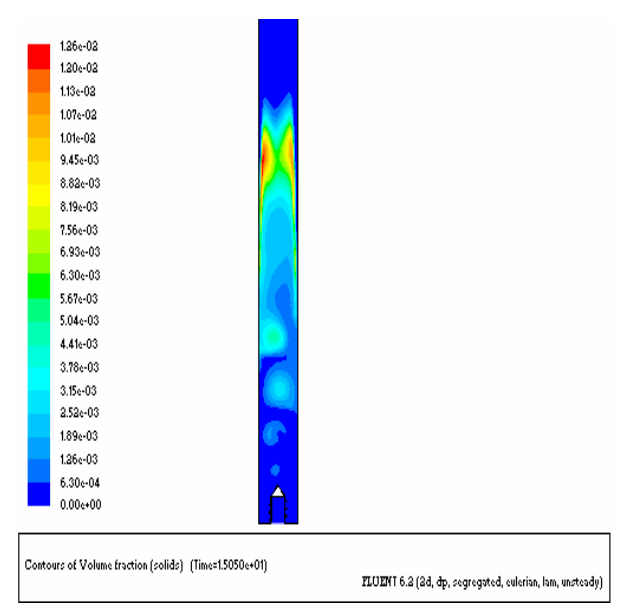

Figure 9. Contours of volume fraction (sand)

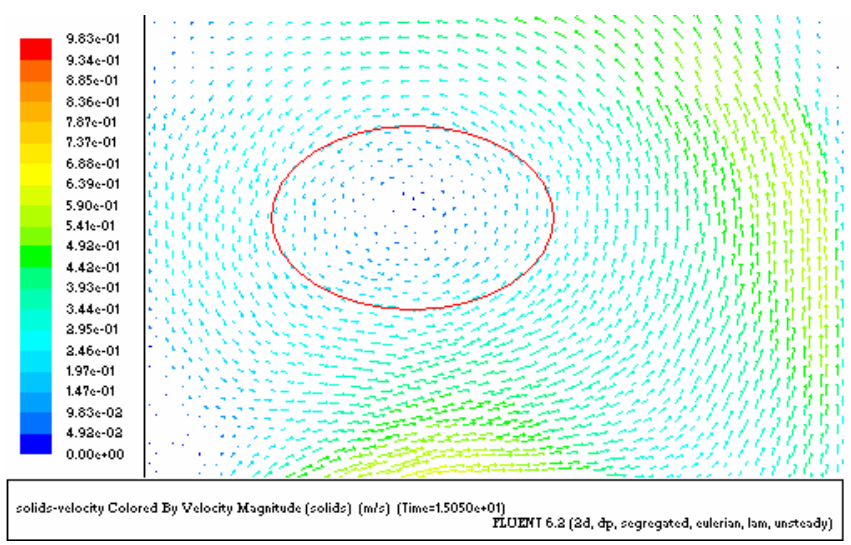

Figure 10 . Velocity vectors and velocity values $(\mathrm{m} / \mathrm{s})$ of sand at $0.25 \mathrm{~m}$ height of CFD result

At the same height i.e. $0.25-0.33 \mathrm{~m}$, results of CFD simulation (Figure 10) and PIV results (Figure 11) show the biggest vortex. Therefore, simulation results were in agreement with experimental results.

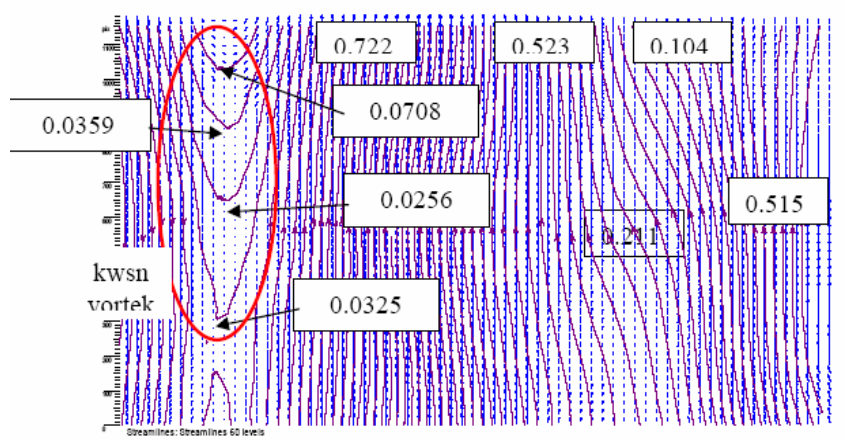

Figure 11 . Velocity vectors and velocity values $(\mathrm{m} / \mathrm{s})$ of sand at $0.25 \mathrm{~m}$ height of PIV result

\section{CONCLUSION}

The results show that silica sand, Gildart's type B particles, have good fluidizing behavior. Both the experimental and the numerical results show that increasing the fluidization velocity and fluidization height is correlated with airflow rate and time. Therefore, the generally accepted PIV method and a drag model were tested for simulation of gassolid flow in FBC by means of the Eulerian multiphase CFD. The validation of the models was based on the experimental results from a 2D modeling. It's applicable to small scale processes and phenomena. However, for simulations of large industrial processes, the models still need to be further developed.

\section{ACKNOWLEDGEMENT}

We gratefully acknowledge the financial assistance from Institutional Grant Universiti Teknologi Malaysia.

\section{REFERENCES}

[1] Gidaspow, D., Jung, J., Singh, R.K., "Hydrodynamics of fluidization using kinetic theory: an emerging paradigm: 2002 Flour-Daniel Lecture, Review article, Powder Technology, Vol. 148, Issues 2-3, pp. 123-141.

[2] Peirano, E., "Modelling and simulation of turbulent gas-solid flows applied to Fluidization", $P h D$ thesis, Chalmers University of Technology, Göteborg, 1998.

[3] D.J. Needham, J.H. Merkin, "The propagation of a voidage disturbance in a uniformly fluidized bed". J. Fluid Mech. 13. 427-454, 1983.

[4] Behringer, RP, "The dynamics of flowing sand". Nonlinear Sci Today. 3: 1-15, 1993.

[5] Jaeger HM; Nagel SR; Behringer RP., "The physics of granular materials". Phys Today. 49: 32-38, 1996.

[6] R. M. Lueptow, A. Akonur, T. Shinbrot, "PIV for granular flows". Experiments in Fluids. Springer-Verlag. 28 (2000). 183-186, 2000.

[7] Dantec Dynamics. "Flow-map PIV Installation \& User's guide". 


\title{
Energy saving in Air-Conditioning System Using a Variable Speed Compressor
}

\author{
Marwan Affandi and Mat Nawi Wan Hassan
}

\begin{abstract}
An energy analysis shows that despite considerable saving of electrical energy, the high cost of variable speed drive and its controller result in a relatively long payback period from 12 years for short operating hours of less than 5 hours daily for set-point of $21^{\circ} \mathrm{C}$. However, for operating hours of 8 hours per day, assuming $80 \%$ of energy saving, the payback period is reduced to 7 and a half years. For operating hours of more than 12 hours per day, the payback period is relatively shorter (less than 4 years for a temperature setting of $21^{\circ} \mathrm{C}$ ).
\end{abstract}

Keywords: air-conditioning systems, variable speed compressor, set-point, energy saving

\section{INTRODUCTION}

Energy savings can be obtained by modulating the speed of the compressor to match a given load on an air conditioning system of a building. In this study an air conditioning system using a constant speed compressor was retrofitted with an inverter and a temperature-speed controller. This system was installed to cool a thermal environmental room of size $4.5 \mathrm{~m}$ by $3.28 \mathrm{~m}$ by $3.43 \mathrm{~m}$. The impact of the proportional control on the performance of the system, the room temperature, the energy consumption, and the motor frequency have been analysed experimentally for both Variable Speed Compressor (VSC) and Constant Speed Compressor (CSC) air conditioning systems. These experiments were conducted in the Thermofluid Laboratory, Faculty of Mechanical Engineering, Universiti Teknologi Malaysia.

\section{EXPERIMENTS AT VARIABLE SPEEDS}

In this kind of experiments, there were three experiments conducted at variable speeds for each setting of room temperature. Each experiment was conducted using different setting that was chosen to give minimum energy consumption. There were several experiments that had been conducted to determine each optimum setting. In the

This work was fully supported by Universiti Teknologi Malaysia (UTM) through an IRPA Project. The first author was very grateful for the fellowship given to him during his study there.

M. Affandi is with The School of Mechatronic Engineering, Universiti Malaysia Perlis (UniMAP), Kangar, Perlis (phone: 049798444; hp: 0124225604; fax: 049798142; e-mail: marwan@ unimap.edu.my).

M.N. Wan Hassan is a retired professor from The Faculty Of Mechanical Engineering, UTM. following, the results of the trials done for each setting of temperature were described.

\section{MOTOR FREQUENCY}

At first the motor ran at high frequency $(50 \mathrm{~Hz})$ and in about 30 minutes it started to decrease. Rapidly the frequency dropped to less than $25 \mathrm{~Hz}$ and after that fluctuated. The temperature controller sent signals to the inverter to vary the frequency as its sensor detected the room temperature. It tried to minimize the error between the set point temperature and the room temperature. The fluctuation became lower as the room temperature approached the set point temperature. Under this proportional control the controlled variable i.e. the frequency became stable at about $22 \mathrm{~Hz}$; see figure 1 .

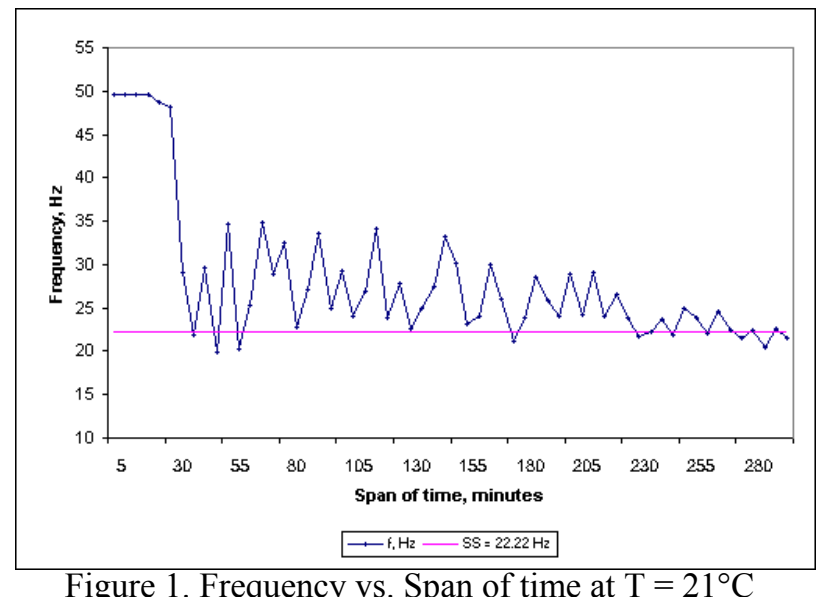

Figure 1 shows the relationship between Average frequency and Span of Time for the set-point of $21^{\circ} \mathrm{C}$; the average was taken from three experiments. Similar measurements for average frequencies were also taken at room temperatures $22^{\circ} \mathrm{C}$ and $23^{\circ} \mathrm{C}$, respectively. Since the lowest frequency is limited to $15 \mathrm{~Hz}$, the final frequency for all setting temperatures will fluctuate in a range from 17 to $25 \mathrm{~Hz}$, or even in a narrower range. For the last forty minutes, the average frequencies are 22.22, 19.71 and 18.05 $\mathrm{Hz}$ for the temperature settings of 21,22 and $23^{\circ} \mathrm{C}$, respectively. If the experiment were extended longer, the fluctuation will be relatively quite small that frequency becomes nearly constant. 


\section{ROOM TEMPERATURES}

Room temperature data for each three experiments were averaged and the results are shown in single graphs. Figure 2 shows the relationship between Room temperature and Span of time for the set-point of $21^{\circ} \mathrm{C}$. The graph reveals that the room temperature dropped $\mathrm{y}$ as the compressor ran at high speed. In less than 25 minutes the temperature decreased to around $21{ }^{\circ} \mathrm{C}$. The room temperature then seemed to fluctuate in a narrow range between 20.5 to $21.7^{\circ} \mathrm{C}$ before approaching $21.1^{\circ} \mathrm{C}$, its steady state temperature.

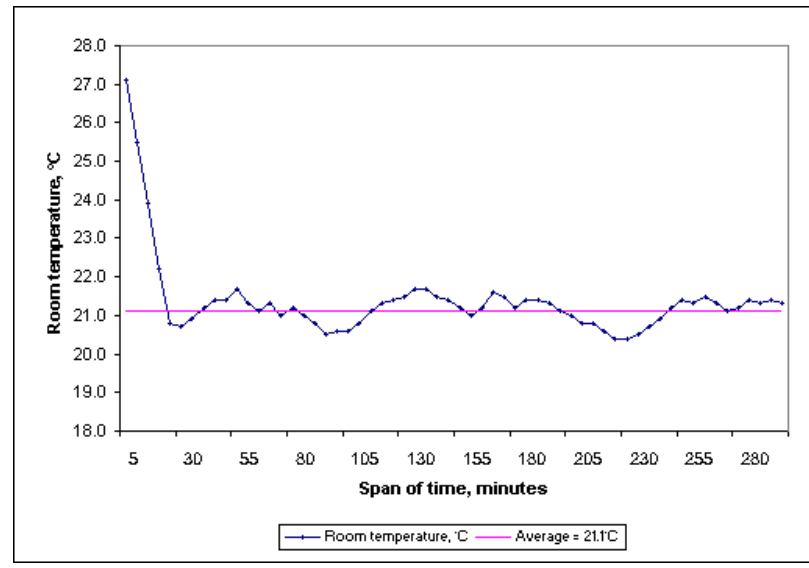

Figure 2. Room temperatures vs. Span of time at $\mathrm{T}=21^{\circ} \mathrm{C}$

\section{Power Consumption at Variable Speeds}

Similar to room temperature data, power consumption data were averaged from the three experiments conducted. Figure 3 shows the relationship between Power consumption vs. Span of time for setting temperature of $21^{\circ} \mathrm{C}$; this curve looks similar to curves for frequency vs. span of time. For the first 30 minutes (or less than 30 minutes for other cases) the power remained constant before it dropped quickly and fluctuated after that. Power consumption also has its steady state value, beyond which, the power will become nearly constant or just fluctuates in a narrow range.

For the setting temperature of $21^{\circ} \mathrm{C}$, it was found that average power consumption for the whole time excluding the first 30 minutes was constant at $1.296 \mathrm{~kW}$. For setting temperature of $22^{\circ} \mathrm{C}$ it is $1.237 \mathrm{~kW}$ (excluding the first 20 minutes) while for setting temperature of $23^{\circ} \mathrm{C}$ it is 1.104 $\mathrm{kW}$ (excluding the first 25 minutes).

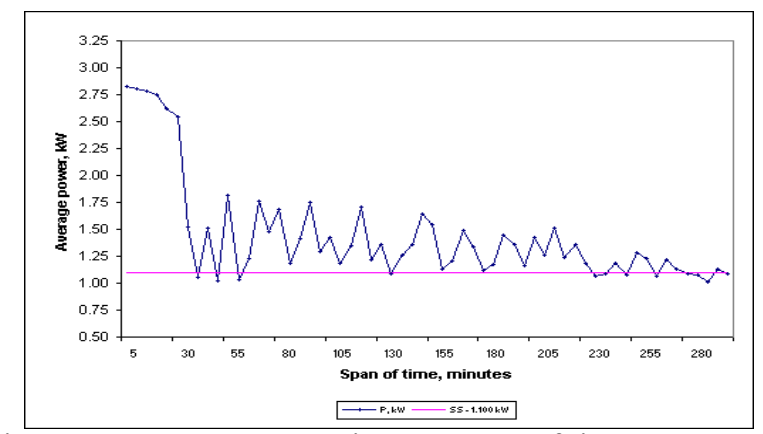

Figure 3. Power consumption vs. Span of time at $\mathrm{T}=21^{\circ} \mathrm{C}$
The steady state power consumption was lower than its average power. For the temperature setting of $21^{\circ} \mathrm{C}$ it was $1.100 \mathrm{~kW}$ while for the temperature settings of $22^{\circ} \mathrm{C}$ and $23^{\circ} \mathrm{C}$ they were 0.974 and $0.881 \mathrm{~kW}$, respectively. All these values were averaged from the last forty minutes. The steady state value of power consumption was useful for extrapolation of operation time beyond five hours.

\section{ENERGY CONSUMPTION}

Energy consumption was calculated cumulatively from the start of the motor using the relationship:

$E_{n+1}=E_{n}+P_{n} \cdot \Delta t / 60$

Here, $\Delta \mathrm{t}=$ time interval in minutes

$\mathrm{P}_{\mathrm{n}}=$ power measured

At $\mathrm{n}=0$ (the start of motor), $\mathrm{E}_{0}=0$. For constant speed, $\mathrm{P}_{\mathrm{n}}$ can be substituted by average power. Since the power is proportional to the frequency, this makes the energy consumption is a linear function of time.

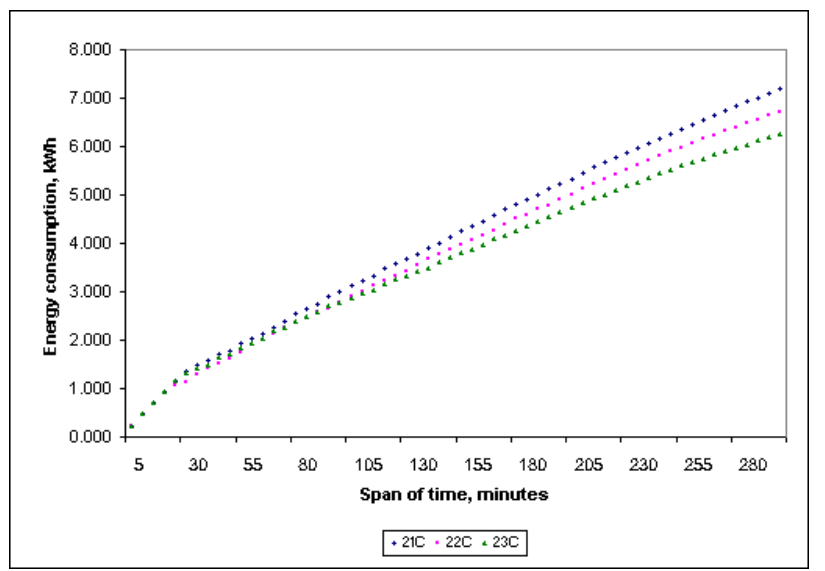

Figure 4. Energy consumption at various temperature settings

Figure 4 shows the relationship between energy consumption and span of time for three temperature settings. Although the power consumption was not constant, the energy consumption seems to be a straight line except at the first forty minutes or less. This should be expected since energy is a cumulative function.

\section{Analysis of Possible EnERgy SAVINGS}

Calculation made [1] shows that energy saving is highest at the lowest room temperature and lowest at the highest room temperature. Assuming that the compressor runs 25 day a month, this means that its monthly operation is 125 hours. For one year, the compressor operates for 1500 hours. Energy saving for a year at each setting temperature can then be estimated as follows:

Energy saving at $21^{\circ} \mathrm{C}=704 \mathrm{kWh}$

Energy saving at $22^{\circ} \mathrm{C}=435 \mathrm{kWh}$

Energy saving at $23^{\circ} \mathrm{C}=174 \mathrm{kWh}$

Cost of necessary equipment and accessories is about RM 3000, including maintenance cost. Payback period for each setting temperature can be calculated from the formula:

Payback period $=\frac{\text { Cost }}{\text { Saving }}$ 
Assume that one $\mathrm{kWh}$ can save RM 0.35. Results of calculations show that at setting temperatures of 21,22 , and $23^{\circ} \mathrm{C}$, Payback periods are 12.2, 19.7, and 49.2 years, respectively. Payback periods for all scenarios (setting temperatures) seem to be very high, particularly for the lowest temperature setting. Furthermore, commercial airconditioning units will not last so long; a 10-year life is acceptable. This will certainly discourage common users to apply variable speed compressors. However, there is still likely further refinement that may produce more attractive results.

In practice daily use of an air-conditioner unit is more than five hours. For an office, for example, the airconditioner operates continuously during office hours, which is about eight hours a day. A home air-conditioner will operate longer; daily operation is often more than ten hours. Since after steady state power achieved the power is relatively constant, energy consumption for extra hours can be estimated. Estimation will be made for energy consumption for 8,10 and 12 hours of daily use, respectively. The principle is the longer the VSC used the bigger the saving.

For extension hours of operation, estimation will be given by extrapolating the power of the unit up to 12 hours. This is of course very subjective but it can still be acceptable since after the steady state is achieved the power consumption will not significantly change. This can be conveniently done by taking the steady state power as the power consumed in the extended hours.

Calculations show that the lowest payback period is achieved by operating the system 12 hours daily at the setting temperature of $21^{\circ} \mathrm{C}$. The payback period, which is 3 years and 8 months, seems low enough to encourage people to use variable speed compressors for their air-conditioner units. For real applications an adjustment will be made that will be more likely achieved.

Previously, it was implicitly assumed that full savings could be obtained. However, due to various reasons such as intermittent operations, drops in the compressor's efficiency and inverter losses, it is assumed that practically only $80 \%$ of the energy saving can be utilized. With this reduction of energy saving the money saved is RM 654. The payback period now increases to 4 years 7 months. However, this should be still acceptable even for ordinary users.

For an office that operates 8 hours daily the payback period is 6 years and 1 month (for the same $21^{\circ} \mathrm{C}$ setting temperature). Assuming that $80 \%$ of energy saving the money saved will be RM 393. Here, the payback period becomes 7 years 6 months. Although this may sound long the office could still afford buy the system. Considering that a big system will be cheaper compared to several small ones, savings in terms of money will be better. For a 100 $\mathrm{kW}$ capacity (typical value for a medium office), the pay back period is likely less than 5 years.

From literature review [2], [3], [4], energy savings found using variable speed were from $10 \%$ to $40 \%$. Previous research [4] showed that for setting temperature of $21^{\circ} \mathrm{C}$ there was opportunity for energy saving $28 \%$ while for the $22^{\circ} \mathrm{C}$ setting temperature energy saving was $33.7 \%$.

\section{CONCLUSION}

It can be concluded that the use of a variable speed compressor to save energy in an air-conditioning system is justified. Although only proportional control that was used, it had produced significant energy saving. More energy savings could be expected using better controls although the improvement would not be very big. The use of a VSC is therefore economically justifiable. In future, further drops in the prices of inverters and temperature controllers will become a catalyst in the promotion of energy conservation through the use of variable speed compressors.

\section{REFERENCES}

[1] M. Affandi, "Energy Saving in An Air-Conditioning System using An Inverter and A Temperature-speed Controller," UTM: PhD Thesis, 2004.

[2] Y. Shima, T. Tateuchi, and H. Sugiura, "Inverter control systems in a residential heat-pump air-conditioner", ASHRAE Trans. 1988, Vol. 94, No. 2, pp. 1541-1552.

[3] T. Senshu, A. Arai, K. Oguni, and F. Harada, "Annual energy saving effect of capacity-modulated air-conditioner equipped with inverterdriven scroll compressor", ASHRAE Trans., 1985, Vol. 91 No. 1, pp. 1569-1584.

[4] S.A. Tassou, C.J. Marquand, and D.R. Wilson, "Comparison of the performance of capacity-controlled and conventional-controlled heatpumps", Applied Energy, 1988, Vol. 14, pp. 241-256.

[5] N.Z. Abidin, "Retrofitting of Compressor Motor in Air Conditioning System for Energy Savings," UTM: Master Thesis, 1995. 


\title{
Numerical Simulation of Plate-Fin Recuperator
}

\author{
A.W. Mazlan, O. Kahar, Y.C. Chuah
}

\begin{abstract}
The aim of this study is to investigate the effect of geometrical variations on the performance of plate-fin recuperator. Solutions for this problem are obtained via numerical approach. Three different types of plate-fin recuperator has been investigated; rectangular, rounded and bullet edges. This study has shown that rounded and bullet edges plate fin configuration exhibits 28 percent increase in surface heat transfer coefficient over its rectangular counterpart but there is no significant difference in the Fanning friction factor.
\end{abstract}

Keywords: simulation, recuperator, micro gas turbine, plate-fin

\section{INTRODUCTION}

Microturbine is a miniature gas turbine with the aim of generating electrical energy from the combustion of hydrocarbon fuel. Typical microturbine system consists of a compressor, combustor, turbine, recuperator and generator. Microturbines as small as $25-75 \mathrm{~kW}$ has been developed to support the energy requirement in office buildings as well as hospitals [1].

According to McDonald [2], the beauty of current microturbines is because of their simplicity, especially the use of single-shaft radial flow turbo machinery. In order to increase the microturbine performance, there are two main parameters that can be manipulated in order to increase the overall microturbine efficiency: turbine inlet temperature and the recuperator effectiveness. With a modest increase on compression ratio, a higher turbine inlet temperature necessitates the use of higher cost supper alloys recuperator.

From a thermo economic perspective, $25 \%-30 \%$ of the total capital cost of a microturbine is belonged to the recuperator. Therefore, recuperator is the one of the most important component in a microturbine in terms of technical and also economic aspect [3].

Offset strip fin [OSF] is one of the most widely used enhanced fin geometries in plate-fin recuperator. As shown in Figure 1, the recuperator fin has a rectangular cross section, and is cut into small strips of length $L_{f}$. Every alternate strip is displaced by about $50 \%$ of the fin pitch in the transverse direction. In addition to the fin spacing and fin height, the major variables are the fin thickness and strip length in the flow direction. According to Shah et. al [4], the heat transfer coefficients for the offset strip fins are 1.5 to 4 times higher than those of plain fin geometries. At the same

A.W. Mazlan, O. Kahar, Y.C. Chuah, are with the Department of Thermo-Fluids, Faculty of Mechanical Engineering, Universiti Teknologi Malaysia, 81310 UTM Skudai, Johor (e-mail: mazlan@fkm.utm.my). pressure drop, the offset strip fin exchanger would require a substantially lower heat transfer surface area than plain fins exchanger.

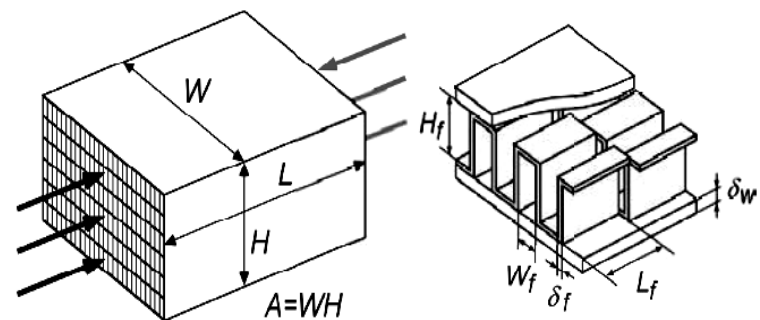

Figure 1. Recuperator and offset strip fin [1]

For specified heat transfer and pressure drop requirements, the offset strip fin requires a higher frontal area than a plain fin, but a shorter flow. The flow passage for offset strip fins are categorized as interrupted flow passage.

Table 1. Operating condition of compact heat exchangers [5]

\begin{tabular}{|l|l|l|l|}
\hline Technology & $\begin{array}{l}\text { Maximal } \\
\text { pressure }\end{array}$ & $\begin{array}{l}\text { Maximal } \\
\text { temperature }\end{array}$ & $\begin{array}{l}\text { Number } \\
\text { of streams }\end{array}$ \\
\hline $\begin{array}{l}\text { Aluminium } \\
\text { plate-fin } \\
\text { heat } \\
\text { exchanger }\end{array}$ & $80-120 \mathrm{bar}$ & $70-200{ }^{\circ} \mathrm{C}$ & $>10$ \\
\hline $\begin{array}{l}\text { Stainless } \\
\text { steel plate- } \\
\text { fin heat } \\
\text { exchanger }\end{array}$ & $80 \mathrm{bar}$ & $650{ }^{\circ} \mathrm{C}$ & $>2$ \\
\hline $\begin{array}{l}\text { Ceramic } \\
\text { plate-fin } \\
\text { heat } \\
\text { exchanger }\end{array}$ & $4 \mathrm{bar}$ & $1300^{\circ} \mathrm{C}$ & 2 \\
\hline
\end{tabular}

The material selection for recuperator depends on its operating conditions such as temperature, pressure, flow rates and also other aspect such as cost. One of the methods to increase microturbine efficiency is to increase the turbine inlet temperature. This in turn will increase the temperature of air entering recuperator, which is beyond $650^{\circ} \mathrm{C}$. Even though stainless steel is widely used material in existing operating machines but due to the temperature requirement, ceramic (silicon nitride and silicon carbide) is more suitable for the proposed $100 \mathrm{~kW}$ recuperator [5]. 

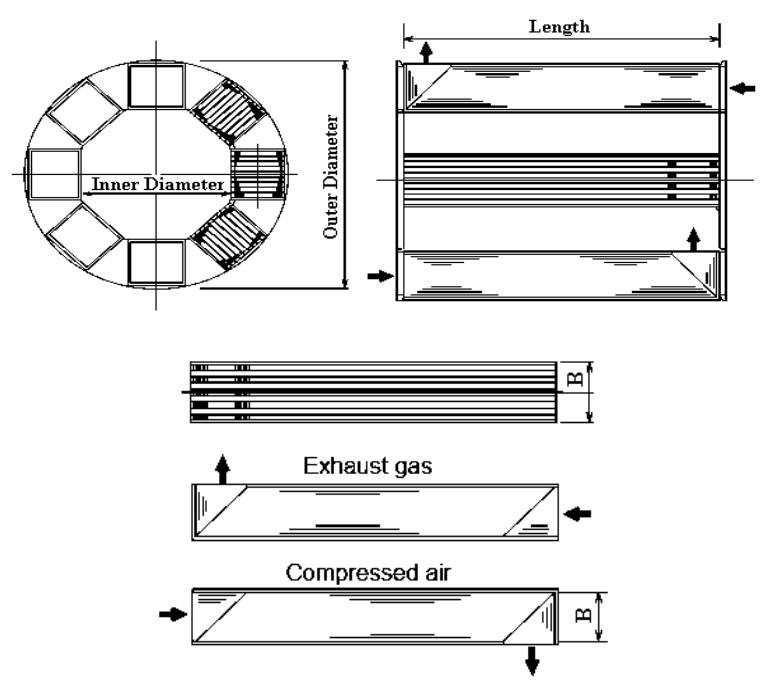

Figure 2. Proposed design of plate-fin recuperator for 100 $\mathrm{kW}[1]$

Figure 2 shows the proposed design of plate-fin recuperator for $100 \mathrm{~kW}$. It consists of annularly arranged 8 box-type recuperators. Such an annular configuration surrounding the turbine can eliminate the external duct, reduce the heat loss from combustor and minimize the overall size of turbogenerator package [1].

\section{CORRELATION FOR OFFSET STRIP FIN}

For the recuperator thermal-hydraulic correlations, first one needs to define flow regime, which is based on

Reynolds Number, $\operatorname{Re}=\frac{\left(\rho U D_{h}\right)}{\mu}$

where $\mathrm{U}$ represent flow velocity $\left(\mathrm{m} \mathrm{s}^{-1}\right), \rho$ is the fluid density $\left(\mathrm{kg} \mathrm{m}^{-3}\right), \mu$ is the dynamic viscosity (Pa.s) and $D_{h}$ is the hydraulic diameter $(\mathrm{m})$. The geometrical parameters for offset strip fin are given in Figure 1 which $L_{f}$ (fin length), $W_{f}$ (fin width), $H_{f}$ (fin height) and (fin thickness). The friction factor $f$ (Fanning factor) is linked to the pressure drops $\Delta p$ per unit length $L$ (length of the heat exchanger) by

$$
f=\frac{\left(\Delta p D_{h}\right)}{\left[4 L\left(\frac{1}{2} \rho U_{o}^{2}\right)\right]}
$$

The heat transfer coefficient $j$ (Colburn factor) is linked to the temperature difference over a heat transfer surface by

$$
j=\frac{N u_{D_{h}}}{\operatorname{Re}_{D_{h}} \operatorname{Pr}^{1 / 3}}
$$

where Nusselt number,

$$
N u_{D_{h}}=\frac{h D_{h}}{\lambda}
$$

Prandtl number, $\operatorname{Pr}=\frac{\mu c_{p}}{\lambda}$ with $c_{p}$ the specific heat capacity $\left(\mathrm{J} \mathrm{kg}^{-1} \cdot{ }^{\circ} \mathrm{C}^{-1}\right), \lambda$ thermal conductivity $\left(\mathrm{W} \cdot \mathrm{m}^{-1} \cdot{ }^{\circ} \mathrm{C}^{-1}\right)$.

The correlations of Manglik \& Bergles [6] have been developed, in the following range:

$$
\begin{aligned}
& 0.646 \leq D_{h} \leq 3.414(\mathrm{~mm}) \\
& 0.135 \leq W_{f} / H_{f} \leq 1.034 \\
& 0.012 \leq \delta_{f} / L_{f} \leq 0.060 \\
& 0.038 \leq \delta_{f} / W_{f} \leq 0.132
\end{aligned}
$$

The hydraulic diameter is defined as follow:

$D_{h}=\frac{4 V}{S}$

where volume, $\mathrm{V}$ and heat transfer area, $\mathrm{S}$

$$
D_{h}=\frac{4 W_{f} H_{f} L_{f}}{2\left(W_{f} L_{f}+H_{f} L_{f}+\delta_{f} H_{f}\right)+\delta_{f} W_{f}}
$$

According to the authors, the range of validity of these correlations is as follows: $300 \leq \operatorname{Re}_{D_{h}} \leq 10000$

$$
\begin{aligned}
f= & 9.6243 \operatorname{Re}_{D_{h}}^{-0.7422}\left(\frac{W_{f}}{H_{f}}\right)^{-0.1856}\left(\frac{\delta_{f}}{L_{f}}\right)^{0.3053}\left(\frac{\delta_{f}}{W_{f}}\right)^{-0.2659} \\
& \times\left[1+7.669 \times 10^{-8} \operatorname{Re}_{D_{h}}^{4.429}\left(\frac{W_{f}}{H_{f}}\right)^{0.920}\left(\frac{\delta_{f}}{L_{f}}\right)^{3.767}\left(\frac{\delta_{f}}{W_{f}}\right)^{0.236}\right]^{0.1} \\
j= & 0.6522 \operatorname{Re}_{D_{h}}^{-0.5403}\left(\frac{W_{f}}{H_{f}}\right)^{-0.1541}\left(\frac{\delta_{f}}{L_{f}}\right)^{0.1499}\left(\frac{\delta_{f}}{W_{f}}\right)^{-0.0678} \\
& \times\left[1+5.269 \times 10^{-5} \operatorname{Re}_{D_{h}}^{1.344}\left(\frac{W_{f}}{H_{f}}\right)^{0.504}\left(\frac{\delta_{f}}{L_{f}}\right)^{0.456}\left(\frac{\delta_{f}}{W_{f}}\right)^{-1.055}\right]^{0.1}
\end{aligned}
$$

These correlations predict the experimental data of 18 test cores within $\pm 20 \%$.

The main objective of heat transfer and pressure drop analysis is to find out the Reynolds number and the physical size of plate-fin recuperator like volume, width and length of recuperator. Basically, the design calculation is based on the cube-shaped recuperator concept. The volume, width and length of the recuperator are taken as $0.03656 \mathrm{~m}^{3}$, $0.2095 \mathrm{~m}$ and $0.4177 \mathrm{~m}$, respectively.

\section{Computational Results}

The numerical simulation is performed by using the CFD software package FLUENT 6.2. Initially two-dimensional computation is carried out using 3217 nodes of triangular mesh in pre-processor GAMBIT. Figure 3 shows the variation of the heat transfer coefficient on the surface of second fin with the gap between fins of $1 \mathrm{~mm}$. The plot shows a similar behavior of the surface heat transfer coefficient $h$, where, 


$$
h=\frac{q}{\left(T_{w}-T_{b}\right)}
$$

The average value of the convective heat transfer coefficient found from this study is $3422.777 \mathrm{~W} / \mathrm{m}^{2} . \mathrm{K}$ while the published data by S. Sundaresan [7, 8] makes up to 3417 which the difference is about $1.7 \%$. The empirical correlation of Manglik and Bergles gives the average value of convective heat transfer coefficient of $2894 \mathrm{~W} / \mathrm{m}^{2} . \mathrm{K}$. Figure 3 shows the comparison between the simulation results and published data.

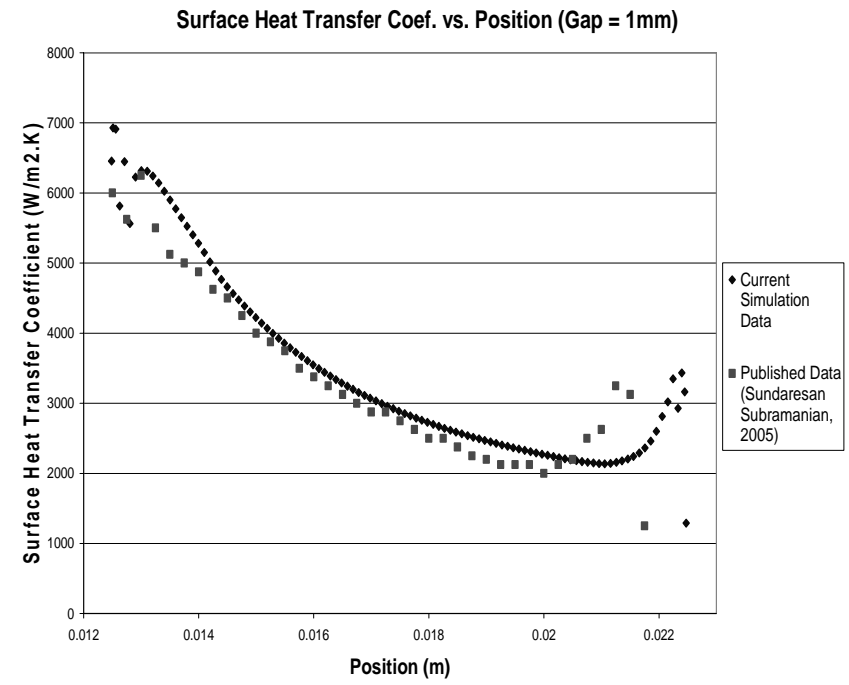

Figure 3. Comparison among validation data and published data $[7,8]$

In three numerical simulations two performance parameters are evaluated: Fanning friction factor and surface heat transfer coefficient. Fanning friction factor is related to the pressure loss while surface heat transfer coefficient is related to the heat transfer rate. The equation for the Fanning friction factor, $f$, is defined as the ratio of wall shear stress $\tau_{\mathrm{w}}$ to the flow kinetic energy per unit volume.

$f=\frac{\tau_{w}}{\rho u_{m}^{2} / 2 g_{c}}$

where $\tau_{w}=$ wall shear stress; $u_{m}=$ fluid mean axial velocity;

$g_{c}=$ proportionality constant in Newton's second law of motion, $g_{c}=1$.

All of the computations are carried out at more then 100000 nodes by using the tet/hybrid mesh in pre-processor GAMBIT. The main purpose in this simulation study is to estimate the performance of the recuperator with three different fin edges namely rectangular, rounded and bullet fin edges. The three-dimensional computations is carried out at 113169 nodes, 116670 nodes and 110215 nodes for rectangular, rounded and bullet fin edge respectively. The meshing size is done by using 0.1 interval size.

The rounded and bullet fin edges cannot be estimated by using the empirical correlation which is only suitable for rectangular fin. The results of Fanning friction factor versus
Reynolds Number at the air side and gas side for the three types of fin edges were shown in Figure 4 and Figure 5, respectively.

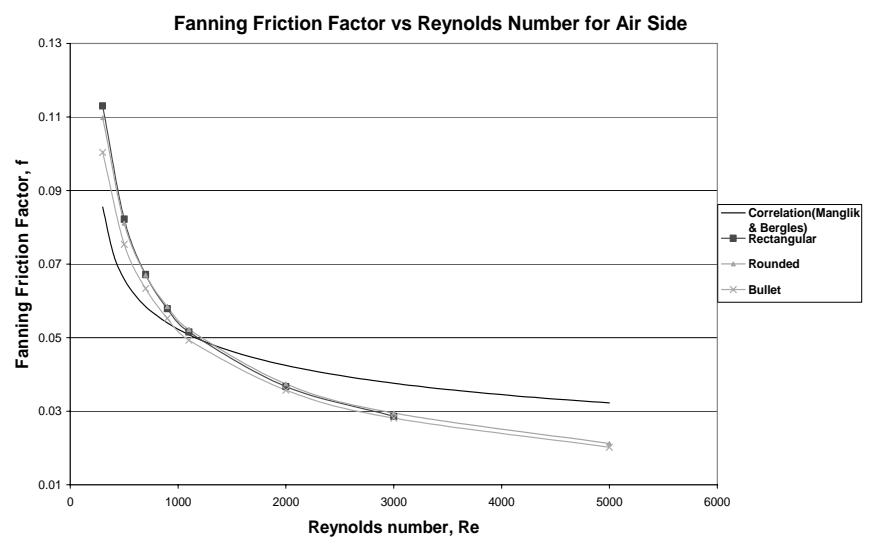

Figure 4. Fanning Friction Factor vs. Reynolds Number for comparison among empirical correlation, rectangular, rounded and bullet fin edge (air side)

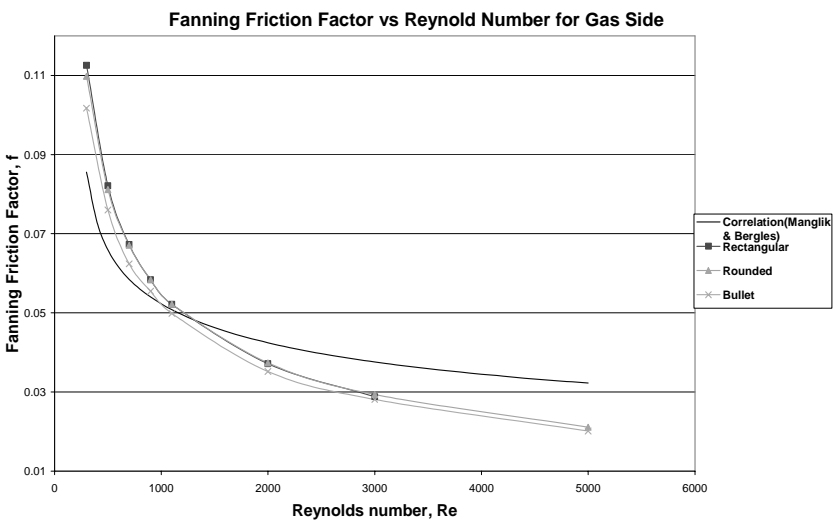

Figure 5. Fanning Friction Factor vs. Reynolds Number for comparison among empirical correlation, rectangular, rounded and bullet fin edge (gas side)

The results of surface heat transfer coefficients versus Reynolds Number for the rectangular, rounded and bullet fin edge for the three types of fin edges are shown in Figure 6 and Figure 7, respectively. As shown in Figure 5 and 6, all the lines plotted are in the upward trend. The surface heat transfer coefficient is shown to become higher with the Reynolds Number for all types of fin edges.

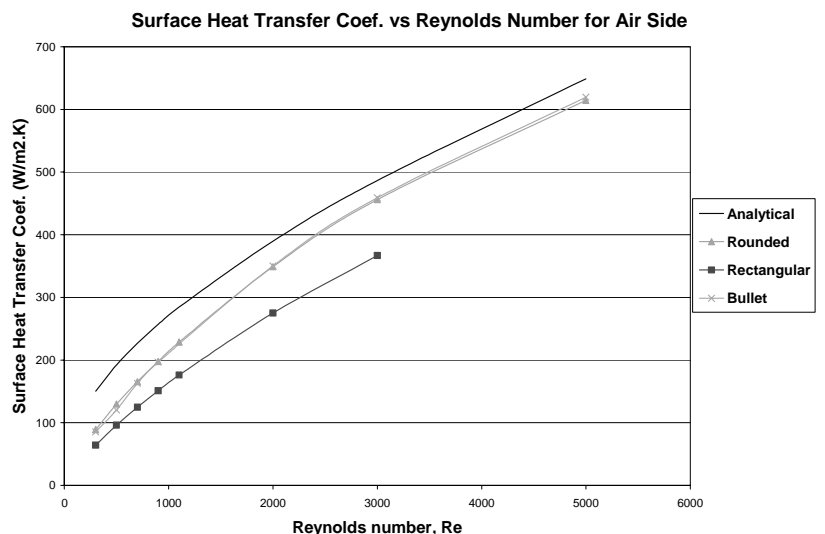

Figure 6. Surface Heat Transfer Coefficients vs. Reynolds Number for comparison among empirical correlation, 
rectangular, rounded and bullet fin edge (air side)

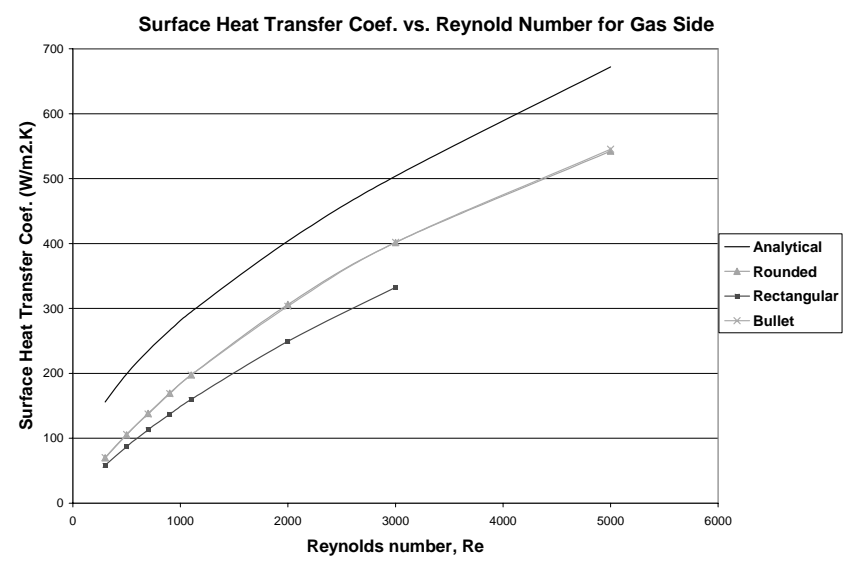

Figure 7: Surface Heat Transfer Coefficients vs. Reynolds Number for comparison among empirical correlation, rectangular, rounded and bullet fin edge (gas side)

From the simulation results, it is noticed that the surface heat transfer coefficient of curve (rounded and bullet) fin edges are higher than rectangular fin edges. The increasing percentage between curve and rectangular fin edges are about $30 \%$. For curve fin edges, the heat transfer performance for rounded and bullet fin edges in terms of surface heat transfer coefficient are almost the similar. The difference between these two curve shapes is not exceeding more than $5 \%$. If we compare the results between the analytical correlation and simulation for rectangular fin edges, the surface heat transfer coefficient for analytical correlation is very high compared with rectangular fin edges. The differences are probably due to two main reasons. The first reason is the boundary conditions that were set in FLUENT simulation. The second reason for the differences is probably to the size chosen for the offset strip fin. The boundary conditions are simplified in order to fit into the FLUENT simulation with certain assumptions. For the second reason, the simulation only can be done on a module of offset strip fins due to the limitation of computer speed.

Although the surface heat transfer coefficient for rounded and bullet fin edges is quite similar but the Fanning friction factor among this two fin edges is difference (Figure 4). The Friction factor for bullet fin edges is slightly lower than rounded fin edges which results in lower pressure loss. In Figure 4, we notice that friction factor between rectangular and rounded fin edges are almost the same. According to the results from the simulation, the friction factor for rounded fin edges is slightly lower than rectangular fin edges in the range of Reynolds number 300-900. After the Reynolds number reached above 900, the friction factor for rounded fin edges is become slightly higher than rectangular fin edges. In Figure 4 and 5, comparison between analytical correlation and the results for rectangular fin edges shows a crossing between the two curves. In the range of Reynolds number 300-1100, the friction factor for rectangular fin edges is higher than analytical correlation while the friction factor for rectangular fin edges is lower than analytical correlation. This situation might be due to the same reason which explained previously. In FLUENT simulation, rectangular fin edges can are be computed until Reynolds Number 3000. For the Reynolds Number above 3000, the results using laminar viscous model diverges.

The same characteristics are shown for the gas side. The surface heat transfer coefficient of curve (rounded and bullet) fin edges are higher than rectangular fin edges (Figure 7). The increasing percentage between curve and rectangular fin edges are up to bout $20 \%$. For curve fin edges, the heat transfer performance for rounded and bullet fin edges in terms of surface heat transfer coefficient are almost the similar. The difference between these two curves is not exceeding more than $1 \%$. Comparing the results between the analytical correlation and simulation for rectangular fin edges, the surface heat transfer coefficient for analytical correlation is very high. The surface heat transfer coefficient for rounded and bullet fin edge is quite similar for gas side but the Fanning friction factor between this two fin edges is not the same (Figure 5). The Friction factor for bullet fin edges is slight lower than rounded fin edges which mean that the pressure loss is lower. In is noticed that the friction factor for rectangular fin edge is similar to rounded fin edge. According to the results from the simulation, the friction factor for rounded fin edges is slightly lower than rectangular fin edges in the range of Reynolds number (300-900). The friction factor for rounded fin edges is slightly higher than rectangular fin edges after the Reynolds number of 900 .

\section{CONClusions}

As a conclusion, the simulation study that estimate the performance of the recuperator for $100 \mathrm{~kW}$ micro turbines with three different fin edges namely rectangular, rounded and bullet fin edges shows promising results. In short, we can conclude that the heat transfer performance for curve fin edges (rounded and bullet) is much better than rectangular fin edges. While in order to reduce the pressure loss, the use of bullet fin edges is recommended.

\section{REFERENCES}

[1] N. Takao, T. Rinzo, K. Shoichi and I. Yutaka, Conceptual Design of Recuperator for Ultramicro Gas Turbine, IGTC, Tokyo OS$102,2003$.

[2] C.F. McDonald, Recuperator Considerations for Future Higher Efficiency Microturbines, Journal of Applied Thermal Engineering, 23, pp. 1463-1487, 2003.

[3] O. O. Omatete, P.J. Maziasz and D.P. Stinton Assesment of Recuperator Materials for Microturbines Oak Ridge, Tennessee: Oak Ridge National Laboratory, 2000.

[4] R.K. Shah and P. S. Dušan , Fundamentals of Heat Exchanger Design, John Wiley \& Sons, Inc, Hoboken, New Jersey, 2003.

[5] B. Thonon, and E. Breuil, Compact Heat Exchangers Technologies for the HTRs Recuperator Application, 1998.

[6] R.M. Manglik, and A.E. Bergles, . Heat Transfer and Pressure Drop Correlations for the Rectangular Offset Strip Fin Compact Heat Exchanger, Experimental Thermal and Fluid Science, 10, pp. 171$180,1995$.

[7] S. Subramanian, "CFD Modeling of Compact Offset Strip-fin High Temperature Heat Exchanger", University of Nevada, Las Vegas, 2003.

[8] S. Subramanian, and R., Akberov,. Development of an Advanced High Temperature Heat Exchanger Design for Hydrogen Production", International Mechanical Engineering Congress and Exposition, University of Nevada, Las Vegas, 2004. 


\title{
Carbon Constrained Energy Planning with Pinch Analysis
}

\author{
Denny K.S. Ng, S.C. Lee, Dominic C.Y. Foo and Raymond R. Tan
}

\begin{abstract}
Due to growing international concern about climate change, the management of carbon dioxide emissions from different human activities has become very important. Many countries now seek to control their carbon dioxide emissions by having proper energy planning. This paper presents an extended graphical targeting approach derived from pinch analysis to locate minimum low-carbon source for energy sector planning. The targeting approach is used to set the energy targets for different sub-sectors in the planning horizon of power generation and transportation. Hypothetical case studies are solved to illustrate the proposed approach.
\end{abstract}

Keywords: carbon emission reduction, energy planning, process integration, pinch analysis.

\section{INTRODUCTION}

There is now a strong consensus in the global community that emissions of greenhouse gases such as carbon dioxide $\left(\mathrm{CO}_{2}\right)$ are causing global warming or climate change. Such changes are projected to cause a rise in average global temperature over the coming decades; at the same time, potential changes in local climatic conditions pose different risks for human communities that rely on predictable weather for their sustenance. To respond to the global warming issue, many countries in the world have ratified the 1997 Kyoto Protocol (UNFCCC, 2007) which is aimed at reducing global emissions of greenhouse gases. Furthermore, preparations are under way for a new international agreement when the Kyoto Protocol expires in 2012. Thus, energy planning is crucial at both national and regional levels. Different planning tools, usually based on optimisation models, are often used by governments to meet emission limits while simultaneously satisfying energy demands of different geographic or economic sectors at

Denny K.S. Ng is $\mathrm{PhD}$ candidate in Chemical Engineering at University of Nottingham Malaysia, Broga Road, 43500 Semenyih, Selangor, Malaysia (phone: +603-89248350; fax: +603-89248017; e-mail: dennynks@yahoo.com).

S.C. Lee undergraduate student in Chemical Engineering at University of Nottingham Malaysia, Broga Road, 43500 Semenyih, Selangor, Malaysia (e-mail: sinc lee@hotmail.com).

Dominic C.Y. Foo is assistnant professor of the School of Chemical and Environmental Engineering of University of Nottingham Malaysia, Broga Road, $43500 \quad$ Semenyih, Selangor, Malaysia (e-mail: Dominic.Foo@nottingham.edu.my).

Raymond R. Tan is associate professor of the Chemical Engineering Department and researcher of the Center for Engineering and Sustainable Development Research at De La Salle University-Manila, 2401 Taft Avenue, 1004 Manila, Philippines (e-mail: tanr_a@dlsu.edu.ph). reasonable costs.

Pinch analysis was developed since 1970s as a systematic technique for the synthesis of heat exchanger network [1][3] for process plants. Analogies between heat and mass transfer in thermodynamic principals have led to the introduction of mass integration, which is well known in the efficient use of industrial resources e.g. solvent, water and utility gases [4]-[16].

Recently, novel applications of pinch analysis in nonconventional areas such as production planning [17], [18], financial management [19], and property-based material recovery network [20]-[23] have been developed and reported in the literature. More recently, Tan and Foo [24] addressed this latter application by utilising the composite curves to locate the minimum amount of zero-carbon energy source during energy planning. However, the work was restricted to cases where mixed zero-carbon energy sources is used to achieve the overall emissions target for a specific country or region, without having to differentiate the various zero-carbon sources. In practice, different energy resources can approach zero net carbon emissions, but their acceptability may depend on other factors such as social acceptability, land or space constraints, and cost. This paper extends the previous works by introducing a revised graphical targeting approach for low-carbon energy source. Furthermore, practical applications of energy planning are introduced in this work. The overall energy planning is broken down into two sub-sectors of power generation and transportation. The proposed approaches are utilised to determine the energy targets for both sub-sectors.

\section{Problem StATEMENT}

The problem definition of a carbon constrained energy planning which is stated as follows:

- $\quad$ A set of energy sources: SOURCES $=\{i \mid i=1,2, \ldots$, $\left.N_{\text {Source }}\right\}$ composed of relatively high carbon content and produce carbon dioxide when the energy is in used. Each source has a available energy, $S_{i}(\mathrm{TJ})$ and is characterised by emission factor, $E F_{i}\left(\mathrm{tCO}_{2} / \mathrm{TJ}\right)$.

- A set of energy demand (region): DEMANDS $=\{j \mid j=$ $\left.1,2, \ldots, N_{\text {Demand }}\right\}$. Energy demands are regions that require the energy sources. Each demand consisted of expected energy consumption, $D_{j}$ (TJ) and the maximum emission limit, $D_{j} E F_{, j}\left(\mathrm{tCO}_{2}\right)$ that can be emitted from the regions.

The emissions limit, (product of $D_{j}$ and $E F_{, j}$ ) of each energy demand must be met by the energy mix supplied to 
it. The objective of this work is to determine the minimum targets for zero and low-carbon energy source while maintain the carbon emission limit.

\section{ENERGy SupPly COMPOSITE CURVE}

Tan and Foo [24] extended the use of material recovery pinch diagram [11], [13], [15] which originally developed for targeting minimum water flowrate for reuse/recycle to locate minimum amount of zero-carbon energy source that is needed to meet the specified emissions limits in energy planning, as shown in 'figure 1 (a)'. The energy supply composite curve is plotted on a cumulative emission limit versus cumulative energy quantity diagram such that the slopes of the segments correspond to the emission factor. The demand and source composite curves are individually plotted in an ascending order of the limiting emission factor. Next, the source composite is moved horizontally until it touches the demand composite with the source composite being below and to the right of the demand composite curve. Minimum zero-carbon energy source and excess carbon-based source are obtained from the overhang of the demand and source composite curves, respectively.

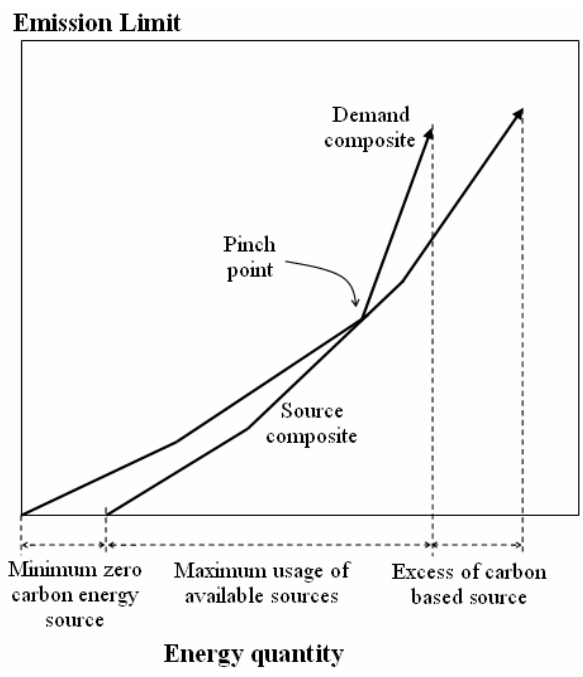

(a)

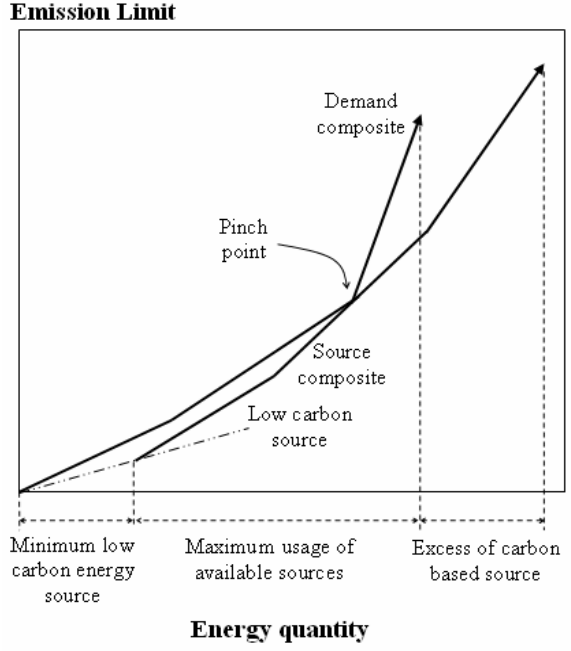

(b)

Figure 1. Energy supply composite curve (a) zero-carbon energy source (b) low-carbon energy source

Note that, in practice, energy sources can only approach carbon neutrality. The method of Tan and Foo [24] assumes that net carbon emissions are so low when compared to fossil energy sources that they can be treated as being approximately zero. A more exact solution can be found by using the true value of carbon intensity; furthermore, such an approach can allow for the differentiation of different low-carbon energy sources from each other. In this work, the composite curve is revised for targeting the minimum low-carbon energy sources ('Figure 1 (b)'). Based on the same concept that used to determine impure fresh water target for water reuse/recycle network [11], [14], [15] a lowcarbon energy source locus is needed which allow the source composite curve slide until it touches the demand composite curve when targeting the low-carbon energy source. The slope of the locus corresponds to the emission factor of the specific low-carbon energy source. The point of contact between both composite curves is known as pinch point. The main purpose of identifying pinch point is to provide valuable insights to allocation of energy sources. A literature example is used to illustrate the proposed approach.

\section{EXAMPLE 1}

'Table 1' shows the data of a hypothetical case study adapted from Tan and Foo [24], where energy is needed to fulfil three regions with five energy sources, i.e. coal, oil, natural gas, biodiesel (treated here as a low-carbon resource) and zero-carbon sources (i.e. hydro, wind, solar and others). As reported by Tan and Foo [24], when only zero-carbon source is used, the minimum energy demand to be fulfilled by the clean source is targeted as $810,000 \mathrm{TJ}$, as shown in the energy supply composite curve in 'figure 2'.

Table 1 Energy limiting data for Example 1 [24]

\begin{tabular}{ccc}
\hline Energy Source & $\begin{array}{c}\text { Emission Factor, } C_{\text {out }, i} \\
\left(\mathrm{t} \mathrm{CO}_{2} / \mathrm{TJ}\right)\end{array}$ & $\begin{array}{c}\text { Available Source, } \\
S_{i}(\mathrm{TJ})\end{array}$ \\
\hline Coal & 105 & 600,000 \\
Oil & 75 & 800,000 \\
Natural gas & 55 & 200,000 \\
Bio-diesel & 16.5 & $>200,000$ \\
Zero-carbon source & 0 & $>200,000$ \\
Total & & $>2,000,000$ \\
\hline
\end{tabular}

\begin{tabular}{cccc}
\hline $\begin{array}{c}\text { Energy } \\
\text { Demand }\end{array}$ & $\begin{array}{c}\text { Expected } \\
\text { Consumption, } \\
D_{j}(\mathrm{TJ})\end{array}$ & $\begin{array}{c}\text { Emission } \\
\text { Limit, } D_{j} C_{\mathrm{in}, j} \\
\left(10^{6} \mathrm{t} \mathrm{CO}_{2}\right)\end{array}$ & $\begin{array}{c}\text { Emission Factor, } \\
C_{\text {out }, j} \\
(\mathrm{t} \mathrm{CO} / \mathrm{TJ})\end{array}$ \\
\hline Region I & $1,000,000$ & 20 & 20 \\
Region II & 400,000 & 20 & 50 \\
Region III & 600,000 & 60 & 100 \\
\hline Total & $2,000,000$ & 100 & \\
\hline
\end{tabular}

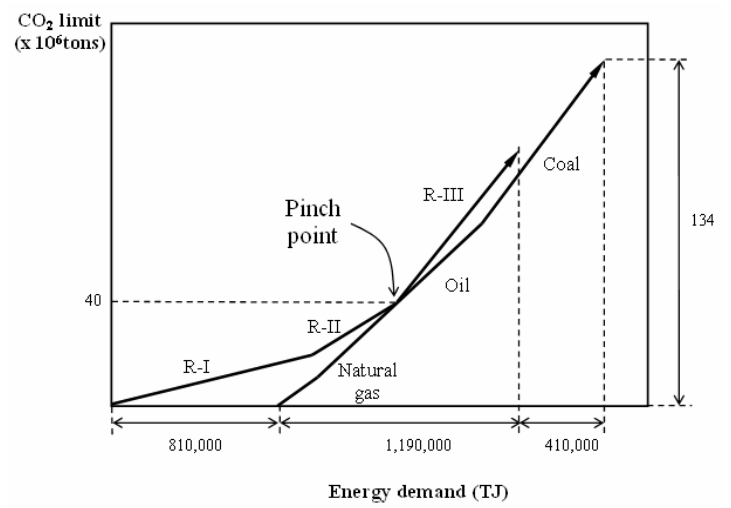

Figure 2. Energy supply composite curve for targeting zerocarbon source [24] 
Since the low-carbon sources are often less expensive than zero-carbon sources, targeting for minimum lowcarbon sources is essential. In this example, biodiesel is chosen as the low-carbon source that used to replace partially or totally the zero-carbon sources. In this case, biodiesel is classified as a low-carbon resource since its production makes use both of biomass (vegetable oil) and fossil fuel (methanol) resources.

The energy supply composite curve is constructed following the above-described procedure, where the demand and source composite curves are plotted individually. Next, a locus of biodiesel with the slope of $16.5 \mathrm{t} \mathrm{CO}_{2} / \mathrm{TJ}$ is plotted in the same graph. The source composite is then slide along the locus until touches the demand composite where a pinch point is formed between the composite curves. Figure 3 shows the energy supply composite curve for this case. As shown, the minimum energy demand to be fulfilled by biodiesel is reported as 1,040,000 TJ, with complete elimination of zero-carbon sources. However, note that the biodiesel demand is higher than the case when zero-carbon sources is used. In addition, the excess of available carbon-based sources (coal) is reported as 640,000 $\mathrm{TJ}$, i.e. an increase of $56 \%$ compared to the case when zerocarbon source is used ('Figure 2'). Both of these phenomenon are due to the use of low-carbon source that limits the use of fossil fuels (i.e. natural gas, oil and coal).

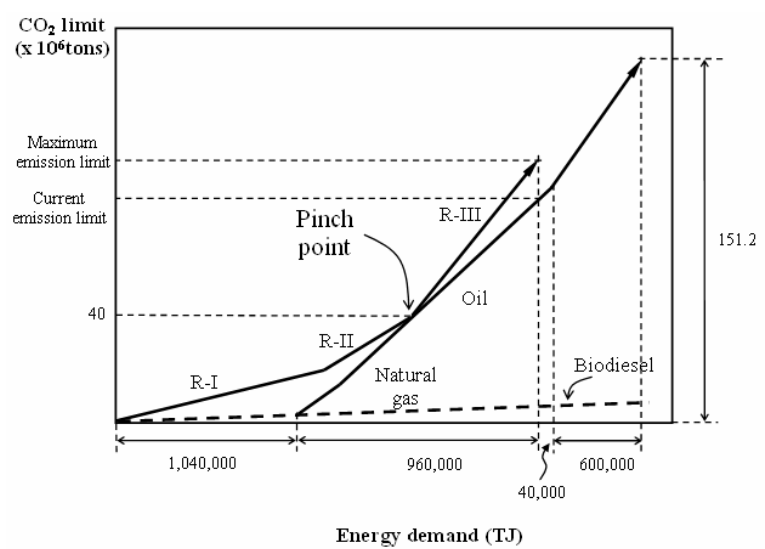

Figure 3. Energy supply composite curve for targeting lowcarbon source

\section{Detail Allocation of Energy Sources}

In this work, a more realistic situation of energy planning is demonstrated where energy demand in each region is further divided into the sub-sectors of power generation (i.e. energy requirement in power plant) and transportation (e. g. buses, cars and trains). The allocation of energy sources into the two sub-sectors is different. The energy sources that may be used in the power generation sector are the conventional sources (e.g. coal, oil and natural gas), as well as the clean energy sources that are categorised as zerocarbon sources, such as renewable energy (e.g. hydropower, solar, wind, etc.) and nuclear energy. The clean energy sources is assumed do not generate greenhouse gases (i.e. carbon dioxide and carbon monoxide) when converted to power. Meanwhile, the conventional fuel power plant with carbon capture technology is categorised as low carbon source that can reduce carbon emissions from the power generation.
On the other hand, different energy sources are used in the transportation sector. Clean sources that may be used in transportation are fuel cell, hydrogen, electricity, etc. which are usually chosen to replace the conventional carbon-based sources in reducing carbon emission. Besides, the lowcarbon energy source such as biodiesel is also available to power vehicles. Biodiesel is considered as a low-carbon source because plants and crops that are used to produce biodiesel will take carbon dioxide from the atmosphere during photosynthesis to balance the carbon cycle.

Although both the zero- and low-carbon energy sources are environmentally attractive, these options are relatively expensive compare with the conventional energy sources. Therefore, conventional carbon-based energy sources should be utilised wisely before considered the clean energy sources or biodiesel in both sub-sectors while maintaining carbon emission limit. Therefore, in this section the energy supply composite curve is utilised to locate the energy supply for both sub-sectors. A hypothetical literature example is solved to illustrate the proposed approach.

\section{EXAMPLE 2}

'Table 2' shows the data for a hypothetical case that is used to illustrate the concept of detailed energy planning. Based on 'table 2', Regions I, II and III are categorised as industrial estate, commercial area, and residential area respectively, with specific energy demand and $\mathrm{CO}_{2}$ emission limits for each region. All regions are further divided into two sub-sectors of power plant and transportation, with the energy demand ratio for the power plant versus transportation given as 8:2, 4:6, and 1:9 respectively. Five energy sources are available for used, i.e. coal, oil, natural gas, biodiesel (zero-carbon source) and zero-carbon source.

Table 2 Detail energy limiting data.

\begin{tabular}{|c|c|c|c|c|c|}
\hline \multicolumn{3}{|c|}{ Energy Source } & \multicolumn{2}{|c|}{$\begin{array}{c}\text { Emission Factor, } C_{\text {out, }} \\
i \\
\left(\mathrm{t} \mathrm{CO}_{2} / \mathrm{TJ}\right)\end{array}$} & $\begin{array}{l}\text { ilable Source, } S_{i} \\
\text { (TJ) }\end{array}$ \\
\hline \multicolumn{2}{|r|}{ Coal } & \multicolumn{3}{|c|}{105} & $1,416,200$ \\
\hline \multicolumn{2}{|r|}{ Oil } & \multicolumn{3}{|c|}{75} & 954,000 \\
\hline \multicolumn{2}{|r|}{ Natural gas } & \multicolumn{3}{|c|}{55} & 629,800 \\
\hline & Biodiesel & \multicolumn{3}{|c|}{16.5} & $>0$ \\
\hline \multicolumn{3}{|c|}{ Zero-carbon source } & \multicolumn{2}{|l|}{0} & $>0$ \\
\hline \multicolumn{3}{|c|}{ Total } & & & $>3,000,000$ \\
\hline \multirow[t]{2}{*}{$\begin{array}{l}\text { Energy } \\
\text { Demand }\end{array}$} & \multicolumn{3}{|c|}{ Expected Consumption, $D_{j}\left(10^{6} \mathrm{TJ}\right)$} & $\begin{array}{c}\text { Limit, } D_{j} C_{\mathrm{in}, j} \\
\left(10^{6}+\mathrm{CO}_{2}\right)\end{array}$ & \multirow[t]{2}{*}{$\begin{array}{c}\text { Emission } \\
\text { Factor, } C_{\text {out }, j} \\
\left(\mathrm{t} \mathrm{CO}_{2} / \mathrm{TJ}\right)\end{array}$} \\
\hline & $\begin{array}{c}\text { Power } \\
\text { Generation }\end{array}$ & Transportation & \multicolumn{2}{|l|}{ Total } & \\
\hline Region I & 1.6 & 0.4 & 2 & 60 & \multirow{2}{*}{$\begin{array}{c}30 \\
37.5\end{array}$} \\
\hline $\begin{array}{l}\text { Region } \\
\text { II }\end{array}$ & 0.32 & 0.48 & 0.8 & 30 & \\
\hline $\begin{array}{c}\text { Region } \\
\text { III } \\
\end{array}$ & 0.02 & 0.18 & 0.2 & 10 & 50 \\
\hline Total & 1.94 & 1.06 & 3 & 100 & \\
\hline
\end{tabular}

In this example, the power generation is assumed to only consume coal, natural gas and zero-carbon energy source in generating power and electricity. Meanwhile, oil, natural gas and biodiesel are used in transportation to power the vehicles. The minimum amount of low- and zero-carbon sources that are needed to meet emissions limits are targeted for each sub-sector.

The energy supply composite curve for power generation is showed in 'figure 4'. As shown, the energy requirement of clean source for power generation is targeted as 
1,109,619 TJ. Besides, it is worth noting that there is an excess of coal $(1,109,619 \mathrm{TJ})$ that cannot be utilised to avoid the violation of $\mathrm{CO}_{2}$ emission constraint.

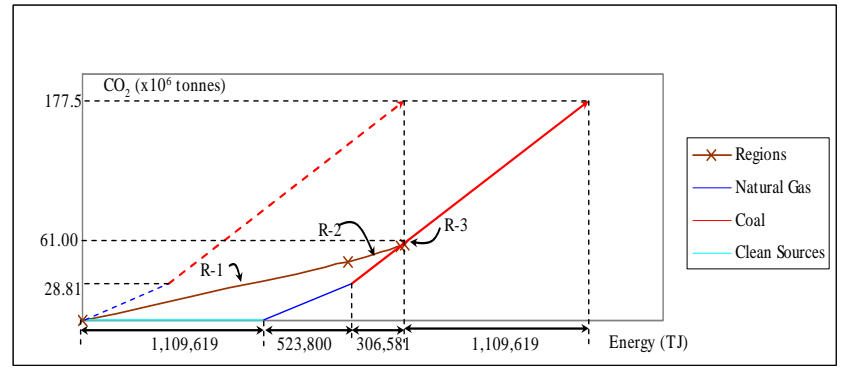

Figure 4. Energy supply composite curve for power generation

However, it is possible to prioritise the use of coal for power generation by having a penalty of additional clean sources, as clean sources are required to compensate the increased emissions that generated from the coal combustion due to its higher emission factor.

On the other hand, the energy supply target for transportation sector is showed in 'figure 5'. In the transportation sector, it is assume that the government policy for maximum allowable usage of natural gas in the transportation sector is set as $10 \%$ out of the total available energy that is generated from oil. Besides, as mentioned earlier, biodiesel may be used as low-carbon source in this case. Based on Figure 5, the overall minimum biodiesel (low-carbon source) that required to fulfil the transportation energy demand while observing the $\mathrm{CO}_{2}$ emission limit is targeted as 656,068 TJ. Similar to the case of power generation, the pinch point for this case is also located at the last point of the demand composite curve.

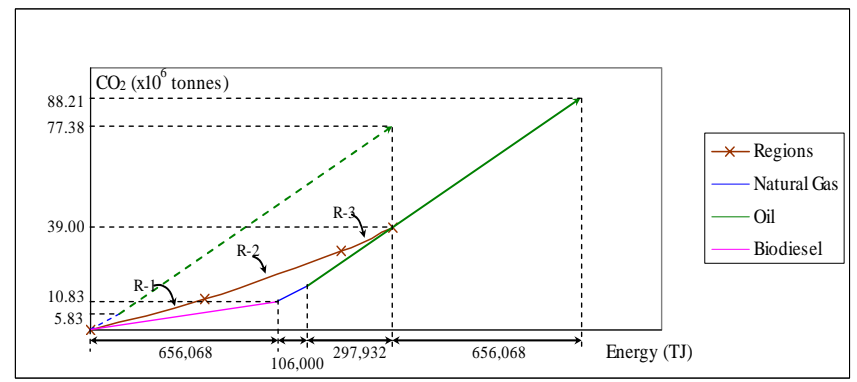

Figure 5. Energy supply composite curve for transportation

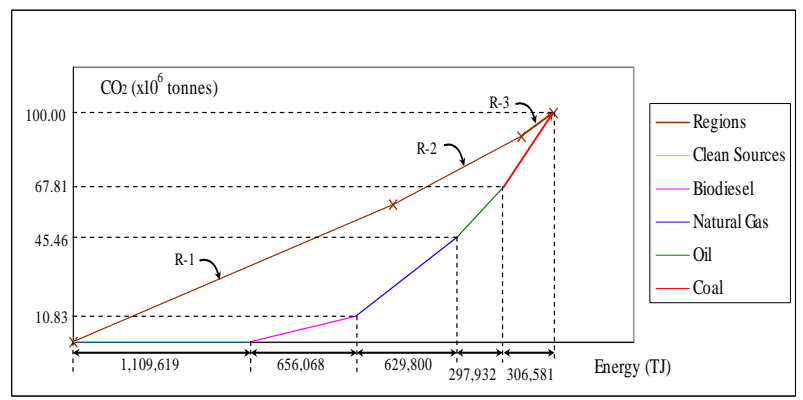

Figure 6. Energy supply composite curve for overall targeting

After segregate targeting is carried out for power generation and transportation sub-sectors respectively, an overall representation is shown in 'figure 6'. Minimum low and zero-carbon sources are targeted to fulfil the overall energy demand; while the $\mathrm{CO}_{2}$ emission limit is observed.

\section{CONCLUSION}

An extended graphical approach for carbon constraint energy planning has been developed based on the previous work of Tan and Foo [24]. In the cases where the energy demands for each region has been divided into power generation and transportation sector, the segregate targeting for minimum energy sources is performed. In addition, zero-carbon energy sources and low-carbon energy sources are separated into different categories and used in different sub-sectors resulting in a more practical approach in utilizing energy source based on their uses.

\section{REFERENCES}

[1] B. Linnhoff, D. W. Townsend, D. Boland, G. F. Hewitt, B. E. A. Thomas, A. R. Guy and R. H. Marshall, "A User Guide on Process Integration for the Efficient Use of Energy,” Rugby: Institute of Chemical Engineers, 1982.

[2] R. Smith, Chemical Process Design. New York: McGraw Hill, 1995.

[3] R. Smith, Chemical Process: Design and Integration. John Wiley \& Sons, Inc, 2005.

[4] M. M. El-Halwagi and V. Manousiothakis, "Synthesis of MassExchange Networks,” AIChE J., vol 35, no. 8, pp. 1233-1244, 1989.

[5] Y. P. Wang and R. Smith, "Wastewater Minimisation," Chem. Eng. Sc., vol 49, no. 7, 981-1006, 1994.

[6] G. P. Towler, R. Mann, A. J-L. Serriere, C. M. D.Gabaude, "Refinery Hydrogen Management: Cost Analysis of Chemically Integrated Facilities,” Ind. Eng. Chem. Res., vol 35, pp. 2378 - 2388, 1996.

[7] M. M. El-Halwagi, Pollution Prevention through Process Integration: Systematic Design Tools, CA: Academic Press, 1997.

[8] N. Hallale and F. Lui, "Refinery Hydrogen Management for Clean Fuels Production,” Adv. Env. Res., vol 6, pp. 81-89, 2001.

[9] J. J. Alves and G. P. Towler, "Analysis of Refinery Hydrogen Distribution Systems,” Ind. Eng. Chem. Res., vol 41, pp. 5759 5769, 2002.

[10] N. Hallale, "A New Graphical Targeting Method for Water Minimisation,” Adv. Env. Res., vol 6, no. 3, pp. 377-390, 2002.

[11] M. M. El-Halwagi, F. Gabriel and D. Harell, "Rigorous Graphical Targeting for Resource Conservation via Material Recycle/Reuse Networks,” Ind. Eng. Chem. Res., vol 42, pp. 4319-4328, 2003.

[12] Z. A. Manan, Y. L. Tan and D. C. Y. Foo, "Targeting the Minimum Water Flowrate Using Water Cascade Analysis Technique,” AIChE J, vol 50, no. 12, pp. 3169-3183, 2004.

[13] R. Prakash and U. V. Shenoy, "Targeting and Design of Water Networks for Fixed Flowrate and Fixed Contaminant Load Operations," Chem. Eng. Sci.,vol 60, no. 1, pp. 255-268, 2005.

[14] A. M. Almutlaq, V. Kazantzi and M. M. El-Halwagi, “An Algebraic Approach to Targeting Waste Discharge and Impure Fresh Usage Via Material Recycle/Reuse Networks,” Clean Tech Environ Policy, vol 7, pp. 294-305, 2005.

[15] El-Halwagi, M. M., Process Integration, Amsterdam: Elsevier Inc., 2006.

[16] D. K. S. Ng, D. C. Y. Foo, Y. L. Tan and R. R. Tan, "Ultimate Flowrate Targeting With Regeneration Placement," Trans IChemE (Part A), vol 85, no. A9, pp. 1253-1267, 2007.

[17] A. Singhvi and U. V. Shenoy, “Aggregate Planning In Supply Chains by Pinch Analysis,” Trans. IChemE (Part A), vol 80, pp. 597 - 605, 2002.

[18] A. Singhvi, K. P. Madhavan and U. V. Shenoy, "Pinch Analysis for Aggregated Production Planning in Supply Chains,” Comp. Chem. Eng., vol 28, pp. 993-999, 2004.

[19] T. K. Zhelev, "On the Integrated Management of Industrial Resources Incorporating Finances,” J. Cleaner Prod., vol 13, pp. 469 - 474, 2005.

[20] M. D. Shelley and M. M. El-Halwagi, "Componentless Design of recovery and allocation systems: a functionality-based clustering approach,” Comp. Chem. Eng., vol 24, pp. 2081-2091, 2000.

[21] X. Qin, F. Gabriel, D. Harell and M. M. El-Halwagi, "Algebraic Techniques for Property Integration via Componentless Design,” Ind. Eng. Chem. Res., vol 43, pp. 3792-3798, 2004. 
[22] V. Kazantzi and M. M. El-Halwagi, "Targeting Material Reuse via Property Integration,” Chem. Eng. Prog., vol 101, no. 8, pp. 28-37, 2005.

[23] D. C. Y. Foo, V. Kazantzi, M. M. El-Halwagi, Z. A. Manan, "Surplus Diagram and Cascade Analysis Technique for Targeting Propertybased Material Reuse Network," Chem. Eng. Sc., vol 61, pp. 26262642, 2006.

[24] R. R. Tan and D. C. Y. Foo, "Pinch Analysis Approach to CarbonConstrained Energy Sector Planning,” Energy, vol 32, no.8, pp. 14221429, 2007. 


\title{
High Reynolds Number Flow past a Two- Dimensional Obstacle
}

\author{
J. Labadin and A.R.H. Rigit
}

\begin{abstract}
This paper produced a preliminary work in the investigations of fluid flow around three-dimensional objects. The focus of the paper is on a two-dimensional object placed within a boundary layer flow. This problem was given tremendous attention due to its application in the studies of wind around tall buildings, hills, terrains as well as in the designing of aero plane's wings. The problem is modeled using the Navier-Stokes equations which then led to the boundary layer equations. The equations governing the flow over even a small object are nonlinear in nature and therefore in general a numerical solution is required. This paper presents the linear analysis of the problem to provide a comparison with the numerical solution. Then a numerical method for the nonlinear problem is proposed and the numerical results obtained are discussed. The results are presented following three cases of two-dimensional obstacles. It was observed that in order for results to be obtained without any difficulties, a restriction on the size of the obstacle must be established.
\end{abstract}

Keywords: boundary layer flows, separation, nonlinear problem

\section{INTRODUCTION}

The problem that considers high Reynolds number flow past a two dimensional object is know as the hump problem. This problem was one of the first problems to which tripledeck theory was applied ([1],[2],[3],[4]). Until today the study of this problem is still on-going due to the its immense application. The work on this paper is strongly related to the Smith \& Walton [5] paper who considered flow past steep hump. They found that the equations governing the flow over even a small hump are nonlinear in nature and therefore in general a numerical solution is required.

We first present the formulation of the governing equation and then presented the linear analysis of the problem, valid for very small hump heights. The corresponding analytic solution provides a comparison with the numerical solution required for larger hump. A numerical method for the nonlinear problem is proposed and the numerical results obtained for three different cases are discussed.

J. Labadin is with the Department of Computational Science and Mathematics, Faculty of Computer Science and Information Technology, Universiti Malaysia Sarawak (phone: +60-82583775; fax: +60-82583764; e-mail: ljane@fit.unimas.my).

A. R. H. Rigit is with the Department of Mechanical Engineering, Faculty of Engineering, Universiti Malaysia Sarawak.(e-mail: arigit@feng.unimas.my).

\section{FORMULATION OF THE GOVERNING EQUATIONS}

A small two-dimensional object of height $h$ and length $l$ is immersed in a boundary layer flow as shown in the schematic diagram figure 1 .

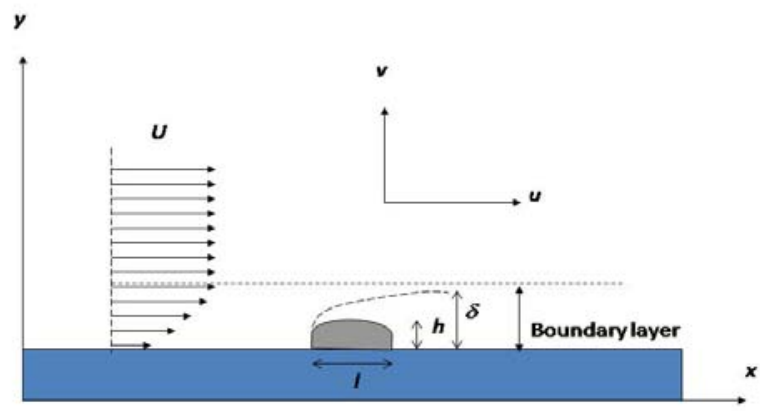

Figure 1. A two-dimensional hump within a boundary layer.

The axis lies parallel to the direction of the flow. The flow is taken to be laminar and steady and the global Reynolds number $R=\frac{U_{D \infty} L_{D}}{v_{D}}$ is assumed to be large, where $L_{D}$ is the typical development length of the incident planar boundary layer present, $U_{D \infty}$ is a representative streamwise speed outside the boundary layer and $v_{D}$ is the kinematic viscosity of the incompressible fluid of density $\rho_{D}$ with $D$ denoting dimensional quantities. The Cartesian coordinates used are $L_{D}(x, y, z)$ streamwise, normal and spanwise, respectively with corresponding velocity components $U_{D \infty}(u, v, w)$. The incident boundary layer has a characteristic thickness of order $R^{-1 / 2}$ in $y$. The hump considered is buried well within the incident boundary layer, thus $h$ is small compared with $O\left(R^{-1 / 2}\right)$. The velocity profile is undisturbed until it reaches the hump, at which point the flow develops a viscous sublayer of thickness $\delta$ on the surface of the hump. The scaled sublayer coordinate near the wall is transformed to $\delta \hat{y}=R^{-1 / 2} y$. The velocity profile near the wall is proportional to $y$ so that $\hat{u} \sim R^{1 / 2} \delta$. Substituting these into the Navier-Stokes equation requires the continuity and momentum balances and leads to the thickness of the sublayer to be $\delta \sim R^{-1 / 2} l^{1 / 3}$. The hump size is sufficiently small that no significant pressure-displacement feedback takes place with the uniform flow outside the boundary layer. This means that the sublayer needs to be kept long and thin so that $\delta \sim R^{-1 / 2} l^{1 / 3}<<l \Rightarrow l>>R^{-3 / 4}$. In this limit the 
streamwise viscous second derivative terms in the momentum equations are small and hence can be neglected. Also, the dimensions are small compared with the tripledeck dimensions which control one form of pressuredisplacement feedback. Thus, the range of concern is $h \sim R^{-1 / 2} l^{1 / 3}$ with $R^{-3 / 4}<<l<R^{-3 / 8}$.

As mentioned, the governing equations for the fluid flow are developed from the Navier-Stokes equations. Since the problem is two-dimensional then the momentum equation in the spanwise direction need not be considered. Implied by the region occupied by the hump, the equations can be rescaled by writing:

$x=l X ; \quad y=R^{-1 / 2} l^{1 / 3} Y ; \quad u=l^{1 / 3} U ; \quad v=R^{-1 / 2} l^{-1 / 3} V ; \quad p=l^{2 / 3} P$ which then leads to the boundary layer equations:

$U_{X}+V_{Y}=0$,
$U_{X}+V U_{Y}=-P^{\prime}(X)+U_{Y Y}$.

This system of equations is subject to the no-slip condition on the hump $U=V=0$ on $Y=h F(X)$ where $F(X)$ is the shape of the hump. The condition to match the outer field is $U \sim Y+o(1)$ as $Y \rightarrow \infty$, in which the $o(1)$ indicates effectively zero displacement. For convenience, the Prandtl shift $\bar{V}=V-h F^{\prime}(X) U, \bar{Y}=Y-h F(X)$ is applied to the governing equations (1) and under this transformation the equations are unaltered, except $V$ now becomes $\bar{V}$ and $Y$ is replaced by $\bar{Y}$. However, the no-slip condition becomes

$$
U=\bar{V}=0 \quad \text { on } \quad \bar{Y}=0
$$

and the outer field condition is

$$
U \sim \bar{Y}+h F(X) \quad \text { as } \quad \bar{Y} \rightarrow \infty
$$

where $F(X)$ is assumed to be zero for $X<0$ and $X>1$. In addition, there is a condition on the shape of the upstream flow specified by the nature of the main boundary layer flow at points upstream of the sublayer. Since the main boundary layer flow exhibits a shear profile near the surface then a suitable initial condition is

$$
(U, \bar{V})=\left(\lambda_{B} \bar{Y}, 0\right) \quad \text { at } \quad X=0,
$$

where $\lambda_{B}$ is a constant that can be removed by another rescaling.

The system of equations (1)-(5) governs the hump problem and they are clearly nonlinear. As is the case with most nonlinear equations, they cannot be solved analytically, and the best way to deal with them for $O(1)$ values of $h$ is by numerical solution. The linear analysis which is valid for $h<<1$, was initially done by Smith \& Walton [5]. The next section the outcome of the linear analysis and the corresponding analysis will be used for validation of the numerical results.

\section{LINEAR ANALYSIS}

The system of equations (1)-(5) is linearized resulting in a system which can be solved analytically using Fourier transforms. From the analysis, the unknown pressure $P$ and the scaled skin friction $\tau=\partial U / \partial \bar{Y}$ can be obtained. Valid for small $h$, these properties are found to be

$$
\begin{gathered}
\tau=\frac{3^{1 / 3}}{\Gamma^{2}\left(\frac{2}{3}\right)} \int_{0}^{X} \frac{F^{\prime}(t)}{(X-t)^{1 / 3}} d t, \\
P=\frac{3^{2 / 3}}{\Gamma^{2}\left(\frac{1}{3}\right)} \int_{0}^{X} \frac{F(t)}{(X-t)^{2 / 3}} d t .
\end{gathered}
$$

Both integrals are valid for $0<X<1$ where $F(X)$ is nonzero. For fluid flow past an obstacle, we expect a region of vorticity downstream of the obstacle which is commonly known as the wake. Therefore, [5] has investigated theoretically how the pressure and the skin-friction are affected when the obstacle gets steeper. They introduced the parameter $\Delta$ as the effective edge lengthscale on the rear face of the obstacle so that the corresponding slope of the rear face is $h / \Delta$. It was found that the critical edge steepness at which nonlinear effects become important is

$$
\Delta \sim h^{3} \text {. }
$$

\section{NumericAl Procedures}

Equation (2) is a parabolic type of partial differential equation and one way to solve it is by using finite difference method. A fully implicit discretization scheme is used for all the terms in the equation and each term is centred at $(n+1, j)$ where the notation $u_{j}^{n}=u(n \Delta x, j \Delta y)$ is adopted. Here the $\Delta x$, and $\Delta y$ are the respective spacings in $x$ and $y$ directions. Thus, the finite difference representation of equation (2) is as follows:

$$
\begin{aligned}
& u\left[\frac{u_{j}^{n+1}-u_{j}^{n}}{\Delta x}\right]+v\left[\frac{u_{j+1}^{n+1}-u_{j-1}^{n+1}}{2 \Delta y}\right] \\
& =-p_{x}+\frac{u_{j+1}^{n+1}-2 u_{j}^{n+1}+u_{j-1}^{n+1}}{\Delta y^{2}}
\end{aligned}
$$

Clearly, the solution will be second order accurate in $y$ and first order accurate in $x$. Second order accuracy in $x$ proves to be difficult because of the existence of a discontinuity in $F^{\prime}(x)$ where $F$ is the shape of the obstacle. The pressure gradient $p_{x}$ is evaluated at the current $x$ location $(n+1, j)$. Rearranging the difference equation above gives the following tridiagonal system of equations associated with the grid line $n+1$ across the boundary layer:

$$
a_{j} u_{j-1}^{n+1}+b_{j} u_{j}^{n+1}+c_{j} u_{j+1}^{n+1}=d_{j},
$$

where

$$
\begin{aligned}
& a_{j}=-\frac{1}{2} v \frac{\Delta x}{\Delta y}-\frac{\Delta x}{\Delta y^{2}}, b_{j}=u+\frac{\Delta x}{\Delta y^{2}} \\
& c_{j}=\frac{1}{2} v \frac{\Delta x}{\Delta y}-\frac{\Delta x}{\Delta y^{2}}, d_{j}=-\Delta x p_{x}+u\left(u_{j}^{n}\right) .
\end{aligned}
$$

To incorporate the no-slip condition (3) and the outer matching condition (4), equation (8) is not applied at $j=1$ (i.e. at $y=0$ ) or $j=\operatorname{JMAX}$ (i.e. at $y=y_{\max }$ ). An equivalent expression for the condition (4) is $U_{Y}=1$ at $Y=Y_{\mathrm{MAX}}$. This can be discretized using a 3-point approximation so that the 
equation formed at $j=$ JMAX-1 is $u_{\mathrm{JMAX}}=4 u_{j}^{n+1}-2 \Delta y-3 u_{j-1}^{n+1}$. Therefore $a j, b j, d j$ in (8) are replaced by $a_{j}-3 c_{j}, b_{j}+4 c_{j}, d_{j}+2 \Delta y c_{j}$ respectively and $c_{j}$ is subsequently set equal to zero. Equation (8) can be solved efficiently using the Thomas algorithm. Once $u_{j}^{n+1}$ is available, $v_{j}^{n+1}$ can then be obtained from (1) in the discretized form

$$
v_{j}^{n+1}=v_{j-1}^{n+1}-\frac{1}{2} \frac{\Delta y}{\Delta x}\left[\left(u_{j}^{n+1}-u_{j}^{n}\right)+\left(u_{j-1}^{n+1}-u_{j-1}^{n}\right)\right],
$$

with $v_{1}^{n+1}=0$. The undifferentiated velocity components, $u$ and $v$, appearing on the left-hand side of (7) are handled using a simple trick. At the start of the iteration in this scheme $u=u_{j}^{k}=u_{j}^{n}$ and $v=v_{j}^{k}=v_{j}^{n}$ for each value of $j$. After the solutions of (8) and (9) are obtained, the current iterative solutions are used then (8) and (9) are resolved. The iteration is terminated when $\left|u_{j}^{k+1}-u_{j}^{k}\right|<\varepsilon$ to some acceptable tolerance $\varepsilon$ and $u_{j}^{n+1}, v_{j}^{n+1}$ are then set to $u_{j}^{k+1}, v_{j}^{k+1}$.

The solution for the pressure is obtained by applying $y \rightarrow \infty$ to (2) using (4) so that $u_{x} \rightarrow h F^{\prime}(x)$. This means that (1) leads to $v_{y} \approx-h F^{\prime}(x) \Rightarrow v \approx-h F^{\prime}(x) y$.

\section{NUMERICAL RESUltS}

The shape of the obstacle considered here is $h F(X)=\left\{\begin{array}{c}h \operatorname{Tanh}\left(\frac{X}{\Delta}\right) \operatorname{Tanh}\left(\frac{1-X}{\Delta}\right), 0 \leq X \leq 1 \\ 0, \quad \text { otherwise }\end{array}\right.$

where $\Delta$ and $h$ are the parameters controlling the edge steepness and height of the obstacle. Clearly, the maximum height of the obstacle is $H=h F(0.5)$.

\section{A. Varying $h$ as $\Delta$ is kept constant}

Here the distribution of the skin-friction, $\tau$, as $h$ increases and $\Delta$ is kept constant (in this case $\Delta=1.0$ ) is considered. The result obtained is shown in Figure 2. When $h>7.0$, the velocity fails to converge even before the flow separates (i.e. when $\tau=0$ ). It is shown here that as $h$ increases the maximum value of $\tau$ is increasing as well and the minimum decreases accordingly until the flow separates. Here the critical height $h_{c}$ for separation is in the range $6.0<h_{c}<6.5$ so that $1.28<H<1.40$.

\section{B. Varying $\Delta$ as $h$ is kept constant}

In this case, $h$ is kept at 0.5 and the critical value of $\Delta$ for separation is found. Figure 3 shows the distribution of the skin-friction, $\tau$. The result shows that when $\Delta$ is relatively large that is $0.1<\Delta<1.0, \tau$ reaches its maximum almost at the top of the obstacle, slowly decreases to its minimum and gradually increases to the value $\sim 1.0$.

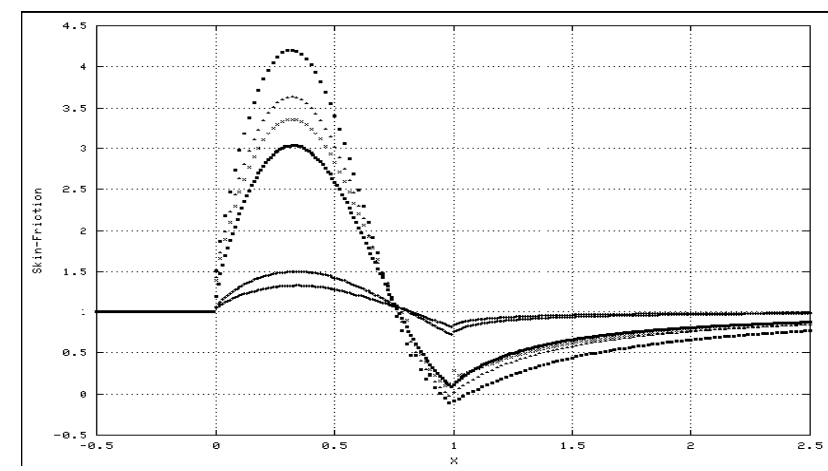

Figure 2. The distribution of the skin-friction keeping $\Delta$ constant $(\Delta=1.0)$ and $h$ increasing $(h=1.0,2.0,5.0,5.5$, 6.0 , and 7.0)

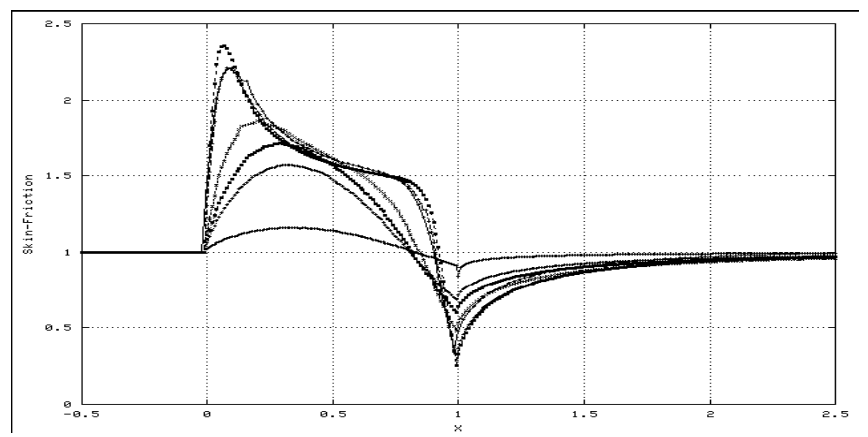

Figure 3. $h$ is kept constant at 0.5 and $\Delta$ is decreasing. Clearly, the shape of the graph changes as $\Delta$ decreases $(\Delta$ $=1.0,0.4,0.3,0.2,0.1,0.05)$

However, when $\Delta$ is reasonably small (i.e. $0.01<\Delta \leq$ $0.1), \tau$ has its maximum at the front-face of the obstacle and finally gradually decreases where the gradient is approximately -1.0. As the flow comes to the rear edge the skin-friction rapidly drops to its minimum and again gradually increases. Thus, when $\Delta$ is small, the front- or rear- face flow reacts to the local shape of the obstacle and hence becomes distinct from the rest of the motion. If the ranges of $\Delta$ above are considered and plot the corresponding obstacle shape accordingly, there are actually three different general shapes of the obstacle.

\section{Smooth edges and rounded top}

The obstacle has smooth edges and rounded top if the range of $\Delta$ is $0.1<\Delta<1.0$. Figure 4 shows the distribution of the skin-friction as $h$ increases and $\Delta$ is kept within the given range (in this case $\Delta=0.6$ ). When $h>3.0$, the velocity fails to converge near the separation point. The critical height at which the flow will experience separation is when $H>1.02$. This means that when the rear face slope if the obstacle is large (i.e. $H / \Delta>1.0$ ), nonlinear effects is felt.

\section{Smooth edges and flat top}

When $\Delta$ is within the range $0.01<\Delta \leq 0.1$, the obstacle has smooth edges and flat top. It is important to note that within this range, $h$ is the maximum height since $F(0.5)=1$ in this range. Figure 5 presents some of the results obtained with $\Delta=0.1$. Initially, the numerical method failed when $h$ $=0.6$. However, when the tolerance is increased from $1 \mathrm{x}$ $10^{-8}$ to $1 \times 10^{-7}$, results were obtained up to $h=0.9$. When $h$ $=1.0$ at $x=0.94$, the value of the skin-friction suddenly shoots up to a very large number $(\approx 460.8)$ from a minimum value of 0.642 at $x=0.92$ showing that there is a grid 
resolution problem. Using a smaller grid (in this case $\delta x=$ 0.01 ) removed the previous discontinuity but the velocity failed to converged at $x=0.98$. The minimum value obtained was $\tau=0.0722$ at $x=0.96$. it is obvious that as the flow reaches the point of separation, adjustment to the program is needed to make sure the velocity converges.

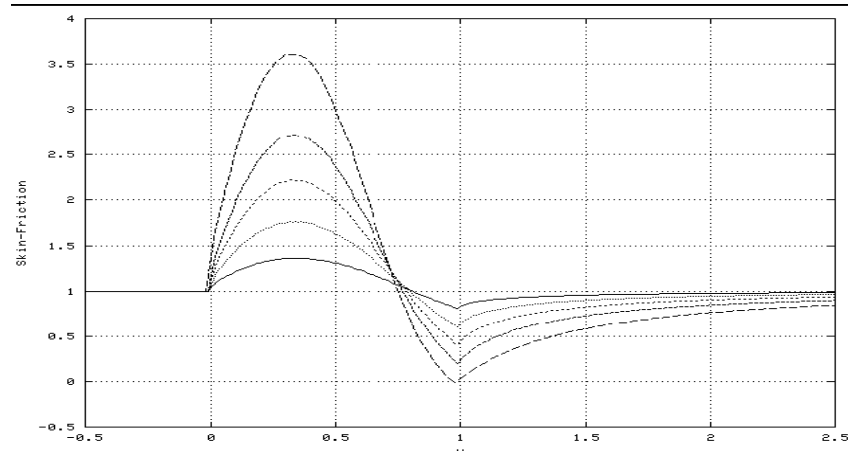

Figure 4. The distribution of the skin-friction as $h$ increases, with $h=0.5,1.0,1.5,2.0,2.75$, and $\Delta=0.6$

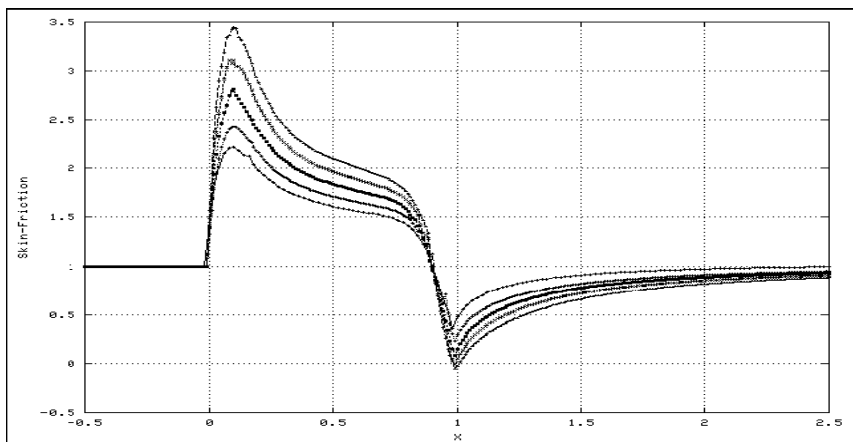

Figure 5. The distribution of the skin-friction as $h$ increases $(h=0.5,0.6,0.7,0.8,0.9)$ and $\Delta=0.1$

\section{E. Steep edges and flat top}

When $\Delta \leq 0.01$, the obstacle has steep edges and a flat top. Unfortunately, the program failed to give any results for this case. Asymptotic analysis can be used to obtain a set of equations independent of $\Delta$ so that results analysis is possible.

From the numerical results presented above, there is obviously relationship between $\Delta$ and $h_{\text {crit. }}$ Table 1 shows some of the results with the corresponding maximum height $H^{c}=h_{\text {crit }} F(0.5)$.

Table 1. Tabulated results from the numerical analysis

\begin{tabular}{|c|c|c|c|}
\hline \multirow{4}{*}{$\begin{array}{l}\text { Smooth edges } \\
\text { and flat top }\end{array}$} & $\Delta$ & $h_{\text {crit }}$ & $H^{c}$ \\
\hline & 0.07 & 0.5 & 0.5 \\
\hline & 0.08 & 0.7 & 0.7 \\
\hline & 0.09 & 0.84 & 0.84 \\
\hline \multirow{10}{*}{$\begin{array}{l}\text { Smooth edges } \\
\text { and rounded top }\end{array}$} & 0.1 & 0.8 & 0.8 \\
\hline & 0.2 & 1.15 & 1.12 \\
\hline & 0.3 & 1.75 & 1.52 \\
\hline & 0.4 & 1.9 & 1.38 \\
\hline & 0.5 & 2.35 & 1.36 \\
\hline & 0.6 & 2.75 & 1.28 \\
\hline & 0.7 & 3.6 & 1.35 \\
\hline & 0.8 & 4.3 & 1.32 \\
\hline & 0.9 & 5.34 & 1.36 \\
\hline & 1.0 & 6.0 & 1.28 \\
\hline
\end{tabular}

Considering the data between $\Delta$ and $h_{\text {crit }}$, a least-square fit as a linear combination of the functions $\Delta$ and $\Delta^{1 / 3}$ can be obtained. The least-square fit for the data between $\Delta$ and $H^{c}$ is found to be $2.3058 \Delta^{1 / 3}-0.9872 \Delta$. Clearly, as $\Delta$ gets small the $\Delta^{1 / 3}$ term is large compared to the $\Delta$ term. Therefore, this suggest that as $\Delta \rightarrow 0, H^{c} \alpha \Delta^{1 / 3}$ which is in agreement with the scaling obtained in (6).

\section{CONCLUSIONS AND FUtURE WORK}

The numerical results have been presented for a full twodimensional obstacle for two (cases (i) and (ii)) of the three cases ((i) smooth edges with rounded top, (ii) smooth edges with flat top, and (iii) steep edges with flat top). It was shown that in order for results to be obtained without any difficulties, there must be a restriction on the size of the obstacle concerned. If $\Delta$ becomes too small (yielding increased face slopes) nonlinear effects become important over a very small lengthscale and the numerical solution of the original equations becomes difficult. By solving the full nonlinear hump problem numerically it was shown that the critical height for separation is related to the edge lengthscale $\Delta$ by $H^{c} \sim \Delta^{1 / 3}$. This is in agreement with the scaling (6) inferred from the solution of the linear hump problem.

Due to the numerical problems mentioned in this paper (especially near the separation point), a better numerical procedure is needed. Dedicated computational fluid dynamics software for example, STAR-CD, COMSOL Multiphysics, etc. may perhaps gives better visualization of the problem. The two-dimensional theory and computation for the hump problem provides a precursor for the study of atmospheric boundary layer from around a tall building, terrain, hill and other similar problem.

\section{REFERENCES}

[1] J. C. R. Hunt. A theory for the laminar wake of a two-dimensional body in a boundary layer. Journal of Fluid Mechanics, 1971, 49:159178.

[2] F. T. Smith. Laminar flow over a small hump on a flat plate. Journal of Fluid Mechanics, 1973, 53:803-824, 1973

[3] F. T. Smith. Flow through constricted or dilated pipes and channels. I, 1976. Quarterly Journal of Mech. Appl. Math., 29:343-364.

[4] F. T. Smith, P. W. M. Brighton, P. S. Jackson, and J. C. R. Hunt. On boundary-layer flow past two-dimensional obstacles. Journal of Fluid Mechanics, 1981, 113:123-152

[5] F. T. Smith and A. G. Walton. Flow past a two- or three-dimensional steep-edged roughness. Proc. Royal Soc. Lond. A., 1998, 454:31-69 


\title{
Single Phase Multilevel Power Inverter
}

\author{
S.M. Bashi, N. Mariun, N.F. Mailah, S. Alhalali
}

\begin{abstract}
This paper deals with conversion of electric energy in form of dc power to an ac power type, which can be connected to the main grid or can be used directly. The out put voltage should be nearly sinusoidal waveform with small distortion or harmonics.

The multilevel voltage source converter typically synthesizes the staircase voltage wave from several levels of dc voltage. This study investigates the performance and discusses advantages and disadvantages of transformer and transformer-less multilevel inverters. In order to generate sinusoidal wave with minimum THD, eliminating harmonic method has been used to calculate conducting angle of each $\mathrm{H}$ bridge. This generates the output wave form waveform with certain voltage and low THD. PIC microcontroller has been used to generate the signals required to operate the system. The proposed circuit was simulated using Pspice / Orcad and the results were compared with the hardware experimental result, and a good agreement has been found.
\end{abstract}

Keywords: harmonic elimination, high voltage inverter, single phase multilevel inverter

\section{INTRODUCTION}

A multilevel inverter converts the electric power from dc to ac form by synthesizing desired sinusoidal voltage from series single-phase, full bridge (H-bridge) inverter units. Separate dc sources are required which may be obtained from batteries, fuel cells, or solar cells [1], or from one dc source synthesizing a combination state of diodes or capacitors, for high power application. Multilevel converter can operate without using a high frequency technique of pulse width modulation (PWM), which has the disadvantage of electromagnetic interface with communication signals and produces high stress on the switches at the high voltage application [2]. Unlike diode clamed and flying-capacitor inverters, Cascade multilevel inverter needs least number of components to achieve the same number of voltage level. Cascade multilevel converters have wide applications especially for High-power Electrical Vehicle (HEV) motor drive because they can convert small dc voltage to high ac voltage. In the recent years so many reports were talking about the disadvantages of the normal inverter especially due to high voltage change rates $\mathrm{dv} / \mathrm{dt}$, which produced a common-mode voltage across the motor winding [3].

High frequency switching can exacerbate the problem because of the numerous times this common-mode voltage

S. M. Bashi, N. Mariun, N. F. Mailah, and S. Alhalali are with the Department of Electrical and Electronic Engineering, Faculty of Engineering, University Putra Malaysia, 43400 Serdang, Selangor, Malaysia. (email: senan@eng.upm.edu.my). is impressed upon the motor each cycle. The main problems reported were "motor bearing failure" and "motor winding insulation breakdown" because of circulating current, dielectric stresses, voltage surge, and corona discharge $[4,5]$. Multilevel inverters overcome these problems since their individual devices has much lower $\mathrm{dv} / \mathrm{dt}$ per switching and they operate at high efficiencies because they can switch on and off at a much lower frequency than PWMcontrolled inverter. The multilevel voltage source inverter is a unique structure that allows them to reach high voltages with low harmonics. For this reason, multilevel inverters can easily provide the high power required for a large electric drives.

\section{A. Cascade Multilevel Inverter}

The cascade H-bridge inverter consists of a number of single phase H-bridge units with or without PWM techniques. Figurel shows the $\mathrm{H}$ - bridge unit and its waveforms, while Figure 2 shows the construction of a single phase cascaded inverter. The main function for each H-bridge is to control the width of its output as shown in Figure 3. Each H-bridge has its conducting angle $(\alpha)$, which means that each $\mathrm{H}$-bridge can produce output waveform with different width.
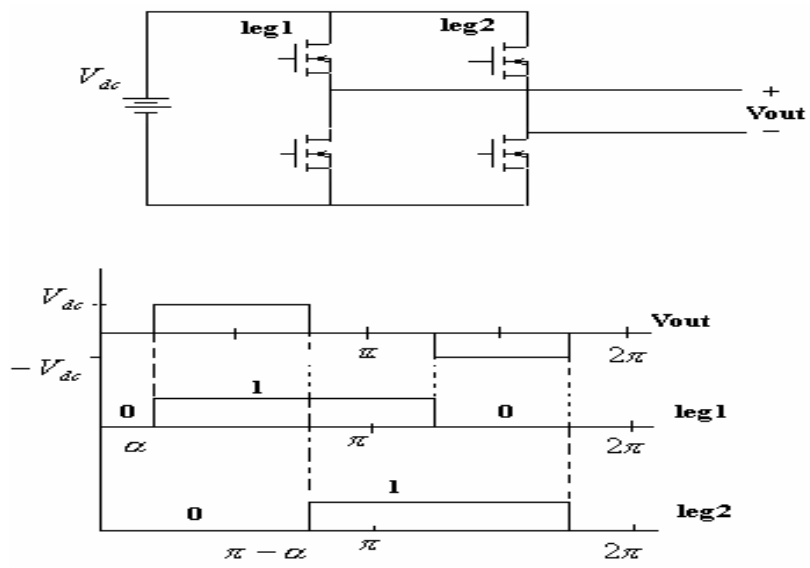

Figure 1. H-bridge unit

The cascade H-bridge multilevel inverter uses a separate dc source for each H-bridge; at any time, the output of each $\mathrm{H}$-bridge has one of three discrete levels; positive, negative or zero, adding them together results in a staircase waveform, $V_{a n}$ as shown in Figure 3. The schematic diagram of transformer type multilevel cascaded inverter is shown in Figure 4. This type usually used in practice to eliminate the high number of dc supplies required to supply each HBridge separately. 


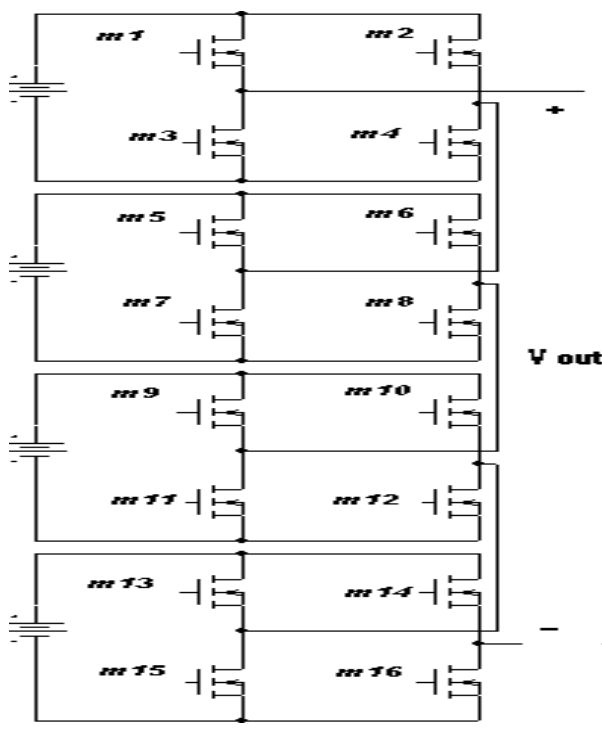

Figure 2. Cascade multilevel circuit

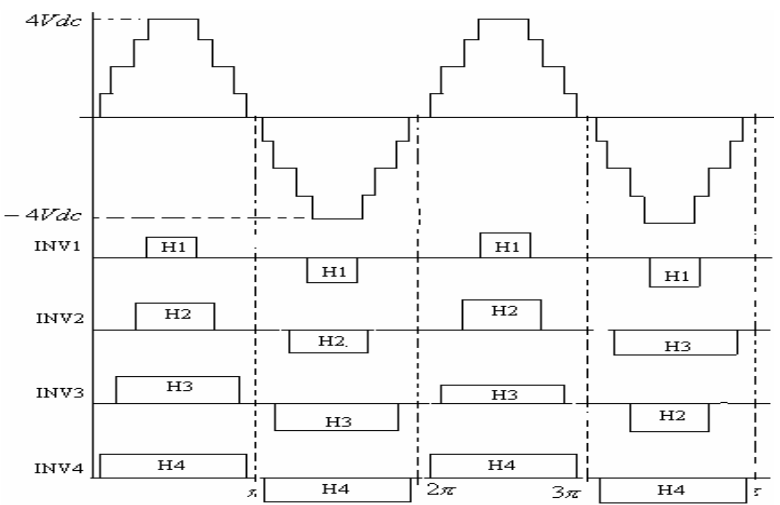

Figure 3. Synthesis of the output voltage

\section{B. Harmonics Eliminations Method}

Choosing the conducting angles of the H-bridges can eliminate a specific harmonic in the output waveform [2]. The required conduction angles can be calculated by analyzing the output phase voltage of cascade inverter assuming four $\mathrm{H}$-Bridges has been chosen, the output voltage $\mathrm{V}_{a 0}$ can be given as:

$\mathrm{V}_{a 0}=\mathrm{V}_{a 1}+\mathrm{V}_{a 2}+\mathrm{V}_{a 3}+\mathrm{V}_{a 4}+\mathrm{V}_{a 5}$

Since the wave is in symmetry along the $\mathrm{x}$-axis, both Fourier coefficient $\mathrm{A}_{0}$ and $\mathrm{A}_{n}$ are zero. Just the analysis of $\mathrm{B}_{n}$ is required.

$\mathrm{B}_{n}=\frac{4 V_{d c}}{n \pi}\left[\sum_{j=1}^{4} \cos \left(n \alpha_{j}\right)\right]$

- $\mathrm{j}$ - number of dc sources

- $\mathrm{n}$ - odd harmonic order

Therefore, to choose the conducting angle of each $\mathrm{H}$ bridge precisely, it is necessary to select the harmonics with a certain amplitude and order, which will be eliminated. To eliminate $5^{\text {th }}, 7^{\text {th }}$, and $11^{\text {th }}$, harmonics and to provide the peak fundamental of the phase voltage equal to $80 \%$ of its maximum value, it needs to solve equation (3), with modulation index $\mathrm{M}=0.8$.

$\cos \left(5 \alpha_{1}\right)+\cos \left(5 \alpha_{2}\right)+\cos \left(5 \alpha_{3}\right)+\cos \left(5 \alpha_{4}\right)=0$

$\cos \left(7 \alpha_{1}\right)+\cos \left(7 \alpha_{2}\right)+\cos \left(7 \alpha_{3}\right)+\cos \left(7 \alpha_{4}\right)=0$

$\cos \left(11 \alpha_{1}\right)+\cos \left(11 \alpha_{2}\right)+\cos \left(11 \alpha_{3}\right)+\cos \left(11 \alpha_{4}\right)=0$

$\cos \left(\alpha_{1}\right)+\cos \left(\alpha_{2}\right)+\cos \left(\alpha_{3}\right)+\cos \left(\alpha_{4}\right)=0.8 * 4$

Solution of this set of nonlinear transcendental equations can be achieved by using numerical methods such as Newton - Raphson method. Table 1 shows a set of conduction angles which produce a minimum harmonics contain, with different modulation indices.

\section{Minimizing Total Harmonic Distortion}

To minimize the THD the following procedure can be followed. The conducting angle to give a certain value of the THD can be determined as follows [5]:

$\operatorname{THD} \%=100 \sqrt{\left(\frac{V(t)_{r m s}}{V_{f}(t)_{r m s}}\right)^{2}}-1$

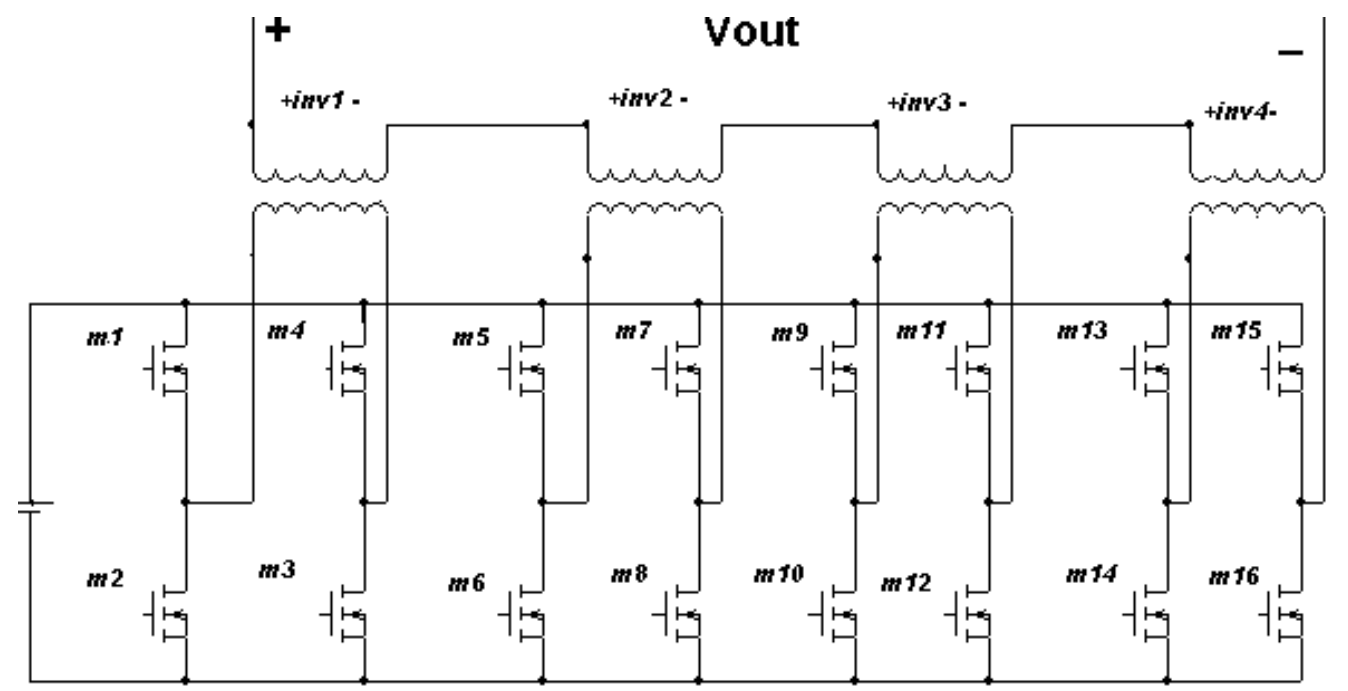

Figure 4. Schematic diagram of transformer multilevel cascaded inverter 
Taking the partial derivative of the THD,

$\frac{\partial T H D^{2}}{\partial \alpha_{j}}=0$

the conducting angle for minimum value of THD can be obtained.

Table1. Sets of the conducting angles with different modulation indices.

\begin{tabular}{|c|c|c|c|c|}
\hline$m_{a}$ & $\alpha_{1}$ & $\alpha_{2}$ & $\alpha_{3}$ & $\alpha_{4}$ \\
\hline 0.1 & 6.103 & 4.156 & 2.672 & 0.58 \\
\hline 0.2 & 14.99 & 1.907 & 6.148 & 0.467 \\
\hline 0.3 & 45.097 & 12.235 & 39.217 & 17.037 \\
\hline 0.4 & 0.667 & 10.98 & 7.245 & 4.973 \\
\hline 0.5 & 7.389 & 6.892 & 4.82 & 0.894 \\
\hline 0.6 & 10.988 & 7.28 & 195.26 & 194.574 \\
\hline 0.7 & 5.024 & 15.05 & 57.35 & 64.52 \\
\hline 0.8 & 18.494 & 24.078 & 0.67 & 0.171 \\
\hline 0.85 & 11.615 & 87.606 & 56.628 & 74.916 \\
\hline
\end{tabular}

\section{Simulation OF THE System}

Pspice/orcade software has been used as tool to simulate the circuit which consists of single phase rectifier and the inverter circuit with and without transformer. Figure 5 shows the simulation result of output voltage for the diode clamped transformer cascade inverter.

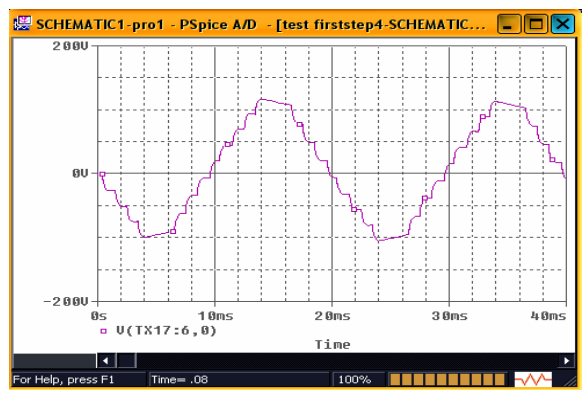

Figure 5. Output voltage of Transformer Cascade Multilevel Inverter

Figure 6 shows the analysis of frequency spectrum for output voltage waveform, it is clear that the harmonics of order $5^{\text {th }}, 7^{\text {th }}$ and $11^{\text {th }}$ have been eliminated, and this give advantage of the multilevel to reduce the total harmonic distortion. THD value must be low especially in the high power application. Multilevel inverter meets the requirement of the high power applications which they can give a high voltage output with low THD; this achievement has been obtained by adjusting the H-bridge conduction angle to eliminate some of high amplitude harmonics.

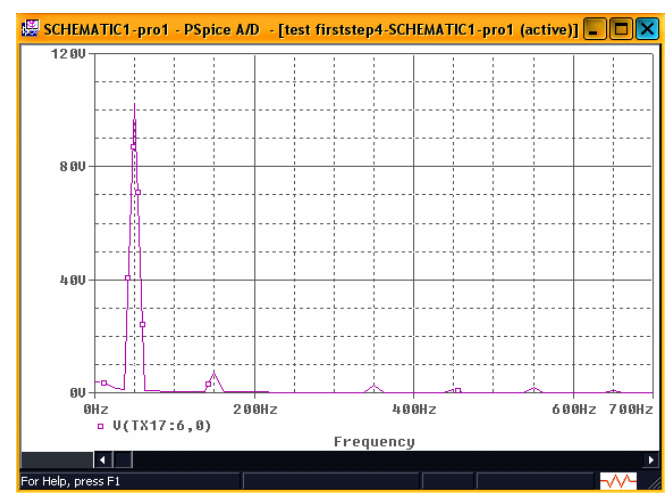

Figure 6. Frequency spectrum analysis of multilevel waveform

\section{EXPERIMENTAL RESULTS}

To verify the validity and superiority of the multilevel inverter, implemented experiments based on a laboratory prototype, which consists of four full-bridge inverter units, four cascaded transformers with same size, and low cost microprocessor PIC16F877A, has been used for the system control.

Figure 7 shows the experimental set up of multilevel cascade inverter, while Figure 8 demonstrates the experimental output voltage of the inverter, with $50 \mathrm{~Hz}$ and modulation index of 0.8 .

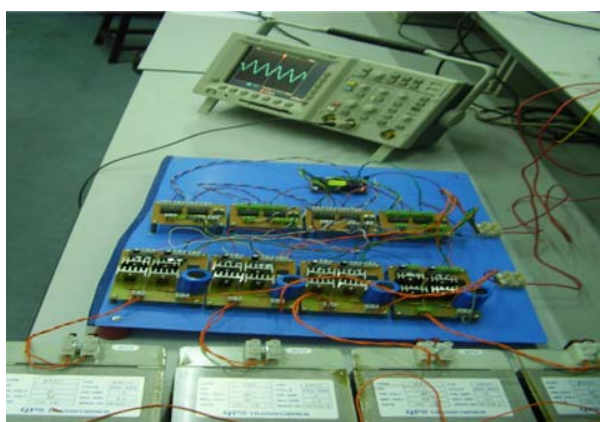

Figure 7. Experimental setup of multilevel cascade inverter

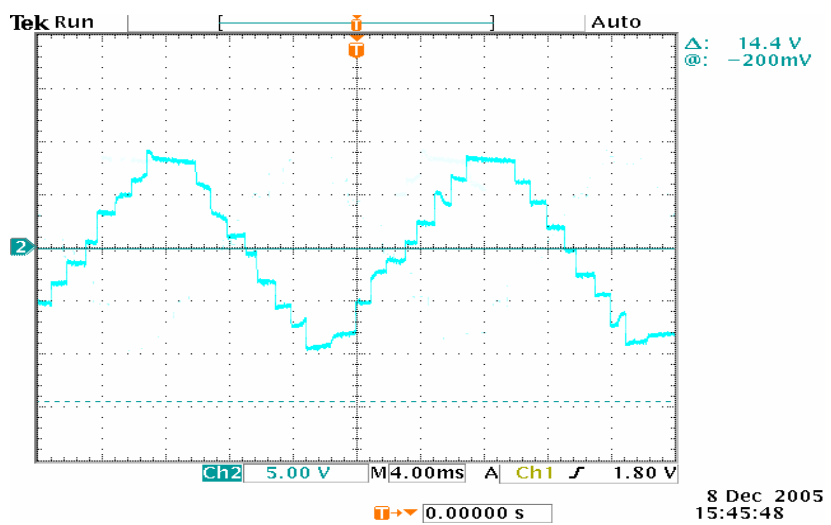

Figure 8. Cascade multilevel inverter output at $\mathrm{M}=0.8$ and $\mathrm{f}=50 \mathrm{~Hz}$

Application of the method of minimizing harmonic with iterative process can provide faster solution to calculate the THD for large number of steps. It has been found in the simulation that the THD is equal to $7.51 \%$ and in experiment is $8.39 \%$ for the same output voltage. And these values can be reduced further if the number of steps is more than 4 . This result confirms the superiority for the multilevel 
inverter with the best result recorded for the PWM inverter topology as $19.7 \%[1,6]$.

Although the transformer is considered as bulky and loose component but utilization of its leakage inductance gives us higher order of harmonic elimination, also the isolation between each bridge is guaranteed.

\section{CONCLUSIONS}

In this work the cascaded multilevel inverter with transformer has been investigated.

Reduction of harmonics and THD can be obtained easily if the conduction angles of H- bridges are properly chosen.

Simulation and experimental results have been obtained which showed a close agreement between them and proved that, with a control strategy of switches peroration at fundamental frequency, these converters have low harmonics or THD and high efficiency.

\section{ACKNOWLEDGMENT}

The authors would like to express his gratitude to Universiti Tun Hussein Onn Malaysia for rewarding a short grant to produce this paper.

\section{REFERENCES}

[1] Leon M. Tolbert, Fang Zheng Peng, , Tim Cunnyngham, and John N. Chiasson, "Charge Balance Control Schemes for Cascade Multilevel Converter in Hybrid Electric Vehicles, IEEE transaction on Industrial Electronics, Vol 49, No.5, October 2002.

[2] Muhammad H. Rashid, "Power Electronics: Circuits, Devices and Application", Prentice Hall, Third Edition, 2004.

[3] Leon M. Tolbert, Fang Zheng Peng, and Thomas G. Habetler, " Multilevel Converters for Large Electric Drives", IEEE Transaction on Industry Application, vol. 35 no. 1, January/February 1999.

[4] Corzine, K; Familiant, Y (2002), "A New Cascaded Multilevel HBridge Drive", IEEE Transactions on Power Electronics, Vol. 17, No.1, January 2002, pp 125-131

[5] Fang Zheng Peng, , John W. McKeever, and Donald J. Adams," A Power Line Conditioner Using Cascade Multilevel Inverters for Distribution Systems", IEEE Transactions on Industrial Electronics, Vol 34, No.6, December 1998.

[6] Jih - Sheng Lai and Fang Zheng Peng, "Multilevel Converters-A New Breed of Power Converters", IEEE Transactions on Industry Application, Vol 32, No.3, May/June 1996. 


\title{
Performance Comparison of PID Controller on SVC and TCSC to the Transmission Line
}

\author{
Shamsul Aizam, Md Zarafi, Rohaiza Hamdan, Nor Anija and Nurul-Aliaa
}

\begin{abstract}
In this paper the common devices where been applied at the transmission line to mitigate the voltage or current sag are Static Var Compensator (SVC) and Thyristor Controller Static Compensator (TCSC). To solve this problem a high response controller is required at the control side. One of the technique is applied the Proportional Integration Derivation (PID) controller, compared to the other controllers due to it simplicity, highly steady state response and less margin error. Here, both Flexible AC Transmission System (FACTS) devices have been applied with the same PID controller in comparing the performance response on the transmission line while having the same industrial load. In determined the response, both transfer functions have been generated and combined with PID controller feedback loop to the transfer function for locate the initial poles and zeros. By changing the poles coordinates the response will change either to have fast response or slow response.
\end{abstract}

Keywords: PID controller, SVC, TCSC, power quality

\section{INTRODUCTION}

Nowadays the ability of utilities to install a power quality conditioner is very appreciated in solving power quality problem in the line. The conditioner can be placed at transmission or distribution line. Common practice used by the utility is by connecting the conditioner on the transmission line. The common power quality conditioners that been installed by the utility are Static Var Compensator (SVC) and Thyristor Controlled Series Compensator (TCSC) due to simple circuit arrangement, no transformer connection between the device to the transmission, reduce cost and easy to maintain [1]-[3]. SVC is a shunt connection to the transmission line while the TCSC is a series connection to the transmission line.

These types of conditioners are very useful in solving power quality problem due too it ability to inject the voltage for SVC or current for TCSC to compensate the power quality problem [4] when occurred on the transmission line. The major power quality problems on the transmission line are voltage sag, voltage swell, flicker, harmonic and unbalanced condition. Both devices have its own responsibility to solve power quality problem such as the $\mathrm{SVC}$ is voltage control that able to solve voltage sag while the TCSC is on transient stability which able to solve

Shamsul Aizam, Md Zarafi, Rohaiza Hamdan, Nor Anija and NurulAliaa are with Department of Electrical Power Engineering, Faculty of Electrical Engineering, University Tun Hussein Onn Malaysia, 86400 Batu Pahat, Johore (email: aizam@uthm.edu.my). unbalanced condition or voltage sag on the transmission line. There are many techniques that available to control the performance of both devices in response to the power quality problem such as the conventional or modern technique. The conventional techniques are easier to develop and it has robust ability compare modern techniques [5].

The conventional techniques are Proportional Integral (PI), Proportional Derivative (PD) and Proportional Integral Derivative (PID) controller. PID controller had been selected because the settling time is faster than PI and PD controller, less percent overshoot, smaller amplitude, fast steady state [6] and the response time is less then PI which is $0.1 \mathrm{sec}$ [7] which this is important parameter to solve power quality problem. Nevertheless the function of pole arrangement controller is used to shift the initial poles from the right to the left of the complex diagram, in order to increase the stability of the system and damping response [7] also been applied to the controller for injecting the voltage or current referring the firing angle. In this work both devices are been applied by PID and pole arrangement controller for response performance analysis.

\section{Modelling OF SVC}

Static VAR compensator is an electrical device in providing fast acting reactive power compensation on high voltage electricity network [8]. It provides a system automated impedance matching device where if load is too capacitive the SVC will inject the current which is proportional to reactive power and vice versa if the load is too inductive [9] and the response time can be in range of $30-60 \mu$ s [10]. The general arrangement of SVC is the fixed capacitor is connected to the thyristor controlled reactor (TCR) or by thyristor-switched capacitor (TSC) with TCR [11] which shown in 'figure 1'.

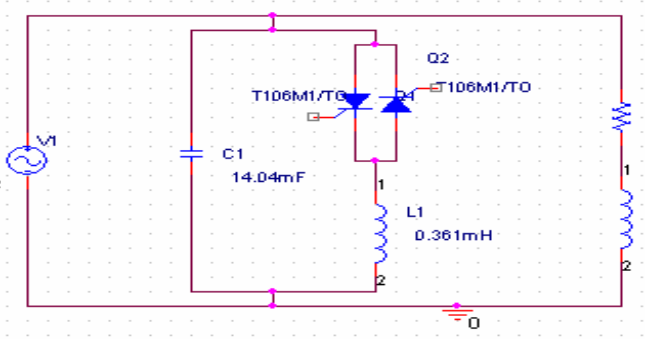

Figure 1. SVC circuit diagram 
In designing the PID controller, the transfer function equation from the SVC circuit must be introduced. The theory of Kirchoff Current Law has been applied to 'figure 1 ', where the SVC is designed in single line diagram which connected to the industrial load. The industrial load is calculated by $\mathrm{S}=666.5 \mathrm{~K}+\mathrm{j} 1154.4 \mathrm{KVar}$. The $\mathrm{C}_{1}$ is a fixed capacitor connected to the thyristor $\mathrm{T}$ which control the operation of load that represent in $\mathrm{R}_{\mathrm{L}}$ and $\mathrm{L}_{\mathrm{L}}$. The equation can be written as

$$
\frac{V_{o}}{I_{i}}=\frac{s^{2} L L_{l}+s L R_{l}}{s^{3} L C L_{l}+s^{2} L C R_{l}+s\left(L+L_{l}\right)+R}
$$

Where $\mathrm{R}_{1}=71.43 \Omega, \mathrm{L}_{1}=0.394 \mathrm{H}, \mathrm{C}=20 \mu \mathrm{F}, \mathrm{L}=0.253 \mathrm{H}$ and this equation will give the initial poles at (113.0882,$34.0778 \pm \mathrm{j} 561.7869)$ with the open loop response having highly damp and frequency where the overshoot is infinity and settling time is $0.115 \mathrm{~s}$ and no steady state response shown in 'figure 2'.

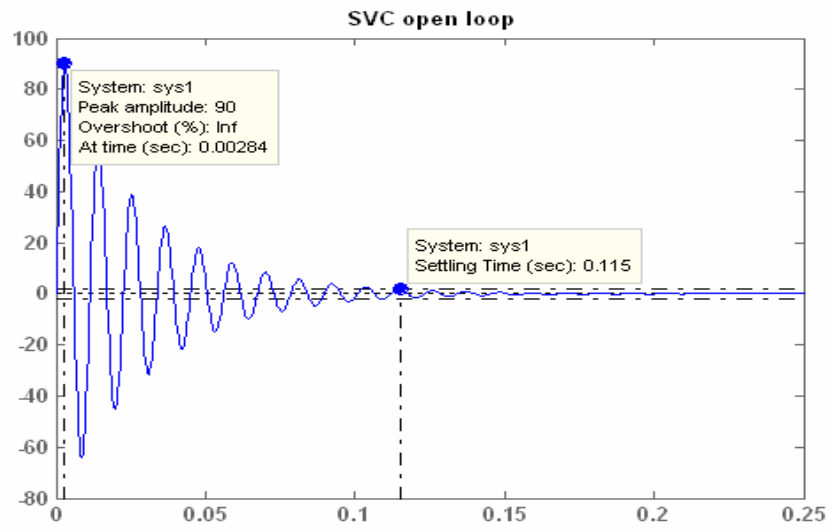

Figure 2. SVC open loop response

\section{Modelling OF TCSC}

TSCS is a combination of capacitor parallel with the tyristors connected in series to the transmission line [4] where it will inject the voltage when the disturbance happen on the transmission line. TCSC is capable to provide a continuous variable capacitor by controlling the firing angle delay of the thyristor [5] and able in mitigating the sub synchronous resonance (SSR) that induced by the generator [4]-[5].

The advantage using TCSC at the transmission line is the thyristor switch allows an unlimited number of operations, exact switching instant can be select by the thyristor to reduce switching transients, no generation of harmonic an very rapid speed of response which is less then half of cycle. 'Figure 3' shows the single line diagram of TCSC.

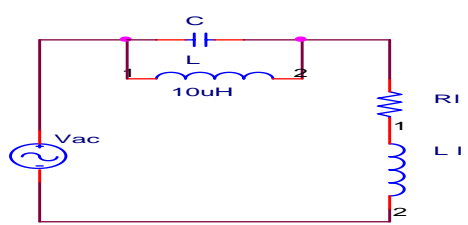

Figure 3. TCSC circuit diagram
Where the $\mathrm{C}$ and $\mathrm{L}$ represent the TCSC with $\mathrm{R}_{\mathrm{L}}$ and $\mathrm{L}_{\mathrm{L}}$ connected at the distribution side. The transfer function from the TCSC is shown in Equation (2) for designing the PID controller of the system.

$$
\frac{V_{\text {in }}}{V_{\text {out }}}=\frac{s^{3} L C L_{L}+s^{2} R_{L} C L+S L_{L}+R_{L}}{s^{3} L C L_{L}+S^{2} R_{L} C L+S\left(L+L_{L}\right)+R_{L}}
$$

The value for $L, C, L_{L}$ and $R_{L}$ are the same which the value applied for the SVC. The initial response of TSCS at the line is shown in 'figure 4' with open loop situations.

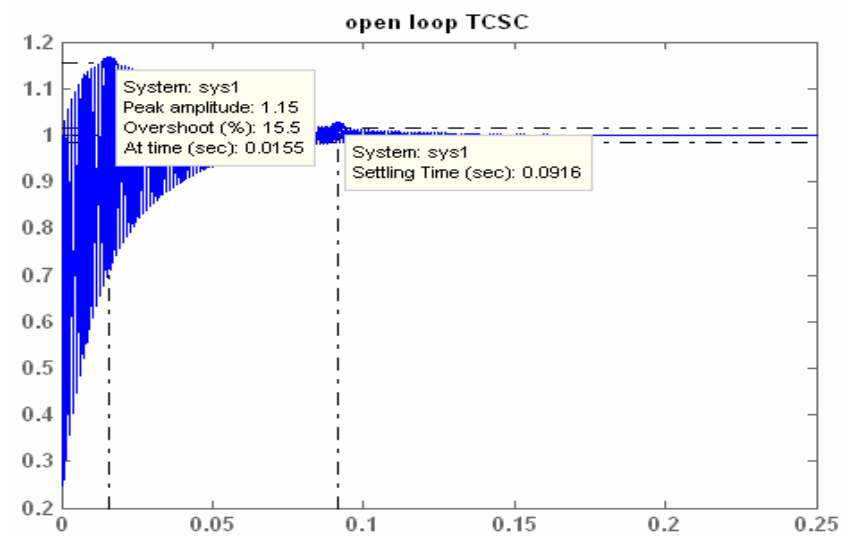

Figure 4. TCSC open loop response

'Figure 4' shows the response of the TCSC without been applied by any controller circuit. From the graph it show that the overshoot is $15.5 \%$ and the settling time is at $0.0916 \mathrm{~s}$ that not suitable to response to the power quality time.

\section{PID Controller Design AND Pole ArRangement TECHNIQUE}

In designing the PID controller, the important parameter is to give fast response compare to the open loop system and with the same the steady state value to control the firing angle to the devices [5]. The transfer function of the PID controller is given by

$K_{p}+\frac{K_{i}}{s}+K_{d} s=\frac{K_{d} s^{2}+K_{p} S+K_{i}}{s}$

In tuning the PID, some variable of $\mathrm{K}_{\mathrm{p}}, \mathrm{K}_{\mathrm{i}}$ or $\mathrm{K}_{\mathrm{d}}$ have to be found. For design the PID controller, MATLAB has been used and combine the Ziegler-Nicholas [6] method to determine the values. The method proposed [5],

- From the transfer function of the devices determined the value $\mathrm{W}_{\mathrm{u}}, \mathrm{K}_{\mathrm{pu}}$ and $\mathrm{P}_{\mathrm{u}}$ by equation

- The gain for $\mathrm{K}_{\mathrm{d}}, \mathrm{K}_{\mathrm{p}}$ and $\mathrm{K}_{\mathrm{i}}$ are determined by $\mathrm{K}_{\mathrm{p}}=$ $0.6\left(\mathrm{~K}_{\mathrm{pu}}\right), \mathrm{T}_{\mathrm{i}}=0.5(\mathrm{Pu}), \mathrm{T}_{\mathrm{d}}=0.125(\mathrm{Pu}), \mathrm{K}_{\mathrm{i}}=\mathrm{K}_{\mathrm{p}} / \mathrm{T}_{\mathrm{i}}, \mathrm{K}_{\mathrm{d}}=$ $\mathrm{K}_{\mathrm{p}} \mathrm{T}_{\mathrm{d}}$

The values of $K_{p}$. $K_{i}$ and $K_{d}$ have been determined by referring to the suggested methods and PID controller will be connected to the devices with the feedback loop show in 'figure 5'.

The M-File Matlab window to determine the new poles location with the pole arrangement method to locate the new pole locations [12] of the controller where combine with the SVC or TCSC which have faster settling time, less 
overshoot, and amplitude reduce compare to original response while having the same steady state value.

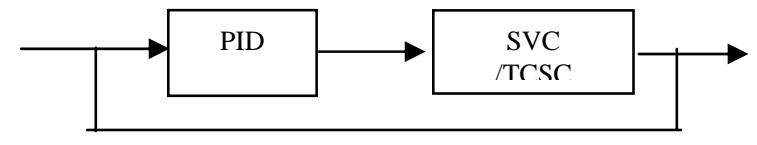

Figure 5. Complete controller circuit.

The program flows in M-File Format is shown in 'figure 6'.

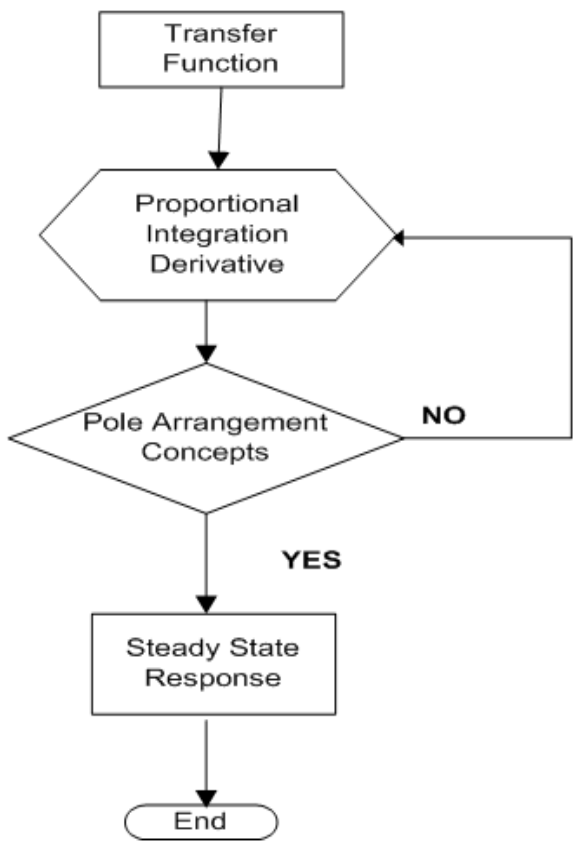

Figure 6. Flow chart of M-File

\section{Simulation Result}

The performance for both components with the PID controller had been simulated in MATLAB environment. 'Figure 7' shows the output for closed loop TCSC without the PID controller.

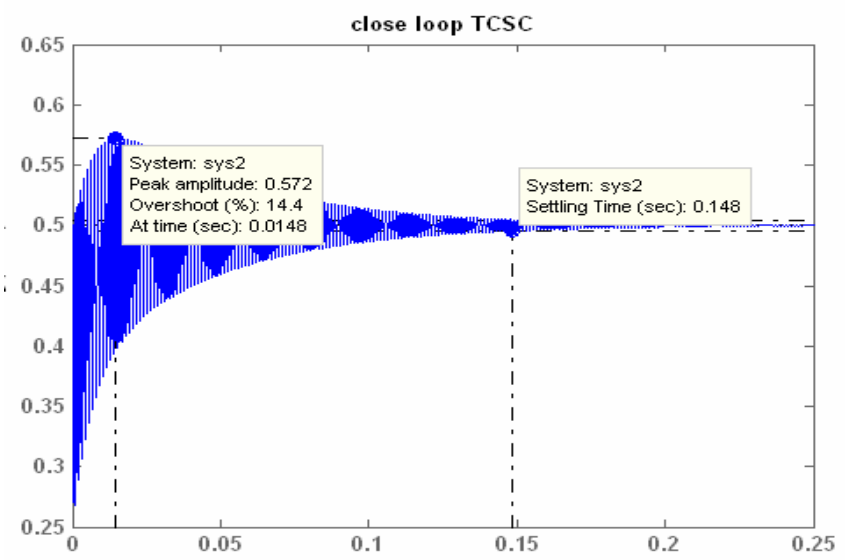

Figure 7. Closed loop TCSC

It shows that the overvoltage of the TCSC has been reduced to $14.4 \%$ compare to the open loop TCSC which is more then $50 \%$ while the settling time is having at $0.14 \mathrm{sec}$ which caused the controller not able to response to the power quality problem which happen less then $20 \mathrm{~ms}$.

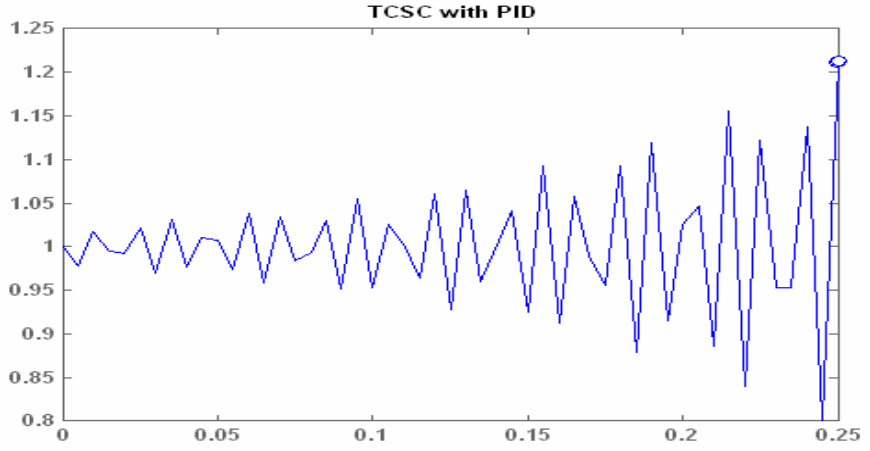

Figure 8. TCSC with PID controller

'Figure 8' shows the TCSC with PID controller. In this graph it shows the response is started at point 1 where same as the open loop response and it means the PID controller is able to maintain the response at 1 . The problem on this graph is the unstable condition for the settling time due to increase oscillation when the time been increase.

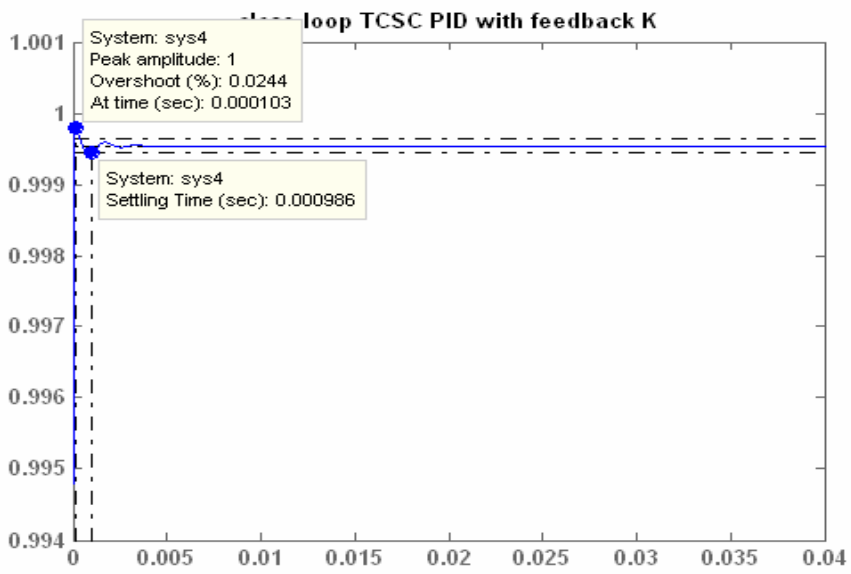

Figure 9. Closed loop TCSC PID controller with feedback

'Figure 9' shows the best response of the PID controller combine with the TCSC with the system having a feedback loop. It shows that the overshoot is reduced to $0.0244 \%$ compare to closed loop TCSC and open loop TCSC. The settling time also been reduced to less the $0.005 \mathrm{sec}$ which is suitable to response the power quality. This response is set when the root locus of the feedback response give the new poles are locations at $1.0 \mathrm{e}+004(-7.9689,-0.0985+0.3758 \mathrm{i}$, $-0.0985-0.3758 \mathrm{i},-0.2458,-0.0181)$ due to pole arrangement application.

The same works had been done for the SVC where the graphs will show the closed loop SVC, SVC with PID and closed loop SVC with feedback loop.

'Figure 10' shows the response of closed loop SVC. In this response, the peak amplitude for the system is started at 0.997, where it not the same with the open loop response where the steady state is about $0.2 \mathrm{sec}$ and also show the decaying time is larger compare to open loop system. 


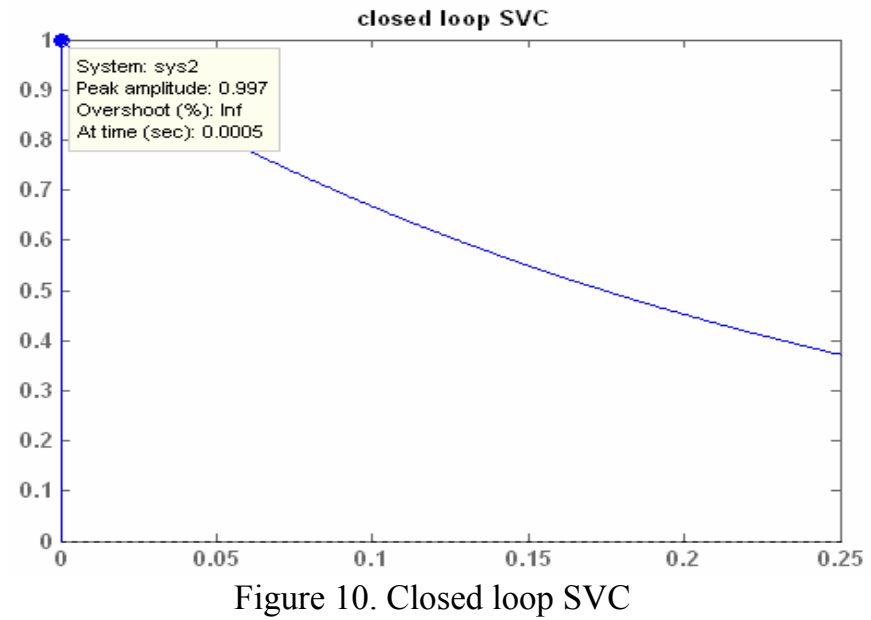

For PID controller with the SVC, the response is shown in 'figure 11'. From the response the overshoot and steady state response is still high from the open loop SVC. This graph gives a conclusion that the system is not in stable condition because the magnitude of steady state is not at 0.2 sec.

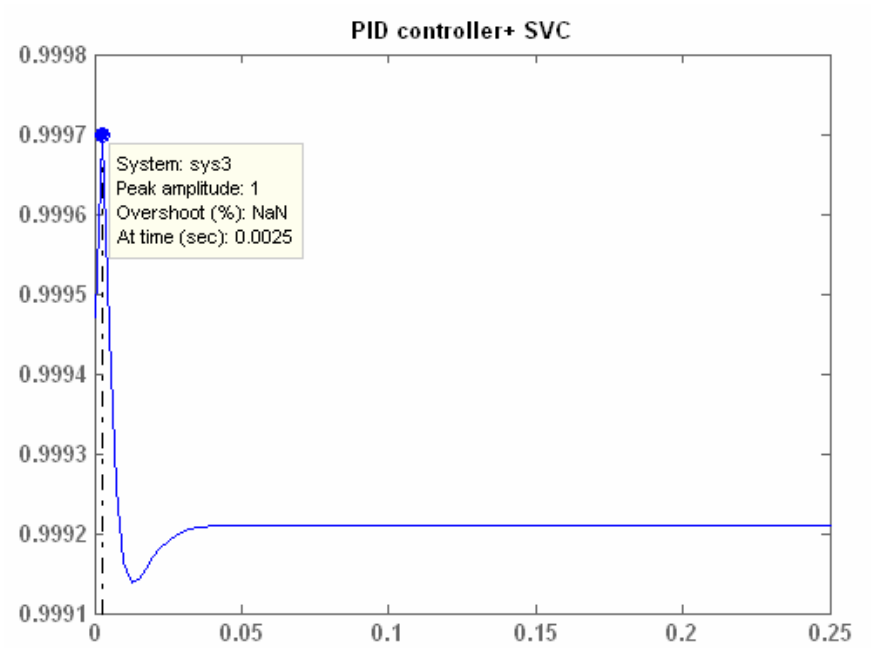

Figure 11. SVC with PID controller

A feedback loop for the PID SVC controller response is shown in 'figure 12'. At figure 12 the maximum amplitude is reduce to 0.3 compare to figure 10 and 11 that will caused the steady state response same with open loop response which is $0.2 \mathrm{sec}$. The settling time for steady state response also had been reduced to about $0.05 \mathrm{~s}$ which is half of the time response of the open loop response. This proved that the system now is in stable condition. This response is perform when the new poles had been located at the new points which are $1.0 \mathrm{e}+002 *(0,-0.8536+5.3749 \mathrm{i},-0.8536-$ 5.3749i, -1.1522).

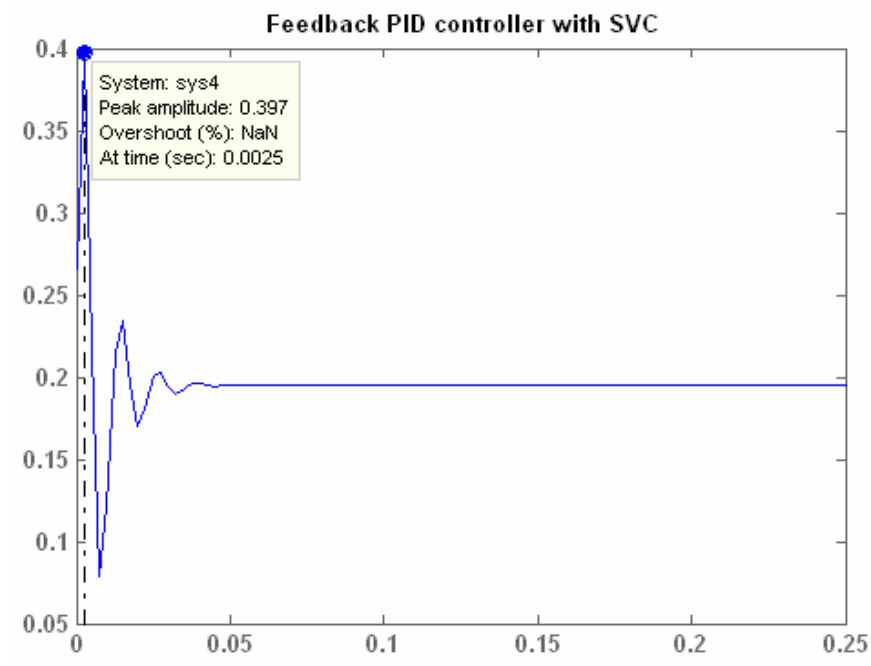

Figure 12. Closed loop TCSC PID controller with feedback

\section{CONCLUSION}

'Table 1' shows the comparison performance of both devices at the same distribution network.

Table 1. Comparison of performance

\begin{tabular}{|c|c|c|c|}
\hline Performance & $\begin{array}{c}\text { Overshoot } \\
(\%)\end{array}$ & $\begin{array}{c}\text { Peak } \\
\text { Amplitude }\end{array}$ & Settling Time \\
\hline SVC (open loop) & Inf & 90 & $0.112 \mathrm{~s}$ \\
\hline TCSC (open loop) & $15.5 \%$ & 1.15 & $0.092 \mathrm{~s}$ \\
\hline $\begin{array}{c}\text { SVC (closed } \\
\text { loop) }\end{array}$ & $\mathrm{NaN}$ & 0.397 & $0.050 \mathrm{~s}$ \\
\hline $\begin{array}{c}\text { TCSC (closed } \\
\text { loop) }\end{array}$ & $0.0244 \%$ & 1 & $0.000986 \mathrm{~s}$ \\
\hline
\end{tabular}

From the table, it shows that the best devices to be apply to the transmission line with the same distribution load is the TCSC compare to the SVC due the settling time and the percentage of the overshoot that in range of the power quality response. As the conclusion the TCSC is able to solve voltage sag due to settling time response.

\section{REFERENCES}

[1] Besanger, Y., Maginier, S., Hadjsaid, N., Feuillet, R., “Thyristor controlled series compensation: some aspects of different circuit parameters and voltage stability margin", 1995 International Conference on Energy Management and Power Delivery, 1995. Proceedings of EMPD '95, Volume 2, 21-23 Nov. 1995 Page(s):753 758 vol. 2.

[2] Keerthivasan, K., Deve, V.S., Jerome, J., Ramanujam, R Modeling of SVC and TCSC for power system dynamic simulation, Power Engineering Conference, 2005. IPEC 2005. The 7th International., 29 Nov.-2 Dec. 2005 Page(s):696 - 700 Vol. 2

[3] Tenorio, A.R.M., Daconti, J.R., "Voltage sag mitigation by means of thyristor controlled series capacitors" 8th International Conference on Harmonics And Quality of Power, 1998. Proceedings, Volume 1, 1416 Oct. 1998 Page(s):572 - 576 vol.1.

[4] Angquist*.L, "Dynamic Performance of TCSC Schemes", CIGRE 1996.

[5] Yolac, .U., Yalcinoz, T., "Comparison of fuzzy logic and PID controllers for TCSC using MATLAB". Universities Power Engineering Conference, 2004. UPEC 2004. 39th International, Volume 1, 6-8 Sept. 2004 Page(s):438 - 442 Vol. 1.

[6] J.P. III Williams, "Control system engineering", John Wiley \& Sons Inc, 1986. 
[7] P. Rao, M. L. Crow, and Z. Yang, "STATCOM Control for Power System Voltage Control Applications," IEEE Transactions on Power Delivery, vol. 5, pp.1311-1317, 2000.

[8] Static VAR Compensator, http://en.wikipedia.org/wiki/static_VAR_Compensator, Access on 15 September 2007.

[9] Lee, S.Y, Bhattacharya, S., Lejonberg, T.,Hammad, A.E.; Lefebvre, S., "Detailed Modeling of Static Var Compensators Using the Electromagnetic Transient Programs", IEEE Transaction on Power Delivery, Volume 7, Issue 2, April 1992 Page(s):836 - 847.

[10] E. Acha, V.G. Agelidis, O. Anaya-Lara, T.J.E. Miller, Power Electronic Control in Electrical Systems, Newnes Power Engineering Series, 2002.

[11] G. Arindam, L.Gerard, "Power quality enhancement using custom power devices", Kluwer Academic Publishers, 2002.

[12] A. Ghosh, A. K. Jindal, and A. Joshi, "Inverter Control Using Output Feedback for Power Compensating Devices," TENCON 2003, Conference on Convergent Technologies for Asia Pacific Region, pp. 48-52.

[13] Matsuki, J., Ikeda, K., Abe, M., Investigations of a thyristorcontrolled series capacitor, Proceedings of the 1996 IEEE IECON 22nd International Conference on Industrial Electronics, Control, and Instrumentation, 1996, Volume 2, 5-10 Aug. 1996 Page(s):683 - 688, vol. 2. 


\title{
Biodiesel: An Eminent and Eco-Friendly Fuel Forever
}

\author{
Lakshmanan Singaram
}

\begin{abstract}
Diesel engines have superior fuel efficiencies, and hence they are predominantly used in commercial transportation and agricultural machinery. Due to the shortage of diesel fuel, its harmful effects on the environment like global warming and the increasing costs, a need for an alternate source of fuel for diesel engines is imminent. Excessive transitional costs and lack of practical alternatives have led to the continued use of petrodiesels despite their shortcomings. This paper investigates the suitability of Biodiesels as a viable alternative to petrodiesel. It reviews the techniques used to produce biodiesel and provides a comprehensive analysis of the benefits of using biodiesel over other fuels. It shows that the use of bio-diesel achieves considerable reduction in various pollutant emission levels, including carbon-mono-oxide, nitrogen oxides, unburned hydrocarbons and particulate matter. Measurements of the various characteristics of biodiesel like Ignition quality, viscosity, heating value and flash-points are reported. Detailed comparisons between the fuel-properties of diesel and biodiesel are used to demonstrate the ease-of transition to biodiesels. Energy-breakdowns of the bio-diesel production process are also provided to analyze the overall energyefficiency of biodiesels. The paper concludes with a summary of the major advantages of using biofuels for both the environment and the economy.
\end{abstract}

Keywords: biodiesel, bio-degradable, non-toxic, renewable, alternative fuel

\section{INTRODUCTION}

The diesel engine was originally developed with the intention of running on a wide-variety of fuels. Due to their superior fuel efficiency, diesel engines have found their way into most transportation systems and agricultural machines. Historically, the availability of large quantities of cheap petrodiesel led to its usage as the primary fuel for diesel engines. These conditions however, have changed over time and there is currently a shortage of cheap diesel fuels. Political instabilities in major oil-producing countries have worsened this situation, forcing an immediate search for an alternative source of fuel for diesel engines. This paper looks at Biodiesels as a viable solution to this impending problem. The eco-friendly, bio-degradable and renewable nature of biodiesel makes it a highly attractive alternative to petrodiesel. In this paper, quantitative measurements are provided to justify these claims and an attempt is made to directly compare the fuel properties of petrodiesel with that of biodiesel [1].

Many other forms of biofuels like keromix and vegetable oils are potential alternatives for replacing petrodiesels. In this paper, a qualitative comparison of biodiesel with these alternatives is also provided and the merits/demerits of using biodiesels are analyzed. It is shown that biodiesels are more practical and viable, compared to these alternatives.

The rest of this paper is organized as follows, first a brief introduction to biofuels is provided, and then the Transesterification process used to produce biofuels is reviewed. Following this, the important characteristics of biodiesel are presented and a detailed analysis of the benefits of using biodiesels is performed. Detailed comparison of the fuel properties of petrodiesel and biodiesel are carried out to demonstrate the viability of the biodiesel solution. The paper finally concludes with a summary of the key benefits of using biodiesel as an alternative fuel for diesel engines.

\section{BIODIESEL}

Biofuels are liquid transportation fuels made from plant matter instead of petroleum. They are renewable, since they can be regenerated from resources such as vegetable oils and recycled restaurant greases. Their ability to reduce toxic gas emissions makes them eco-friendly. These fuels are also easy to dispose, and hence bio-degradable. These fuels also have the potential to substantially reduce the dependence on non-renewable oil and support the rural economies.

The closed-carbon cycle of biofuels ensures that the carbon-di-oxide emissions from the engine exhausts are perfectly balanced by the plants producing bio-fuels. This property could be exploited to virtually eliminate excessive exhaust of greenhouse gases and help mitigate the effects of additional global warming. Experimental studies suggest that bio-diesels produce $78 \%$ less carbon-di-oxide emissions when compared to conventional petrodiesels.

Primary biofuels used today are bioethanol and biodiesel. Biodiesel can be used as an alternative to diesel in engines. The major objective of this paper is to evaluate the suitability of biodiesel as such an alternative, and illustrate the benefits of using biodiesel using a quantitative analysis. 
$0-\mathrm{OCR}_{1}$
$-0-\mathrm{OCR}_{2}$
$\mathrm{o}-\mathrm{OCR}_{3}$

Triciplyceride

\section{cat. base \\ $3 \mathrm{EtOH}$
Ethyl esters of fatty acids
Glycerol \\ $\mathrm{R}_{1} \mathrm{COOEt}+\mathrm{R}_{2} \mathrm{COOEt}+\mathrm{R}_{3} \mathrm{COOEt}+\mathrm{C}_{3} \mathrm{H}_{5}(\mathrm{OH})_{3}$}

Figure 1. Transesterification process used in biodiesel production

The primary advantage of using biodiesel is that, it does not require any modification to the standard diesel engine. Other alternatives that require such modifications are not practical, since they are costly and involve a huge effort to redesign existing engines. These exorbitantly high transitional costs have been the major reason for the continued dependence on conventional sources of fuel like diesel. Biodiesels do not suffer from this problem, and therefore represent both a viable and practical alternative to petrodiesel.

Biodiesel can be obtained from a variety of renewable sources including soybean oil, canola oil, sunflower oil, cottonseed oil, and animal fats. These sources can be obtained directly from agricultural feed stocks or by recycling used oil such as cooking grease. The pure form of biodiesel is called as "neat biodiesel" (B100). It can also blended with petrodiesel in various proportions (common form - B20 i.e. $20 \%$ biodiesel and $80 \%$ petrodiesel). It can also be used as a sulfur-free lubricating fuel additive in smaller percentages (Typically $2 \%$ ). Ability to mix different proportions of biodiesel with petrodiesel and still achieve the fuel properties, eases the transition to biofuels since the users can get accustomed to biodiesel in gradual increments. A detailed review of the technique used to produce biodiesel is presented in the following section.

\section{BIODIESEL PRODUCTION}

Biodiesel is commonly synthesized through a process known as Transesterification [3]. This involves a reaction between a short-chain alcohol and a glyceride containing oil (can be plant oil, vegetable oil, fat, or grease). The reaction is shown in Figure 1.

Two-stage Transesterification processes are used to convert inedible/ high FFA (free fatty acid) oils. The first stage consists of an acid catalyzed Transesterification to reduce the FFA content to less than 2\%. Second stage involves the conversion of the intermediary products to mono-esters and glycerol.
The catalyst is usually sodium or potassium hydroxide (Lye or Caustic soda) that has already been mixed with methanol. $R_{1}, R_{2}, R_{3}$ indicate the fatty acid chains associated with the oil or fat, which are largely palmitic, stearic, oleic or linoleic in nature. This base catalyzed reaction is the most economical way to produce biodiesel since it requires only low temperatures and pressures. It is also highly efficient, since it can produce more than $98 \%$ conversion yield. The entire process may be carried out in the biodiesel processor described below.

\section{A. Biodiesel Processor}

The biodiesel processor consists of a $1 / 2 \mathrm{HP}$ electric motor and 55 gallon metal drum. The metal drum is used as a mixer and there are 2 pulleys connecting the mixer blade to the motor. A propeller made from 2 L-Shaped brackets is connected to the mixing shaft. The mixing process is carried out from 250 to $400 \mathrm{rpm}$ and a brass bail value is used to drain the glycerin obtained from the biodiesel manufacturing process, which is a useful byproduct.

\section{B. Manufacturing Process}

Each batch of biodiesel typically requires $100 \mathrm{ml}$ of vegetable oil, $20 \mathrm{ml}$ of methanol and $0.35-0.55 \mathrm{gm}$ of Lye. Unused-oil requires lower levels of catalyst concentration, while used-oil usually requires more. Mixing the Lye and methanol produces sodium methoxide, which is mixed with the vegetable oil for an hour. The mixture is allowed to settle for 8 hours and then filtered using a 5micron filter. The filtered glycerin can also be used in the production of soap. The entire process is illustrated in Figure 2.

Test batches are usually prepared to estimate the correct concentration of Lye, which depends on the quality of the vegetable oil and is hard to characterize apriori. After preparation, the mixture is allowed to settle for 8 hours before usage.

Table 1. Energy consumption at the various stages of production

\begin{tabular}{|l|l|l|l|l|}
\hline MJ per MJ fuel & Biodiesel Prod. Stage. & Energy & Petrodiesel Prod. Stage & Energy \\
\hline 0 & Soya bean agriculture & 0.0573 & Domestic crude conversion & 0.0461 \\
\hline 0.05 & Soya bean transport & 0.0034 & Foreign crude oil conversion & 0.0223 \\
\hline 0.1 & Soya bean crushing & 0.0794 & Domestic crude transport & 0.0033 \\
\hline 0.15 & Soya oil transport & 0.0072 & Foreign crude transport & 0.0131 \\
\hline 0.2 & Soya oil conversion & 0.0801 & Crude oil refining & 0.1198 \\
\hline 0.25 & Biodiesel transport & 0.0044 & Diesel fuel transport & 0.0063 \\
\hline 0.3 & Total & 0.2318 & Total & 0.2009 \\
\hline
\end{tabular}




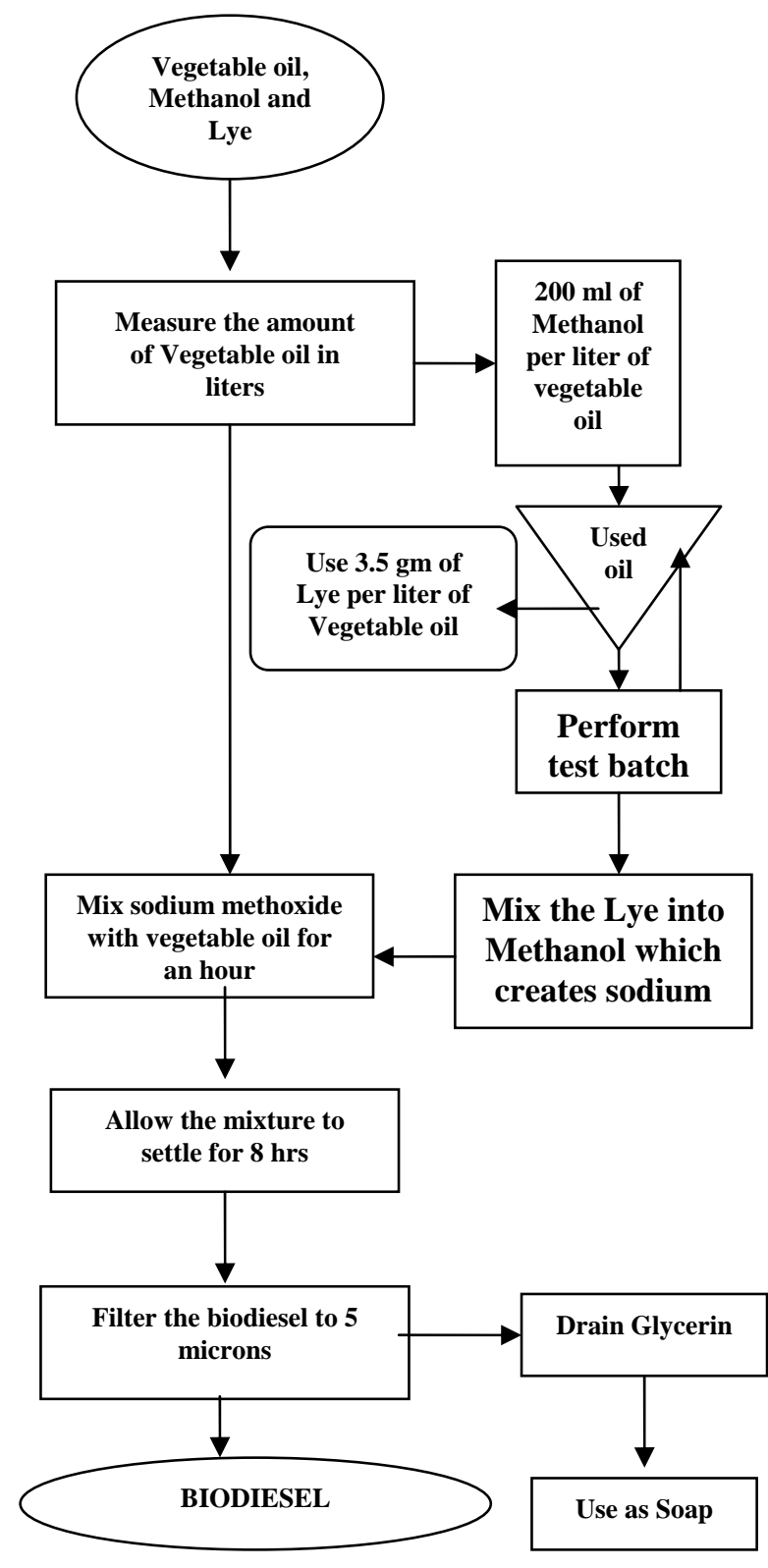

Figure 2. Manufacturing biodiesel

\section{Energy Demands}

It is useful to compare the energy demands of the various stages in the biodiesel production process with those of the conventional petrodiesel production process [2]. A summary of the energy consumption is provided in Table. 1 .

It is important to note here, that agriculture accounts for most of the energy demands in the biodiesel production process (almost 25\%). Therefore, the overall energy demand is much lesser in the case of biodiesel, when compared to the demands of petrodiesel.

The other important observation to be made here is that, crude oil refining dominates energy demands of petrodiesel production and soy oil conversion dominates biodiesel production.

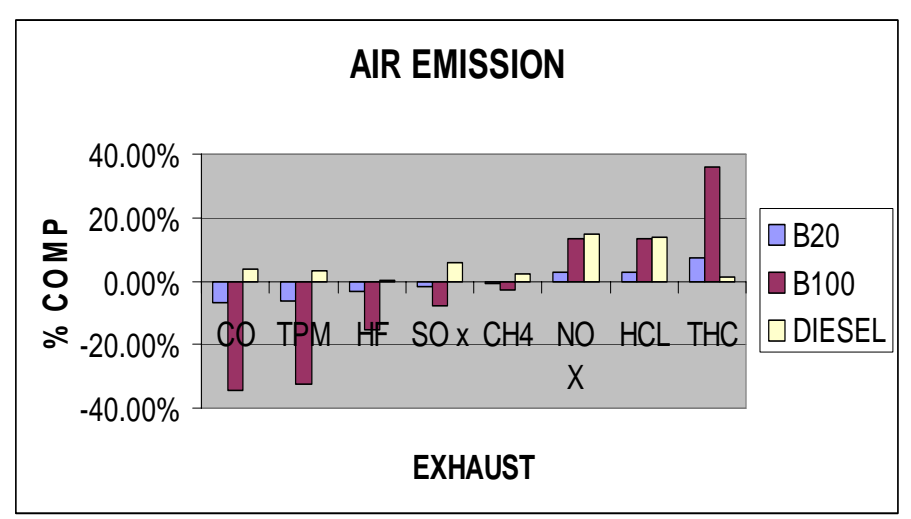

(i) Comparison of emission rates of B20 and B100 with petrodiesel

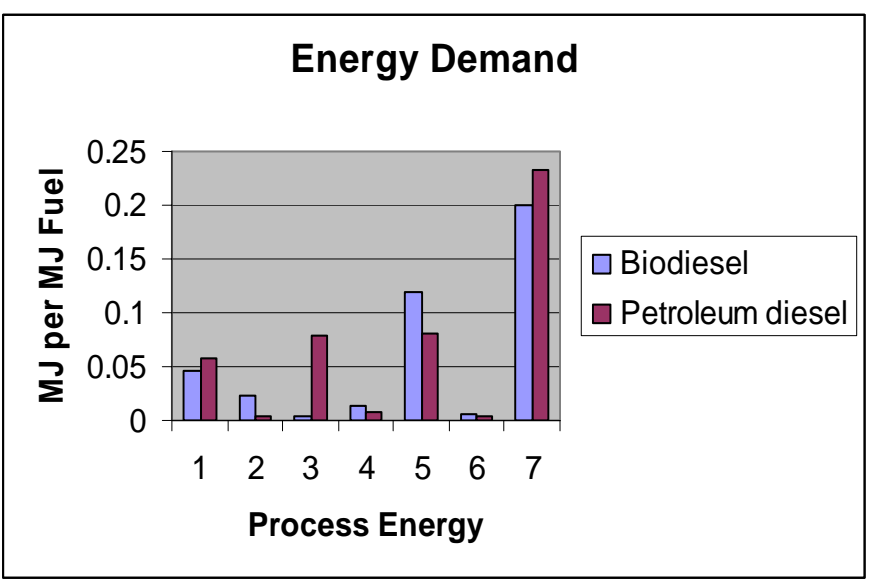

(ii) Comparison of process energy demands at different stages of production

Figure 3.

\section{BIODIESEl CHARACTERISTICS}

The interesting characteristics of biodiesel, which make it a suitable alternative source of fuel for diesel engines, can be summarized as follows.

\section{A. Ignition Quality}

Diesel combustion demands self-ignition of the fuel, because it is sprayed near TDC into a hot, swirling cylinder. Long ignition may also lead to knocking. It is therefore desirable, that the cetane number be between 40 and 60 . Biodiesels have a cetane number in this range and are therefore suitable alternatives in this aspect.

\section{B. Viscosity}

The ease of combustion and thermal efficiency depend on fuel viscosity. Too low viscosity leads to internal pump leakage. Viscosities of biodiesels are comparable to that of diesel.

\section{Heating Value}

Although the diesel combustion process can accommodate a wide variation in heating value, it is desirable that the C.V of the fuels is nearer to that of diesel. Practical systems demand high C.V values since it reduces the quantity of fuel to be handled. Biodiesels are closer to diesel in this respect too. 


\section{D.Pour-Cold-Flash Points}

Pour point and Cold point are important for cold weather operation. These values should be below the freezing point of the oil used. The flash point is important from a safety point of view. These values for commercial vegetable oils range from 50 to 300 degree Celsius.

A quantitative analysis of these properties may be obtained from Table 2 .
Table 2. Biodiesel characteristics

\begin{tabular}{|l|l|l|l|l|}
\hline FUEL & $\begin{array}{l}\text { DYN. } \\
\text { VISC. } \\
\mathbf{2 0}^{\circ} \mathbf{C}\end{array}$ & $\begin{array}{l}\text { DENSITY } \\
\text { (gm/cc) }\end{array}$ & $\begin{array}{l}\text { C.V } \\
\text { (kJ/ } \\
\text { kg) }\end{array}$ & $\begin{array}{l}\text { CETAN } \\
\text { E NO. }\end{array}$ \\
\hline Diesel oil & 4.8 & .845 & 44870 & 49.6 \\
\hline Peanut oil & 81.8 & .9173 & 39638 & 36.4 \\
\hline Sunflower & 65.6 & .923 & 39320 & 33.4 \\
\hline $\begin{array}{l}\text { Methyl ester } \\
\text { sovbean }\end{array}$ & 4.5 & .883 & 37700 & 51.3 \\
\hline Soybean & 66.4 & .9239 & 38000 & 34.8 \\
\hline
\end{tabular}

Table 3. Comparison of petrodiesel and biodiesel properties

\begin{tabular}{|l|l|l|}
\hline Property & Petrodiesel (ASTMD975) & Biodiesel(ASTMPS121) \\
\hline Lower Heating Value, Btu/lb & 250 & 910 \\
\hline Kinetic Viscosity, @ $40^{\circ} \mathrm{C}$ & $1.3-4.1$ & $1.9-6.0$ \\
\hline Specific Gravity kg/1 @ $60^{\circ} \mathrm{F}$ & 0.85 & 0.88 \\
\hline Water, ppm by wt & 161 & $0.05 \% \max$ \\
\hline Carbon, wt. \% & 87 & 77 \\
\hline Hydrogen, wt. \% & 13 & 12 \\
\hline Oxygen, by dif. wt. \% & 0 & 11 \\
\hline Sulfur, wt. \% & $0.05 \%$ max & 0 \\
\hline Boiling Point ${ }^{\circ} \mathrm{C}$ & 188 to 343 & 182 to 338 \\
\hline Flash Point ${ }^{\circ} \mathrm{C}$ & 60 to 80 & 100 to 170 \\
\hline Cloud Point ${ }^{\circ} \mathrm{C}$ & -15 to 5 & -3 to 12 \\
\hline Pour Point ${ }^{\circ} \mathrm{C}$ & -35 to -15 & -15 to 16 \\
\hline Cetane Number & 40 to 55 & 48 to 60 \\
\hline
\end{tabular}

\section{E. Comparison with Petrodiesel Characteristics}

In order to fully establish the claim that biodiesels can be used as a direct substitute for petrodiesels, it is necessary to directly compare the physical and chemical properties of petrodiesel with those of biodiesel. Table 3 provides such a comparison of these properties and shows that the properties of biodiesel are very similar to that of petrodiesel, thereby making biodiesels a practical substitute for petrodiesel in diesel engines.

\section{F. Analysis of Biodiesel Benefits}

Biodiesels have the potential to reduce net $\mathrm{CO}_{2}$ emissions by more than $78 \%$. Even a preliminary transition to using $\mathrm{B} 20$ in urban buses can reduce $\mathrm{CO}_{2}$ emissions by almost $16 \%$. A complete transition to $\mathrm{B} 100$ can also significantly reduce the emission-levels of particulate matter $(32 \%), \mathrm{CO}$ $(35 \%)$ and $\mathrm{SO}_{\mathrm{x}}(8 \%)$.

Although biodiesels significantly reduce the emission levels of most harmful gases, they produce more $\mathrm{NO}_{\mathrm{x}}$ than petrodiesel [4]. The use of B100 however, increases the $\mathrm{NO}_{\mathrm{x}}$ life cycle emissions by $13.35 \%$ and $\mathrm{B} 20$ results in a $2.67 \%$ increase. This increase is mainly due to the tail pipe emissions. Biodiesels however, can reduce the tailpipe emission of small particulates ( $<10$ microns) by $68 \%$, tailpipe

CO emission by $46 \%$ and completely eliminate tailpipe $\mathrm{SO}_{\mathrm{x}}$ emissions. A summary of this comparison of B20 and B100 with petrodiesel maybe obtained from Figure3(i).

Table 4. Emission rates of B20, B100, petrodiesel

\begin{tabular}{|c|c|c|c|}
\hline COMPOSITION & B20 & B100 & DIESEL \\
\hline $\mathrm{CO}$ & $-6.90 \%$ & $-34.50 \%$ & $3.94 \%$ \\
\hline $\mathrm{TPM}$ & $-6.48 \%$ & $-32.41 \%$ & $3.40 \%$ \\
\hline $\mathrm{HF}$ & $-3.10 \%$ & $-15.51 \%$ & $0.01 \%$ \\
\hline $\mathrm{SO} \times$ & $-1.61 \%$ & $-8.03 \%$ & $5.66 \%$ \\
\hline $\mathrm{CH}_{4}$ & $-0.51 \%$ & $-2.57 \%$ & $2.08 \%$ \\
\hline $\mathrm{NO}_{\mathrm{X}}$ & $2.67 \%$ & $13.35 \%$ & $14.82 \%$ \\
\hline $\mathrm{HCL}$ & $2.71 \%$ & $13.54 \%$ & $13.98 \%$ \\
\hline $\mathrm{THC}$ & $7.19 \%$ & $35.96 \%$ & $1.48 \%$ \\
\hline
\end{tabular}

Biodiesels do not require modifications to the infrastructure used to transport or store conventional petrodiesels. High stability of biodiesels at room temperature and similarity of physical properties with petrodiesel, make it easier to handle biodiesels compared to other alternatives .As can be shown in Figure 4 the flash 
point of biodiesels is pretty high and they are relatively safe to under normal operating conditions [5].

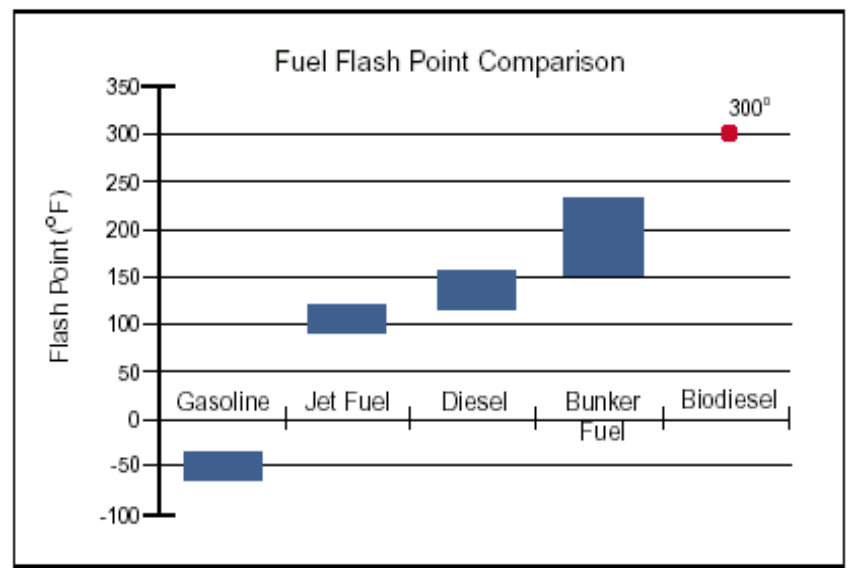

Figure 4. Comparison of flash points of various fuels

Another useful property to analyze is the cloud point of biodiesel. It can be seen from Figure 5 that the cloud point of diesel increases with concentration of biodiesels (as \% in petro diesel). The implications of this behavior are that only lower concentrations of biodiesel are feasible in cold regions where the temperature drops below the freezing point.

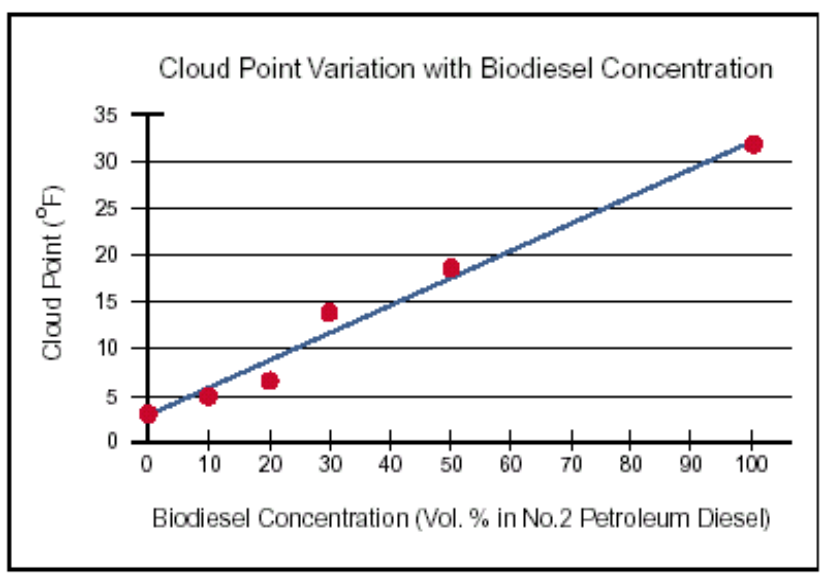

Figure 5. Cloud point vs biodiesel concentration

\section{G. Comparison with other Biofuels}

Keromix and Vegetable oil are comparable alternatives to biodiesels. Biodiesels are usually created by mixing 20 parts of methanol with 80 parts of vegetable oil. Keromix on the other hand, employs kerosene instead of methanol. Vegetable oils can be used as a fuel, provided the temperature of the oil is maintained at $70^{\circ} \mathrm{C}$ at every stage in the fuel tank, fuel hose and the fuel filter.

Some of the key differences between these forms are:

1. Biodiesels can be used as a lubrication additive to normal diesel while the other forms cannot be used.

2. Vehicle modification is required for keromix and vegetable oil. The major advantage of biodiesel is that it can be used in unmodified diesel engines.

3. Stability of bio-diesels at room temperature is much higher compared to the other forms.

4. Biodiesels are good as a startup fuel while the others require a separate startup fuel.
The common properties of these alternative forms are that they all provide better lubrication than diesel and do not affect the engine torque, power or fuel-mileage.

\section{CONCLUSION}

Biodiesels are the most-practical, renewable, biodegradable and eco-friendly substitutes for petrodiesel. They are economically viable as they do not require changes to the conventional diesel engine. Existing diesel infrastructure including pumps, tanks and transport trucks can also be used without any modifications. The mileage of the diesel engine is also uncompromised with biodiesels. The transition from petrodiesel to biodiesel is also easier, since the proportion of the latter can be gradually increased (B2 to B100).

A preliminary analysis of the emission rates shows us that the use of biodiesels can reduce the emission of green-house gases significantly. The closed-carbon life cycle of biodiesel will recycle the carbon-di-oxide present the biosphere and avoid artificially inflating the carbon-di-oxide levels. The natural lubricating property of biodiesel helps alleviate dependence on sulphur that is a major contributor of acidrain. Biodiesel emissions have the potential to decrease the emission-levels of polycyclic aromatic hydrocarbons (PAHs) and their other carcinogenic variants. Studies show that these carcinogens may be reduced by up to $75 \%$ with the introduction of biodiesels.

Practicality of biodiesels has been shown using a quantitative analysis of their physical properties and presenting a detailed comparison with those of petrodiesel. The flash-point comparisons also show that biodiesel is potentially much safer to handle than conventional fuels and requires no special changes to the storage/transportation infrastructures.

Biodiesels are safer to handle, since their high flash point increases their ignition temperature, thereby making them easier to transport. They also have a pleasant aroma compared to petrodiesels, which makes them more aesthetically appealing. Biodiesels are thus a highlypromising solution, to the search for an alternative fuel for diesel engines.

\section{REFERENCES}

[1] Shaine Tyson, K., "Clean Alternative Fuels: Biodiesel", United States EPA Fact Sheet, May 2002.

[2] Karl Lang, "Biodiesel : On the road to fueling the future", HART/IRI Fuels Information Services 2001.

[3] Hideku Fukuda, Akihiko Kondo and Hideo Noda, "Biodiesel Fuel production by Transesterification of Oils", Journal of Bioscience and Bioengineering, Vol. 92, 405-416 (2001).

[4] Shaine Tyson, K., "Biodiesel for the global environment", NREL, May 2000

[5] Joshua and Kia Tickell, "Fuel for the new millenium: One low-tech solution to a high-tech problem", Home Power, Aug 1999. 


\title{
Simultaneous Reduction of Energy and Water - A Special Case on Chilled Water Network Synthesis
}

\author{
Irene M.L. Chew, Denny K.S. Ng, Dominic C.Y. Foo and M. Subramaniam
}

\begin{abstract}
Resource conservation has been an active area of research throughout the past decades. Various techniques have been proposed to reduce energy and water consumption in process plants, ranging from insight-based to mathematicalbased optimisation approaches. In this paper, a special case of simultaneous reduction of energy and water, i.e. chilled water network system is analysed. A chilled water network is essential for semiconductor manufacturing, as it is distributed in a piping system and connected to air condition cooling units, to create a constant temperature environment. Adverse effects of various environmental conditions would impact the manufacturing of integrated circuits greatly. For instance, 'cleanroom' temperature, humidity and moisture control play pivotal roles in the semiconductor wafer fabrication industry for optimum processing. Conventionally, chilled water is sent to each cooling unit in all buildings in parallel arrangement for heat removal from the ambient air. In this work, a mixed series/parallel arrangement is proposed, where chilled water exiting certain cooling and dehumidification coils in the heating, ventilating and air-conditioning (HVAC) system may be redirected to other units that tolerate higher inlet temperature. An algebraic targeting approach known as the chilled water cascade analysis (CWCA) technique is proposed to locate the minimum chilled water flowrate needed in removing the given amount of heat load. Reduction of chilled water flowrate is observed through effective reuse/recycle scheme within chilled water network. This eventually leads to dual utility cost saving through less chilled water pumping cost.
\end{abstract}

This work is financially supported by University of Nottingham Research Committee through New Researcher Fund (NRF 3822/A2RBR9), Research Studentship and Research Assistantships are gratefully acknowledged. Scholarship from World Federation of Scientists (WFS) is also appreciated.

Irene M. L. Chew is a PhD candidate at the University of Nottingham Malaysia. Her area of research is in water minimization through process integration. (phone: +60389248358, fax: +60389248017, email: Irene.Chew@nottingham.edu.my).

Denny K. S. Ng is a PhD candidate at the University of Nottingham Malaysia. His area of research is in water minimization through process integration. (email: dennynks@yahoo.com).

Dominic C. Y. Foo is an Assistant Professor at the University of Nottingham Malaysia. His specializes in waste minimization through process integration techniques, as well as modeling and optimization involving chemical, environmental and biochemical processes. (email: Dominic.Foo@nottingham.edu.my).

Mahendran Subramaniam is a Facilities Systems Engineer, in a semiconductor company, Intel Products Malaysia. (e-mail: indran80@yahoo.com). This work is based on his dissertation for his MSc. studies on the chilled water network at his previous company, SilTerra Malaysia, an electronic wafer fabrication company.
Keywords: process integration, energy reduction, water minimisation, pinch analysis, chilled water network

\section{INTRODUCTION}

CHILLED water system for building air conditioning has been one of the most critical utility systems in semiconductor and electronic wafer fabrication plants. A constant temperature is essentially important in electronic wafer fabrication 'cleanroom' operations, mainly to control the air-borne particulates distribution, process control especially on the wet processes of etching and cleaning, and finally to ensure equipment operator's comfort [1]. According to Chang [2], in the semiconductor industry, among the biggest users of cooling load are the chiller units, where $27 \%$ of the overall power consumption is used to drive the chilled water network distribution.

Various mathematical optimisation methods have been explored to optimise chiller loadings for energy saving [2][5]. The proposed works are generally dealt with energy management, in which the desirable outcome is to operate each chiller unit with the highest efficiency and most importantly with the lowest power consumption.

Chilled water is one of the unique resources where it comprises the elements of water and energy, and acts as a transport medium to remove heat of the targeted premises. A great deal of savings through pinch analysis has been done on heat [6]-[8]; water [9]-[19]; hydrogen [20]; and finance [21]. Pinch analysis has been known as a powerful tool in minimum resource targeting (heat, water, hydrogen, etc.) through resource reuse/recycle.

As of today, no work has been done on the chilled water system using pinch analysis. A simultaneous power and water savings will be observed from the new proposed chilled water cascade analysis (CWCA) by re-utilizing the used chilled water to another air-conditioning unit which tolerates a higher chilled water supply temperature. An industrial case study from a semiconductor chilled water system is used to illustrate the proposed approach.

\section{Chilled Water System IN Semiconductor Plant}

Chilled water system is important as it is the main beat of the air-handling units and heat exchangers. 'Figure 1' illustrated a typical chiller system. The purpose of the chillers is to transfer heat from chilled water system to condenser water system, using Freon refrigerant. Freon is 
compressed to high pressure and condensed as a liquid in a condenser. Liquid Freon is channelled to the evaporator/cooler, where the pressure is relieved to atmospheric pressure. Through evaporation, liquid Freon will then absorb heat from the water and this generates chilled water. Chilled water from the Utility Building will then be supplied to each building in at a supply temperature of, $T_{\mathrm{CHW}, \mathrm{s}}$ for cooling purpose, and the heated chilled water will return to the evaporator/chiller at a return temperature, $T_{\mathrm{CHW}, \mathrm{r}}$ to be cooled down by exchanging heat with the coolant. As chillers service multiple numbers of AHUs and MAUs, $T_{\mathrm{CHW}, \mathrm{r}}$ is an estimated average temperature comprises the returned chilled water from all these units.

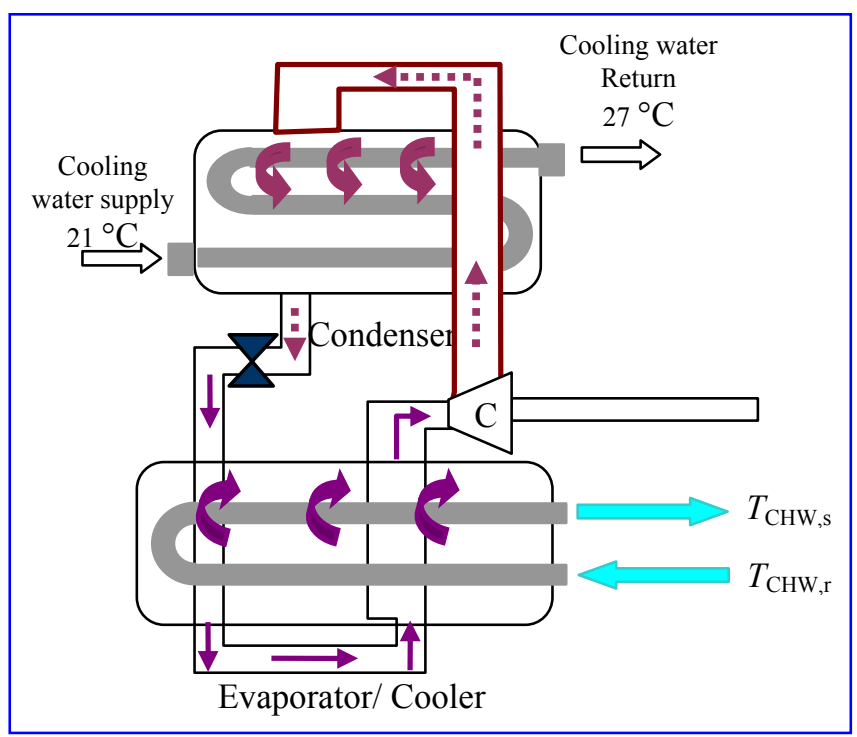

Figure 1 Chiller Work System.

Chilled water is needed for the heat exchangers of the Process Cooling Water (PCW), Low Temperature Process Cooling Water (LTPCW) and Process Vacuum Systems, as well as the Compressed Dry Air (CDA) System. However, the main usage is to cool air entering each building in the plant, via the heating, ventilating and air-conditioning (HVAC) system. The plant consists of four main blocks, i.e. Fabrication (Fab), Office, Chemical Storage and Utility buildings, with a number of Air-Handling Units (AHU) to provide cooling and ventilation for different levels in each building. Besides, Make-up Air-Handling Units (MAU) are installed in Fab building to top up the AHU, while AirConditioning unit (ACU) located at the Office Building. In this work, an industrial chilled water network case study taken from Subramaniam [22] is used to illustrate the proposed approach. 'Table 1' summarised chilled water limiting flowrate, $F$, as well as heat capacity flowrate, $F C_{\mathrm{P}}$, for each $\mathrm{AHU}$ and MAU, assuming chilled water heat capacity, $C_{P}$ at $4.18 \mathrm{~kJ} \mathrm{~kg}^{-1}{ }^{\circ} \mathrm{C}^{-1}$. It is noted that there are a total of six MAUs and seven AHUs for cooling and dehumidification coils in the Fab building. The AHU and MAU enthalpy difference, $\Delta H_{\text {unit }}$ were calculated by substituting the entering water temperature, EWT and leaving water temperature, LWT into (1).

$$
\Delta H_{\text {unit }}=F \times \mathrm{C}_{\mathrm{P}} \times(\mathrm{LWT}-\mathrm{EWT})
$$

The total flow rate enthalpy, $F_{\text {Total }} C_{\mathrm{P}}$ without considering the chilled water integration is amounted at $3115.02 \mathrm{~kJ}^{\circ} \mathrm{C}^{-1}$, while the total enthalpy difference, $\Delta H_{\text {Total }}$ for the chilled water network is $22634.66 \mathrm{~kW}$.

\section{Chilled Water CAscade Analysis}

In the following section, a chilled water cascade analysis (CWCA) is demonstrated. The main objective of the chilled water cascade analysis is to establish the baseline for chilled water targets, i.e. minimum fresh chilled water (chilled water from chiller) for a process upon utilising available chilled water sources discharged from multiple units of AHU and MAU to meet its heat sinks. In order to achieve the targets, one has to fulfil, both the flow rate (in Cumulative Net Flowrate Heat Capacity) and heat load requirements (in temperature). This is to be done for all chilled water-using processes, which are being considered in the fulfilment of the respective heat sinks required.

In order to carry out a CWCA, the $F C_{\mathrm{P}}$ of EWT and LWT for each unit are listed in the sink column, $F_{j} \mathrm{C}_{\mathrm{P}}$ and source column, $F_{i} \mathrm{C}_{\mathrm{P}}$, respectively in an ascending order of temperature (shows in 'table 2'). By setting the chilled water supply temperature, $T_{\mathrm{CHW}, \mathrm{s}}$ at $6.67{ }^{\circ} \mathrm{C}$ as shown in the first row of temperature, $T$ column, minimum chilled water flowrate enthalpy $F_{\min } \mathrm{C}_{\mathrm{P}}$ is targeted at $2553.37 \mathrm{~kJ}{ }^{\circ} \mathrm{C}^{-1}$ with zero cumulative heat load. The chilled water pinch point temperature is located at $10{ }^{\circ} \mathrm{C}$. Equation (1) which was originally developed to calculate the AHU and MAU may be modified to calculate the chilled water return temperature, $T_{\mathrm{CHW}, \mathrm{r}}$ for the chilled water network system:

$$
\Delta H_{\text {system }}=F_{\min } \times \mathrm{C}_{\mathrm{P}} \times\left(T_{\mathrm{CHW}, \mathrm{s}}-T_{\mathrm{CHW}, \mathrm{r}}\right)
$$

By substituting $\Delta H=22634.66 \mathrm{~kW}$, the calculated $T_{\mathrm{CHW}, \mathrm{r}}$ is at $15.53^{\circ} \mathrm{C}$ when chilled water integration is considered. The fresh chilled water requirement is reduced from $745.22 \mathrm{~kg} / \mathrm{s}$ to $610.85 \mathrm{~kg} / \mathrm{s}$. Concurrently, energy savings is observed as less pumping work is required in the chilled water network since the total chilled water flowrate has been reduced.

\section{Chilled Water Network Design}

A network design that achieved the minimum chilled water flowrate target is shown in 'figure 2'. In this work, the chilled water network is designed based on the revised Nearest Neighbour Algorithm (NNA) that was originally developed to synthesise water network [15].

'Figure 3' also illustrates the chilled water network design in a conventional layout in the studied semiconductor plant, where fresh chilled water (CHW) and reuse/recycle chilled water flowrate distribution is illustrated.

\section{CONCLUSION}

In this work, a dual savings of water and energy, are observed once reuse/recycle chilled water scheme is implemented in the chilled water network. As the chilled water flowrate reduced in the chilled water network, the pumping cost of the chilled water is also reduced proportionally. However, further study is required to optimise the network design, which considers topological constraints of all AHUs and MAUs. As such, simultaneous optimization of chiller system and chilled water network could be carried out in future works. 
Table 1. AHU and MAU Chilled Water Limiting Data

\begin{tabular}{|c|c|c|c|c|c|c|c|}
\hline Building & Types & Unit & $\begin{array}{c}\text { EWT } \\
\left({ }^{\circ} \mathbf{C}\right)\end{array}$ & $\begin{array}{c}\text { LWT } \\
\left({ }^{\circ} \mathbf{C}\right)\end{array}$ & $\begin{array}{c}F \\
(\mathrm{~kg} / \mathrm{s})\end{array}$ & $\begin{array}{c}F C_{\mathbf{P}} \\
\left(\mathbf{k J}^{\circ} \mathrm{C}^{-1}\right)\end{array}$ & $\begin{array}{c}\Delta H \\
(\mathbf{k W})\end{array}$ \\
\hline \multirow{9}{*}{$\begin{array}{l}\text { Fabrication } \\
\qquad(\text { Fab })\end{array}$} & \multirow{5}{*}{ Cooling Coils } & F4-MAU 1 thru 6 & 6.67 & 17.7 & 228.66 & 955.80 & 10542.46 \\
\hline & & F3-AHU 1 & 6.67 & 16.67 & 19.27 & 80.55 & 805.49 \\
\hline & & F3-AHU 2 & 6.67 & 16.67 & 32.39 & 135.39 & 1353.90 \\
\hline & & F3-AHU 3 & 6.67 & 17.7 & 12.62 & 52.75 & 581.85 \\
\hline & & F3-AHU 4 & 6.67 & 16.67 & 13.14 & 54.93 & 549.25 \\
\hline & \multirow{4}{*}{$\begin{array}{l}\text { Dehumidification } \\
\text { coils }\end{array}$} & F4-MAU 7 thru 12 & 6.67 & 10.00 & 224.82 & 939.75 & 3129.36 \\
\hline & & F3-AHU 5 & 6.67 & 11.11 & 16.18 & 67.63 & 300.29 \\
\hline & & F3-AHU 6 & 6.67 & 10.5 & 31.24 & 130.58 & 500.13 \\
\hline & & F3-AHU 7 & 6.67 & 11.11 & 15.14 & 63.29 & 280.99 \\
\hline \multirow{3}{*}{ Chemical } & \multirow{3}{*}{ Cooling Coils } & C3-AHU 1 & 10.00 & 17.7 & 6.83 & 28.55 & 219.83 \\
\hline & & C3-AHU 2 & 8.00 & 17.7 & 9.98 & 41.72 & 404.65 \\
\hline & & C3-AHU 3 & 10.00 & 20.88 & 35.15 & 146.93 & 1598.57 \\
\hline \multirow{10}{*}{ Office } & \multirow{5}{*}{ Cooling Coils } & O4-AHU 1 & 15.00 & 20.00 & 18.20 & 76.08 & 380.38 \\
\hline & & O4-AHU 2 & 15.00 & 19.00 & 13.40 & 56.01 & 224.05 \\
\hline & & O4-AHU 3 & 15.00 & 20.00 & 10.10 & 42.22 & 211.09 \\
\hline & & O4-AHU 4 & 15.00 & 19.00 & 8.30 & 34.69 & 138.78 \\
\hline & & O3-AHU 5 & 15.00 & 20.00 & 6.20 & 25.92 & 129.58 \\
\hline & \multirow{5}{*}{ Cooling Coils } & O1-ACU 6 & 6.67 & 16.67 & 2.80 & 11.70 & 117.04 \\
\hline & & O1-ACU 7 & 6.67 & 16.67 & 2.80 & 11.70 & 117.04 \\
\hline & & O1-ACU 8 & 6.67 & 16.67 & 2.80 & 11.70 & 117.04 \\
\hline & & O1-ACU 9 & 6.67 & 16.67 & 1.50 & 6.27 & 62.70 \\
\hline & & O1-ACU 10 & 6.67 & 16.67 & 1.50 & 6.27 & 62.70 \\
\hline \multirow{2}{*}{ Utility } & \multirow[t]{2}{*}{ Cooling Coils } & U3-AHU 1 & 17.00 & 22.60 & 6.40 & 26.75 & 149.81 \\
\hline & & U3-AHU 2 & 17.00 & 24.01 & 25.80 & 107.84 & 755.64 \\
\hline Total & & & & & & $\begin{array}{c}F_{\text {Total }} C_{P} \\
=3115.02\end{array}$ & $\begin{aligned} & \Delta H_{\text {Total }} \\
= & 22634.66\end{aligned}$ \\
\hline
\end{tabular}

Table 2 Chilled Water Cascade Analysis

\begin{tabular}{|c|c|c|c|c|c|c|c|}
\hline$T$ & $\Delta T$ & $\Sigma \boldsymbol{F}_{j} \mathrm{C}_{\mathbf{P}}$ & $\Sigma F_{i} \mathrm{C}_{\mathrm{P}}$ & $\Sigma F_{i} \mathbf{C}_{\mathbf{P}}-\Sigma \boldsymbol{F}_{j} \mathbf{C}_{\mathbf{P}}$ & $\operatorname{Cum} F C_{P}$ & $\Delta H$ & $\operatorname{Cum} \Delta H$ \\
\hline \multirow[b]{2}{*}{6.67} & & & \multicolumn{5}{|c|}{$F_{\min } C_{P}=2553.37$} \\
\hline & \multirow{2}{*}{1.33} & 2528.31 & & -2528.31 & & \multirow{2}{*}{33.32} & \multirow[b]{2}{*}{33.32} \\
\hline 8 & & 41.72 & & -41.72 & 25.00 & & \\
\hline 10 & \multirow{2}{*}{$\begin{array}{c}2 \\
0.5\end{array}$} & 175.48 & 939.75 & 764.27 & -16.66 & -33.32 & \multirow{2}{*}{$\begin{array}{c}\mathbf{0 . 0 0} \\
\text { (PINCH) } \\
373.81\end{array}$} \\
\hline 10.5 & & & 130.58 & 130.58 & 747.61 & 373.81 & \\
\hline 11.11 & 0.61 & & 130.92 & 130.92 & 878.19 & 535.70 & 909.50 \\
\hline 15 & 3.89 & \multirow{2}{*}{234.92} & & -234.92 & 1009.11 & 3925.44 & 4834.94 \\
\hline 16.67 & 1.67 & & 318.52 & 318.52 & 774.19 & 1292.91 & 6127.85 \\
\hline 17 & 0.33 & \multirow{2}{*}{134.60} & & -13460 & 1092.71 & 360.59 & 6488.44 \\
\hline 17.7 & 0.7 & & 1078.82 & 1078.82 & 958.11 & 670.68 & \multirow{2}{*}{7159.13} \\
\hline 17.1 & 1.3 & & $10 / 0.02$ & 1070.02 & 2036.93 & 2648.01 & \\
\hline & \multirow[t]{2}{*}{1} & & 90.11 & $90 . / 1$ & 2127.64 & 2127.64 & 9807.14 \\
\hline 20 & & & 144.21 & 144.21 & F & & 11934.77 \\
\hline 20.88 & 0.88 & & 146.93 & 146.93 & 2271.85 & 1999.23 & 13934.00 \\
\hline & 1.72 & & & & 2418.77 & 4160.29 & \\
\hline 22.6 & & & 26.75 & 26.75 & & & 18094.29 \\
\hline 24.01 & 1.41 & & 107.84 & 107.84 & 2445.53 & 3448.19 & 21542.48 \\
\hline & & & & & 2553.37 & & \\
\hline
\end{tabular}




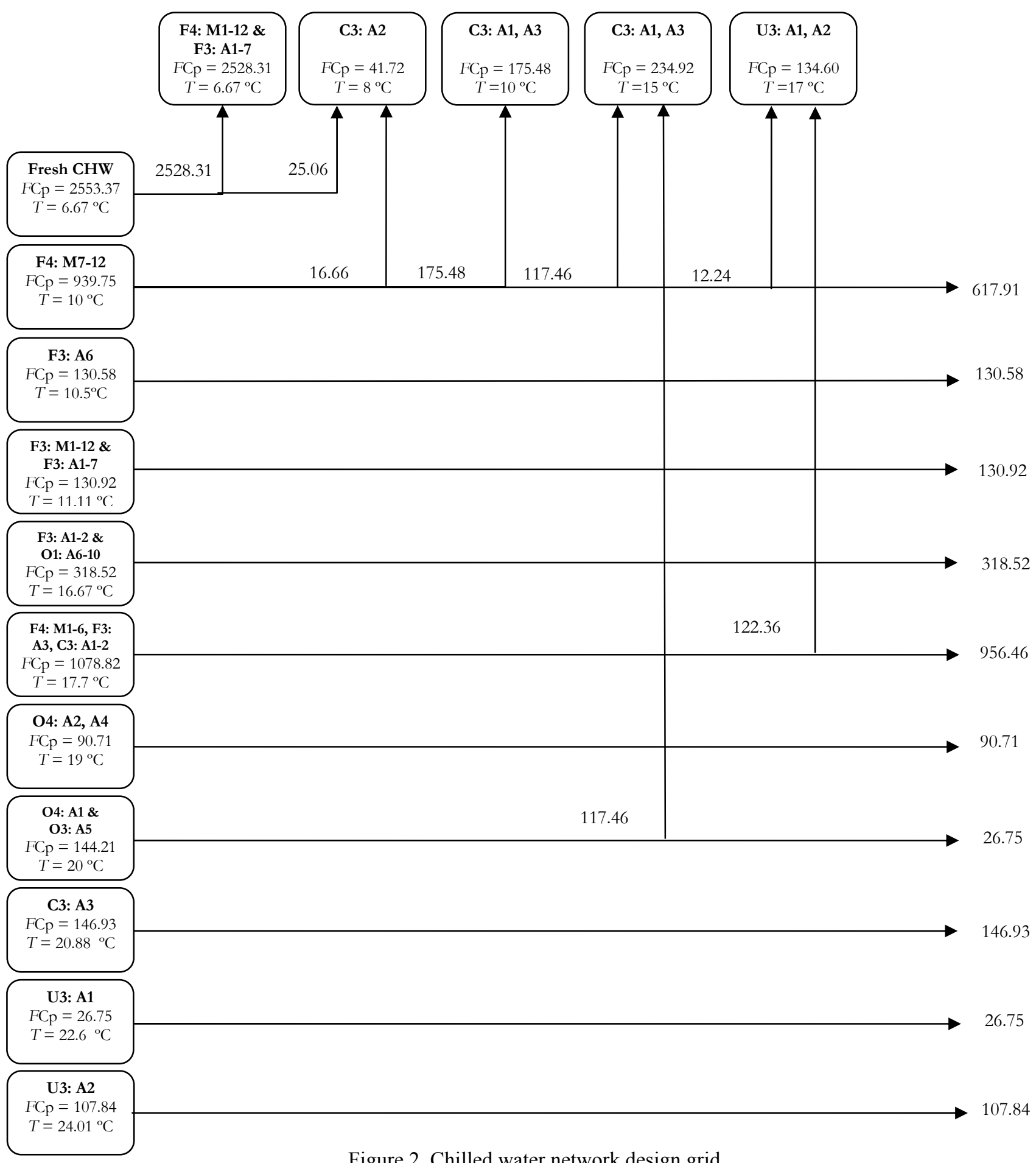

Figure 2. Chilled water network design grid 


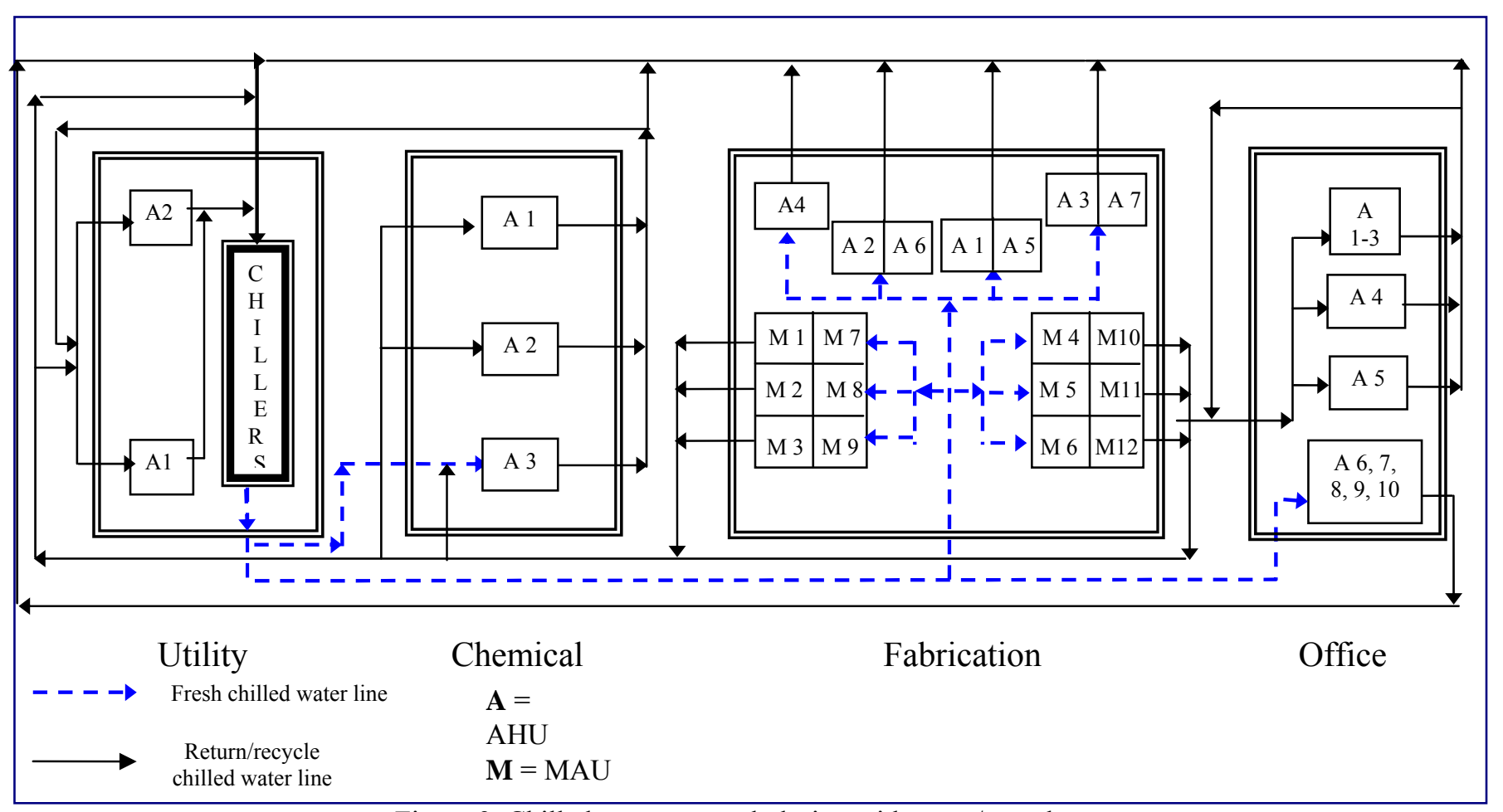

Figure 3. Chilled water network design with reuse/recycle

\section{ACKNOWLEDGEMENT}

The corporation and assistance from various technical personnel and management of SilTerra Malaysia, Kulim, Kedah, is highly appreciated in providing the field data for this research study.

\section{REFERENCES}

[1] Microchip Fabrication, $5^{\text {th }}$ ed., McGraw-Hill, New York, 2004.

[2] Y. C. Chang. (2006, Sept). "An innovative approach for demand side management-optimal chiller loading by simulated annealing." Energy, vol. 31, pp. 1883-1896.

[3] K.F. Fong, V. I. Hanby, T. T. Chow. (2006). "HVAC system optimization for energy management by evolutionary programming," Energy and Buildings, vol. 38, pp. 220-231.

[4] Y. C. Chang. (2007). "Optimal chiller loading by evolution strategy for saving energy," Energy and Buildings, vol. 39, pp. 437-444.

[5] Y. C. Chang. (2005). "Genetic algorithm based optimal chiller loading for energy conservation," Applied Thermal Engineering, vol. 25, pp. 2800-2815.

[6] B. Linhoff, and E. Hindmarsh. (1983). "The pinch design method for heat exchanger network," Chemical Engineering Science, vol. 38, no. 5, pp. 745-763.

[7] F. Marechal, and B. Kalitventzeff. (1996). "Targeting the minimum cost energy requirements. A new graphical technique for evaluating the integration of utility systems," Computers chemical Engineering. vol. 20, pp. S225-S230.

[8] L. Matijaseviæ, and H. Otmaeiæ. (2002). "Energy recovery by pinch technology," Applied Thermal Engineering, vol. 22, pp. 477-484.

[9] V. Agrawal, and U. V. Shenoy. (2006). "Unified Conceptual Approach to Targeting and design of water and hydrogen networks," AIChE Journal, vol. 52, no. 3, pp. 1071-1081.

[10] A. M. Almutlaq, V. Kazantzi, and M. M. El-Halwagi. (2005). “An algebraic approach to targeting waste discharge and impure fresh usage via material recycle/reuse networks," Clean Technologies and Environmental Policy, vol. 7, no. 4, pp. 294-305.

[11] S. Bandyopadhyay, M. D. Ghanekar, and H. K. Pillai. (2006). "Process water management," Industrial and Engineering Chemistry Research, vol. 45, pp. 5287-5297.

[12] S. Bandyopadhyay. (2006). "Source composite curve for waste reduction," Chemical Engineering Journal, vol. 125, pp. 99-110.

[13] Z. A. Manan, Y. L. Tan, and D. C. Y. Foo. (2004). "Targeting the minimum water flowrate using water cascade analysis technique," AIChE Journal, vol. 50, no. 12, pp. 3169-3183.
[14] D. C. Y. Foo, Z. A. Manan, and Y. L. Tan. (2006, July). "Use cascade analysis to optimize water networks," Chemical Engineering Progress, vol. 102, no. 7, pp. 45-52.

[15] R. Prakash, and U. V. Shenoy. (2005). "Targeting and design of water networks for fixed flowrate and fixed contaminant load operations," Chemical Engineering Science, vol. 60, no. 1, pp. 255-268.

[16] Y. P. Wang, and R. Smith. (1994). "Wastewater minimisation," Chemical Engineering Science, vol. 49, pp. 981-1006.

[17] M. M. El-Halwagi, F. Gabriel, and D. Harell. (2003). "Rigorous graphical targeting for resource conservation via material recycle/reuse networks," Industrial \& Engineering Chemistry Research, vol. 42, pp. 4319-4328.

[18] D. C. Y. Foo. (2007). "Cascade analysis for impure fresh feed and multiple external water sources," Chemical Engineering Research and Design, vol. 85, no. A8, pp. 1169-1177.

[19] N. Hallale. (2002). "A new graphical targeting method for water minimisation," Advances in Environmental Research, vol. 6, no. 3, pp. 377-390.

[20] J. J. Alves, and G. P. Towler, (2002). "Analysis of refinery hydrogen distribution systems," Industrial and Engineering Chemistry Research, vol. 41, 5759- 5769 .

[21] T. K. Zhelev. (2004). "On the integrated management of industrial resources incorporating finances. Sustainables systems theory: ecological and other aspects," Journal of Cleaner Production, vol. 12, pp. 469-74.

[22] M. Subramaniam, "Synthesis of chilled water network," MSc. dissertation, Dept. Chemical. Eng., University Technology of Malaysia, 2007. 


\title{
Tensile Behavior of Hybrid Natural/Glass Fibers Reinforced Composites at Different Temperatures
}

\author{
Wan Noor Azira Wan Yaakob and Al Emran Ismail
}

\begin{abstract}
Nowadays, natural fiber reinforced composites are given bright opportunities to replace synthetic fiber reinforced composites especially in automotive sectors. Natural fibers offer considerable high strength, low weight and less abrasive effect. But this fiber is incompatible to polymeric material which leads to interfacial bonding problems. To encounter this problem, hybridization between natural and synthetic fibers are formulated and tested. In this work, coir and glass fibers are blended mechanically with polypropylene granular to homogenize the composite mixture. Both fibers are chemically treated to enhance the bonding with matrix material. It is then extruded into identical dimensions and shape (dog-bone tensile specimen). Several different temperatures are selected ranging from room temperature to $100^{\circ} \mathrm{C}$ and the specimens are quasi-statically stressed at constant cross-head displacement $1.5 \mathrm{~mm} / \mathrm{min}$. It is found that the interaction between fiber hybridizations and temperatures played an important role in determining the tensile behavior of this composite. Scanning electron microscope (SEM) is used to observe the fracture surfaces of these samples after tensile tests. Different toughening mechanisms are observed such as fiber pull-out and interfacial detachments which are depend on the temperature and fiber volume fraction.
\end{abstract}

Keywords: natural reinforced composites, fiber hybridizations, tensile behavior, high temperatures, interfacial debonding

\section{INTRODUCTION}

The utilization of the natural fiber reinforced composites in the automotive and furniture sectors are not new and this composite is increasingly used from time to time. Most of these composites are used as load bearing application parts. This is because the composites have considerable low strength of mechanical strength. The low strength is due to the lack of interfacial bonding between cellulosic fiber and polymeric matrix material [1]-[3]. Generally to enhance the interface bonding between fibers and matrix, coupling agent is used. The natural fibers are treated with the chemical solution in order to create a layer of silane on the fibers surface.

Therefore it will increase the wetability behavior of the

A. E Ismail is the staff of the Faculty of Mechanical \& Manufacturing Engineering, Tun Hussein Onn University of Malaysia. Parit Raja, Batu Pahat, 86400 Johor, Malaysia. (e-mail: emran@uthm.edu.my).

W. N. A. W Yaakob is a student study for a degree of mechanical engineering at this University. matrix resin with the fibers. On the other hand, hybridization of the both natural and glass fibers is introduced to overcome the low composite strength if the composite is reinforced with natural fiber alone. This process is hoped to increase the composites sustainability under any loading condition. This present work explores the effect of hybridization of both natural and glass fibers at considerable high temperatures. Different temperature ranges are selected in order to study the tensile response of the hybrid composites. Fracture surface of the samples are also investigated to study the toughening mechanisms of the fibers for all temperatures and fiber hybridization differences.

\section{MATERIAL AND METHODS}

Coir fibers are obtained locally in Batu Pahat, Johor, Malaysia. These fibers experienced a purification process where the fibers were soaked in 5\% sodium solution and mechanically stirred for 1 hour. This process was conducted in order to remove the unwanted layers on the fiber surfaces. Then, treated fibers were dried in furnace environment at constant temperature $80^{\circ} \mathrm{C}$ for 24 hour to make sure the cellulosic fibers were completely dried. The removal of unwanted fiber layers is to improve the stress transfer from the matrix to the fibers. In order to enhance the wetability behavior upon the fibers, fibers were treated again with silane coupling agent. $10 \%$ silane solution was prepared with methanol in weight. Coupling agent is normally used to enhance the compatibility between the fibers and matrix. Sodium treated fibers were immersed into silane solution for 30 minutes at room temperature and it was mechanically stirred at constant speed. Stirring process was carried out to homogenize the distribution of the silane onto the fiber surfaces. Then, they were dried in furnace at temperature $50^{\circ} \mathrm{C}$ for 12 hours. The as-received glass fibers do not experienced any chemical treatment and it was assumed that the fiber surfaces were free from grease or oil. Both fibers (natural and glass fibers) were cut into specific length of about $10 \mathrm{~mm}$. Fibers and polypropylene granular were mixed together using ball milling for 3 hours to get homogenized composite mixture and melted before they were feed into extrusion machine. In this work, extrusion parameters were kept constant throughout the process such feeding and extrusion rate and temperature. Then, it was extruded into identical standard samples shape and 
dimension. After 24 hours, the samples were removed from the mould and they were cured in an atmospheric environment and under controlled relative humidity about $55 \%$ for another 24 hours in order to have better composite hardness and shrinkage. Different fiber volume fractions are selected such as 5, 10, 15 and 20\%. Hybridization of the fibers is conducted by using half of natural fibers and other half for glass fibers. Tensile test was conducted using universal testing machine using constant cross-head displacement $1.5 \mathrm{~mm} / \mathrm{min}$. Four heating temperatures were selected including room temperature, 50, 75 and $100^{\circ} \mathrm{C}$. Before any tensile test, the samples were heated for 1 hour. The samples were stressed until the fracture to occur and stress-strain curves were extracted from these tests. The results obtained from the tests were related to temperatures, fiber volume fractions and fracture mechanisms. Scanning electron microscope (SEM) was used to observe the fracture surfaces of the samples under quasi-static loading.

\section{RESULTS AND DISCUSSION}

\section{A. The Effect of Temperature on the Elastic Modulus}

'Figure 1.0' shows the influence of temperature on the elastic modulus. At room temperature $\left(25^{\circ} \mathrm{C}\right)$, the effect of fiber volume fractions are not very significant. Increasing the fiber fractions reduce the elastic modulus, this finding is well agreed with previous research works [1]-[3]. When the heating temperature increases up to $50^{\circ} \mathrm{C}$, large elastic modulus reduction is detected. The behavior may be due to breaking of the Si-O-Si bond on the fibers to the matrix as reported by M.M Thwe and Kin L. (2003). When the interfacial bonding was break, the efficiency of the stress transfer is lowered and therefore reducing the composite strength. 'Figure 2.0' reveals the fracture surface of the composite heated at $50^{\circ} \mathrm{C}$ under tensile loading. At low magnification, longer pull-out fibers are observed and indicated the ineffectiveness of the stress transfer between matrix to the fibers.

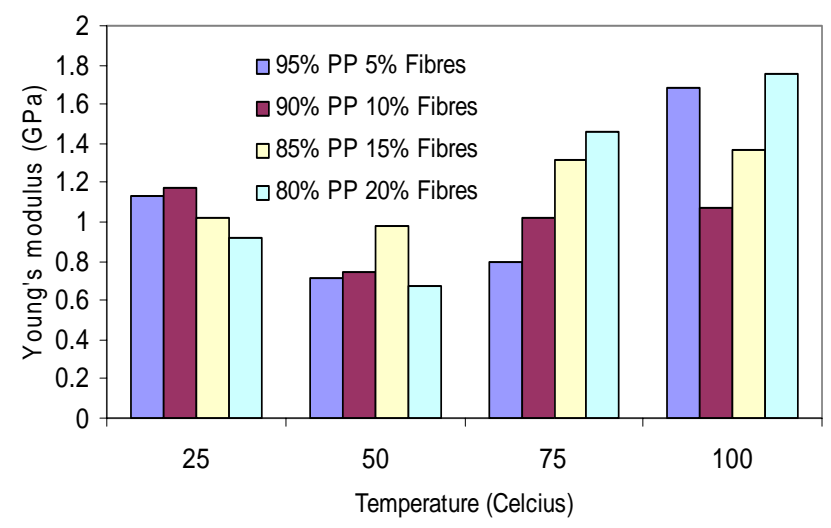

Figure 1. The effect of temperature on the modulus of elasticity at different fiber volume fractions

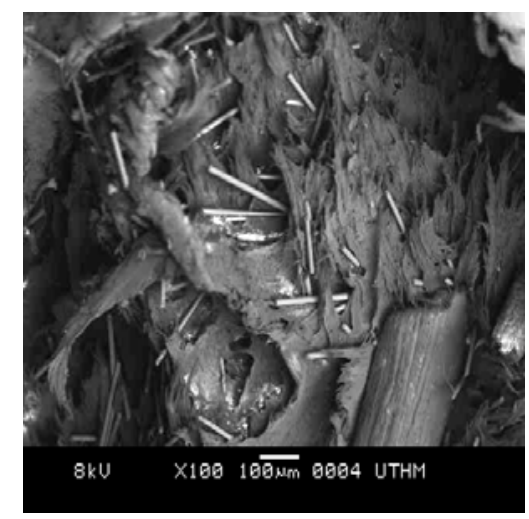

Figure 2. Fracture surface of $2.5 \%$ fiber volume fraction at $50^{\circ} \mathrm{C}$ fiber

\section{B. The Effect of Temperature on the Maximum Load}

'Figure 3.0' shows the effect of temperatures on the maximum load for different fiber volume fractions. It can be seen here that, the temperatures have played an important role in increasing the mechanical strength. But at higher temperature $\left(100^{\circ} \mathrm{C}\right)$, significant composite strength reduction occurred especially for high content of fiber fractions. When the fibers are exposed to the high temperature, they tend to shrink themselves and this will leave some spaces between matrix and fibers interfaces. Stress would concentrate at these sites and degrade the composite integrity. 'Figure 3.0' also indicates that when the $10 \%$ fiber volume fraction composites are stressed at temperature $75^{\circ} \mathrm{C}$, the composite strength suddenly increased. The increment can be related to the toughening mechanism that is observed in 'figure 4.0'. From this figure obviously show that shorter pull out fibers are observed indicating the significance of stress transfer from the matrix to the fibers.

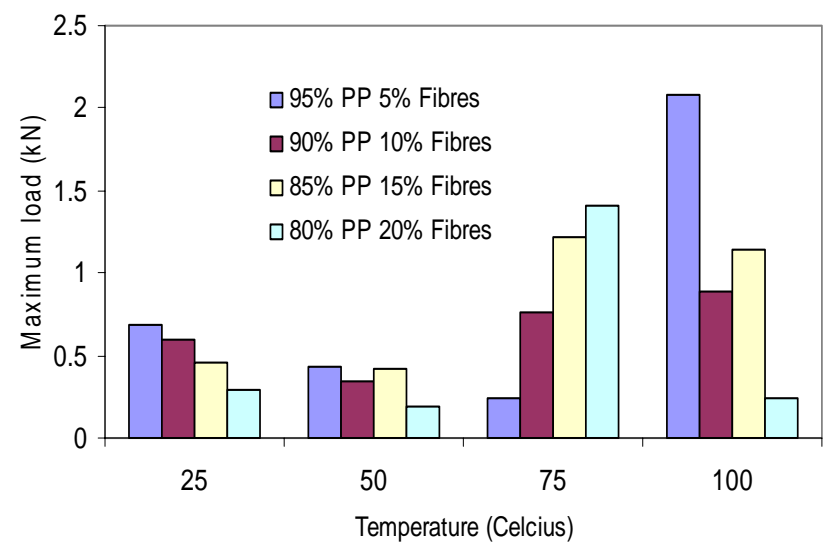

Figure 3. The effect of temperature on the maximum load for different fiber volume fractions 


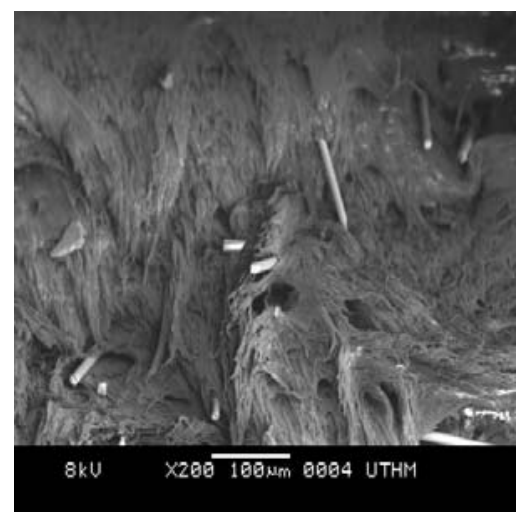

Figure 4. The fracture surface of the $10 \%$ fiber volume fraction reinforced composites at $75^{\circ} \mathrm{C}$

\section{The Effect of Fiber Volume Fraction on the Ultimate Tensile Strength.}

'Figure 5.0' shows that the significant role of fiber volume fractions on the ultimate tensile strength. It can be seen here that at lower temperatures (less than $50^{\circ} \mathrm{C}$ ), heating temperatures not played to modify the tensile strength of the composites. While when the composites are exposed to the higher temperatures large tensile strength reduction occurred when the fiber volume fractions increased. High temperature responsible to reduce the moisture content of the fibers and therefore reducing the bonding at the interfacial boundary between the matrix and the fibers. 'Figure 6.0' reveals the fracture surface of the $20 \%$ fiber volume fraction reinforced composites. From this micrograph, glass fibers are well embedded in the matrix while for the natural fiber (bigger in size), the bonding incompatibility between fibers and matrix is not strong enough to support shear force from the matrix to the fibers.

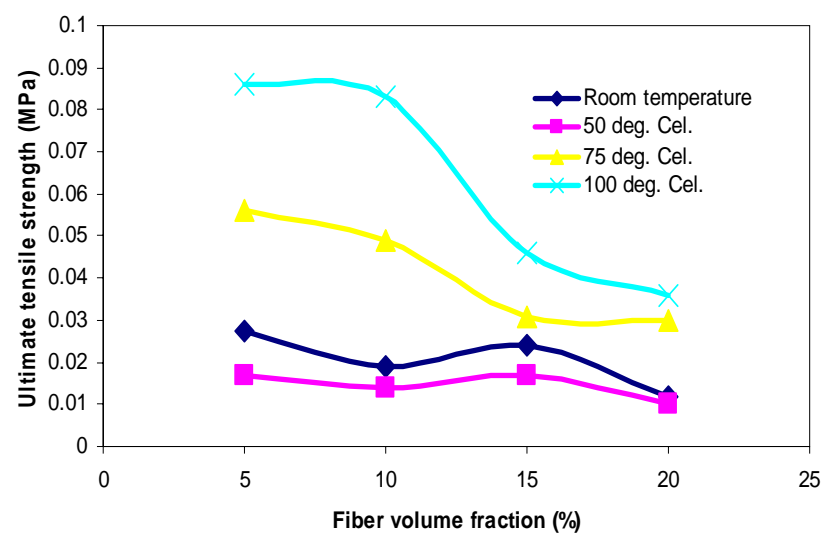

Figure 5. The effect of fiber volume fraction on the ultimate tensile strength for different heating temperatures

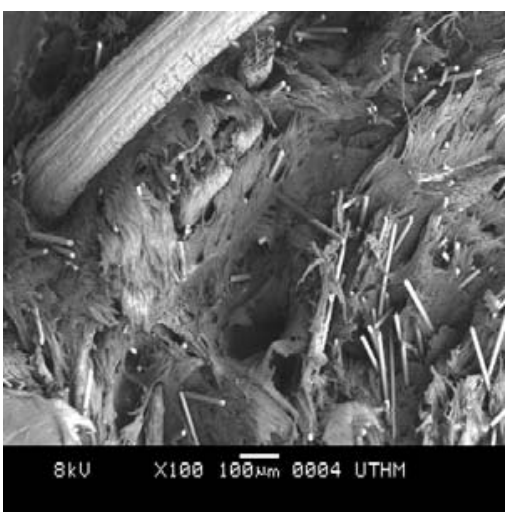

Figure 6 . The fracture surface of the $20 \%$ fiber volume fraction reinforced composites at $100^{\circ} \mathrm{C}$

\section{CONCLUSION}

The main objective of this study is to gage the effect of hybridization of natural and glass fibers reinforced polypropylene using extrusion technique. The conclusions based on this study are as follows:

1. It was found that a threshold temperature was $50^{\circ} \mathrm{C}$, below this temperature the mechanical strength is reduced while higher that this temperature, the mechanical strength is increased.

2. The composite strength increased when the fiber volume fraction is increased especially at higher temperatures.

3. From the SEM observation, it was found that the bonding compatibility between natural fibers and the matrix was very poor but addition of glass fibers give an advantage of increasing the composite strength.

4. Glass fiber worked as micro-toughening effect in the composite to prevent sudden failure.

\section{REFERENCES}

[1] Gayer U. and Schuh T.H. (1996). Automotive Application of Natural Fibers Composite, First International Symposium on Lignocellulosic Composites, UNESP-Sao Paulo State University Schlösser Th., Gayer U. and Karrer G. (1999). Technischer Bericht 0003-98 DaimlerChrysler AG

[2] Thwe M.M, Kin Liao. (2003). Durability of bamboo-glass fiber reinforced polymer matrix hybrid composites. Composites Science and Technology Vol. 63:375 - 387.

[3] Chand N., Hashmi S.A.R. (1993). Mechanical properties of sisal fiber at elevated temperature. Journal of Material Science Vol. 28:6724 6732 . 


\title{
A Comparison of Wastewater Treatment Systems for Small Sites in Sarawak
}

\author{
F.E. Tang, C.L.I. Ho and A.H. Tan
}

\begin{abstract}
Feasible, affordable and sustainable solutions for wastewater management in Sarawak need to be explored in order to prevent high additional loads to water bodies such as rivers. Wastewater should be managed in an efficient and optimized way, appropriate to the location or site. Environmental sustainability, including energy use and performance of the technology in removing conventional wastewater constituents need to be considered to determine feasibility of adopting a particular technology to a community. Wastewater generation patterns for small sites may be different from the established domestic generation patterns. This would help the design and planning of the wastewater treatment system. In this study, the wastewater generation pattern for an institution of higher learning was performed by monitoring water consumption. Various existing technologies adopted in Sarawak for both domestic and commercial wastewater treatment are also explored and compared. Several treatment systems are found to be appropriate and effective for small sites, with varying costs and energy use. The data obtained from this study could be a useful reference data for other similar institutions of higher learning in designing their wastewater treatment system.
\end{abstract}

Keywords: maximum wastewater generation, wastewater treatment, treatment system characteristics

\section{INTRODUCTION}

Malaysia's 26 million people generate about six million tons of sewage annually (Asian Development Bank 2006). This sewage is treated or partially treated and discharged into the rivers, from which $98 \%$ of Malaysians' fresh water supply comes from. A more effective and sustainable wastewater management is urgently required to avoid further damage to water resources.

While the capital of the State of Sarawak, Kuching city is experiencing explosive development, this has progressivel degraded the quality of its surrounding environment. A river quality baseline study for Sarawak River in Kuching showed that the river was seriously polluted with faecal y(Larsen \& Lynghus 2004). This study should be an alarm call to the authorities to scrutinise and re-consider the current wastewater management system for the city and for the whole state of Sarawak. Therefore there is a need to

F.E. Tang and C.L..I. Ho are with Curtin University of Technology Sarawak Campus, CDT 250, 98009 Miri Sarawak. (Tel: +60 85443939 fax: +60 85 443837:e-mail: tang.fu.ee@ curtin.edu.my).

A.H. Tan was an undergraduate student at Curtin University of Technology Sarawak Campus. explore all affordable, sustainable and feasible solutions for wastewater management in Sarawak, to improve hygienic and environment quality by using least possible cost and resources. It is important to have a wastewater treatment system that is appropriate in scale, cost, efficiency and flexibility.

This paper investigates different wastewater treatment systems used in Sarawak, particularly in the cities of Kuching, Miri and Bintulu. The methods here include nonmechanical treatment systems such as oxidation ponds, and electro-mechanical systems such as open aeration. For the principle treatment processes for each method, please refer to Tan, et al. (2007). The various systems are presented and compared in terms of cost, capacity and land use; operation and maintenance; environmental impact and energy utilization. Even though public health is often the priority in wastewater treatment for developing countries (Williams et al., 1995), the suitable allocation of optional treatment systems and the energy saving technology are important issues that must be considered for wastewater treatments on a small scale or in lower-density areas (Ukita et al., 1993).

In addition, this paper demonstrates quantification of wastewater for an institution of higher learning in Malaysia based on its water consumption. For new institutions of similar type in Malaysia, the data developed from this project will be a useful reference for estimating wastewater flow in the planning and design of their wastewater treatment facilities.

\section{Methodology}

A desktop study of several wastewater treatment systems currently being used in Sarawak to the authors' knowledge was undertaken. In addition, site visits were made to investigate the different wastewater treatment systems in Kuching, Miri and Bintulu. This included sites such as Wisma Sesco, Normah Medical Specialist, and University Malaysia Sarawak campus in Kuching, Curtin University Sarawak Campus, in Miri and Bintulu City. In addition, to collect data and information, interviews have been carried out with staff from local councils, government regulatory bodies and the like. The engineers, maintenance technicians or personnel in-charge of the wastewater treatment plant were approached and queried.

A study into wastewater generation was also carried out at Curtin University Sarawak Campus. Water consumption is used in many international surveys as an indicator of wastewater generation (Larsen \& Lynghus 2004, 
Tchobanoglous, Burton \& Stensel 2004). The fundamental assumption is that tap water consumed equals wastewater that will be discharged into the wastewater treatment system. Therefore, this same approach is adopted in the wastewater generation study in Curtin University Sarawak Campus. The water meters in the campus were monitored, and readings from the water meters, in units of litres, were taken every day from morning to night (7am to $9 \mathrm{pm}$ ), for time intervals of $1-3$ hours. These readings were collected everyday for the duration of a week, representing one wastewater generation data set. A total of three data sets were collected from the campus water meters for the study, during two typical teaching weeks and one semester break. The three periods are chosen in order to investigate the difference in water consumption during regular sessions when classes are carried out as well as when classes are not in session to show seasonal variations in water consumption. The collected readings were then presented.

\section{COMPARISON OF VARIOUS WASTEWATER TREATMENT SYSTEMS}

The various wastewater treatment systems explored in this study include Individual Septic Tanks (ISTS), Imhoff tanks, oxidation and aerated ponds, open extended activated sludge system, open aeration system, ecological sanitation (ecosan) system, Hi-Kleen system and Intermittent Decanted Extended Aeration (IDEA) system. The selection of a suitable wastewater treatment system should include such criteria as cost, capacity, land requirements, environmental aspects such as environmental impact, and energy and chemical usage, and efficiency and reliability of the plant. Principle treatment processes for each of these methods are presented in Tan, 2006 and Tan, et al., 2007. These systems have been individually studied, and a summary of their characteristics are presented in Tables 1 to 3 .

\section{ENERGY UTILIZATION AND SUSTAINABILITY}

Energy utilization for the various wastewater systems used in Sarawak can range from minimal energy consumption to a significant level depending on the intensity of mechanical aeration. Mechanical systems such as activated sludge systems utilize physical, chemical and biological mechanisms to treat wastewater, which generally requires high operational and energy costs. Pond systems primarily utilize physical and biological processes, and land treatment systems utilizes soil and plants (Metcalf and Eddy, 2003).

The method adopted must be cost effective and in addition, the environmental impact of the system also needs to be considered to determine sustainability of the system. As seen from Tables 1, 2 and 3, a comparison between the various treatment systems was presented.
Table 1. Comparison of different wastewater treatment systems in terms of cost, capacity and land use

\begin{tabular}{|c|c|c|}
\hline $\begin{array}{l}\text { Wastewater } \\
\text { treatment facility }\end{array}$ & $\begin{array}{l}\text { Capital, } \\
\text { operational and } \\
\text { maintenance costs }\end{array}$ & $\begin{array}{l}\text { Capacity and } \\
\text { land use }\end{array}$ \\
\hline \begin{tabular}{l}
\multicolumn{2}{l}{ Individual } \\
Septic Tanks \\
(ISTs)
\end{tabular} & $\begin{array}{l}\text { - Most } \\
\text { economic, low } \\
\text { capital costs } \\
\text { - High durability } \\
\text { - Maintenance } \\
\text { cost mainly } \\
\text { due to } \\
\text { desludging }\end{array}$ & $\begin{array}{l}\text { - Up to } 150 \mathrm{PE} \\
\text { - Little space } \\
\text { required as it } \\
\text { is } \\
\text { constructed } \\
\text { underground }\end{array}$ \\
\hline Imhoff Tanks & $\begin{array}{l}\text { - Slightly higher } \\
\text { capital and } \\
\text { maintenance } \\
\text { costs than IST }\end{array}$ & $\begin{array}{l}\text { - Up to } 1000 \mathrm{PE} \\
\text { - Little space } \\
\text { required }\end{array}$ \\
\hline Oxidation ponds & $\begin{array}{l}\text { - Cost effective } \\
\text { - Simple } \\
\text { construction } \\
\text { - Minimum } \\
\text { maintenance } \\
\text { and operations } \\
\text { costs }\end{array}$ & $\begin{array}{l}\text { - Can serve all } \\
\text { PE } \\
\text { - Large areas of } \\
\text { reasonably } \\
\text { flat, low } \\
\text { permeability } \\
\text { areas required }\end{array}$ \\
\hline $\begin{array}{l}\text { Ecological } \\
\text { Sanitation } \\
\text { System (ecosan) }\end{array}$ & $\begin{array}{l}\text { - Economic } \\
\text { capital, } \\
\text { operation and } \\
\text { maintenance } \\
\text { costs } \\
\text { - Reasonably } \\
\text { simple and low- } \\
\text { cost } \\
\text { construction } \\
\text { - Desludging } \\
\text { expenses } \\
\text { required for off- } \\
\text { site blackwater } \\
\text { treatment }\end{array}$ & $\begin{array}{l}\text { - Little space } \\
\text { required, with } \\
\text { underground } \\
\text { sludge holding } \\
\text { tank }\end{array}$ \\
\hline Aerated pond & $\begin{array}{l}\text { - Similar to } \\
\text { Oxidation pond, } \\
\text { with higher } \\
\text { operation and } \\
\text { maintenance } \\
\text { costs due to } \\
\text { electricity } \\
\text { consumption }\end{array}$ & $\begin{array}{l}\text { - Lower land } \\
\text { requirement } \\
\text { than oxidation } \\
\text { pond }\end{array}$ \\
\hline Hi-Kleen system & $\begin{array}{l}\text { - High capital, } \\
\text { operation and } \\
\text { maintenance } \\
\text { costs }\end{array}$ & $\begin{array}{ll}\text { - Flexibility in } \\
\text { upgrading } \\
\text { plant } \\
\text { increasing } & \text { to } \\
\text { population } & \end{array}$ \\
\hline $\begin{array}{l}\text { Open Extended } \\
\text { Activated } \\
\text { Sludge System, } \\
\text { Open Aeration } \\
\text { System and } \\
\text { Intermittent } \\
\text { Decanted } \\
\text { Extended } \\
\text { Aeration (IDEA) } \\
\text { System }\end{array}$ & $\begin{array}{l}\text { - High capital, } \\
\text { operation and } \\
\text { maintenance } \\
\text { costs } \\
\text { - Maintenance } \\
\text { expenses } \\
\text { contribute to the } \\
\text { key life-span } \\
\text { expenses }\end{array}$ & $\begin{array}{l}\text { - Can serve all } \\
\text { PE }\end{array}$ \\
\hline
\end{tabular}


Table 2. Comparison of different wastewater treatment systems in terms of environmental aspects; energy and chemical consumption

\begin{tabular}{|c|c|c|}
\hline $\begin{array}{l}\text { Wastewater } \\
\text { treatment facility }\end{array}$ & $\begin{array}{l}\text { Energy and } \\
\text { Chemical } \\
\text { Consumption }\end{array}$ & \begin{tabular}{l}
\multicolumn{2}{l}{ Environmental } \\
and social \\
impacts
\end{tabular} \\
\hline \begin{tabular}{l}
\multicolumn{2}{l}{ Individual } \\
Septic Tanks \\
(ISTs)
\end{tabular} & $\begin{array}{l}\text { - Zero energy } \\
\text { consumption } \\
\text { - No mechanical } \\
\text { equipment nor } \\
\text { chemical } \\
\text { usage }\end{array}$ & $\begin{array}{l}\text { - Discharging } \\
\text { partially } \\
\text { treated } \\
\text { effluent into } \\
\text { local drains } \\
\text { create odour } \\
\text { nuisance and } \\
\text { health risk, } \\
\text { and pollute } \\
\text { receiving } \\
\text { water body }\end{array}$ \\
\hline Imhoff Tanks & $\begin{array}{l}\text { - Similar to IST } \\
\text { unless pump is } \\
\text { used }\end{array}$ & - Same as IST \\
\hline Oxidation ponds & $\begin{array}{l}\text { - Zero energy } \\
\text { consumption } \\
\text { - Suited to } \\
\text { tropical climates } \\
\text { - No chemical } \\
\text { usage }\end{array}$ & $\begin{array}{l}\text { - Odour } \\
\text { nuisance, } \\
\text { potential } \\
\text { mosquito } \\
\text { breeding } \\
\text { habitat } \\
\text { - Not suitable } \\
\text { near housing } \\
\text { areas } \\
\end{array}$ \\
\hline $\begin{array}{l}\text { Ecological } \\
\text { Sanitation } \\
\text { System (ecosan) }\end{array}$ & $\begin{array}{l}\text { - Zero energy } \\
\text { consumption } \\
\text { - No chemical } \\
\text { usage } \\
\text { - For blackwater, } \\
\text { zero energy } \\
\text { consumption for } \\
\text { sludge holding } \\
\text { tank, but } \\
\text { electricity } \\
\text { consumed for } \\
\text { off-site sludge } \\
\text { treatment plant } \\
\text { - Potential for } \\
\text { generating } \\
\text { energy if biogas } \\
\text { generation or } \\
\text { similar plants } \\
\text { are used }\end{array}$ & $\begin{array}{l}\text { - } \text { No odour } \\
\text { nuisance, no } \\
\text { visual } \\
\text { pollution, zero } \\
\text { emission of } \\
\text { greenhouse } \\
\text { gases } \\
\text { - Reduces } \\
\text { pollution of } \\
\text { receiving } \\
\text { water body } \\
\text { - Resources } \\
\text { conserved via } \\
\text { recycling } \\
\text { - Eliminates } \\
\text { large } \\
\text { quantities of } \\
\text { blackwater }\end{array}$ \\
\hline Aerated pond & $\begin{array}{l}\text { - Higher energy } \\
\text { consumption } \\
\text { than Oxidation } \\
\text { Pond }\end{array}$ & $\begin{array}{l}\text { - Similar to } \\
\text { Oxidation } \\
\text { Pond }\end{array}$ \\
\hline Hi-Kleen system & $\begin{array}{l}\text { - Electro- } \\
\text { mechanical } \\
\text { treatment } \\
\text { technology, } \\
\text { with high } \\
\text { energy } \\
\text { consumption } \\
\text { - Chlorine may } \\
\text { be used for } \\
\text { disinfection }\end{array}$ & $\begin{array}{l}\text { - } \text { Enclosed } \\
\text { system with no } \\
\text { odour and } \\
\text { insect } \\
\text { problems }\end{array}$ \\
\hline
\end{tabular}

\begin{tabular}{|c|c|c|}
\hline & process & \\
\hline $\begin{array}{l}\text { Open Extended } \\
\text { Activated } \\
\text { Sludge System, } \\
\text { Open Aeration } \\
\text { System and } \\
\text { Intermittent } \\
\text { Decanted } \\
\text { Extended } \\
\text { Aeration (IDEA) } \\
\text { System }\end{array}$ & $\begin{array}{l}\text { - Electro- } \\
\text { mechanical } \\
\text { treatment } \\
\text { technology, } \\
\text { with high } \\
\text { energy } \\
\text { consumption } \\
\text { - Chlorine may } \\
\text { be used for } \\
\text { disinfection }\end{array}$ & $\begin{array}{l}\text { - Create odour } \\
\text { and noise } \\
\text { nuisances } \\
\text { - Visually } \\
\text { unappealing } \\
\text { - Partially } \\
\text { enclosed } \\
\text { (IDEA) }\end{array}$ \\
\hline
\end{tabular}

Table 3. Comparison of different wastewater treatment systems in terms of efficiency; operation and maintenance

\begin{tabular}{|c|c|c|}
\hline $\begin{array}{l}\text { Wastewater } \\
\text { treatment facility }\end{array}$ & $\begin{array}{ll}\text { Efficiency } & \text { and } \\
\text { reliability } & \text { of } \\
\text { treatment } & \end{array}$ & $\begin{array}{l}\text { Operation, } \\
\text { maintenance and } \\
\text { sustainability }\end{array}$ \\
\hline $\begin{array}{l}\text { Individual } \\
\text { Septic Tanks } \\
\text { (ISTs) }\end{array}$ & $\begin{array}{l}\text { - Only primary } \\
\text { treatment, } \\
\text { with low } \\
\text { efficiency and } \\
\text { inconsistent } \\
\text { performance } \\
\text { - Dissolved and } \\
\text { suspended } \\
\text { solids leaves } \\
\text { the tank } \\
\text { untreated } \\
\text { - Efficiency } \\
\text { dependent on } \\
\text { capacity of } \\
\text { community } \\
\text { served and } \\
\text { quantity of } \\
\text { sludge settled } \\
\text { in tank } \\
\text { - Produces a } \\
\text { large quantity } \\
\text { of sludge }\end{array}$ & $\begin{array}{l}\text { - Simplest } \\
\text { facility and } \\
\text { maintenance, } \\
\text { without } \\
\text { needing } \\
\text { technical } \\
\text { supervision } \\
\text { - Only } \\
\text { maintenance } \\
\text { required is } \\
\text { desludging, } \\
\text { carried out } \\
\text { annually or } \\
\text { biennially, } \\
\text { requiring } \\
\text { labour } \\
\text { - High } \\
\text { durability }\end{array}$ \\
\hline Imhoff Tanks & $\begin{array}{l}\text { - Similar to ISTs } \\
\text { but bigger in } \\
\text { size } \\
\text { - Low treatment } \\
\text { efficiency } \\
\text { - Performance } \\
\text { depends on } \\
\text { desludging and } \\
\text { maintenance } \\
\text { works }\end{array}$ & $\begin{array}{l}\text { - Requires more } \\
\text { frequent } \\
\text { desludging } \\
\text { than ISTs } \\
\text { - Requires very } \\
\text { little operator } \\
\text { skills: scum } \\
\text { removal, } \\
\text { reverse flow } \\
\text { direction and } \\
\text { sludge } \\
\text { removal } \\
\end{array}$ \\
\hline Oxidation ponds & $\begin{array}{l}\text { - Degree } \\
\text { treatment } \\
\text { dependent of } \\
\text { weather } \\
\text { - Effective but } \\
\text { inconsistent } \\
\text { standard of } \\
\text { effluent } \\
\text { achieved } \\
\text { - Excessive algae } \\
\text { growth will be }\end{array}$ & $\begin{array}{l}\text { - Simple } \\
\text { operation, } \\
\text { requiring } \\
\text { regular } \\
\text { maintenance } \\
\text { due to } \\
\text { variation in } \\
\text { performance } \\
\text { - Routine } \\
\text { maintenance } \\
\text { does not }\end{array}$ \\
\hline
\end{tabular}




\begin{tabular}{|c|c|c|}
\hline & $\begin{array}{l}\text { detrimental to } \\
\text { effluent BOD }\end{array}$ & $\begin{array}{l}\text { require skilled } \\
\text { labour } \\
\text { - Minimal } \\
\text { sludging } \\
\text { handing, } \\
\text { desludging } \\
\text { takes place } \\
\text { every } 10 \text { years }\end{array}$ \\
\hline $\begin{array}{l}\text { Ecological } \\
\text { Sanitation } \\
\text { System (ecosan) }\end{array}$ & $\begin{array}{l}\text { - High treatment } \\
\text { efficiency } \\
\text { - Sensitive } \\
\text { towards } \\
\text { wastewater } \\
\text { constituents, ie } \\
\text { must be free } \\
\text { from pollutants } \\
\text { or chemicals } \\
\text { - High flexibility }\end{array}$ & $\begin{array}{l}\text { - Low } \\
\text { technology, } \\
\text { easy to operate } \\
\text { and } \\
\text { maintaining, } \\
\text { not requiring } \\
\text { skilled labour } \\
\text { - Does not } \\
\text { require } \\
\text { frequent } \\
\text { maintenance }\end{array}$ \\
\hline Aerated pond & $\begin{array}{l}\text { - More efficient } \\
\text { than Oxidation } \\
\text { pond }\end{array}$ & $\begin{array}{l}\text { - More } \\
\text { maintenance } \\
\text { required due } \\
\text { to aeration } \\
\text { system }\end{array}$ \\
\hline Hi-Kleen system & $\begin{array}{l}\text { - High efficiency } \\
\text { with high } \\
\text { quality effluent } \\
\text { - High resistance } \\
\text { to variations in } \\
\text { loads } \\
\text { - Independent } \\
\text { from climatic } \\
\text { conditions }\end{array}$ & $\begin{array}{l}\text { - Sophisticated } \\
\text { operation with } \\
\text { a high level of } \\
\text { mechanism } \\
\text { - Need complete } \\
\text { sludge } \\
\text { treatment and } \\
\text { disposal } \\
\text { - Requires } \\
\text { skilled labour } \\
\text { to operate, } \\
\text { supervise and } \\
\text { maintain }\end{array}$ \\
\hline $\begin{array}{l}\text { Open Extended } \\
\text { Activated } \\
\text { Sludge System, } \\
\text { Open Aeration } \\
\text { System and } \\
\text { Intermittent } \\
\text { Decanted } \\
\text { Extended } \\
\text { Aeration (IDEA) } \\
\text { System }\end{array}$ & $\begin{array}{l}\text { - } \text { Reasonable } \\
\text { efficiency and } \\
\text { resistance to } \\
\text { variations in } \\
\text { load } \\
\text { - Require regular } \\
\text { maintenance } \\
\text { and inspection }\end{array}$ & $\begin{array}{l}\text { - Sophisticated } \\
\text { operation with } \\
\text { a high level of } \\
\text { mechanism } \\
\text { - Need complete } \\
\text { sludge } \\
\text { treatment and } \\
\text { disposal } \\
\text { - Requires } \\
\text { skilled labour } \\
\text { to operate, } \\
\text { supervise and } \\
\text { maintain }\end{array}$ \\
\hline
\end{tabular}

In general, activated sludge systems requires more energy than either pond or land treatment systems (Muga and Mihelcic, 2007). In Sarawak, at the low end of the spectrum, individual septic tanks (ISTs), Imhoff tanks, and the Ecosan system utilizes zero or minimal energy. For ISTs, the practice of source separation has the potential for the development of a sustainable wastewater system (Dallas et al., 2004), which has led to a pilot project incorporating the Ecosan principles for a residential area in Kuching, Sarawak. The Ecosan system is a land treatment system that incorporates a small constructed wetland to treat the greywater component of wastewater. The underlying aim of the Ecosan system is to treat and reuse wastewater with as little expenditure and energy as possible (Langergraber and Muellegger, 2004). This method of incorporating a wetland system to treat domestic sewage is low in cost, and easy to maintain and operate. It has also been studied in developing countries such as Brazil (Philippi et al., 1999) and Costa Rica (Dallas et al., 2004). At the high end of the spectrum, aerated ponds and systems that utilize electro-mechanical treatment technologies including Hi-Kleen, EAAS, and IDEA can consume up to $600 \mathrm{~W} \mathrm{~h} / \mathrm{m}^{3}$ of wastewater. HiKleen and IDEA are currently used in large commercial sites, such as a university campus, and EAAS is used mostly in newly developed residential areas that incorporated a sewer network in the planning and design of the development.

The feasibility of installing a particular technology to a community or area also depends on the energy resources available. Open aeration, Hi-Kleen, EAAS and the IDEA systems are small-scale wastewater systems that highly depend on civil infrastructure such as electricity supply to run its equipments. Thus, these systems are insufficiently robust for rural areas in terms of energy resource, but can be considered for commercial sites in town areas. The highly automated and mechanized nature of the systems requires high maintenance and frequent breakdowns of the pumps or control systems can easily render the treatment system inefficient. In addition, to overcome clogging of the sewer networks, substantial amount of potable water is required to flush the system which leads to an increased in energy to treat the additional wastewater (Lier and Lettinga, 1999).

For Sarawak, in areas where population density is very low, the ponds system can be a good option in terms of energy efficiency for wastewater treatment systems. Ponds system, whether mechanically aerated or not, usually requires low energy consumption and is easy to operate and maintain. Properly designed ponds system has the capability of removal efficiencies comparable to mechanical systems (Muga and Mihelcic, 2007).

\section{WASTEWATER GENERATION}

Quantification of wastewater generation is a fundamental step in designing the collection and treatment of wastewater. For a small, commercial sites such as an institution of higher learning, the diurnal wastewater generation patterns may be different from the typical diurnal domestic wastewater generation patterns (as presented in McGhee, 1991). This may affect the design of the sewerage and treatment of wastewater for such sites.

Wastewater flow rates can be estimated from water consumption records (Larsen \& Lynghus 2004; Tchobanoglous, Burton \& Stensel 2004). The data collected from water consumption records are very useful for areas where the water usage for landscape irrigation is insignificant and 90 percent or more of water consumed becomes wastewater. Wastewater generation for Curtin University Sarawak Campus is quantified by monitoring the main water meter for the campus. Three sets of data have been collected, during two typical teaching weeks (Weeks 11 and 2) and one semester break (Week free)

The water consumption or wastewater flows varies according to the time of day, different days throughout the 
week and during different periods or seasons in a year. The first and second variations are referred to as short-term variations in this paper, while the third variation is referred to as seasonal variation.

\section{Week 11 of Semester One}

The average cumulative water consumption in a day for Curtin University Sarawak Campus for week 11 of semester one is presented in 'figure 1'. The legend shown in 'figure 1 ' is applicable to all the other figures.

From 'figure 1', the water consumption during the weekends is significantly lower $(38 \%)$ than the water consumption during the weekdays. This variation in water consumption between weekdays and weekends is expected, as classes are not held during the weekends. There is also a lag time of 1 hour between the occurrences of the peak average hourly water consumption during the weekdays and weekends of week 11 of semester one as seen in 'figure 2'. The peak average hourly water consumption during the weekends is about $44 \%$ less than that during the weekdays.

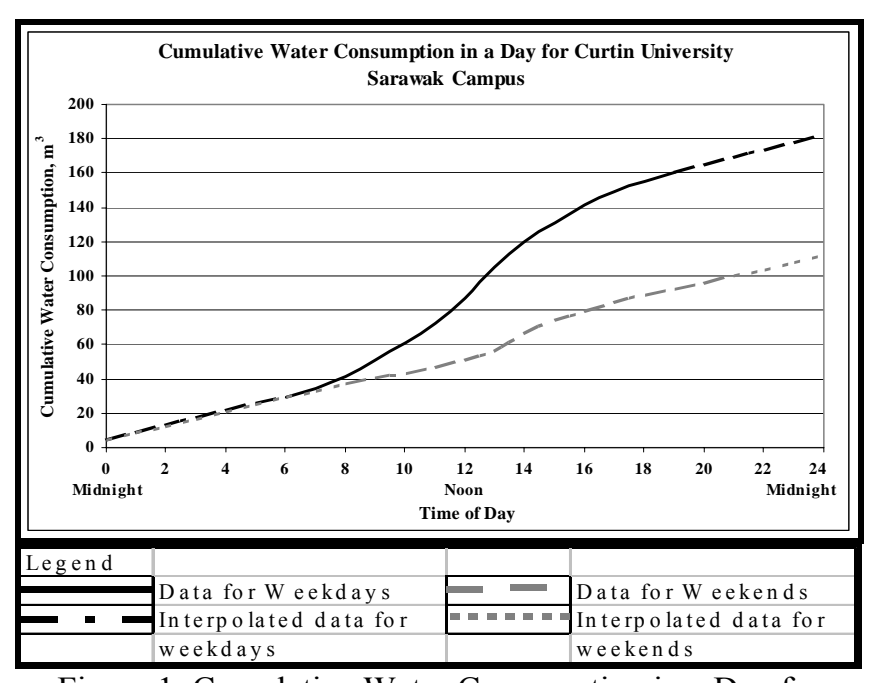

Figure 1. Cumulative Water Consumption in a Day for Curtin University Sarawak Campus for Week 11 of Semester One

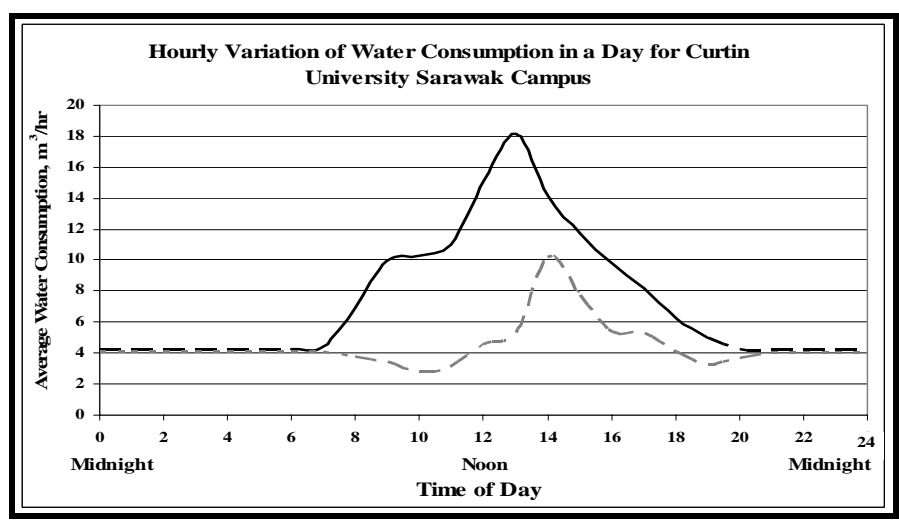

Figure 2. Cumulative Water Consumption in a Day for Curtin University Sarawak Campus for Week 2 of Semester Two. Week 2 of Semester Two

\section{Week 2 of Semester Two}

For this week, the generation patterns were similar to 'figures 1 and 2'. It was found that the total average water consumption for Curtin University Sarawak Campus during the weekdays for week 2 of semester two $\left(145 \mathrm{~m}^{3}\right)$ is about
$29 \%$ more than that during the weekends of the week (105 $\mathrm{m}^{3}$ ). Similar to the average hourly variation of water consumption for week 11 of semester one ('figure 2'), a lag of time for the occurrence of peak hourly water consumption for weekdays and weekends is observed for week 2 of semester two. The peak of average hourly water consumption during the weekends $\left(11 \mathrm{~m}^{3}\right)$ is $32 \%$ less than weekdays $\left(7.5 \mathrm{~m}^{3}\right)$.

\section{Week Free (Semester Break)}

'Figure 3' shows the average cumulative water consumption in a day for Curtin University Sarawak Campus for a week free during semester break. The average total water consumption during weekends for a week free during semester break is about $6 \%$ than during the weekdays. The peak average hourly water consumption during the weekdays and weekends for the week free occur at 12 noon, with the peak average hourly water consumption during weekends $25 \%$ less than that during weekdays.

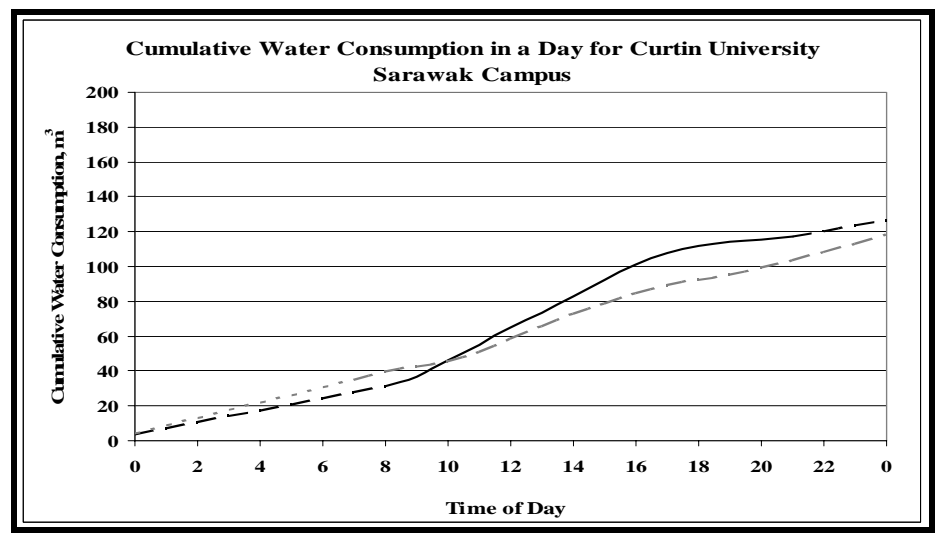

Figure 3. Cumulative Water Consumption in a Day for Curtin University Sarawak Campus for a Week Free

\section{Short-term Variations}

From the figures presented, water consumption changes during the course of a day. The main finding from the research is that this pattern for an institution of higher learning is different from the typical diurnal pattern of domestic water consumption.

From the observation of the typical domestic water consumption or wastewater generation curve, there are two peaks in a day: the first peak generally occurs around 8 a.m. in the morning, while the second peak arises in the early evening between 6 p.m. to 8 p.m. (Tchobanoglous, Burton $\&$ Stensel 2004). However, the hourly variations in water consumption in Curtin Sarawak Campus during the teaching weeks of semester 1 and 2 present another type of pattern: the maximum peak usage occurs around 1 p.m. during weekdays and 2 p.m. during weekends. In addition, the peak average hourly water consumption occurs around 12 noon during the weekdays and weekends of the week free of July's semester break. The water consumption for Curtin University Sarawak Campus during weekdays starts to increase around 8 a.m. in the morning when all the activities are just starting and reaches the maximum during the lunch break in the midday. The water consumption decreases during late evening when the classes end, and there are less people in the campus.

Also, from 'figures 1 and 2', it can be noted that the water consumption in the campus during the weekends is 
significantly less than during the weekdays. Therefore, it can be seen from these two main observations that the population in the campus has direct influence on the quantity of water consumed during the day in a week. It is observed that the water consumption during the semester break ('figure 3') did not vary significantly between weekdays and weekends. Thus, the extent of water consumption variation during a day in week is dependent on the activities carried out and the population size of the particular institution.

\section{Seasonal Variations}

Seasonal variation is observed to be dependent on the activities in the campus. The extent of seasonal variation is expected to be dependent on the size of the community and the seasonal activities (Tchobanoglous, Burton \& Stensel 2004). For an institution of higher learning like Curtin University Sarawak Campus, the variation would be the holiday period before commencement of classes. It is shown that the water consumption during a non-teaching week is as expected, less than the water consumption during a teaching week due to significant decrease in students' population during the non-teaching week.

\section{CONCLUSION}

The various treatment systems in use in Sarawak have varying degree of energy consumption, but selection of the treatment system is not only an energy use issue, but also involves resource availability, costs and treatment performance. However, the sustainability of a treatment system is not only a function of economic and environmental aspects, but must also include a social dimension, influenced by an area's geographic and demographic situation (Muga and Mihelcic, 2007). Therefore, to wholly evaluate the treatment systems in place, various indicators would have to be developed to study the treatment technologies and its sustainability. In addition, from analysis of water consumption data, diurnal variations in water consumption for an institution of higher learning are different from the diurnal variations for domestic water consumption. The main factors that influence the variations in water consumption or wastewater generation in an educational institution are found to be the size of the population and the activities carried out in the institution. The data collected in quantifying wastewater generation in Curtin University Sarawak Campus could be used as a useful reference data for other similar institutions of higher learning in Malaysia.

\section{ACKNOWLEDGMENT}

The help an assistance of numerous staff members from various government and private organizations in Kuching, Miri and Bintulu in collecting information for this project is gratefully acknowledged.

\section{REFERENCES}

[1] Asian Development Bank 2006, Country Water Action: Malaysia. Retrieved July 25, 2006, from http://www.adb.org/water/actions/mal/water-treatment.asp.

[2] Bjerregaard, D. 2004, Urban Ecological Sanitation-Kuching is Paving the Way, The NREB and Danida Copenhagen, Kuching.
[3] Crites, R. \& Tchobanoglous, G. 1998, Small and Decentralized Wastewater Management Systems, WCB/McGraw-Hill, United States of America.

[4] Dallas, S., Scheffe, B., Ho, G., 2004. "Reedbeds for greywater treatment - case study in Santa Elena-Monteverde, Costa Rica, Central America". Ecological Engineering Vol. 23, pp55-61.

[5] Department of Irrigation and Drainage Sarawak n.d., Wastewater Management. Retrieved: March 8, 2006, from http://www.did.sarawak.gov.my/urban/html/wasterwater.htm.

[6] Guidelines for Developers: Sewage Treatment Plants 1998, vol. 4, $2^{\text {nd }}$ edn, Sewerage Services Department, Ministry of Housing and Local Government, Kuala Lumpur.

[7] Indah Water Konsortium Sdn. Bhd. n.d., Sludge Treatment. Retrieved July 23, 2006, from http://www.iwk.com.my/services-sludgetreatment.html.

[8] Langergraber, G., Muellegger, E., 2004. "Ecological sanitation - a way to solve global sanitation problems?" Environment International Vol. 31, 433-444.

[9] Larsen, Ib \& Lynghus, H. 2004, Framework Plan for Integrated Wastewater Management for the City of Kuching, Sarawak, Sarawak Government and DANIDA, Kuching.

[10] Lier, J.B., Lettinga, G., 1999. "Appropriate technologies for effective management of industrial and domestic wastewaters: the decentralized approach." Water Science and Technology Vol. 40 (7), pp171-183.

[11] Mara, D. 2003, 'Chapter 1: What is Domestic Wastewater and Why Treat It?', Domestic Wastewater Treatment in Developing Countries, Earthscan, UK, pp. 1-6.

[12] Metcalf and Eddy, 2003. Wastewater Engineering: Treatment and Reuse. McGraw-Hill, Boston.

[13] McGhee, T.J. 1991, Water Supply and Sewerage, $6^{\text {th }}$ edn, McGrawHill Inc., Singapore.

[14] Muga, H.E., Mihelcic, J.R., 2007. "Sustainability of wastewater treatment technologies." Journal of Environmental Management.

[15] Philippi, L.S., Costa, R.H.R., Sezerino, P.H., 1999. "Domestic effluent treatment through integrated system of septic tank and root zone." Water Science and Technology Vol. 40 (3), pp125-131.

[16] PJBumi Berhad 2005, Company Profile, PJBumi Berhad, Kuala Lumpur.

[17] V. Quisenberry, P. Brown, B. Smith and D. F. Hallahan, 2006; 'InSitu Liquid Storage Capacity Measurement of Subsurface Wastewater Absorption System Products', Journal of Environmental Health; Vol. 69, No. 4;

[18] K. Rainwater, A. Jackson, W. Ingram, Y.L. Chang, et al., 2005; 'Field Demonstration of the Combined Effects of Absorption and Evapotranspiration on Septic System Drainfield Capacity', Water Environment Research, Vol. 77, Iss. 2.

[19] Standards \& Industrial Research Institute of Malaysia 1991, Malaysian Standard Code of Practice for Design and Installation of Sewerage Systems, MS 1228:1991.

[20] Tan, A.H., 2006, 'A Study into a Viable Wastewater Treatment System for a Commercial Site in Sarawak', Final Year Project Report, Curtin University of Technology.

[21] Tan, A.H., Tang, F.E., Ho, C.L.I., 2007 "A Study into a Viable Wastewater Treatment System for a Commercial Site in Sarawak", Proceedings of the $3^{\text {rd }}$ World Engineering Congress, IEM, Penang, Malaysia.

[22] Tchobanoglous, G., Burton, F.L. \& Stensel, H.D. 2004, 'Analysis and selection of wastewater flow rates and constituent loadings', Wastewater Engineering: Treatment and Reuse, international edn, McGraw Hill, Singapore, pp. 153-214.

[23] Ukita, M., Shirota, H., Nakaishi, H., 1993. "Option of appropriate system for wastewater treatment in low-density areas." Water Science and Technology Vol. 28 (10), pp49-56.

[24] Weida Environmental Technology Sdn. Bhd. n.d. Operation and Maintenance Manual: Intermittently Decanted Extended Aeration (IDEA) Activated Sludge Sewage Treatment Plant for Curtin University of Technology, Sarawak Campus, Miri, Sarawak, Kuching.

[25] Williams, J., Bahgat, M., May, E., Ford, M., Butler, J., 1995. "Mineralisation and pathogen removal in gravel bed hydroponic constructed wetlands for wastewater treatment." Water Science and Technology Vol. 32 (3), pp49-58. 


\title{
The Effect of Staged Combustion in Reduction of Noxious Emissions for Agricultural Residues Using a Fluidized Bed Combustor
}

\author{
Mohamad Shaiful Ashrul Ishak, Hayati Abdullah, and Mohammad Nazri Mohd. Ja'afar
}

\begin{abstract}
Fluidized bed technologies have been shown to play a key role in the area of solid fuel combustion. This study was carried out to investigate the reduction of $\mathrm{NO}_{\mathrm{x}}$ emission by using staged combustion in a small laboratory scale fluidized bed combustor. The experiments were carried out using palm shell as fuel and silica sand as the bed material. The experimental rig has a maximum capacity of $25 \mathrm{~kW}$. Fluidizing air was supplied through a distributor and secondary air was introduced into the freeboard via an ejector. Flue gas samples were collected and analyzed for a fluidizing velocity of up to $2 \mathrm{~m} / \mathrm{s}$ and a bed temperature of $700^{\circ} \mathrm{C}$. Various series of tests were conducted to determine the influence of fuel-related and operation dependent parameters on the formation and reduction of $\mathrm{NO}_{\mathrm{x}}$. The study shows that a much lower emissions of oxides of nitrogen $\left(\mathrm{NO}_{\mathrm{x}}\right)$ were achieved when compared to the unstaged combustion and this represents a significant achievement where more than 25 percent of $\mathrm{NO}_{\mathrm{x}}$ emissions reduction was obtained.
\end{abstract}

Keywords: fluidized bed combustor, air staging, palm shell, combustion, emissions

\section{INTRODUCTION}

Combustion is a process to produce energy chemically and widely practiced in the industry. This happens when combustible substance reacts with oxygen from air [1]. Fossil fuels such as natural gas, petroleum and coal are the major sources of combustible material currently used. Because of the limited resources, new alternatives must be sought and introduced to complement these energy sources. One way is by using biomass energy [2]. Biomass fuel is a source of energy produced from the combustion of biomass waste.

Waste combustion implicates bad effect to the environment because of the emissions it produces. Therefore, improving combustion efficiency will be valuable for the environment. The combustion efficiency

Mohamad Shaiful Ashrul Ishak is with the School of Manufacturing Engineering, Universiti Malaysia Perlis, Blok B, Kompleks Pusat Pengajian Jejawi 1, 02600 Jejawi, Perlis, Malaysia. (Tel: +604-9798398, Fax: +6049798160, E-mail: mshaiful@unimap.edu.my).

Hayati Abdullah is with the Department of Aeronautics \& Automotive, Faculty of Mechanical Engineering, Universiti Teknologi Malaysia, 81310 UTM, Johor, Malaysia.

Mohammad Nazri Mohd. Ja'afar is with the Department of Aeronautics \& Automotive, Faculty of Mechanical Engineering, Universiti Teknologi Malaysia, 81310 UTM, Johor, Malaysia. depends on temperature and an increase in temperature will increase the combustion efficiency [3]. The different composition of the biomass fuel will affect the combustion efficiency because of the different chemical content of the combustible substance. As an example, the proximate analysis for palm shell shows that it contains $9.7 \%$ moisture, $67 \%$ volatile, $21.2 \%$ fixed carbon and $2.1 \%$ ash. The ultimate analysis carried out for palm shell indicates that it consists of $47.62 \%$ carbon, $6.2 \%$ hydrogen, $0.7 \%$ nitrogen and the gross calorific value is $19.1 \mathrm{MJ} / \mathrm{kg}$. The principal reactions for the combustion of the fuel are as follows:

$$
\begin{aligned}
& \mathrm{C}+\mathrm{O}_{2}+3.76 \mathrm{~N}_{2} \rightarrow \mathrm{CO}_{2}+3.76 \mathrm{~N}_{2} \\
& \mathrm{H}_{2}+1 / 2 \mathrm{O}_{2} \rightarrow \mathrm{H}_{2} \mathrm{O}
\end{aligned}
$$

To ensure that the combustion product is less polluting, numerous on-going research has been carried out effectively and are consistent with coherent legislation law. One practical method applied is staged combustion and a number of research in this area has been carried out to study the effect of reducing pollution $[4,5]$.

\section{FUNDAMENTAL OF FLUIDIZATION}

Fluidization bed combustion mode (including bubbling fluidized bed and circulating fluidized bed) is playing more and more important role in energy conversion field of coal due to its advantages of good fuel flexibility and easier control of $\mathrm{NO}_{\mathrm{x}}$ and $\mathrm{SO}_{\mathrm{x}}$ emission over many other combustion methods. Fluidization is the levitation of a mass (bed) of particles by a fluid [6]. In this state, the particle mass behaves like a fluid in that it tends to establish a level and flow in response to the pressure drop. A gas that passes through a bed of particles will create a drag force at the surface of the particle and act upwards on the particle. As the air flow rate increases, the drag force will increase too. When the force is equal to the weight of the particle, the particle will float in the combustion chamber.

The bed is considered just fluidized and is referred to as an incipiently fluidized bed or a bed at minimum fluidization. There is intense mixing and gas contact in this condition. These properties make it easy to have an isothermal system with good mass transfer. Figure 1 shows the mechanism of the fluidized bed. 


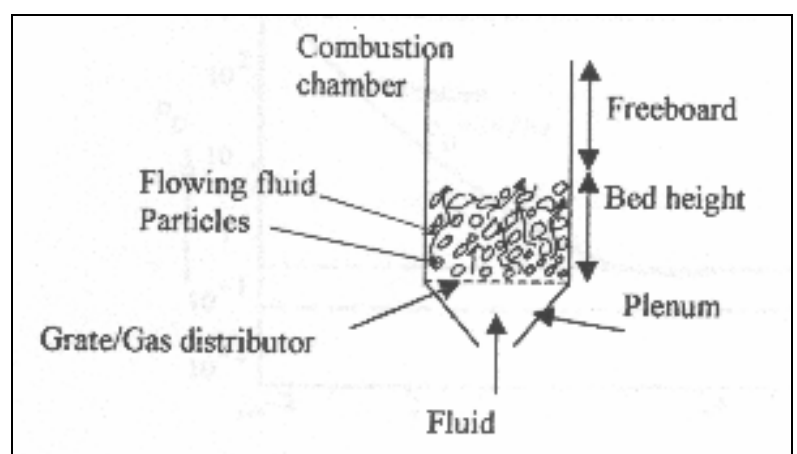

Figure1. Fluidized bed combustion

\section{Staged COMBUStion}

Staged combustion involves introducing either the combustion air or fuel into the flame in different stages. With air staging a portion of the combustion air, typically about 50 to $75 \%$ is supplied to a primary combustion zone with all of the fuel. This produces a fuel rich zone and due to this substoichimetric combustion condition, the formation of thermal and fuel $\mathrm{NO}_{\mathrm{X}}$ is inhibited. The remainder of the air is injected upstream, forming a secondary flame zone, where the combustion is completed. $\mathrm{NO}_{\mathrm{X}}$ formation in this secondary flame zone is also reduced due to reduction of flame temperature, which occurs due to the mixing of inerts form the primary flame zone. $\mathrm{NO}_{\mathrm{X}}$ reduction attainable with this method usually ranges from 30 to $40 \%$ lower, than that resulting from conventional single stage combustors [7]. Staging requires rapid mixing of fuel and air and generally longer residences times to ensure the burnout of $\mathrm{CO}$ and hydrocarbon in the final fuel lean stage [8]. Air staging can control both thermal and fuel $\mathrm{NO}_{\mathrm{X}}$, however it is more effective in reducing fuel $\mathrm{NO}_{\mathrm{X}}$ and in fact, is the best method for controlling the conversion of fuel bound nitrogen to fuel $\mathrm{NO}_{\mathrm{X}}$ emissions.

Figure 2 shows the schematic diagram for air staging. The applied air to fluidize the bed sand was introduced from the main blower. The supplied air is a significant factor in influencing the gas solid contact during the turbulence action of the bed sand. It also affects the quality of the combustion process. The combustion quality could be accessed from the flue gas emission level that leaves the cyclone outlet. Thus, the supplied air has an effect on the emission level.

The secondary air into the combustion chamber was introduced to reduce the emissions, which are the $\mathrm{NO}_{\mathrm{x}}, \mathrm{CO}$ and smoke. Air staging is one of the techniques for emission control.

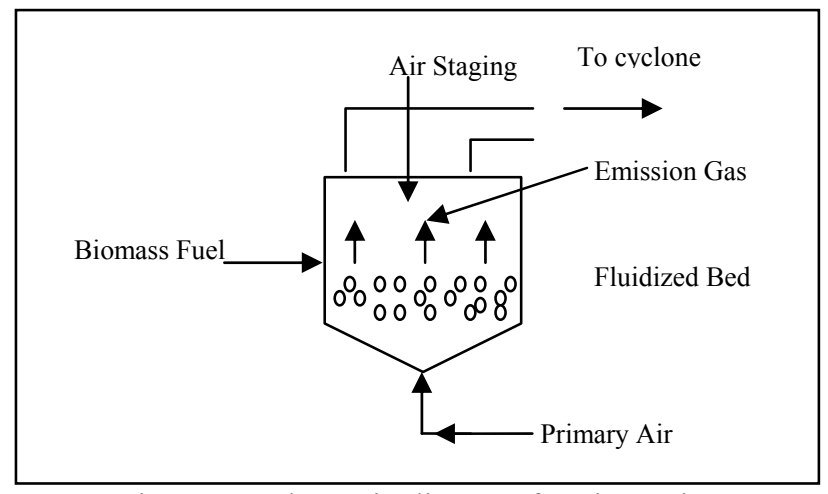

Figure 2. Schematic diagram for air staging

\section{EXPERIMENTAL SET UP}

The experimental test rig consists of 5 main components, which includes the combustion reactor, fuel screw feeder, cyclone, exhaust pipe and air blower as shown in figure 3. The function of the exhaust is to direct the gas out. The fuel used in this experiment is palm shell and the particle size is between $1.25 \mathrm{~mm}$ and $4.25 \mathrm{~mm}$. The combustion process starts with preheating using auxiliary fuel. The biomass fuel is then charged into the combustor and the preheating process ends when the temperature attained is $700^{\circ} \mathrm{C}$.

The primary air supply will then be increased at the desired flow rate. The fuel flow rates are then fixed when the bed temperature is constant. Secondary air was introduced at $100 \mathrm{~mm}$ upstream of the bed height with 10 $\mathrm{mm}$ inside diameter mild steel tube. The air staging experiments were carried out with secondary air to total air ratio various from 0 to 0.4 or 0 to $40 \%$ of primary air are diverted to secondary air. The exhaust sampling probe is mounted at the end of the freeboard. The gas analyser used is the portable Kane May gas analyzer (Quintox model, United Kingdom)

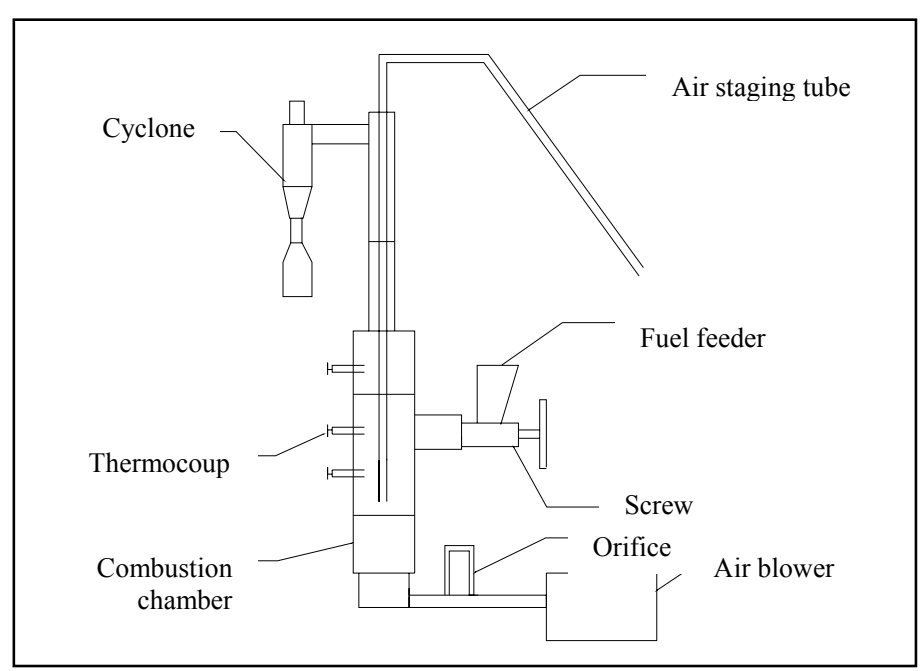

Figure 3 Experimental test rig

\section{Results AND Discussions}

Figures 4 to 6 show the result of air staging tests carried out on reducing exhaust emissions from the fluidized bed combustor. Tests on exhaust emissions were carried out using various secondary air flow rates at stoichiometric equivalence ratio $(\phi)$.

Figure 4 shows a large reduction in oxides of nitrogen $\left(\mathrm{NO}_{\mathrm{X}}\right)$ emissions when air staging is applied to the fluidized bed combustor. This is apparent from 20 percent of air staging supplied to a primary combustion zone. Emissions level below $25 \mathrm{ppm}$ was obtained for the whole range of operating air ratio. $\mathrm{NO}_{\mathrm{X}}$ emissions reduction of approximately more than 25 percent was obtained at the rtion of the combustion air of 20 percent when using air Iging as compared to non-air staging test supplied at 100 $\mathrm{mm}$ upstream to the bed height. Thermal $\mathrm{NO}_{\mathrm{X}}$ produced by oxidation of atmospheric nitrogen in post flame gas can also be reduced by using air staging method. The secondary air will decrease the temperature in the combustor and reduce the $\mathrm{NO}_{\mathrm{X}}$ emission. The secondary air also reduces the 
prompt NO produced by high-speed reaction at the flame front during the combustion by reducing the speed of the flame.

The principal reactions for the combustion of the fuel are as follows:

$$
\begin{aligned}
& N_{2}+O \leftrightarrow N O+N \\
& N+O_{2} \leftrightarrow N O+O \\
& N_{2}+O_{2} \leftrightarrow 2 N O \\
& N+O H \leftrightarrow N O+H
\end{aligned}
$$

Figure 5 shows the plot of carbon monoxide (CO) emissions at different secondary air to total air ratio. CO emission reduction of more than 10 percent was achieved by using air staging compared to non-air staging test at the portion of the combustion air of 20 percent. CO emission will be emitted from the combustion process due to the lack of sufficient oxygen to complete the reaction from $\mathrm{CO}$ to $\mathrm{CO}_{2}$ and also because of low bed temperature [9]. When the secondary air is added into the combustor to supply the oxygen to complete the combustion, the combustor temperature will drop and this will cause an increase in CO emission. Anyway, this situation only happens at the beginning of the process.

Figure 6 shows a plot of carbon dioxide $\left(\mathrm{CO}_{2}\right)$ emissions versus air ratio when air staging is applied to the fluidized bed combustor. There was a slight increase in carbon dioxide emissions when air staging method is applied. This was observed throughout the whole range of operating air ratio. $\mathrm{CO}_{2}$ increases about 20 percent when 20 percent of air staging supplied to a primary combustion zone. However, this increase is not significant as compared to the reduction of $\mathrm{NO}_{\mathrm{X}}$ emissions. When secondary air was introduced, oxygen from the air will complete the combustion and CO will decrease (Figure 5). Furthermore, carbon dioxide emissions are more stable and non-toxic. However, $\mathrm{CO}_{2}$ is a greenhouse gas and can contribute to global climate change. The principal reactions for the combustion of the fuel are as follows:

$$
2 \mathrm{CO}+\mathrm{O}_{2} \rightarrow 2 \mathrm{CO}_{2}
$$

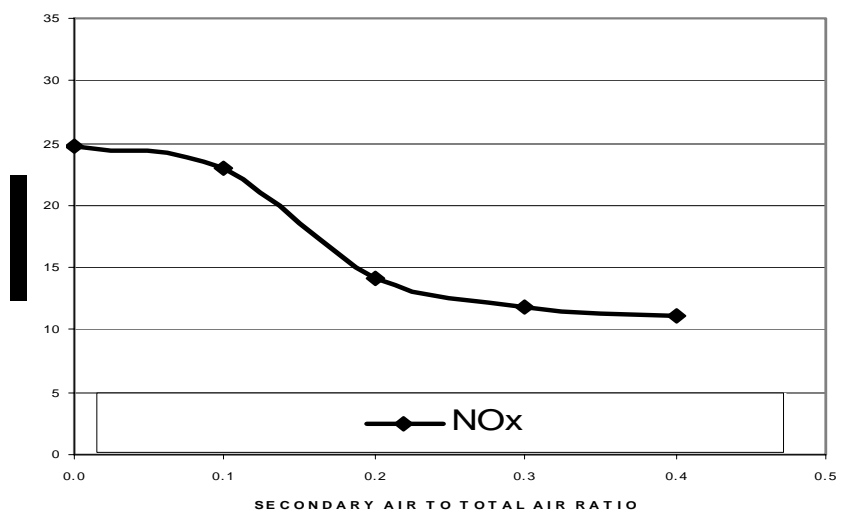

Figure 4. $\mathrm{NO}_{\mathrm{X}}$ vs air ratio

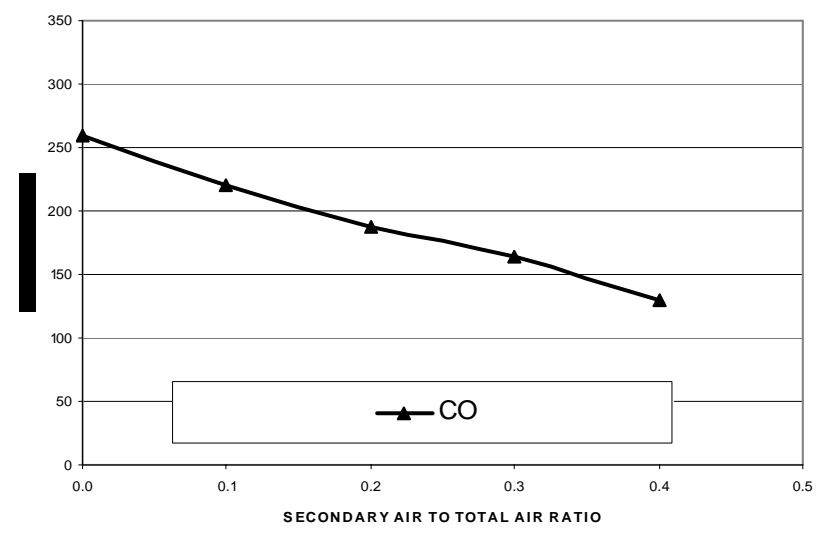

Figure 5. CO vs air ratio

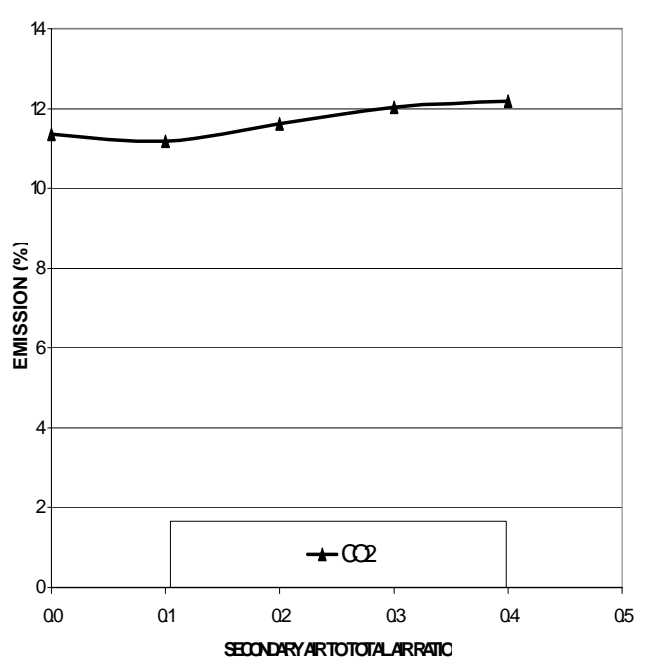

Figure 6. $\mathrm{CO}_{2}$ vs air ratio

\section{CONCLUSION}

The use of air staging for combustion is to reduce emissions from the fluidized bed combustor especially the oxides of nitrogen $\left(\mathrm{NO}_{\mathrm{x}}\right)$ and carbon monoxide (CO). $\mathrm{NO}_{\mathrm{x}}$ emissions reduction of more than 25 percent was obtained at the portion of the combustion air of 20 percent air staging compared to the non-air staging test. Air staging method also helps to decrease the CO emission and to increase the formation of carbon dioxide from the fluidized bed combustor. A number of contributing factors to the effectiveness of the combustion process include bed materials, fluidizing velocity, size of fuel particle and feeding rate. Further research would be required to enhance the understanding of these factors.

\section{ACKNOWLEDGMENT}

The authors would like to thank to Ministry of Higher Education (MOHE) for supporting this research under Fundamental Research Grant Scheme (FRGS 9003- 00057) and also School of Manufacturing Engineering, Universiti Malaysia Perlis for providing research facilities to undertake this work. 


\section{REFERENCES}

[1] Howard J. R., 'Fludized Bed Combustion and Application', Applied Science Publisher, New York, 1983.

[2] Adi Surjosatyo, 'Fluidized Bed Incinerator of Palm Shell and Oil Sludge Waste’, Tesis Sarjana, Universiti Teknologi Malaysia, 1998.

[3] Noel De Nevers, 'Air Pollution Control Engineering', Mc Graw-Hill Inc., New York, 1995.

[4] Mohammad Nazri Mohd. Jaafar, Farid Nasir Ani, Ainul Hayati Yunus,' Aplikasi Udara Berperingkat Bagi Mengurangkan Pancaran Dalam Pembakar Lapisan Terbendalir', Proceedings of Malaysian Science \& Technology Congress 2000, Genting Highland, 2000.

[5] Mohammad Nazri Mohd. Jaafar, Farid Nasir Ani, Loh Kar Yee,' Pengurangkan Emisi Dari Pembakar Lapisan Terbendalir Melalui Udara Berperingkat Bagi', Proceedings of Malaysian Science \& Technology Congress 2001, Melaka, 2001.

[6] Pell, M., 'Gas Fluidization', Elsevier Science Publisher Company Inc, 1990.

[7] Waibel, R. T., 1993, Ultra Low $\mathrm{NO}_{\mathrm{X}}$ Burners for Industrial Process Heaters. Second International Conference on Combustion Technologies for a Clean Environment. Lisbon

[8] Streichsbier, M., 1998, Non-Catalytic $\mathrm{NO}_{x}$ Removal from Gas Turbine Exhaust with Cyanuric Acid in a Recirculating Reactor. University of California, Berkeley: Ph. D. Thesis.

[9] William C. Gardiner Jr., 'Combustion Chemistry', SpringerVerlag Inc., New York, 1984. 
ENCON2007-121

\title{
Simulation of a Three-Phase UPFC
}

\author{
N.F. Mailah, S.M. Bashi, N. Mariun and I. Aris
}

\begin{abstract}
FACTS or Flexible Alternating Current Transmission Systems is a system that based on the power electronics devices and other static equipment to provide control of one or more AC transmission system parameters to enhance controllability and increase power transfer capability. UPFC is one of the FACTS devices which was first proposed by Laszlo Gyugyi in 1992, with the objectives of controlling, simultaneously or selectively, all the parameters affecting power flow in the transmission line (i.e. voltage, impedance and phase angle). UPFC consists of two switching power converters connected to each other back-to-back through a DC link capacitor and connected to the $\mathrm{AC}$ system by a shunt and series transformers. The systems are modeled and simulated using MATLAB/SIMULINK software. Shunt inverter or STATCOM is modeled as a 3-phase multipulse converter and the series inverter or SSSC is modeled as a 3-phase, 3-level multilevel converter. Faults are set to the system to observe the operation of STATCOM and phase shift, $\phi$ of the SSSC is varied to observe the operation of SSSC. Simulations are carried out and the results obtained agree with the theory of operation of the UPFC.
\end{abstract}

Keywords: STATCOM, SSSC, UPFC, modeling, operation

\section{INTRODUCTION}

Flexible Alternating Current Transmission System or FACTS uses power electronic based systems and others static equipment to provide control of one or more AC transmission system parameters to enhance controllability and increase power transfer capability. It was first introduced in 1980s by Dr. Narain G. Hingorani [1] and since then, the technology has been progressing at an increasing pace.

Traditionally, power flow control is gained with the use of a phase shifter and mechanically changing tap setting of a transformer. However this method is not flexible enough to cope with the increasing needs. Following the trend of

N.F. Mailah is with the Department of Electrical and Electronic Engineering, Faculty of Engineering, Universiti Putra Malaysia, 43400 UPM Serdang, Selangor, Malaysia (phone: 603-8946-6316; fax: 603-89466327; e-mail: nashiren@eng.upm.edu.my).

S.M. Bashi is with the Department of Electrical and Electronic Engineering, Faculty of Engineering, Universiti Putra Malaysia, 43400 UPM Serdang, Selangor, Malaysia (phone: 603-8946-6326; fax: 603-89466327; e-mail: senan@eng.upm.edu.my).

N. Mariun is with the Department of Electrical and Electronic Engineering, Faculty of Engineering, Universiti Putra Malaysia, 43400 UPM Serdang, Selangor, Malaysia (phone: 603-8946-6321; fax: 603-89466327; e-mail: norman@eng.upm.edu.my).

I. Aris is with the Department of Electrical and Electronic Engineering, Faculty of Engineering, Universiti Putra Malaysia, 43400 UPM Serdang, Selangor, Malaysia (phone: 603-8946-6324; fax: 603-8946-6327; e-mail: ishak@eng.upm.edu.my). deregulating the electric power industry, a demand for flexible power load flow is becoming a technical need feasibly achievable by the innovative power electronics [2], thus the use of FACTS devices. This technology is based on the used of high voltage and high current power electronics devices in association with communication links and local automatic controllers.

UPFC concept was first proposed by L. Gyugyi in 1992, with the objectives of controlling, simultaneously or selectively, all the parameters affecting power flow in the transmission line (i.e. voltage, impedance and phase angle). It can also independently control both real and reactive power flow in the transmission line [3]. Other than that, it has the capabilities of improving transient stability, mitigating system oscillations and providing voltage support [4].

Since the introduction of the UPFC, many studies and investigation of its performance have been carried out either by simulation and hardware model. Zheng et. al. [5] in their work has developed a simulation model of UPFC with 12pulse converters using Matlab and Simulink software. Static and dynamic characteristics of the developed UPFC simulation model in a power system are investigated under normal operating condition (i.e. no disturbance) and under severe disturbance. The developed model reflects precisely the operation characteristics of the practical devices. It shows that the UPFC can control the voltage and power flow of the system effectively.

Toufan and Annakkage [6] investigate in their paper the performance of a UPFC constructed by a back-to-back connection of a hysteresis current forced (HCF) converter and a pulse width modulated (PWM) inverter. The model has been developed at a component level and simulated using PSCAD/EMTDC software. From the investigation, the UPFC model can maintain an almost constant DC bus voltage and has the ability to pass the real power bidirectionally. It has also been shown that using quadrate or inphase voltage injection, the UPFC can enhance the dynamic stability of the power systems effectively.

A current injection model of the UPFC is developed for transient stability studies in Meng and So [7]. The effect of UPFC can be represented by an equivalent circuit with a shunt current source and a series connected voltage source. The series voltage source can be solved into in-phase and quadrature components with respect to the line current, and the current injection model is obtained by replacing the voltage source with the current source. The controller of the UPFC is based on optimal control strategies in a singlemachine infinite-bus system (SMIB). In their paper, they proposed that the controller coordinates input signals to 
control the two components of the UPFC series voltage and the shunt compensation of the UPFC, in order to maintain the system bus voltage. The eigenvalue analysis and nonlinear results show that the proposed model and control method can significantly improve system dynamic performance.

New control approach combining the traditional control technique with an artificial intelligence technique such as Genetic Algorithm (GA) has been studied by Faried and Aldamaty [8]. The GA based UPFC is designed using eigenvalue shifting technique and the effectiveness of the new controller is demonstrated through time-domain simulations using Matlab. From the results, it shows that this new control approach give the UPFC more flexibility and increase capabilities in damping the power system oscillations when compared to the fixed power injection UPFC.

In this paper, the work concentrates on the designing and developing a simulation model of a three phase UPFC using SimPowerSystem Blockset of Matlab/Simulink software. The shunt converter is developed using the traditional 6pulse three-phase bridge topology, while the series converter is developed using multilevel, 3-level topology. A simple single switching $\mathrm{ON}$ and $\mathrm{OFF}$ is adopted as the control strategy of the UPFC. Results obtained from the simulation model are then compared with the theory of operation of the UPFC. Faults are set to the system to observe the operation of STATCOM and phase shift, $\phi$ of the SSSC is varied to observe the operation of SSSC. A good working simulation model has been obtained for the UPFC.

\section{UNIFIED POWER FlOW CONTROLLER}

The Unified Power Flow Controller (UPFC) is the cornerstone of Electric Power Research Institute's (EPRI's) Flexible Alternating Current Transmission System (FACTS) program. UPFC was proposed for real-time control and dynamic compensation of AC transmission systems and offers multiple compensation functions [9].

UPFC consists of two switching power converters connected to each other back-to-back through a DC link capacitor as shown in figure 1 . The converters are connected to the AC system by a shunt and series transformers. This arrangement functions as an ideal ac-toac power converter in which real power can freely flow in either direction between the two inverters.

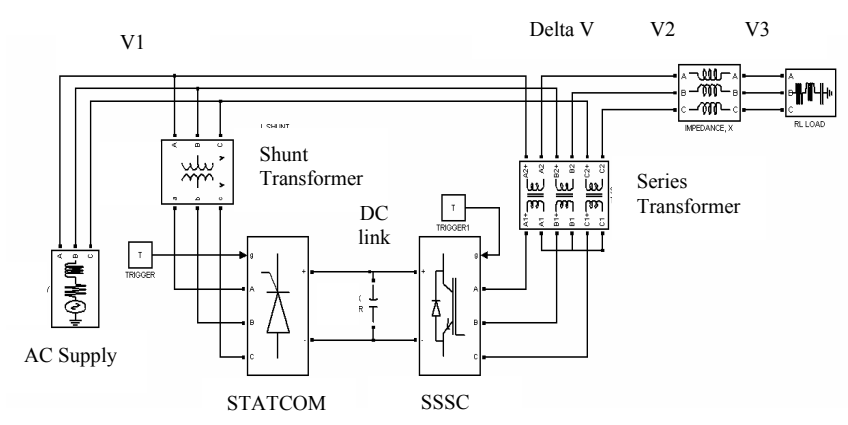

Figure 1. Three-phase diagram of UPFC

The shunt inverter or STATCOM is connected to the system via a shunt transformer. It injects an almost sinusoidal controlled current of variable magnitude at the point of connection. STATCOM compensates reactive power flow in the transmission line and at the same time keeps the dc voltage constant across the dc link capacitor (i.e. regulates the active power flow between shunt and series converters).

The series converter or SSSC operates by adding a series voltage, $\Delta \mathrm{V}$ of a variable magnitude and phase angle, and thus forcing the power to flow to a desired value. This voltage addition has a controllable magnitude of range, $\delta \mathrm{V}$ $\left(0 \leq \delta \mathrm{V} \leq \delta \mathrm{V}_{\max }\right)$ and phase angle, $\phi\left(0^{\circ} \leq \phi \leq 360^{\circ}\right)$ and can be considered as asynchronous ac voltage source [10]. It is connected to the system through a series transformer.

\section{Methodology}

The main interest in this paper is to design and develop a simulation model of a three phase UPFC using SimPowerSystem Blockset of Matlab/Simulink software. The designing of the UPFC takes two steps. The shunt converter or STATCOM is designed first and then followed by the designing of the series converter or SSSC.

The STATCOM is developed using the traditional 6pulse, three-phase bridge topology and consists of two converters; rectifier and inverter as illustrated in figure 2 . The rectifier is constructed using diode and the inverter using thyristors. A capacitor connects the dc side of the rectifier and inverter and a shunt transformer connects both ac side of the converters to the ac supply. Under normal operation, the rectifier will charge up the capacitor and when there is a voltage drop or sag occurs to the system such as a fault, the inverter operates and the capacitor gets discharged. As the charge in the capacitor depletes, the rectifier will operate and charge back the capacitor.

SSSC is developed based on the three-level multilevel structure using Insulated Gate Bipolar Transistors (IGBTs) as the power switches. Figure 3 shows the simulation model of the SSSC. By controlling the phase shift, $\phi$, the angle of the output voltage of the SSSC, $\Delta \mathrm{V}$ can be controlled and by varying the value of $\mathrm{dc}$ link capacitor voltage, $\mathrm{V}_{\mathrm{dc}}$, the magnitude of the output voltage of the SSSC, $\delta \mathrm{V}$ can be varied. This two parameters; phase shift, $\phi$ and dc link capacitor voltage, $\mathrm{V}_{\mathrm{dc}}$, provide the control of series voltage with variable magnitude and phase angle for the SSSC. By combining the STATCOM and the SSSC as in figure 1, a complete system of UPFC is constructed.

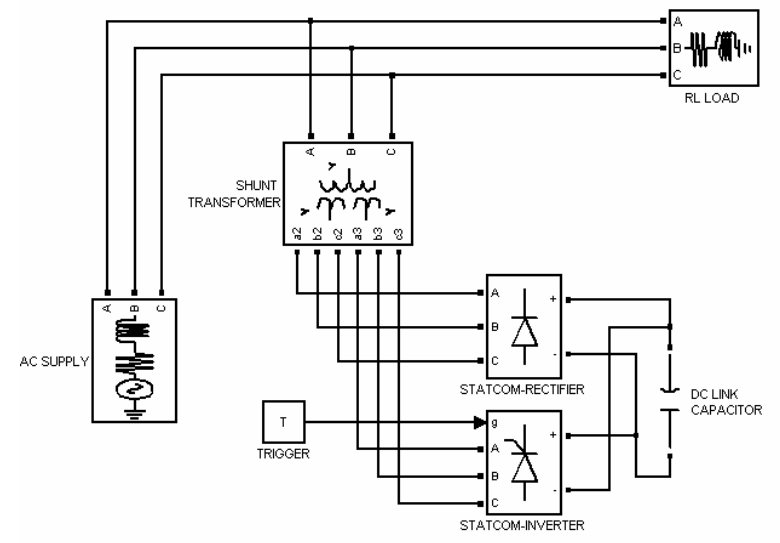

Figure 2. Simulation model of STATCOM 


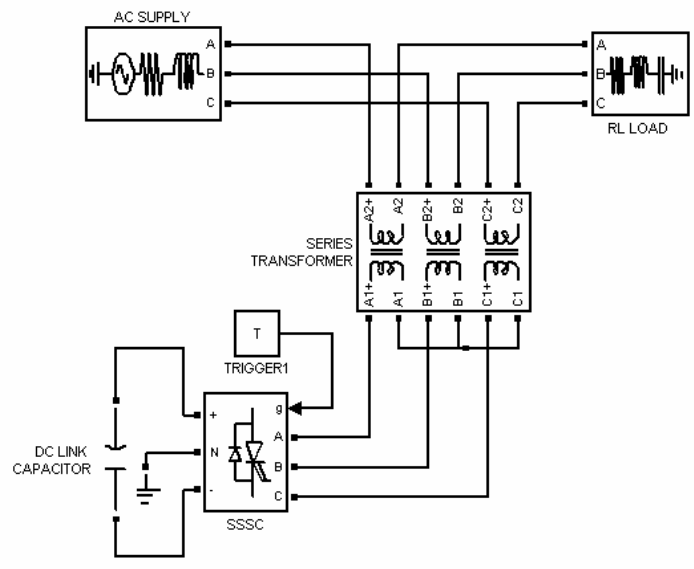

Figure 3. Simulation model of SSSC

\section{RESUlts AND Discussions}

The operation of the STATCOM is studied by applying two separate three phase solid faults at two separate times on the same transmission lines. The first fault occurs at time $0.5 \mathrm{~s}$ to $0.7 \mathrm{~s}$ for a duration of $0.2 \mathrm{~s}$ and the second fault occurs at time $1.2 \mathrm{~s}$ to $1.3 \mathrm{~s}$ for a duration of $0.1 \mathrm{~s}$.

As seen from the figure 4 , when a fault occurs, the voltage of the ac supply line drops and returns to normal after the fault is removed. The drop of sag value depends on fault and system impedances. A slight transient is noticed after the removal of fault.

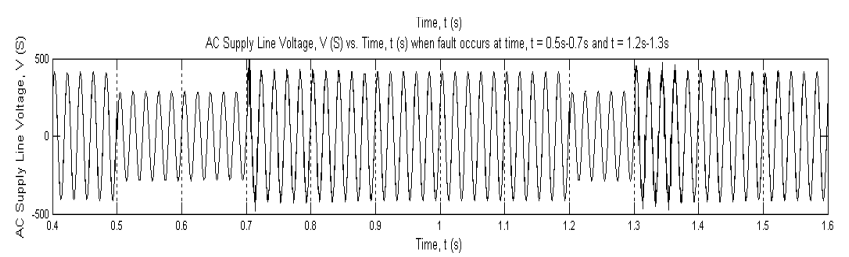

Figure 4. AC supply line voltage

Figure 5 shows the ac current waveform of the inverter and rectifier. As illustrated, the current is of pulsed type due to the reason of the switching OFF and ON of the power devices when the converter operates. When faults occurred, the ac voltage of the supply lines will dropped, so, in order to mitigate the voltage drop, the inverter starts to operate. By using the dc link capacitor as power supply, the inverter discharges the capacitor to inject current to the ac supply. As the capacitor discharges and its voltage decreases, the rectifier starts to charge the capacitor back and injecting a reactive power into the ac system.

Figure 6 shows the real power and reactive power transferred from the STATCOM to the AC supply system during fault. It shows that during the duration of fault, the real power is absorbed by the STATCOM and the reactive power is generated by the STATCOM.

The SSSC operates by changing the phase shift, $\phi$, in order to change the angle of the output voltage of the SSSC, $\Delta V$. Figure 7 shows the SSSC ac output line voltage for phase shift, $\phi$ equal to zero. When this voltage is added to the ac supply line voltage, a new $\mathrm{V}_{2}$ is achieved. This way, the active power and reactive power can be controlled.
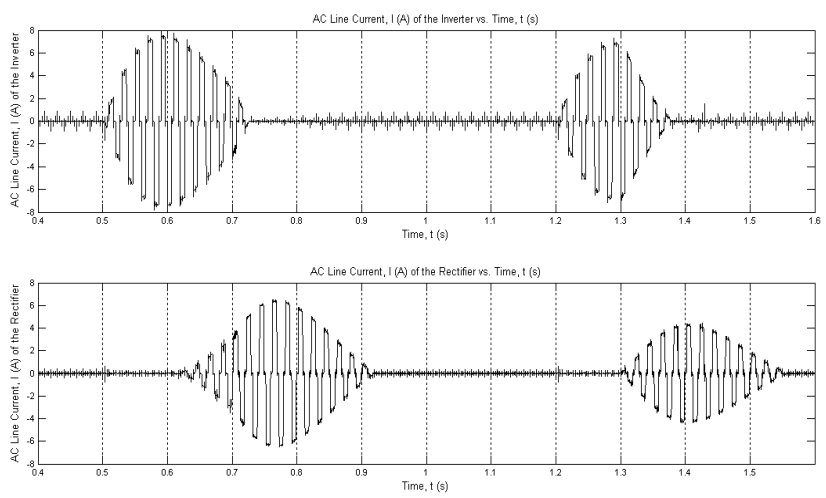

Figure 5. AC line current of the rectifier (Top) $\mathrm{AC}$ line current of the inverter (Bottom)
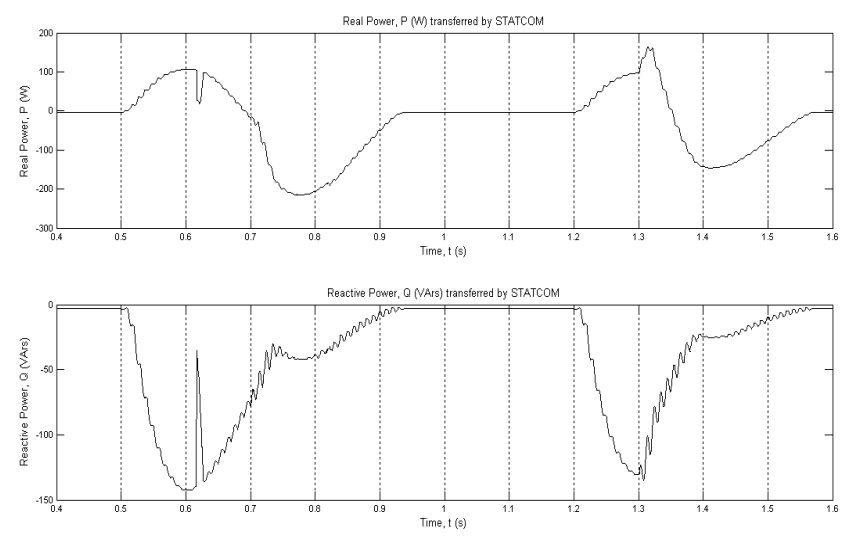

Figure 6. Real power (top) and reactive power (bottom) of the STATCOM

Figure 7 shows the line-to-neutral voltage and line-to-line voltage of the three-level neutral-point-clamped SSSC. It differs from the conventional two-level inverter as it is now has three voltage level i.e. $+200 \mathrm{~V}, 0 \mathrm{~V}$ and $-200 \mathrm{~V}$, as compared to the two-level where it only has two voltage level i.e. $+200 \mathrm{~V}$ and $-200 \mathrm{~V}$. This zero voltage value is obtained by switching $\mathrm{ON}$ two power switches that are connected to the neutral point of the phase leg. Table 1 gives the switching states of the power switches.

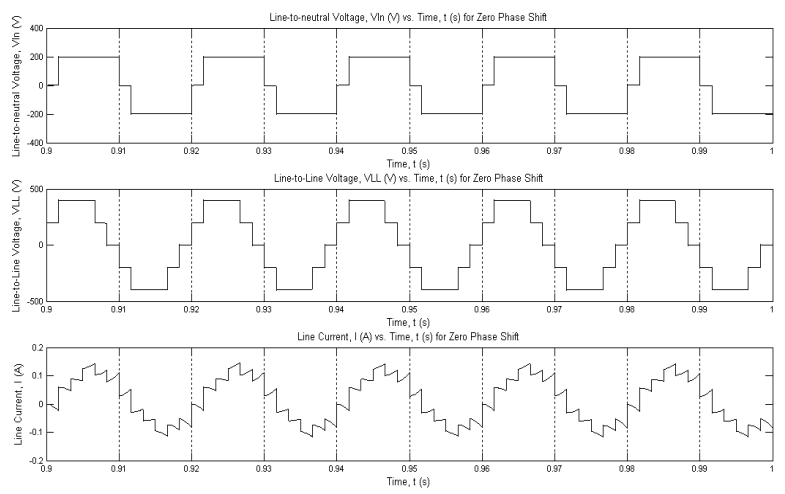

Figure 7. SSSC line-to-neutral voltage (top), line-to-line voltage (middle) and line current (bottom)

Table 1. Switching states for three-level neutral-pointclamped inverter

\begin{tabular}{|c|c|c|c|c|}
\hline Line Neutral Voltage & S1 & S2 & S3 & S4 \\
\hline$+\mathbf{V}$ & On & On & Off & Off \\
\hline $\mathbf{0}$ & Off & On & On & Off \\
\hline$-\mathbf{V}$ & Off & Off & On & On \\
\hline
\end{tabular}


Figure 8 shows the voltage of $\mathrm{V} 1, \mathrm{~V} 2$ and $\Delta \mathrm{V}$. In this case the voltage V1 and V2 are of different magnitude and in-phase to each other as the phase shift is zero.

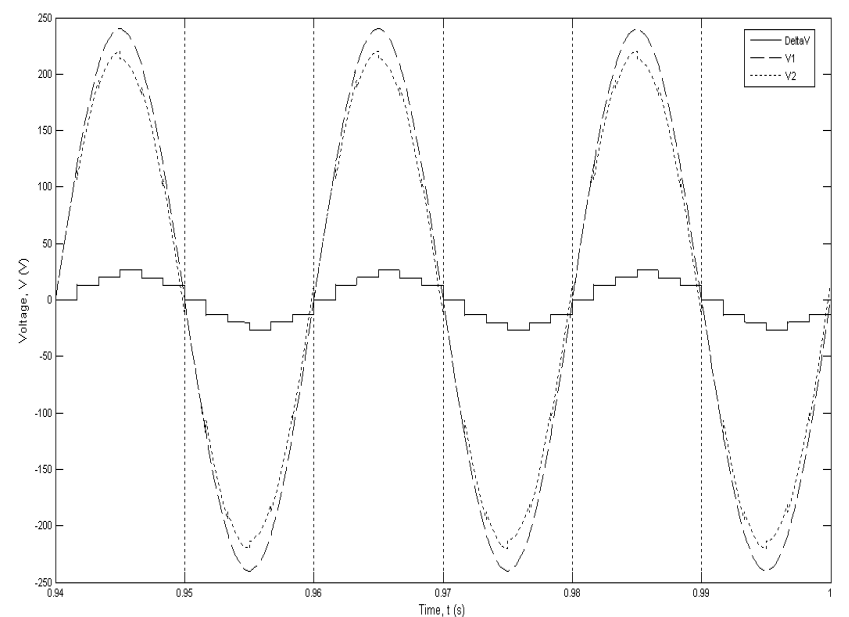

Figure 8. Voltage V1, V2 and $\Delta \mathrm{V}$

The phase shift, $\phi$ is varied, but with the same magnitude of $\delta \mathrm{V}$. As the phase shift, $\phi$ is varied, the voltage of the SSSC is also shifting. When this output voltage, $\Delta \mathrm{V}$ is added to V1, a new V2 is obtained. If phase shift, $\phi$ leads, voltage, $\Delta \mathrm{V}$ also leads and the resultant voltage, $\mathrm{V} 2$ will has a leading phase shift, and vice versa. This phase shift, $\phi$, also effect the amount of real power and reactive power transferred from the SSSC. This shows by specifying certain value of phase shift, $\phi$, the control of power can be achieved.

\section{CONCLUSION}

Designing and developing a simulation model of a three phase UPFC using SimPowerSystem Blockset is presented in this paper. The STATCOM is modeled based on the traditional 6-pulse three-phase bridge and the SSSC is modeled based on three-level multilevel structure. Simulation is carried out using a simple $\mathrm{ON}$ and $\mathrm{OFF}$ switching of the switching devices.

The control of power in the transmission system can be achieved by controlling the phase shift, $\phi$, of the SSSC.

\section{REFERENCES}

[1] P. Moore and R. Ashmole, "Flexible AC transmission systems," in Power Engineering Journal, Volume 9, Issue 6,1995 pp. 282-286.

[2] H. W. Wang and W. Fang, "Coordiated power control strategy for flexible AC transmission system," in IEEE International Conference on Power Electronics and Drive Systems 19999, pp. 677-682.

[3] N. G Hingorani and L. Gyugyi, Understanding FACTS. IEEE Press 2000.

[4] L.Y. Dong, L. Zhang, and M.L. Crow, "A new control strategy for the unified power flow controller," in IEEE Power Engineering Society Winter Meeting 2002, Volume 1, pp. 562-566.

[5] S. Zheng, L.T. Yoke, S. Cheng and Y. Zhu, "Dynamic characteristic study of UPFC based on a detailed simulation model," in IEEE Power Engineering Society Winter Meeting 2000, Vol. 2, pp. 1360-1364.

[6] M. Toufan and U. D. Annakkage, "Simulation of the unified power flow controller performance using PSCAD/EMTDC," in Electric Power Systems Research, Volume 46, Issue 1, 1998, pp. 67-75.

[7] Z.J. Meng and P.L. So, "A current injection UPFC model for enhancing power system dynamic performance," in IEEE Power Engineering Society Winter Meeting 2000, Vol. 2, pp. 1544-1549.

[8] S.O. Faried, and A.A. Eldamaty, "Damping power system oscillations using a genetic algorithm based unified power flow controller" in
Canadian Conference on Electrical and Computer Engineering, Vol.1, 2004, pp. 65-68.

[9] A. Edris, A.S. Mehraban, M.Rahman, L. Gyugyi, S. Arabi and T. Reitman, "Controlling the flow of real and reactive power," in IEEE Computer Applications in Power, Volume 11, Issue 1, 1998, pp. 20 25.

[10] J. Wang and F.Z. Peng, "Unified power flow controller using the cascade multilevel inverter," in IEEE Trans. On Power Electronics, Vol. 19, No.4, 2004, pp. 1077-1084. 


\title{
Co-Combustion of Agricultural Residues with Coal in a Fluidised Bed
}

\author{
W.A. Wan Ab Karim Ghani, A.B. Alias, R.M. Savory, M. A.M. Salleh and K.R. Cliffe
}

\begin{abstract}
Power generation from biomass is an attractive technology which utilizes agricultural residue waste. In order to explain the behavior of biomass-fired fluidized bed incinerator, biomass sources from agricultural residues (rice husk and palm kernel) was co-fired with coal in a $0.15 \mathrm{~m}$ diameter and $2.3 \mathrm{~m}$ high fluidised bed combustor. The combustion efficiency and $\mathrm{CO}$ emissions were studied and compared with those for pure coal combustion. Co-combustion of mixture of biomass with coal in a fluidised bed combustor designed for coal combustion increased combustion efficiency up to $20 \%$ depending upon excess air levels. CO levels fluctuated between $200-900 \mathrm{ppm}$ were observed when coal is added. It is evident from this research that efficient co-firing of biomass with coal can be achieved with minimum modification of existing coal-fired boilers.
\end{abstract}

Keywords: co-combustion, biomass, coal, fluidised bed, agricultural residue

\section{INTRODUCTION}

Waste-to-energy is gaining more and more attention as landfill costs and environmental concerns increase in many developed countries including Malaysia. A recent study shows that biomass in Malaysia contributes about 14\% approximately 340 million barrel of oil equivalent (boe), of the energy used every year. One of the major sectors that contribute waste to the biomass energy utilization in Malaysia was paddy cultivation and oil palm plantation. Based on production of 2,194 thousand tonne and 60,000 thousand tonne in year 2000, energy potential from these two resources are 16.4 and $229 \mathrm{PJ}$, respectively [1]. This implied about total of $375 \mathrm{MW}$ potential electric can be generated.

Combustion of agricultural residues is commonly used in industries for energy recovery. However, many researchers found that stand alone biomass firing is difficult to get high efficiency [2 and 3]. Thus, co-firing biomass with coal in industrial and utility boilers could offer an alternative

W.A. Wan Ab Karim Ghani is with the Dept. of Chemical and Environmental Engineering, University Putra Malaysia, 43400 UPM, Serdang, Malaysia (Tel: +603-89466287, Fax: +603-86567120, email: wanaz@eng.upm.edu.my).

A.B. Alias, R.M. Savory and A.M. Salleh are with the Faculty of Chemical Engineering, Universiti Teknologi MARA, 40450 Shah Alam, Selangor, Malaysia.

K.R. Cliffe is with the Dept. of Chemical and Process Engineering, University of Sheffield, Mappin Street, S1 3JD, Sheffield, United Kingdom (email: k.cliffe@sheffield.ac.uk). approach with improved combustion efficiency, lower-cost and reduced risk technology. Fluidised bed combustion (FBC) has been shown to be a versatile technology capable of burning practically any waste combination with low emissions [4]. The significant advantages of fluidised bed combustors over conventional combustors include their compact furnace, simple design, effective burning of a wide variety of fuels, relatively uniform temperature, and the ability to reduce emissions nitrogen oxide and sulphur dioxide gas [5].

This research was performed with the objective to characterise the biomass properties that affect cocombustion of biomass with coal in an FBC, particularly biomass that is available in large quantities in Malaysia (e.g. rice husk and palm kernel shell).

\section{EXPERIMENTAL}

\section{A. Fuel Characterization}

In this study British coal, rice husk and palm kernel shell originated from Perlis were employed as fuel. These fuels were open air dried for 2 to 3 days to remove moisture. The proximate and ultimate analyses were performed on coal and rice husk are summarized in Table 1. In comparison with coal, Table 1 shows that the main characteristics of the dry biomass are low calorific value $(14-18 \mathrm{MJ} / \mathrm{kg})$, high volatile matter content (60-75\%), ash content (10-25\%), low carbon content (35-45\%) and most importantly high oxygen content $(27-40 \%)$. This is of particular importance since it influences the stoichiometric air requirement for combustion. It is also important to note that the biomass has a lower particle density and varies in size with a great variation in shape than coal.

\section{B. Experimental Apparatus and Operating Procedures}

Figure 1, is a schematic diagram of the Atmospheric Fluidised Bed combustor used in this investigation. The system comprises of a $0.15 \mathrm{~m}$ diameter and $2.3 \mathrm{~m}$ high combustion chamber, allows for bed depths of up to $0.3 \mathrm{~m}$ using $850 \mu \mathrm{m}$ sand, cyclone, screw feeder and gas analyzer. The combustor body is constructed from $1 \mathrm{~cm}$ thick 306 stainless steel and covered in Kaowool insulation to prevent excessive heat loss during operation. Fluidising air was introduced at the base of the bed through a nozzle distributor and provided fluidisation and combustion air. Start up of the bed was achieved using an in-bed technique; 
Propane was introduced directly into the distributor plate by injectors and mixed with air in the nozzles, providing a combustible mixture at the nozzle exit. Bed and freeboard temperatures were measured at 8 different heights above the distributor plate by means of sheathed $\mathrm{Ni} / \mathrm{Cr}-\mathrm{Ni}$ thermocouples (TC) type K. Fuel was fed pneumatically into the bed surface from a sealed hopper through an inclined feeding pipe the flowrate for which flow rate was controlled by a screw-feeder. A cyclone was fitted to the combustor exit and the carryover from the bed was collected for analysis. $\mathrm{CO}$ and $\mathrm{O}_{2}$ were measured using a Xentra 4904 $\mathrm{B} 1$ continuous emissions analyzer, whereas $\mathrm{CO}_{2}$ was measured using a non-dispersive infrared absorption spectrometry analyser.

Table 1. Coal and biomass characterization

\begin{tabular}{|l|c|c|c|}
\hline \multicolumn{1}{|c|}{ Fuel } & $\begin{array}{c}\text { British } \\
\text { Coal }\end{array}$ & Rice husk & $\begin{array}{c}\text { Palm } \\
\text { kernel } \\
\text { shell }\end{array}$ \\
\hline $\begin{array}{l}\text { Proximate analysis } \\
\text { (\% wt, dry basis) }\end{array}$ & 58.90 & 15.00 & 18.60 \\
Fixed carbon & 38.20 & 60.70 & 72.50 \\
Volatile matter & 2.90 & 24.30 & 8.90 \\
Ash & & & \\
& & & \\
Ultimate analysis & & & \\
(\%wt, dry basis.) & & & \\
Carbon & 80.10 & 36.20 & 49.5 \\
Hydrogen & 5.30 & 5.71 & 6.74 \\
Nitrogen & 0.90 & 0.10 & 1.85 \\
Sulphur & 0.70 & 0.00 & 0.00 \\
Oxygen & 13.00 & 57.99 & 41.91 \\
& & & \\
\hline Calorific value & 31.1 & 13.5 & 18.0 \\
(MJ/kg) & & & \\
\hline Particle size (mm) & $1.4-4.8$ & $0.8 \times 1.00$ & $3 \times 6$ \\
& mm & mm & mm \\
& & (cyclindrical & (recta \\
& & shape) & ngle) \\
\hline Particle density & 1200 & 98 & 435 \\
(kg/m ${ }^{3}$ ) & & & \\
\hline
\end{tabular}

The percentage of combustion efficiency was computed using the following relation:

$E=\frac{\mathrm{B}+\text { unburned carbon in ash }}{\mathrm{C}} \times 100$

where $\mathrm{B}$ and $\mathrm{C}$ are the mass fractions of burnt and total carbon in the fuel, respectively. B can be determined from the flue gas composition, fractional excess air and the fuel ultimate analyses.

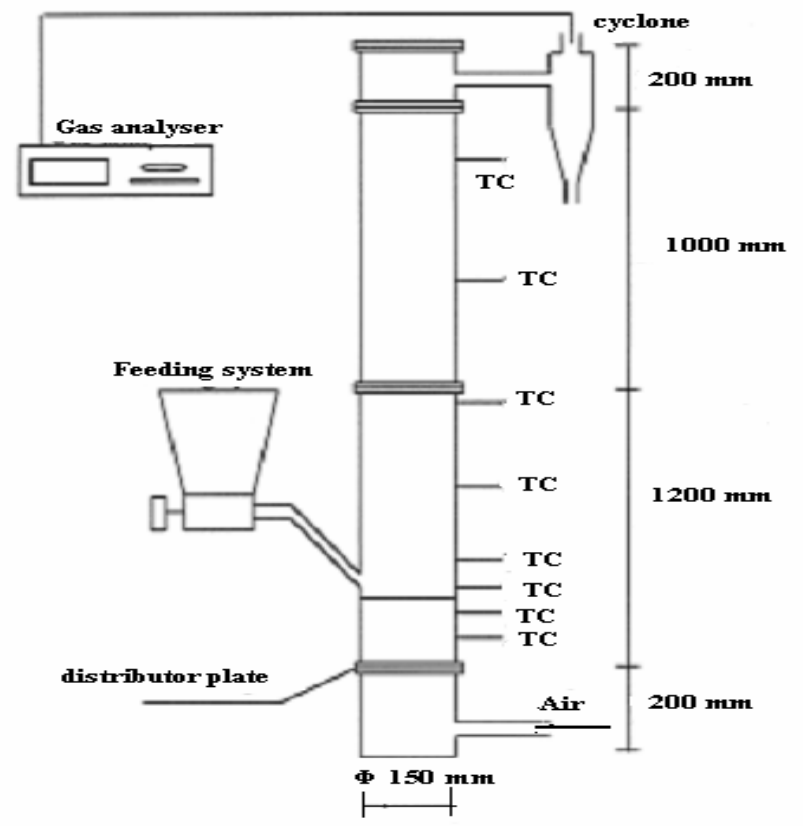

Figure 1. A schematic diagram of the laboratory scale fluidised bed combustor $(\mathrm{Tc}=$ Thermocouple $)$

\section{RESULTS AND DISCUSSION}

\section{A. Carbon Combustion Efficiencies}

The combustion tests were performed using different coal mass fraction; 0, 50 and $100 \%$, corresponding to heat input of $10 \mathrm{~kW}$ under optimum excess air conditions. Figure 2 shows the effect of different mixtures of rice husk and palm kernel shell with coal on carbon combustion efficiency with the same heat input. Generally, Carbon combustion efficiency for single biomass (rice husk and palm kernel shell) but increases with increasing coal addition and experimental runs. The following carbon combustion efficiencies, from Eq. (1), range between $67-75 \%$ for burning $100 \%$ rice husk and $80-83 \%$ for burning $100 \%$ palm kernel shell, $83-88 \%$, and $86-92 \%$ for $50 \%$ of coal addition to rice husk and palm kernel shell, respectively. The improved carbon combustion efficiency by cocombustion of rice husk with coal can be attributed to an increase in bed temperature, Figure 3, which is caused by the addition of fixed carbon content in the mixture. This fixed carbon, from coal, burns in the bed while the volatile gas burns in the freeboard region. Thus, there is more chance for fuel conversion carbon to carbon dioxide as the coal fraction increases and less volatile and tend to escape combustion, because of the reduced biomass concentration [6]. In addition, increasing the fluidizing velocity increases the turbulence in the bed leading to better solid mixing and gas-solid contacting and so as the amount of carbon in the bed is burnt at higher rate. Consequently, higher carbon burn out obtained leads to higher carbon combustion efficiency. However, when the combustion is stabilized, increasing fluidizing velocity contributed to a greater particle elutriation rate than the carbon to $\mathrm{CO}$ conversion rate and hence increased the unburned carbon [6]. In addition, increasing the fluidizing velocity increases the turbulence in the bed burn in a faster rate. Consequently, higher carbon burn out leads to higher carbon combustion 
efficiency. However, when the combustion is stabilized, increasing the fluidizing velocity contributes to a greater particle elutriation rate than carbon to carbon monoxide conversion rate and increases the amount of unburned carbon. This phenomenon can be seen in Figure 2 where the carbon combustion efficiency is lower than expected for $50 \%$ rice husk mixtures when the fluidizing velocity increases beyond the optimum value. Apart from solid mixing, increasing the fluidizing velocity also influences the fuel particle settling time during the combustion process in the FBC. Increasing fluidizing velocity drives the lighter fuel particles upwards and into the freeboard region, which is indicated by higher freeboard temperatures. Thus, the settling time for the biomass to reach the bed will be greater and a significant portion of the combustion will be completed before the particles return to the bed is reached, although this is dependent upon fuel particle size and density. This settling time depends on the fuel particle size and particle density. The greater settling time the higher the freeboard temperature due to greater volatile combustion contributing to higher combustion efficiency providing the bed temperature is maintained within the range of 800 $900^{\circ} \mathrm{C}$.

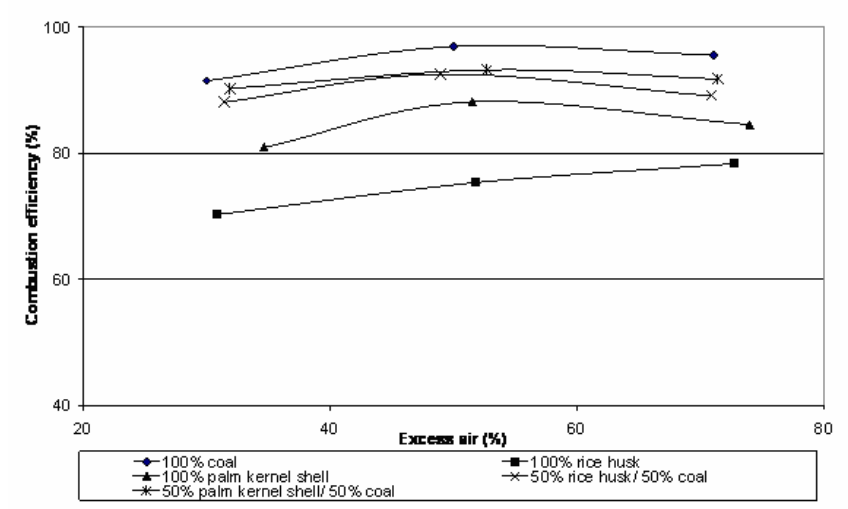

Figure 2. Carbon combustion efficiency during cocombustion of coal with rice husk and palm kernel shell as a function of excess air.

\section{B. Temperature Profiles}

Figure 3 illustrates the axial temperature distributions along the FBC height for fuel studied at 50\% excess air. As can be seen from the figure, coal combustion gives higher bed temperature $(\mathrm{y}=0-40 \mathrm{~cm})$ but lower freeboard temperature $(\mathrm{y}=450-120 \mathrm{~cm})$ in comparison to biomass. Then, all the temperatures shows start to fall from $120 \mathrm{~cm}$ above distributor plate indicating that most of the combustion was completed. This significant combustion behavior can be explained by the devolatilization process of the fuel (Sami et al, 2001). With high volatility (more than $50 \%$ ) and low ignition temperature $\left(250-350^{\circ} \mathrm{C}\right)$, biomass (rice husk and palm kernel shell) will start to devolatilize upon feeding at $45 \mathrm{~cm}$ of the FBC height (freeboard region) and was mostly burned before it reached the bed region. While coal with low volatility $(30 \%)$ and higher ignition temperature $\left(400-600^{\circ} \mathrm{C}\right)$ will travel down to the bed and completed combustion in the bed region. This was also greatly influenced by settling velocity of the fuel particles which correspond to the fuel particle size and fluidizing velocity [6]. Those explain why palm kernel shell has higher bed temperature than rice husk even though the volatility is almost similar (see Table 1). This was due to the fact that greater particle size contributed to a greater devolatilisation time and settling time. The greater settling time, the higher the freeboard temperature due to greater volatile combustion contributing to higher combustion efficiency providing the bed temperature is maintained within the range of 800 $900^{\circ} \mathrm{C}$.

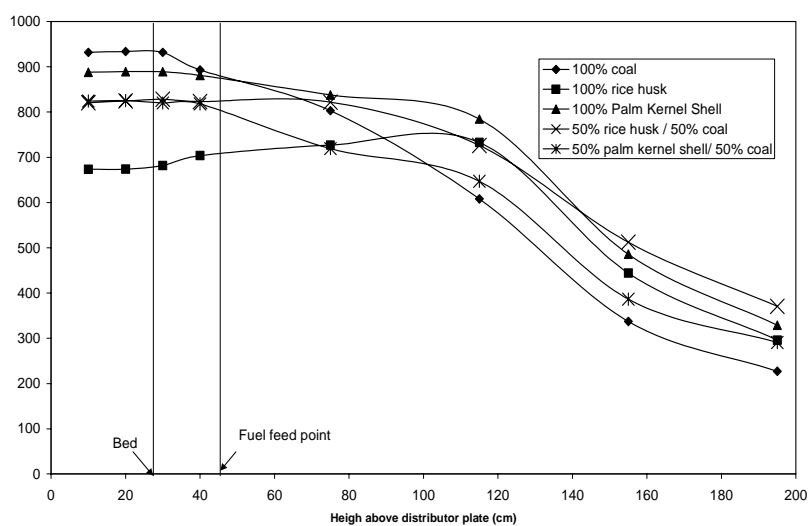

Figure 3. Axial temperature profile for co-combustion of coal with rice husk and palm kernel shell combustion in the case of excess air $=50 \%$

Significant increment of carbon combustion efficiencies was noted with coal addition to biomass fraction (see Fig. 2). The improvement can be attributed to an increase in bed temperature, Figure 3, which is caused by the addition of fixed carbon content in the mixture. This fixed carbon, from coal, burns in the bed while the volatile gas burns in the freeboard region. Thus, there is more chance for fuel conversion carbon to carbon dioxide as the coal fraction increases and less volatile and tend to escape combustion, because of the reduced biomass concentration. Furthermore, this can be explain by the fact that biomass fuels with lower density (about half) compared to coal tend to burn in freeboard and coal tends to burn in the bed region. Therefore, the addition of coal in biomass increases the amount of fixed carbon reaching the bed resulting in higher bed temperatures. This observation agrees with the results of Abelha et al, 2003 and Suksankraisorn et al, 2003 who investigated the co-firing of coal and chicken litter and cofiring of lignite with municipal solid waste in a fluidised bed combustor, respectively.

\section{Carbon Monoxide (CO) Reading}

In order to enable comparison of $\mathrm{CO}$ from all tests were converted to $\mathrm{CO}$ emitted $6 \%$ flue gas oxygen. Figure 4 , it is evident that there are significant fluctuations in $\mathrm{CO}$ emissions, which between 200 and 900 ppm under the same conditions. The orders of fluctuation were similar to those observed by Abelha et al.[7] and Sami et al. [8]. The fluctuations are caused by slight variations in feed composition and this effect is reflected in the temperature profiles. It is noted that the addition of coal has no significant influence on $\mathrm{CO}$ emissions during all cocombustion cases, except at coal $(50 \%) /$ rice husk $(50 \%)$ where emissions tend to be lower than expected in reference to the other rice husk fractions. This phenomenon is due to 
the synergistic nature of the coal and rice husk mixture, which enhances the fuel reactivity and lowers the $\mathrm{CO}$ emissions [9]. In most cases the emission of $\mathrm{CO}$ seems relatively insensitive to changes in excess and fluidising air. This insensitivity to increase excess air due to increased segregation of fuels in the combustor between the feed point and the bed. If the combustor receives a batch with a relatively high amount of fuel pellets, then as burning $\mathrm{CO}_{2}$ is produced since the pellets need to be heated and dried first. While this occurs, oxygen is not consumed and results in high $\mathrm{CO}$ emissions. The decrease in $\mathrm{CO}$ levels at low percentages of excess air, not below than $50 \%$, can be attributed to low excess air, relatively high bed temperatures (about $900^{\circ} \mathrm{C}$ ) causing rapid enhances and ignition of volatiles from rice husk. Thus, higher $\mathrm{CO}$ to $\mathrm{CO}_{2}$ conversion rates and enhancing the reactivity of the mixture [6].

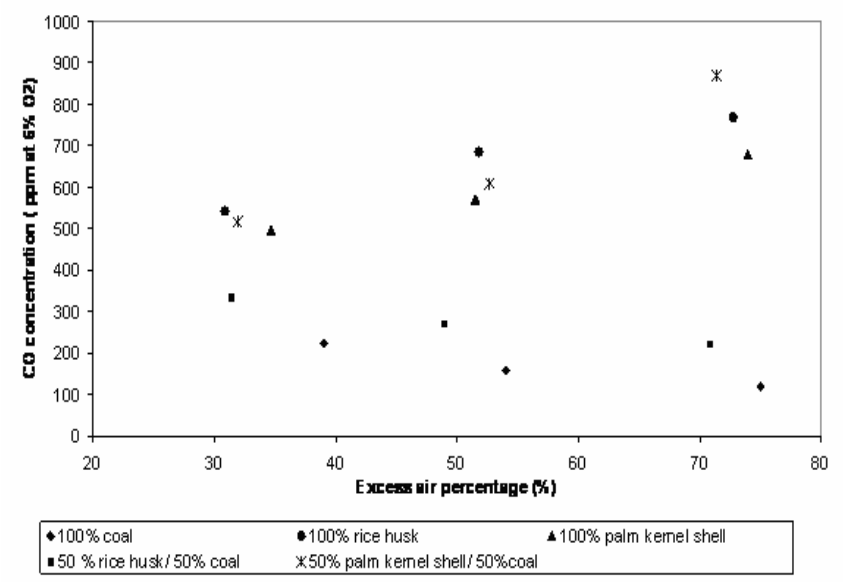

Figure 4. $\mathrm{CO}$ emissions as a function of excess air and Rice husk fraction combustion at heat input $10 \mathrm{KW}$

\section{CONCLUSION}

The ultimate conclusion is that stand alone biomass (rice husk and palm kernel shell) combustion could not achieve satisfactory combustion efficiency (more than $85 \%$ ) even at higher excess air (more than 70\%). However, additional of coal of $50 \%$ mass fraction could increases the carbon combustion efficiency up to $80 \%$ with acceptable $\mathrm{CO}$ emission limit $(<2500 \mathrm{ppm})$. As a conclusion, the combination factors of settling velocity and bed temperature are the major factors attributed to the resulting effects of biomass co-combustion.

\section{ACKNOWLEDGMENT}

This work was performed within the University of Sheffield, a research project supported by the Ministry of Science, Technology and Innovation (MOSTI), Malaysia. Special acknowledgement to Dr. Khudzir Ismail from Universiti Teknologi MARA for supplying the raw materials.

\section{REFERENCES}

[1] BIOGEN (2004). BIOGEN information Sheet, November, 2004

[2] Natrajan, R., Nordin, A., and Rao, A. N., 'Overview of combustion and gasification of rice husk in fluidised bed reactors', Biomass and bioenergy, 14,1998, pp.533- 546.
[3] Bhattacharya, S.C. and Wu, W., 'Fluidised bed combustion of rice husk for disposal and energy recovery', Energy from Biomass and Wastes XII, 1989, 591- 601.

[4] Tillman, D. A. 'Biomass cofiring: the technology, the experience, the combustion consequences', Biomass and Bioenergy, 19(6), 2000, pp. 365-384.

[5] Saxena SC and Jotshi CK. 'Fluidized bed incineration of waste materials'. Progress in Energy and Combustion Science 1994; 20:281.

[6] Suksankraisorn, K, Patumsawad, S. and Fungtammasan, B., 'Combustion studies of high moisture content waste in a fluidised bed', Waste Management, 23, 2003; 433-439.

[7] Abelbha, P., Gulyurthu, I., Boavida, D., Seabra Barros, J., Cabrita, I., Leahy, J.,Kelleher,B.,and Leahy M, 'Combustion of poultry litter in fluidised bed combustor', Fuel, 82, 2003, 687-692.

[8] Sami M, Annamalai K, and Woodridge M. 'Co-combustion of coal and biomass fuel blends', Progress Energy Combustion Science, 27, 2001;171-214.

[9] Kuprianov, V. I and Pemchart, W. 'Emissions from a conical FBC fired with a biomass fuel', Applied Energy, 74, 2003, pp. 383-392. 


\title{
Photovoltaic Power System for Tropical Greenhouse Cooling
}

\author{
Desa Ahmad and Faisal Mohammed Seif Al-Shamiry
}

\begin{abstract}
Natural ventilation in tropical greenhouse is common method for ventilation which gives higher inside temperatures compared to the outside temperatures. In addition, this type of ventilation is not enough to reduce high temperature inside the structure in low land areas. Thus the requirement of cooling is increased. Use of the fossil fuel to run the cooling fans are not economically viable due to increasing of fuel cost and greenhouses are not always located near the electrical grid. This paper presents a study on the installation and testing of a complete photovoltaic hybrid system for cooling a tropical greenhouse. The hybrid photovoltaic system consists of two photovoltaic sub-systems connected to each other. This system includes 48 photovoltaic solar panels with 18.75 watt each, an inverter, a charge controller and a battery bank (including 12 batteries). The PV system is located at Universiti Putra Malaysia Research Park. The national electricity grid was used as a backup unit. The load consisted of two misting fans for cooling greenhouse with 400 Watt electric power and five hours (11:00 am to $16: 00 \mathrm{pm})$ daily operation. The results obtained showed that the maximum current drawn from the array was found to be 14.9 ampere at 13:00 pm (with load). The voltage of array was found to be 26.9 volt while the voltage and current of battery bank were found to be 26.2 volt and 23.0 ampere respectively. The total load for 6 months was found to be $12.37 \mathrm{kWh}$. This study highlighted the advantages and disadvantages of PV hybrid energy systems for tropical greenhouse cooling as an application of renewable energy in agriculture. The results showed that PV system would be suitable to supply electricity to cover the load requirement demands without using energy from the national electrical grid.
\end{abstract}

Keywords: PID controller, SVC, TCSC, power quality

\section{INTRODUCTION}

Malaysia lies in the tropical region between $1^{\circ} \mathrm{N}$ and $7^{\circ} \mathrm{N}$, and $100^{\circ} \mathrm{E}$ and $119^{\circ} \mathrm{E}$ and is made up of Peninsular Malaysia and the states of Sabah and Sarawak on the island of Borneo. The climate is hot and humid throughout the year with heavy rainfall. Rainfall range is between 2032$2540 \mathrm{~mm}$, temperature $21-32^{\circ} \mathrm{C}$, relative humidity $80-90 \%$,

Desa Ahmad is with the Department of Biological and Agricultural Engineering, Faculty of Engineering, Universiti Putra Malaysia, 43400 Serdang, Selangor,

(phone:03-89464455, fax:03-89466425, e-mail: desa@eng.upm.edu.my)

Faisal Mohammed Seif Al-Shamiry is with the Agricultural Engineering Department, Faculty of Agriculture, Sana'a University, P.O.Box 13383, Sana'a, Republic of Yemen (e-mail: faisalupm@yahoo.com) solar radiation $12-20 \mathrm{MJ} / \mathrm{m}^{2}$ and wind speed $2-22 \mathrm{~m} / \mathrm{s}$. There are approximately 6 hours of sunshine per day [1].

Renewable energy plays an increasingly important role in the energy supply of the developing and industrializing countries. Developing a clean and renewable energy has become one of the most important tasks assigned to modern science and engineering. Photovoltaic (device that converts sunlight directly into electrical energy) energy comes along as the most promising one as a future energy resource. The ultimate objective of any design procedure employed is to obtain the sizes and ratings of the various energy conversion and energy storage devices needed to supply energy to different loads [2].

A hybrid system can be generally defined as an electricity production and distribution system which consists of a combination of two or more types of electricity generating sources (examples are solar photovoltaic panels and wind turbine generators,). Usually it also includes an energy storage.

Batteries provide storage of energy, which is delivered at the time when the solar radiation is low, in cloudy periods or at night-time. Lead-acid batteries operation is characterized by the limited range of depth of discharge (DOD), the value currently being limited to a maximum of $80 \%$ at the best operating conditions. If this value is exceeded, the battery suffers from over-discharge, and permanent damage may result if the battery remains at this state for long. The state of charge (SOC) had been shown to be an influencing parameter onto the battery voltage evaluation, especially during the charging and overcharging process [3]. The inverter has to be capable of handling the maximum expected power AC loads [4].

AC utility back-up would incorporate an automatic charger wired to the battery bank which would regulate charge when batteries are low and automatically cut off when batteries have sufficient operating power.

The PV energy systems differ from conventional energy systems in that they have high initial and low operating costs, and using PV systems is very important, especially when their life cycle costs are competitive with the other types of conventional energy sources [5],[6].

The price of PV system and its installation are important factors in the economics of PV systems. These include the prices of PV modules, storage batteries, the control unit, the inverter, installation and all other auxiliaries. The photovoltaic (PV) module prices have dropped from $\$ 500 / \mathrm{W}$ peak in 1972 to approximately $\$ 4-\$ 5 / \mathrm{W}$ peak in 
1990 [7]. Realistic economic analysis of PV systems is difficult to translate into an accurate long-term cost projection because of the number of complex factors involved [8], [9].

This paper presents a study on the installation and testing of a complete photovoltaic hybrid system for cooling a tropical greenhouse.

\section{MATERIALS AND METHOD}

\section{A. Electric Load Estimation}

The load consists of two fans with total power of $520 \mathrm{~W}$. Each fan requires $260 \mathrm{~W}$ and $240 \mathrm{VAC}$. The total average daily load is $2.6 \mathrm{kWh}$ /day. Fan consumption at 260 Watts and at $240 \mathrm{VAC}$ through an inverter would require 21.7 Amps/Hour at 12 VDC load demand with operation of 5 Hours/day (11:00-16:00) and 3 days autonomy. The total load demand is 217 Amps.

\section{B. System Configuration}

The PV system includes 48 photovoltaic solar panels, one inverter, charge controller and a battery bank as shown in 'figures 1 and 2'. The national electricity grid is used as a backup unit. Each PV panel generates $18.75 \mathrm{~W}_{\mathrm{p}}$ and 1.14 Amps/hour. Therefore to provide electric power for 217 Amps total, at least 38 PV Modules would be required. The total energy generation from 48 modules (48*1.14Amps/hour*5 hours of sunshine/day) is 273.6 Amps. This energy covers the load demand (217 Amps) consumption and 56.6Amps to further offset weather and inverter efficiency inherent with PV system. Turn-on and turn-off functions were carried out by means of timer, temperature and humidity sensors. The system was turnedon at 11:00 am and turned-off at 16:00 pm by means of the timer sensor. When the inside temperature dropped to $\leq 30^{\circ} \mathrm{C}$ the system could be turned-off, when the temperature reached $>30^{\circ} \mathrm{C}$ the system was turned-on. This process was carried out by means of temperature sensor. When the inside humidity reached $85 \%$ the water supply was stopped by means of water solenoid valve and humidity sensor. Grid is used to charge the battery during cloudy days if battery voltage falls below preset levels in the controller. In case of total PV system failure it would change over to the grid system.

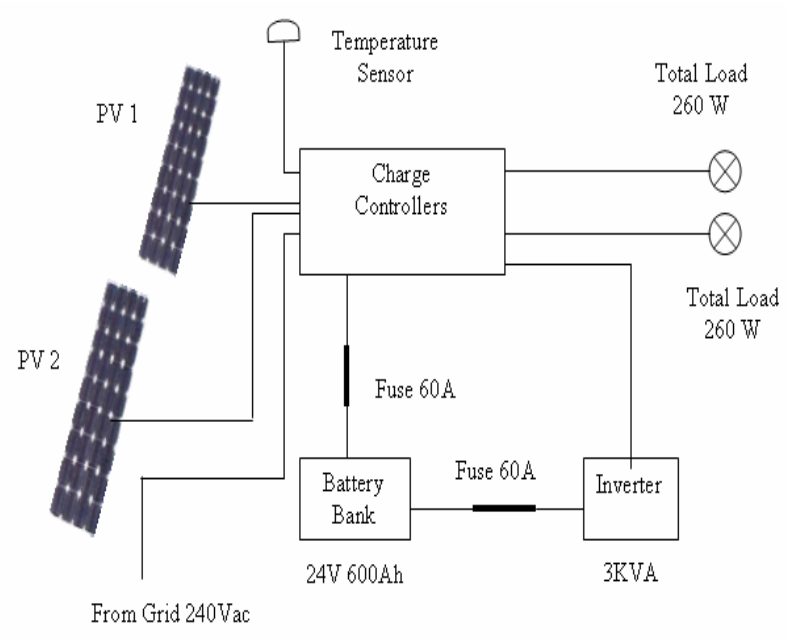

Figure 1. Schematic diagram of the PV energy system

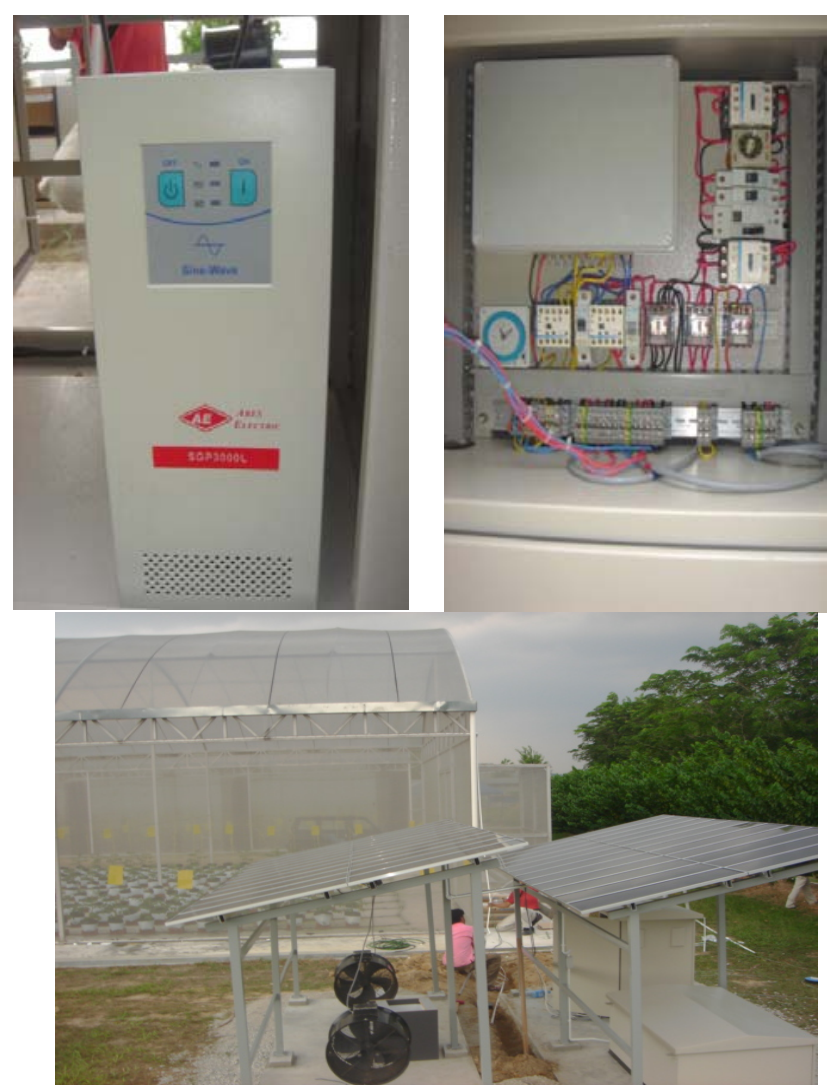

Figure 2. PV array structure, controller and inverter.

\section{RESUltS AND DiscusSION}

\section{A. PV System}

'Figure 3' shows the outside and cell temperatures. From this figure, the cell temperature was found to be $49^{\circ} \mathrm{C}$ while the outside temperature was found to be $36.9^{\circ} \mathrm{C}$ at $14: 00$ $\mathrm{pm}$. The maximum PV array voltage and current were found to be $26.9 \mathrm{~V}$ and 14.9 Amps while the maximum battery voltage and current were found to be $26.2 \mathrm{~V}$ and 23 Amps as shown in 'figure 4'. 'Figure 5' shows the daily electric power from PV system. The PV system power was found to be 340.2 Watt at 11:00 am. This value increased to 400.81 at 13:00 pm and decreased to $275.6 \mathrm{Watt}$ at $16: 00 \mathrm{pm}$. The total energy per day (from 8:00 am to 6:00 pm), given by the PV modules to the battery bank was calculated to be 2.8 $\mathrm{kWh}$. From the load data, the consumption of energy per day was $2.6 \mathrm{kWh}$, which is about $92.86 \%$ of the PV supplied energy. These results showed that PV system would be suitable to supply electricity to cover the load requirement demands without using energy from the electrical grid.

\section{B. Battery Test}

To test the battery bank, the PV arrays were switched off, cutting the electric power supply from PV arrays to the battery. The battery bank was sized for 3 days autonomy and consisted of 12 batteries of 100 Ah each with total of 1200 Ah. The depth of discharge (DOD) was $70 \%$ equivalent to 840 Ah for usage with 3 days autonomy. 'Figure 6' shows the battery bank voltage against time. The fans were switched on for three days from 11:00 am to 16:00 pm. On the third day and when the battery voltage 
reduced to $20.9 \mathrm{~V}$ the fans automatically switched off. The inverter was also switched off and the system changed to the grid supply system.

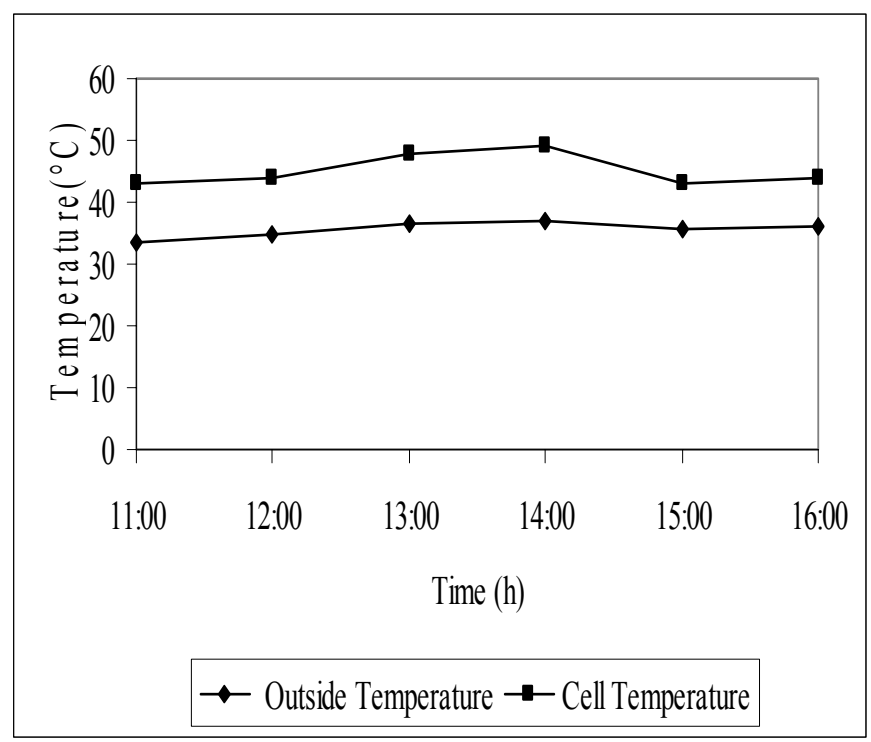

Figure 3. Outside and cell temperatures

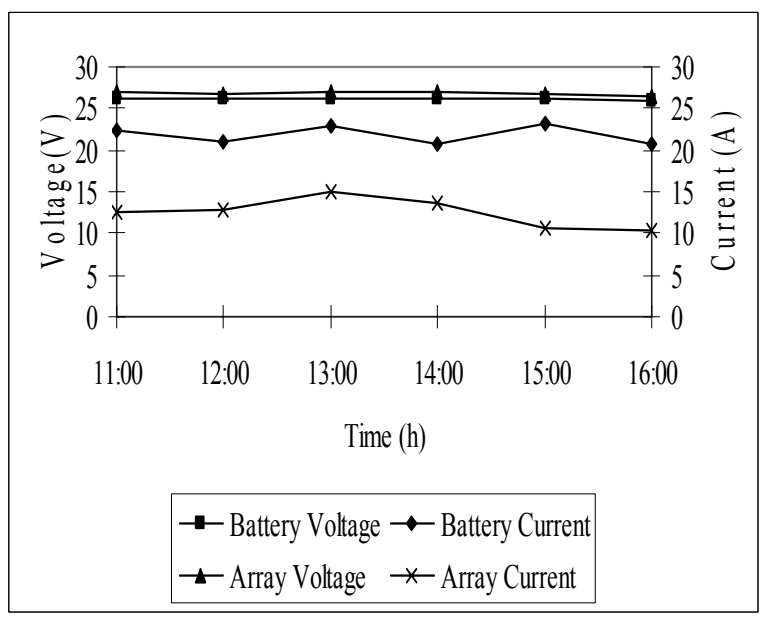

Figure 4. DC system output

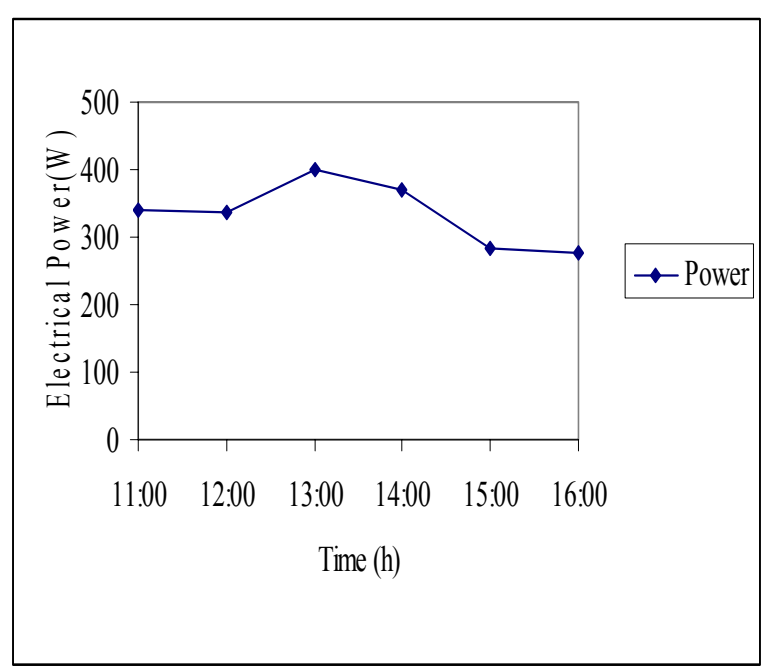

Figure 5. System power

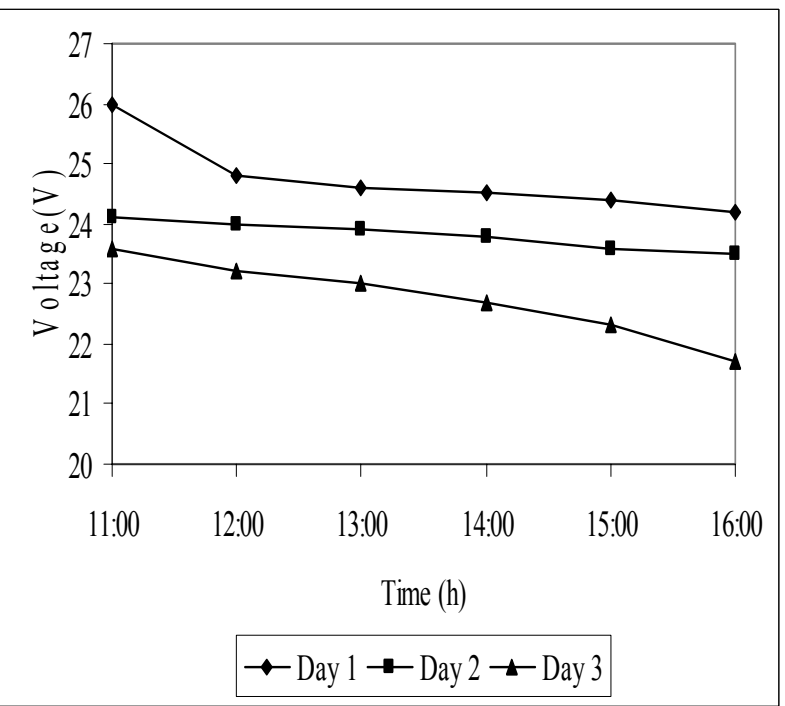

Figure 6. Battery voltage

\section{CONCLUSION}

The photovoltaic hybrid system for cooling a tropical greenhouse was installed and tested. The load requirement compared favourably with the actual requirement using the grid system whereby the evaporative (misting fans) cooling system in a selected greenhouse was operated with energy generated by the electric grid. This study showed that the designed and tested PV system operated satisfactorily. As PV systems are clean and do not cause pollution of any type during their use while the price of PV decreases every year and their efficiencies and reliability are increased, there is a good potential for this research finding to be used for various agricultural applications.

\section{ACKNOWLEDGEMENT}

This project was funded by MOSTI under IRPA Project No. 09-02-04-0873 EA001.

\section{REFERENCES}

[1] R. Kamaruddin, B.J.Bailey and J.I Montero. A naturally ventilation Greenhouse for temperate vegetable production in the tropics. Acta Horticulturae, 578, .2002.

[2] R.Ramakumar, I.Abouzahr and K. Ashenayi. A Knowledge-Based Approach to the design of integrated renewable energy system, IEEE Transaction on Energy Conversion, vol.7, No.4: 648-659, 1992.

[3] C.Armenta-Deu, Prediction of battery behaviour in SAPV applications. Renewable Energy 28, 2003, 1671-1684.

[4] S.R.Wenham, M.A Green and M.E.Watt Applied photovoltaics. Australia: Center for Photovoltaic Devices and Systems, 1994.

[5] G.E. Ahmad. Photovoltaic-powered rural zone family house in Egypt, Renewable Energy, 26,2002, 379-390.

[6] M.Dakak A.Hirata, R.Muhida, and Z.Kawasaki.Operation strategy of residential centralized photovoltaic system in remote areas, Renewable Energy, 28, 2003 997-1012.

[7] M.Kolhe, S.Kolhe and J.C.Joshi Economic viability of stand-alone solar photovoltaic system in comparison with diesel-powered system for India. Energy Economies 24, pp. 155-165., 2002

[8] R.A.Wagdy, R.P.Mertens, and R.Van Overstraeten. Economic feasibility of photovoltaic system in the developing contries. Solar Wind Technology 2 (1), 9-14. 1985.

[9] R.Haas. The value of photovoltaic electricity for society. Solar Energy 54 (1), 25-31. 1995. 


\title{
Initial Performance Testing of an Airboat for Sarawak Rural Riverine Ecological and Flood- Rescue Applications
}

\author{
Peter Nai Yuh Yek, Mohammad Omar Abdullah, Ervina Junaidi, Rusli Muslimen, Sinin Hamdan, \\ Khairuddin Ad Hamid and Peter Kuek
}

\begin{abstract}
This paper describes the construction of a prototype airboat intended for riverine ecological study and flood-rescue application in the rural areas of Sarawak, Malaysia. The boat is designed for 2 - 5 passengers with a 2stroke 3-cylinder 540cc engine. Many local riverine area transportation and ecological study are limited because of tideline dependent; however, the airboat can be considered as one of the promising means of riverine transportation vehicles as the boat can easily ply at the local everglades areas or shallow waters during low tides. In addition, it is also useful during flooding period for the rescue purpose as the airboat is able to operate at many terrain conditions.
\end{abstract}

Keywords: riverine ecology, airboat, riverine transportation, mangrove ecology, engine performance

\section{INTRODUCTION}

The usage of water transportation is very important in the riverine area of Sarawak, Malaysia, especially in the rural area that could not be assessed by normal means of car transportation. Many parts of these areas are swamp and the rivers are shallow especially at upstream and delta regions, shown at figure 1 .

The conventional blade type marine engine is not feasible for use in those areas especially during low tide. This problem can be solved by using airboat ${ }^{1}$ for the means of transportation. This is because unlike conventional boats, airboat's propeller is above the water level thus it can easily move around on the swampy, shallow and everglade areas. As such, the airboat can be used anytime even during low tide situation and beneficial especially during emergency

Peter Nai Yuh Yek is a MEng research Student at the Faculty of Engineering, Universiti Malaysia Sarawak, 94300 Kota Samarahan, Sarawak, Malaysia (email: peteryeknaiyuh@gmail.com)

Mohammad Omar Abdullah is with the Faculty of Engineering, Universit Malaysia Sarawak (Corresponding author Tel: +60 82679301 (Direct line). Fax:+6082672317 e-mail: amomar@feng.unimas.my).

Ervina Juanidi is with the Faculty of Engineering, Universiti Malaysia Sarawak (e-mail: jervina@feng.unimas.my).

Sinin Hamdan is with the Faculty of Engineering, Universiti Malaysia Sarawak (e-mail: hsinin@feng.unimas.my).

Khairuddin Ab Hamid is with the Universiti Malaysia Sarawak (e-mail khair@cans.unimas.my).

Peter Kuek is with the Malaysian Airboat Company (e-mail: ochem@tm.net.my, sze0@streamyx.com).

Airboats are essentially flat-bottomed vessels propelled in a forward direction by a aircraft type propeller and powered by either an aircraft or automotive engine cases.

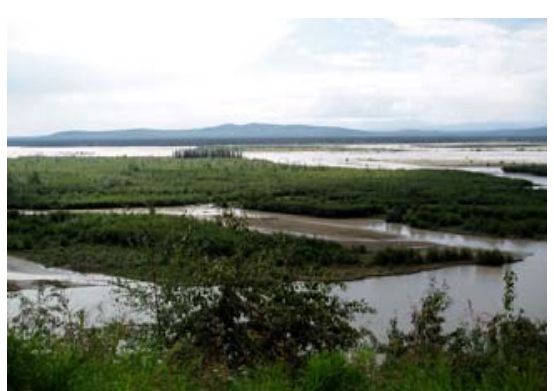

Figure 1. A typical delta view during the low tide

The use of airboat in ice and water rescued emergencies environments [1]. It had been found that airboats could minimize risk to responding personnel and reduce the time required to perform life saving operations by a quantifiable figure of $50-80 \%$. Compared with the cost of an ambulance, the author concluded that an airboat, which cost around US\$ $25,000-40,000$, can be considered as an inexpensive investment.

Airboats have been used and expanding in Alaska, U.S.A., particularly in the interior [2]. This is because the remote wetland area is hardly accessible on foot or by conventional boat. The capability of the airboats to traverse wetlands with sensitive vegetation and soils makes them different from ordinary watercraft. Thus they should be considered as a separate category of off-road vehicle by the managers of public lands. Beside that, airboats are also used for hunting, recreational travel, cabin access, and even commercial sightseeing tours. The number of airboat users has increased dramatically. In additional that airboat is able to bounce over broken ice at frozen Mississippi River for hydro survey application [3]. During the winter months, solid or broken ice becomes too hazardous for the hulls of the conventional survey boats but airboat has the ability to navigate over it. Airboat has also been used for backwater surveys that are too shallow for outboard powered boats.

The working principle of airboat and hovercraft are quite similar, they are different only in the base construction to provide air cushion in the later. However, the airboat is more stable and less maintenance cost than a hovercraft. Airboats are usually designed to travel very fast and stable over almost any wet terrain. It performs according to the 
Newton's Laws of Motion, viz. to every action there is always an equal and opposite reaction. The wind thrust acting backward generated by the propeller causes the boat to move forward [1].

For the current work, one of the primary aims is to design for used for the shallow river transportation in the riverine areas, especially during low-tide period. In the Faculty of Engineering, recently our research team had commissioned and conducted two initial tests on the airboat as shown in Figure.2 Results have shown that the existing airboat together with the old Samurai petrol jeep 540 cc engine, after some modifications, able to achieve a speed of around $15 \mathrm{~km} / \mathrm{h}$ with acceptable balancing capability [4]. However, the speed and balancing of the airboat can be further improved through a careful and better detail design work.

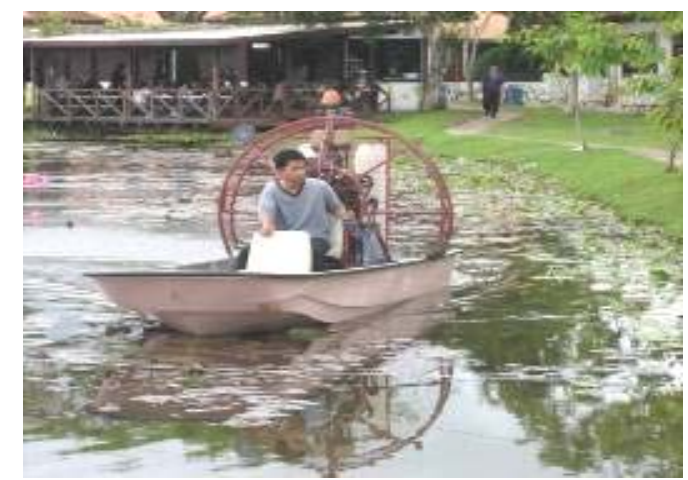

Figure2. The airboat constructed can move freely in the swampy area

\section{THE SARAWAK RIVERINE AND ECOLOGICAL CONDITIONS}

The Rajang River delta plain shown at figure 1, covers an area of $6500 \mathrm{~km}^{2}$ and peat deposits can up to $15 \mathrm{~m}$ thick, occur in this delta plain [5]. During low tide period, large areas of delta with peat along the river bank make conventional boat hardly to operate. Tides are semidiurnal within the delta plain of the Rejang River (figure.1) and its influence extends about $120 \mathrm{~km}$ inland. Conventional boat is only can be used for 12 hours per day and this condition is very inconvenient to the riverine area peoples. Tides along the Sarawak coast are corresponding to the widening of the shelf [5]. As an example in the Bako National Park, the conventional boats are not able to reach the jetty during the low tide period. They are only able to stop at the beachside to fetch the visitor. As a result, it creates a great inconvenience to the visitor and effect the tourism sector in the Sarawak. This situation could be possibly solved by airboat application.

Sarawak Kanan River, on the other hand, is one of the tidal rivers frequently affected by flood in Sarawak State, Malaysia. Over the years, physical development has been concentrated in the middle valley town of Bau, where it is the center of administration and commercial for the district. The town of Bau and surrounding areas are well known flood-prone area; figure 3 for it is geographically located in the floodplain of Sarawak Kanan River flood stricken. For instance back in the Year 2005, thousands of people were affected and a lot of properties were damaged [6]. Therefore, airboat could be very useful means of emergency usage for life saving, especially during the flood incident. This is because during the flood, the conventional boat's pilot is very difficult to estimate the terrain condition and this is very harmful and dangerous. Further more, the floating objects or shallow areas can damage the blades of the conventional boats.

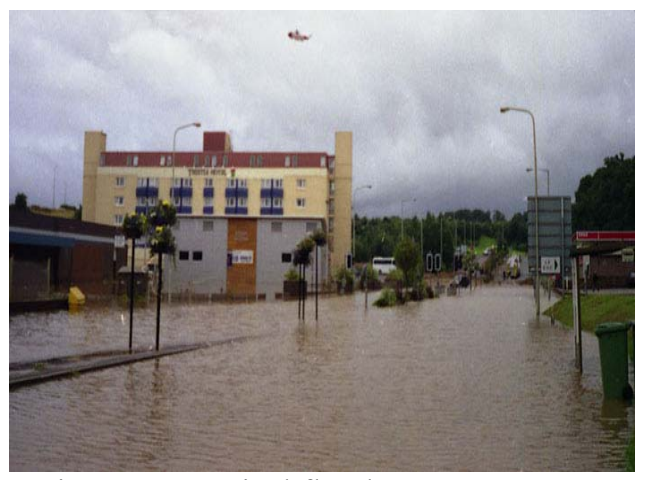

Figure3. A typical flood-prone areas at Bau

Mangroves are a group of evergreen shrubs as shown at Figure 4, found in swamps and forests along the coastal areas in tropical Kuching for instance areas adjacent to Bako National Park. The mangrove forests are an essential component of the tropical coastlines for many reasons. The forests acts as an intermediary between the open ocean and the coast helping to prevent erosion, filter nutrients, and provide protection from severe weather. Mangrove forests also form an incredibly diverse and complex habitat, which includes nursery grounds for fish/marine invertebrates, offer shelter for a variety of organisms, and are nesting areas for coastal birds. The mangroves and the ecosystem they created also have many uses for humans, but have tragically been exploited like so many other resources [7].

An investigation on the ecology protection and species richness in a mangrove ecology reported by Tomlinson elsewhere appears to be influenced by temperature, tidal amplitude, rainfall, catchments area, freshwater seepage, and frequency of cyclones [8]. Due to the important and roles of the mangrove ecology, as far as the local situations are concerned, investigation and researches need to be done toward the ecology prevention and protection. The airboat can play a very crucial part to carry out the study locally here in Sarawak. The mangrove ecology is wide and hardly reaches by the normal boat because some portion of the roots of the mangrove shrubs are high above the water level, shown at Figure 4, and the river bank is shallow. Airboat with a specific design are need to suit for this usage. The detail of the current boat design is discussed in section III .

Airboat is effectively used for sampling fishes in shallow, vegetated habitats by electrofishing [9]. It has been used successfully to sample fishes in a variety of fresh habitats. Two anodes suspended in fronts of the boat and used the hull of the airboat for the cathode. Electrofishing units mounted to the airboat are considered effective for sampling large fishes and can be used more frequently over larger spatial areas than block nets. Such method may well be feasible to be modified and adopted for local investigation.

Yet another possible ecological investigation in Brazil, airboat could be developed with spraying equipment, DGPS navigation system and flow control system used for weed survey and the application of precise and accurate rates of herbicides in aquatic ecosystems [10]. Profuse growth of aquatic plants has been observed in numerous lakes and 
rivers as consequence of the contamination of these ecosystems by wastewater and the nutrients and sediments brought from agricultures areas by erosion. The author reported that in Brazil, the availability of airboat as a means of transportation has improved the study of the chemical control of aquatics plants and less time consuming. Similar method may be used if needed for future investigation.

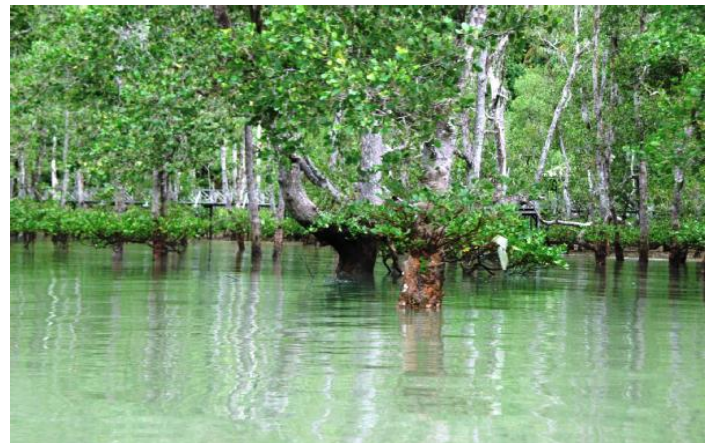

Figure 4. Typical ecology of mangrove in Kuching, Sarawak

For the above purposes and for initial educational training, an airboat is assembled, designed and built locally. The design is assisted by using CATIA as shown in figure 5 .

\section{DESIGN OF THE AIRBOAT}

During the design stage, numerous factors including application types, stabilization (balancing), vibration, speed and cost of construction of the boat needs to be considered [11]. The size of the propeller and engine must be appropriate to make airboat perform well. The size of the boat thus capacity matching is also one of the important factors in sizing such requirement.

\section{A. Hull Design}

This hull is designed with shallow $\mathrm{V}$ shape at the front as shown in figure.5. This would reduce pounds in wave for the comfortability of the passengers [11]. Size of the airboat is $4.3 \times 1.8$ meter that can accommodate 2 to 5 persons with total load capacity of $650 \mathrm{~kg}$ to $700 \mathrm{~kg}$. The $143.5 \mathrm{~kg}$ hull, is made from light weight fiberglass to minimize the load. Lighter boat will cause less water resistance thus easier to move around and has minimum impact on the ecology.

\section{B. Rail and Frame Design and Construction}

Steel rail is designed and built along the boat so that engine frames is easily to adjust to suitable distance viz. whether forward or backward. Adjustment can be done $60 \mathrm{~cm}$ forward and $40 \mathrm{~cm}$ backward from the present distance. During the testing, the distance of the central gravity and central buoyancy can be adjusted easily so that the performances of the airboat can be improved with respect of center gravity and center of buoyancy. Frame which is also made from steel is very important to safeguard the pilot and the passengers from the propeller. Central gravity of the airboat has been modified to the lowest level; this is to prevent the boat from capsizing. Propeller used for the airboat is fixed pitch with two blades wooden aircraft types. The diameter length of the propeller is $141.5 \mathrm{~cm}$. Diameter of the propeller is very important to determine the thrust produce by the engine. Larger or longer the propeller is able to produce stronger thrust with the same engine rpm. Propeller of the airboat is installed about $90.5 \mathrm{~cm}$ from the bottom of the boat to generate powerful thrust.

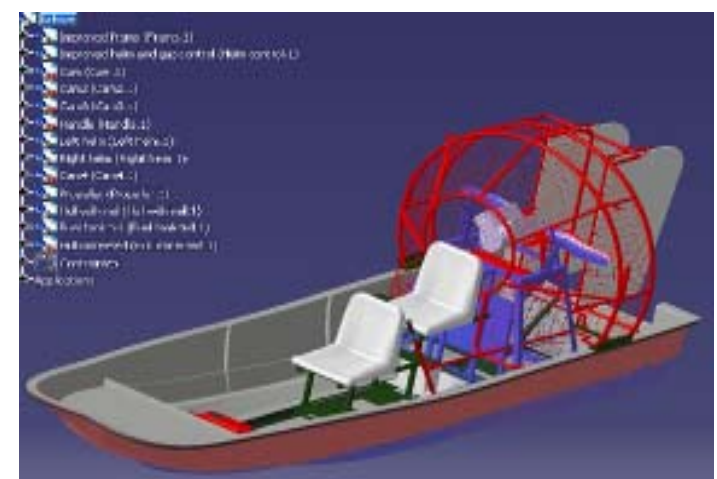

Figure 5. The Water level is analyzed by the CATIA design software program

\section{INITIAL PERFORMANCES EVALUATION}

The engine is an old, small 4WD, Suzuki 539cc engine obtained from the junk yard. After extensive browsing on web pages, it was found that the engine was the third edition of Samurai: i.e. the LJ50 built back in 1974. It is a 33 horsepower and $24 \mathrm{KW}$ at $5,500 \mathrm{rpm}$. Its idling position was around $950 \mathrm{rpm}$ whereas the top speed is near $6500 \mathrm{rpm}$.

It is understood that most of the available engines' operation are not perform at the optimum capability and can causes energy loses as well as other mechanical losses. Normally, different engine may need different combination of operating conditions in order to perform at their best. The operating parameters such as fuel type, fuel-air ratio, temperature, pressure, and air humidity may influence the test result. In the current study, as we are lacking of advance engine testing apparatus such as engine test bed, only simple test had been performed and reported therein.

\section{A. Engine Performance \\ 1) RPM measurement}

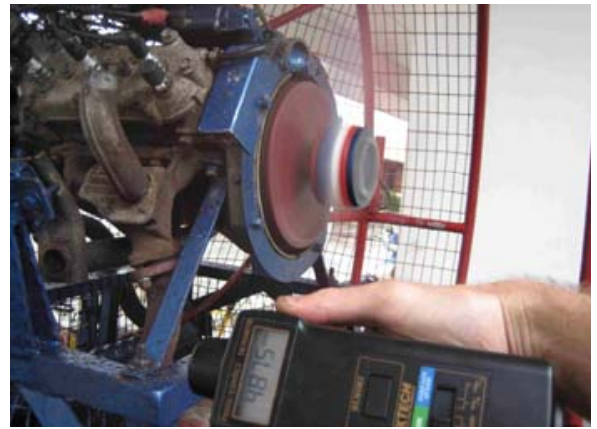

Figure 6 . The propeller RPM measured by tachometer

Tachometer is used to measures engine rpm by checking the speed rotation of crankshaft. Tachometer could measure the rpm by both mechanical and optical means. Rpm for the propeller is measured by optical sensor which is showed at figure 6 . Rpm measurement is very important to keep the speed within the allowable range for engine operation so that it can reduce the failure or longevity of the engine life [12]. 
Table 1.Specification of tachometer

\begin{tabular}{ll}
\hline Model & Extech Instrument 461895 \\
Measuring range & Contact: $5-99999 \mathrm{rpm}$ \\
& Non-contact: $1-20000 \mathrm{rpm}$ \\
Sampling Time & Contact: $1 \mathrm{sec}$ (over $6 \mathrm{rpm})$ \\
& Non-contact: 1 sec (over $60 \mathrm{rpm})$ \\
Temperature & $-20^{\circ} \mathrm{C}$ to $70{ }^{\circ} \mathrm{C}$ \\
Sensing Distance & up to $0.6 \mathrm{~m}$ \\
Accuracy & $\pm 0.05 \%$ of reading or \pm 1 digit \\
\hline
\end{tabular}

\section{2) Fuel consumption measurement}

Fuel efficiency is actually the rate of fuel consumption over a distance traveled and is dependent on all the foregoing factors as well as vehicle weight, size and load [13]. We measured the fuel consumption in 2 conditions, i.e. without load and with propeller. This allowed comparisons to be made. Fuel efficiency depend on the best combination of power, fuel economy and emission control [14].

\section{B. Airboat Performance}

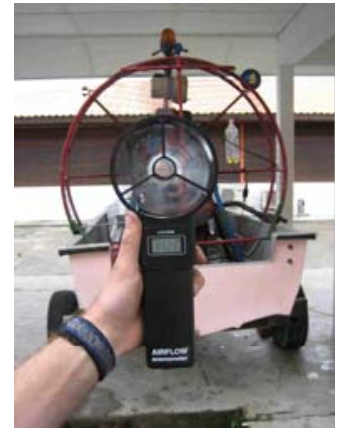

Figure 7 . Wind velocity measured by using anemometer

Table 2. Specification of anemometer

\begin{tabular}{ll}
\hline Model & AF-VA 6 \\
Velocity Resolution & $0.01 \mathrm{~m} / \mathrm{sec}$ \\
Weight (less batteries) & $31.0 \mathrm{~g}$ \\
Operating Environment & $0^{\circ} \mathrm{C}$ to $80^{\circ} \mathrm{C}$ \\
Accuracy & $\pm 1 \%$ of reading \pm 1 digit \\
\hline
\end{tabular}

Thrust generated by the wind is related to the propeller design and engine Rpm. Larger thrust of the wind can contribute to increase the speed of the airboat. The wind speed is measured using a simple anemometer as shown in figure7. Speed radar could be used to measure the speed of the airboat. The specification is given in Table 2.

\section{RESULTS AND DISCUSSION}

\section{A. RPM Measurement}

On idling position i.e. without propeller, the engine was tested to be able to run approximately $900 \mathrm{rpm}$ on average, whereas on top speed, it could reach up to $6000 \mathrm{rpm}$. These results came close to the data given in the Suzuki manual, which are $950 \mathrm{rpm}$ for the idling position and $6500 \mathrm{rpm}$ for the top speed. This indicates that the engine is still quite intake despite old age! Also, as the RPM results acquired were quite similar for both mechanical and the optical method, we therefore opted for the later in our subsequent measurements which are less dangerous especially when working with the propeller.

\section{B. Fuel Consumption Measurement}

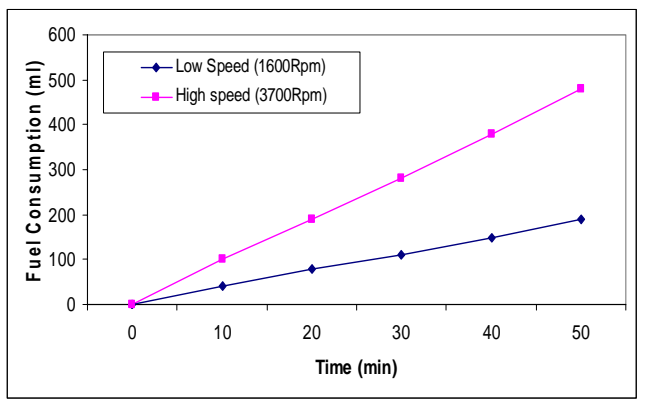

Figure 8. Fuel consumption vs. propeller at low speed (1600 RPM) condition and high speed (3700 RPM)

The fuel consumption vs. time at low speed and high speed conditions are recorded and presented as in figure 8 . Fuel consumption is increased with time of operation. It is clearly shown that the fuel consumption at high speed is higher than at lower speed. When rpm of the engine increase to 2.3 times from $1600 \mathrm{rpm}$ to $3700 \mathrm{rpm}$, the fuel consumption increased to about 2.5 times as far as the present testing time is concerned. The fuel consumption for the high speed of this engine is about $31.13 \mathrm{ml} / \mathrm{s}$. Although the 2-stroke engine has better power-to-weight ratio, it do not use fuel efficiently. Generally, the 2-stroke engine has greater fuel consumption due to incomplete combustion. Four strokes engine or diesel engine could generate a larger torque with more complete and clean combustion, but with size and weight disadvantages.

\section{Wind Velocity Measurement}

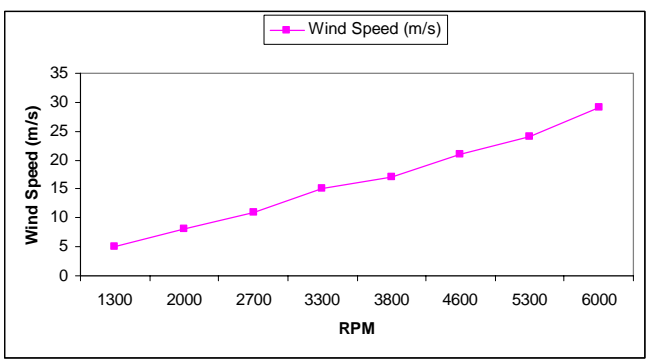

Figure 9. Wind speed vs. RPM

Figure 9 shows the plot of wind speed vs. RPM. The wind speed generated by the propeller was found almost linearly proportional to the RPM of the engine's shaft. The minimum wind speed was around $5 \mathrm{~m} / \mathrm{s}$ (or $18 \mathrm{~km} / \mathrm{h}$ ) whereas the maximum was around $30 \mathrm{~m} / \mathrm{s}(65 \mathrm{~km} / \mathrm{h})$. Larger size of propeller or better aerodynamic-designed propeller could possibly generate larger thrust to move the boat. 


\section{D.Airboat Speed Measurement}

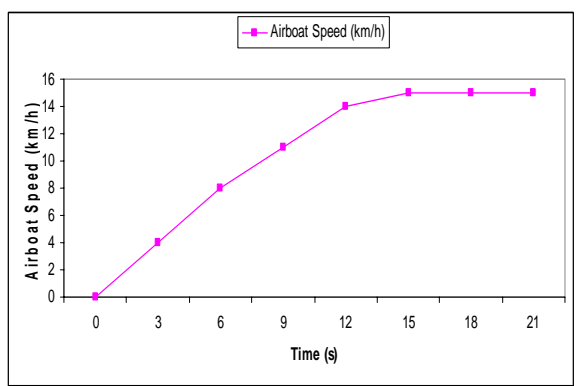

Figure10. Airboat speed vs. time.

Figure 10 shows that the airboat speed increases with time and reach maximum at $15 \mathrm{~km} / \mathrm{h}$. According to Newton second and third laws, airboat speed is depending on the thrust generate by the propeller to the backward. Load of the boat, water and air friction have limited the airboat speed.

\section{RECOMMENDATION FOR FURTHER WORKS}

A. Simulation of airboat hull's stability with Prolines 7 Pro software.

B. Flows investigation with computational softwares such as finite element method (FEM) or computational fluids dynamics (CFD) software; two softwares have been proposed i.e. FEMLAB and FLUENT respeectively.

C. Further study of using local biofuel, such as palm-diesel or coconut diesel, for powering the airboat.

The feasibility of using local biofuel, such as palm-diesel or coconut diesel, for powering the airboat is suggested for further study. For that purpose, there will be a need to replace the current gasoline (petrol) engine with a diesel engine and to test the associated performance with a standard engine test bed. A suitable choice of working fuel is important too. Appendix 1 lists the general important properties of a few conventional biofuel properties compared to that of petrol and No. 2 diesel oil [15]. Some physical characteristics of palm-diesel (a typical local biofuel) as presented by the author in Appendix 2 has shown that palm derivatives are having some promising characteristics including environmentally friendly with zero aromatic content and high flash point that could minimise fire-hazard [16].

\section{CONCLUSION}

An affordable airboat had been constructed and assembled from scratch. This research is done to provide an alternative transportation method to the area which hardly can be reached by vehicle. The main conclusions are summarized as follows:

(i) The current airboat constructed functions very well and can play a very important role for the ecology researches as far as the local riverine and mangrove ecology conditions are concerned.

(ii) From the initial study conducted, the airboat could also be useful means of emergency usage for life saving, especially during the flood at Sarawak Kanan River and other tidal rivers frequently affected by flood.

(iii) It was found that when rpm of the engine increase to 2.3 times from $1600 \mathrm{rpm}$ to $3700 \mathrm{rpm}$, the fuel consumption increased to about 2.5 times. Wind speed generated by the propeller was proportional to the RPM of the engine's shaft. The maximum wind speed generate is about $65 \mathrm{~km} / \mathrm{h}$. In a typical test, the speed of the airboat can easily reached about $15 \mathrm{~km} / \mathrm{h}$ as far as the present design is concerned. This speed is quite reasonable for normal application.

\section{APPENDIX}

Appendix 1. Comparison between gasoline, diesel and biodiesel

\begin{tabular}{|c|c|c|c|}
\hline & Gasoline & $\begin{array}{l}\text { No. } 2 \\
\text { Diesel }\end{array}$ & Biodiesel \\
\hline $\begin{array}{l}\text { Chemical } \\
\text { Structure }\end{array}$ & $\mathrm{C} 4$ to $\mathrm{C} 12$ & $\mathrm{C} 10$ to $\mathrm{C} 20$ & $\begin{array}{l}\text { Methyl esters of } \\
\text { C16-C18 fatty } \\
\text { acids }\end{array}$ \\
\hline Cetane number & 5 to 20 & 40 to 55 & 46 to 60 \\
\hline Octane number & 86 to 94 & 8 to 15 & $\sim 25$ \\
\hline Main fuel source & Crude Oil & Crude Oil & $\begin{array}{l}\text { Soy bean oil, } \\
\text { waste cooking } \\
\text { oil, animal fats, } \\
\text { and } \\
\text { rapeseed oil }\end{array}$ \\
\hline $\begin{array}{l}\text { Energy Content } \\
\text { per Gallon }\end{array}$ & $\begin{array}{l}109,000- \\
125,000 \\
\text { Btu }\end{array}$ & $\begin{array}{l}128,000- \\
130,000 \\
\text { Btu }\end{array}$ & $\begin{array}{l}117,000- \\
120,000 \text { Btu } \\
\text { (compared to } \\
\text { diesel \#2) }\end{array}$ \\
\hline $\begin{array}{l}\text { Energy Ratio } \\
\text { Compared to } \\
\text { Gasoline and } \\
\text { Diesel }\end{array}$ & & & $\begin{array}{l}1.1 \text { to } 1 \text { (relative } \\
\text { to gasoline) or } \\
90 \% \text { (relative to } \\
\text { diesel) }\end{array}$ \\
\hline Physical State & Liquid & Liquid & Liquid \\
\hline $\begin{array}{l}\text { Types of vehicles } \\
\text { available today }\end{array}$ & $\begin{array}{l}\text { All types of } \\
\text { vehicle } \\
\text { classes. }\end{array}$ & $\begin{array}{l}\text { Many types } \\
\text { of } \\
\text { vehicles } \\
\text { classes. }\end{array}$ & $\begin{array}{l}\text { Any vehicle that } \\
\text { runs on diesel } \\
\text { today-no } \\
\text { modifications } \\
\text { are needed for } \\
\text { up to } 5 \% \text { blends. } \\
\text { Many } \\
\text { engines also } \\
\text { compatible with } \\
\text { Up to } 20 \% \\
\text { blends. }\end{array}$ \\
\hline $\begin{array}{l}\text { Environmental } \\
\text { Impacts of } \\
\text { Burning the Fuel }\end{array}$ & $\begin{array}{l}\text { Produces } \\
\text { harmful } \\
\text { emissions; } \\
\text { however, } \\
\text { gasoline } \\
\text { and gasoline } \\
\text { vehicles are } \\
\text { rapidly } \\
\text { improving } \\
\text { and } \\
\text { emissions are } \\
\text { being } \\
\text { reduced. }\end{array}$ & $\begin{array}{l}\text { Produces } \\
\text { harmful } \\
\text { emissions; } \\
\text { however, } \\
\text { diesel and } \\
\text { diesel } \\
\text { vehicles are } \\
\text { rapidly } \\
\text { improving } \\
\text { and } \\
\text { emissions } \\
\text { are } \\
\text { being } \\
\text { reduced } \\
\text { especially } \\
\text { with } \\
\text { after- } \\
\text { treatment } \\
\text { devices. }\end{array}$ & $\begin{array}{l}\text { Reduces } \\
\text { particulate } \\
\text { matter } \\
\text { and global } \\
\text { warming gas } \\
\text { emissions } \\
\text { compared to } \\
\text { conventional } \\
\text { diesel; however, } \\
\text { NOx emissions } \\
\text { may be } \\
\text { increased. }\end{array}$ \\
\hline
\end{tabular}


Appendix 2. Physical characteristics of palm-diesel

\begin{tabular}{|l|c|c|l|l|}
\hline Property & \multicolumn{2}{|c|}{$\begin{array}{c}\text { Palm diesel } \\
\text { (methyl ester) }\end{array}$} & $\begin{array}{l}\text { Diesel } \\
\text { oil }\end{array}$ & $\begin{array}{l}\text { Test } \\
\text { method }\end{array}$ \\
\hline & CPO & PFAD & & \\
\hline $\begin{array}{l}\text { Specific } \\
\text { gravity }\end{array}$ & 0.8730 & 0.8730 & 0.6364 & $\begin{array}{l}\text { ASTM } \\
\text { D1250 }\end{array}$ \\
\hline Pour point , ${ }^{\circ} \mathrm{C}$ & 14 & 13.5 & -45 & ASTM D97 \\
\hline Flash point , ${ }^{\circ} \mathrm{C}$ & 172 & 169 & 76 & ASTM D93 \\
\hline Fire point, ${ }^{\circ} \mathrm{C}$ & 180 & 178 & 108 & ASTM D93 \\
\hline $\begin{array}{l}\text { Aromatic } \\
\text { content, } \%\end{array}$ & Nil & Nil & 30 & $\begin{array}{l}\text { ASTM } \\
\text { D1319 }\end{array}$ \\
\hline $\begin{array}{l}\text { Kinematic } \\
\text { viscosity @ } 40 \\
{ }^{\circ} \mathrm{C}, \mathrm{cST}\end{array}$ & 4.6 & 4.7 & 3.4 & $\begin{array}{l}\text { ASTM } \\
\text { D445 }\end{array}$ \\
\hline
\end{tabular}

$\mathrm{CPO}=$ crude palm oil; $\mathrm{PFAD}=$ palm fatty acid

\section{ACKNOWLEDGMENT}

The authors indebted to Cyril Joubert from the Universite de Technologie de Belfort-Montbéliard (UTB), France, who conducted some initial works during his internship training here at the Faculty of Engineering, UNIMAS in the year 2006 for a 6 -month period.

\section{REFERENCES}

[1] Dumment.R. (2004), The Use of Airboat in Ice and Water Rescue Emergencies. Fire Engineering, march 2004, pp. 113-126.

[2] Racine C.H, Walters J.C and Jorgenson M.T., 1998, Airboat Use and Disturbance of Floating Mat Fen Wetlands in Interior Alaska, U.S.A. Arctic VOL. 51, NO.4. December 1998. pp. 371- 377

[3] James.R , 1999. Winter Survey By Airboat On The Frozen Mississippi River. DPC: Dredging \& Port Construction, Vol. 26, Issue 2. pp. 12

[4] Jourbert. C. , P.N.Y.Yek, M.O.Abdullah, E. Junaidi, S. Hamdan, A. Rahim, R. Muslimen, K. Ab Hamid and P. Kuek. 2006. Design, building and initial performance of an airboat for Sarawak rural riverine applications. Paper presented at the First Regional Conference on Vehicle Engineering and Technology. (Rivert 2006). Putra World Trade Centre (PWTC), Kuala Lumpur 03-05 July 2006.

[5] Staub J.R, Among H.L \& Gastaldo R.A., 2000, Seasonal sediment transport and deposition in the Rajang River delta, Sarawak, East Malaysia. Sedimentary Geology 133 (2000), pp. 249-264

[6] Frederik J. Putuhenaa, Y. S. Mah, \& Saida.S., 2005, Floodplanin Mapping and Visualization For Flood Risk Assessment and Decision Support Tool in The Sarawak Kanan River. International Conference on Energy, Environment and Disasters - INCEED2005, Charlotte, NC, USA

[7] Chapman, V.J., "Mangrove Biogeography", in Hydrobiology of the Mangal, The Ecosystem of the Mangrove Forest", the Hebrew University of Jerusalem, 1984

[8] Tomlinson, P.B., 1986, The Botany of Mangroves, Cambridge University Press, Cambridge, United Kingdom

[9] Chick J. H. (1999), Effectiveness of the Airboat Electrofishing for Sampling Fishes in Shallow, Vegetated Habitats. North American Journal of fisheries Management. pp. 957-967

[10] Velini, E.D., 2003, Development of an Airboat for Weed Survey and Aquatic Herbicide Application Using DGPS and Flow Control System. ASAE Annual International Meeting, Las Vegas, Nevada, USA, 27-30 July 2003.

[11] Brewer T, Edward S., 1994, Understanding Boat Design, International Marine, Camden. pp 9-21

[12] Hamilton H. Mabie and Charles F. Reinholtz, 1987, Mechanics and Dynamics of Machinery. John Willey $14^{\text {th }}$ Edition. USA

[13] Thiessen F. J and Dales D. N., 1994, Automotive Principle \& Service, Prentice Hall Career \& Technology Englewood Cliff, New Jersey.

[14] Layne. K.,1993, Automotive Engine Performance. Tuneup, Testing, and Service. Volume 1, Regents/Prentice Hall, Englewood Cliffs, New Jersey.

[15] DOE (2006). US Department of Energy (DOE), 2006, Energy efficiency and renewable energy (EERE): Fuel Property Database is available at: http:/www.eere.energy.gov/afdc/pdfs/fueltable.pdf. Accessed on 15 April 2006.

[16] Yassin, A.A., Kamis, A. and Abdullah, M.O. (1991). Formulation of an environmentally save palm-diesel oil-based drilling fluid. Society of Petroleum Engineers (SPE) Technical Papers Microfiche No. 23001 


\title{
Mukah Coal-fired Power Project (MPG): Environmental Considerations
}

\author{
Johari Atok
}

\begin{abstract}
Both environmental and pollution problems create a force direct or indirect to transform our economy. They not only help us shape the legal and economic context of management in our country and abroad, but also the technological innovation. This paper introduces environmental considerations in the implementation of the Mukah Coal-Fired Power Project and discusses the challenges that they pose to managers through experiences gained.
\end{abstract}

\section{INTRODUCTION}

The range of environmental issues is broad, and the impact is huge. To manage the environment successfully requires attention to efficiency, effectiveness and long-term goals. Environmental management must consider a mix of ethical, social, technical and competitive issues. Two environmental considerations are addressed. At the early development stage of the project, the use of advance technology to improve environmental performance has been implanted in the conceptual design that appear to have good opportunities for additional improvements in technology integration for the construction of Mukah Coal-Fired Power Plant, a 2 x 135MW project by Mukah Power Generation Sdn Bhd (MPG).

The second aspect is in the area of MPG's experience, where environmental actions have been developed and applied to pollution problems. The actions companies can take enable them to solve environmental problems with appropriate and practical environmental management plan and monitoring program. This is to ensure the project will not pose long-term adverse environmental impacts during its post construction stage. The environmental evaluation work addressed in this paper is on-going.

\section{THE NATURAL ENVIRONMENT}

Business used to look at environmental issues as a no-win situation: You either help the environment and hurt your business, or help your business only at cost to the environment. Fortunately, things have changed. Businesses produce waste in the process of extracting raw material from nature, transforming the raw materials into useful products and transporting the finished products to markets. These essential business activities yield by-products, which have undesirable qualities that have to be absorbed by nature. Thus, the physical environment not only provides goods and

Johari Atok is with the Sarawak Energy Berhad (Formerly known as Sarawak Enterprise Corporation Berhad). materials to the economy, but the goods and materials flow back again to the environment as wastes or residuals. A simple materials balance model illustrates the relationship between the economy and the environment (see Figure 1).

The production sector, which consists of mines and factories, extracts materials from nature and processes them into goods. Transportation and distribution networks move and store the finished products before they reach the point of consumption. The environment provides the material inputs needed to sustain economic activity and carries away the wastes generated by it.

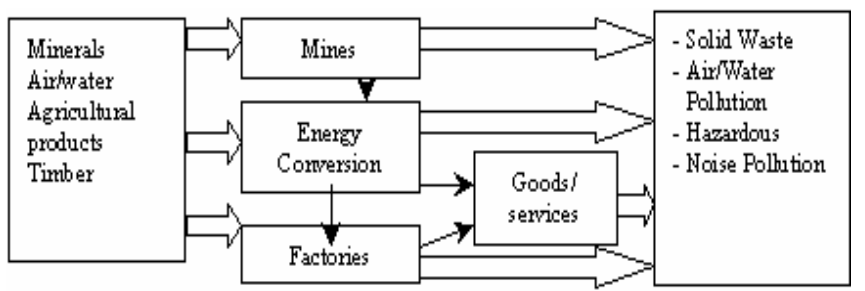

Figure 1. From nature to nature: the flow of material (SOURCE: A. Freeman, R. Myrick, A. Haveman, and V. Kneese, The Economics of Environmental Policy, New York: John Wiley, 1973)

\section{MPG'S EXPERIENCE - ENVIRONMENTAL CONSIDERATIONS}

\section{A. The Company and Project Brief}

Mukah Power Generation Sdn Bhd (MPG) is a whollyowned subsidiary of Sarawak Energy Berhad - SEB (formerly known as Sarawak Enterprise Corporation Berhad). MPG is presently in the process of constructing the $2 \times 135 \mathrm{MW}$ coal-fired power plant in Mukah, Sarawak. The greenfield 270MW Mukah Coal-Fired Power Plant project is located about $2.5 \mathrm{~km}$ east of the road junction where the Sibu-Mukah Road intersects the Balingian-Mukah Road. MPGs project site covers an area of 865 -acre. MPG is built with a total installed capacity of $270 \mathrm{MW}$ consisting of 2x135MW boiler-turbine-generator units using indigenous coal, which is also available in Mukah. Site clearing commenced in March 2006. The construction work at MPG is now in the second year. The construction is about $77 \%$ completed as end of August 2007. The first unit is scheduled to come into commercial operation in November 2008 and the second one 5 months later, i.e., in April 2009. 


\section{B. Design for Environment (DFE)}

At MPG we focused on corporate social responsibilities, which led our stakeholders and employees to form a strong culture in caring for environment. We formed a task force to create design for environment (DFE), that is, a tool for improving environmental performance. The initial stage of MPG's project involved selection of experienced team members. Our team stressed that the principal measures in MPG's project is to implement the environment quality standard beyond that of regulatory requirements like Malaysian Environmental Quality Acts.

Recent advances in technology permit the construction of cost-competitive advanced technology coal-fired plants that emits fewer pollutants. At MPG, the plant is built as a conventional two unit pulverized coal-fired, steam-electric generating station. Coal will be used as the primary fuel while light fuel oil will be used for start-up, flame stabilization and during low load operations. The two units of high-pressure boiler are of single reheat, single drum, single furnace, balanced draft and forced circulation type. The boiler is designed for automatic operation over the full range of operating conditions and complies with specified number of start-up, shut downs and load changes. The steam turbine is designed with two units of high-pressure heaters, four units of low-pressure heaters and condensing tandem compound type. On the environmental equipment, low $\mathrm{NO}_{\mathrm{x}}$ burner will be used for reduction of $\mathrm{NO}_{\mathrm{x}}$. The MukahBalingian coal has low sulphur content of less than $0.5 \%$. In view of the low sulphur content, flue gas desulphurization (FGD) facility to remove sulphur is not required and other particulates are reduced by the electrostatic precipitator.

\section{1) Technology background - process flow chart}

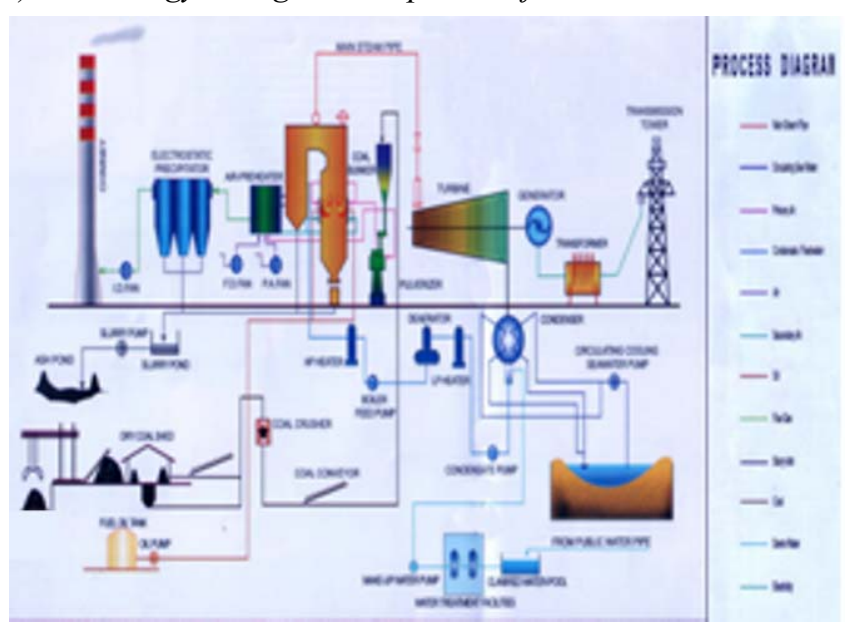

Figure 2. Process flow chart

Coal Sources: The boiler was designed to suit the Mukah Coal properties whilst the Balingian coal was considered as the examined coal. Both types of coal were being analyzed for volatile matter, ash analysis and resistivity of the ash fly as in Table 1, Table 2 and Table 3 respectively.
Table 1. Summary of Mukah and Balingian coal analysis

\begin{tabular}{|c|l|c|c|c|c|}
\hline No. & \multicolumn{1}{|c|}{ Item } & Symbol & Unit & (Mukah Coal) & $\begin{array}{c}\text { (Balingian } \\
\text { Coal) }\end{array}$ \\
\hline 1 & Carbon & $\mathrm{C}^{y}$ & $\%$ & 43.4 & 35.46 \\
\hline 2 & Hydrogen & $\mathrm{H}^{y}$ & $\%$ & 3.1 & 6.64 \\
\hline 3 & Oxygen & $\mathrm{O}^{y}$ & $\%$ & 14.57 & 5.47 \\
\hline 4 & Nitrogen & $\mathrm{N}^{y}$ & $\%$ & 0.914 & 0.51 \\
\hline 5 & Sulphur & $\mathrm{S}^{y}$ & $\%$ & 0.16 & 0.27 \\
\hline 6 & A sh & $\mathrm{A}^{y}$ & $\%$ & 8 & 7.63 \\
\hline 7 & Total moisture & $\mathrm{W}^{y}$ & $\%$ & 30 & 44.03 \\
\hline 8 & Volatile matter & $\mathrm{V}^{y}$ & $\%$ & 28.8 & 25.52 \\
\hline 9 & Lower heating value & $\mathrm{Q}^{y} \mathrm{Dw}$ & $\mathrm{Kj} / \mathrm{kg}$ & 17257 & 12127 \\
\hline 10 & Hard grove grindability index & $\mathrm{HGI}$ & $/$ & 45 & 44 \\
\hline 11 & A sh iritial deformation temperature & $\mathrm{t} 1$ & ${ }^{\circ} \mathrm{C}$ & 1200 & 1173 \\
\hline 12 & A sh softening temperature & $\mathrm{t} 2$ & ${ }^{\circ} \mathrm{C}$ & 1280 & 1216 \\
\hline 13 & A sh hemisphicaler temperature & $\mathrm{t} 3$ & ${ }^{\circ} \mathrm{C}$ & 1310 & 1276 \\
\hline
\end{tabular}

Table 2. Ash analysis

\begin{tabular}{|c|c|c|}
\hline Chemical Properties & Design Coal & Examined Coal \\
\hline $\mathrm{SiO}_{2}$ - Silicon Dioxide & 43.94 & 30.36 \\
\hline $\mathrm{Fe}_{2} \mathrm{O}_{3}$ - Iron OxideFerric Oxide & 14.40 & 28.10 \\
\hline $\mathrm{Al}_{2} \mathrm{O}_{3}$-Aluminium Oxide & 26.95 & 14.91 \\
\hline $\mathrm{MgO}$ - Magnesium Oxide & 3.25 & 7.26 \\
\hline $\mathrm{CaO}$-Calcium Oxide & 6.00 & 8.95 \\
\hline $\mathrm{TiO}_{2}$ - Titanium Dioxide & 0.80 & 0.79 \\
\hline $\mathrm{SO}_{3}$ - Sulphur Trioxide & 2.63 & 5.94 \\
\hline $\mathrm{P}_{2} \mathrm{O}_{5}$ - Phosphorus Pentoxide & 0.11 & 1.11 \\
\hline $\mathrm{K}_{2} \mathrm{O}$ - Potassium Oxide & 1.45 & 1.06 \\
\hline $\mathrm{Na}_{2} \mathrm{O}$ - Sodium Oxide & 0.31 & 0.65 \\
\hline $\mathrm{Mn}_{3} \mathrm{O}_{4}$ - Hausmannite & 0.16 & 0.165 \\
\hline
\end{tabular}

Table 3. Resistivity data of fly ash (based on the design coal)

\begin{tabular}{|c|l|l|}
\hline Temperature $\left({ }^{\circ} \mathrm{C}\right)$ & Unit & Resistivity \\
\hline 100 & $\Omega \mathrm{cm}$ & $1.62 \times 10^{11}$ \\
\hline 120 & $\Omega \mathrm{cm}$ & $7.81 \times 10^{11}$ \\
\hline 150 & $\Omega \mathrm{cm}$ & $9.26 \times 10^{11}$ \\
\hline 180 & $\Omega \mathrm{cm}$ & $2.78 \times 10^{11}$ \\
\hline
\end{tabular}

Light diesel shall be used as fuel support for boiler ignition as well as combustion support. Its contents and properties are as follows:

$\begin{array}{ll}\text { Fuel } & \text { High speed diesel } \\ \text { Gross Calorific Value } & 44080 \mathrm{~kg} / \mathrm{kg} \\ \text { Kinematic Visco sity } & 2.0-5.8 \mathrm{cst} \\ \text { Ash } & 0.01 \% \text { by vot } \\ \text { Sulphur } & 0.05 \% \text { by wt. } \\ \text { P.M. } & 66^{\circ} \mathrm{C} \\ \text { Cloud Foint } & 55^{\circ} \mathrm{C} \\ \text { Density } & 859 \mathrm{~kg} / \mathrm{m}^{3}\end{array}$

Another component that contributed to the environmental performance of the plant is the combustion system of the boiler technology. At MPG, we adopted the Advanced Pollution Minimum burners and the Advanced Combustion Technology-ACT combustion system. The burners use a "staged combustion" system whereby the fuel burns more slowly and at a lower peak temperature. This increases burn time and reduced flame temperature resulting in relative reduction in the formation of Nitrogen Oxides during coal combustion. The ACT technology also tends to reduce NOx emissions from the combustion of coal by further slowing of the coal combustion by staged introduction of air through additional ports in the furnace known as Additional Air Ports. 
On the coal handling aspect, the coal unloading facility is adjacent to the power plant and uses semi-closed to closed mechanical belt system and water spray to prevent the spread of dust. Coal stockpile will be compacted, water sprays and rows of tree planted for wind and dust protection. The use of proven air pollution control technology ensures that environmental performances comply with or are better than DOE's environmental standards. MPG introduced Environmental Management Plan (EMP) during the early stage of construction for all the contractors to comply and adopt on site. During the construction stage, air quality and water quality around site is monitored by installing "Air Quality Monitoring System" at different locations on site and Water Quality Monitoring System" in the waters and silt traps around the plant respectively. Figure 3, figure 4 and figure 5 show the result in July 2007 including monitoring results since May 2007 related to ambient TSP, ambient PM-10 which are all significantly better than the relevant DOE standards. During and after the construction periods, air quality and water quality is continuously monitored with On-site Continuous Emission Monitoring Systems (CEMS). It is expected that MPG's designs will result in controlling air quality better than DOE's standard; reducing $\mathrm{NO}_{\mathrm{x}}$ and $\mathrm{SO}_{\mathrm{x}}$ better than DOE's standard. The result for TSP and PM10 sampling are tabulated in the tables and figures as shown below.

\section{2) Ambient air quality monitoring}

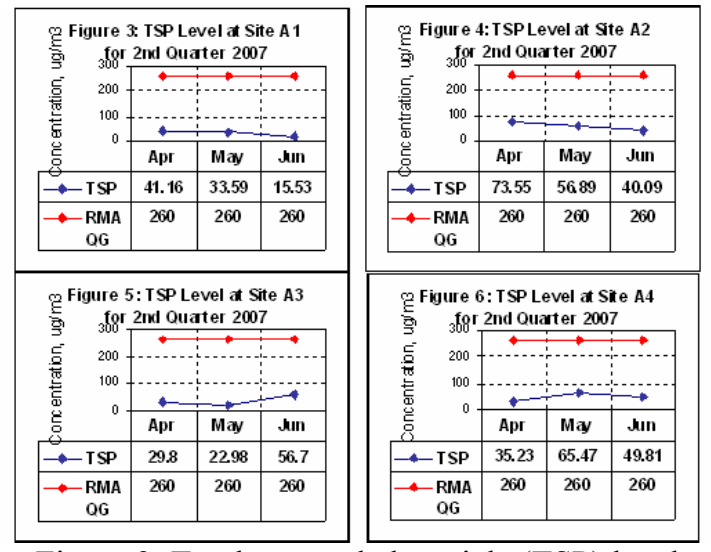

Figure 3. Total suspended particle (TSP) levels

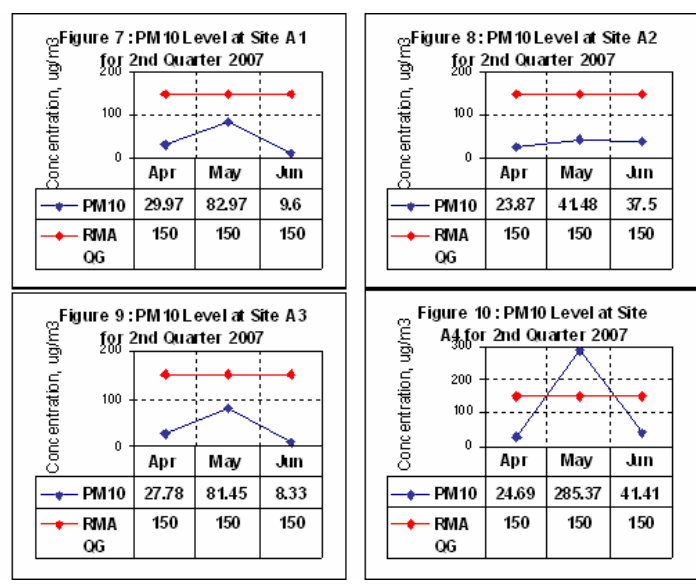

Figure 4. $\mathrm{PM}_{10}$ levels

*RMAQG - Recommended Malaysian Ambient Quality Guidelines

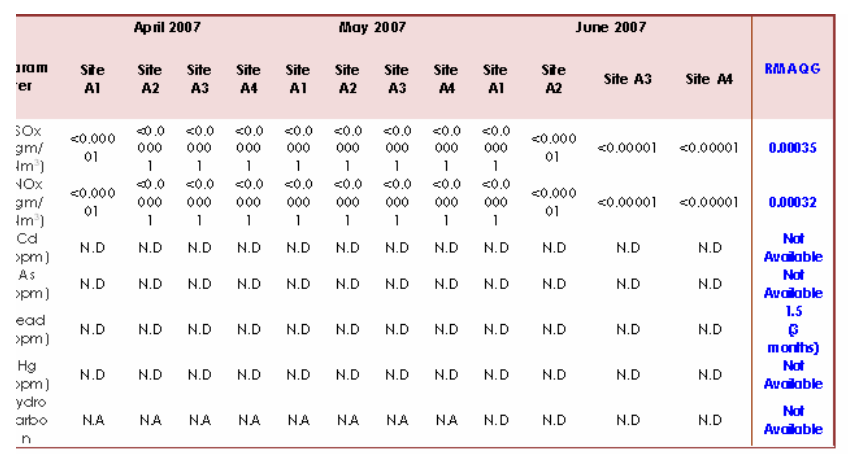

Figure 5. Result of ambient air sampling for $2^{\text {nd }}$ quarter 2007

Note: N.D. - Not Detected

N.A - Not Available

RMAQG - Recommended Malaysian Air Quality Guidelines

The environmental monitoring carried out at Mukah Power Generation during its construction shows that:

i. TSP level measured for 24-hours sampling at all the sampling sites are below the recommended level of $260 \mu \mathrm{g} / \mathrm{m}^{3}$. The lowest level recorded is $15.53 \mu \mathrm{g} / \mathrm{m}^{3}$ at site A1 in June and the highest level is $73.55 \mu \mathrm{g} / \mathrm{m}^{3}$ at site A3 in April.

ii. PM10 level measured for 24-hours sampling was also below the recommended level of $150 \mu \mathrm{g} / \mathrm{m}^{3}$. The lowest level recorded is $8.33 \mu \mathrm{g} / \mathrm{m}^{3}$ at site A3 in June and the highest level is $285.37 \mu \mathrm{g} / \mathrm{m}^{3}$ at site A4 in May.

iii. Concentrations of $\mathrm{SO}_{\mathrm{x}}$ and $\mathrm{NO}_{\mathrm{x}}$ in ambient air were less than $0.00001 \mathrm{gm} / \mathrm{Nm}^{3}$ at all the four sites for the whole of $2^{\text {nd }}$ quarter monitoring period. Thus they comply with the Recommended Malaysian Air Quality Guidelines (RMAQG).

iv. Hydrocarbon measurement has only been implemented starting in June. No hydrocarbon was detected in ambient air at all four sites for June monitoring.

v. Both W1 and W2 sites monitored for water quality were well below the baseline data and the recommended limit of the Interim National Water Quality Standards for Malaysia, except for $\mathrm{BOD}_{5}$, COD, salinity, suspended solid and ammoniacal nitrogen. This is due to rain occurrence prior to water sampling. Meanwhile, water quality results for both W3 and W4 sites showed compliance to Interim Marine Water Quality Standards for Malaysia and were slightly similar to the baseline data.

vi. $\mathrm{pH}$ level for rainwater in all sites were acceptable as all sites showed a measurement of more than $\mathrm{pH}$ 5.6, which is the normal $\mathrm{pH}$ for rain water.

vii. The daytime noise levels measured were all within the DOE recommended guidelines of $65 \mathrm{~dB}(\mathrm{~A})$. Daytime noise levels for all sites were ranging from $56.7 \mathrm{~dB}(\mathrm{~A})$ to $62.9 \mathrm{~dB}(\mathrm{~A})$. For nighttime noise, levels at N1 and N4 sites slightly exceeded the DOE recommended guidelines of $55 \mathrm{~dB}(\mathrm{~A})$. Noise levels for nighttime at all sites were ranging from $49.1 \mathrm{~dB}(\mathrm{~A})$ to $60.1 \mathrm{~dB}(\mathrm{~A})$.

Water quality will also be continuously monitored to ensure compliance with DOE's regulations. Wastewater treatment in the power plant is strictly controlled. The temperature and $\mathrm{pH}$ of cooling water is controlled before being discharged to the sea. 


\section{What COMPANIES CAN Do}

Pollution and environmental problems posed numerous challenges for business. These challenges occurred at the highest levels in the firm; business strategy and organization. They also affected corporate staff in the public affairs and legal departments and influenced people in traditional functional areas such as operations, and finance.

Environmental considerations must be part of companywide decision making from the beginning; they are not something to be considered only at the end. Companies may have to think long term even if profits suffer in the short term. Management support at the highest level is essential because changes of the magnitude called for by environmental issues cannot be accomplished without it.

SEB subsidiary companies such as Sejingkat Power Corporation Sdn Bhd $(2 \times 55 \mathrm{MW}$ coal-fired power plant in Sejingkat, Kuching), PPLS Power Generation Sdn Bhd (2x55MW coal-fired power plant in Sejingkat), Sarawak Power Generation Sdn Bhd (2x110MW gas turbines in Bintulu), and Tg. Kidurong Power Station (192MW gas turbines) have made well-regarded responses to environmental expectations in the past. Their responses may have relevance for other companies. Based on these responses, we organized a list of actions companies can take as appended in Table 4.

Table 4. List of action.

\begin{tabular}{|c|c|}
\hline Subject & Actions \\
\hline $\begin{array}{l}\text { Strategy and } \\
\text { Organization }\end{array}$ & $\begin{array}{l}\text { - Cut back on environmentally } \\
\text { unsafe operation; } \\
\text { - Carry out R\&D on environmentally } \\
\text { safe activities. }\end{array}$ \\
\hline Public Affairs & $\begin{array}{l}\text { - Avoid losses caused by appearing } \\
\text { insensitive to environmental issues; } \\
\text { - Attempt to gain environmental } \\
\text { legitimacy and credibility. }\end{array}$ \\
\hline The Legal Area & $\begin{array}{l}\text { - Prevent confrontation with state or } \\
\text { federal pollution control agencies; } \\
\text { - Comply early; and } \\
\text { - Take advantage of innovative } \\
\text { compliance program. }\end{array}$ \\
\hline Operations & $\begin{array}{l}\text { - Promote new manufacturing } \\
\text { technologies; } \\
\text { - Encourage technological advances } \\
\text { that reduces pollution from } \\
\text { products and manufacturing } \\
\text { processes; } \\
\text { - Develop new product formulations; } \\
\text { - Modify production equipment and } \\
\text { change manufacturing operations to } \\
\text { achieve source reduction; } \\
\text { - Eliminate manufacturing waste; } \\
\text { - Find alternative uses for wastes; } \\
\text { and } \\
\text { - Recycle wastes. }\end{array}$ \\
\hline Finance & $\begin{array}{l}\text { - Gain the respect of the socially } \\
\text { responsible investment community. }\end{array}$ \\
\hline
\end{tabular}

\section{CONCLUSIONS}

Pollution problems are worldwide, and businesses operate in setting throughout the world where environmental conditions and environmental laws vary. Managers must be aware of the differences because awareness may provide them with opportunities for gain and the potential to avoid liabilities. Advanced coal power technology provides a costeffective, environmentally-preferred approach to using widely available and relatively price-stable coal resources. Today, these plants produce relatively clean emissions. Equally important, the design of these plants offers the cheapest route for capturing $\mathrm{CO} 2$, and reducing the future economic risk associated with greenhouse gas regulations.

Our state government can encourage development of advanced coal technology power plants by requiring considerations of Integrated Combined-Cycle Technology (ICCT) in the clean air permitting process and by developing financial incentives to foster development of ICCT.

\section{REFERENCES}

[1] A.A. Marcus, "Business And Society: Strategy, Ethics, and the Global Society", $2^{\text {nd }}$ Edition. Irwin, New York, 1996, pp 403-404, 15 pp 449453.

[2] B.C. Field, "Environmental Economics: An Introduction", $2^{\text {nd }}$ Edition. New York: McGraw-Hill, 1997.

[3] A.E. Boyle, "Environmental Regulation and Economic Growth". Oxford: Clarendon Press, 1994.

[4] C.E. Reese, "Deregulation and Environmental Quality". Quorum Books: Westport, CT, 1983.

[5] Department of Environment (DOE), Malaysia, "Environmental Quality Act 1974”. Kuala Lumpur: International Law Book Services, 2006.

[6] Department of Environment (DOE), Malaysia, "Interim Marine Water Quality Standards (IMWQS)".

[7] Department of Environment (DOE), Malaysia, "Malaysian Ambient Air Quality Recommended Guideline (MAAQRG)".

[8] M.E. Guy, "Ethical Decision Making in Everyday Works Situation". New York: Quorum Books, 1990.

[9] Sarawak Enterprise Corporation Berhad (2005), Detail Environmental Impact Assessment (DEIA) for the Proposed Mukah Coal-Fired Power Station, Mukah Division, Sarawak. Report prepared by EPR (Kuching) Sdn. Bhd. Approved by Department of Environment, Malaysia on 21 December 2005. pp 13-20.

[10] Sarawak Enterprise Corporation Berhad (2007), Environmental Monitoring Report - July 2007 for Mukah Coal-Fired Power Station, Mukah Division, Sarawak. Report prepared by Sekitar Ceria Environmental Services Sdn. Bhd.

[11] TSDA Haji Abdul Aziz B. Dato Haji Hussain and Yong Kiong Choon (2007), "Mukah Coal-Fired Power Project: A Fast Track and Innovative Approach in Power Plant Implementation in Sarawak" presented at The Asia Business Forum 16-17 April 2007, Kuala Lumpur. Periodical Publication - LIRIK Wartawarga Sarawak Energy Berhad, July 2007. pp 6-8.

[12] T.S. Bateman, and S.A. Snell, "Management: Building Competitive Advantage". $4^{\text {th }}$ Edition. New York: McGraw-Hill, 1999. pp 176-178. 


\title{
Untreated and Treated Coconut Coir Reinforced Polyester Composite
}

\author{
Mahshuri Yusof and Bruno Steven Engang
}

\begin{abstract}
The mechanical properties of coconut coir reinforced polyester composites were studied. Cocos nucifera coir fibres were divided into three main groups which were untreated, treated with silane and treated with $\mathrm{NaOH}$. Then, the random orientation fibres with different volume fractions $(5 \%, 10 \%$ and $15 \%)$ were mixed with unsaturated polyester in the mould The results showed that specimens treated with silane gives better mechanical properties as stiffness, tensile strength and toughness are higher compared to untreated and treated with $\mathrm{NaOH}$. Silane is a good surface treatment agent works to roughen the fibre surface and produced waterresistant coating between polyester and coconut fibres surfaces. This reaction results in higher wettability, provides higher surface area for bonding, and increase the energy needed for fracture.
\end{abstract}

Keywords: cocos nufera, silane, unsaturated polyester maximum

\section{INTRODUCTION}

Natural fibres are cellulose-based fibres and also known as green fibres. They are widely used as reinforcements in natural fibre-reinforced matrix composites to enhance the mechanical properties of the resulting composites [1]. Natural fibres are grouped based on their origins whether from plant, animal or mineral. Fibres from plant's fruit, leaf, trunk and skin are usually used in reinforcing plastics material in the composites industry. Generally, the origin of the plant fibres is mainly grouped into some categories such as leaf fibre, bast fibre, seed hair fibres and fibres from fruits. Leaf fibres are fibres which run the length of leaves. Some examples of leaf fibres are sisal, abaca, pineapple, banana and oil plum fibres. Flax, jute hemp, ramie, stem and kenaf are examples of bast fibres. Bast or stem fibres are fibrous bundles in the inner bark of the plant stem and run the length of the stem. Another source of natural fibre is from seed. Some popular seed hair fibres are cotton, coir and kapok. Fibres also can be obtained from fruits. Coir such as coconut coir is one example of fibres from fruits.

Coconut palm tree (Cocos nucifera) is a tropical plant of the Arecaceae (Palmae) family and is predominantly in Sri Lanka and India [2]. It is found distributed in over 85

Mdm Mahshuri Yusof is now a lecturer of Department of Mechanical and Manufacturing Engineering, Universiti Malaysia Sarawak, 94300 Kota Samarahan, Sarawak, Malaysia. (email: ymashun@feng.unimas.my).

Mr Bruno Steven Engang is an undergraduate student of Mechanical and Manufacturing Departmant of Universiti Malaysia Sarawak and will finish his study at the end of November 2007. countries. These palm trees can grow to the heights of over 100 feet, requiring the nut to survive tremendous falls. Various sources suggested that the husk, composed of lignin and cellulose based coir fibre and coir pith serves as a defence mechanism to protect the nut during that fall. The coir fibre can be extracted from any part of the tree, including the long leaf sheath, the midribs of the leaves, the bark of the stalk and the fruit crust. Coconut fruit fibres or coir fibres is extracted from both the exocarp and the endocarp of the fruit [3]. These coir fibres are currently used in the form of mats in applications such as substrates for ornamental plants, soil support and seat cushion for automobiles. The traditional way of processing coir starts with curing the coconut husks in water for 6-7 months. Large curing pits are generally used and husks are added in one end and cured husks are retrieved from the other end. Curing of husks in water increases the flexibility and durability of coir fibres [4]

The coupling agents are used to improve the fibre/matrix interfacial strength through physical and chemical bond and to protect the fibre surface from moisture and reactive fluids. Silane is one of excellent coupling agent [5]. Herrera and Valadez (2005) observed that the failure mode of henequen natural fibre treated with the $\mathrm{NaOH}$ solution and silane coupling agent is mainly due to shear yielding rather than tearing. With increasing fibre-matrix interaction, the failure mode changed from interfacial failure to matrix failure [6]. The objective of fibre chemical treatment is to remove the lignin, hemicellulose, silica and pith from the fibre to have a better impregnation between fibre and matrix and improving surface roughness for better interaction [7][8]. There is no definite research conclusion about concentration and time of treatment with respect to the optimum mechanical properties. Prasad et al (1983) found that 72 hours soaking in $5 \% \mathrm{NaOH}$ solution gives best UTS and 96 hours soaking in the same concentration give best tensile modulus ${ }^{7}$. However, Mohanty (2000) investigated that soaking in $2 \% \mathrm{NaOH}$ solution is the best treatment for the optimum UTS while soaking in $5 \%$ of $\mathrm{NaOH}$ solution for 1 hour gives the best flexural properties [8]. Calado et al (2006) stated that soaking in $\mathrm{Na}_{2} \mathrm{SO}_{3}$ is another method available for chemical treatment of coir fibre [9].

\section{MAterials AND SAMPle PREPARAtion}

The coconut husks were submerged into the lake within 1 to 2 months under constant temperature of $30 \pm 2^{\circ} \mathrm{C}$. The 
retted coir was washed with water jet to remove dirt and unwanted substances. Then, they were dried in the force air concentration oven with the temperature of $60 \pm 2^{\circ} \mathrm{C}$. In order to ensure the fibres were purely dried and free from lignin or other contaminants, the weight of the tows of fibre have been measured periodically by digital analytical balance with $0.01 \mathrm{~g}$ precision until a constant weight was attained.

The coir was divided into 3 groups; untreated, treated with $\mathrm{NaOH}$ and treated with silane $\left(\mathrm{C}_{10} \mathrm{H}_{20} \mathrm{O}_{5} \mathrm{Si}\right)$.

Master batch solutions of $4.5\left(10^{-3}\right) \mathrm{mol} / \mathrm{L}$ total silane were prepared since this concentration is the optimum level for fibre surface treatment ${ }^{5}$. The silane solution was stirred for 1 hour at room temperature. The coupling agent was then coated on clean coir fibres by soaking them in silane solution for 2 minutes. Then, the tow was removed and allowed to dry overnight in the oven at temperature of $60 \pm 2{ }^{\circ} \mathrm{C}$

In this study, $0.5 \mathrm{~mol} / \mathrm{L}$ solution of $\mathrm{NaOH}$ was prepared. The clean untreated coir was then soaked in the $\mathrm{NaOH}$ for 30 minutes. The treated coir was then removed and dried in the forced air concentration oven at $60 \pm 2^{\circ} \mathrm{C}$.

Test specimens in form of random orientations were chosen for fibre reinforced composites in this research. Fibres with the particular volume fractions $(5 \%, 10 \%$ and $15 \%$ ) were aligned in the random orientation in the mould. Releasing agent was smeared evenly onto the mould surface before it. Then, a mixture of unsaturated polyester and Butanox M-50 (hardener) in weight ratio of 1:0.015, are poured onto it, in zig-zag form After that, each layer of fibres was applied with unsaturated polyester. The laminate was then pressed under room temperature until the thickness of the specimen become $2 \mathrm{~mm}$ for 4 hours. The composites were then placed in an oven at $80^{\circ} \mathrm{C}$ for 12 hours for post curing. Finally, the laminate was cut into the dimension of $240 \mathrm{~mm}$ length $\times 25.4 \mathrm{~mm}$ width $\times 2 \mathrm{~mm}$ thickness according to ASTM D3039-79. The specimen shall have a constant rectangular cross section with a specimen width variation of no more than $\pm 1 \%$ and a specimen thickness variation of no more than $\pm 4 \%$.

\section{MechanicAl TeSting}

Twenty experiments were done for each group of tests and the average readings were taken for more accurate results. The experiments were carried out according to ASTM D3039-79 in air-conditioned room at $23{ }^{\circ} \mathrm{C}$ temperature and at $2 \mathrm{~mm} / \mathrm{min}$ crosshead speed. Tabs were glued at the ends of the samples to avoid fibre pull-out. The tab used in the testing was made from E-glass fibrereinforced polymer matrix materials (woven) in a $0^{\circ} / 90^{\circ}$ laminate configuration.

\section{RESUlTS AND DisCUSSION}

\section{A. Tensile Test}

'Figure 1' shows that higher fibre volume fraction had higher tensile strength and higher modulus of elasticity. The same shapes of curves were discovered for the coconut coir reinforced unsaturated polymer composite ('Figure 2') which has been treated with $\mathrm{NaOH}$ solution. The difference between these two graphs is that the composite treated with $\mathrm{NaOH}$ has higher values for tensile strength and modulus of elasticity in all types of fibre content.

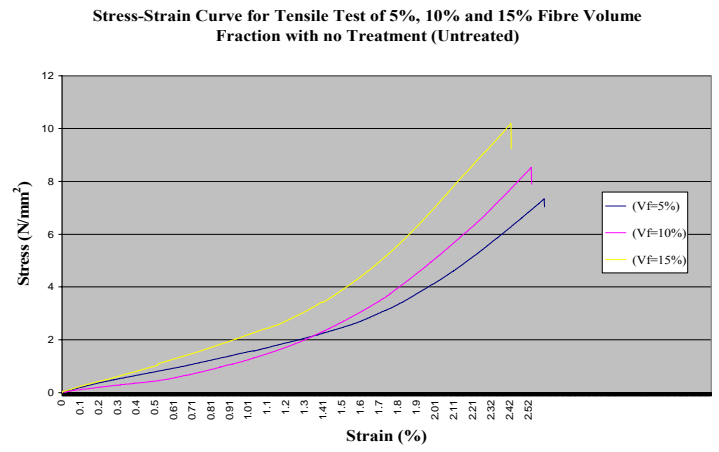

Figure 1. Stress-strain curves for tensile test of 5\%, 10\% and $15 \%$ fibre volume fraction with no treatment (untreated)

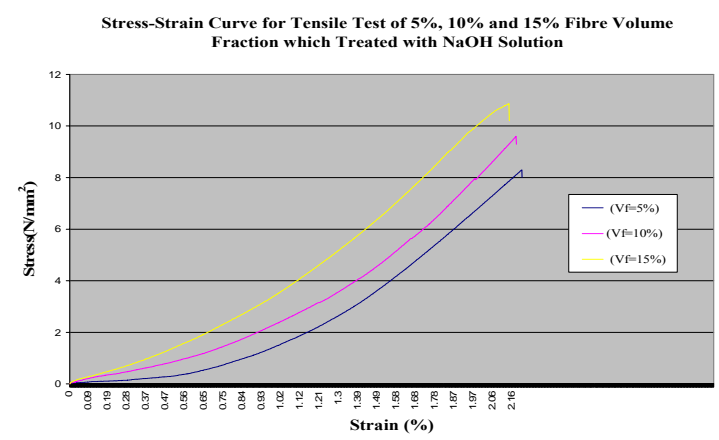

Figure 2. Stress-strain curves for tensile test of 5\%, 10\% and $15 \%$ fibre volume fraction treated with $\mathrm{NaOH}$ solution

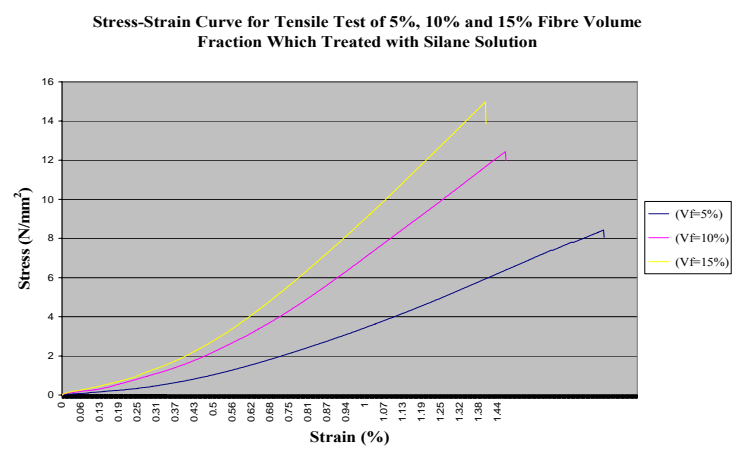

Figure 3. Stress-strain curves for tensile test of 5\%, 10\% and $15 \%$ fibre volume fraction treated with silane solution.

When comparing all of the graphs, the coconut coir reinforced unsaturated polyester composite specimens which are treated with silane solution had the highest values of tensile strength and modulus of elasticity. This shows that the coir that undergoes the silane treatment will produce stronger bond with matrix. When the penetration at the interface between the matrix and resin is stronger, this will produce the better tensile strength characteristic of the composites. The tensile strength of the composites will increase respect to the increasing percentage in the fibre volume fraction. Higher fibre content will produce higher strength to the composite.

\section{B. Tensile Strength}

'Figure 4' shows that the tensile strength of composite will increases as the volume fraction of the fibre increases. When the fibre surface was modified with an aqueous 
$\mathrm{NaOH}$ solution, the tensile strength of the composite shows a small improvement. The improvements are about $13 \%$, $12.5 \%$ and $7 \%$ for fibre volume fractions of $5 \%, 10 \%$ and $15 \%$ respectively. However, when the silane coupling agent is used, the increment of tensile strength seems to improve noticeably which are about $14.8 \%, 45.8 \%$ and $47 \%$ for $5 \%$, $10 \%$ and $15 \%$ of fibre volume fraction respectively.

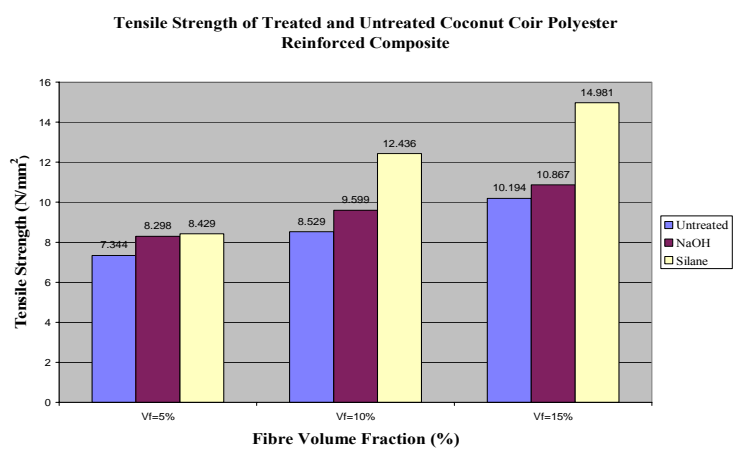

Figure 4. Tensile strength of treated and untreated coconut coir polyester reinforced composite

The results show that for coconut coir fibre, the use of silane coupling agent posses better adhesion of fibre-matrix compared to treated with $\mathrm{NaOH}$. This is also proves that by using silane as a surface treatment, will improve the interfacial bonding by giving rise to additional sites of mechanical interlocking, and thus promoting more resin/fibre interpenetration at the interface.

Generally, the silane coupling agent produces the strong bonding between the thermosetting polymer matrix and the natural fibre due to the production of water-resistant coating between both surfaces. Water resistant coating is produced when silane is hydrolysed to the silanol in the aqueous solution to which the fibres are exposed, and thus silanol molecules will compete with water molecules to form hydrogen bonds with the hydroxyl groups bound to the fibre surface. This will be driven off during the fibre drying process to produce polysiloxane layer which is water resistant. As a result, the resin will be easily penetrates into fibre surface and the contact area between fibre and matrix is increases.

\section{Stiffness}

'Figure 5' shows the stiffness of coconut coir polyester reinforced composite treated with silane gained higher value when comparing to the untreated and treated with $\mathrm{NaOH}$. This is clearly shown especially for fibre volume fractions of $10 \%$ and $15 \%$ which show the stiffness of $379.939 \mathrm{MPa}$ and $425.276 \mathrm{MPa}$ for specimen treated with $\mathrm{NaOH}$ and 559.176 $\mathrm{MPa}$ and $721.32 \mathrm{MPa}$ for specimen treated with silane respectively. However the stiffness between treated with $\mathrm{NaOH}$ and silane shows small difference for $5 \%$ fibre volume fraction.

\section{D.Energy at Break}

Energy at break refers to the toughness of the materials, means the amount of energy needed for fracture. It is represented by the area under tress-strain curve up to the point of fracture. 'Figure 6' reveals that the specimen with fibre treated with silane resist the fracture better compared to fibre treated with $\mathrm{NaOH}$ and untreated specimen. The surface treatment agent work to roughen the fibre surface. The rough surface would allow higher wettability and provide higher surface area for bonding and thus, increase the energy needed for fracture.

Stiffness of Treated and Untreated Coconut Coir Polyester Reinforced Composite According to the Fibre Volume Fraction

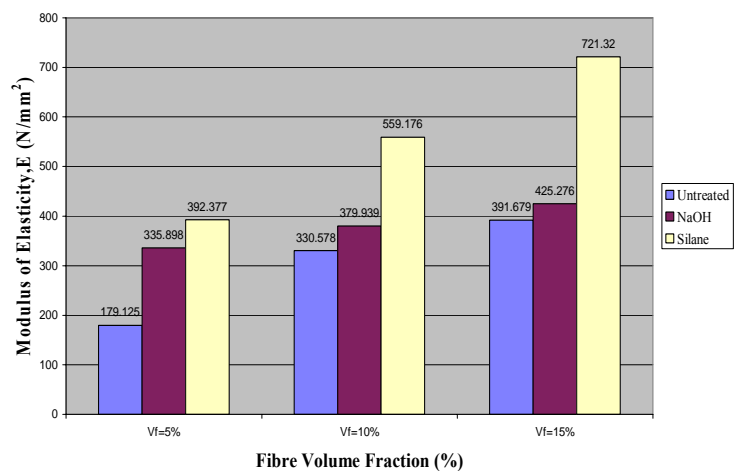

Figure 5: Stiffness of treated and untreated coconut coir polyester reinforced composite according to the fibre volume fraction

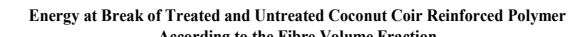
According to the Fibre Volume Fraction

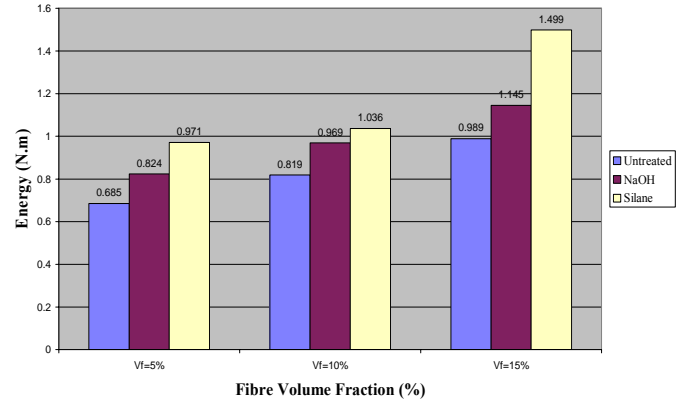

Figure 6: Energy at break of treated and untreated coconut coir reinforced polymer according to the fibre volume fraction.

'Table 1' lists the data on the tensile strength, modulus of elasticity and the energy at break of untreated, treated with $\mathrm{NaOH}$ and treated with silane coconut coir reinforced unsaturated polyester composites with different fibre volume fractions.

\section{CONCLUSION}

It was found that the strongest composite is treated with silane, and then followed by treated with $\mathrm{NaOH}$ and the weakest is untreated composite. The strength of the composites is also increases with the increasing of the volume fraction content in the composite. Besides, the coconut fibre reinforced polyester composite treated with silane with $15 \%$ fibre volume fraction gained higher stiffness and toughness.

It can be concluded that the coconut coir fibre would be tightly bonded with polyester if it is treated with silane. Therefore, silane is the better coupling agent for coconut coir fibre compared to $\mathrm{NaOH}$. 
Table 1. Summary of tensile properties of coconut coir unsaturated polyester composites

\begin{tabular}{|c|c|c|c|c|c|c|c|c|c|}
\hline \multirow[t]{2}{*}{$\begin{array}{l}\text { Fibre Volume Fraction } \\
\qquad\left(\mathrm{V}_{\mathrm{F}}\right)\end{array}$} & \multicolumn{3}{|c|}{ Tensile Strength (MPA) } & \multicolumn{3}{|c|}{$\begin{array}{c}\text { Modulus of Elasticity } \\
(\mathrm{MPa})\end{array}$} & \multicolumn{3}{|c|}{ Energy at Break (Joule) } \\
\hline & $5 \%$ & $10 \%$ & $15 \%$ & $5 \%$ & $10 \%$ & $15 \%$ & $5 \%$ & $10 \%$ & $15 \%$ \\
\hline Untreated & 7.3 & 8.5 & 10.2 & 179.1 & 330.6 & 391.7 & 0.7 & 0.8 & 1.0 \\
\hline Treated with $\mathrm{NaOH}$ & 8.3 & 9.6 & 10.9 & 335.9 & 380.0 & 425.3 & 0.8 & 1.0 & 0.1 \\
\hline Treated with silane & 8.4 & 12.4 & 15.0 & 392.4 & 559.2 & 721.3 & 1.0 & 1.1 & 1.5 \\
\hline
\end{tabular}

\section{ACKNOWLEDGMENT}

The authors gratefully acknowledge the support by Department of Mechanical and Manufacturing Engineering, Faculty of Engineering, Universiti Malaysia Sarawak.

\section{REFERENCES}

[1] F. L. Matthews, and R. D. Rawlings,"Composite Materials: Engineering and Science". Cambridge: Woodhead Publishing Limited, 1994

[2] Jafferjee Brothers, "Composites Applications Using Coir Fibres_in Sri Lanka.”_Thesis Ph.D., Delft University of Technology, Netherlands, 2003

[3] N. S. Monteiro, L. A. H. Terrones., F. P. D .Lopes, and J. R. M. Almeida, "Mechanical Strength of Polyester Matrix Composites Reinforced with Coconut Fiber Wastes". Revista Materia, 2005;10:.571-576.

[4] A. H. Charles, "Modern Plastic Handbook: Modern Plastics", McGraw-Hill, New York, 2000

[5] J. Rout, S .S. Nayal, M. Misra, A. K. Mohanty, "Scanning Electron Microscopy Study of Chemically Modified Coir Fibres", Journal of Applied Polymer Science, 2001:79,1169-1177

[6] P. J. Herrera-Franco, and A. Valadez-Gonzalez,(2005) A Study of the Mechanical Properties of Short natural Fibre Reinforced Composites Composites: Part B. 2005;36:597-608

[7] S. V. Prasad, C. Pavithran, P. K. Rohatgi, Alkali treatment for coir fibres for coir-polyester composites, Research regional laboratory, India, 1983:1443-1454

[8] S. K. Nayak, S. S. Tripahy., J. Rout, A. K. Mohanty, "Coir-Polyester composites: Effect on fibre surface treatment on mechanical properties of composite" International Plastics Engineering and Technology", 2000;.4: 79-86.

[9] V. Calado, D. W. Barreto, J. R. M. D'almeida, "The effect of a chemical treatment on the structure and morphology of coir fibres", Journal of Materials Science Letters, 2000;19: 2151-2153. 


\title{
Numerical Modelling of ROCLA VersaTrap Type W
}

\author{
M. Ismail and H. Mikraz
}

\begin{abstract}
This study investigates the performance of a hydrodynamic separator, VersaTrap type $W$, intended for sedimentation applications. A scale model of VersaTrap type $W$ is numerically analyzed to establish the hydraulic characteristics and pollutant removal efficiencies (PRE). 0\% blocked screen condition was used during analysis. Comparing Computational Fluid Dynamic simulation and experimental results suggest that CFD software is an effective tool for assessing the outcomes of the hydraulic separation system. Data analysis has demonstrated that the headloss increases in proportion to flow rates. The separation efficiencies are inversely proportional to flow rates. The study findings have capabilities to optimize any other types of wastewater treatment systems.
\end{abstract}

Keywords: computational fluid dynamics; hydraulic characteristics; pollutant removal efficiency

\section{INTRODUCTION}

Computational Fluid Dynamics (CFD) software is one of the most popular commercial flow simulation programs and is increasingly used in many engineering fields. It is also implicating in water industry, drainage systems and processes [8]-[9]-[13]-[20]. The program solves fluid flow equations including continuity and momentum. When applied within a control volume based finite difference framework, predicts the characteristics of the flow within complex fluid dynamic systems. Flow velocity and direction throughout the analytical domain can be predicted. It can also trace the path of particles of different sizes as they flow through the system. One major advantage of CFD is that a model can be created and evaluated within a week and at less than $20 \%$ of the cost of physical prototyping [1]. In addition, CFD provides far more information about the reasons behind the performance of a design concept. Many studies were focused on the prediction of particle behaviour in the field of sewer and drainage systems designed to facilitate their removal, for instance, (Stovin et al. [23], Faram and Harwood [10], Faram and Harwood [11] and Slack et al. [21].

\section{TREATMENT MECHANISM}

The VersaTrap scale model type W (VTW) has been designed to remove suspended solids and floatables,

M. Ismail and H. Nikraz are with the Civil Engineering Department Curtin University of Technology, Perth, Western Australia (phone: 61421199443; fax: 6189266 4511; e-mail: msahgiar@gmail.com). sediments and oil from wastewater to prevent reentrainment. As shown in figure 1, the unit has two cylindrical chambers (e.g. internal and external). The internal chamber is called the separation or treatment chamber, which has screen at the bottom. Water enters into chamber through tangential inlet and initiates the swirling motion that causes an apparent centrifugal force on the fluid and pollutants within the separator. The hydrodynamic separator has no moving parts and is designed to operate under low flow conditions. Suspended solids and sediments are captured at the bottom of the basket whilst floatables and oil contaminants are collected at the water surface in the separation chamber [15].

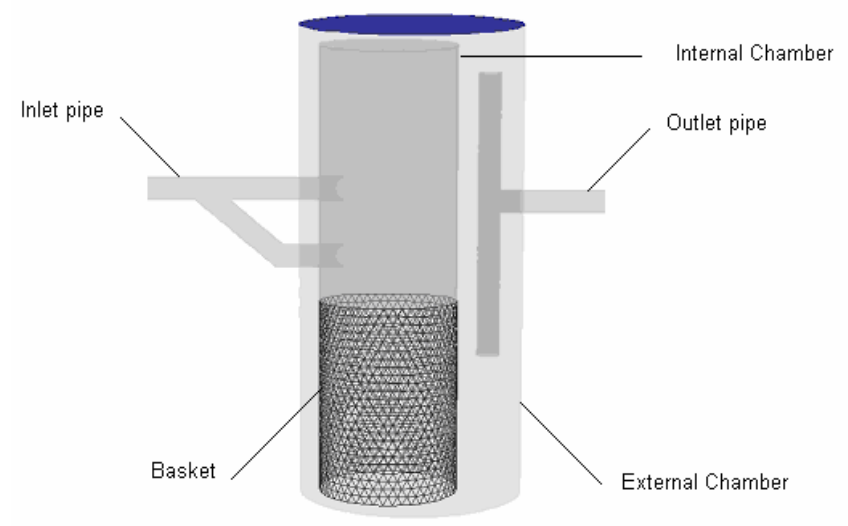

Figure 1. Schematic of the original VTW considered

\section{CFD AsSESSMENT Methodology}

Throughout the study, the Fluent CFD software, (version 6.2.16) was used in conjunction with the associated Gambit, (version 2.3.16). The model was simulated at inlet flow rates of $0.6,1.25$ and $2.5 \mathrm{~L} / \mathrm{s}$; corresponding to the design treatment flow (DTF), design peak flow (DPF) and double design peak flow (DDPF) of VTW respectively. Three dimensional (3D) model was structured using tetrahedral meshes comprising of 212000 computational cells. Using an unstructured grid helps not only to eliminate the occurrence of singularities but provides full geometrical facility [6].

Inlet flow rate was defined by uniform velocities across the inlet plane of the system. System outlets were defined with a pressure outlet corresponding to atmospheric pressure, representing a free discharge. The fluid free surfaces in each chamber were approximated by fixed 
friction wall boundaries, locations of which were determined experimentally. Unsteady state flow field predictions were obtained and solutions were converged. By using data of the static pressure, velocity head of the inlet and outlet, the headloss was determined (equation 1). Also, by comparing the volume fraction of sand at time ( $t$ ) to the volume that was introduced, the efficiency (\%) is obtained (equation 2).

$\frac{v_{1}^{2}}{2 g}+\frac{p_{1}}{\rho g}+z_{1}=\frac{v_{2}^{2}}{2 g}+\frac{p_{2}}{\rho g}+z_{2}+H L$

where:

$P_{1}$ is the pressure head at the inlet pipe

$P_{2}$ is the pressure head at the outlet pipe

$V_{1}$ is the velocity head at the inlet pipe

$V_{2}$ is the velocity head at the outlet pipe

$Z_{1}$ is the elevation level at the inlet pipe

$Z_{2}$ is the elevation level at the outlet pipe

$H L$ is the total headloss (energy loss)

Efficiency $(\%)$ at time $(t)=$

$100 \% x \frac{\text { volume fraction remaining in the system }}{\text { volume fraction injected }}$

where time (t) can be taken as the time from entry of particles into the model to the time at which it exits.

To replicate the experimental method presented by Ismail et al. [15], two models were used in the project namely Volume of Fluid (VOF) and Eularian-Lagrangian model. VOF model was used to determine the hydraulic characteristics of the model and Eularian-Lagrangian model was used to obtain the efficiency of the model at three different flow rates. The sand that has been injected into the flow domain was granular with a density of $2500 \mathrm{~kg} / \mathrm{m}^{3}$. The particle sizes that have been injected were ranged from 50 to 2360 micrometer. A validation study performed yielded good comparisons between experimental data and predictions for headloss and particle removal efficiency.

\section{Velocity Profile}

Since the separation mechanism is associated with the velocity field, its exact determination should be considered crucial for an increased understanding of the separation process and future modifications to the separator design. The definition of velocity components is based on the geometry of the hydrocyclone. Many experimental laboratory and pilot plant studies have been performed on separators/hydrocyclones. The majority covers the influence of operating parameters or geometry changes on the separation result. Very few studies pay attention to the effect that a difference in geometry causes on the internal flow field. For example, Yamamoto and Jiao [24] presented a hydrocyclone with a perforated inner cylinder that improved the separation efficiency. In many other studies, the researchers have indicated that axial and tangential velocity components are the most significant velocities which are responsible for particle separation [14]-[22].

In the following sections, measurement results of tangential, axial and radial flow velocity are presented.

\section{A. Tangential Velocity}

The tangential velocity component is the dominant component of fluid flow in cyclones which results in centrifugal force for particle separation. Tangential velocity profiles of the model are presented in figures $2 \mathrm{a}$ and $2 \mathrm{~b}$. It has frequently been studied. Kelsall [17] used a microscope tracking technique, where he visually tracked small aluminum particles in a transparent hydrocyclone. The hydrocyclone was operated with an air-core at varying underflow. Kelsall found that the tangential velocity increased from the hydrocyclone wall towards the centre, reached a maximum value and then rapidly decreased. The maximum value was located at a radius that was similar to that of the hydrocyclone wall. The tangential velocity at the centre is always zero for the case of axisymmetrical flow. The predicted results qualitatively compared with the experimental results of Fisher and Flack [12], who showed that the shear stresses at the wall caused the velocity to approach or drop below zero. This agrees to the present study results. In the model, the tangential velocity found to increase rapidly from the lowest value that close to internal chamber wall, towards to the centre [7]. This was found in the seven selected surfaces starting from the middle downwards to the bottom of internal/treatment chamber. Although trends for the tangential velocity profiles are similar, the magnitudes of local velocities are significantly different (see figure $2 \mathrm{~b}$ ).

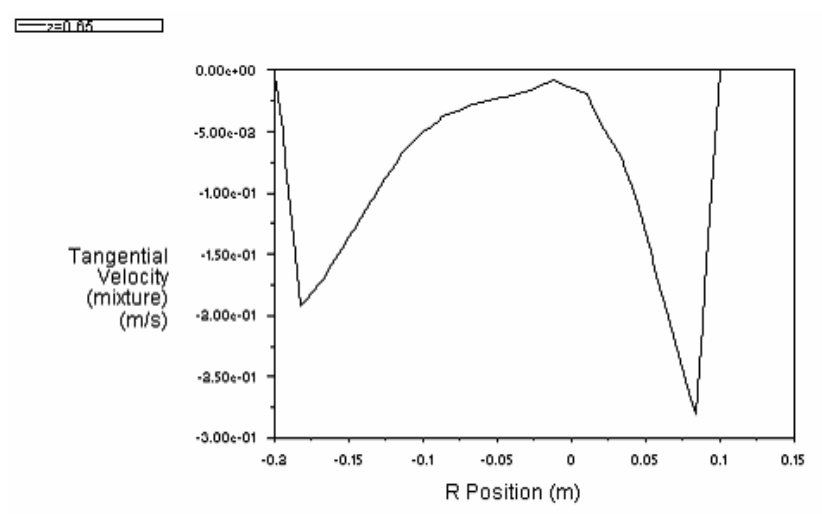

Figure $2 \mathrm{a}$. Tangential velocity profile at $\mathrm{z}=0.65 \mathrm{~m}$ from the bottom of internal chamber

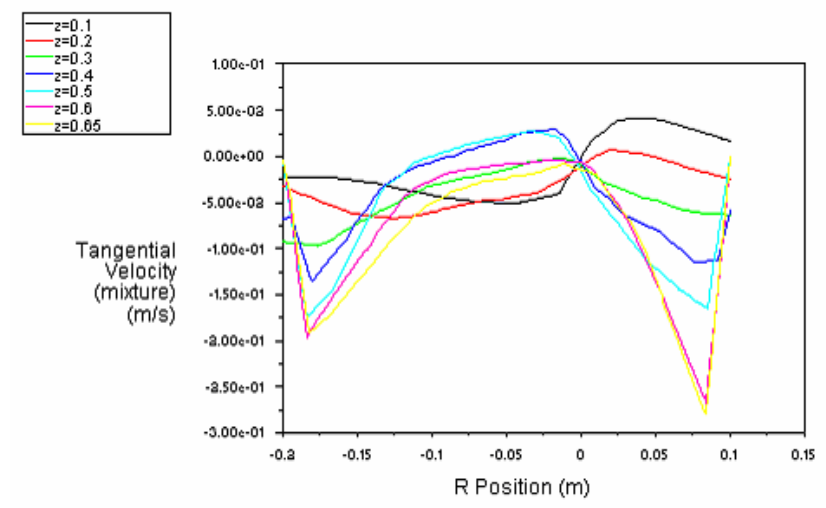

Figure $2 b$. Tangential velocity profile at all selected surfaces

\section{B. Axial Velocity}

The corresponding axial velocity distributions of the model are presented in figures $3 \mathrm{a}$ and $3 \mathrm{~b}$. The radial 
positions from the central axis (marked $(-0.05)$ ), up to the outer wall of the hydrocyclone (internal chamber) were analyzed. Many studies showed similar results for the tangential velocity component. At the axial velocity component, the results are more diversified. Most studies showed a downward velocity close to the wall and an upward velocity in the centre. However, some studies reported a heavily varying axial velocity over short radii close to the centreline. Dissimilarities in measurement results between various studies are often greater than similarities. However, the studies of Bergstrom and Vomhoff [2] agree on an increasing inward radial velocity towards the hydrocyclone centre.

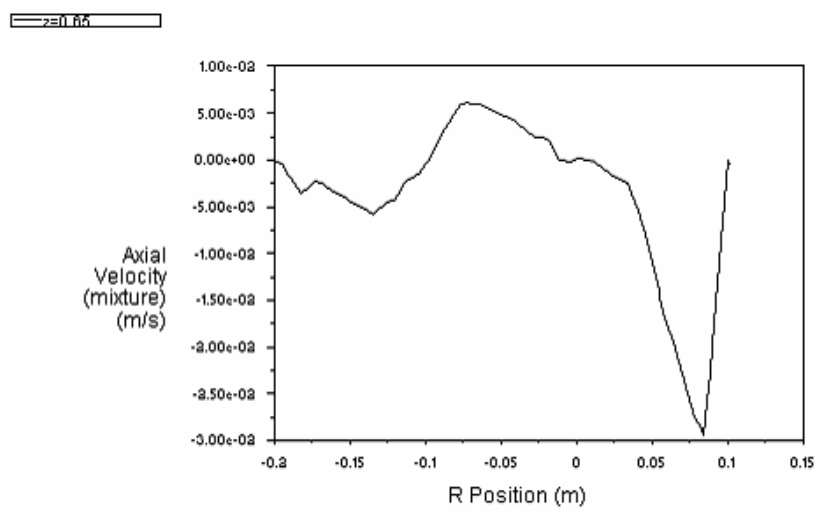

Figure 3a. Axial velocity profile at $\mathrm{z}=0.65 \mathrm{~m}$

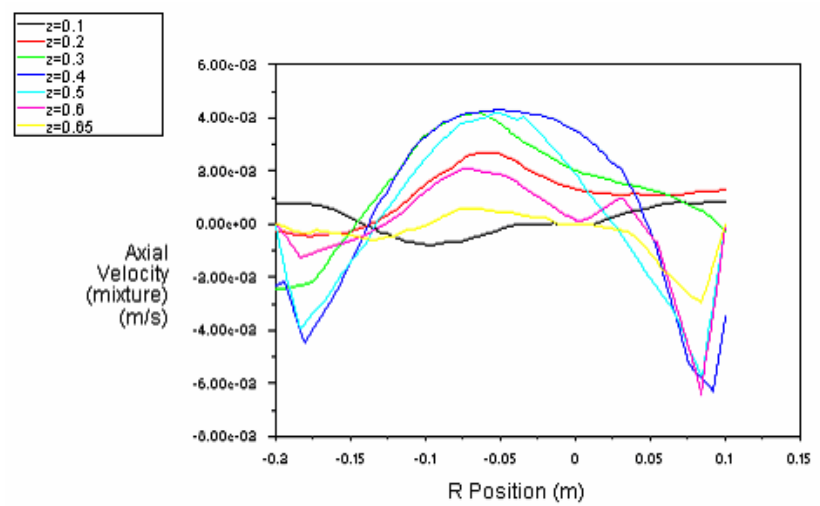

Figure $3 b$. Axial velocity profile at all selected surfaces

Kelsall [17] measured radial profiles of the axial velocity at different vertical levels. The axial velocity was downward, close to the cyclone wall and upward towards the centre. He also presented an envelope of zero axial velocity. Knowles et al. [18] measured the vertical velocity and discovered an undulating behaviour of the axial velocity inside the vortex finder. In this study, the axial velocity profiles of VTW model are similar to Kelsall [17], Jordan [16] and Ismail \& Nikraz [7]. The velocity was negative close to the treatment chamber wall and increased towards the centre. The velocity values are not identical in both sides at selected levels. This is because of the instability of the flow inside the treatment chamber.

\section{Radial Velocity}

The radial velocities are rarely measured experimentally or reported numerically. Their small magnitudes and large oscillations make them difficult to measure or simulate. Instead, (Hsieh and Rajamani [14]; Chang and Dhir [3]) they are often calculated using the continuity equation. The numerical radial velocity distributions are presented in figures $4 \mathrm{a}$ and $4 \mathrm{~b}$.

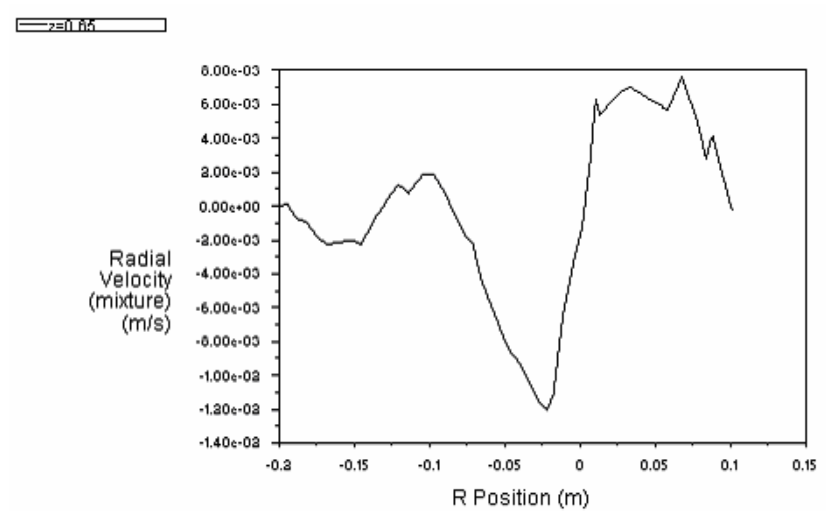

Figure 4a. Radial velocity profile at $\mathrm{z}=0.65 \mathrm{~m}$

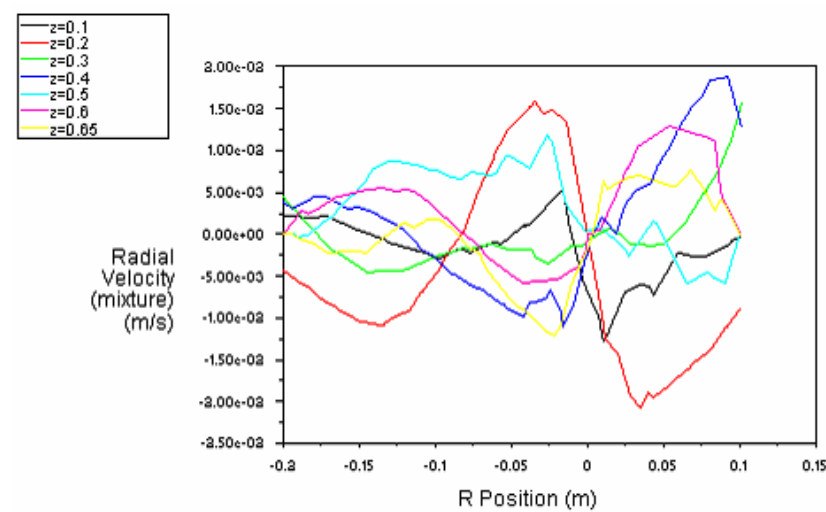

Figure 4 b. Radial velocity profile at all selected surfaces

The radial velocity component appears to be the most problematic to measure [2]. A few researchers have tried to measure the radial velocity; they have obtained profiles that are hard to interpret. An explanation for this could be that the radial velocity in absolute terms is much smaller than the tangential and axial velocity, and hence becomes difficult to measure [19].

Kelsall [16] stated that the inward velocity was greatest close to the cyclone wall and decreased as the radius decreased. Also, Chu and Chen, [4] measured the radial velocity using styrene particles with a particle dynamics analyzer, PDA. The velocity assumed its maximum value close to the center of the cyclone. The results of the previous studies disagree considerably. Closer to the center of the cyclone the flow was directed outward. The radial velocity in VTW separation chamber was found similar to Knowles et al [18]. The radial velocity pattern is a bit different from one level to another. This suggests that the radial velocity is the most difficult velocity component to measure.

\section{Pressure Profile}

Very few studies performed pressure investigations inside the hydrocyclones. Chu et al. [5] did measurements with a pressure probe inside a hydrocyclone. They studied the fluctuations of the pressure at several axial and radial positions. The highest fluctuations were found to be close to the axis. However, they didn't study their periodicity. These findings are not in the current research. This could be 
related to the difference of the geometries. The highest pressure fluctuations were found close to the wall [7]. The fluctuations were inclined proportionally inclined as $\mathrm{z}$ level positions inclined (see figures $5 \mathrm{a}$ and $5 \mathrm{~b}$ ).

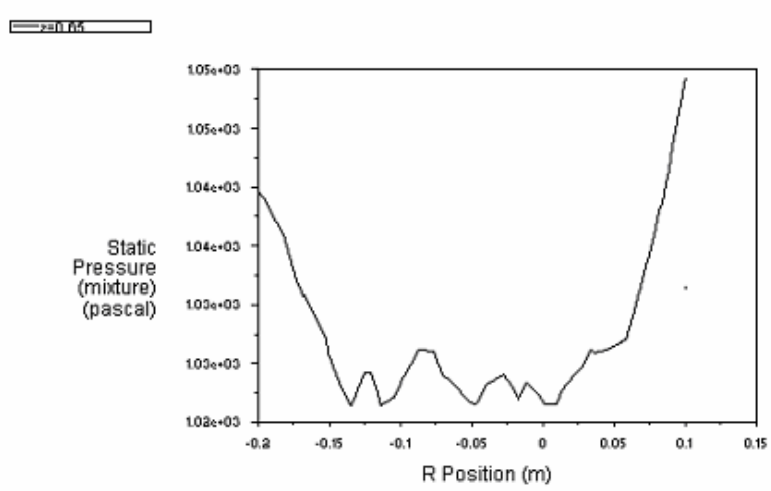

Figure 5a. The static pressure fluctuation at the inlet level $(0.65 \mathrm{~m})$ in the treatment chamber of VTW model
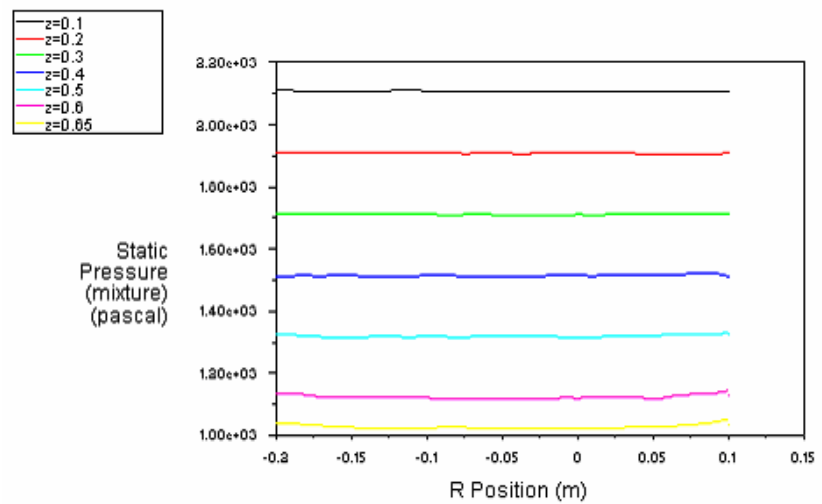

Figure $5 \mathrm{~b}$. The static pressure fluctuations at seven levels in the treatment chamber of VTW model

\section{HEADLOSS RESULTS}

Three tests were performed to accomplish the headloss at the selected flow rates with $0 \%$ blocked screen condition. CFD resulted very similar outputs as of the experimental results; as the increase of the flow rate leads to the increase of the headloss. The headloss as found were $270.95 \mathrm{~mm}$ for DDPF, $131.766 \mathrm{~mm}$ for DPF and $64.8 \mathrm{~mm}$ for DTF $\mathrm{L} / \mathrm{s}$. CFD results suggests similarity to the experimental results with error percentages of $7.4 \%$ for DTF, $11.4 \%$ for DDPF, and $29.4 \%$ for DPF. These minor error percentages resulted from the fluctuations in the manometer. Figure 6 shows very good correspondence between the CFD predictions and the experimental data.

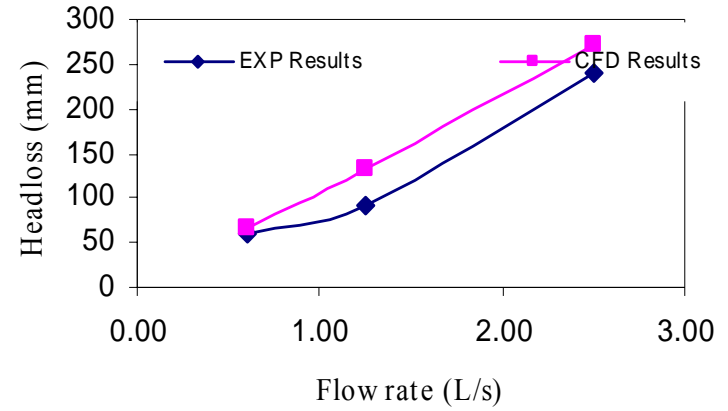

Figure 6. Numerical validation of headloss and flow rate at $0 \%$ conditions

\section{EFFICIENCY RESULTS}

Eularian-Eularian model was used to get the efficiency of VTG. Three tests were carried for three selected flow rates; 5,10 and $20 \mathrm{~L} / \mathrm{s}$. The total trapped efficiency was found to decrease as the flow rates increased ('figure 8'). For example, comparing to sand tests, the highest trapped efficiency was found $86 \%$ at the DTF $(5 \mathrm{~L} / \mathrm{s})$ as compared to the experimental result of $80.5 \%$. This was also well above the $80 \%$ level required to achieve certification [1]. Similarly, the captured rate increased with the increase of particle sizes at each flow rate (e.g. it was almost $100 \%$ for 2360 micrometers at all flow rates). These close results of experimental and CFD methods, increased the confidence to use CFD as an alternate technique to that of experimental work (see 'figure 9').

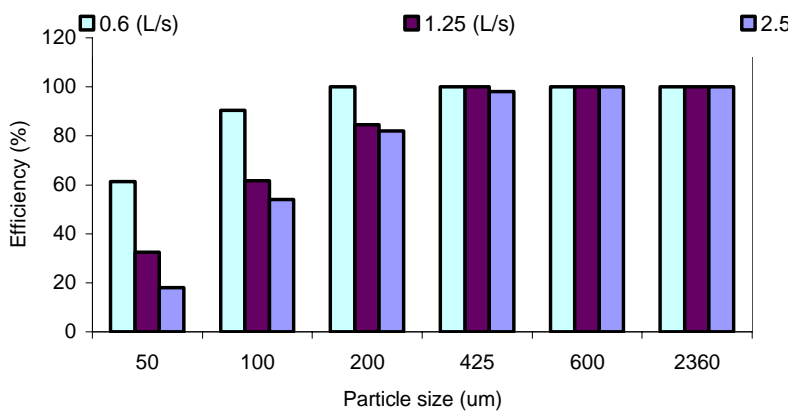

Figure 7. Particle removal efficiency predictions for different inlet flow rates

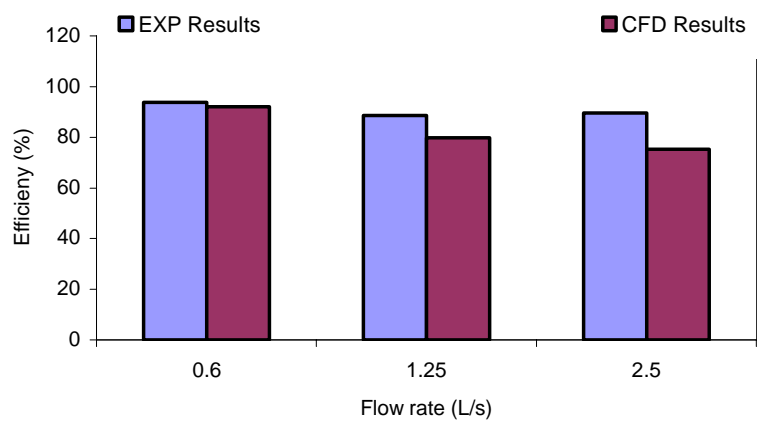

Figure 8. Numerical validation of particle removal efficiency 


\section{Time DePendent Particle Retention PREDiction}

Figure 9 illustrates how during the period following initial injection of 10 micron of sediment particles into the sediment storage region of the simulated chamber, the particle retention efficiency is predicted to decay with the time. This data that corresponds to an inlet flow rate of 1.25 $\mathrm{L} / \mathrm{s}$ highlights the importance of taking time factors into account when performing such a study experimentally or numerically.

It has been reported that the predicted rates of particle retention efficiency decay are not as high as those predicted for particle removal efficiency [9]. For VTW, the decay rates are marginally lower than VTG that Ismail and Nikraz [7] have tested. This significantly suggests better particle retention capability. This can be explained by considering the physical location of the sediment storage region relating to the way of the flow fields impinge on this area.

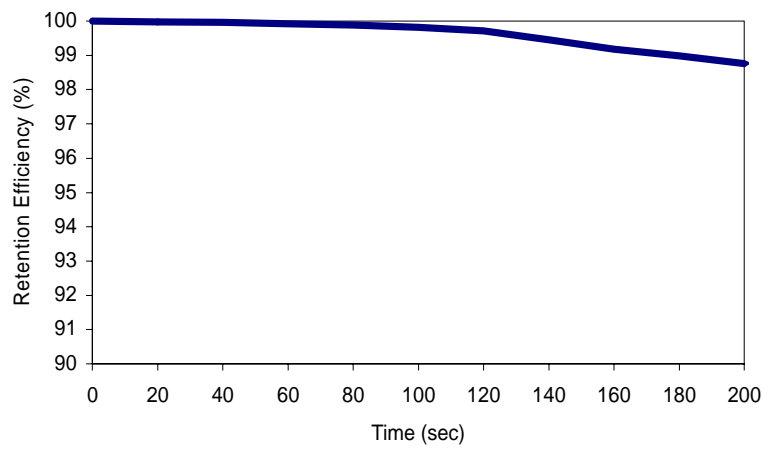

Figure 9. Time dependent efficiency prediction of VTW for 10 micron sediment particle size at an inlet flow rate of 1.25 $\mathrm{L} / \mathrm{s}(\mathrm{DPF})$

\section{CONCLUSION}

The hydraulic performance and pollutant removal efficiency (PRE) of VersaTrap type W was numerically investigated in this paper. The study showed a downward tangential and axial velocity close to the wall and an upward in the centre. Hydraulic results found that the head losses increase as the flow rates increase in each configuration. PREs were found inversely proportional with the increase of flow rates. Particle retention efficiency is likely to be high in chambers where collected sediments/pollutants isolated and protected from the main flow region activity.

The study demonstrated that CFD simulation could be used to assess the relative impact of design change on a hydrodynamic separator, yielding direct savings in fabrication costs. Comparisons between headloss and efficiency curves produced by a well validated experimental model and those produced by the CFD simulation suggest that CFD is an effective tool for predicting the relative impact of change on the outputs of the hydraulic treatment systems.

\section{REFERENCES}

[1] R. Andoh, "CFD Saves \$50,000 in Design of Stormwater Separator". Hydro International, Portland, Maine, USA. Fluent News Summer. 2006.

[2] J. Bergstr"om, and H. Vomhoff, "Velocity measurements in a cylindrical hydrocyclone operated with an opaque fiber suspension". Minerals Engineering 17 (5), 2004. 599-604.
[3] F. Chang, and V. K Dhir, "Turbulent flow field in tangentially injected swirl flows in tubes". International Journal of Heat and Fluid Flow 15 (5), 1994. 346-356.

[4] L.Y. Chu, W.M. Chen, "Research on the motion of solid particles in a hydrocyclone”, Sep. Sci. Technol. 28 (10), 1993. 1875-1886.

[5] L.Y. Chu, J.J. Qin, W.M. Chen, X.-Z. Lee, "Energy consumption and its reduction in the hydrocyclone separation process. Part II. Timeaveraged and fluctuating characteristics of the turbulent pressure in a hydrocyclone", Sep. Sci. Technol. 35 (15), 2000. 2543-2560.

[6] M. J. Doby, W. Kraipech, and A. F. Nowakowski, "Numerical prediction of outlet velocity patterns in solid-liquid separators". Chemical Engineering Journal 111, pp173-180, 2005.

[7] M. Ismail and H. Nikraz "Numerical modelling of Rocla VersaTrap type G", (unpublished work style) unpublished.

[8] M. G. Faram, and R. Y. G. Andoh, "Application of Simulation and Predictive Techniques for the Evaluation of Hydrodynamic Separators", Wastewater Treatment: Standards and Technologies to Meet the Challenges of the 21st Century, CIWEM/AETT Millennium Conf., Leeds, UK, 4-6, April. 2000.

[9] M. G. Faram, and R. Harwood, "A Method for the Numerical Assessment of Sediment Interceptors", 3rd International Conference on Sewer Processes \& Networks, Paris, France, 15-17, April. 2002.

[10] M. G. Faram, and R. Harwood, "Assessment of the Effectiveness of Stormwater Treatment Chambers using Computational Fluid Dynamics". 9th International Conference on Urban Drainage, Portland, Oregon, USA, 9-13, September. 2002.

[11] M. G. Faram, and R. Harwood, "Investigation Into The Sediment Removal and Retention Capabilities of Stormwater Treatment Chambers". StormCon Conference, San Antonio, Texas, USA. July 28-31, 2003

[12] M. Fisher, R. Flack, "Velocity distributions in a hydrocyclone separator", Exp. Fluids 32, 2002. 302-312.

[13] R. Harwood, "CSO Modelling Strategies Using Computational Fluid Dynamics", 9ICUD conference, Portland, Oregon, USA, 2002.

[14] K.T. Hsieh, and R.K. Rajamani, "Mathematical model of the hydrocyclone based on physics of fluid flow". AIChE Journal 37 (5), 1991. 735-746.

[15] M. Ismail, H. Nikraz, A. Khan, and W. Hepburn, "The laboratory test performance of two gross pollutant traps". International Conference on Environment 2006 (ICENV 2006). Penang, Malaysia.13-15 November, 2006.

[16] K. Jordan, "Numerical modeling of highly swirling flows in a cylindrical through-flow hydrocyclone". SE-100 44 Stockholm, Sweden, 2005.

[17] D.F. A Kelsall, "Study of the motion of solid particles in a hydraulic cyclone, Trans". Inst. Chem. Eng. 30. 1952. 87-108.

[18] S.R. Knowles, D.R. Woods, and I.A. Feuerstein, "The velocity distribution within a hydrocyclone operating without an air-core", Can. J. Chem. Eng. 51. 1973. 263-271.

[19] T.C. Monredon, K.T. Hsieh, and R.K. Rajamani,. "Fluid flow of the hydrocyclone: an investigation of device dimensions", Int. J. Miner. Process. 35. 1992. 68-83.

[20] Y. Okamoto, M. Konugi, and H. Tsuchiya, "Numerical Simulation of the Performance of a Hydrodynamic Separator", 9ICUD conference, Portland, Oregon, USA, 2002

[21] M. D. Slack, S. Del Porte, and M. S. Engelman, "Designing Automated Computational Fluid Dynamics Modelling Tools for Hydrocyclone Design". Minerals Engineering 17 (5), 2004. 543-547.

[22] G. Solero, and A. Coghe, "Experimental fluid dynamic characterization of a cyclone chamber". Experimental Thermal and Fluid Science 27, 2002. 87-96.

[23] V. R. Stovin, J. P. Grimm, and A. J. Saul, "The retention of fine sediments in storage chambers: an assessment of time-dependent effects". Proceedings of the Novatech 01 Conference, 25-27 June, Lyon, France, 2001. 973-980.

[24] K. Yamamoto, X. Jiao, "Hydrocyclone with a perforated inner cylinder", Nippon Kikai Gakkai Ronbunshu B 63 (615), 1997. 3623 3628 . 


\title{
Communication Network Infrastructure for Power Quality Management System (PQMS)
}

\author{
Ahmad Najib Habeb
}

\begin{abstract}
The paper will begin by briefly explore the subsystems in the PQMS and it's communication requirements which is reflected by the implementation design and objectives, PQ parameters to be monitored as well as the location of remote instruments placement. It will then go on to focus on integrated communication infrastructure via high speed narrowband powerline communication on the medium voltage (MV) and low voltage (LV) electrical distribution network. The paper is set out to determine that the utilization of one communication medium at last-mile for multiple energy management applications such as PQMS, SCADA, AMR and DSM application is the ideal approach to optimize investment and operational expenditure. A case study of powerline project's undertaking in Malaysia and misconceptions about powerline communication is also being discussed.
\end{abstract}

Keywords: power quality, power quality monitoring system, PQMS, integrated communication infrastructure, powerline communication

\section{INTRODUCTION}

With the electric industry undergoing changes, increased attention is being focused on power supply reliability and power quality. Utilities and customers especially the commercial and industrial customers are concerned about reliable power, whether the focus is on interruptions and disturbances or extended outages. Power quality issues have become a controversial topic since modern electronic equipment and computer-controlled systems are very sensitive to power quality parameters especially voltage sags and harmonics. The PQ challenge to a power utility is both technical and strategic planning. Many utilities around the world are already laying the framework for a service contract in which utilities may promise their large customers a certain level of "quality" in delivered power.

One of the most critical elements in analyzing power quality is Power Quality Monitoring System shortly known as PQMS. It collects PQ information in 'real-time' which is helpful in identifying the cause of power system disturbances. The detailed analysis and report can be used to determine a requirement for power quality solutions and further evaluate whether the solution implemented is effective. The report is also useful for the utilities from receiving erroneous complaints regarding bad power quality

Ahmad Najib Habeb. are in PowerQ Sdn. Bhd., Suite 910, Block A, Phileo Damansara 2, 15, Jalan 16/11, Off Jln Damansara, 46350, Petaling Jaya, Selangor, Malaysia (tel: 037660 0380/ 037956 7698/ 03 7956 7740, fax: 037660 0383/ 037956 7128, e-mail: najib@powerq.com.my). by their customers.

\section{EXPLORING SUB-SYSTEMS IN PQMS}

The remote instruments can comprise of various brands, different locations and each with own data source type. This includes PQ monitors, DA RTUs, protection fault recorders and revenue meter with built-in PQ functions which are located at remotes customer premises, secondary substation as well as at the main intake substations. The remote instruments will correspond with the PQ Data Concentrators that will create, update and edit PQ databases. The PQ Data Concentrators which are possibly located at the main intake substation will communicate with the central databases and PQ Master System to generate reports and perform data analysis.

There are two distinct locations that require communication network infrastructure. While the communication of PQ Data Concentrator to Central Database could utilize the WAN, the remote instruments will require another communication network infrastructure to communicate with the PQ Data Concentrator. This is the communication linkage in which this paper will propose to use DLC system that will be explored in the later section.

\section{COMMUNICATION REQUIREMENTS FOR PQMS}

In several past projects, [4], [6], [8] and [9] have revealed that monitoring location can be placed at the main intake substations, secondary substations or main entrance of key customers. Location is important to determine the availability of communication channel. Many utilities already have existing Point of Presents (PoP) of corporate Wide Area Network (WAN) at the main intake substations, hence no issue. In contrast, if monitors are installed at the secondary substations or main entrances of key customers, an alternative third party communication network infrastructure is required, thus incur additional cost and complexity.

While project [8] focus only at main intake substations which fully utilized the corporate WAN communication channel, project [4] includes monitoring at randomly selected remote sites via mix communication mediums to achieved the primary objective; to characterize power quality on primary distribution feeders to obtain project results which represent power quality on typical distribution feeders nationwide. Similar approach was done in project [6] where monitors were also installed at main entrances of key customer. 
Project [4] suggested that a good compromise approach is to monitor at the substation and at selected customer service entrance locations. The substation is important because it is the point of common coupling for most RMS voltage variations. The voltage sag experienced at the substation during a feeder fault is experienced by all the customers on other feeders supplied from the same substation bus. Customer equipment sensitivity and location on a feeder together determine the service entrance locations for monitoring. For instance, it is valuable to have a location immediately down line from each protective device on the feeder.

Power quality monitoring involves measurement of various parameters waveform, RMS samples and characteristic of PQ parameters such as voltage sags, transients, flickers and harmonics. In addition to the steady state logs, the amount of data sources to be transferred also depend on how often power quality events occur. Generally, small bandwidth of $9.6 \mathrm{kbps}$ is adequate, however, in dialup and shared communication system such as PSTN, wireless and GSM network, the actual throughput that can be achieved may be lesser that required and the multiple accesses from various remote instruments may congest the communication network traffic.

\section{INTEGRATED COMMUNICATION INFRASTRUCTURE}

Recent project [1] has rigorously conducted a research to define various applications within the utilities, communication mediums and protocols, and how a mix and integrated communication infrastructure can support multiple applications. Since it was revealed that many utility applications only require relatively small bandwidth, by mean of multiplexing or channel tunneling, one communication medium support more than one channel each supporting respective application such AMR, SCADA/DA, DSM, Protection, and PQMS. Project [1] suggests to integrate with the existing IP network of corporate WAN, not only in the backbone network mostly via Fibre optic but also for backhaul and last-mile which may utilize other hybrid communication mediums. This will optimize investment (CAPEX) and less operational expenditure (OPEX).

However, possibly due to geographic or commercial reasons, the research finding in project [1] which based in USA has overlooks the high possibility of high speed narrowband powerline communication or DLC system being conducted relatively at the same time by project [2], a project based in Europe. DLC system and its' potential will be explained in the later section.

\section{InTRODUCTION TO POWERLINE COMMUNICATION}

While PLC is generally refers to Powerline communication, there are other terms being used by different people or refers to different segments:

- PLC - Powerline Carrier, is a terminology being used by the utilities in the earlier day that refers to narrowband powerline communication on the high voltage transmission line i.e $132 \mathrm{kV}$ or $275 \mathrm{kV}$. The technology is being used to control switchgear, telephone system, and protection of transmission line over a very long distance.

- $\mathrm{PLT} / \mathrm{BPL}$ - Broadband over Powerline is to provide broadband access through ordinary power lines within the houses/building or to the substation. It utilizes frequency range from $2-30 \mathrm{Mhz}$ with data rate up to $200 \mathrm{Mbps}$ targeting high-speed Internet access, VoIP or VoD services.

- DLC - Distribution Line Carrier is high speed narrowband powerline communication that utilizes a frequency range from $9-500 \mathrm{Khz}$ with data rate up to $576 \mathrm{kbps}$. DLC system is very suitable even for very large network for multiple Real-time Energy Managements applications

Low speed powerline such as Lonworks, PowerBUS, $\mathrm{X} 10$, INSTEON, a proprietary system that use narrowband frequency but only achieve data rate of less than 9.6kbps suitable for individual application i.e AMR, home or building automation.

\section{CASe Study in Malaysia}

While Powerline Carrier on high voltage (transmission line) has been utilized in the early days, BPL, DLC system and narrowband powerline communication on the distribution network is only explored starting year 2000 . Several departments were involved such as TNB Research, Fibrecomm, TNB ICT Division, TNB Engineering, TNB Distribution. Various activities involves engagement of consultancy and joint-study that measure the powerline channel characteristics such as noises, line impedance and EMC radiation. The distribution network design is being studied to understand different type of cables, switchgears and transformers for suitable installation method of couplers in Malaysia. Several readily available products in the market were tested for performance verification and implemented in smale-scale trials in several locations.

One of the significant activities, with support from respective department, the Powerline unit of ICT division has come out with a safety and installation guideline. This guideline was formulated and planned for the nationwide implementation. However, in late 2005, TNB has ceased Powerline unit due to the company direction. Subsequently, a private company has acquired necessary licenses to carry out BPL commercialization. In addition, PowerQ Sdn. Bhd. is a company that promotes the DLC system for an integrated communication infrastructure for real-time energy management applications.

\section{Distribution Line CARRIER - DLC SyStem}

DLC System was designed to have scalable, modular, robust and reliable real-time communication infrastructure on heavily hostile powerline environment that covers long distance and complex medium voltage and low voltage electrical distribution network. With useable (programmable) frequency range of 9 to $500 \mathrm{kHz}$, a maximum speed of $576 \mathrm{Kbps}$ can be achieved. DLC System is compliant with the Standards EN 50065 (CENELEC) in Europe, IEC 61000-3 and FCC part 15 subpart B in USA.

Similar technical and implementation concept as BPL system, DLC System, however, posses significant differences and offer many advantages with respective to the usage for energy management applications. By using inductive or capacitive coupler, DLC signal can be injected onto the existing electrical cable, MV and LV distribution 
network which act as a communication medium. Recent technological development has overcomes many obstacles and enable DLC to become very reliable and cost effective communication infrastructure solutions. It integrated well with other communication medium such as Fibre optic or leased-line in corporate Wide Area Network (WAN), the ideal way of implementing a real-time energy management application such as PQMS.

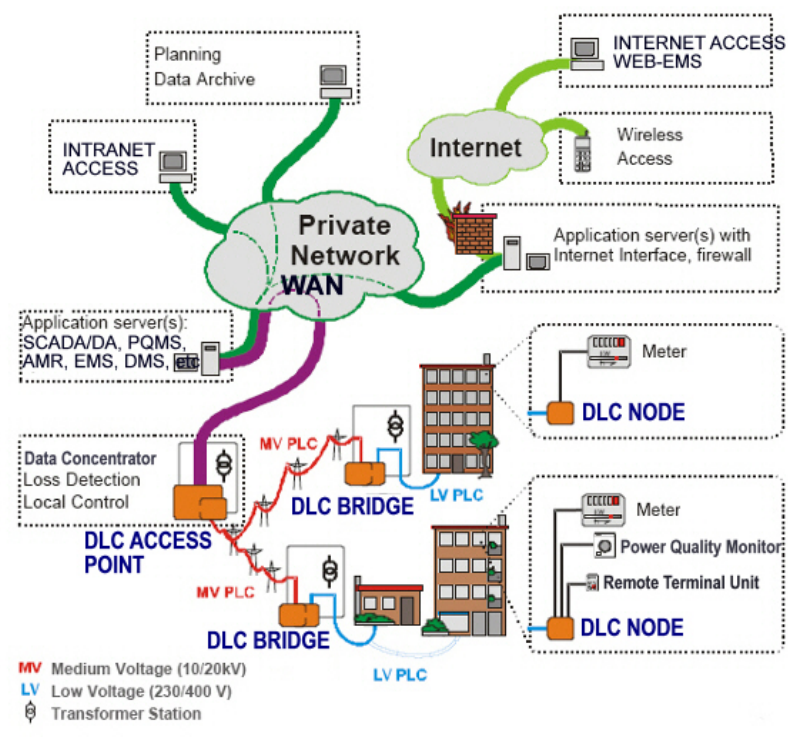

Figure 1. Overall communication network infrastructure

The overall communication network architecture shown in 'figure 1' is implemented using a cascaded powerline communication system in the last-mile over MV-LV distribution network. Network integration with the WAN, an existing IP network is done at the main intake substation. In the more typical case, communication both at midvoltage and low-voltage sides is Master/Slave based. The DLC Bridge is installed at the secondary transformer station to act as a repeater and interconnect MV and LV network.

The communication gateway or transition between powerline network and IP network communication environments is carried out by the DLC Access Point, installed at the main intake substation. In addition an Access Point also performs a number of application-specific tasks, such as data concentration, logging, failure detection, various maintenance procedures, etc.

The other side of the communication infrastructure is primarily comprised of DLC Nodes, each coupled with a low-voltage DLC slave. The DLC Node is installed at the consumer site, e.g. inside building or apartment which is connected to the end equipment such as PQ instruments, energy meter, remote terminal unit (RTU) and others. Some model is also equipped with built-in digital input/output, metering inputs and relays.

For example, the PQMS Master system will communicate with the PQ Data Concentrator at the main intake substation over IP network. DLC Access Point will extend the communication network for PQ Data Concentrators to interrogate the remote instruments located at secondary substations or customer buildings in which communication channel is being tunneled over the powerline network.

The universal communication infrastructure based on the DLC system, as well as the open architecture of the DLC
Access Points, allow for integrating with various kinds of add-on services, such as security alarms, domotic control, building and smart home automation.

\section{POWERLINE MISCONCEPTION}

Over years of involvement in the powerline communication evaluation, trial, installation and weigh against project [1], [3] and [10], various questions and misconceptions about this evolving technology are gathered. Due to the long history and overwhelmed introduction of BPL system, it is not surprising that many people are confused and not able to make clear differentiation of different powerline communication segments and understand each advantages or disadvantages thus making a quick conclusion.

The following is some of the list and brief clarification judged against the DLC system:

- DLC system does not need an electricity to work. However, DLC equipments need be powered via DC supply to continuously operating even during the electricity breakdown.

- The latest DLC system supports ad-hoc networking and network redundancy that automatically select another route should a power switching or cable fault occur.

- The latest DLC system uses complex and innovative multi-carrier OFDM method with excellent error correction to establish a stable communication in a noisy and hostile powerline environment.

- Unlike BPL system which can only work in a short distance, DLC system uses much lower frequency, thus the signal attenuation is lesser and able to reach over $10 \mathrm{~km}$.

- There is no electromagnetic field radiation, interference and licensing issues related to DLC system. These issues are a concern in BPL system that have not been resolved and highly debated in many countries.

- Latest DLC system can reach over 500Kbps. Although, it is not satisfying for high speed internet access, this bandwidth can be shared and meets many energy management applications.

- The latest DLC system uses Single Frequency Network (SFN) that allows a scalable and easier network commissioning. It can even be done and monitor remotely.

- Unlike BPL system that requires coupler and repeater at every substation, DLC signal can go through several ring-substations without anything or with only a bypassing couplers.

- Unlike capacitive coupler, which is expensive and difficult to install, most inductive coupler can be safely installed in various methods for live environment with good performance.

- $\quad$ DLC system can use XLPE, PILC or any 2-wires cable via capacitive or correct inductive coupler method.

- DLC system should in MV or LV distribution network, not only limited within a building or a house.

\section{CONCLUSION}

Power quality monitoring is fast becoming an integral component being implemented by utilties. As a support system, communication network infrastructure is a critical 
matter in determining a successful PQMS deployment. Depending on the objectives of the PQMS, location and availability of corporate WAN, back haul and last-mile communication infrastructure would be considered as very important.

DLC System is a viable communication options for fulfilling communication gap. Without mistakenly put side by side with other powerline segment, DLC system can be fully utilized for a cost-effective integrated communication infrastructure in real-time energy management applications. A concentrated effort and support by different departments who are responsible in various applications within the utilities is crucial to ensure successful implementation.

\section{REFERENCES}

[1] EPRI, Palo Alto, CA and Electricity Innovation Institute, Palo Alto, CA, "The Integrated Energy And Communication Systems Architecture", Volume II \& IV, 2003.

[2] REMPLI, NNE5-2001-825, Real-time Energy Management via Powerlines and Internet, "REMPLI System Overview", 2004.

[3] REMPLI, NNE5-2001-825, Real-time Energy Management via Powerlines and Internet, "Application Requirements Report", 2003

[4] D. Sabin, M. McGranaghan, A. Sundaram, "A Systems Approach to Power Quality Monitoring for Performance Assessment"

[5] C. L. Su, C. F. Chen, C. N. Lu, E. Liu, "Communication Network Performance Study of a High-Tech Park Power Quality Monitoring System" IEEE.

[6] YK. Ho, T. Li, P. Dolg, "Considerations and Requirements for an Integrated PQ Monitoring System” Power Quality Applications, 2004

[7] Electrotek Concepts, "PQView - Power Quality Database Management and Analysis Software" http://www.pqview.com.

[8] Hamdan Ali, "Power Quality Monitoring System (PQMS) In Malaysia", PQ Synergy Pattaya, Thailand, 2005.

[9] Channarong Sorndit "Provincial Electricity Authority - PEA (Thailand) Power quality Programs", PQ Synergy Pattaya, Thailand, 2005.

[10] Ahmad Najib Habeb, TNB ICT, "Defining Technical Consideration in Deploying Broadband Powerline Communication", TNB Technica Conference, University Tenaga Nasional, Malaysia, 2004. 


\title{
Control and Testing of a Pepper Solar Tumble Dryer
}

\author{
A. Rahim Md. Amin, Mahbub Hasan and Ong Chin Tsien
}

\begin{abstract}
Solar radiation is the energy emitted by the sun in the form of electromagnetic waves. Applications of solar energy include solar heating, cooling and direct conversion of solar radiation into electricity by photovoltaic cells. A solar dryer is an equipment that uses solar energy for drying foods. Traditional sun drying method involves spreading the material in open sun, which is economical. However open sun drying often yields poor quality, since the product is not protected from dust, rain, wind. insects, birds, rodents and other domestic animals. Besides it is impossible to control the drying rate during open drying. Current work describes control and testing of a solar tumble dryer used to overcome the disadvantages of traditional sun drying method. Pepper berries were used as the product to be dried. Several testing on fresh green pepper berries were conducted under certain variable settings; such as controller humidity set point, ventilation fan, tumbler speed and fin. The performance and control of the tumble dryer was found to be better than open drying method. The peak temperature inside the tumbler was approximately $14^{0} \mathrm{C}$ above the ambient temperature under good weather condition. Experimental results obtained from the work provide useful information for further development of the dryer in terms of various variables mentioned above.
\end{abstract}

Keywords: control, testing, pepper solar tumble dryer

\section{INTRODUCTION}

During food drying or dehydration, the essence is to reduce the moisture content of the product to a level that prevents deterioration within a certain period [1]. During the drying process, moisture is initially removed from the interior to the surface of the material that leads to final removal of moisture to external environment [2]. If the temperature is too low at the start of the drying process, micro-organisms may grow before the food is adequately dried. If the temperature is too high and the humidity is too low, the food may harden on the surface. This makes it more difficult for moisture to escape and subsequently food does not dry properly [3]. So proper control of the drying process

A. Rahim Md. Amin, Department of Mechanical and Manufacturing Engineering, Faculty of Engineering, Universiti Malaysia Sarawak, 94300 Kota Samarahan, Sarawak, Malaysia (phone: +6082583318; fax: +6082583410; e-mail: arahim@feng.unimas.my).

Mahbub Hasan, Department of Mechanical and Manufacturing, Faculty of Engineering, Universiti Malaysia Sarawak, 94300 Kota Samarahan, Sarawak, Malaysia (e-mail: hmahbub@feng.unimas.my).

Ong Chin Tsien, Department of Mechanical and Manufacturing Engineering, Faculty of Engineering, Universiti Malaysia Sarawak, 94300 Kota Samarahan, Sarawak, Malaysia. is a necessity. Sun drying is the oldest and traditional method of preparing spices. However it lacks control on drying rate and yields poor quality. Solar drying can be employed effectively to control drying rate and produce good quality products.

Current study describes the control and testing procedure of a pepper solar tumble dryer. Certain equipments suitable for control and data collection were installed here to ensure sufficient information and data collection for subsequent analysis. Those equipments included temperature and humidity sensors and a ventilation fan that were used to control the moisture in the system. Besides a motor and its speed controller were also installed in order to control the speed of the tumbler during experiment.

\section{MethodOLOGY}

The relative humidity and velocity of air inside a solar dryer are important factors affecting the drying rate in the initial stage of drying. Without adequate air movement in the early stages of drying, there is a danger of development of mould in the product. In current work, a pepper tumble dryer previously designed in UNIMAS was used during experiment [4]. A special control system was developed here that involved sacrifice of temperature rise in the early stages in favour of air movement to prevent mould formation. At the later stages the temperature was recovered using a different algorithm. Description of the control system and other methodologies involved are described below.

\section{A. Control System Setup}

A control system is compulsory for any active space heating system whether it is liquid or air based. In current study a variable speed motor, speed controller, humidity and temperature transmitter and controller and display unit were setup in order to collect various data during experimental trials of the dryer for its performance evaluation. All systems were centralized into one control panel. Besides ventilation fan was also installed in order to control the humidity of the dryer. Before data collection, the display controller was set according to parameters needed to meet required control function for temperature and humidity transmitter. These settings included temperature and humidity limits, output setting, units, resolution, range etc. 


\section{B. Preparation of Pepper Berries}

The preparation of pepper berries in the early stage of experiment is considered an important task during solar drying process. Initially pepper berries were separated from its stalk. Then they were washed thoroughly to remove dirt before loading into the tumbler for drying.

\section{C.Measurement of Temperature and Moisture Content}

Initially the tumbler was run without any load in order to compare tumbler temperature with ambient temperature. After that pepper was loaded and temperature was measured at various tumbler rotations. The ventilation fan was activated at various stages of the experiment. The temperature display on the controller indicated the temperature inside the tumbler, while the ambient temperature was measured using a thermometer.

During drying process, pepper berries moisture content was measured using Electrical moisture meter, Distillation method and weighing method [5]. The Electrical moisture meter can be used to measure moisture not more than $20 \%$ $\mathrm{RH}$. While the Distillation method is capable of measuring moisture content at any level, it is a time consuming process.

\section{RESULTS}

\section{A. No Load Trial}

In the initial stage of the investigation, tumbler and ambient temperature measurement was carried out in order to determine the effectiveness of the tumbler to absorb and trap heat. No pepper was loaded inside the tumbler and the speed of the tumbler was set at $1 / 2 \mathrm{rpm}$. Results are shown in 'table 1'.

Table 1. Results of temperature and humidity measurement without using any pepper.

\begin{tabular}{|c|c|c|c|c|}
\hline $\begin{array}{c}\text { Solar } \\
\text { Time } \\
(\mathrm{H})\end{array}$ & $\begin{array}{c}\text { Ambient } \\
\text { Temp. } \\
\left({ }^{\mathrm{C}} \mathrm{C}\right)\end{array}$ & $\begin{array}{c}\text { Dryer } \\
\text { Temp. } \\
\left({ }^{0} \mathrm{C}\right)\end{array}$ & $\begin{array}{c}\text { Ambient } \\
\text { Air } \\
\text { Humidity } \\
(\% \mathrm{RH})\end{array}$ & $\begin{array}{c}\text { Dryer } \\
\text { Humidity } \\
(\% \mathrm{RH})\end{array}$ \\
\hline 10 & 28.6 & 30.7 & 78 & 59 \\
\hline 11 & 30.1 & 36.3 & 62 & 38 \\
\hline 12 & 30.6 & 37.0 & 63 & 36 \\
\hline 13 & 31.8 & 37.2 & 57 & 34 \\
\hline 14 & 30.0 & 36.6 & 73 & 35 \\
\hline 15 & 29.6 & 34.8 & 72 & 40 \\
\hline 16 & 29 & 34.1 & 73 & 42 \\
\hline 17 & 28.5 & 32.5 & 71 & 46 \\
\hline
\end{tabular}

Based on 'table 1', in the first hour there was a sharp rise of temperature from $(30.7 \text { to } 36.3)^{0} \mathrm{C}$ inside the tumbler. At certain time during the day, the tumbler temperature reached $39.4^{\circ} \mathrm{C}$, which was $9^{0} \mathrm{C}$ above the ambient temperature. The humidity inside the tumbler decreased drastically from $59 \%$ $\mathrm{RH}$ to $38 \% \mathrm{RH}$ on the first hour of testing. It remained stable around $35 \% \mathrm{RH}$ until 14.00 and then cloudy weather slowly brought the humidity up in a steady rate. From the data collected, the humidity was set at $50 \% \mathrm{RH}$ for the following experiments. In order to monitor the influence of ventilation fan on dryer's temperature, the fan was activated manually. The temperature decreased by $(1-3)^{0} \mathrm{C}$ indicating that not much heat was given up while the fan was in intermittent operation. However continuous operation somehow delays or hinders the temperature rise inside the dryer.

\section{B. Fresh Pepper Berries Experiment}

Fresh pepper berries experiment was carried out in three different trials. Each trial consisted of three days experiment. Besides temperature and moisture content measurement, observation on pepper berries was also conducted. The moisture content on the berries was taken at the end of each day. In order to compare the drying rate under the same condition, same amount of berries were dried in open air also. 'Table 2' shows the results of moisture content at the end of each day for the first trial.

Table 2. Moisture content comparison between tumbler and open drying during first trial

\begin{tabular}{|c|c|c|}
\hline \multirow{2}{*}{ Trial 1 } & \multicolumn{2}{|c|}{ Moisture Content (\% RH) } \\
\cline { 2 - 3 } & Tumbler Drying & Open Drying \\
\hline End of day 1 & 68.7 & 41.9 \\
\hline End of day 2 & 64.5 & 28.9 \\
\hline End of day 3 & 59.3 & 12.3 \\
\hline
\end{tabular}

According to 'table 2', under the same condition, the dryer was unable to dry the peppers properly compared to open drying. An observation was made on the pepper berries at the end of each day. It was noted that mould infestation took place on the $3^{\text {rd }}$ day, as the peppers were inadequately dried inside the tumbler. Due to this shortcoming, decision was made to reduce the fin inside the tumbler to 1 .

The same procedure was followed in the second trial. Results of the second trial are shown in 'table 3'. Initial moisture content was $70.3 \% \mathrm{RH}$. After the first day of the trial, the moisture content decreased to $52.35 \% \mathrm{RH}$. Even the moisture content decreased to $31 \% \mathrm{RH}$ and $20.5 \% \mathrm{RH}$ on the second and third day of the trials respectively. These results show that fin had a great influence on the drying process.

Table 3. Moisture content comparison between tumbler and open drying during second trial

\begin{tabular}{|c|c|c|}
\hline \multirow{2}{*}{ Trial 1 } & \multicolumn{2}{|c|}{ Moisture Content (\% RH) } \\
\cline { 2 - 3 } End of day 1 & Tumbler Drying & Open Drying \\
\hline End of day 2 & 52.3 & 53.8 \\
\hline End of day 3 & 31.0 & 33.3 \\
\hline
\end{tabular}

In order to see the effect of tumbler speed on the rate of drying, the rotation of tumbler was set at $1 / 2,2$ and $3 \mathrm{rpm}$ respectively during the third trial. For one set experiments, the ventilation fan was disabled during tumbler rotation. The weight of the sample was taken after each hour of experiment. Results of third trial for tumbler drying for different rotations and settings are shown in 'figure 1' and appendix ('tables A1 to A4'). According to 'figure 1', three (3) rpm rotation of the dryer was most efficient in drying the pepper berries, while experiments with no ventilation fan yielded lowest drying rate. However faster rotation of the tumbler consumes more energy. At the same time it may degrade peppers quality. 


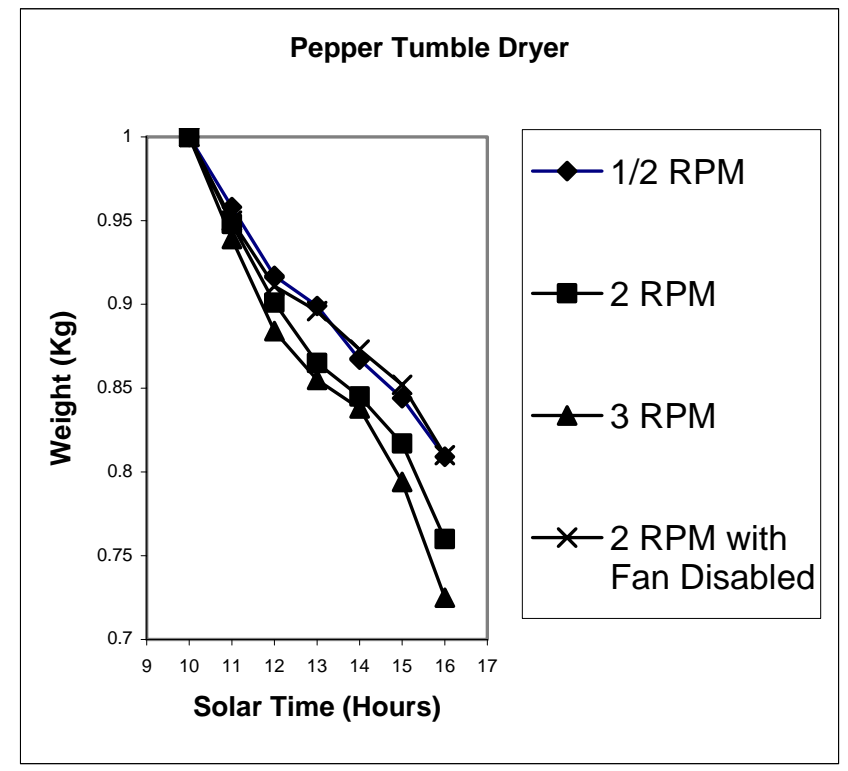

Figure 1. Comparison of drying rate at different speed and setting

Comparison of drying rate between tumbler and open drying is shown in appendix ('tables A1 to A4'). The tumble dryer gave better performance in drying peppers compared to open drying at each rotation.

\section{DiscusSION}

\section{A. Ventilation Fan}

The objective of the control system was to minimize fan operation to avoid conspicuous temperature drop. As the air in the dryer starts to warm up, its moisture carrying capacity increases and relative humidity decreases. Based on relative humidity of each trial, it can be said that ventilation fan can remove the moist air from the dryer. Only $(1-3)^{0} \mathrm{C}$ temperature drop was observed when the fan was in intermittent operation. When the fan was disabled in trial 3, the temperature was at the high side compared to other trials, but the drying rate was directly affected.

\section{B. Temperature and Humidity}

The temperature within the solar dryer varied approximately between $(28 \text { to } 45)^{0} \mathrm{C}$ during the trials. The maximum air temperature measured in the dryer occurred between noon and $4 \mathrm{pm}$. The average moisture content inside the tumbler was approximately 50.2\% RH compared to ambient air humidity that was $61.2 \% \mathrm{RH}$.

\section{Tumbler Speed}

The tumbler speed was varied between $1 / 2$ to $3 \mathrm{rpm}$ during the trial. Different rotation of the tumble dryer gave different drying rate, with $3 \mathrm{rpm}$ rotation yielded the highest drying rate compared to other rotations of the dryer.

\section{D.Pepper Berries}

During the experiment, it was observed that infestation of mould on the pepper berries took place on the $3^{\text {rd }}$ day of testing when the berries were not properly dried. On the other hand, extra caution needs to be taken to prevent over drying of the samples in order to prevent of flavour of the product. This means that the drying process is one of the critical areas that determined the quality of the pepper berries.

\section{E. Fin}

From trial 1 and 2, it was clear that fin clearly affected the drying rate of the pepper. In order to prevent mould formation, the fin was reduced to one in trial 2. As a result moisture content of the berries substantially dropped during the second trial.

\section{CONCLUSION}

Current study describes control system and testing of a pepper solar tumble dryer. A ventilation fan was also installed in the control system. According to the data gathered, operation of the ventilation fan in order to remove humid air was successful. However the fan was only required at intermittent times when high humidity was sensed inside the tumbler. Even though the temperature inside the dryer rose to a daily average of $37^{\circ} \mathrm{C}$, the peak dryer air temperature measured was $45^{\circ} \mathrm{C}$. The fin and speed of the tumbler played an important role in determining the drying rate. Faster rotation of the tumbler provides higher drying rate. However it consumes more energy and at the same time may degrade peppers quality. Thus continuous rotation is inevitable in this type of dryer. As a whole, the performance and control of the pepper tumble dryer was found to be better than open drying method. Experimental results obtained from the work provide useful information for further development of the dryer. Authors recommend that some sort of insulator can be applied to the inner and outer surfaces of the dryer designed before [4] in order to trap heat for longer period. Heat collector can be introduced into the design in a quest to increase air temperature inside the dryer. For overnight storage, continuous rotation, fan operation and heating can be implemented to avoid reabsorption of moisture and mould growth.

\section{REFERENCES}

[1] O. V. Ekechukwu, Experimental Studies of Integral-Type Natural Circulation Solar Energy Tropical Crop Dryers, Ph.D. Thesis, Cranfield Institute of Technology, UK, 1987.

[2] H. P. Garg and J. Prakash, Solar Energy Fundamental \& Applications, Tata McGraw-Hill Publishing Company Limited, New Delhi, India, 2000.

[3] P. Kendall and L. Allen, Drying Vegetables, Food and Nutrition Series-Preparation, Colorado State University, USA, 1998.

[4] L. Y. Han, Design and Fabrication of a Pepper Solar Tumble Dryer, Bachelor Thesis, Universiti Malaysia Sarawak, Sarawak, Malaysia, 2004.

[5] M. C. Gough, Moisture Meter Calibration, a Practical Guide, TDRI Storage Department, Slough, UK, 1993. 


\section{APPENDIX}

Table A1: Results of tumbler drying at a rotation of $1 / 2 \mathrm{rpm}$.

\begin{tabular}{|c|c|c|c|c|c|c|}
\hline $\begin{array}{c}\text { Solar } \\
\text { Time } \\
(\text { Hour })\end{array}$ & $\begin{array}{c}\text { Ambient } \\
\text { Temperature }\left({ }^{0} \mathrm{C}\right)\end{array}$ & $\begin{array}{c}\text { Dryer } \\
\text { Temperature } \\
\left({ }^{0} \mathrm{C}\right)\end{array}$ & $\begin{array}{c}\text { Ambient Air } \\
\text { Humidity }(\% \mathrm{RH})\end{array}$ & $\begin{array}{c}\text { Dryer Humidity } \\
(\% \mathrm{RH})\end{array}$ & $\begin{array}{c}\text { Weight after Open } \\
\text { Drying }(\mathrm{Kg})\end{array}$ & $\begin{array}{c}\text { Weight after Tumbler } \\
\text { Drying (Kg) }\end{array}$ \\
\hline 10.00 & 28.7 & 30.1 & 80 & 67 & 1.000 & 1.000 \\
\hline 11.00 & 29.1 & 32.1 & 79 & 65 & 0.975 & 0.958 \\
\hline 12.00 & 30.1 & 39.1 & 67 & 49 & 0.945 & 0.917 \\
\hline 13.00 & 30.2 & 39.4 & 67 & 48 & 0.929 & 0.899 \\
\hline 14.00 & 28.8 & 34.8 & 67 & 55 & 0.912 & 0.867 \\
\hline 15.00 & 29.3 & 36.0 & 67 & 51 & 0.890 & 0.844 \\
\hline 16.00 & 29.0 & 35.4 & 67 & 50 & 0.871 & 0.809 \\
\hline
\end{tabular}

Table A2: Results of tumbler drying at a rotation of $2 \mathrm{rpm}$.

\begin{tabular}{|c|c|c|c|c|c|c|}
\hline $\begin{array}{c}\text { Solar } \\
\text { Time } \\
(\text { Hour })\end{array}$ & $\begin{array}{c}\text { Ambient } \\
\text { Temperature }\left({ }^{\circ} \mathrm{C}\right)\end{array}$ & $\begin{array}{c}\text { Dryer } \\
\text { Temperature } \\
\left({ }^{0} \mathrm{C}\right)\end{array}$ & $\begin{array}{c}\text { Ambient Air } \\
\text { Humidity }(\% \mathrm{RH})\end{array}$ & $\begin{array}{c}\text { Dryer Humidity } \\
(\% \mathrm{RH})\end{array}$ & $\begin{array}{c}\text { Weight after Open } \\
\text { Drying }(\mathrm{Kg})\end{array}$ & $\begin{array}{c}\text { Weight after Tumbler } \\
\text { Drying }(\mathrm{Kg})\end{array}$ \\
\hline 10.00 & 28.2 & 30.8 & 78 & 67 & 1.000 & 1.000 \\
\hline 11.00 & 29.6 & 31.2 & 79 & 66 & 0.980 & 0.948 \\
\hline 12.00 & 29.8 & 32.3 & 67 & 58 & 0.968 & 0.901 \\
\hline 13.00 & 30.2 & 37.6 & 69 & 49 & 0.932 & 0.865 \\
\hline 14.00 & 30.0 & 38.0 & 67 & 48 & 0.901 & 0.845 \\
\hline 15.00 & 28.7 & 33.4 & 67 & 50 & 0.873 & 0.817 \\
\hline 16.00 & 30.1 & 34.3 & 64 & 48 & 0.858 & 0.760 \\
\hline
\end{tabular}

Table A3: Results of tumbler drying at a rotation of $3 \mathrm{rpm}$.

\begin{tabular}{|c|c|c|c|c|c|c|}
\hline $\begin{array}{c}\text { Solar } \\
\text { Time } \\
(\text { Hour })\end{array}$ & $\begin{array}{c}\text { Ambient } \\
\text { Temperature }\left({ }^{0} \mathrm{C}\right)\end{array}$ & $\begin{array}{c}\text { Dryer } \\
\text { Temperature } \\
\left({ }^{0} \mathrm{C}\right)\end{array}$ & $\begin{array}{c}\text { Ambient Air } \\
\text { Humidity }(\% \mathrm{RH})\end{array}$ & $\begin{array}{c}\text { Dryer Humidity } \\
(\% \mathrm{RH})\end{array}$ & $\begin{array}{c}\text { Weight after Open } \\
\text { Drying }(\mathrm{Kg})\end{array}$ & $\begin{array}{c}\text { Weight after Tumbler } \\
\text { Drying (Kg) }\end{array}$ \\
\hline 10.00 & 28.2 & 32.4 & 72 & 62 & 1.000 & 1.000 \\
\hline 11.00 & 29.0 & 33.8 & 72 & 52 & 0.973 & 0.939 \\
\hline 12.00 & 30.1 & 35.3 & 67 & 52 & 0.957 & 0.884 \\
\hline 13.00 & 30.3 & 35.8 & 73 & 53 & 0.936 & 0.855 \\
\hline 14.00 & 29.1 & 37.1 & 67 & 48 & 0.912 & 0.838 \\
\hline 15.00 & 30.2 & 37.5 & 67 & 50 & 0.897 & 0.794 \\
\hline 16.00 & 30.0 & 36.7 & 67 & 49 & 0.867 & 0.725 \\
\hline
\end{tabular}

Table A4: Results of tumbler drying at a rotation of $2 \mathrm{rpm}$ with ventilation fan disabled.

\begin{tabular}{|c|c|c|c|c|c|c|}
\hline $\begin{array}{c}\text { Solar } \\
\text { Time } \\
(\text { Hour })\end{array}$ & $\begin{array}{c}\text { Ambient } \\
\text { Temperature }\left({ }^{0} \mathrm{C}\right)\end{array}$ & $\begin{array}{c}\text { Dryer } \\
\text { Temperature } \\
\left({ }^{0} \mathrm{C}\right)\end{array}$ & $\begin{array}{c}\text { Ambient Air } \\
\text { Humidity }(\% \mathrm{RH})\end{array}$ & $\begin{array}{c}\text { Dryer Humidity } \\
(\% \mathrm{RH})\end{array}$ & $\begin{array}{c}\text { Weight after Open } \\
\text { Drying (Kg) }\end{array}$ & $\begin{array}{c}\text { Weight after Tumbler } \\
\text { Drying (Kg) }\end{array}$ \\
\hline 10.00 & 28.6 & 29.5 & 75 & 68 & 1.000 & 1.000 \\
\hline 11.00 & 30.0 & 33.3 & 68 & 64 & 0.973 & 0.950 \\
\hline 12.00 & 30.1 & 35.7 & 70 & 65 & 0.965 & 0.911 \\
\hline 13.00 & 29.0 & 37.6 & 73 & 64 & 0.933 & 0.896 \\
\hline 14.00 & 30.4 & 44.4 & 62 & 52 & 0.907 & 0.873 \\
\hline 15.00 & 31.9 & 40.4 & 71 & 59 & 0.863 & 0.852 \\
\hline 16.00 & 30.1 & 35.8 & 67 & 59 & 0.822 & 0.810 \\
\hline
\end{tabular}




\title{
Series Connection of High Voltage Lines Characteristics
}

\author{
Nurlailie Izani Azizan and Erwan Sulaiman
}

\begin{abstract}
Electrical power stations and transmission networks are necessary if the electrical power supply to an entire area is reliable. Transmission networks require a multitude of lines and transformers as well as the corresponding switchgear and substations. Because of the enormous economic importance of electrical power, special attention is given to guaranteeing the smooth operations of the transmission devices so that the supply to the consumer remains as interruption-free as possible. This research is to determine the characteristics of series connection of high voltage lines and the effect of the operating capacitance on the voltages and currents. The researches are using DE LORENZO equipments to conduct an experiment related to series connection of the high voltage lines. As a result, it is important that in the case of short-circuit; the disconnection of the network elements involved must be guaranteed by the corresponding circuit breakers.
\end{abstract}

Keywords: series, high voltage

\section{INTRODUCTION}

Various voltage levels are used for the transmission of electrical power, the levels are determined by considerations regarding the amount of power to be transmitted and the distance over which it is to be transmitted. The higher the choice of transmission voltage, the lower the currents and correspondingly to the transmission losses, which as is wellknown, increase with the square of the current. On the other hand, network investment costs increase as the voltage increases.

For reasons of standardization, specific rated voltages have been agreed upon, which have been allocated the following ranges:

(i) Low voltage $(230 / 400 \mathrm{~V})$

(ii) Medium voltage $(10 / 20 / 30 \mathrm{kV})$

(iii) High voltage $(110 \mathrm{kV})$ and

(iv) Ultra-high-voltage $(220 / 380 \mathrm{kV})$

The cross-section of overhead power transmission lines is selected according to two criteria [1]:

1. An excessive current density, which would lead to impermissible heating of the conductor material, must be prevented.

2. A minimum cross-section must be maintained, in consideration of the expected voltage drop along the

Nurlailie Izani Azizan and Erwan Sulaiman are currently with Department of Electrical Power Engineering, University of Tun Hussein Onn Malaysia, 86400, Batu Pahat, Johor. (Phone: 07-4537608; Fax: 074536060; email: 1ly_blues@yahoo.com, erwan@uthm.edu.my). line.

As regards networks, a distinction is drawn between radial, ringed and meshed networks, examples of which are given in figure 1 [1].
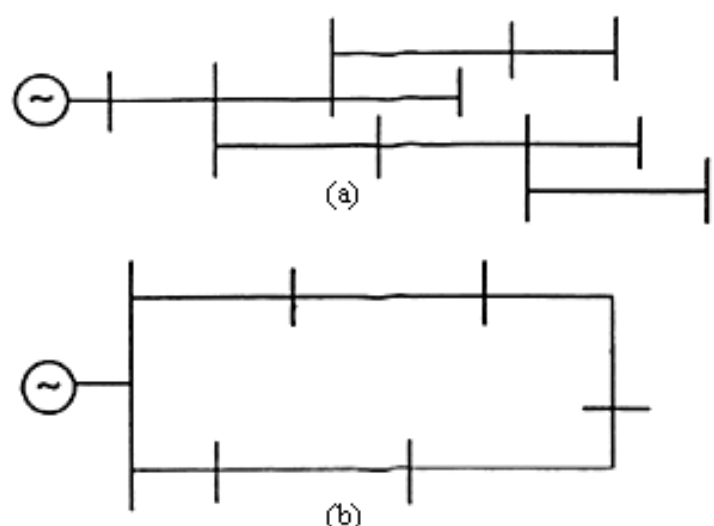

(b)

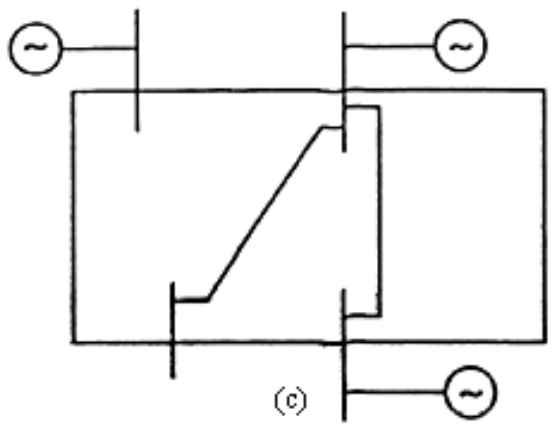

Figure 1. (a) Radial (b) Ringed and (c) Meshed networks

The radial network represents the simplest form of a supply network. When an item of operating equipment which are in feed, transformer and lines fails, all consumers situated downstream from it are affected. For ringed network, each consumer is fed from the both sides. This provides a better guarantee of supply. When a fault occurs, only the faulty section is isolated, so that the supply however only from one side to a large number of consumers can be maintained. Finally the meshed network is the most reliable supply system, since each consumer can be reached via different transmission paths. In addition, the failure of one of the supply in feed can usually be taken over by the remaining power generating blocks.

\section{ELECTRICAL SUPPLY NETWORKS}

Before the principles underlying the basic circuit can be 
deduced, the equivalent circuit diagrams of the operating equipments to be considered are introduced. In a healthy system, the three phases are loaded symmetrically and so a single-phase treatment is adequate. The equivalent circuit diagram of an overhead line is given in figure 2 .

In standard practice, the leakage losses are not considered in the equivalent circuit diagram. The quantities $R, L$ and $C_{B}$ are quantities per unit length. The equivalent circuit diagram represents the condition adequately when the line length does not greatly exceed $200 \mathrm{~km}$ and when only steady-state conditions are considered [2].

\section{SERIES CONNECTION}

The series connection of two lines was conducted in order to measure the voltage distribution in the series connection of two lines and also to investigate the effect of the operating capacitances on the voltages and currents.

The arrangement, where the individual units of operating equipment are connected in series, is the basis of the radial network. The relations are deduced first for the simplified representation for without shunt elements, and then a more exact treatment, in which the shunt elements are considered, is carried out. A characteristic feature of a series circuit is that approximately the same current flows through all branches [3]. Thus, the cross-section of the weakest line is decisive in determining the extent to which a series circuit can be loaded. The simplified equivalent circuit diagram of two lines in series is in figure 3.

\section{Methodology}

The developments of this research have been planned with detail. The experiment was conducted using DELORENZO equipments for series connection of two lines in two conditions i.e., without operating capacitances and with operating capacitances. Figure 4 and figure 5 show the topographic diagram of two connection lines in series for both situations. The effects of the operating capacitance on the voltages and currents are analyzed.

In figure 4 , the bridging plugs connecting the capacitances to both line models are removed while the three-phase balanced resistive load is used as a load. The line voltage, $\mathrm{U}_{\mathrm{B}}(\mathrm{V})$, load voltage, $\mathrm{U}_{\mathrm{E}}(\mathrm{V})$ and load current, $\mathrm{I}_{\mathrm{E}}(\mathrm{A})$ are measured to see its characteristics for the voltage curve as a function of the load current.

Meanwhile, in figure 5, the bridging plugs connecting the capacitance were inserted and the value of the line voltage, $\mathrm{U}_{\mathrm{B}}(\mathrm{V})$, load voltage, $\mathrm{U}_{\mathrm{E}}(\mathrm{V})$, load current, $\mathrm{I}_{\mathrm{E}}(\mathrm{A})$, line current, $\mathrm{I}_{1}(\mathrm{~A})$ and line current, $\mathrm{I}_{2}(\mathrm{~A})$ are measured respectively.

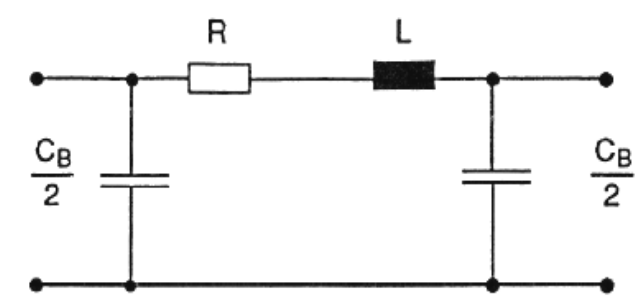

Figure 2. Single-Phase Equivalent Circuit of a Line

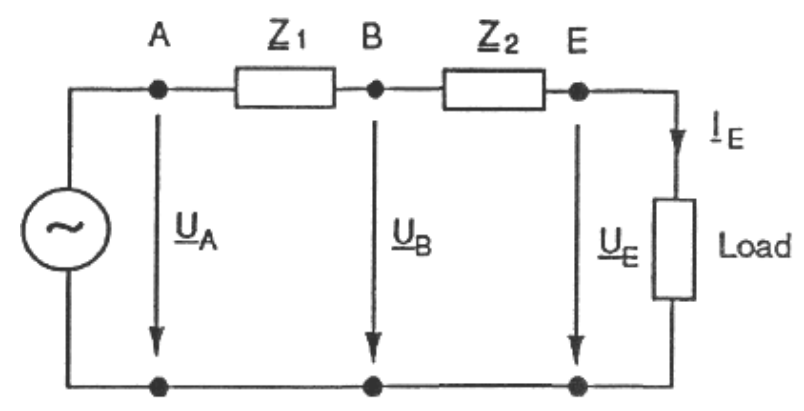

Key:

Longitudinal impedance of line $1, \underline{Z}_{1}=R_{1}+j \omega L_{1}$ $=\mathrm{R}_{1}+\mathrm{j} \mathrm{X}_{1}$

Longitudinal impedance of line $2, \underline{Z}_{2}=R_{2}+j \omega L_{2}=$ $\mathrm{R}_{2}+\mathrm{jX}$

Feeding voltage at the beginning of line 1, $\underline{\mathrm{U}}_{\mathrm{A}}$

Voltage at the beginning of line $2, \underline{U}_{2}$

Load voltage at the end of line $2, \underline{\mathrm{U}}_{\mathrm{E}}$

Load current, also the current through the two lines, $\underline{\mathrm{I}}_{\mathrm{E}}$

Figure 3. Simplified Equivalent Circuit Diagrams of Two Lines in Series

\section{RESUlts}

Table 1 and Table 2 show the result of series connection lines for both situations respectively.

Table 1. Experiment Results for Series Connection Lines without Operating Capacitance

\begin{tabular}{|c|c|c|c|c|}
\hline \hline $\begin{array}{c}\text { Voltage } \\
\underline{\mathrm{U}}_{\mathrm{A}}(\mathrm{V})\end{array}$ & Load & $\underline{\mathrm{U}}_{\mathrm{B}}(\mathrm{V})$ & $\underline{\mathrm{U}}_{\mathrm{E}}(\mathrm{V})$ & $\underline{\mathrm{I}}_{\mathrm{E}}(\mathrm{A})$ \\
\hline 220 & $\mathrm{R}_{1}$ & 228.6 & 219.4 & 0.447 \\
\hline 220 & $\mathrm{R}_{2}$ & 218.9 & 204.5 & 0.451 \\
\hline 220 & $\mathrm{R}_{3}$ & 197.0 & 171.1 & 0.458 \\
\hline 220 & $\mathrm{R}_{4}$ & 177.7 & 139.5 & 0.464 \\
\hline 220 & $\mathrm{R}_{5}$ & 161.8 & 112.3 & 0.465 \\
\hline 220 & $\mathrm{R}_{6}$ & 147.7 & 84.8 & 0.466 \\
\hline \hline
\end{tabular}

Table 2. Experimental Results for Series Connection Lines with Operating Capacitance

\begin{tabular}{|c|c|c|c|c|c|c|}
\hline \hline $\begin{array}{c}\text { Voltag } \\
\mathrm{e} \\
\left(\mathrm{U}_{\mathrm{A}}^{\mathrm{V}}\right)\end{array}$ & $\begin{array}{c}\text { Loa } \\
\mathrm{d}\end{array}$ & $\underline{\mathrm{I}}_{1}(\mathrm{~A})$ & $\underline{\mathrm{I}}_{2}(\mathrm{~A})$ & $\underline{\mathrm{U}}_{\mathrm{B}}(\mathrm{V})$ & $\underline{\mathrm{U}}_{\mathrm{E}}(\mathrm{V})$ & $\underline{\mathrm{I}}_{\mathrm{E}}(\mathrm{A})$ \\
\hline \hline 220 & $\mathrm{R}_{1}$ & 0.994 & 0.548 & 267.4 & 270.8 & 0.606 \\
\hline 220 & $\mathrm{R}_{2}$ & 1.004 & 0.554 & 246.5 & 240.9 & 0.615 \\
\hline 220 & $\mathrm{R}_{3}$ & 1.012 & 0.561 & 209.9 & 187.2 & 0.625 \\
\hline 220 & $\mathrm{R}_{4}$ & 1.016 & 0.564 & 183.5 & 144.7 & 0.628 \\
\hline 220 & $\mathrm{R}_{5}$ & 1.017 & 0.565 & 165.3 & 112.5 & 0.629 \\
\hline 220 & $\mathrm{R}_{6}$ & 1.018 & 0.566 & 150.8 & 185.7 & 0.630 \\
\hline \hline
\end{tabular}

The graph for $U(V)$ versus $I_{E}(A)$ and $I(A)$ versus $I_{E}(A)$ were plotted to show the graphical different in terms of the characteristics for both series connection with and without using the operating capacitance as shown in figure 6 , figure 7 and figure 8 . 


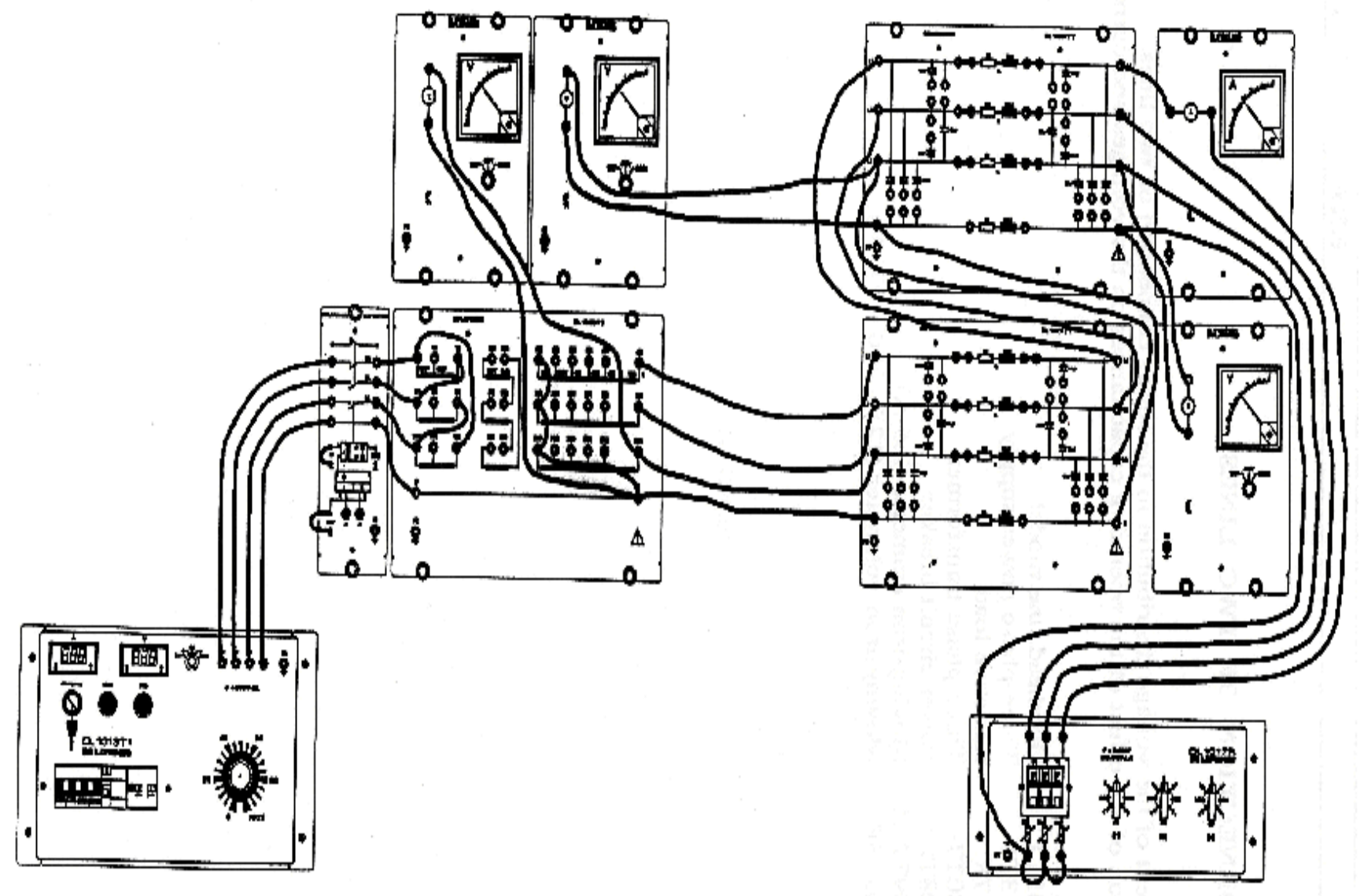

Figure 4. Topographic Diagram of Two connection lines in Series without Operating Capacitance

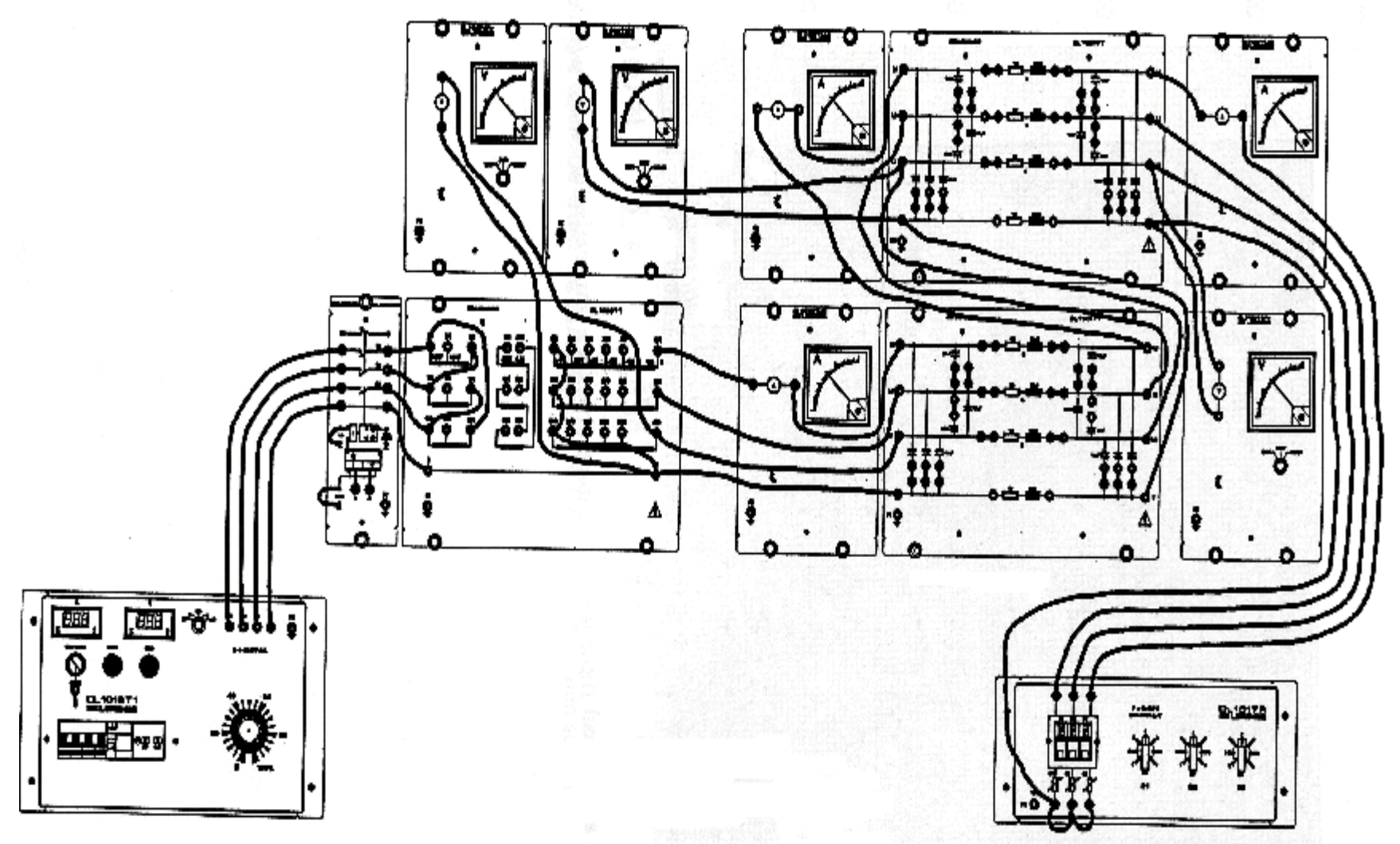

Figure 5. Topographic Diagram of Two connection lines in Series without Operating Capacitance 
Graph U (V) versus IE(A)

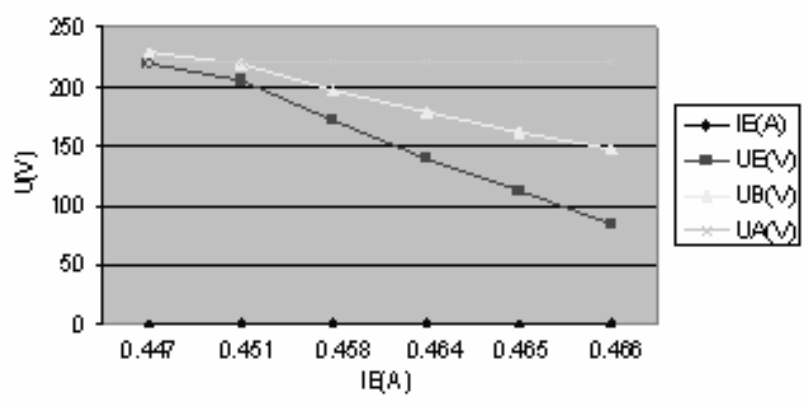

Figure 6. Voltage, U(V) Curves As A Function Of The Load Current, $\mathrm{I}_{\mathrm{E}}(\mathrm{A})$ Without Operating Capacitance

In figure 6, when the operating capacitance is not used, the voltage values at the load show that the voltage drops are impermissibly large with very long line.

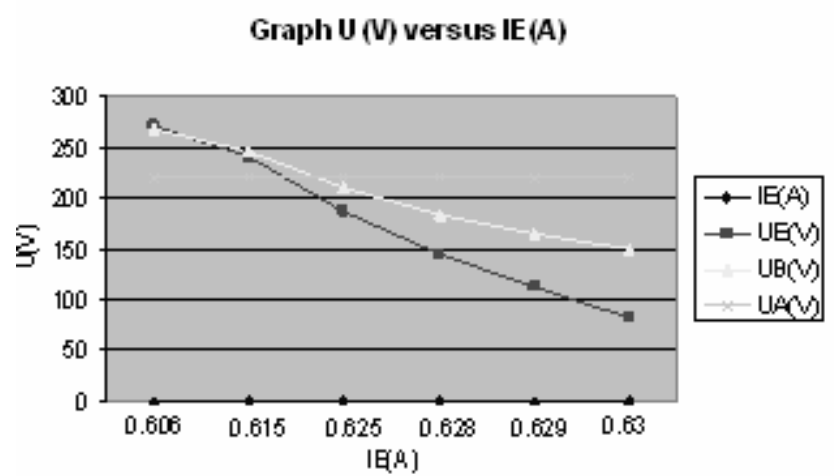

Figure 7. Voltage, U(V) Curves as a function of the Load Current, $\mathrm{I}_{\mathrm{E}}(\mathrm{A})$ with operating capacitance

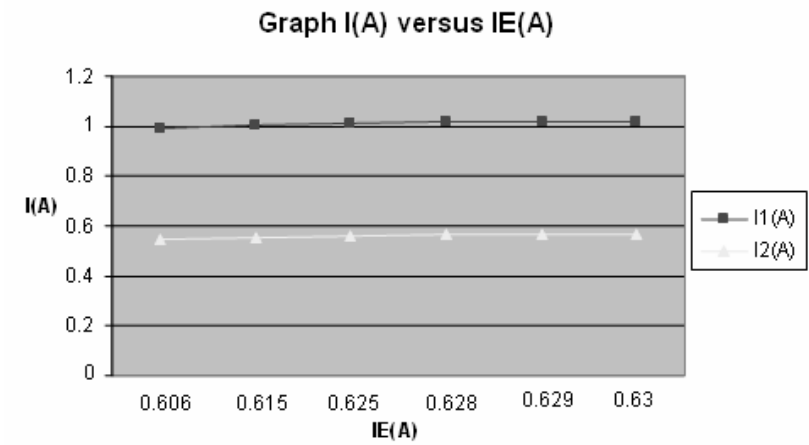

Figure 8. Line Current, I(A) Curves as a function of the Load Current, $\mathrm{I}_{\mathrm{E}}(\mathrm{A})$ with operating capacitance

In Figure 7 and Figure 8, when the line capacitances are taken into consideration the line currents flow at beginning and end points of the two line models. More over the influence of the line inductances on the voltage drop is partially compensated by the effect of the cross capacitances.

\section{CONCLUSION}

From this study, it is conclude that a better result can be achieved when the operating capacitance are connected and the voltage drop at load voltage can be reduced connected it. The differences of characteristics regarding the associated voltages and currents between those two connections have been addressed.

\section{ACKNOWLEDGEMENT}

The authors wish to express their gratitude to the staff of Power Laboratory, Universiti Tun Hussein Onn Malaysia for their encouragement and assistance in the laboratory works.

\section{REFERENCES}

[1] DE LORENZO GROUP Manual DL GTU 102.3, Teaching Systems for Technical Training.

[2] E. Kuffel, W.S. Zaengl and J.Kuffel "High voltage engineering : Fundamentals" $2^{\text {nd }}$ ed. Oxford: Newnes, 2000.

[3] Jan Machowski, Janusz W Bialek, James R Bumby. "Power system dynamics and stability” Chichester: John Wiley, 1997. 


\title{
Prospects of Photovoltaic Powered Crop Production Greenhouse - An Optimistic Case Study for Aqaba in Jordan
}

\author{
M.A. Alghoul, K. Sopian, N. Amin, and M.A.M. Teridi
}

\begin{abstract}
A stand-alone photovoltaic power system is designed to operate crop production greenhouse in an exemplary place of Aqaba in Jordan, which is located at latitude of $29 \mathrm{~N}$ and longitude of $35 \mathrm{E}$ with an altitude of $51 \mathrm{~m}$ above sea level. The sizing of photovoltaic power system requires the plant size, electrical consumption load, and meteorological data conditions as inputs. The total load in summer and the total load in winter are estimated for daily operation. Monthly average daily values of global solar radiation on a horizontal surface, maximum and minimum temperatures, relative humidity, and sunshine duration data for 10 years (1989-1998) are also used in this study. The PV system is designed according to total load in summer (higher load) and the minimum sunshine duration in winter to supply electric power, which can operate two electric fans $(1.5 \mathrm{hp}) \&$ one cooling pump $(0.75 \mathrm{hp})$ and other electrical accessories of the greenhouse $(39 \mathrm{~m} \times 9 \mathrm{~m} \times 3 \mathrm{~m})$. The array size of 120 Watt, 16.9 $\mathrm{V}$, and 7.1 Amp. The battery is sized considering different factors that affect battery efficiency to reliably operate the estimated loads during a sequence of below average insolation. The storage battery size is chosen to be of $6 \mathrm{~V}$ and $360 \mathrm{Ah}$. The case study in Aqaba shows all the prospects of using standalone PV systems to be recognized as viable alternatives.
\end{abstract}

Keywords: solar photovoltaics, stand-alone PV system, greenhouse environment, crop production

\section{NOMENCLATURE}

$L C=$ Load Current (measured in amps, $A$ )

$L V=$ Load Voltage (measured in volts, $V$ )

$L P=$ Load power (measured in Watt, $V$ )

$D D C=$ Daily Duty Cycle

$W D C=$ Weekly Duty Cycle

$A(A)=$ Amperage required by the system

$P C E F=$ power conversion efficiency factor (decimal)

$N S V=$ the nominal system voltage $(V)$

AHD = Amp-Hours Load per Day

M.A. Alghoul is with the Solar Energy Research Institute, Universiti Kebangsaan Malaysia, 43600 UKM Bangi, Selangor, Malaysia (Corresponding author:e-mail: Alghoul@vlsi.eng.ukm.my).

K. Sopian is with the Solar Energy Research Institute, Universiti Kebangsaan Malaysia, 43600 UKM Bangi, Selangor, Malaysia.

N. Amin is with the Department of Electrical, Electronic and System Engineering, Faculty of Engineering, 43600 UKM Bangi, Selangor, Malaysia

M.A.M. Teridi is with the Solar Energy Research Institute, Universiti Kebangsaan Malaysia, 43600 UKM Bangi, Selangor, Malaysia.
$A H D_{T}=$ the total amp-hour load per Day

$W E F=$ the wire efficiency factor (Decimal)

$B E F=$ the battery efficiency factor (Decimal)

$A H D_{C}=$ the corrected amp-hour load

$P C D=$ Peak Current Draw

$L P_{T D C}=$ Total DC Load Power

$L P_{T A C}=$ Total AC Load Power

$D C=$ design current

$P S=$ peak sun

$R B C=$ required battery capacity

$S D=$ storage days

$D O D_{\max }=$ maximum depth of discharge of battery type

$D T=$ derate for temperature

$B_{P}=$ number of batteries in parallel

$B C=$ capacity of selected battery of selected battery type

$B_{S}=$ number of batteries in series

$N B V=$ nominal battery voltage

$B_{T}=$ total number of batteries

$B C_{S}=$ system battery capacity

$B C_{U}=$ usable battery capacity

$D C_{D}=$ the derated design current

$M D F=$ module derate factor

$M_{P}=$ the number of parallel-connected modules

$M_{C}=$ rated module current

$V R C B=$ voltage required to charge the batteries

$M_{S}=$ the number of series connected modules

$M_{V H T}=$ module voltage at highest temperature

$M_{T}=$ the total number of modules required

$A_{C}=$ the rated array current

\section{INTRODUCTION}

Aqaba is bordered by Palestine, Jordan, Egypt and Saudi Arabia and is one of the hinges connecting the Asian and African continents. Aqaba is blessed with the abundance of solar radiation in summer and warm climate in winter [1] along with the availability of shipping facilities by sea and main roads to encourage the utilization of greenhouse vegetable production. The advantage of greenhouse vegetable production lies in the fact that relatively small amount of area is required as compared to the field-grown produce and the return on investment can be good enough when requisite markets can be found nearby.

The observed data of Aqaba from (1989-1998) used in this study is collected from Jordan Meteorological Department are shown in fig 2, and table 1. Aqaba is very attractive for solar applications [2], where the minimum and maximum sunshine duration is $6.19-12.19$ hours while the 
yearly average daily is 9.38 hours. Global solar radiation data on horizontal surface is ranged from 12.43 MJ in December and 29.66 MJ in June while the yearly average daily $21.18 \mathrm{MJ}(5.88 \mathrm{kWh})$, which is considered very attractive for solar applications.

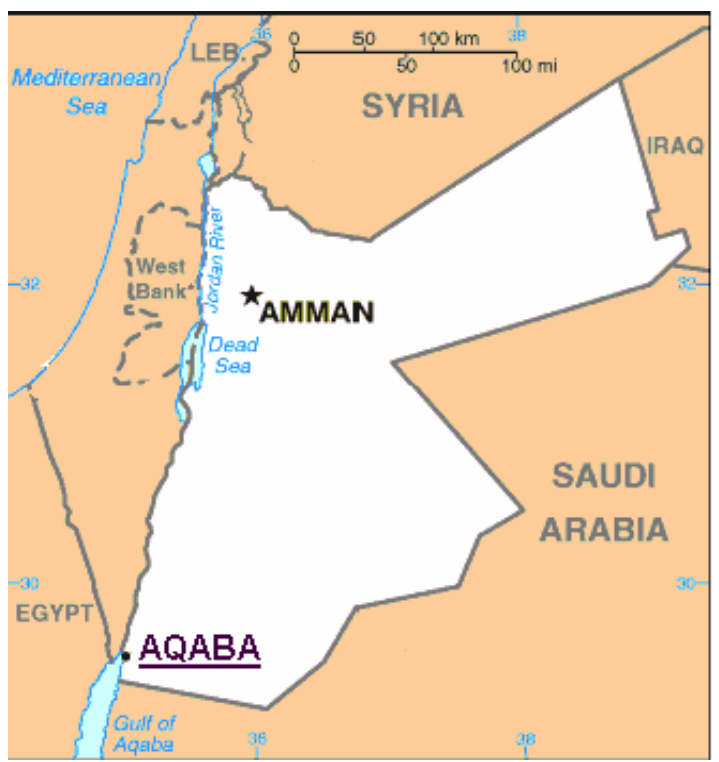

Figure 1. Geographical location of Aqaba at the southwest part of Jordan

Monthly average daily of maximum ambient temperature during daytime is high in summer $\left(30.5-39.3^{\circ} \mathrm{C}\right)$ and ranges from 19.4 to $26.8^{0} \mathrm{C}$ in winter, while the yearly average daily is $30.48^{0} \mathrm{C}$. Monthly average daily of minimum ambient temperature during night-time is ranged from 16.7 to $25^{\circ} \mathrm{C}$ in summer and in winter ranged from 8.4 to $15^{\circ} \mathrm{C}$, while the yearly average daily is $17.61{ }^{\circ} \mathrm{C}$. Monthly average daily relative humidity is ranged from $36.7-43.5 \%$ in summer and $50-57 \%$ in winter, while the yearly average daily is $47.22 \%$.

Thus, utilizing solar photovoltaic to power agriculture applications in Aqaba is considered very significant. The principle objective of this study is to design a stand-alone photovoltaic (PV) system that can power the load of the greenhouse. Moreover, it is to estimate the roughly cost of the main subsystems such as modules and batteries to quantify the applicability of powering the load of the greenhouse by PV system.

\section{GREENHOUSE ENVIRONMENT}

The inside temperature and relative humidity of the greenhouse rises to extreme levels above the ambient temperatures that damages of the crops $[3,4,5$, and 6]. The introduction of greenhouse shading reduces the thermal stress on greenhouse crops where shading is used to control light and lower interior temperatures [7-16]. Shading may be required from the late spring until the early fall if daytime temperatures rise too high. Proper ventilation is also important not only for temperature control, but also to replenish carbon dioxide and control relative humidity, where relative humidity above 90 percent will encourage disease problems. However, air temperatures and relative humidity may still far from the suitable ranges. When natural convection is used alone, the inside temperature of the greenhouse still can rise to critical levels above ambient temperatures. Forced convection using fans can lower air temperatures more than greenhouses cooled by natural convection only. But forced convection alone may not reduce temperatures to the desired levels. Evaporative cooling can be used to produce lower temperatures when this is the case where it is the most efficient and economical way to reduce greenhouse temperatures. The most commonly used system of evaporative cooling is the fan and pad system.

As the sun rises, greenhouse temperature begins to rise until it reaches for example $26^{\circ} \mathrm{C}$, during which, the fans start to operate and consequently the water pump too. Water is applied to the pad material by the pump as air is being drawn through the pads by fans located on the opposite side of the greenhouse. Evaporative cooling produces two changes in the condition of the air exiting the pads. The air is cooler and its humidity has been raised. The wet-bulb temperature of the entering air is the limiting factor in the amount of cooling that can be accomplished. As the sun sets, the solar radiation intensity and ambient temperature drop, and hence the temperature inside the greenhouse drops accordingly to below $26^{\circ} \mathrm{C}$ causing the operation of the fans and pump to stop for the rest of the day. In general range of air temperatures inside the greenhouse should be more than $10^{\circ} \mathrm{C}$ and less than $35^{\circ} \mathrm{C}$ and relative humidity less than $80 \%$.

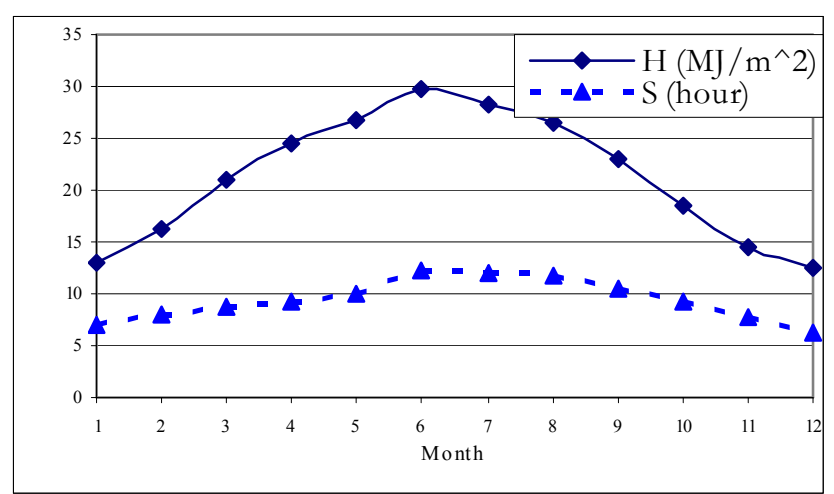

Figure 2. Monthly average daily global solar radiation and sunshine duration 
Table 1. Monthly daily average of some observed meteorological parameters for Aqaba from 1989-1998

\begin{tabular}{cccccccccc}
\hline Aqaba & $H$ & $S$ & $H O$ & $S O$ & $H / H O$ & $S / S 0$ & $T_{\max }$ & $T_{\min }$ & Humidity \\
\hline & $\left(M J / \mathrm{m}^{2}\right)$ & $($ hour $)$ & $\left(\mathrm{MJ} / \mathrm{m}^{2}\right)$ & (hour) & & & $\left({ }^{\circ} \mathrm{C}\right)$ & $\left({ }^{\circ} \mathrm{C}\right)$ & $(\%)$ \\
Jan & 12.97 & 6.95 & 21.53 & 10.33 & 0.60 & 0.67 & 19.43 & 8.39 & 57.14 \\
Feb & 16.20 & 8.00 & 26.07 & 10.98 & 0.62 & 0.73 & 21.41 & 9.59 & 53.11 \\
Mar & 21.00 & 8.79 & 31.74 & 11.82 & 0.66 & 0.74 & 25.41 & 12.79 & 50.93 \\
Apr & 24.58 & 9.14 & 36.93 & 12.74 & 0.67 & 0.72 & 30.50 & 16.74 & 39.81 \\
May & 26.77 & 10.03 & 40.01 & 13.50 & 0.67 & 0.74 & 35.14 & 20.95 & 36.21 \\
Jun & 29.66 & 12.19 & 41.05 & 13.86 & 0.72 & 0.88 & 38.29 & 23.50 & 37.48 \\
Jul & 28.24 & 12.01 & 40.39 & 13.68 & 0.70 & 0.88 & 39.32 & 25.24 & 40.44 \\
Aug & 26.45 & 11.75 & 37.85 & 13.02 & 0.70 & 0.90 & 39.13 & 25.45 & 43.46 \\
Sep & 23.01 & 10.52 & 33.29 & 12.14 & 0.69 & 0.87 & 36.22 & 23.32 & 49.62 \\
Oct & 18.42 & 9.33 & 27.48 & 11.23 & 0.67 & 0.83 & 32.69 & 20.18 & 49.58 \\
Nov & 14.46 & 7.63 & 22.49 & 10.49 & 0.64 & 0.73 & 26.78 & 14.97 & 52.40 \\
Dec & 12.43 & 6.19 & 20.17 & 10.14 & 0.62 & 0.61 & 21.44 & 10.17 & 56.46 \\
Annual & 21.18 & 9.38 & 31.58 & 11.99 & 0.66 & 0.77 & 30.48 & 17.61 & 47.22 \\
\hline
\end{tabular}

Table 2. Optimum, maximum and minimum temperatures for growing various fruit and vegetable crops. All temperatures are in degrees Celsius [6]

\begin{tabular}{cccc}
\hline Crop & $\begin{array}{c}\text { Optimum Temperature } \\
\text { Range }\end{array}$ & $\begin{array}{c}\text { Minimum } \\
\text { Temperature }\end{array}$ & $\begin{array}{c}\text { Maximum } \\
\text { Temperature }\end{array}$ \\
\hline Rock melon, Cucumber & $18-24$ & 15 & 32 \\
Pimpkin, Squash & $18-24$ & 10 & 32 \\
Tomato, Capsicum & $21-24$ & 18 & 27 \\
Mangos, Bananas & $22-30$ & 12 & 40 \\
Wine Grapes & $15-25$ & 0 & 40 \\
Onions And Garlic & $13-24$ & 7 & 29 \\
Broccoli, Cabbage, Spanish & $15-18$ & 4.5 & 24 \\
Carrot, Potato & $15-18$ & 7 & 24 \\
Lettuce, Cauliflower, Celery & $15-18$ & 7 & 24 \\
Beans & $15-21$ & 10 & 27 \\
Sweet corn & $15-24$ & 10 & 35 \\
Watermelon, Sweet Potato & $21-29$ & 18 & 35 \\
Strawberry & $12-24$ & 5 & 30 \\
\hline
\end{tabular}

Table 3. Greenhouse AC Load profile for the Cold Season (Winter)

\begin{tabular}{|l|l|l|l|l|}
\hline AC Load (Winter) & Watt & hr/Day & Day/Week & Watt $\boldsymbol{h r}$ \\
\hline Electric Heater (1) & 2240 & 10 & 7 & 156800 \\
\hline Air Fan 1.5 hp (1) & 1120 & 1 & 7 & 7840 \\
\hline Lighting 35W (10 items) & 350 & 10 & 7 & 24500 \\
\hline AC Weekly Load (Watt hour) & & 189140 \\
\hline Daily Energy Load (Watt hour) & 32424 \\
\hline
\end{tabular}

Table 4. Greenhouse AC Load profile for the Hot Summer Season

\begin{tabular}{|l|c|c|c|c|}
\hline AC Load (Summer) & Watt & hr/Day & Day/Week & Watt hr \\
\hline Air Fans 1.5 hp (2) & 2240 & 10 & 7 & 156800 \\
\hline Circulation Pump 0.75 hp (1) & 840 & 10 & 7 & 58800 \\
\hline Lighting 35W (10 items) & 350 & 10 & 7 & 24500 \\
\hline Battery cabinet ventilation fan (2) & 200 & 10 & 7 & 14000 \\
\hline System cabinet ventilation fan (2) & 200 & 10 & 7 & 14000 \\
\hline Office ventilation fan (2) & 200 & 10 & 7 & 14000 \\
\hline AC Weekly Load (Watt hour) & & & 282100 \\
\hline Daily Energy Load (Watt hour) & & 48360 \\
\hline
\end{tabular}

\section{GREenhouse LoAD ProfiLe}

Heating equipments in winter dominate the demand for electricity in greenhouses. One electric fan may be used one hour only during the day to accommodate the humidity of the greenhouse. The greenhouse heating system balances the heat loss under cold conditions with sufficient output. In cold days, it is essential to know the minimum temperature requirements for the crop, the lowest outdoor temperature that might be expected, and the surface area of the greenhouse. Heat loss also will be affected by wind and site 
exposure. Forced-air heaters can be installed in the ridge area of the greenhouse take up a minimum of space.

Table 3 shows the greenhouse load profile in winter. It is obvious that the load is only AC loads; the minimum daily energy load budget of the year is $32.424(\mathrm{kWh})$.

Cooling and pumping equipments in summer dominate the demand for electricity in greenhouses. The demand for electricity occurs during the times when solar radiation is available and in abundance; an excellent harmony between demand and supply which elucidate the feasibility of using solar energy. Therefore, a stand-alone PV power supply should be utilized and in demand.

Table 4 shows the greenhouse load profile in summer. From this table, it is obvious that the maximum daily energy load of the year is $48.36 \mathrm{kWh}$.

\section{Methodology}

Sizing stand Alone PV system [17-22] involves finding the cheapest combination of array size and storage capacity that will meet the anticipated load requirements with the minimum acceptable level of security. It can vary widely depending on the application and the choices of the user or procurement agency. The following information is required for system sizing:

- The daily or hourly load requirement during a typical year.

- Typical current and voltage characteristics of the selected type of module at various irradiances and temperatures.

- The mean daily irradiation on the plane of the array for every month of a typical year.

- The tilt factors for different tilt and azimuth angles.

- The maximum number of consecutive sunless days likely to be experienced.

- The mean daily ambient temperature for every month of a typical year.

- The estimated cell temperature rises above ambient of the modules in the array.

- The required security of supply.

- The selected DC bus voltage.

- The estimated percentage of energy losses in the battery, power conditioning equipment and control system.

- The estimated losses in the array due to module mismatch, cable, dust and shading and voltage drop across blocking diodes.

\section{A. Load Calculation}

The month that has the highest load demand and the lowest solar insolation should be examined first. Typically this "worst-case" month will be used for the system sizing. The first step in calculating the load is to figure out the DC and/or AC load power required by each item to be powered by the PV system. The load power (LP), measured in watts (W) is

$$
\mathrm{LP}(\mathrm{W})=\mathrm{LC}(\mathrm{A}) \times \mathrm{LV}(\mathrm{V})
$$

where:

$\mathrm{LC}=$ Load Current $(\mathrm{A})$

$\mathrm{LV}=$ Load Voltage $(\mathrm{V})$
The next calculation performed converts the load power from watts to amp-hours per day. This conversion takes into account the duty cycle of the load, any power conditioning efficiency losses (due to inverters or converters), and the desired PV system voltage (nominal system voltage). The first step in the conversion is to find the average number of hours a day period (AVG) that the load requires power.

$$
\operatorname{AVG}(\mathrm{HRS} / \mathrm{DAY})=[\mathrm{DDC} \times \mathrm{WDC} \div 7]
$$

where:

$\mathrm{DDC}=$ Daily Duty Cycle

$\mathrm{WDC}=$ Weekly Duty Cycle

The second step in the conversion of the load power in watts to amp-hours per day is to find the amperage required by the system A (A).

$$
\mathrm{A}(\mathrm{A})=[\mathrm{LP}(\mathrm{W}) \div \mathrm{NSV}(\mathrm{V})] \div \mathrm{PCEF}(\text { Decimal })
$$

In equation 3 , the power conversion efficiency factor (PCEF) accounts for losses that will occur within the PV system with the use of power conditioning devices such as inverters or converters. The nominal system voltage (NSV) equals the voltage that the system is being designed to operate at, typically 12 volts dc, 24 volts dc, or 120 volts ac.

Performing the calculations in equation 2 and 3 result in units of hours per day and amps, respectively. The conversion of load power in watts to amp-hours per day (AHD) is given by:

$$
\operatorname{AHD}(\mathrm{AH} / \mathrm{DAY})=\mathrm{AVG}(\mathrm{HRS} / \mathrm{DAY}) \times \mathrm{A}(\mathrm{A})
$$

Once the load in amp-hours per day has been calculated for each item to be powered by the PV system, the total amp-hour load $\left(\mathrm{AHD}_{\mathrm{T}}\right)$ of the system can be figured by simply summing the individual amp-hour per day loads. This value is then divided by the wire and battery efficiency factors (WEF and BEF) of the equipment selected for the system to calculate the corrected amp-hour load. The result of these calculations is the corrected amp-hour load $\left(\mathrm{AHD}_{\mathrm{C}}\right)$ as shown in equation 5 .

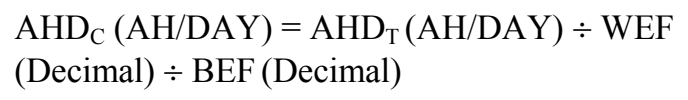

This calculation takes into account efficiency losses that will occur within the system. It is essential that these losses are factored into the load; otherwise, the system may be sized incorrectly, resulting in poor performance. The wire efficiency factor is equal to 1-wire loss. The battery efficiency factor is equal to the ampere-hours out divided by ampere-hours into the selected battery type of the PV system.

To calculate the peak current draw, this value is equal to the current required if all the loads are operating simultaneously. The peak current draw is used for sizing fuses, wires, etc. The first step in figuring the peak current draw is to compute the total load power in watts for both the $\mathrm{dc}$ and ac loads. Once these values are calculated, they are 
summed and divided by the nominal system voltage to arrive at the peak current draw.

$\operatorname{PCD}(\mathrm{A})=\left(\mathrm{LP}_{\mathrm{TDC}}(\mathrm{W})+\mathrm{LP}_{\mathrm{TAC}}(\mathrm{W})\right) \div \mathrm{NSV}(\mathrm{V})$

where

$\mathrm{PCD}=$ Peak Current Draw

$\mathrm{LP}_{\mathrm{TDC}}=$ Total DC Load Power

$\mathrm{LP}_{\mathrm{TAC}}=$ Total AC Load Power

\section{B. Design Current and Array Tilt Angle}

To calculate the design current, the current required meeting the system load, and the array tilt angle. The season or month in which the load is the greatest will generally dictate the array tilt angle. The corrected amp-hour load for each month is divided by the corresponding peak sun value for that month at the array tilt angles of latitude $-15^{\circ}$, latitude, and latitude $+15^{\circ}$. The peak sun value is equal to the average number of hours each day when solar insolation is equal to $1000 \mathrm{w} / \mathrm{m}^{2}$. The result of dividing the load by the peak sun is the design current, the current required to meet the system load.

$\mathrm{DC}(\mathrm{A})=\mathrm{AHD}_{\mathrm{C}}(\mathrm{AH} / \mathrm{DAY}) \div \mathrm{PS}(\mathrm{HRS} / \mathrm{DAY})$

where:

$\mathrm{DC}=$ Design Current

$\mathrm{PS}=$ Peak Sun

Once the design current is found for each of the three tilt angles for all 12 months, the largest design current and corresponding peak sun value is recorded for each tilt angle. These values represent the period when the amount of solar insolation is at its lowest for each tilt angle. In other words, these are the worst-case scenarios throughout a year. Of the three design currents recorded, the tilt angle associated with the smallest design current and corresponding peak sun value is selected. Following this procedure allows for the selection of the tilt angle that results in the smallest current required to meet the system load during the worst-case solar insolation scenario.

\section{Battery Sizing}

The first step in sizing the batteries for the system is to calculate required battery capacity of the PV system. This is given by:

$$
\begin{aligned}
& \mathrm{RBC}(\mathrm{AH})=\left[\mathrm{AHD}_{\mathrm{C}}(\mathrm{AH} / \mathrm{DAY}) \times \mathrm{SD}(\mathrm{DAYS})\right] \div \\
& {\left[\mathrm{DOD}_{\max }(\text { Decimal }) \div \mathrm{DT}(\text { Decimal })\right]}
\end{aligned}
$$

where:

$\mathrm{RBC}=$ Required Battery Capacity

$\mathrm{SD}=$ Storage Days

$\mathrm{DOD}_{\max }=$ Maximum Depth of Discharge of Battery Type

DT $=$ Derating for Temperature

Storage days are defined as the number of consecutive days the battery subsystem is required to meet the load with no energy production by the array. The number of storage days for a system is dependent on the desired system availability. Availability is the term used to express the percentage of time that a power system is capable of meeting load requirements. A higher availability demand will increase the number of storage days required, and therefore increase the required battery capacity.

In order to take into account the limitations of the battery type selected for the PV system, the maximum depth of discharge and the derating for temperature factor of the selected battery are considered. For reasons such as extending battery life, the depth of discharge may be downgraded from the manufacturer's claim. Downgrading will also result in a more conservative design. The derating for temperature factor is utilized to account for performance losses in cold operating temperatures.

Once the required battery capacity is found, the next step is to find the number of batteries in parallel needed for the PV system. Connecting batteries in parallel increases the current output of a battery subsystem; i.e. connecting two $12 \mathrm{~V}, 100 \mathrm{amp}$ rated batteries in parallel will produce 200 amps at $12 \mathrm{~V}$. To ensure that there will be enough batteries to meet the power demands, round to the next highest complete battery if the calculation results in a decimal.

$\mathrm{B}_{\mathrm{P}}=\mathrm{RBC}(\mathrm{AH}) \div \mathrm{BC}(\mathrm{AH})$

where:

$B_{P}=$ Number of Batteries in Parallel

$\mathrm{BC}=$ Capacity of Selected Battery of Selected Battery Type

Next, the number of batteries required in series is calculated. Connecting batteries in series increases the voltage output of a battery subsystem; i.e. connecting two $12 \mathrm{~V}, 100 \mathrm{amp}$ batteries in series will produce $100 \mathrm{amps}$ at $24 \mathrm{~V}$. The number of batteries required in series is given by:

$\mathrm{B}_{\mathrm{S}}=\mathrm{NSV}(\mathrm{V}) \div \mathrm{NBV}(\mathrm{V})$

where:

$\mathrm{B}_{\mathrm{S}}=$ Number of Batteries in Series

$\mathrm{NBV}=$ Nominal Battery Voltage

After figuring the number of batteries required in series and in parallel, the total number of batteries $\left(\mathrm{B}_{\mathrm{T}}\right)$ required by the PV system is calculated.

$\mathrm{B}_{\mathrm{T}}=\mathrm{B}_{\mathrm{S}} \times \mathrm{B}_{\mathrm{P}}$

The final value in sizing the battery is the usable battery capacity. It is the amount of ampere-hours that can safely be used from the installed batteries. To find the usable battery capacity, it is first required that the system battery capacity $\left(\mathrm{BC}_{\mathrm{S}}\right)$ be calculated. This is given by:

$\mathrm{BC}_{\mathrm{S}}(\mathrm{AH})=\mathrm{B}_{\mathrm{P}} \times \mathrm{BC}(\mathrm{AH})$

Once the system battery capacity is known, the usable battery capacity $\left(\mathrm{BC}_{\mathrm{U}}\right)$ is found by the equation:

$\mathrm{BC}_{\mathrm{U}}(\mathrm{AH})=\mathrm{BC}_{\mathrm{S}}(\mathrm{AH}) \times \mathrm{DOD}_{\max }($ Decimal $)$ 


\section{D.Array Sizing}

The first step in calculating the array size is to find the derated design current $\left(\mathrm{DC}_{\mathrm{D}}\right)$, the minimum array current required to supply the average daily load.

$\mathrm{DC}_{\mathrm{D}}(\mathrm{A})=\mathrm{DC}(\mathrm{A}) \div \mathrm{MDF}($ Decimal $)$

where:

$\mathrm{MDF}=$ Module Derate Factor

The module derate factor is used to adjust module current from the manufacturer's testing data, typically conducted at $1000 \mathrm{w} / \mathrm{m}^{2}$ and $25^{\circ} \mathrm{C}$, to field conditions.

After finding the derated design current, the number of parallel-connected modules $\left(\mathrm{M}_{\mathrm{P}}\right)$ required to provide the array current is calculated. To ensure that there will be enough modules to meet the power demands, round to the next highest complete module if the calculation results in a decimal

$\mathrm{M}_{\mathrm{P}}=\mathrm{DC}_{\mathrm{D}}(\mathrm{A}) \div \mathrm{M}_{\mathrm{C}}(\mathrm{A})$

where:

$\mathrm{M}_{\mathrm{C}}=$ Rated Module Current

The next step is to calculate the number of modules required to be connected in series. To find the number of modules to be connected in series, the voltage required to charge the batteries of the system must first be found because the PV modules must be capable of producing enough voltage to charge the batteries of the system. The voltage required to charge the batteries (VRCB) of the system is given by:

$\operatorname{VRCB}(\mathrm{V})=1.2 \times \mathrm{NSV}(\mathrm{V}) \times \mathrm{B}_{\mathrm{S}}$

The factor of 1.2 is used to build conservatism into the system. It ensures that there will be enough modules in series to produce the required system voltage.

The number of series connected modules $\left(\mathrm{M}_{\mathrm{S}}\right)$ required to produce the system voltage is found by equation 17 . Similarly to the procedure followed after finding the number of modules in parallel, the number of modules in series is rounded to the next highest complete module.

$\mathrm{M}_{\mathrm{S}}=\mathrm{VRCB}(\mathrm{V}) \times \mathrm{M}_{\mathrm{VHT}}(\mathrm{V})$

where:

$\mathrm{M}_{\mathrm{VHT}}=$ Module Voltage at Highest Temperature

Next, the total number of modules required $\left(\mathrm{M}_{\mathrm{T}}\right)$ by the PV system is calculated. This is given by:

$\mathrm{M}_{\mathrm{T}}=\mathrm{M}_{\mathrm{S}} \times \mathrm{M}_{\mathrm{P}}$

Once the total number of modules required for the PV system is found, it is necessary to perform several simple calculations in order to find the rated array current, array short circuit current, array rated voltage, and array open circuit voltage. These specifications are used to size BOS components. The rated array current $\left(\mathrm{A}_{\mathrm{C}}\right)$ is given by:
$A_{C}(A)=M_{P} \times M_{C}(A)$

The array short circuit current $\left(\mathrm{A}_{\mathrm{SCC}}\right)$ is given by:

$\mathrm{A}_{\mathrm{SCC}}(\mathrm{A})=\mathrm{M}_{\mathrm{P}} \times \mathrm{M}_{\mathrm{SCC}}(\mathrm{A})$

where:

$\mathrm{M}_{\mathrm{SCC}}=$ module short circuit current

The array rated voltage $\left(A_{V}\right)$ is given by:

$\mathrm{A}_{\mathrm{V}}(\mathrm{V})=\mathrm{M}_{\mathrm{S}} \times \mathrm{M}_{\mathrm{V}}(\mathrm{V})$

where:

$\mathrm{M}_{\mathrm{V}}=$ rated module voltage

The array open circuit voltage $\left(\mathrm{A}_{\mathrm{OCV}}\right)$ is given by:

$\mathrm{A}_{\mathrm{OCV}}(\mathrm{V})=\mathrm{M}_{\mathrm{S}} \times \mathrm{M}_{\mathrm{OCV}}(\mathrm{V})$

where

$\mathrm{M}_{\mathrm{OCV}}=$ module open circuit voltage.

\section{E. Charge Controllers and Inverter Sizing}

The sizing for the charge controller is simple and straightforward. The calculations can also be performed several times to find the best controller for the system. The first step in sizing the charge controller is to calculate the minimum current $\left(\mathrm{C}_{\min }\right)$ that the charge controller must be able to handle.

$\mathrm{C}_{\min }(\mathrm{A})=\mathrm{A}_{\mathrm{SCC}}(\mathrm{A}) \times 1.25$

The array short circuit current is multiplied by a factor of 1.25 to build conservatism into the system. It oversize the controller by $25 \%$ so that the controller will be capable of handling current production at times of abnormally high solar irradiance conditions.

Next, the minimum charge controller current value is divided by the rated controller current $\left(\mathrm{C}_{\mathrm{C}}\right)$ to find the amount of controllers to be connected in parallel $\left(\mathrm{C}_{\mathrm{P}}\right)$.

$\mathrm{C}_{\mathrm{P}}=\mathrm{C}_{\min }(\mathrm{A}) \div \mathrm{C}_{\mathrm{C}}(\mathrm{A})$

In case the calculation results in decimal values, we round up to be on safe side. In addition to the sizing of the controller, there are many characteristics to consider when selecting a charge controller for a PV system. Some controllers have features such as temperature compensation and reverse current protection. Other possible options include adjustable set points (high voltage disconnect, high voltage re-connect, low voltage disconnect, low voltage reconnect) and meters (battery voltage, array current, and load current). Depending on the needs of the PV system, some of these features may be necessary or helpful. In sizing the inverter, it should have sufficiently large capacity compared to the load capacity. 


\section{RESUlTS AND Discussion}

For study case, the array tilt angle for the $21^{\text {st }}$ day of every month is calculated. The tilt angle for optimum performance of PV system is dependent on the geographical location i.e. latitude, longitude and season to be determined experimentally. In Aqaba, tilt angle is $6^{\circ}$ for June (summer) and $52^{\circ}$ for December (winter) as shown in table 5 .

As a general rule, an array set at an angle equal to the latitude of the site will receive the maximum annual solar radiation. If there is a high wintertime load demand, an array tilt angle of latitude plus $15^{\circ}$ is usually best in the northern hemisphere. For a large summer load (like our case) in the northern hemisphere, an array tilt angle of latitude minus $15^{\circ}$ is recommended. Table 6 describes well global solar radiation at different tilted angles.

The theoretical calculations for the design of modules and batteries have been done. In this design, 102 batteries are recommended when battery autonomy is taken to be 3 days. The PV array of 115 modules can generate power of 13.7 $\mathrm{kW}$ to operate the greenhouse load with $4.03 \mathrm{~kW}$ daily as shown in tables $7(a-b)$.

The bill of quantities - modules and batteries prices as reported in references [23 and 24] - and the cost of PV rate $\$$ are listed in table 8 . Assuming 30 years as design life of the PV system; the PV rate is $\$ 0.43(\mathrm{kWh})$ while a current utility rate is $\$ 0.07(\mathrm{kWh})$. If we add the cost of inverters, solar charge controller and balance of system components (wiring systems, mounting hardware, installation, etc...), the PV rate may exceed the double of this initial estimate, depending on the time of purchase in a ever changing economy.

\section{CONCLUSION}

A chronological methodology that allows the design estimation of the PV system components is accomplished according to environmental condition, greenhouse size, electrical load of the greenhouse and the subsystem characteristics in an exemplary location of Aqaba in Jordan. A number of $115 \mathrm{PV}$ modules and 102 storage batteries are recommended to meet maximum daily demand load of the greenhouse and these 102 specified batteries can supply power to the load for three days when there is no solar radiation. Utilization of solar photovoltaic systems to power agriculture applications such as greenhouses is still costly as compared to the utility prices due to the high initial cost of the PV components and the high load of greenhouses. However, this case study could be an attractive model for the strategic locations of agriculture with self-sufficient power supplies for the years of alternative energy usage to come.

Table 5. The altitude angle and the array angle at the $21^{\text {st }}$ day of every month

\begin{tabular}{cccc}
\hline Month & (n) & Altitude Angle & Tilt Array \\
\hline Jan & 17 & 40.13 & 49.87 \\
Feb & 47 & 48.09 & 41.91 \\
Mar & 75 & 58.63 & 31.37 \\
Apr & 105 & 70.46 & 19.54 \\
May & 135 & 79.84 & 10.16 \\
Jun & 162 & 84.13 & 5.87 \\
Jul & 198 & 82.23 & 7.77 \\
Aug & 228 & 74.50 & 15.5 \\
Sep & 258 & 63.26 & 26.74 \\
Oct & 288 & 51.45 & 38.55 \\
Nov & 318 & 42.13 & 47.87 \\
Dec & 344 & 38.00 & 52 \\
\hline
\end{tabular}

Table 6. Aqaba global solar radiation $\left(\mathrm{MJ} / \mathrm{m}^{2}\right)$ at different tilt angles

\begin{tabular}{|c|c|c|c|c|c|c|c|}
\hline Month & $\begin{array}{l}\boldsymbol{H}_{\text {Tilt }} \\
\left(0^{\circ}\right)\end{array}$ & $\begin{array}{c}\boldsymbol{H}_{\text {Tilt }} \\
\left(15^{\circ}\right)\end{array}$ & $\begin{array}{c}\boldsymbol{H}_{\text {Tilt }} \\
\left(25^{\circ}\right)\end{array}$ & $\begin{array}{c}\boldsymbol{H}_{\text {Tilt }} \\
\left(29^{\circ}\right)\end{array}$ & $\begin{array}{c}\boldsymbol{H}_{\text {Tilt }} \\
\left(35^{\circ}\right)\end{array}$ & $\begin{array}{c}\boldsymbol{H}_{\text {Tilt }} \\
\left(\mathbf{4 5 ^ { \circ }}\right)\end{array}$ & $\begin{array}{c}\boldsymbol{H}_{\text {Tilt }} \\
\left(60^{\circ}\right)\end{array}$ \\
\hline Jan & 12.97 & 16.22 & 17.98 & 18.57 & 19.31 & 20.17 & 20.51 \\
\hline Feb & 16.20 & 19.02 & 20.42 & 20.84 & 21.32 & 21.72 & 21.32 \\
\hline Mar & 21.00 & 22.88 & 23.57 & 23.69 & 23.72 & 23.32 & 21.74 \\
\hline Apr & 24.58 & 25.01 & 24.71 & 24.44 & 23.86 & 22.49 & 19.54 \\
\hline May & 26.77 & 26.00 & 24.94 & 24.36 & 23.31 & 21.19 & 17.22 \\
\hline Jun & 29.66 & 28.23 & 26.63 & 25.81 & 24.37 & 21.57 & 16.50 \\
\hline Jul & 28.24 & 27.12 & 25.78 & 25.08 & 23.82 & 21.35 & 16.81 \\
\hline Aug & 26.45 & 26.42 & 25.74 & 25.30 & 24.46 & 22.64 & 18.99 \\
\hline Sep & 23.01 & 24.45 & 24.77 & 24.73 & 24.51 & 23.69 & 21.45 \\
\hline Oct & 18.42 & 21.10 & 22.35 & 22.70 & 23.07 & 23.24 & 22.46 \\
\hline Nov & 14.46 & 17.95 & 19.79 & 20.39 & 21.14 & 21.96 & 22.14 \\
\hline Dec & 12.43 & 15.79 & 17.71 & 18.36 & 19.20 & 20.20 & 20.74 \\
\hline Annual $\left(M J / \mathrm{m}^{2}\right)$ & 21.18 & 22.52 & 22.87 & 22.86 & 22.67 & 21.96 & 19.95 \\
\hline Annual $\left(\mathrm{kWh} / \mathrm{m}^{2}\right)$ & 5.88 & 6.25 & 6.35 & 6.35 & 6.30 & 6.10 & 5.54 \\
\hline
\end{tabular}


Table 7-a. Design values of batteries depending on the environment of the location and battery characteristics

\begin{tabular}{ll}
\hline Battery Size & \\
\hline Total daily amp hours & 4030 \\
\hline Days of autonomy & 3 \\
Normal storage capacity in amp hours & 12090 \\
Maximum drawdown, typically $50 \%, 80 \%$ & 0.8 \\
Required battery capacity in amp hours & 15113 \\
Derating factor for cold weather: lowest temperature $\left(8.39{ }^{\circ} \mathrm{C}\right)$ & 1.2 \\
Total rated battery capacity in amp hours & 18125 \\
Single battery capacity in amp hours & 360 \\
Number of batteries in parallel & 51 \\
System volts & 12 \\
Rated battery voltage & 6 \\
Number of batteries in series & 2 \\
Number of batteries in parallel & 51 \\
Total number of batteries & 102 \\
\hline
\end{tabular}

Table 7-b. Design values of modules depending on the environment of the location and modules specification

\begin{tabular}{cc}
\hline $\boldsymbol{P V}$ Array Size & \\
\hline Total daily amp hours & 4030 \\
\hline Module high-temperature inefficiency, & 1.25 \\
battery charger and wiring losses factor & \\
Corrected total daily amp hours & 5037.5 \\
Bright sunshine hours & 6.19 \\
Array current in amps & 813.81 \\
Module current in amps & 7.1 \\
Number of modules in parallel & 115 \\
Nominal system volts & 12 \\
Module voltage & 16.9 \\
Number of modules in series & 1 \\
Number of modules in parallel & 115 \\
Total number of PV modules & 115 \\
\hline
\end{tabular}

\begin{tabular}{ll}
\hline Solar charge controller sizing & \\
\hline Peak amps produced by solar module & 7.1 \\
\hline $\begin{array}{l}\text { Safety factor to handling current production at times of abnormally } \\
\text { high solar irradiance conditions }\end{array}$ & 1.25 \\
Total number of PV modules & 115 \\
Minimum rated charge control capacity (Amp) & 1020.63 \\
\hline & \\
\hline Inverter sizing & 4030 \\
\hline Total watts of power needed for the maximum load & 1.2 \\
\hline Inverter loss factor & 4836 \\
\hline Total watts of power match with inverter specification & \\
\hline
\end{tabular}


Table 8. Summarized initial cost of the PV system assuming 30 years of design life (an example)

\begin{tabular}{cccc}
\hline Type & No of Items & Unit Price & Item Total \\
\hline PV modules: Astropower AP-120 & 115 & $\$ 590.40$ & $\$ 67,896.00$ \\
120 Watts, 16.9 Vmp, 7.1 Imp, 21 Voc, 7.7 & & & \\
Batteries: L16P & 102 & $\$ 196.00$ & $\$ 19,992.00$ \\
6V, 360AH (20HR) Flooded Lead Acid & & & \\
Inverter: SW4024 & 1 & $\$ 1,285.00$ & $\$ 1,285.00$ \\
Charge Controller: CC-60 & 1 & $\$ 300.00$ & $\$ 300.00$ \\
Total Initial Cost Estimate & & & $\$ 89,473.00$ \\
Maintenance & & & $\$ 26,841.90$ \\
KW System & & & 4.03 \\
Sunshine Duration/Day & & & 6.19 \\
kWh's/Day & & & 24.9457 \\
Annual Output (kWh's) & & 9105.1805 \\
Design Life (Years) & & 30 \\
Total Expenditure & & $\$ 116,314.90$ \\
PV Rate (kWh) & & $\$ 0.43$ \\
Current Utility Rates (kWh) & & $\$ 0.07$ \\
\hline
\end{tabular}

\section{REFERENCES}

[1] Jordan Meteorological Department. Jordan Climatological Data handbook (1988). Amman-Jordan

[2] The Potential of Solar Energy Application in Jordan, Vol.3, Assessment and Analysis of Available Energy Resources, Royal Scientific Society, (1983), Amman-Jordan.

[3] Hanan J J; Holley W L; Goldberry K L (1978). Greenhouse management. Advances Series in Agricultural Sciences, Vol. 5. Springer-Verlag, Berlin

[4] Jolliet O; Bailey B J (1994). The effect of climate on tomato transpiration in greenhouses: measurements and models comparison. Agricultural and Forest Meteorology, 58, 43-62

[5] Yang X; Short T H; Fox R D; Bauerle W L (1990). Transpiration, leaf temperature and stomatal resistance of a greenhouse cucumber crop. Agriculture and Forest Meteorology, 51, 197-209

[6] http://agspsrv34.agric.wa.gov.au/agency/education/pdf/climate_sci.pd $\mathrm{f}$

[7] Montero J I; Short T; Curry R B; Bauerle WL (1981). Influence of evaporative cooling systems on greenhouse environments. ASAE Paper no 81-4027

[8] Kittas C; Bartzanas T; Jaffrin A (2001). Greenhouse evaporative cooling: measurements and data analysis. Transactions of the ASAE, 44 (3), 683-689

[9] Giacomelli G A; Ginigers M S; Krass A E; Mears D R (1985) Improved methods of greenhouse evaporative cooling. Acta Horticulturae, 174, 49-55

[10] Landsberg J I; White B; Thorpe M R (1979). Computer analysis of the efficiency of evaporative cooling for glasshouses in a high-energy environments. Journal of Agricultural Engineering Research, 24, 29 39

[11] Montero J I; Anton A; Biel C; Franquet A (1990). Cooling of greenhouse with compressed air fogging nozzles. Acta Horticulturae, 281, 199-209

[12] Nijskens J; Deltour J; Coutisse S; Nisen A (1984). Heat transfer through covering materials of greenhouses. Agricultural and Forest Meteorology, 33 (2), 193-214

[13] Willits D H (2000). Constraints and limitation in greenhouse cooling. Challenges for the next decade. Acta Horticulturae, 534, 57-66

[14] Willits D H; Peet M M (1993). The effect of evaporative cooling on the efficiency of external greenhouse shade cloths. ASAE Paper no. $93-4042$

[15] http://www1.agric.gov.ab.ca/\$department/deptdocs.nsf/all/opp2892?o pendocument $\# 4$

[16] http://www.cahe.nmsu.edu/pubs/_circulars/circ556.html

[17] SANDIA: "Stand-alone photovoltaic systems. A handbook of recommended design practices", Sandia National Laboratories, Report No. SAND87-7023, Updated March 1995.

[18] http://www.isat.jmu.edu/common/projects/UNPEPP/PVChapters.doc.

[19] L. Barra et al., An analytical method to determine the optimal size of a PV plant. Solar Energy 33 (1984), pp. 509-514.
[20] M. Egido and E. Lorenzo, The sizing of stand alone PV systems: a review and a proposed model. Solar Energy Materials and Solar Cells 26 (1992), pp. 51-69.

[21] Sidrach, A general multivariate model for sizing stand alone PV systems. Solar Energy Materials \& Solar Cells 59 (1999), pp. 185197.

[22] E. Lorenzo and L. Narvarte, On the usefulness of stand alone sizing methods. Progress in Photovoltaics 8 (2000), pp. 391-409.

[23] http://www.windpowerunlimited.com/solar_modules/module_pricing. htm

[24] http://www.absak.com/catalog/product_info.php/cPath/23_112/produ cts $\mathrm{id} / 177$ 


\title{
Commercial Production Trial of Empty Fruit Bunch based Briquettes
}

\author{
A.B. Nasrin, A.N. Ma, Y.M. Choo, S. Mohamad, Z. Zainal and A.M. Fadzil
}

\begin{abstract}
Palm oil industry generates vast amount of palm biomass. Converting palm biomass into a uniform and solid fuel through briquetting process appears to be an attractive solution in upgrading its properties and add value. In this study, raw materials including empty fruit bunch (EFB), in powder and fibre forms, and sawdust were densified into briquettes at high temperature and pressure using screw extrusion technology. The briquettes were analysed to determine its physical and chemical properties, mechanical strength and burning characteristics. It was found that briquettes made either from $100 \%$ pulverized EFB or mixed with sawdust exhibited good burning properties. EFB fibre, due to their physical properties, were recommended to be blended with sawdust in producing quality briquettes. In overall, converting palm biomass into briquettes has increased its energy content and reduced moisture content about minimum of $5 \%$ and $38 \%$ respectively compared to its raw materials. The properties of palm biomass briquettes obtained from the study were compared to the commercial sawdust briquettes properties and to the minimum requirements of DIN 51731. The details of the study were highlighted in this paper. Palm biomass briquettes can become an important renewable energy fuel source in the future.
\end{abstract}

Keywords: briquettes, biomass fuel, screw extrusion technology

\section{INTRODUCTION}

Malaysian palm oil industry has grown tremendously over the last 4 decades and since then, it has maintained its position as the leading world's country in the production of palm oil. Nevertheless, the industry has also generated vast quantities of palm biomass, mainly from milling and crushing palm kernel. The types and amounts of these biomass generated in year 2006 are tabulated in Table 1.

Generally, most of these biomass can be used as combustion fuels. Currently, the shell and fibre are the main sources of energy in palm oil mills. These fuels are burnt in boiler to produce steam for electricity generation to be used in the milling process. However, EFB and PKC, due to its

This work was supported by Malaysian Palm Oil Board.

A.B. Nasrin, A.N. Ma, Y.M. Choo, S. Mohamad are with the Engineering \& Processing Research Division,MPOB, No. 6, Persiaran Institusi,B.B.Bangi, 43000 Kajang. (corresponding author: 0387694479; fax: 0389262971; e-mail: nasrin@mpob.gov.my).

Z. Zainal is with ${ }^{2}$ School of Mechanical Engineering, Universiti Sains Malaysia, 14300 Nibong Tebal, Pulau Pinang (e-mail: mezainal@yahoo.com).

A.M. Fadzil is with the Faculty of Chemical Engineering, Universiti Teknologi MARA, 40450 Shah Alam, Selangor. (e-mail: mfadzi1740@salam.uitm.edu.my). physical properties and demands, are not normally utilized as fuel. In order to make use of the resource and expand its usage as fuel in various industries and applications, raw oil palm biomass should be treated and upgraded into uniform and useful fuels. Upgrading the properties of these abundantly available palm biomass can be done in many ways and one of the proven methods is via briquetting process.

Densification or briquetting is the process of compacting the biomass residue into a uniform solid fuel called briquettes. It has higher density and energy content, and less moist compared to its raw materials. Briquetting of biomass can be done using various techniques, either with or without binder addition. Biomass briquettes are mostly used for cooking, heating, barbequeing and camping in the countries such as USA, EU, Australia, Japan, Korea and Taiwan. In the developing countries, biomass briquettes are mainly for household usage only. For larger commercial scale, it can be used as fuel in producing steam, district heating and electricity generation. In Malaysia, the briquette industry was started with wood wastes, mainly in the form of sawdust ${ }^{[3]}$. Most of the local sawdust briquettes or charcoal briquettes are exported for oversea markets ${ }^{[3]}$. The products are rarely used in the local market as it could not compete with the availability of cheap fuels such as wood, charcoal and kerosene which are widely used in the rural areas and restaurants ${ }^{[3]}$. This industry has grown impressively for the past few years and indicated that there is good return for investment ${ }^{[3]}$.

Briquetting of palm biomass is new to the millers. The low supply of sawdust and wood residues for briquettes and charcoal briquettes has put palm biomass in the position to be used as substitute for sawdust. Palm biomass can be used $100 \%$ or can be blended with sawdust for the production of briquettes. Therefore, this is an opportunity for the industry to make use of all these wastes for value added products.

Table 1. Palm biomass generated in year 2007

\begin{tabular}{lcccc}
\hline Biomass & $\begin{array}{c}\text { Quantity, } \\
\text { million } \\
\text { tonnes, } *\end{array}$ & $\begin{array}{c}\text { Moisture } \\
\text { Content, } \\
\%^{* *}\end{array}$ & $\begin{array}{c}\text { Calorific } \\
\text { Value, } \\
\mathrm{kJ} / \mathrm{kg}^{* *}\end{array}$ & $\begin{array}{c}\text { Main } \\
\text { uses }\end{array}$ \\
\hline Fibre & 11.15 & 37.00 & 19068 & Fuel \\
\hline Shell & 5.18 & 12.00 & 20108 & Fuel \\
\hline $\begin{array}{l}\text { Empty } \\
\text { Fruit } \\
\text { Bunches }\end{array}$ & 18.32 & 67.00 & 18838 & Mulch \\
\hline Source: ${ }^{*}[1], * *[2]$ & & & \\
\end{tabular}




\section{Methodology}

Empty fruit bunch (EFB) and sawdust were used as raw materials. Raw EFB was converted into pulverized and fibre forms. PKC was collected and used as a raw material without any pre-treatment process. The properties of the raw materials used were determined and presented in Table 3.

Palm biomass briquettes were prepared by using commercial screw extrusion technology available in the sawdust briquettes plant. Through this technology, the biomass was extruded continuously by a screw through a taper die which is heated externally ${ }^{[5]}$. No binder is required as the lignin of biomass that fluidized during the process helps in binding ${ }^{[5]}$. The pressure and temperature applied for the process were about $7 \mathrm{MPa}$ and $150-250^{\circ} \mathrm{C}$ respectively. The typical screw extrusion briquetting machine is illustrated in Figure 1.

The production trials of palm biomass briquettes were carried out either by using $100 \%$ palm biomass or blending with sawdust. At the beginning, 100\% palm biomass were used as raw material, however if the briquettes could not be formed, sawdust will be gradually added to the palm biomass at the minimum percentage till quality briquettes were produced. The briquettes produced then are analyzed to determine their physical, chemical, mechanical and combustion properties. The results are compared to the commercial sawdust briquettes and to the minimum requirements of DIN $51731^{[6]}$. The descriptions of the study and method or standard used are shown as follow in Table 2. The production process trials of palm biomass briquettes can be summarized in Figure 2.

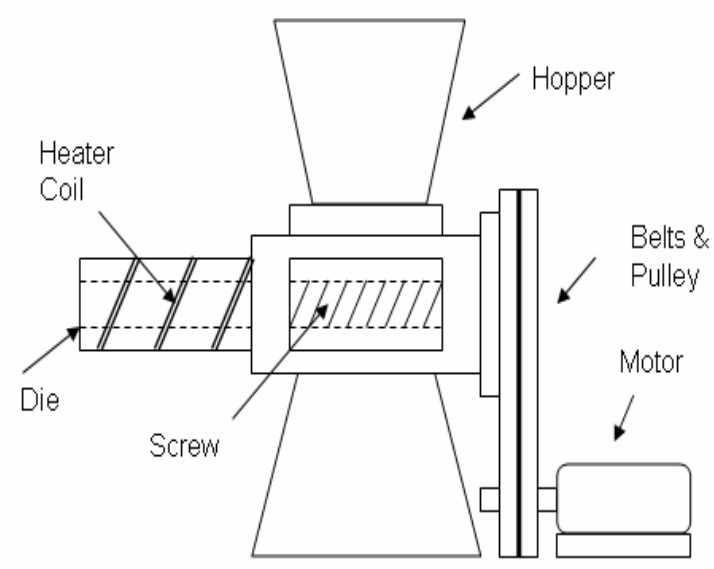

Figure 1. The typical commercial screw extrusion technology (source: modified from www.ait.ac.th ${ }^{[4]}$ )

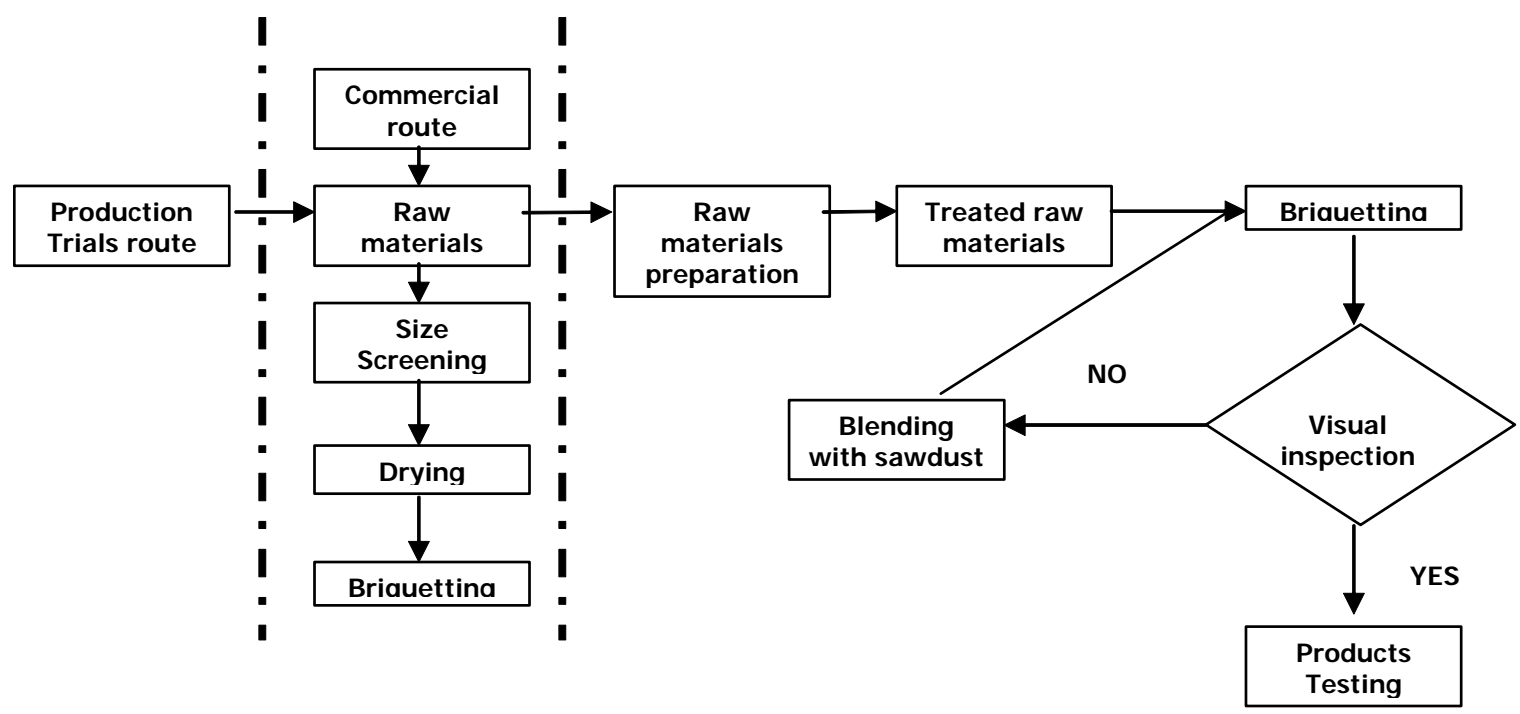

Figure 2. The palm biomass briquetting production trials approach

Table 2. Methodology and standard for properties study of raw materials and products

Experimental Standard Method / Methodology

\begin{tabular}{ll}
\hline Moisture Content & BS 4289 part 3: 1978 \\
Calorific Value & ASTM 2015 \\
Ash Content & 1SO 1171-97 \\
Mechanical Strength & Compressive and Elasticity Strengths \\
Combustion Analysis & Burning profile \& combustion rate \\
\hline
\end{tabular}


Table 3. Properties of raw materials used for the production trials

\begin{tabular}{|c|c|c|c|c|}
\hline $\begin{array}{c}\text { Raw } \\
\text { Material }\end{array}$ & $\begin{array}{c}\text { Average } \\
\text { Size of } \\
\text { materials }\end{array}$ & $\begin{array}{c}\text { Calorific } \\
\text { Value, } \\
\mathrm{kJ} / \mathrm{kg}\end{array}$ & $\begin{array}{c}\text { Moisture } \\
\text { Content, } \\
\% \\
\end{array}$ & $\begin{array}{c}\text { Ash } \\
\text { Content, } \\
\% \\
\end{array}$ \\
\hline $\begin{array}{l}\text { Pulverized } \\
\text { EFB,( } \\
\text { PEFB) }\end{array}$ & $\begin{array}{c}<212 \\
\mu \mathrm{m}\end{array}$ & 17000 & 12.0 & 2.41 \\
\hline $\begin{array}{l}\text { EFB Fibre, } \\
\text { (FEFB) }\end{array}$ & $3 \mathrm{~cm}$ & 16641 & 16.0 & 4.70 \\
\hline $\begin{array}{l}\text { EFB Fibre, } \\
\text { (FEFB) }\end{array}$ & $2.5 \mathrm{~mm}$ & 16641 & 14.0 & 4.60 \\
\hline $\begin{array}{l}\text { Sawdust, } \\
\text { (SD) }\end{array}$ & $\begin{array}{c}<500 \\
\mu \mathrm{m}\end{array}$ & 18506 & 10.0 & 2.31 \\
\hline
\end{tabular}

Table 4. The properties and its blending ratios of palm biomass briquettes

\begin{tabular}{lccc}
\hline \multicolumn{1}{c}{$\begin{array}{c}\text { Products } \\
\text { (Blending } \\
\text { ratio) }\end{array}$} & $\begin{array}{c}\text { Calorific } \\
\text { Value, kJ/kg }\end{array}$ & $\begin{array}{c}\text { Moisture } \\
\text { content, } \%\end{array}$ & $\begin{array}{c}\text { Ash } \\
\text { content, } \\
\%\end{array}$ \\
\hline $\begin{array}{l}100 \% \\
\text { Pulverized }\end{array}$ & 17823 & 7.39 & 2.85 \\
$\begin{array}{l}\text { EFB } \\
\text { Pulverized }\end{array}$ & 18273 & 7.22 & 2.22 \\
$\begin{array}{l}\text { EFB } \\
\text { Sawdust } \\
\text { (50:50) }\end{array}$ & & & \\
$\begin{array}{l}\text { Fibre EFB }+ \\
\text { sawdust } \\
\text { (40:60) }\end{array}$ & 18775 & 7.32 & 2.99 \\
$\begin{array}{l}\text { Sawdust } \\
\text { (control }\end{array}$ & 18936 & 6.81 & 1.63 \\
product) & & & \\
DIN 51731 & 17500 & $<10.0$ & 0.7 \\
(minimum) & & & \\
\hline
\end{tabular}

\section{RESUlTS AND DisCUSSION}

\section{A. Production Trial and Product Properties}

The production trials were carried out using EFB in pulverized and powder forms, and sawdust. The aims of the production are to produce binderless palm briquettes without jeopardizing the overall quality of briquettes and production performance. Figure 3 shows the photo of palm biomass briquettes.

The general physical properties of produced palm briquettes can be summarized as follows:

- A cylindrical shape with $55 \mathrm{~mm}$ of heptagon or hexagon diameter, $500 \mathrm{~mm}$ in length, bulk density of $1300 \mathrm{~kg} / \mathrm{m}^{3}$ and average weight of $1.1 \mathrm{~kg}$.

- The briquettes outer surface is partially carbonized for easy ignition, combustion and as a protection from ambient moisture.

- A concentric hole in the briquette is designed for a better circulation of combustion air.

From the production trials conducted using screw extrusion technology, it was found that $100 \%$ palm biomass briquettes can be made from pulverized EFB. Other palm biomass raw materials needed to be blended with sawdust in order to form the binderless palm based biomass briquettes. This is due to their fibrous physical properties such as low density and less surface area that hinder the smooth flow ability of biomass and in producing natural lignin as binder. They were easily broken into pieces during briquetting or produce a low physical quality briquettes as shown in Figure $3 \mathrm{a}$, if sawdust was not added. It is recommended that the feeding part of the machine be modified or binder added if briquettes are made $100 \%$ from these palm based raw materials.

The properties of palm biomass briquettes and its blending ratios are tabulated in the Table 4.

Results obtained were comparable to the local sawdust briquettes and meet the minimum requirements of DIN 51731. The briquetting process of palm biomass increased its energy content and reduced moisture contents to about $5 \%$ and $38 \%$ respectively compared to its raw materials The study also showed that in order to have good physical appearance of briquettes, the minimum blending ratios of sawdust must be at $50 \%$ of total weight.

The quality of produced palm briquettes is shown in Figure $3 b \& 3 c$.

\section{B. Proximate \& Ultimate Analysis}

Results for proximate and ultimate analysis for palm based biomass briquettes are tabulated in Table 5. Data for its raw materials were also included for comparison. Results were for briquettes made from treated EFB and sawdust. Volatile matter and fixed carbon are parameters that contribute to good combustion property. Briquetting has improved these parameters by lowering the volatile value and increasing the fixed carbon for higher calorific values. There was no significant increment on the chemical elements on the products as the process is more on the mechanical treatment for physical improvement of palm biomass.

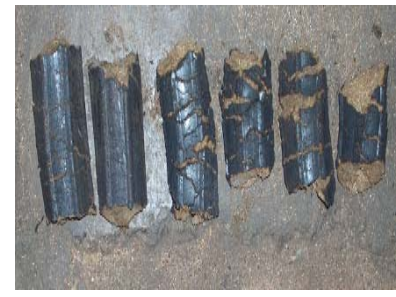

(a)

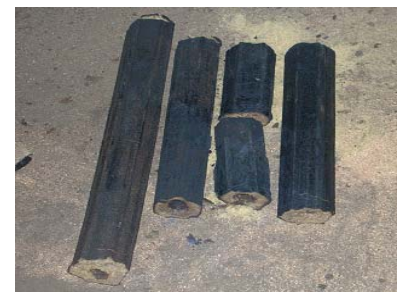

(b)
Figure 3. (a) Unacceptable palm briquettes quality during production trials; (b) \& (c) Quality palm briquettes for commercial purposes

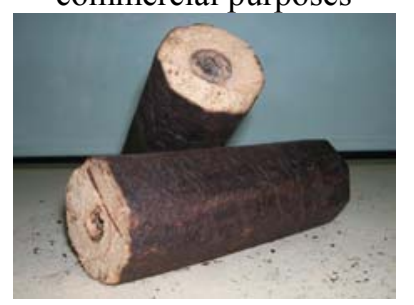

(c)

Figure 3. Continued 
Table 5. Proximate and ultimate analysis for palm based briquettes

\begin{tabular}{|l|c|c|c|c|}
\hline \multicolumn{1}{|c|}{ Parameters, \% } & $\begin{array}{c}\text { Treated EFB } \\
\text { (Raw Material) }\end{array}$ & Sawdust Briquettes & EFB briquettes & EFB +Sawdust briquettes \\
\hline Volatile Matter & 87.04 & 20.1 & 70.5 & 70.9 \\
\hline Fixed Carbon & 18 & 19.8 & 19.1 & 19.2 \\
\hline Carbon & 45.9 & 48.6 & 4.6 & 46.1 \\
\hline Hydrogen & 5.7 & 6.1 & 6.15 & 5.77 \\
\hline Nitrogen & 0.8 & 0.3 & 0.6 & 0.5 \\
\hline
\end{tabular}

\section{Mechanical Strength Analysis}

Vertical and horizontal forces were applied to the briquettes. Vertical force or compressive strength analysis of briquettes were determined using a hydraulic press machine. Horizontal force was analyzed using Zwick $100-$ Tensile Machine. The average compressive and horizontal strength for palm biomass briquettes were $7.2 \mathrm{MPa}$ and 0.37 MPa respectively. It was found that the addition of sawdust improved palm briquettes strength and its outer physical surface condition. The mechanical strength analysis of palm briquettes is shown in Table 6 .

Table 6. Mechanical strength of palm biomass

\begin{tabular}{cccc}
\hline $\begin{array}{c}\text { Strength } \\
\text { Analysis, } \\
\text { MPa }\end{array}$ & $\begin{array}{c}\mathbf{1 0 0 \%} \\
\text { EFB } \\
\text { Briquettes }\end{array}$ & $\begin{array}{c}\text { Pulverized } \\
\text { EFB }+ \\
\text { Sawdust } \\
\text { Briquettes }\end{array}$ & $\begin{array}{c}\text { EFB Fibre + } \\
\text { Sawdust } \\
\text { Briquette }\end{array}$ \\
\hline Compressive & 7.5 & 7.5 & 6.5 \\
Horizontal & 0.34 & 0.52 & 0.24 \\
\hline
\end{tabular}

\section{Combustion Analysis}

The combustion process of palm briquettes was studied using a typical open air stove as an attempt to simulate the actual combustion situation for domestic heating or cooking. From the analysis, it was found that palm briquettes were not easy to ignite. Therefore, firestarter medium such as kerosene was used to ease this process. The burning profile of palm briquettes is illustrated in Figure 4. The average burning rate was recorded at $0.43 \mathrm{~g} / \mathrm{min}$. The analysis also showed that at the normal surrounding air, palm biomass briquettes are capable to generate about $0.13 \mathrm{~kW}$ of thermal output. Although amber and less level of smoke conditions dominated most of the combustion process, however with adequate supply of air, there was a higher tendency of the fuel to re-flaming.

\section{E. Commercial Benefits}

Due to the depletion of sawdust supply and the global interest for renewable based fuels, palm biomass is a potential raw material to be used in briquette production. It also can become a significant alternative for local energy production especially in the industrial sector. The process improves oil palm biomass properties in many ways compared to its raw materials, and later the fuels are suitable to be used in any large scale of thermochemical devices.

The production cost of briquetting depends on the sizes used for raw materials. As the major cost is contributed for the pre-treatment process of EFB, it is recommended that the EFB treatment plant and briquette plant be integrated in palm oil mills to make the project more economically viable. Through this commercialization approach, the costs for raw materials, treatment process, logistics and utility could be obtained at no or very minimal charges.

Based on the economic analysis to integrate the briquette plant in $60 \mathrm{t} / \mathrm{hr}$ palm oil mill, it was found that the investment cost is at RM 4.0 million with the pay back period is less than 5 years. The specific energy requires to produce 1 tonne/hour briquette is $125 \mathrm{kwhr}$. The processing cost is estimated at RM 250/tonne and the selling prices for export market vary from a minimum of RM 350 to RM600/ per tonne. These figures are based on the selling prices for overseas market. Besides gaining extra profits from the selling of oil palm biomass, it also helps millers in disposing their solid waste in a very profitable approach.

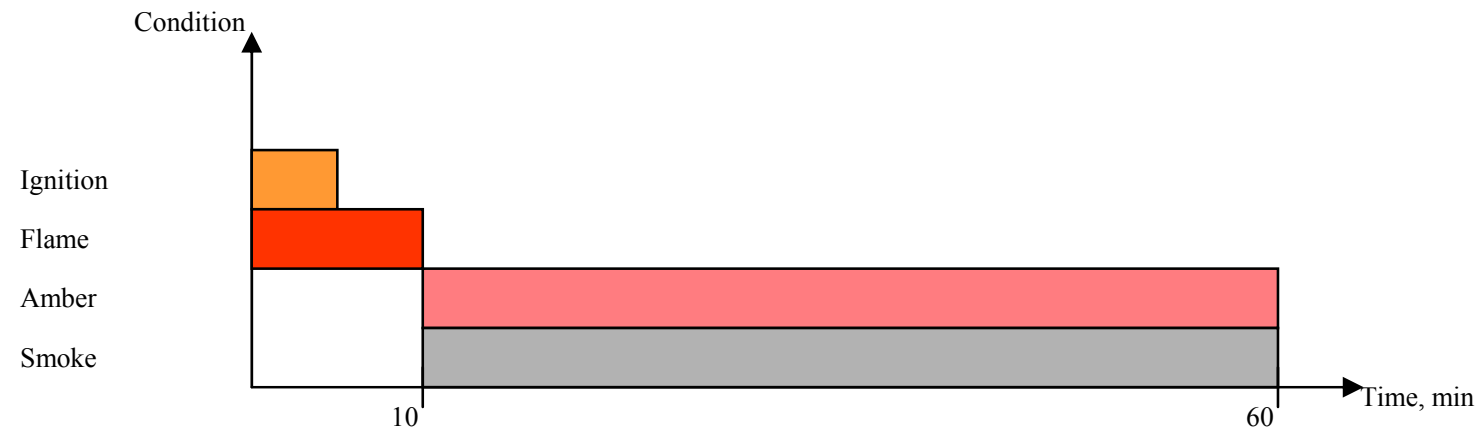

Figure 4. Burning profile of palm briquettes (source: modified from Pentananunt et al. ${ }^{[7]}$ ) 


\section{CONCLUSION}

From the experiments carried out, it was generally found that the characteristics of palm biomass briquettes produced from screw extrusion technology, were satisfactory compared to commercial sawdust briquettes. Nevertheless, the results meet the international standards requirements for export markets. It is recommended that the modification of the machine and addition of binder are necessary in producing quality briquettes. The blending of palm biomass with sawdust can improve its physical, mechanical and combustion properties. Therefore, palm biomass is suitable for substitution in certain ratios of sawdust briquettes production.

Production cost is a barrier for local market. More R\&D activities are still required to make it more cost effective as renewable energy source and for oversea markets.

\section{ACKNOWLEDGEMENT}

The authors wish to thank Director-General of MPOB for his permission to publish this paper. Thanks are also due to all staff of Energy \& Environment Unit and Biomass \& Technology Unit of MPOB for their technical assistance. The cooperation from those industrial partners is also greatly appreciated.

\section{REFERENCES}

[1] Economics \& Industry Development Division, MPOB, 2006 Malaysian Oil Palm Statistics 2005, 25 ${ }^{\text {th }}$ Edition, MPOB Publisher, pp: $23-40$.

[2] Vijaya, S., Chow, M.C. and Ma. A.N.,2004. Energy Database of the Oil Palm. MPOB Palm Oil Engineering Bulletin, 70:15-22

[3] Hoi Why Kong, 1995. Wood and Charcoal Briquetting in Malaysia. In: Proceedings of International Workshop on Biomass Briquetting, (eds P.D.Grover and S.K Mishra). pp 68-74. FAO Regional Wood Energy Development Programme

[4] www.ait.ac.th

[5] P.D.Grover and S.K. Mishra, 1996. Biomass Briquetting: Technology and Practices. FAO Report, United Nations, Bangkok,

[6] Deutsches Institut für Normung e.V, 1996 DIN 51731 Testing On Solid Fuels - Compresses Untreated Wood- Requirements and Testing

[7] Pentananunt, R., Rahman A.N.M.M. and Bhattacharya, S.C., 1990. Upgrading of Biomass by Means of Torrefaction. Energy, 15(12): 1175 - 


\title{
Review of Recycling and Its Techniques
}

\author{
Azizul Mohamad, Abdul Sattar Megat Husin and Daniel William
}

\begin{abstract}
Recycling is the reprocessing of materials into new products. Recycling is a key concept of modern waste management and becoming more and more important lately. Level of recycling varied substantially from one industry or country to the others. This paper discussed briefly on the history of recycling as well as on major benefits and drawbacks of recycling. Emphasis on current recycling techniques for various common materials and international universal recycling codes are thoroughly addressed.
\end{abstract}

Keywords: recycling, sustainable engineering, environment

\section{INTRODUCTION}

Recycling is the reprocessing of materials into new products. Recycling prevents useful material resources being wasted, reduces the consumption of raw materials and reduces energy usage, and hence greenhouse gas emissions, compared to virgin production. Recycling is a key concept of modern waste management and is the third component of the waste hierarchy.

Recyclable materials, also called recyclables or recyclates, may originate from a wide range of sources including the home and industry. They include glass, paper, aluminium, asphalt, iron, textiles and plastics. Biodegradable waste such as food waste or garden waste is also recyclable with the assistance of micro-organisms through composting or anaerobic digestion [1].

Recyclates need to be sorted and separated into material types. Contamination of the recylates with other materials must be prevented to increase the recyclates value and facilitate easier reprocessing for the ultimate recycling facility. This sorting can be performed either by the producer of the waste or within semi- or fully-automated materials recovery facilities.

There are two common household methods of helping increase recycling. In curbside collection, consumers leave presorted materials for recycling in front of their property, typically in boxes or sacks to be collected by a recycling vehicle. Alternatively, with a "bring" or carry-in system, the householder may take the materials to collection points,

Azizul Mohamad is with Mechanical Engineering Program, School of Mechatronic Engineering, Universiti Malaysia Perlis, 01000 Kangar, Perlis, MALAYSIA (phone: 604-979-8679; fax: 604-979-8142; e-mail: azizul@unimap.edu.my).

Abdul Sattar Megat Husin is currently a student of Bachelor of Mechatronic Engineering, School of Mechatronic Engineering, Universiti Malaysia Perlis, 01000 Kangar, Perlis, MALAYSIA

Daniel William is currently a student of Bachelor of Mechatronic Engineering, School of Mechatronic Engineering, Universiti Malaysia Perlis, 01000 Kangar, Perlis, MALAYSIA such as transfer stations or civic amenity sites, where recyclates are placed into recycling bins based on the type of material.

Recycling does not include reuse where items retain their existing form for other purposes without the need for reproducing a new product [1].

\section{HISTORY}

Recycling has been a common practice throughout human history. In pre-industrial times, scrap made of bronze and other precious metals were collected in Europe and melted down for perpetual reuse, and in Britain dust and ash from wood and coal fires was down cycled as a base material in brick making.

The main driver for these types of recycling was the economic advantage of obtaining recycled feedstock instead of acquiring virgin material, as well as a lack of public waste removal in ever more-populated sites.

Paper recycling began in Britain in 1921, when the British Waste Paper Association (now Confederation of Paper Industries) was established to encourage trade in waste paper recycling [1].

Resource shortages caused by the world wars, and other such world-changing occurrences greatly encouraged recycling. Massive government promotion campaigns were carried out in World War II in every country involved in the war, urging citizens to donate metals and conserve fiber, as a matter of significant patriotic importance.

Resource conservation programs established during the war were continued in some countries without an abundance of natural resources, such as Japan, after the war ended.

The next big investment in recycling occurred in the 1970 s, due to rising energy costs (recycling aluminum uses only $5 \%$ of the energy required by virgin production; glass, paper and metals have less dramatic but very significant energy savings when recycled feedstock is used [1].

\section{BENEFITS}

Recycling is beneficial in two ways: it reduces the inputs (energy and raw materials) to a production system and reduces the amount of waste produced for disposal.

Some materials like aluminum can be recycled indefinitely as there is no change to the materials. Other recycled materials like paper require a percentage of raw materials (wood fibers) to be added to compensate for the degradation of existing fibers.

The resources being processed are purer, less energy is needed to process them and less energy is needed to transport from the place of extraction (e.g. 
bauxite/aluminium ore mines in Brazil or coniferous forests in Scandinavia).

This reduces the environmental, social, and usually the economic costs of manufacturing. For example, bauxite mines in Brazil displace indigenous people, create noise pollution from blasting, machinery and transport, and create air pollution in the form of particulates (dust). The habitat loss and visual destruction is also negative both to the aesthetic qualities of the areas and the local environment. However, the mines do provide employment and revenue to the local population and economy, promoting development of the country as a whole.

Recycling aluminium saves $95 \%$ of the energy cost of processing new aluminium because the melting temperature is reduced from $900{ }^{\circ} \mathrm{C}$ to $600{ }^{\circ} \mathrm{C}$. It is by far the most efficient material to recycle.

The most commonly used methods for waste disposal such as landfill, pyrolysis, and incineration may be environmentally damaging and unsustainable. Therefore any way to reduce the volume of waste being disposed in this fashion may be beneficial. The maximum environmental benefit is gained by waste minimization i.e. reducing the amount of waste produced, and reusing items in their current form such as refilling bottles.

\section{DRAWBACKS}

All recycling techniques consume energy for transportation and processing, and some also use considerable amounts of water.

There may also be drawbacks with the collection methods associated with recycling. Increasing collections of separated wastes adds to vehicle movements and the production of carbon dioxide.

This may be negated however by centralized facilities such as some advanced material recovery facilities of mechanical biological treatment systems for the separation of mixed wastes. However, this is almost never the case for urban areas, taking into account the massive number of workers, machines, and vehicles needed for the recycling process.

\section{RECYCLING TECHNIQUES}

There are many different materials can be recycled, but each type requires a different technique to recycle them. Some of the most common materials are further discussed.

\section{A. Batteries}

The large variation in size and type of batteries makes their recycling extremely difficult: they must first be sorted into similar kinds and each kind requires an individual recycling process. Additionally, older batteries contain mercury and cadmium, harmful materials which must be handled with care.

Lead-acid batteries, like those used in automobiles, are relatively easy to recycle and many new lead-acid batteries contain a high percentage of recycled material.

\section{B. Electronics Disassembly and Reclamation}

The direct disposal of electrical equipment such as old computers and mobile phones is banned in many areas due to the toxic contents of certain components. The recycling process works by mechanically separating the metals, plastics and circuit boards contained in the appliance. When this is done on a large scale at an electronic waste recycling plant, component recovery can be achieved in a costeffective manner.

Electronic devices, including audio-visual components like televisions, VCRs, stereo equipment, mobile phones and other hand-held devices, and computer components, contain valuable elements and substances suitable for reclamation, including lead, copper, and gold.

They also contain a plethora of toxic substances, such as dioxins, PCBs, cadmium, chromium, radioactive isotopes, and mercury. Additionally, the processing required to reclaim the precious substances including incineration and acid treatments release, generate and synthesize further toxic by-products.

In the United States, an estimated $70 \%$ of heavy metals in landfills come from discarded electronics. Some regional governments are attempting to curtail the accumulation of electronics in landfills by passing laws obligating manufacturers and consumers to recycle these devices.

However, as in many cases, safe dismantlement of these devices in accordance with first world safety standards is unprofitable, historically much of the electronic waste has been shipped to countries with lower or less rigorouslyenforced safety protocols. Places like Guiyu, China dismantle tons of electronics every year, profiting from the sale of precious metals, but at the cost of the local environment and the health of its residents.

Mining to produce the same metals, to meet demand for finished products in the west, also occurs in the same countries, and the United Nations Conference on Trade and Development (UNCTAD) has recommended that restrictions against recycling exports be balanced against the environmental costs of recovering those materials from mining.

\section{C.Ferrous Metals}

Iron and steel are the world's most recycled materials, and among the easiest materials to recycle, as they can be separated magnetically from the waste stream. Recycling is via a steelworks: scrap is either remelted in an Electric Arc Furnace (90-100\% scrap), or used as part of the charge in a Basic Oxygen Furnace (around 25\% scrap).

Any grade of steel can be recycled to top quality new metal, with no 'downgrading' from prime to lower quality materials as steel is recycled repeatedly. $42 \%$ of crude steel produced is recycled material.

\section{D.Non-Ferrous Metals}

Aluminium is shredded and ground into small pieces or crushed into bales. These pieces or bales are melted in an aluminium smelter to produce molten aluminium. By this stage the recycled aluminium is indistinguishable from virgin aluminium and further processing is identical for both.

Due to the high melting point of aluminum ore, large amounts of energy are required to extract aluminum from ore, making the environmental benefits of recycling aluminium enormous.

Approximately $5 \%$ of the $\mathrm{CO}_{2}$ is produced during the recycling process compared to producing raw aluminium 
and an even smaller percentage when considering the complete cycle of mining and transporting the aluminium. Also, as open-cut mining most often used for obtaining aluminium ore, mining destroys large sections of natural land.

An aluminium can is $100 \%$ recyclable every time it is recycled; it saves enough energy to watch television for about three hours as compared to mining and producing a new can.

\section{E. Glass}

Glass bottles and jars are accumulated via curbside collection schemes and bottle banks, where the glass may be sorted into color categories, namely flint/clear, green and brown/amber glasses. The collected glass cullet is taken to a glass recycling plant where it is monitored for purity and contaminants are removed. The term "cullet" derives from the practice of remelting flawed containers which have been "culled" from production lines. The cullet is crushed and added to a raw material mix in a melting furnace. It is then mechanically blown or molded into new jars or bottles [2].

Glass cullet is also used in the construction industry for aggregate and glassphalt. Glassphalt is a road-laying material which comprises around $30 \%$ recycled glass. Glass can be recycled indefinitely as its structure does not deteriorate when reprocessed.

\section{F. Paper}

Recycled paper is made from waste paper, usually mixed with fresh wood pulp. If the paper contains ink, it must be deinked. This also removes fillers, clays, and fiber fragments.

At present, almost all paper can be recycled, but some types are harder to recycle than others. Kraft paper, papers coated with plastic or aluminum foil, and papers that are waxed, pasted, or gummed are usually not recycled because the process is too expensive. Gift wrap paper also cannot be recycled. Different types of paper are usually sorted before recycling, such as newspapers and cardboard boxes.

Different grades of paper are recycled into different types of new products. Old newspapers are usually made into new newsprint, egg cartons, or paperboard. Old corrugated boxes are made into new corrugated boxes or paperboard. Highgrade white office paper can be made into almost any new paper product: stationery, newsprint, magazines, or books [3].

Sometimes recyclers ask for the removal of the glossy inserts from newspapers because they are a different type of paper. Glossy inserts have a heavy clay coating that some paper mills cannot accept. Since the paper is weighed down by the clay coating, a paper mill gets more recyclable fibers from a ton of pure newsprint.

Paper can only be recycled a finite number of times due to the shortening of paper fibers making the material less versatile. Often it will be mixed with a quantity of virgin material, referred to as downcycling. This does not however exclude the material from being used in other processes such as composting or anaerobic digestion, where further value can be extracted from the material in the form of compost or biogas.

\section{G.Plastic}

Plastic recycling is the process of recovering scrap or waste plastics and reprocessing the material into useful products. Compared to glass or metallic materials, plastic poses unique challenges - because of the massive number of types of plastic, they each carry a resin identification code, and must be sorted before they can be recycled.

The recycling process of plastic can be costly, due to the fact that while metals can be sorted using electromagnets, no such 'easy sorting' capability exists for plastics. In addition to this, while labels do not need to be removed from bottles for recycling, lids are often made from a different kind of non-recyclable plastic.

Plastic have low entropy of mixing, which is due to the high molecular weight of large polymer chains. Another way of stating this problem is that, since a macromolecule interacts with its environment along its entire length, its enthalpy of mixing is very, very large compared to that of a small organic molecule with a similar structure; thermal excitations are often not enough to drive such a huge molecule into solution on their own. Due to this uncommon influence of mixing enthalpy, polymers must often be of nearly identical composition in order to mix with one another [4].

\section{INTERNATIONAL CODES}

The communication and identification are laid out in International Universal Recycling Codes. These codes outline what material an item is made from, to facilitate easier reprocessing.

Table 1 outlines the International Universal Recycling Codes.

\section{CONCLUSION}

Recycling is a very crucial process in both energy and environmental terms. Its huge benefits are undeniable. Various techniques for recycling had and will be always be introduced and improvised. A common code for recycling and sharing of recycling technology are some major steps in achieving successful and comprehensive recycling goals.

Recycling has becoming a global agenda and should be tackled at an international level through continuous research, education and cooperation. 
Table 1. The International Universal Recycling Codes

\begin{tabular}{|c|c|c|}
\hline Symbol & Code & Description \\
\hline \multirow{8}{*}{ Plastics } & \#1 PET(E) & $\begin{array}{l}\text { Polyethylene } \\
\text { terephthalate }\end{array}$ \\
\hline & \#2 PE-HD & $\begin{array}{l}\text { High-density } \\
\text { polyethylene }\end{array}$ \\
\hline & \#3 PVC & Polyvinyl chloride \\
\hline & \#4 PE-LD & $\begin{array}{l}\text { Low-density } \\
\text { polyethylene }\end{array}$ \\
\hline & \#5 PP & Polypropylene \\
\hline & \#6 PS & Polystyrene \\
\hline & \#7 O(ther) plastic & All other plastics \\
\hline & \#ABS & $\begin{array}{l}\text { Acrylonitrile Butadiene } \\
\text { Styrene: Monitor/TV } \\
\text { cases, coffee makers } \\
\text { Cell Phones, most } \\
\text { computer plastic }\end{array}$ \\
\hline \multirow{7}{*}{ Batteries } & \#8 Lead & Lead-acid battery \\
\hline & \#9 Alkaline & Alkaline battery \\
\hline & $\# 10 \mathrm{NiCD}$ & $\begin{array}{l}\text { Nickel-cadmium } \\
\text { battery }\end{array}$ \\
\hline & \#11 NiMH & $\begin{array}{l}\text { Nickel metal hydride } \\
\text { battery }\end{array}$ \\
\hline & $\# 12 \mathrm{Li}$ & Lithium battery \\
\hline & $\# 13 \mathrm{SO}(\mathrm{Z})$ & Silver-oxide battery \\
\hline & $\# 14 \mathrm{CZ}$ & Zinc-carbon battery \\
\hline \multirow{3}{*}{ Paper } & \#20 C PAP (PCB) & Cardboard \\
\hline & \#21 PAP & $\begin{array}{l}\text { Other paper, mixed } \\
\text { paper (magazines, } \\
\text { mail) }\end{array}$ \\
\hline & \#23 PBD (PPB) & $\begin{array}{l}\text { Paperboard: Greeting } \\
\text { cards, frozen food } \\
\text { boxes, book covers }\end{array}$ \\
\hline \multirow{2}{*}{ Metals } & $\# 40 \mathrm{FE}$ & Steel \\
\hline & \#41 ALU & Aluminium \\
\hline
\end{tabular}

\section{REFERENCES}

[1] Internet. Recycling. http://en.wikipedia.com/wiki/Recycling. Accessed 1 August 2007.

[2] Internet. Glass Recycling Issues: Its More than Mayonnaise Jars. http://www.waste-management-information.org.uk/. Accessed 11 August 2007.

[3] Internet. Paper University - EarthAnswers - How is Paper Recycled? http://www.tappi.org/paperu/all_about_paper/earth_answers/Recycle1 .htm. Accessed 21 August 2007.

[4] "Polymer Recycling: Science, Technology and Applications", John Scheirs, Wiley, 1998. 


\title{
A Study on a Hybrid Solar Thermoelectric and Adsorption Cooling System
}

\author{
M.O. Abdullah, K. Abd. Hamid, S.L. Leo and J.L. Ngui
}

\begin{abstract}
A Solar Peltier-Adsorption cooler has been fabricated and laboratory tested. The system consists of a collector reservoir, a condenser, an evaporator, a flat plat photovoltaic (12V), cooper tubes, a cooling box with build-in thermoelectric element, a flat plat collector and a support frame. The solar flat plate collector consists of seven adsorbent beds, which can accommodate $3.5 \mathrm{~kg}$ of adsorbent. The working pair used is palm activated carbon and methanol. In this system, adsorption cycle is operates intermittently with one cycle per day. The cooling effect occurs during the adsorption process at night. Meanwhile, photovoltaic panel provides electricity for peltier cooler to generate cooling effect during the day time. As a result, cooling is achieved in the cooling box continuously. Experimental results showed that, the lowest temperature can be achieved in daytime is $10^{\circ} \mathrm{C}$, and at night at $22^{\circ} \mathrm{C}$ by using $300 \mathrm{ml}$ load of water. The COP of the hybrid system calculated to be 0.05 , i.e. cooling effect of $5 \%$. On-going works are currently in progress aim to optimize the system by computer modeling and to improve the overall system efficiency.
\end{abstract}

Keywords: adsorption; thermoelectric; activated carbon; solar energy

\section{INTRODUCTION}

Solar cooling seems to be the most promising application for solar energy [1]. It is an important use of the solar energy because the supply of solar energy and the demand of cooling are greatest during the same period [2]. Researchers over the past few decades have developed and invented a number of additional solar thermal applications, such as refrigeration and air-conditioning, drying and curing of agricultural products and electric power production by solar thermal conversion.

Normally there are two ways to achieve cooling by mean of solar energy. The first method is by converting the solar energy to electricity which by means of photovoltaic cells to

M.O. Abdullah is the Head of Chemical Engineering \& Energy Sustainability Dept, Faculty of Engineering, Universiti Malaysia Sarawak (corresponding author, e-mail: amomar@feng.unimas.my)

Professor K. Abd. Hamid is the Deputy Vice Chancellor and Director of Center of Excellence for Rural Informative (CoERI), UNIMAS (e-mail:

khair@adm.unimas.my).

S.L. Leo is a postgraduate candidate with the Faculty of Engineering, Universiti Malaysia Sarawak (UNIMAS), 94300 Kota Samarahan, Sarawak (e-mail: leo9421@yahoo.com).

J.L. Ngui is a postgraduate candidate with the Faculty of Engineering, Universiti Malaysia Sarawak (UNIMAS), 94300 Kota Samarahan, Sarawak (e-mail: jialin83@yahoo.com). run the domestic cooler. The second method is by using the solar thermal radiation directly to generate cooling through sorption process. The one that are being studied in this research is a combination of both methods by hybridization peltier cooler using PV power source and solar heat to desorb refrigerant in the adsorption system.

\section{Hybrid SOlar PELTIER-AdSORPTION COOLER}

Hybrid solar peltier-adsorption cooler is actually a combination of peltier cooler and adsorption cooler.

\section{A. Peltier Cooler}

Peltier cooler is a device that is cold on one side and hot on the other. It is resulted from the passage of a DC current through junctions of two different materials. Heat is "pumped" from the cold side to the hot side. The direction of current flow determines which side to be cold or hot.

\section{B. Adsorption Cooler}

The adsorption cooler is mainly consisting of a collector containing the adsorbers, a condenser, an evaporator and a reservoir. Adsorption is the fixation of gas molecules (adsorbate) on the surface of a solid (adsorbent), according to van der Waals interaction. Since the interaction is weak, adsorption is reversible and exothermic. This process induces neither changes of solid volume nor does it involve any chemical reaction.

A complete adsorption cycle is the succession of two phases. The first phase is regeneration of the adsorbent to release adsorbate, which is condensed in the condenser and accumulated in the reservoir. The second phase occurs during the night when the evaporation of the adsorbate in the evaporator and the adsorption process take place.

\section{SYSTEM DESCRIPTION}

The hybrid cooling system ('figure 1') consists of a few main assembly parts, which involved analysis, conceptualize, visualize, fabrication of the parts and assembly process. A feasible analysis of the system has been carried out. Analysis is done by calculating the capacity of the adsorbent bed and estimates the quantity of activated carbon that can be filled by using density properties calculation. Then, the volume of reservoir can be predetermining for designing process. 


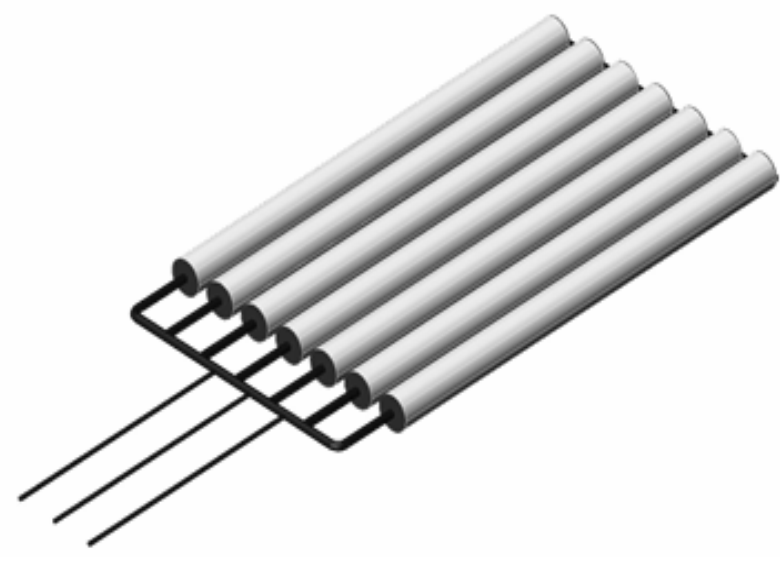

Figure 1. Absorbers

\section{A. Construction of Components}

1) Absorber: As shown in 'figure 1', absorber plays as an important part for this system because it acts as the heart of the system. Construction of the absorber involved welding operation that contributes the most complex operation because it needs special precaution of handling and experience skills in order to avoid the leakage and unsuitable heat of operation. Firstly, seven copper cylinders with diameter of $5 \mathrm{~cm}$ and $75 \mathrm{~cm}$ in length each are prepared. Fourteen round copper plates with diameter of 6 $\mathrm{cm}$ are fabricated to cover the cylinder both end. Seven pieces of the plate are then drilled with a hole $(1.27 \mathrm{~cm}$ in diameter) in the centre as refrigerant path. After that, seven perforated copper tubes with diameter of $0.95 \mathrm{~cm}$ are located at the centre of each cylinder and acting as mass flow channel for the refrigerant vapor. $0.5 \mathrm{~kg}$ of palm activated carbon in granular form is then filled into each absorber cylinder, respectively. Welding process is finally applied using silver rod to complete the absorbers.

2) Heat Trap Collector: Heat trap collector is the module use to collect the heat in the collector box. The trapped heat is supplied to heat the absorbers during the regeneration phase. Compare to solar evacuated collector, the flat-plate heat trap is of simple construction, low relative cost, no moving part relatively ease to repair and also durable. Heat trap collector generally is a box covered with a single glazing and insulated by glass wool inside the box, as shown in 'figure 2'. The heat trap outer box is painted with black paint to increase the heat adsorb into the box while the inner side is painted with aluminum paint to reduce heat lost to surrounding.

3) Reservoir: Reservoir, as shown in 'figure 3', is constructed with a combination of three copper tubes of 3.5 $\mathrm{mm}$ diameter and height $40 \mathrm{~cm}$. The tubes are assembled together via connection pipes connected with an interval of $2 \mathrm{~cm}$ to each other.

\section{B. Inspection Test}

As mentioned by Lemmini and Errougani [3], the cooling system must be airtight in order for the system to work properly because the present of air in the system would affect the thermodynamic property of refrigerant. Thus, the

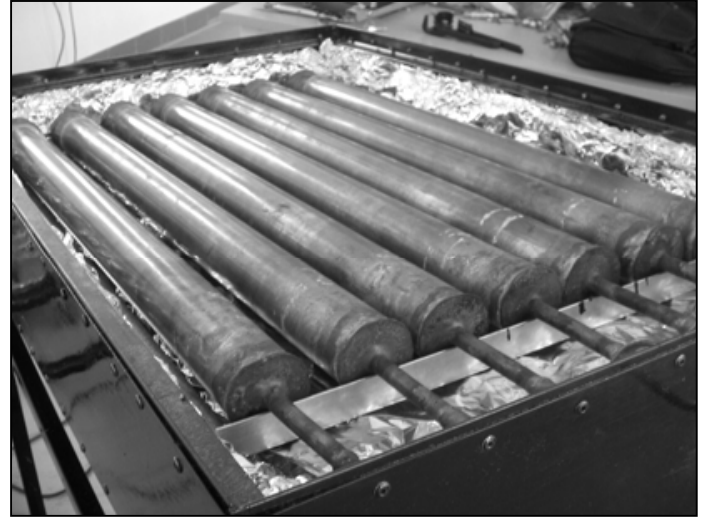

Figure 2. Heat trap collector with absorbers

prototype (as shown in 'figure 4') is checked for vacuum proof before it ready to be tested. A Robinair vacuum pump is used to evacuate the system until the pressure reached below 0.1 bars. More precaution is taken to ensure no leakage occurs especially at the welded parts and the tubing joints. After one week of evacuation, the pressure showed no variations, which confirmed that system is vacuum proof. A total of $500 \mathrm{ml}$ of methanol was then charged to the system.

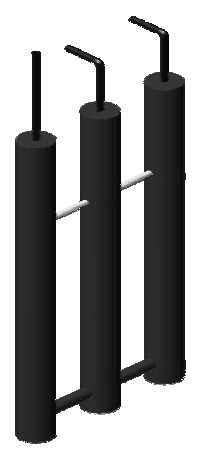

Figure 3. Reservoir

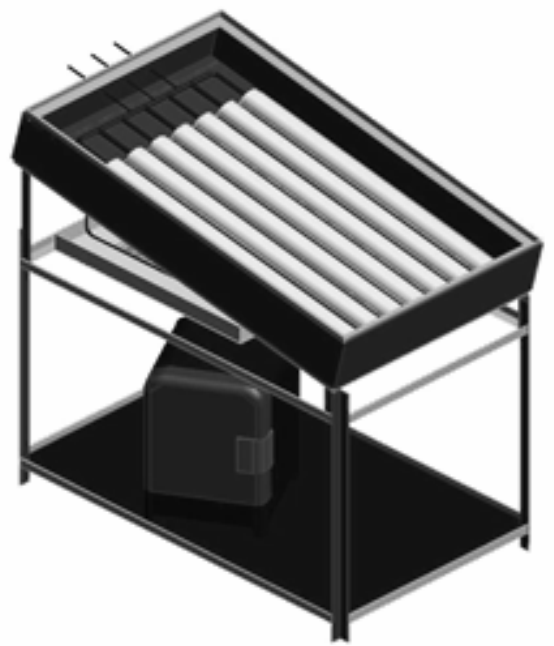

Figure 4. Hybrid Peltie-Adsorption Cooling System

\section{Operational of the Hybrid Cooler}

During the day light, peltier cooler operates using electricity generated from photovoltaic (PV) panel. The thermoelectric module in the cooling box acts as a heat pump, where it transmits the heat from the cooling plate to 
the heat sink. The heat around the heat sink then released to the environment by the fan built under the module. The operation of peltier cooler is performed continuously until the solar radiation is not available during the day.

Adsorption cycle consists of four stages; these stages are (i) heating and pressurization, (ii) heating and desorption with condensation, (iii) cooling and depressurization and (iv) cooling and adsorption with evaporation. During the heating and pressurization stage, the adsorbers are heated by using solar heat while being closed. The activated carbon temperature in the adsorber increased and induces the pressure to increase.

At the second stage, the adsorbers continue receiving heat while being connected to the condenser. The adsorbent temperature is continuously increasing, which induces desorption of methanol vapor. The desorbed vapor is then condensed to form a liquid in the condenser. In the following stage, the adsorbers temperature decrease at night while being closed. The decrement in temperature induces the pressure reduces from the condensation pressure down to the evaporation pressure. At last stage, the adsorbers continue to release heat while being connected to the evaporator. The activated carbon temperature and pressure continues decreasing, which induce the adsorption of methanol vapor. This adsorbed methanol vapor is vaporized through the evaporator, which produces cooling effect in the cooling box at night.

\section{RESUlts AND Discussions}

During the experiment, $300 \mathrm{ml}$ of pure water is put into the cooling box as a load. The results obtained are presented graphically in 'figures 5 and 6'. The cooling effect generated by the peltier cooler is instantaneous when electricity is supplied by the photovoltaic during daytime. At the same time, adsorbers temperature is observed to reach up to $106{ }^{\circ} \mathrm{C}$. The initial temperature of the water recorded is $28.0^{\circ} \mathrm{C}$ in the morning and chilled to $10.9{ }^{\circ} \mathrm{C}$ in the evening by the peltier cooler. At night, the load temperature is decreased to approximately $5{ }^{\circ} \mathrm{C}$ after adsorption process started. The coefficient of performance (COP) of the hybrid system is calculated to approximately 0.05 or $5.0 \%$.

\section{CONClusions}

Hybrid solar peltier-adsorption cooler has been successfully designed, fabricated and tested in laboratory. The experimental results showed the hybrid cooler produces cooling effect in the evaporator continuously. The COP of the system is calculated as $5.0 \%$. The advantages of hybrid peltier-adsorption cooling system compare to conventional cooling system are it do not consume any electricity, environmental friendly and suitable for rural area applications.

\section{RECOMMENDATION FOR FURTHER WORKS}

A new upgraded version of the hybrid solar thermoelectricadsorption cooling system aimed to achieve better efficiency and working fluids to the system is proposed herewith. With the introduction of more adsorbents and working fluids in the system, the adsorption cycle will inevitably performed better. Further experimental test to be obtained from a new construction or modification of the apparatus could be made. With the introduction of modeling and simulation using Fortran and MATLAB software, further validations and computational works can be made. Such endeavors are on-going here at UNIMAS.

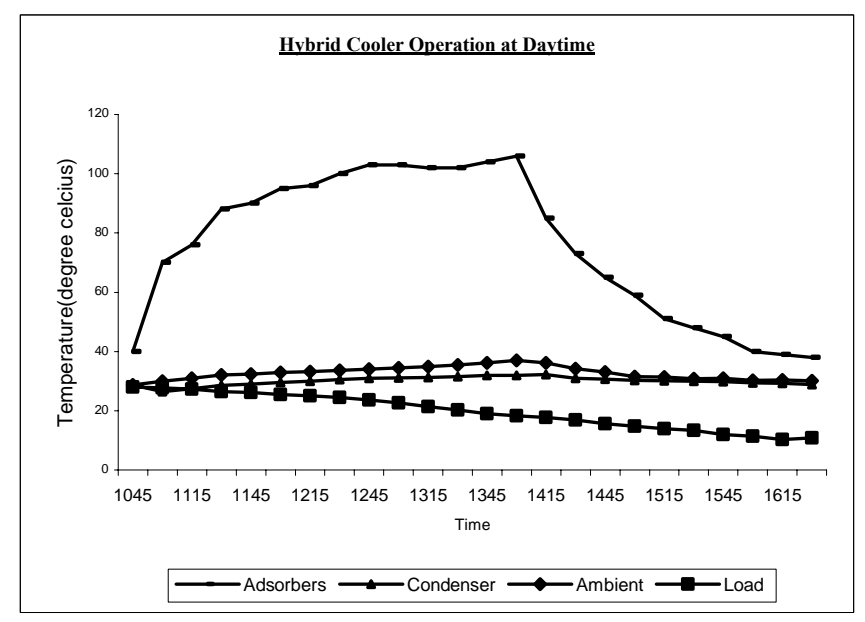

Figure 5. Operation of the hybrid cooler at daytime

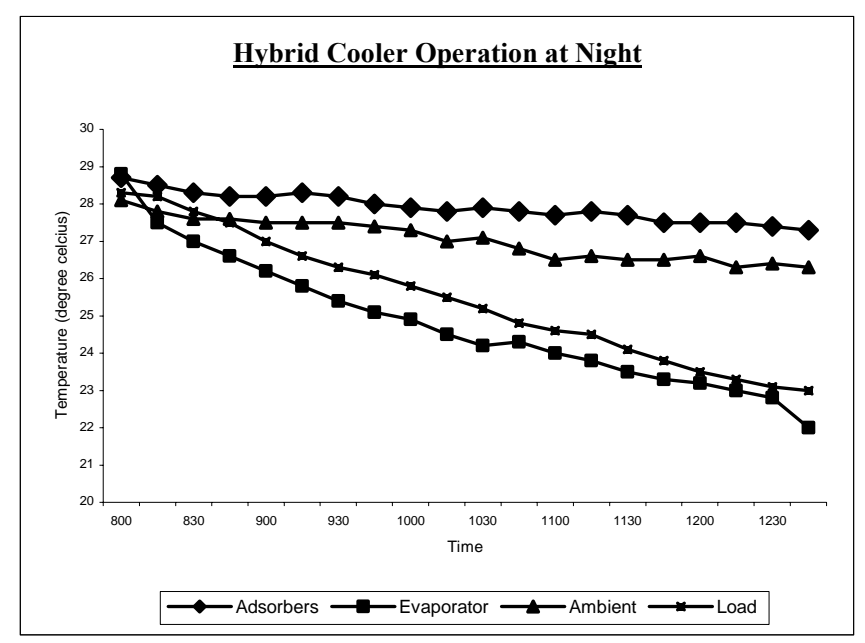

Figure 6. Operation of the hybrid cooler at Night

\section{ACKNOWLEDGEMENTS}

The authors would like to acknowledge the ASHRAE and the Ministry of Science, Technology \& Innovation, Malaysia for funding the project. The research has been carried out under the ASHRAE Fund and the eSience Fund project no 03-01-09-SF0018. Also, we appreciate the helps from a few of our final year project students in the early phase of this study.

\section{REFERENCES}

[1] F. Lemmini and F. Meunier, "Simulation of an adsorptive solar refrigerator operating in Morocco," Journal of Islamic Academy of Science, vol. 3, no. 4, pp. 273-279, 1990.

[2] J. Eric, "A study of thermal decomposition of methanol in solar powered adsorption refrigeration systems," Solar Energy, Vol. 62, No. 5, pp. 325-329, 1998.

[3] F. Lemmini and A. Errougani, "Building and experimentation of a solar powered adsorption refrigerator," Renewable Energy, Vol. 30, pp. 1989-2003, 2005. 


\title{
Biogas Production from Animal Waste by Anaerobic Digestion
}

\author{
Shanker Kumar Sinnaudan, Caroline Marajan and Sahol Hamid Abu Bakar
}

\begin{abstract}
Currently, farm wastes such as swine, poultry manure and cattle dungs are directly disposed or discharged in to the open channels and river systems. These problems are clearly evident among the small scale farmers who are cannot affort to have a sophisticated and costly waste treatment plants. It causes an alarming rate of enviromental pollution, expecially water pollution. Thus, a methodology for the treatment farm waste namely anaerobic digester is proposed to maintain the water quality. At the same time this system also designed to produce biogas for domestic use and organic fertilizer which can be use for domestic or industrial purpose. Odour control, renewable energy production, pathogen reduction, greenhouse reduction, reduction in total oxygen demand of the treated manure are some of the key expected benefits of an anaerobic digester. The research started by designing a anaerobic digester where it was found that the digester successfully yielded gas. From the study it is recommended that the best performing digesters are the digesters that are exposed to high temperature and contained higher solid concentrations. The system suggest if implemented at the field scale may help the farmers, reduce pollution and even more give profit to farmers.
\end{abstract}

Keywords: animal waste, biogas, anaerobic digestion, methane, water quality

\section{INTRODUCTION}

One of the biggest challenges that livestock producers face is managing manure and process water in a way that controls odors and protects environmental quality. With livestock and poultry production that has increased rapidly over the past decades, livestock producers are finding solutions on how to manage animal waste as it has becomes serious problem to the environmental. Improperly managed manure can result in severe consequences to the environment such as ground water contamination and surface water runoff. Manure contains pathogen and the nutrients phosphorus and nitrogen. When properly managed

Dr Shanker Kumar Sinnaudan is with the Water Resources Engineering and Management Research Centre (WAREM), Universiti Teknologi MARA Kampus Pulau Pinang, 13500 Permatang Pauh, Pulau Pinang, Malaysia (Tel: +604-382 2714/3462, Fax: +604-382 3460, email: drsshan@yahoo.com).

Caroline Marajan is with the Faculty of Civil Engineering, Universiti Teknologi MARA Kampus Pulau Pinang, 13500 Permatang Pauh, Pulau Pinang, Malaysia (Tel:+604-382 3446, Fax: +604-382 2717, email: darabajik@yahoo.com).

Dato' Prof. Ir. Dr Sahol Hamid Abu Bakar is with the Faculty of Civil Engineering, Universiti Teknologi MARA Malaysia, 40000 Shah Alam, Selangor, Malaysia (email: saho199@yahoo.com). and applied, growing plants use these nutrients, and a healthy soil and water can absorb limited pathogens. Spreading more manure than can be used by growing plants can result in the extra nutrients leaching into and contaminating groundwater. As well, an improperly designed or damaged storage facility can leak manure, where it can enter the groundwater. Improper application of manure to fields can pollute rivers and lakes with runoff nitrates, phosphorus and pathogens. Manure in water consumes oxygen required by fish and other aquatic life. If too much oxygen in the water is used to break down manure, natural stream life will suffer or to be killed. Taking shortcut by dischaging animal waste into waterways is a typical phemenon which can be seen among the farming communities in the rural areas in Malaysia. Figure 1, 2 and 3 shows the contamination of sources of water due to this phenomenon.

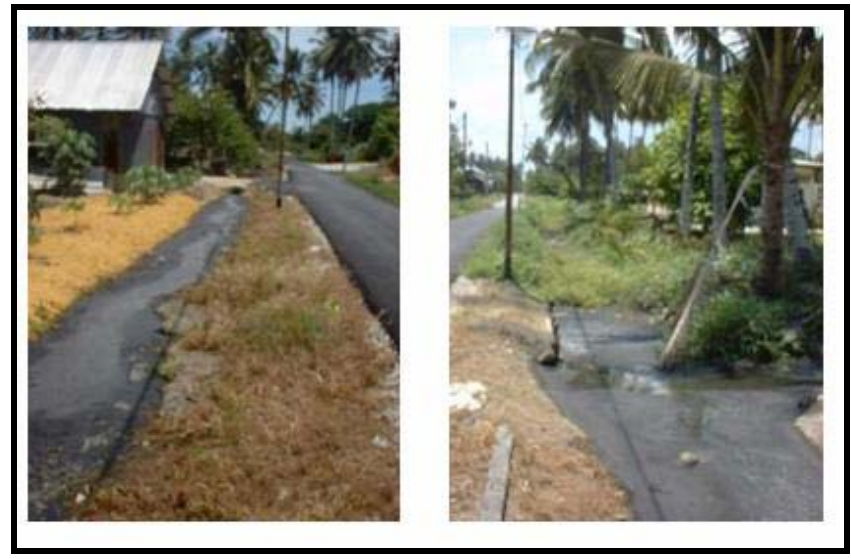

Figure 1. Waterways along the road are being congested with sludge

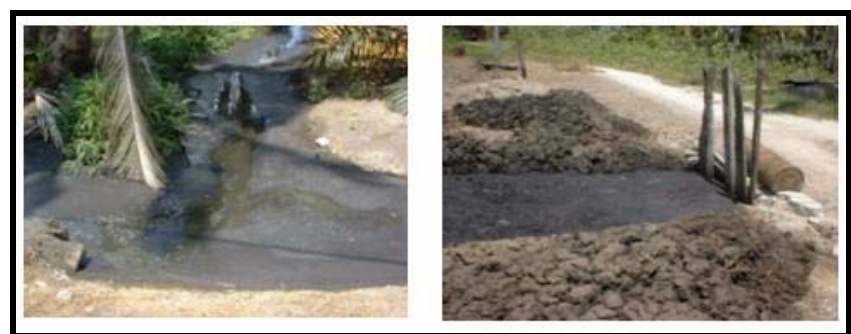

Figure 2. Local drainage and waterways are being clogged with animal sludge 


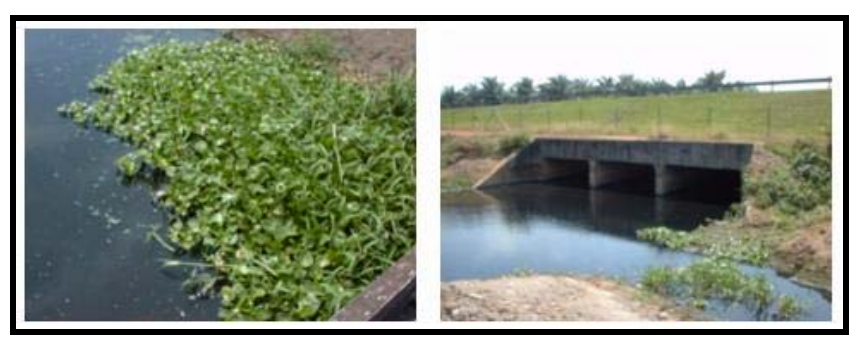

Figure 3. Eichornia Crassipes indicates that area are high in BOD level due to the waterways are heavily polluted with farm waste

The continuous fermentation of farm waste which was discharge illegally into waterways will produce green house gases such as methane and sulphur. This may lead to an increase in vectors of human and animal diseases and loss of asthetic values of waterways as shown in Figures 4, 5 and 6.

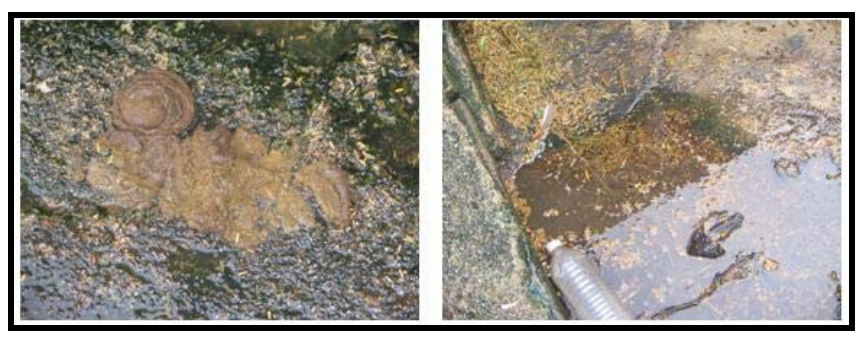

Figure 4. Unmanaged animal waste and untreated cattle urine all over the breeding area

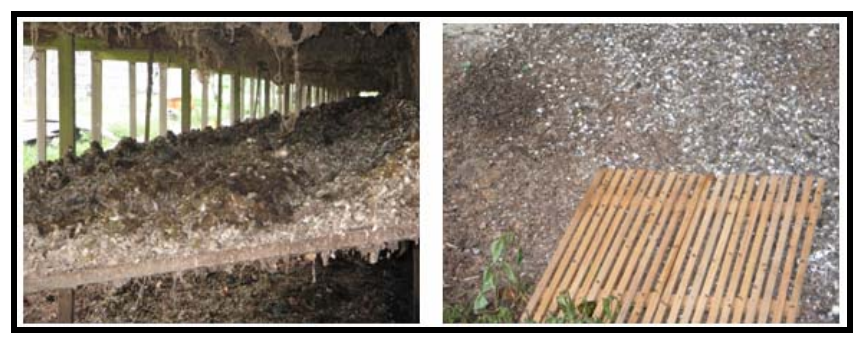

Figure 5. Breeding ground for fly due to the uncollected chicken waste result

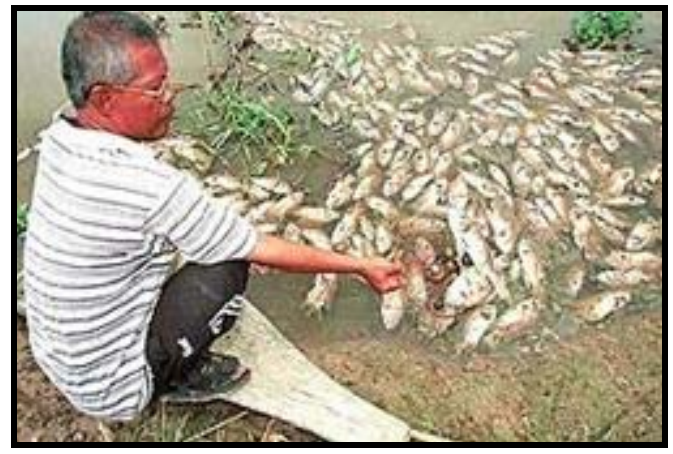

Figure 6. Aquatic life died due to high level of BOD

Farn waste mismanagement can also induce other environmental problems. One of the adverse problems is global warming as a result of emissions of carbon dioxide. This will later affect the thinning of ozone layer which release emissions of methane, nitrogen oxide and CFCs. Farm waste mismanagement also can affect the publics' health condition where diseases such as Japanese encephalities (JE) virus, Nipah virus, etc can be fatal. However, not many are realise that the waste can be transfered in to wealth. The potential of generating Methane from farm waste for domestic and industrial use should be take note. Methane was first recognized in 1890s as having practical and commercial value for lighting purposes in England (Cheremisinoff et al. 1980). Then, the anaerobic biogas system was first setup in Bombory, India in 1900's (Gottas, 1956). Successful production of methane gas to meet the energy needs in rural areas are evident, particularly in India and China and are more recently are being installed in Vietnam (Rodriguez et al. 1997). The system was further refined and finetuned into large scale and more advanced systems available located at Japan, China, Taiwan (Sheen \& Hong, 1999), Europe and The United States (Minor, 1999).

Initial reactors were build as a single brick-line reactor and iron gas holder. However due to high level of corrosion, the technology later improved in 1972 in Taiwan with fiberreinforced plastic. Then later bag-type fermentor was invented. However, due to difficulties in withdrawing the sludge settled, the tent-type was introduced to market. The issue of setup a miniature of methane production unit at the small scale farms to convert waste into something more valuable is still researchable. In Malaysia, there are few guidelines for cattle and poultry farming which also suggest of integration of anaerobic digester for waste management (DVP, 2001). However, this system is not favoured by small scale farmers since the fossil energy or fuels are much more cheaper, lack of enviromental consciousness and general believe that it is ok to discharge farm waste into waterways because it was practiced through many generations.

Thus, it is high time to setup a pilot study in Penang Malaysia where there is a population of more than 300, 000 pigs and Cattles recorded (DVP, 2001).Based on recent studies conducted by Jenagi (2001), a general conclusion can be drawn that there are simpler version of plug-flow digesters being studied in University of Adelaide in Roseworthy and Tokyo University of Agriculture and Technology. This system is considered suitable to be developed, however need to be further studied to suit Malaysian weather condition and farming techniques. These treatment facilities mainly use anaerobic process in order to digest the waste content. Anaerobic process involves a series of reactions by several kinds of anaerobic bacteria feeding on the raw organic matter without oxygen. As these different kinds of bacteria (hydrolytic, transitional and methanogenic) become active, the by products of one type of bacteria provide the food for another bacterial population. There are two stages of an anaerobic digestion namely liquefaction and gasification. From these two stages, about $70 \%$ of the methane produced during fermentation comes from acetic acid with the balance coming from direct reduction of carbon dioxide (Schulte et al. 1975).

\section{MATERIALS AND METHODS}

The present research mainly conducted in the environmental laboratory of Universiti Teknologi MARA Penang. The samples of manure are collected from Abdul Razak Feed Lot Farm located in Simpang Empat, Seberang Perai Tengah, Penang approximately $25 \mathrm{~km}$ from UiTM Penang.

A simple laboratory scale anaerobic digester incorporated with biogas generator facilities was derived. In order to minimize the cost of model construction, many recyclable parts are used with custom made design. From this, the 
apparatus can be classified into three major components which are the digester (low density polyethylene tank), aspirator or sucker and gas collection container. Figure 7 shows the initial design of the biogas generator and Figure 8 show the fully-functional laboratory apparatus after the trial runs and modifications:

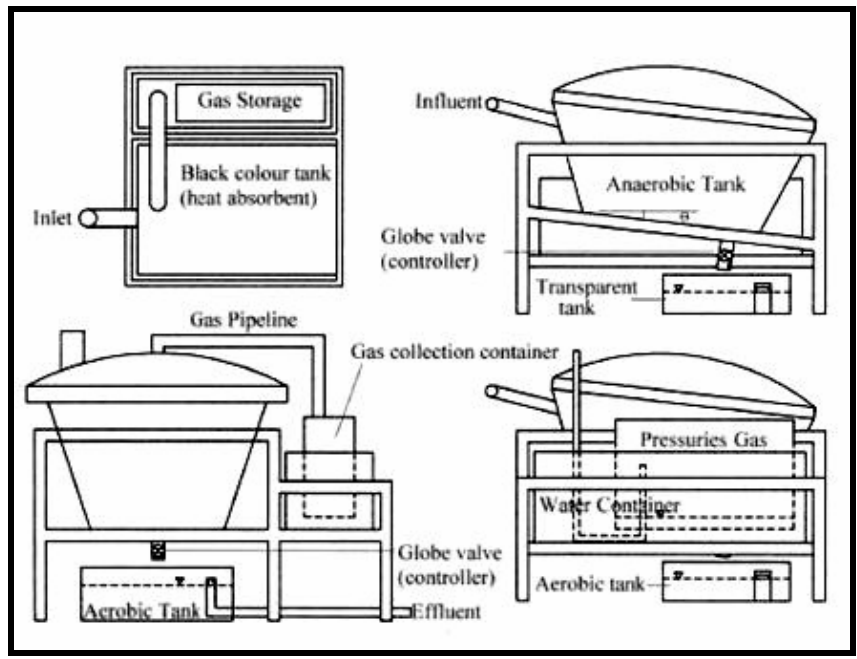

Figure 7. Initial design for the laboratory experiment apparatus

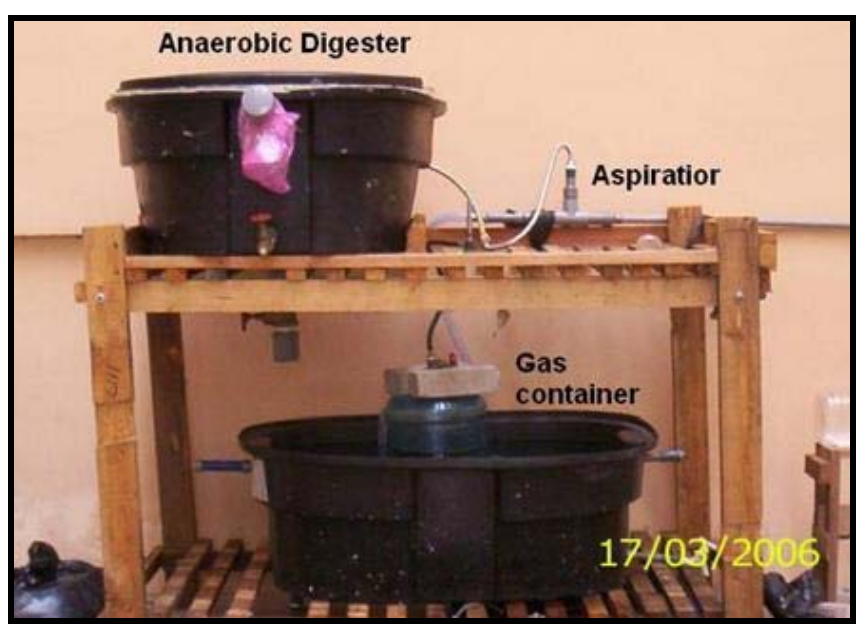

Figure 8. Laboratory biogas generator

Collected manure from the feedlot was mixed thoroughly with water before it was placed into the digester through influent duct. The slurry is mixed with a proportion of a waste and two part of water (1 kg manure with $2 \mathrm{~kg}$ water). The manure was left in the digester at least 24 hours without any disturbance so that fermentation process can take place. Gas can be collected using aspirator/sucker which was connected from the digester to the gas collection container. Aspirator was left to operate for two minutes before the reading is taken in each inspection. Starting from the first inspection, it has to be continuously inspected for 3 times each day in order to analyze the biogas production performance. Figure 9 show the gas level reading of the inspection.

When the gas collection container is full, gas yield test is then conducted to verify the properties of the gas yielded. The results of the biogas production are presented in Figure 10 and Figure 11.

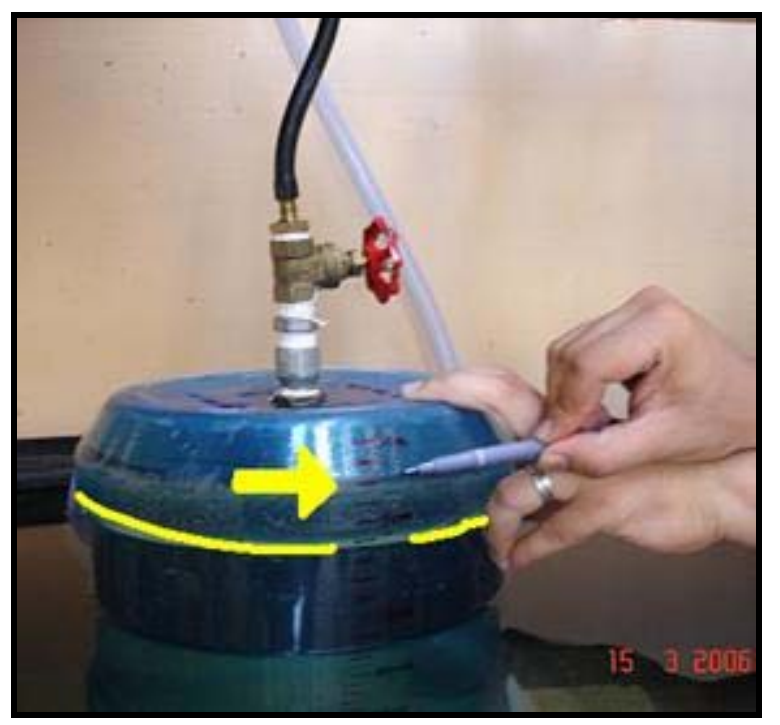

Figure 9 Gas level reading

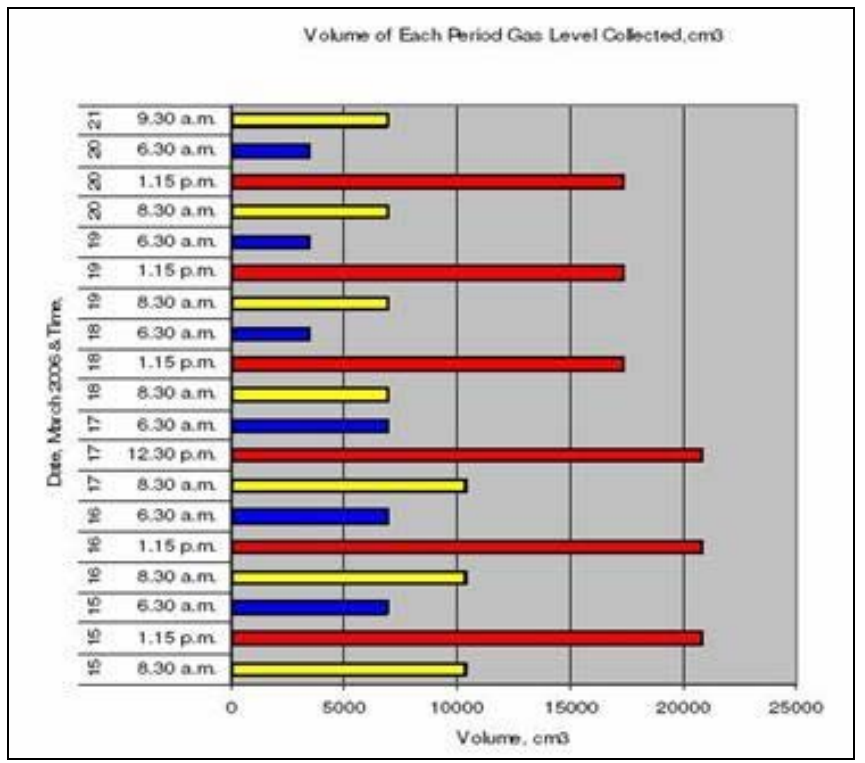

Figure 10. The recorded gas level reading (volume)

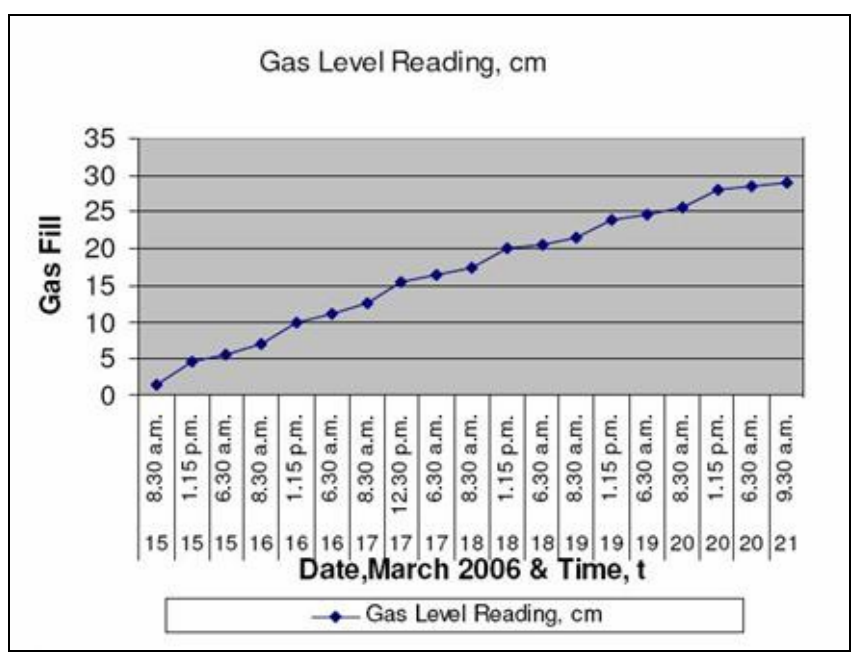

Figure 11. Graph for gas level reading versus time

\section{DISCUSSION}

The results of present study show that the ratio of a day production of methane is 1.5:3:1 for morning, afternoon and evening. Gas production in the afternoon is more rapid than 
the other period of time. This indicates that warm or hot environment induces the production of the biogas. The gas production ratio also agrees with general theory of methane gas production which is recorded most rapidly occurs between the ranges from temperatures $29^{\circ} \mathrm{C}$ to $41^{\circ} \mathrm{C}$.

On the other hand, gas yield test was conducted at day time and night time to prove that the collected gas is methane which is odourless, colourless and burnt with blue flame. Due to the source of methane that came directly from manure, it is a pure heat substance. It can be observe from the transparent flame tested during the day and blue flame during the night. Figure 12 and Figure 13 shows the attempt the test the gas during day and night time.

\section{FUTURE WORK}

The current research is to be extended to field scale and detailed studies will be conducted in the near future. The prototype developed can be tested in the field scale to verify its functional suitability. Figure 14 shows the recommended design of the biogas generator to be tested in the field scale.

\section{CONCLUSIONS}

It is a shocking finding that the awareness of proper farm waste management practices among the small-scale cattle and poultry farmers still lacking. Thus, an attractive farm waste management methodology is needed. The present study successfully introduces a simple yet functional farm waste management system that incorporates an anaerobic digester and biogas (methane) collection system which can be extended to field scale. The environmental problems in the country especially the water resources pollution can be reduced. Beside that, the proposed prototype may give benefit to all farmers and open a new research and development avenue to all engineers.

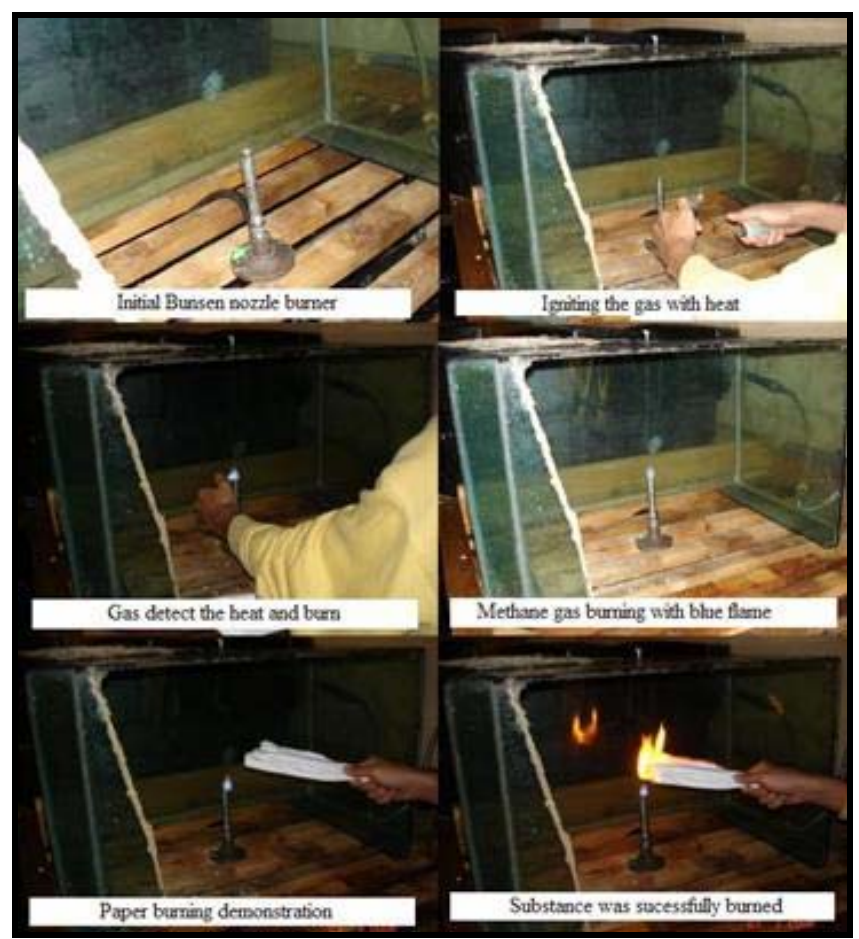

Figure 12. Gas tested during the night

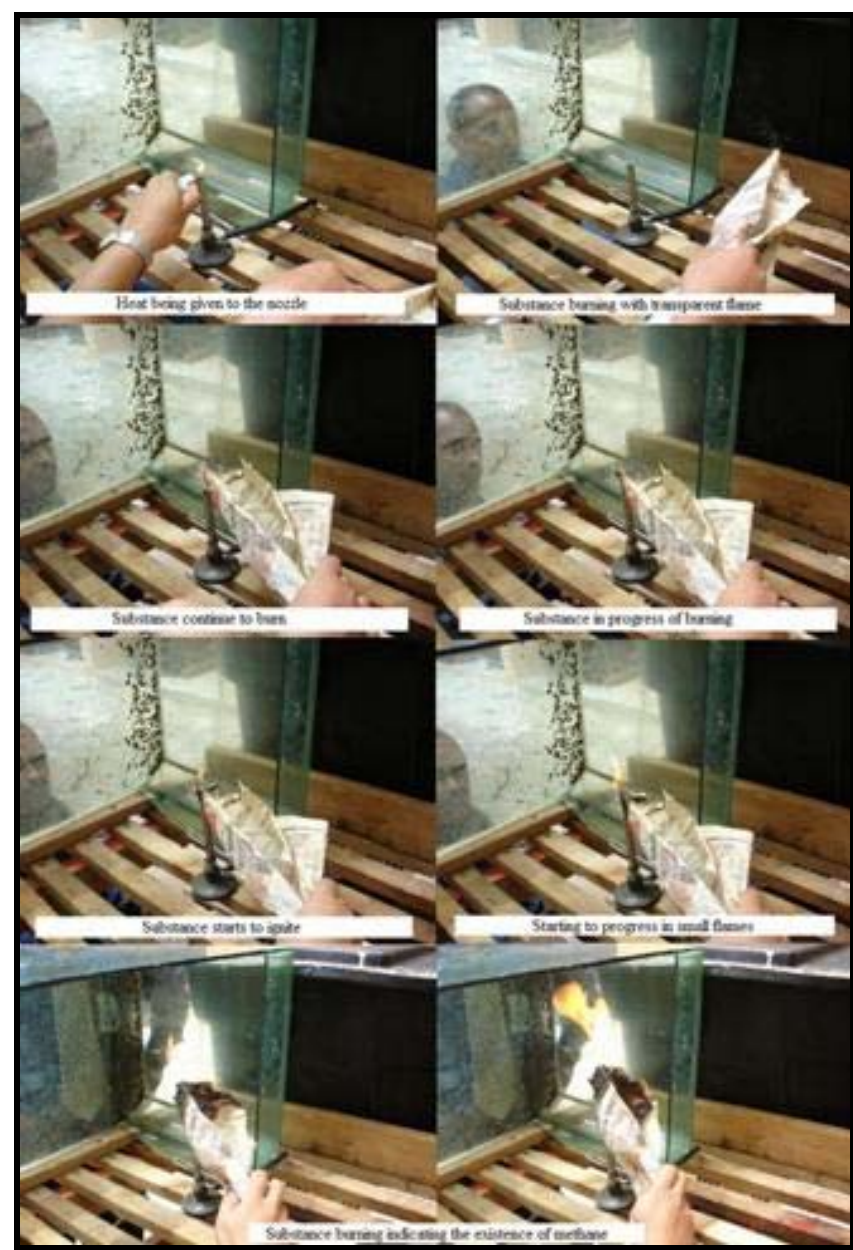

Figure 13. Gas tested during the day

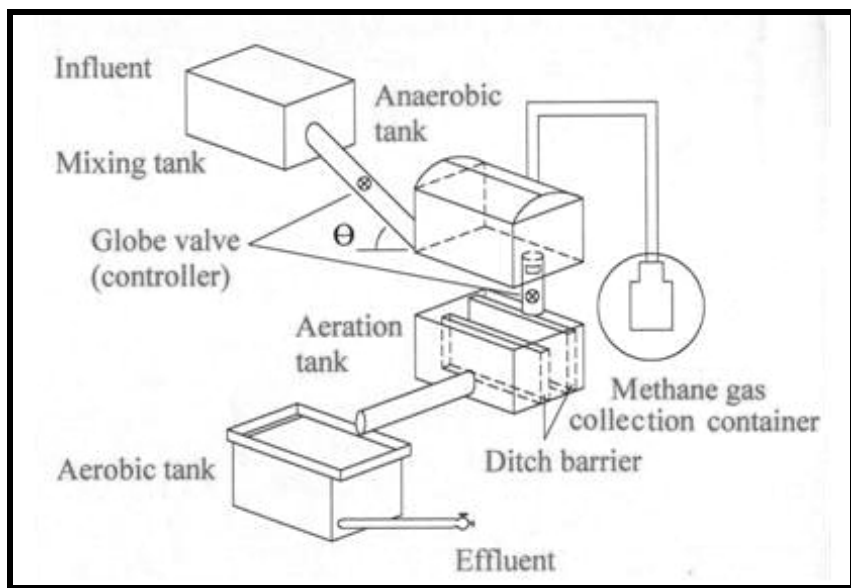

Figure 14. Proposed field scale low cost plug-flow anaerobic digester

\section{ACKNOWLEDGEMENT}

The authors would like to acknowledge the people who had directly or indirectly contributed in this research expecially from the Seberang Perai Minicipal Council, Department of Environment and Mr. Abdul Razak Din Feed Lot Farm. A special thanks due to Mr. Hairul Izwan Amri who had sponsored the recyclable model construction materials which were used in this study. 


\section{REFERENCES}

[1] Akta Kualiti Alam Sekeliling 1974 (Akta 127) dan PeraturanPeraturan dan Perintah-Perintah (2005). International Law Book Services. Malaysia.

[2] Cheremisinoff, N.P. \& Ellerbusch, R. (1980). Biomass: Applications, Technology, and Production. Marcel Decker, Inc., New York, New York, pg. 22.

[3] Davis, M. L. and Masten, S. J. (2004). Principle of Environmental Engineering and Science. Mc Graw Hill: New York.

[4] Estimating Manure Nutrients from Livestock and Poultry (webpage) http:// ianrpubs.unl.edu/wastemgt/g531.htm (accessed on 4 September 2005, 8.45 p.m.).

[5] Jabatan Perkhidmatan Haiwan (2003). Manual Perternakan Lembu Fidlot. Malaysia Ministry of Agriculture.

[6] Jabatan Perkhidmatan Veterinar Negeri Pulau Pinang (2001). Garis Panduan Penternakan Khinzir Negeri Pulau Pinang. Penang State Government. Malaysia.

[7] Jenagi, Luke (2001). Producing Methane Gas from Effluent. Adelaide University.

[8] NRCS Agricultural Waste Management Handbook (webpage) http://www.sc.nrcs.usda.gov/technical/awmbdh.html (accessed on 1 September 2005, 1.00 p.m.).

[9] Ong, H.K., Zulkifli, I, Tee T.P. and Liang J.B. (2002). Global Perspective in Livestock Waste Management. Malaysian Society of Animal Production.

[10] Rodriquez, L. \& An, B. X. (1997). Installation and performance of low cost polyethylene tube digesters on small scale farms. World Animal Review 88, pg $38-47$.

[11] Schulte, D.D. \& Lapp H.M. (1975). Methane Production from Animal Wastes. Fundamental Considerations. Canadian Agricultural Engineering 17(2).

[12] Schulte, D.D. and V.Luis. 1983. Kinetic analysis of anaerobic fermentation of dry beef cattle manure. ASAE Paper No. 83-4057. St. Joseph, Mich.

[13] The Star (webpage) http://www.ecologyasia.com/newsarchives/2003/feb-03/thestar 20030208 3.htm (accessed on 12 September 2005, 9.00 p.m.).

[14] World Health Organization (webpage) http://www.who.int/mediacentre/factsheets /fs262/en (accessed on 11 September $2005,8.30$ p.m.). 


\title{
Clathrate Hydrate Phase Equilibria for Gas Mixtures Containing Hydrogen and Carbon Dioxide in Aqueous Solutions of Water and Tetrahydrofuran
}

\author{
Khalik M. Sabil, Nadia Oujamaa, Johannes M. Bruining, Geert-Jan Witkamp, and Cor J. Peters
}

\begin{abstract}
Hydrate - aqueous liquid - vapor equilibrium conditions of hydrate formed from mixtures containing carbon dioxide and hydrogen in aqueous tetrahydrofuran solutions (5.6 mol\%) at different mixing ratios of $\mathrm{CO}_{2}$ and $\mathrm{H}_{2}$ are presented. All the phase equilibrium measurements are performed by using a Cailletet apparatus. The experimental temperature range is from 275 up to $295 \mathrm{~K}$ and the pressure range is from 1 up to $10 \mathrm{MPa}$. The addition of THF has been proven to significantly reduce the hydrate equilibrium pressure at a specified temperature. Moreover, the hydrate formed in the aqueous THF solutions is found to be more thermally stable compared to that of in water only. Also, the equilibrium conditions are dependent on the ratio of $\mathrm{CO}_{2}$ and $\mathrm{H}_{2}$ in the mixture.
\end{abstract}

Keywords: clathrate hydrate, phase equilibria, carbon dioxide, hydrogen, tetrahydrofuran

\section{INTRODUCTION}

The promising technology enhancement in fuel cells development and problems related with our dependency on fossil fuels are leading the world towards what is broadly known as the hydrogen economy. If predictions will become reality over the next several decades, we will begin to see an amazing shift from today's fossil fuel economy towards a much cleaner hydrogen economy future. One of the more interesting problems with the hydrogen economy is how to produce hydrogen on a commercial scale. There are two viable options for hydrogen production, i.e. electrolysis of water and reforming/gasification of fossil fuels. Although

K.M. Sabil is with Physcial Chemistry and Molecular Thermodynamics, DelftChemTech, Delft University of Technology, Delft 2628 BL The Netherlands (phone: +31-624839581; e-mail: k.sabil@, tudelft.nl).

N. Oujamaa was with Physcial Chemistry and Molecular Thermodynamics, DelftChemTech, Delft University of Technology, Delft 2628 BL The Netherlands.

J.M. Bruining is with Faculty of Civil Engineering and Geosciences, Department of Geotechnology, Delft University of Technology, Delft 2628 RX The Netherlands (e-mail: J.Bruining@citg.tudelft.nl).

G.-J. Witkamp is with Process \& Energy Laboratory, Delft University of Technology, Delft 2628 CA The Netherlands (e-mail: G.J.Witkamp@tudelft.nl ).

C.J. Peters is with Physcial Chemistry and Molecular Thermodynamics, DelftChemTech, Delft University of Technology, Delft 2628 BL The Netherlands (e-mail: C.J.Peters@tudelft.nl). the first option will completely remove the need of fossil fuels for energy production, it is still not feasible for mass production of hydrogen. Although slightly perverse as fossil fuel, the second option is used as the source of hydrogen for the hydrogen economy as it may offer a good temporary step during the transition to the full hydrogen economy.

In the reforming/gasification of fossils fuels, coal is gasified or natural gas is reformed to produce synthesis gas which is a mixture of $\mathrm{CO}$ and $\mathrm{H}_{2}$. Next, $\mathrm{CO}$ is converted to $\mathrm{CO}_{2}$ by the water shift reaction process. Then, $\mathrm{CO}_{2}$ can be separated from the mixture and the $\mathrm{H}_{2}$ can be collected as the main product of the process. Currently, separation of $\mathrm{CO}_{2}$ and $\mathrm{H}_{2}$ is achieved by absorption and pressure swing adsorption techniques. However, due to the high cost to perform such operations due to high pressure requirements and recycling of chemicals used, new processes are being considered. One such process is based on gas hydrate technology. The basis for the separation is the selective partition of the target component between the hydrate phase and the gaseous phase [1].

Gas clathrate hydrates are non-stoichiometric solid state inclusion compounds that are formed by a combination of water and small gas or liquid molecules. In gas hydrates, guest molecules are trapped inside the cavities of a crystal lattice of water molecules. The presence of these molecules stabilizes the crystal structure. Carbon dioxide $\left(\mathrm{CO}_{2}\right)$ is known to form structure sI hydrate at moderate pressures, typically in the range of a few MPa. Mao et al. [2] reported that hydrogen $\left(\mathrm{H}_{2}\right)$ forms structure sII hydrate at very high pressures, typically up to $200 \mathrm{MPa}$ or at very low temperature (about $80 \mathrm{~K}$ ). The presence of additives known as promoters such as tetrahydrofuran, tetrahydropyran and 1,4-dioxane can reduce the pressure required for hydrate formation [3]. Kumar et al. [4] studied the gas hydrate formation conditions of binary gas mixtures of $\mathrm{H}_{2}$ and $\mathrm{CO}_{2}$ and of ternary gas mixtures of $\mathrm{H}_{2}, \mathrm{CO}_{2}$ and propane. They found that the presence of carbon dioxide lowers the hydrate formation pressure substantially compared to that of pure carbon dioxide. Addition of propane as a third substance reduces the pressure even further. Zhang et al. [5] studied the gas hydrate formation of binary gas mixtures of $\mathrm{H}_{2}$ and $\mathrm{CH}_{4}$ in the presence of $6 \mathrm{~mol} \%$ tetrahydrofuran (THF). The results showed that the presence of THF in water drastically 
reduced the hydrate formation pressure of pure $\mathrm{CH}_{4}$ or of the $\mathrm{H}_{2} / \mathrm{CH}_{4}$ mixtures. Similarly, Florusse et al. [6] studied the $\mathrm{H}_{2}$ hydrate formation in presence of THF. They reported that hydrate clusters of $\mathrm{H}_{2}$ can be stabilized and stored at low pressure in structure sII binary clathrate hydrates in the presence of THF.

As a result, it is interesting to determine the pressuretemperature conditions that are required for $\mathrm{CO}_{2} / \mathrm{H}_{2}$ mixtures to form hydrate in presence of a promoter to decrease the hydrate formation pressure. Such information will be useful for the development of gas hydrate based processes as a potential $\mathrm{CO}_{2} / \mathrm{H}_{2}$ separation process. The objective of the present work is to present phase equilibrium data for gas hydrates formed by $\mathrm{CO}_{2} / \mathrm{H}_{2}$ mixtures in tetrahydrofuran containing aqueous solutions.

\section{EXPERIMENTAL}

The gas-rack apparatus is used for the sample preparation. The working principle of the gas rack equipment is based on the principles of communicating vessels. A schematic drawing of the gas-rack equipment is shown in Figure 1.

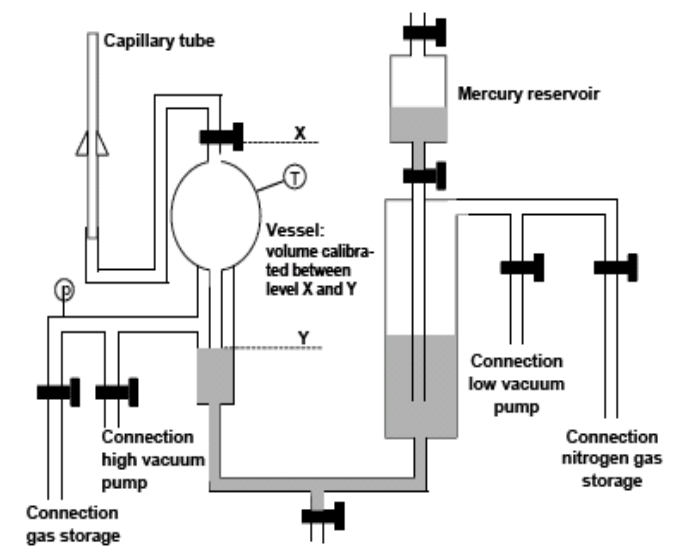

Figure 1. Schematic overview of gas-rack equipment

Firstly, a capillary tube containing a known amount of liquid component is connected to the gas-rack. Then, the sample is degassed under high vacuum conditions while the sample is kept frozen with liquid nitrogen. Additional degassing of the liquid sample is achieved by successive freezing and melting of the sample, also under high vacuum. A predetermined amount of a gas is then dosed volumetrically at known temperature and pressed into the tube using mercury. When only one gas component is needed, the tube is directly sealed with a mercury column. If more gases are needed then the procedure mentioned above is repeated before the tube is sealed with mercury.

A Cailletet apparatus is used for the measurement of the phase equilibra. The operating principle of this equipment is based on visual observation of the phases and their transitions. In Figure 2, a schematic overview of the Cailletet equipment is shown. The operating pressure range for this equipment is from 1 up to $15 \mathrm{MPa}$, while the temperature range is largely dependent on the heat transferring fluid used.

The main part of this equipment is the thick-walled capillary tube. The length of the tube is approximately 50 $\mathrm{cm}$ and the inner diameter is 2 to $4 \mathrm{~mm}$. The sample with fixed composition is located in the top of the tube, while the rest of the column is sealed with mercury and connected to a mercury reservoir. The capillary tube is placed into an autoclave, where the mercury reservoir is connected to a hydraulic oil system that can be pressurized with a screwtype hand-pump. The autoclave and the pressure generating system are filled with hydraulic oil (Shell Tellus-22 hydraulic oil). Pressure is generated hydraulically in the Cailletet equipment with a dead-weight pressure gauge (de Wit). The accuracy of the dead-weight pressure gauge is equal to the smallest weight, which is $0.005 \mathrm{MPa}$.

The stirring device consists of two magnets, each of them is located at one side of the capillary tube and a steel ball, which is present inside the capillary tube. By moving the stirring device up and down the sample is mixed homogeneously. The stirring device is driven by an electric motor, which provides a speed from 0 to $3500 \mathrm{rpm}$. A double-walled jacket is placed around the capillary tube. In this jacket, a heat-transferring fluid is circulated to keep the sample at a constant temperature. In this work ethanol is used as the heat-transferring fluid. The fluid is circulated by using a thermostatic bath (Lauda). The temperature can be controlled with an accuracy of $0.01 \mathrm{~K}$. The actual temperature is measured by using a Platinum resistance thermometer.

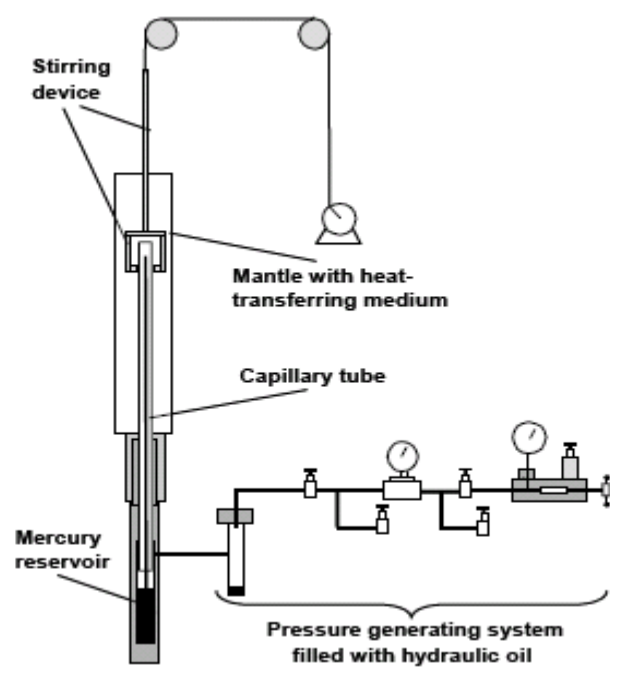

Figure 2. Schematic overview of the Cailletet equipment

For the measurement of clathrate hydrate equilibrium dissociation lines, i.e. the phase transition $\mathrm{H}+\mathrm{Lw}+\mathrm{V} \rightarrow$ $\mathrm{Lw}+\mathrm{V}$, the pressure is fixed at a constant value by means of the dead-weight pressure gauge. Subsequently, the temperature is elevated in small steps, typically at a rate of $0.5 \mathrm{~K}$ per 10 minutes, until the dissociation of the hydrate phase can be observed. Small bubbles appear around the hydrate crystals and the temperature is kept constant as long as these bubbles are observed. When the bubbles disappear and the hydrate phase is still present, the temperature is increased by small steps of $0.01 \mathrm{~K}$ until no hydrate crystals are observed anymore. The temperature where the hydrate phase disappears is taken as the phase transition temperature.

\section{RESUlTS AND DISCUSSION}

To study the effect of THF on the clathrate hydrate equilibrium pressure of the $\mathrm{CO}_{2} / \mathrm{H}_{2}$ mixed hydrate, a comparison is made between a system with and without THF. The results are presented in Figure 3. For both 
systems, the mixing ratio of $\mathrm{CO}_{2} / \mathrm{H}_{2}$ is $57.1: 42.9$ and the concentration of the THF solution is $5.6 \mathrm{~mol} \%$.

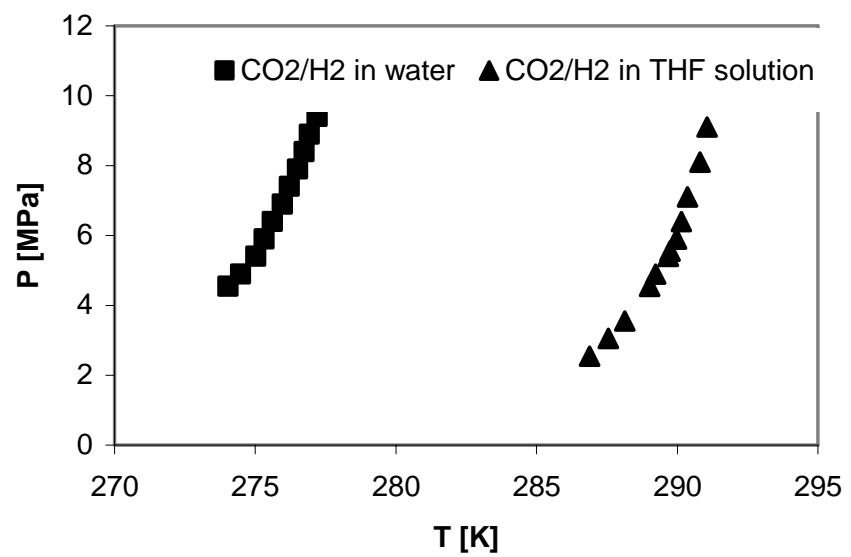

Figure 3. $\mathrm{H}-\mathrm{Lw}-\mathrm{V}$ equilibrium line for $\mathrm{CO}_{2} / \mathrm{H}_{2}$ in water and $\mathrm{CO}_{2} / \mathrm{H}_{2}$ in a $5.6 \mathrm{~mol} \%$ THF aqueous solution

As shown in Figure 3, the inclusion of THF in the hydrate formation system resulted in a drastic decrease of the equilibrium pressure. Moreover, the gas hydrate equilibrium line is significantly shifted towards higher temperatures. For example, at $5.9 \mathrm{MPa}$, the equilibrium temperature of $\mathrm{CO}_{2} / \mathrm{H}_{2}$ in water is $275.32 \mathrm{~K}$ and in the aqueous THF system the equilibrium temperature is as high as $289.98 \mathrm{~K}$. The significant decrease in pressure requirement and increase of the equilibrium temperature expand the hydrate stability region of the system. This will make the hydrate based process for separation of $\mathrm{CO}_{2}$ and $\mathrm{H}_{2}$ attractive since the process can be performed at almost ambient pressure and temperature conditions.

The experimental results obtained in this study indicate that the hydrate equilibrium pressure of the $\mathrm{CO}_{2} / \mathrm{H}_{2}$ mixture is higher than that of the pure $\mathrm{CO}_{2}$ system, as shown in Figure 4. However, the equilibrium pressure is notably lower compared to that of the THF-stabilized $\mathrm{H}_{2}$-system.

Figure 5 shows the hydrate equilibrium lines for $\mathrm{CO}_{2} / \mathrm{H}_{2}$ mixtures in $5.6 \mathrm{~mol} \% \mathrm{THF}$ for different gas mixture ratios. It is found that as the amount of $\mathrm{H}_{2}$ relative to $\mathrm{CO}_{2}$ increases in the gas mixture, the equilibrium line shifts to higher pressure for a fixed value of the temperature. The trend observed agrees well with those reported by Kumar et al. [4] for $\mathrm{CO}_{2} / \mathrm{H}_{2}$ mixtures in water and for $\mathrm{CO}_{2} / \mathrm{H}_{2} /$ Propane mixtures in water. Since $\mathrm{H}_{2}$ forms more difficult hydrate in comparison to $\mathrm{CO}_{2}$, as shown in Figure 2, higher pressures or lower temperatures are needed for the gas hydrate to form and to remain stable at the given conditions. Thus, as the amount of $\mathrm{H}_{2}$ increases in the mixture, higher equilibrium pressures can be observed for the mixture.

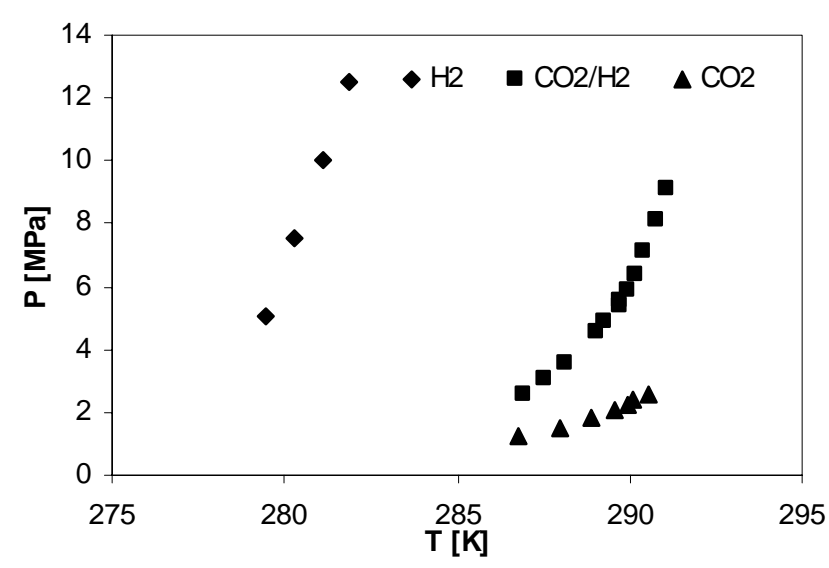

Figure 4. $\mathrm{H}-\mathrm{Lw}-\mathrm{V}$ equilibrium line for $\mathrm{H}_{2}, \mathrm{CO}_{2} / \mathrm{H}_{2}$ and $\mathrm{CO}_{2}$ hydrate system in an aqueous THF solution ( $5.6 \mathrm{~mol} \%)$

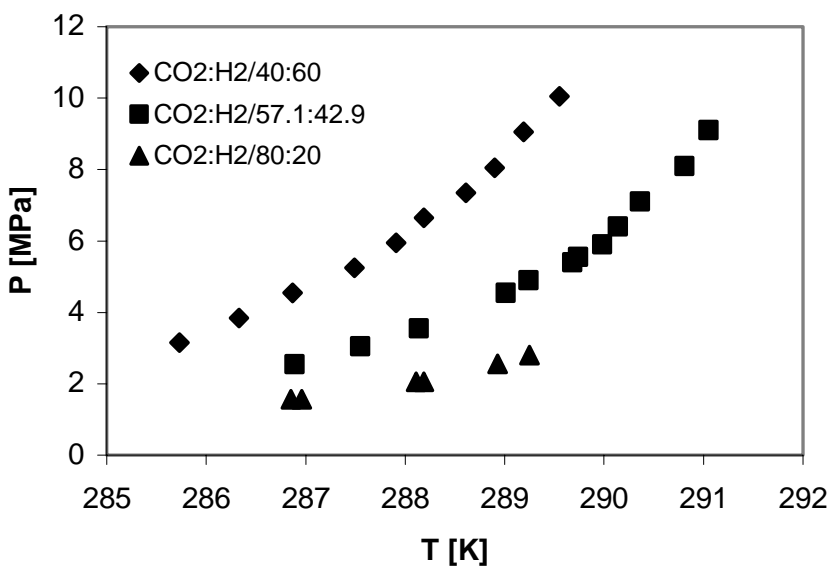

Figure 5. H-Lw-V equilibrium line for various $\mathrm{CO}_{2} / \mathrm{H}_{2}$ mixtures in $5.6 \mathrm{~mol} \%$ THF aqueous solutions

\section{CONCLUSIONS}

The H-Lw-V equilibrium line for various $\mathrm{CO}_{2} / \mathrm{H}_{2}$ mixtures in $5.6 \mathrm{~mol} \%$ THF aqueous solutions is determined. The results show that addition of THF in the systems gives a significant equilibrium pressure reduction along with an increase of the equilibrium temperature of about $10 \mathrm{~K}$. The results also demonstrate that the gas hydrate equilibrium pressures are substantially lower than that of the THFstabilized $\mathrm{H}_{2}$-system and slightly higher than that of the corresponding $\mathrm{CO}_{2}$-system. Also in was established that the equilibrium conditions depend on the mixing ratio of $\mathrm{CO}_{2} / \mathrm{H}_{2}$.

\section{REFERENCES}

[1] S,-P. Kang, H. Lee, Environ. Sci. Technol. 34 (2000) pp.4397-4400.

[2] W.L. Mao, H.K. Mao, A.F. Goncharov, V.V.Struzhkin, Q.Z. Guo, J.Z. Hu, J.F. Shu, R.J. Hemley, M. Somayazulu, Y.S. Zhao, Science 297, (2002) pp. 2247-2249.

[3] M.D. Jager, R.M. de Deugd, C.J. Peters, J. de Swaan Arons, E.D. Sloan, Fluid Phase Equilibr. 165 (1999) 209-223.

[4] R. Kumar, H.-J. Wu, P. Englezos. Fluid Phase Equilibr. 244 (2006) pp. 167-171.

[5] S.X. Zhang, G.J. Chen, C.F. Ma, L.Y. Yang and T.M. Guo, J. Chem. Eng. Data 45 (2000), pp. 908-911.

[6] L.J. Florusse, C.J. Peters, J. Schoonman, K.C. Hester, C.A. Koh, S.F. Dec, K.N. Marsh and E.D. Sloan, Science 306 (2004), pp. 469-471. 


\title{
Thermodynamic Analysis of a Closed Rankine Cycle Solar Power Generator
}

\author{
Rajamohan Ganesan
}

\begin{abstract}
An alternative method of heat extraction from solar energy resource is investigated with the use of Flat Plate Solar Collector for Closed Rankine Cycle Solar Power Generator. The system uses the concept of harnessing power generation from solar heat source. The closed Rankine power cycle configuration consists of evaporator, turbine, condenser, feed pump and R-134A is used as the working fluid. The temperature gradient of the Closed Rankine Cycle Solar Power system is utilized to run the turbine and generates mechanical energy. The turbine is placed in between the evaporator and condenser section. The solar energy collected in the solar collector is considered as the heat source for evaporator and the water acts as heat sink for the condenser. An attempt is made to analysis the thermodynamic aspects of the cycle and the net work generations by the system are elaborated. The report presents the analysis of an alternative method of heat extraction from solar energy thermal resource is investigated with the use of solar flat plate collector.
\end{abstract}

Keywords: renewable energy, flat plate solar collector, refrigerant, Rankine cycle, low-grade heat source

\section{INTRODUCTION}

Solar energy plays an important role towards achieving long lasting, sustainable, environment friendly renewable energy resources to fulfill the energy needs for mankind. The use of non-renewable fuels, such as fossil fuels has many side effects. Their combustion products produce pollution, acid rain and global warming. Conversion to clean energy sources such as solar energy would enable the world to improve the quality of life throughout the planet. Therefore, extensive research and development in solar energy utilization technologies must be carried out.

In this system, a liquid working fluid is vaporized in an evaporator (solar collector tubes); the heat required for vaporization is transferred from the solar flat plate collector by means of a heat exchanger. The high-pressure vapor leaving from the evaporator drives an expansion turbine, similar to a steam turbine that is designed to operate at a lower inlet pressure. The turbine is connected to an electric generator in the usual manner. The low-pressure exhaust from the turbine is cooled and converted back into the liquid in the condenser. The cooling is achieved by passing cold water through a heat exchanger. The liquid working fluid is

Rajamohan Ganesan is with School of Engineering and Science, Curtin University of Technology, Sarawak Campus, CDT 250, 98009, Miri, Sarawak, Malaysia (E-mail: rajamohan.g@curtin.edu.my). then pumped back as high-pressure liquid to the evaporator, thus closing the cycle [3].

There are many advantages of using solar based power plants from ref [2], a few benefits are noted such as, no pollution, it is renewable energy resource, there are no costs associated with fuel, the low operating temperatures and pressure reduces the component costs and disadvantages of using solar based power plants, it has a day time potential. The report presents the analysis of an alternative method of low-grade heat extraction from solar energy thermal resource, which is investigated with the use of solar flat plate collector.

\section{RENEWABLE ENERGY RESOURCES}

The daily average insolation in Sarawak is $4.85 \mathrm{kWh} / \mathrm{m}^{2}$ as shown in figure 1 [9]. Per day minimum insolation is 4.32 $\mathrm{kWh} / \mathrm{m}^{2}$ in December and the maximum is $5.342 \mathrm{kWh} / \mathrm{m}^{2}$ in April. The figure also shows that there is a slight variation of the insolation over the year. This indicates that the electrical power output from solar is stable throughout the whole year and Malaysia is located in the moderately feasible belts for solar application. As such solar energy is the preferable choice when compared to other sources of energy such as wind and ocean energy. Therefore, solar energy source is considered as the renewable component in this design.

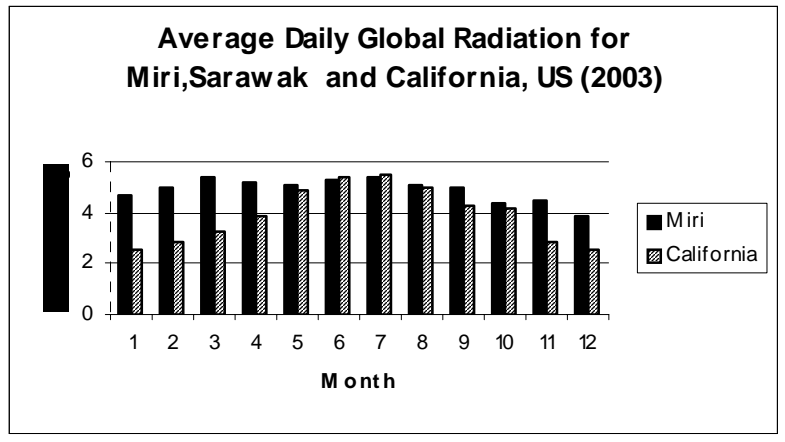

Figure 1. Average Daily Solar Radiation for Miri and California

\section{Flat Plate SOlar COLlector}

Figure 2 shows the schematic diagram of a solar collector. A flat-plate collector consists of an absorber, a transparent cover, a frame, and insulation. Usually an iron-poor solar safety glass is used as a transparent cover, as it transmits a great amount of short-wave light spectrum. Simultaneously, 
only very little of the heat emitted by the absorber escapes from the cover (greenhouse effect). In addition, the transparent cover prevents wind and breezes from carrying the collected heat away (convection). Together with the frame, the cover protects the absorber from adverse weather conditions. Typical frame materials include aluminum and galvanized steel; sometimes fiberglass-reinforced plastic is used [10]. The insulation on the back of the absorber and on the side walls lessens the heat loss through conduction. Flat collectors demonstrate a good price-performance ratio, as well as a broad range of mounting possibilities.

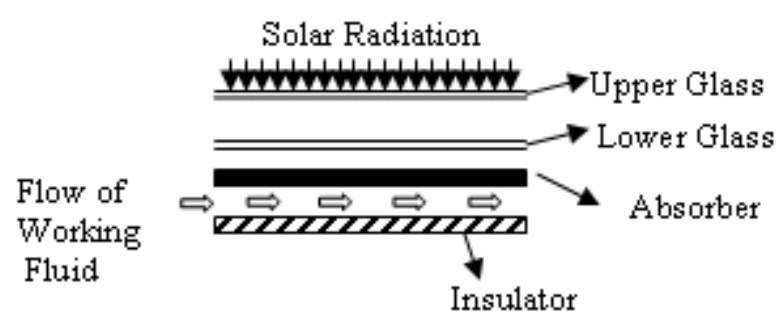

Figure 2. Schematic Diagram of Solar Collector

\section{Closed Rankine Cycle Solar Power Generator}

The heat transfer mechanism that drives a closed Rankine power cycle is the recirculation of a working fluid through a cycle of evaporation, vapor transfer, condensation, and liquid return [4]. The power cycle gets the energy from absorbing the heat from the solar flat plate collector. The heat that is absorbed increases the temperature of a working fluid inside of the solar collector tubes and causes the working fluid to evaporate at an elevated pressure. The less dense vapor then rises. As the vapor is rising, it is expanding from a higher pressure to a lower pressure. Useful work can be extracted from this expansion process. As the working fluid flows from the evaporator to the condenser in the vapor phase, the kinetic energy of the working fluid is increased. When a turbine and generator are incorporated into the high velocity vapor stream, electric power can be extracted. When the vapor passes through the turbine it loses kinetic energy and the pressure and temperature of the vapor decreases. This leads to condensation of the vapor back to liquid. The working fluid returns back to the pump of the Rankine cycle through a return tube [4].

A solar Thermal Energy conversion using closed Rankine power cycle is schematically shown in figure 3. In this system heat is transferred from the solar flat plate collector to the working fluids refrigerant in an evaporator. Due to working fluids low boiling point, it becomes a saturated vapour at state 3 expands, which turns a turbine attached to a generator for producing electricity. The vapour at state 4 is then sent to the second heat exchanger (condenser) where heat is transferred from the vapour to cold water and thus working fluids returns to a saturated liquid at state 2 back to the first heat exchanger where the process is repeated [2].

The system analyzed here is a minimal amount of power generation plant, which assumes a source of heat from solar collector and cold water entering and exiting temperature of $24^{\circ} \mathrm{C}$ and $18^{\circ} \mathrm{C}$. Also temperature differences across both heat exchangers are modeled as isobaric (both hot and cold side). In addition, the heat exchangers are assumed to have an overall coefficient (U) of $1.2 \mathrm{Kw} / \mathrm{m}^{2} \mathrm{~K}$ [2].

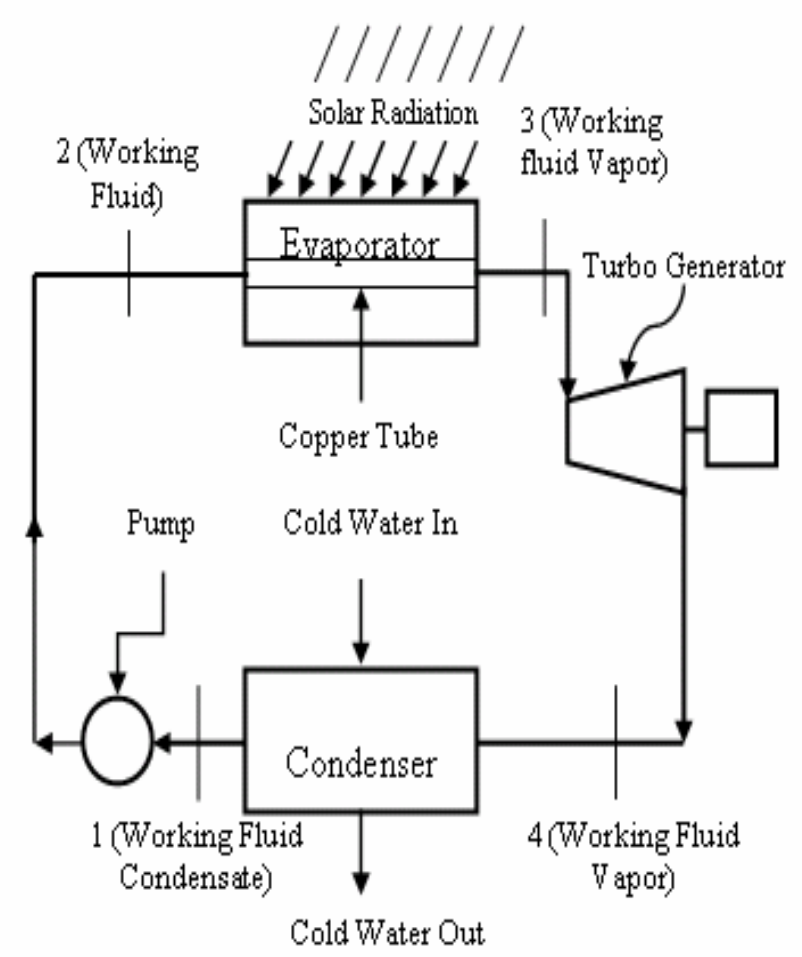

Figure 3. Schematic Diagram of a Closed Rankine Cycle Solar Power Generator

\section{Useful heat gain}

The performance of flat-plate solar collector could be expressed by an energy balance that indicates the distribution of incident solar radiation into the useful energy gain and thermal losses. In order that the performance of the solar collector be as high as economically practical design and operating factors that increase the solar heat flux absorbed and reduce the thermal losses are determined. Under steady condition, the useful heat collected by solar collector can be stated below [11].

Heat collected $=$ Energy absorbed - Heat losses

$Q_{c}=A_{c}\left[H R(\tau \alpha)-U\left(T_{p}-T_{a}\right)\right]$

The collector efficiency is, $\eta=Q_{c} / A_{c} H R$

An attempt is made to derive the mathematical equations which may be used to describe the thermal performance of flat-plate solar collector of the tube-and-sheet type. Temperature distribution between any two tubes across the width of the plate is considered as the classical fin problem. The useful heat gain is the heat transfer rate to the fluid after considering all resistances and heat losses.

Total heat transfer rate from the plate for a fin of length $L$, is,

$Q_{U}=L[(W-D) F+D]\left[S-U\left(T_{b}-T_{a}\right)\right]$

Introducing the collector plate efficiency factor, $F_{p}=1 /\left(U W L\left(r_{c}+r_{b}+r_{t}+r_{s}+r_{f}\right)\right)$

The useful heat gained by the fluid obtained is, $Q_{u}=W L F_{p}\left(S-U\left(T_{f}-T_{a}\right)\right)$

Useful heat gain collection efficiency is, 
$\eta=Q_{u} / A H R$

\section{R-134 as the working fluid for the Rankine cycle}

Water is commonly used as the working fluid in a Rankine power cycle, due to its environmental acceptability. In this investigation, R-134A is used as working fluid because of its superior high thermal efficiency and conductivity [4]. The cycle is assumed to be an idealized Rankine cycle. The figure 4 shows the different steps of the Rankine cycle according to Nguyen et al. [5]. The first process (From 1-2) consists of adiabatic compression of the liquid. During the second process (From 2-3) heat is added isobarically to convert the liquid to a vapor. The third process (From 3-4) consists of adiabatic expansion of the vapor to lower pressure. During the fourth process (From 41) there is isobaric heat rejection that condenses the vapor back to a liquid.

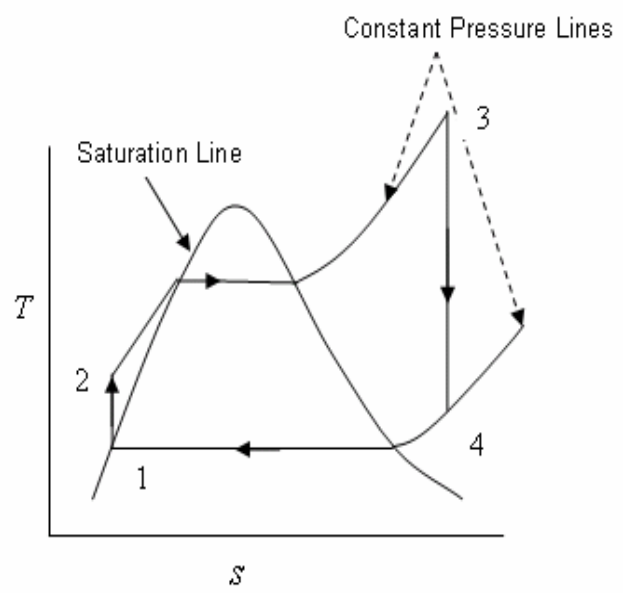

Figure 4. Ideal Rankine Cycle T-s Diagram

The above four processes are repeated over and over again to produce the Rankine cycle. The state point process steps are as follows.

\section{State 1-2}

(Reversible adiabatic pumping process in the pump)

$\mathrm{T}_{1}=20^{\circ} \mathrm{C}$ (saturated liquid)

$\mathrm{P}_{1}=571.59 \mathrm{kPa}$ [from table]

$\mathrm{h}_{1}=227.4 \mathrm{~kJ} / \mathrm{kg}$

Form state 1 to state 2, Specific Entropy remains constant

$\mathrm{S}_{1}=\mathrm{S}_{2}=1.096 \mathrm{~kJ} / \mathrm{kg} . \mathrm{K}$

$\mathrm{P}_{2}=1600 \mathrm{kPa}$

$w_{\text {Pump }}=37.054 \mathrm{~kJ} / \mathrm{kg}$

$\mathrm{h}_{2}=264.45 \mathrm{~kJ} / \mathrm{kg}$

State 2-3

Heat supplied from evaporator at constant temperature to change state 2 into saturated R-134a at constant pressure.

$q_{\text {in }}=161.80 \mathrm{~kJ} / \mathrm{kg}$

Since $\mathrm{P}_{2}=\mathrm{P}_{3}=1600 \mathrm{kPa}$

Assumed volume flow rate

$\mathrm{V}=0.02 \mathrm{~m}^{3} / \mathrm{s}$

Energy needed $=267.05 \mathrm{~W}$.

\section{State 3-4}

Isentropic expansion of the vapor across the turbine, including partial condensation.

Energy from the solar collector $=161.809 \mathrm{~kJ} / \mathrm{kg}$

$\mathrm{S}_{3}=\mathrm{S} 4=1.7046 \mathrm{~kJ} / \mathrm{kg} . \mathrm{K}$
$W_{\text {turb }}=53.69 \mathrm{~kJ} / \mathrm{kg}$

$W_{\text {net }}=16.64 \mathrm{~kJ} / \mathrm{kg}$

State 4-1

Complete condensation in the condenser at constant temperature.

$h_{4}=372.565 \mathrm{~kJ} / \mathrm{kg}$

$q_{R}=145.165 \mathrm{~kJ} / \mathrm{kg}$

$\eta_{t h}=10.28 \%$

\section{Formulae}

From the state point enthalpies, the specific heat transferred to the working fluid in the boiler and the specific heat transferred from the working fluid in the condenser are found using the following formulas and assumptions.

Assumptions:

Warm water $=24^{0} \mathrm{C}$

Cooling water $=18^{0} \mathrm{C}$

Tubes required for condenser

$N=\frac{A \times 10^{5}}{(\pi d) L}=\frac{1.1538 \times 10^{5} \times 10^{-5}}{\pi(0.05) 10}=3.64$

So 4 tubes are needed.

Heat added $(\mathrm{kJ} / \mathrm{kg}) \quad q_{\text {in }}=h_{3}-h_{2}$

Heat rejected $(\mathrm{kJ} / \mathrm{kg}) \quad q_{R}=h_{4}-h_{1}$

Turbine work $(\mathrm{kJ} / \mathrm{kg}) \quad W_{\text {turb }}=h_{3}-h_{4}$

Pump work $(\mathrm{kJ} / \mathrm{kg}) \quad W_{\text {pump }}=h_{2}-h_{1}$

Cycle Network $(\mathrm{kJ} / \mathrm{kg}) \quad W_{n e t}=W_{\text {turb }}-W_{\text {pump }}$

Thermal Efficiency

$$
\eta_{\text {thermal }}=\frac{W_{\text {net }}}{q_{\text {in }}}=\frac{\left[h_{3}-h_{4}\right]+\left[h_{2}-h_{1}\right]}{\left[h_{3}-h_{2}\right]}
$$

Mass flow rate of cold water $(\mathrm{kg} / \mathrm{s})$ entering inside the condenser

$$
m_{\text {cold }}=\frac{Q_{\text {cond }}}{\left[C p_{\text {water }}\left(T_{c 2}-T_{C 1}\right)\right]}
$$

\section{Nomenclature}

$R \quad$ Refrigerant

$R-134 a$ Tetrafluroethane

$h \quad$ Enthalpy at specific point

$T$ Temperature of specific Point

Subscripts

$1,2,3,4$ refer to the specific points $1,2,3,4$ respectively

$P \quad$ Pressure of specific point

$v$ Specific volume

$\mathrm{V}$ Volume flow rate

$R$ Conversion factor

$A_{c} \quad$ Area of collector, $m^{2}$

$H$ Radiation incident rate, $W / m^{2}$

$S \quad$ Solar heat flux, $W / \mathrm{m}^{2}$

$U$ Overall heat loss coefficient, $W / m^{2} K$

$W$ Tube pitch, $m$ 
$Q_{U} \quad$ Rate of heat energy at bond-sheet, $W$

$Q_{u}$ Rate of useful heat gain, $W$

$T_{p} \quad$ Plate temperature, ${ }^{\circ} \mathrm{C}$

$T_{a}$ Ambient temperature, ${ }^{\circ} \mathrm{C}$

$T_{f} \quad$ Fluid temperature, ${ }^{\circ} \mathrm{C}$

\section{CONCLUSION}

The analysis used in this paper is an alternative method of low-grade heat extraction from solar energy resource with the use of closed Rankine cycle. It has been concluded that the generation of maximum thermal efficiency of $10.28 \%$ can be achieved by using the effective Closed Rankine Cycle Solar Power Generator. To work out minimal amount of power generation from solar energy source, a Closed Rankine Cycle Solar Power Generator system is an extremely attractive option. Further investigations are needed in order to test sensitivity of the theoretical efficiency gain from solar temperature gradient heat extraction to move practical assumptions in these areas.

\section{ACKNOWLEDGEMENTS}

Authors are very much thankful to A/Prof Chua Han Bing, A/Prof Kaniraj Jeyachandran Shenbaga and Dr Khaled, Senior Lecturer, School of Engineering and Science, Curtin Sarawak Malaysia for their constant support and valuable guidance to carryout this research.

\section{REFERENCES}

[1] Patrick.K.Takahashi and Andrew Trenka, Ocean Energy conversion its promises as a total resource system, Pergamon Press Ltd, UK, 1998

[2] $\mathrm{Wu}$ and T.J.Burke, Intelligent computer aided optimization on specific power of an OTEC Rankine power plant, Elsevier Science Ltd, UK, 1998

[3] G.D.Rai, Non-Conventional Energy Sources, Khanna Publishers, India, 2002

[4] A.Akbarzadeh, P.Johnson, T.Nguyen, M.Mochiziki, M.Mashiko, I.Sauciuc. S.Kusaba, H.Suzuki, Formulation and analysis of the heat pipe turbine for production of power from renewable sources, Journal of applied thermal engineering, Elsevier Science Ltd, UK, 2001

[5] T.Nguyen, P.Johnson, A.Akbarzadeh, K.Gibson, M.Mochiziki, Design, Manufacture and testing of a closed cycle thermosyphon engine, Journal of Heat Recovery System and CHP 15 (4) (1994) 333.

[6] A.S.Domkundwar, Power plant Engineering, Dhanpat Raj \& Sons, India, 2000

[7] http://www.solarsavers.co.uk/intro.asp\#Flat\%20plate\%20collectors

[8] W.haaf, Solar Chimney, Part II, Preliminary test result from the pilot plant Manzanares, Solar energy, Volume 2, PP 141-161, 1983

[9] K.Ghosh, Measurement of wind velocity created by a solar chimney and hybridization of wind and solar thermal power, British wind energy association, Indian institute of technology, Kanpur 208016, India

[10] Hazami M, Kooli S, Lazaar M, Farhat A \& Belghith A 2005, 'Performance of a solar storage collector' Elsevier, Tunisia, 2001

[11] Sen Z, 'Solar energy in process and future research trends', Science Direct, pp 367-416, 2004. 


\title{
Removal of Silt and Clay Particles from Water using Inclined Circular Coalescence Plates Oil
}

\author{
L.H. Ngu, K.K. Wong, Y.W. Oon, P.L. Law and A.A.R. Pgn Hj Yuosf
}

\begin{abstract}
A novel circular separation tank with inclined coalescence plates was recently developed, and performance tests were carried out to determine its efficiency in removing suspended solids from wastewaters. The primary component of the separator consists of series of inverted and upright frustums-shaped coalescence plates to form a multiple angle plate arrangement for enhance gravity separation and coagulation of suspended solid particles. It applies the principle of Boycott effect whereby the presence of inclined frustums medium promotes faster settling of suspended solid particles and their coagulation. The separator takes the shape of a circular tank to take advantage of the continual decreasing in horizontal velocity, $v_{h}$ as surface area increases. It is designed with a center-feed vertical perforated-pipe distributor inlet directing flow to the inclined coalescence conical frustums to provide co-current flow. The effluent Total Suspended Solids, TSS Concentration, $C_{e}$ was found to be directly proportional to influent flowrate, $Q_{i}$. The influent flowrate, $Q_{i}$ can be expressed as a function of effluent TSS concentration, $C_{e}$ for different influent TSS concentration, $C_{s s}$ by a series of power equations. These power equations were used to predict the required flowrate, $Q$ for a given influent TSS concentration, $C_{s s}$ to achieved desired effluent TSS concentration, $C_{e}$ of $50 \mathrm{mg} / \mathrm{L}$ (Class II) and $150 \mathrm{mg} / \mathrm{L}$ (Class III) to meet environmental discharge requirement under the Proposed Interim National Water Quality Standards for Malaysia. Power equation $Q_{50}=3423.6 C_{s s}^{-2.8525}$ and $Q_{150}=$ $63688 C_{s s}{ }^{-2.9533}$ express the required flowrate needed to achieved effluent TSS concentration, $C_{e}$ of $50 \mathrm{mg} / \mathrm{L}$ and $150 \mathrm{mg} / \mathrm{L}$ respectively. The separation tank smallest particle size removable, $d$ can be predicted for different influent flowrate, $Q_{i}$ using power equation $d=85.664 Q_{i}{ }^{0.7338}\left(\mathrm{R}^{2}=9448\right)$. The separator can separated solids particles of $2.92 \mu \mathrm{m}$ and above at design flowrate of $1.0 \times 10^{-5} \mathrm{~m}^{3} / \mathrm{s}$.
\end{abstract}

Keywords: voltage sag, clearing time, sag severity, sensitive load

\section{INTRODUCTION}

Silts in the form of suspended solids, dissolved solids and turbidity in wastewater are result of erosion due to land clearing and earthworks, i.e. clearing of forests and other vegetation for agricultural activities, for urban and infrastructure development and logging of timber. Malaysia

L.H. Ngu and K.K. Wong are PhD candidates with the Department of Civil Engineering, UNIMAS, 94300, Kota Samarahan, Sarawak, Malaysia (email: lockhei@yahoo.co.uk).

P.L. Law and A.A.R. Pgn Hj Yuosf are Lecturers with Faculty of Engineering, UNIMAS, 94300, Kota Samarahan, Sarawak, Malaysia (Tel: 6082-583282, email: puonglaw@feng.unimas.my).
Environmental Quality (DOE) Report 2004 reported that $25.8 \%$ of Malaysia's river basins are polluted by suspended solids due to earthworks and land-clearing activities. Suspended solids can lead to the development of sludge deposits and anaerobic conditions when untreated wastewater is discharged in the aquatic environment (Tchobanoglous et al., 2004).

Under the Interim National Water Quality Standards for Malaysia the amount of total suspended solids for Class II (water bodies of good quality which required conventional treatment as water supply) and Class III (defined with primary objective of protecting common and moderately tolerant aquatic species of economic value and required extensive treatment as water supply) is $50 \mathrm{mg} / \mathrm{L}$ and 150 mg/L respectively. For Interim Marine Water Quality Standards $50 \mathrm{mg} / \mathrm{L}$ or less of TSS is allowable.

\section{SEPARATION TANK DESIGN}

A circular phase separation tank with inclined coalescence plates was recently developed by Law et al. (2006) to removed physically emulsified and free oil from wastewaters. The separation tank separation techniques can also separate solids from water and hence the separation tank suspended solids removal efficiency was evaluated through a series of performance test. The separation tank (1) required flowrate, $Q$ to achieve desirable effluent TSS concentration, $C_{e}$ of $50 \mathrm{mg} / \mathrm{L}$ and $150 \mathrm{mg} / \mathrm{L}$ for different influent TSS concentration, $C_{s s}$ and (2) smallest droplets size separable at design flowrate were to be determined.

The primary component of the separation system consists of a series of inverted and upright frustums (Fig. 1) to enhance gravity separation and coagulation of suspended solid particles in wastewaters. It applies the principle of Boycott effect whereby the presence of inclined frustums medium promotes faster settling of solid particles and coagulation of solid particles (Rensselear, 2002). The separator comes in the shape of a circular basin by taking advantage of the continual decrease in horizontal velocity, $v_{h}$ as surface area increases (Deininger et al., 1996). The inlet of the separation system is equipped with an upflow center-feed perforated-pipe distributor to direct flow to the parallel inclined frustums to enhance solid-water separation. 


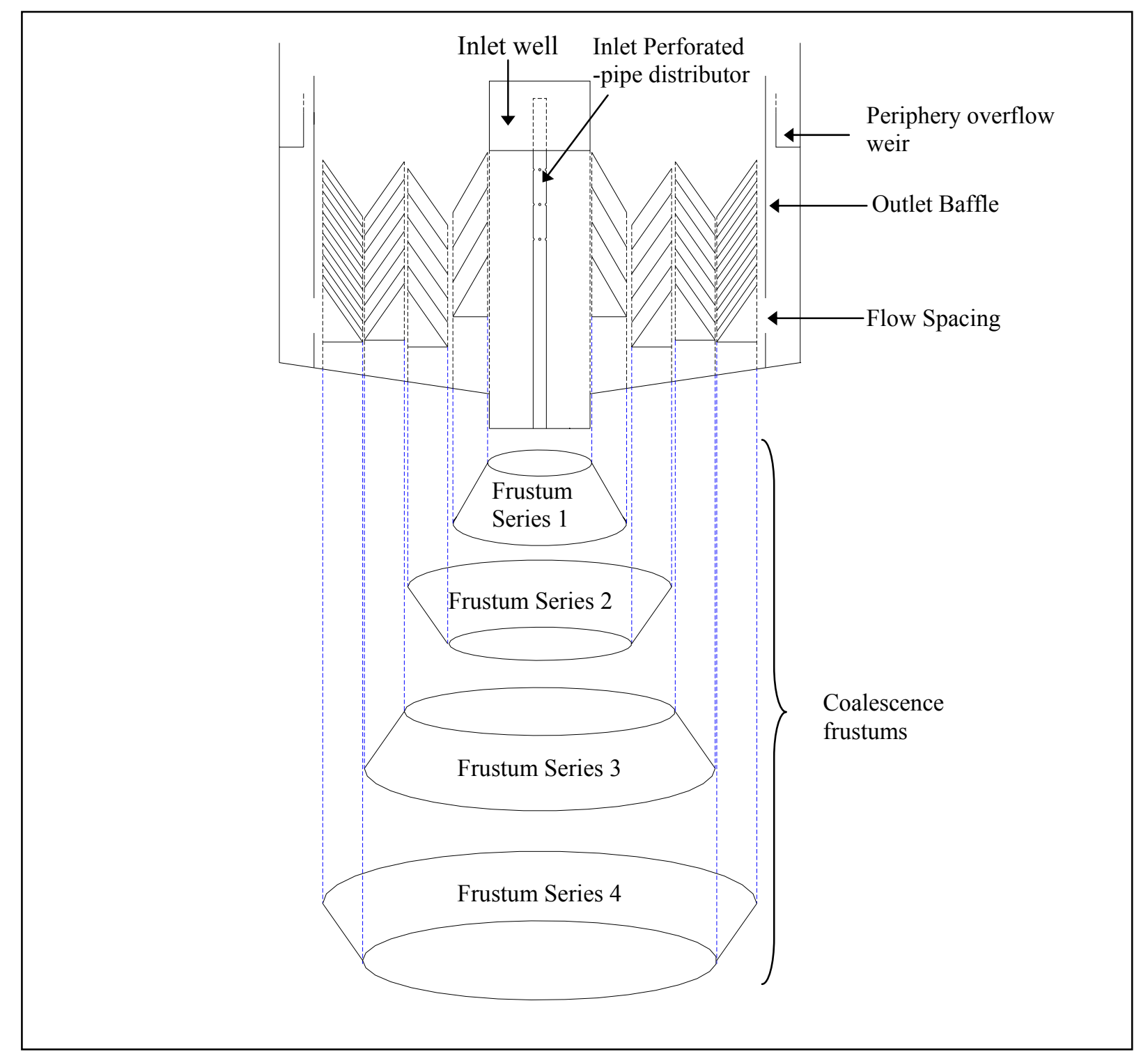

Figure 1. Circular phase separation tank with parallel inclined coalescence frustums (consisting of up-right and inverted series of conical frustums)

\section{A. Applicability of Coalescence Frustums}

The installation of embedded successive layers of parallel coalescing frustums was expected to serve two purposes, i.e., 1) to promote laminar flow, and 2) to promote optimum solid-water separation efficiency. By using the principles of a) a maximum amount of frustum surface area provided for solid particles coagulation to take place, and b) a minimum distance (spacing inversely proportionally to distance from inlet) for heavier solid particles to settle and hit the top side of the frustums as shown in Fig. 2. The coalescing frustums assist solid particles to coagulate and settle to the bottom to be collected.

The use of coalescence frustum facilitates the capture of solid particles from water, and easy removal of settle solid from the frustums to the bottom of the tank. Settled solid spreads on the surface of the frustums and coagulates into larger particles and eventually forms a film of sludge on the frustums. It was necessary, having captured the solid on the frustums, to remove it from the frustums in an orderly manner that does not re-entrain the solid into the wastewater stream. The design of coalescence frustums was such that coagulated particles were required to travel $10 \mathrm{~cm}$ (maximum) before they encountered a sludge port. These sludge ports were vertically aligned so that when the solid particles are released from the frustums, they settle directly to the bottom.

\section{EXPERIMENTAL PROCEDURE}

This section outlines the research areas and experimental procedure of carrying out the performance tests of the system in an attempt to determine its efficiency and capability for removal of suspended solids from wastewaters.

\section{A. Mixing Tank Set up and Sample Preparation}

The primary function of the mixing tank in this experimental setup was to mix silt and clay with water to form influent of known TSS concentrations and to pump the mixture at designed flowrate as influent to the separation system. The mixing tank was designed as a typical agitation process vessel or a cylindrical vessel with a vertical axis at a 
capacity of 200 liters (L). The mixing tank is intended as a rapid mixing tank using mechanical agitators to impart power to the water to produce high shear, turbulence and velocity gradient, $G$. The mixing tank was designed and made from fiberglass material. This mixing tank has a submersible pump at the bottom of the tank and a single impeller shaft system installed with a $0.25 \mathrm{HP}$ motor as shown in Fig. 3.

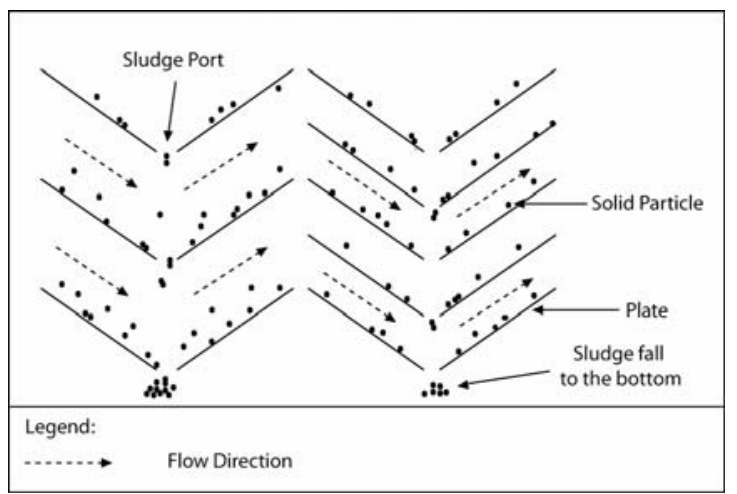

Figure 2. Parallel inclined coalescing frustums: mechanism of solid-water separation

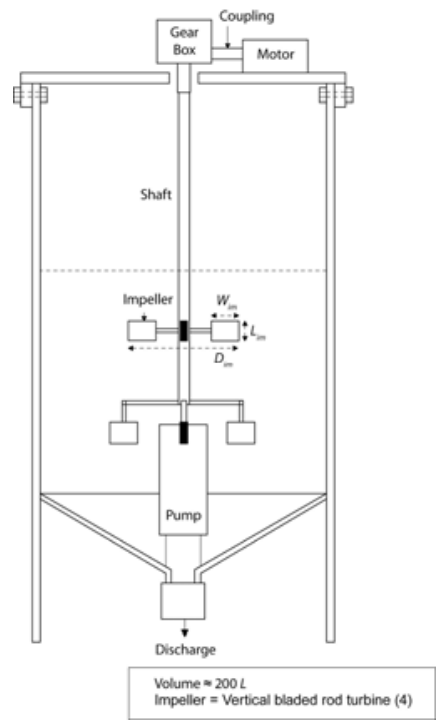

Figure 3. Mixing tank equiped with bladed turbine and submersible pump

A volume of 200 liters of silt/clay in water mixture at known concentrations were prepared with sieved silt and clay passing through $0.063 \mathrm{~mm}$ sieve and specific gravity of 2.72 (Geospec, 2007). Mixing tank was filled with $200 \mathrm{~L}$ of water and silt and clay as illustrated in Table 1, and the water and silt/clay was mixed for 5 minutes by switching on the motor-impeller system. The mixture is continually mixed for 2 minutes in every interval of 10 minutes to maintain the suspended solids concentration and mixture. A schematic diagram of the separation system and mixing tank connected in series is illustrated in Fig. 4.

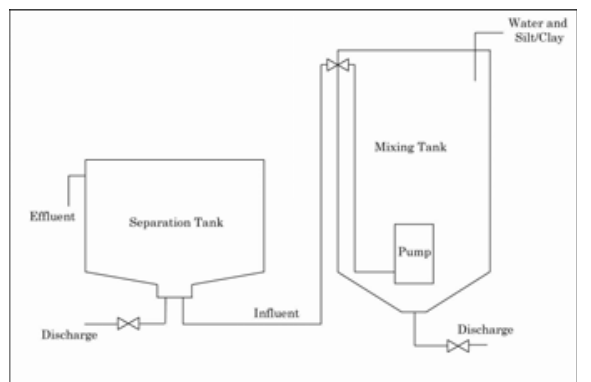

Figure 4. Schematic diagram of mixing and separation system

\section{B. Suspended Solids Removal Efficiencies}

Separation tank suspended solids removal efficiency and capability would vary with different influent flowrates, $Q_{i}$, influent Total Suspended Solids (TSS) concentrations, $C_{s s}$, and separation tank retention times, $t$. Different influent TSS concentrations tested were $100 \mathrm{mg} / \mathrm{L}, 150 \mathrm{mg} / \mathrm{L}, 200$ $\mathrm{mg} / \mathrm{L}, 250 \mathrm{mg} / \mathrm{L}, 300 \mathrm{mg} / \mathrm{L}, 350 \mathrm{mg} / \mathrm{L}$ and $400 \mathrm{mg} / \mathrm{L}$, which were 1.33 to 8 times higher than the desired separator effluent concentration of $50 \mathrm{mg} / \mathrm{L}$ and $150 \mathrm{mg} / \mathrm{L}$. In this research study, a total of twenty eight (28) tests were carried out, and the details are illustrated in Table 2.

Influent TSS concentration of Test 1 as stated in Table 2 (the sample was prepared based on the details shown in Table 1). A sample of influent was collected from the mixing tank and influent TSS concentration was measured with a spectrophotometer (HACH DR-2400). The influent was pumped to the separation tank at $0.5 \times 10^{-5} \mathrm{~m}^{3} / \mathrm{s}$. The mixture from the mixing tank was continuously pumped into the separation tank for a period of 288 minutes (which was also the retention time in the separator).

Table 1. Ratio of water and silt/clay

\begin{tabular}{|c|c|c|}
\hline $\begin{array}{c}\text { Total Suspended Solids } \\
\text { Concentration, } \mathrm{C}_{s s} \\
(\mathrm{mg} / \mathrm{L})\end{array}$ & $\begin{array}{c}\text { Water } \\
(\mathrm{L})\end{array}$ & Silt and Clay (g) \\
\hline 100 & 200 & 33 \\
\hline 150 & 200 & 50 \\
\hline 200 & 200 & 67 \\
\hline 250 & 200 & 84 \\
\hline 300 & 200 & 100 \\
\hline 350 & 200 & 117 \\
\hline 400 & 200 & 133 \\
\hline
\end{tabular}

Table 2. Test condition influent TSS concentrations, $C_{s s}$, retention time, $t$ and influent flowrates, $Q_{i}$

\begin{tabular}{|c|c|c|c|c|c|c|c|c|}
\hline \multirow{2}{*}{$\begin{array}{c}\text { Flowra } \\
\text { te } \\
\left(\mathrm{m}^{3} / \mathrm{s}\right)\end{array}$} & \multirow{2}{*}{$\begin{array}{l}\text { Retenti } \\
\text { on } \\
\text { time, } t \\
(\mathrm{~min})\end{array}$} & \multicolumn{7}{|c|}{$\begin{array}{l}\text { Influent Total Suspended Solids Concentration, } \mathrm{C}_{s s} \\
(\mathrm{mg} / \mathrm{L})\end{array}$} \\
\hline & & 100 & 150 & 200 & 250 & 300 & 350 & 400 \\
\hline $\begin{array}{c}0.5 \times \\
10^{-5}\end{array}$ & 288 & $\begin{array}{c}\text { Test } \\
1\end{array}$ & $\begin{array}{c}\text { Test } \\
5\end{array}$ & $\begin{array}{c}\text { Test } \\
9\end{array}$ & $\begin{array}{c}\text { Test } \\
13\end{array}$ & $\begin{array}{c}\text { Test } \\
17\end{array}$ & $\begin{array}{c}\text { Test } \\
21\end{array}$ & $\begin{array}{c}\text { Test } \\
25\end{array}$ \\
\hline $\begin{array}{c}0.7 \times \\
10^{-5}\end{array}$ & 205 & $\begin{array}{c}\text { Test } \\
2\end{array}$ & $\begin{array}{c}\text { Test } \\
6\end{array}$ & $\begin{array}{c}\text { Test } \\
10\end{array}$ & $\begin{array}{c}\text { Test } \\
14\end{array}$ & $\begin{array}{c}\text { Test } \\
18\end{array}$ & $\begin{array}{c}\text { Test } \\
22\end{array}$ & $\begin{array}{c}\text { Test } \\
26\end{array}$ \\
\hline $\begin{array}{c}1.0 \times \\
10^{-5}\end{array}$ & 144 & $\begin{array}{c}\text { Test } \\
3\end{array}$ & $\begin{array}{c}\text { Test } \\
7\end{array}$ & $\begin{array}{c}\text { Test } \\
11\end{array}$ & $\begin{array}{c}\text { Test } \\
15\end{array}$ & $\begin{array}{c}\text { Test } \\
19\end{array}$ & $\begin{array}{c}\text { Test } \\
23\end{array}$ & $\begin{array}{c}\text { Test } \\
27\end{array}$ \\
\hline $\begin{array}{c}2.0 \times \\
10^{-5}\end{array}$ & 72 & $\begin{array}{c}\text { Test } \\
4\end{array}$ & $\begin{array}{c}\text { Test } \\
8\end{array}$ & $\begin{array}{c}\text { Test } \\
12\end{array}$ & $\begin{array}{c}\text { Test } \\
16\end{array}$ & $\begin{array}{c}\text { Test } \\
20\end{array}$ & $\begin{array}{c}\text { Test } \\
24\end{array}$ & $\begin{array}{c}\text { Test } \\
28\end{array}$ \\
\hline
\end{tabular}

The mixture in the mixing tank was topped up by addition of $100.0 \mathrm{~L}$ of water and the required silt/clay to produce an influent TSS concentration of $100 \mathrm{mg} / \mathrm{L}$ whenever necessary. The mixture was thoroughly agitated for 2 
minutes for every 10 minutes interval. Effluent from the separation tank was collected every 30 minutes and its TSS concentration measured with a spectrophotometer until the end of the experiment (after the retention time). The procedure was then repeated for Test 2 to Test 28 (Table 2).

\section{RESUlTS AND DisCUSSION}

Fig. 5 illustrates the effluent TSS concentration, $C_{e}$ at different influent flowrate, $Q_{i}$ and influent TSS concentration, $C_{s s}$. The effluent TSS concentration, $C_{e}$ was found to be directly proportional to influent flowrate, $Q_{i}$. The influent flowrate, $Q_{i}$ can be expressed as a function of effluent TSS concentration, $C_{e}$ for different influent TSS concentration, $C_{s s}$ by a series of Power equations which was obtained through curve fitting exercise. These power equations are shown in Table 3.

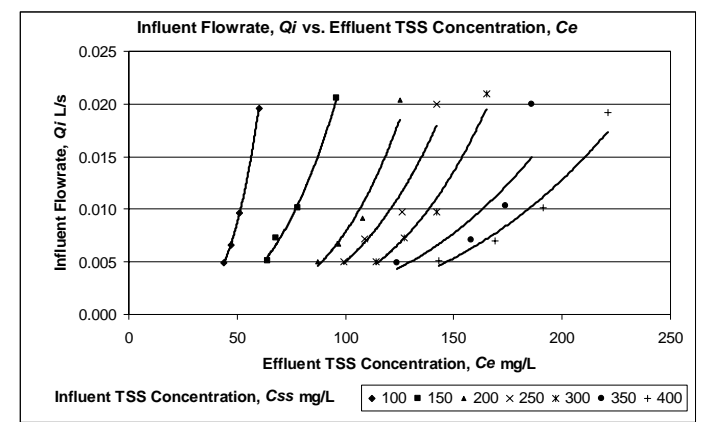

Figure 5. Effluent TSS concentration, $C_{e}$ at different influent flowrate, $Q_{i}$ and influent TSS concentration, $C_{s s}$

Table 3 influent flowrate, $Q_{i}$ as a function of effluent TSS concentration, $C_{e}$ power equations were used to calculate the required flowrate, $Q$ for a given influent TSS concentration, $C_{s s}$ to achieved desired effluent TSS concentration, $C_{e}$ of 50 $\mathrm{mg} / \mathrm{L}$ (Class II) and $150 \mathrm{mg} / \mathrm{L}$ (Class III) to meet environmental discharge requirement under the Proposed Interim National Water Quality Standards for Malaysia. Required flowrate to achieved desired effluent TSS concentration of $50 \mathrm{mg} / \mathrm{L}$ and $150 \mathrm{mg} / \mathrm{L}$ were denoted $Q_{50}$ and $Q_{150}$ respectively. Required flowrate, $Q_{75}, Q_{100}$ and $Q_{125}$ were also calculated using the same method.

Table 3. Influent flowrate, $Q_{i}$ as a function of effluent TSS concentration, $C_{e}$ for different influent TSS concentration,

$$
C_{s S}
$$

\begin{tabular}{|l|l|l|}
\hline $\begin{array}{l}\text { Influent Total } \\
\text { Suspended Solids } \\
\text { Concentration, } \\
\mathrm{C}_{s s}(\mathrm{mg} / \mathrm{L})\end{array}$ & Relationship & $\mathrm{R}^{2}$ \\
\hline 100 & $Q_{i}=2.0 \times 10^{-10} C_{e}{ }^{4.4616}$ & \\
\hline 150 & $Q_{i}=7.0 \times 10^{-9} C_{e} 3.2704$ & 0.9999 \\
\hline 200 & $Q_{i}=2.0 \times 10^{-10} C_{e}^{3.8446}$ & 0.9868 \\
\hline 250 & $Q_{i}=3.0 \times 10^{-10} C_{e}^{3.6248}$ & 0.9667 \\
\hline 300 & $Q_{i}=7.0 \times 10^{-11} C_{e}^{3.8005}$ & 0.9786 \\
\hline 350 & $Q_{i}=2.0 \times 10^{-9} C_{e}^{3.0474}$ & 0.8174 \\
\hline 400 & $Q_{i}=1.0 \times 10^{-9} C_{e} 3.0305$ & 0.9604 \\
\hline
\end{tabular}

Fig. 6 illustrates the required flowrate, $Q$ to achieve desired effluent TSS Concentration, $C_{e}$ at different influent TSS concentration, $C_{s s}$ obtained through the calculation using the power equations given in Table 3 . The required flowrate, $Q$ can also be expressed as a function of influent
TSS concentration, $C_{S S}$ to achieve different desired effluent TSS concentration, $C_{e}$ through curve fitting exercise. These power equations are listed in Table 4. Power equation $Q_{50}=$ $3423.6 C_{s s}^{-2.8525}$ and $Q_{150}=63688 C_{s s}^{-2.9533}$ express the required flowrate needed to achieved effluent TSS concentration, $C_{e}$ of $50 \mathrm{mg} / \mathrm{L}$ and $150 \mathrm{mg} / \mathrm{L}$ respectively.

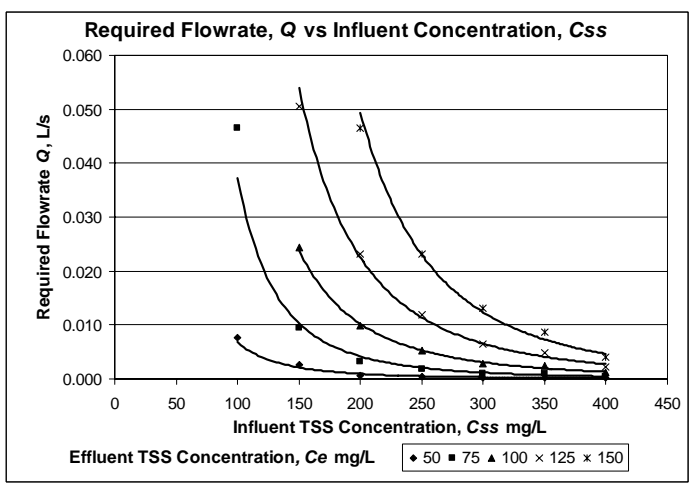

Figure 6. Required flowrate, $Q$ to achieve desired effluent TSS concentration, $C_{e}$ at different influent TSS concentration, $C_{s s}$

Table 4 Required flowrate, $Q$ as a function of influent TSS concentration, $C_{s S}$ to achieve different desired effluent TSS concentration, $C_{e}$

\begin{tabular}{|c|c|c|}
\hline $\begin{array}{l}\text { Desired Effluent } \\
\text { Total Suspended } \\
\text { Solids } \\
\text { Concentration, } \mathrm{C}_{e} \\
(\mathrm{mg} / \mathrm{L})\end{array}$ & Relationship & $\mathrm{R}^{2}$ \\
\hline 50 & $Q_{50}=3423.6 C_{s s}^{-2.8525}$ & 0.9571 \\
\hline 75 & $Q_{75}=77708 C_{S s}{ }^{-3.1601}$ & 0.9787 \\
\hline 100 & $Q_{100}=63688 C_{s s}^{-2.9533}$ & 0.9850 \\
\hline 125 & $Q_{125}=223066 C_{s s}^{-3.0408}$ & 0.9879 \\
\hline 150 & $Q_{150}=3.0 \times 10^{6} C_{s s}-3.4121$ & 0.9836 \\
\hline
\end{tabular}

Fig. 7 illustrates the particle size distribution of the silt/clay sample (Geospec, 2007) used in the experiment. The smallest particle size removed, $d$ at different $C_{s s}$ and $Q_{i}$ can be obtained from Fig. 7 using the percentage removal achieved at different $C_{s s}$ and $Q_{i}$ as listed in Table 5. The average percentage removal and smallest particle size removed, $d$ at different $Q_{i}$ as listed in Table 5 are $43.45 \%$ and $4.51 \mu \mathrm{m}$ for $Q_{i}=2.0 \times 10^{-5} \mathrm{~m}^{3} / \mathrm{s}, 50.06 \%$ and $3.28 \mu \mathrm{m}$ for $Q_{i}=1.0 \times 10^{-5} \mathrm{~m}^{3} / \mathrm{s}, 54.91 \%$ and $2.39 \mu \mathrm{m}$ for $Q_{i}=0.7 \times$ $10^{-5} \mathrm{~m}^{3} / \mathrm{s}$ and $60.70 \%$ and $1.58 \mu \mathrm{m}$ for $Q_{i}=0.5 \times 10^{-5} \mathrm{~m}^{3} / \mathrm{s}$.

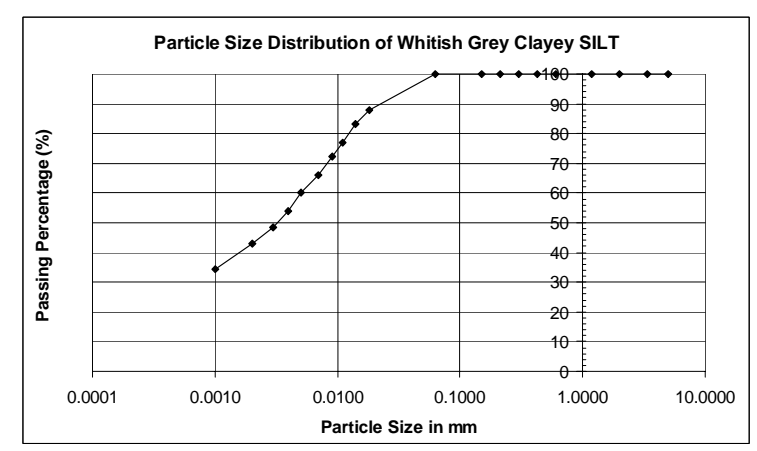

Figure 7. Particle size distribution of silt/clay sample

Fig. 8 show the smallest particle size removed, $d$ at different $Q_{i}$. The separation tank smallest particle size removable, $d$ can be predicted for different influent 
flowrate, $Q_{i}$ using power equation $d=85.664 Q_{i}{ }^{0.7338}\left(\mathrm{R}^{2}=\right.$ 9448) obtain through curve fitting exercise. Using this equation the separator can separated solids particles of 2.92 $\mu \mathrm{m}$ and above at design flowrate of $1.0 \times 10^{-5} \mathrm{~m}^{3} / \mathrm{s}$.

The separator system was designed to separate solid particles sizes of more than $10 \mu \mathrm{m}$ in diameter. From experimental result and interpretation, the separation tank can removed solid particles size of $2.92 \mu \mathrm{m}$ and above at design flowrate of $1.0 \times 10^{-5} \mathrm{~m}^{3} / \mathrm{s}$.

Table 5. Percentage removal at different influent TSS concentration, $C_{s s}$ and influent flowrate, $Q_{i}$ and the respective smallest particle size removed

\begin{tabular}{|c|c|c|c|c|c|}
\hline \multirow[b]{2}{*}{$\begin{array}{l}\text { Influent } \\
\text { Total } \\
\text { Suspended } \\
\text { Solids } \\
\text { Concentra } \\
\text { tion, C } C_{s s} \\
(\mathrm{mg} / \mathrm{L})\end{array}$} & & \multicolumn{4}{|c|}{ Flowrate $\left(\mathrm{m}^{3} / \mathrm{s}\right)$} \\
\hline & & $\begin{array}{ll}2.0 & \times \\
10^{-5}\end{array}$ & $\begin{array}{ll}1.0 & \times \\
10^{-5} & \end{array}$ & $\begin{array}{ll}0.7 & \times \\
10^{-5} & \end{array}$ & $\begin{array}{ll}0.5 \\
10^{-5}\end{array}$ \\
\hline \multirow[t]{2}{*}{100} & \% Removal & 41.75 & 50.00 & 53.47 & 60.00 \\
\hline & $\begin{array}{l}>\text { particle size } \\
\text { removed }(\mu \mathrm{m})\end{array}$ & 4.70 & 3.29 & 2.64 & 1.65 \\
\hline \multirow[t]{2}{*}{150} & \% Removal & 36.84 & 48.34 & 54.05 & 57.33 \\
\hline & $\begin{array}{l}>\text { particle size } \\
\text { removed }(\mu \mathrm{m})\end{array}$ & 6.06 & 3.60 & 2.54 & 1.96 \\
\hline \multirow[t]{2}{*}{200} & \% Removal & 42.92 & 46.53 & 50.51 & 56.28 \\
\hline & $\begin{array}{l}>\text { particle size } \\
\text { removed }(\mu \mathrm{m})\end{array}$ & 4.52 & 3.93 & 3.20 & 2.12 \\
\hline \multirow[t]{2}{*}{250} & \% Removal & 43.87 & 49.80 & 56.40 & 60.56 \\
\hline & $\begin{array}{l}>\text { particle size } \\
\text { removed }(\mu \mathrm{m})\end{array}$ & 4.37 & 3.33 & 2.10 & 1.59 \\
\hline \multirow[t]{2}{*}{300} & \% Removal & 45.90 & 52.67 & 57.09 & 62.00 \\
\hline & $\begin{array}{l}>\text { particle size } \\
\text { removed }(\mu \mathrm{m})\end{array}$ & 4.04 & 2.79 & 1.98 & 1.43 \\
\hline \multirow[t]{2}{*}{350} & \% Removal & 47.16 & 50.85 & 55.11 & 64.47 \\
\hline & $\begin{array}{l}>\text { particle size } \\
\text { removed }(\mu \mathrm{m})\end{array}$ & 3.82 & 3.13 & 2.34 & 1.15 \\
\hline \multirow[t]{2}{*}{400} & \% Removal & 45.70 & 52.25 & 57.75 & 64.25 \\
\hline & $\begin{array}{l}>\text { particle size } \\
\text { removed }(\mu \mathrm{m})\end{array}$ & 4.08 & 2.87 & 1.91 & 1.17 \\
\hline \multicolumn{2}{|c|}{ Average \% Removal } & 43.45 & 50.06 & 54.91 & 60.70 \\
\hline \multicolumn{2}{|c|}{$\begin{array}{l}\text { Average of smallest particle } \\
\text { size removed }(\mu \mathrm{m})\end{array}$} & 4.51 & 3.28 & 2.39 & 1.58 \\
\hline
\end{tabular}

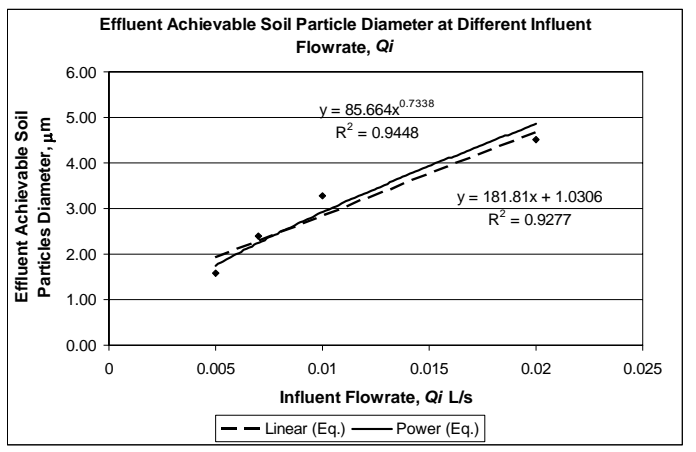

Figure 8. Smallest particles size removable, $d$ at different influent flowrate, $Q_{i}$

\section{CONCLUSIONS}

At present, the circular enhanced gravity separator with inclined coalescing frustums had not being extensively developed and used so far for treating oil and grease and suspended solids. Patent search of a circular phase separation tank with inclined coalescence plate arranges in multiple angle by Intellectual Property Services, SIRIM Berhad, Malaysia confirmed the novelty of such separator [SIRIM, 2004]. The primary components of the separation system consist of a few series of inverted and upright frustums to form multiple angle plate arrangement to enhance gravity separation and coagulation of solid particles by taking advantage of principle of Boycott effect whereby the presence of inclined frustums medium promotes faster settling of solid particles. A circular basin structure equipped with a center-feed vertical perforatedpipe distributor inlet to direct flow to the inclined coalescence conical frustums to provide an even and continual decreasing in horizontal velocity, $v_{h}$ (as surface area increases). The number of conical frustums in each series would successively increase in number by decreasing the spaces between frustums so as to provide more coalescing area. This enables more separation of solid particles when horizontal velocity, $v_{h}$ reduces with increasing radius and coalescing area.

The following conclusions can be drawn from the separator performance tests. The influent flowrate, $Q_{i}$ can be expressed as a function of effluent TSS concentration, $C_{e}$ for different influent TSS concentration, $C_{s s}$ by a series of power equations. These power equations can be used to predict the required flowrate, $Q$ for a given influent TSS concentration, $C_{s s}$ to achieved desired effluent TSS concentration, $C_{e}$ of $50 \mathrm{mg} / \mathrm{L}$ (Class II) and $150 \mathrm{mg} / \mathrm{L}$ (Class III) to meet environmental discharge requirement under the Proposed Interim National Water Quality Standards for Malaysia. Power equation $Q_{50}=3423.6 C_{s s}^{-2.8525}$ and $Q_{150}=$ $63688 C_{S S}^{-2.9533}$ express the required flowrate needed to achieved effluent TSS concentration, $C_{e}$ of $50 \mathrm{mg} / \mathrm{L}$ and 150 $\mathrm{mg} / \mathrm{L}$ respectively. The separation tank smallest particle size removable, $d$ can be predicted for different influent flowrate, $Q_{i}$ using power equation $d=85.664 Q_{i}{ }^{0.7338}\left(\mathrm{R}^{2}=\right.$ 9448). The separator can separated solids particles of 2.92 $\mu \mathrm{m}$ and above at design flowrate of $1.0 \times 10^{-5} \mathrm{~m}^{3} / \mathrm{s}$ which met its design criteria of separating solid particles of more than $10 \mu \mathrm{m}$ in diameter at design flowrate.

\section{REFERENCES}

[1] A Deininger, F.W. Günthert and P.A. Wilderer, "The Influence of Currents on Circular Secondary Clarifier Performance and Design", Wat. Sci. Tech., vol. 34, no. 3-4, pp. 405-412, 1996.

[2] Geospec Sdn. Bhd., Specific Gravity and Particle Size Distribution Test, GSB/LAB/8/07, 2007.

[3] HACH DR-1600

[4] Law, P.L., Ngu, L.H., Wong, K.K., and Abdul Rahman, A. (2006). Development and Performance Tests of a Separator for Removal of Physically Emulsified and Free Oils from Wastewaters. Journal of the Institution of Engineers, Malaysia, 67, No. 2, pp. 10-19.

[5] Malaysia Environmental Quality Report 2004. Department of Environmental, Ministry of Science, Technology and the Environment, Malaysia.

[6] Rensselear Polytechnic Institute, http://www.rpi.edu/dept/chemeng/Biotech-Environ/SEDIMENT/boycott.htm. Accessed on 27/11/2002.

[7] SIRIM Berhad, Intellectual Property Services, Search Report, Circular Phase Separation Tank With Inclined Coalescence Plates for Removal of Oil and Grease from Wastewaters. Ref. No. [Job No.] ISD 426/13/1(NS/2004-03/49). Malaysia. 2004.

[8] Tchobanoglous, G., Burton, F.L. and Stensel, H.D. (2004). Wastewater Engineering: Treatment and Reuse, $4^{\text {th }}$ Ed. McGraw-Hill, Boston, USA. 


\title{
A 3-stage Wastewater Treatment System: Part I. Development
}

\author{
Yin Wee Oon, Puong Ling Law, Chee Khoon Ng, Lock Hei Ngu and Kien Kuok Wong
}

\begin{abstract}
This research focuses on the design and development of 3 -staged micro-scale wastewater treatment system applicable to a typical household of four to five persons. The three-stage system consists of a hair-based spiral oil\& grease removal tank, a plastic medium mixed flow trickling filter and water hyacinth-based free surface water (FSW) wetland system operating in series. The first stage consists of an oil and grease removal tank filled with hair blocks and operated in a spiral plane, which is aimed to remove physically emulsified free oils in wastewater .This continual removal system followed by a circular trickling filter filled with perforated cylindrical plastic tubes in horizontal and vertical arrangements to provide alternate cross and vertical flows. And, this filter is suitable for treating small volume domestic effluents loaded with suspended solids (SS), ammonia-nitrogen $\left(\mathrm{NH}_{3}-\mathrm{N}\right)$, and biochemical oxygen demand (BOD). The last stage consists of a hyacinth-based free water surface (FWS) wetland system filled with Pistia Stratiotes (a species of tropical water hyacinth) for removal of nutrients such as nitrogen $(\mathrm{N})$, phosphorous $(\mathrm{P})$, and potassium (K) from wastewaters.
\end{abstract}

Keywords: hair, filter, oil and grease, trickling, free surface water wetland, Pistia Stratiotes

\section{INTRODUCTION}

In the past, some major conventional methods used in oil pollution are such as using chemical dispersants, mechanical collection or natural removal. However these methods are restricted by many factors especially time and economical factors [1]. This has led to the rapid blooming removal system researches and development. The ability of hair solution in removing oil \& grease from water has been proven when McCrory"s finding arisen in 1989, whereas he found that oil adsorped onto the hair immediately in his experiments. This finding had convinced The National Aeronautic and Space Administration's (NASA's) Marshall Center in Huntsville, Alabama and the time being onwards researches were carried out in schools and research institutions world widely. The proposed oil and grease removal system was operated in a spiral plane due to the factors of surface area of contacts and reaction duration. A

Yin Wee Oon, Puong Ling Law, Chee Khoon Ng, Lock Hei Ngu and Kien Kuok Wong are with the Department of Civil Engineering, Faculty of Engineering, Universiti Malaysia Sarawak, 94300 Kota Samarahan, Malaysia (Tel: 6082-583282, Fax: 6082-583410, email: puonglaw@feng.unimas.my). spiral movement separation technique, which enables the wastewater to flow into the system and the horizontal velocity, $v_{h}$ decelerates with distance from the inlet as a result of continual increase of surface area due to spiral angle movement, is used. The reduction in horizontal velocity, $v_{h}$ would enhance the adsorption rate of most oil droplets. At the same time, the presence hair blocks in both sides of the flow path promotes optimum oil-liquid separation by principles of McCrory's findings that hair fabrics has an unbelievable ability in oil slick adsorption. The system promotes a maximum amount of oil droplets collision onto the hair block surfaces which provided for oil droplets adsorption to take place, while minimizing the flow clogging by introducing a gap in between both the hair block sides along the movement path (spacing of the gap is proportionally to the spiral path). The tiny or small oil and grease droplets would collide and caught by the hair fabric as they move along the spiral path of the system. It's believed that the enhancements below would lead to a "promising result" in oil and grease removal:

1) Installation of hair blocks at both sides of the flow path. Hair blocks successively installed along both sides of the flow path promote optimum adsorption efficiency when the oil drops passing through along the system. The occurrence of collision between hair blocks and oil drops would be raised when the contact surface area increased. More oil drops would be trapped.

2) Installation of hair blocks in spiral plane. Movement in spiral plane acquires rapid and continual turning and reflection of flow. This promote more adsorption occurrence between hair blocks and oil drops instead of moving constantly throughout the system as in other system that employs rectangular or non curving movement plane.

Hence, the separation of oil and grease from wastewater would be achieved.

Aerobic wastewater treatment system utilizes microorganisms for removal of suspended and dissolved organic matters from wastewaters, and the nutrients in a free surface water (FWS) wetland could be assimilated by water hyacinth growing on it [2]. The traditional trickling filter consists of a bed of a highly permeable medium to which microorganisms are attached and through which wastewater is percolated or trickled. The filter media usually consist of either rock or a variety of plastic packing materials. Rock filter beds are usually circular, and wastewaters are distributed over the top of the bed by a rotary distributor [3]. 
As wastewater trickles downwards over the slime layer, organic matters and dissolved oxygen are extracted, metabolic end products such as carbon dioxide are released, and organic content would decrease to the point where microorganisms in the lower zone are in a state of starvation (Figure 2). Thus, majority of BOD is extracted from the wastewater after passing through the filter media [3].

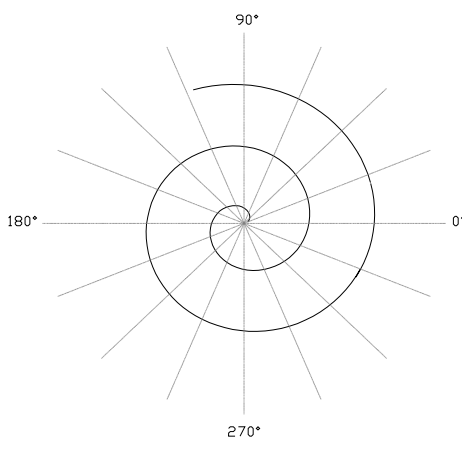

Figure 1. Separation system in spiral plane (Archimedean' spiral curve plane)

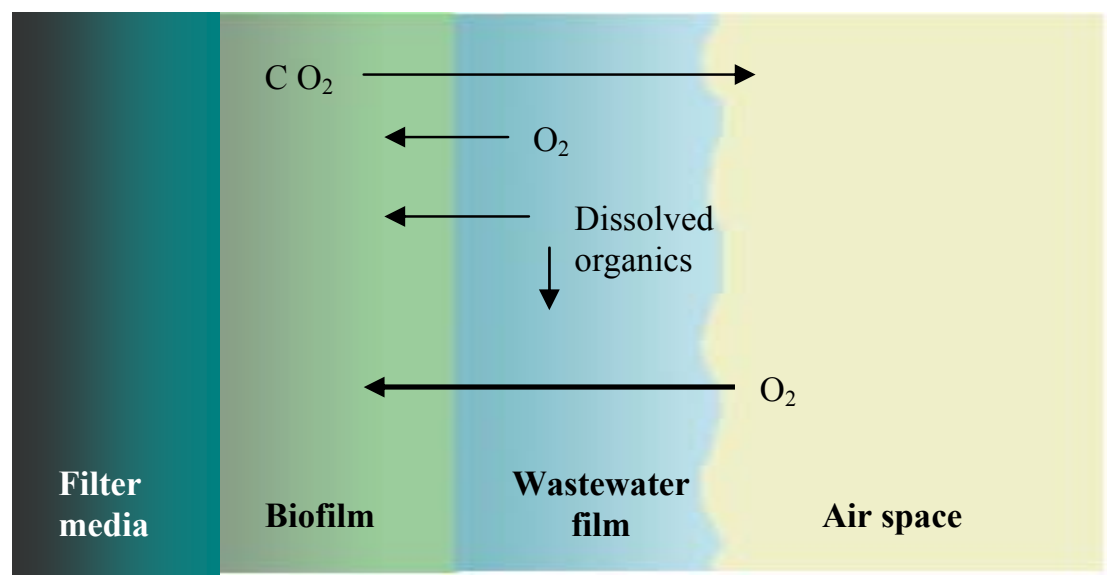

Figure 2. Biological Activity of Attached-Growth Biological Microorganism [3]

These bacterial populations would carry out nitrification process sequentially and oxidize ammonium growing on the slime layer to nitrate with intermediate formation of nitrite carried out by nitrosomanas and nitrobacter. The two steps in nitrification process and their equations are as follows [3]:

1) Ammonia is oxidized to nitrite $\left(\mathrm{NO}_{2}^{-}\right)$by Nitrosomanas bacteria.

$2 \mathrm{NH}_{4}^{+}+3 \mathrm{O}_{2} \rightarrow 2 \mathrm{NO}_{2}^{-}+4 \mathrm{H}^{+}+2 \mathrm{H}_{2} \mathrm{O}$

2) The nitrite is converted to nitrate $\left(\mathrm{NO}_{3}{ }^{-}\right)$by Nitrobacter bacteria.

$2 \mathrm{NO}_{2}^{-}+\mathrm{O}_{2} \rightarrow 2 \mathrm{NO}_{3}$

Recently, rock trickling filters are continuously being upgraded and rehabilitated with plastic sheet media and synthetic media to replace the use of stone. As compared to rock, plastic sheet media has 2 to 3 times higher in specific surface area that provides proportionally more area for biomass attachment [4;5]. Thus, void volume would be substantially increased that improves ventilation and hydraulic loading capacity. At present, primary types of plastic flow media widely used include cross flow media, vertical flow media and mixed flow media. In cross flow media design, down-flowing liquid is split at each cross point creating more redistribution points, and its major drawbacks are its inferior bio-solids flushing action and biomass fouling problem. Vertical flow media has vertical channels with cross flow at module interface by making use of its limited inner surface area of the channels that results in low specific surface area available for biomass attachment and low organic loading. Commercially available mixed media takes advantage of its uniformity in distribution of wastewater over the surface of the filter by alternating cross flow and vertical flow media layers [6]. Additionally, mixed media filter reduces excessive biomass build up of shearing materials from vertical layers. This medium type and arrangement could provide approximately $16 \%$ more surface area per volume from the recommended plastic cross flow media of $15.84 \mathrm{~m}^{2} / \mathrm{m}^{3}$ [7].

Water hyacinth grow profusely in the tropics and the use of hyacinth as one of the functional units in municipal wastewater treatment has increasingly gained its potential [2;8]. For instance, duckweed-based wastewater treatment has been implemented by the city of San Diego, USA to produce a treated effluent attaining quality standards that would be expected from advanced secondary treatment processes [2]. Water hyacinth could potentially be used in both tertiary treatment systems for the removal of nutrients, and in integrated secondary and tertiary treatment systems for removal of both organics and nutrients [2;6]. Hyacinthbased system is a free surface water (FSW) wetland system typically formed by shallow channels or basins where the water surface is open to the atmosphere and a suitable medium exists to support the growth of emergent or submerged aquatic plants $[6 ; 3]$. Wastewater treatment takes place as the hyacinth assimilate nutrients from the effluents [2]. Recently, two floating aquatic "macrophyte" plants have been used in wastewater treatment including 
Eichhornia crassipes (a type of water hyacinth) and Lemnacea spirodella (duckweed) [7]. As the influents move along the system, organics are biodegraded either aerobically or anaerobically. Nutrients are reduced through a variety of biological, physical and chemical processes $[2 ; 8]$. It is believed that two major mechanisms for ammonia reduction in hyacinth systems are bacterial nitrification and plant uptake [9].

\section{DESIGN AND DEVELOPMENT}

This research involves the development and field tests of a three-stage micro-scale wastewater treatment system for small volume domestic wastewaters loaded with physically emulsified free oils, $\mathrm{SS}, \mathrm{BOD}, \mathrm{NH}_{3}-\mathrm{N}$, and nutrients. As illustrated in Figure 3, the system consists of 1) a hair-based spiral separation tank, 2) a plastic medium mixed flow trickling filter, and 3) Pistia Stratiotes-based FWS wetland system operating in series.

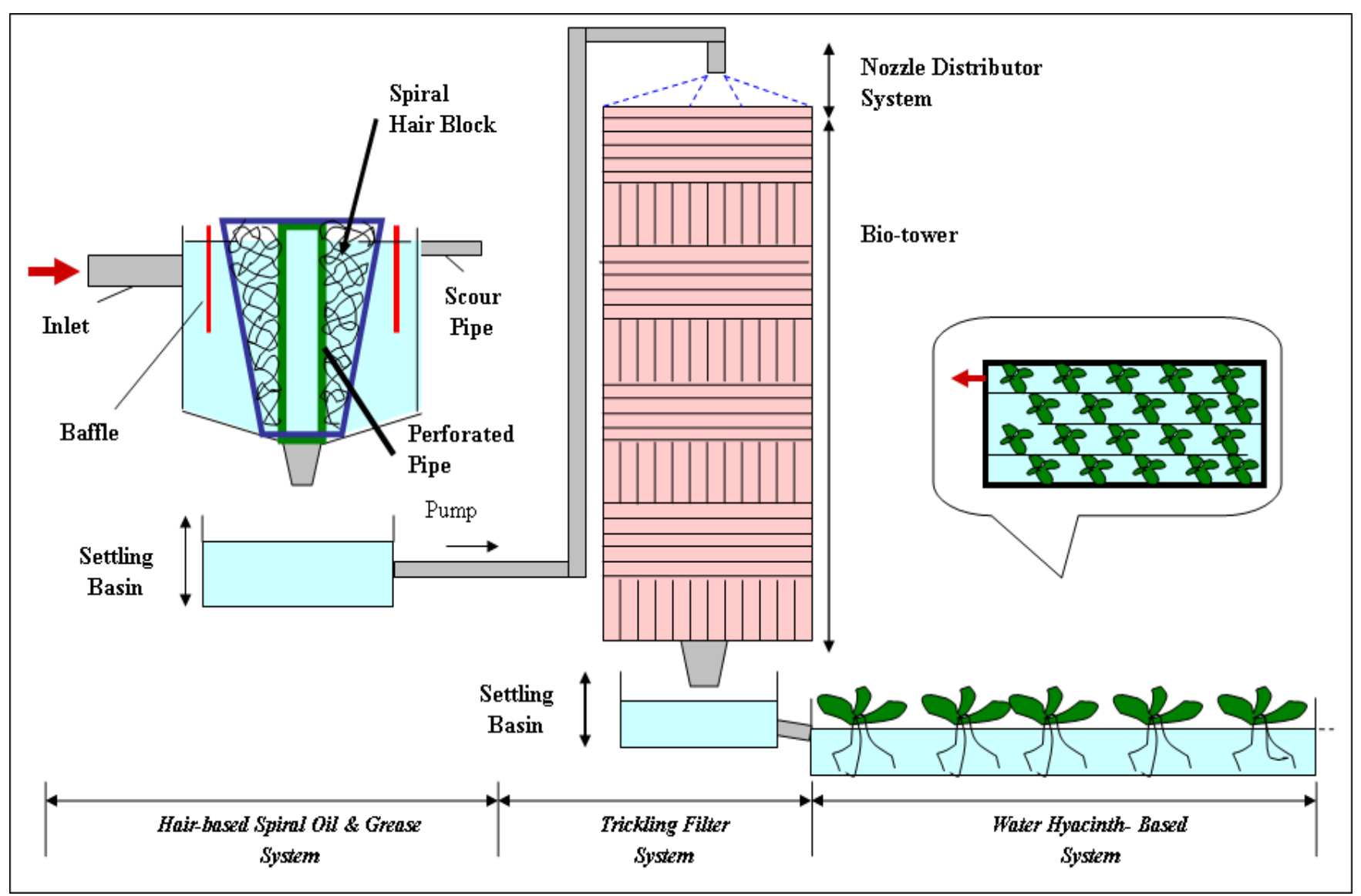

Figure 3. Schematic diagram of the overall wastewater treatment system operated in series

\section{A. Hair-Based Spiral Separation System}

The proposed hair based oil and grease separation system operated in spiral plane movement enables the horizontal velocity, $v_{h}$ decelerates along the distance from the inlet to the perimeter baffle zone, as a result of continual increase of surface area due to spiral angle movement. As the horizontal velocity, $v_{h}$ decreased, the adsorption rate of most oil droplets would increase. The usage of double sided hair blocks along the flow path would enhance the system to an optimum level by the principles of McCrory's findings that hair fabrics has an unbelievable ability in oil slick adsorption. An optimum amount of oil droplets would collide onto the hair block surfaces where adsorption take place and the gap in between both the hair block sides would minimize the occurrence of flow clogging along the movement path.

The proposed separation technique is very much dependent on the arrangement or orientation of the hair blocks thickness, gap spacing and the spiral angles of the movement path. However, other factors such as influent concentration, flow rates, viscosity and specific gravity, volumes of the systems and temperature are undoubtedly playing important roles in removal or separation efficiency of oil and grease from wastewater.

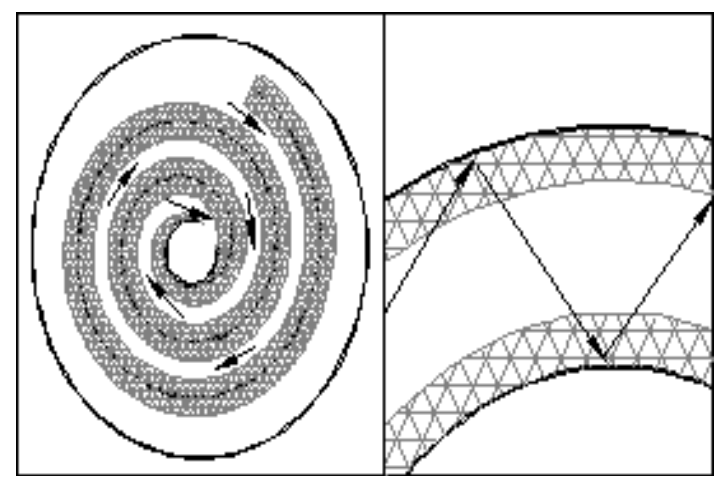

Figure 4. Hair-based separation tank in spiral plane

The proposed oil and grease hair based separation systems (refer to Figures 5(a) to (c)) features included a $750 \mathrm{~mm}$ diameter and $460 \mathrm{~mm}$ height circular separation tank was used to take advantage of the continual decreasing in horizontal velocity as surface area increases. Other systems 
employ rectangular tank whereby the horizontal velocity, $v_{h}$ is constant throughout. The center of system was a $150 \mathrm{~mm}$ diameter inlet well. The $25 \mathrm{~mm}$ thick hair blocks, which was formed by hair caging with wire mesh, were attached to both sides of a spiral-planed alumminium plate with a gap of $110 \mathrm{~mm}$ to each other and the gap distance decreased proportionally along the moving path .The outlet baffle, which was a $50 \mathrm{~mm}$ periphery overflow outlet weir channel was used to give uniform flow removal and outlet launder to direct effluent out of the separator.

The effluent of the first stage system are then collected prior discharge into the next stage system, which is the trickling filter system that in charged of removing the organic matters such as ammoniacal nitrogen, BOD and suspended solids from wastewater.

\section{B. Mixed Flow Trickling Filter}

In this research, a micro-scale trickling filter was designed and developed from conventional design with the differences in its filter medium design characteristics and media arrangements. As shown in Figure 3, the major components of the system consists of 1) circular tower for filter medium storage, 2) hydraulic nozzle distributor equipped with high pressure hydraulic pump, and 3) filter medium consists of hollow perforated plastic tubes arranged in alternating cross and vertical parallel manners. The first layer of the filter makes up of $40 \mathrm{~mm}$ diameter spherical plastic (Figure 6) to achieve better uniformity of water distribution for the following layer of plastic tube medium. The hollow tubular structure as filter medium enables the usage of the inner surface area of the hollow tubes and thus providing higher specific surface area. Additionally, the alternating vertical and cross flows would raise the void spaces to increase the biological growth for waste treatment. The details of specific surface area and percent void space for filter fill or selected media are shown in Table 1.

The filter medium tubes measure $24.13 \mathrm{~mm}$ on outer diameter, and $21.59 \mathrm{~mm}$ on inner diameter are alternatively placed in a plastic circular basin of $292.1 \mathrm{~mm}$ in diameter and $777.24 \mathrm{~mm}$ in height as illustrated in Figure 6 and Figure 7. As shown in Figure 7, the cross flow perforated tubes are arranged in four sub-layers at $45^{\circ}$ between each other to enhance oxygen transfer and wetting rates inside the filter. The perforated holes in cross flow media layer tubes are approximately $7.62 \mathrm{~mm}$ in diameter at approximately $65 \mathrm{~mm}$ apart on top and bottom of the tubes. For the vertical flow media layers, each layer consists of 121 units of $101.60 \mathrm{~mm}$ vertical tubes.

This system is equipped with a fixed nozzle distributor controlled by a valve to permit a desired flow rate at approximately 185 liters/day (L/day). Flow is applied to the trickling filter through a hydraulic pump before distributed by the nozzle. Because of the hydraulic gradient factor, a 6metre pressure head hydraulic pump is selected for this purpose to provide uniform distribution of wastewater on top of trickling filter. The under drain collects the filtrate and solids, and serves as a source of air for the microorganisms to maintain aerobic conditions in the filter. Sufficient air is being provided to the filter medium by natural draft and wind forces through numerous of ventilation ports located at the bottom of the filter.
Effluents from the filter are channeled to a clarifier whereby solid particles would be settled out before overflowing to the hyacinth-based FSW wetland system.

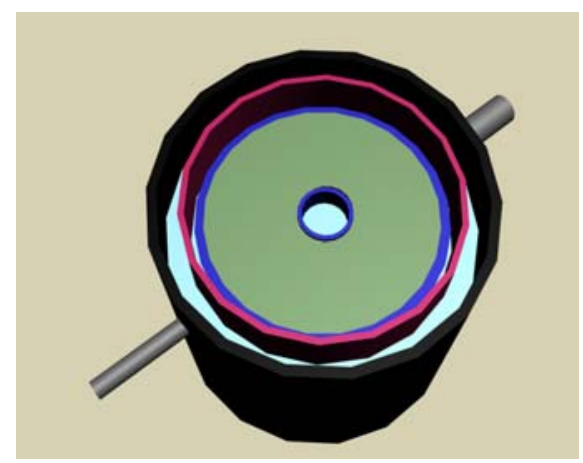

(a) Perspective view

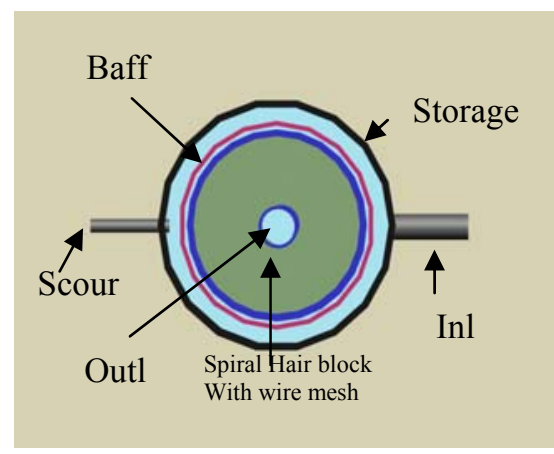

(b) Top view

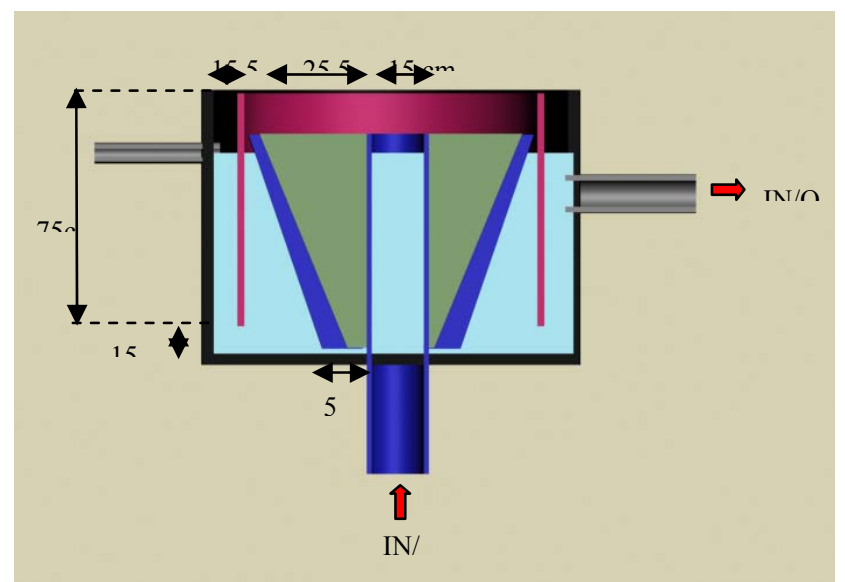

(c) Side view

Figure 5. Schematic diagrams of the proposed oil and grease hair based separation systems
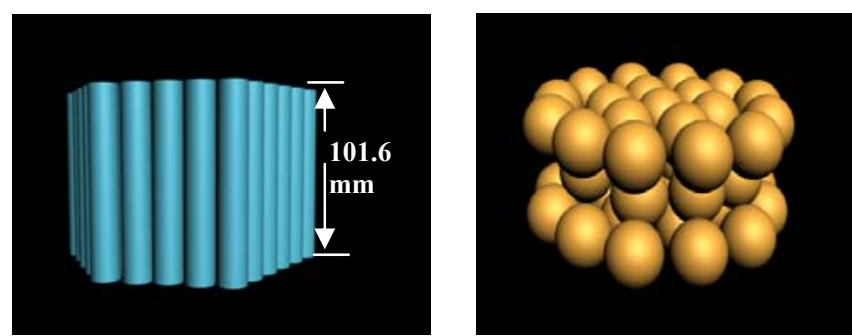

Figure 6. Top layer of trickling filter and filter vertical flow media 


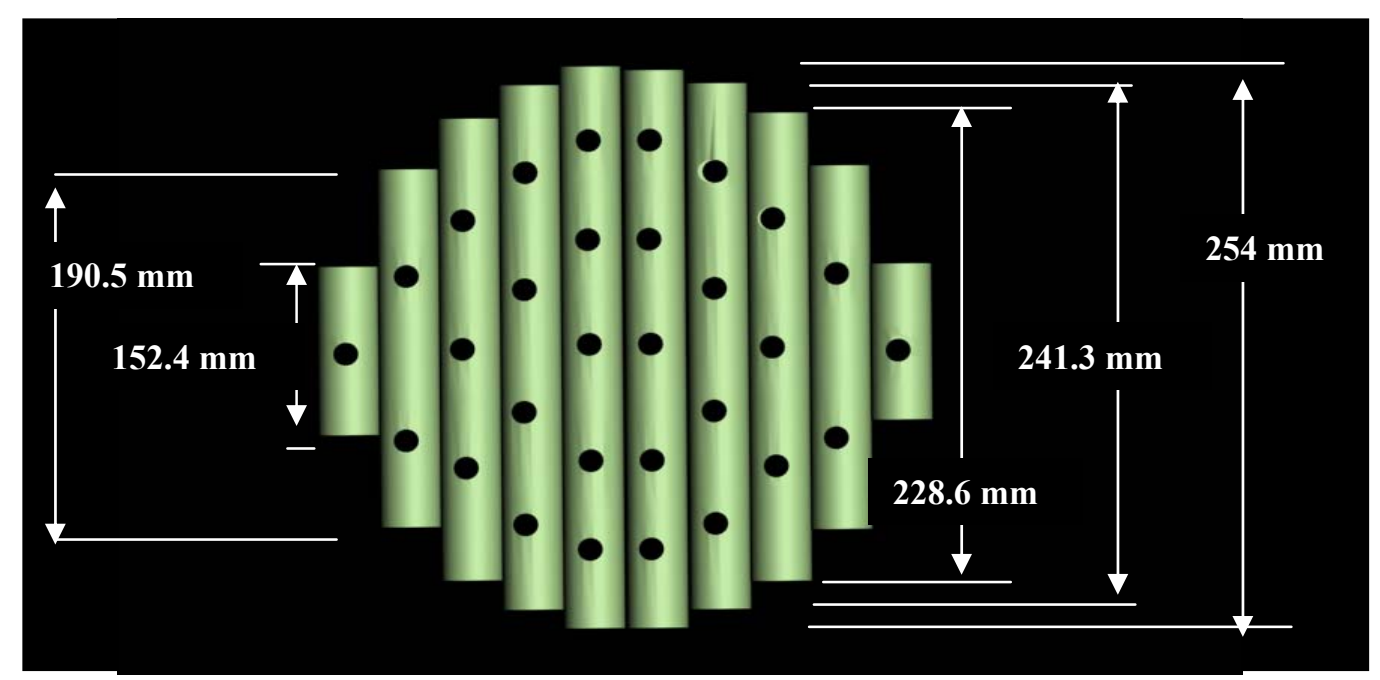

Figure 7. Filter cross flow media

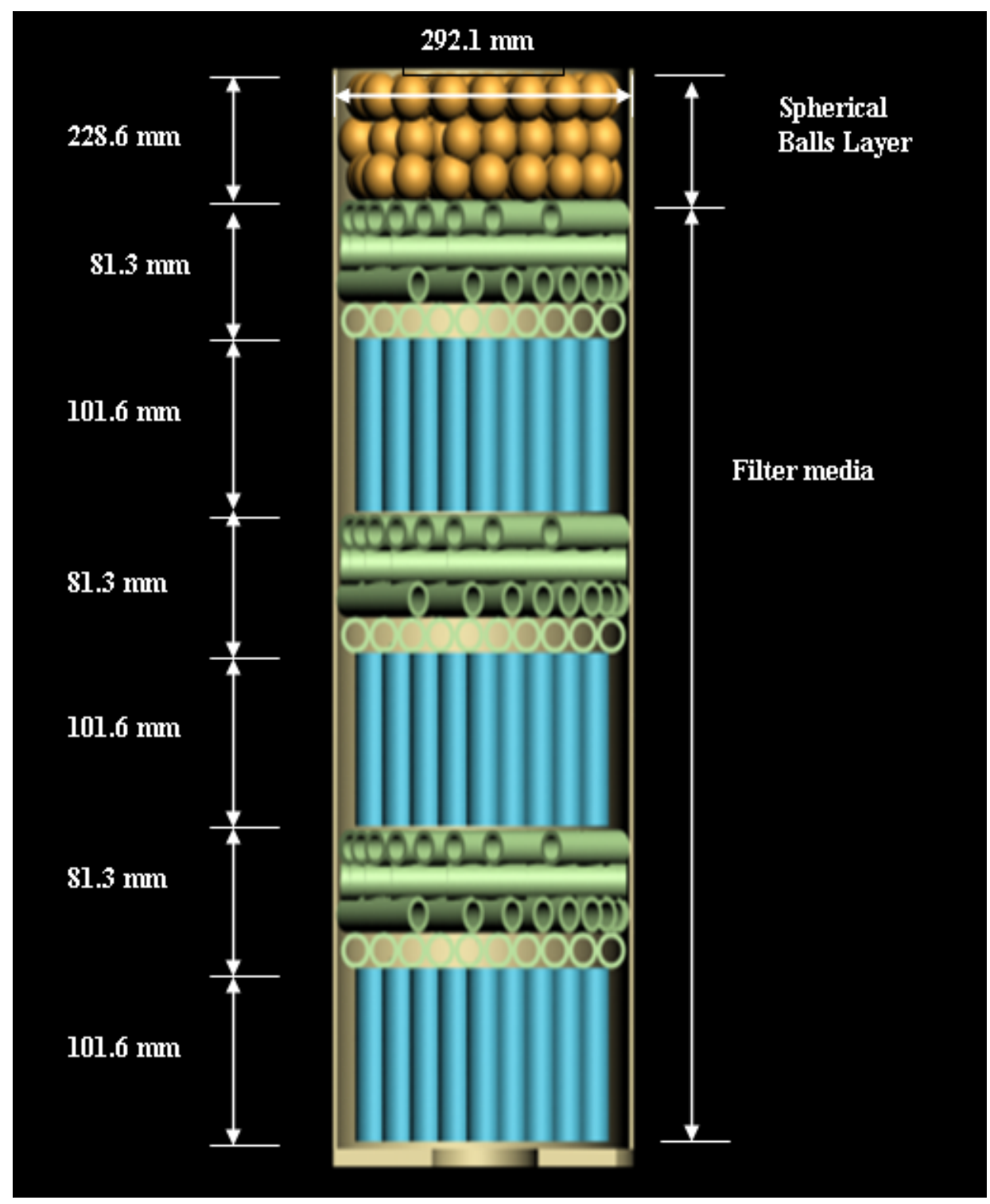

Figure 8. Mixed flow trickling filter filled with plastic medium

\section{Hyacinth-Based FSW Wetland System}

The hyacinth-based FSW wetland system consists of a plastic basin with approximately 250 liters in volume (length $=1220 \mathrm{~mm}$, width $=910 \mathrm{~mm}$, and depth $=230 \mathrm{~mm}$ as illustrated in Figure 9. The basin is partitioned into four compartments to enhance the uniformity flow throughout the basin and two holes are provided for influents from trickling filter and final effluents. Operational parameters 
such as surface overflow rate (SOR), detention time (DT), and horizontal velocity $\left(\mathrm{V}_{\mathrm{h}}\right)$ are shown in Table 2 .

Table 1. Specific surface area vs. percent void space

\begin{tabular}{|l|l|}
\hline Volume & $0.05 \mathrm{~m}^{3}\left(1.84 \mathrm{ft}^{3}\right)$ \\
\hline Specific Surface Area & $190.08 \mathrm{~m}^{2} / \mathrm{m}^{3}\left(57.50 \mathrm{ft}^{2} / \mathrm{ft}^{3}\right)$ \\
\hline Void Space, \% & $55.60 \%$ \\
\hline
\end{tabular}

The proposed hyacinth-based FSW wetland system is filled with a type of floating tropical water hyacinth called, "Pistia Stratiotes" (Figure 10). Pistia Stratiotes is characterized by numerous number of suspended roots of over one foot long $(>30 \mathrm{~cm})$, and the above-water portion consisting of leaves are traditionally used as animal feeds frequently practiced by local farmers in the tropics. Pistia Stratiotes is a free-floating aquatic plant commonly used as an ornamental plant in water gardens. This tropical hyacinth produces attractive rosettes of bright green, wedged shaped, overlapping, deeply veined velvety leaves that grow up to 10 inches $(25.4 \mathrm{~cm})$ long and 4 inches $(10.16 \mathrm{~cm})$ wide. This species of hyacinth grows well outdoors all yeararound in the tropics with water $\mathrm{pH}$ between 6.2 and 7.2 [10;11]. Pistia Stratiotes, because of it floating capabilities, is believed to have filtration capability for ponds with high nitrogen and phosphate levels. This type of water hyacinth grows vegetatively by production of daughter plants that allows intertwining of the plants, thereby forming large contiguous mats of floating vegetation $[10 ; 11]$. These mats are one of the most important growth characteristics contributing to the removal of nutrients from wastewaters. Details on system design outlining the dimensions, settling basin and hyacinth-based system are summarized in Table 3.

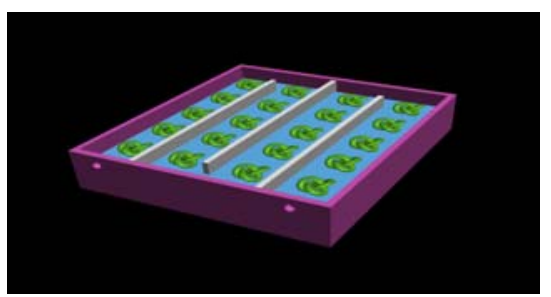

Figure 9. Hyacinth-based system

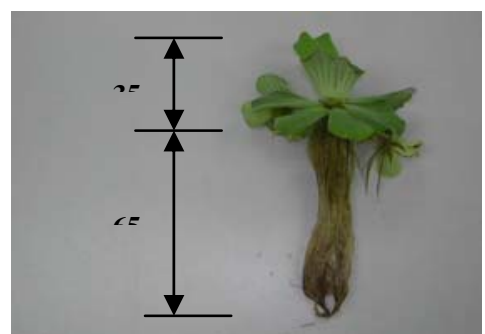

Figure 10. Pistia Stratiotes and leave-to-root length ratio
Table 3. System design of the hyacinth-based system

\begin{tabular}{|l|l|}
\hline $\begin{array}{l}\text { Surface Overflow Rate, } \\
\text { SOR }\end{array}$ & $\begin{array}{l}165.70 \text { liters/day.m }{ }^{2}(15.4 \\
\text { L/day.ft }\end{array}$ \\
\hline Detention Time, DT & 24 hours $(1.0$ day $)$ \\
\hline Horizontal Velocity, $\mathrm{V}_{\mathrm{h}}$ & $5.15\left(10^{-2}\right) \mathrm{m} / \mathrm{hr}(0.17 \mathrm{ft} / \mathrm{hr})$ \\
\hline
\end{tabular}

\section{CONCLUSION}

At present, all oil and grease separation system, trickling filter systems and hyacinth-based FSW wetland systems are applied individually for treating wastewaters. A 3-stage system that operates in series had not been extensively developed and used for removing emulsified free oil, organics and nutrients from wastewaters. The proposed hair-based spiral separation tank is believed to perform well in removing physically emulsified free oils when moves along the spiral plane and baffle zone. Meanwhile, the filter provides alternated cross and vertical flows would provide an even and thorough flow in addition to increasing total media surface area and void space in the system. The proposed hyacinth-based FSW wetland system filled with a species of tropical long-rooted water hyacinth (Pistia Stratiotes) would further enhance the removal of nutrients and suspended solids from domestic wastewaters.

\section{REFERENCES}

[1] National Research Council, (1989). Using oil pollution dispersants on the sea. National Academy Press, Washington, D.C.

[2] Brix, H. \& Schierup, H. (1989). The use of aquatic Macrophytes in water-pollution control. Ambio, 18(2), 100-107.

[3] Tchobangoglous, G and Burton, F.L. (1991). Wastewater Engineering: Treatment, Disposal, and Reuse, Third Edition. Metcalf \& Eddy, Inc., USA.

[4] United States Environmental Protection Agency (USEPA), Sept 2000. Wastewater Technology Fact Sheet Trickling Filters, EPA 832-F-00014, Office of Water, Washington, D.C.

[5] United States Environmental Protection Agency (USEPA), Sept 2000. Wastewater Technology Fact Sheet Trickling Filter Nitrification, EPA 832-F-00-015, Office of Water, Washington, D.C.

[6] Middlebrooks, E. J. (1995). Upgrading pond effluents: An overview. Water, Science \& Technology, 31(12), 353-368.

[7] Brentwood Industries (2004).Brentwood AccuPac Cross Flow media. Available at URL:

http://www.brentw.com/water/crossflow_main.html --> June 2004

[8] Mandi, L. (1994). Marrakesh wastewater purification experiment using vascular aquatic plants Eichhornia Crassipes and Lemna Gibba. Water, Science \& Technology, 29(4), 283-287.

[9] Hauser, J. R. (1984). Use of wastewater aquatic treatment system for ammonia control and effluent polishing. Research Journal of the Water Pollution Control Federation, 56(3), 219-225.

[10] Kumar, P. \& Garde, R. J. (1989). Potentials of water hyacinth for sewage treatment. Research Journal of the Water Pollution Control Federation, 61 (11/12), 1702-1706.

[11] Mars, R. (2004). Greywater Treatment with Macrophytes, Greywater Reuse Systems. Washington: Midland Business Centre. 
Table 3. Dimensions of system design

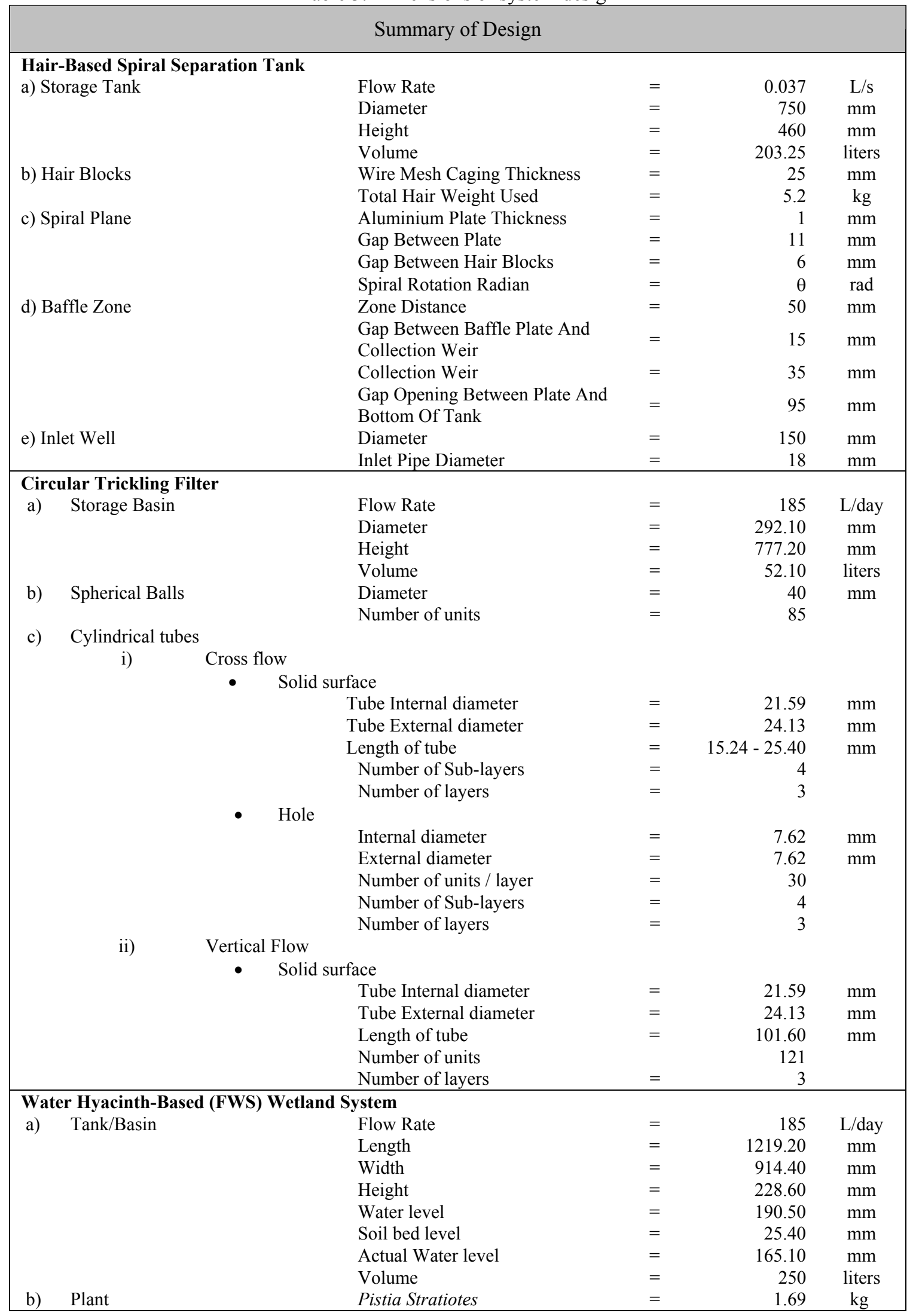




\title{
A 3-stage Wastewater Treatment System: Part II. Performance Evaluation
}

\author{
Yin Wee Oon, Puong Ling Law, Chee Khoon Ng, Lock Hei Ngu and Kien Kuok Wong
}

\begin{abstract}
A 3 stage micro-scale wastewater treatment system consists of 1) a hair-based spiral oil\& grease removal tank, 2) a mixed flow (vertical flow alternated with cross flow) filter, and 3) a hyacinth-based free surface water (FSW) wetland system filled with Pistia Stratiotes operating in series was recently developed. Field tests were carried out to determine the efficiencies of the system in removal of physically emulsified free oils, organic matters such as biochemical oxygen demand (BOD), ammonia-nitrogen $\left(\mathrm{NH}_{3}-\right.$ $\mathrm{N})$, suspended solids (SS), and nutrients such as nitrogen (N), phosphorous (P), and potassium (K) from wastewaters. From this study, it was found that the hair-based spiral system has successfully retained $53 \%$ of physically emulsified free oils from wastewater; meanwhile the mixed flow filter was capable of reducing approximately $33.2 \%$ in $\mathrm{BOD}, 59.9 \%$ in $\mathrm{NH}_{3}-\mathrm{N}$, $51.6 \%$ in $\mathrm{N}, \mathbf{1 4 . 7 \%}$ in $\mathrm{P}, \mathbf{2 0 . 1} \%$ in $\mathrm{K}, 24.4 \%$ in turbidity level; and the Pistia Stratiotes-based FSW wetland system was capable of removing approximately $30.8 \%$ in BOD, $30.3 \%$ in $\mathrm{NH}_{3}-\mathrm{N}, 40.9 \%$ in $\mathrm{N}, 42.2 \%$ in $\mathrm{P}, 53.2 \%$ in $\mathrm{K}$, and $29.7 \%$ in reduction in turbidity levels. When the mixed flow filter and hyacinth-based FSW wetland system operated in series, the removal efficiencies of the system were approximately $64.1 \%$ for BOD, $\mathbf{9 0 . 2} \%$ for $\mathrm{NH}_{3}-\mathrm{N}, \mathbf{9 2 . 5 \%}$ for $\mathrm{N}, \mathbf{5 6 . 9 \%}$ for $\mathrm{P}, \mathbf{7 3 . 3 \%}$ for $\mathrm{K}$, and $\mathbf{5 4 . 1 \%}$ for turbidity. Experimental data also showed that daily uptake rates or removal rates $(\mathrm{mg} / \mathrm{kg}$-day) of organics and nutrients by per kilogram of Pistia Stratiotes were approximately $1,460 \mathrm{mg}$ for $\mathrm{BOD}, 942 \mathrm{mg}$ for $\mathrm{NH}_{3}-\mathrm{N}$, $1,134 \mathrm{mg}$ for $\mathrm{N}, 1,361 \mathrm{mg}$ for $P$, and $5,884 \mathrm{mg}$ for $K$. Experimental data also showed that daily uptake rates or removal rates $(\mathrm{mg} / \mathrm{kg}$-day) of organics and nutrients by per kilogram of Pistia Stratiotes were approximately $1,460 \mathrm{mg}$ for BOD, $942 \mathrm{mg}$ for $\mathrm{NH}_{3}-\mathrm{N}, 1,134 \mathrm{mg}$ for $\mathrm{N}, 1,361 \mathrm{mg}$ for $\mathrm{P}$, and $5,884 \mathrm{mg}$ for $\mathrm{K}$.
\end{abstract}

Keywords: hair, spiral, trickling, hyacinth-based, Pistia Stratiotes, organics, nutrients

\section{INTRODUCTION}

The main process in the 3 stage system is a hair-based adsorption system flowing in spiral plane that followed by the mixed flow filter, which is an aerobic attached-growth biological activities by nitrification [1;2;3], while the hyacinth-based FSW wetland process applies the principles of biodegradation of organic matters by bacteria and

Yin Wee Oon, Puong Ling Law, Chee Khoon Ng, Lock Hei Ngu and Kien Kuok Wong are with the Department of Civil Engineering, Faculty of Engineering, Universiti Malaysia Sarawak, 94300 Kota Samarahan, Malaysia (Tel: 6082-583282, Fax: 6082-583410, email: puonglaw@feng.unimas.my). hyacinth uptake of nutrients [4;5;6]. This applied experimental research focuses on the performance tests of the previously developed 3-stage micro-scale domestic wastewater treatment system consisting of a hair-based spiral removal tank, a mixed flow filter connected in series with a hyacinth-based FSW wetland system. Field tests were carried out to determine the removal efficiencies of 1) physically emulsified free oils; 2) $\mathrm{BOD}, \mathrm{NH}_{3}-\mathrm{N}$, and $\mathrm{SS}$ by the mixed flow filter, 3) $\mathrm{BOD}, \mathrm{NH}_{3}-\mathrm{N}, \mathrm{SS}, \mathrm{N}, \mathrm{P}$, and $\mathrm{K}$ by the hyacinth-based FSW wetland system, and 4) combined removal efficiencies, i.e., oil\& grease removal tank, filter and hyacinth-based FWS wetland system operating in series.

\section{MATERIALS AND EXPERIMENTAL PROCEDURE}

\section{A. Influent Wastewater Source}

Figure 1 schematically illustrates the overall experimental setup details, process flow and wastewater sampling points. In this study, raw wastewater samples were collected from one of the facultative ponds at Universiti Malaysia Sarawak (UNIMAS) campus. The pond water contains a mixture of septic tank effluents and wastewaters generated from food courts, pantries, laboratories, and storm waters that contain a relatively big amount of emulsified free oils, $\mathrm{BOD}, \mathrm{NH}_{3}-\mathrm{N}$, SS, N, P, and K.

Raw wastewaters were intensified with oils before pumped into the hair-based spiral filter by a hydraulic stirring pump. Two patterns of flow were tested, which are 1) Core-Baffle-Perimeter (Figure 2), 2) Perimeter-BaffleCore (Figure 3), to compare the efficiency of the filter system. Control valves were used to stabilize system flows meanwhile controlling the system flow rate. The flow rate of the system was maintained at 0.0227 liters/second $(\mathrm{L} / \mathrm{s})$ to $0.0526 \mathrm{~L} / \mathrm{s}$. Then the effluent was pumped by a hydraulic pump to the mixed flow trickling filter and applied onto the top layer of the filter by a fixed nozzle distributor. Because of the hydraulic gradient factor, a 6-metre pressure head submersible hydraulic pump was chosen for this experimental work. Flow rate through the fixed nozzle distributor was controlled by a valve to permit a desired flow at approximately 185 liters/day (L/day) to minimize the impact of shear velocity on the attached growth. This system has an under drain that collects the filtrate and solids, which also serves as a source of air for the microorganisms to maintain aerobic conditions in the filter. Sufficient air supply would be provided by natural draft and 
wind forces through ventilation ports at the bottom of the filter. Filter effluents were gravitated to the settling basin.

The Pistia Stratiotes-based FSW wetland system was designed to provide a surface overflow rate of approximately 167.70 liters/day.m ${ }^{2} \quad\left(15.4 \quad\right.$ L/day. $\left.\mathrm{ft}^{2}\right)$, detention time of 24 hours (1.0 day), and horizontal velocity of $5.15\left(10^{-3}\right) \mathrm{m} / \mathrm{hr}(0.17 \mathrm{ft} / \mathrm{hr})$. In this study, a total of 47 units of equally sized Pistia Stratiotes were used with the total plant mass of approximately $1.69 \mathrm{~kg}$. This FSW wetland system was placed outdoor sheltered by a transparent plastic sheet to avoid external disturbances such as rain waters. Pistia Stratiotes is characterized by having high number of suspended roots of over one foot long and longer roots are believed to have higher uptake capacity.

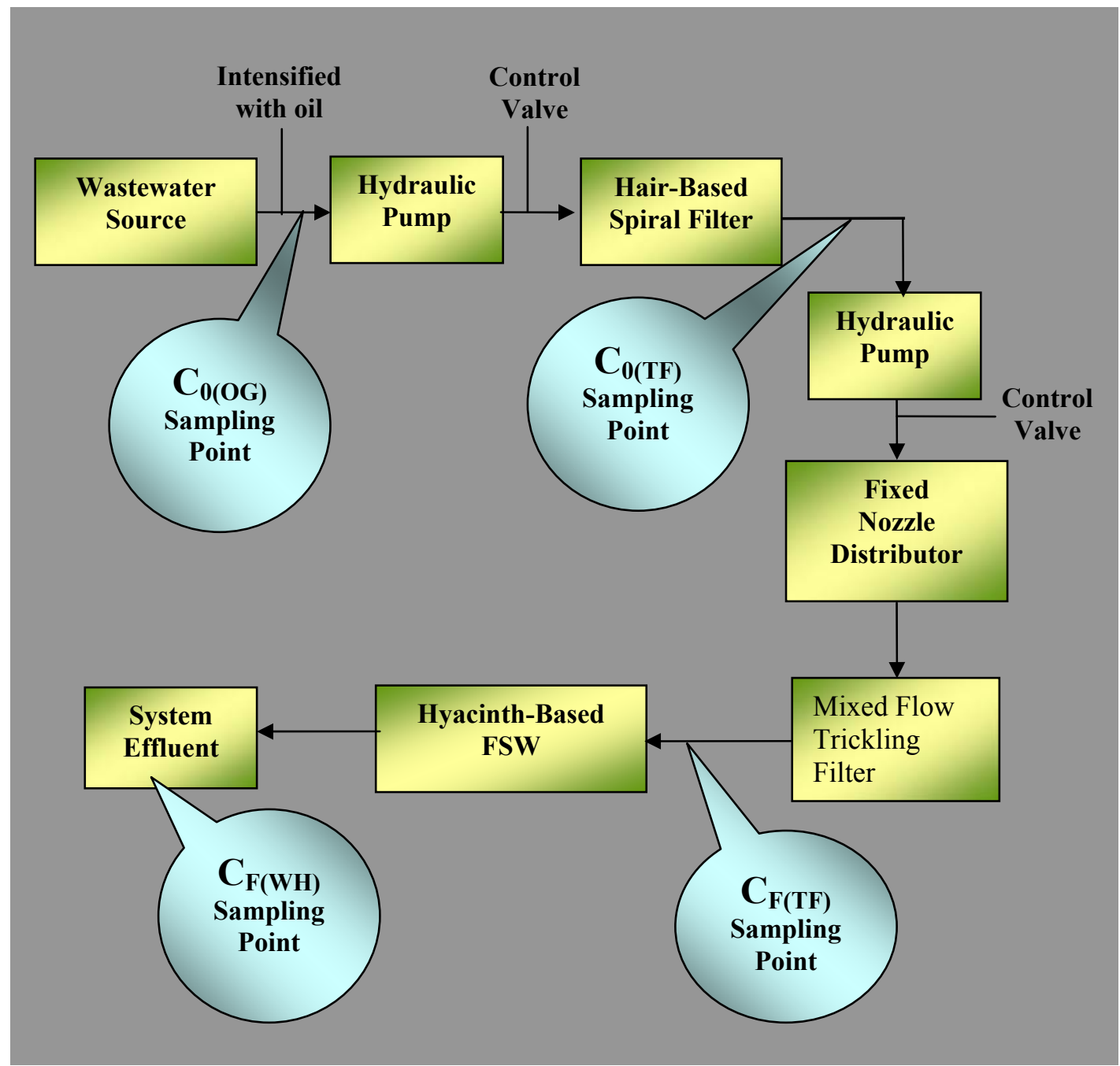

Figure 1. Schematic diagram of the overall process flow and water sampling points

\section{B. Sampling and Analysis}

In this study, the intensified raw wastewater samples abbreviated as $\mathrm{C}_{\mathrm{o}(\mathrm{OG})}$ (filter influent) were collected at location prior to influent to the hair-based spiral filter. A valve is located between the hydraulic stirring pump and filter to regulate flow rate. The influents were either discharged into the system though the system core or the perimeter weir as the inlet and flowed through a spiral plane filled with hair blocks and an intermediated a baffle zone before reaching the outlet (perimeter or core, vice versaly)as shown in Figure 2 and Figure 3.The effluent of the hairbased spiral tank was taken as $\mathrm{C}_{0(\mathrm{TF})}$ as it was also act as the influent to the next stage of filtration system which connect in series to the previous. Before discharging directly into the mixed flow trickling filter, the influent $\mathrm{C}_{0 \text { (TF) }}$ flow was controlled by a valve at a flowing rate of $185 \mathrm{~L} /$ day and a spraying nozzle was used to provide uniform spray onto the top layer of the filter. The wastewaters would trickle down from the top layer to the bottom by gravitation and collected in the settling basin. Samples were collected from the settling tank and abbreviated as $\mathrm{C}_{\mathrm{F}(\mathrm{TF})}$ (filter effluent). Filter effluents $\left(\mathrm{C}_{\mathrm{F}(\mathrm{TF}}\right)$ were then channeled to the hyacinth-based FSW wetland system as influent. Samples were then collected from the effluent of the hyacinth-based FSW

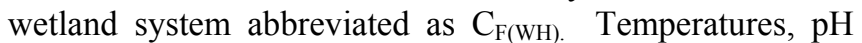
values, and turbidity at the sampling points were also monitored regularly and recorded throughout this experimental period. The experimental work of this research study was carried out for a period of 30 days. Trial run period extended from $2^{\text {nd }}$ to $10^{\text {th }}$ of February 2006 to make sure that stabilized conditions were attained. Actual sampling and analysis started on $11^{\text {th }}$ February 2006 and ended on $2^{\text {nd }}$ March 2006, and a total of 6 batches (for hairbased spiral system) and 9 batches (for mixed flow trickling filter \& water hyacinth-based system) of data were collected at the individual points and analyzed. Samples drawn at

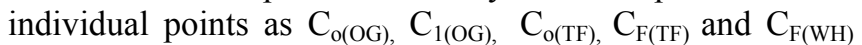
(Figures 1 to 3 ) were then analyzed at Environmental 
Engineering Laboratory, Department of Civil Engineering, Univeristi Malaysia Sarawak (UNIMAS). Some of the major analysis equipments and methods used in this study included HACH DR/4000 Spectrophotometer (8038, 8075,
8048 and 8237; 2), Hanna Dissolved Oxygen (DO) meter (APHA 5210B), Atomic Absorption (AA) Spectrophotometer (APHA $3500 \mathrm{~KB}$ ).

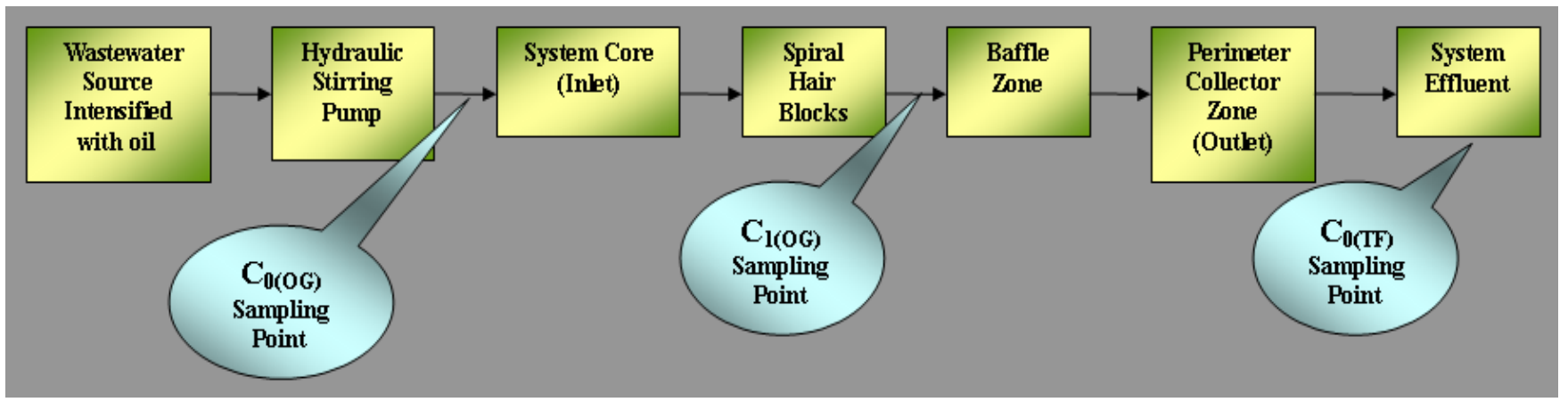

Figure 2. Schematic diagram of process flow and sampling point for "core-baffle-perimeter" pattern system

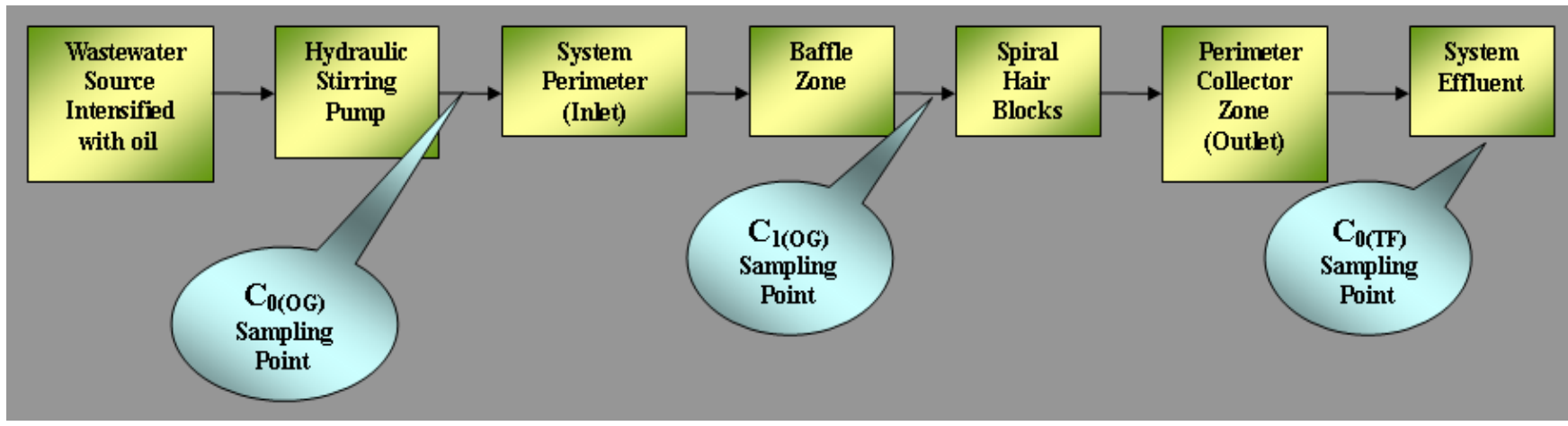

Figure 3. Schematic diagram of process flow and sampling point for "perimeter-baffle-core" pattern system

\section{RESULTS AND DISCUSSION}

Figure 4(a) and Figure 5(a) are the illustrations of the comparison of concentration and removal efficiencies of emulsified free oils of the hair based spiral removal system in the pattern flow "Core-Baffle-Perimeter" respectively. The system achieves an average reduction in concentration of $52.9 \mathrm{mg} / \mathrm{l}$ for "Core-Baffle Zone" and $22.8 \mathrm{mg} / \mathrm{l}$ for "Baffle-Perimeter Zone." The removal efficiencies for the overall "Core-Baffle-Perimeter" system was 53\% whereas $36.5 \%$ for Core-Baffle Zone and $15.8 \%$ for "BafflePerimeter Zone." It shows that the efficiencies of "BafflePerimeter Zone" are relatively low compared to "CoreBaffle Zone."

Figure 4(b) show the reduction of concentration of the hair based spiral removal system in the pattern flow "Perimeter-Baffle-Core" while Figure 5(b) described its removal efficiencies of emulsified free oils. An average reduction of $26.1 \mathrm{mg} / \mathrm{l}$ for Perimeter-Baffle Zone and 9.2 $\mathrm{mg} / \mathrm{l}$ for Baffle-Core Zone were summarized in the concentration of emulsified free oils. Meanwhile, the total removal efficiencies was $37 \%$ whereas $27.3 \%$ (PerimeterBaffle Zone) and 9.7\% (Baffle-Core Zone) for the overall "Perimeter- Baffle-Core" system. This pattern concluded a better performance in the removal efficiencies of "Perimeter-Baffle Zone" compared to "Baffle-Core Zone."

Figure 6(a) and Figure 7 illustrate the BOD removal efficiencies of the mixed flow trickling filter and hyacinthbased FSW wetland system by observing the amount of reduction in BOD levels during the experimental period. As shown, the filter demonstrated a very consistent removal rate $(\%)$ regardless of influent concentrations with an average removal efficiency of approximately $33.2 \%$. Similarly, the hyacinth-based FSW wetland system recorded a consistent removal rate with an average of approximately $30.8 \%$. It is shown that the performances of the two components of the system were quite close with a deviation of approximately $2.4 \%$. The combined BOD removal rate of the two components, i.e., the mixed flow filter and hyacinthbased system operated in series was approximately $64.0 \%$.

Figure 6(a) and Figure 7 show the observed removal efficiencies of $\mathrm{NH}_{3}-\mathrm{N}$ by trickling filter and hyacinth-based FSW wetland system from wastewaters during the study period. The average $\mathrm{NH}_{3}-\mathrm{N}$ removal efficiency achieved by the filter recorded $59.9 \%$ and the reduction rate $(\mathrm{mg} / \mathrm{L})$ was rather consistent throughout the experimental period regardless of influent $\mathrm{NH}_{3}-\mathrm{N}$ levels. As shown in Figure 6(a), hyacinth-based FSW wetland system demonstrated a similar consistent achievable effluent level to less than $2 \mathrm{mg} / \mathrm{L}$ regardless of the influent $\mathrm{NH}_{3}-\mathrm{N}$ levels, which indicates that Pistia Stratiotes has an extremely high uptake capacity of $\mathrm{NH}_{3}-\mathrm{N}$. As observed, it was demonstrated that $\mathrm{NH}_{3}-\mathrm{N}$ levels would substantially be reduced by both the mixed flow filter and hyacinth-based system. When the mixed flow filter and hyacinth-based system were to operate in series, the overall achievable removal efficiency of the system could be as high as $90.2 \%$.

Figure 6(a) and Figure 7 show the differences in phosphorous $(\mathrm{P})$ levels between $\mathrm{C}_{0(\mathrm{TF})}$ and $\mathrm{C}_{\mathrm{F}(\mathrm{TF})}$, and between $\mathrm{C}_{\mathrm{F}(\mathrm{TF})}$ and $\mathrm{C}_{\mathrm{F}(\mathrm{WH})}$. The amount of $\mathrm{P}$ reduced by the mixed filter was approximately $14.7 \%$, while the hyacinthbased FSW wetland system achieved $42.2 \%$. This indicates that the removal of $\mathrm{P}$ by hyacinth-based system was $27.5 \%$ 
higher that the mixed flow filter. When the performances are expressed in $\mathrm{mg} / \mathrm{L}$ of $\mathrm{P}$ reduced in the wastewaters, the observed data collected during experimental period demonstrated that, the filter could achieve an average of 0.6 $\mathrm{mg} / \mathrm{L}$ reduction of $\mathrm{P}$ while the hyacinth-based FSW system achieved an average of $1.14 \mathrm{mg} / \mathrm{L}$. When the filter and hyacinth-based system were to operate in series, the combined overall achievable removal efficiency of the system was approximately $56.9 \%$.

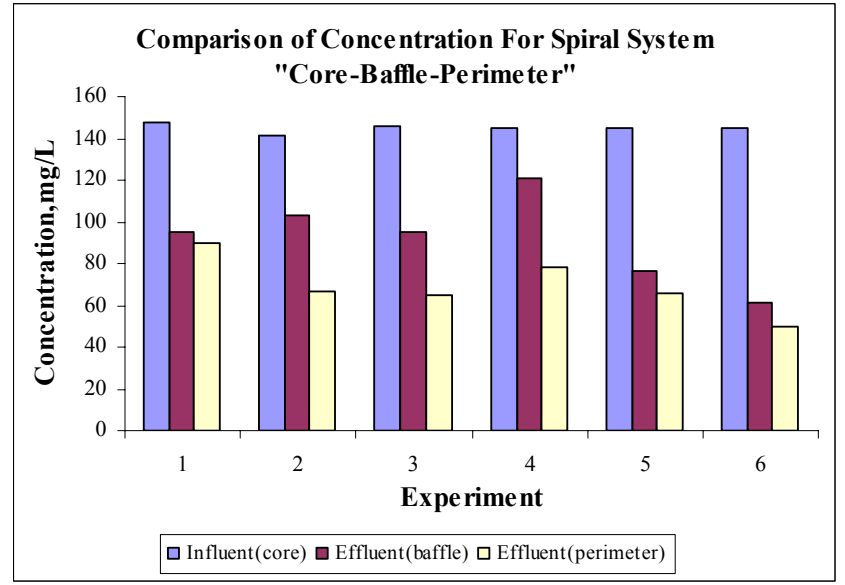

(a) "Core-Baffle-Perimeter"

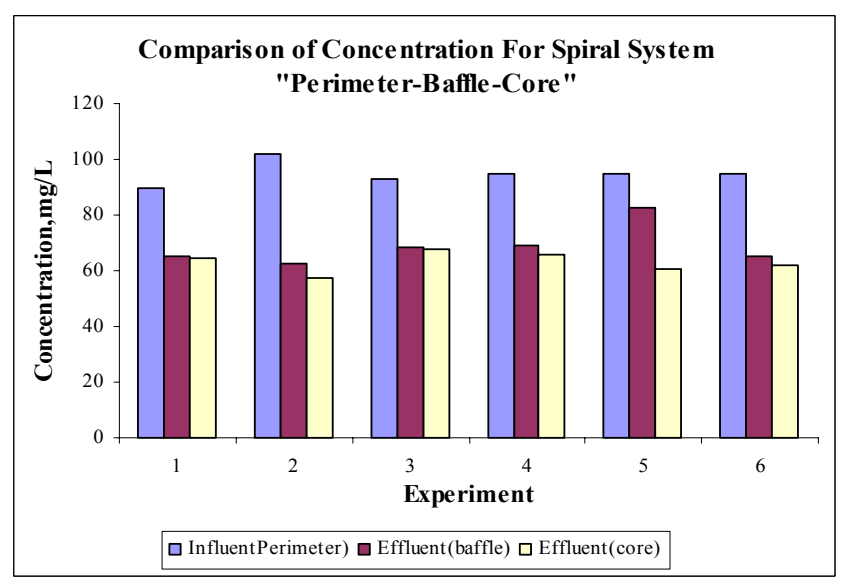

(b) "Perimeter-Baffle-Core"

Figure 4. Comparison of concentration for hair-based spiral filter system

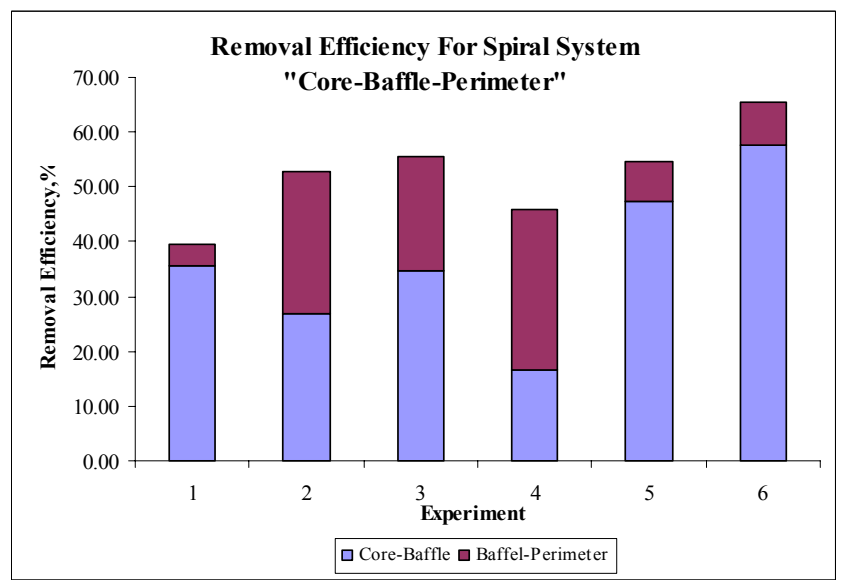

(a) "Core-Baffle-Perimeter"

Figure 5. Removal efficiency for hair-based spiral filter system

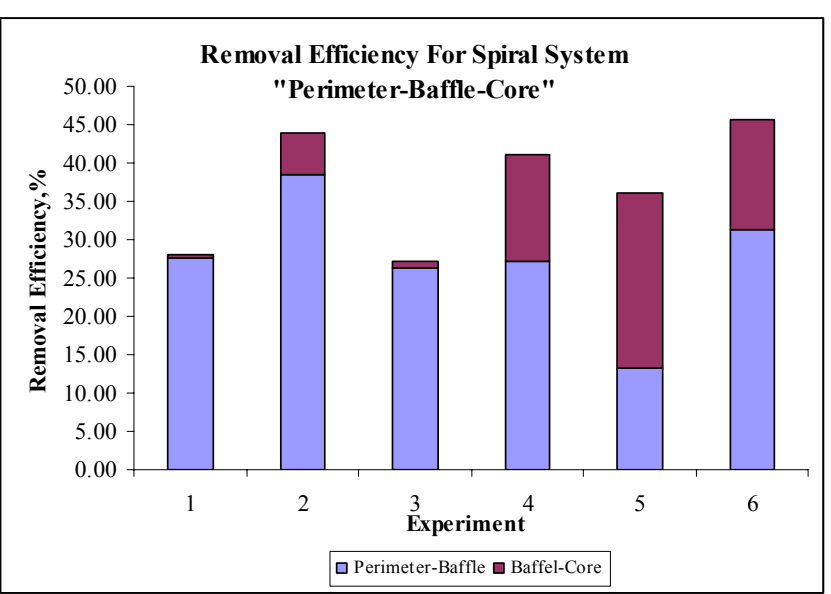

(b) "Perimeter-Baffle-Core" Figure 5. Continued

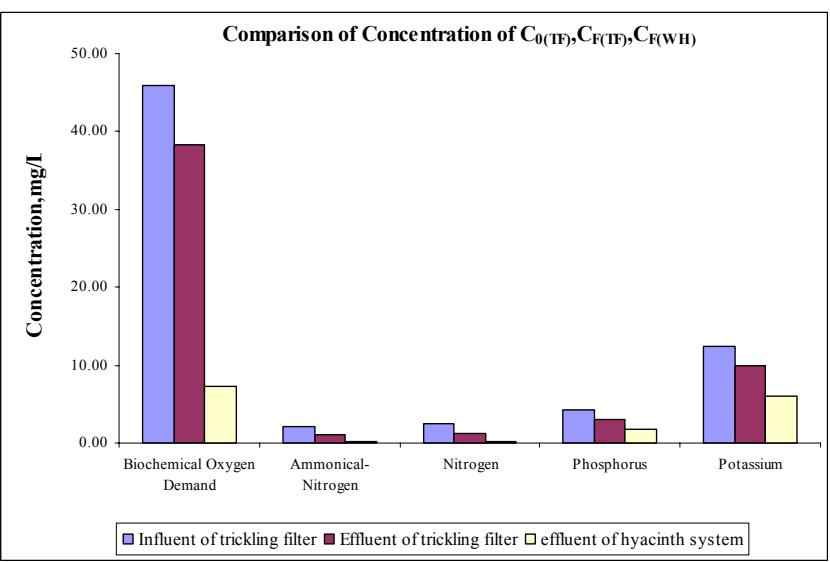

(a)

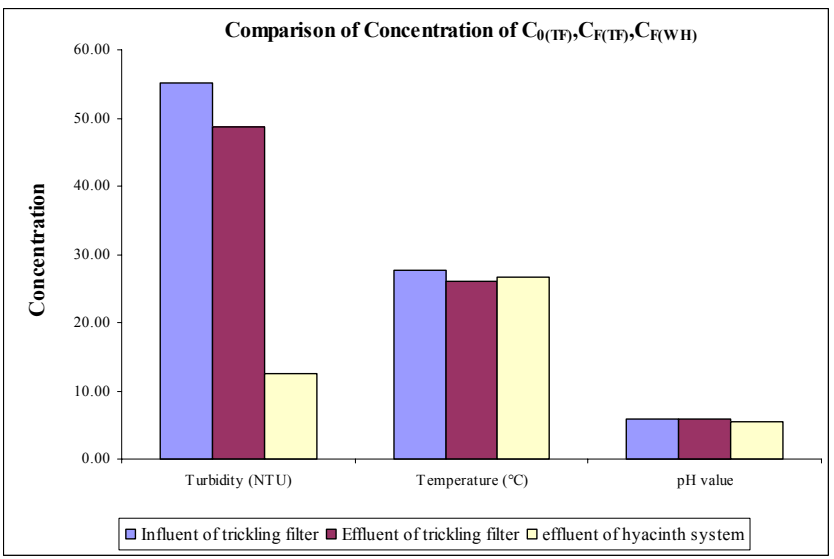

(b)

Figure 6. Comparison of concentration of effluents for trickling filter and hyacinth system

Plots of total nitrogen $(\mathrm{N})$ measured at the influents and effluents of the filter and hyacinth-based system with respect to time are illustrated in Figure 6(a) and Figure 7. The average amount of reduction in total nitrogen recorded between $\mathrm{C}_{0(\mathrm{TF})}$ and $\mathrm{C}_{\mathrm{F}(\mathrm{TF})}$ was approximately $51.6 \%$ by mixed flow filter, and between $\mathrm{C}_{\mathrm{F}(\mathrm{TF})}$ and $\mathrm{C}_{\mathrm{F}(\mathrm{WH})}$ indicated a $40.9 \%$ reduction by hyacinth-based system. When the filter and hyacinth-based system were to operate in series, the overall average total nitrogen removal efficiency could be as high as $92.5 \%$. When expressed amount of $\mathrm{N}$ removed from per liter $(\mathrm{mg} / \mathrm{L})$ of wastewater, the mixed flow filter attained 
an average of $0.88 \mathrm{mg} / \mathrm{L}$ while the hyacinth-based system achieved approximately $0.95 \mathrm{mg} / \mathrm{L}$. An analysis of the plots in Figure 6(a) shows that the patterns of total nitrogen reduction in the wastewater samples observed were similar to $\mathrm{NH}_{3}-\mathrm{N}$. This could be due to the direct relationship of $\mathrm{NH}_{3}-\mathrm{N}$ and total nitrogen because $\mathrm{NH}_{3}-\mathrm{N}$ molecules are formed by the nitrogen atoms after combining with hydrogen atoms to achieve stabilization. In the nitrogen cycle, the $\mathrm{NH}_{3}-\mathrm{N}$ would be transformed to nitrite and nitrate or backward through nitrification or denitrification processes. Thus, a reduction in $\mathrm{NH}_{3}-\mathrm{N}$ would give a lower total nitrogen level.

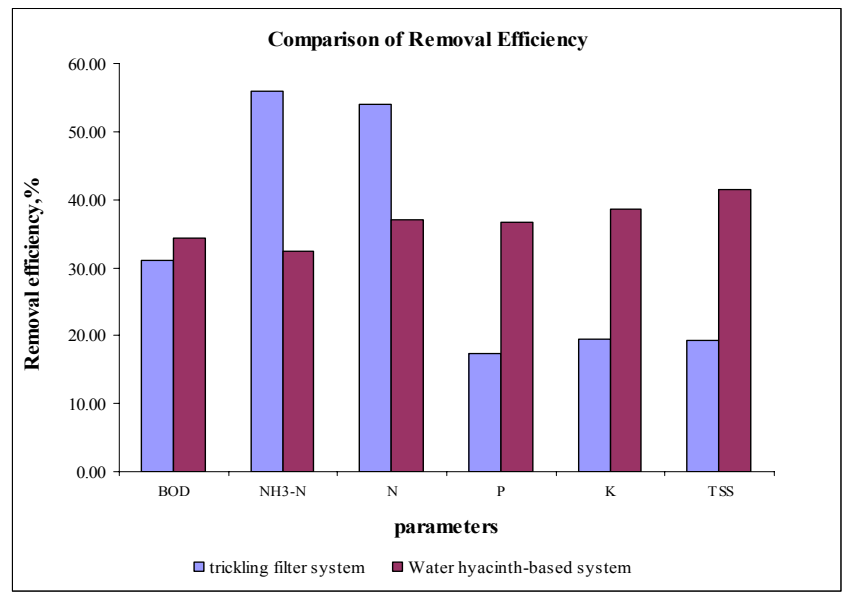

Figure 7. Comparison of removal efficiency for trickling filter and hyacinth system
In this experimental work, the potassium $(\mathrm{K})$ levels in the raw wastewater influents of the trickling filter recorded were in the range of 3.14 and $18.86 \mathrm{mg} / \mathrm{L}$. As illustrated in Figure 6(a) and Figure 7, the trickling filter was capable of removing an average of approximately $20.1 \%$ and water hyacinth-based FSW wetland system attained an average of $53.2 \%$ of $\mathrm{K}$ from wastewaters, which indicates that the $\mathrm{K}$ uptake rate of Prista Stratiotes is approximately $33.1 \%$ higher than the mixed flow filter. It was also demonstrated that when the filter and hyacinth-based FSW wetland system were to operate in series, the combined overall system removal efficiency system could achieve approximately $73.3 \%$.

In this study, effluent turbidity levels are measured in Nephelometry Turbidity Unit (NTU) for all water samples collected. The turbidity readings showed a decreasing trend as the wastewater moved through the treatment system. In Figure 6(b) and Figure 7, it is shown that the hyacinth-based FSW wetland system was capable of bringing down the average turbidity level to about 4 NTU, while the trickling filter was capable of reducing average turbidity level to approximately 8 NTU. The average reduction in turbidity levels were approximately $24.4 \%$ achieved by the mixed flow trickling filter, $29.7 \%$ by hyacinth-based system, and $54.1 \%$ when the filter and hyacinth-based system operated in series.

From Table 1, an value of $45 \%$ of removal efficiencies for the hair-based spiral system was recorded as the average value of the 2 patterns flow conducted in the experiment. The "Core-Baffle-Perimeter" and "Perimeter-Baffle-Core" patterns recorded $53 \%$ and $37 \%$ respectively.

Table 1. Overall removal efficiencies, $\%$ (when 3 stage water treatment systems operated in series)

\begin{tabular}{|c|c|c|c|c|}
\hline \multirow[b]{2}{*}{ Parameters } & \multicolumn{4}{|c|}{ Removal Efficiencies (\%) } \\
\hline & $\begin{array}{l}\text { Hair-based } \\
\text { Spiral Filter } \\
(\%)\end{array}$ & $\begin{array}{l}\text { A. Trickling } \\
\text { B. Filter } \\
\text { C. }(\%)\end{array}$ & $\begin{array}{l}\text { Hyacinth-Based } \\
\text { System } \\
(\%)\end{array}$ & $\begin{array}{c}\text { D. } 3 \text { Stage Treatment } \\
\text { System Operating in Series } \\
(\mathbf{\%})\end{array}$ \\
\hline Emulsified Free Oils & $45.0(*$ average $)$ & 1 & 1 & 45.0 \\
\hline Biochemical Oxygen Demand & 1 & 33.2 & 30.8 & 64.0 \\
\hline Ammoniacal Nitrogen & 1 & 59.9 & 30.3 & 90.2 \\
\hline Nitrogen & 1 & 51.6 & 40.9 & 92.5 \\
\hline Phosphorus & 1 & 14.7 & 42.2 & 56.9 \\
\hline Potassium & 1 & 20.1 & 53.2 & 73.3 \\
\hline Turbidity & 1 & 24.4 & 29.7 & 54.1 \\
\hline
\end{tabular}

Note: (*average) is an average value for patterns "Perimeter-Baffle-Core" and "Core-Baffle-Perimeter"

As shown in Table 1, the observed average removal efficiencies of trickling filter were $33.2 \%$ for BOD, $59.9 \%$ for ammoniacal nitrogen, $51.6 \%$ for total nitrogen, $14.7 \%$ for phosphorus, $20.1 \%$ for potassium, and $24.4 \%$ reduction on turbidity levels. The hyacinth-based FSW wetland system demonstrated to be capable of removing an average of $30.8 \%$ of BOD, $30.3 \%$ of ammoniacal-nitrogen, $40.9 \%$ of nitrogen, $42.2 \%$ of phosphorus, $53.2 \%$ of potassium, and $29.7 \%$ reduction on turbidity levels. It is found that hyacinth-based FSW wetland system was relatively more efficient than trickling filter in terms of phosphorus and potassium removal, while the trickling filter was relatively more efficient in BOD and total nitrogen removal. When filters and hyacinth-based system were to operate in series, the removal efficiencies (Table 1 ) were $45 \%$ for emulsified free oils, $64.1 \%$ for BOD, $90.2 \%$ for ammoniacal nitrogen, $92.5 \%$ for total nitrogen, 56.9 for phosphorous, $73.3 \%$ for potassium, and $54.1 \%$ reduction on turbidity levels.

In this research, water hyacinth (Pistia Stratiotes) mass increment versus nutrient uptake rates by the hyacinth was studied. Measured field tests data showed that daily uptake rate of organics and nutrients expressed in $\mathrm{mg} / \mathrm{kg}$-day, i.e., $\mathrm{mg}$ of organics and nutrients removed by one $\mathrm{kg}$ of Pistia Stratiotes per day were approximately $1,460 \mathrm{mg}$ for BOD, $942 \mathrm{mg}$ for $\mathrm{NH}_{3}-\mathrm{N}, 1,134 \mathrm{mg}$ for $\mathrm{N}, 1,361 \mathrm{mg}$ for $\mathrm{P}$, and $5,884 \mathrm{mg}$ for $\mathrm{K}$. 


\section{CONCLUSION}

A three-stage treatment system consisting of a spiralframed filter, a mixed flow filter and hyacinth-based FSW wetland was developed and field tests were carried out to determine system efficiencies in removal of oil and grease, organic matters and nutrients. For the hair-based spiral system, the flow pattern Core-Baffle-Perimeter has achieved an average removal efficiency of $53 \%$ while the pattern Perimeter-Baffle-Core has concluded an average efficiency of $37 \%$.For the Bio-tower and Water-hyacinth based system, it is concluded that the removal efficiencies achieved by the mixed flow filter were approximately $33.2 \%$ for BOD, $59.9 \%$ for $\mathrm{NH}_{3}-\mathrm{N}, 51.6 \%$ for $\mathrm{N}, 14.7 \%$ for $\mathrm{P}, 20.1 \%$ for $\mathrm{K}$, and $24.4 \%$ reduction in turbidity level, whilst the Pistia Stratiotes-based FSW system achieved $30.8 \%$ for BOD, $30.3 \%$ for $\mathrm{NH}_{3}-\mathrm{N}, 40.9 \%$ for $\mathrm{N}, 42.2 \%$ for $\mathrm{P}, 53.2 \%$ for $\mathrm{K}$, and $29.7 \%$, reduction in turbidity level. The combined overall system removal efficiencies (i.e., mixed flow filter and Pistia Stratiotes-based system operating in series) were approximately $64.1 \%$ for BOD, $90.2 \%$ for $\mathrm{NH}_{3}-\mathrm{N}, 92.5 \%$ for $\mathrm{N}, 56.9 \%$ for $\mathrm{P}, 73.3 \%$ for $\mathrm{K}$, and $54.1 \%$ reduction on turbidity levels. It is also concluded that daily uptake or removal rates ( $\mathrm{mg} / \mathrm{kg}$-day) of organics and nutrients by one $\mathrm{kg}$ of Pistia Stratiotes per day were approximately 1,460 mg for BOD, $942 \mathrm{mg}$ for $\mathrm{NH}_{3}-\mathrm{N}$, $1,134 \mathrm{mg}$ for $\mathrm{N}, 1,361 \mathrm{mg}$ for $\mathrm{P}$, and 5,884 $\mathrm{mg}$ for $\mathrm{K}$.

\section{REFERENCES}

[1] Tchobangoglous, G and Burton, F.L. (1991). Wastewater Engineering: Treatment, Disposal, and Reuse, Third Edition. Metcalf \& Eddy, Inc., USA.

[2] United States Environmental Protection Agency (USEPA), Sept 2000 Wastewater Technology Fact Sheet Trickling Filters, EPA 832-F-00014, Office of Water, Washington, D.C.

[3] United States Environmental Protection Agency (USEPA), Sept 2000. Wastewater Technology Fact Sheet Trickling Filter Nitrification, EPA 832-F-00-015, Office of Water, Washington, D.C.

[4] Brix, H. \& Schierup, H. (1989). The use of aquatic Macrophytes in water-pollution control. Ambio, 18(2), 100-107.

[5] Kumar, P. \& Garde, R. J. (1989). Potentials of water hyacinth for sewage treatment. Research Journal of the Water Pollution Control Federation, 61 (11/12), 1702-1706.

[6] Mars, R. (2004). Greywater Treatment with Macrophytes, Greywater Reuse Systems. Washington: Midland Business Centre.

\section{NOMENCLATURE}

$\mathrm{C}_{\mathrm{o}(\mathrm{OG})} \quad$ Oil\&Grease Filter Influent

$\mathrm{C}_{1(\mathrm{OG})} \quad$ Oil\&Grease Filter Intermediate Effluent

$\mathrm{C}_{\mathrm{o}(\mathrm{TF})} \quad$ Oil\&Grease Filter Effluent/Trickling Filter Influent

$\mathrm{C}_{\mathrm{F}(\mathrm{TF})} \quad$ Trickling Filter Effluent/Hyacinth-Based FSW Influent

$\mathrm{C}_{\mathrm{F}(\mathrm{WH})} \quad$ Hyacinth-Based FSW Effluent 


\title{
Oil Droplets Removal Efficiencies Using Inclined Coalescence Plates: A Comparison between Co- and Counter-Current Flow
}

\author{
L.H. Ngu, K.K. Wong, P.L. Law and A.A.R. Pgn Hj Yuosf
}

\begin{abstract}
Circular separation tank with inclined coalescence plates applies the principle of Boycott effect whereby the presence of inclined medium promotes faster rising of oil droplets and their coalescence. The separator takes the shape of a circular tank to take advantage of the continual decreasing in horizontal velocity, $v_{h}$ as surface area increases. It is designed with a center-feed vertical perforatedpipe distributor inlet directing flow to the inclined coalescence plates to provide either co-current or counter current flow depending on the arrangement of inclined coalescence plate. This research is conducted to study the effect of different flow on oil-water separation efficiency. Co-current flow for the circular separation tank was achieved with 4 series of inverted and upright frustums-shaped coalescence plates. Counter current flow was achieved with 6 sets of coalescing plates, extending along the radius and each covering $60^{\circ}$ of the separation tank with plates inclined at $55^{\circ}$ and $125^{\circ}$. The effluent oil concentration, $C_{e}$ was found to be directly proportional to influent flowrate, $Q_{i}$. $Q_{i}$ can be expressed as a function of $C_{e}$ for different influent oil concentration, $C_{i}$ by a series of power equations. These power equations were used to predict the required flowrate, $Q_{10}$ for a given $C_{i}$ to achieved desired $C_{e}$ of $10 \mathrm{mg} / \mathrm{L}$ to meet environmental discharge requirement under the Malaysia Environmental Quality Act and Regulation. Different power equation $Q_{10}=22.851 C_{i}^{-1.6225}$ and $Q_{10}=166.19 C_{i}^{-1.4314}$. Using these equations, $Q_{10}$ for counter current flow is 18.6 times higher than co-current flow. Results were obtained through curve fitting exercise to express the $Q_{10}$ for co-current and counter current flow respectively. This indicates that co-current flow required a lower flowrate to achieve $C_{e}$ of $10 \mathrm{mg} / \mathrm{L}$. At $C_{i}=100 \mathrm{mg} / \mathrm{L}$ counter current flow achieved $15 \%$ higher oil removal as compare to co-current flow.
\end{abstract}

Keywords: oil-water separation, inclined coalescence plates

\section{INTRODUCTION}

Production of wastewater loaded with excessive amount of oil and grease is a common and long-existing problem in both the industrial and municipal sectors. Under the Malaysian Environmental Quality Act and Regulation, the

L.H. Ngu and K.K. Wong are PhD candidates with the Department of Civil Engineering, UNIMAS, 94300, Kota Samarahan, Sarawak, Malaysia (email: lockhei@yahoo.co.uk).

P.L. Law and A.A.R. Pgn Hj Yuosf are Lecturers with Faculty of Engineering, UNIMAS, 94300, Kota Samarahan, Sarawak, Malaysia (Tel: 6082-583282, email: puonglaw@feng.unimas.my). amount of oil and grease content allowable in sewage and industrial effluent discharge is $10 \mathrm{mg} / \mathrm{L}$ or less. For Interim Marine Water Quality Standards no amount $(0 \mathrm{mg} / \mathrm{L})$ of oil and grease is allowable. Malaysia Environmental Quality Report 2004 reported Malaysia's marine water oil and grease quality of $34.7 \mathrm{mg} / \mathrm{L}$ in 2001, $50.5 \mathrm{mg} / \mathrm{L}$ in 2002, $34.7 \mathrm{mg} / \mathrm{L}$ in 2003 and $49.1 \mathrm{mg} / \mathrm{L}$ in 2004.

A circular phase separation tank with inclined coalescence plates was recently developed by Law et al. (2006) to removed physically emulsified and free oil from wastewaters. The primary component of the separation system consists of 4 series of inverted and upright frustums (Fig. 1) to enhance gravity separation and coalescence of oil droplets in wastewaters. It applies the principle of Boycott effect whereby the presence of inclined frustums medium promotes faster rising of oil droplets and coalescence of oil (Rensselear, 2002). The separator comes in the shape of a circular basin by taking advantage of the continual decrease in horizontal velocity, $v_{h}$ as surface area increases (Deininger et al., 1996). The inlet of the separation system is equipped with an upflow center-feed perforated-pipe distributor to direct flow to the parallel inclined frustums to give a co-current flow.

\section{INCLINED Plate ARRANGEMENT FOR CO-CURRENT AND COUNTER CURRENT FLOW}

\section{A. Applicability of Inclined Coalescence Plates}

The inclined coalescence plates were expected to serve two purposes, i.e., (1) to promote laminar flow, and (2) to promote optimum oil-water separation efficiency. By using the principles of (a) a maximum amount of plate surface area provided for oil droplets coalescence to take place, and (b) a minimum distance (spacing inversely proportionally to distance from inlet) for lighter oil droplets to rise and hit the bottom side of the inclined plates as shown in Fig. 3. The coalescing plates assist oil droplets to coalescence and rise to the surface to be collected.

The use of coalescence plate facilitates the capture of oil droplets from water, and easy removal of captured oil from the plates to the surface of the tank. Captured oil spreads on the surface of the plates and coalescences into larger droplets and eventually forms a film on the plates. It was necessary, having captured the oil on the plates, to remove it 
from the plates in an orderly manner that does not re-entrain the oil into the wastewater stream. The design of coalescence plates was such that coalescence droplets were required to travel a maximum of $10 \mathrm{~cm}$ (co-current flow) or $5.66 \mathrm{~cm}$ (counter-current) before they encountered an oil port. These oil ports were vertically aligned so that when the oil particles are released from the plates, they rise directly to the surface.

\section{B. Inclined Plate Arrangement for Co-Current Flow}

Figure 1 illustrates the inclined coalescence plate's arrangement to give a co-current flow for oil-water separation. The arrangement consist of (a) four series of parallel inclined frustums, (b) all series consist of several parallel successive layer of frustums, (c) series of frustums are placed up-right and inverted positions that subsequently form a multiple-angle arrangement, (d) frustums are inclined at an angle, $\theta$ between $50^{\circ}$ to $60^{\circ}$, (e) all frustums have an inclined length of $10.0 \mathrm{~cm}$, (f) number of frustums would increase with each subsequent series, and (g) interval between frustums decreases with each subsequent series. Fig. 3a shows the mechanism of oil-water separation for cocurrent flow.

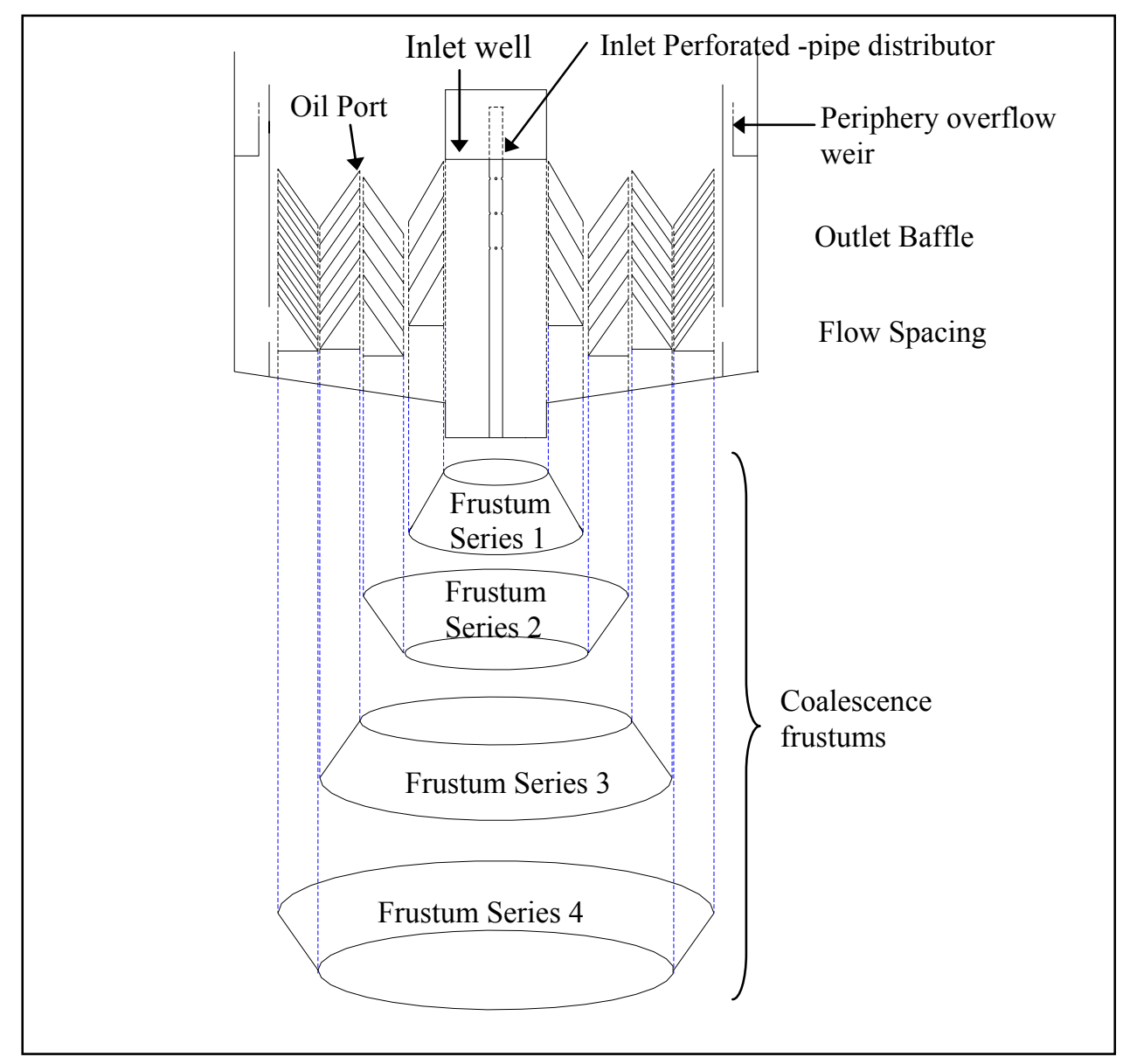

Figure 1. Circular phase separation tank with parallel inclined coalescence frustums (consisting of up-right and inverted series of conical frustums) to achieved co-current flow

\section{Inclined Plate Arrangement for Counter Current Flow}

Figure 2 illustrates the inclined coalescence plate's arrangement to give a counter current flow for oil water separation. The arrangement consist of (a) 6 set of coalescing plates, each covering $60^{\circ}$ of the circular separation tank, (b) each coalescing plates set consist of several parallel successive layer of plates, (c) plates inclined at angle, $\theta 55^{\circ}$ and $125^{\circ}$ to form the dual angle coalescing plates arrangement, (d) coalescence plates, CP1 extend along radius of separation tank, and (e) interval between inclined plates form the oil port. Fig. $3 b$ shows the mechanism of oil-water separation for counter current flow.

\section{EXPERIMENTAL PROCEDURE}

This section outlines the research areas and experimental procedure of carrying out the experiment to determine the relation between influent flowrate, $Q_{i}$ and effluent oil concentration, $C_{e}$ at different influent oil concentration, $C_{i}$ in an attempt to determine the required flowrate, $Q$ to achieve desirable effluent oil concentration, $C_{e}$ of $10 \mathrm{mg} / \mathrm{L}$. Influent flowrate, $Q_{i}$ relation with effluent oil concentration, $C_{e}$ for inclined plate arrangement with co-current flow had being carried out by Law et al., 2006 at $C_{i}$ of $100 \mathrm{mg} / \mathrm{L}$ and 1000 $\mathrm{mg} / \mathrm{L}$. Similar procedure at different $C_{i}$ will be carried out for inclined plate arrangement with counter current flow to compare the effect of different flow pattern on oil-water separation efficiency. 


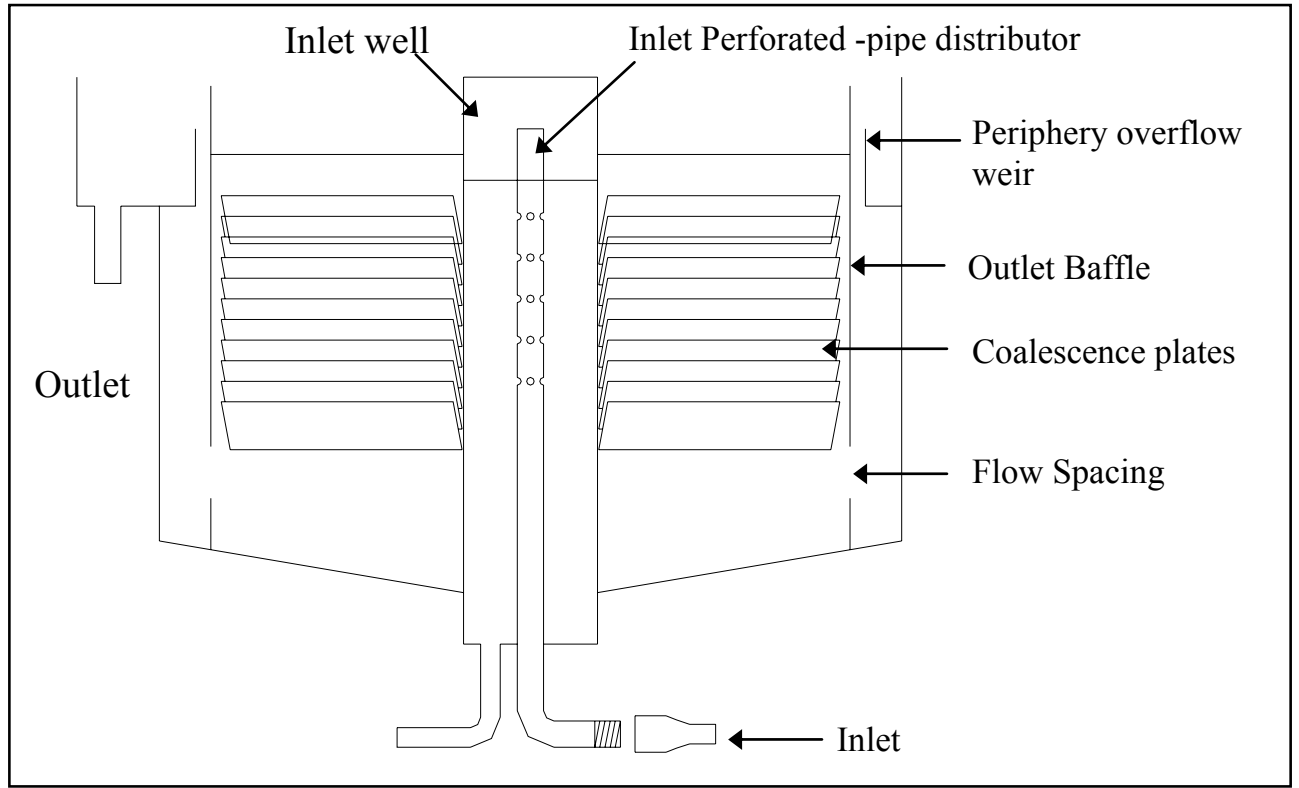

(a) Side view

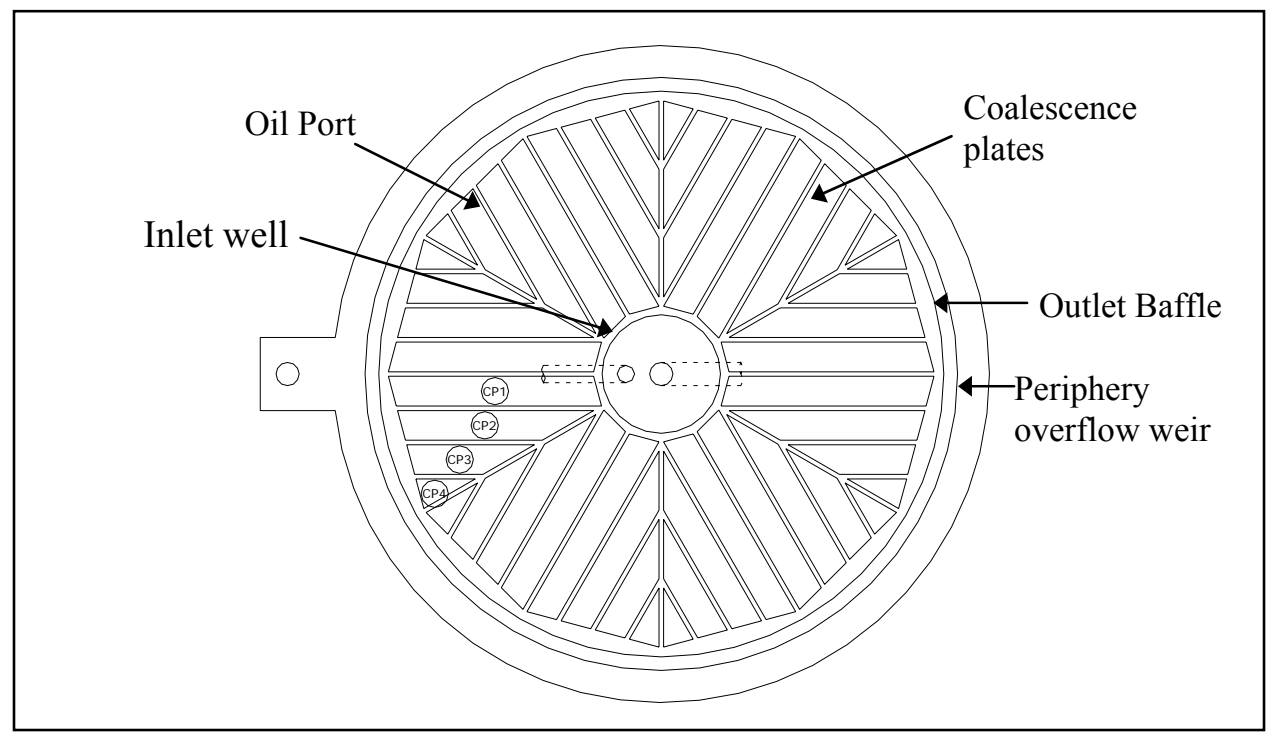

(b) Top view

Figure 2. Circular phase separation tank with inclined coalescence plates arrangement to achieved counter current flow

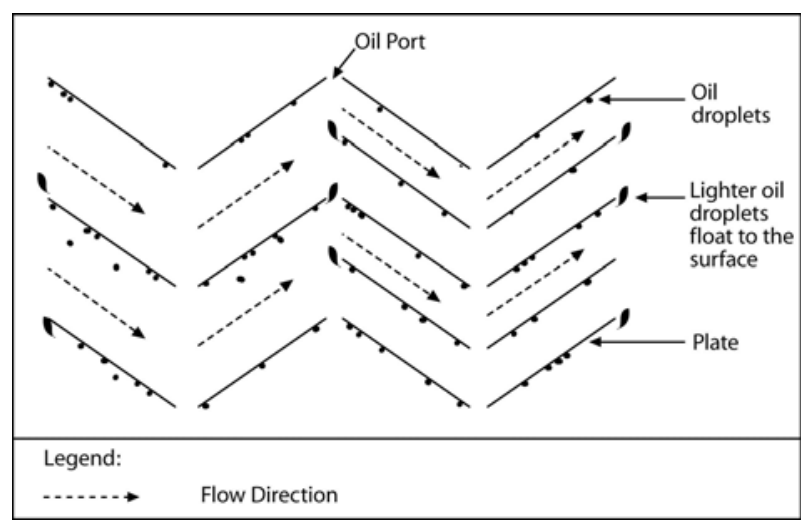

(a) Co-current flow

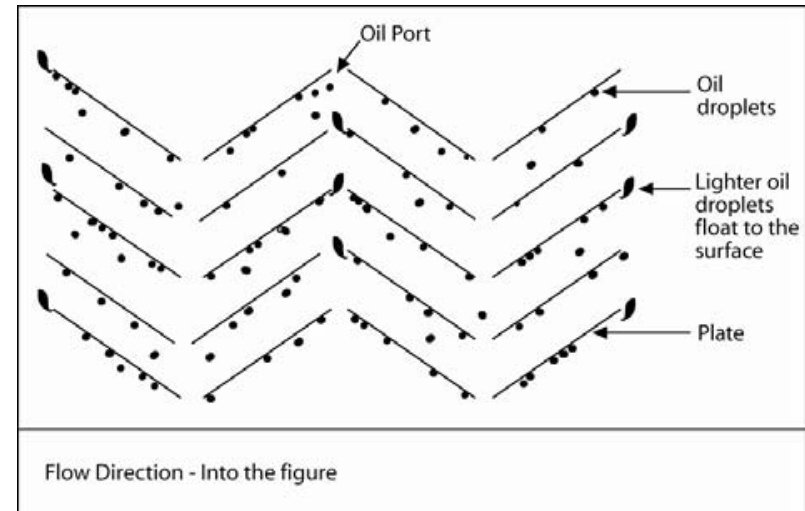

(b) Counter current flow

Figure 3. Parallel inclined coalescing plates: mechanism of oil-water separation 


\section{A. Mixing Tank Set Up and Sample Preparation}

The primary function of the mixing tank in this experimental setup was to mix oil with water to form influent of known oil concentrations and to pump the mixture at designed flowrate as influent to the separation system. The mixing tank was designed as a typical agitation process vessel or a cylindrical vessel with a vertical axis at a capacity of 200 liters (L).

A volume of 200 liters of oil water mixture at known concentrations were prepared with palm olein oil with mass density, $\rho_{o}$ given as $890 \mathrm{~kg} / \mathrm{m}^{3}$. Mixing tank was filled with $200 \mathrm{~L}$ of water and oil as illustrated in Table 1, and the water and oil was mixed for 5 minutes by switching on the motor-impeller system. A schematic diagram of the separation system and mixing tank connected in series is illustrated in Fig. 4.

\section{B. Oil Removal Relation with Influent Flowrate}

Separation tank oil removal efficiency and capability would vary with different influent flowrates, $Q_{i}$, influent oil concentrations, $C_{i}$, and separation tank retention times, $t$. Different influent oil concentrations tested were $100 \mathrm{mg} / \mathrm{L}$, $200 \mathrm{mg} / \mathrm{L}, 300 \mathrm{mg} / \mathrm{L}$ and $400 \mathrm{mg} / \mathrm{L}$, which were 10 to 40 times higher than the desired separator effluent concentration of $10 \mathrm{mg} / \mathrm{L}$. In this research study, a total of sixteen (16) tests were carried out, and the details are illustrated in Table 2.
Influent oil concentration of Test 1 as stated in Table 2 (the sample was prepared based on the details shown in Table 1). 1L sample of influent was collected from the mixing tank and oil concentration in water was measured with an oil water analyzer, Model OCMA-310 of Horiba. The influent was pumped to the separation tank at $0.5 \times 10^{-5}$ $\mathrm{m}^{3} / \mathrm{s}$. The mixture from the mixing tank was continuously pumped into the separation tank for a period of 288 minutes (which was also the retention time in the separator).

The mixture in the mixing tank was topped up by addition of $100.0 \mathrm{~L}$ of water and the required oil to produce an influent oil concentration of $100 \mathrm{mg} / \mathrm{L}$ whenever necessary. The mixture was thoroughly agitated for 2 minutes. A volume of $1.0 \mathrm{~L}$ of effluent was collected and measured with the HORIBA oil water analyzer. The procedure was then repeated for Test 2 to Test 16 (Table 2).

Table 1. Ratio of water and oil

\begin{tabular}{|c|c|c|}
\hline $\begin{array}{c}\text { Influent Oil } \\
\text { Concentration, } \mathrm{C}_{i} \\
(\mathrm{mg} / \mathrm{L})\end{array}$ & Water (L) & Oil (mL) \\
\hline 100 & 200 & 22.74 \\
\hline 200 & 200 & 45.48 \\
\hline 300 & 200 & 68.22 \\
\hline 400 & 200 & 90.96 \\
\hline
\end{tabular}

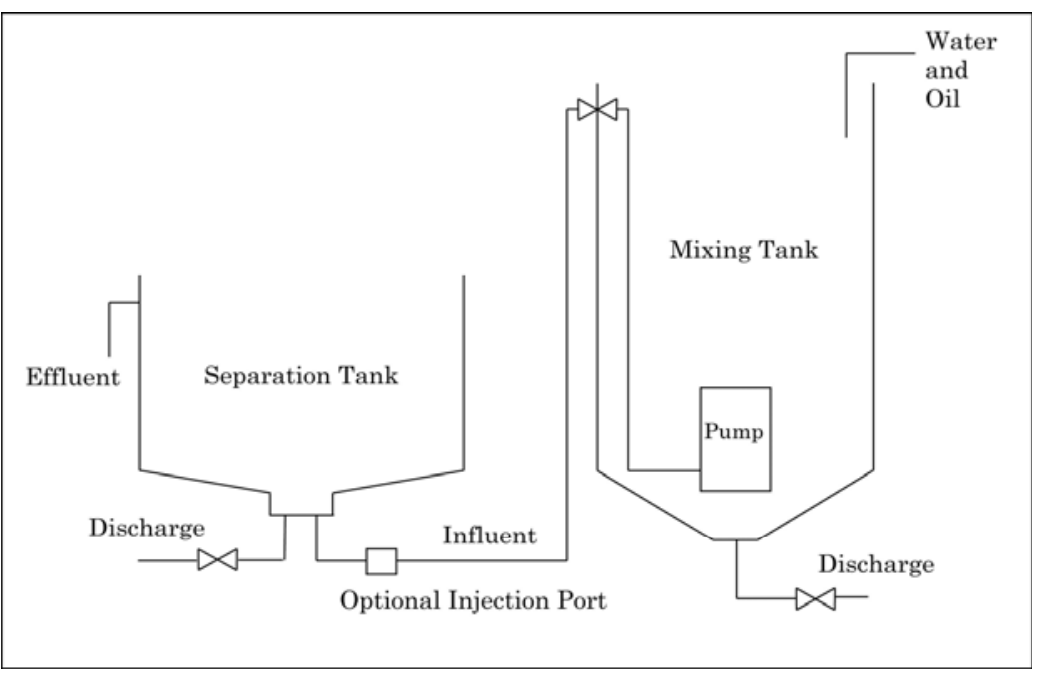

Figure 4. Schematic diagram of mixing and separation system

Table 2. Test condition influent oil concentrations, $C_{i}$, retention time, $t$ and influent flowrates, $Q_{i}$

\begin{tabular}{|c|c|c|c|c|}
\hline $\begin{array}{c}\text { Flowrate } \\
\left(\mathrm{m}^{3} / \mathrm{s}\right)\end{array}$ & Retention time, $\mathrm{t}(\mathrm{min})$ & \multicolumn{3}{|c|}{ Influent Oil Concentration, $\mathrm{C}_{i}(\mathrm{mg} / \mathrm{L})$} \\
\cline { 2 - 5 } & & 100 & 200 & 300 \\
\hline $0.5 \times 10^{-5}$ & 288 & Test 1 & Test 5 & Test 9 \\
\hline $0.7 \times 10^{-5}$ & 205 & Test 2 & Test 6 & Test 10 \\
\hline $1.0 \times 10^{-5}$ & 144 & Test 3 & Test 7 & Test 14 \\
\hline $2.0 \times 10^{-5}$ & 72 & Test 4 & Test 8 & Test 15 \\
\hline
\end{tabular}

\section{RESUlTS AND DISCUSSION}

Law et al., 2006 phase separator inclined coalescence plate arrangement with co-current flow influent flowrate, $Q_{i}$ relation with effluent oil concentration, $C_{e}$ at influent oil concentration, $C_{i}$ of $100 \mathrm{mg} / \mathrm{L}$ and $1000 \mathrm{mg} / \mathrm{L}$ is illustrated in Fig. 5. The effluent oil concentration, $C_{e}$ was found to be directly proportional to influent flowrate, $Q_{i}$. The influent flowrate, $Q_{i}$ can be expressed as a function of effluent oil concentration, $C_{e}$ for different influent oil concentration, $C_{i}$ by a series of power equations, $Q_{i}=1 \times 10^{-5} C_{e}^{3.1139}\left(\mathrm{R}^{2}=\right.$ $0.7616)$ for $C_{i}=100 \mathrm{mg} / \mathrm{L}$ and $Q_{i}=1 \times 10^{-6} C_{e}^{2.4914}\left(\mathrm{R}^{2}=\right.$ 0.9896 ) for $C_{i}=1000 \mathrm{mg} / \mathrm{L}$ which was obtained through curve fitting exercise. These relation were used to calculate 
the required flowrate, $Q_{10}$ for a given influent oil concentration, $C_{i}$ to achieved desired effluent oil concentration, $C_{e}$ of $10 \mathrm{mg} / \mathrm{L}$ to meet environmental discharge requirement.

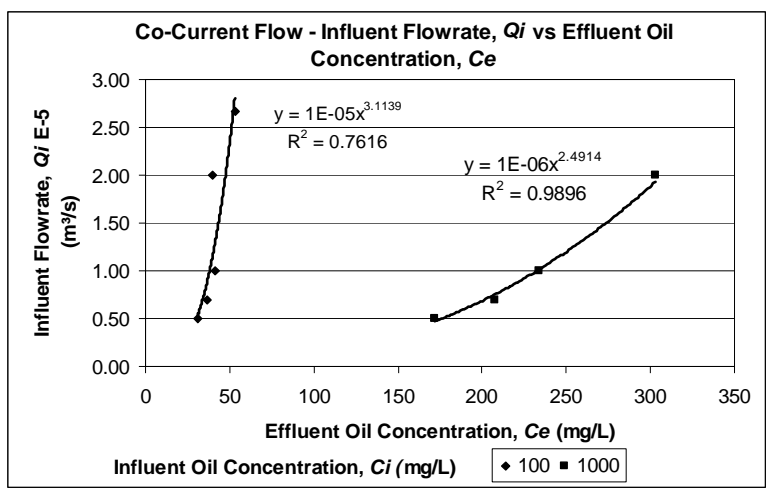

Figure 5. Effluent oil concentration, $C_{e}$ at different influent flowrate, $Q_{i}$ and influent oil concentration, $C_{i}$ of $100 \mathrm{mg} / \mathrm{L}$ and $1000 \mathrm{mg} / \mathrm{L}$ for co-current flow

Fig. 6 illustrates the required flowrate, $Q_{10}$ relation to influent oil concentration, $C_{i}$. Power equation $Q_{10}=22.851$ $C_{i}^{-1.6225}$ express the required flowrate needed to achieved effluent oil concentration, $C_{e}$ of $10 \mathrm{mg} / \mathrm{L}$ obtained through curve fitting exercise.

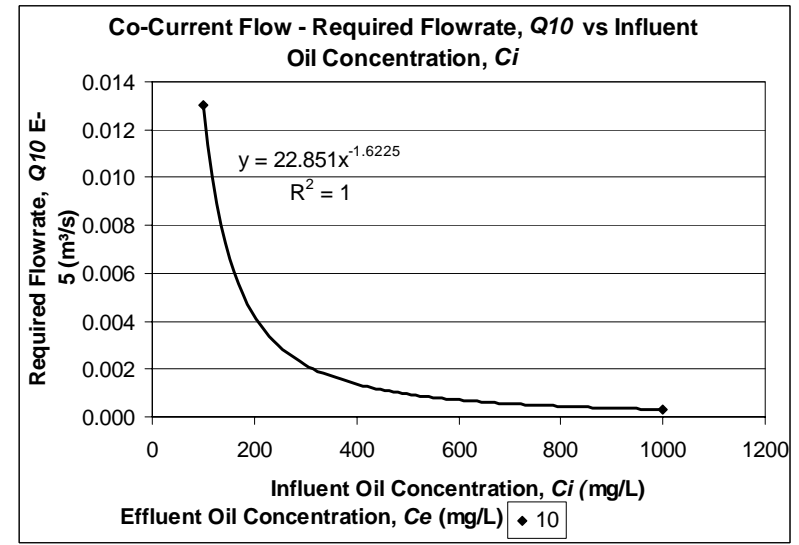

Figure 6. Required flowrate, $Q_{10}$ to achieve desired oil concentration, $C_{e}$ of $10 \mathrm{mg} / \mathrm{L}$ for influent oil concentration,

$C_{i}$ of $100 \mathrm{mg} / \mathrm{L}$ and $1000 \mathrm{mg} / \mathrm{L}$ for co-current flow

Fig. 7 illustrated the phase separator inclined coalescence plate arrangement with counter current flow influent flowrate, $Q_{i}$ relation with effluent oil concentration, $C_{e}$ at different influent oil concentration, $C_{i}$. The effluent oil concentration, $C_{e}$ was found to be directly proportional to influent flowrate, $Q_{i}$. The influent flowrate, $Q_{i}$ can be expressed as a function of effluent oil concentration, $C_{e}$ for different influent oil concentration, $C_{i}$ by a series of power equations as listed in Table 3 obtained through curve fitting exercise. These relation were used to calculate the required flowrate, $Q_{10}$ for a given influent oil concentration, $C_{i}$ to achieved desired effluent oil concentration, $C_{e}$ of $10 \mathrm{mg} / \mathrm{L}$ to meet environmental discharge requirement.

Fig. 8 illustrates the required flowrate, $Q_{10}$ relation to influent oil concentration, $C_{i}$. Power equation $Q_{10}=166.19$ $C_{i}^{-1.4313}\left(\mathrm{R}^{2}=0.7819\right)$ express the required flowrate needed to achieved effluent oil concentration, $C_{e}$ of $10 \mathrm{mg} / \mathrm{L}$ obtained through curve fitting exercise.

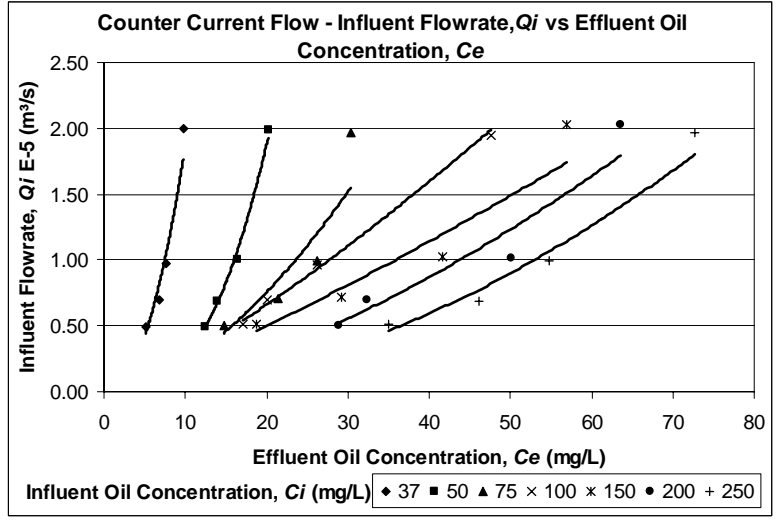

Figure 7. Effluent oil concentration, $C_{e}$ at different influent flowrate, $Q_{i}$ and influent oil concentration, $C_{i}$ for counter current flow

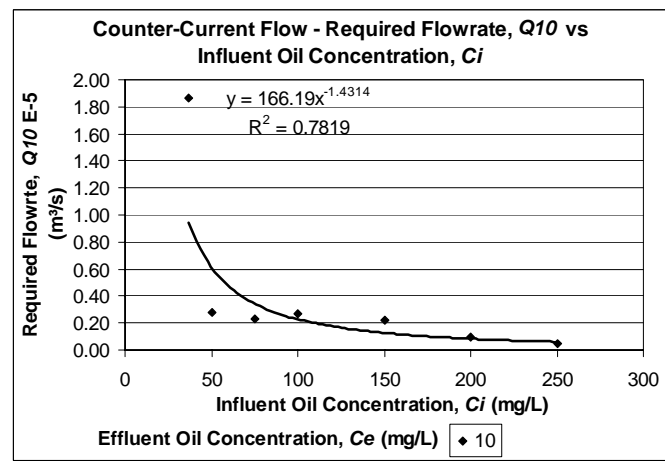

Figure 8. Required flowrate, $Q_{10}$ to achieve desired oil concentration, $C_{e}$ of $10 \mathrm{mg} / \mathrm{L}$ for influent oil concentration, $C_{i}$ for counter current flow

Power equation $Q_{10}=22.851 C_{i}^{-1.6225}$ and $Q_{10}=166.19 C_{i}^{-}$ 1.4314 express the $Q_{10}$ for co-current and counter current flow respectively. Using these equations, $Q_{10}$ for counter current flow is 18.6 times higher than co-current flow. This indicates that co-current flow required a lower flowrate to achieve $C_{e}$ of $10 \mathrm{mg} / \mathrm{L}$. At $C_{i}=100 \mathrm{mg} / \mathrm{L}$ counter current flow achieved $15 \%$ higher oil removal as compare to cocurrent flow.

Table 3. Influent flowrate, $Q_{i}$ as a function of effluent oil concentration, $C_{e}$ for different influent oil concentration, $C_{i}$

\begin{tabular}{|l|l|c|}
\hline $\begin{array}{l}\text { Influent Oil } \\
\text { Concentration, } \mathrm{C}_{i} \\
(\mathrm{mg} / \mathrm{L})\end{array}$ & Relationship & $\mathrm{R}^{2}$ \\
\hline 37 & $Q_{i}=0.0133 C_{e}{ }^{2.1472}$ & 0.9443 \\
\hline 50 & $Q_{i}=0.0004 C_{e}{ }^{2.8367}$ & 0.9953 \\
\hline 75 & $Q_{i}=0.0044 C_{e}{ }^{1.7212}$ & 0.8619 \\
\hline 100 & $Q_{i}=0.0146 C_{e}{ }^{1.272}$ & 0.9928 \\
\hline 150 & $Q_{i}=0.014 C_{e}{ }^{1.1936}$ & 0.936 \\
\hline 200 & $Q_{i}=0.0027 C_{e}{ }^{1.5609}$ & 0.9369 \\
\hline 250 & $Q_{i}=0.0006 C_{e}{ }^{1.8586}$ & 0.9636 \\
\hline
\end{tabular}

Both the separator system was designed to separate oil droplets sizes of more than $10 \mu \mathrm{m}$ in diameter to meet effluent requirements (Malaysia Environmental Quality Act and Regulation) of less than $10 \mathrm{mg} / \mathrm{L}$.

With reference to the experimental results in Fig. 5 and Fig 7, oil removal efficiency at different flowrates and influent concentrations, the desirable effluent oil concentration of $10 \mathrm{mg} / \mathrm{L}$ had only being achieved for inclined coalescence plate arrangement with counter current 
flow for $C_{i}$ of $37 \mathrm{mg} / \mathrm{L}$. However, this does not imply that the separator could not separate oil droplets down to $10 \mu \mathrm{m}$ in diameter for other $C_{i}$. The possible factor that might have contributed to this occurrence was due to a different influent oil droplets distribution spectrum. In this experiment, the mixing influent oil droplets distribution spectrum had not been determined. The emulsion of oil in water could contain a large portion of its oil in the form of oil droplets with diameter below $10 \mu \mathrm{m}$, which the separator was not designed to separate. If a large portion of the oil is in the form of oil droplets of diameter below $10 \mu \mathrm{m}$, then theoretically the separator is incapable of separating these oil droplets which result in higher concentration of oil in the effluent which could not meet the regulatory requirement and separator design requirement of $10 \mathrm{mg} / \mathrm{L}$.

\section{CONCLUSIONS}

The following conclusions can be drawn from the separator performance tests. The influent flowrate, $Q_{i}$ can be expressed as a function of effluent oil concentration, $C_{e}$ for different influent oil concentration, $C_{i}$ by a series of power equations. These power equations can be used to predict the required flowrate, $Q_{10}$ for a given influent oil concentration, $C_{i}$ to achieved desired effluent oil concentration, $C_{e}$ of $10 \mathrm{mg} / \mathrm{L}$ to meet environmental discharge requirement under the Malaysia Environmental Quality Act and Regulation. Power equation $Q_{10}=22.851$ $C_{i}^{-1.6225}$ and $Q_{10}=166.19 C_{i}^{-1.4314}$ express the $Q_{10}$ for cocurrent and counter current flow respectively. Using these equations, $Q_{10}$ for counter current flow is 18.6 times higher than co-current flow. This indicates that co-current flow required a lower flowrate to achieve $C_{e}$ of $10 \mathrm{mg} / \mathrm{L}$. This indicates that inclined coalescence plate arrangement with counter current flow is more effective for oil droplets removal. At $C_{i}=100 \mathrm{mg} / \mathrm{L}$ counter current flow achieved $15 \%$ higher oil removal as compare to co-current flow.

\section{REFERENCES}

[1] A Deininger, F.W. Günthert and P.A. Wilderer, "The Influence of Currents on Circular Secondary Clarifier Performance and Design", Wat. Sci. Tech., vol. 34, no. 3-4, pp. 405-412, 1996.

[2] HORIBA. Instruction Manual: Oil Content Analyzer, OCMA-310, Japan, 1995.

[3] Law, A.T. (1996). Oil Pollution in the Malaysian Seas. Consumer Association of Penang (CAP) -Sahabat Alam Malaysia (SAM) National Conference, State of the Environment in Malaysia, Penang, 1996.

[4] Law, P.L., Ngu, L.H., Wong, K.K., and Abdul Rahman, A. (2006) Development and Performance Tests of a Separator for Removal of Physically Emulsified and Free Oils from Wastewaters. Journal of the Institution of Engineers, Malaysia, 67, No. 2, pp. 10-19.

[5] Malaysia Environmental Quality Report 2004. Department of Environmental, Ministry of Science, Technology and the Environment, Malaysia.

[6] Mohr, K.S. (2000). An Overview of US and International Regulations Regarding Hydrocarbons in Water Effluents. Water Environment Federation Industrial Wastes Technical Conference, St. Louis, Missouri.

[7] Rensselear Polytechnic Institute, http://www.rpi.edu/dept/chemeng/Biotech-Environ/SEDIMENT/boycott.htm. Accessed on $27 / 11 / 2002$. 


\title{
Optimum Coalescence Plate Arc Length for Removal of Oil Droplets from Wastewaters
}

\author{
Haidar S. Almarouf, Puong Ling Law, Heing Ho Lau and Azhaili Baharun
}

\begin{abstract}
Aspects on the arc length of coalescence plates of an oil-water separator as determinant factors for removal of oil droplets from wastewaters were investigated. The primary component of the separator consists of a series of concave and convex shaped coalescence plates to form multiple angles plate arrangement for enhancement of oil droplet coalescence onto the plates. Experimental results were obtained using different overflow rates and different arc lengths of coalescence plates, and were statistically evaluated. As a result, a series of mathematical equations pertaining to oil removal efficiencies from wastewaters were formulated. The predicted and observed data were compared, and optimal coalescence plate arc lengths for maximum oil droplets removal from wastewater at different flowrates were determined. It was also found that coalescence plate arc length had a more significant effect on oil removal efficiency at high overflow rate than at low overflow rate, i.e. oil removal efficiency dropped with reducing coalescence plate arc length. It was also concluded that removal efficiency of emulsified oil droplets from wastewater was strongly correlated to the system's overflow rate and horizontal projection of arc coalescence plate area.
\end{abstract}

Keywords: optimum, coalescence, arc length, oil droplets, wastewaters

\section{INTRODUCTION}

Continuous growth of population and rapid industrial development leads to rapid increase of oil and grease (O\&G) discharges into the receiving waters. As a result, more stringent effluent discharge standards were introduced in recent years in the ASEAN region [1]. At present, simple O\&G removal systems are often inadequate and more complex or sophisticated systems are either too expansive or too maintenance intensive [2].

Gravity separation is one of the widely used unit operations for removal of O\&G from wastewaters. The performance of a gravity oil-water separator is dependent on the rising velocity of the individual oil droplets and system surface overflow rate [3]. Generally, a typical coalescence plate separator has a relatively low overflow rate and is attained by the application of parallel plates whereby oil droplets can be collected after having risen in a short

Haidar S. Almarouf, Puong Ling Law and Azhaili Baharun are with the Department of Civil Engineering, University of Malaysia Sarawak, Kota Samarahan, Malaysia. (Tel: 6082-583282, Fax: 6082-583410, email: puonglaw@feng.unimas.my).

Hieng Ho Law is with Department of Civil and Construction Engineering, Curtin University of Technology (Sarawak Campus), Miri, Malaysia (e-mail: lau.hieng.ho@curtin.edu.my). distance. Coalescence of oil droplets will then occur and are usually removed by using tilted plates. Theoretically, efficiencies of the process were investigated by Yao [4] and Tikhe [5] who demonstrated that removal efficiencies were partially dependant by system's overflow rates. Recently, Law, et al. developed a separator for removal of physically emulsified and free oils from wastewaters by using a series of coalescence frustums [6]. This is a center-feed upflow circular separator equipped with a specially designed a vertical perforated inlet pipe distributor [7].

A number of different plate shapes and designs are commercially available ranging from simple flat type to complicated corrugated structures. From the vast body of available literature with regard to oil-liquid separation technologies, a few aspects not thoroughly investigated or under-studied are the ones related to plate separators. Firstly, the relation between separation efficiency and plate shape geometry was not studied, and secondly, there appears to be a critical value for the droplet diameter, whereby smaller droplets cannot be separated at all by a plate separator. In contrast to the plate separators, a flat plate further complicates the understanding of oil removal efficiency due to 1) surface slip, 2) effects of shear with the oil droplets experiencing re-suspension, and 3) dispersion of oil droplets. In this study, the horizontal projection of the plate area was considered since theoretically, inclination of coalescence plate leads to a marginal increase in overflow rate.

This paper reports on the investigation outcomes resulted from further theoretical considerations and experimental works on multiple angles parallel arc coalescence plate separators. Some constructive details including innovative variations in the inlet and outlet construction were investigated with respect to oil droplets or emulsified oils removal efficiencies from wastewaters.

\section{HYPOTHESIS}

The mechanisms of oil droplets removal from a liquid by gravity separation include the principles of Stockes' Law [8] and Boycott effect [9]. Separation of oil droplets from water is a liquid-liquid separation carried out almost exclusively by gravity separation using floatation of the oil droplets to remove it from water, either natural or enhanced. Natural gravitational separation is carried out in American Petroleum Institute (API) separators in large tanks [10]. Oil in water is characterized by a spectrum of droplet sizes. The droplet size that must be removed to attain a given effluent 
concentration depends on the oil specific gravity, concentration and average droplet size present. Design of an enhanced gravity separator size employs the mechanism of the rise velocity $v_{r}$, is given by Stockes' Law [8]:

$$
v_{r}=\frac{g}{18 \mu}\left(\rho_{o}-\rho\right) d^{2}
$$

In the 1920's, Boycott noticed that blood cells settled faster in test tubes that were inclined than in tubes that were straight up or vertical. Acrivos and co-workers developed a theoretical basis, but the general concept is not difficult to grasp [9]. In the event that settler is inclined, the falling particles and rising liquid get out of each other's way (Fig. 1). In a vertical tube, particles settling displace fluid that must rise. An element of this fluid passes past more particles and has to accelerate and decelerate depending on whether its path is wide or narrow [9].

Fluid elements in the inclined tube escape quicker from the particles and flow more easily, and as the particle suspension gets more concentrated it also gets denser [9]. This provides more driving force for settling. The net effect is that the particles slide down along the wall while the clear liquid flows upward with less interference than in the vertical tube [9]. The vector arrows for the enlarged view are the same at the start, but eventually the inclined tube gets the particles near the wall where their direction changes. In this region, they are denser than in the vertical tube, and the liquid has a shorter distance to escape from them [9].

In horizontal separation tank, unlike an up flow one, some percentage of the particles with the $v_{r}$ less than $v_{o}$ will be removed [11]. The geometrical arrangements (concave and convex) plate shapes would enhance the coalescence of oil droplets and therefore lead to improved separation efficiency as well as once the straight plate been arched then the curved plate will provide extra plate length which consequently leading an improvement on removal efficiency.

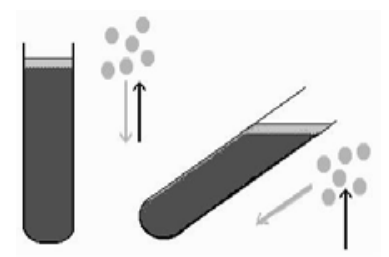

Fig.1. Comparison of particle settling in a vertical and an inclined tube [9]

\section{EXPERIMENTAL}

A schematic laboratory experimental setup is illustrated in Fig. 2. The proposed system took the shape in rectangular settling basin embedded with multiple angles parallel arc coalescence plates. The system was designed to remove oil droplets with sizes $\geq 10 \mu \mathrm{m}$ in diameter. The primary function of the mixing tank in this experiment was to physically mix the oil droplets with water to produce a solution containing physically emulsified and free oils. The mixing tank was equipped with a typical agitation process vessel or a cylindrical vessel with a vertical axis having a capacity of approximately 200 liters per day (L/d). The mixing tank acted as a rapid mixing tank using mechanical agitators to impart power to the water to produce high shear, turbulence with velocity gradient of approximately $700 \mathrm{sec}^{-}$ 1 .

The influent oil concentrations were prepared in the range of 100 to $1,000 \mathrm{mg} / \mathrm{L}\left(0.1\right.$ to $\left.1.0 \mathrm{~kg} \cdot \mathrm{m}^{-3}\right)$. The oil concentrations in water were determined using a precalibrated oil-in-water analyzer (Model OCMA-310, HORIBA) [12]. The oil content analyzer deploys infrared adsorption method to measure oil content in water, and oil dispersed in water is extracted by a solvent with its molecular structure as $\mathrm{CI}\left(\mathrm{CF}_{2}-\mathrm{CFCI}\right)_{2} \mathrm{CL}$. The oil concentration in a sample was measured by the changes in the amounts of infrared adsorption in the 3.4 to $3.5 \mu \mathrm{m}$ wavelength range of the extracted liquid. The oil analyzer is a non-dispersive infrared analysis meter that allows a relatively very sensitive analysis since the cell length is adjustable the equipment has the ability to take measurements without losing elements with low boiling points [12].

The oil-water separator (length $=0.8 \mathrm{~m}$, width $=0.4 \mathrm{~m}$ and height $=0.4 \mathrm{~m})$ consists of an inlet zone and baffle $(0.16 \mathrm{~m}$ in length), the oil-water separation zone consisting of series of inclined parallel arc coalescence plates, and the outlet zone outlet and baffle $(0.16 \mathrm{~m})$. The inlet and outlet baffles were designed in accordance the American Petroleum Institute (API) manual [13], which consists of a slot baffle at the entry, an oil retention baffle and outlet weir (Fig. 3). The coalescence arc plates embedded in the oil-water separation tank were inclined at an angle $\theta$ of $55^{\circ}$ from horizontal. The coalescence plates were constructed using aluminum material. Experiment works were carried out using different plate arc lengths in an attempt to configure and determine the optimum plate lengths at different overflow rates. The design parameters and dimensions of arc coalescence plates are shown in Table 1. The governing equations for computation of arc geometry parameters were listed in Appendix I.

In this experimental work, the ratio of chord length (c) to middle ordinate $(\mathrm{m})$ was $\mathrm{c} / \mathrm{m}=4$ while $\mathrm{R}=0.57 \mathrm{c}[10]$, and the flowrates ranged from $5.6 \times 10^{-6}$ to $2 \times 10^{-5} \mathrm{~m}^{3} / \mathrm{s}$. Additionally, the horizontal projection of the total plate area was correlated with overflow rate with respect to oil removal efficiency. Table 2 shows the number of arc plates versus horizontal projection area for the individual sets of arc length. During the experimental runs, removal efficiencies $(\mathrm{E}, \%)$ were determined based on the following relationship.

$\mathrm{E}(\%)=\frac{C_{i}-C_{o}}{C_{i}}$

Where $C_{i}$ and $C_{o}$ are oil droplet concentrations in the water samples collected at inlet (A) and outlet (B) (Fig. 1). The values of $\mathrm{v}_{\mathrm{o}} / \mathrm{v}_{\mathrm{r}}$ ratio $(\mathrm{v})$ were correlated with the relative length $\mathrm{L}_{\text {rel }}$, i.e. ratio of plate length-to-plate spacing (Table $2)$. In this experimental work, the plate spacing was theoretically determined to be most appropriate at $50 \mathrm{~mm}$, ratio $\mathrm{V}_{\mathrm{o}} / \mathrm{v}_{\mathrm{r}}(r)$ was in the range of 0.6 and 3 , design ratio at 0.8 , and the relative lengths were between 1.7 and 4.4. The horizontal projection area would be the arc plate area multiplied by cosine $\theta$, and this area was used for all cases to determine the effects of arc plate geometries on oil 
droplet removal efficiencies.

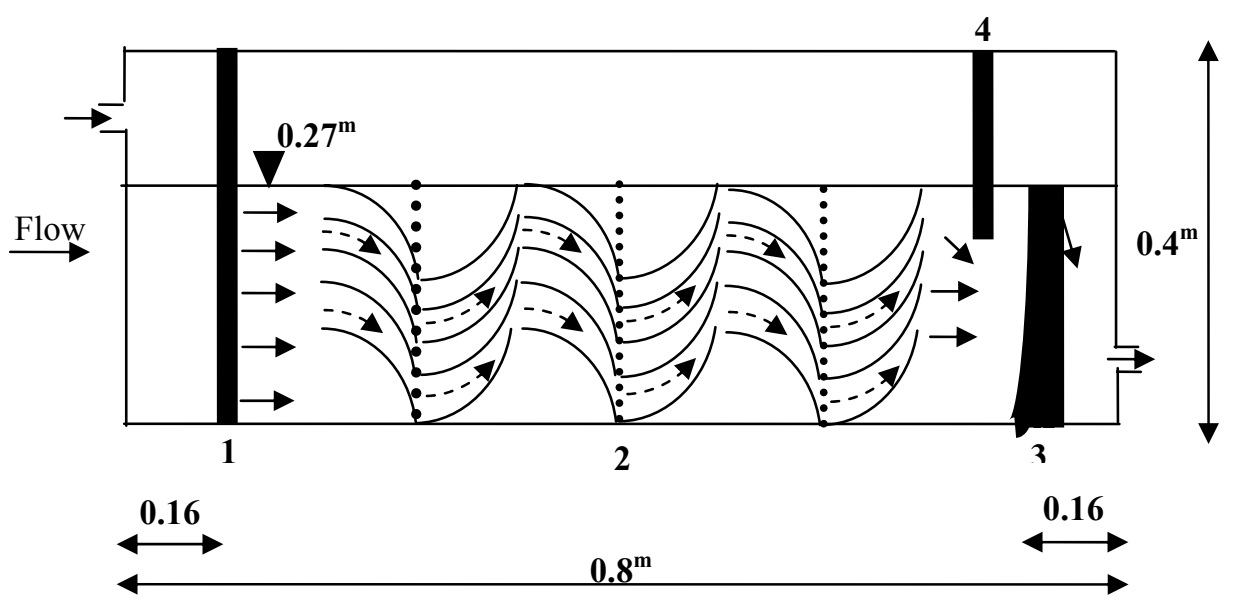

(a) Inlet \& outlet designs (1 - vertical slot baffle, 2 - plate pack, 3 - effluent weir, 4 - oil retention baffle)

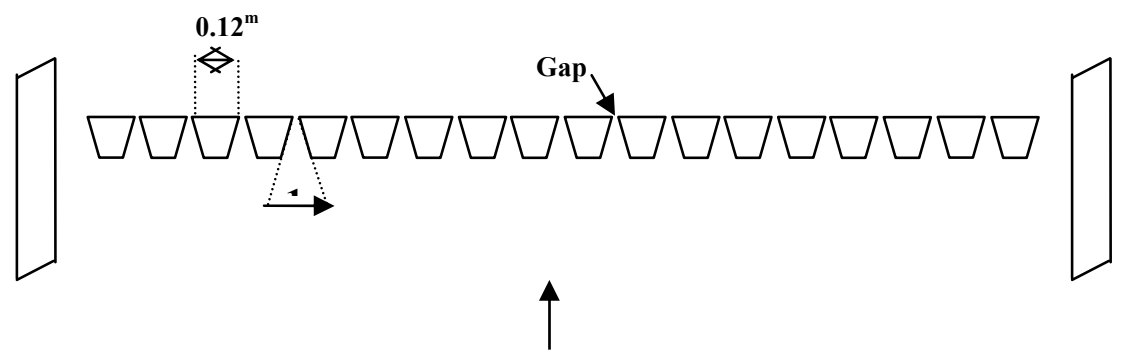

Flow

(b) Top view of vertical slot baffle

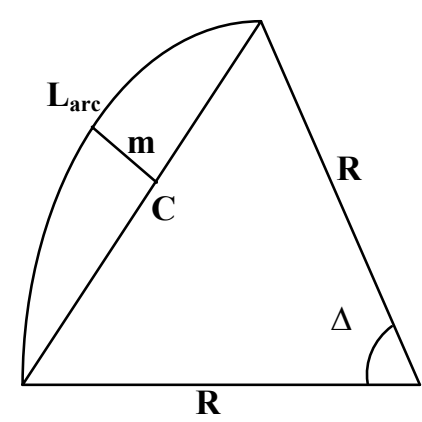

(c) Details of arc coalescence plate

Fig. 3. Schematic diagram

Table 1. Design parameters of arc coalescence plate

\begin{tabular}{|l|l|l|l|l|l|}
\hline No. & $\begin{array}{c}\text { Arc } \\
\text { length, } \\
\mathbf{m}\end{array}$ & $\begin{array}{c}\text { Arc } \\
\text { radius, } \\
\mathbf{m}\end{array}$ & $\begin{array}{c}\text { Long } \\
\mathbf{c h o r d} \\
\mathbf{( c )} \mathbf{m}\end{array}$ & $\begin{array}{c}\text { Middle } \\
\text { ordinate } \\
\mathbf{( M ) ~} \mathbf{~ m}\end{array}$ & $\begin{array}{c}\text { Central } \\
\text { angle }(\boldsymbol{\Delta}) \\
\mathbf{0}\end{array}$ \\
\hline 1 & 0.85 & 0.04 & 0.07 & 0.0175 & 122.6 \\
\hline 2 & 0.098 & 0.0456 & 0.08 & 0.02 & 122.6 \\
\hline 3 & 0.12 & 0.057 & 0.1 & 0.025 & 122.6 \\
\hline 4 & 0.146 & 0.0684 & 0.12 & 0.03 & 122. \\
\hline 5 & 0.17 & 0.0798 & 0.14 & 0.035 & 122.6 \\
\hline 6 & 0.19 & 0.0912 & 0.16 & 0.04 & 122. \\
\hline 7 & 0.22 & 0.1026 & 0.18 & 0.045 & 122.6 \\
\hline
\end{tabular}

\section{RESUlts AND Discussion}

Experimentally gathered data are presented in Table 3, and Figure 3 illustrates the variations in removal efficiencies, E (\%) with respect to different plate relative lengths $\left(\mathrm{L}_{\mathrm{rel}}\right)$. It was demonstrated that the oil droplet removal efficiencies with respect to the ratio of $\mathrm{v}_{0} / \mathrm{v}_{\mathrm{r}}$ appeared to be nonlinear, and could be expressed in the form of a second degree polynomial equations, whereby the parameters were dependant on the relative length $\left(\mathrm{L}_{\text {rel. }}\right)$. An equation representing second degree polynomial for a given $\mathrm{L}_{\text {rel }}$ can be written as follow.

$$
E\left(L_{r e l .}\right)=a\left(L_{r e l .}\right) r^{2}+b\left(L_{r e l .}\right) r+c\left(L_{r e l .}\right)
$$

Where as $a\left(\mathrm{~L}_{\text {rel. }}\right), b\left(\mathrm{~L}_{\text {rel. }}\right)$ and $\mathrm{c}\left(\mathrm{L}_{\text {rel. }}\right)$ are the model parameters. These parameters were calculated or estimated based on the available experimental data with respect to the individual coalescence plate arc lengths (Table 2). To determine model parameter values, a two-stage analysis were carried out. First stage focused on the formulation of a set of E-r relationship holding relative plate lengths as 
constant, while the second stage involved the simplification or reduction of the complex equation to a simple E-r relationship equation. The analysis on the observed data for the individual plate arc lengths resulted in the formulation of the following equations.

$$
\begin{aligned}
& E(1.7)=1.6 r^{2}+13.3 r-87.4 \\
& E(1.96)=6 r^{2}+0.54 r-81.3 \\
& E(2.4)=4.9 r^{2}+0.7 r-80.6 \\
& E(2.92)=4.4 r^{2}+1.1 r-82.4 \\
& E(3.4)=1.8 r^{2}+10.4 r-88.8 \\
& E(3.8)=3 r^{2}+14.3 r-89.5 \\
& E(4.4)=6 r^{2}+6.8 r-83.1
\end{aligned}
$$

From these equations, it was demonstrated that coefficients of $r$ and $r^{2}$ could all result in negative values, which indicated that it was not possible to attain $100 \%$ removal efficiency. It was also noted that non-linearity could play a dominant role in determining the level of efficiency, especially at higher $r$ values, i.e. when $r$ is greater than 2. Model coefficients shown in Table 4 are plotted in Figure 4 with respect to $a\left(\mathrm{~L}_{\text {rel. }}\right)$ and $b\left(\mathrm{~L}_{\text {rel. }}\right)$ or variation of $\mathrm{a}$ and $\mathrm{b}$ coefficients with $\mathrm{L}_{\text {rel. }}$, and in Figure 5 with respect to $c\left(\mathrm{~L}_{\text {rel. }}\right)$ or variation of c coefficients versus

\begin{tabular}{|c|c|c|c|c|c|c|c|c|c|c|c|c|c|c|}
\hline \multicolumn{15}{|c|}{ Relative Length } \\
\hline & \multicolumn{2}{|c|}{1.7} & \multicolumn{2}{|c|}{1.96} & \multicolumn{2}{|c|}{2.4} & \multicolumn{2}{|c|}{2.92} & \multicolumn{2}{|c|}{3.4} & \multicolumn{2}{|c|}{3.8} & \multicolumn{2}{|c|}{4.4} \\
\hline Run & $r$ & $\mathrm{E}$ & $r$ & $E$ & $r$ & $\mathrm{E}$ & $r$ & $\mathrm{E}$ & $r$ & $\mathrm{E}$ & $r$ & $\mathrm{E}$ & $r$ & $\mathrm{E}$ \\
\hline 1 & 0.6 & 78 & 0.6 & 79 & 0.6 & 78 & 0.6 & 80 & 0.6 & 80 & 0.6 & 80 & 0.6 & 76 \\
\hline 2 & 1 & 74 & 1 & 75 & 1 & 75 & 1 & 77 & 1 & 79 & 1 & 72 & 1 & 72 \\
\hline 3 & 1.4 & 65 & 1.4 & 68 & 1.4 & 71 & 1.4 & 73 & 1.4 & 73 & 1.4 & 64 & 1.4 & 63 \\
\hline 4 & 1.8 & 57 & 1.8 & 61 & 1.8 & 63 & 1.8 & 65 & 1.8 & 63 & 1.8 & 53 & 1.8 & 51 \\
\hline 5 & 2.2 & 47 & 2.2 & 51 & 2.2 & 54 & 2.2 & 60 & 2.2 & 56 & 2.2 & 44 & 2.2 & 39 \\
\hline 6 & 2.6 & 49 & 2.6 & 40 & 2.6 & 45 & 2.6 & 49 & 2.6 & 49 & 2.6 & 32 & 2.6 & 26 \\
\hline 7 & 3 & 30 & 3 & 25 & 3 & 34 & 3 & 40 & 3 & 43 & 3 & 19 & 3 & 10 \\
\hline
\end{tabular}
$\mathrm{L}_{\text {rel }}$. From the plots (Figs. 4 and 5), it was found that the parameters tended to change in accordance to a third-degree polynomial with respect to $\mathrm{L}_{\text {rel }}$.

Table 2. Arrangement of arc coalescence plates

\begin{tabular}{|c|c|c|c|}
\hline $\begin{array}{c}\text { Arc Length } \\
\mathbf{m}\end{array}$ & Plates No. & $\begin{array}{c}\text { Plate Area } \\
\mathbf{M}^{\mathbf{2}}\end{array}$ & $\begin{array}{c}\text { Horizontal } \\
\text { projection Area } \\
\mathbf{m}^{\mathbf{2}}\end{array}$ \\
\hline 0.85 & 40 & 2.04 & 1.17 \\
\hline 0.098 & 52 & 2.04 & 1.17 \\
\hline 0.12 & 43 & 2.06 & 1.18 \\
\hline 0.146 & 35 & 2.04 & 1.17 \\
\hline 0.17 & 30 & 2.04 & 1.17 \\
\hline 0.19 & 27 & 2.05 & 1.17 \\
\hline 0.22 & 23 & 2.02 & 1.16 \\
\hline
\end{tabular}

Table 3. Experimental removal efficiency (E) versus $r$

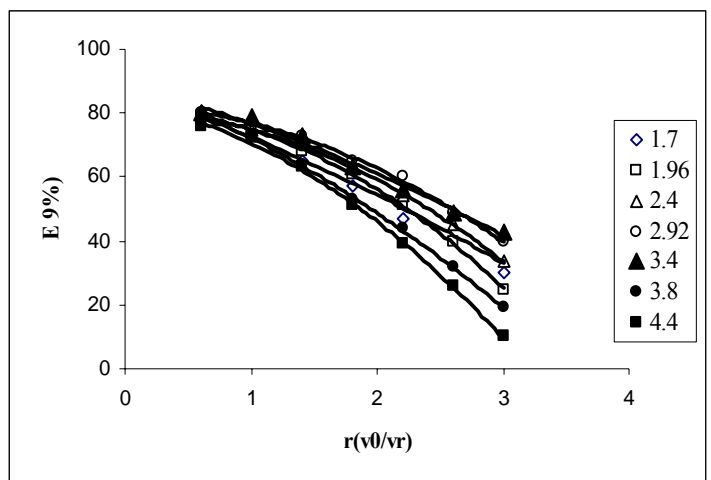

Fig. 4. Removal efficiency (E, \%) versus relative lengths $\left(\mathrm{L}_{\mathrm{rel}}\right)$

Table 4. Second degree polynomials: coefficients a, b \& c

\begin{tabular}{|c|c|c|c|}
\hline $\mathrm{L}_{\text {rel. }}$ & $\mathrm{a}$ & $\mathrm{b}$ & $\mathrm{c}$ \\
\hline 1.7 & -1.6 & -13.3 & 87.4 \\
\hline 1.96 & -6 & -0.54 & 81.3 \\
\hline 2.4 & -4.9 & -0.7 & 80.6 \\
\hline 2.92 & -4.4 & -1.1 & 82.4 \\
\hline 3.4 & -1.8 & -10.4 & 88.8 \\
\hline 3.8 & -3 & -14.3 & 89.5 \\
\hline 4.4 & -6 & -6.8 & 83.1 \\
\hline
\end{tabular}

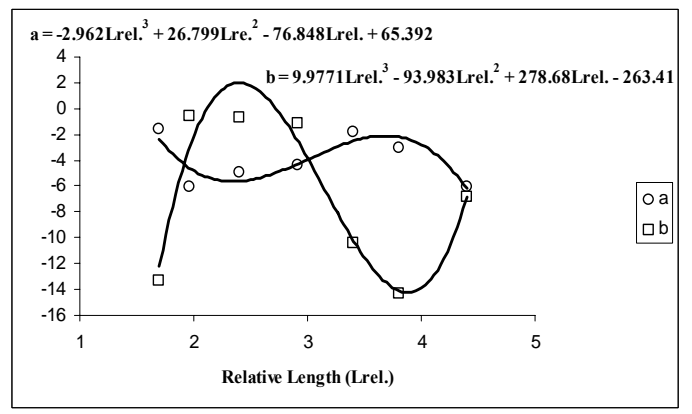

Fig. 5. Variations of $a$ and $b$ coefficients with $\mathrm{L}_{\text {rel }}$

The relationships of the three parameters and relative plate arc lengths $\left(\mathrm{L}_{\text {rel..) }}\right.$ are illustrated in the following equations (Equations 11, 12, 13 and 14):

$$
\begin{aligned}
& a\left(L_{\text {rel }}\right)=-2.96 \mathcal{L}_{\text {rel. }}^{3}+26.79 \mathcal{L}_{\text {rel. }}^{2}-7684 \mathcal{L}_{\text {rel. }}+63.392 \\
& b\left(L_{\text {rel }}\right)=9.977 \mathcal{L}_{\text {rel. }}^{3}-93.983 \mathcal{L}_{\text {rel. }}^{2}+27868 L_{\text {rel. }}-26341 \\
& c\left(L_{\text {rel }}\right)=-6.588 \mathcal{Z}_{\text {rel. }}^{3}+60.53 \mathcal{L}_{\text {rel. }}^{2}-17489 L_{\text {rel }}+24186
\end{aligned}
$$

Substitute Equations 11, 12, and 13 into Equation 3 leads to the following expression: 


$$
\begin{aligned}
& E\left(L_{r e l}\right)=\left(-2.96 \mathcal{Z}_{r e l}^{3}+2679 \mathcal{L}_{r e l}^{2}-76848+65.39\right)^{2} r^{2} \\
& +\left(9.977 \mathcal{L}_{r e l}^{3}-93.98 \mathcal{Z}_{r e l}^{2}+27868 \mathcal{L}_{r e l}-26341\right) r \\
& -6.588 \mathcal{Z}_{r e l}^{3}+6053 \mathcal{Z}_{r e l}^{2}-17489 L+34186
\end{aligned}
$$

Where $\mathrm{L}_{\text {rel }}$ is the relative plate length and Equation 14 is valid for $\mathrm{L}_{\text {rel }}$ values ranging from 1.7 to 4.4 and $\mathrm{r}$ represents $v_{\mathrm{o}} / v_{r}$ ratio. The experimentally observed and theoretically calculated values with respect to removal efficiencies of oil droplets from wastewaters by using Equation 14 are given in Table 5 for $r$ value equals to 2 .

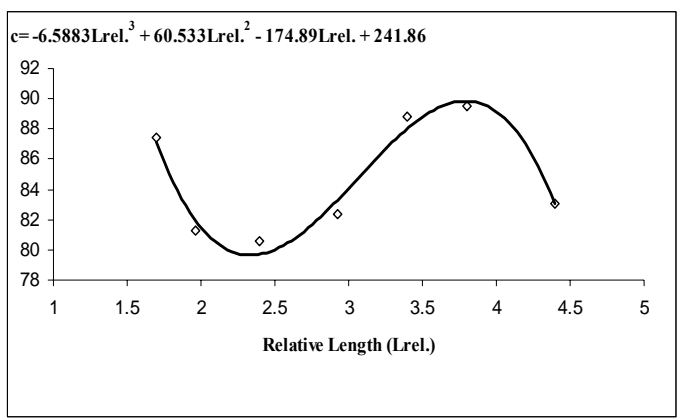

Fig. 6. Coefficient, c versus relative arc length, $\mathrm{L}_{\text {rel }}$.

Table 5. Removal efficiencies: observed versus predicted

\begin{tabular}{|l|l|l|l|}
\hline $\mathrm{L}_{\text {rel. }}$ & $\begin{array}{l}\text { Calculated E } \\
(\%)\end{array}$ & $\begin{array}{l}\text { Experimental E } \\
(\%) \text { values }\end{array}$ & $\begin{array}{l}\text { Average } \\
\text { error (9\%) }\end{array}$ \\
\hline 1.7 & 53 & 52.6 & 0.75 \\
\hline 1.96 & 57.6 & 58.4 & 1.4 \\
\hline 2.4 & 61 & 60.5 & 0.82 \\
\hline 2.92 & 61.4 & 60.8 & 0.9 \\
\hline 3.4 & 58 & 56 & 3.4 \\
\hline 3.8 & 53.5 & 67.2 & 20.4 \\
\hline 4.4 & 45 & 43 & 4.4 \\
\hline & & & $=4.6$ \\
\hline
\end{tabular}

As shown in Table 5, the estimated or predicted removal efficiency values obtained based on general equations showed good agreement with measured or experimental data. The average relative error percentage falls far below the practically acceptable limit of $5 \%$. Based on the relationship between the removal efficiency, E (\%) and the plate relative lengths $\left(\mathrm{L}_{\text {rel. }}\right)$, the optimum plate length that would result in highest removal efficiency of oil droplets from wastewater can be determined from different $r$ values. Plots of removal efficiencies versus relative plate lengths $\left(\mathrm{L}_{\text {rel. }}\right)$ are illustrated in Figure 6 , whereby the optimum relative plate length that provided the highest removal efficiency was approximately 2.92. It was demonstrated that relative plate lengths would have a significant effect on removal efficiency at higher $r\left(\mathrm{v}_{\mathrm{o}} / \mathrm{v}_{\mathrm{r}}\right)$ values, and that at low $r$ values, the effect of plate relative plate length on removal efficiency was relatively less significant.

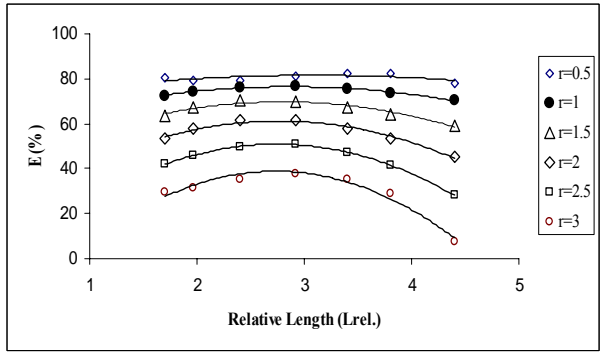

Fig. 7. Observed removal efficiencies for various relative lengths $\left(\mathrm{L}_{\mathrm{rel}}\right)$

\section{CONCLUSIONS}

In this study, it was concluded that 1) in a gravity-based phase oil-water separator by using the equally spaced inclined multiple parallel arc coalescence plates, oil droplets removal efficiency, E (\%) can be expressed as a function of relative plate lengths $\left(\mathrm{L}_{\text {rel. }}\right)$ and the $r$ value in the following expression.

$$
\begin{aligned}
& E\left(L_{r e l}\right)=\left(-2.96 \mathcal{L}_{r e l}^{3}+2679 \mathcal{L}_{r e l}^{2}-76848+65.392 r^{2}\right. \\
& +\left(9.977 \mathcal{L}_{r e l}^{3}-93.98 \mathcal{Z}_{r e l}^{2}+27868 \mathcal{L}_{r e l}-26341\right) r \\
& -6.588 \mathcal{Z}_{r e l}^{3}+6053 \mathcal{Z}_{r e l}^{2}-17489 L+34186
\end{aligned}
$$

The above equation can be rewritten to yield;

$$
\begin{aligned}
& E\left(L_{r e l .}\right)=\left(241.86-263.4 r+65.392 r^{2}\right) \\
& +\left(-174.89+278.68 r-76.848 r^{2}\right) L_{r e l .} \\
& +\left(60.533-93.983 r+26.799 r^{2}\right) L^{2} \\
& +\left(-6.5883+9.9771 r-2.962 r^{2}\right) L^{3}
\end{aligned}
$$

For optimum (most efficient) removal of oil droplets from wastewaters for a given overflow rate, the derivative of the above expression can be used with respect to $\mathrm{L}_{\text {rel }}$ that results in the following expression.

$$
\begin{aligned}
& \left(-174.89+278.68 r-76.848 r^{2}\right) \\
& +\left(121.066-187.966 r+53.598 r^{2}\right) L_{r e l} \\
& +\left(-19.7649+29.9313 r-8.886 r^{2}\right) L_{r e l}^{2}
\end{aligned}
$$

It is possible to determine the relative plate length with a given $r$ from this equation. For example, if $r$ equals to 3 , then

$$
9.945 L_{r e l .}^{2}-39.55 L_{r e l}+30.482=0
$$

This is based on the fact that both $r=\mathrm{v}_{0} / \mathrm{v}_{\mathrm{r}}$ and $\mathrm{L}_{\mathrm{rel}}=\mathrm{L}_{\text {arc }} / \mathrm{s}$, $\mathrm{L}_{\text {rel }}$ are estimated to be 2.92. In this study, it was concluded that the optimum arc plate length for this specific oil-water separator was estimated to be $140 \mathrm{~mm}$ at an overflow rate of approximately $1.8 \times 10^{-5} \mathrm{~m} / \mathrm{sec}$ and plates spacing of $50 \mathrm{~mm}$. It was also concluded that arc lengths would have a more significant effect on oil removal efficiency at high overflow rates than at low overflow rate, and the oil removal efficiency was strongly correlated to system's overflow rate and horizontal projection plate area. 


\section{REFERENCES}

[1] Hwa, T. J. and Kean, C.K. (1982). Study of the performance of an inclined Corrugated plate oil separator. Engineering Journal of Singapore, 9(1), 3-9.

[2] Mohr, K.S. (1995). Stormwater Treatment for Contaminant Removal. International Symposium on Public Works and the Human Environment, Washington D.C.

[3] Zeevalkink, J.A. and. Brunsmann, J.J. (1983). Oil Removal from Water in Parallel Plate Gravity Type Separators. Water Research, 17 (4), 365-373.

[4] Yao, K.M. (1970). Theoretical Study of High Rate Sedimentation. Journal of Water Pollution Control, 42 (1), 218-228

[5] Tikhe, M.L. (1974). Some Theoretical Aspects of Tube Settlers. Indian Journal Environmental Health 16(2), 26-33.

[6] Law, P.L., Ngu, L.H., Wong, K.K. and Rahman, A.A. (2006), "Development and Performance Tests of a Separator for Removal of Physically Emulsified and Free Oils from Wastewaters", Journal of the Institution of Engineers, Malaysia, Vol. 67, No. 2, pp 10-19.

[7] Ngu, L.H, Law, P.L. and Wong, K.K. (2004), "A Study on Flow Characteristics of a Vertical Perforated-Pipe Distributor in a Circular Separator", Journal of Civil Engineering (IEB), Vol. CE 32, No. 2, pp 121-132.

[8] Perry, R.H. and Green, D.W. (1997). Chemical Engineers' Handbook. $7^{\text {th }} E d$., Australia, McGraw-Hill.

[9] Rensselear Polytechinc Institute (2002). http://www.rpi.edu/chemeng/Biotech- Ennviron/SEDIMENT/boycott.htm. Accessed on 27/11/2002

[10] Chen, C.L. and Cheng, C.H. (2002). Buoyancy Induced Flow and Convective Heat Transfer in an Inclined Arc-shape Enclosure. International Journal of Heat and Fluid Flow, 23(2), 823-830.

[11] Arizona Department of Environmental Quality (1996). Best Available Demonstrated Control Technology Guidance Document for Pretreatment with Oil/Water Separators. State of Arizona, USA.

[12] HORIBA (1995). Instruction Manual, Oil Content Analyzer, OCMA310, Japan. Pledel, (1-1 - 10-5).

[13] American Petroleum Institute (API) (1990). Manual on Disposal of Refinery Wastes, American Petroleum Institute (API), Chapter 5 (3$13)$. 


\title{
Energy Saving Additive Added Ammonia Absorption Refrigeration System
}

\author{
A. Mohammad, R. Mohd Yunus, Z. Hassan, A. Abdullah, M.H. Ab Rahim
}

\begin{abstract}
In an energy and environmentally conscious world, a cost effective and environmental friendly refrigeration and cooling system is essential especially for countries with a hot climate like Malaysia. A conventional compressor airconditioning system would consume a sizeable amount of energy to operate and this is reflected in the monthly energy bills. A more energy efficient system is the compressor free absorption refrigeration system. However, the existing absorption system has a lower cooling efficiency compared to the compressor system. An innovative system which uses additive (absorbent agent) in the refrigerant solution has been developed and was found to perform $10 \%$ better than the conventional compressor based system. This innovative system also runs by using waste heat or solar energy replacing the electricity power to supply heat to vaporize the refrigerant solution. The use of waste heat or solar energy will reduce the electricity bills tremendously.
\end{abstract}

Keywords: energy saving, absorption refrigeration system, waste heat, cooling efficiency

\section{INTRODUCTION}

In an energy and enviromentally conscious world, a cost effective and environmental friendly refrigeration and cooling system is essential especially for countries with a hot climate like Malaysia. A conventional compressor airconditioning system would consume a sizeable amount of energy to operate and this is reflected in the monthly energy bills. A more energy efficient system is the compressor less absorption chiller system.

However, the existing absorption system has a lower cooling efficiency compared to the compressor system. An innovative system which uses additive in the refrigerant solution has been developed and was found to perform $10 \%$ better than the conventional compressor based system.

\section{A. Absorption Refrigeration System}

An absorption refrigeration system basically consists of an evaporator, a condenser, a generator, an absorber and an economizer (Romero, Rivera, 2000). A quantity of waste heat is added at a relatively high temperature to the generator to vaporize the working fluid from the solution. The vaporized working fluid goes to the condenser where it is condensed delivering an amount of heat at an intermediate temperature.

The liquid leaving the condenser passes through a valve to the evaporator where it is evaporated by means of a quantity of heat at a low temperature, producing the cooling effect. The vaporized working fluid then goes to the absorber where it is absorbed by the solution coming from the generator, delivering an amount of heat at an intermediate temperature. Finally, the solution leaving the absorber is pre-heated in the economizer and returns to the generator, starting the cycle again.

\section{B. Zeolite A}

According to the IUPAC, zeolite A belongs to the LTA group (Linde Type A). The fully hydrated Na-exchanged LTA structure, of general formula $\mathrm{Na}_{12}\left(\mathrm{Si}_{12} \mathrm{Al}_{12} \mathrm{O}_{48}\right) \bullet 27 \mathrm{H}_{2} \mathrm{O}$ is built up by truncated cubooctahedron units known as sodalite or $\beta$-cages (diameter $0.66 \mathrm{~nm}$ ), which are linked together via the cubic D4R unit to form larger cavities $\alpha$-cages (diameter $1.23 \mathrm{~nm}$ ) with the shape of a trubcated cubo-octahedron as shown in Figure 1.3. The structure are interconnected by windows formed by rings consisting of 8 atoms either Si or Al of 0.41 x $0.41 \mathrm{~nm}$ generating a uniform $3 \mathrm{D}$ pore system according to the cubic system at which zeolite $\mathrm{NaA}$ crystallizes. When fully hydrated, the $\alpha$-cages and $\beta$-cages have space for 20 and 4 water molecules respectively, while the 8 rings contains 3 molecules of water.

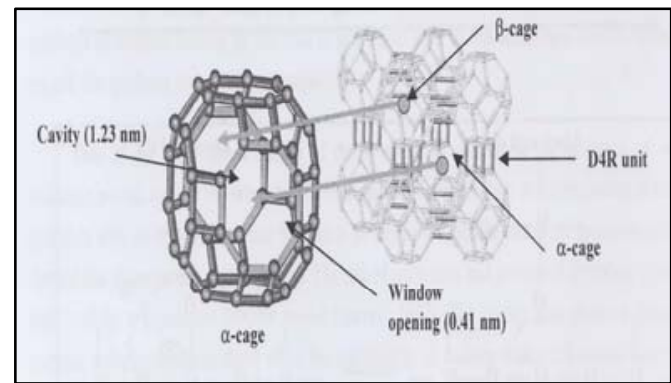

Figure 1. Unit cell of structure LTA (zeolite $\mathrm{NaA}$ ) 


\section{RESEARCH Methodology}

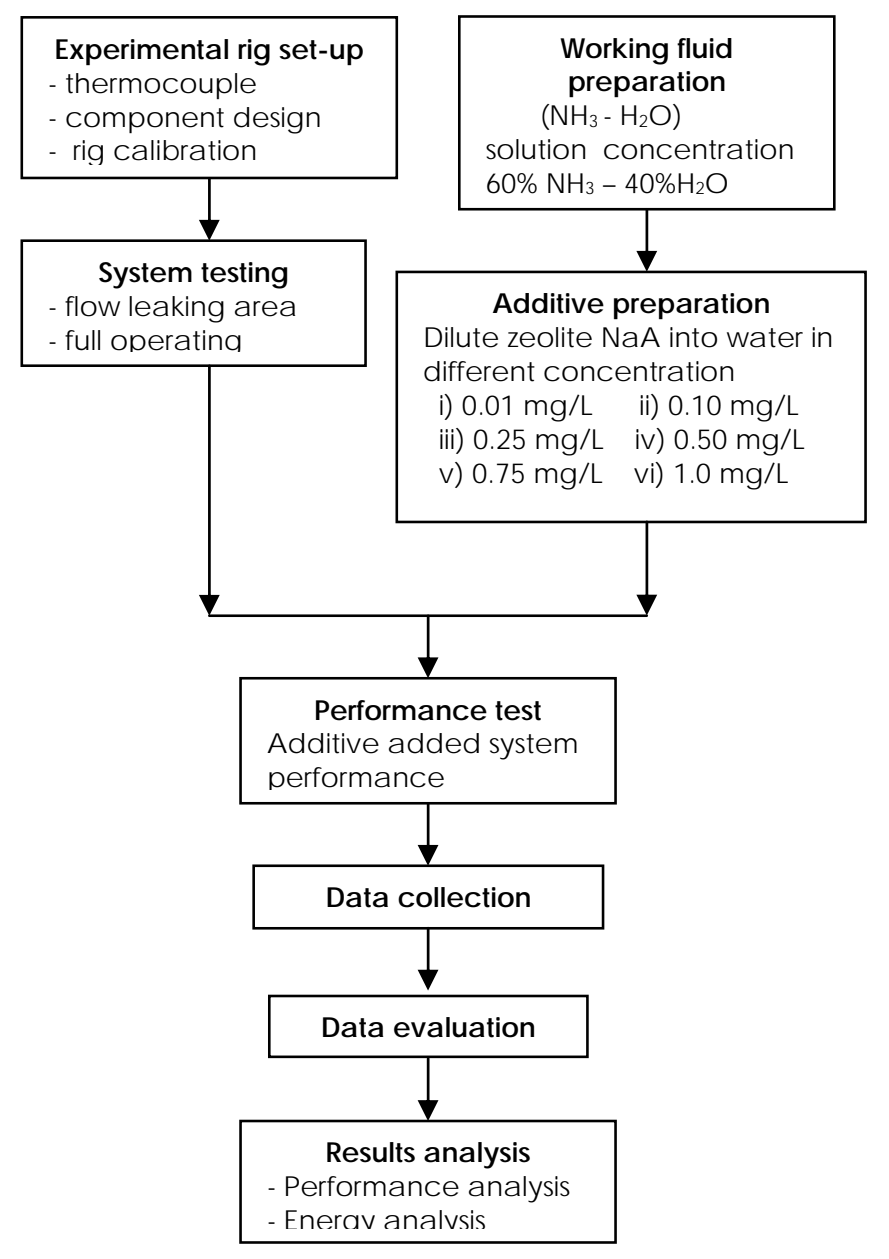

III. RESUlTS AND DiSCUSSION

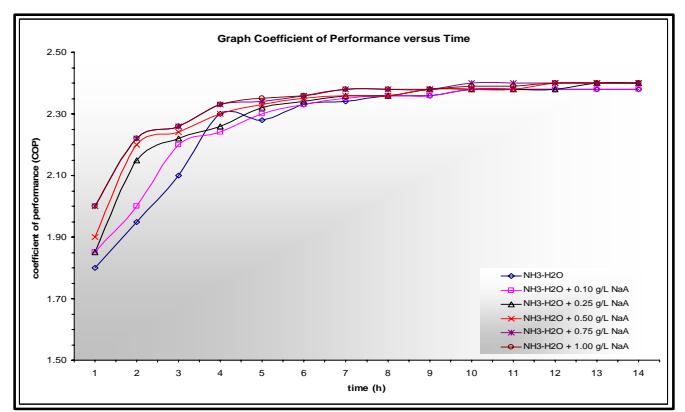

Figure 2. Graph coefficient of performance (COP) versus time (h)

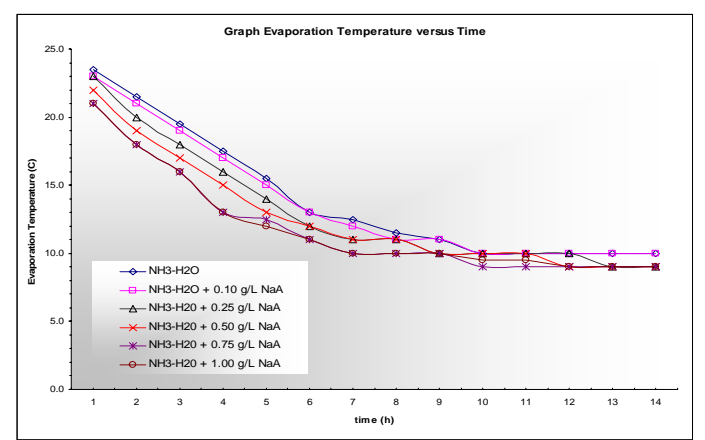

Figure 3. Graph evaporation temperature $\left({ }^{\circ} \mathrm{C}\right)$ versus time (h)

\section{CONCLUSIONS}

At the end of this study, the effect and the new performance of the ammonia-water absorption refrigeration using zeolite $\mathrm{A}$ as an additive will be identified. The coefficient of performance of the new develop system is very important for future where it enable us to improve the system. Here, we also can perform the refrigeration unit not only with better performance also consider on the energy saving aspect which ammonia absorption refrigeration system just used a small quantity of energy as fuel sources either electricity, gas or other sources of energy.. For the next step of research, some improvement will do to the refrigeration system to study another effect to improving the performance of the system.

\section{REFERENCES}

[1] Anyanwu, E.E.,Oteh, U.U., Ogueke, N.V. (2001). "Simulation of a solid adsorption solar refrigerator using activated carbon/methanol adsorbent/refrigerant pair.” Energy Conversion and Management. 42. 899-915.

[2] Da We Sun, (1999). "Comparative Study of the Performance of Ejector Refrigeration Cycle Operating With Various Refrigerants". Energy Conversion and Management. 40. 873-884

[3] Fatouh, M., Murthy, S.S. (1995). "Performance of an HCFC22-based Vapour Absorption Refrigeration System.” International Journal of Refrigeration. 18. 7. 465-476.

[4] Florides,G.A., Kaloqiron,S.A., Tassou,S.A., Wrobel,L.C., (2002). "Modelling, Simulation and Warming Impact Assessment of a Domestic-Size Absorption Solar Cooling System”. Applied Thermal Engineering. 22. 1313-1325

[5] Horuz, I., (1998). "A Comparison between Ammonia-Water and Water-Lithium Bromide Solutions in Vapor Absorption Refrigeration Systems.” Int. Comm. Heat Mass Transfer..25. 5. 711-721.

[6] Jose, F.S., Manuel,V. (2001). "Study and control of the optimal generation temperature in $\mathrm{NH}_{3}-\mathrm{H}_{2} \mathrm{O}$ absorption refrigeration systems." Applied Thermal Engineering, 21. 343-357.

[7] Lee, B.K., Chun, B.H. et. al. (2002). “ Experimental analysis of bubble mode in a plate type absorber.” Chemical Engineering Science. 57. 1923-1929.

[8] Ponbsid Srikhirin, et. al. (2001). “ A Review of Absorption Refrigeration Technologies.” Renewable and Sustainable Energy Reviews. 5. 343-372.

[9] Phonglumchiag, K. (1995). “ Fabrication and Testing of The Modeling Absorption Refrigeration using Zeolite/Water.” $\mathrm{PhD}$ Thesis.

[10] Rogdakis, E., Stegou-Sagia, A. (1996). “ Neutral Gas Absorption Refrigeration Units with Methyl Amine as Refrigerant and Hydrogen as Inert Gas.” Energy Conversion Management. 37.1609-1622.

[11] Romero,R.J., Rivera,W., Best,R., (2000). “ Comparison of The Theoretical Performance of A Solar Air Conditioning System Operating With Water/lithium Bromide and an Aqueous Ternary Hydroxide”, Solar Energy Materials \& Solar Cells. 63. 387-399

[12] Selim, A.M., Elsayed, M.M. (1999). " Performance of a packed bed absorber for aqua ammonia absorption refrigeration system.” International Journal of Refrigeration. 22. 283-292

[13] Wu, C. (1996). "Performance of an Endoreversible Carnot Refrigerator.” Energy Conversion and Management. 37. No.10. 15091512

[14] Yoon, J., Kwon, O. (1999). "Cycle analysis of air-cooled absorption chillers using a new working solution.” Energy. 24. 795-809. 